This document was prepared in conjunction with work accomplished under Contract No. DE-AC09-96SR18500 with the U. S. Department of Energy.

\title{
DISCLAIMER
}

This report was prepared as an account of work sponsored by an agency of the United States Government. Neither the United States Government nor any agency thereof, nor any of their employees, makes any warranty, express or implied, or assumes any legal liability or responsibility for the accuracy, completeness, or usefulness of any information, apparatus, product or process disclosed, or represents that its use would not infringe privately owned rights. Reference herein to any specific commercial product, process or service by trade name, trademark, manufacturer, or otherwise does not necessarily constitute or imply its endorsement, recommendation, or favoring by the United States Government or any agency thereof. The views and opinions of authors expressed herein do not necessarily state or reflect those of the United States Government or any agency thereof.

This report has been reproduced directly from the best available copy.

Available for sale to the public, in paper, from: U.S. Department of Commerce, National Technical Information Service, 5285 Port Royal Road, Springfield, VA 22161, phone: (800) 553-6847, fax: (703) 605-6900

email: orders@ntis.fedworld.gov

online ordering: http://www.ntis.gov/help/index.asp

Available electronically at http://www.osti.gov/bridge

Available for a processing fee to U.S. Department of Energy and its contractors, in paper, from: U.S. Department of Energy, Office of Scientific and Technical Information, P.O. Box 62, Oak Ridge, TN 37831-0062,

phone: (865)576-8401,

fax: (865)576-5728

email: $\underline{\text { reports@ adonis.osti.gov }}$ 
Key Words:

Treated LAW Feed Evaporation

Properties Modeling

\title{
Retention:
}

Permanent

Key WTP R\&T References:

Test Specification:

24590-PTF-TSP-RT-01-008, Rev. 0

Task Plan: WSRC-TR-2003-00095

R\&T Focus Area: Treated Feed Evaporation

Test Scoping Statement(s): S-93A, S-69

\section{MODELING TREATED LAW FEED EVAPORATION}

\author{
W. E. Daniel, 999-W
}

\section{OCTOBER 2003}

Westinghouse Savannah River Company

Savannah River Site

Aiken, SC 29808

Prepared for the U.S. Department of Energy Under Contract Number DE-AC09-96SR18500

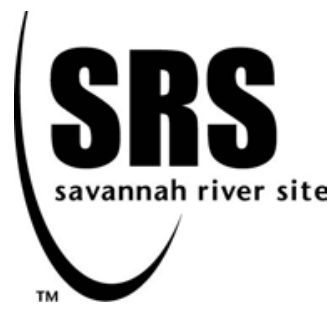


This page was intentionally left blank 


\section{TABLE OF CONTENTS}

LIST OF FIGURES ..................................................................................................................... iv

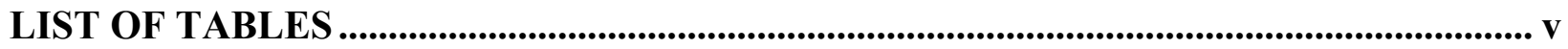

LIST OF ACRONYMS ............................................................................................ vi

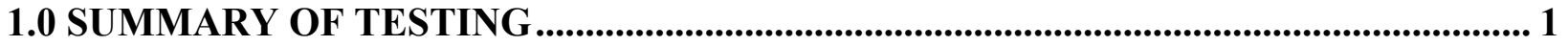

1.1 OBJECTIVES........................................................................................................................1

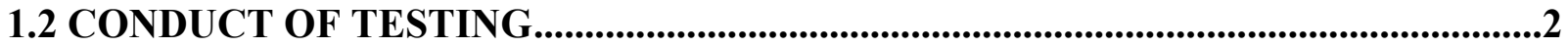

1.3 RESULTS AND PERFORMANCE AGAINST OBJECTIVES ..........................................3

1.4 QUALITY REQUIREMENTS..............................................................................................14

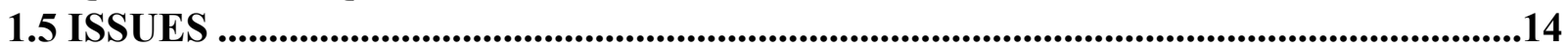

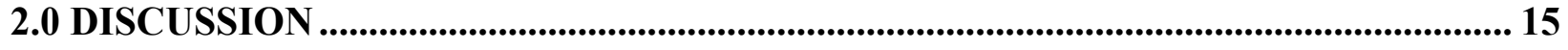

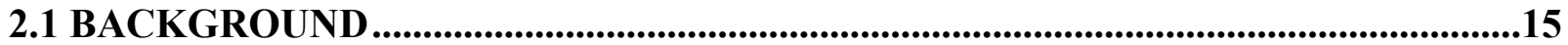

2.2 DESCRIPTION OF THE PROCESS ......................................................................................16

2.3 OLI/ESP MODEL FLOWSHEET AND CHEMISTRY MODEL ..................................16

2.4 DETERMINATION OF THE FACTOR SPACE FOR THE DESIGNS OF

EXPERIMENT ............................................................................................................21

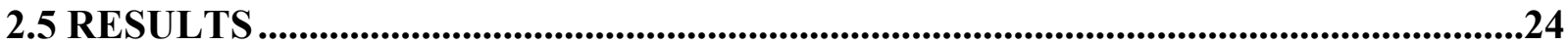

2.5.1 Predictive Physical Property Models and Validation Phase 1 .................................... 24

2.5.1.1 Envelope A Predictive Models ...................................................................................... 24

2.5.1.2 Envelope B Predictive Models ........................................................................................ 31

2.5.1.3 Envelope C Predictive Models ...................................................................................... 38

2.5.2 Comparison of Simulation Results with Experimental Results .................................. 46

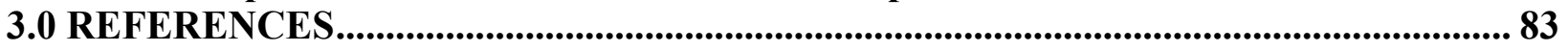

APPENDIX A. DESIGN MATRICES FOR ENVELOPES A, B, AND C ............................85

APPENDIX B. SOLIDS PREDICTIONS FOR ENVELOPES A, B, AND C ..................... 117 


\section{LIST OF FIGURES}

Figure 1. OLI Treated Feed Evaporator Model ………….................................................... 3

Figure 2. Simulated vs. Predicted Viscosity for Envelope A ………...................................... 4

Figure 3. \% NASGEL versus Na M for Bottoms Concentrate of Envelope A............................. 8

Figure 4. \% NASGEL versus Temperature for Bottoms Concentrate of Envelope A .................. 8

Figure 5. \% NASGEL versus SBS/Feed for $10 \mathrm{Na} \mathrm{M}$ Bottoms Concentrate of Envelope A....... 9

Figure 6. \% NASGEL versus SBS/Feed for $8 \mathrm{Na} \mathrm{M}$ Bottoms Concentrate of Envelope A......... 9

Figure 7. \% NASGEL versus SBS/Feed for $6 \mathrm{Na}$ M Bottoms Concentrate of Envelope A....... 10

Figure 8. \% NASGEL versus Na M for Bottoms Concentrate of Envelope C............................ 11

Figure 9. \% NASGEL versus Na M for Bottoms Temperature of Envelope C.......................... 12

Figure 10. \% NASGEL versus SBS/Feed for $10 \mathrm{Na}$ M Bottoms Concentrate of Envelope C.. 12

Figure 11. \% NASGEL versus SBS/Feed for $8 \mathrm{Na}$ M Bottoms Concentrate of Envelope C..... 13

Figure 12. \% NASGEL versus SBS/Feed for $6 \mathrm{Na}$ M Bottoms Concentrate of Envelope C..... 13

Figure 13. Simulated Density versus Predicted Density for Envelope A ..................................... 26

Figure 14. Simulated Conductivity versus Predicted Conductivity for Envelope A ................... 27

Figure 15. Simulated Cp versus Predicted Cp for Envelope A.................................................. 28

Figure 16. Simulated $[\mathrm{Na}]$ versus Predicted $[\mathrm{Na}]$ for Envelope A ............................................. 29

Figure 17. Envelope A Bottoms Solids Fraction versus Na Molarity and Temperature ............. 30

Figure 18. Simulated Density versus Predicted Density for Envelope B ……………............... 32

Figure 19. Simulated Viscosity versus Predicted Viscosity for Envelope B .............................. 33

Figure 20. Simulated Conductivity versus Predicted Conductivity for Envelope B ................... 34

Figure 21. Simulated Cp versus Predicted Cp for Envelope B................................................... 35

Figure 22. Simulated [Na] versus Predicted [Na] for Envelope B ......................................... 36

Figure 23. Envelope B Bottoms Solids Fraction versus Na Molarity and Temperature ............. 37

Figure 24. Simulated Density versus Predicted Density for Envelope C ................................... 39

Figure 25. Simulated Viscosity versus Predicted Viscosity for Envelope C................................ 40

Figure 26. Simulated Conductivity versus Predicted Conductivity for Envelope C ................... 41

Figure 27. Simulated $\mathrm{Cp}$ versus Predicted $\mathrm{Cp}$ for Envelope C................................................. 42

Figure 28. Simulated [Na] versus Predicted [Na] for Envelope C ........................................... 43

Figure 29. Envelope C Bottoms Solids Fraction versus Na Molarity and Temperature ............. 44

Figure 30. Envelope C Bottoms Solids Fraction versus Na Molarity and SBS/Feed.................. 45 


\section{LIST OF TABLES}

Table 1. Comparison between Measured and Predicted Physical Properties .................................. 6

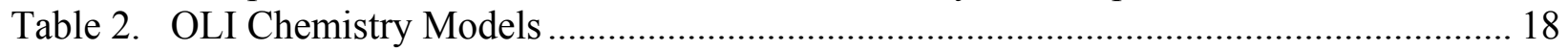

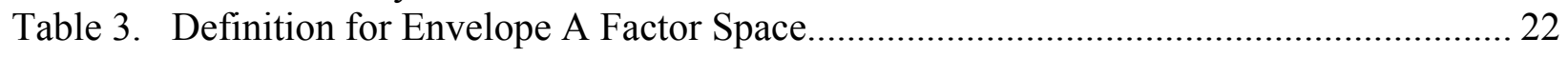

Table 4. Definition for Envelope B Factor Space...................................................................... 23

Table 5. Definition for Envelope C Factor Space....................................................................... 23

Table 6. Valid Variable Ranges for Envelope A Models ............................................................ 25

Table 7. Valid Variable Ranges for Envelope B Models............................................................. 31

Table 8. Valid Variable Ranges for Envelope C Models.............................................................. 38

Table 9. Comparison of Measured and Predicted Densities for Envelope A .............................. 46

Table 10. Comparison of Measured versus Predicted Viscosities for Envelope A ..................... 48

Table 11. Comparison of Measured versus Predicted Conductivity for Envelope A.................. 49

Table 12. Comparison of Measured versus Predicted Cp for Envelope A ..................................50

Table 13. Comparison of Measured versus Predicted Na M for Envelope A.............................. 52

Table 14. Predicted Solids for Envelope A Simulations - Part 1 .............................................. 54

Table 15. Predicted Solids from Envelope A Simulations - Part 2 ………............................. 57

Table 16. Comparison of Measured and Predicted Densities for Envelope B............................. 60

Table 17. Comparison of Measured versus Predicted Viscosities for Envelope B ..................... 61

Table 18. Comparison of Measured versus Predicted Conductivity for Envelope B ................... 62

Table 19. Comparison of Measured versus Predicted Cp for Envelope B ………….................. 63

Table 20. Comparison of Measured versus Predicted Na M for Envelope B................................ 64

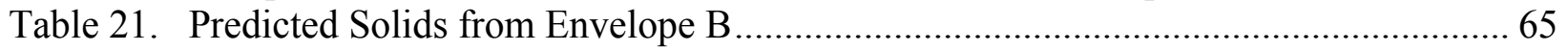

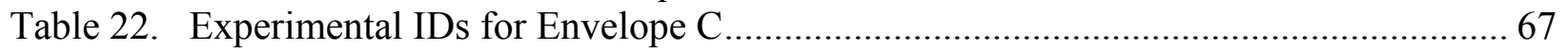

Table 23. Comparison of Measured and Predicted Densities for Envelope C............................. 68

Table 24. Comparison of Measured versus Predicted Viscosities for Envelope C .................... 69

Table 25. Comparison of Measured versus Predicted Conductivity for Envelope C .................. 70

Table 26. Comparison of Measured versus Predicted $\mathrm{Cp}$ for Envelope C .................................. 71

Table 27. Comparison of Measured versus Predicted Na M for Envelope C.............................. 71

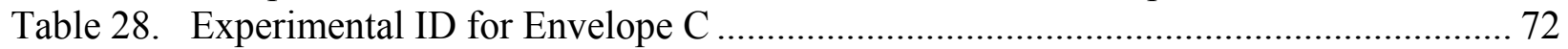

Table 29. Predicted Solids from Envelope C Simulations - Part 1 ............................................. 74

Table 30. Predicted Solids from Envelope C Simulations - Part 2 ……………......................... 78

Table 31. Comparisons of AW-101 Radioactive Experimental Measured Values versus

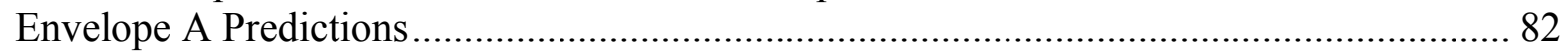

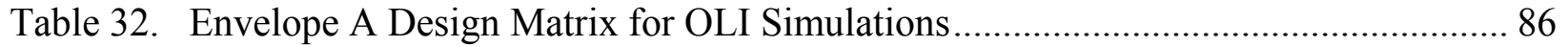

Table 33. Envelope B Design Matrix for OLI Simulations ........................................................ 99

Table 34. Envelope C Design Matrix for OLI Simulations ......................................................... 108

Table 35. Predicted Solids for Envelope A Simulations - Part 1 ……...................................... 118

Table 36. Predicted Solids for Envelope A Simulations - Part 2 ………………................... 135

Table 37. Predicted Solids for Envelope B Simulations - Part 1 ........................................... 153

Table 38. Predicted Solids for Envelope B Simulations - Part 2 ………………...................... 166

Table 39. Predicted Solids for Envelope C Simulations - Part 1 .......................................... 179

Table 40. Predicted Solids for Envelope C Simulations - Part 2 ………................................. 193 


\section{LIST OF ACRONYMS}

$\begin{array}{ll}\text { HLW } & \text { High Level Waste } \\ \text { IX } & \text { Ion Exchange } \\ \text { LAW } & \text { Low Activity Waste } \\ \text { NASGEL } & \text { Sodium Aluminosilicate gelatin } \\ \text { OLH } & \text { Orthogonal Latin Hypercube } \\ \text { OLI/ESP } & \text { OLI Environmental Simulation Package Software } \\ \text { RPP } & \text { River Protection Project } \\ \text { SBS } & \text { Submerged Bed Scrubber } \\ \text { Sr/TRU } & \text { Strontium/Transuranic } \\ \text { TFE } & \text { Hanford RPP-WTP Treated Feed Evaporator } \\ \text { TF-COUP } & \text { Tank Farm - Contractor Operation and Utilization Plan } \\ \text { UF } & \text { Ultra-Filtration }\end{array}$


WSRC-TR-2003-00269, REVISION 0

SRT-RPP-2003-00125, REVISION 0

\subsection{SUMMARY OF TESTING}

Previous Hanford-RPP evaporator modeling has focused on the treated LAW feed and eluate evaporation systems without the inclusion of secondary waste recycles. ${ }^{1-4}$ This task investigates the potential impact that secondary-waste recycle streams may have on the operation of the treated LAW evaporator. The treated LAW evaporator will concentrate the treated waste effluent streams from the Cs ion exchange blended with the LAW melter offgas scrubbing recycle stream. The Tc ion exchange system (originally part of the test specification) has since been eliminated from the flowsheet. The LAW melter offgas scrubbing recycle stream is the major contributor to the overall recycle volume that is to be mixed with the treated waste feed prior to evaporation. Based on experience at Savannah River Site, the introduction of silica from melter offgas recycle into high sodium/aluminum feeds can produce sodiumaluminum-silicate precipitates upon concentration. These sodium-aluminum-silicates can cause operational shutdowns due to plugging of lines and fouling of heat transfer surfaces.

This task examines the potential of the treated waste feed blends to form sodium-aluminumsilicate precipitates when evaporated using the zeolite database. To investigate the behavior of the blended pretreated waste feed, an OLI ESP model of the treated LAW evaporator was built. A range of waste feed compositions representative of Envelope A, B, and C were then fed into the OLI model to predict various physical and chemical properties of the evaporator concentrates. Additional runs with treated LAW evaporator were performed to compare chemical and physical property model predictions and experimental results for small scale radioactive tests (S-69) of the treated feed (AW 101) evaporation process.

\subsection{OBJECTIVES}

The objectives of this task were to develop physical property correlations of the concentrated treated feed evaporator bottoms as stated in the Treated LAW Feed: Simulant Evaporation and Physical Properties Modeling test specification from RPP. ${ }^{5}$ The model was to simulate the treated LAW evaporator operating at $50^{\circ} \mathrm{C}$ at the bubble point vacuum with Envelope A, B, or $\mathrm{C}$ wastes blended with LAW melter offgas scrubbing recycle (SBS) as feed and the evaporator concentrate or bottoms stream being varied between $15^{\circ} \mathrm{C}$ and $66^{\circ} \mathrm{C}$. The physical property correlations were to be expressed in terms of the waste feed compositions, LAW SBS to waste feed volumetric flow ratio, and the evaporator bottoms temperature and sodium concentration. Simulation results would then be regressed to generate predictive equations for density, heat capacity, thermal conductivity, viscosity, and solubility of the Treated LAW evaporator bottoms concentrate. Simulation results were to be validated with experimental results which had already been completed. 


\section{WSRC-TR-2003-00269, REVISION 0 SRT-RPP-2003-00125, REVISION 0}

Development of these equations was successful based on the goal of developing physical property correlations for each waste envelope with an error no greater than $\pm 15 \%$ between calculated and modeled physical properties. The equation to predict solubility or the amount of total solids in the Treated LAW evaporator bottoms concentrate could not be developed to satisfy this goal. There was not enough information captured by the chosen model inputs to adequately describe the complicated nature of solids precipitation occurring in bottoms concentrate. However, general trends in solids formation were identified with respect to the bottoms Na molarity and temperature, which will be discussed later.

Another objective of this task was to verify the derived physical property equations with experimental tests with simulated blended LAW feed solutions ${ }^{6}$. The predicted physical properties were compared with experimental results documented in the report Treated $L A W$ Feed Evaporation: Physical Properties and Solubility Determination ${ }^{7}$ and with radioactive experiments for AW-101 documented in the report Evaporation of Pretreated Hanford Tank $A W-101$ Sample Mixed with Recycle ${ }^{8}$. The predicted densities and heat capacities were within $+/-15 \%$ of the measured values for Envelopes A, B, and C. The Na molarity predictions for Envelope A and B were also within $+/-15 \%$. The other predicted physical properties were outside $+/-15 \%$ of the measured values. This mismatch with measured values was due in part to being outside the range of the predictions, comparing a measured slurry property with a predicted supernate property, and the exclusion of solids in the predictions. More details about this comparison are given in section 2.5.2.

\subsection{CONDUCT OF TESTING}

The treated feed evaporator process was simulated using the OLI Environmental Simulation Program (OLI/ESP) version 6.6 using the CARBONAT, HNO3DB, SILICA, and ZEOLITE private databases, along with the public database. These databases were developed and validated in prior work. ${ }^{9}$ A schematic of the OLI treated feed evaporator model is shown in Figure 1. The model fits were done using JMP ${ }^{\circledR}$ version 5.0.1 ${ }^{10}$ using linear and nonlinear least squares fit routines.

Distinct physical property models were developed for each waste of the envelopes, A, B, and C. The model variables were the waste feed compositions, the evaporator bottoms temperature $\left(15-66^{\circ} \mathrm{C}\right)$ and sodium concentration $(6,8$, and $10 \mathrm{M})$, and the volumetric flow ratio of LAW SBS recycle relative to the treated waste feed $(0-2)$. The test matrices were generated assuming that the physical properties are linear functions in composition, and up to second order with respect to the process variables (i.e., temperature, flow ratio, etc.). Additional simulation points not included in the model fits were used to validate these assumptions.

Since this task was to be validated using results of prior experimental work, ${ }^{7}$ the waste envelope definitions from this prior work were used for this task. Envelope A waste formulations came from a matrix developed by R. Eibling and T. Edwards for the Waste Feed Evaporation R\&T Task S-90 based upon a review of TFCOUP 3A. ${ }^{11}$ For Envelope B a best basis mass inventory of 10/1/1998 was used to develop a composition for an AZ101 simulant. ${ }^{12}$ This composition was used in the experimental work as well as this modeling work. 
A recipe for AZ102 that was based on the analysis of a radioactive sample was used in the experimental work and this modeling work as well. ${ }^{13}$ For envelope, C an AN-102 simulant was prepared using a Sr/TRU precipitation procedure outlined in a separate document. ${ }^{14}$ This simulant was then filtered and used to represent AN-102 in the earlier experimental work ${ }^{7}$ and this modeling study. The AN-107 simulant was the product of a pilot-scale filtration study by Duignan $^{14}$ at SRTC's Thermal Fluids Laboratory. This simulant underwent Sr/TRU removal and cross-flow filtration. The filtered supernate of this simulant was then used for the earlier experimental work as well as this modeling work. ${ }^{7}$

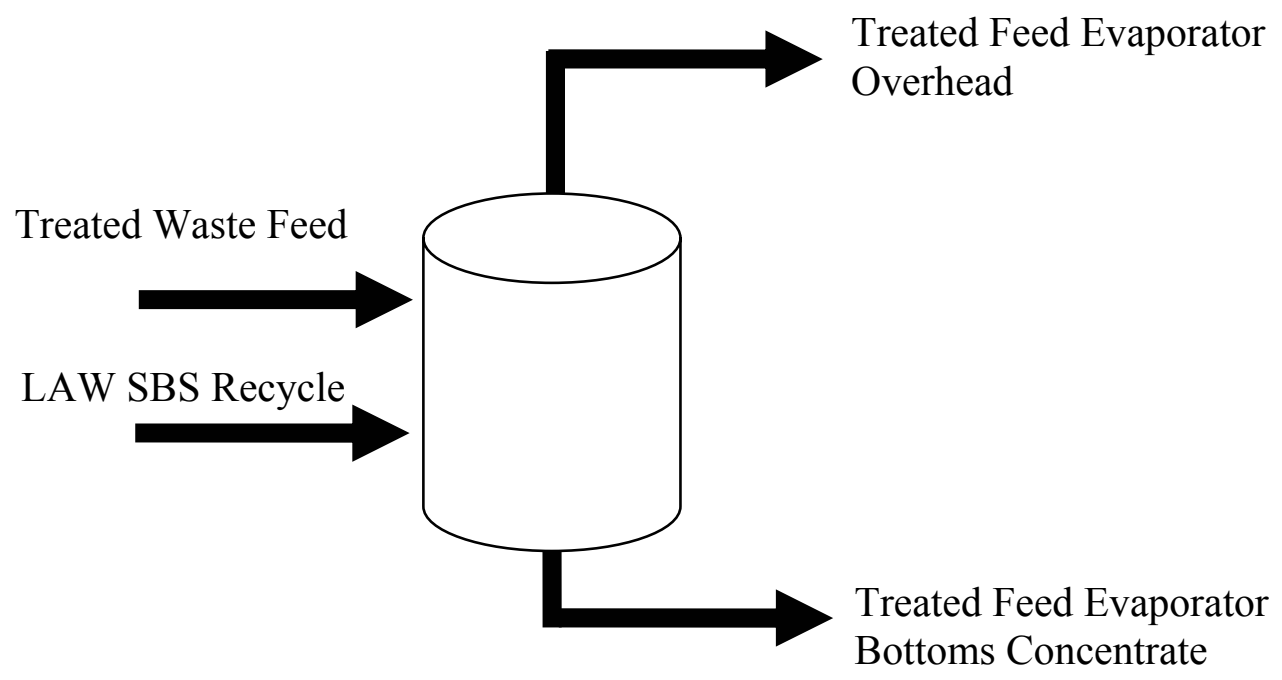

Figure 1. OLI Treated Feed Evaporator Model

Validation of the models was done in two parts; the physical property model predictions were compared to those of the simulation software using design points not included in the model fit, and the chemistry and physical properties predicted by the simulation software were compared to experimental results for selected simulation design points.

\subsection{RESULTS AND PERFORMANCE AGAINST OBJECTIVES}

Figure 2 is an example of the type of plot used to validate the physical property models against the simulation results. This figure shows the simulated viscosity from OLI versus the fitted model prediction of viscosity. The figure shows the predicted viscosity as a dotted black line, the predicted viscosity plus $15 \%$ as a red solid line, and the predicted viscosity minus $15 \%$ as the solid green line. The blue circles represent the simulated data points used to fit the model and the red asterisks represent the simulated points used as validation of the model. The complete set of results can be found in section 2.5 . 
As shown in Figure 2, all the validation points (red asterisks) fall between the $\pm 15 \%$ prediction curves, exceeding the $80 \%$ confidence acceptance criteria, indicating that the fitted model is able to predict the simulation results within the acceptable error. The equation for the fitted viscosity model is shown immediately following the figure.

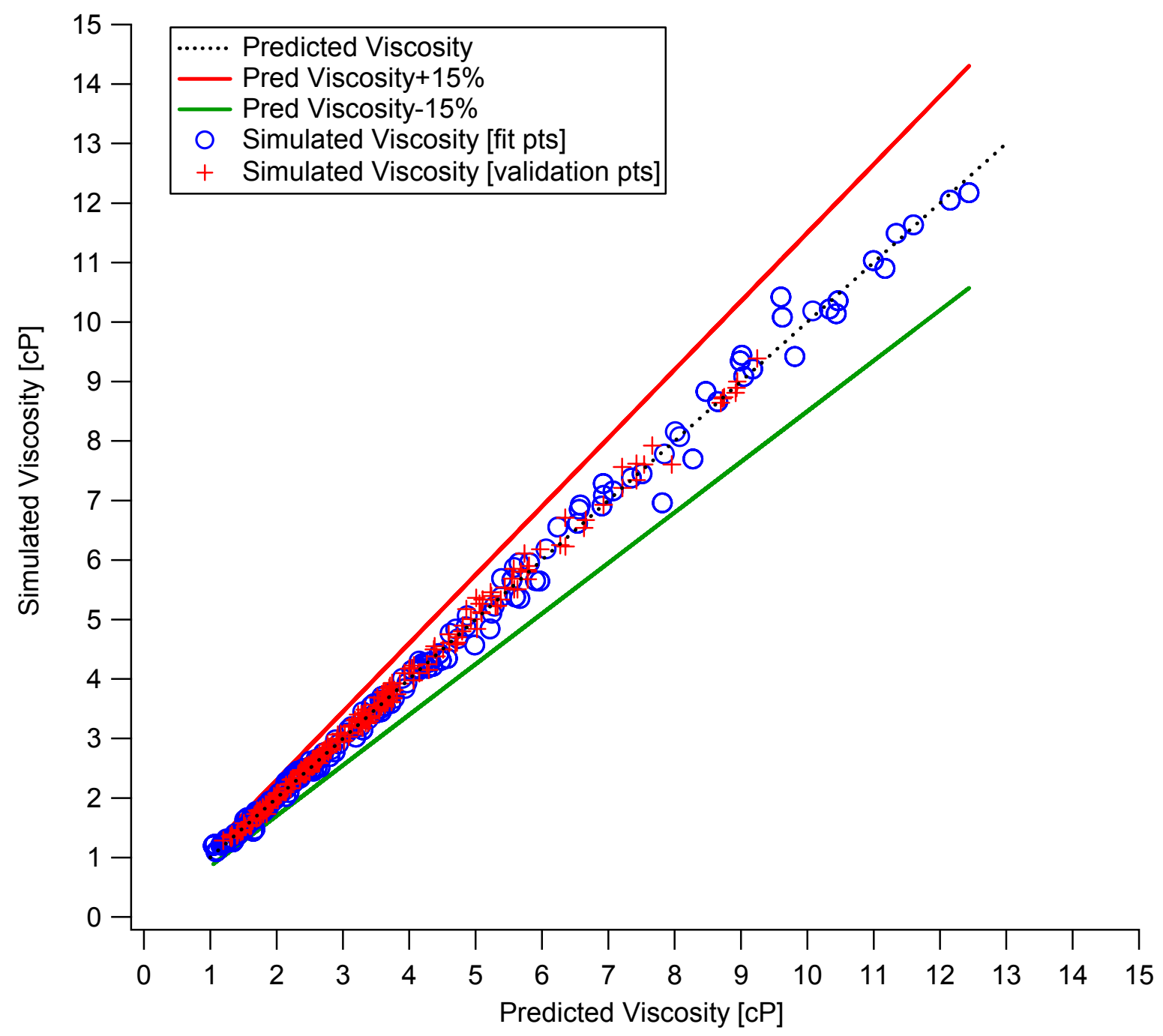

Figure 2. Simulated vs. Predicted Viscosity for Envelope A 


\section{Equation 1}

$$
\begin{aligned}
\text { viscosity }_{\mathrm{EnvA}}[\mathrm{cP}]= & 0.3118 \cdot[\mathrm{Na}]+0.4547 \cdot x_{\mathrm{OH}}+1.148 \cdot x_{\mathrm{AlO} 2}+2.117 \cdot x_{\mathrm{CO} 3}+0.7458 \cdot x_{\mathrm{NO} 2}+ \\
& 0.02692 \cdot \mathrm{SBS} / \mathrm{Feed}-2.437 \mathrm{E}-3 \cdot \mathrm{Temp} \cdot[\mathrm{Na}]- \\
& 4.213 \mathrm{E}-4 \cdot \mathrm{Temp} \cdot \mathrm{SBS} / \mathrm{Feed}-0.3334+ \\
& \exp \left(\begin{array}{l}
\frac{530.9}{73.43+\mathrm{Temp}}+0.3041 \cdot[\mathrm{Na}]+2.080 \cdot x_{\mathrm{OH}}+0.4869 \cdot x_{\mathrm{AlO} 2}+0.6207 \cdot x_{\mathrm{CO} 3} \\
-0.8173 \cdot x_{\mathrm{NO} 2}-0.0479 \cdot \mathrm{SBS} / \text { Feed }-7.266
\end{array}\right)
\end{aligned}
$$

Where

$x_{\mathrm{OH}}, x_{\mathrm{AlO} 2}, x_{\mathrm{CO} 3}, x_{\mathrm{NO} 2}$ are the relative mass fractions of $\mathrm{OH}-, \mathrm{AlO} 2-\mathrm{CO} 3--$, and $\mathrm{NO} 2-$ in the waste feed;

$S B S / F e e d$ is the volume ratio of SBS recycle to treated waste feed flow;

$[\mathrm{Na}]$ is the Na molarity

Temp is the temperature in degrees Celsius of the evaporator concentrate bottoms stream

Some of the predicted physical properties of treated feed evaporate bottoms stream matched with the experimental measurements completed in prior work ${ }^{7}$ and some properties did not. Table 1 summarizes the comparison between measured and predicted physical properties.

The $+/-55 \%$ discrepancy in the measured versus predicted $\mathrm{Na} \mathrm{M}$ for Envelopes $\mathrm{A}$ and $\mathrm{B}$ is in part due to the fact that the prediction equation was derived from simulated data between 6 and $10 \mathrm{Na} \mathrm{M}$ only. It is not surprising that when the prediction is extrapolated down to $2 \mathrm{Na} \mathrm{M}$ the measured values do not match more than $+/-55 \%$. Another reason for the large discrepancy is that it is difficult to derive a predictive relationship relating treated feed waste composition (dry basis), SBS/Feed ratio, bottoms temperature, and bottoms density to bottoms Na molarity.

Four out of the 31 heat capacity measurements for Envelope A did not fall within $+/-15 \%$ of the predictions. Part of the reason for this discrepancy is that the experimental heat capacities are for the supernate while those predicted are for the slurry (supernate plus solids). Analyses of the experimental samples show evidence of solids which in turn helps explain some of the deviation from predicted values.

The thermal conductivity predictions for envelopes $\mathrm{A}, \mathrm{B}$, and $\mathrm{C}$ were within $+/-25 \%$ of most of the measured values for the bottoms concentrate with a Na molarity between 2 and 8 . This large discrepancy is due in part that the simulated conductivity is for the supernate only where as the measured conductivity is for the entire slurry (supernate plus solids). Prior modeling work $^{9}$ showed that the experimental conductivity has a standard deviation about the value of water of $6.5 \%$. The simulated conductivities are based on correction factors for the conductivity of water using various anions/cations as defined in Perry's Chemical Handbook. ${ }^{15}$ 
The simulated conductivities for all envelopes fell within a $3 \%$ standard deviation of the value of water. Since the measurement error is higher than this value, the predicted conductivities cannot be distinguished from those of water. Therefore there is no need to use a complicated prediction equation for conductivity when statistically a prediction for the conductivity of water is just as accurate.

Table 1. Comparison between Measured and Predicted Physical Properties

\begin{tabular}{|c|c|c|c|c|}
\hline Property & Molarity Range & Envelopes & $\begin{array}{l}\text { Measured within } \\
\text { Predicted }\end{array}$ & Comments \\
\hline \multirow{3}{*}{ Na M } & $6-10$ & A, B & $+/-15 \%$ & none \\
\hline & 2 & A, B & $+/-55 \%$ & none \\
\hline & $2-10$ & $\mathrm{C}$ & $+/-32 \%$ & none \\
\hline \multirow{3}{*}{ Density } & $2-10$ & $\mathrm{~A}$ & $+/-6 \%$ & none \\
\hline & $1.2-8$ & $\mathrm{~B}$ & $+/-5 \%$ & none \\
\hline & $2-10$ & $\mathrm{C}$ & $+/-11 \%$ & none \\
\hline \multirow{3}{*}{ Heat Capacity } & $2-8$ & A & $+/-15 \%$ & $\begin{array}{l}4 \text { out of the } 31 \text { samples } \\
\text { did not fall within }+/-15 \%\end{array}$ \\
\hline & $1.2-5$ & $\mathrm{~B}$ & $+/-15 \%$ & \\
\hline & $2-8$ & $\mathrm{C}$ & $+/-10 \%$ & \\
\hline \multirow{3}{*}{$\begin{array}{c}\text { Thermal } \\
\text { Conductivity }\end{array}$} & $2-8$ & A & $+/-25 \%$ & $\begin{array}{l}3 \text { out of the } 21 \text { samples } \\
\text { did not fall within }+/-25 \%\end{array}$ \\
\hline & $1.2-5$ & B & $+/-25 \%$ & $\begin{array}{l}4 \text { out of the } 12 \text { samples } \\
\text { did not fall within }+/-25 \%\end{array}$ \\
\hline & $2-8$ & $\mathrm{C}$ & $+/-20 \%$ & $\begin{array}{l}3 \text { out of the } 12 \text { samples } \\
\text { did not fall within }+/-25 \%\end{array}$ \\
\hline \multirow{6}{*}{ Viscosity } & $2-9$ & A & $+/-20 \%$ & $\begin{array}{c}100 \% 25^{\circ} \mathrm{C} \\
\text { measurements }\end{array}$ \\
\hline & $2-8$ & A & $+/-50 \%$ & $\begin{array}{c}40 \% \text { of } 15^{\circ} \mathrm{C}, 60^{\circ} \mathrm{C} \\
\text { measurements (other } 60 \% \\
\text { within }+/-20 \% \text { ) }\end{array}$ \\
\hline & $1.2-9$ & $\mathrm{~B}$ & $+/-60 \%$ & $15^{\circ} \mathrm{C}$ measurements \\
\hline & $1.2-9$ & B & $+/-70 \%$ & $25^{\circ} \mathrm{C}$ measurements \\
\hline & $1.2-9$ & B & $+/-50 \%$ & $60^{\circ} \mathrm{C}$ measurements \\
\hline & $2-9$ & $\mathrm{C}$ & $+/-20$ to $70 \%$ & $\begin{array}{l}15^{\circ} \mathrm{C}-60^{\circ} \mathrm{C} \\
\text { measurements }\end{array}$ \\
\hline
\end{tabular}




\section{WSRC-TR-2003-00269, REVISION 0 SRT-RPP-2003-00125, REVISION 0}

The viscosity predictions for all envelopes had large errors when compared to the measured values. It is expected that the lower and higher temperature extremes produced solids that are not accounted for by the viscosity prediction since it is based only on the supernate phase of the bottoms concentrate. Another factor contributing to the difference is that the viscosity prediction models were based on the bottoms concentrate ranging from 6 to $10 \mathrm{M}$ Na while the test data covered 2 to $10 \mathrm{M} \mathrm{Na}$. Since the WTP is likely to operate the evaporator at a concentration between 6 and $8 \mathrm{Na} \mathrm{M}$ then the correlation will provide a more accurate prediction within this range. To obtain a better predictor of viscosity at the low and high sodium molarities, an accurate count of the solids in each sample plus a solids correction term derived from experimental results would need to be included. Prior modeling work ${ }^{9}$ for the waste feed evaporator showed that when the supernate viscosity was measured experimentally and compared with modeling predictions there was relatively good agreement (within $+/-15 \%$ ).

No accurate prediction equations for the solubility of the evaporator bottoms stream in terms of the total insoluble solids present could be derived for Envelopes A, B, and C. Several attempts were made to include nonlinear and linear terms in the prediction fits, but the waste feed compositions, SBS to treated Waste Feed ratio, the bottoms temperature and Na molarity did not provide enough data to characterize these phenomena. The inability to derive a linear equation to predict solubility or solids behavior is not surprising due to the discontinuities present regarding solubility of various salts. For example, it is difficult to predict solids behavior when temperature-induced phase transitions occur, like for mono-hepta-deca hydrated sodium carbonate, where each phase has a different solubility. It is also difficult to capture the inverse solubility behavior of species like sulfates which can appear and disappear in large quantities with a small temperature change. Sodium nitrate also will suddenly drop out in large quantities with shifts in the sodium molarity. Despite these obstacles some general observations were made from the simulations.

For Envelope A, about 35\% of the simulated values (116 out of 336 total - including fit and validation points) had bottoms insoluble solids greater than $1 \mathrm{wt} \%$ but only about $10 \%$ of the simulated values (33 points) had insoluble solids greater than $2 \mathrm{wt} \%$. Of this $10 \%$ segment, $64 \%$ of the values were between $2 \mathrm{wt} \%$ and $5 \mathrm{wt} \%, 9 \%$ of the values were between $5 \mathrm{wt} \%$ and $10 \mathrm{wt} \%$, and $27 \%$ were between $10 \mathrm{wt} \%$ and $20 \mathrm{wt} \%$. A complete listing of the solids predicted for Envelope A is shown in Table 35 and Table 36. The high weight percent insoluble solids were only observed at bottoms temperatures less than $20^{\circ} \mathrm{C}$ and sodium molarities greater than 8 . The primary salts were sodium oxalate, sodium carbonate, sodium sulfate-carbonate, sodium fluoride, sodium aluminosilicate gelatin (NASGEL), and sodium nitrate. The sodium nitrate only came out at $10 \mathrm{M} \mathrm{Na}$ concentrations. For the simulated runs, NASGEL solids appear in the bottoms concentrate stream over a wider \% range at $6 \mathrm{Na} \mathrm{M}$ than $8 \mathrm{Na} \mathrm{M}$ and $10 \mathrm{Na} \mathrm{M}$ as shown in Figure 3. In fact at $10 \mathrm{Na} \mathrm{M}$, no NASGEL forms for the simulated runs performed. The NASGEL solids appear at all tested bottoms temperatures as shown in Figure 4. The SBS/Feed ratio has no effect on NASGEL formation at $10 \mathrm{Na} \mathrm{M}$ as shown in Figure 5. Increasing the SBS/Feed ratio above 1.75 slightly increases the chance of NASGEL formation as shown in Figure 6. Increasing the SBS/feed ratio above 0.5 greatly increases the chance of NASGEL formation at $6 \mathrm{Na} \mathrm{M}$ as shown in Figure 7. Please note that due to the variability in the data, a low $\mathrm{Na} M$ like 6 and a high SBS/Feed ratio does not guarantee that NASGEL will form. 
WSRC-TR-2003-00269, REVISION 0

SRT-RPP-2003-00125, REVISION 0

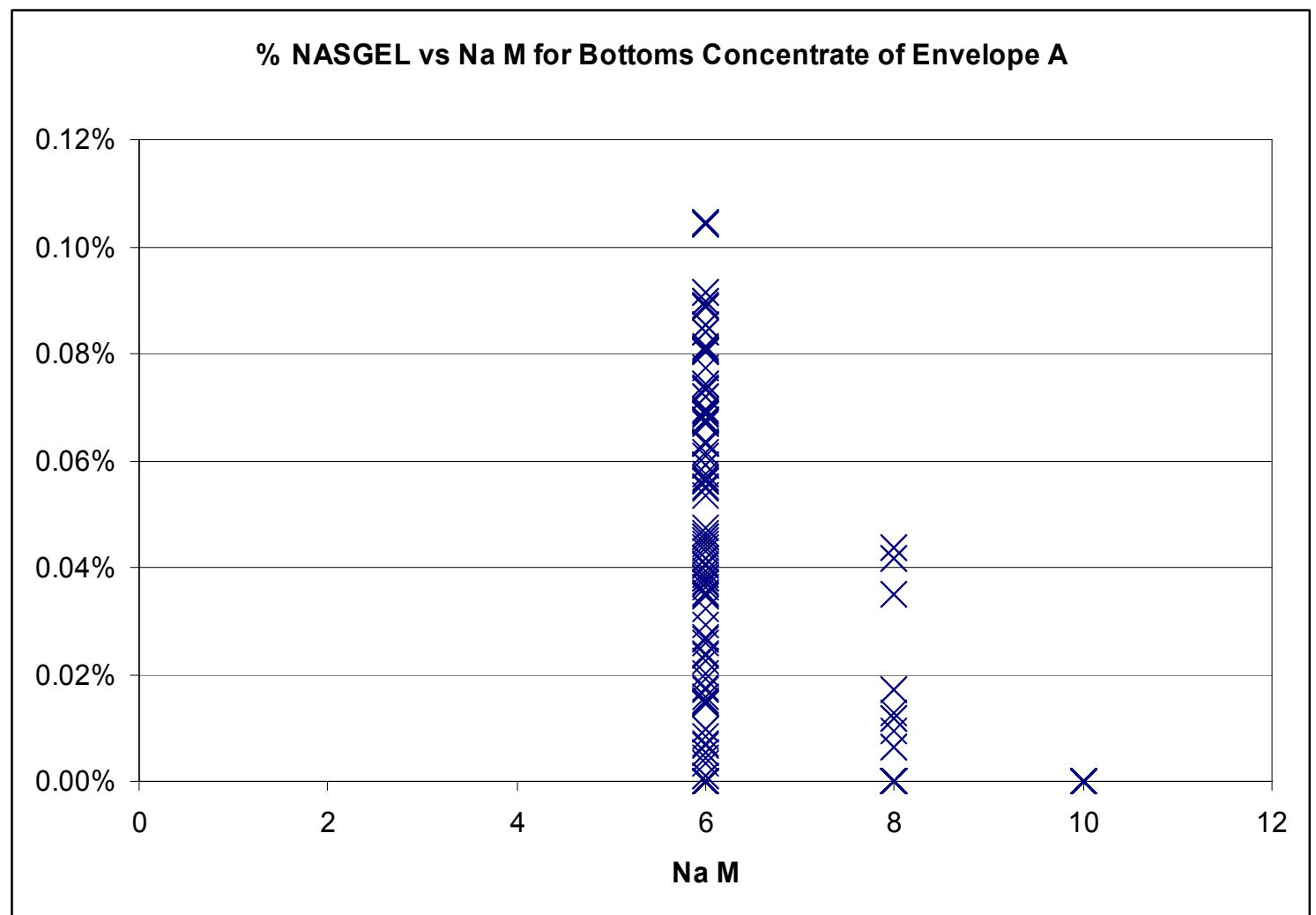

Figure 3. \% NASGEL versus Na M for Bottoms Concentrate of Envelope A

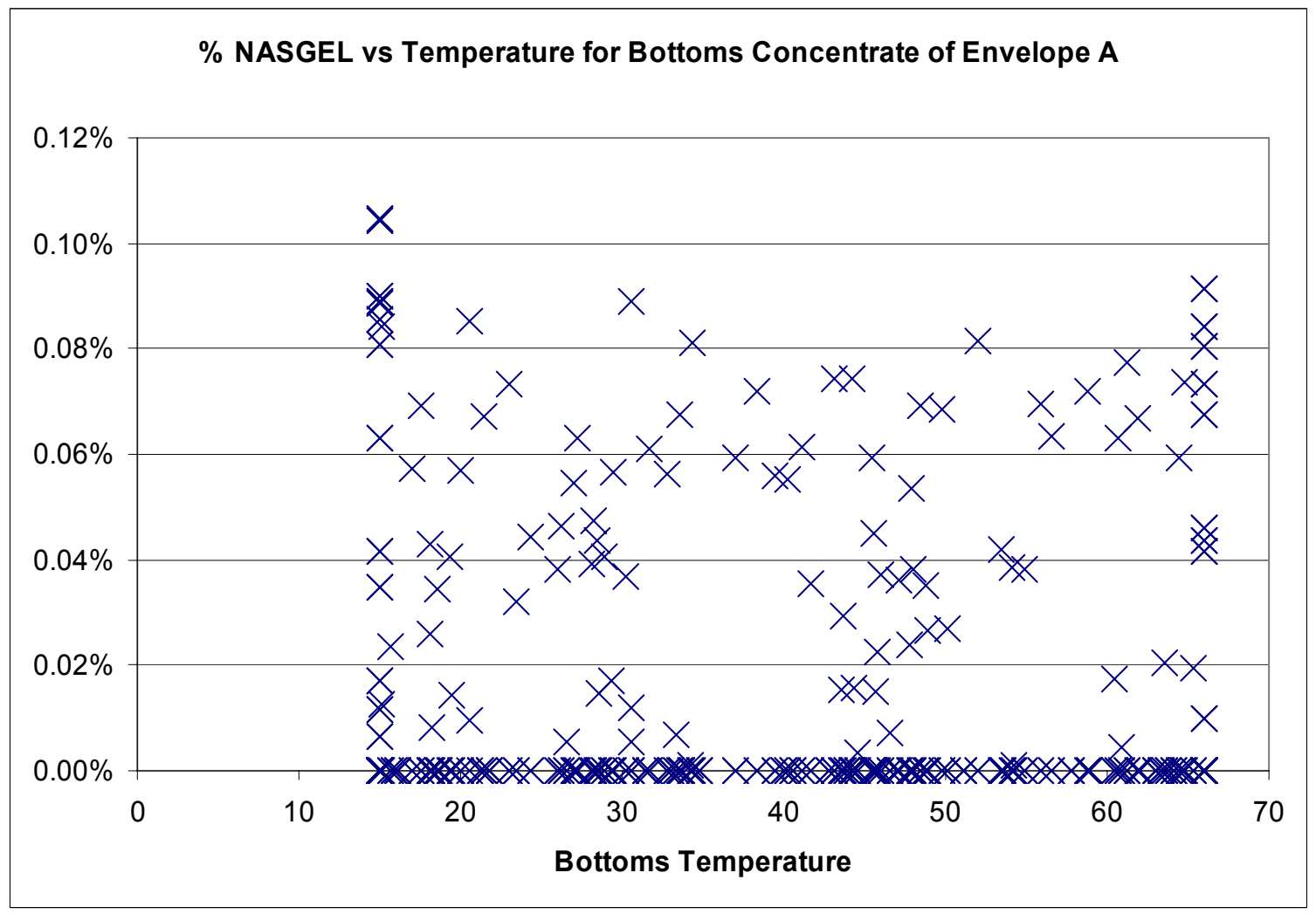

Figure 4. \% NASGEL versus Temperature for Bottoms Concentrate of Envelope A 
WSRC-TR-2003-00269, REVISION 0

SRT-RPP-2003-00125, REVISION 0

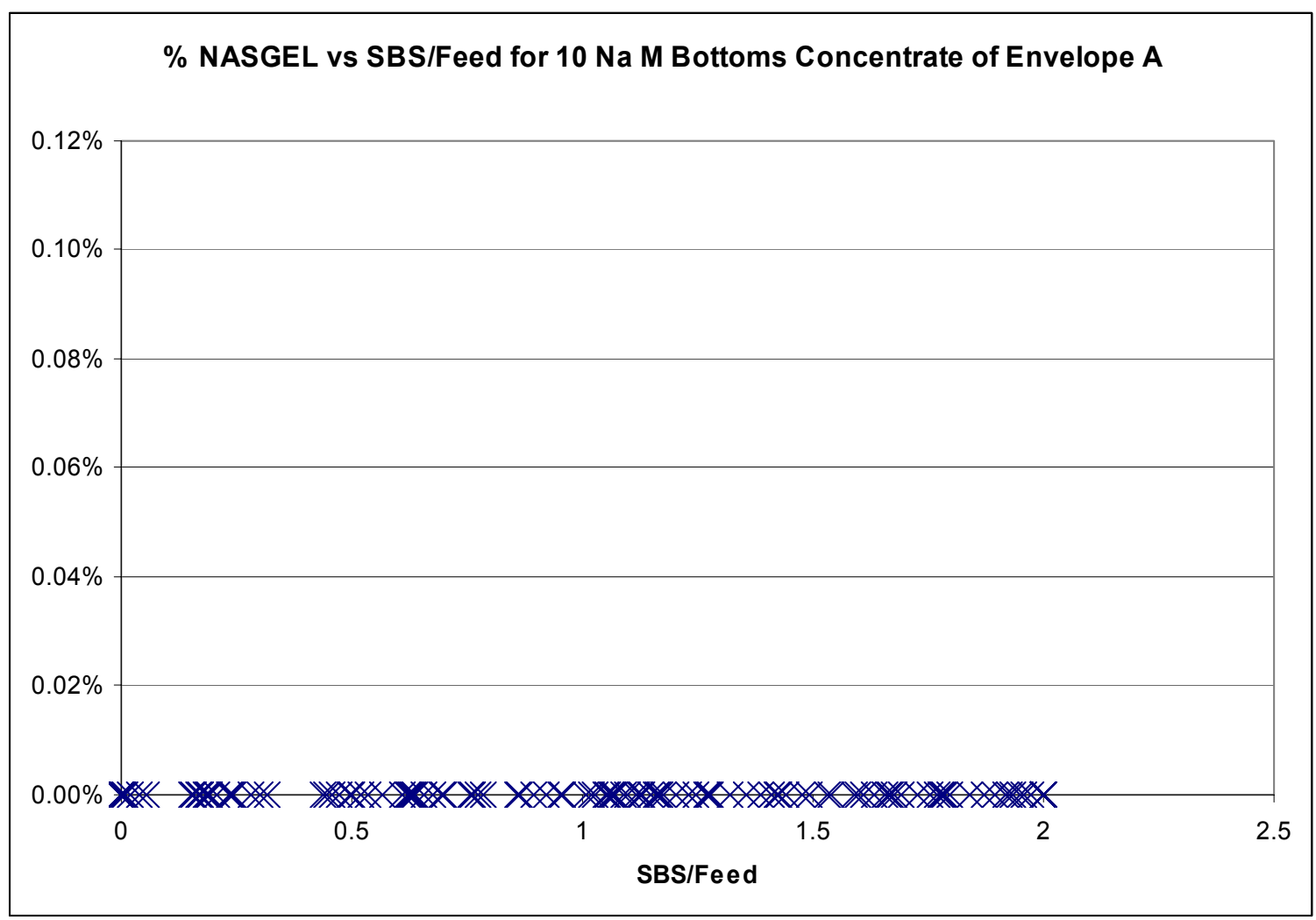

Figure 5. \% NASGEL versus SBS/Feed for 10 Na M Bottoms Concentrate of Envelope A

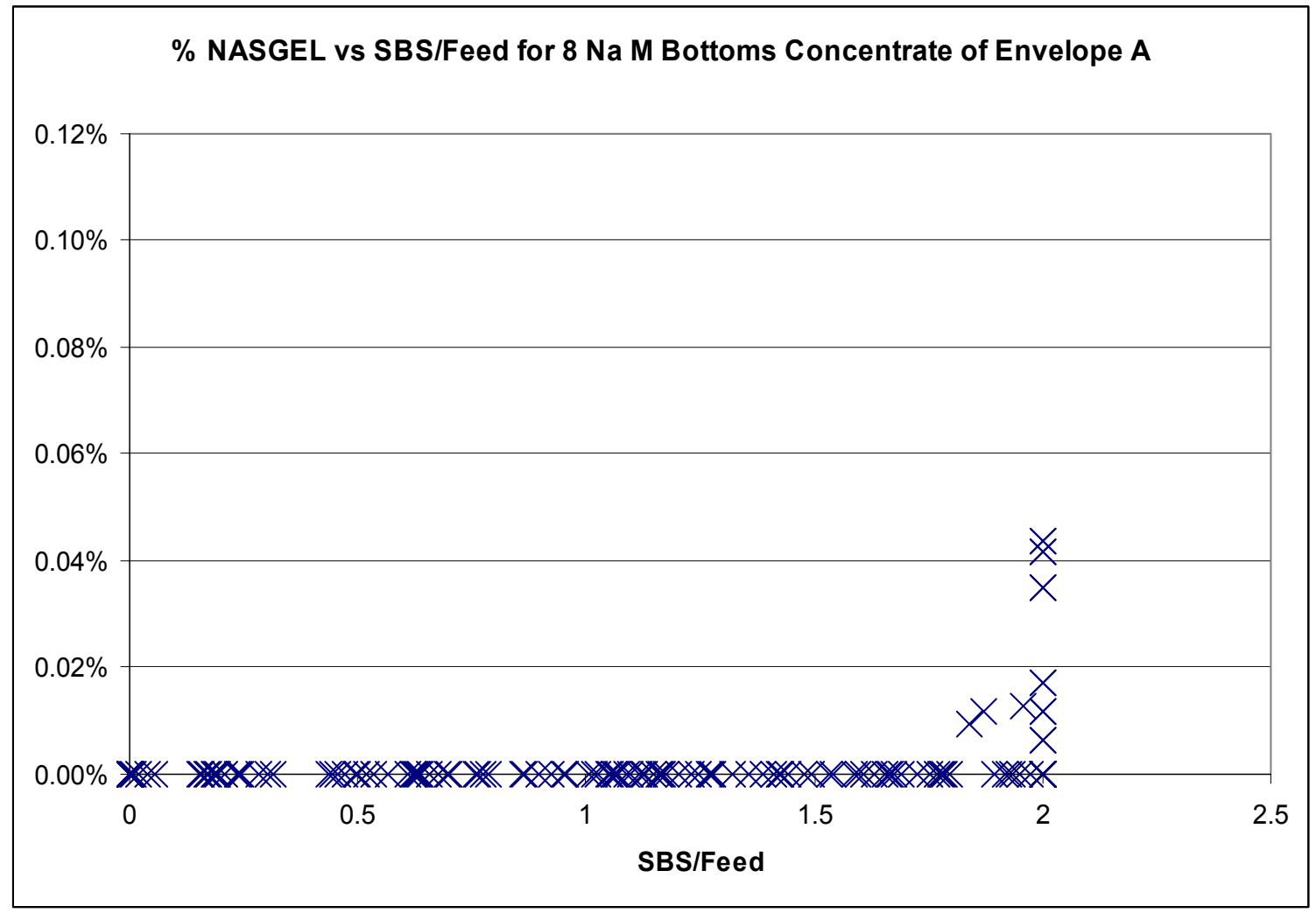

Figure 6. \% NASGEL versus SBS/Feed for 8 Na M Bottoms Concentrate of Envelope A 
WSRC-TR-2003-00269, REVISION 0

SRT-RPP-2003-00125, REVISION 0

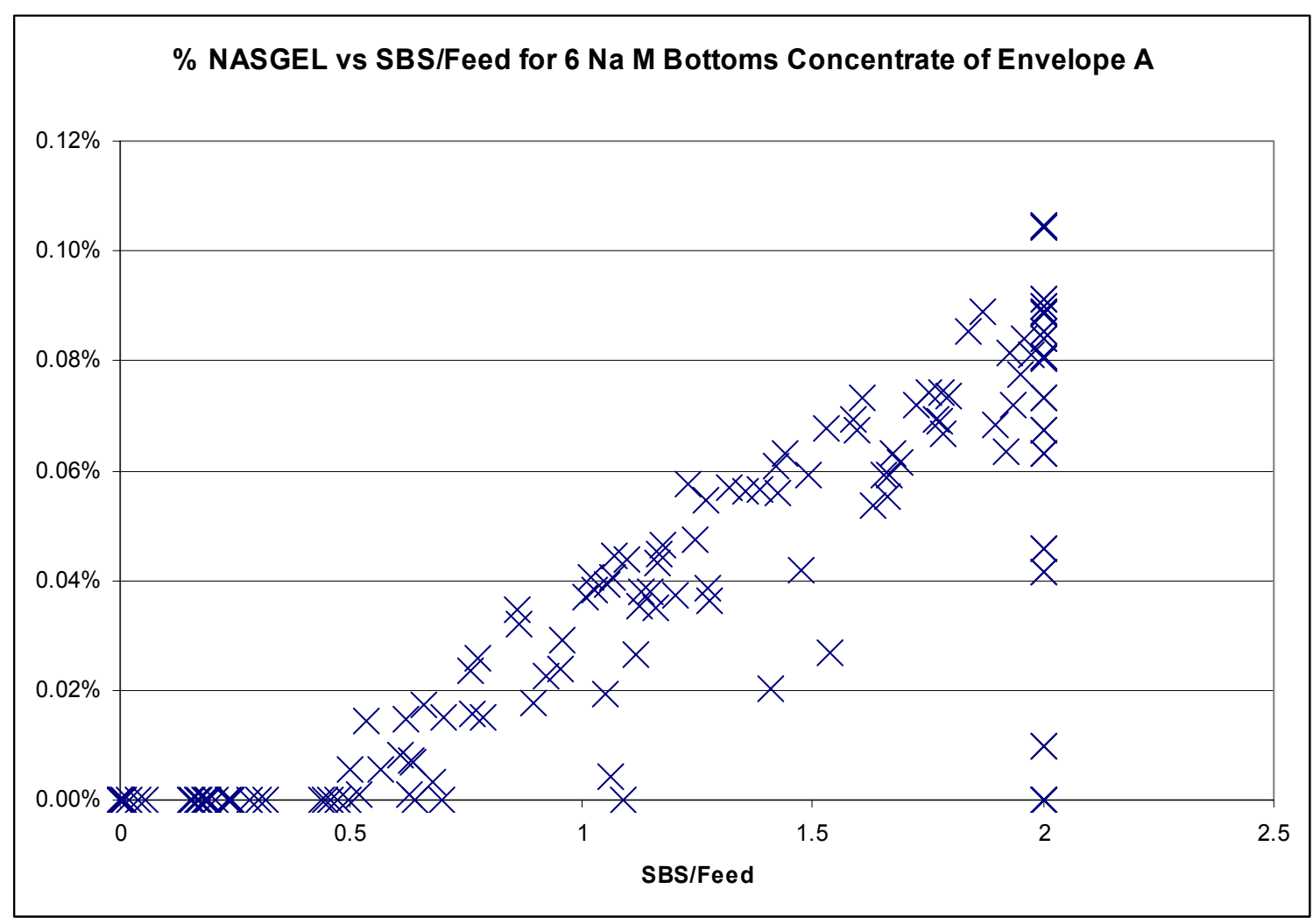

Figure 7. \% NASGEL versus SBS/Feed for 6 Na M Bottoms Concentrate of Envelope A

For Envelope B, about $21 \%$ of the simulated values ( 83 out of 399 total - including fit and validation points) had bottoms insoluble solids greater than $1 \mathrm{wt} \%$ but only about $11 \%$ of the simulated values (42 points) had insoluble solids greater than $2 \mathrm{wt} \%$. None of the predicted values went over $3 \mathrm{wt} \%$. The insoluble solids only went above $2 \mathrm{wt} \%$ when the Na molarity went above $8 \mathrm{M} \mathrm{Na}$. At $6 \mathrm{M} \mathrm{Na}$ the solids stayed below $1 \mathrm{wt} \%$, at $8 \mathrm{M} \mathrm{Na}$ the solids stayed below $2 \mathrm{wt} \%$, and at $10 \mathrm{M} \mathrm{Na}$ the solids stayed below $3 \mathrm{wt} \%$. More solids appear to come out between 20 and $40{ }^{\circ} \mathrm{C}$ than the other temperatures. The primary salts were aluminum hydroxide, sodium oxalate, sodium fluorosulfate, and sodium fluoride. None of the runs indicated that NASGEL forms.

For envelope C, about $60 \%$ of the simulated values (242 out of 405 points - including fit and validation points) had bottoms insoluble solids greater than $1 \mathrm{wt} \%$ but only about $36 \%$ of the simulated values (145 points) had insoluble solids greater than $2 \mathrm{wt} \%$. Of this $36 \%$ segment, $83 \%$ of the values were between $2 \mathrm{wt} \%$ and $5 \mathrm{wt} \%$ while the remaining $17 \%$ of the values were between $5 \mathrm{wt} \%$ and $7 \mathrm{wt} \%$. The higher weight percents were observed at NA M greater than 8 . Also temperatures have a much smaller impact on the solubility than the Na molarity. The primary salts were calcium fluoride, hydrosodalite, sodium oxalate, sodium carbonate, sodium fluorosulfate, sodium sulfate-carbonate, sodium fluoride, sodium aluminosilicate gelatin (NASGEL), and sodium nitrate. 
For the simulated runs, NASGEL solids appear in the bottoms concentrate stream over a wider $\%$ range at $10 \mathrm{Na} \mathrm{M}$ than $8 \mathrm{Na} \mathrm{M}$ and $6 \mathrm{Na} \mathrm{M}$ as shown in Figure 8 . For all sodium molarities simulated, the $\%$ NASGEL in the bottoms stream stays below $1.2 \%$. The NASGEL solids appear range 0 and $1.2 \%$ for bottoms temperatures between $15^{\circ} \mathrm{C}$ and $66^{\circ} \mathrm{C}$ as shown in Figure 9. Increasing the SBS/Feed ratio above 0.5 for the $10 \mathrm{Na} \mathrm{M}$ bottoms concentrate increases the chance of NASGEL formation as shown in Figure 10. Increasing the SBS/Feed ratio above 0.25 for the $8 \mathrm{Na} \mathrm{M}$ bottoms concentrate increases the chance of NASGEL formation as shown in Figure 11. For the $8 \mathrm{Na} \mathrm{M}$ bottoms concentrate NASGEL begins to form at lower SBS/Feed ratios but the \% NASGEL at the highest SBS/Feed ratio is less than at $10 \mathrm{Na} \mathrm{M}$. The $6 \mathrm{Na} \mathrm{M}$ bottoms concentrate shows NASGEL formation starting around $\mathrm{SBS} /$ Feed of 0.15 and increasing as SBS/Feed ratio increases. Going from $10 \mathrm{Na} \mathrm{M}$ bottoms concentrate down to 8 a M then $6 \mathrm{Na} \mathrm{M}$, the maximum amount of NASGEL formed at the highest SBS/Feed ratio decreases along with the $\mathrm{Na} \mathrm{M}$. Please note that due to the variability in the data, a low $\mathrm{Na} M$ like 6 and a high SBS/Feed ratio like 2 can still form more NASGEL than a higher $\mathrm{Na} \mathrm{M}$ at the same SBS/Feed ratio.

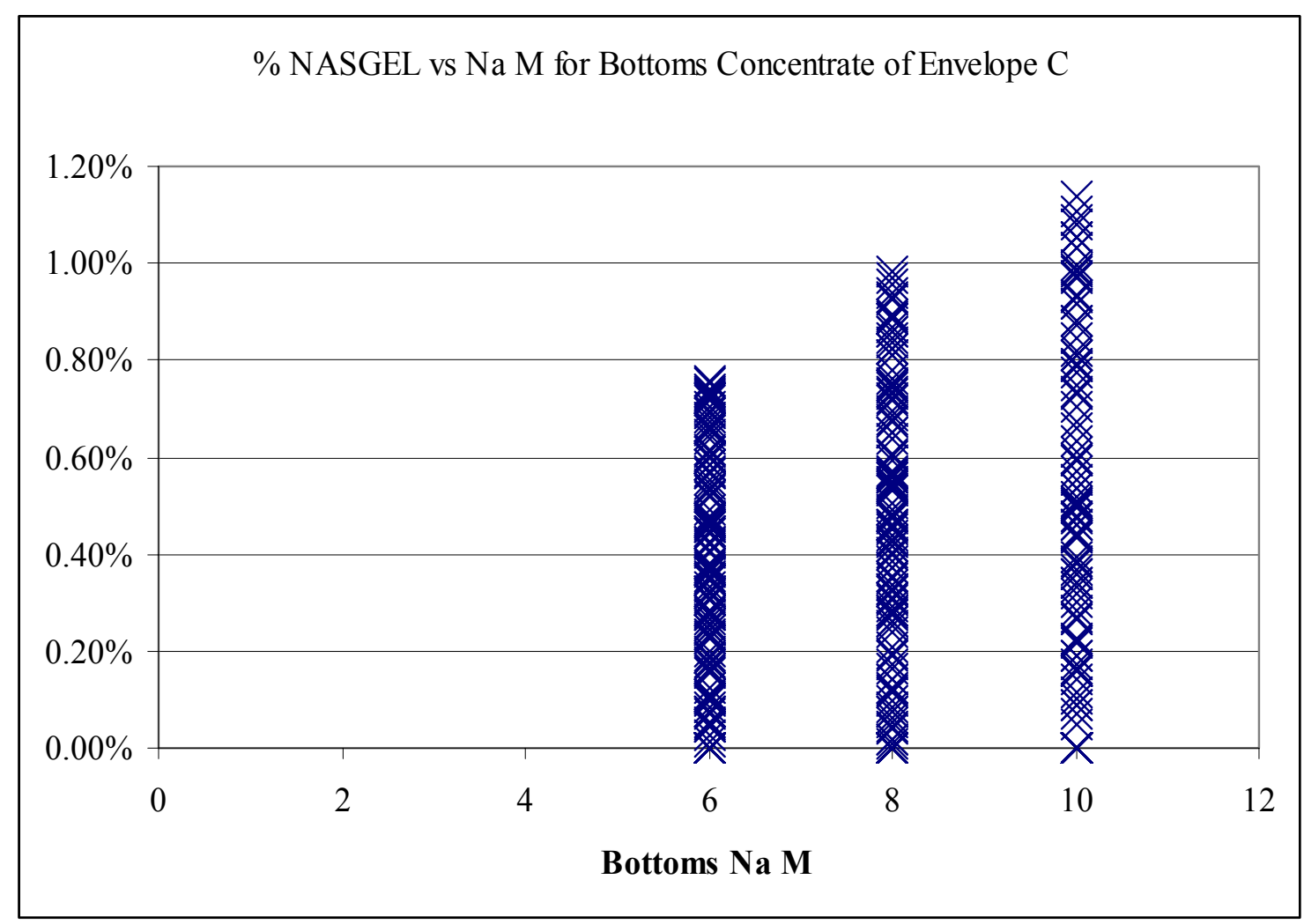

Figure 8. \% NASGEL versus Na M for Bottoms Concentrate of Envelope C 
WSRC-TR-2003-00269, REVISION 0

SRT-RPP-2003-00125, REVISION 0

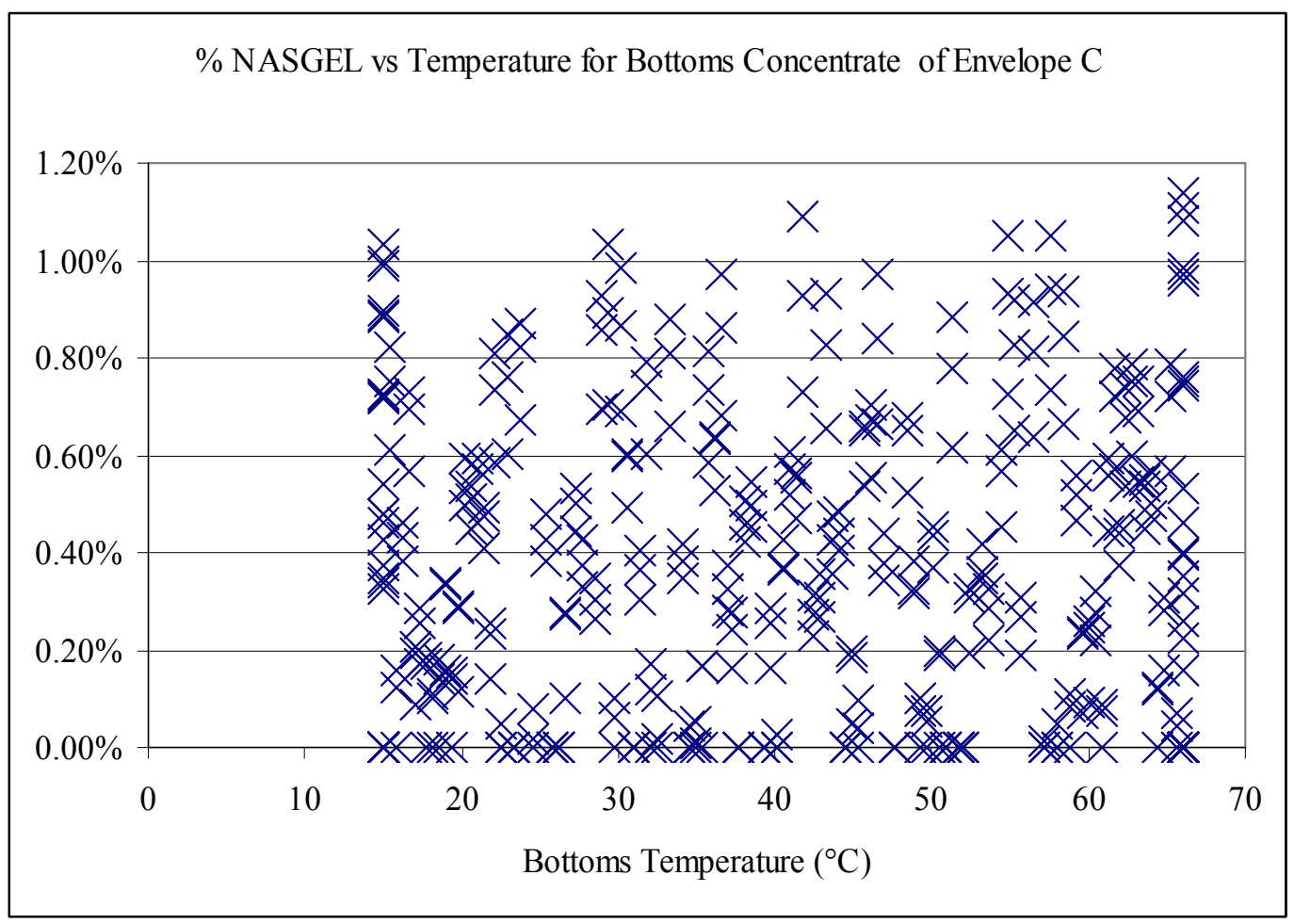

Figure 9. \% NASGEL versus Na M for Bottoms Temperature of Envelope C

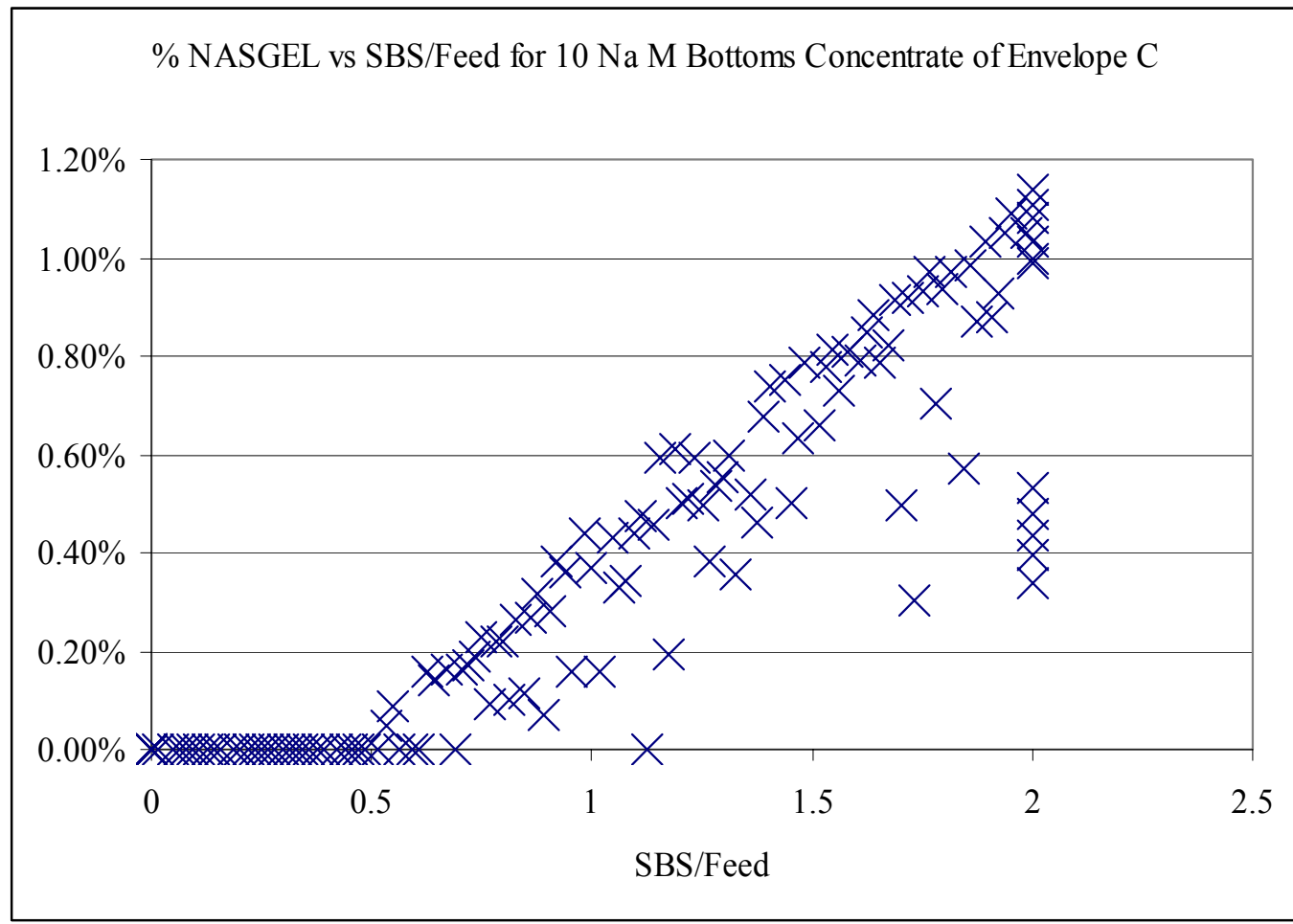

Figure 10. \% NASGEL versus SBS/Feed for 10 Na M Bottoms Concentrate of Envelope C 
WSRC-TR-2003-00269, REVISION 0

SRT-RPP-2003-00125, REVISION 0

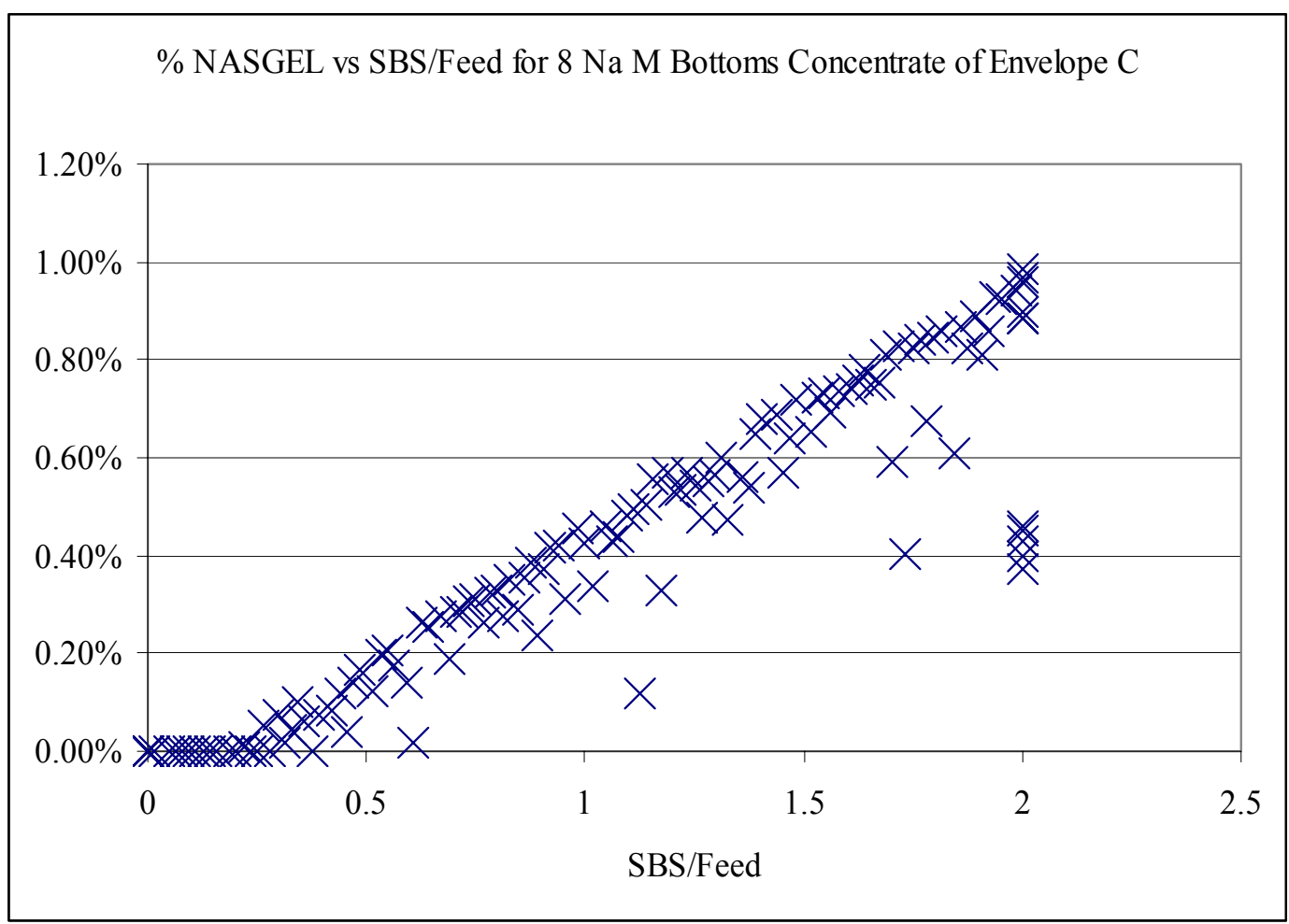

Figure 11. \% NASGEL versus SBS/Feed for 8 Na M Bottoms Concentrate of Envelope C

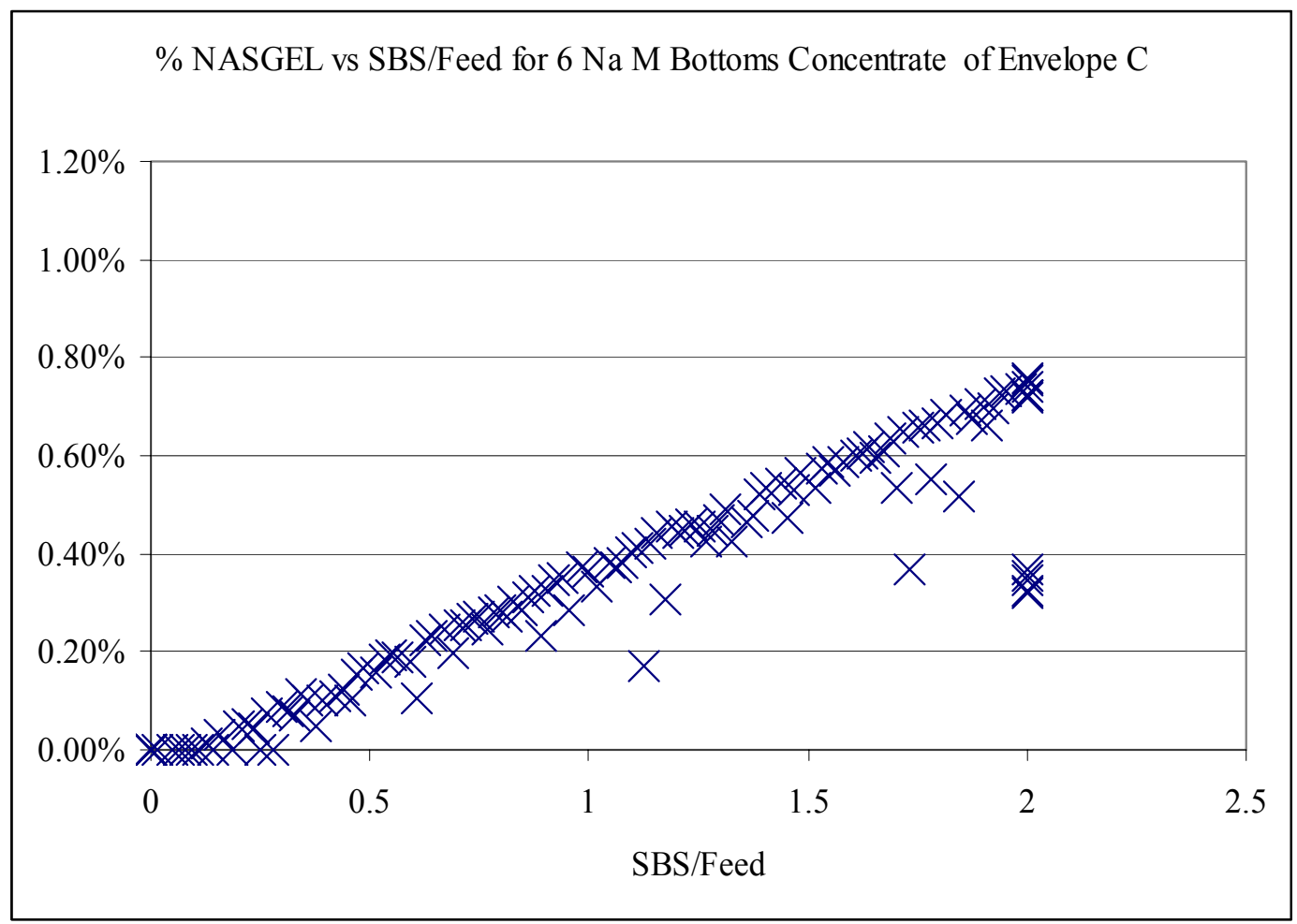

Figure 12. \% NASGEL versus SBS/Feed for 6 Na M Bottoms Concentrate of Envelope C 


\subsection{QUALITY REQUIREMENTS}

This work was conducted in accordance with the RPP-WTP QA requirements specified for work conducted by SRTC as identified in DOE IWO MOSRLE60. SRTC has provided matrices to WTP demonstrating compliance of the SRTC QA program with the requirements specified by WTP. Specific information regarding the compliance of the SRTC QA program with RW-0333P, Revision 10, NQA-1 1989, Part 1, Basic and Supplementary Requirements and NQA-2a 1990, Subpart 2.7 is contained in these matrices. The quality assurance plan is documented in the Task Technical and Quality Assurance Plan for Modeling Treated LAW Feed Evaporation and Validation of Evaporation Pilot Testing. ${ }^{16}$

The quality requirements pertaining to OLI/ESP simulation software have been addressed in the document "Software Quality Assurance Plan for Hanford RPP-WTP Evaporator Modeling"17. OLI/ESP version 6.6 was used with the private databases CARBONAT, HNO3DB, SILICA, and ZEOLITE. JMP version 5.0.1 was used to fit the simulation physical property data to the mathematical forms.

\subsection{ISSUES}

None 
WSRC-TR-2003-00269, REVISION 0

SRT-RPP-2003-00125, REVISION 0

\subsection{DISCUSSION}

\subsection{BACKGROUND}

This report describes the treated feed evaporator (TFE) modeling work as requested in Treated LAW Feed: Simulant Evaporation and Physical Properties Modeling test specification from $\mathrm{RPP}^{5}$ and specified in the Task Technical and Quality Assurance Plan for Modeling Treated LAW Feed Evaporation and Validation of Evaporation Pilot Testing ${ }^{16}$ for the R\&T Test Scoping Statement S-93A in support of the Hanford River Protection Project (RPP) Waste Treatment Plant (WTP) project.

Previous Hanford-RPP evaporator modeling has focused on the treated LAW feed and eluate evaporation systems without the inclusion of secondary waste recycles. ${ }^{1-4}$ This task investigates the potential impact that secondary-waste recycle streams may have on the operation of the treated LAW evaporator. The treated LAW evaporator will concentrate the treated waste effluent streams from the Cs ion exchange blended with the LAW melter offgas scrubbing recycle stream. The Tc ion exchange system that was originally part of the test specification has since been eliminated. The LAW melter offgas scrubbing recycle stream is by far the major contributor to the overall recycle volume that is to be mixed with the treated waste feed prior to evaporation and known to contain silica from the LAW melter. Based on experience at Savannah River Site, the introduction of silica from melter offgas recycle into high sodium feeds can produce sodium-aluminum-silicate precipitates upon concentration. These sodium-aluminum-silicates can cause operational shutdowns due to plugging of lines and fouling of heat transfer surfaces. This task will examine the potential of the treated waste feed blends to form sodium-aluminum-silicate precipitates when evaporated using the zeolite database.

To investigate the behavior of the blended pretreated waste feeds, an OLI ESP model of the treated LAW evaporator was built. The model was to simulate the treated LAW evaporator operating at $50^{\circ} \mathrm{C}$ under the appropriate vacuum with Envelope A, B, and $\mathrm{C}$ wastes blended with LAW melter offgas scrubbing recycle (SBS) as feed and the evaporator concentrate or bottoms stream being varied between $15^{\circ} \mathrm{C}$ and $66^{\circ} \mathrm{C}$. The treated LAW evaporator model was run at 6,8 , and 10 endpoint $\mathrm{Na}$ molarities for the bottoms concentrate stream to generate data about the density, heat capacity, thermal conductivity, viscosity, and solubility of this stream. This run data was then regressed to generate predictive equations describing the physical properties of the Treated LAW evaporator bottoms concentrate. Additional runs with treated LAW evaporator were performed to compare chemical and physical property model predictions and experimental results for small scale radioactive tests (S-69) of the treated feed (AW 101) evaporation processes. 
Equations for the sodium molarity, density, heat capacity, thermal conductivity, and viscosity of the Treated LAW evaporator bottoms concentrate were developed. These equations were successful based on the goal of developing physical property correlations for each waste envelope with an error no greater than $\pm 15 \%$ between calculated and modeled physical properties. The equation to predict solubility or the amount of total solids in the Treated LAW evaporator bottoms concentrate could not be developed to satisfy this goal. Not enough information was captured by the chosen model inputs to accurately describe the bottom concentrate solids content. However, general trends were identified with respect to the bottoms $\mathrm{Na}$ molarity and temperature, which will be discussed later.

\subsection{DESCRIPTION OF THE PROCESS}

The Waste Treatment Plant process is described in detail in the document "WTP Material Balance and Process Flowsheet Bases, Requirements, and Results"18. To summarize, evaporation is used throughout the Waste Treatment Plant pretreatment process to minimize the volume of waste that must vitrified on the back side of the process. Evaporation is used throughout the process to help reduce the volume of waste, as in the first unit process in pretreatment (Waste Feed Evaporators) which has been modeled in earlier work. ${ }^{9}$ Evaporation is also employed before the LAW vitrification (Treated Feed Evaporator) step. The treated LAW evaporator will concentrate the treated waste effluent stream from the Cs ion exchange blended with the LAW melter offgas scrubbing recycle stream. The goal of the Treated Feed Evaporation process is removal of the as much water as possible while maintaining a reasonably low insoluble solids content.

A major goal of this modeling task was to validate the experimental work on the treated feed evaporator. ${ }^{7}$ To that end, the waste feeds for envelopes A, B, and $\mathrm{C}$ were taken from this prior report. ${ }^{7}$ To simulate the pretreatment of these waste feeds, they were first diluted to a density of $1.22 \mathrm{~g} / \mathrm{ml}$ for the aqueous phase. Additionally, as in the experimental work, the waste feeds were filtered to simulate the pretreatment process. This filtering action was duplicated in the modeling. The OLI modeling is described next.

\subsection{OLI/ESP MODEL FLOWSHEET AND CHEMISTRY MODEL}

The treated feed evaporation process was simulated using the OLI Environmental Simulation Program (OLI/ESP) version 6.6 using the CARBONAT, HNO3DB, SILICA, URANIUM, and ZEOLITE private databases, along with the public database. OLI ESP only performs steady state calculations.

The treated feed evaporator was modeled as a flash calculation. ${ }^{15}$ To simulate the pretreatment of LAW waste feeds, all waste feeds were first diluted to reach the UF permeate target density of $1.22 \mathrm{~g} / \mathrm{ml}$, which corresponds to a sodium molarity of about 5. The waste feeds were then filtered to approximate the Cs effluent resulting from the pre-treatment steps. The SBS feed rate was controlled to match a specified volume ratio between 0 and 2. A range of SBS/Feed ratios were chosen to accommodate the expected variations in flow ratio during actual plant operations. 
The supernate from the pretreatment waste feeds along with the ratioed SBS stream were then evaporated at $50^{\circ} \mathrm{C}$ at the bubble point pressure. The evaporator bottoms stream was then cooled at $1 \mathrm{~atm}$ to a temperature in the desired range of 15 to $66^{\circ} \mathrm{C}$. The $\mathrm{Na} \mathrm{M}$ of this cooled evaporator bottoms (product) stream was then checked against desired values of 6,8 , or 10 . If the desired $\mathrm{Na} \mathrm{M}$ was not reached, the evaporator molar vapor fraction was adjusted until the desired setpoint was achieved.

The waste feed flow rates used in the OLI simulations were based on a LAW glass production rate of 30 metric tons per day (two melters) at a $\mathrm{Na}_{2} \mathrm{O}$ loading of $19.5,5.0$, and $11.2 \mathrm{wt} \%$ for Envelope A, B, and C respectively (from Table A-15 of the TF COUP document ${ }^{19}$ and Min Prod Rate from Flowsheet Bases Assumptions and Requirements 24590-WTP-RPT-PT-02005), where only the sodium content of the waste stream was used to calculate the $\mathrm{Na}_{2} \mathrm{O}$ loading. This production rate was chosen as a matter of convenience and any mass flow rate could have been used.

The LAW SBS flow rate is not tied directly to the treated feed evaporator process but to the downstream LAW vitrification off gas treatment. Based on run data from Duratek SBS Run Data from VSL-02R8800-2 Rev 0 and input from the customer, the SBS flow rate was treated as an independent variable for the physical property models developed here. It is expressed in terms of its volumetric flow relative to that of the treated waste feed stream, having a range of 0 -2 (i.e., up to twice the volumetric flow rate of the treated waste feed stream). The composition was based on the analytical results from the VSL melter pilot testing, Mark Crowder's AW-101 testing ${ }^{8}$ and from prior experimental work. ${ }^{7}$

OLI/ESP does not have the ability to calculate the heat capacity of a stream directly, so this was accomplished for the evaporator bottoms concentrate stream using the OLI Scratch Pad tool in OLI/Express. The scratch pad tool was used to generate an enthalpy vs. temperature plot at each calculated steady-state composition for the temperature range $15-66^{\circ} \mathrm{C}$ in one-degree increments.

The chemistry models used for all the simulations are shown in Table 2. 
WSRC-TR-2003-00269, REVISION 0

SRT-RPP-2003-00125, REVISION 0

Table 2. OLI Chemistry Models

\begin{tabular}{|c|c|c|c|c|c|c|}
\hline $\begin{array}{c}\text { Species } \\
\text { (OLI Name) }\end{array}$ & $\begin{array}{c}\text { Species } \\
\text { (Common name) }\end{array}$ & $\begin{array}{l}\text { Species } \\
\text { Formula }\end{array}$ & $\begin{array}{l}\text { AW101 } \\
\text { Chemistry } \\
\text { model }\end{array}$ & $\begin{array}{c}\text { Envelope A } \\
\text { Chemistry } \\
\text { Model }\end{array}$ & $\begin{array}{c}\text { Envelope B } \\
\text { Chemistry } \\
\text { Model }\end{array}$ & $\begin{array}{c}\text { Envelope C } \\
\text { Chemistry } \\
\text { Model }\end{array}$ \\
\hline AGOH & Silver Hydroxide & $\mathrm{AgOH}$ & $\mathrm{X}$ & $\mathrm{n} / \mathrm{a}$ & $\mathrm{n} / \mathrm{a}$ & $\mathrm{n} / \mathrm{a}$ \\
\hline AL2O3 & Aluminum Oxide & $\mathrm{Al} 2 \mathrm{O} 3$ & $\mathrm{X}$ & $\mathrm{n} / \mathrm{a}$ & $\mathrm{n} / \mathrm{a}$ & $\mathrm{n} / \mathrm{a}$ \\
\hline AL2O5H5CL & $\begin{array}{l}\text { Dialuminum } \\
\text { Chloride } \\
\text { Pentahydroxide }\end{array}$ & $\mathrm{Al} 2(\mathrm{OH}) 5 \mathrm{Cl}$ & $\mathrm{n} / \mathrm{a}$ & $\mathrm{X}$ & $\mathrm{n} / \mathrm{a}$ & $\mathrm{n} / \mathrm{a}$ \\
\hline $\mathrm{ALOOH}$ & $\begin{array}{c}\text { Aluminum Oxide } \\
\text { Hydroxide }\end{array}$ & $\mathrm{AlO}(\mathrm{OH})$ & $\mathrm{n} / \mathrm{a}$ & $\mathrm{X}$ & $\mathrm{n} / \mathrm{a}$ & $\mathrm{n} / \mathrm{a}$ \\
\hline ALBR3 & Aluminum Bromide & $\mathrm{AlBr} 3$ & $\mathrm{X}$ & $\mathrm{n} / \mathrm{a}$ & $\mathrm{n} / \mathrm{a}$ & $\mathrm{n} / \mathrm{a}$ \\
\hline $\mathrm{BOH} 3$ & Boric Acid & $\mathrm{B}(\mathrm{OH}) 3$ & & $\mathrm{X}$ & $\mathrm{n} / \mathrm{a}$ & $\mathrm{n} / \mathrm{a}$ \\
\hline $\mathrm{BAOH} 2$ & Barium Hydroxide & $\mathrm{Ba}(\mathrm{OH}) 2$ & $\mathrm{X}$ & $\mathrm{n} / \mathrm{a}$ & $\mathrm{n} / \mathrm{a}$ & $X$ \\
\hline CAF2 & Calcium Fluoride & $\mathrm{CaF} 2$ & $\mathrm{X}$ & $\mathrm{n} / \mathrm{a}$ & $\mathrm{n} / \mathrm{a}$ & $\mathrm{n} / \mathrm{a}$ \\
\hline $\mathrm{CAOH} 2$ & Calcium Hydroxide & $\mathrm{Ca}(\mathrm{OH}) 2$ & $\mathrm{X}$ & $\mathrm{X}$ & $\mathrm{X}$ & $\mathrm{X}$ \\
\hline $\mathrm{CDOH} 2$ & $\begin{array}{l}\text { Cadmium } \\
\text { Hydroxide }\end{array}$ & $\mathrm{Cd}(\mathrm{OH}) 2$ & $\mathrm{X}$ & $\mathrm{n} / \mathrm{a}$ & $\mathrm{n} / \mathrm{a}$ & $\mathrm{n} / \mathrm{a}$ \\
\hline CEOH3 & $\begin{array}{l}\text { Cerium (III) } \\
\text { Hydroxide }\end{array}$ & $\mathrm{Ce}(\mathrm{OH}) 3$ & $\mathrm{X}$ & $\mathrm{n} / \mathrm{a}$ & $\mathrm{n} / \mathrm{a}$ & $\mathrm{n} / \mathrm{a}$ \\
\hline CITRAC & Citric Acid & $\mathrm{C} 6 \mathrm{H} 8 \mathrm{O} 7$ & $\mathrm{n} / \mathrm{a}$ & $\mathrm{n} / \mathrm{a}$ & $\mathrm{X}$ & $\mathrm{X}$ \\
\hline $\mathrm{CO} 2$ & Carbon Dioxide & $\mathrm{CO} 2$ & $\mathrm{n} / \mathrm{a}$ & $\mathrm{X}$ & - & - \\
\hline CROH3 & $\begin{array}{l}\text { Chromium (III) } \\
\text { Hydroxide }\end{array}$ & $\mathrm{Cr}(\mathrm{OH}) 3$ & $\mathrm{X}$ & $\mathrm{X}$ & $\mathrm{X}$ & $\mathrm{X}$ \\
\hline $\mathrm{CSOH}$ & Cesium Hydroxide & $\mathrm{CsOH}$ & $\mathrm{X}$ & $\mathrm{n} / \mathrm{a}$ & $\mathrm{n} / \mathrm{a}$ & $\mathrm{n} / \mathrm{a}$ \\
\hline CUOH2 & $\begin{array}{l}\text { Copper (II) } \\
\text { Hydroxide }\end{array}$ & $\mathrm{Cu}(\mathrm{OH}) 2$ & $\mathrm{X}$ & $\mathrm{n} / \mathrm{a}$ & $\mathrm{X}$ & $\mathrm{X}$ \\
\hline \begin{tabular}{l|l} 
FEIIIOH3 & 1 \\
\end{tabular} & Iron (III) Hydroxide & $\mathrm{Fe}(\mathrm{OH}) 3$ & $\mathrm{X}$ & $\mathrm{X}$ & $\mathrm{X}$ & $\mathrm{X}$ \\
\hline FETIO3 & Iron (II) Titanate & FeTiO3 & $\mathrm{X}$ & $\mathrm{n} / \mathrm{a}$ & $\mathrm{n} / \mathrm{a}$ & $\mathrm{n} / \mathrm{a}$ \\
\hline FORM2 & $\begin{array}{l}\text { Formic Acid } \\
\text { (dimer) }\end{array}$ & $\mathrm{C} 2 \mathrm{H} 4 \mathrm{O} 4$ & $\mathrm{X}$ & $\mathrm{n} / \mathrm{a}$ & $\mathrm{n} / \mathrm{a}$ & $\mathrm{X}$ \\
\hline GDOH3 & $\begin{array}{l}\text { Gadolinium (III) } \\
\text { Hydroxide }\end{array}$ & $\mathrm{Gd}(\mathrm{OH}) 3$ & $\mathrm{X}$ & $\mathrm{n} / \mathrm{a}$ & $\mathrm{n} / \mathrm{a}$ & $\mathrm{n} / \mathrm{a}$ \\
\hline $\mathrm{H} 2 \mathrm{CO} 3$ & Carbonic Acid & $\mathrm{H} 2 \mathrm{CO} 3$ & $\mathrm{X}$ & $\mathrm{n} / \mathrm{a}$ & $\mathrm{n} / \mathrm{a}$ & $\mathrm{n} / \mathrm{a}$ \\
\hline $\mathrm{H} 2 \mathrm{CRO} 4$ & Chromic (VI) Acid & $\mathrm{H} 2 \mathrm{CrO} 4$ & $\mathrm{X}$ & $\mathrm{n} / \mathrm{a}$ & $\mathrm{n} / \mathrm{a}$ & $\mathrm{n} / \mathrm{a}$ \\
\hline $\mathrm{H} 2 \mathrm{MOO} 4$ & $\begin{array}{c}\text { Molybdenic (VI) } \\
\text { Acid }\end{array}$ & $\mathrm{H} 2 \mathrm{MoO} 4$ & $\mathrm{X}$ & $\mathrm{n} / \mathrm{a}$ & $X$ & $\mathrm{X}$ \\
\hline $\mathrm{H} 2 \mathrm{O}$ & Water & $\mathrm{H} 2 \mathrm{O}$ & $\mathrm{X}$ & $\mathrm{X}$ & $\mathrm{X}$ & $\mathrm{X}$ \\
\hline $\mathrm{H} 2 \mathrm{SO} 4$ & Sulfuric Acid & $\mathrm{H} 2 \mathrm{SO} 4$ & $\mathrm{n} / \mathrm{a}$ & $\mathrm{X}$ & $\mathrm{n} / \mathrm{a}$ & $\mathrm{n} / \mathrm{a}$ \\
\hline $\mathrm{H} 3 \mathrm{PO} 4$ & Phosphoric Acid & H3PO4 & $\mathrm{X}$ & $\mathrm{X}$ & $\mathrm{n} / \mathrm{a}$ & $\mathrm{n} / \mathrm{a}$ \\
\hline $\mathrm{H} 4 \mathrm{SIO} 4$ & Silicic Acid & $\mathrm{H} 4 \mathrm{SiO} 4$ & $\mathrm{X}$ & $\mathrm{X}$ & $\mathrm{X}$ & $\mathrm{X}$ \\
\hline
\end{tabular}

$\mathrm{n} / \mathrm{a}=$ not applicable 
WSRC-TR-2003-00269, REVISION 0

SRT-RPP-2003-00125, REVISION 0

Table 2. OLI Chemistry Models (cont'd)

\begin{tabular}{|c|c|c|c|c|c|c|}
\hline $\begin{array}{c}\text { Species } \\
\text { (OLI Name) }\end{array}$ & $\begin{array}{c}\text { Species } \\
\text { (Common name) }\end{array}$ & $\begin{array}{l}\text { Species } \\
\text { Formula }\end{array}$ & $\begin{array}{c}\text { AW101 } \\
\text { Chemistry } \\
\text { model }\end{array}$ & $\begin{array}{l}\text { Envelope A } \\
\text { Chemistry } \\
\text { Model }\end{array}$ & $\begin{array}{l}\text { Envelope B } \\
\text { Chemistry } \\
\text { Model }\end{array}$ & $\begin{array}{c}\text { Envelope C } \\
\text { Chemistry } \\
\text { Model }\end{array}$ \\
\hline H6F6 & $\begin{array}{c}\text { Hydrogen Fluoride } \\
\text { (Hexamer) }\end{array}$ & (HF)6 & $\mathrm{X}$ & $\mathrm{X}$ & $\mathrm{X}$ & $\mathrm{X}$ \\
\hline HCL & Hydrogen Chloride & $\mathrm{HCl}$ & $\mathrm{X}$ & $\mathrm{X}$ & $\mathrm{X}$ & $\mathrm{X}$ \\
\hline $\mathrm{HF}$ & Hydrofluoric Acid & $\mathrm{HF}$ & $\mathrm{n} / \mathrm{a}$ & $\mathrm{X}$ & $\mathrm{n} / \mathrm{a}$ & $\mathrm{n} / \mathrm{a}$ \\
\hline HNO2 & Nitrous (III) Acid & HNO2 & $\mathrm{X}$ & $\mathrm{X}$ & $\mathrm{X}$ & $\mathrm{X}$ \\
\hline HNO3 & Nitric Acid & HNO3 & $\mathrm{X}$ & $X$ & $\mathrm{X}$ & $\mathrm{X}$ \\
\hline K1ALSO4 & $\begin{array}{c}\text { Potassium } \\
\text { Aluminum Sulfate }\end{array}$ & $\mathrm{KAl}(\mathrm{SO} 4) 2$ & $\mathrm{X}$ & $\mathrm{n} / \mathrm{a}$ & $\mathrm{n} / \mathrm{a}$ & $\mathrm{n} / \mathrm{a}$ \\
\hline $\mathrm{K} 2 \mathrm{CRO} 4$ & $\begin{array}{c}\text { Potassium } \\
\text { Chromate (VI) }\end{array}$ & $\mathrm{K} 2 \mathrm{CrO} 4$ & $\mathrm{X}$ & $\mathrm{n} / \mathrm{a}$ & $\mathrm{X}$ & $\mathrm{X}$ \\
\hline KMGCL3 & $\begin{array}{l}\text { Potassium } \\
\text { Magnesium } \\
\text { Chloride } \\
\end{array}$ & $\mathrm{KMgCl} 3$ & $\mathrm{X}$ & $\mathrm{X}$ & $\mathrm{X}$ & $\mathrm{X}$ \\
\hline $\mathrm{KOH}$ & $\begin{array}{l}\text { Potassium } \\
\text { Hydroxide }\end{array}$ & $\mathrm{KOH}$ & $\mathrm{X}$ & $\mathrm{X}$ & $\mathrm{X}$ & $\mathrm{X}$ \\
\hline LAOH3 & $\begin{array}{l}\text { Lanthanum (III) } \\
\text { Hydroxide }\end{array}$ & $\mathrm{La}(\mathrm{OH}) 3$ & $\mathrm{X}$ & $\mathrm{n} / \mathrm{a}$ & $\mathrm{n} / \mathrm{a}$ & $\mathrm{n} / \mathrm{a}$ \\
\hline $\mathrm{LIOH}$ & Lithium Hydroxide & $\mathrm{LiOH}$ & $X$ & $X$ & $\mathrm{X}$ & $\mathrm{X}$ \\
\hline $\mathrm{MGOH} 2$ & $\begin{array}{l}\text { Magnesium } \\
\text { Hydroxide }\end{array}$ & $\mathrm{Mg}(\mathrm{OH}) 2$ & $\mathrm{X}$ & $\mathrm{n} / \mathrm{a}$ & $\mathrm{n} / \mathrm{a}$ & $\mathrm{n} / \mathrm{a}$ \\
\hline $\mathrm{MNOH} 2$ & $\begin{array}{l}\text { Manganese (II) } \\
\text { Hydroxide }\end{array}$ & $\mathrm{Mn}(\mathrm{OH}) 2$ & $\mathrm{X}$ & $\mathrm{n} / \mathrm{a}$ & $\mathrm{X}$ & $\mathrm{X}$ \\
\hline $\mathrm{NA} 2 \mathrm{C} 2 \mathrm{O} 4$ & Sodium Oxalate & $\mathrm{Na} 2 \mathrm{C} 2 \mathrm{O} 4$ & $X$ & $\mathrm{X}$ & $\mathrm{X}$ & $\mathrm{X}$ \\
\hline $\mathrm{NA} 2 \mathrm{CO} 3$ & Sodium Carbonate & $\mathrm{Na} 2 \mathrm{CO} 3$ & $X$ & $X$ & $X$ & $X$ \\
\hline NA2MOO4 & $\begin{array}{c}\text { Sodium Molybdate } \\
\text { (VI) }\end{array}$ & $\mathrm{Na} 2 \mathrm{MoO} 4$ & $\mathrm{X}$ & $\mathrm{n} / \mathrm{a}$ & $\mathrm{n} / \mathrm{a}$ & $\mathrm{n} / \mathrm{a}$ \\
\hline NA2SIO3 & Sodium Metasilicate & $\mathrm{Na} 2 \mathrm{SiO} 3$ & $\mathrm{X}$ & $\mathrm{X}$ & $\mathrm{n} / \mathrm{a}$ & $\mathrm{n} / \mathrm{a}$ \\
\hline NA2SO4 & Sodium Sulfate & $\mathrm{Na} 2 \mathrm{SO} 4$ & $\mathrm{X}$ & $X$ & $X$ & $X$ \\
\hline $\mathrm{NA} 2 \mathrm{U} 2 \mathrm{O} 7$ & $\begin{array}{c}\text { Sodium Pyrouranate } \\
(\mathrm{VI})\end{array}$ & $\mathrm{Na} 2 \mathrm{U} 2 \mathrm{O} 7$ & $\mathrm{n} / \mathrm{a}$ & $\mathrm{X}$ & $\mathrm{n} / \mathrm{a}$ & $\mathrm{n} / \mathrm{a}$ \\
\hline NA3HSO42 & $\begin{array}{l}\text { Sodium Sulfate } \\
\text { Bisulfate }\end{array}$ & $\begin{array}{l}\mathrm{Na} 2 \mathrm{SO} 4 . \mathrm{Na} \\
\mathrm{HSO} 4\end{array}$ & $\mathrm{X}$ & $\mathrm{X}$ & $\mathrm{X}$ & $\mathrm{X}$ \\
\hline NA3PO4 & Sodium Phosphate & $\mathrm{Na} 3 \mathrm{PO} 4$ & $\mathrm{X}$ & $\mathrm{X}$ & $\mathrm{X}$ & $\mathrm{X}$ \\
\hline NAALO2 & Sodium Aluminate & $\mathrm{NaAlO} 2$ & $X$ & $X$ & $\mathrm{X}$ & $X$ \\
\hline NAALOH4 & $\begin{array}{c}\text { Sodium Aluminum } \\
\text { Hydroxide }\end{array}$ & $\mathrm{NaAl}(\mathrm{OH}) 4$ & $\mathrm{X}$ & $\mathrm{X}$ & $\mathrm{X}$ & $\mathrm{X}$ \\
\hline NABO2 & Sodium Metaborate & $\mathrm{NaBO} 2$ & $\mathrm{X}$ & $\mathrm{n} / \mathrm{a}$ & $\mathrm{n} / \mathrm{a}$ & $\mathrm{n} / \mathrm{a}$ \\
\hline NABOH4 & $\begin{array}{l}\text { Sodium Boron } \\
\text { Hydroxide }\end{array}$ & $\mathrm{NaB}(\mathrm{OH}) 4$ & $\mathrm{X}$ & $\mathrm{X}$ & $\mathrm{X}$ & $\mathrm{X}$ \\
\hline NACL & Sodium Chloride & $\mathrm{NaCl}$ & $X$ & $\mathrm{X}$ & $\mathrm{n} / \mathrm{a}$ & $\mathrm{X}$ \\
\hline
\end{tabular}

$\mathrm{n} / \mathrm{a}=$ not applicable 
WSRC-TR-2003-00269, REVISION 0

SRT-RPP-2003-00125, REVISION 0

Table 2. OLI Chemistry Models (cont'd)

\begin{tabular}{|c|c|c|c|c|c|c|}
\hline $\begin{array}{c}\text { Species } \\
\text { (OLI Name) }\end{array}$ & $\begin{array}{c}\text { Species } \\
\text { (Common name) }\end{array}$ & $\begin{array}{l}\text { Species } \\
\text { Formula }\end{array}$ & $\begin{array}{l}\text { AW101 } \\
\text { Chemistry } \\
\text { model }\end{array}$ & $\begin{array}{l}\text { Envelope A } \\
\text { Chemistry } \\
\text { Model }\end{array}$ & $\begin{array}{l}\text { Envelope B } \\
\text { Chemistry } \\
\text { Model }\end{array}$ & $\begin{array}{c}\text { Envelope C } \\
\text { Chemistry } \\
\text { Model }\end{array}$ \\
\hline $\mathrm{NACOOH}$ & Sodium Formate & $\mathrm{NaCOOH}$ & $\mathrm{X}$ & & $\mathrm{X}$ & $\mathrm{X}$ \\
\hline NAF & Sodium Fluoride & $\mathrm{NaF}$ & $\mathrm{X}$ & $\mathrm{X}$ & $\mathrm{X}$ & $\mathrm{X}$ \\
\hline $\begin{array}{c}\text { NAGLYCOL } \\
\text { AT }\end{array}$ & Sodium Glycolate & $\mathrm{Na}[\mathrm{C} 2 \mathrm{H} 3 \mathrm{O} 3]$ & $\mathrm{n} / \mathrm{a}$ & $\mathrm{n} / \mathrm{a}$ & $\mathrm{X}$ & $\mathrm{X}$ \\
\hline NANO2 & Sodium Nitrite & $\mathrm{NaNO} 2$ & $\mathrm{X}$ & $\mathrm{X}$ & $\mathrm{X}$ & $\mathrm{X}$ \\
\hline NANO3 & Sodium Nitrate & NaNO3 & $\mathrm{X}$ & $\mathrm{X}$ & $\mathrm{X}$ & $\mathrm{X}$ \\
\hline $\mathrm{NAOH}$ & Sodium Hydroxide & $\mathrm{NaOH}$ & $\mathrm{X}$ & $\mathrm{X}$ & $\mathrm{X}$ & $X$ \\
\hline $\begin{array}{c}\text { NAPHOH.12 } \\
\text { H2O }\end{array}$ & $\begin{array}{l}\text { Sodium } \\
\text { Orthophosphate } \\
\text { Hydroxide } \\
\text { Dodecahydrate }\end{array}$ & \begin{tabular}{|c|}
$\mathrm{Na} 3 \mathrm{PO} 4 . \mathrm{O} .2$ \\
$5 \mathrm{NaOH} .12 \mathrm{H}$ \\
$2 \mathrm{O}$
\end{tabular} & $\mathrm{n} / \mathrm{a}$ & $\mathrm{X}$ & $\mathrm{n} / \mathrm{a}$ & $\mathrm{n} / \mathrm{a}$ \\
\hline NATCVIIO4 & \begin{tabular}{|c|} 
Sodium \\
Pertechnetate (VII) \\
\end{tabular} & $\mathrm{NaTcO} 4$ & $\mathrm{X}$ & $\mathrm{n} / \mathrm{a}$ & $\mathrm{n} / \mathrm{a}$ & $\mathrm{n} / \mathrm{a}$ \\
\hline $\mathrm{NDOH} 3$ & $\begin{array}{c}\text { Neodymium (III) } \\
\text { Hydroxide }\end{array}$ & $\mathrm{Nd}(\mathrm{OH}) 3$ & $\mathrm{X}$ & $\mathrm{n} / \mathrm{a}$ & $\mathrm{n} / \mathrm{a}$ & $\mathrm{n} / \mathrm{a}$ \\
\hline NIOH2 & $\begin{array}{l}\text { Nickel (II) } \\
\text { Hydroxide }\end{array}$ & $\mathrm{Ni}(\mathrm{OH}) 2$ & $\mathrm{X}$ & $\mathrm{n} / \mathrm{a}$ & $\mathrm{X}$ & $\mathrm{X}$ \\
\hline OXALAC & Oxalic Acid & $\mathrm{C} 2 \mathrm{H} 2 \mathrm{O} 4$ & $\mathrm{X}$ & $\mathrm{n} / \mathrm{a}$ & $\mathrm{X}$ & $\mathrm{X}$ \\
\hline $\mathrm{PBOH} 2$ & Lead (II) Hydroxide & $\mathrm{Pb}(\mathrm{OH}) 2$ & $\mathrm{X}$ & $\mathrm{n} / \mathrm{a}$ & $\mathrm{n} / \mathrm{a}$ & $\mathrm{n} / \mathrm{a}$ \\
\hline $\mathrm{P} 2 \mathrm{O} 5$ & $\begin{array}{l}\text { Phosphorus } \\
\text { Pentoxide }\end{array}$ & $\mathrm{P} 2 \mathrm{O} 5$ & $\mathrm{n} / \mathrm{a}$ & $\mathrm{X}$ & $\mathrm{n} / \mathrm{a}$ & $\mathrm{n} / \mathrm{a}$ \\
\hline SBOH3 & $\begin{array}{l}\text { Antimony (III) } \\
\text { Hydroxide }\end{array}$ & $\mathrm{Sb}(\mathrm{OH}) 3$ & $\mathrm{X}$ & $\mathrm{n} / \mathrm{a}$ & $\mathrm{n} / \mathrm{a}$ & $\mathrm{n} / \mathrm{a}$ \\
\hline $\mathrm{SIO} 2$ & Silicon Dioxide & $\mathrm{SiO} 2$ & $\mathrm{X}$ & $\mathrm{X}$ & $\mathrm{n} / \mathrm{a}$ & $\mathrm{n} / \mathrm{a}$ \\
\hline SNIVOH4 & Tin (IV) Hydroxide & $\mathrm{Sn}(\mathrm{OH}) 4$ & $X$ & - & $\mathrm{n} / \mathrm{a}$ & $\mathrm{n} / \mathrm{a}$ \\
\hline $\mathrm{SO} 3$ & Sulfur Trioxide & $\mathrm{SO} 3$ & & $\mathrm{X}$ & $\mathrm{n} / \mathrm{a}$ & $\mathrm{n} / \mathrm{a}$ \\
\hline $\mathrm{SROH} 2$ & $\begin{array}{l}\text { Strontium } \\
\text { Hydroxide }\end{array}$ & $\mathrm{Sr}(\mathrm{OH}) 2$ & $\mathrm{X}$ & $\mathrm{n} / \mathrm{a}$ & $\mathrm{n} / \mathrm{a}$ & $\mathrm{X}$ \\
\hline TCIVOOH22 & $\begin{array}{c}\text { Oxytechnetium (IV) } \\
\begin{array}{c}\text { Dihydroxide } \\
\text { (dimer) }\end{array} \\
\end{array}$ & $\begin{array}{c}(\mathrm{TcO}(\mathrm{OH}) 2) \\
2\end{array}$ & $\mathrm{X}$ & $\mathrm{n} / \mathrm{a}$ & $\mathrm{n} / \mathrm{a}$ & $\mathrm{n} / \mathrm{a}$ \\
\hline TIIVOH4 & $\begin{array}{l}\text { Titanium (IV) } \\
\text { Hydroxide }\end{array}$ & $\mathrm{Ti}(\mathrm{OH}) 4$ & $\mathrm{n} / \mathrm{a}$ & $\mathrm{X}$ & $\mathrm{X}$ & $\mathrm{X}$ \\
\hline UIVOH4 & $\begin{array}{l}\text { Uranium (IV) } \\
\text { Hydroxide }\end{array}$ & $\mathrm{U}(\mathrm{OH}) 4$ & $\mathrm{X}$ & $\mathrm{n} / \mathrm{a}$ & $\mathrm{n} / \mathrm{a}$ & $\mathrm{n} / \mathrm{a}$ \\
\hline VOOH3 & $\begin{array}{l}\text { Vanadate }(\mathrm{V}) \\
\text { Hydroxide }\end{array}$ & $\mathrm{VO}(\mathrm{OH}) 3$ & $\mathrm{X}$ & $\mathrm{n} / \mathrm{a}$ & $\mathrm{n} / \mathrm{a}$ & $\mathrm{n} / \mathrm{a}$ \\
\hline $\mathrm{ZNOH} 2$ & Zinc Hydroxide & $\mathrm{Zn}(\mathrm{OH}) 2$ & $\mathrm{X}$ & $\mathrm{X}$ & $X$ & $\mathrm{X}$ \\
\hline ZRBR4 & Zirconium Bromide & $\mathrm{ZrBr} 4$ & $\mathrm{X}$ & $\mathrm{n} / \mathrm{a}$ & $\mathrm{n} / \mathrm{a}$ & $\mathrm{n} / \mathrm{a}$ \\
\hline ZROH4 & $\begin{array}{l}\text { Zirconium } \\
\text { Hydroxide }\end{array}$ & $\mathrm{Zr}(\mathrm{OH}) 4$ & $\mathrm{X}$ & $\mathrm{X}$ & $\mathrm{X}$ & $\mathrm{X}$ \\
\hline
\end{tabular}

$\mathrm{n} / \mathrm{a}=$ not applicable 
Sodium aluminosilicate gelatin (NASGEL) is a necessary precursor to the formation of crystalline aluminosilicates (zeolite A, hydroxysodalite, and cancrinite). For this reason, only the precipitation of NASGEL was modeled in these OLI simulations. These solids should not form in the evaporator bottoms due to the relative short residence time of evaporator bottoms compared to the kinetics of crystalline aluminosilicate formation. If OLI predicts NASGEL in the evaporator bottoms, then that may indicate that its other crystalline forms could precipitate later in the process such as in holding tanks.

\subsection{DETERMINATION OF THE FACTOR SPACE FOR THE DESIGNS OF EXPERIMENT}

The simulation design matrices were derived from a model factor space defined by certain model variables and their ranges. The design matrices are given in Appendix A and described briefly below. Their derivation is more fully described in other documents ${ }^{20-22}$.

The design matrices consist of two types of design points - fit points and validation points. The fit points are used for the model fits and consist of the extreme (minimum and maximum) values of each of the variable ranges. This minimum/maximum choice assumes a linear response. The validation points are generated using the Orthogonal Latin Hypercube (OLH) technique $^{23}$, which produces points uniformly distributed over the linear factor space. The validation points are used to validate the property model predictions against the simulation results or they are used to improve the model fit when the response appears to be non-linear. The model variables, their constraints, and the corresponding factor spaces used to derive the design matrices are described next.

The physical property models are expressed the in terms of two variable types, 1) mixture variables, which define the composition of the waste feed stream, and 2) process variables which define the "state" of the process, the evaporator bottoms temperature and Na molarity and the SBS to Feed volume ratio.

For all envelopes, the temperature range used for the bottoms concentrate was $15-66^{\circ} \mathrm{C}$ and the LAW SBS to treated waste feed volume flow ratio was 0 to 2 . The bottoms concentrate $\mathrm{Na}$ molarity was varied between 6 and 10 molarity based on the prior experimental work. ${ }^{7}$

Given the broad range of compositions between Envelopes A, B, and C, physical property models were developed for each envelope. The significant species and concentration ranges for each of the envelopes were based on the characterization done in the parallel experimental work. ${ }^{7}$

Envelope A was based on prior work done for the Waste Feed Evaporator; ${ }^{9}$ Envelope B was represented by the tanks AZ-101 and AZ-102; and Envelope C was represented by the tanks AN-102 and AN-107, as described in the prior experimental work. ${ }^{7}$ 


\section{WSRC-TR-2003-00269, REVISION 0 SRT-RPP-2003-00125, REVISION 0}

The treated waste feed will be concentrated to various endpoint $\mathrm{Na}$ molarities depending on operational and processing constraints. From prior modeling work ${ }^{9}$ it was shown that the waste feed should be expressed in terms of the mass of its non-water species since the amount of water evaporated will change depending on the dilution of the waste feed via pre-treatment and/or the addition of Law SBS recycle. However, the non-water species will always be present and thus have the greatest impact on the properties of the bottoms concentrate.

Using the recipes and tank characterizations from the prior experimental work, ${ }^{7}$ the waste feed compositions for each envelope were analyzed and the significant species which varied the most were chosen to define the waste feed composition factor space. The waste feed composition factor space for Envelope A was defined in earlier modeling work ${ }^{9}$ and is shown in Table 3. The constraints were defined such that $\left[\mathrm{AlO}_{2}\right] /[\mathrm{OH}] \leq 0.7,\left[\mathrm{PO}_{4}\right]+0.07[\mathrm{~F}] \leq 0.05$, and the sum of the molar charge of the mixture variables must be equal to 4.73648 . The concentrations are in terms of a $5 \mathrm{M} \mathrm{Na}$ solution (i.e., a concentration of $0.1 \mathrm{M} \mathrm{OH}$ in a $7 \mathrm{M} \mathrm{Na}$ solution is adjusted to $0.1 \times 5 / 7=0.0714 \mathrm{M} \mathrm{OH}$ ).

Table 3. Definition for Envelope A Factor Space

\begin{tabular}{|c|c|c|c|c|c|c|c|c|c|c|}
\hline \multicolumn{8}{|c|}{$\begin{array}{c}\text { Mixture Variable Molar Ranges } \\
\text { Normalized to equivalent molarity at 5M Na }\end{array}$} & \multicolumn{3}{|c|}{ Process Variables } \\
\hline Variable & $\begin{array}{c}{\left[\mathrm{AlO}_{2}\right]} \\
(\mathrm{molar} \\
\text { at } 5 \mathrm{M} \\
\mathrm{Na}) \\
\end{array}$ & $\begin{array}{c}{\left[\mathrm{CO}_{3}\right]} \\
(\mathrm{molar} \\
\text { at 5M } \\
\mathrm{Na}) \\
\end{array}$ & $\begin{array}{c}{[\mathrm{F}]} \\
\text { (molar at } \\
5 \mathrm{M} \mathrm{Na} \text { ) }\end{array}$ & $\begin{array}{c}{\left[\mathrm{NO}_{2}\right]} \\
(\mathrm{molar} \\
\text { at 5M } \\
\mathrm{Na}) \\
\end{array}$ & $\begin{array}{c}{\left[\mathrm{NO}_{3}\right]} \\
(\mathrm{molar} \\
\text { at 5M } \\
\mathrm{Na}) \\
\end{array}$ & $\begin{array}{c}{[\mathrm{OH}]} \\
\text { (molar } \\
\text { at 5M } \\
\mathrm{Na}) \\
\end{array}$ & $\underset{\text { (molar at }}{\left[\mathrm{PO}_{4}\right]}$ & $\begin{array}{l}{[\mathrm{Na}]} \\
\text { (molar) }\end{array}$ & $\begin{array}{l}\text { SBS / Feed } \\
\text { (volumetric) }\end{array}$ & $\underset{\left({ }^{\circ} \mathrm{C}\right)}{\text { Temp }}$ \\
\hline minimum & 0.207 & 0.326 & 0.00927 & 0.731 & 0.991 & 0.983 & 0.00632 & 6 & 0.0 & 15 \\
\hline maximum & 1.12 & 0.686 & 0.236 & 1.59 & 2.08 & 2.89 & 0.0436 & 10 & 2.0 & 66 \\
\hline constraints & \multicolumn{2}{|c|}{$\left[\mathrm{AlO}_{2}\right] /[\mathrm{OH}] \leq 0.7$} & \multicolumn{2}{|c|}{$\left[\mathrm{PO}_{4}\right]+0.07[\mathrm{~F}] \leq 0.05$} & \multicolumn{6}{|c|}{ charge equivalent: $\left[\mathrm{AlO}_{2}\right]+2\left[\mathrm{CO}_{3}\right]+[\mathrm{F}]+\left[\mathrm{NO}_{3}\right]+\left[\mathrm{NO}_{2}\right]+[\mathrm{OH}]+3\left[\mathrm{PO}_{4}\right]=4.73648$} \\
\hline \multicolumn{11}{|c|}{ Fixed Molar Concentrations } \\
\hline \multicolumn{3}{|c|}{$\left[\mathrm{SO}_{4}\right]($ molar at $5 \mathrm{M} \mathrm{Na})$} & \multicolumn{3}{|c|}{$\left[\mathrm{C}_{2} \mathrm{O}_{4}\right]$ (molar at $\left.5 \mathrm{M} \mathrm{Na}\right)$} & \multicolumn{2}{|c|}{$[\mathrm{Cl}]$ (molar at 5M Na) } & & \multicolumn{2}{|c|}{$\left[\mathrm{SiO}_{3}\right]$ (molar at $\left.5 \mathrm{M} \mathrm{Na}\right)$} \\
\hline \multicolumn{3}{|c|}{0.0544} & \multicolumn{3}{|c|}{0.02} & \multicolumn{2}{|c|}{0.102} & & \multicolumn{2}{|l|}{0.00636} \\
\hline
\end{tabular}

The composition factor space for Envelope B is shown in Table 4, along with the concentration of the species that were held fixed relative to that of sodium. Note that the Envelope B waste feed input to OLI was targeted at a Na molarity of 6.5 to avoid convergence issues with the dilution to $1.22 \mathrm{~g} / \mathrm{ml}$ step. To calculate the molarity of the fixed species at another initial $\mathrm{Na}$ Molarity simply multiply the ratio term shown in Table 4 by the new desired initial $\mathrm{Na}$ molarity. 
WSRC-TR-2003-00269, REVISION 0

SRT-RPP-2003-00125, REVISION 0

Table 4. Definition for Envelope B Factor Space

\begin{tabular}{|c|c|c|c|c|c|c|c|c|c|}
\hline & \multicolumn{6}{|c|}{$\begin{array}{c}\text { Mixture Variable Mass Fraction Ranges } \\
\text { Mass fraction relative to total mass of mixture variables }\end{array}$} & \multicolumn{3}{|c|}{ Process Variables } \\
\hline Variable & $\begin{array}{c}\mathrm{AlO}_{2}^{-1} \\
\text { mass } \\
\text { fraction }\end{array}$ & $\begin{array}{c}\mathrm{CO}_{3}^{-2} \\
\text { mass } \\
\text { fraction }\end{array}$ & $\begin{array}{c}\mathrm{NO}_{3}^{-1} \\
\text { mass } \\
\text { fraction }\end{array}$ & $\begin{array}{c}\mathrm{OH}^{-1} \\
\text { mass } \\
\text { fraction }\end{array}$ & $\begin{array}{c}\mathrm{SO}_{4}^{-2} \\
\text { mass } \\
\text { fractio } \\
\mathbf{n}\end{array}$ & $\begin{array}{c}\mathrm{C}_{2} \mathrm{O}_{4}^{-2} \\
\text { mass } \\
\text { fraction }\end{array}$ & $\begin{array}{c}{[\mathrm{Na}]} \\
\text { (molar) }\end{array}$ & $\begin{array}{l}\text { SBS / Feed } \\
\text { (volumetric) }\end{array}$ & $\begin{array}{c}\text { Temp } \\
\left.{ }^{\circ} \mathrm{C}\right)\end{array}$ \\
\hline Min & 0.0380 & 0.2529 & 0.3603 & 0.0928 & 0.1438 & 0.0000 & 6 & 0.0 & 15 \\
\hline Max & 0.1107 & 0.2785 & 0.3755 & 0.1074 & 0.2152 & 0.0248 & 10 & 2.0 & 66 \\
\hline $\begin{array}{c}\text { charge } \\
\text { constraint }\end{array}$ & \multicolumn{9}{|c|}{$\begin{array}{c}-0.0270 \leq-0.016955 \cdot \mathrm{AlO}_{2}^{-1}-0.033328 \cdot \mathrm{CO}_{3}^{-2}-0.016128 \cdot \mathrm{NO}_{3}^{-1}-0.058798 \cdot \mathrm{OH}^{-1}- \\
0.02082 \cdot \mathrm{SO}_{4}^{-2}-0.02272 \cdot \mathrm{C}_{2} \mathrm{O}_{4}^{-2} \leq-0.0251\end{array}$} \\
\hline \multicolumn{10}{|c|}{ Molar Ratios Fixed Relative to Sodium Molarity } \\
\hline$\left[\mathrm{NO}_{2}\right]$ & {$[\mathrm{K}]$} & {$[\mathrm{F}]$} & & $\mathrm{OOH}]$ & {$[\mathrm{Cr}$} & & $\begin{array}{l}\left.\mathrm{H}_{7} \mathrm{O}_{7}\right] \\
\text { trate) }\end{array}$ & $\begin{array}{c}\left.\mathrm{HOCH}_{2} \mathrm{COO}\right] \\
\text { (glycolate) }\end{array}$ & {$\left[\mathrm{PO}_{4}\right]$} \\
\hline 0.267 & 0.0271 & 0.019 & & 0190 & 0.00 & & 0423 & 0.0212 & 0.00262 \\
\hline
\end{tabular}

The composition factor space for Envelope $\mathrm{C}$ is shown in Table 5, along with the concentration of the species that were held fixed relative to that of sodium. Note that the Envelope $\mathrm{C}$ waste feed input to OLI was targeted at a Na molarity of 6.5 to avoid convergence issues with the dilution to $1.22 \mathrm{~g} / \mathrm{ml}$ step. To calculate the molarity of the fixed species at another initial $\mathrm{Na}$ Molarity simply multiply the ratio term shown in Table 4 by the new desired initial $\mathrm{Na}$ molarity.

Table 5. Definition for Envelope C Factor Space

\begin{tabular}{|c|c|c|c|c|c|c|c|c|c|}
\hline \multirow[b]{2}{*}{ Variable } & \multicolumn{5}{|c|}{$\begin{array}{c}\text { Mixture Variable Mass Fraction Ranges } \\
\text { Mass fraction relative to total mass of mixture variables }\end{array}$} & \multicolumn{4}{|c|}{ Process Variables } \\
\hline & $\begin{array}{c}\mathrm{AlO}_{2}^{-1} \\
\text { mass } \\
\text { fraction }\end{array}$ & $\begin{array}{c}\mathrm{CO}_{3}^{-2} \\
\text { mass } \\
\text { fraction }\end{array}$ & $\begin{array}{c}\mathrm{NO}_{2}^{-1} \\
\text { mass } \\
\text { fraction }\end{array}$ & $\begin{array}{c}\mathrm{OH}^{-1} \\
\text { mass } \\
\text { fraction }\end{array}$ & $\begin{array}{c}\mathrm{SO}_{3}^{-2} \\
\text { mass } \\
\text { fraction }\end{array}$ & $\underset{\text { (molar) }}{[\mathrm{Na}]}$ & \multicolumn{2}{|c|}{$\begin{array}{c}\text { SBS / } \\
\text { Feed } \\
\text { (volumetric) }\end{array}$} & $\underset{\left({ }^{\circ} \mathrm{C}\right)}{\text { Temp }}$ \\
\hline minimum & 0.0052 & 0.2984 & 0.3926 & 0.0828 & 0.0644 & 6 & \multicolumn{2}{|c|}{0.0} & 15 \\
\hline maximum & 0.1319 & 0.3282 & 0.5131 & 0.1099 & 0.0733 & 10 & \multicolumn{2}{|c|}{2.0} & 66 \\
\hline $\begin{array}{l}\text { charge } \\
\text { constraint }\end{array}$ & \multicolumn{9}{|c|}{$\begin{array}{r}-0.0310 \leq-0.016955 \cdot \mathrm{AlO}_{2}^{-1}-0.0333 \cdot \mathrm{CO}_{3}^{-2}-0.02174 \cdot \mathrm{NO}_{2}^{-1} \\
-0.058798 \cdot \mathrm{OH}^{-1}-0.02082 \cdot \mathrm{SO}_{4}^{-2}\end{array}$} \\
\hline \multicolumn{10}{|c|}{ Molar Ratios Fixed Relative to Sodium Molarity } \\
\hline$[\mathrm{Cl}]$ & {$[\mathrm{F}]$} & \multicolumn{2}{|c|}{$\left[\mathrm{PO}_{4}\right]$} & {$\left[\mathrm{C}_{2} \mathrm{O}_{4}\right]$} & {$[\mathrm{K}]$} & \multicolumn{2}{|c|}{$\left[\mathrm{NO}_{3}\right]$} & \multicolumn{2}{|c|}{$[\mathrm{COOH}]$} \\
\hline 0.0114 & 0.0168 & \multicolumn{2}{|c|}{0.00336} & 0.00241 & 0.00732 & \multicolumn{2}{|c|}{0.394} & \multicolumn{2}{|c|}{0.0238} \\
\hline
\end{tabular}




\section{WSRC-TR-2003-00269, REVISION 0 SRT-RPP-2003-00125, REVISION 0}

In the treated feed evaporator experimental work, the waste feeds for all envelopes were created from recipes and then filtered. Since the state of the treated evaporator waste feed could be characterized in a number of ways, the choice was made to dilute the characterized waste feeds to $1.22 \mathrm{~g} / \mathrm{ml}$ density to provide a common basis for the modeling. It is expected that the treated waste feed will be at or near this density after the pre-treatment. This density corresponds to a feed roughly at $5 \mathrm{Na} \mathrm{M}$. The filtration step just simulates the pre-treatment filtration and precipitation removal with the outlet streams ultimately representing the treated waste feeds.

\subsection{RESULTS}

The results are given in two sections, 1) the model fits with plots comparing model predictions with simulation results and 2) the comparison of simulation with experimental results.

Computer simulations have no inherent random error like real experimental work so statistics normally used to gauge the success of an experiment could not be used here. Therefore, the physical property models were first validated using a portion of the design points (OLH or Orthogonal Latin Hypercube). ${ }^{23}$ If the physical property models adequately predicted a majority of the OLH points within $+/-15 \%$, the model was considered to be adequate in terms of predicting the OLI simulation results. As a final part of the validation, the experimental results from the prior work on Treated Feed Evaporation ${ }^{7}$ were compared with model predictions.

\subsubsection{Predictive Physical Property Models and Validation Phase 1}

This section shows the predictive models derived from the OLI simulations along with graphs of the simulation results versus the physical model predictions for each waste envelope. In each graph the design points used to fit the model are identified with blue open circles, the validation $(\mathrm{OLH})$ points are identified by red asterisks, a solid red line identifies the predicted value $+15 \%$, and a solid green line identifies the predicted value $-15 \%$.

The physical property models for all the waste envelopes were able to meet the $\pm 15 \%$ of predicted values criterion except for the Na molarity prediction for Envelope C. Even in this case, $96 \%$ of OLH points still fell within the $\pm 15 \%$ border. So the model still does a fair job at predicting the sodium molarity for most cases.

\subsubsection{Envelope A Predictive Models}

All concentrations are in terms of molarity as shown in Table 6 . The concentrations of the mixture variables (all species except $\mathrm{Na}$ ) are expressed relative to a stream at a $5 \mathrm{M} \mathrm{Na}$ concentration. (e.g., an $\left[\mathrm{AlO}_{2}\right]$ concentration of $2 \mathrm{M}$ in an $8 \mathrm{M} \mathrm{Na}$ stream is first adjusted to an equivalent concentration in a $5 \mathrm{M}$ stream: $\left.2 \mathrm{M} \mathrm{AlO}_{2} *(5 \mathrm{M} \mathrm{Na} / 8 \mathrm{M} \mathrm{Na})=1.25 \mathrm{M} \mathrm{AlO}_{2}\right)$ and then applied to the equation. The three process variables are $[\mathrm{Na}]$ (the bottoms concentrate $\mathrm{Na}$ molarity, as opposed to the treated evaporator feed stream Na concentration just mentioned), SBS/Feed (volumetric ratio of LAW SBS to treated waste feed flow, and Temp (the bottoms concentrate temperature). 
WSRC-TR-2003-00269, REVISION 0

SRT-RPP-2003-00125, REVISION 0

Table 6. Valid Variable Ranges for Envelope A Models

\begin{tabular}{|c|c|c|c|c|c|c|c|c|c|c|}
\hline & \multicolumn{7}{|c|}{$\begin{array}{c}\text { Mixture Variable Molar Ranges } \\
\text { Normalized to equivalent molarity at } 5 \mathrm{M} \mathrm{Na}\end{array}$} & \multicolumn{3}{|c|}{ Process Variables } \\
\hline Variable & $\begin{array}{c}{\left[\mathrm{AlO}_{2}\right]} \\
(\mathrm{molar} \\
\text { at } 5 \mathrm{M} \\
\mathrm{Na})\end{array}$ & $\begin{array}{c}{\left[\mathrm{CO}_{3}\right]} \\
(\mathrm{molar} \\
\text { at 5M } \\
\mathrm{Na})\end{array}$ & $\begin{array}{c}{[\mathrm{F}]} \\
\text { (molar at } \\
5 \mathrm{M} \mathrm{Na})\end{array}$ & $\begin{array}{c}{\left[\mathrm{NO}_{2}\right]} \\
(\mathrm{molar} \\
\text { at 5M } \\
\mathrm{Na})\end{array}$ & $\begin{array}{c}{\left[\mathrm{NO}_{3}\right]} \\
(\mathrm{molar} \\
\text { at 5M } \\
\mathrm{Na})\end{array}$ & $\begin{array}{c}{[\mathrm{OH}]} \\
(\mathrm{molar} \\
\text { at 5M } \\
\mathrm{Na})\end{array}$ & $\begin{array}{c}{\left[\mathrm{PO}_{4}\right]} \\
(\mathrm{molar} \text { at } \\
5 \mathrm{M} \mathrm{Na})\end{array}$ & $\begin{array}{c}{[\mathrm{Na}]} \\
\text { (molar) }\end{array}$ & $\begin{array}{c}\text { SBS / Feed } \\
\text { (volumetric) }\end{array}$ & $\underset{\left({ }^{\circ} \mathrm{C}\right)}{\text { Temp }}$ \\
\hline minimum & 0.207 & 0.326 & 0.00927 & 0.731 & 0.991 & 0.983 & 0.00632 & 6 & 0.0 & 15 \\
\hline maximum & 1.12 & 0.686 & 0.236 & 1.59 & 2.08 & 2.89 & 0.0436 & 10 & 2.0 & 66 \\
\hline
\end{tabular}


Envelope A bottoms concentrate slurry density at its steady state endpoint condition is represented by Equation 2 and shown in Figure 13.

\section{Equation 2}

$$
\begin{aligned}
\operatorname{density}_{\mathrm{EnvA}}\left[\frac{\mathrm{g}}{\mathrm{ml}}\right]= & 1.117 \cdot x_{\mathrm{AlO2}}+1.110 \cdot x_{\mathrm{CO} 3}+1.075 \cdot x_{F}+1.072 \cdot x_{\mathrm{NO2}}+1.096 \cdot x_{\mathrm{NO3}}+ \\
& 0.9813 \cdot x_{\mathrm{OH}}+1.141 \cdot \mathrm{x}_{\mathrm{PO} 4}-1.119 \mathrm{E}-3 \cdot \text { Temp }+7.046 \mathrm{E}-3 \cdot \mathrm{SBS} / \text { Feed }+ \\
& 0.03258 \cdot[\mathrm{Na}]-4.438 \mathrm{E}-5 \cdot(\mathrm{SBS} / \text { Feed }-1) \cdot(\text { Temp }-40.5)- \\
& 1.150 \mathrm{E}-4 \cdot([\mathrm{Na}]-8) \cdot(\text { Temp }-40.5)
\end{aligned}
$$

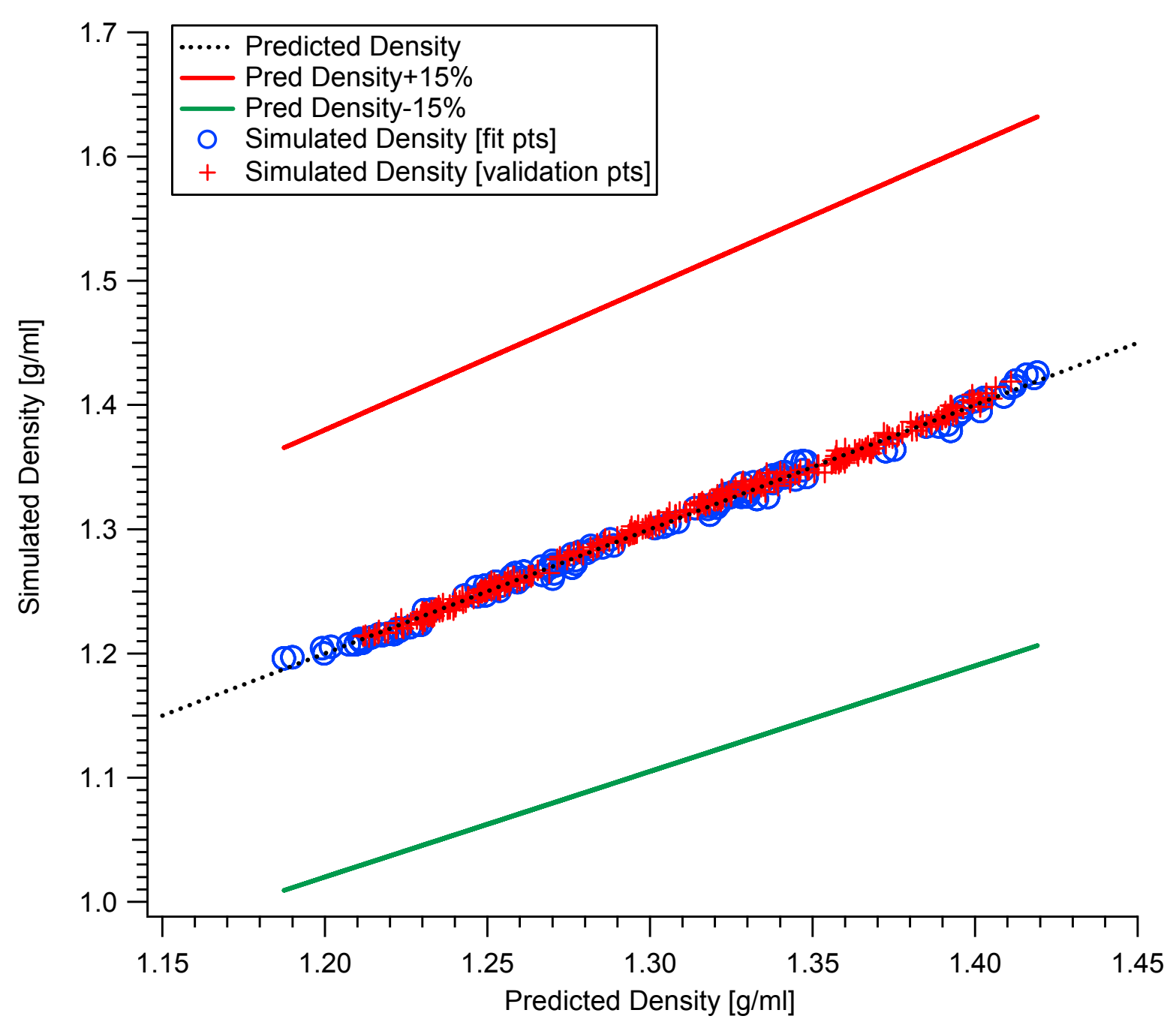

Figure 13. Simulated Density versus Predicted Density for Envelope A 
Envelope A bottoms concentrate slurry conductivity at its steady state endpoint condition is represented by Equation 3 and shown in Figure 14.

\section{Equation 3}

$$
\begin{aligned}
\text { conductivity }_{\mathrm{EnvA}}\left[\frac{\mathrm{cal}}{\mathrm{s} \cdot \mathrm{cm} \cdot \mathrm{K}}\right]= & 1.271 \mathrm{E}-3 \cdot x_{\mathrm{AlO} 2}+1.326 \mathrm{E}-3 \cdot x_{\mathrm{CO} 3}+1.392 \mathrm{E}-3 \cdot x_{F}+1.304 \mathrm{E}-3 \cdot x_{\mathrm{NO} 2}+ \\
& 1.272 \mathrm{E}-3 \cdot \mathrm{x}_{\mathrm{NO} 3}+1.863 \mathrm{E}-3 \cdot x_{\mathrm{OH}}+1.402 \mathrm{E}-3 \cdot x_{P O 4}+3.087 \mathrm{E}-6 \cdot \mathrm{Temp}- \\
& 5.159 \mathrm{E}-6 \cdot \mathrm{SBS} / \mathrm{Feed}-3.590 \mathrm{E}-6 \cdot[\mathrm{Na}]+ \\
& 1.639 \mathrm{E}-6 \cdot(\mathrm{Temp}-40.5) \cdot\left(x_{\mathrm{OH}}-0.1422\right)+ \\
& 4.555 \mathrm{E}-4 \cdot\left(x_{\mathrm{NO} 3}-0.4156\right) \cdot\left(x_{\mathrm{NO} 3}-0.4156\right)
\end{aligned}
$$

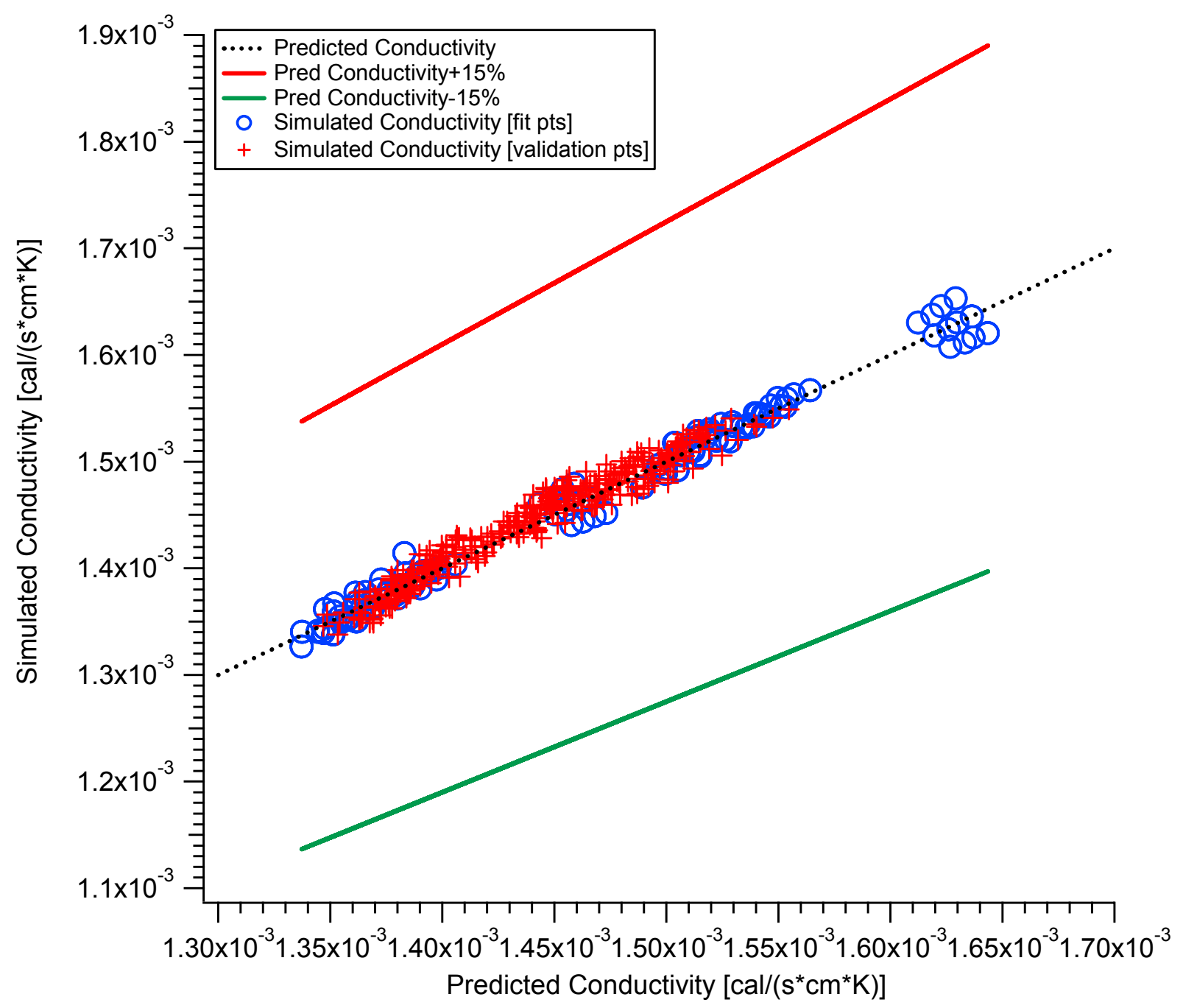

Figure 14. Simulated Conductivity versus Predicted Conductivity for Envelope A 
Envelope A bottoms concentrate slurry heat capacity at its steady state endpoint condition is represented by Equation 4 and shown in Figure 15.

\section{Equation 4}

$$
\begin{aligned}
\mathrm{Cp}_{\mathrm{EnvA}}\left[\frac{\mathrm{cal}}{\mathrm{g}{ }^{\mathrm{o}} \mathrm{C}}\right]= & 0.8824 * x_{\mathrm{AlO} 2}+0.7165 * x_{\mathrm{CO} 3}+0.9227 * x_{\mathrm{F}}+0.7351 * x_{\mathrm{NO} 2}+ \\
& 0.8640 * x_{\mathrm{NO} 3}+0.9586 * x_{\mathrm{OH}}+0.8870 * x_{\mathrm{PO} 4}-3.443 \mathrm{E}-3 * \mathrm{SBS} / \mathrm{Feed}- \\
& 2.020 \mathrm{e}-2 *[\mathrm{Na}]-0.05588 *\left(\mathrm{x}_{\mathrm{CO} 3}-0.0510\right) *([\mathrm{Na}]-8)+ \\
& -0.03794 *\left(x_{\mathrm{NO} 2}-0.2331\right) *([\mathrm{Na}]-8)+0.02999 *\left(x_{\mathrm{NO} 3}-0.4156\right) *([N a]-8)+ \\
& 0.002053 *\left(x_{\mathrm{NO} 3}-0.4156\right) *(\mathrm{Temp}-40.5)+9.093 \mathrm{e}-5 *([\mathrm{Na}]-8) *(\mathrm{Temp}-40.5)
\end{aligned}
$$

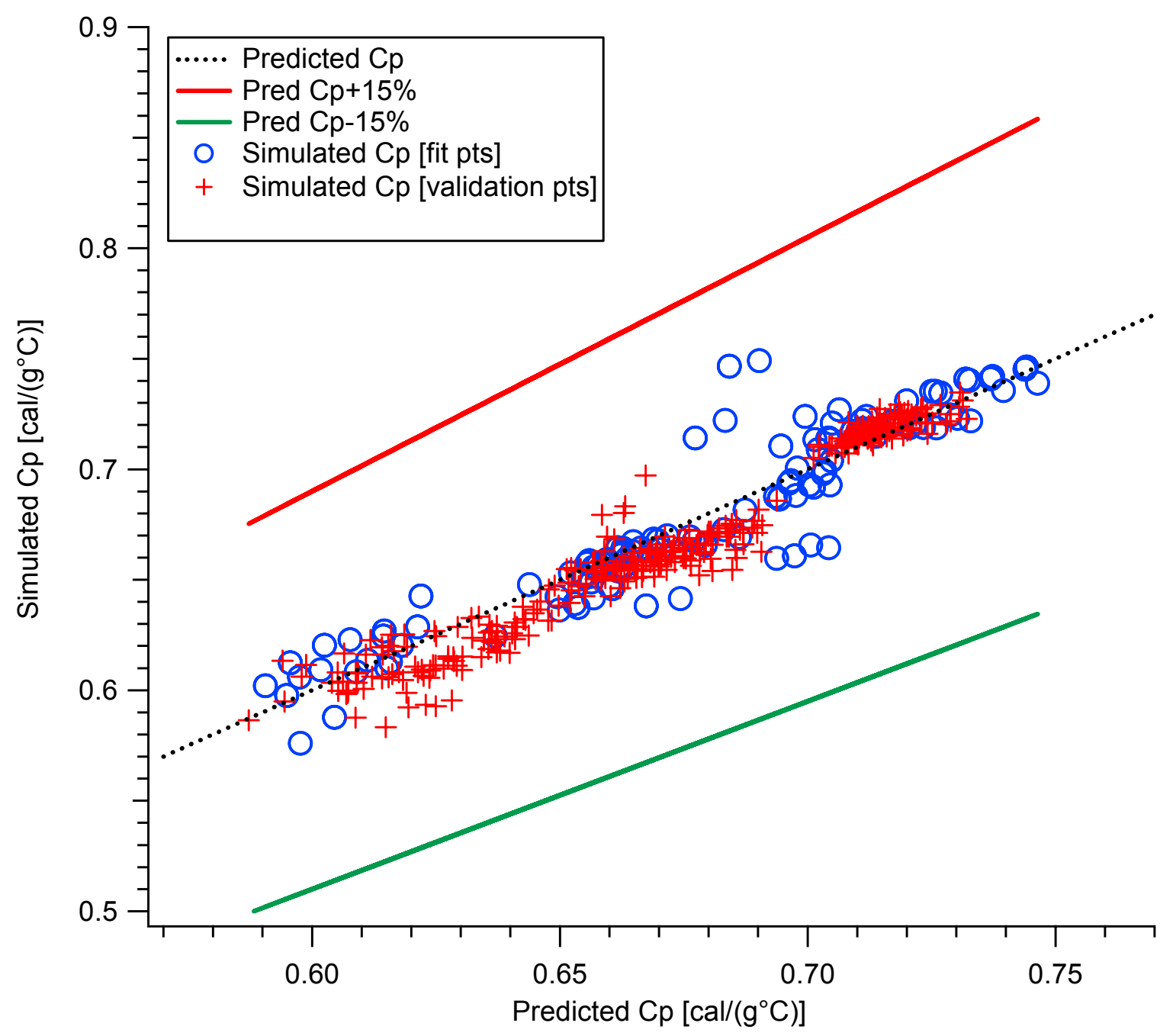

Figure 15. Simulated Cp versus Predicted Cp for Envelope A 
Envelope A bottoms concentrate slurry Na molarity at its steady state endpoint condition is represented by Equation 5 and shown in Figure 16.

\section{Equation 5}

$$
\begin{aligned}
{[\mathrm{Na}]_{\mathrm{EnNA}}\left[\frac{\mathrm{gmol}}{\mathrm{L}}\right]=} & -34.36 \cdot x_{A l O 2}-34.10 \cdot x_{\mathrm{CO} 3}-33.09 \cdot x_{F}-32.95 \cdot x_{\mathrm{NO} 2}-33.72 \cdot x_{\mathrm{NO} 3}-30.24 \cdot x_{O H}- \\
& 35.14 \cdot x_{P O 4}+0.03444 \cdot \mathrm{Temp}-0.2139 \cdot \mathrm{SBS} / \text { Feed }+30.82 \cdot \text { Density }+ \\
& 0.1106 \cdot(\text { Density }-1.300) \cdot(\text { Temp }-40.5)- \\
& 0.9262 \cdot(\text { Density }-1.300) \cdot(\text { SBS } / \text { Feed }-1)
\end{aligned}
$$

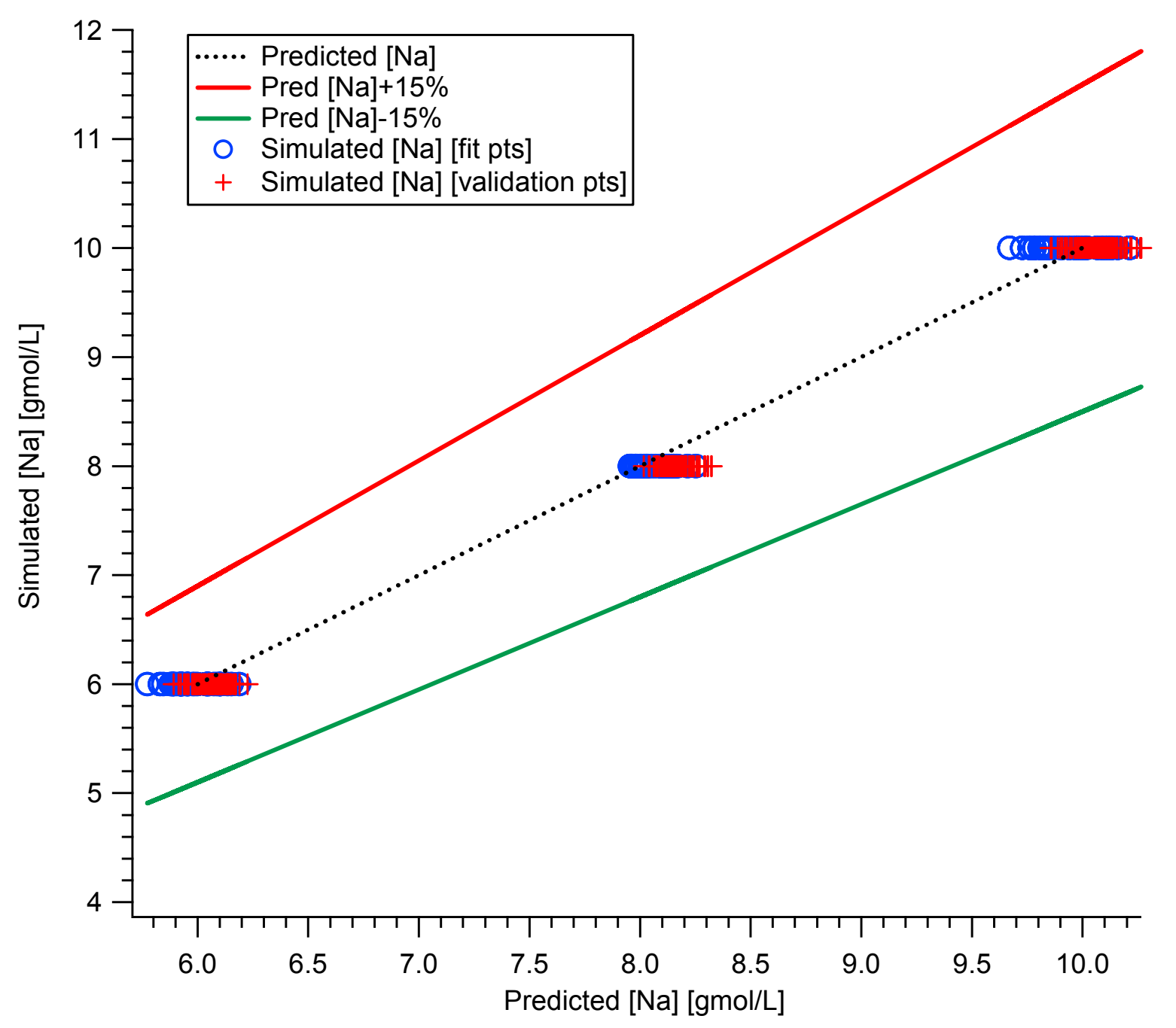

Figure 16. Simulated [Na] versus Predicted [Na] for Envelope A 
Although no valid solids prediction model could be developed for the bottoms concentrate stream for Envelope A, the general behavior of the simulated points can be seen in Figure 17. As is evident from the graph, insoluble solids in the bottoms concentrate should not be a concern unless a high Na molarity like $10 \mathrm{M}$ and a low temperature like $20^{\circ} \mathrm{C}$ are targeted simultaneously.

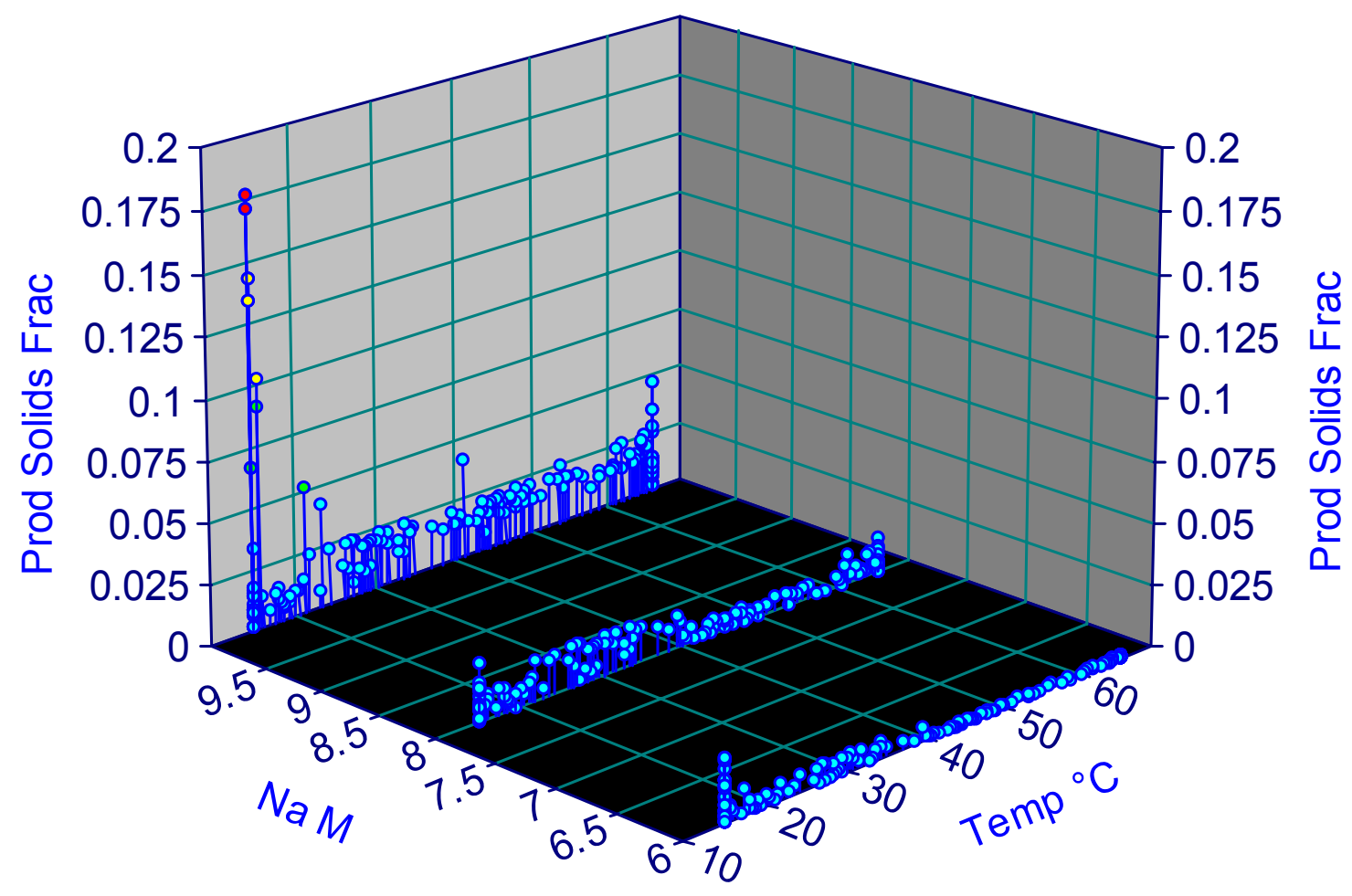

Figure 17. Envelope A Bottoms Solids Fraction versus Na Molarity and Temperature 


\subsubsection{Envelope B Predictive Models}

All concentrations for the Envelope B model mixture variables listed in Table 7 are in terms of weight fraction relative to the total mass of all the mixture variables. The three process variables are [Na] (the bottoms concentrate Na molarity), SBS/Feed (volumetric ratio of LAW SBS to treated waste feed flow, and Temp (the bottoms concentrate temperature).

Table 7. Valid Variable Ranges for Envelope B Models

\begin{tabular}{|c|c|c|c|c|c|c|c|c|c|}
\hline \multirow[b]{2}{*}{ Variable } & \multicolumn{6}{|c|}{$\begin{array}{c}\text { Mixture Variable Mass Fraction Ranges } \\
\text { Mass fraction relative to total mass of mixture variables }\end{array}$} & \multicolumn{3}{|c|}{ Process Variables } \\
\hline & $\begin{array}{c}\mathrm{AlO}_{2}^{-1} \\
\text { mass } \\
\text { fraction }\end{array}$ & $\begin{array}{c}\mathrm{CO}_{3}^{-2} \\
\text { mass } \\
\text { fraction }\end{array}$ & $\begin{array}{c}\mathrm{NO}_{3}^{-1} \\
\text { mass } \\
\text { fraction }\end{array}$ & $\begin{array}{c}\mathrm{OH}^{-1} \\
\text { mass } \\
\text { fraction }\end{array}$ & $\begin{array}{c}\mathrm{SO}_{4}^{-2} \\
\text { mass } \\
\text { fraction }\end{array}$ & $\begin{array}{c}\mathrm{C}_{2} \mathrm{O}_{4}^{-2} \\
\text { mass } \\
\text { fraction }\end{array}$ & $\begin{array}{c}{[\mathrm{Na}]} \\
\text { (molar) }\end{array}$ & $\begin{array}{c}\text { SBS / Feed } \\
\text { (volumetric) }\end{array}$ & $\underset{\left({ }^{\circ} \mathrm{C}\right)}{\operatorname{Temp}}$ \\
\hline Min & 0.0380 & 0.2529 & 0.3603 & 0.0928 & 0.1438 & 0.0000 & 6 & 0.0 & 15 \\
\hline Max & 0.1107 & 0.2785 & 0.3755 & 0.1074 & 0.2152 & 0.0248 & 10 & 2.0 & 66 \\
\hline
\end{tabular}


The Envelope B evaporator bottoms concentrate slurry density at its steady state endpoint condition is represented by Equation 6 and shown in Figure 18.

\section{Equation 6}

$$
\begin{aligned}
\operatorname{density}_{\mathrm{EnvB}}\left[\frac{\mathrm{g}}{\mathrm{ml}}\right] & =3.463 \mathrm{E}-3 \cdot(\text { SBS / Feed }-1)-0.02774 \cdot(\text { Temp }-45) / 25)+1.034 \cdot x_{A l O 2}+ \\
& 1.009 \cdot x_{\mathrm{CO} 3}+0.9938 \cdot x_{\mathrm{NO3}}+0.9362 \cdot x_{\mathrm{OH}}+1.068 \cdot x_{\mathrm{SO} 4}+1.020 \cdot x_{\mathrm{C} 2 \mathrm{O} 4}+ \\
& 0.04177 \cdot[\mathrm{Na}]
\end{aligned}
$$

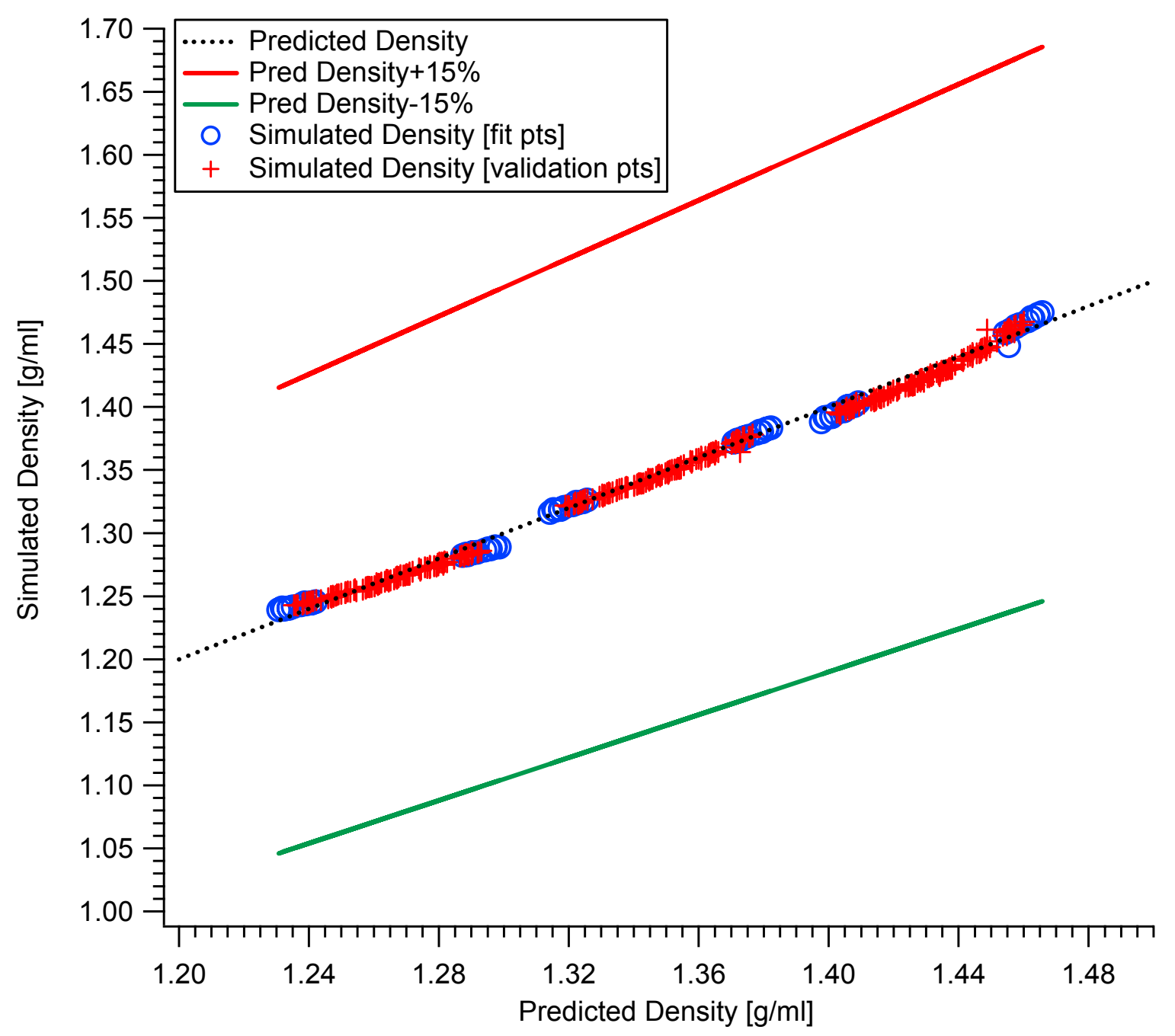

Figure 18. Simulated Density versus Predicted Density for Envelope B 
The Envelope B evaporator bottoms supernate viscosity at its steady state endpoint condition is represented by Equation 7 and shown in Figure 19.

\section{Equation 7}

$$
\begin{aligned}
& \operatorname{visc}_{\mathrm{EnvB}}[\mathrm{cP}]=\operatorname{Exp}\left(\frac{2122}{137.5+\mathrm{Temp}}+0.2318 \cdot[\mathrm{Na}]+5.162 \cdot x_{\mathrm{OH}}-15.24\right)+ \\
& 0.5203 \cdot[\mathrm{Na}]-4.817 \cdot x_{\mathrm{OH}}+0.08783 \cdot x_{\mathrm{OH}} \cdot \mathrm{Temp}-4.700 \mathrm{E}-3 \cdot[\mathrm{Na}] \cdot \mathrm{Temp}
\end{aligned}
$$

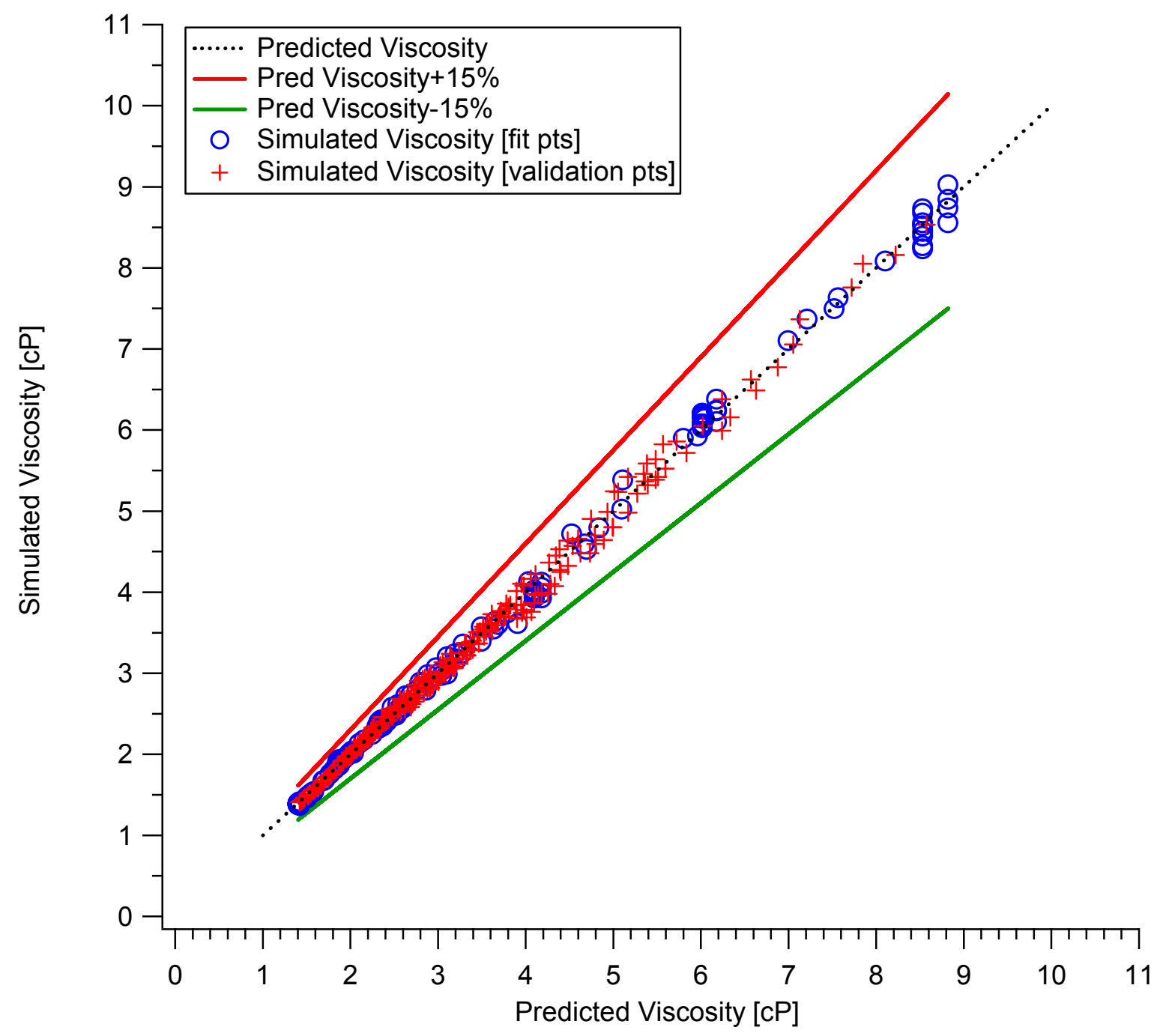

Figure 19. Simulated Viscosity versus Predicted Viscosity for Envelope B 
The Envelope B evaporator bottoms concentrate slurry conductivity at its steady state endpoint condition is represented by Equation 8 and shown in Figure 20.

\section{Equation 8}

$$
\begin{aligned}
\text { conductivity }_{\mathrm{EnvB}}\left[\frac{\mathrm{cal}}{\mathrm{s} \cdot \mathrm{cm} \cdot \mathrm{K}}\right]= & 1.274 \mathrm{E}-3 \cdot x_{\mathrm{AlO2}}+1.355 \mathrm{E}-3 \cdot x_{\mathrm{CO} 3}+1.432 \mathrm{E}-3 \cdot x_{\mathrm{NO} 3}+1.674 \mathrm{E}-3 \cdot x_{\mathrm{OH}}+ \\
& 1.373 \mathrm{E}-3 \cdot x_{\mathrm{SO} 4}+1.418 \mathrm{E}-3 \cdot x_{\mathrm{C} 2 O 4}-1.041 \mathrm{E}-6 \cdot(\mathrm{SBS} / \mathrm{Feed}-1)- \\
& 6.550 \mathrm{E}-6 \cdot[\mathrm{Na}]-1.785 \mathrm{E}-5 \cdot([\mathrm{Na}]-8) \cdot\left(x_{\mathrm{AlO} 2}-0.06883\right)+ \\
& 3.853 \mathrm{E}-5 \cdot([\mathrm{Na}]-8) \cdot\left(x_{\mathrm{OH}}-0.09767\right)
\end{aligned}
$$

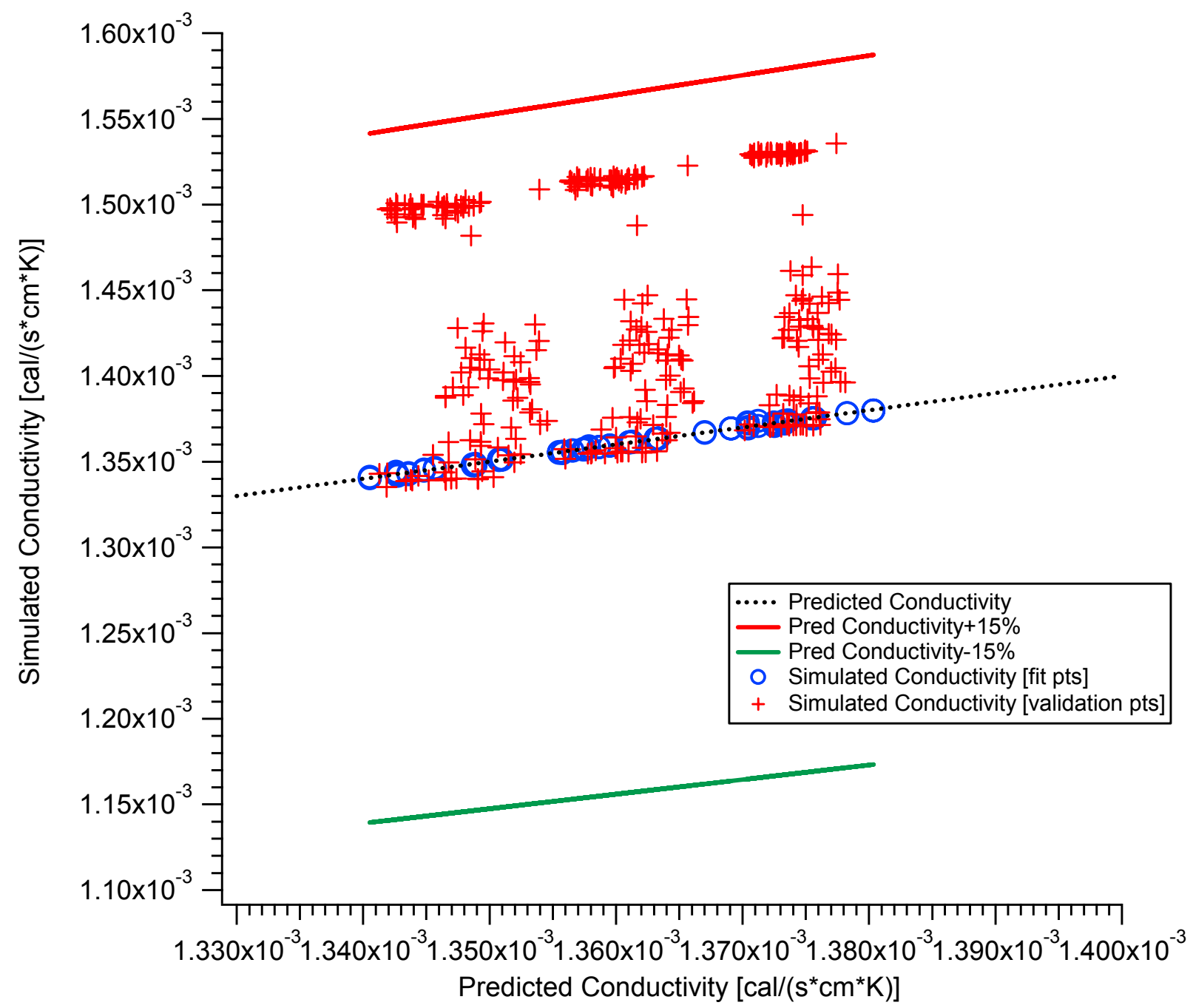

Figure 20. Simulated Conductivity versus Predicted Conductivity for Envelope B 
Envelope B bottoms concentrate slurry heat capacity at its steady state endpoint condition is represented by Equation 9 and shown in Figure 21.

\section{Equation 9}

$$
\begin{aligned}
\mathrm{Cp}_{\mathrm{EnvB}}\left[\frac{\mathrm{cal}}{\mathrm{g}{ }^{\mathrm{O}} \mathrm{C}}\right]= & -0.01526 \cdot x_{A l O 2}+1.168 \cdot x_{\mathrm{CO} 3}+1.149 \cdot x_{F}+0.9522 \cdot x_{\mathrm{NO} 2}+ \\
& 1.398 \cdot x_{\mathrm{NO} 3}+1.188 \cdot x_{\mathrm{OH}}+1.228 \cdot x_{\mathrm{PO} 4}-7.754 \mathrm{E}-2 \cdot \mathrm{SBS} / \mathrm{Feed}- \\
& 2.020 \mathrm{e}-2 \cdot[\mathrm{Na}]-0.05588 \cdot\left(\mathrm{x}_{\mathrm{CO} 3}-0.0510\right) \cdot([\mathrm{Na}]-8)+ \\
& -0.03794 \cdot\left(x_{\mathrm{NO} 2}-0.2331\right) \cdot([\mathrm{Na}]-8)+0.02999 \cdot\left(x_{\mathrm{NO}}-0.4156\right) \cdot([\mathrm{Na}]-8)+ \\
& 0.002053 \cdot\left(x_{\mathrm{NO} 3}-0.4156\right) \cdot(\mathrm{Temp}-40.5)+9.093 \mathrm{e}-5 \cdot([\mathrm{Na}]-8) \cdot(\mathrm{Temp}-40.5)
\end{aligned}
$$

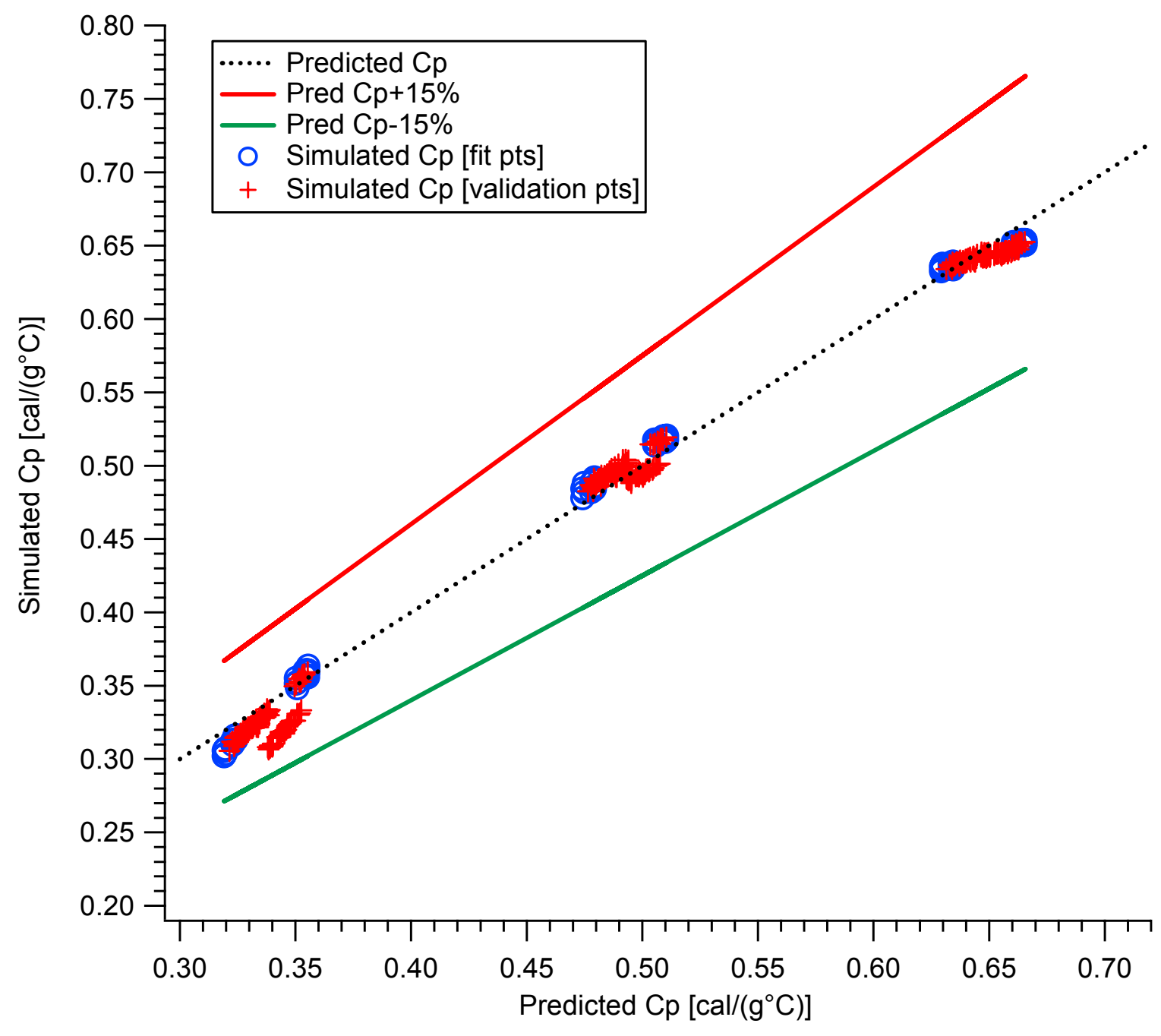

Figure 21. Simulated Cp versus Predicted Cp for Envelope B 
The Envelope B evaporator bottoms concentrate slurry Na molarity at its steady state endpoint condition is represented by Equation 10 and shown in Figure 22.

\section{Equation 10}

$$
\begin{aligned}
{[\mathrm{Na}]_{\mathrm{EnvB}}\left[\frac{\mathrm{gmol}}{\mathrm{L}}\right]=} & -0.08268 \cdot(\mathrm{SBS} / \text { Feed }-1)+0.6680 \cdot \frac{(\text { Temp }-45)}{25}-25.34 \cdot x_{A l O 2}-24.70 \cdot x_{C O 3}- \\
& 24.41 \cdot x_{\mathrm{NO3}}-22.97 \cdot x_{\mathrm{OH}}-26.14 \cdot x_{\mathrm{SO} 4}-25.03 \cdot x_{\mathrm{C} 204}+24.41 \cdot \text { Density }- \\
& 0.3649 \cdot(\mathrm{SBS} / \text { Feed }-1) \cdot(\text { Density }-1.348)+ \\
& 1.854 \cdot \frac{(\text { Temp }-45)}{25} \cdot(\text { Density }-1.348)
\end{aligned}
$$

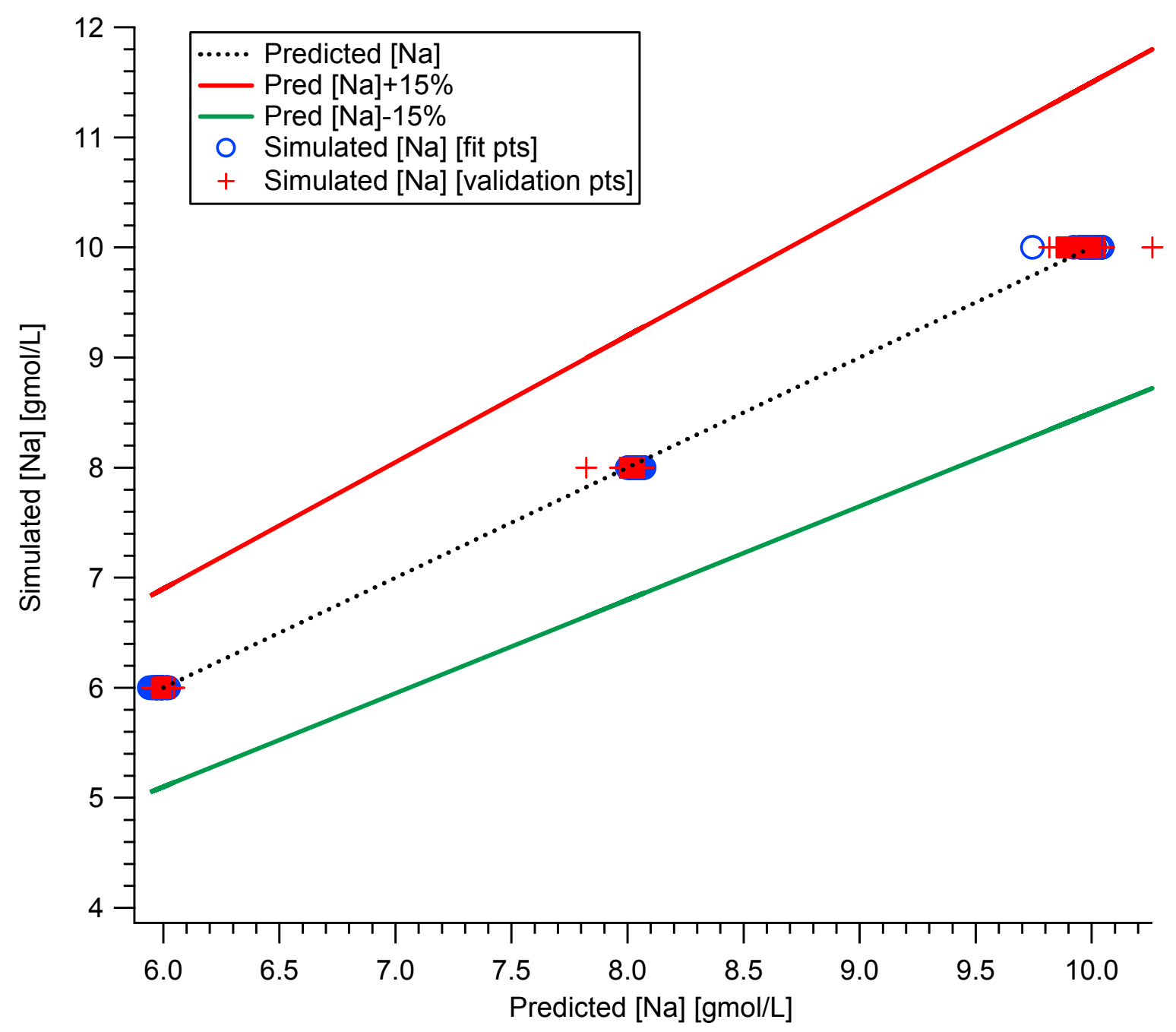

Figure 22. Simulated [Na] versus Predicted [Na] for Envelope B 
Although no valid solids prediction model could be developed for the bottoms concentrate stream for Envelope B, the general behavior of the simulated points can be seen in Figure 23. The graph shows that for a temperature range of 15 to $66^{\circ} \mathrm{C}$ and a Na molarity range of 6 to 10 $\mathrm{Na} \mathrm{M}$, the fraction of insoluble solids in the bottoms stream is always below $3 \mathrm{wt}-\%$. The graph also shows that the bottoms Na molarity concentration has the biggest impact on the amount solids that forms compared to temperature. The graph also shows that the majority of solids precipitate out between 20 and $40^{\circ} \mathrm{C}$.

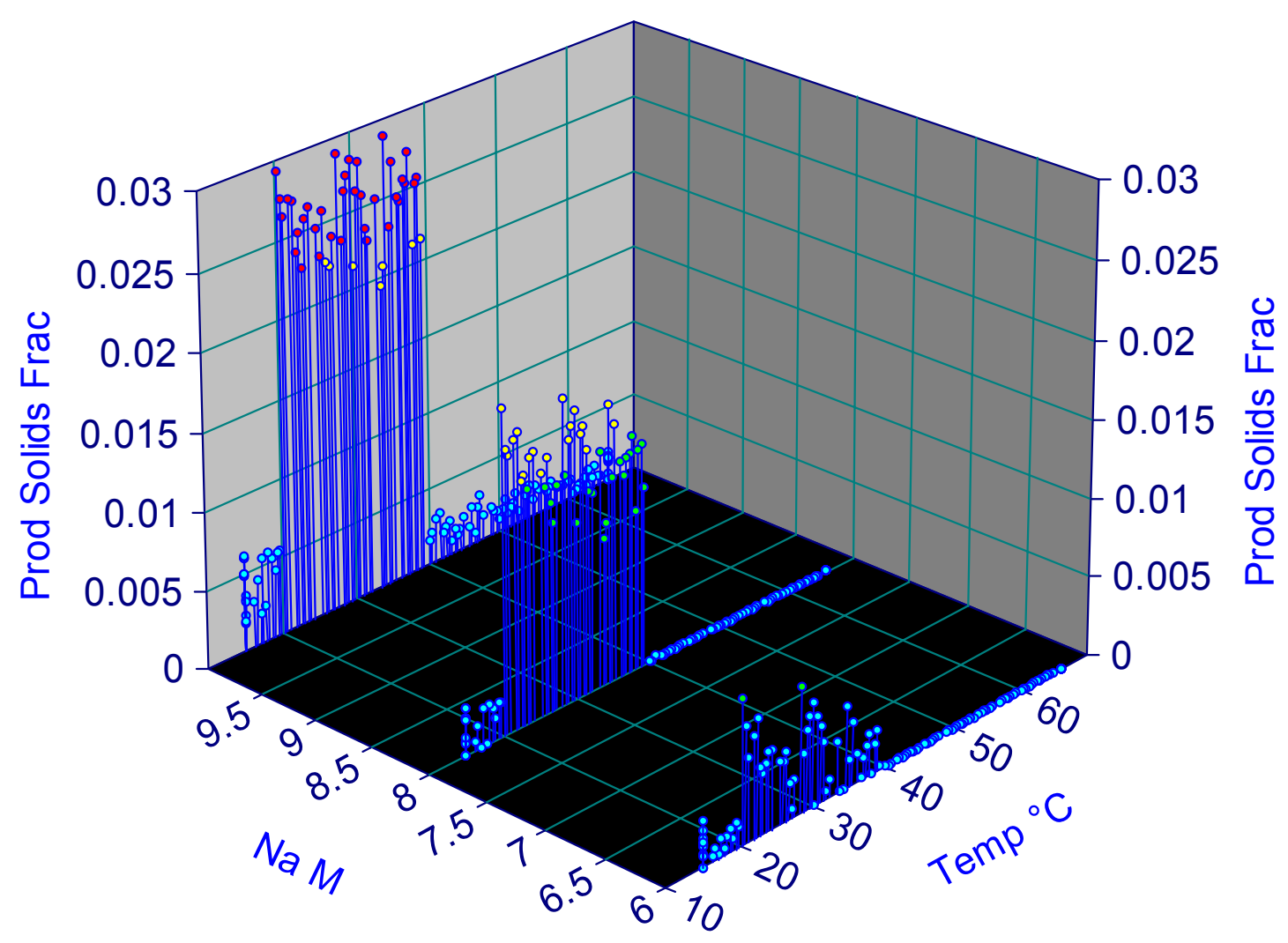

Figure 23. Envelope B Bottoms Solids Fraction versus Na Molarity and Temperature 


\subsubsection{Envelope C Predictive Models}

Concentrations for the Envelope $\mathrm{C}$ model mixture variables listed in Table 8 are in terms of weight fraction relative to the total mass of all the mixture variables. The three process variables are $[\mathrm{Na}]$ (the bottoms concentrate Na molarity), SBS/Feed (volumetric ratio of LAW SBS to treated waste feed flow, and Temp (the bottoms concentrate temperature).

Table 8. Valid Variable Ranges for Envelope C Models

\begin{tabular}{|c|c|c|c|c|c|c|c|c|}
\hline & \multicolumn{4}{|c|}{$\begin{array}{c}\text { Mixture Variable Mass Fraction Ranges } \\
\text { Mass fraction relative to total mass of mixture variables }\end{array}$} & \multicolumn{3}{c|}{ Process Variables } \\
\hline \multirow{2}{*}{ Variable } & $\begin{array}{c}\mathrm{AlO}_{2}^{-1} \\
\text { mass } \\
\text { fraction }\end{array}$ & $\begin{array}{c}\mathrm{CO}_{3}^{-2} \\
\text { mass } \\
\text { fraction }\end{array}$ & $\begin{array}{c}\mathrm{NO}_{2}^{-1} \\
\text { mass } \\
\text { fraction }\end{array}$ & $\begin{array}{c}\mathrm{OH}^{-1} \\
\text { mass } \\
\text { fraction }\end{array}$ & $\begin{array}{c}\mathrm{SO}_{3}^{-2} \\
\text { mass } \\
\text { fraction }\end{array}$ & $\begin{array}{c}{[\mathbf{N a}]} \\
(\text { molar) }\end{array}$ & $\begin{array}{c}\text { SBS / } \\
\text { Feed } \\
(\text { volumetric) }\end{array}$ & $\begin{array}{c}\text { Temp } \\
\left({ }^{\mathbf{0}} \mathbf{C}\right)\end{array}$ \\
\hline minimum & 0.0052 & 0.2984 & 0.3926 & 0.0828 & 0.0644 & 6 & 0.0 & 15 \\
\hline maximum & 0.1319 & 0.3282 & 0.5131 & 0.1099 & 0.0733 & 10 & 2.0 & 66 \\
\hline
\end{tabular}


The Envelope $\mathrm{C}$ evaporator bottoms concentrate slurry density at its steady state endpoint condition is represented by Equation 11 and shown in Figure 24.

\section{Equation 11}

$$
\begin{aligned}
\text { density }_{\mathrm{EnVC}}\left[\frac{\mathrm{g}}{\mathrm{ml}}\right]= & 0.01257 \cdot(\mathrm{SBS} / \text { Feed }-1)-0.03233 \cdot \frac{\text { Temp }-45}{25}- \\
& 1.765 \mathrm{E}-3 \cdot(\mathrm{SBS} / \text { Feed }-1) \cdot \frac{\text { Temp }-45}{25}+ \\
& 1.074 \cdot \mathrm{x}_{\mathrm{AlO} 2}+1.051 \cdot \mathrm{x}_{\mathrm{CO} 3}+1.042 \cdot \mathrm{x}_{\mathrm{NO} 2}+1.018 \cdot \mathrm{x}_{\mathrm{OH}}+1.132 \cdot \mathrm{x}_{\mathrm{SO} 4}+ \\
& 0.03275 \cdot[\mathrm{Na}]-2.685 \mathrm{E}-3 \cdot([N a]-8) \cdot \frac{\text { Temp }-45}{25}
\end{aligned}
$$

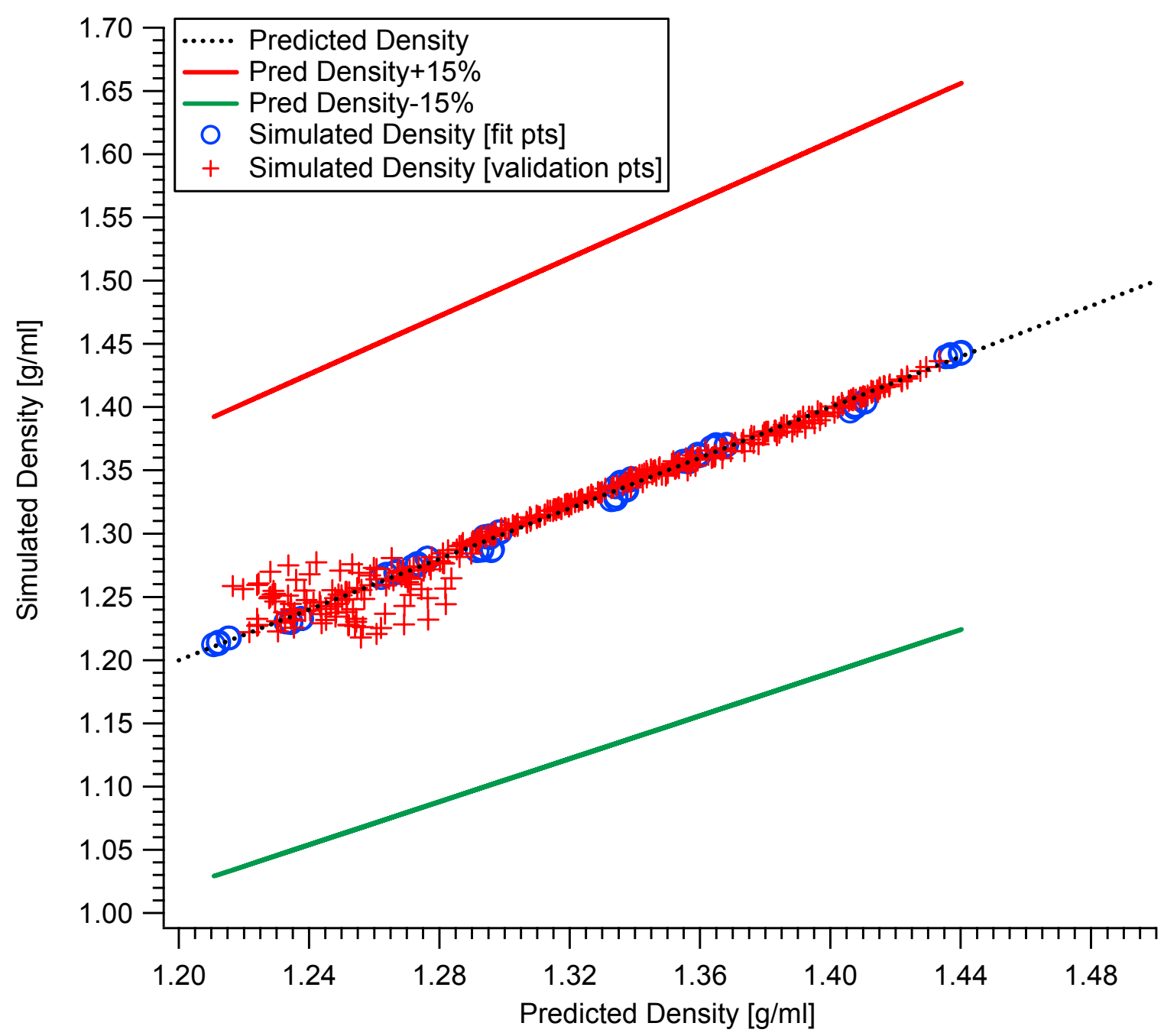

Figure 24. Simulated Density versus Predicted Density for Envelope C 
The Envelope $\mathrm{C}$ evaporator bottoms supernate viscosity at its steady state endpoint condition is represented by Equation 12 and shown in Figure 25.

\section{Equation 12}

$$
\begin{aligned}
\operatorname{visc}_{\mathrm{EnvC}_{\mathrm{C}}[\mathrm{cP}]}= & \operatorname{Exp}\left(\begin{array}{l}
\frac{7418}{31.70+\text { Temp }}+0.2390 \cdot[\mathrm{Na}]-0.7285 \cdot x_{\mathrm{NO} 2}+ \\
-22.96+-0.08727 \cdot \mathrm{SBS} / \mathrm{Feed}
\end{array}\right)+ \\
& 0.4093 \cdot[\mathrm{Na}]-0.4685 \cdot x_{\mathrm{NO} 2}-2.672 \mathrm{E}-3 \cdot[\mathrm{Na}] \cdot \text { Temp }-0.04042 \cdot \mathrm{SBS} / \text { Feed }
\end{aligned}
$$

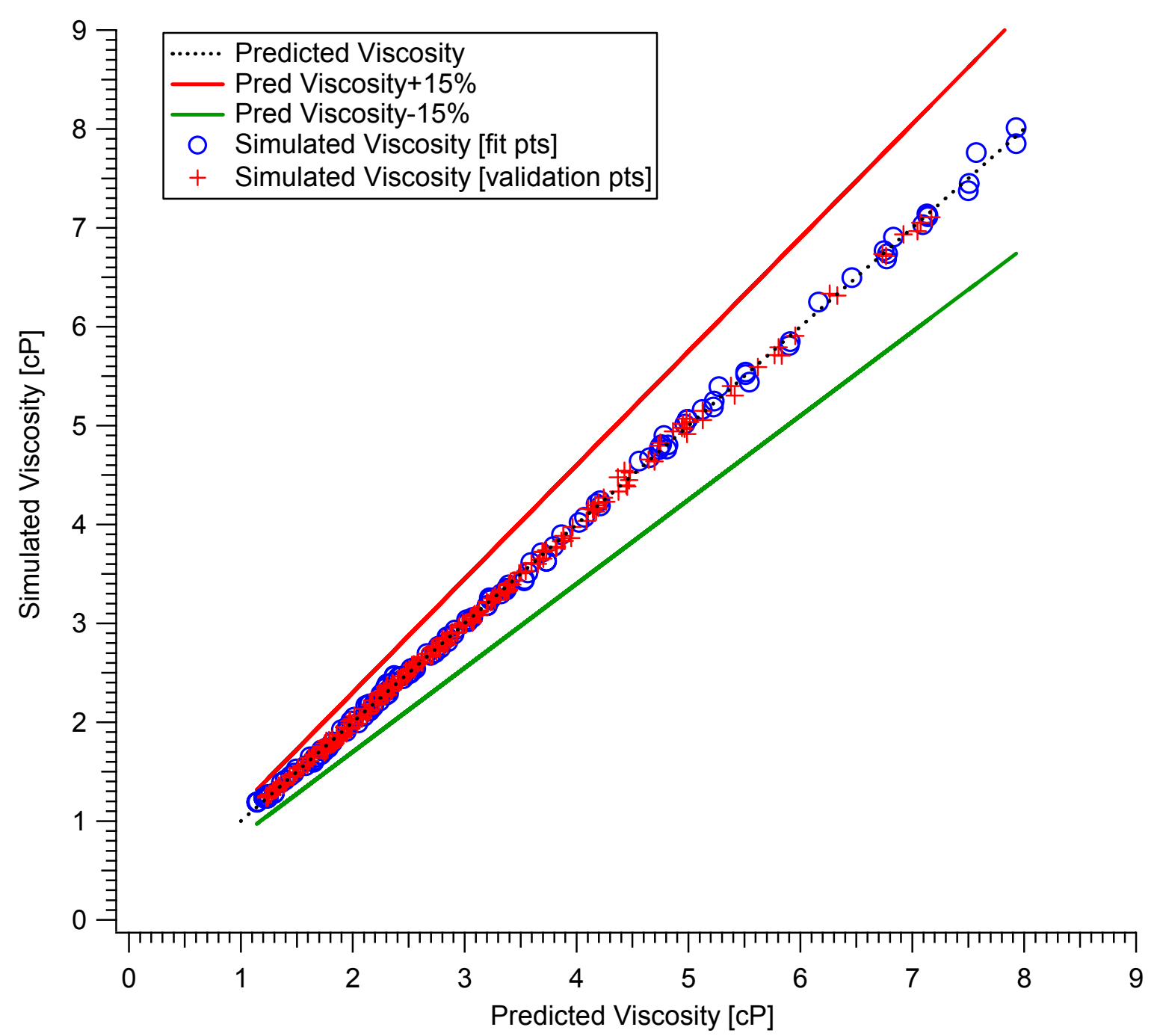

Figure 25. Simulated Viscosity versus Predicted Viscosity for Envelope C 
The Envelope C evaporator bottoms concentrate slurry conductivity at its steady state endpoint condition is represented by Equation 13 and shown in Figure 26.

\section{Equation 13}

$$
\begin{aligned}
\text { conductivity }_{\mathrm{EnvC}}\left[\frac{\mathrm{cal}}{\mathrm{s} \cdot \mathrm{cm} \cdot \mathrm{K}}\right] & =-7.282 \mathrm{E}-6 \cdot(\mathrm{SBS} / \text { Feed }-1)-1.498 \mathrm{E}-6 \cdot \frac{\mathrm{Temp}-45}{25}+ \\
& -9.488 \mathrm{E}-7 \cdot(\mathrm{SBS} / \text { Feed }-1) \cdot \frac{\text { Temp }-45}{25}+1.318 \mathrm{E}-3 \cdot x_{\mathrm{AlO} 2}+ \\
& 1.468 \mathrm{E}-3 \cdot x_{\mathrm{CO} 3}+1.385 \mathrm{E}-3 \cdot x_{\mathrm{NO} 2}+1.602 \mathrm{E}-3 \cdot x_{\mathrm{OH}}+ \\
& 1.258 \mathrm{E}-3 \cdot x_{\mathrm{SO} 4}-1.110 \mathrm{E}-5 \cdot[\mathrm{Na}]
\end{aligned}
$$

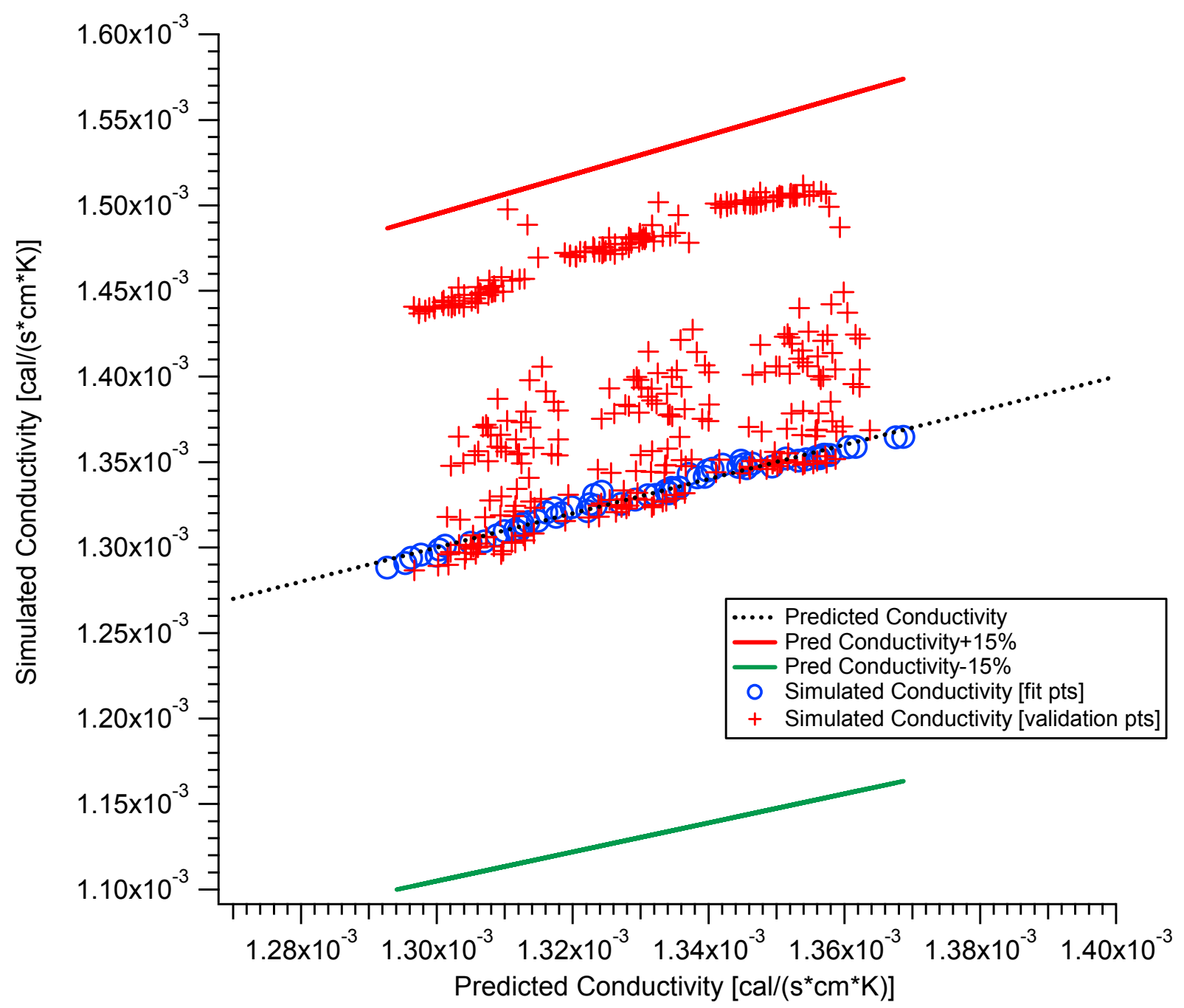

Figure 26. Simulated Conductivity versus Predicted Conductivity for Envelope C 
Envelope $\mathrm{C}$ bottoms concentrate slurry heat capacity at its steady state endpoint condition is represented by Equation 14 and shown in Figure 27.

\section{Equation 14}

$$
\begin{aligned}
\mathrm{Cp}_{\mathrm{EnvC}}\left[\frac{\mathrm{cal}}{\mathrm{g}{ }^{\circ} \mathrm{C}}\right]= & -0.008923 \cdot \frac{\mathrm{Temp}-45}{25}+0.9093 \cdot x_{\mathrm{AlO} 2}+0.8587 * x_{\mathrm{CO} 3}+0.8621 \cdot x_{\mathrm{NO} 2}+ \\
& 1.005 \cdot x_{\mathrm{OH}}+0.9629 \cdot x_{\mathrm{SO} 4}-0.03087 \cdot[\mathrm{Na}]
\end{aligned}
$$

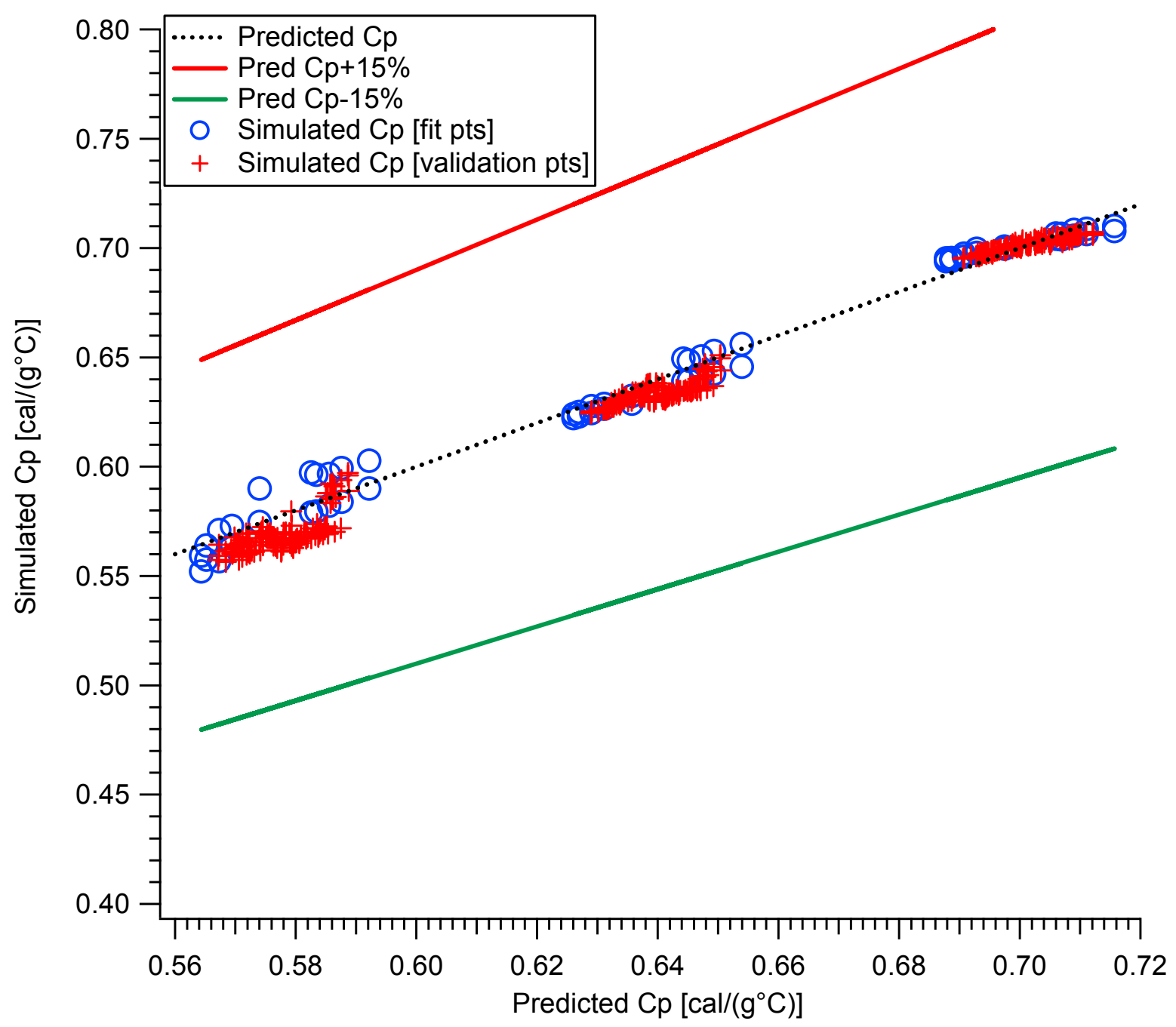

Figure 27. Simulated Cp versus Predicted $C p$ for Envelope $C$ 
The Envelope $\mathrm{C}$ evaporator bottoms concentrate slurry Na molarity at its steady state endpoint condition is represented by Equation 15 and shown in Figure 28.

\section{Equation 15}

$$
\begin{aligned}
{[\mathrm{Na}]_{\mathrm{EnvC}}\left[\frac{\mathrm{gmol}}{\mathrm{L}}\right]=} & -0.3663 \cdot(\mathrm{SBS} / \text { Feed }-1)+0.9644 \cdot \frac{\text { Temp }-45}{25}-33.00 \cdot x_{A l O 2}- \\
& 31.33 \cdot x_{\mathrm{CO} 3}-31.88 \cdot \mathrm{x}_{\mathrm{NO} 2}-32.29 \cdot \mathrm{x}_{\mathrm{OH}}-34.16 \cdot \mathrm{x}_{\mathrm{SO} 4}+30.49 \cdot \text { Density }- \\
& 1.764 \cdot(\mathrm{SBS} / \text { Feed }-1) \cdot(\text { Density }-1.322)+ \\
& 2.327 \cdot \frac{\text { Temp }-45}{25} \cdot(\text { Density }-1.322)
\end{aligned}
$$

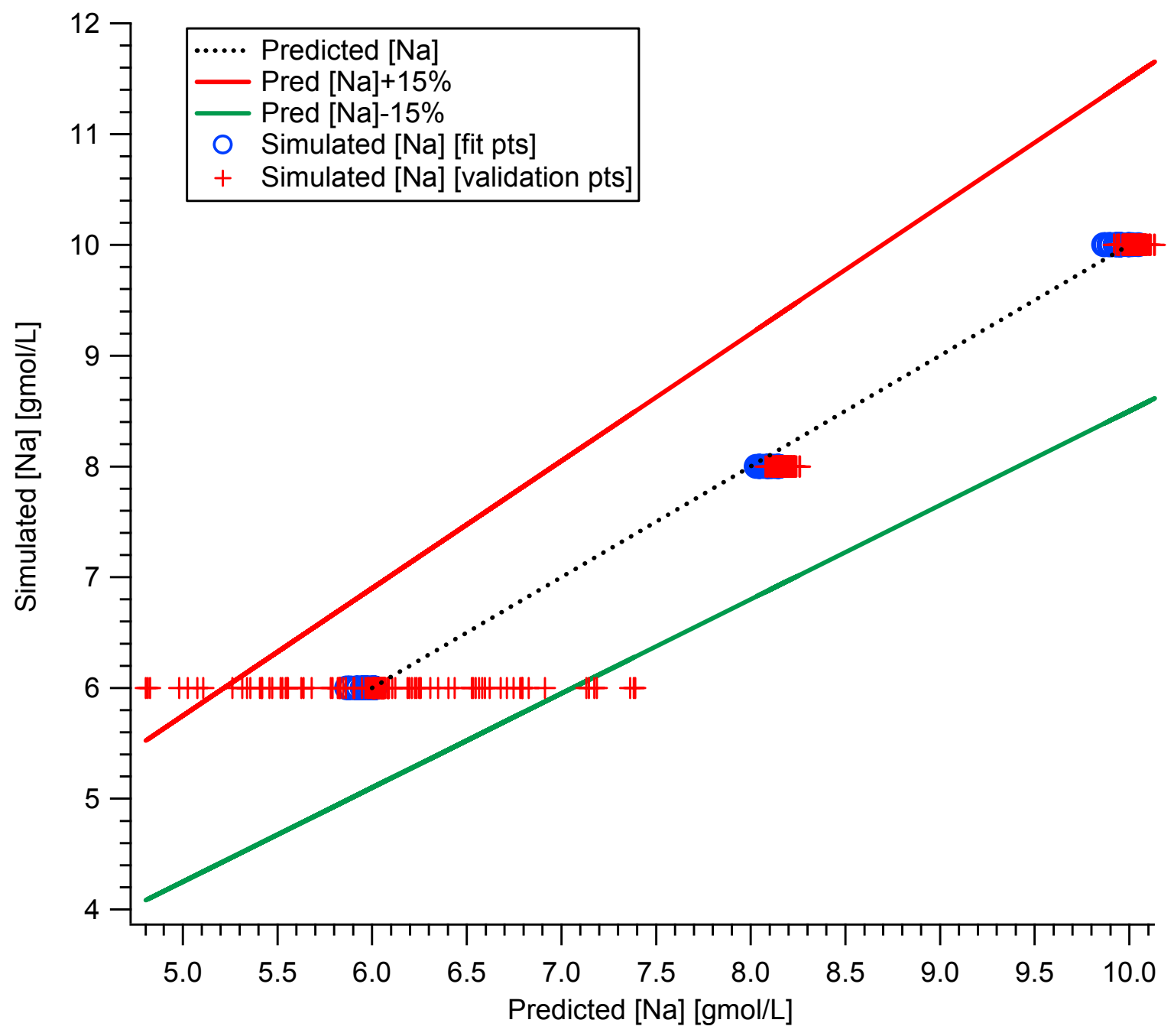

Figure 28. Simulated [Na] versus Predicted [Na] for Envelope C 
Although no valid solids prediction model could be developed for the bottoms concentrate stream for Envelope C, the general behavior of the simulated points can be seen in Figure 29 and Figure 30. The graphs show that the bottoms Na molarity concentration and the SBS/Feed ratio have the biggest impact on the amount solids that form compared to temperature. As the $\mathrm{Na}$ molarity or the SBS/Feed ratio increase the amount of insoluble solids also increases.

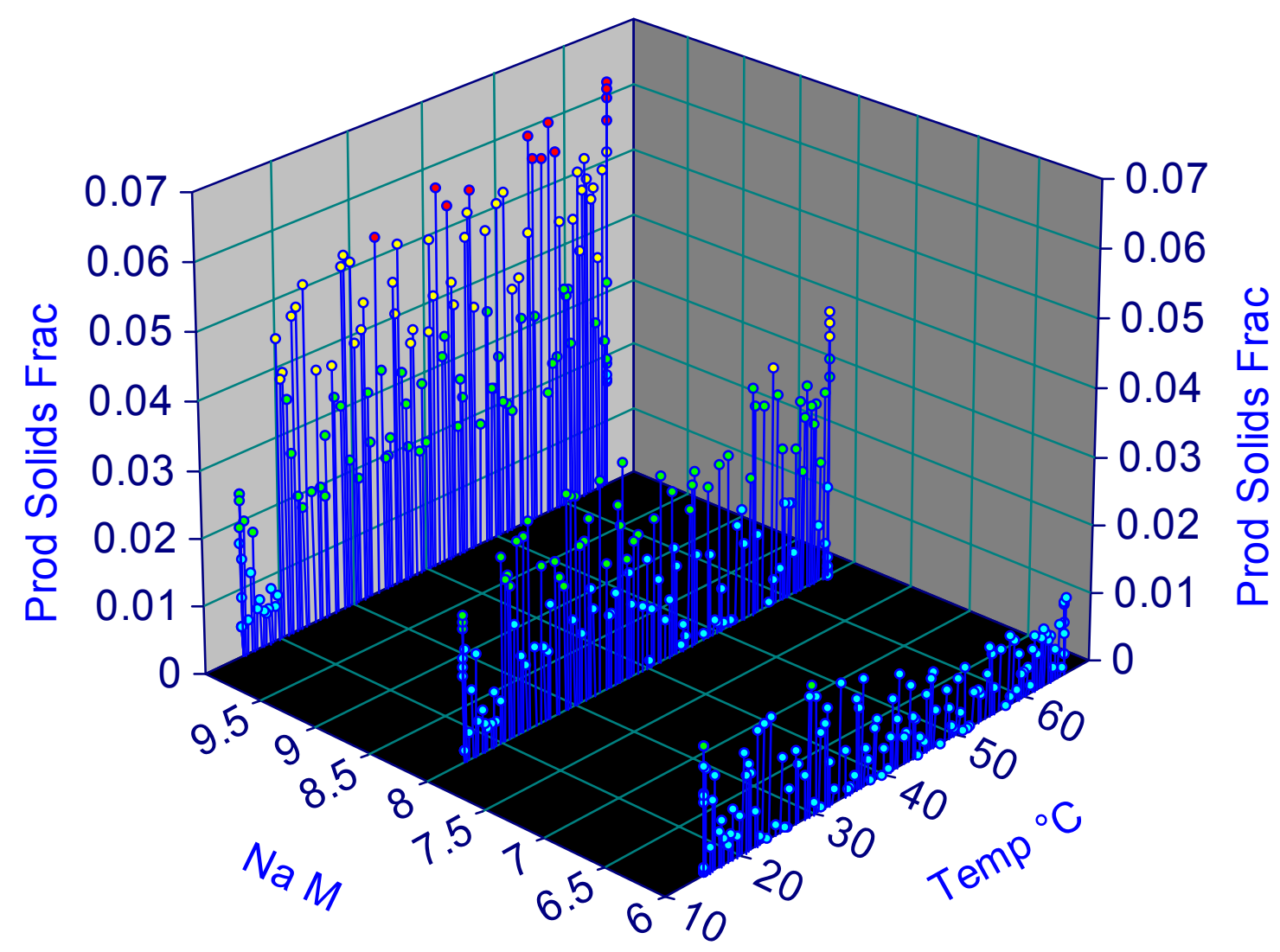

Figure 29. Envelope C Bottoms Solids Fraction versus Na Molarity and Temperature 


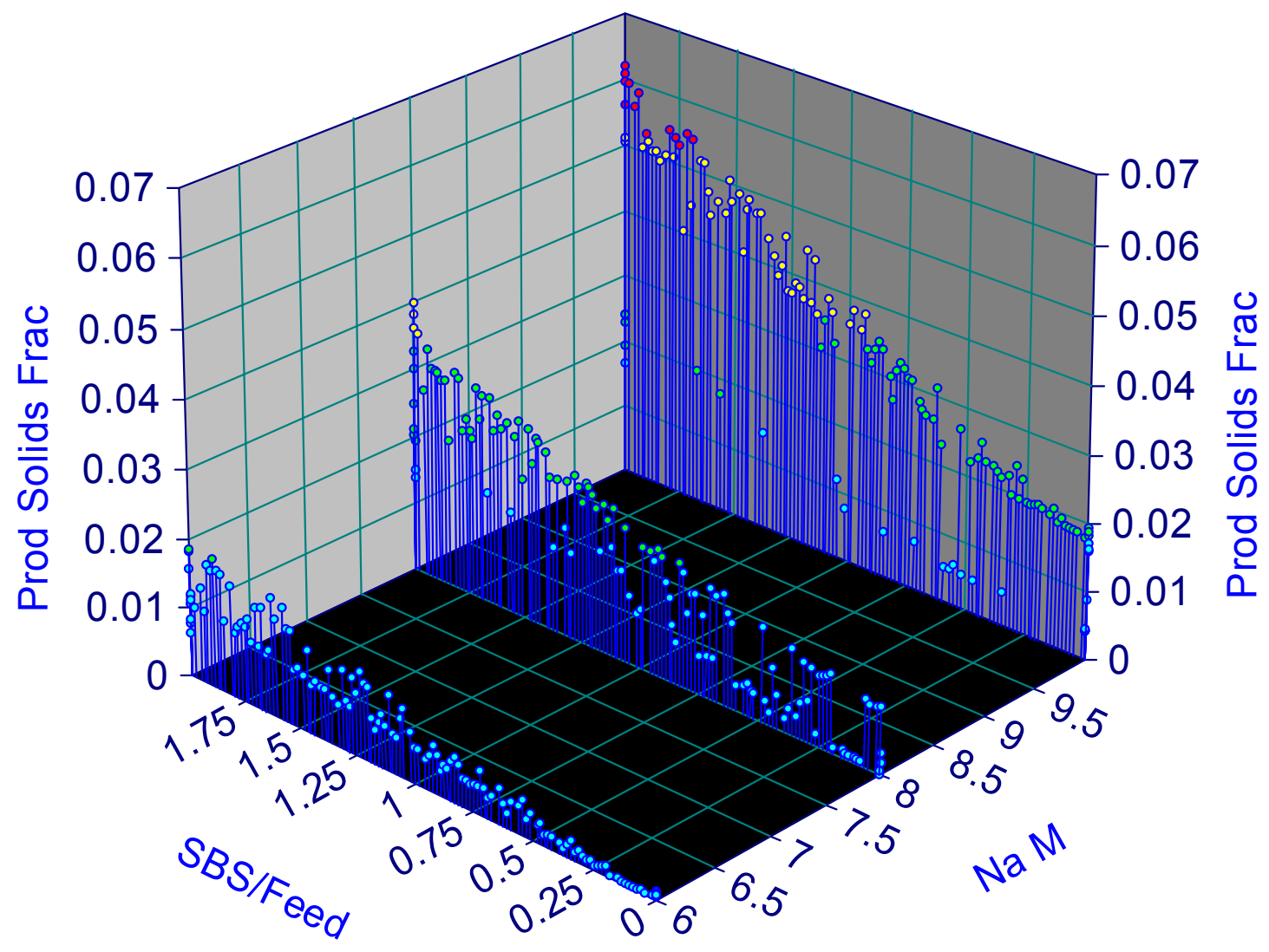

Figure 30. Envelope C Bottoms Solids Fraction versus Na Molarity and SBS/Feed 
WSRC-TR-2003-00269, REVISION 0

SRT-RPP-2003-00125, REVISION 0

\subsubsection{Comparison of Simulation Results with Experimental Results}

Experimental work was completed as part of Task S-94 to generate data concerning the evaporation of the LAW treated wastes for Envelopes A, B, and C. ${ }^{7}$ Several physical properties were measured for the various waste feeds against which the prediction models discussed earlier can be compared.

The density predictions for Envelope A were within $+/-6 \%$ of the measured values for the bottoms concentrate with a Na molarity between 2 and 10. The predicted density for Envelope A versus measured values is shown in Table 9. As is evidenced from the table, the model prediction for density does a good job at matching the experimental values.

Table 9. Comparison of Measured and Predicted Densities for Envelope A

\begin{tabular}{|c|c|c|c|c|c|c|c|c|c|c|c|c|}
\hline \multirow{2}{*}{$\begin{array}{c}\text { Temperature } \\
\text { (Degrees C) }\end{array}$} & \multicolumn{4}{|c|}{ Measured Density [g/ml] } & \multicolumn{4}{|c|}{ Predicted Density $[\mathrm{g} / \mathrm{ml}]$} & \multicolumn{4}{|c|}{ \% Difference $b / n$ Meas \& Pred Density } \\
\hline & 15 & 25 & 50 & 65 & 15 & 25 & 50 & 65 & 15 & 25 & 50 & 65 \\
\hline SM-01-INIT-A & 1.110 & 1.106 & 1.093 & 085 & 1.145 & .141 & 1.129 & 1.122 & $-3.2 \%$ & $-3.2 \%$ & $-3.3 \%$ & $3.4 \%$ \\
\hline SM-02-INIT-A & .100 & 1.096 & 1.084 & 1.075 & 1.136 & 1.132 & 1.121 & 1.114 & $-3.3 \%$ & $-3.3 \%$ & $-3.4 \%$ & $-3.6 \%$ \\
\hline SM-03-INIT-A & 100 & 1.096 & 1.083 & 1.074 & 1.125 & 1.120 & 1.109 & 1.102 & $-2.2 \%$ & $-2.2 \%$ & $-2.4 \%$ & $-2.6 \%$ \\
\hline SM-04-INIT-A & 1.093 & 1.089 & 1.076 & 1.068 & 1.103 & 1.098 & 1.087 & 1.080 & $-0.9 \%$ & $-0.9 \%$ & $-1.0 \%$ & $-1.2 \%$ \\
\hline SM-05-INIT-A & 1.096 & 1.091 & 1.079 & 1.070 & 1.106 & 1.102 & 1.090 & 1.083 & $-1.0 \%$ & $-0.9 \%$ & $-1.0 \%$ & $-1.2 \%$ \\
\hline SM-06-INIT-A & 1.103 & 1.099 & 1.086 & 1.077 & 1.131 & 1.127 & 1.115 & 1.109 & $-2.5 \%$ & $-2.5 \%$ & $-2.7 \%$ & $-2.9 \%$ \\
\hline SM-07-INIT-A & 1.114 & 1.110 & 1.097 & 1.089 & 1.137 & 1.132 & 1.120 & 1.113 & $-2.1 \%$ & $-2.0 \%$ & $-2.1 \%$ & $-2.2 \%$ \\
\hline & 1.100 & 1.096 & & 1.074 & & 1.122 & 1.111 & 1.104 & $-2.4 \%$ & $-2.4 \%$ & $-2.5 \%$ & $-2.7 \%$ \\
\hline SM-09-INIT-A & 1.108 & 1.104 & 1.091 & 1.082 & 1.134 & 1.129 & 1.118 & 1.111 & $-2.3 \%$ & $-2.3 \%$ & $-2.5 \%$ & $-2.7 \%$ \\
\hline SM-10-INIT-A & 1.106 & 1.102 & 1.089 & 1.080 & 1.121 & 1.116 & 1.105 & 1.098 & $-1.3 \%$ & $-1.3 \%$ & $-1.5 \%$ & $-1.7 \%$ \\
\hline SM-11-INIT-A & 1.105 & 1.100 & 1.088 & 1.079 & 1.130 & 1.126 & 1.114 & $\mid 1.107$ & $-2.3 \%$ & $-2.3 \%$ & $-2.4 \%$ & $-2.6 \%$ \\
\hline SM-12-INIT-A & 1.105 & 1.100 & 1.088 & 1.079 & 1.130 & 1.125 & 1.114 & 1.107 & $-2.3 \%$ & $-2.2 \%$ & $-2.4 \%$ & $-2.6 \%$ \\
\hline SM-01-06-A & 1.293 & 1.286 & 1.270 & 1.260 & 1.284 & 1.275 & 1.252 & 1.238 & $0.7 \%$ & $0.9 \%$ & $1.4 \%$ & $1.7 \%$ \\
\hline SM-02-06-A & 1.268 & 1.262 & 1.246 & 1.235 & 1.272 & 1.263 & 1.241 & 1.228 & $-0.3 \%$ & $-0.1 \%$ & $0.4 \%$ & $0.6 \%$ \\
\hline SM-03-06-A & 1.268 & 1.262 & 1.245 & 1.235 & 1.260 & 1.251 & 1.229 & 1.215 & $0.6 \%$ & $0.8 \%$ & $1.3 \%$ & $1.6 \%$ \\
\hline SM-04-06-A & 1.243 & 1.238 & 1.222 & 1.212 & 1.232 & 1.224 & 1.202 & 1.189 & $0.9 \%$ & $1.1 \%$ & $1.6 \%$ & $1.9 \%$ \\
\hline SM-05-06-A & & 1.245 & & & & & & 1.200 & $0.4 \%$ & $0.7 \%$ & $.3 \%$ & $1.6 \%$ \\
\hline SM-06-06-A & 1.278 & 1.272 & 1.256 & 1.245 & 1.267 & 1.258 & 1.236 & 1.222 & $0.9 \%$ & $1.1 \%$ & $1.6 \%$ & $1.9 \%$ \\
\hline SM-07-06-A & 1.297 & 1.291 & 1.275 & 1.265 & 1.282 & 1.273 & 1.249 & 1.235 & $1.1 \%$ & $1.4 \%$ & $2.0 \%$ & $2.4 \%$ \\
\hline SM-08-06-A & 1.271 & 1.265 & 1.248 & 1.238 & 1.260 & 1.252 & 1.229 & 1.216 & $0.9 \%$ & $1.1 \%$ & $1.5 \%$ & $1.8 \%$ \\
\hline SM-09-06-A & 1.294 & 1.288 & 1.271 & 1.258 & 1.273 & 1.264 & 1.241 & 1.227 & $1.6 \%$ & $1.9 \%$ & $2.3 \%$ & $2.4 \%$ \\
\hline SM-10-06-A & 1.283 & 1.277 & 1.261 & 1.251 & 1.262 & 1.253 & 1.230 & 1.216 & $1.7 \%$ & $1.9 \%$ & $2.5 \%$ & $2.8 \%$ \\
\hline SM-11-06-A & 1.282 & 1.276 & 1.260 & 1.250 & 1.274 & 1.264 & 1.241 & 1.227 & $0.7 \%$ & $0.9 \%$ & $1.5 \%$ & $1.8 \%$ \\
\hline SM-12-06-A & 1.282 & 1.276 & 1.260 & 1.250 & 1.271 & 1.261 & 1.239 & 1.225 & $0.9 \%$ & $1.1 \%$ & $1.7 \%$ & $2.0 \%$ \\
\hline SM-01-08-A & 1.341 & 1.335 & 1.318 & 1.308 & 1.330 & 1.319 & 1.292 & 1.276 & $0.9 \%$ & $1.2 \%$ & $1.9 \%$ & $2.4 \%$ \\
\hline SM-02-08-A & 1.335 & 1.328 & 1.310 & 1.298 & 1.329 & 1.318 & 1.292 & 1.275 & $0.4 \%$ & $0.7 \%$ & $1.4 \%$ & $1.7 \%$ \\
\hline SM-03-08-A & 1.340 & \begin{tabular}{|l|}
1.334 \\
\end{tabular} & 1.317 & 1.309 & 1.324 & 1.313 & 1.286 & \begin{tabular}{|l|}
1.269 \\
\end{tabular} & $1.2 \%$ & $1.6 \%$ & $2.4 \%$ & $3.0 \%$ \\
\hline SM-04-08-A & 1.307 & 1.301 & 1.285 & 1.276 & 1.294 & 1.283 & 1.256 & 1.240 & $1.1 \%$ & $1.4 \%$ & $2.3 \%$ & $2.8 \%$ \\
\hline
\end{tabular}


WSRC-TR-2003-00269, REVISION 0

SRT-RPP-2003-00125, REVISION 0

Table 9. Comparison of Measured and Predicted Densities for Envelope A (cont'd)

\begin{tabular}{|c|c|c|c|c|c|c|c|c|c|c|c|c|}
\hline \multirow{2}{*}{$\begin{array}{c}\text { Temperature } \\
\text { (Degrees C) }\end{array}$} & \multicolumn{4}{|c|}{ Measured Density [g/ml] } & \multicolumn{4}{|c|}{ Predicted Density [g/ml] } & \multicolumn{4}{|c|}{$\%$ Difference b/n Meas \& Pred Density } \\
\hline & 15 & 25 & 50 & 65 & 15 & 25 & 50 & 65 & 15 & 25 & 50 & 65 \\
\hline SM-05-08-A & 1.318 & 1.312 & 1.295 & 1.285 & 1.312 & 1.300 & 1.272 & 1.255 & $0.5 \%$ & $.9 \%$ & $1.8 \%$ & $2.3 \%$ \\
\hline SM-06-08-A & 1.353 & 1.346 & 1.328 & 1.317 & 1.331 & 1.320 & 1.292 & 1.276 & $1.6 \%$ & $2.0 \%$ & $2.7 \%$ & $3.1 \%$ \\
\hline SM-07-08-A & 375 & 1.368 & 1.351 & 1.341 & 1.351 & 1.339 & 1.310 & 1.292 & $1.8 \%$ & $.1 \%$ & $3.1 \%$ & $3.6 \%$ \\
\hline SM-08-08-A & 1.346 & 1.339 & 1.321 & 1.310 & 1.323 & 1.312 & 1.285 & 1.269 & $1.7 \%$ & $2.0 \%$ & $2.7 \%$ & $3.2 \%$ \\
\hline SM-09-08-A & 1.376 & 1.369 & 1.350 & 1.332 & 1.338 & 1.327 & 1.299 & 1.282 & $\%$ & $3.0 \%$ & $3.8 \%$ & $8 \%$ \\
\hline SM-10-08-A & 1.361 & 1.354 & 1.336 & 1.325 & 1.328 & 1.316 & 1.288 & 1.271 & $2.4 \%$ & $2.8 \%$ & $3.6 \%$ & $4.0 \%$ \\
\hline SM-11-08-A & 1.354 & 1.347 & 1.331 & 1.321 & 1.340 & 1.328 & 1.300 & 1.283 & $1.0 \%$ & $1.4 \%$ & $2.3 \%$ & $2.9 \%$ \\
\hline SM-12-08-A & 1.356 & 1.350 & 1.334 & 1.325 & 1.336 & 1.325 & 1.297 & 1.280 & $1.4 \%$ & $1.8 \%$ & $2.8 \%$ & $3.4 \%$ \\
\hline SM-01-10-A & 1.502 & 1.495 & 1.479 & 1.472 & 1.486 & 1.470 & 1.431 & 1.408 & $1.0 \%$ & $1.6 \%$ & $3.2 \%$ & $4.4 \%$ \\
\hline SM-02-10-A & 1.445 & 1.438 & 1.423 & 1.414 & 1.435 & 1.421 & 1.386 & 1.364 & $0.7 \%$ & $1.2 \%$ & $2.6 \%$ & $3.5 \%$ \\
\hline SM-03-10-A & 1.455 & 1.448 & 1.429 & 1.416 & 1.433 & 1.419 & 1.382 & 1.360 & $1.5 \%$ & $2.0 \%$ & $3.3 \%$ & $3.9 \%$ \\
\hline SM-04-10-A & 1.415 & 1.409 & 1.391 & 1.380 & 1.402 & 1.388 & 1.353 & 1.331 & $0.9 \%$ & $1.5 \%$ & $2.8 \%$ & $3.5 \%$ \\
\hline SM-05-10-A & 1.424 & 1.418 & 1.401 & 1.392 & 1.426 & 1.411 & 1.374 & 1.351 & $-0.1 \%$ & $0.5 \%$ & $2.0 \%$ & $3.0 \%$ \\
\hline SM-06-10-A & 1.468 & 1.462 & 1.445 & 1.434 & 1.439 & 1.424 & 1.388 & 1.366 & $2.0 \%$ & $2.6 \%$ & $3.9 \%$ & $4.7 \%$ \\
\hline SM-07-10-A & 1.499 & 1.492 & 1.474 & 1.463 & 1.465 & 1.450 & 1.411 & 1.388 & $2.3 \%$ & $2.9 \%$ & $4.2 \%$ & $5.1 \%$ \\
\hline SM-08-10-A & 1.455 & 1.451 & 1.435 & 1.423 & 1.432 & 1.417 & 1.381 & 1.359 & $1.6 \%$ & $2.3 \%$ & $3.7 \%$ & $4.4 \%$ \\
\hline SM-09-10-A & 1.477 & 1.472 & 1.458 & 1.447 & 1.488 & 1.472 & 1.432 & 1.407 & $-0.7 \%$ & $0.1 \%$ & $1.8 \%$ & $2.7 \%$ \\
\hline SM-10-10-A & 1.481 & 1.474 & 1.456 & 1.445 & 1.440 & 1.425 & 1.388 & 1.365 & $2.8 \%$ & $3.3 \%$ & $4.7 \%$ & $5.5 \%$ \\
\hline SM-11-10-A & 1.476 & 1.469 & 1.451 & 1.439 & 1.454 & 1.439 & 1.401 & 1.379 & $1.5 \%$ & $2.0 \%$ & $3.4 \%$ & $4.2 \%$ \\
\hline SM-12-10-A & 1.476 & 1.469 & 1.452 & 1.441 & 1.449 & 1.434 & 1.396 & 1.374 & $1.9 \%$ & $2.4 \%$ & $3.8 \%$ & $4.6 \%$ \\
\hline
\end{tabular}

The comparison of the measured versus predicted viscosities was not as good as for the densities as shown in Table 10. The best predictions were for $25^{\circ} \mathrm{C}$ and for $\mathrm{Na}$ molarities between 6 and 10 . The viscosity predictions for Envelope A were within $+/-20 \%$ for the $25^{\circ} \mathrm{C}$ measurements but only $+/-50 \%$ for $15^{\circ} \mathrm{C}$ and $60^{\circ} \mathrm{C}$ tests. It is expected that the lower and higher temperature extremes produced solids that are not accounted for by the viscosity prediction since it is based only on the supernate phase of the bottoms concentrate. For the $15^{\circ} \mathrm{C}$ and $60^{\circ} \mathrm{C}$ viscosity measurements versus predictions, less than $40 \%$ of the test points had the large $+/-50 \%$ error while the remaining $60 \%$ of the test points fell within $+/-20 \%$. Another factor contributing to the difference is that the viscosity prediction model was based on bottoms concentrate ranging from 6 to $10 \mathrm{M} \mathrm{Na}$ while the test data covered 2 to $10 \mathrm{M} \mathrm{Na}$. 
WSRC-TR-2003-00269, REVISION 0

SRT-RPP-2003-00125, REVISION 0

Table 10. Comparison of Measured versus Predicted Viscosities for Envelope A

\begin{tabular}{|c|c|c|c|c|c|c|c|c|c|c|c|}
\hline $\begin{array}{c}\text { Envelope } \\
\text { A } \\
\text { Sample }\end{array}$ & \begin{tabular}{|c|} 
Measured \\
Na $M$ at \\
$15^{\circ} \mathrm{C}$ and \\
$60^{\circ} \mathrm{C}$
\end{tabular} & \begin{tabular}{|c|} 
Measured \\
Viscosity \\
{$[\mathrm{cP}]$ at } \\
$15^{\circ} \mathrm{C}$
\end{tabular} & \begin{tabular}{|c|} 
Predicted \\
Viscosity \\
{$[\mathrm{cP}]$ at } \\
$15^{\circ} \mathrm{C}$
\end{tabular} & \begin{tabular}{|c|}
$\%$ \\
Diff \\
b/n \\
Meas \\
$\&$ \\
Pred \\
Visc. \\
at \\
$1^{\circ} \mathrm{C}$
\end{tabular} & $\begin{array}{c}\text { Measured } \\
\mathrm{Na} \mathrm{M} \text { at } \\
25^{\circ} \mathrm{C}\end{array}$ & \begin{tabular}{|c|} 
Measured \\
Viscosity \\
{$[\mathrm{cP}]$ at } \\
$25^{\circ} \mathrm{C}$
\end{tabular} & \begin{tabular}{|c|} 
Predicted \\
Viscosity \\
{$[\mathrm{cP}]$ at } \\
$25^{\circ} \mathrm{C}$
\end{tabular} & \begin{tabular}{|c|}
$\%$ \\
Diff \\
b/n \\
Meas \\
$\&$ \\
Pred \\
Visc. \\
at \\
$25^{\circ} \mathrm{C}$
\end{tabular} & $\begin{array}{c}\text { Measured } \\
\text { Viscosity } \\
{[\mathrm{cP}] \text { at }} \\
60^{\circ} \mathrm{C}\end{array}$ & \begin{tabular}{|c|} 
Predicted \\
Viscosity \\
{$[\mathrm{cP}]$ at } \\
$60^{\circ} \mathrm{C}$
\end{tabular} & $\begin{array}{c}\% \text { Diff } \\
\text { b/n } \\
\text { Meas \& } \\
\text { Pred } \\
\text { Visc. at } \\
60^{\circ} \mathrm{C}\end{array}$ \\
\hline $1-2$ & 2.10 & 1.90 & 1.75 & $8.0 \%$ & 2.01 & 1.41 & 1.40 & $0.0 \%$ & 0.73 & 1.00 & $-36.3 \%$ \\
\hline $1-6$ & 6.01 & 6.41 & 4.12 & $35.7 \%$ & 6.70 & 4.31 & 3.31 & $4.0 \%$ & 1.87 & 1.68 & $10.3 \%$ \\
\hline $1-8$ & 7.29 & 10.22 & 5.36 & $47.5 \%$ & 8.94 & 6.28 & 4.95 & $5.3 \%$ & 2.47 & 1.94 & $21.4 \%$ \\
\hline $2-2$ & 1.97 & 1.68 & 1.53 & $9.0 \%$ & 2.10 & 1.27 & 1.05 & $0.9 \%$ & 0.67 & 0.51 & $24.1 \%$ \\
\hline $2-6$ & 5.80 & 4.50 & 3.69 & $18.0 \%$ & 6.00 & 3.14 & 2.99 & $0.6 \%$ & 1.44 & 1.36 & $5.3 \%$ \\
\hline $2-8$ & 7.40 & 7.06 & 5.14 & $27.2 \%$ & 8.00 & 4.79 & 4.04 & $3.0 \%$ & 2.04 & 1.77 & $13.5 \%$ \\
\hline $3-2$ & 2.02 & 1.62 & 1.50 & $7.6 \%$ & 2.00 & 1.22 & 1.30 & $-0.3 \%$ & 0.65 & 0.83 & $-27.7 \%$ \\
\hline $3-6$ & 5.83 & 3.99 & 3.66 & $8.2 \%$ & 6.09 & 2.92 & 2.48 & $1.7 \%$ & 1.39 & 1.25 & $10.4 \%$ \\
\hline $3-8$ & 7.63 & 6.63 & 5.35 & $19.3 \%$ & 8.00 & 4.51 & 4.12 & $1.6 \%$ & 2.07 & 1.85 & $10.8 \%$ \\
\hline $4-2$ & 1.97 & 1.65 & 1.39 & $15.6 \%$ & 2.20 & 1.25 & 1.05 & $0.8 \%$ & 0.66 & 0.60 & $8.4 \%$ \\
\hline $4-6$ & 5.62 & 3.89 & 3.90 & $-0.3 \%$ & 6.46 & 2.83 & 2.89 & $-0.2 \%$ & 1.33 & 1.29 & $3.1 \%$ \\
\hline $4-8$ & 7.34 & 6.44 & 5.91 & $8.3 \%$ & 8.43 & 4.22 & 4.65 & $-1.7 \%$ & 1.91 & 1.91 & $-0.1 \%$ \\
\hline $5-2$ & 2.09 & 1.65 & 1.46 & $11.2 \%$ & 2.00 & 1.26 & 1.40 & $-0.5 \%$ & 0.65 & 0.99 & $-52.8 \%$ \\
\hline $5-6$ & 6.02 & 4.12 & 4.33 & $-5.0 \%$ & 6.28 & 3.08 & 3.07 & $0.1 \%$ & 1.40 & 1.68 & $-19.9 \%$ \\
\hline $5-8$ & 7.87 & 7.07 & 6.76 & $4.4 \%$ & 8.33 & 4.74 & 4.41 & $1.3 \%$ & 2.06 & 2.10 & $-2.1 \%$ \\
\hline $6-2$ & 2.01 & 1.54 & 1.30 & $15.7 \%$ & 2.18 & 1.21 & 1.08 & $0.5 \%$ & 0.64 & 0.52 & $19.3 \%$ \\
\hline $6-6$ & 5.83 & 3.78 & 3.55 & $6.1 \%$ & 6.00 & 2.76 & 2.99 & $-0.9 \%$ & 1.32 & 1.37 & $-3.9 \%$ \\
\hline $6-8$ & 7.62 & 6.48 & 5.31 & $18.1 \%$ & 8.66 & 4.31 & 4.61 & $-1.2 \%$ & 2.05 & 1.83 & $10.9 \%$ \\
\hline $7-2$ & 2.14 & 1.92 & 1.73 & \begin{tabular}{|l|}
$9.9 \%$ \\
\end{tabular} & 2.00 & 1.44 & 1.30 & $0.6 \%$ & 0.72 & 0.85 & $-18.5 \%$ \\
\hline $7-6$ & 6.25 & 6.76 & 4.62 & $31.7 \%$ & 6.00 & 4.67 & 2.44 & $8.9 \%$ & 1.88 & 1.34 & $28.7 \%$ \\
\hline $7-8$ & 8.17 & 13.40 & 7.10 & $47.0 \%$ & 8.00 & 8.47 & 4.12 & $17.4 \%$ & 3.27 & 2.01 & $38.5 \%$ \\
\hline $8-2$ & 2.02 & 1.50 & 1.13 & $24.9 \%$ & 2.16 & 1.15 & 1.04 & $0.5 \%$ & 0.62 & 0.61 & $1.0 \%$ \\
\hline $8-6$ & 5.79 & 3.20 & 3.13 & $2.2 \%$ & 6.60 & 2.39 & 2.97 & $-2.3 \%$ & 1.21 & 1.33 & $-9.7 \%$ \\
\hline $8-8$ & 7.56 & 5.09 & 4.63 & $9.1 \%$ & 8.38 & 3.52 & 4.61 & $-4.3 \%$ & 1.74 & 1.97 & $-13.5 \%$ \\
\hline $9-2$ & 2.03 & 1.63 & 1.33 & $18.3 \%$ & 2.13 & 1.25 & 1.44 & $-0.8 \%$ & 0.66 & 0.98 & $-48.8 \%$ \\
\hline $9-6$ & 5.95 & 4.37 & 3.73 & $14.6 \%$ & 6.66 & 3.13 & 3.28 & $-0.6 \%$ & 1.44 & 1.66 & $-15.5 \%$ \\
\hline $9-8$ & 7.79 & 7.42 & 5.66 & $23.7 \%$ & 7.74 & 4.95 & 3.95 & $4.0 \%$ & 2.17 & 2.08 & $4.2 \%$ \\
\hline $10-2$ & 2.07 & 1.70 & 1.27 & $25.5 \%$ & 2.13 & 1.30 & 1.06 & $1.0 \%$ & 0.67 & 0.53 & $21.2 \%$ \\
\hline $10-6$ & 6.04 & 4.96 & 3.41 & $31.3 \%$ & 6.55 & 3.55 & 3.38 & $0.7 \%$ & 1.59 & 1.43 & $10.2 \%$ \\
\hline $10-8$ & 7.90 & 9.51 & 5.05 & $46.9 \%$ & 8.48 & 5.55 & 4.45 & $4.4 \%$ & 2.47 & 1.91 & $22.7 \%$ \\
\hline $11-2$ & 2.10 & 1.65 & 1.29 & $21.6 \%$ & 2.25 & 1.19 & 1.39 & $-0.8 \%$ & 0.67 & 0.85 & $-26.2 \%$ \\
\hline $11-6$ & 6.14 & 4.69 & 3.70 & $21.2 \%$ & 6.83 & 3.55 & 2.88 & $2.7 \%$ & 1.54 & 1.32 & $14.6 \%$ \\
\hline $11-8$ & 8.00 & 8.01 & 5.59 & $30.2 \%$ & 8.44 & 5.39 & 4.50 & $3.5 \%$ & 2.38 & 1.96 & $17.7 \%$ \\
\hline $12-2$ & 2.07 & 1.66 & 1.50 & $9.8 \%$ & 2.24 & 1.31 & 1.06 & $1.0 \%$ & 0.67 & 0.62 & $7.1 \%$ \\
\hline $12-6$ & 6.04 & 4.68 & 3.96 & $15.3 \%$ & 6.77 & 3.43 & 3.07 & $1.4 \%$ & 1.54 & 1.39 & $10.0 \%$ \\
\hline $12-8$ & 7.89 & 7.95 & 5.95 & $25.1 \%$ & 8.00 & 5.37 & 4.27 & $4.4 \%$ & 2.21 & 2.07 & $6.2 \%$ \\
\hline
\end{tabular}


The thermal conductivity predictions for Envelope A were within $+/-25 \%$ of all the measured values for the bottoms concentrate with a Na molarity between 2 and 8 except for 3 samples. This large discrepancy is due in part to the fact that the simulated conductivity is for the supernate only, whereas the measured conductivity is for the entire slurry (supernate plus solids). Prior modeling work ${ }^{9}$ showed that the experimental conductivity has a standard deviation of $6.5 \%$ (about the value of water). The simulated conductivities are based on correction factors for the conductivity of water using various anions/cations as defined in Perry's Chemical Handbook. ${ }^{15}$ The simulated conductivities for all envelopes fell within a 3\% standard deviation of the value of water. Since the measurement error is higher than this value, the predicted conductivities cannot be distinguished from those of water. Therefore, there is no need to use the complicated prediction equation for conductivity when statistically a prediction for the conductivity of water is just as accurate. A complete list of the measured versus predicted conductivities is shown in Table 11.

Table 11. Comparison of Measured versus Predicted Conductivity for Envelope A

\begin{tabular}{|c|c|c|c|}
\hline $\begin{array}{c}\text { Envelope A } \\
\text { Sample }\end{array}$ & $\begin{array}{c}\text { Measured } \\
\text { Conductivity } \\
{\left[\mathrm{W} / \mathbf{m}^{\circ} \mathbf{C}\right] \text { at } 25^{\circ} \mathrm{C}}\end{array}$ & $\begin{array}{c}\text { Predicted } \\
\text { Conductivity } \\
{\left[\mathrm{W} / \mathbf{m}^{\circ} \mathrm{C}\right] \text { at } 25^{\circ} \mathrm{C}}\end{array}$ & $\begin{array}{c}\% \text { Diff b/n } \\
\text { measured \& } \\
\text { Predicted } \\
\text { Conductivity }\end{array}$ \\
\hline SM-55-01 & 0.531 & 0.571 & $-7.4 \%$ \\
\hline SM-55-02 & 0.539 & 0.627 & $-16.5 \%$ \\
\hline SM-55-04 & 0.474 & 0.591 & $-24.8 \%$ \\
\hline SM-55-05 & 0.482 & 0.571 & $-18.5 \%$ \\
\hline SM-55-06 & 0.512 & 0.627 & $-22.6 \%$ \\
\hline SM-55-07 & 0.603 & 0.594 & $1.4 \%$ \\
\hline SM-55-09 & 0.584 & 0.571 & $2.4 \%$ \\
\hline SM-55-10 & 0.519 & 0.627 & $-21.0 \%$ \\
\hline SM-55-12 & 0.576 & 0.591 & $-2.6 \%$ \\
\hline SM-01-6M & 0.445 & 0.564 & $-26.6 \%$ \\
\hline SM-02-6M & 0.547 & 0.623 & $-13.7 \%$ \\
\hline SM-10-6M & 0.486 & 0.622 & $-27.8 \%$ \\
\hline SM-01-8M & 0.513 & 0.585 & $-14.1 \%$ \\
\hline SM-03-8M & 0.504 & 0.572 & $-13.5 \%$ \\
\hline SM-04-8M & 0.348 & 0.579 & $-66.3 \%$ \\
\hline SM-05-8M & 0.523 & 0.586 & $-12.0 \%$ \\
\hline SM-06-8M & 0.485 & 0.575 & $-18.6 \%$ \\
\hline SM-07-8M & 0.559 & 0.572 & $-2.3 \%$ \\
\hline SM-08-8M & 0.499 & 0.579 & $-16.0 \%$ \\
\hline SM-10-8M & 0.500 & 0.576 & $-15.2 \%$ \\
\hline SM-12-8M & 0.542 & 0.580 & $-6.9 \%$ \\
\hline
\end{tabular}


The heat capacity predictions for Envelope A were within $+/-15 \%$ of all the measured values for the bottoms concentrate with a Na molarity between 2 and 8 except for 4 samples. Part of the reason for the 4 points that exceeded $+/-15 \%$ is that the experimental heat capacities are for the supernate, while those predicted are for the slurry (supernate plus solids). Analyses of the experimental samples show evidence of solids which in turn helps explain some of the deviation from predicted values. A complete listing of the measured versus predicted heat capacities is shown in Table 12.

Table 12. Comparison of Measured versus Predicted $\mathrm{Cp}$ for Envelope A

\begin{tabular}{|c|c|c|c|}
\hline Envelope A Sample & $\begin{array}{c}\text { Measured Cp } \\
{\left[\mathrm{cal} / \mathrm{g}^{*} \mathrm{~K}\right]} \\
\end{array}$ & $\begin{array}{c}\text { Predicted Cp } \\
{\left[\mathrm{cal} / \mathrm{g}^{*} \mathrm{~K}\right]} \\
\end{array}$ & $\begin{array}{c}\% \text { Diff } \mathbf{b} / \mathbf{n} \text { measured } \\
\text { \& Predicted Cp }\end{array}$ \\
\hline SM-55-01 & 0.849 & 0.840 & $1.1 \%$ \\
\hline SM-55-02 & 0.862 & 0.812 & $5.8 \%$ \\
\hline SM-55-04 & 0.865 & 0.829 & $4.2 \%$ \\
\hline SM-55-05 & 0.876 & 0.841 & $4.0 \%$ \\
\hline SM-55-06 & 0.860 & 0.811 & $5.7 \%$ \\
\hline SM-55-07 & 0.872 & 0.831 & $4.8 \%$ \\
\hline SM-55-08 & 0.858 & 0.830 & $3.2 \%$ \\
\hline SM-55-09 & 0.994 & 0.837 & $15.8 \%$ \\
\hline SM-55-10 & 0.871 & 0.812 & $6.8 \%$ \\
\hline SM-55-12 & 0.857 & 0.828 & $3.4 \%$ \\
\hline SM-01-6M & 0.731 & 0.677 & $7.3 \%$ \\
\hline SM-02-6M & 0.836 & 0.735 & $12.0 \%$ \\
\hline SM-03-6M & 0.769 & 0.694 & $9.8 \%$ \\
\hline SM-04-6M & 0.780 & 0.705 & $9.7 \%$ \\
\hline SM-05-6M & 0.788 & 0.690 & $12.4 \%$ \\
\hline SM-06-6M & 0.388 & 0.735 & $-89.7 \%$ \\
\hline SM-07-6M & 0.792 & 0.696 & $12.1 \%$ \\
\hline SM-08-6M & 0.762 & 0.702 & $7.9 \%$ \\
\hline SM-09-6M & 0.759 & 0.678 & $10.7 \%$ \\
\hline SM-10-6M & 0.776 & 0.724 & $6.7 \%$ \\
\hline SM-11-6M & 0.773 & 0.679 & $12.2 \%$ \\
\hline SM-01-8M & 0.768 & 0.611 & $20.4 \%$ \\
\hline SM-03-8M & 0.736 & 0.680 & $7.7 \%$ \\
\hline SM-04-8M & 0.506 & 0.656 & $-29.6 \%$ \\
\hline SM-05-8M & 0.761 & 0.633 & $16.9 \%$ \\
\hline SM-06-8M & 0.734 & 0.649 & $11.7 \%$ \\
\hline SM-07-8M & 0.732 & 0.680 & $7.1 \%$ \\
\hline SM-08-8M & 0.712 & 0.657 & $7.8 \%$ \\
\hline SM-10-8M & 0.739 & 0.652 & $11.7 \%$ \\
\hline SM-11-8M & 0.764 & 0.673 & $11.9 \%$ \\
\hline SM-12-8M & 0.726 & 0.667 & $8.2 \%$ \\
\hline
\end{tabular}


The sodium molarity predictions for Envelope A were within $+/-15 \%$ of the measured values for the bottoms concentrate with a Na molarity between 6 and 10. However, for the initial samples at 2 molar, the predicted values are within $+/-50 \%$. This discrepancy is due to the prediction being derived from data with $\mathrm{Na} \mathrm{M}$ between 6 and 10, and thus should not be expected to do well when extrapolated down to $2 \mathrm{M}$. Another reason for the large discrepancy is that it is difficult to derive a predictive relationship relating waste feed composition (dry basis), SBS/Feed ratio, bottoms temperature, and bottoms density to bottoms Na molarity. The measured sodium molarities versus model predictions are shown in Table 13.

No accurate prediction equations for the solubility of the evaporator bottoms stream in terms of the total insoluble solids present could be derived in either linear or nonlinear forms for Envelopes A, B, or C. Several attempts were made to include nonlinear and linear terms in the prediction fits, but the waste feed compositions, SBS to waste Feed ratio, the bottoms temperature, and $\mathrm{Na}$ molarity did not provide enough data about this phenomena. However, some general observations were made from the simulations.

For Envelope A, about 35\% of the simulated values (16 out of 336 total - including fit and validation points) had bottoms insoluble solids greater than $1 \mathrm{wt} \%$ but only about $10 \%$ of the simulated values (33 points) had insoluble solids greater than $2 \mathrm{wt} \%$. Of this $10 \%$ segment, $64 \%$ of the values were between $2 \mathrm{wt} \%$ and $5 \mathrm{wt} \%, 9 \%$ of the values were between $5 \mathrm{wt} \%$ and $10 \mathrm{wt} \%$, and $27 \%$ were between $10 \mathrm{wt} \%$ and $20 \mathrm{wt} \%$. The high weight percent insoluble solids were only observed at bottoms temperatures less than $20^{\circ} \mathrm{C}$ and sodium molarities greater than 8. The primary salts were sodium oxalate, sodium carbonate, sodium sulfate-carbonate, sodium fluoride, sodium aluminosilicate gelatin (NASGEL), and sodium nitrate. The sodium nitrate only came out at $10 \mathrm{M} \mathrm{Na}$ concentrations. For the simulated runs, NASGEL solids appear in the bottoms concentrate stream over a wider \% range at $6 \mathrm{Na} \mathrm{M}$ than $8 \mathrm{Na} \mathrm{M}$ and $10 \mathrm{Na} \mathrm{M}$ as shown in Figure 3. In fact at $10 \mathrm{Na} \mathrm{M}$, no NASGEL forms for the simulated runs performed. The NASGEL solids appear at all tested bottoms temperatures as shown in Figure 4. The $\mathrm{SBS} /$ Feed ratio has no effect on NASGEL formation at $10 \mathrm{Na} \mathrm{M}$ as shown in Figure 5. Increasing the SBS/Feed ratio above 1.75 slightly increases the chance of NASGEL formation as shown in Figure 6 . Increasing the SBS/feed ratio above 0.5 greatly increases the chance of NASGEL formation at $6 \mathrm{Na} \mathrm{M}$ as shown in Figure 7. Please note that due to the variability in the data, a low Na M like 6 and a high SBS/Feed ratio does not guarantee that NASGEL will form. The experimental solids measurements from prior work ${ }^{7}$ were only qualitative and not quantitative. This fact makes comparison of simulated solids predictions with experimental results difficult. However, Table 14 shows some simulated solids predictions for runs that have similar waste composition, SBS/Feed ratio, and $\mathrm{Na} \mathrm{M}$ as some experimental runs as identified by the column Experimental ID. The table also shows various trends as $\mathrm{Na} \mathrm{M}$ is increased with the same waste feed. A complete listing of all the simulated solids predictions for Envelope A is shown in Appendix B. 
WSRC-TR-2003-00269, REVISION 0

SRT-RPP-2003-00125, REVISION 0

Table 13. Comparison of Measured versus Predicted Na M for Envelope A

\begin{tabular}{|c|c|c|c|}
\hline Experimental ID & Measured Na M & Predicted Na M & $\begin{array}{c}\text { \%Diff Meas Na M\& } \\
\text { Pred Na M } @ 25^{\circ} \mathrm{C}\end{array}$ \\
\hline 1-INIT-A & 2.10 & 1.23 & $41.5 \%$ \\
\hline 2-INIT-A & 1.97 & 1.08 & $44.9 \%$ \\
\hline 3-INIT-A & 2.02 & 1.49 & $26.5 \%$ \\
\hline 4-INIT-A & 1.97 & 1.87 & $5.1 \%$ \\
\hline 5-INIT-A & 2.09 & 1.98 & $5.4 \%$ \\
\hline 6-INIT-A & 2.01 & 1.35 & $32.7 \%$ \\
\hline 7-INIT-A & 2.14 & 1.64 & $23.5 \%$ \\
\hline 8-INIT-A & 2.02 & 1.42 & $29.5 \%$ \\
\hline 9-INIT-A & 2.03 & 1.44 & $29.3 \%$ \\
\hline 10-INIT-A & 2.07 & 1.83 & $11.7 \%$ \\
\hline 11-INIT-A & 2.10 & 1.52 & $27.5 \%$ \\
\hline 12-INIT-A & 2.07 & 1.51 & $27.0 \%$ \\
\hline 1-6-A & 6.01 & 6.39 & $-6.4 \%$ \\
\hline $2-6-A$ & 5.80 & 5.84 & $-0.6 \%$ \\
\hline $3-6-A$ & 5.83 & 6.23 & $-6.8 \%$ \\
\hline 4-6-A & 5.62 & 6.13 & $-9.2 \%$ \\
\hline $5-6-A$ & 6.02 & 6.38 & $-5.9 \%$ \\
\hline $6-6-A$ & 5.83 & 6.31 & $-8.2 \%$ \\
\hline $7-6-A$ & 6.25 & 6.83 & $-9.2 \%$ \\
\hline $8-6-A$ & 5.79 & 6.26 & $-8.1 \%$ \\
\hline $9-6-A$ & 5.95 & 6.69 & $-12.5 \%$ \\
\hline $10-6-A$ & 6.04 & 6.85 & $-13.4 \%$ \\
\hline $11-6-\mathrm{A}$ & 6.14 & 6.55 & $-6.6 \%$ \\
\hline $12-6-\mathrm{A}$ & 6.04 & 6.52 & $-8.0 \%$ \\
\hline $1-8-A$ & 7.29 & 7.78 & $-6.7 \%$ \\
\hline $2-8-A$ & 7.40 & 7.71 & $-4.2 \%$ \\
\hline $3-8-A$ & 7.63 & 8.29 & $-8.7 \%$ \\
\hline 4-8-A & 7.34 & 7.96 & $-8.4 \%$ \\
\hline 5-8-A & 7.87 & 8.28 & $-5.2 \%$ \\
\hline $6-8-A$ & 7.62 & 8.42 & $-10.5 \%$ \\
\hline 7-8-A & 8.17 & 9.03 & $-10.5 \%$ \\
\hline 8-8-A & 7.56 & 8.38 & $-10.8 \%$ \\
\hline 9-8-A & 7.79 & 9.01 & $-15.6 \%$ \\
\hline 10-8-A & 7.90 & 9.04 & $-14.5 \%$ \\
\hline 11-8-A & 8.00 & 8.58 & $-7.3 \%$ \\
\hline $12-8-\mathrm{A}$ & 7.89 & 8.64 & $-9.6 \%$ \\
\hline
\end{tabular}


WSRC-TR-2003-00269, REVISION 0

SRT-RPP-2003-00125, REVISION 0

Table 13. Comparison of Measured versus Predicted Na M for Envelope A (cont'd)

\begin{tabular}{|c|c|c|c|}
\hline Experimental ID & Measured Na M & Predicted Na M & $\begin{array}{c}\text { \%Diff Meas Na M\& } \\
\text { Pred Na M } \mathbf{a}^{\circ} \mathbf{C}\end{array}$ \\
\hline 1-10-A & 11.70 & 12.36 & $-5.6 \%$ \\
\hline 2-10-A & 10.39 & 10.88 & $-4.7 \%$ \\
\hline 3-10-A & 10.70 & 11.56 & $-8.0 \%$ \\
\hline 4-10-A & 10.40 & 11.03 & $-6.0 \%$ \\
\hline 5-10-A & 11.09 & 11.31 & $-2.0 \%$ \\
\hline 6-10-A & 10.67 & 11.73 & $-10.0 \%$ \\
\hline 7-10-A & 11.39 & 12.57 & $-10.4 \%$ \\
\hline 8-10-A & 10.61 & 11.57 & $-9.0 \%$ \\
\hline 9-10-A & 12.00 & 11.97 & $0.2 \%$ \\
\hline $10-10-\mathrm{A}$ & 11.06 & 12.49 & $-12.9 \%$ \\
\hline $11-10-\mathrm{A}$ & 11.22 & 12.06 & $-7.5 \%$ \\
\hline 12-10-A & 11.05 & 12.06 & $-9.2 \%$ \\
\hline
\end{tabular}


WSRC-TR-2003-00269, REVISION 0 SRT-RPP-2003-00125, REVISION 0

Table 14. Predicted Solids for Envelope A Simulations - Part 1

\begin{tabular}{|c|c|c|c|c|c|c|c|c|c|c|c|c|c|c|c|c|c|c|c|c|}
\hline $\begin{array}{c}\text { Experi- } \\
\text { mental ID }\end{array}$ & $\begin{array}{l}\text { Sim. } \\
\text { Point } \\
\text { Type }\end{array}$ & $\begin{array}{l}\text { Sim. } \\
\text { ID }\end{array}$ & Temp & $\begin{array}{l}\text { SBS/ } \\
\text { Feed }\end{array}$ & $\begin{array}{l}\mathbf{N a} \\
\mathbf{M}\end{array}$ & $\begin{array}{l}\text { ALO } \\
\text { H3 } \\
{[\mathrm{g}]}\end{array}$ & $\begin{array}{c}\text { CA3P } \\
\text { O42 } \\
{[\mathrm{g}]}\end{array}$ & $\begin{array}{c}\text { CACO } \\
3[\mathrm{~g}]\end{array}$ & $\begin{array}{l}\text { CAF } \\
2 \text { [g] }\end{array}$ & $\begin{array}{c}\text { CAOH } \\
2[\mathrm{~g}]\end{array}$ & $\begin{array}{c}\text { CATIO } \\
3[\mathrm{~g}]\end{array}$ & $\begin{array}{c}\text { CROH } \\
3 \text { [g] }\end{array}$ & $\begin{array}{c}\text { HYDR } \\
\text { OSOD } \\
\text { [g] }\end{array}$ & $\begin{array}{c}\text { MGOH } \\
2[\mathrm{~g}]\end{array}$ & $\begin{array}{c}\text { NA2C2O } \\
4[\mathrm{~g}]\end{array}$ & \begin{tabular}{|} 
NA2CO \\
$3.1 \mathrm{H} 2 \mathrm{O}$ \\
{$[\mathrm{g}]$}
\end{tabular} & $\begin{array}{c}\text { NA2U2O } \\
7[\mathrm{~g}]\end{array}$ & $\begin{array}{c}\text { NA3FSO } \\
4[\mathrm{~g}]\end{array}$ & $\begin{array}{l}\text { NA6S } \\
\text { O42C } \\
\text { O3 [g] }\end{array}$ & $\begin{array}{c}\text { NAF } \\
{[\mathrm{g}]}\end{array}$ \\
\hline SM-55-10 & MPV & 10EA035 & 15.0 & 2.0 & 10 & 0 & 0 & 0 & 0 & 321 & 51 & 0 & 0 & 75 & 727 & 0 & 0 & 0 & 0 & 7542 \\
\hline SM-55-10 & MPV & $8 \mathrm{EA} 035$ & 15.0 & 2.0 & 8 & 0 & 0 & 0 & 336 & 0 & 50 & 0 & 1448 & 75 & 607 & 0 & 0 & 0 & 0 & 5532 \\
\hline SM-55-10 & MPV & 6EA035 & 15.0 & 2.0 & 6 & 0 & 0 & 0 & 338 & 0 & 51 & 0 & 0 & 75 & 303 & 0 & 0 & 0 & 0 & 1841 \\
\hline SM-55-09 & OLH & 10EA084 & 15.9 & 0.3 & 10 & 0 & 0 & 0 & 0 & 53 & 8 & 0 & 0 & 12 & 1127 & 0 & 0 & 0 & 0 & 9985 \\
\hline SM-55-09 & OLH & 8EA084 & 15.9 & 0.3 & 8 & 0 & 0 & 0 & 55 & 0 & 8 & 0 & 0 & 12 & 972 & 0 & 0 & 0 & 0 & 8017 \\
\hline SM-55-09 & OLH & 6EA084 & 15.9 & 0.3 & 6 & 3318 & 0 & 0 & 56 & 0 & 8 & 0 & 0 & 12 & 618 & 0 & 0 & 0 & 0 & 4038 \\
\hline SM-55-09 & $\mathrm{OLH}$ & 10EA100 & 23.0 & 1.6 & 10 & 0 & 0 & 0 & 0 & 287 & 45 & 0 & 0 & 67 & 1089 & 0 & $4.24 \mathrm{E}-07$ & 6894 & 0 & 0 \\
\hline SM-55-09 & $\mathrm{OLH}$ & $8 \mathrm{EA} 100$ & 23.0 & 1.6 & 8 & 0 & 0 & 0 & 0 & 285 & 45 & 0 & 0 & 67 & 907 & 0 & 4.04E-07 & 3393 & 0 & 0 \\
\hline SM-55-09 & OLH & 6EA100 & 23.0 & 1.6 & 6 & 0 & 375 & 0 & 11 & 0 & 45 & 0 & 0 & 67 & 429 & 0 & $2.46 \mathrm{E}-07$ & 0 & 0 & 0 \\
\hline SM-55-12 & MPV & 10EA037 & 15.0 & 2.0 & 10 & 0 & 0 & 0 & 0 & 362 & 57 & 0 & 0 & 85 & 1394 & 0 & $3.09 \mathrm{E}-05$ & 0 & 0 & 0 \\
\hline SM-55-01 & MPV & 8EA037 & 15.0 & 2.0 & 8 & 0 & 0 & 0 & 0 & 360 & 57 & 0 & 0 & 85 & 1220 & 5020 & $3.04 \mathrm{E}-05$ & 0 & 0 & 0 \\
\hline SM-55-12 & MPV & 6EA037 & 15.0 & 2.0 & 6 & 6421 & 0 & 484 & 0 & & 57 & 0 & 0 & 85 & 861 & 25304 & $2.90 \mathrm{E}-05$ & 0 & 0 & 0 \\
\hline SM-55-01 & MPV & 10EA067 & 66.0 & 2.0 & 10 & 0 & 0 & 0 & 0 & 361 & 57 & 0 & 0 & 85 & 931 & 16857 & 0 & 0 & 18151 & 0 \\
\hline SM-55-12 & MPV & 8EA067 & 66.0 & 2.0 & 8 & 0 & 0 & 486 & 0 & 0 & 57 & 12 & 0 & 85 & 309 & 0 & 0 & 0 & 10990 & 0 \\
\hline SM-55-01 & MPV & 6ЕA067 & 66.0 & 2.0 & 6 & 0 & 0 & 486 & 0 & 0 & 57 & 28 & 0 & 85 & 0 & 0 & 0 & 0 & 0 & 0 \\
\hline SM-55-12 & MPV & 10EA077 & 66.0 & 2.0 & 10 & 0 & 0 & 0 & 0 & 361 & 57 & & 0 & 85 & 931 & 16857 & 0 & 0 & 18151 & 0 \\
\hline SM-55-01 & MPV & 8EA077 & 66.0 & 2.0 & 8 & 0 & 0 & 486 & 0 & 0 & 57 & 12 & 0 & 85 & 309 & 0 & 0 & 0 & 10990 & 0 \\
\hline SM-55-12 & MPV & 6ЕA077 & 66.0 & 2.0 & 6 & 0 & 0 & 486 & 0 & 0 & 57 & 28 & 0 & 85 & 0 & 0 & 0 & 0 & 0 & 0 \\
\hline SM-55-02 & MPV & 10EA066 & 66.0 & 2.0 & 10 & 0 & 0 & 0 & 373 & 0 & 56 & 5 & 0 & 83 & 969 & 0 & 0 & 0 & 17478 & 9692 \\
\hline SM-55-03 & MPV & 8EA066 & 66.0 & 2.0 & 8 & 0 & 0 & 0 & 374 & 0 & 56 & 25 & 0 & 83 & 332 & 0 & 0 & 0 & 8824 & 6829 \\
\hline SM-55-02 & MPV & 6ЕA066 & 66.0 & 2.0 & 6 & 0 & 0 & 0 & 375 & 0 & 56 & 37 & 0 & 83 & 0 & 0 & 0 & 0 & 0 & 418 \\
\hline SM-55-03 & MPV & $10 \mathrm{EA} 076$ & 66.0 & 2.0 & 10 & 0 & 0 & 0 & 373 & 0 & 56 & 5 & 0 & 83 & 969 & 0 & 0 & 0 & 17478 & 9692 \\
\hline SM-55-02 & MPV & 8EA076 & 66.0 & 2.0 & 8 & 0 & 0 & 0 & 374 & 0 & 56 & 25 & 0 & 83 & 332 & 0 & 0 & 0 & 8824 & 6829 \\
\hline SM-55-03 & MPV & 6EA076 & 66.0 & 2.0 & 6 & 0 & 0 & 0 & 375 & 0 & 56 & 37 & 0 & 83 & 0 & 0 & 0 & 0 & 0 & 418 \\
\hline SM-55-04 & MPV & 10EA061 & 66.0 & 2.0 & 10 & 0 & 0 & 0 & 0 & 315 & 50 & 0 & 0 & 74 & 214 & 0 & 0 & 0 & 13876 & 0 \\
\hline SM-55-05 & MPV & $8 \mathrm{EA} 061$ & 66.0 & 2.0 & 8 & 0 & 0 & 0 & 0 & 313 & 50 & 0 & 0 & 74 & 0 & 0 & 0 & 0 & 4085 & 0 \\
\hline SM-55-04 & MPV & $6 \mathrm{EA} 061$ & 66.0 & 2.0 & 6 & 0 & 435 & 0 & 0 & 0 & 50 & 0 & 0 & 74 & 0 & 0 & 0 & 0 & 0 & 0 \\
\hline SM-55-05 & MPV & 10EA062 & 66.0 & 2.0 & 10 & 0 & 0 & 0 & 0 & 319 & 50 & 0 & 0 & 75 & 202 & 0 & 0 & 0 & 13992 & 0 \\
\hline SM-55-04 & MPV & 8EA062 & 66.0 & 2.0 & 8 & 0 & 0 & 0 & 0 & 318 & 50 & 0 & 0 & 75 & 0 & 0 & 0 & 0 & 4339 & 0 \\
\hline
\end{tabular}


WSRC-TR-2003-00269, REVISION 0 SRT-RPP-2003-00125, REVISION 0

Table 14. Predicted Solids from Envelope A Simulations - Part 1 (cont'd)

\begin{tabular}{|c|c|c|c|c|c|c|c|c|c|c|c|c|c|c|c|c|c|c|c|c|}
\hline $\begin{array}{c}\text { Experi- } \\
\text { mental ID }\end{array}$ & $\begin{array}{l}\text { Sim. } \\
\text { Point } \\
\text { Type }\end{array}$ & $\begin{array}{c}\text { Sim. } \\
\text { ID }\end{array}$ & Temp & $\begin{array}{l}\text { SBS/ } \\
\text { Feed }\end{array}$ & $\begin{array}{c}\mathbf{N a} \\
\mathbf{M}\end{array}$ & $\begin{array}{l}\text { ALO } \\
\text { H3 } \\
{[\mathrm{g}]}\end{array}$ & $\begin{array}{c}\text { CA3P } \\
\text { O42 } \\
\text { [g] }\end{array}$ & $\begin{array}{c}\text { CACO } \\
3[\mathrm{~g}]\end{array}$ & $\begin{array}{l}\text { CAF } \\
2 \text { [g] }\end{array}$ & $\begin{array}{c}\text { CAOH } \\
2[\mathrm{~g}]\end{array}$ & $\begin{array}{c}\text { CATIO } \\
3 \text { [g] }\end{array}$ & $\begin{array}{c}\text { CROH } \\
3[\mathrm{~g}]\end{array}$ & $\begin{array}{c}\text { HYDR } \\
\text { OSOD } \\
{[\mathrm{g}]}\end{array}$ & $\begin{array}{c}\text { MGOH } \\
2 \text { [g] }\end{array}$ & $\begin{array}{c}\mathrm{NA2C2O} \\
4[\mathrm{~g}]\end{array}$ & $\begin{array}{c}\text { NA2CO } \\
3.1 H 2 O \\
{[g]}\end{array}$ & $\begin{array}{c}\text { NA2U2O } \\
7[\mathrm{~g}]\end{array}$ & $\begin{array}{c}\text { NA3FSO } \\
4[\mathrm{~g}]\end{array}$ & $\begin{array}{l}\text { NA6S } \\
\text { O42C } \\
\text { O3 [g] }\end{array}$ & $\begin{array}{c}\text { NAF } \\
{[\mathrm{g}]}\end{array}$ \\
\hline SM-55-05 & MPV & 6EA062 & 66.0 & 2.0 & 6 & 0 & 440 & 0 & 0 & 0 & 50 & 0 & 0 & 75 & 0 & 0 & 0 & 0 & 0 & 0 \\
\hline SM-55-04 & MPV & 10EA071 & 66.0 & 2.0 & 10 & 0 & 0 & 0 & 0 & 315 & 50 & 0 & 0 & 74 & 214 & 0 & 0 & 0 & 13876 & 0 \\
\hline SM-55-05 & MPV & 8EA071 & 66.0 & 2.0 & 8 & 0 & 0 & 0 & 0 & 313 & 50 & 0 & 0 & 74 & 0 & 0 & 0 & 0 & 4085 & 0 \\
\hline SM-55-04 & MPV & 6EA071 & 66.0 & 2.0 & 6 & 0 & 435 & 0 & 0 & 0 & 50 & 0 & 0 & 74 & 0 & 0 & 0 & 0 & 0 & 0 \\
\hline SM-55-05 & MPV & 10EA072 & 66.0 & 2.0 & 10 & 0 & 0 & 0 & 0 & 319 & 50 & 0 & 0 & 75 & 202 & 0 & 0 & 0 & 13992 & 0 \\
\hline SM-55-04 & MPV & 8EA072 & 66.0 & 2.0 & 8 & 0 & 0 & 0 & 0 & 318 & 50 & 0 & 0 & 75 & 0 & 0 & 0 & 0 & 4339 & 0 \\
\hline SM-55-05 & MPV & 6EA072 & 66.0 & 2.0 & 6 & 0 & 440 & 0 & 0 & 0 & 50 & 0 & 0 & 75 & 0 & 0 & 0 & 0 & 0 & 0 \\
\hline SM-55-06 & MPV & 10EA064 & 66.0 & 2.0 & 10 & 0 & 0 & 0 & 0 & 340 & 54 & 0 & 0 & 80 & 471 & 0 & 0 & 0 & 4966 & 7849 \\
\hline SM-55-06 & MPV & 8EA064 & 66.0 & 2.0 & 8 & 0 & 0 & 0 & 358 & 0 & 54 & 0 & 0 & 80 & 0 & 0 & 0 & 0 & 0 & 4794 \\
\hline SM-55-06 & MPV & 6EA064 & 66.0 & 2.0 & 6 & 0 & 0 & 0 & 359 & 0 & 54 & 18 & 0 & 80 & 0 & 0 & 0 & 0 & 0 & \\
\hline SM-55-06 & MPV & 10EA074 & 66.0 & 2.0 & 10 & 0 & 0 & 0 & 0 & 340 & 54 & 0 & 0 & 80 & 471 & 0 & 0 & 0 & 4966 & 7849 \\
\hline SM-55-06 & MPV & $8 \mathrm{EA} 074$ & 66.0 & 2.0 & 8 & 0 & 0 & 0 & 358 & 0 & 54 & 0 & 0 & 80 & 0 & 0 & 0 & 0 & 0 & 4794 \\
\hline SM-55-06 & MPV & 6ЕA074 & 66.0 & 2.0 & 6 & 0 & 0 & 0 & 359 & 0 & 54 & 18 & 0 & 80 & 0 & 0 & 0 & 0 & 0 & 0 \\
\hline SM-55-06 & $\mathrm{OLH}$ & 10EA138 & 43.2 & 1.8 & 10 & 0 & 0 & 0 & 335 & 0 & 50 & 0 & 0 & 75 & 1113 & 0 & $8.69 \mathrm{E}-06$ & 0 & 15423 & 1190 \\
\hline SM-55-06 & $\mathrm{OLH}$ & $8 \mathrm{EA} 138$ & 43.2 & 1.8 & 8 & 0 & 0 & 0 & 335 & 0 & 50 & 0 & 0 & 75 & 705 & 0 & 0 & 0 & 380 & 0 \\
\hline SM-55-06 & $\mathrm{OLH}$ & 6EA138 & 43.2 & 1.8 & 6 & 0 & 0 & 0 & 333 & 0 & 50 & 0 & 0 & 75 & 0 & 0 & 0 & 0 & 0 & 0 \\
\hline SM-55-07 & MPV & 10EA070 & 66.0 & 2.0 & 10 & 0 & 0 & 0 & 0 & 337 & 53 & 0 & 0 & 79 & 414 & 0 & 0 & 0 & 7758 & 6630 \\
\hline SM-55-07 & MPV & 8EA070 & 66.0 & 2.0 & 8 & 0 & 0 & 0 & 354 & 0 & 53 & 0 & 0 & 79 & 27 & 0 & 0 & 0 & 0 & 3986 \\
\hline SM-55-07 & MPV & \begin{tabular}{|l|}
$6 \mathrm{EA} 070$ \\
\end{tabular} & 66.0 & 2.0 & 6 & 0 & 0 & 0 & 355 & 0 & 53 & 0 & 0 & 79 & 0 & 0 & 0 & 0 & 0 & 0 \\
\hline SM-55-07 & MPV & 10EA080 & 66.0 & 2.0 & 10 & 0 & 0 & 0 & 0 & 337 & 53 & 0 & 0 & 79 & 414 & 0 & 0 & 0 & 7758 & 6630 \\
\hline SM-55-07 & MPV & $8 \mathrm{EA} 080$ & 66.0 & 2.0 & 8 & 0 & 0 & 0 & 354 & 0 & 53 & 0 & 0 & 79 & 27 & 0 & 0 & 0 & 0 & 3986 \\
\hline SM-55-07 & MPV & 6EA080 & 66.0 & 2.0 & 6 & 0 & 0 & 0 & 355 & 0 & 53 & 0 & 0 & 79 & 0 & 0 & 0 & 0 & 0 & 0 \\
\hline SM-55-08 & MPV & 10EA063 & 66.0 & 2.0 & 10 & 0 & 0 & 0 & 0 & 352 & 56 & 0 & 0 & 83 & 494 & 0 & 0 & 0 & 2511 & 0 \\
\hline SM-55-08 & MPV & 8EA063 & 66.0 & 2.0 & 8 & 0 & 491 & 0 & 0 & 0 & 56 & 21 & 0 & 83 & 0 & 0 & 0 & 0 & 0 & 0 \\
\hline SM-55-08 & MPV & 6EA063 & 66.0 & 2.0 & 6 & 0 & 490 & 0 & 0 & 0 & 56 & 35 & 0 & 83 & 0 & 0 & 0 & 0 & 0 & 0 \\
\hline SM-55-08 & MPV & 10EA073 & 66.0 & 2.0 & 10 & 0 & 0 & 0 & 0 & 352 & 56 & 0 & 0 & 83 & 494 & 0 & 0 & 0 & 2511 & 0 \\
\hline SM-55-08 & MPV & 8EA073 & 66.0 & 2.0 & 8 & 0 & 491 & 0 & 0 & 0 & 56 & 21 & 0 & 83 & 0 & 0 & 0 & 0 & 0 & 0 \\
\hline SM-55-08 & MPV & 6EA073 & 66.0 & 2.0 & 6 & 0 & 490 & 0 & 0 & 0 & 56 & 35 & 0 & 83 & 0 & 0 & 0 & 0 & 0 & 0 \\
\hline SM-55-09 & MPV & 10EA068 & 66.0 & 2.0 & 10 & 0 & 498 & 0 & 0 & 0 & 57 & 0 & 0 & 84 & 584 & 0 & 0 & 0 & 7050 & 0 \\
\hline
\end{tabular}


WSRC-TR-2003-00269, REVISION 0 SRT-RPP-2003-00125, REVISION 0

Table 14. Predicted Solids from Envelope A Simulations - Part 1 (cont'd)

\begin{tabular}{|c|c|c|c|c|c|c|c|c|c|c|c|c|c|c|c|c|c|c|c|c|}
\hline $\begin{array}{c}\text { Experi- } \\
\text { mental ID }\end{array}$ & $\begin{array}{l}\text { Sim. } \\
\text { Point } \\
\text { Type }\end{array}$ & $\begin{array}{l}\text { Sim. } \\
\text { ID }\end{array}$ & Temp & $\begin{array}{l}\text { SBS/ } \\
\text { Feed }\end{array}$ & $\begin{array}{l}\mathbf{N a} \\
\mathbf{M}\end{array}$ & $\begin{array}{c}\text { ALO } \\
\text { H3 } \\
\text { [g] } \\
\end{array}$ & $\begin{array}{c}\text { CA3P } \\
\text { O42 } \\
\text { [g] }\end{array}$ & $\begin{array}{c}\text { CACO } \\
3[\mathrm{~g}]\end{array}$ & $\begin{array}{l}\text { CAF } \\
2 \text { [g] }\end{array}$ & $\begin{array}{c}\text { CAOH } \\
2 \text { [g] }\end{array}$ & $\begin{array}{c}\text { CATIO } \\
3[\mathrm{~g}]\end{array}$ & $\begin{array}{c}\text { CROH } \\
3 \text { [g] }\end{array}$ & $\begin{array}{c}\text { HYDR } \\
\text { OSOD } \\
{[\mathrm{g}]}\end{array}$ & $\begin{array}{c}\text { MGOH } \\
2 \text { [g] }\end{array}$ & $\begin{array}{c}\text { NA2C2O } \\
4[g]\end{array}$ & $\begin{array}{c}\text { NA2CO } \\
3.1 H 2 O \\
{[g]}\end{array}$ & $\begin{array}{c}\text { NA2U2O } \\
7[\mathrm{~g}]\end{array}$ & $\begin{array}{c}\text { NA3FSO } \\
4[\mathrm{~g}]\end{array}$ & $\begin{array}{l}\text { NA6S } \\
\text { O42C } \\
\text { O3 [g] }\end{array}$ & $\begin{array}{c}\text { NAF } \\
{[\mathrm{g}]}\end{array}$ \\
\hline SM-55-09 & MPV & 8EA068 & 66.0 & 2.0 & 8 & 0 & 498 & 0 & 0 & 0 & 57 & 5 & 0 & 84 & 51 & 0 & 0 & 0 & 0 & 0 \\
\hline SM-55-09 & MPV & 6EA068 & 66.0 & 2.0 & 6 & 0 & 496 & 0 & 0 & 0 & 57 & 24 & 0 & 84 & 0 & 0 & 0 & 0 & 0 & 0 \\
\hline SM-55-09 & MPV & 10EA078 & 66.0 & 2.0 & 10 & 0 & 498 & 0 & 0 & 0 & 57 & 0 & 0 & 84 & 584 & 0 & 0 & 0 & 7050 & 0 \\
\hline SM-55-09 & MPV & 8EA078 & 66.0 & 2.0 & 8 & 0 & 498 & 0 & 0 & 0 & 57 & 5 & 0 & 84 & 51 & 0 & 0 & 0 & 0 & 0 \\
\hline SM-55-09 & MPV & 6ЕA078 & 66.0 & 2.0 & 6 & 0 & 496 & 0 & 0 & 0 & 57 & 24 & 0 & 84 & 0 & 0 & 0 & 0 & 0 & 0 \\
\hline SM-55-09 & $\mathrm{OLH}$ & 10EA166 & 54.1 & 1.3 & 10 & 0 & 0 & 0 & 0 & 224 & 36 & 0 & 0 & 53 & 823 & 0 & $1.19 \mathrm{E}-07$ & 0 & 13589 & 547 \\
\hline SM-55-09 & $\mathrm{OLH}$ & 8EA166 & 54.1 & 1.3 & 8 & 0 & 48 & 0 & 198 & 0 & 36 & 0 & 0 & 53 & 399 & 0 & 0 & 0 & 0 & 0 \\
\hline SM-55-09 & $\mathrm{OLH}$ & 6EA166 & 54.1 & 1.3 & 6 & 0 & 309 & 0 & 0 & 0 & 36 & 0 & 0 & 53 & 0 & 0 & 0 & 0 & 0 & 0 \\
\hline SM-55-10 & MPV & 10EA065 & 66.0 & 2.0 & 10 & 0 & 0 & 0 & 0 & 320 & 51 & 0 & 0 & 75 & 381 & 0 & 0 & 0 & 5419 & 6439 \\
\hline SM-55-10 & MPV & 8EA065 & 66.0 & 2.0 & 8 & 0 & 0 & 0 & 337 & 0 & 51 & 0 & 0 & 75 & 0 & 0 & 0 & 0 & 0 & 3634 \\
\hline SM-55-10 & MPV & 6EA065 & 66.0 & 2.0 & 6 & 0 & 0 & 0 & 338 & 0 & 51 & 9 & 0 & 75 & 0 & 0 & 0 & 0 & 0 & 0 \\
\hline SM-55-10 & MPV & 10EA075 & 66.0 & 2.0 & 10 & 0 & 0 & 0 & 0 & 320 & 51 & 0 & 0 & 75 & 381 & 0 & 0 & 0 & 5419 & 6439 \\
\hline SM-55-10 & MPV & $8 \mathrm{EA} 075$ & 66.0 & 2.0 & 8 & 0 & 0 & 0 & 337 & 0 & 51 & 0 & 0 & 75 & 0 & 0 & 0 & 0 & 0 & 3634 \\
\hline SM-55-10 & MPV & 6EA075 & 66.0 & 2.0 & 6 & 0 & 0 & 0 & 338 & 0 & 51 & 9 & 0 & 75 & 0 & 0 & 0 & 0 & 0 & 0 \\
\hline SM-55-10 & $\mathrm{OLH}$ & 10EA142 & 44.2 & 1.8 & 10 & 0 & 0 & 0 & 322 & 0 & 48 & 0 & 0 & 72 & 913 & 0 & 0 & 0 & 5681 & 8866 \\
\hline SM-55-10 & $\mathrm{OLH}$ & 8EA142 & 44.2 & 1.8 & 8 & 0 & 0 & 0 & 322 & 0 & 48 & 0 & 0 & 72 & 563 & 0 & 0 & 0 & 0 & 6371 \\
\hline SM-55-10 & $\mathrm{OLH}$ & 6EA142 & 44.2 & 1.8 & 6 & 0 & 0 & 0 & 322 & 0 & 48 & 0 & 0 & 72 & 0 & 0 & 0 & 0 & 0 & 931 \\
\hline SM-55-11 & MPV & 10EA069 & 66.0 & 2.0 & 10 & 0 & 483 & 0 & 0 & 0 & 55 & 0 & 0 & 82 & 528 & 0 & 0 & 0 & 9773 & 0 \\
\hline SM-55-11 & MPV & 8EA069 & 66.0 & 2.0 & 8 & 0 & 482 & 0 & 0 & 0 & 55 & 0 & 0 & 82 & 60 & 0 & 0 & 0 & 0 & 0 \\
\hline SM-55-11 & MPV & 6EA069 & 66.0 & 2.0 & 6 & 0 & 481 & 0 & 0 & 0 & 55 & 17 & 0 & 82 & 0 & 0 & 0 & 0 & 0 & 0 \\
\hline SM-55-11 & MPV & 10EA079 & 66.0 & 2.0 & 10 & 0 & 483 & 0 & 0 & 0 & 55 & 0 & 0 & 82 & 528 & 0 & 0 & 0 & 9773 & 0 \\
\hline SM-55-11 & MPV & 8EA079 & 66.0 & 2.0 & 8 & 0 & 482 & 0 & 0 & 0 & 55 & 0 & 0 & 82 & 60 & 0 & 0 & 0 & 0 & 0 \\
\hline SM-55-11 & MPV & \begin{tabular}{|l|} 
6EA079 \\
\end{tabular} & 66.0 & 2.0 & 6 & 0 & 481 & 0 & 0 & 0 & 55 & 17 & 0 & 82 & 0 & 0 & 0 & 0 & 0 & 0 \\
\hline SM-55-11 & $\mathrm{OLH}$ & 10EA153 & 47.1 & 1.3 & 10 & 0 & 0 & 0 & 0 & 220 & 35 & 0 & 0 & 52 & 753 & 0 & 0 & 0 & 10556 & 7013 \\
\hline SM-55-11 & $\mathrm{OLH}$ & 8EA153 & 47.1 & 1.3 & 8 & 0 & 0 & 0 & 231 & 0 & 35 & 0 & 0 & 52 & 456 & 0 & 0 & 0 & 0 & 4599 \\
\hline SM-55-11 & $\mathrm{OLH}$ & 6EA153 & 47.1 & 1.3 & 6 & 0 & 0 & 0 & 232 & 0 & 35 & 0 & 0 & 52 & 0 & 0 & 0 & 0 & 0 & 0 \\
\hline
\end{tabular}


WSRC-TR-2003-00269, REVISION 0 SRT-RPP-2003-00125, REVISION 0

Table 15. Predicted Solids from Envelope A Simulations - Part 2

\begin{tabular}{|c|c|c|c|c|c|c|c|c|c|c|c|c|c|}
\hline $\begin{array}{c}\text { Experimental } \\
\text { ID }\end{array}$ & $\begin{array}{l}\text { Simulation } \\
\text { Point Type }\end{array}$ & $\begin{array}{c}\text { Simulation } \\
\text { ID }\end{array}$ & Temp & SBS/Feed & $\begin{array}{l}\mathrm{Na} \\
\mathrm{M}\end{array}$ & $\begin{array}{c}\text { NAFPO4.19H2O } \\
{[\mathrm{g}]}\end{array}$ & $\begin{array}{c}\text { NANO3 } \\
{[\mathrm{g}]}\end{array}$ & $\begin{array}{c}\text { NAPHOH.12H2O } \\
{[\mathrm{g}]}\end{array}$ & $\begin{array}{c}\text { NASGEL.15.5H2O } \\
{[\mathrm{g}]}\end{array}$ & $\begin{array}{c}\text { ZRO2 } \\
{[\mathrm{g}]}\end{array}$ & $\begin{array}{c}\text { Total } \\
\text { Insoluble } \\
\text { Solids [g] }\end{array}$ & $\begin{array}{c}\text { Total } \\
\text { Soln } \\
\text { Mass [g] }\end{array}$ & $\begin{array}{c}\text { Insoluble } \\
\text { Solids \% } \\
\text { of Total } \\
\text { Soln }\end{array}$ \\
\hline SM-55-10 & MPV & 10EA035 & 15.0 & 2.0 & 10 & 0 & 0 & 442 & 0 & 0 & 9157 & 1063410 & $0.9 \%$ \\
\hline SM-55-10 & MPV & $8 \mathrm{EA} 035$ & 15.0 & 2.0 & 8 & 0 & 0 & 1693 & 0 & 0 & 9741 & 1260550 & $0.8 \%$ \\
\hline SM-55-10 & MPV & 6EA035 & 15.0 & 2.0 & 6 & 0 & 0 & 598 & 1014 & 0 & 4219 & \begin{tabular}{|l|}
1606820 \\
\end{tabular} & $0.3 \%$ \\
\hline SM-55-09 & $\mathrm{OLH}$ & 10EA084 & 15.9 & 0.3 & 10 & 0 & 78089 & 2551 & 0 & 0 & 91825 & 1010770 & $9.1 \%$ \\
\hline SM-55-09 & OLH & 8EA084 & 15.9 & 0.3 & 8 & 0 & 0 & 1852 & 0 & 0 & 10918 & 1267330 & $0.9 \%$ \\
\hline SM-55-09 & $\mathrm{OLH}$ & 6ЕA084 & 15.9 & 0.3 & 6 & 0 & 0 & 818 & 0 & 0 & 8868 & 1614340 & $0.5 \%$ \\
\hline SM-55-09 & $\mathrm{OLH}$ & 10EA100 & 23.0 & 1.6 & 10 & 0 & 0 & 0 & 0 & 0 & 8383 & 1111410 & $0.8 \%$ \\
\hline SM-55-09 & $\mathrm{OLH}$ & 8 EA100 & 23.0 & 1.6 & 8 & 1288 & 0 & 0 & 0 & 0 & 5986 & \begin{tabular}{|l|}
1327270 \\
\end{tabular} & $0.5 \%$ \\
\hline SM-55-09 & $\mathrm{OLH}$ & 6EA100 & 23.0 & 1.6 & 6 & 0 & 0 & 0 & 1231 & 0 & 2160 & 1680660 & $0.1 \%$ \\
\hline SM-55-12 & MPV & 10EA037 & 15.0 & 2.0 & 10 & 0 & 0 & 0 & 0 & 0 & 1897 & 1146220 & $0.2 \%$ \\
\hline SM-55-01 & MPV & 8EA037 & 15.0 & 2.0 & 8 & 0 & 0 & 0 & 473 & 0 & 7216 & 1358540 & $0.5 \%$ \\
\hline SM-55-12 & MPV & 6EA037 & 15.0 & 2.0 & 6 & 0 & 0 & 0 & 1513 & 0 & 34725 & 1682650 & $2.1 \%$ \\
\hline SM-55-01 & MPV & 10EA067 & 66.0 & 2.0 & 10 & 0 & 0 & 0 & 0 & 2 & 36444 & \begin{tabular}{|l|}
1040280 \\
\end{tabular} & $3.5 \%$ \\
\hline SM-55-12 & MPV & $8 \mathrm{EA} 067$ & 66.0 & 2.0 & 8 & 0 & 0 & 0 & 0 & 1 & 11940 & 1278210 & $0.9 \%$ \\
\hline SM-55-01 & MPV & 6EA067 & 66.0 & 2.0 & 6 & 0 & 0 & 0 & 1380 & 1 & 2036 & 1640640 & $0.1 \%$ \\
\hline SM-55-12 & MPV & 10EA077 & 66.0 & 2.0 & 10 & 0 & 0 & 0 & 0 & 2 & 36444 & 1040280 & $3.5 \%$ \\
\hline SM-55-01 & MPV & $8 \mathrm{EA} 077$ & 66.0 & 2.0 & 8 & 0 & 0 & 0 & 0 & 1 & 11940 & 1278210 & $0.9 \%$ \\
\hline SM-55-12 & MPV & 6ЕA077 & 66.0 & 2.0 & 6 & 0 & 0 & 0 & 1380 & 1 & 2036 & 1640640 & $0.1 \%$ \\
\hline SM-55-02 & MPV & 10EA066 & 66.0 & 2.0 & 10 & 0 & 0 & 0 & 0 & 2 & 28659 & 1024260 & $2.8 \%$ \\
\hline SM-55-03 & MPV & $8 \mathrm{EA} 066$ & 66.0 & 2.0 & 8 & 0 & 0 & 0 & 542 & 2 & 17069 & 1244260 & $1.4 \%$ \\
\hline SM-55-02 & MPV & 6EA066 & 66.0 & 2.0 & 6 & 0 & 0 & 0 & 1476 & 2 & 2447 & 1615790 & $0.2 \%$ \\
\hline SM-55-03 & MPV & 10EA076 & 66.0 & 2.0 & 10 & 0 & 0 & 0 & 0 & 2 & 28659 & 1024260 & $2.8 \%$ \\
\hline SM-55-02 & MPV & $8 \mathrm{EA} 076$ & 66.0 & 2.0 & 8 & 0 & 0 & 0 & 542 & 2 & 17069 & 1244260 & $1.4 \%$ \\
\hline SM-55-03 & MPV & 6ЕA076 & 66.0 & 2.0 & 6 & 0 & 0 & 0 & 1476 & 2 & 2447 & 1615790 & $0.2 \%$ \\
\hline SM-55-04 & MPV & 10EA061 & 66.0 & 2.0 & 10 & 0 & 0 & 0 & 0 & 0 & 14528 & 1024620 & $1.4 \%$ \\
\hline SM-55-05 & MPV & 8EA061 & 66.0 & 2.0 & 8 & 0 & 0 & 0 & 0 & 0 & 4521 & 1239210 & $0.4 \%$ \\
\hline SM-55-04 & MPV & 6EA061 & 66.0 & 2.0 & 6 & 0 & 0 & 0 & 0 & 0 & 558 & 1580500 & $0.0 \%$ \\
\hline SM-55-05 & MPV & 10EA062 & 66.0 & 2.0 & 10 & 0 & 0 & 0 & 0 & 0 & 14637 & 1041660 & $1.4 \%$ \\
\hline SM-55-04 & MPV & $8 \mathrm{EA062}$ & 66.0 & 2.0 & 8 & 0 & 0 & 0 & 0 & 0 & 4781 & 1259580 & $0.4 \%$ \\
\hline SM-55-05 & MPV & 6ЕA062 & 66.0 & 2.0 & 6 & 0 & 0 & 0 & 0 & 0 & 565 & 1607210 & $0.0 \%$ \\
\hline
\end{tabular}

-57 - 
WSRC-TR-2003-00269, REVISION 0 SRT-RPP-2003-00125, REVISION 0

Table 15. Predicted Solids from Envelope A Simulations - Part 2 (cont'd)

\begin{tabular}{|c|c|c|c|c|c|c|c|c|c|c|c|c|c|}
\hline $\begin{array}{c}\text { Experimental } \\
\text { ID } \\
\end{array}$ & $\begin{array}{l}\text { Simulation } \\
\text { Point Type }\end{array}$ & $\begin{array}{c}\text { Simulation } \\
\text { ID }\end{array}$ & Temp & SBS/Feed & $\begin{array}{l}\mathbf{N a} \\
\mathbf{M} \\
\end{array}$ & \begin{tabular}{|c|} 
NAFPO4.19H2O \\
{$[\mathrm{g}]$}
\end{tabular} & $\begin{array}{c}\text { NANO3 } \\
{[\mathrm{g}]} \\
\end{array}$ & $\begin{array}{c}\text { NAPHOH.12H2O } \\
{[\mathrm{g}]}\end{array}$ & $\begin{array}{c}\text { NASGEL.15.5H2O } \\
{[\mathrm{g}]}\end{array}$ & $\begin{array}{c}\mathrm{ZRO2} \\
{[\mathrm{g}]}\end{array}$ & $\begin{array}{c}\text { Total } \\
\text { Insoluble } \\
\text { Solids [g] }\end{array}$ & $\begin{array}{c}\text { Total } \\
\text { Soln } \\
\text { Mass [g] } \\
\end{array}$ & \begin{tabular}{|c|}
$\begin{array}{c}\text { Insoluble } \\
\text { Solids \% } \\
\text { of Total } \\
\text { Soln }\end{array}$ \\
\end{tabular} \\
\hline SM-55-04 & MPV & 10EA071 & 66.0 & 2.0 & 10 & 0 & 0 & 0 & 0 & 0 & 14528 & 1024620 & $1.4 \%$ \\
\hline SM-55-05 & MPV & $8 \mathrm{EA} 071$ & 66.0 & 2.0 & 8 & 0 & 0 & 0 & 0 & 0 & 4521 & 1239210 & $0.4 \%$ \\
\hline SM-55-04 & MPV & 6EA071 & 66.0 & 2.0 & 6 & 0 & 0 & 0 & 0 & 0 & 558 & 1580500 & $0.0 \%$ \\
\hline SM-55-05 & MPV & 10EA072 & 66.0 & 2.0 & 10 & 0 & 0 & 0 & 0 & 0 & 14637 & 1041660 & $1.4 \%$ \\
\hline SM-55-04 & MPV & $8 \mathrm{EA} 072$ & 66.0 & 2.0 & 8 & 0 & 0 & 0 & 0 & 0 & 4781 & 1259580 & $0.4 \%$ \\
\hline SM-55-05 & MPV & 6EA072 & 66.0 & 2.0 & 6 & 0 & 0 & 0 & 0 & 0 & 565 & 1607210 & $0.0 \%$ \\
\hline SM-55-06 & MPV & 10EA064 & 66.0 & 2.0 & 10 & 0 & 0 & 0 & 0 & 2 & 13760 & 1037530 & $1.3 \%$ \\
\hline SM-55-06 & MPV & $8 \mathrm{EA064}$ & 66.0 & 2.0 & 8 & 0 & 0 & 0 & 0 & 1 & 5286 & 1251370 & $0.4 \%$ \\
\hline SM-55-06 & MPV & 6EA064 & 66.0 & 2.0 & 6 & 0 & 0 & 0 & 733 & 0 & 1244 & 1599360 & $0.1 \%$ \\
\hline SM-55-06 & MPV & 10EA074 & 66.0 & 2.0 & 10 & 0 & 0 & 0 & 0 & 2 & 13760 & 1037530 & $1.3 \%$ \\
\hline SM-55-06 & MPV & 8EA074 & 66.0 & 2.0 & 8 & 0 & 0 & 0 & 0 & 1 & 5286 & 1251370 & $0.4 \%$ \\
\hline SM-55-06 & MPV & 6EA074 & 66.0 & 2.0 & 6 & 0 & 0 & 0 & 733 & 0 & 1244 & 1599360 & $0.1 \%$ \\
\hline SM-55-06 & $\mathrm{OLH}$ & 10EA138 & 43.2 & 1.8 & 10 & 0 & 0 & 0 & 0 & 0 & 18185 & 1077310 & $1.7 \%$ \\
\hline SM-55-06 & $\mathrm{OLH}$ & 8EA138 & 43.2 & 1.8 & 8 & 0 & 0 & 0 & 0 & 0 & 1545 & 1311840 & $0.1 \%$ \\
\hline SM-55-06 & $\mathrm{OLH}$ & 6EA138 & 43.2 & 1.8 & 6 & 0 & 0 & 0 & 1232 & 0 & 1690 & 1659720 & $0.1 \%$ \\
\hline SM-55-07 & MPV & 10EA070 & 66.0 & 2.0 & 10 & 0 & 0 & 0 & 0 & 0 & 15272 & 1030910 & $1.5 \%$ \\
\hline SM-55-07 & MPV & $8 \mathrm{EA} 070$ & 66.0 & 2.0 & 8 & 0 & 0 & 0 & 0 & 0 & 4500 & 1248220 & $0.4 \%$ \\
\hline SM-55-07 & MPV & 6EA070 & 66.0 & 2.0 & 6 & 0 & 0 & 0 & 664 & 0 & 1151 & 1592410 & $0.1 \%$ \\
\hline SM-55-07 & MPV & 10EA080 & 66.0 & 2.0 & 10 & 0 & 0 & 0 & 0 & 0 & 15272 & 1030910 & $1.5 \%$ \\
\hline SM-55-07 & MPV & $8 \mathrm{EA} 080$ & 66.0 & 2.0 & 8 & 0 & 0 & 0 & 0 & 0 & 4500 & 1248220 & $0.4 \%$ \\
\hline SM-55-07 & MPV & 6ЕA080 & 66.0 & 2.0 & 6 & 0 & 0 & 0 & 664 & 0 & 1151 & 1592410 & $0.1 \%$ \\
\hline SM-55-08 & MPV & 10EA063 & 66.0 & 2.0 & 10 & 0 & 0 & 0 & 0 & 3 & 3498 & 1075780 & $0.3 \%$ \\
\hline SM-55-08 & MPV & $8 \mathrm{EA} 063$ & 66.0 & 2.0 & 8 & 0 & 0 & 0 & 0 & 2 & 653 & 1287540 & $0.1 \%$ \\
\hline SM-55-08 & MPV & 6EA063 & 66.0 & 2.0 & 6 & 0 & 0 & 0 & 1310 & 2 & 1975 & 1629160 & $0.1 \%$ \\
\hline SM-55-08 & MPV & 10EA073 & 66.0 & 2.0 & 10 & 0 & 0 & 0 & 0 & 3 & 3498 & 1075780 & $0.3 \%$ \\
\hline SM-55-08 & MPV & $8 \mathrm{EA} 073$ & 66.0 & 2.0 & 8 & 0 & 0 & 0 & 0 & 2 & 653 & 1287540 & $0.1 \%$ \\
\hline SM-55-08 & MPV & 6ЕA073 & 66.0 & 2.0 & 6 & 0 & 0 & 0 & 1310 & 2 & 1975 & 1629160 & $0.1 \%$ \\
\hline SM-55-09 & MPV & 10EA068 & 66.0 & 2.0 & 10 & 0 & 0 & 0 & 0 & 2 & 8275 & 1081440 & $0.8 \%$ \\
\hline SM-55-09 & MPV & 8EA068 & 66.0 & 2.0 & 8 & 0 & 0 & 0 & 0 & 1 & 696 & 1298520 & $0.1 \%$ \\
\hline SM-55-09 & MPV & 6ЕA068 & 66.0 & 2.0 & 6 & 0 & 0 & 0 & 1201 & 1 & 1864 & 1640040 & $0.1 \%$ \\
\hline
\end{tabular}


WSRC-TR-2003-00269, REVISION 0 SRT-RPP-2003-00125, REVISION 0

Table 15. Predicted Solids from Envelope A Simulations - Part 2 (cont'd)

\begin{tabular}{|c|c|c|c|c|c|c|c|c|c|c|c|c|c|}
\hline $\begin{array}{c}\text { Experimental } \\
\text { ID }\end{array}$ & $\begin{array}{l}\text { Simulation } \\
\text { Point Type }\end{array}$ & $\begin{array}{c}\text { Simulation } \\
\text { ID }\end{array}$ & Temp & SBS/Feed & $\begin{array}{l}\mathrm{Na} \\
\mathrm{M}\end{array}$ & \begin{tabular}{|c|} 
NAFPO4.19H2O \\
{$[\mathrm{g}]$}
\end{tabular} & $\begin{array}{c}\text { NANO3 } \\
{[\mathrm{g}]} \\
\end{array}$ & $\begin{array}{c}\text { NAPHOH.12H2O } \\
{[\mathrm{g}]}\end{array}$ & $\begin{array}{c}\text { NASGEL.15.5H2O } \\
{[\mathrm{g}]}\end{array}$ & $\begin{array}{c}\text { ZRO2 } \\
{[g]}\end{array}$ & $\begin{array}{c}\text { Total } \\
\text { Insoluble } \\
\text { Solids [g] }\end{array}$ & $\begin{array}{c}\text { Total } \\
\text { Soln } \\
\text { Mass [g] }\end{array}$ & \begin{tabular}{|c} 
Insoluble \\
Solids \% \\
of Total \\
Soln \\
\end{tabular} \\
\hline SM-55-09 & MPV & 10EA078 & 66.0 & 2.0 & 10 & 0 & 0 & 0 & 0 & 2 & 8275 & 1081440 & $0.8 \%$ \\
\hline SM-55-09 & MPV & $8 \mathrm{EA} 078$ & 66.0 & 2.0 & 8 & 0 & 0 & 0 & 0 & 1 & 696 & 1298520 & $0.1 \%$ \\
\hline SM-55-09 & MPV & 6EA078 & 66.0 & 2.0 & 6 & 0 & 0 & 0 & 1201 & 1 & 1864 & 1640040 & $0.1 \%$ \\
\hline SM-55-09 & $\mathrm{OLH}$ & 10EA166 & 54.1 & 1.3 & 10 & 0 & 0 & 0 & 0 & & 15271 & 1056870 & $1.4 \%$ \\
\hline SM-55-09 & $\mathrm{OLH}$ & $8 \mathrm{EA} 166$ & 54.1 & 1.3 & 8 & 0 & 0 & 0 & 0 & & 733 & 1282420 & $0.1 \%$ \\
\hline SM-55-09 & $\mathrm{OLH}$ & 6EA166 & 54.1 & 1.3 & 6 & 0 & 0 & 0 & 625 & & 1022 & 1622340 & $0.1 \%$ \\
\hline SM-55-10 & MPV & 10EA065 & 66.0 & 2.0 & 10 & 0 & 0 & 0 & 0 & 1 & 12686 & 1003030 & $1.3 \%$ \\
\hline SM-55-10 & MPV & 8EA065 & 66.0 & 2.0 & 8 & 0 & 0 & 0 & 0 & 0 & 4097 & 1214500 & $0.3 \%$ \\
\hline SM-55-10 & MPV & 6EA065 & 66.0 & 2.0 & 6 & 0 & 0 & 0 & 152 & 0 & 625 & 1552640 & $0.0 \%$ \\
\hline SM-55-10 & MPV & 10EA075 & 66.0 & 2.0 & 10 & 0 & 0 & 0 & 0 & 1 & 12686 & 1003030 & $1.3 \%$ \\
\hline SM-55-10 & MPV & $8 \mathrm{EA} 075$ & 66.0 & 2.0 & 8 & 0 & 0 & 0 & 0 & 0 & 4097 & 1214500 & $0.3 \%$ \\
\hline SM-55-10 & MPV & 6EA075 & 66.0 & 2.0 & 6 & 0 & 0 & 0 & 152 & 0 & 625 & 1552640 & $0.0 \%$ \\
\hline SM-55-10 & OLH & 10EA142 & 44.2 & 1.8 & 10 & 0 & 0 & 0 & 0 & 0 & 15901 & 1065420 & $1.5 \%$ \\
\hline SM-55-10 & $\mathrm{OLH}$ & 8 EA142 & 44.2 & 1.8 & 8 & 0 & 0 & 0 & 0 & 0 & 7376 & 1284360 & $0.6 \%$ \\
\hline SM-55-10 & OLH & 6EA142 & 44.2 & 1.8 & 6 & 0 & 0 & 0 & 1220 & 0 & 2593 & 1642590 & $0.2 \%$ \\
\hline SM-55-11 & MPV & 10EA069 & 66.0 & 2.0 & 10 & 0 & 0 & 0 & 0 & 1 & 10921 & 1062980 & $1.0 \%$ \\
\hline SM-55-11 & MPV & $8 \mathrm{EA} 069$ & 66.0 & 2.0 & 8 & 0 & 0 & 0 & 0 & 1 & 679 & 1284020 & $0.1 \%$ \\
\hline SM-55-11 & MPV & 6ЕA069 & 66.0 & 2.0 & 6 & 0 & 0 & 0 & 1096 & 0 & 1730 & 1624750 & $0.1 \%$ \\
\hline SM-55-11 & MPV & 10EA079 & 66.0 & 2.0 & 10 & 0 & 0 & 0 & 0 & 1 & 10921 & 1062980 & $1.0 \%$ \\
\hline SM-55-11 & MPV & 8EA079 & 66.0 & 2.0 & 8 & 0 & 0 & 0 & 0 & 1 & 679 & 1284020 & $0.1 \%$ \\
\hline SM-55-11 & MPV & 6EA079 & 66.0 & 2.0 & 6 & 0 & 0 & 0 & 1096 & 0 & 1730 & 1624750 & $0.1 \%$ \\
\hline SM-55-11 & $\mathrm{OLH}$ & 10EA153 & 47.1 & 1.3 & 10 & 0 & 0 & 0 & 0 & 0 & 18628 & 1036670 & $1.8 \%$ \\
\hline SM-55-11 & $\mathrm{OLH}$ & $8 \mathrm{EA} 153$ & 47.1 & 1.3 & 8 & 0 & 0 & 0 & 0 & 0 & 5373 & 1257080 & $0.4 \%$ \\
\hline SM-55-11 & $\mathrm{OLH}$ & 6EA153 & 47.1 & 1.3 & 6 & 0 & 0 & 0 & 581 & 0 & 899 & 1605420 & $0.1 \%$ \\
\hline
\end{tabular}


The density predictions for Envelope B were within $+/-5 \%$ of the measured values for the bottoms concentrate with a Na molarity between 1.2 and 8 . All the simulated and measured values are shown in Table 16.

Table 16. Comparison of Measured and Predicted Densities for Envelope B

\begin{tabular}{|c|c|c|c|c|c|c|c|c|c|c|c|c|}
\hline \multirow{2}{*}{$\begin{array}{c}\text { Temperature } \\
\text { Degrees C }\end{array}$} & \multicolumn{6}{|c|}{ Measured Density [g/ml] } & Predicted Density [g/ml] & \multicolumn{3}{|c|}{ Difference b/n Meas \& Pred Density } \\
\cline { 2 - 13 } & $\mathbf{1 5}$ & $\mathbf{2 5}$ & $\mathbf{5 0}$ & $\mathbf{6 5}$ & $\mathbf{1 5}$ & $\mathbf{2 5}$ & $\mathbf{5 0}$ & $\mathbf{6 5}$ & $\mathbf{1 5}$ & $\mathbf{2 5}$ & $\mathbf{5 0}$ & $\mathbf{6 5}$ \\
\hline 1-INIT & 1.090 & 1.086 & 1.074 & 1.065 & 1.083 & 1.072 & 1.044 & 1.028 & $0.7 \%$ & $1.3 \%$ & $2.8 \%$ & $3.5 \%$ \\
\hline 2-INIT & 1.067 & 1.064 & 1.052 & 1.044 & 1.095 & 1.084 & 1.056 & 1.040 & $-2.6 \%$ & $-1.9 \%$ & $-0.4 \%$ & $0.4 \%$ \\
\hline 3-INIT & 1.092 & 1.088 & 1.076 & 1.067 & 1.105 & 1.094 & 1.067 & 1.050 & $-1.2 \%$ & $-0.6 \%$ & $0.9 \%$ & $1.6 \%$ \\
\hline 4-INIT & 1.069 & 1.065 & 1.054 & 1.045 & 1.113 & 1.102 & 1.074 & 1.057 & $-4.1 \%$ & $-3.4 \%$ & $-1.9 \%$ & $-1.1 \%$ \\
\hline 1-3-B & 1.157 & 1.153 & 1.139 & 1.127 & 1.158 & 1.147 & 1.119 & 1.103 & $-0.1 \%$ & $0.5 \%$ & $1.7 \%$ & $2.2 \%$ \\
\hline 2-3-B & 1.149 & 1.145 & 1.134 & 1.125 & 1.162 & 1.151 & 1.123 & 1.106 & $-1.1 \%$ & $-0.5 \%$ & $1.0 \%$ & $1.6 \%$ \\
\hline $3-3-\mathrm{B}$ & 1.157 & 1.152 & 1.138 & 1.129 & 1.176 & 1.165 & 1.138 & 1.121 & $-1.7 \%$ & $-1.2 \%$ & $0.0 \%$ & $0.7 \%$ \\
\hline $4-3-\mathrm{B}$ & 1.149 & 1.144 & 1.132 & 1.124 & 1.180 & 1.169 & 1.141 & 1.125 & $-2.7 \%$ & $-2.2 \%$ & $-0.8 \%$ & $-0.1 \%$ \\
\hline 1-5-B & 1.238 & 1.236 & 1.226 & 1.209 & 1.242 & 1.231 & 1.203 & 1.186 & $-0.3 \%$ & $0.5 \%$ & $1.9 \%$ & $1.9 \%$ \\
\hline $2-5-\mathrm{B}$ & 1.247 & 1.243 & 1.225 & 1.214 & 1.263 & 1.252 & 1.225 & 1.208 & $-1.4 \%$ & $-0.7 \%$ & $0.0 \%$ & $0.5 \%$ \\
\hline $3-5-\mathrm{B}$ & 1.248 & 1.242 & 1.226 & 1.216 & 1.260 & 1.249 & 1.221 & 1.204 & $-1.0 \%$ & $-0.6 \%$ & $0.4 \%$ & $0.9 \%$ \\
\hline $4-5-\mathrm{B}$ & 1.235 & 1.229 & 1.215 & 1.206 & 1.291 & 1.279 & 1.252 & 1.235 & $-4.5 \%$ & $-4.1 \%$ & $-3.0 \%$ & $-2.4 \%$ \\
\hline 1-8-B & 1.392 & 1.383 & 1.364 & 1.350 & 1.367 & 1.356 & 1.328 & 1.312 & $1.8 \%$ & $1.9 \%$ & $2.6 \%$ & $2.8 \%$ \\
\hline $2-8-\mathrm{B}$ & 1.367 & 1.363 & 1.348 & 1.341 & 1.395 & 1.384 & 1.357 & 1.340 & $-2.1 \%$ & $-1.6 \%$ & $-0.6 \%$ & $0.1 \%$ \\
\hline $3-8-\mathrm{B}$ & 1.407 & 1.402 & 1.381 & 1.368 & 1.385 & 1.374 & 1.346 & 1.330 & $1.6 \%$ & $2.0 \%$ & $2.5 \%$ & $2.8 \%$ \\
\hline $4-8-\mathrm{B}$ & 1.360 & 1.362 & 1.356 & 1.348 & 1.401 & 1.390 & 1.363 & 1.346 & $-3.0 \%$ & $-2.1 \%$ & $-0.5 \%$ & $0.2 \%$ \\
\hline
\end{tabular}

The viscosity predictions for Envelope B did not match the experimental values as well as those for Envelope A. At a temperature of $15^{\circ} \mathrm{C}$ the predicted viscosity varied $+/-60 \%$ of the measured value. At a temperature of $25^{\circ} \mathrm{C}$ the predicted viscosity varied $+/-70 \%$ of the measured value. At a temperature of $60^{\circ} \mathrm{C}$ the predicted viscosity varied $+/-50 \%$ of the measured value. These bad matches are all at points less than $6 \mathrm{Na} \mathrm{M}$ and the viscosity prediction model was based on bottoms concentrate ranging from 6 to $10 \mathrm{M} \mathrm{Na}$. It is also expected that the lower and higher temperature extremes produced solids that are not accounted for by the viscosity prediction since it is based only on the supernate phase of the bottoms concentrate. To obtain a better predictor of viscosity, an accurate count of the solids in each sample plus a solids correction term derived from experimental results would need to be included. Prior modeling work ${ }^{9}$ for the waste feed evaporator showed that when the supernate viscosity was measured experimentally and compared with modeling predictions there was relatively good agreement (within $+/-15 \%$ ). A complete listing of the measured versus predicted viscosities is shown in Table 17. 
WSRC-TR-2003-00269, REVISION 0

SRT-RPP-2003-00125, REVISION 0

Table 17. Comparison of Measured versus Predicted Viscosities for Envelope B

\begin{tabular}{|c|c|c|c|c|c|c|c|c|c|c|}
\hline $\begin{array}{l}\text { Envelope } \\
\text { B Sample }\end{array}$ & $\begin{array}{c}\text { Measured } \\
\text { Na M }\end{array}$ & $\begin{array}{c}\text { Measured } \\
\text { Viscosity } \\
{[\mathrm{cP}] \text { at }} \\
15^{\circ} \mathrm{C}\end{array}$ & $\begin{array}{c}\text { Predicted } \\
\text { Viscosity } \\
{[\mathrm{cP}] \text { at }} \\
15^{\circ} \mathrm{C}\end{array}$ & $\begin{array}{c}\% \text { Diff } \\
\text { b/n Meas } \\
\text { \& Pred } \\
\text { Visc. at } \\
15^{\circ} \mathrm{C}\end{array}$ & $\begin{array}{c}\text { Measured } \\
\text { Viscosity } \\
\text { [cP] at } \\
25^{\circ} \mathrm{C}\end{array}$ & $\begin{array}{c}\text { Predicted } \\
\text { Viscosity } \\
{[\mathrm{cP}] \text { at }} \\
25^{\circ} \mathrm{C}\end{array}$ & $\begin{array}{c}\% \text { Diff } b / n \\
\text { Meas \& } \\
\text { Pred Visc. } \\
\text { at } 25^{\circ} \mathrm{C}\end{array}$ & $\begin{array}{c}\text { Measured } \\
\text { Viscosity } \\
{[\mathrm{cP}] \text { at }} \\
60^{\circ} \mathrm{C}\end{array}$ & $\begin{array}{c}\text { Predicted } \\
\text { Viscosity } \\
{[\mathrm{cP}] \text { at }} \\
60^{\circ} \mathrm{C}\end{array}$ & $\begin{array}{c}\% \text { Diff } \\
\text { b/n Meas } \\
\text { \& Pred } \\
\text { Visc. at } \\
60^{\circ} \mathrm{C}\end{array}$ \\
\hline $1-2$ (INIT) & 1.40 & 1.33 & 0.97 & $27.1 \%$ & 1.05 & 0.48 & $54.7 \%$ & 0.59 & 0.47 & $20.3 \%$ \\
\hline $1-3$ & 3.00 & 1.88 & 2.17 & $-15.2 \%$ & 1.42 & 1.32 & $6.8 \%$ & 0.74 & 0.87 & $-17.9 \%$ \\
\hline $1-5$ & 5.48 & 2.80 & 4.47 & $-59.6 \%$ & 2.11 & 2.83 & $-33.9 \%$ & 1.04 & 1.51 & $-45.4 \%$ \\
\hline $1-8$ & 8.59 & 6.94 & 8.75 & $-26.0 \%$ & 4.80 & 5.30 & $-10.4 \%$ & 2 & 2.37 & $-18.7 \%$ \\
\hline 2-2 (INIT) & 1.39 & 1.40 & 0.91 & $35.3 \%$ & 1.08 & 0.60 & $44.5 \%$ & 0.57 & 0.38 & $32.5 \%$ \\
\hline $2-3$ & 3.00 & 2.01 & 1.87 & $7.0 \%$ & 1.56 & 1.35 & $13.5 \%$ & 0.8 & 0.78 & $2.7 \%$ \\
\hline $2-5$ & 5.64 & 3.60 & 3.70 & $-2.8 \%$ & 2.64 & 2.69 & $-1.8 \%$ & 1.29 & 1.44 & $-11.2 \%$ \\
\hline $2-8$ & 8.30 & 7.47 & 6.10 & $18.4 \%$ & 5.19 & 4.27 & $17.8 \%$ & 2.14 & 2.12 & $1.0 \%$ \\
\hline 3-2 (INIT) & 1.20 & 1.43 & 0.83 & $41.7 \%$ & 1.14 & 0.38 & $67.0 \%$ & 0.61 & 0.42 & $30.9 \%$ \\
\hline $3-3$ & 3.00 & 1.93 & 2.17 & $-12.2 \%$ & 1.52 & 1.32 & $13.0 \%$ & 0.8 & 0.87 & $-9.0 \%$ \\
\hline $3-5$ & 5.00 & 3.00 & 3.97 & $-32.4 \%$ & 2.32 & 2.51 & $-8.3 \%$ & 1.11 & 1.39 & $-25.0 \%$ \\
\hline $3-8$ & 8.00 & 8.07 & 7.77 & $3.7 \%$ & 5.52 & 4.76 & $13.7 \%$ & 2.33 & 2.20 & $5.4 \%$ \\
\hline 4-2 (INIT) & 1.30 & 1.54 & 0.86 & $44.4 \%$ & 1.42 & 0.56 & $60.6 \%$ & 0.66 & 0.36 & $45.0 \%$ \\
\hline $4-3$ & 3.00 & 2.04 & 1.87 & $8.4 \%$ & 1.59 & 1.35 & $15.1 \%$ & 0.83 & 0.78 & $6.2 \%$ \\
\hline $4-5$ & 5.00 & 3.46 & 3.22 & $7.0 \%$ & 2.65 & 2.35 & $11.4 \%$ & 1.3 & 1.27 & $2.0 \%$ \\
\hline $4-8$ & 8.00 & 8.61 & 5.79 & $32.8 \%$ & 5.64 & 4.07 & $27.8 \%$ & 2.44 & 2.04 & $16.4 \%$ \\
\hline
\end{tabular}


The thermal conductivity predictions for Envelope B were within $+/-25 \%$ of the measured values for the bottoms concentrate with a Na molarity between 1.2 and 5 except for four samples. This large discrepancy is due in part that the simulated conductivity is for the supernate only, whereas the measured conductivity is for the entire slurry (supernate plus solids). Prior modeling work ${ }^{9}$ showed that the experimental conductivity has a standard deviation of $6.5 \%$ (about the value of water). The simulated conductivities are based on correction factors for the conductivity of water using various anions/cations as defined in Perry's Chemical Handbook. ${ }^{15}$ The simulated conductivities for all envelopes fell within a $3 \%$ standard deviation of the value of water. Since the measurement error is higher than this value, the predicted conductivities cannot be distinguished from those of water. Therefore there is no need to use the complicated prediction equation for conductivity when statistically a prediction for the conductivity of water is just as accurate. A complete list of the measured versus predicted conductivities is shown in Table 18.

Table 18. Comparison of Measured versus Predicted Conductivity for Envelope B

\begin{tabular}{|c|c|c|c|}
\hline Envelope B Sample & $\begin{array}{c}\text { Measured Conductivity } \\
{\left[\mathbf{W} / \mathbf{m}^{\circ} \mathbf{C}\right] \text { at } \mathbf{2 5}^{\circ} \mathbf{C}}\end{array}$ & $\begin{array}{c}\text { Predicted Conductivity } \\
{\left[\mathbf{W} / \mathbf{m}^{\circ} \mathbf{C}\right] \text { at } \mathbf{2 5}^{\circ} \mathbf{C}}\end{array}$ & $\begin{array}{c}\text { \% Diff b/n measured \& } \\
\text { Predicted Conductivity }\end{array}$ \\
\hline 1-IN-B & 0.514 & 0.592 & $-15.3 \%$ \\
\hline 2-IN-B & 0.474 & 0.592 & $-24.8 \%$ \\
\hline $3-\mathrm{IN}-\mathrm{B}$ & 0.504 & 0.583 & $-15.7 \%$ \\
\hline $4-\mathrm{IN}-\mathrm{B}$ & 0.472 & 0.582 & $-23.2 \%$ \\
\hline 1-3-B & 0.428 & 0.590 & $-37.8 \%$ \\
\hline $2-3-\mathrm{B}$ & 0.466 & 0.589 & $-26.5 \%$ \\
\hline $3-3-\mathrm{B}$ & 0.466 & 0.578 & $-23.9 \%$ \\
\hline $4-3-\mathrm{B}$ & 0.475 & 0.577 & $-21.5 \%$ \\
\hline $1-5-\mathrm{B}$ & 0.434 & 0.587 & $-35.3 \%$ \\
\hline $2-5-\mathrm{B}$ & 0.444 & 0.585 & $-31.8 \%$ \\
\hline $3-5-\mathrm{B}$ & 0.538 & 0.572 & $-6.4 \%$ \\
\hline $4-5-\mathrm{B}$ & 0.512 & 0.570 & $-11.4 \%$ \\
\hline
\end{tabular}


The heat capacity predictions for Envelope B were within $+/-15 \%$ of the measured values for the bottoms concentrate with a Na molarity between 1.2 and 5. Table 19 shows a complete listing of the measured versus predicted values for Envelope B.

Table 19. Comparison of Measured versus Predicted Cp for Envelope B

\begin{tabular}{|c|c|c|c|}
\hline Envelope B Sample & $\begin{array}{c}\text { Measured Conductivity } \\
{\left[\mathbf{W} / \mathbf{m}^{\circ} \mathbf{C}\right] \text { at } \mathbf{2 5}^{\circ} \mathbf{C}}\end{array}$ & $\begin{array}{c}\text { Predicted Conductivity } \\
{\left[\mathbf{W} / \mathbf{m}^{\circ} \mathbf{C}\right] \mathbf{~ a t ~}^{\circ} \mathbf{~}^{\circ}}\end{array}$ & $\begin{array}{c}\text { \% Diff b/n measured } \\
\text { \& Predicted } \\
\text { Conductivity }\end{array}$ \\
\hline 1-IN-B & 0.514 & 0.592 & $-15.3 \%$ \\
\hline 2-IN-B & 0.474 & 0.592 & $-24.8 \%$ \\
\hline 3-IN-B & 0.504 & 0.583 & $-15.7 \%$ \\
\hline $4-I N-B$ & 0.472 & 0.582 & $-23.2 \%$ \\
\hline 1-3-B & 0.428 & 0.590 & $-37.8 \%$ \\
\hline $2-3-B$ & 0.466 & 0.589 & $-26.5 \%$ \\
\hline $3-3-B$ & 0.466 & 0.578 & $-23.9 \%$ \\
\hline $4-3-B$ & 0.475 & 0.577 & $-21.5 \%$ \\
\hline $1-5-B$ & 0.434 & 0.587 & $-35.3 \%$ \\
\hline $2-5-B$ & 0.444 & 0.585 & $-31.8 \%$ \\
\hline $3-5-B$ & 0.538 & 0.572 & $-6.4 \%$ \\
\hline $4-5-B$ & 0.512 & 0.570 & $-11.4 \%$ \\
\hline
\end{tabular}

The sodium molarity predictions for Envelope B were within $+/-15 \%$ of the measured values for the bottoms concentrate with a Na molarity between 6 and 10. . However, for the initial samples at 2 molar, the predicted values are within $+/-55 \%$. This discrepancy is again due to the fact that the model prediction was based on simulated data with Na M between 6 and 10 . Another reason for the large discrepancy is that it is difficult to derive a predictive relationship relating waste feed composition (dry basis), SBS/Feed ratio, bottoms temperature, and bottoms density to bottoms Na molarity. A complete listing of the measured versus predicted Na molarities is shown in Table 20. 
WSRC-TR-2003-00269, REVISION 0

SRT-RPP-2003-00125, REVISION 0

Table 20. Comparison of Measured versus Predicted Na M for Envelope B

\begin{tabular}{|c|c|c|c|}
\hline Experimental ID & Measured Na M & Predicted Na M & $\begin{array}{c}\text { \%Diff Meas Na M \& } \\
\text { Pred Na M a 25 } \mathbf{C}^{\circ}\end{array}$ \\
\hline 1-INIT & 1.2 & 1.9 & $-54.4 \%$ \\
\hline 2-INIT & 1.4 & 1.8 & $-26.9 \%$ \\
\hline 3-INIT & 1.3 & 1.9 & $-47.4 \%$ \\
\hline 4-INIT & 1.4 & 1.3 & $4.0 \%$ \\
\hline 1-3-B & 3.0 & 3.7 & $-24.0 \%$ \\
\hline 2-3-B & 3.0 & 3.1 & $-2.0 \%$ \\
\hline 3-3-B & 3.0 & 3.3 & $-8.5 \%$ \\
\hline 4-3-B & 3.0 & 3.0 & $0.0 \%$ \\
\hline 1-5-B & 5.0 & 5.5 & $-9.5 \%$ \\
\hline 2-5-B & 5.4 & 5.3 & $1.9 \%$ \\
\hline 3-5-B & 5.0 & 5.2 & $-3.1 \%$ \\
\hline $4-5-\mathrm{B}$ & 5.6 & 4.8 & $15.1 \%$ \\
\hline 1-8-B & 8.0 & 8.6 & $-7.5 \%$ \\
\hline 2-8-B & 8.6 & 8.1 & $5.6 \%$ \\
\hline 3-8-B & 8.0 & 8.6 & $-7.6 \%$ \\
\hline $4-8-\mathrm{B}$ & 8.3 & 7.7 & $7.7 \%$ \\
\hline & & & \\
\hline
\end{tabular}

No accurate prediction equations for the solubility of the evaporator bottoms stream in terms of the total insoluble solids present could be derived for Envelopes A, B, or C. Several attempts were made to include nonlinear and linear terms in the prediction fits, but the waste feed compositions, SBS to waste feed ratio, the bottoms temperature and Na molarity did not provide enough data to characterize these phenomena. However, some general observations were made from the simulations. For Envelope B, about $21 \%$ of the simulated values ( 83 out of 399 total - including fit and validation points) had bottoms insoluble solids greater than $1 \mathrm{wt} \%$ but only about $11 \%$ of the simulated values ( 42 points) had insoluble solids greater than $2 \mathrm{wt} \%$. None of the predicted values went over $3 \mathrm{wt} \%$. The insoluble solids only went above $2 \mathrm{wt} \%$ when the Na molarity went above $8 \mathrm{M}$. At $6 \mathrm{M} \mathrm{Na}$ the solids stayed below $1 \mathrm{wt} \%$, at $8 \mathrm{M}$ the solids stayed below $2 \mathrm{wt} \%$, and at $10 \mathrm{M} \mathrm{Na}$ the solids stayed below $3 \mathrm{wt} \%$. More solids appear to come out between $20^{\circ} \mathrm{C}$ and $40{ }^{\circ} \mathrm{C}$ than the other temperatures. The primary salts were aluminum hydroxide, sodium oxalate, sodium fluorosulfate, and sodium fluoride. For further comparison with experimental results, Table 21 was created which shows simulated run data that is similar to the experimental run points in terms of waste composition, SBS/Feed ratio, and $\mathrm{Na} \mathrm{M}$. The table also shows various trends as $\mathrm{Na} \mathrm{M}$ is increased with the same waste feed. Please send Appendix B for a complete listing of all solids that formed for all the simulated runs for Envelope B. 
WSRC-TR-2003-00269, REVISION 0 SRT-RPP-2003-00125, REVISION 0

Table 21. Predicted Solids from Envelope B

\begin{tabular}{|c|c|c|c|c|c|c|c|c|c|c|c|c|c|c|c|c|c|}
\hline Experimental ID & $\begin{array}{c}\text { Simulation } \\
\text { Point } \\
\text { Type }\end{array}$ & $\begin{array}{c}\text { Simulation } \\
\text { ID }\end{array}$ & \begin{tabular}{|c|} 
Temp \\
${ }^{\circ} \mathrm{C}$
\end{tabular} & SBS/Feed & $\begin{array}{c}\text { Na } \\
\text { Molarity }\end{array}$ & $\begin{array}{c}\text { ALOH3 } \\
{[\mathrm{g}]}\end{array}$ & $\begin{array}{r}\text { CAF } \\
{[\mathrm{g}]} \\
\end{array}$ & $\begin{array}{c}\text { CATIO3 } \\
{[\mathrm{g}]}\end{array}$ & $\begin{array}{c}\text { MGOH2 } \\
{[\mathrm{g}]}\end{array}$ & $\mid \begin{array}{c}\mathrm{NA2C2O} \\
{[\mathrm{g}]}\end{array}$ & $\begin{array}{c}\text { NA3FSO } \\
{[\mathrm{g}]}\end{array}$ & $\begin{array}{c}\text { NAF } \\
\text { [g] }\end{array}$ & \begin{tabular}{c|c} 
NIOH2 \\
{$[\mathrm{g}]$}
\end{tabular} & $\begin{array}{c}\text { ZRO2 } \\
\text { [g] }\end{array}$ & $\begin{array}{c}\text { Total } \\
\text { Solids } \\
\text { [g] } \\
\end{array}$ & $\begin{array}{c}\text { Total } \\
\text { Soln } \\
\text { Mass [g] }\end{array}$ & \begin{tabular}{|c|c|}
$\begin{array}{c}\text { Solids } \\
\% \text { of }\end{array}$ \\
Total \\
Soln \\
\end{tabular} \\
\hline Initial AZ101- 70.7\%,60\% & EV & 10TB001 & 15 & 0 & 10 & 0 & 0 & 0 & 0 & 830 & 0 & 583 & 0 & 0 & $1.41 \mathrm{E}+03$ & $32.91 \mathrm{E}+05$ & $54.86 \mathrm{E}-03$ \\
\hline Initial AZ102- 70.7\%,60\% & $\mathrm{EV}$ & $10 \mathrm{~TB} 005$ & 15 & 0 & 10 & 0 & 0 & 0 & 0 & 878 & 0 & 565 & 0 & 0 & $1.44 \mathrm{E}+03$ & $32.91 \mathrm{E}+05$ & $54.96 \mathrm{E}-03$ \\
\hline Initial AZ101- 70.7\%,60\% & EV & 10TB007 & 66 & 0 & 10 & 0 & 0 & 0 & 0 & 0 & 0 & 0 & 0 & 0 & 0 & $2.79 \mathrm{E}+05$ & 0 \\
\hline Initial AZ102- 70.7\%,60\% & EV & 10TB011 & 66 & 0 & 10 & 0 & 0 & 0 & 0 & 66 & 0 & 38 & 0 & 0 & $1.05 \mathrm{E}+02$ & $22.79 \mathrm{E}+05$ & $3.75 \mathrm{E}-04$ \\
\hline Initial AZ101- 70.7\%,60\% & EV & 10TB013 & 15 & 2 & 10 & 0 & 4 & 1 & 10 & 793 & 0 & 999 & 0 & 0 & $1.81 \mathrm{E}+03$ & $33.01 \mathrm{E}+05$ & $56.00 \mathrm{E}-03$ \\
\hline Initial AZ102- 70.7\%,60\% & $\mathrm{EV}$ & 10TB017 & 15 & 2 & 10 & 0 & 4 & 1 & 10 & 842 & 0 & 983 & 0 & 0 & $1.84 \mathrm{E}+03$ & $33.02 \mathrm{E}+05$ & $56.10 \mathrm{E}-03$ \\
\hline Initial AZ101- 70.7\%,60\% & $\mathrm{EV}$ & 10TB019 & 66 & 2 & 10 & 0 & 4 & 1 & 10 & 0 & 0 & 405 & 1 & 0 & $4.21 \mathrm{E}+02$ & $22.89 \mathrm{E}+05$ & $51.46 \mathrm{E}-03$ \\
\hline Initial AZ102- 70.7\%,60\% & $\mathrm{EV}$ & 10TB023 & 66 & 2 & 10 & 0 & 4 & 1 & 10 & 0 & 0 & 448 & 1 & 0 & $4.64 \mathrm{E}+02$ & $22.89 \mathrm{E}+05$ & $51.61 \mathrm{E}-03$ \\
\hline Initial AZ101- 70.7\%,60\% & $\mathrm{OLH}$ & 10TB025 & 40 & 1 & 10 & 0 & 2 & 1 & 4 & 0 & 0 & 441 & 0 & 0 & $4.48 \mathrm{E}+02$ & $22.91 \mathrm{E}+05$ & $51.54 \mathrm{E}-03$ \\
\hline Initial AZ101- 70.7\%,60\% & $\mathrm{OLH}$ & 10TB029 & 23 & 1 & 10 & 0 & 1 & $3.63 \mathrm{E}-01$ & 3 & 0 & 6810 & 0 & 0 & 0 & $6.81 \mathrm{E}+03$ & $32.86 \mathrm{E}+05$ & $52.39 \mathrm{E}-02$ \\
\hline Initial AZ101- 70.7\%,60\% & $\mathrm{OLH}$ & 10TB037 & 32 & 1 & 10 & 0 & 1 & $3.63 \mathrm{E}-01$ & 3 & 495 & 6175 & 0 & 0 & 0 & $6.67 \mathrm{E}+03$ & $32.83 \mathrm{E}+05$ & $52.36 \mathrm{E}-02$ \\
\hline Initial AZ101- 70.7\%,60\% & $\mathrm{OLH}$ & 10TB048 & 37 & 2 & 10 & 0 & 3 & 1 & 8 & 327 & 6998 & 0 & $6.13 \mathrm{E}-02$ & 0 & $7.34 \mathrm{E}+03$ & $32.85 \mathrm{E}+05$ & $52.58 \mathrm{E}-02$ \\
\hline Initial AZ101- 70.7\%,60\% & $\mathrm{OLH}$ & 10TB058 & 51 & 1 & 10 & 0 & 1 & $3.03 \mathrm{E}-01$ & 2 & 0 & 0 & 290 & 0 & 0 & $2.94 \mathrm{E}+02$ & $22.85 \mathrm{E}+05$ & $51.03 \mathrm{E}-03$ \\
\hline Initial AZ101- 70.7\%,60\% & $\mathrm{OLH}$ & 10 TB063 & 53 & 0 & 10 & 0 & 1 & $1.82 \mathrm{E}-01$ & 1 & 35 & 0 & 185 & 0 & 0 & $2.22 \mathrm{E}+02$ & $22.84 \mathrm{E}+05$ & $57.80 \mathrm{E}-04$ \\
\hline Initial AZ101- 70.7\%,60\% & $\mathrm{OLH}$ & 10TB070 & 66 & 2 & 10 & 0 & 4 & 1 & 9 & 0 & 0 & 483 & 1 & 0 & $4.98 \mathrm{E}+02$ & $22.89 \mathrm{E}+05$ & $51.72 \mathrm{E}-03$ \\
\hline Initial AZ102- 70.7\%,60\% & $\mathrm{OLH}$ & 10TB084 & 58 & 1 & 10 & 0 & 3 & 1 & 7 & 0 & 0 & 313 & 1 & 0 & $3.25 \mathrm{E}+02$ & $2.88 \mathrm{E}+05$ & $51.13 \mathrm{E}-03$ \\
\hline Initial AZ102- 70.7\%,60\% & $\mathrm{OLH}$ & 10TB094 & 62 & 1 & 10 & 0 & 1 & 4.24E-01 & 3 & 0 & 0 & 191 & $2.17 \mathrm{E}-01$ & 0 & $1.97 \mathrm{E}+02$ & $2.85 \mathrm{E}+05$ & $56.91 \mathrm{E}-04$ \\
\hline Initial AZ102- 70.7\%,60\% & $\mathrm{OLH}$ & $10 \mathrm{~TB} 100$ & 45 & 0 & 10 & 0 & 1 & $2.43 \mathrm{E}-01$ & 2 & 325 & 0 & 288 & 0 & 0 & $6.16 \mathrm{E}+02$ & $2.86 \mathrm{E}+05$ & $52.15 \mathrm{E}-03$ \\
\hline Initial AZ101- 70.7\%,60\% & EV & 8TB001 & 15 & 0 & 8 & 0 & 0 & 0 & 0 & 767 & 0 & 0 & 0 & 0 & $7.67 \mathrm{E}+02$ & $23.43 \mathrm{E}+05$ & $52.24 \mathrm{E}-03$ \\
\hline Initial AZ102- 70.7\%,60\% & EV & $8 \mathrm{~TB} 005$ & 15 & 0 & 8 & 0 & 0 & 0 & 0 & 795 & 0 & 0 & 0 & 0 & $7.95 \mathrm{E}+02$ & $23.44 \mathrm{E}+05$ & $52.31 \mathrm{E}-03$ \\
\hline Initial AZ101- 70.7\%,60\% & EV & 8TB007 & 66 & 0 & 8 & 0 & 0 & 0 & 0 & 0 & 0 & 0 & 0 & 0 & 0 & $3.30 \mathrm{E}+05$ & 0 \\
\hline Initial AZ102- 70.7\%,60\% & EV & 8TB011 & 66 & 0 & 8 & 0 & 0 & 0 & 0 & 0 & 0 & 0 & 0 & 0 & 0 & $3.31 \mathrm{E}+05$ & 0 \\
\hline Initial AZ101- 70.7\%,60\% & EV & $8 \mathrm{~TB} 013$ & 15 & 2 & 8 & 0 & 4 & 1 & 10 & 728 & 0 & 325 & 0 & 0 & $1.07 \mathrm{E}+03$ & $33.55 \mathrm{E}+05$ & $53.01 \mathrm{E}-03$ \\
\hline Initial AZ102- 70.7\%,60\% & EV & $8 \mathrm{~TB} 017$ & 15 & 2 & 8 & 0 & 4 & 1 & 10 & 755 & 0 & 282 & 0 & 0 & $1.05 \mathrm{E}+03$ & $33.56 \mathrm{E}+05$ & $52.95 \mathrm{E}-03$ \\
\hline Initial AZ101- 70.7\%,60\% & EV & 8TB019 & 66 & 2 & 8 & 0 & 4 & 1 & 10 & 0 & 0 & 0 & 1 & 0 & $1.63 \mathrm{E}+01$ & $13.43 \mathrm{E}+05$ & $54.75 \mathrm{E}-05$ \\
\hline Initial AZ102- 70.7\%,60\% & EV & $8 \mathrm{~TB} 023$ & 66 & 2 & 8 & 0 & 4 & 1 & 10 & 0 & 0 & 0 & 1 & $4.12 \mathrm{E}-03$ & $31.64 \mathrm{E}+01$ & $13.43 \mathrm{E}+05$ & $54.76 \mathrm{E}-05$ \\
\hline Initial AZ101- 70.7\%,60\% & $\mathrm{OLH}$ & $8 \mathrm{~TB} 025$ & 40 & 1 & 8 & 0 & 2 & 1 & 4 & 0 & 0 & 0 & $6.75 \mathrm{E}-02$ & 0 & $6.93 \mathrm{E}+00$ & $03.44 \mathrm{E}+05$ & $52.01 \mathrm{E}-05$ \\
\hline Initial AZ101- 70.7\%,60\% & $\mathrm{OLH}$ & 8TB029 & 23 & 1 & 8 & 0 & 1 & 3.63E-01 & 3 & 0 & 5170 & 0 & 0 & 0 & $5.17 \mathrm{E}+03$ & $33.38 \mathrm{E}+05$ & $51.53 \mathrm{E}-02$ \\
\hline Initial AZ101- 70.7\%,60\% & $\mathrm{OLH}$ & 8TB037 & 32 & 1 & 8 & 0 & 1 & $3.63 \mathrm{E}-01$ & 3 & 352 & 3916 & 0 & 0 & 0 & $4.27 \mathrm{E}+03$ & $33.37 \mathrm{E}+05$ & $51.27 \mathrm{E}-02$ \\
\hline Initial AZ101- 70.7\%,60\% & $\mathrm{OLH}$ & 8TB048 & 37 & 2 & 8 & 0 & 3 & 1 & 8 & 155 & 4474 & 0 & 4.09E-01 & 0 & $4.64 \mathrm{E}+03$ & $33.39 \mathrm{E}+05$ & $1.37 \mathrm{E}-02$ \\
\hline
\end{tabular}

$-65-$ 
WSRC-TR-2003-00269, REVISION 0 SRT-RPP-2003-00125, REVISION 0

Table 21. Predicted Solids from Envelope B (cont'd)

\begin{tabular}{|c|c|c|c|c|c|c|c|c|c|c|c|c|c|c|c|c|c|}
\hline Experimental ID & \begin{tabular}{|c|} 
Simulation \\
Point \\
Type
\end{tabular} & $\begin{array}{c}\text { Simulation } \\
\text { ID }\end{array}$ & \begin{tabular}{|c|} 
Temp \\
oC
\end{tabular} & SBS/Feed & $\begin{array}{c}\text { Na } \\
\text { Molarity }\end{array}$ & $\begin{array}{c}\text { ALOH3 } \\
{[\mathrm{g}]}\end{array}$ & $\begin{array}{c}\text { CAF2 } \\
{[\mathrm{g}]}\end{array}$ & $\begin{array}{c}\text { CATIO3 } \\
{[\mathrm{g}]}\end{array}$ & $\begin{array}{c}\text { MGOH2 } \\
{[\mathrm{g}]}\end{array}$ & $\begin{array}{c}\mathrm{NA2C2O} 4 \\
{[\mathrm{~g}]} \\
\end{array}$ & $\begin{array}{c}\text { NA3FSO4 } \\
{[\mathrm{g}]} \\
\end{array}$ & $\begin{array}{c}\text { NAF } \\
{[\mathrm{g}]}\end{array}$ & $\begin{array}{c}\text { NIOH2 } \\
{[\mathrm{g}]}\end{array}$ & $\begin{array}{c}\text { ZRO2 } \\
{[\mathrm{g}]}\end{array}$ & $\begin{array}{c}\text { Total } \\
\text { Solids } \\
{[\mathrm{g}]} \\
\end{array}$ & \begin{tabular}{|c|} 
Total \\
Soln \\
Mass [g] \\
\end{tabular} & \begin{tabular}{|c|}
$\begin{array}{c}\text { Solids } \\
\text { \% of } \\
\text { Total } \\
\text { Soln }\end{array}$ \\
\end{tabular} \\
\hline Initial AZ101- 70.7\%,60\% & $\mathrm{OLH}$ & $8 \mathrm{~TB} 052$ & 44 & 1 & 8 & 0 & 2 & 1 & 5 & 0 & 0 & 0 & $1.98 \mathrm{E}-01$ & 0 & $7.80 \mathrm{E}+00$ & $3.44 \mathrm{E}+05$ & $2.27 \mathrm{E}-05$ \\
\hline Initial AZ101- 70.7\%,60\% & $\mathrm{OLH}$ & 8TB054 & 46 & 1 & 8 & 0 & 2 & $4.85 \mathrm{E}-01$ & 4 & 0 & 0 & 0 & $1.09 \mathrm{E}-01$ & 0 & $6.17 \mathrm{E}+00$ & $3.42 \mathrm{E}+05$ & $1.81 \mathrm{E}-05$ \\
\hline Initial AZ101- 70.7\%,60\% & $\mathrm{OLH}$ & $8 \mathrm{~TB} 058$ & 51 & 1 & 8 & 0 & 1 & $3.03 \mathrm{E}-01$ & 2 & 0 & 0 & 0 & $5.20 \mathrm{E}-03$ & 0 & $3.76 \mathrm{E}+00$ & $3.38 \mathrm{E}+05$ & $1.11 \mathrm{E}-05$ \\
\hline Initial AZ101- 70.7\%,60\% & $\mathrm{OLH}$ & $8 \mathrm{~TB} 063$ & 53 & 0 & 8 & 0 & 1 & $1.82 \mathrm{E}-01$ & 1 & 0 & 0 & 0 & 0 & 0 & $2.23 \mathrm{E}+00$ & $3.37 \mathrm{E}+05$ & $6.61 \mathrm{E}-06$ \\
\hline Initial AZ101- 70.7\%,60\% & $\mathrm{OLH}$ & $8 \mathrm{~TB} 070$ & 66 & 2 & 8 & 0 & 4 & 1 & 9 & 0 & 0 & 0 & 1 & 0 & $1.47 \mathrm{E}+01$ & $3.43 \mathrm{E}+05$ & $4.27 \mathrm{E}-05$ \\
\hline Initial AZ102- 70.7\%,60\% & $\mathrm{OLH}$ & 8TB084 & 58 & 1 & 8 & 0 & 3 & 1 & 7 & 0 & 0 & 0 & 1 & 0 & $1.13 \mathrm{E}+01$ & $3.41 \mathrm{E}+05$ & $3.32 \mathrm{E}-05$ \\
\hline Initial AZ102- 70.7\%,60\% & $\mathrm{OLH}$ & 8TB094 & 62 & 1 & 8 & 0 & 1 & 4.24E-01 & 3 & 0 & 0 & 0 & $2.92 \mathrm{E}-01$ & 0 & $5.55 \mathrm{E}+00$ & $3.37 \mathrm{E}+05$ & $1.64 \mathrm{E}-05$ \\
\hline Initial AZ102- 70.7\%,60\% & $\mathrm{OLH}$ & $8 \mathrm{~TB} 100$ & 45 & 0 & 8 & 0 & 1 & $2.43 \mathrm{E}-01$ & 2 & 19 & 0 & 0 & 0 & 0 & $2.21 \mathrm{E}+01$ & $3.39 \mathrm{E}+05$ & $6.51 \mathrm{E}-05$ \\
\hline Initial AZ101- 70.7\%,60\% & $\mathrm{EV}$ & 6TB001 & 15 & 0 & 6 & 341 & 0 & 0 & 0 & 608 & 0 & 0 & 0 & 0 & $9.49 \mathrm{E}+02$ & $4.28 \mathrm{E}+05$ & $2.22 \mathrm{E}-03$ \\
\hline Initial AZ102- 70.7\%,60\% & $\mathrm{EV}$ & $6 \mathrm{~TB} 005$ & 15 & 0 & 6 & 0 & 0 & 0 & 0 & 626 & 0 & 0 & 0 & 0 & $6.26 \mathrm{E}+02$ & $4.28 \mathrm{E}+05$ & $1.46 \mathrm{E}-03$ \\
\hline Initial AZ101- 70.7\%,60\% & EV & $6 \mathrm{~TB} 007$ & 66 & 0 & 6 & 0 & 0 & 0 & 0 & 0 & 0 & 0 & 0 & 0 & 0 & $4.14 \mathrm{E}+05$ & 0 \\
\hline Initial AZ102- 70.7\%,60\% & EV & 6TB011 & 66 & 0 & 6 & 0 & 0 & 0 & 0 & 0 & 0 & 0 & 0 & 0 & 0 & $4.15 \mathrm{E}+05$ & 0 \\
\hline Initial AZ101- 70.7\%,60\% & EV & 6ТВ013 & 15 & 2 & 6 & 714 & 4 & 1 & 10 & 567 & 0 & 0 & $8.13 \mathrm{E}-02$ & 0 & $1.30 \mathrm{E}+03$ & $4.44 \mathrm{E}+05$ & $2.92 \mathrm{E}-03$ \\
\hline Initial AZ102- 70.7\%,60\% & $\mathrm{EV}$ & 6TB017 & 15 & 2 & 6 & 0 & 4 & 1 & 10 & 579 & 0 & 0 & $2.82 \mathrm{E}-01$ & 0 & $5.95 \mathrm{E}+02$ & $4.44 \mathrm{E}+05$ & $1.34 \mathrm{E}-03$ \\
\hline Initial AZ101- 70.7\%,60\% & EV & 6ТВ019 & 66 & 2 & 6 & 0 & 4 & 1 & 10 & 0 & 0 & 0 & 1 & 0 & $1.62 \mathrm{E}+01$ & $4.30 \mathrm{E}+05$ & $3.77 \mathrm{E}-05$ \\
\hline Initial AZ102- 70.7\%,60\% & EV & 6TB023 & 66 & 2 & 6 & 0 & 4 & 1 & 10 & 0 & 0 & 0 & 1 & $3.78 \mathrm{E}-02$ & $1.63 \mathrm{E}+01$ & $4.30 \mathrm{E}+05$ & $3.79 \mathrm{E}-05$ \\
\hline Initial AZ101- 70.7\%,60\% & $\mathrm{OLH}$ & $6 \mathrm{~TB} 025$ & 40 & 1 & 6 & 0 & 2 & 1 & 4 & 0 & 0 & 0 & $2.40 \mathrm{E}-01$ & 0 & $7.04 \mathrm{E}+00$ & $4.31 \mathrm{E}+05$ & $1.64 \mathrm{E}-05$ \\
\hline Initial AZ101- 70.7\%,60\% & $\mathrm{OLH}$ & 6ТВ029 & 23 & 1 & 6 & 0 & 1 & $3.63 \mathrm{E}-01$ & 3 & 0 & 1801 & 0 & 0 & 0 & $1.81 \mathrm{E}+03$ & $4.29 \mathrm{E}+05$ & $4.21 \mathrm{E}-03$ \\
\hline Initial AZ101- 70.7\%,60\% & $\mathrm{OLH}$ & 6ТВ037 & 32 & 1 & 6 & 0 & 1 & $3.63 \mathrm{E}-01$ & 3 & 104 & 113 & 0 & 0 & 0 & $2.22 \mathrm{E}+02$ & $4.30 \mathrm{E}+05$ & $5.16 \mathrm{E}-04$ \\
\hline Initial AZ101- 70.7\%,60\% & $\mathrm{OLH}$ & 6TB048 & 37 & 2 & 6 & 0 & 3 & 1 & 8 & 0 & 555 & 0 & 1 & 0 & $5.67 \mathrm{E}+02$ & $4.33 \mathrm{E}+05$ & $1.31 \mathrm{E}-03$ \\
\hline Initial AZ101- 70.7\%,60\% & $\mathrm{OLH}$ & 6TB052 & 44 & 1 & 6 & 0 & 2 & 1 & 5 & 0 & 0 & 0 & $3.46 \mathrm{E}-01$ & 0 & $7.88 \mathrm{E}+00$ & $4.30 \mathrm{E}+05$ & $1.83 \mathrm{E}-05$ \\
\hline Initial AZ101- 70.7\%,60\% & $\mathrm{OLH}$ & 6TB054 & 46 & 1 & 6 & 0 & 2 & $4.85 \mathrm{E}-01$ & 4 & 0 & 0 & 0 & $2.43 \mathrm{E}-01$ & 0 & $6.23 \mathrm{E}+00$ & $4.28 \mathrm{E}+05$ & $1.46 \mathrm{E}-05$ \\
\hline Initial AZ101- 70.7\%,60\% & $\mathrm{OLH}$ & 6TB058 & 51 & 1 & 6 & 0 & 1 & $3.03 \mathrm{E}-01$ & 2 & 0 & 0 & 0 & $1.08 \mathrm{E}-01$ & 0 & $3.76 \mathrm{E}+00$ & $4.24 \mathrm{E}+05$ & $8.86 \mathrm{E}-06$ \\
\hline Initial AZ101- 70.7\%,60\% & $\mathrm{OLH}$ & 6ТВ063 & 53 & 0 & 6 & 0 & 4.64E-01 & $1.82 \mathrm{E}-01$ & 1 & 0 & 0 & 0 & $8.02 \mathrm{E}-03$ & 0 & $2.12 \mathrm{E}+00$ & $4.22 \mathrm{E}+05$ & $5.03 \mathrm{E}-06$ \\
\hline Initial AZ101- 70.7\%,60\% & $\mathrm{OLH}$ & 6ТВ070 & 66 & 2 & 6 & 0 & 4 & 1 & 9 & 0 & 0 & 0 & 1 & 0 & $1.46 \mathrm{E}+01$ & $4.30 \mathrm{E}+05$ & $3.38 \mathrm{E}-05$ \\
\hline Initial AZ102- 70.7\%,60\% & $\mathrm{OLH}$ & 6TB084 & 58 & 1 & 6 & 0 & 3 & 1 & 7 & 0 & 0 & 0 & 1 & 0 & $1.13 \mathrm{E}+01$ & $4.27 \mathrm{E}+05$ & $2.64 \mathrm{E}-05$ \\
\hline Initial AZ102- 70.7\%,60\% & $\mathrm{OLH}$ & 6ТВ094 & 62 & 1 & 6 & 0 & 1 & 4.24E-01 & 3 & 0 & 0 & 0 & $3.40 \mathrm{E}-01$ & 0 & $5.45 \mathrm{E}+00$ & $4.23 \mathrm{E}+05$ & $1.29 \mathrm{E}-05$ \\
\hline Initial AZ102- 70.7\%,60\% & $\mathrm{OLH}$ & $6 \mathrm{~TB} 100$ & 45 & 0 & 6 & 0 & 1 & $2.43 \mathrm{E}-01$ & 2 & 0 & 0 & 0 & $2.89 \mathrm{E}-03$ & 0 & $2.94 \mathrm{E}+00$ & $4.25 \mathrm{E}+05$ & $6.92 \mathrm{E}-06$ \\
\hline
\end{tabular}


The density predictions for Envelope $\mathrm{C}$ were within $+/-11 \%$ of the measured values for the bottoms concentrate with a Na molarity between 2 and 10. The experimental runs that were compared are shown in Table 22. The measured and predicted value comparison is shown in Table 23.

Table 22. Experimental IDs for Envelope C

\begin{tabular}{|c|c|}
\hline Experimental Description & \begin{tabular}{|c|} 
Experimental \\
ID
\end{tabular} \\
\hline $1=55 \%$ SBS $(\mathrm{C} 2), 43 \%$ AN102 & 1-INIT \\
\hline $2=55 \%$ SBS $(\mathrm{C} 2), 43 \%$ AN107 & 2-INIT \\
\hline $3=43 \%$ SBS $(\mathrm{C} 2), 55 \%$ AN102 & 3-INIT \\
\hline $4=43 \%$ SBS $(\mathrm{C} 2), 55 \%$ AN107 & 4-INIT \\
\hline $1=55 \%$ SBS $(\mathrm{C} 2), 43 \%$ AN102 conc to $6 \mathrm{Na} \mathrm{M}$ & $\# 1-6$ \\
\hline $2=55 \% \mathrm{SBS}(\mathrm{C} 2), 43 \% \mathrm{AN} 107$ conc to $6 \mathrm{Na} \mathrm{M}$ & $\# 2-6$ \\
\hline $3=43 \%$ SBS (C2), $55 \%$ AN102 conc to $6 \mathrm{Na} \mathrm{M}$ & $\# 3-6$ \\
\hline $4=43 \%$ SBS (C2), $55 \%$ AN107 conc to $6 \mathrm{Na} \mathrm{M}$ & $\# 4-6$ \\
\hline $1=55 \%$ SBS (C2), $43 \%$ AN102 conc to $8 \mathrm{Na} \mathrm{M}$ & $\# 1-8$ \\
\hline $2=55 \%$ SBS (C2), $43 \%$ AN107 conc to $8 \mathrm{Na} \mathrm{M}$ & $\# 2-8$ \\
\hline $3=43 \%$ SBS (C2), $55 \%$ AN102 conc to $8 \mathrm{Na} \mathrm{M}$ & $\# 3-8$ \\
\hline $4=43 \%$ SBS (C2), $55 \%$ AN107 conc to $8 \mathrm{Na} \mathrm{M}$ & $\# 4-8$ \\
\hline $1=55 \%$ SBS $(\mathrm{C} 2), 43 \%$ AN102 conc to $10 \mathrm{Na} \mathrm{M}$ & $\# 1-10$ \\
\hline $2=55 \%$ SBS (C2), $43 \%$ AN107 conc to $10 \mathrm{Na} \mathrm{M}$ & $\# 2-10$ \\
\hline $3=43 \%$ SBS $(\mathrm{C} 2), 55 \%$ AN102 conc to $10 \mathrm{Na} \mathrm{M}$ & $\# 3-10$ \\
\hline $4=43 \%$ SBS (C2), $55 \%$ AN107 conc to $10 \mathrm{Na} \mathrm{M}$ & $\# 4-10$ \\
\hline
\end{tabular}


WSRC-TR-2003-00269, REVISION 0

SRT-RPP-2003-00125, REVISION 0

Table 23. Comparison of Measured and Predicted Densities for Envelope C

\begin{tabular}{|c|c|c|c|c|c|c|c|c|c|c|c|c|}
\hline \multirow[b]{2}{*}{ Temperature } & \multicolumn{4}{|c|}{ Measured Density [g/ml] } & \multicolumn{4}{|c|}{ Predicted Density [g/ml] } & \multicolumn{4}{|c|}{$\begin{array}{c}\% \text { Difference } b / n \text { Meas \& Pred } \\
\text { Density }\end{array}$} \\
\hline & 15 & 25 & 50 & 65 & 15 & 25 & 50 & 65 & 15 & 25 & 50 & 65 \\
\hline 1 -INIT & 1.143 & 1.138 & 1.124 & 1.112 & 1.150 & 1.143 & 1.126 & 1.115 & $-0.62 \%$ & $-0.44 \%$ & $-0.11 \%$ & $-0.30 \%$ \\
\hline 2-INIT & 1.128 & 1.123 & 1.109 & 1.099 & 1.147 & 1.139 & 1.122 & 1.111 & $-1.66 \%$ & $-1.46 \%$ & $-1.12 \%$ & $-1.13 \%$ \\
\hline 3-INIT & 1.176 & 1.171 & 1.157 & 1.149 & 1.165 & 1.157 & 1.139 & 1.128 & $0.98 \%$ & $1.17 \%$ & $1.55 \%$ & $1.79 \%$ \\
\hline 4-INIT & 160 & 1.155 & 1.141 & 1.132 & 1.158 & 1.151 & 1.133 & 1.122 & $0.17 \%$ & $0.36 \%$ & $0.74 \%$ & $0.89 \%$ \\
\hline$\# 1-6$ & 1.383 & 1.376 & 1.356 & 1.343 & 1.313 & 1.301 & 1.271 & 1.253 & $5.05 \%$ & $5.43 \%$ & $6.25 \%$ & $6.66 \%$ \\
\hline$\# 2-6$ & 1.359 & 1.351 & 1.328 & 1.313 & 1.306 & 1.294 & 1.265 & 1.247 & $3.86 \%$ & $4.20 \%$ & $4.73 \%$ & $5.03 \%$ \\
\hline$\# 3-6$ & 1 & 5 & 9 & 43 & 1 & 7 & 1 & 1. & $5.33 \%$ & 0 & 0 & 0 \\
\hline$\# 4-6$ & 1.332 & 1.325 & 1.308 & 1.298 & 1.289 & 1.278 & 1.250 & 1.233 & $3.23 \%$ & $3.59 \%$ & $4.45 \%$ & $4.95 \%$ \\
\hline$\# 1-8$ & 1.487 & 1.479 & 1.460 & 1.445 & 1.391 & 1.377 & 1.341 & 1.320 & $6.45 \%$ & $6.95 \%$ & $8.16 \%$ & $8.69 \%$ \\
\hline$\# 2-8$ & 1.448 & 1.440 & 1.421 & 1.409 & 1.382 & 1.368 & 1.333 & 1.312 & $4.56 \%$ & $5.00 \%$ & $6.18 \%$ & $6.89 \%$ \\
\hline$\# 3-8$ & 1.476 & 1.470 & 1.460 & 1.456 & 1.377 & 1.363 & 1.329 & 1.308 & $6.73 \%$ & $7.26 \%$ & $8.98 \%$ & $10.17 \%$ \\
\hline$\# 4-8$ & 1.429 & 1.422 & 1.405 & 1.396 & 1.366 & 1.353 & 1.319 & 1.299 & $4.38 \%$ & $4.87 \%$ & $6.12 \%$ & $6.92 \%$ \\
\hline$\# 1-10$ & 1.577 & 1.569 & 1.549 & 1.537 & 1.455 & 1.439 & 1.399 & 1.374 & $7.70 \%$ & $8.26 \%$ & $9.70 \%$ & $10.58 \%$ \\
\hline$\# 2-10$ & 1.501 & 1.492 & 1.473 & 1.460 & 1.443 & 1.427 & 1.387 & 1.363 & $3.89 \%$ & $4.39 \%$ & $5.81 \%$ & $6.64 \%$ \\
\hline$\# 3-10$ & $\mathrm{n} / \mathrm{a}$ & 1.510 & $\mathrm{n} / \mathrm{a}$ & $\mathrm{n} / \mathrm{a}$ & 1.433 & 1.418 & 1.379 & 1.356 & $\mathrm{n} / \mathrm{a}$ & $6.12 \%$ & $\mathrm{n} / \mathrm{a}$ & $\mathrm{n} / \mathrm{a}$ \\
\hline$\# 4-10$ & $\mathrm{n} / \mathrm{a}$ & 1.488 & 1.469 & 1.458 & 1.411 & 1.397 & 1.360 & 1.338 & $\mathrm{n} / \mathrm{a}$ & $6.16 \%$ & $7.46 \%$ & $8.25 \%$ \\
\hline
\end{tabular}


The viscosity predictions for Envelope $\mathrm{C}$ were not good as Envelope A, with errors between the measured and predicted values ranging from $20 \%$ to $70 \%$. It is estimated that part of this error is due to the prediction being based on simulated values between 6 and 10 molar. It is also expected that the lower and higher temperature extremes produced solids that are not accounted for by the viscosity prediction since it is based only on the supernate phase of the bottoms concentrate. A better predictor of viscosity could be obtained by getting an accurate count of the solids in each sample plus a solids correction term derived from experimental results would need to be included. Prior modeling work ${ }^{9}$ for the waste feed evaporator showed that when the supernate viscosity was measured experimentally and compared with modeling predictions, relatively good agreement (within $+/-15 \%$ ) was obtained. A complete listing of the measured versus predicted viscosities is shown in Table 24.

Table 24. Comparison of Measured versus Predicted Viscosities for Envelope $\mathbf{C}$

\begin{tabular}{|c|c|c|c|c|c|c|c|c|c|c|}
\hline $\begin{array}{l}\text { Envelope } \\
\text { C Sample }\end{array}$ & \begin{tabular}{|c|} 
Measured \\
Na $M$
\end{tabular} & $\begin{array}{c}\text { Measured } \\
\text { Viscosity } \\
{[\mathrm{cP}] \text { at }} \\
15^{\circ} \mathrm{C}\end{array}$ & $\begin{array}{c}\text { Predicted } \\
\text { Viscosity } \\
{[\mathrm{cP}] \text { at }} \\
15^{\circ} \mathrm{C}\end{array}$ & \begin{tabular}{|c|}
$\%$ Diff \\
b/n Meas \\
\& Pred \\
Visc. at \\
$15^{\circ} \mathrm{C}$
\end{tabular} & $\begin{array}{c}\text { Measured } \\
\text { Viscosity } \\
{[\mathrm{cP}] \text { at }} \\
25^{\circ} \mathrm{C}\end{array}$ & $\begin{array}{c}\text { Predicted } \\
\text { Viscosity } \\
{[\mathrm{cP}] \text { at }} \\
25^{\circ} \mathrm{C}\end{array}$ & \begin{tabular}{|c|}
$\%$ Diff \\
b/n Meas \\
\& Pred \\
Visc. at \\
$25^{\circ} \mathrm{C}$ \\
\end{tabular} & \begin{tabular}{|c|} 
Measured \\
Viscosity \\
{$[\mathrm{cP}]$ at } \\
$60^{\circ} \mathrm{C}$
\end{tabular} & $\begin{array}{c}\text { Predicted } \\
\text { Viscosity } \\
{[\mathrm{cP}] \text { at }} \\
60^{\circ} \mathrm{C}\end{array}$ & $\begin{array}{c}\% \text { Diff b/n } \\
\text { Meas \& } \\
\text { Pred Visc. } \\
\text { at } 60^{\circ} \mathrm{C}\end{array}$ \\
\hline $1-2$ & 2.19 & 1.77 & 1.12 & $36.6 \%$ & 1.46 & 0.79 & $-46.2 \%$ & 0.76 & 0.32 & $58.0 \%$ \\
\hline $1-6$ & 6.71 & 7.52 & 3.92 & $47.8 \%$ & 4.97 & 2.92 & $-41.2 \%$ & 2.39 & 1.52 & $36.2 \%$ \\
\hline $1-8$ & 8.87 & 15.31 & 5.88 & $61.6 \%$ & 12.80 & 4.26 & $-66.7 \%$ & 5.04 & 2.14 & $57.5 \%$ \\
\hline $2-2$ & 2.16 & 1.73 & 1.14 & $34.0 \%$ & 1.37 & 0.80 & $-41.6 \%$ & 0.67 & 0.33 & $50.6 \%$ \\
\hline $2-6$ & 6.60 & 6.33 & 3.90 & $38.4 \%$ & 4.35 & 2.90 & $-33.2 \%$ & 1.99 & 1.51 & $23.9 \%$ \\
\hline $2-8$ & 8.71 & 13.68 & 5.80 & $57.6 \%$ & 9.80 & 4.21 & $-57.0 \%$ & 3.71 & 2.12 & $42.9 \%$ \\
\hline $3-2$ & 2.84 & 2.13 & 1.52 & $28.5 \%$ & 1.72 & 1.10 & $-35.8 \%$ & 0.88 & 0.52 & $41.4 \%$ \\
\hline $3-6$ & 6.55 & 7.38 & 3.91 & $47.0 \%$ & 4.87 & 2.90 & $-40.4 \%$ & 2.23 & 1.51 & $32.3 \%$ \\
\hline $3-8$ & 8.73 & 15.02 & 5.91 & $60.7 \%$ & 11.29 & 4.27 & $-62.2 \%$ & 3.73 & 2.14 & $42.7 \%$ \\
\hline $4-2$ & 2.72 & 2.03 & 1.50 & $26.2 \%$ & 1.57 & 1.08 & $-31.1 \%$ & 0.86 & 0.51 & $41.3 \%$ \\
\hline $4-6$ & 6.37 & 5.95 & 3.84 & $35.5 \%$ & 4.16 & 2.85 & $-31.5 \%$ & 1.85 & 1.48 & $19.9 \%$ \\
\hline $4-8$ & 8.51 & 11.97 & 5.78 & $51.8 \%$ & 7.84 & 4.18 & $-46.7 \%$ & 2.99 & 2.10 & $29.9 \%$ \\
\hline
\end{tabular}


The thermal conductivity predictions for Envelope $C$ were within $+/-20 \%$ of the measured values for the bottoms concentrate with a Na molarity between 2 and 8 except for 3 samples. The discrepancy for the 3 samples outside the $+/-20 \%$ range is due in part to the fact that the simulated conductivity is for the supernate only where as the measured conductivity is for the entire slurry (supernate plus solids). Prior modeling work ${ }^{9}$ showed that the experimental conductivity has a standard deviation of $6.5 \%$ (about the value of water). The simulated conductivities are based on correction factors for the conductivity of water using various anions/cations as defined in Perry's Chemical Handbook. ${ }^{15}$ The simulated conductivities for all envelopes fell within a 3\% standard deviation of the value of water. Since the measurement error is higher than this value, the predicted conductivities cannot be distinguished from those of water. Therefore there is no need to use the complicated prediction equation for conductivity when statistically a prediction for the conductivity of water is just as accurate. A complete listing of the measured versus predicted conductivities is shown in Table 25.

Table 25. Comparison of Measured versus Predicted Conductivity for Envelope C

\begin{tabular}{|c|c|c|c|}
\hline Envelope C Sample & $\begin{array}{c}\text { Measured } \\
\text { Conductivity } \\
{\left[\mathbf{W} / \mathbf{m}^{\circ} \mathbf{C}\right] \text { at } \mathbf{2 5}^{\circ} \mathbf{C}}\end{array}$ & $\begin{array}{c}\text { Predicted } \\
\text { Conductivity } \\
{\left[\mathbf{W} / \mathbf{m}^{\circ} \mathbf{C}\right] \text { at } \mathbf{2 5}^{\circ} \mathbf{C}}\end{array}$ & $\begin{array}{c}\text { \% Diff b/n measured } \\
\text { \& Predicted } \\
\text { Conductivity }^{\circ}\end{array}$ \\
\hline 1-in-C & 0.229 & 0.581 & $-154.1 \%$ \\
\hline 2-in-C & 0.209 & 0.590 & $-182.6 \%$ \\
\hline 3-in-C & 0.587 & 0.580 & $1.2 \%$ \\
\hline 4-in-C & 0.634 & 0.590 & $7.0 \%$ \\
\hline 1-6-C & 0.403 & 0.560 & $-39.0 \%$ \\
\hline 2-6-C & 0.638 & 0.570 & $10.7 \%$ \\
\hline 3-6-C & 0.672 & 0.563 & $16.2 \%$ \\
\hline 4-6-C & 0.713 & 0.573 & $19.7 \%$ \\
\hline 1-8-C & 0.643 & 0.550 & $14.5 \%$ \\
\hline 2-8-C & 0.601 & 0.560 & $6.8 \%$ \\
\hline 3-8-C & 0.675 & 0.553 & $18.1 \%$ \\
\hline 4-8-C & 0.603 & 0.563 & $6.6 \%$ \\
\hline
\end{tabular}


The heat capacity predictions for Envelope $\mathrm{C}$ were within $+/-10 \%$ of the measured values for the bottoms concentrate with a Na molarity between 2 and 8 . A complete listing of the measured versus predicted values is shown in Table 26.

Table 26. Comparison of Measured versus Predicted $\mathrm{Cp}$ for Envelope $\mathrm{C}$

\begin{tabular}{|c|c|c|c|}
\hline Envelope B Sample & $\begin{array}{c}\text { Measured Cp } \\
{\left[\mathbf{c a l} / \mathbf{g}^{*} \mathbf{K}\right]}\end{array}$ & $\begin{array}{c}\text { Predicted Cp } \\
{[\mathbf{c a l} / \mathbf{g} \text { *K] }}\end{array}$ & $\begin{array}{c}\text { \% Diff b/n measured } \\
\text { \& Predicted Cp }\end{array}$ \\
\hline 1-in-C & 0.836 & 0.837 & $-0.2 \%$ \\
\hline 2-in-C & 0.837 & 0.823 & $1.7 \%$ \\
\hline $2-6-\mathrm{C}$ & 0.728 & 0.686 & $5.8 \%$ \\
\hline 1-8-C & 0.683 & 0.631 & $7.6 \%$ \\
\hline 2-8-C & 0.679 & 0.621 & $8.6 \%$ \\
\hline
\end{tabular}

The sodium molarity predictions for envelope $\mathrm{C}$ were worse than for the other envelopes only coming within $+/-32 \%$ of the measured values for the bottoms concentrate with a $\mathrm{Na}$ molarity between 2 and 10 . This poor comparison was expected since the Na molarity prediction did not satisfy the $+/-15 \%$ of the predicted values criterion even after adding some of the validation points to the fits to try to capture some nonlinear behavior. One reason for the large discrepancy is because it is difficult to derive a predictive relationship relating waste feed composition (dry basis), SBS/Feed ratio, bottoms temperature, and bottoms density to bottoms Na molarity. Table 27 shows a complete list of the measured versus predicted Na molarities.

Table 27. Comparison of Measured versus Predicted Na M for Envelope C

\begin{tabular}{|c|c|c|c|}
\hline Experimental ID & Measured Na M & Predicted Na M & \%Diff Meas Na M \& Pred Na M @25 ${ }^{\circ}$ \\
\hline 1-INIT & 2.2 & 2.1 & $4.1 \%$ \\
\hline 2-INIT & 2.2 & 2.0 & $6.4 \%$ \\
\hline 3 -INIT & 2.8 & 3.1 & $-8.1 \%$ \\
\hline $4-$ INIT & 2.7 & 2.9 & $-7.9 \%$ \\
\hline$\# 1-6$ & 6.7 & 8.7 & $-29.3 \%$ \\
\hline$\# 2-6$ & 6.6 & 8.3 & $-26.4 \%$ \\
\hline$\# 3-6$ & 6.5 & 8.6 & $-32.1 \%$ \\
\hline$\# 4-6$ & 6.4 & 7.9 & $-23.3 \%$ \\
\hline$\# 1-8$ & 8.9 & 11.5 & $-30.1 \%$ \\
\hline$\# 2-8$ & 8.7 & 10.8 & $-24.0 \%$ \\
\hline$\# 3-8$ & 8.7 & 11.7 & $-33.6 \%$ \\
\hline$\# 4-8$ & 8.5 & 10.6 & $-24.9 \%$ \\
\hline$\# 1-10$ & 10.7 & 14.0 & $-31.5 \%$ \\
\hline$\# 2-10$ & 10.4 & 12.2 & $-17.8 \%$ \\
\hline$\# 3-10$ & 10.3 & 12.8 & $-24.6 \%$ \\
\hline$\# 4-10$ & 9.8 & 12.5 & $-28.3 \%$ \\
\hline
\end{tabular}


Table 29 and Table 30 show the predicted solids for Envelope $\mathrm{C}$ and identify experimental run samples that are similar in composition, SBS/Feed ratio, and endpoint Na molarity. Because of slight differences in experimental runs and simulation runs no points are exact matches. However, the points listed in the table come close. Note that the experimental ID is described in Table 28. For Envelope C, about $60 \%$ of the simulated values (242 our of 405 points - including fit and validation points) had bottoms insoluble solids greater than $1 \mathrm{wt} \%$, but only about $36 \%$ of the simulated values (145 points) had insoluble solids greater than 2 $\mathrm{wt} \%$. Of this $36 \%$ segment, $83 \%$ of the values were between $2 \mathrm{wt} \%$ and $5 \mathrm{wt} \%$, while the remaining $17 \%$ of the values were between $5 \mathrm{wt} \%$ and $7 \mathrm{wt} \%$. The higher solids weight percents were observed at NA M greater than 8 . Temperatures have a much smaller impact on the solubility than the Na molarity. The primary salts were calcium fluoride, hydrosodalite, sodium oxalate, sodium carbonate, sodium fluorosulfate, sodium sulfatecarbonate, sodium fluoride, sodium aluminosilicate gelatin (NASGEL), and sodium nitrate. For the simulated runs, NASGEL solids appear in the bottoms concentrate stream over a wider \% range at $10 \mathrm{Na} \mathrm{M}$ than $8 \mathrm{Na} \mathrm{M}$ and $6 \mathrm{Na} \mathrm{M}$ as shown in Figure 8. For all sodium molarities simulated, the \% NASGEL in the bottoms stream stays below $1.2 \%$. The NASGEL solids range between 0 and $1.2 \%$ for bottoms temperatures between $15^{\circ} \mathrm{C}$ and 66 ${ }^{\circ} \mathrm{C}$ as shown in Figure 9. Increasing the SBS/Feed ratio above 0.5 for the $10 \mathrm{Na} \mathrm{M}$ bottoms concentrate increases the chance of NASGEL formation as shown in Figure 10. Increasing the SBS/Feed ratio above 0.25 for the $8 \mathrm{Na} \mathrm{M}$ bottoms concentrate increases the chance of NASGEL formation as shown in Figure 11. For the $8 \mathrm{Na} \mathrm{M}$ bottoms concentrate NASGEL begins to form at lower SBS/Feed ratios but the \% NASGEL at the highest SBS/Feed ratio is less than at $10 \mathrm{Na} \mathrm{M}$. The $6 \mathrm{Na} \mathrm{M}$ bottoms concentrate shows NASGEL formation starting around SBS/Feed of 0.15 and increasing as SBS/Feed ratio increases. Going from $10 \mathrm{Na} \mathrm{M}$ bottoms concentrate down to $8 \mathrm{Na} \mathrm{M}$ then $6 \mathrm{Na} \mathrm{M}$, the maximum amount of NASGEL formed at the highest SBS/Feed ratio decreases along with the $\mathrm{Na} \mathrm{M}$. Please note that due to the variability in the data, a low Na M like 6 and a high SBS/Feed ratio like 2 can still form more NASGEL than a higher $\mathrm{Na} \mathrm{M}$ at the same SBS/Feed ratio. For a complete listing of the solids predicted for Envelope C please see Appendix B.

Table 28. Experimental ID for Envelope C

\begin{tabular}{|c|c|}
\hline ID & Experimental Description \\
\hline 1 & $55 \%$ SBS (C2), 43\% AN102 \\
\hline 2 & $55 \%$ SBS (C2), 43\% AN107 \\
\hline 3 & $43 \%$ SBS (C2), 55\% AN102 \\
\hline 4 & $43 \%$ SBS (C2), 55\% AN107 \\
\hline
\end{tabular}

Additional experiments were performed with AW-101 radioactive simulant. ${ }^{8}$ The physical property prediction models for Envelope A were used to compare measured versus predicted values from this experimental work. These comparisons are shown in Table 31. As can be seen the $\mathrm{Na} \mathrm{M}$ predictions for the experimental samples other than the feed are not very good. Since the experimental samples were taken as the process was still being evaporated and not at steady state, the $\mathrm{Na} \mathrm{M}$ predictions would not be expected to be very good since they are based on steady state simulated conditions. The density predictions are within $+/-$ $15 \%$ which is a good indicator of the ability of OLI to predict density, at least within an acceptable range. 
The predicted viscosities are off by as much as 50\% from the measured values. Since the viscosity prediction has a strong dependence on $\mathrm{Na}$ molarity and represents the equilibrium value, it is not surprising the predicting values are off as indicated. In the experiment, samples were pulled at various bottom concentrations which most likely did not represent the process at equilibrium. OLI is a steady state simulator and thus any predictions derived from its results represent steady state points. Also the viscosity can be strongly influenced by solids which can cause the large discrepancies seen here. The predicted heat capacities are within $+/-20 \%$. 
WSRC-TR-2003-00269, REVISION 0 SRT-RPP-2003-00125, REVISION 0

Table 29. Predicted Solids from Envelope C Simulations - Part 1

\begin{tabular}{|c|c|c|c|c|c|c|c|c|c|c|c|c|c|c|c|c|c|}
\hline \begin{tabular}{|c|} 
Experimental \\
ID
\end{tabular} & \begin{tabular}{|l|} 
Simulation \\
Point Type
\end{tabular} & $\begin{array}{c}\text { Simulation } \\
\text { ID }\end{array}$ & \begin{tabular}{|c|} 
Temp \\
${ }^{\circ} \mathrm{C}$ \\
$(\mathbf{P} 2)$
\end{tabular} & $\begin{array}{c}\text { SBS/Feed } \\
\text { (P1) }\end{array}$ & \begin{tabular}{l|}
$\mathrm{Na}$ \\
$\mathrm{M}$
\end{tabular} & $\begin{array}{c}\text { ALOH3 } \\
{[\mathrm{g}]}\end{array}$ & $\begin{array}{c}\text { BASO4 } \\
\text { [g] }\end{array}$ & $\begin{array}{c}\text { CA3PO42 } \\
{[\mathrm{g}]}\end{array}$ & $\begin{array}{c}\mathrm{CACO3} \\
{[\mathrm{g}]}\end{array}$ & $\begin{array}{c}\text { CAF2 } \\
\text { [g] }\end{array}$ & $\begin{array}{c}\text { CAOH2 } \\
\text { [g] }\end{array}$ & $\begin{array}{c}\text { CATIO3 } \\
{[\mathrm{g}]}\end{array}$ & $\begin{array}{c}\text { CROH3 } \\
{[\mathrm{g}]}\end{array}$ & $\begin{array}{c}\text { FEIIIOH3 } \\
{[\mathrm{g}]}\end{array}$ & $\begin{array}{c}\text { HYDROSOD } \\
{[\mathrm{g}]}\end{array}$ & $\begin{array}{c}\text { MGF2 } \\
\text { [g] }\end{array}$ & $\begin{array}{c}\text { MGOH2 } \\
\text { [g] }\end{array}$ \\
\hline 1 & EV & 10TC001 & 15 & 0 & \begin{tabular}{|l|}
10 \\
\end{tabular} & 0 & 0 & 0 & 0 & 0 & 0 & 0 & 0 & 0 & 0 & 0 & 0 \\
\hline 1 & EV & $8 \mathrm{TC} 001$ & 15 & 0 & 8 & 0 & 0 & 0 & 0 & 0 & 0 & 0 & 0 & 0 & 0 & 0 & 0 \\
\hline 1 & EV & 6TC001 & 15 & 0 & 6 & 371 & 0 & 0 & 0 & 0 & 0 & 0 & 0 & 0 & 0 & 0 & 0 \\
\hline 1 & EV & 10TC002 & 15 & 0 & \begin{tabular}{|l|}
10 \\
\end{tabular} & 0 & 0 & 0 & 0 & 0 & 0 & 0 & 0 & 0 & 0 & 0 & 0 \\
\hline 1 & EV & $8 \mathrm{TC} 002$ & 15 & 0 & 8 & 0 & 0 & 0 & 0 & 0 & 0 & 0 & 0 & 0 & 0 & 0 & 0 \\
\hline 1 & EV & 6TC002 & 15 & 0 & 6 & 515 & 0 & 0 & 0 & 0 & 0 & 0 & 0 & 0 & 0 & 0 & 0 \\
\hline 2 & $\mathrm{EV}$ & $10 \mathrm{TC} 004$ & 15 & 0 & \begin{tabular}{|l|}
10 \\
\end{tabular} & 0 & 0 & 0 & 0 & 0 & 0 & 0 & 0 & 0 & 0 & 0 & 0 \\
\hline 2 & EV & 8TC004 & 15 & 0 & 8 & 0 & 0 & 0 & 0 & 0 & 0 & 0 & 0 & 0 & 0 & 0 & 0 \\
\hline 2 & EV & 6TC004 & 15 & 0 & 6 & 0 & 0 & 0 & 0 & 0 & 0 & 0 & 0 & 0 & 0 & 0 & 0 \\
\hline 2 & EV & 10TC005 & 15 & 0 & \begin{tabular}{|l|}
10 \\
\end{tabular} & 0 & 0 & 0 & 0 & 0 & 0 & 0 & 0 & 0 & 0 & 0 & 0 \\
\hline 2 & EV & $8 \mathrm{TC} 005$ & 15 & 0 & 8 & 0 & 0 & 0 & 0 & 0 & 0 & 0 & 0 & 0 & 0 & 0 & 0 \\
\hline 2 & EV & 6TC005 & 15 & 0 & 6 & 0 & 0 & 0 & 0 & 0 & 0 & 0 & 0 & 0 & 0 & 0 & 0 \\
\hline 1 & EV & 10TC006 & 66 & 0 & \begin{tabular}{|l|}
10 \\
\end{tabular} & 0 & 0 & 0 & 0 & 0 & 0 & 0 & 0 & 0 & 0 & 0 & 0 \\
\hline 1 & EV & 8TC006 & 66 & 0 & 8 & 0 & 0 & 0 & 0 & 0 & 0 & 0 & 0 & 0 & 0 & 0 & 0 \\
\hline 1 & EV & 6TC006 & 66 & 0 & 6 & 0 & 0 & 0 & 0 & 0 & 0 & 0 & 0 & 0 & 0 & 0 & 0 \\
\hline 1 & $\mathrm{EV}$ & $10 \mathrm{TC} 007$ & 66 & 0 & \begin{tabular}{|l|}
10 \\
\end{tabular} & 0 & 0 & 0 & 0 & 0 & 0 & 0 & 0 & 0 & 0 & 0 & 0 \\
\hline 1 & EV & $8 \mathrm{TC} 007$ & 66 & 0 & 8 & 0 & 0 & 0 & 0 & 0 & 0 & 0 & 0 & 0 & 0 & 0 & 0 \\
\hline 1 & EV & 6TC007 & 66 & 0 & 6 & 0 & 0 & 0 & 0 & 0 & 0 & 0 & 0 & 0 & 0 & 0 & 0 \\
\hline 2 & EV & $10 \mathrm{TC} 009$ & 66 & 0 & \begin{tabular}{|l|l|}
10 \\
\end{tabular} & 0 & 0 & 0 & 0 & 0 & 0 & 0 & 0 & 0 & 0 & 0 & 0 \\
\hline 2 & EV & 8TC009 & 66 & 0 & 8 & 0 & 0 & 0 & 0 & 0 & 0 & 0 & 0 & 0 & 0 & 0 & 0 \\
\hline 2 & $\mathrm{EV}$ & 6ТC009 & 66 & 0 & 6 & 0 & 0 & 0 & 0 & 0 & 0 & 0 & 0 & 0 & 0 & 0 & 0 \\
\hline 2 & EV & $10 \mathrm{TC} 010$ & 66 & 0 & \begin{tabular}{|l|}
10 \\
\end{tabular} & 0 & 0 & 0 & 0 & 0 & 0 & 0 & 0 & 0 & 0 & 0 & 0 \\
\hline 2 & EV & $8 \mathrm{TC} 010$ & 66 & 0 & 8 & 0 & 0 & 0 & 0 & 0 & 0 & 0 & 0 & 0 & 0 & 0 & 0 \\
\hline 2 & EV & 6TC010 & 66 & 0 & 6 & 0 & 0 & 0 & 0 & 0 & 0 & 0 & 0 & 0 & 0 & 0 & 0 \\
\hline 1 & EV & 10TC011 & 15 & 2.0 & \begin{tabular}{|l|}
10 \\
\end{tabular} & 0 & 0 & 0 & 0 & 2036 & 0 & 289 & 0 & 471 & 4113 & 0 & 374 \\
\hline 1 & $\mathrm{EV}$ & $8 \mathrm{TC} 011$ & 15 & 2.0 & 8 & 1507 & 1 & 0 & 0 & 2036 & 0 & 289 & 0 & 512 & 4468 & 0 & 374 \\
\hline 1 & EV & 6TC011 & 15 & 2.0 & 6 & 3177 & 4 & 0 & 0 & 2036 & 0 & 289 & 0 & 537 & 4684 & 0 & 374 \\
\hline 1 & EV & $10 \mathrm{TC} 012$ & 15 & 2.0 & 10 & 0 & 0 & 0 & 0 & 2028 & 0 & 288 & 0 & 429 & 3739 & 0 & 372 \\
\hline 1 & EV & $8 \mathrm{TC} 012$ & 15 & 2.0 & 8 & 1162 & 1 & 0 & 0 & 2028 & 0 & 288 & 0 & 487 & 4251 & 0 & 372 \\
\hline 1 & EV & 6TC012 & 15 & 2.0 & 6 & 3389 & 4 & 0 & 0 & 2028 & 0 & 288 & 0 & 520 & 4535 & 0 & 372 \\
\hline
\end{tabular}


WSRC-TR-2003-00269, REVISION 0 SRT-RPP-2003-00125, REVISION 0

Table 29. Predicted Solids from Envelope C Simulations - Part 1 (cont'd)

\begin{tabular}{|c|c|c|c|c|c|c|c|c|c|c|c|c|c|c|c|c|c|}
\hline $\begin{array}{c}\text { Experimental } \\
\text { ID }\end{array}$ & \begin{tabular}{|l|} 
Simulation \\
Point Type
\end{tabular} & \begin{tabular}{|c|} 
Simulation \\
ID
\end{tabular} & $\begin{array}{c}\text { Temp } \\
{ }^{\circ} \mathbf{C} \\
(\mathrm{P} 2)\end{array}$ & $\begin{array}{c}\text { SBS/Feed } \\
\text { (P1) }\end{array}$ & $\begin{array}{l}\mathrm{Na} \\
\mathrm{M}\end{array}$ & $\begin{array}{c}\text { ALOH3 } \\
{[\mathrm{g}]}\end{array}$ & $\begin{array}{c}\text { BASO4 } \\
{[\mathrm{g}]}\end{array}$ & $\begin{array}{c}\text { CA3PO42 } \\
{[\mathrm{g}]}\end{array}$ & $\begin{array}{c}\mathrm{CACO3} \\
{[\mathrm{g}]}\end{array}$ & $\begin{array}{c}\text { CAF2 } \\
\text { [g] }\end{array}$ & \begin{tabular}{|} 
CAOH2 \\
{$[\mathrm{g}]$}
\end{tabular} & $\begin{array}{c}\text { CATIO3 } \\
{[\mathrm{g}]}\end{array}$ & \begin{tabular}{|c|} 
CROH3 \\
{$[\mathrm{g}]$}
\end{tabular} & $\begin{array}{c}\text { FEIIIOH3 } \\
{[\mathrm{g}]}\end{array}$ & $\begin{array}{c}\text { HYDROSOD } \\
{[\mathrm{g}]}\end{array}$ & $\begin{array}{c}\text { MGF2 } \\
{[\mathrm{g}]}\end{array}$ & $\begin{array}{c}\text { MGOH2 } \\
\text { [g] }\end{array}$ \\
\hline 2 & EV & 10TC014 & 15 & 2.0 & \begin{tabular}{|l|}
10 \\
\end{tabular} & 0 & 0 & 0 & 0 & 1991 & 0 & 283 & 0 & 461 & 4024 & 0 & 365 \\
\hline 2 & EV & $8 \mathrm{TC} 014$ & 15 & 2.0 & 8 & 0 & 1 & 0 & 0 & 1991 & 0 & 283 & 0 & 503 & 4390 & 0 & 365 \\
\hline 2 & EV & 6TC014 & 15 & 2.0 & 6 & 0 & 4 & 0 & 0 & 1991 & 0 & 283 & 18 & 529 & 4612 & 0 & 365 \\
\hline 2 & EV & $10 \mathrm{TC} 015$ & 15 & 2.0 & \begin{tabular}{|l|}
10 \\
\end{tabular} & 0 & 0 & 0 & 0 & 2007 & 0 & 285 & 0 & 517 & 4512 & 530 & 368 \\
\hline 2 & EV & $8 \mathrm{TC} 015$ & 15 & 2.0 & 8 & 0 & 0 & 0 & 0 & 2007 & 0 & 285 & 58 & 540 & 4707 & 101 & 368 \\
\hline 2 & EV & 6TC015 & 15 & 2.0 & 6 & 0 & 4 & 0 & 0 & 2007 & 0 & 285 & 136 & 552 & 4820 & 0 & 368 \\
\hline 1 & $\mathrm{EV}$ & 10TC016 & 66 & 2.0 & \begin{tabular}{|l|}
10 \\
\end{tabular} & 0 & 3 & 0 & 0 & 2035 & 0 & 289 & 264 & 0 & 0 & 1227 & 374 \\
\hline 1 & EV & $8 \mathrm{TC} 016$ & 66 & 2.0 & 8 & 0 & 3 & 0 & 0 & 2035 & 0 & 289 & 268 & 0 & 0 & 248 & 374 \\
\hline 1 & EV & 6TC016 & 66 & 2.0 & 6 & 0 & 3 & 0 & 0 & 2034 & 0 & 289 & 271 & 0 & 0 & 0 & 374 \\
\hline 1 & EV & $10 \mathrm{TC} 017$ & 66 & 2.0 & \begin{tabular}{|l|}
10 \\
\end{tabular} & 0 & 3 & 0 & 0 & 2027 & 0 & 288 & 259 & 0 & 0 & 1042 & 372 \\
\hline 1 & EV & $8 \mathrm{TC} 017$ & 66 & 2.0 & 8 & 0 & 3 & 0 & 0 & 2027 & 0 & 288 & 265 & 0 & 0 & 73 & 372 \\
\hline 1 & $\mathrm{EV}$ & 6TC017 & 66 & 2.0 & 6 & 0 & 3 & 0 & 0 & 2026 & 0 & 288 & 268 & 0 & 0 & 0 & 372 \\
\hline 2 & EV & 10TC019 & 66 & 2.0 & \begin{tabular}{|l|}
10 \\
\end{tabular} & 0 & 3 & 0 & 0 & 1990 & 0 & 283 & 258 & 0 & 0 & 1134 & 365 \\
\hline 2 & EV & 8TC019 & 66 & 2.0 & 8 & 0 & 3 & 0 & 0 & 1990 & 0 & 283 & 262 & 0 & 0 & 133 & 365 \\
\hline 2 & EV & 6TC019 & 66 & 2.0 & 6 & 0 & 3 & 0 & 0 & 1989 & 0 & 283 & 264 & 0 & 0 & 0 & 365 \\
\hline 2 & $\mathrm{EV}$ & $10 \mathrm{TC} 020$ & 66 & 2.0 & \begin{tabular}{|l|}
10 \\
\end{tabular} & 0 & 4 & 0 & 0 & 2006 & 0 & 285 & 266 & 0 & 0 & 1362 & 368 \\
\hline 2 & EV & $8 \mathrm{TC} 020$ & 66 & 2.0 & 8 & 0 & 3 & 0 & 0 & 2006 & 0 & 285 & 268 & 0 & 0 & 400 & 368 \\
\hline 2 & EV & 6TC020 & 66 & 2.0 & 6 & 0 & 2 & 0 & 0 & 2005 & 0 & 285 & 269 & 118 & 1033 & 0 & 368 \\
\hline 1 & $\mathrm{OLH}$ & $10 \mathrm{TC} 025$ & 31 & 0 & \begin{tabular}{|l|l|}
10 \\
\end{tabular} & 0 & 0 & 0 & 0 & 0 & 0 & 0 & 0 & 0 & 0 & 0 & 0 \\
\hline 1 & $\mathrm{OLH}$ & $8 \mathrm{TC} 025$ & 31 & 0 & 8 & 0 & 0 & 0 & 0 & 0 & 0 & 0 & 0 & 0 & 0 & 0 & 0 \\
\hline 1 & $\mathrm{OLH}$ & 6TC025 & 31 & 0 & 6 & 0 & 0 & 0 & 0 & 0 & 0 & 0 & 0 & 0 & 0 & 0 & 0 \\
\hline 3 & $\mathrm{OLH}$ & $10 \mathrm{TC} 035$ & 49 & 0.9 & \begin{tabular}{|l|}
10 \\
\end{tabular} & 0 & 1 & 0 & 0 & 888 & 0 & 126 & 34 & 0 & 0 & 0 & 163 \\
\hline 3 & OLH & $8 \mathrm{TC} 035$ & 49 & 0.9 & 8 & 0 & 0 & 0 & 0 & 889 & 0 & 126 & 67 & 0 & 0 & 0 & 163 \\
\hline 3 & $\mathrm{OLH}$ & 6TC035 & 49 & 0.9 & 6 & 0 & 0.3 & 0 & 0 & 888 & 0 & 126 & 85 & 0 & 0 & 0 & 163 \\
\hline 1 & $\mathrm{OLH}$ & $10 \mathrm{TC} 040$ & 50 & 0.3 & \begin{tabular}{|l|}
10 \\
\end{tabular} & 0 & 0 & 0 & 0 & 270 & 0 & 38 & 0 & 0 & 0 & 0 & 50 \\
\hline 1 & $\mathrm{OLH}$ & $8 \mathrm{TC} 040$ & 50 & 0.3 & 8 & 0 & 0 & 0 & 0 & 270 & 0 & 38 & 0 & 0 & 0 & 0 & 50 \\
\hline 1 & $\mathrm{OLH}$ & 6ТC040 & 50 & 0.3 & 6 & 0 & 0 & 0 & 0 & 269 & 0 & 38 & 0 & 0 & 0 & 0 & 50 \\
\hline 1 & $\mathrm{OLH}$ & $10 \mathrm{TC} 046$ & 55 & 1.7 & 10 & 0 & 3 & 0 & 0 & 1746 & 0 & 248 & 199 & 0 & 0 & 469 & 320 \\
\hline 1 & $\mathrm{OLH}$ & $8 \mathrm{TC} 046$ & 55 & 1.7 & 8 & 0 & 2 & 0 & 0 & 1746 & 0 & 248 & 213 & 0 & 0 & 0 & 320 \\
\hline 1 & $\mathrm{OLH}$ & 6TC046 & 55 & 1.7 & 6 & 0 & 2 & 0 & 0 & 1745 & 0 & 248 & 221 & 0 & 0 & 0 & 320 \\
\hline
\end{tabular}


WSRC-TR-2003-00269, REVISION 0 SRT-RPP-2003-00125, REVISION 0

Table 29. Predicted Solids from Envelope C Simulations - Part 1 (cont'd)

\begin{tabular}{|c|c|c|c|c|c|c|c|c|c|c|c|c|c|c|c|c|c|}
\hline $\begin{array}{c}\text { Experimental } \\
\text { ID }\end{array}$ & \begin{tabular}{|l|} 
Simulation \\
Point Type
\end{tabular} & $\begin{array}{c}\text { Simulation } \\
\text { ID }\end{array}$ & \begin{tabular}{|c|} 
Temp \\
${ }^{\circ} \mathrm{C}$ \\
$(\mathbf{P} 2)$
\end{tabular} & $\begin{array}{c}\text { SBS/Feed } \\
\text { (P1) }\end{array}$ & \begin{tabular}{l|}
$\mathrm{Na}$ \\
$\mathrm{M}$
\end{tabular} & \begin{tabular}{|} 
ALOH3 \\
[g]
\end{tabular} & $\begin{array}{c}\text { BASO4 } \\
{[\mathrm{g}]}\end{array}$ & $\begin{array}{c}\text { CA3PO42 } \\
{[\mathrm{g}]}\end{array}$ & $\begin{array}{c}\mathrm{CACO3} \\
{[\mathrm{g}]}\end{array}$ & $\begin{array}{c}\text { CAF2 } \\
\text { [g] }\end{array}$ & $\begin{array}{c}\text { CAOH2 } \\
\text { [g] }\end{array}$ & $\begin{array}{c}\text { CATIO3 } \\
{[\mathrm{g}]}\end{array}$ & $\begin{array}{c}\text { CROH3 } \\
{[\mathrm{g}]}\end{array}$ & $\begin{array}{c}\text { FEIIIOH3 } \\
{[\mathrm{g}]}\end{array}$ & $\begin{array}{c}\text { HYDROSOD } \\
{[\mathrm{g}]}\end{array}$ & $\begin{array}{c}\text { MGF2 } \\
\text { [g] }\end{array}$ & $\begin{array}{c}\text { MGOH2 } \\
\text { [g] }\end{array}$ \\
\hline 1 & $\mathrm{OLH}$ & 10TC048 & 48 & 1.4 & \begin{tabular}{|l|}
10 \\
\end{tabular} & 0 & 2 & 0 & 0 & 1412 & 0 & 201 & 110 & 0 & 0 & 0 & 259 \\
\hline 1 & $\mathrm{OLH}$ & $8 \mathrm{TC} 048$ & 48 & 1.4 & 8 & 0 & 1 & 0 & 0 & 1412 & 0 & 201 & 141 & 0 & 0 & 0 & 259 \\
\hline 1 & $\mathrm{OLH}$ & 6TC048 & 48 & 1.4 & 6 & 0 & 1 & 0 & 0 & 1412 & 0 & 201 & 159 & 0 & 0 & 0 & 259 \\
\hline 1 & $\mathrm{OLH}$ & 10TC050 & 40 & 0.2 & \begin{tabular}{|l|}
10 \\
\end{tabular} & 0 & 0 & 0 & 0 & 158 & 0 & 23 & 0 & 0 & 0 & 0 & 29 \\
\hline 1 & $\mathrm{OLH}$ & $8 \mathrm{TC} 050$ & 40 & 0.2 & 8 & 0 & 0 & 0 & 0 & 158 & 0 & 23 & 0 & 0 & 0 & 0 & 29 \\
\hline 1 & $\mathrm{OLH}$ & 6TC050 & 40 & 0.2 & 6 & 0 & 0 & 0 & 0 & 158 & 0 & 23 & 0 & 0 & 0 & 0 & 29 \\
\hline 1 & $\mathrm{OLH}$ & 10TC052 & 35 & 0.2 & \begin{tabular}{|l|}
10 \\
\end{tabular} & 0 & 0 & 0 & 0 & 113 & 102 & 32 & 0 & 0 & 0 & 0 & 41 \\
\hline 1 & $\mathrm{OLH}$ & $8 \mathrm{TC} 052$ & 35 & 0.2 & 8 & 0 & 0 & 0 & 0 & 221 & 0 & 32 & 0 & 0 & 0 & 0 & 41 \\
\hline 1 & $\mathrm{OLH}$ & 6TC052 & 35 & 0.2 & 6 & 0 & 0 & 0 & 0 & 221 & 0 & 32 & 0 & 0 & 0 & 0 & 41 \\
\hline 3 & $\mathrm{OLH}$ & $10 \mathrm{TC} 055$ & 29 & 0.8 & \begin{tabular}{|l|}
10 \\
\end{tabular} & 0 & 1 & 0 & 0 & 0 & 796 & 120 & 0 & 0 & 0 & 0 & 154 \\
\hline 3 & $\mathrm{OLH}$ & $8 \mathrm{TC} 055$ & 29 & 0.8 & 8 & 0 & 0 & 0 & 0 & 840 & 0 & 120 & 0 & 0 & 0 & 0 & 154 \\
\hline 3 & $\mathrm{OLH}$ & 6TC055 & 29 & 0.8 & 6 & 0 & 1 & 0 & 0 & 841 & 0 & 120 & 0 & 43 & 376 & 0 & 154 \\
\hline 1 & $\mathrm{OLH}$ & 10TC060 & 21 & 1.1 & \begin{tabular}{|l|}
10 \\
\end{tabular} & 0 & 1 & 0 & 0 & 0 & 1067 & 160 & 0 & 31 & 274 & 0 & 207 \\
\hline 1 & $\mathrm{OLH}$ & $8 \mathrm{TC} 060$ & 21 & 1.1 & 8 & 0 & 1 & 0 & 0 & 1125 & 0 & 160 & 0 & 139 & 1214 & 0 & 207 \\
\hline 1 & $\mathrm{OLH}$ & 6TC060 & 21 & 1.1 & 6 & 959 & 2 & 0 & 0 & 1126 & 0 & 160 & 0 & 210 & 1830 & 0 & 207 \\
\hline 1 & $\mathrm{OLH}$ & 10TC062 & 37 & 1.8 & \begin{tabular}{|l|}
10 \\
\end{tabular} & 0 & 3 & 511 & 1866 & 0 & 0 & 262 & 87 & 0 & 0 & 0 & 338 \\
\hline 1 & $\overline{\mathrm{OLH}}$ & $8 \mathrm{TC} 062$ & 37 & 1.8 & 8 & 0 & 2 & 143 & 792 & 1116 & 0 & 262 & 151 & 208 & 1812 & 0 & 338 \\
\hline 1 & $\mathrm{OLH}$ & 6TC062 & 37 & 1.8 & 6 & 0 & 2 & 0 & 0 & 1843 & 0 & 262 & 187 & 326 & 2845 & 0 & 338 \\
\hline 1 & $\mathrm{OLH}$ & $10 \mathrm{TC} 067$ & 56 & 0.7 & \begin{tabular}{|l|l|}
10 \\
\end{tabular} & 0 & 0.49 & 0 & 0 & 745 & 0 & 106 & 47 & 0 & 0 & 0 & 137 \\
\hline 1 & $\mathrm{OLH}$ & $8 \mathrm{TC} 067$ & 56 & 0.7 & 8 & 0 & 0.03 & 0 & 0 & 745 & 0 & 106 & 67 & 0 & 0 & 0 & 137 \\
\hline 1 & $\mathrm{OLH}$ & 6TC067 & 56 & 0.7 & 6 & 0 & 0.00 & 0 & 0 & 744 & 0 & 106 & 79 & 0 & 0 & 0 & 137 \\
\hline 1 & $\mathrm{OLH}$ & 10TC068 & 61 & 0.3 & \begin{tabular}{|l|}
10 \\
\end{tabular} & 0 & 0 & 0 & 0 & 301 & 0 & 43 & 6 & 0 & 0 & 0 & 55 \\
\hline 1 & OLH & 8TC068 & 61 & 0.3 & 8 & 0 & 0 & 0 & 0 & 301 & 0 & 43 & 19 & 0 & 0 & 0 & 55 \\
\hline 1 & $\mathrm{OLH}$ & 6TC068 & 61 & 0.3 & 6 & 0 & 0 & 0 & 0 & 300 & 0 & 43 & 26 & 0 & 0 & 0 & 55 \\
\hline 1 & $\mathrm{OLH}$ & $10 \mathrm{TC} 074$ & 43 & 1.8 & \begin{tabular}{|l|}
10 \\
\end{tabular} & 0 & 3 & 0 & 0 & 1778 & 0 & 253 & 140 & 0 & 0 & 0 & 326 \\
\hline 1 & $\mathrm{OLH}$ & $8 \mathrm{TC} 074$ & 43 & 1.8 & 8 & 0 & 1 & 0 & 0 & 1778 & 0 & 253 & 179 & 3 & 30 & 0 & 326 \\
\hline 1 & $\mathrm{OLH}$ & 6ТC074 & 43 & 1.8 & 6 & 0 & 2 & 0 & 0 & 1777 & 0 & 253 & 201 & 181 & 1580 & 0 & 326 \\
\hline 2 & $\mathrm{OLH}$ & $10 \mathrm{TC} 083$ & 50 & 2.0 & 10 & 0 & 3 & 0 & 0 & 1991 & 0 & 283 & 225 & 0 & 0 & 854 & 365 \\
\hline 2 & $\mathrm{OLH}$ & $8 \mathrm{TC} 083$ & 50 & 2.0 & 8 & 0 & 2 & 0 & 0 & 1991 & 0 & 283 & 241 & 0 & 0 & 17 & 365 \\
\hline 2 & $\mathrm{OLH}$ & 6TC083 & 50 & 2.0 & 6 & 0 & 2 & 0 & 0 & 1991 & 0 & 283 & 250 & 167 & 1453 & 0 & 365 \\
\hline
\end{tabular}


WSRC-TR-2003-00269, REVISION 0 SRT-RPP-2003-00125, REVISION 0

Table 29. Predicted Solids from Envelope C Simulations - Part 1 (cont'd)

\begin{tabular}{|c|c|c|c|c|c|c|c|c|c|c|c|c|c|c|c|c|c|}
\hline $\begin{array}{c}\text { Experimental } \\
\text { ID }\end{array}$ & $\begin{array}{l}\text { Simulation } \\
\text { Point Type }\end{array}$ & \begin{tabular}{|c|} 
Simulation \\
ID
\end{tabular} & $\begin{array}{c}\text { Temp } \\
{ }^{\circ} \mathrm{C} \\
(\mathrm{P} 2)\end{array}$ & \begin{tabular}{|c|} 
SBS/Feed \\
(P1)
\end{tabular} & \begin{tabular}{l|}
$\mathrm{Na}$ \\
$\mathrm{M}$
\end{tabular} & $\begin{array}{c}\text { ALOH3 } \\
\text { [g] }\end{array}$ & $\begin{array}{c}\text { BASO4 } \\
\text { [g] }\end{array}$ & $\begin{array}{c}\text { CA3PO42 } \\
\text { [g] }\end{array}$ & $\begin{array}{c}\mathrm{CACO3} \\
{[\mathrm{g}]}\end{array}$ & $\begin{array}{c}\text { CAF2 } \\
\text { [g] }\end{array}$ & $\begin{array}{c}\text { CAOH2 } \\
\text { [g] }\end{array}$ & $\begin{array}{c}\text { CATIO3 } \\
{[\mathrm{g}]}\end{array}$ & $\begin{array}{c}\text { CROH3 } \\
{[\mathrm{g}]}\end{array}$ & $\begin{array}{c}\text { FEIIIOH3 } \\
{[\mathrm{g}]}\end{array}$ & $\begin{array}{c}\text { HYDROSOD } \\
{[\mathrm{g}]}\end{array}$ & $\begin{array}{c}\text { MGF2 } \\
\text { [g] }\end{array}$ & $\begin{array}{c}\text { MGOH2 } \\
\text { [g] }\end{array}$ \\
\hline 4 & $\mathrm{OLH}$ & 10TC093 & 32 & 1.1 & 10 & 0 & 1 & 289 & 1153 & 0 & 0 & 159 & 0 & 0 & 0 & 0 & 206 \\
\hline 4 & $\mathrm{OLH}$ & $8 \mathrm{TC} 093$ & 32 & 1.1 & 8 & 0 & 1 & 0 & 0 & 1119 & 0 & 159 & 0 & 0 & 0 & 0 & 206 \\
\hline 4 & $\mathrm{OLH}$ & 6TC093 & 32 & 1.1 & 6 & 0 & 1 & 0 & 0 & 1120 & 0 & 159 & 33 & 111 & 968 & 0 & 206 \\
\hline 2 & $\mathrm{OLH}$ & 10TC098 & 31 & 1.7 & 10 & 0 & 3 & 444 & 1778 & 0 & 0 & 245 & 0 & 90 & 786 & 0 & 317 \\
\hline 2 & OLH & $8 \mathrm{TC} 098$ & 31 & 1.7 & 8 & 0 & 2 & 0 & 0 & 1722 & 0 & 245 & 73 & 239 & 2087 & 0 & 317 \\
\hline 2 & $\mathrm{OLH}$ & 6TC098 & 31 & 1.7 & 6 & 0 & 2 & 0 & 0 & 1724 & 0 & 245 & 131 & 331 & 2891 & 0 & 317 \\
\hline
\end{tabular}


WSRC-TR-2003-00269, REVISION 0

SRT-RPP-2003-00125, REVISION 0

Table 30. Predicted Solids from Envelope C Simulations - Part 2

\begin{tabular}{|c|c|c|c|c|c|c|c|c|c|c|c|c|c|c|c|c|c|}
\hline \begin{tabular}{|c|} 
Experimental \\
ID
\end{tabular} & $\begin{array}{c}\text { Simulation } \\
\text { Point } \\
\text { Type }\end{array}$ & \begin{tabular}{|c|} 
Simulation \\
ID
\end{tabular} & \begin{tabular}{|c|} 
Temp \\
oC \\
(P2)
\end{tabular} & $\begin{array}{c}\text { SBS/Feed } \\
\text { (P1) }\end{array}$ & $\begin{array}{l}\mathrm{Na} \\
\mathrm{M}\end{array}$ & $\begin{array}{c}\text { NA2C2O4 } \\
{[\mathrm{g}]}\end{array}$ & $\begin{array}{c}\text { NA3FSO4 } \\
{[\mathrm{g}]}\end{array}$ & $\begin{array}{c}\text { NA6SO42CO3 } \\
{[\mathrm{g}]}\end{array}$ & $\begin{array}{c}\mathbf{N A F} \\
{[\mathrm{g}]}\end{array}$ & $\begin{array}{c}\text { NANO3 } \\
{[\mathrm{g}]}\end{array}$ & $\begin{array}{c}\text { NASGEL.15.5H2O } \\
{[\mathrm{g}]}\end{array}$ & $\underset{[\mathrm{g}]}{\mathrm{NIOH} 2}$ & $\begin{array}{c}\text { SRCO3 } \\
{[\mathrm{g}]}\end{array}$ & $\begin{array}{c}\text { ZRO2 } \\
{[\mathrm{g}]}\end{array}$ & $\begin{array}{c}\text { Total } \\
\text { Solids } \\
{[\mathrm{g}]}\end{array}$ & $\begin{array}{c}\text { Total } \\
\text { Soln } \\
\text { Mass }[g]\end{array}$ & \begin{tabular}{|c|} 
Solids \\
\% of \\
Total \\
Soln \\
\end{tabular} \\
\hline 1 & $\mathrm{EV}$ & $10 \mathrm{TC} 001$ & 15 & 0 & 10 & 1165 & 0 & 0 & 1690 & 0 & 0 & 0 & 0 & 0 & $2.85 \mathrm{E}+03$ & $6.29 \mathrm{E}+05$ & $0.45 \%$ \\
\hline 1 & $\mathrm{EV}$ & $8 \mathrm{TC} 001$ & 15 & 0 & 8 & 1018 & 0 & 0 & 364 & 0 & 0 & 0 & 0 & 0 & $1.38 \mathrm{E}+03$ & $7.56 \mathrm{E}+05$ & $0.18 \%$ \\
\hline 1 & $\mathrm{EV}$ & 6TC001 & 15 & 0 & 6 & 687 & 0 & 0 & 0 & 0 & 0 & 0 & 0 & 0 & $1.06 \mathrm{E}+03$ & $9.55 \mathrm{E}+05$ & $0.11 \%$ \\
\hline 1 & $\mathrm{EV}$ & $10 \mathrm{TC} 002$ & 15 & 0 & 10 & 1099 & 0 & 0 & 1737 & 2711 & 0 & 0 & 0 & 0 & $5.55 \mathrm{E}+03$ & $6.27 \mathrm{E}+05$ & $0.89 \%$ \\
\hline 1 & EV & $8 \mathrm{TC} 002$ & 15 & 0 & 8 & 960 & 0 & 0 & 441 & 0 & 0 & 0 & 0 & 0 & $1.40 \mathrm{E}+03$ & $7.55 \mathrm{E}+05$ & $0.19 \%$ \\
\hline 1 & $\mathrm{EV}$ & 6TC002 & 15 & 0 & 6 & 647 & 0 & 0 & 0 & 0 & 0 & 0 & 0 & 0 & $1.16 \mathrm{E}+03$ & $9.54 \mathrm{E}+05$ & $0.12 \%$ \\
\hline 2 & $\mathrm{EV}$ & $10 \mathrm{TC} 004$ & 15 & 0 & 10 & 1063 & 0 & 0 & 1684 & 0 & 0 & 0 & 0 & 0 & $2.75 \mathrm{E}+03$ & $6.25 \mathrm{E}+05$ & $0.44 \%$ \\
\hline 2 & $\mathrm{EV}$ & $8 \mathrm{TC} 004$ & 15 & 0 & 8 & 920 & 0 & 0 & 381 & 0 & 0 & 0 & 0 & 0 & $1.30 \mathrm{E}+03$ & $7.52 \mathrm{E}+05$ & $0.17 \%$ \\
\hline 2 & EV & 6TC004 & 15 & 0 & 6 & 597 & 0 & 0 & 0 & 0 & 0 & 0 & 0 & 0 & $5.97 \mathrm{E}+02$ & $9.51 \mathrm{E}+05$ & $0.06 \%$ \\
\hline 2 & $\mathrm{EV}$ & $10 \mathrm{TC} 005$ & 15 & 0 & 10 & 1144 & 0 & 0 & 1639 & 0 & 0 & 0 & 0 & 0 & $2.78 \mathrm{E}+03$ & $6.27 \mathrm{E}+05$ & $0.44 \%$ \\
\hline 2 & $\mathrm{EV}$ & $8 \mathrm{TC} 005$ & 15 & 0 & 8 & 990 & 0 & 0 & 284 & 0 & 0 & 0 & 0 & 0 & $1.27 \mathrm{E}+03$ & $7.54 \mathrm{E}+05$ & $0.17 \%$ \\
\hline 2 & $\overline{E V}$ & 6ТC005 & 15 & 0 & 6 & 649 & 0 & 0 & 0 & 0 & 0 & 0 & 0 & 0 & $6.49 \mathrm{E}+02$ & $9.52 \mathrm{E}+05$ & $0.07 \%$ \\
\hline 1 & EV & $10 \mathrm{TC} 006$ & 66 & 0 & 10 & 673 & 0 & 8455 & 986 & 0 & 0 & 0 & 0 & 0 & $1.01 \mathrm{E}+04$ & $5.91 \mathrm{E}+05$ & $1.71 \%$ \\
\hline 1 & EV & $8 \mathrm{TC} 006$ & 66 & 0 & 8 & 246 & 0 & 310 & 0 & 0 & 0 & 0 & 0 & 0 & $5.55 \mathrm{E}+02$ & $7.22 \mathrm{E}+05$ & $0.08 \%$ \\
\hline 1 & EV & 6TC006 & 66 & 0 & 6 & 0 & 0 & 0 & 0 & 0 & 0 & 0 & 0 & 0 & 0 & $9.16 \mathrm{E}+05$ & 0 \\
\hline 1 & EV & $10 \mathrm{TC} 007$ & 66 & 0 & 10 & 648 & 0 & 9837 & 1038 & 0 & 0 & 0 & 0 & 0 & $1.15 \mathrm{E}+04$ & $5.90 \mathrm{E}+05$ & $1.95 \%$ \\
\hline 1 & $\mathrm{EV}$ & $8 \mathrm{TC} 007$ & 66 & 0 & 8 & 238 & 0 & 1984 & 0 & 0 & 0 & 0 & 0 & 0 & $2.22 \mathrm{E}+03$ & $7.19 \mathrm{E}+05$ & $0.31 \%$ \\
\hline 1 & $\mathrm{EV}$ & 6TC007 & 66 & 0 & 6 & 0 & 0 & 0 & 0 & 0 & 0 & 0 & 0 & 0 & 0 & $9.16 \mathrm{E}+05$ & 0 \\
\hline 2 & $\overline{E V}$ & $10 \mathrm{TC} 009$ & 66 & 0 & 10 & 598 & 0 & 7838 & 968 & 0 & 0 & 0 & 0 & 0 & $9.40 \mathrm{E}+03$ & $5.88 \mathrm{E}+05$ & $1.60 \%$ \\
\hline 2 & $\mathrm{EV}$ & $8 \mathrm{TC} 009$ & 66 & 0 & 8 & 181 & 0 & 0 & 0 & 0 & 0 & 0 & 0 & 0 & $1.81 \mathrm{E}+02$ & $7.18 \mathrm{E}+05$ & $0.03 \%$ \\
\hline 2 & $\mathrm{EV}$ & 6ТC009 & 66 & 0 & 6 & 0 & 0 & 0 & 0 & 0 & 0 & 0 & 0 & 0 & 0 & $9.12 \mathrm{E}+05$ & 0 \\
\hline 2 & $\mathrm{EV}$ & $10 \mathrm{TC} 010$ & 66 & 0 & 10 & 639 & 0 & 9535 & 948 & 0 & 0 & 0 & 0 & 0 & $1.11 \mathrm{E}+04$ & $5.87 \mathrm{E}+05$ & $1.90 \%$ \\
\hline 2 & $\mathrm{EV}$ & $8 \mathrm{TC} 010$ & 66 & 0 & 8 & 208 & 0 & 1000 & 0 & 0 & 0 & 0 & 0 & 0 & $1.21 \mathrm{E}+03$ & $7.18 \mathrm{E}+05$ & $0.17 \%$ \\
\hline 2 & EV & 6TC010 & 66 & 0 & 6 & 0 & 0 & 0 & 0 & 0 & 0 & 0 & 0 & 0 & 0 & $9.13 \mathrm{E}+05$ & 0 \\
\hline 1 & $\mathrm{EV}$ & 10TC011 & 15 & 2.0 & 10 & 1160 & 0 & 0 & 1232 & 0 & 7344 & 55 & 5 & 45 & $1.71 \mathrm{E}+04$ & $7.11 \mathrm{E}+05$ & $2.41 \%$ \\
\hline 1 & EV & $8 \mathrm{TC} 011$ & 15 & 2.0 & 8 & 985 & 0 & 0 & 0 & 0 & 7595 & 56 & 5 & 44 & $1.79 \mathrm{E}+04$ & $8.47 \mathrm{E}+05$ & $2.11 \%$ \\
\hline 1 & $\mathrm{EV}$ & 6TC011 & 15 & 2.0 & 6 & 609 & 0 & 0 & 0 & 0 & 7673 & 57 & 5 & 43 & $1.95 \mathrm{E}+04$ & $1.06 \mathrm{E}+06$ & $1.84 \%$ \\
\hline 1 & EV & $10 \mathrm{TC} 012$ & 15 & 2.0 & 10 & 1094 & 0 & 0 & 1279 & 0 & 7020 & 54 & 5 & 41 & $1.63 \mathrm{E}+04$ & $7.10 \mathrm{E}+05$ & $2.30 \%$ \\
\hline 1 & EV & 8ТC012 & 15 & 2.0 & 8 & 929 & 0 & 0 & 0 & 0 & 7478 & 55 & 5 & 40 & $1.71 \mathrm{E}+04$ & $8.46 \mathrm{E}+05$ & $2.02 \%$ \\
\hline 1 & $\mathrm{EV}$ & 6TC012 & 15 & 2.0 & 6 & 572 & 0 & 0 & 0 & 0 & 7596 & 56 & 5 & 39 & $1.94 \mathrm{E}+04$ & $1.06 \mathrm{E}+06$ & $1.83 \%$ \\
\hline
\end{tabular}


WSRC-TR-2003-00269, REVISION 0 SRT-RPP-2003-00125, REVISION 0

Table 30. Predicted Solids from Envelope C Simulations - Part 2 (cont'd)

\begin{tabular}{|c|c|c|c|c|c|c|c|c|c|c|c|c|c|c|c|c|c|}
\hline \begin{tabular}{|} 
Experimental \\
ID
\end{tabular} & $\begin{array}{c}\text { Simulation } \\
\text { Point } \\
\text { Type }\end{array}$ & \begin{tabular}{|c|} 
Simulation \\
ID
\end{tabular} & \begin{tabular}{|c|} 
Temp \\
oC \\
(P2)
\end{tabular} & $\begin{array}{c}\text { SBS/Feed } \\
\text { (P1) }\end{array}$ & $\begin{array}{l}\mathrm{Na} \\
\mathrm{M}\end{array}$ & \begin{tabular}{|} 
NA2C2O4 \\
{$[g]$}
\end{tabular} & \begin{tabular}{|} 
NA3FSO4 \\
{$[\mathrm{g}]$}
\end{tabular} & \begin{tabular}{|c|} 
NA6SO42CO3 \\
{$[\mathrm{g}]$}
\end{tabular} & \begin{tabular}{|c|} 
NAF \\
{$[g]$} \\
$g]$
\end{tabular} & $\begin{array}{c}\text { NANO } \\
3 \text { [g] }\end{array}$ & $\begin{array}{c}\text { NASGEL.15.5H2O } \\
{[\mathrm{g}]}\end{array}$ & \begin{tabular}{|c|} 
NIOH2 \\
{$[\mathrm{g}]$}
\end{tabular} & \begin{tabular}{|c|} 
SRCO3 \\
[g]
\end{tabular} & $\begin{array}{c}\text { ZRO2 } \\
{[\mathrm{g}]}\end{array}$ & $\begin{array}{c}\text { Total } \\
\text { Solids } \\
{[\mathrm{g}]}\end{array}$ & 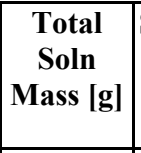 & \begin{tabular}{|c|} 
Solids \\
$\%$ of \\
Total \\
Soln \\
\end{tabular} \\
\hline 2 & $\mathrm{EV}$ & 10TC014 & 15 & 2.0 & \begin{tabular}{|l|}
10 \\
\end{tabular} & 1042 & 0 & 0 & 1195 & 0 & 2398 & 54 & 5 & 44 & $1.19 \mathrm{E}+04$ & $7.06 \mathrm{E}+05$ & $1.68 \%$ \\
\hline 2 & $\overline{E V}$ & $8 \mathrm{TC} 014$ & 15 & 2.0 & 8 & 864 & 0 & 0 & 0 & 0 & 3147 & 55 & 5 & 43 & $1.16 \mathrm{E}+04$ & $8.41 \mathrm{E}+05$ & $1.38 \%$ \\
\hline 2 & $\mathrm{EV}$ & 6TC014 & 15 & 2.0 & 6 & 483 & 0 & 0 & 0 & 0 & 3425 & 56 & 5 & 43 & $1.18 \mathrm{E}+04$ & $1.05 \mathrm{E}+06$ & $1.12 \%$ \\
\hline 2 & EV & 10TC015 & 15 & 2.0 & 10 & 1122 & 0 & 0 & 778 & 0 & 3397 & 56 & 5 & 49 & $1.36 \mathrm{E}+04$ & $7.09 \mathrm{E}+05$ & $1.92 \%$ \\
\hline 2 & EV & $8 \mathrm{TC} 015$ & 15 & 2.0 & 8 & 932 & 0 & 0 & 0 & 0 & 3612 & 56 & 5 & 49 & $1.27 \mathrm{E}+04$ & $8.44 \mathrm{E}+05$ & $1.51 \%$ \\
\hline 2 & EV & 6TC015 & 15 & 2.0 & 6 & 531 & 0 & 0 & 0 & 0 & 3676 & 57 & 5 & 49 & $1.25 \mathrm{E}+04$ & $1.06 \mathrm{E}+06$ & $1.18 \%$ \\
\hline 1 & EV & 10TC016 & 66 & 2.0 & \begin{tabular}{|l|}
10 \\
\end{tabular} & 686 & 0 & 26982 & 0 & 0 & 7342 & 58 & 5 & 56 & $3.93 \mathrm{E}+04$ & $6.46 \mathrm{E}+05$ & $6.09 \%$ \\
\hline 1 & $\mathrm{EV}$ & $8 \mathrm{TC} 016$ & 66 & 2.0 & 8 & 250 & 0 & 18490 & 0 & 0 & 7642 & 58 & 5 & 56 & $2.97 \mathrm{E}+04$ & $7.77 \mathrm{E}+05$ & $3.83 \%$ \\
\hline 1 & EV & 6TC016 & 66 & 2.0 & 6 & 0 & 0 & 0 & 0 & 0 & 7696 & 58 & 5 & 56 & $1.08 \mathrm{E}+04$ & $1.01 \mathrm{E}+06$ & $1.06 \%$ \\
\hline 1 & $\mathrm{EV}$ & 10TC017 & 66 & 2.0 & 10 & 657 & 0 & 28196 & 68 & 0 & 6965 & 58 & 5 & 55 & $4.00 \mathrm{E}+04$ & $6.44 \mathrm{E}+05$ & $6.21 \%$ \\
\hline 1 & EV & $8 \mathrm{TC} 017$ & 66 & 2.0 & 8 & 240 & 0 & 19975 & 0 & 0 & 7506 & 58 & 5 & 55 & $3.09 \mathrm{E}+04$ & $7.74 \mathrm{E}+05$ & $3.99 \%$ \\
\hline 1 & $\overline{E V}$ & 6TC017 & 66 & 2.0 & 6 & 0 & 0 & 1038 & 0 & 0 & 7610 & 58 & 5 & 55 & $1.17 \mathrm{E}+04$ & $1.01 \mathrm{E}+06$ & $1.16 \%$ \\
\hline 2 & EV & 10TC019 & 66 & 2.0 & 10 & 562 & 0 & 25684 & 0 & 0 & 2532 & 57 & 5 & 54 & $3.29 \mathrm{E}+04$ & $6.42 \mathrm{E}+05$ & $5.13 \%$ \\
\hline 2 & EV & $8 \mathrm{TC} 019$ & 66 & 2.0 & 8 & 133 & 0 & 16990 & 0 & 0 & 3088 & 57 & 5 & 54 & $2.34 \mathrm{E}+04$ & $7.74 \mathrm{E}+05$ & $3.02 \%$ \\
\hline 2 & EV & 6ТC019 & 66 & 2.0 & 6 & 0 & 0 & 0 & 0 & 0 & 3212 & 57 & 5 & 54 & $6.23 \mathrm{E}+03$ & $1.01 \mathrm{E}+06$ & $0.62 \%$ \\
\hline 2 & EV & 10TC020 & 66 & 2.0 & 10 & 609 & 0 & 27567 & 0 & 0 & 3421 & 57 & 5 & 55 & $3.60 \mathrm{E}+04$ & $6.41 \mathrm{E}+05$ & $5.62 \%$ \\
\hline 2 & $\overline{E V}$ & $8 \mathrm{TC} 020$ & 66 & 2.0 & 8 & 167 & 0 & 18216 & 0 & 0 & 3559 & 57 & 5 & 55 & $2.54 \mathrm{E}+04$ & $7.73 \mathrm{E}+05$ & $3.28 \%$ \\
\hline 2 & $\mathrm{EV}$ & 6ТC020 & 66 & 2.0 & 6 & 0 & 0 & 0 & 0 & 0 & 3571 & 57 & 5 & 55 & $7.77 \mathrm{E}+03$ & $1.01 \mathrm{E}+06$ & $0.77 \%$ \\
\hline 1 & OLH & 10TC025 & 31 & 0 & \begin{tabular}{|l|l|}
10 \\
\end{tabular} & 1060 & 10131 & 0 & 0 & 0 & 0 & 0 & 0 & 0 & $1.12 \mathrm{E}+04$ & $6.10 \mathrm{E}+05$ & $1.84 \%$ \\
\hline 1 & OLH & $8 \mathrm{TC} 025$ & 31 & 0 & 8 & 857 & 6462 & 0 & 0 & 0 & 0 & 0 & 0 & 0 & $7.32 \mathrm{E}+03$ & $7.34 \mathrm{E}+05$ & $1.00 \%$ \\
\hline 1 & $\overline{\mathrm{OLH}}$ & $6 \mathrm{TC} 025$ & 31 & 0 & 6 & 363 & 0 & 0 & 0 & 0 & 0 & 0 & 0 & 0 & $3.63 \mathrm{E}+02$ & $9.43 \mathrm{E}+05$ & $0.04 \%$ \\
\hline 3 & OLH & $10 \mathrm{TC} 035$ & 49 & 0.9 & 10 & 890 & 0 & 16372 & 1156 & 0 & 1986 & 25 & 2 & 23 & $2.17 \mathrm{E}+04$ & $6.24 \mathrm{E}+05$ & $3.47 \%$ \\
\hline 3 & $\mathrm{OLH}$ & $8 \mathrm{TC} 035$ & 49 & 0.9 & 8 & 550 & 0 & 4471 & 0 & 0 & 2936 & 25 & 2 & 22 & $9.25 \mathrm{E}+03$ & $7.63 \mathrm{E}+05$ & $1.21 \%$ \\
\hline 3 & OLH & $6 \mathrm{TC} 035$ & 49 & 0.9 & 6 & 0 & 0 & 0 & 0 & 0 & 3160 & 25 & 2 & 22 & $4.47 \mathrm{E}+03$ & $9.73 \mathrm{E}+05$ & $0.46 \%$ \\
\hline 1 & OLH & $10 \mathrm{TC} 040$ & 50 & 0.3 & 10 & 897 & 0 & 11269 & 1227 & 0 & 0 & 7 & 1 & 5 & $1.38 \mathrm{E}+04$ & $6.06 \mathrm{E}+05$ & $2.27 \%$ \\
\hline 1 & OLH & $8 \mathrm{TC} 040$ & 50 & 0.3 & 8 & 554 & 0 & 0 & 0 & 0 & 394 & 7 & 1 & 5 & $1.32 \mathrm{E}+03$ & $7.45 \mathrm{E}+05$ & $0.18 \%$ \\
\hline 1 & OLH & $6 \mathrm{TC} 040$ & 50 & 0.3 & 6 & 0 & 0 & 0 & 0 & 0 & 702 & 7 & 1 & 5 & $1.07 \mathrm{E}+03$ & $9.43 \mathrm{E}+05$ & $0.11 \%$ \\
\hline 1 & $\mathrm{OLH}$ & $10 \mathrm{TC} 046$ & 55 & 1.7 & 10 & 823 & 0 & 25005 & \begin{tabular}{|l|}
645 \\
\end{tabular} & 0 & 5895 & 49 & 4 & 47 & $3.55 \mathrm{E}+04$ & $6.43 \mathrm{E}+05$ & $5.52 \%$ \\
\hline 1 & $\mathrm{OLH}$ & $8 \mathrm{TC} 046$ & 55 & 1.7 & 8 & 448 & 0 & 14622 & 0 & 0 & 6428 & 50 & 4 & 47 & $2.41 \mathrm{E}+04$ & $7.78 \mathrm{E}+05$ & $3.10 \%$ \\
\hline 1 & $\mathrm{OLH}$ & 6ТC046 & 55 & 1.7 & 6 & 0 & 0 & 0 & 0 & 0 & 6544 & 50 & 4 & 47 & $9.18 \mathrm{E}+03$ & $1.01 \mathrm{E}+06$ & $0.91 \%$ \\
\hline
\end{tabular}


WSRC-TR-2003-00269, REVISION 0

SRT-RPP-2003-00125, REVISION 0

Table 30. Predicted Solids from Envelope C Simulations - Part 2 (cont'd)

\begin{tabular}{|c|c|c|c|c|c|c|c|c|c|c|c|c|c|c|c|c|c|}
\hline \begin{tabular}{|c|} 
Experimental \\
ID
\end{tabular} & $\begin{array}{c}\text { Simulation } \\
\text { Point } \\
\text { Type }\end{array}$ & \begin{tabular}{|c|} 
Simulation \\
ID
\end{tabular} & $\begin{array}{c}\text { Temp } \\
\text { oC } \\
(\mathbf{P 2})\end{array}$ & $\begin{array}{c}\text { SBS/Feed } \\
\text { (P1) }\end{array}$ & $\begin{array}{l}\mathbf{N a} \\
\mathrm{M}\end{array}$ & $\begin{array}{c}\text { NA2C2O4 } \\
{[g]}\end{array}$ & $\begin{array}{c}\text { NA3FSO4 } \\
{[\mathrm{g}]}\end{array}$ & $\begin{array}{c}\text { NA6SO42CO3 } \\
{[\mathrm{g}]}\end{array}$ & $\begin{array}{c}\text { NAF } \\
{[g]}\end{array}$ & $\begin{array}{c}\text { NANO3 } \\
{[\mathrm{g}]}\end{array}$ & $\begin{array}{c}\text { NASGEL.15.5H2O } \\
{[\mathrm{g}]}\end{array}$ & $\underset{[\mathrm{g}]}{\mathrm{NIOH} 2}$ & $\begin{array}{c}\text { SRCO3 } \\
{[\mathrm{g}]}\end{array}$ & $\begin{array}{c}\text { ZRO2 } \\
{[\mathrm{g}]}\end{array}$ & $\begin{array}{c}\text { Total } \\
\text { Solids } \\
{[\mathrm{g}]}\end{array}$ & $\begin{array}{c}\text { Total } \\
\text { Soln } \\
\text { Mass }[g]\end{array}$ & \begin{tabular}{|c|} 
Solids \\
$\%$ of \\
Total \\
Soln \\
\end{tabular} \\
\hline 1 & $\mathrm{OLH}$ & $10 \mathrm{TC} 048$ & 48 & 1.4 & 10 & 894 & 0 & 21644 & 1112 & 0 & 4303 & 40 & 3 & 37 & $3.00 \mathrm{E}+04$ & $6.37 \mathrm{E}+05$ & $4.71 \%$ \\
\hline 1 & $\mathrm{OLH}$ & $8 \mathrm{TC} 048$ & 48 & 1.4 & 8 & 556 & 0 & 9719 & 0 & 0 & 5048 & 40 & 3 & 37 & $1.74 \mathrm{E}+04$ & $7.77 \mathrm{E}+05$ & $2.24 \%$ \\
\hline 1 & $\mathrm{OLH}$ & 6TC048 & 48 & 1.4 & 6 & 0 & 0 & 0 & 0 & 0 & 5224 & 40 & 3 & 37 & $7.34 \mathrm{E}+03$ & $9.99 \mathrm{E}+05$ & $0.73 \%$ \\
\hline 1 & $\mathrm{OLH}$ & $10 \mathrm{TC} 050$ & 40 & 0.2 & 10 & 979 & 0 & 9185 & 1389 & 0 & 0 & 3 & 0.3 & 0.5 & $1.18 \mathrm{E}+04$ & $6.10 \mathrm{E}+05$ & $1.93 \%$ \\
\hline 1 & $\mathrm{OLH}$ & $8 \mathrm{TC} 050$ & 40 & 0.2 & 8 & 701 & 0 & 0 & 0 & 0 & 0 & 4 & 0.3 & 0.3 & $9.15 \mathrm{E}+02$ & $7.46 \mathrm{E}+05$ & $0.12 \%$ \\
\hline 1 & $\mathrm{OLH}$ & 6TC050 & 40 & 0.2 & 6 & 124 & 0 & 0 & 0 & 0 & 269 & 4 & 0.3 & 0.2 & $6.07 \mathrm{E}+02$ & $9.44 \mathrm{E}+05$ & $0.06 \%$ \\
\hline 1 & $\mathrm{OLH}$ & $10 \mathrm{TC} 052$ & 35 & 0.2 & 10 & 995 & 10924 & 0 & 0 & 0 & 0 & 4 & 0.4 & 0.4 & $1.22 \mathrm{E}+04$ & $6.14 \mathrm{E}+05$ & $1.99 \%$ \\
\hline 1 & $\mathrm{OLH}$ & $8 \mathrm{TC} 052$ & 35 & 0.2 & 8 & 776 & 6910 & 0 & 0 & 0 & 54 & 5 & 0.4 & 0.1 & $8.04 \mathrm{E}+03$ & $7.40 \mathrm{E}+05$ & $1.09 \%$ \\
\hline 1 & $\mathrm{OLH}$ & 6TC052 & 35 & 0.2 & 6 & 242 & 0 & 0 & 0 & 0 & 500 & 5 & 0.4 & 0 & $1.04 \mathrm{E}+03$ & $9.51 \mathrm{E}+05$ & $0.11 \%$ \\
\hline 3 & $\mathrm{OLH}$ & $10 \mathrm{TC} 055$ & 29 & 0.8 & 10 & 1050 & 15455 & 0 & 0 & 0 & 1680 & 21 & 2 & 16 & $1.93 \mathrm{E}+04$ & $6.36 \mathrm{E}+05$ & $3.03 \%$ \\
\hline 3 & $\mathrm{OLH}$ & $8 \mathrm{TC} 055$ & 29 & 0.8 & 8 & 853 & 9189 & 0 & 0 & 0 & 2675 & 22 & 2 & 16 & $1.39 \mathrm{E}+04$ & $7.67 \mathrm{E}+05$ & $1.81 \%$ \\
\hline 3 & $\mathrm{OLH}$ & 6TC055 & 29 & 0.8 & 6 & 362 & 1395 & 0 & 0 & 0 & 2992 & 23 & 2 & 16 & $6.32 \mathrm{E}+03$ & $9.83 \mathrm{E}+05$ & $0.64 \%$ \\
\hline 1 & $\mathrm{OLH}$ & $10 \mathrm{TC} 060$ & 21 & 1.1 & 10 & 1096 & 16765 & 0 & 0 & 0 & 3089 & 29 & 3 & 21 & $2.27 \mathrm{E}+04$ & $6.50 \mathrm{E}+05$ & $3.50 \%$ \\
\hline 1 & $\mathrm{OLH}$ & $8 \mathrm{TC} 060$ & 21 & 1.1 & 8 & 924 & 9535 & 0 & 0 & 0 & 3862 & 30 & 3 & 19 & $1.72 \mathrm{E}+04$ & $7.84 \mathrm{E}+05$ & $2.20 \%$ \\
\hline 1 & $\mathrm{OLH}$ & 6TC060 & 21 & 1.1 & 6 & 503 & 1768 & 0 & 0 & 0 & 4108 & 31 & 3 & 19 & $1.09 \mathrm{E}+04$ & $1.00 \mathrm{E}+06$ & $1.09 \%$ \\
\hline 1 & $\mathrm{OLH}$ & $10 \mathrm{TC} 062$ & 37 & 1.8 & 10 & 1024 & 20066 & 2798 & 0 & 0 & 6419 & 52 & 4 & 48 & $3.35 \mathrm{E}+04$ & $6.60 \mathrm{E}+05$ & $5.07 \%$ \\
\hline 1 & $\mathrm{OLH}$ & $8 \mathrm{TC} 062$ & 37 & 1.8 & 8 & 753 & 12968 & 0 & 0 & 0 & 6855 & 52 & 4 & 48 & $2.55 \mathrm{E}+04$ & $7.98 \mathrm{E}+05$ & $3.20 \%$ \\
\hline 1 & $\mathrm{OLH}$ & 6TC062 & 37 & 1.8 & 6 & 118 & 3168 & 0 & 0 & 0 & 6972 & 52 & 4 & 48 & $1.62 \mathrm{E}+04$ & $1.02 \mathrm{E}+06$ & $1.58 \%$ \\
\hline 1 & $\mathrm{OLH}$ & $10 \mathrm{TC} 067$ & 56 & 0.7 & 10 & 795 & 0 & 14957 & 1077 & 0 & 1165 & 21 & 2 & 19 & $1.91 \mathrm{E}+04$ & $6.16 \mathrm{E}+05$ & $3.10 \%$ \\
\hline 1 & OLH & $8 \mathrm{TC} 067$ & 56 & 0.7 & 8 & 432 & 0 & 5072 & 0 & 0 & 2300 & 21 & 2 & 19 & $8.90 \mathrm{E}+03$ & $7.52 \mathrm{E}+05$ & $1.18 \%$ \\
\hline 1 & OLH & 6ТC067 & 56 & 0.7 & 6 & 0 & 0 & 0 & 0 & 0 & 2556 & 21 & 2 & 19 & $3.66 \mathrm{E}+03$ & $9.60 \mathrm{E}+05$ & $0.38 \%$ \\
\hline 1 & $\mathrm{OLH}$ & 10TC068 & 61 & 0.3 & 10 & 744 & 0 & 12402 & 1044 & 0 & 0 & 8 & 1 & 7 & $1.46 \mathrm{E}+04$ & $6.00 \mathrm{E}+05$ & $2.43 \%$ \\
\hline 1 & $\mathrm{OLH}$ & $8 \mathrm{TC} 068$ & 61 & 0.3 & 8 & 348 & 0 & 3218 & 0 & 0 & 539 & 8 & 1 & 7 & $4.54 \mathrm{E}+03$ & $7.33 \mathrm{E}+05$ & $0.62 \%$ \\
\hline 1 & $\mathrm{OLH}$ & 6TC068 & 61 & 0.3 & 6 & 0 & 0 & 0 & 0 & 0 & 806 & 8 & 1 & 7 & $1.25 \mathrm{E}+03$ & $9.35 \mathrm{E}+05$ & $0.13 \%$ \\
\hline 1 & OLH & $10 \mathrm{TC} 074$ & 43 & 1.8 & 10 & 957 & 0 & 23824 & 1120 & 0 & 6080 & 50 & 4 & 47 & $3.46 \mathrm{E}+04$ & $6.52 \mathrm{E}+05$ & $5.31 \%$ \\
\hline 1 & $\mathrm{OLH}$ & $8 \mathrm{TC} 074$ & 43 & 1.8 & 8 & 630 & 0 & 9562 & & 0 & 6576 & 50 & 4 & 47 & $1.94 \mathrm{E}+04$ & $7.97 \mathrm{E}+05$ & $2.44 \%$ \\
\hline 1 & OLH & 6ТC074 & 43 & 1.8 & 6 & 0 & 0 & 0 & & 0 & 6695 & 50 & 4 & 47 & $1.11 \mathrm{E}+04$ & $1.02 \mathrm{E}+06$ & $1.09 \%$ \\
\hline 2 & $\mathrm{OLH}$ & 10TC083 & 50 & 2.0 & 10 & 785 & 0 & 25189 & 345 & 0 & 2850 & 56 & 5 & 54 & $3.30 \mathrm{E}+04$ & $6.52 \mathrm{E}+05$ & $5.06 \%$ \\
\hline 2 & $\mathrm{OLH}$ & $8 \mathrm{TC} 083$ & 50 & 2.0 & 8 & 424 & 0 & 12638 & & 0 & 3574 & 57 & 5 & 54 & $1.97 \mathrm{E}+04$ & $7.92 \mathrm{E}+05$ & $2.48 \%$ \\
\hline 2 & $\mathrm{OLH}$ & 6TC083 & 50 & 2.0 & 6 & 0 & 0 & 0 & & 0 & 3780 & 57 & 5 & 54 & $8.41 \mathrm{E}+03$ & $1.02 \mathrm{E}+06$ & $0.82 \%$ \\
\hline
\end{tabular}


WSRC-TR-2003-00269, REVISION 0 SRT-RPP-2003-00125, REVISION 0

Table 30. Predicted Solids from Envelope C Simulations - Part 2 (cont'd)

\begin{tabular}{|c|c|c|c|c|c|c|c|c|c|c|c|c|c|c|c|c|c|}
\hline \begin{tabular}{|} 
Experimental \\
ID
\end{tabular} & \begin{tabular}{|c|} 
Simulation \\
Point \\
Type
\end{tabular} & \begin{tabular}{|c|} 
Simulation \\
ID
\end{tabular} & \begin{tabular}{|c|} 
Temp \\
oC \\
(P2)
\end{tabular} & $\begin{array}{c}\text { SBS/Feed } \\
\text { (P1) }\end{array}$ & \begin{tabular}{l|}
$\mathrm{Na}$ \\
$\mathrm{M}$
\end{tabular} & \begin{tabular}{|c|} 
NA2C2O4 \\
{$[\mathrm{g}]$}
\end{tabular} & $\begin{array}{c}\text { NA3FSO4 } \\
{[\mathrm{g}]}\end{array}$ & $\begin{array}{c}\text { NA6SO42CO3 } \\
{[\mathrm{g}]}\end{array}$ & $\begin{array}{c}\text { NAF } \\
{[g]}\end{array}$ & $\begin{array}{c}\text { NANO3 } \\
\text { [g] }\end{array}$ & $\begin{array}{c}\text { NASGEL.15.5H2O } \\
{[\mathrm{g}]}\end{array}$ & $\begin{array}{c}\text { NIOH2 } \\
{[\mathrm{g}]}\end{array}$ & \begin{tabular}{|c|} 
SRCO3 \\
{$[\mathrm{g}]$}
\end{tabular} & $\begin{array}{c}\text { ZRO2 } \\
{[\mathrm{g}]}\end{array}$ & $\begin{array}{c}\text { Total } \\
\text { Solids } \\
{[\mathrm{g}]}\end{array}$ & \begin{tabular}{|c|} 
Total \\
Soln \\
Mass $[g]$
\end{tabular} & \begin{tabular}{|c|} 
Solids \\
\% of \\
Total \\
Soln \\
\end{tabular} \\
\hline 4 & $\mathrm{OLH}$ & 10TC093 & 32 & 1.1 & \begin{tabular}{|l|}
10 \\
\end{tabular} & 955 & 16732 & 0 & 0 & 0 & 0 & 30 & 3 & 26 & $1.96 \mathrm{E}+04$ & $6.40 \mathrm{E}+05$ & $3.06 \%$ \\
\hline 4 & $\overline{\mathrm{OLH}}$ & $8 \mathrm{TC} 093$ & 32 & 1.1 & 8 & 722 & 9227 & 0 & 0 & 0 & 917 & 31 & 3 & 26 & $1.24 \mathrm{E}+04$ & $7.73 \mathrm{E}+05$ & $1.60 \%$ \\
\hline 4 & OLH & 6TC093 & 32 & 1.1 & 6 & 181 & 1574 & 0 & 0 & 0 & 1705 & 31 & 3 & 26 & $6.12 \mathrm{E}+03$ & $9.92 \mathrm{E}+05$ & $0.62 \%$ \\
\hline 2 & $\mathrm{OLH}$ & 10TC098 & 31 & 1.7 & \begin{tabular}{|l|l|}
10 \\
\end{tabular} & 943 & 19874 & 0 & 0 & 0 & 1985 & 48 & 4 & 44 & $2.66 \mathrm{E}+04$ & $6.59 \mathrm{E}+05$ & $4.03 \%$ \\
\hline 2 & OLH & 8TC098 & 31 & 1.7 & 8 & 702 & 9953 & 0 & 0 & 0 & 3216 & 48 & 4 & 43 & $1.87 \mathrm{E}+04$ & $7.98 \mathrm{E}+05$ & $2.34 \%$ \\
\hline 2 & $\mathrm{OLH}$ & 6TC098 & 31 & 1.7 & 6 & 159 & 2855 & 0 & 0 & 0 & 3741 & 49 & 4 & 43 & $1.25 \mathrm{E}+04$ & $1.02 \mathrm{E}+06$ & $1.23 \%$ \\
\hline
\end{tabular}


WSRC-TR-2003-00269, REVISION 0 SRT-RPP-2003-00125, REVISION 0

Table 31. Comparisons of AW-101 Radioactive Experimental Measured Values versus Envelope A Predictions

\begin{tabular}{|c|c|c|c|c|c|c|c|c|c|c|c|c|c|c|c|c|}
\hline AW101 Test ID & $\begin{array}{c}\text { Meas } \\
\text { Temp }\end{array}$ & $\begin{array}{c}\text { Meas } \\
\text { Press } \\
{[\mathrm{mmHg}]}\end{array}$ & SBS/Feed & $\begin{array}{c}\text { Measured } \\
\text { Na M }\end{array}$ & $\begin{array}{c}\text { Predicted } \\
\text { Na M }\end{array}$ & $\begin{array}{c}\% \text { Diff } \\
\text { b/n } \\
\text { Meas/ } \\
\text { \& Pred } \\
\text { Na M } \\
\end{array}$ & $\begin{array}{c}\text { Measured } \\
\text { Density } \\
{[\mathrm{g} / \mathrm{ml}]}\end{array}$ & $\begin{array}{c}\text { Predicted } \\
\text { Density } \\
{[\mathrm{g} / \mathrm{ml}]}\end{array}$ & $\begin{array}{c}\% \text { Diff b/n } \\
\text { Meas/Pred } \\
\text { Density }\end{array}$ & $\begin{array}{c}\text { Meas } \\
\text { visc } \\
\text { [cP] }\end{array}$ & $\begin{array}{l}\text { Pred } \\
\text { Visc } \\
{[\mathrm{cP}]}\end{array}$ & $\begin{array}{c}\% \text { Diff b/n } \\
\text { Meas/Pred } \\
\text { Visc }\end{array}$ & $\begin{array}{c}\text { Meas Cp } \\
{[\text { supernate] }} \\
{\left[\mathbf{c a l} / \mathbf{g}^{\circ} \mathbf{C}\right]}\end{array}$ & $\begin{array}{c}\text { Meas Cp } \\
\text { [slurry] } \\
{\left[\text { cal } / \mathbf{g}^{\circ} \mathbf{C}\right]}\end{array}$ & $\begin{array}{c}\text { Pred Cp } \\
\text { [supernate] } \\
{\left[\text { cal } / \mathbf{g}^{\circ} \mathbf{C}\right]}\end{array}$ & $\begin{array}{c}\% \text { Diff b/n } \\
\text { Meas/Pred } \\
\text { Cp }\end{array}$ \\
\hline AW101007 & 44.9 & 64.0 & 1.2 & 7.0 & 10.3 & $-48.5 \%$ & 1.36 & 1.25 & $7.8 \%$ & $\mathrm{n} / \mathrm{a}$ & $\mathrm{n} / \mathrm{a}$ & $\mathrm{n} / \mathrm{a}$ & $\mathrm{n} / \mathrm{a}$ & $\mathrm{n} / \mathrm{a}$ & $\mathrm{n} / \mathrm{a}$ & $\mathrm{n} / \mathrm{a}$ \\
\hline AW101008 & 47.1 & 72.9 & 1.2 & 7.0 & 10.4 & $-49.8 \%$ & 1.36 & 1.25 & $8.0 \%$ & $\mathrm{n} / \mathrm{a}$ & $\mathrm{n} / \mathrm{a}$ & $\mathrm{n} / \mathrm{a}$ & $\mathrm{n} / \mathrm{a}$ & $\mathrm{n} / \mathrm{a}$ & $\mathrm{n} / \mathrm{a}$ & $\mathrm{n} / \mathrm{a}$ \\
\hline AW101009 & 50.1 & 80.5 & 1.2 & 7.0 & 10.6 & $-51.6 \%$ & 1.36 & 1.25 & $8.2 \%$ & $\mathrm{n} / \mathrm{a}$ & $\mathrm{n} / \mathrm{a}$ & $\mathrm{n} / \mathrm{a}$ & $\mathrm{n} / \mathrm{a}$ & $\mathrm{n} / \mathrm{a}$ & $\mathrm{n} / \mathrm{a}$ & $\mathrm{n} / \mathrm{a}$ \\
\hline AW101010 & 52.3 & 60.2 & 1.9 & 7.6 & 11.1 & $-46.0 \%$ & 1.38 & 1.27 & $7.9 \%$ & $\mathrm{n} / \mathrm{a}$ & $\mathrm{n} / \mathrm{a}$ & $\mathrm{n} / \mathrm{a}$ & $\mathrm{n} / \mathrm{a}$ & $\mathrm{n} / \mathrm{a}$ & $\mathrm{n} / \mathrm{a}$ & $\mathrm{n} / \mathrm{a}$ \\
\hline feed & 25 & 760.0 & 1.9 & 2.1 & 1.4 & $33.2 \%$ & 1.08 & 1.11 & $-3.1 \%$ & $\mathrm{n} / \mathrm{a}$ & $\mathrm{n} / \mathrm{a}$ & $\mathrm{n} / \mathrm{a}$ & $\mathrm{n} / \mathrm{a}$ & $\mathrm{n} / \mathrm{a}$ & $\mathrm{n} / \mathrm{a}$ & $\mathrm{n} / \mathrm{a}$ \\
\hline AW101011 & 55.6 & 71.6 & 1.9 & 7.6 & 11.2 & $-47.9 \%$ & 1.38 & 1.27 & $8.2 \%$ & $\mathrm{n} / \mathrm{a}$ & $\mathrm{n} / \mathrm{a}$ & $\mathrm{n} / \mathrm{a}$ & $\mathrm{n} / \mathrm{a}$ & $\mathrm{n} / \mathrm{a}$ & $\mathrm{n} / \mathrm{a}$ & $\mathrm{n} / \mathrm{a}$ \\
\hline AW101012 & 58.4 & 80.5 & 1.9 & 7.6 & 11.4 & $-49.5 \%$ & 1.38 & 1.26 & $8.4 \%$ & $\mathrm{n} / \mathrm{a}$ & $\mathrm{n} / \mathrm{a}$ & $\mathrm{n} / \mathrm{a}$ & $\mathrm{n} / \mathrm{a}$ & $\mathrm{n} / \mathrm{a}$ & $\mathrm{n} / \mathrm{a}$ & $\mathrm{n} / \mathrm{a}$ \\
\hline AW101013 & 62.7 & 75.4 & 1.2 & 10.4 & 18.0 & $-73.2 \%$ & 1.57 & 1.34 & $14.7 \%$ & $\mathrm{n} / \mathrm{a}$ & $\mathrm{n} / \mathrm{a}$ & $\mathrm{n} / \mathrm{a}$ & $\mathrm{n} / \mathrm{a}$ & $\mathrm{n} / \mathrm{a}$ & $\mathrm{n} / \mathrm{a}$ & $\mathrm{n} / \mathrm{a}$ \\
\hline feed & 25 & 760.0 & 1.2 & 2.2 & 2.0 & $8.8 \%$ & 1.10 & 1.11 & $-1.0 \%$ & $\mathrm{n} / \mathrm{a}$ & $\mathrm{n} / \mathrm{a}$ & $\mathrm{n} / \mathrm{a}$ & $\mathrm{n} / \mathrm{a}$ & $\mathrm{n} / \mathrm{a}$ & $\mathrm{n} / \mathrm{a}$ & $\mathrm{n} / \mathrm{a}$ \\
\hline visc pt & 25 & 760.0 & 1.2 & $\mathrm{n} / \mathrm{a}$ & $\mathrm{n} / \mathrm{a}$ & $\mathrm{n} / \mathrm{a}$ & $\mathrm{n} / \mathrm{a}$ & $\mathrm{n} / \mathrm{a}$ & $\mathrm{n} / \mathrm{a}$ & 7.1 & 3.7 & $47.9 \%$ & $\mathrm{n} / \mathrm{a}$ & $\mathrm{n} / \mathrm{a}$ & $\mathrm{n} / \mathrm{a}$ & $\mathrm{n} / \mathrm{a}$ \\
\hline visc pt 2 & 25 & 760.0 & 1.9 & $\mathrm{n} / \mathrm{a}$ & $\mathrm{n} / \mathrm{a}$ & $\mathrm{n} / \mathrm{a}$ & $\mathrm{n} / \mathrm{a}$ & $\mathrm{n} / \mathrm{a}$ & $\mathrm{n} / \mathrm{a}$ & 8.7 & 4.2 & $52.2 \%$ & $\mathrm{n} / \mathrm{a}$ & $\mathrm{n} / \mathrm{a}$ & $\mathrm{n} / \mathrm{a}$ & $\mathrm{n} / \mathrm{a}$ \\
\hline Cp pt-Blend 1 & 50 & 760.0 & 1.2 & $\mathrm{n} / \mathrm{a}$ & $\mathrm{n} / \mathrm{a}$ & $\mathrm{n} / \mathrm{a}$ & $\mathrm{n} / \mathrm{a}$ & $\mathrm{n} / \mathrm{a}$ & $\mathrm{n} / \mathrm{a}$ & $\mathrm{n} / \mathrm{a}$ & $\mathrm{n} / \mathrm{a}$ & $\mathrm{n} / \mathrm{a}$ & 0.78 & 0.81 & 0.64 & $20.97 \%$ \\
\hline Cp pt 2-Blend 2 & 50 & 760.0 & 1.9 & $\mathrm{n} / \mathrm{a}$ & $\mathrm{n} / \mathrm{a}$ & $\mathrm{n} / \mathrm{a}$ & $\mathrm{n} / \mathrm{a}$ & $\mathrm{n} / \mathrm{a}$ & $\mathrm{n} / \mathrm{a}$ & $\mathrm{n} / \mathrm{a}$ & $\mathrm{n} / \mathrm{a}$ & $\mathrm{n} / \mathrm{a}$ & 0.79 & 0.76 & 0.65 & $14.70 \%$ \\
\hline
\end{tabular}


WSRC-TR-2003-00269, REVISION 0

SRT-RPP-2003-00125, REVISION 0

\subsection{REFERENCES}

1. Choi, A.S., Preliminary Modeling Results of Pretreated LAW Evaporator, Westinghouse Savannah River Company: Aiken, SC (1999).

2. $\quad$ Saito, H.H., et al., AN-107(C) Simulant Bench-Scale LAW Evaporation with Organic Regulatory Analysis, Westinghouse Savannah River Company: Aiken, SC (2001).

3. Calloway, T.B., Evaporation of Hanford Envelope A Simulant (AN-105), Westinghouse Savannah River Company: Aiken, SC (2000).

4. Calloway, T.B., A.S. Choi, and P.R. Monson, Evaporation of Hanford Envelope B Simulant (AZ-101) Preliminary Report, Westinghouse Savannah River Company: Aiken, SC (2000).

5. Longwell, R.L., Treated LAW Feed: Simulant Evaporation and Physical Properties Modeling, Washington Group International: Richland, WA (2002).

6. Calloway, T.B. and M.A. Stone, Task Technical and Quality Assurance Plan for Treated LAW Feed: Evaporation and Physical Properties Determination, Westinghouse Savannah River Co.: Aiken, SC (2002).

7. Josephs, J.E., Michael E. Stone, T. B. Calloway, R. E. Eibling, C. D. Barnes, E. K. Hansen, Treated LAW Feed Evaporation: Physical Properties and Solubility Determination, Westinghouse Savannah River Co.: Aiken, SC (2003).

8. Crowder, M.L., C. L. Crawford, E. K. Hansen, Fernando Fondeur, Evaporation of Pretreated Hanford Tank AW-101 Sample Mixed with Recycle, Westinghouse Savannah River Co.: Aiken, SC 29808 (2003).

9. $\quad$ Barnes, C.D., W E Daniel, J E Laurinat, Waste Feed Evaporation Physical Properties Modeling, Westinghouse Savannah River Company: Aiken, SC (2003).

10. JMP ${ }^{\circledR}$ Statistics and Graphics Guide, SAS Institute, Inc. (2002).

11. Edwards, T.B., A Statistical Design to Support RPP LAW Feed Simulant Testing (U), Savannah River Site: Aiken, SC 29808 (2002).

12. Eibling, R.E., C. A. Nash, Hanford Waste Simulant Created to Support the Research and Development on the River Protection Project - Waste Treatment Plant, Westinghouse Savannah River Company: Aiken, SC 29808. p. 26-32 (2000).

13. Crawford, C.L., Interim Report for Crucible Scale Active Vitrification of Waste Envelope B (AZ-102) (U), Westinghouse Savannah River Company: Aiken, SC 29808 (2001).

14. Bannochie, C.J., Standardized Baseline Precipitation Procedure for Hanford RPP Studies, Westinghouse Savannah River Company: Aiken, SC, 29808 (2001).

15. Perry, R.H. and D.W. Green, Perry's Chemical Engineer's Handbook. 6th ed, New York: McGraw-Hill Book Company. 13-25, 13-26 (1984).

16. Daniel, W.E., Task Technical and Quality Assurance Plan for Modeling Treated LAW Feed Evaporation and Validation of Evaporation Pilot Testing, Westinghouse Savannah River Co.: Aiken, SC 29808 (2003).

17. Choi, A.S., Software Quality Assurance Plan for Hanford RPP-WTP Evaporator Modeling, Westinghouse Savannah River Co.: Aiken, SC 29808 (2001).

18. Bergman, I.M., et al., WTP Material Balance and Process Flowsheet Bases, Requirements, and Results, Bechtel: Richland, WA 99352 (2001). 


\section{WSRC-TR-2003-00269, REVISION 0 \\ SRT-RPP-2003-00125, REVISION 0}

19. Kirkbride, R.A., et al., Tank Farm Contractor Operation and Utilization Plan, Numatec Hanford Corp. and CH2MHill Hanford Group, Inc: Richland, WA. (2001).

20. Edwards, T.B., A Statistically Designed Test Matrix for a Computer Study of Treated Feed Evaporator Runs for Envelope B (U), Savannah River Site: Aiken, SC 29808 (2003).

21. Edwards, T.B., A Statistically Designed Test Matrix for a Computer Study of Treated Feed Evaporator Runs for Envelope C (U), Savannah River Site: Aiken, SC 29808 (2003).

22. Edwards, T.B., A Statistically Designed Test Matrix for a Computer Study of Waste Feed Evaporation for Envelope A (U), Savannah River Site: Aiken, SC 29808 (2002).

23. Ye, K.Q., Orthogonal Column Latin Hypercubes and Their Application in Computer Experiments. J. American Statistical Association, 93: p. 1430-1439 (1998). 


\section{APPENDIX A. DESIGN MATRICES FOR ENVELOPES A, B, AND C}

The following tables list the compositions of the waste feed streams, SBS/Feed volumetric flow ratio, bottoms concentrate temperature, and bottoms concentrate target Na molarity used in the OLI/ESP computer simulations. The waste feed compositions are in terms of mass fractions to one another. In other words, the mass fractions of the species shown must sum to one. As described earlier, a dry basis is used to represent those species in the waste feeds that have significant variance across the various tanks of each envelope. The remaining species in the waste feeds have fixed compositions.

The Run No. indicates the simulation run number. The Test ID indicates the type of run with the first 1-2 digits represent the target Sodium Molarity (10,8 or 6) and then EA, TB, or TC for Envelope A, B, and C respectively followed by the 3 digits that represent the run number. The Type describes the type of design point. MPV and EV indicate model design points used in the property model fits and are derived using only the minimum and maximum values of the factor space. OLH indicates a design point used to validate the model (though some were occasionally used in the model fits as described in the results section and are marked by asterisk in the following tables), and were generated to be uniformly distributed over the factor space (as opposed to only the extreme vertices of the MPV points). The columns $x_{\mathrm{AlO} 2}, x_{\mathrm{CO} 3}, x_{\mathrm{F}}, x_{\mathrm{NO} 2}, x_{\mathrm{NO} 3}, x_{\mathrm{OH}}, x_{\mathrm{PO}_{4}} x_{\mathrm{SO} 4}$, and $x_{\mathrm{C2O}}$, are the relative waste feed mass fractions of $\mathrm{AlO}_{2}^{-}, \mathrm{CO}_{3}^{-2}, \mathrm{~F}^{-}, \mathrm{NO}_{2}^{-}, \mathrm{NO}_{3}^{-}, \mathrm{OH}^{-}, \mathrm{PO}_{4}^{-3}, \mathrm{SO}_{4}^{-2}$, and $\mathrm{C}_{2} \mathrm{O}_{4}{ }^{-2}$ respectively. Note that this is the complete list of mass feed fractions for all envelopes and only certain species were varied per envelope. SBS/Feed is the volumetric feed flow ratio of the LAW SBS recycle stream to the treated waste feed stream. Temp is the temperature in ${ }^{\circ} \mathrm{C}$ of the bottoms concentrate stream. Na $\mathbf{M}$ is the Na molarity of the bottoms concentrate stream. 
Table 32. Envelope A Design Matrix for OLI Simulations

\begin{tabular}{|c|c|c|c|c|c|c|c|c|c|c|c|c|}
\hline $\begin{array}{l}\text { Run } \\
\text { No. }\end{array}$ & Test ID & Type & $\mathrm{x}_{\mathrm{AlO} 2}$ & $\mathrm{x}_{\mathrm{CO} 3}$ & $\mathrm{x}_{\mathrm{F}}$ & $\mathrm{x}_{\mathrm{NO} 2}$ & $\mathrm{x}_{\mathrm{NO} 3}$ & $\mathrm{x}_{\mathrm{OH}}$ & $\mathrm{X}_{\mathrm{PO} 4}$ & SBS/Feed & Temp & $\mathrm{Na} \mathrm{M}$ \\
\hline 1 & 10EA001 & MPV & 0.0774 & .0124 & 0.0011 & 0.2132 & 0.3895 & 0.2810 & 0.0254 & 0 & 15 & 10 \\
\hline 2 & 10EA002 & IPV & 0.0782 & 0125 & .0011 & 0.2153 & 0.3935 & 0.2956 & 0.0038 & - & 15 & 10 \\
\hline 3 & 10EA003 & PV & 0.0552 & 0089 & 0.0008 & 0.3208 & 0.5360 & 0.0756 & 0.0027 & 0 & 15 & 10 \\
\hline 4 & 10EA004 & MPV & 0.0602 & 0.0097 & 0.0214 & 0.1659 & 0.6167 & 0.1232 & 0.0030 & 0 & 15 & 10 \\
\hline 5 & 10EA005 & MPV & 0.0673 & 0.0108 & 0.0240 & 0.3911 & 0.3389 & 0.1501 & 0.0178 & 0 & 15 & 10 \\
\hline 6 & 10EA006 & MPV & 0.0672 & 2197 & 0.0239 & 0.1852 & 0.3941 & 0.0920 & 0.0178 & 0 & 15 & 10 \\
\hline 7 & 10EA007 & MPV & 0.2066 & 0.2077 & 0.0009 & 0.1750 & 0.3197 & 0.0870 & 0.0031 & 0 & 15 & 10 \\
\hline 8 & 10EA008 & MPV & 0.1920 & 0.0087 & 0.0008 & 0.1487 & 0.5530 & 0.0791 & 0.0178 & 0 & 15 & 10 \\
\hline 9 & 10EA009 & MPV & 0.2347 & 0.0094 & 0.0009 & 0.3423 & 0.2966 & 0.0967 & 0.0194 & 0 & 15 & 10 \\
\hline 10 & 10EA010 & $\mathrm{IPV}$ & 0.3310 & 0.0101 & 0.0225 & 0.1738 & 0.3176 & 0.1420 & 0.0031 & 0 & 15 & 10 \\
\hline 11 & 10EA011 & APV & 0.0774 & .0124 & 0.0011 & 0.2132 & 0.3895 & 0.2810 & 0.0254 & 0 & 15 & 10 \\
\hline 12 & 10ЕA012 & MPV & 0.0782 & 0.0125 & 0.0011 & 0.2153 & 0.3935 & 0.2956 & 0.0038 & 0 & 15 & 10 \\
\hline 13 & 10EA013 & MPV & 0.0552 & 0.0089 & 0.0008 & 0.3208 & 0.5360 & 0.0756 & 0.0027 & 0 & 15 & 10 \\
\hline 14 & 10EA014 & PPV & 0.0602 & .0097 & 0.0214 & 0.1659 & 0.6167 & 0.1232 & 0.0030 & $\sigma$ & 15 & 10 \\
\hline 15 & 10EA015 & MPV & 0.0673 & .0108 & 0.0240 & 0.3911 & 0.3389 & 0.1501 & 0.0178 & 0 & 15 & 10 \\
\hline 16 & 10EA016 & MPV & 0.0672 & 0.2197 & 0.0239 & 0.1852 & 0.3941 & 0.0920 & 0.0178 & 0 & 15 & 10 \\
\hline 17 & 10EA017 & PV & 0.2066 & 2077 & 0.0009 & 0.1750 & 0.3197 & 0.0870 & 0.0031 & 0 & 15 & 10 \\
\hline 18 & 10EA018 & MPV & 0.1920 & 0.0087 & 0.0008 & 0.1487 & 0.5530 & 0.0791 & 0.0178 & 0 & 15 & 10 \\
\hline 19 & 10EA019 & MPV & 0.2347 & 0.0094 & 0.0009 & 0.3423 & 0.2966 & 0.0967 & 0.0194 & 0 & 15 & 10 \\
\hline 20 & 10EA020 & MPV & 0.3310 & 0.0101 & 0.0225 & 0.1738 & 0.3176 & 0.1420 & 0.0031 & 0 & 15 & 10 \\
\hline 21 & 10EA021 & $\mathrm{PV}$ & 0.0774 & 0.0124 & 0.0011 & 0.2132 & 0.3895 & 0.2810 & 0.0254 & 2 & 15 & 10 \\
\hline 22 & 10EA022 & MPV & 0.0782 & 0.0125 & 0.0011 & 0.2153 & 0.3935 & 0.2956 & 0.0038 & 2 & 15 & 10 \\
\hline 23 & 10EA023 & PV & 0.0552 & 0.0089 & 0.0008 & 0.3208 & 0.5360 & 0.0756 & 0.0027 & 2 & 15 & 10 \\
\hline 24 & 10EA024 & PV & 0.0602 & 0.0097 & 0.0214 & 0.1659 & 0.6167 & 0.1232 & 0.0030 & 2 & 15 & 10 \\
\hline 25 & 10EA025 & IPV & 0.0673 & 0.0108 & 0.0240 & 0.3911 & 0.3389 & 0.1501 & 0.0178 & 2 & 15 & 10 \\
\hline 26 & 10EA026 & MPV & 0.0672 & 0.2197 & 0.0239 & 0.1852 & 0.3941 & 0.0920 & 0.0178 & 2 & 15 & 10 \\
\hline 27 & 10EA027 & MPV & 0.2066 & 0.2077 & 0.0009 & 0.1750 & 0.3197 & 0.0870 & 0.0031 & 2 & 15 & 10 \\
\hline 28 & 10EA028 & MPV & 0.1920 & 0.0087 & 0.0008 & 0.1487 & 0.5530 & 0.0791 & 0.0178 & 2 & 15 & 10 \\
\hline 29 & 10EA029 & MPV & 0.2347 & 0.0094 & 0.0009 & 0.3423 & 0.2966 & 0.0967 & 0.0194 & 2 & 15 & 10 \\
\hline 30 & 10EA030 & MPV & 0.3310 & 0.0101 & 0.0225 & 0.1738 & 0.3176 & 0.1420 & 0.0031 & 2 & 15 & 10 \\
\hline 31 & 10EA031 & MPV & 0.0774 & 0.0124 & 0.0011 & 0.2132 & 0.3895 & 0.2810 & 0.0254 & 2 & 15 & 10 \\
\hline 32 & 10EA032 & MPV & 0.0782 & 0.0125 & 0.0011 & 0.2153 & 0.3935 & 0.2956 & 0.0038 & 2 & 15 & 10 \\
\hline 33 & 10EA033 & MPV & 0.0552 & 0.0089 & 0.0008 & 0.3208 & 0.5360 & 0.0756 & 0.0027 & 2 & 15 & 10 \\
\hline 34 & 10EA034 & MPV & 0.0602 & 0.0097 & 0.0214 & 0.1659 & 0.6167 & 0.1232 & 0.0030 & 2 & 15 & 10 \\
\hline 35 & 10EA035 & MPV & 0.0673 & 0.0108 & 0.0240 & 0.3911 & 0.3389 & 0.1501 & 0.0178 & 2 & 15 & 10 \\
\hline 36 & 10EA036 & MPV & 0.0672 & 0.2197 & 0.0239 & 0.1852 & 0.3941 & 0.0920 & 0.0178 & 2 & 15 & 10 \\
\hline 37 & 10EA037 & MPV & 0.2066 & 0.2077 & 0.0009 & 0.1750 & 0.3197 & 0.0870 & 0.0031 & 2 & 15 & 10 \\
\hline 38 & 10EA038 & MPV & 0.1920 & 0.0087 & 0.0008 & 0.1487 & 0.5530 & 0.0791 & 0.0178 & 2 & 15 & 10 \\
\hline 39 & 10EA039 & MPV & 0.2347 & 0.0094 & 0.0009 & 0.3423 & 0.2966 & 0.0967 & 0.0194 & 2 & 15 & 10 \\
\hline 40 & 10EA040 & MPV & 0.3310 & 0.0101 & 0.0225 & 0.1738 & 0.3176 & 0.1420 & 0.0031 & 2 & 15 & 10 \\
\hline 41 & 10EA041 & MPV & 0.0774 & 0.0124 & 0.0011 & 0.2132 & 0.3895 & 0.2810 & 0.0254 & 0 & 66 & 10 \\
\hline 42 & 10EA042 & MPV & 0.0782 & 0.0125 & 0.0011 & 0.2153 & 0.3935 & 0.2956 & 0.0038 & 0 & 66 & 10 \\
\hline 43 & 10EA043 & MPV & 0.0552 & 0.0089 & 0.0008 & 0.3208 & 0.5360 & 0.0756 & 0.0027 & 0 & 66 & 10 \\
\hline 44 & 10EA044 & MPV & 0.0602 & 0.0097 & 0.0214 & 0.1659 & 0.6167 & 0.1232 & 0.0030 & 0 & 66 & 10 \\
\hline 45 & 10EA045 & MPV & 0.0673 & 0.0108 & 0.0240 & 0.3911 & 0.3389 & 0.1501 & 0.0178 & 0 & 66 & 10 \\
\hline 46 & 10EA046 & MPV & 0.0672 & 0.2197 & 0.0239 & 0.1852 & 0.3941 & 0.0920 & 0.0178 & 0 & 66 & 10 \\
\hline
\end{tabular}


Table 32. Envelope A Design Matrix for OLI Simulations (cont'd)

\begin{tabular}{|c|c|c|c|c|c|c|c|c|c|c|c|c|}
\hline $\begin{array}{l}\text { Run } \\
\text { No. }\end{array}$ & \begin{tabular}{|l|} 
Test ID \\
\end{tabular} & Type & $\mathrm{x}_{\mathrm{AlO} 2}$ & $\mathrm{x}_{\mathrm{CO} 3}$ & $\mathrm{x}_{\mathrm{F}}$ & $\mathrm{x}_{\mathrm{NO} 2}$ & $\mathrm{x}_{\mathrm{NO} 3}$ & $\mathrm{x}_{\mathrm{OH}}$ & $\mathrm{X}_{\mathrm{PO} 4}$ & SBS/Feed & Temp & $\mathrm{Na} \mathrm{M}$ \\
\hline 47 & 10EA047 & MPV & 2066 & 2077 & 0009 & 0.1750 & 3197 & .0870 & 0.0031 & 0 & 66 & 10 \\
\hline 48 & 10EA048 & MPV & 1920 & 0087 & 0008 & 1487 & 5530 & 0791 & 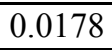 & 0 & 66 & 10 \\
\hline 49 & 10EA049 & MPV & 0.2347 & 0094 & 0009 & 0.3423 & 2966 & 0967 & 0194 & 0 & 66 & 10 \\
\hline 50 & 10EA050 & MPV & 0.3310 & 0.0101 & 0.0225 & 0.1738 & 3176 & 0.1420 & 0.0031 & 0 & 66 & 10 \\
\hline 51 & 10EA051 & MPV & 0.0774 & 0.0124 & 0011 & 0.2132 & .3895 & 0.2810 & 0.0254 & 0 & 66 & 10 \\
\hline 52 & 10EA052 & MPV & 0782 & 0125 & 0011 & 2153 & 3935 & 0.2956 & 0.0038 & 0 & 66 & 10 \\
\hline 53 & 10EA053 & MPV & 0.0552 & 0.0089 & 0.0008 & 0.3208 & 0.5360 & 0.0756 & 0.0027 & 0 & 66 & 10 \\
\hline 54 & 10EA054 & MPV & 0.0602 & 0.0097 & 0.0214 & 0.1659 & 0.6167 & 0.1232 & 0.0030 & 0 & 66 & 10 \\
\hline 55 & 10EA055 & MPV & 0.0673 & 0.0108 & 0.0240 & 0.3911 & 0.3389 & 0.1501 & 0.0178 & 0 & 66 & 10 \\
\hline 56 & 10EA056 & MPV & 0.0672 & 2197 & 0.0239 & 1852 & 3941 & 0.0920 & 0.0178 & 0 & 66 & 10 \\
\hline 57 & 10EA057 & MPV & 0.2066 & 2077 & 0.0009 & 0.1750 & 0.3197 & 0.0870 & 0.0031 & 0 & 66 & 10 \\
\hline 58 & 10EA058 & MPV & 0.1920 & 0.0087 & 0.0008 & 0.1487 & 0.5530 & 0.0791 & 0.0178 & 0 & 66 & 10 \\
\hline 59 & 10EA059 & MPV & 0.2347 & 0.0094 & 0.0009 & 0.3423 & 0.2966 & 0.0967 & 0.0194 & 0 & 66 & 10 \\
\hline 60 & 10EA060 & MPV & 3310 & 0101 & .0225 & 738 & 3176 & .1420 & 31 & 0 & 66 & 10 \\
\hline 61 & 10EA061 & MPV & 0.0774 & 0.0124 & 0.0011 & 0.2132 & 0.3895 & 0.2810 & 0.0254 & 2 & 66 & 10 \\
\hline 62 & 10EA062 & MPV & 0.0782 & 0.0125 & 0.0011 & 0.2153 & 0.3935 & 0.2956 & 0.0038 & 2 & 66 & 10 \\
\hline 63 & 10EA063 & MPV & 0552 & 0089 & 0.0008 & 08 & 360 & 56 & 27 & 2 & 66 & 10 \\
\hline 64 & 10EA064 & MPV & 0.0602 & 0097 & 0.0214 & 0.1659 & 6167 & 0.1232 & 0.0030 & 2 & 66 & 10 \\
\hline 65 & 10EA065 & MPV & 0.0673 & 0.0108 & 0.0240 & 911 & 0.3389 & 0.1501 & 0.0178 & 2 & 66 & 10 \\
\hline 66 & 10EA066 & MPV & 0.0672 & 0.2197 & 0.0239 & 0.1852 & 0.3941 & 0.0920 & 0.0 & 2 & 66 & 10 \\
\hline 67 & $10 \mathrm{E}$ & MPV & 066 & 077 & 009 & 50 & 97 & 70 & 0 . & 2 & 66 & 10 \\
\hline 68 & 10EA068 & MPV & 0.1920 & 0.0087 & 0.0008 & 487 & 0.5530 & 0.0791 & 0.0178 & 2 & 66 & 10 \\
\hline 69 & 10EA069 & MPV & 0.2347 & 0.0094 & 0.0009 & & 0.2966 & 0.0967 & 0.0 & 2 & 66 & 10 \\
\hline 70 & 10EA070 & MPV & 0.3310 & 0101 & 0.0225 & 0.1738 & 0.3176 & 0.1420 & 0.0 & 2 & 66 & 10 \\
\hline 71 & 10EA071 & MPV & 0.0774 & 0124 & 0011 & 0.2132 & 0.3895 & 0.2810 & 0.0254 & 2 & 66 & 10 \\
\hline 72 & 10EA072 & MPV & 0.0782 & 0125 & 0.0011 & 53 & 0.3935 & 56 & & 2 & 66 & 10 \\
\hline 73 & 10EA073 & MPV & 0.0552 & 0.0089 & 0.0008 & 0.3208 & 0.5360 & 0.0756 & 0.0 & 2 & 66 & 10 \\
\hline 74 & 10EA074 & MPV & 0.0602 & 0.0097 & 0.0214 & 659 & 0.6167 & 0.1232 & 0.0030 & 2 & 66 & 10 \\
\hline 75 & 10EA075 & MPV & 0.0673 & 0.0108 & 0.0240 & 0.3911 & 0.3389 & 0.1501 & 0.0178 & 2 & 66 & 10 \\
\hline 76 & 10EA076 & MPV & 0.0672 & 0.2197 & 0.0239 & 52 & 0.3941 & 0. & 78 & 2 & 66 & 10 \\
\hline 77 & 10EA077 & MPV & 0.2066 & 0.2077 & 0.0009 & 50 & 97 & 0.0870 & 31 & 2 & 66 & 10 \\
\hline 78 & 10EA078 & MPV & 0.1920 & 0.0087 & 0.0008 & 87 & 30 & 791 & 78 & 2 & 66 & 10 \\
\hline 79 & 10EA079 & MPV & 0.2347 & 0.0094 & 0.0009 & 0.3423 & 0.2966 & 0.0967 & 94 & 2 & 66 & 10 \\
\hline 80 & 10EA080 & MPV & 0.3310 & 0.0101 & 0.0225 & 0.1738 & 0.3176 & 0.1420 & 0.0031 & 2 & 66 & 10 \\
\hline 81 & 10EA081 & $\mathrm{OLH}$ & 1918 & 1138 & 0232 & 382 & 0.3215 & 52 & 163 & 0.0391 & 15 & 10 \\
\hline 82 & 10EA082 & $\mathrm{OLH}$ & 0.1843 & 0.0414 & 0.0223 & 0.2884 & 0.3481 & 0.1047 & 0.0108 & 1.957 & 15.102 & 10 \\
\hline 83 & 10EA083 & $\mathrm{OLH}$ & 0.1542 & 0.0297 & 0.0155 & 0.1729 & 0.5433 & 0.0790 & 0.0053 & 0.7578 & 15.714 & 10 \\
\hline 84 & 10EA084 & $\mathrm{OLH}$ & 0.1966 & 0.0295 & 0.0198 & 0.1654 & 0.4987 & 0.0840 & 0.0060 & 0.3008 & 15.918 & 10 \\
\hline 85 & 10EA085 & $\mathrm{OLH}$ & 0.0956 & 0.1002 & 0.0214 & 0.3327 & 0.3348 & 0.0982 & 0.0171 & 0.0039 & 16.122 & 10 \\
\hline$* 86$ & 10EA086 & OLH & 0.0998 & 0.0861 & 0.0117 & 0.3543 & 0.3352 & 0.1095 & 0.0033 & 1.2305 & 17.04 & 10 \\
\hline 87 & 10EA087 & $\mathrm{OLH}$ & 0.1262 & 0.1404 & 0.0048 & 0.2244 & 0.3738 & 0.1118 & 0.0186 & 1.5898 & 17.55 & 10 \\
\hline 88 & 10EA088 & OLH & 0.1821 & 0.1572 & 0.0199 & 0.1779 & 0.3597 & 0.0908 & 0.0123 & 0.7734 & \begin{tabular}{|l|l|}
18.06 \\
\end{tabular} & 10 \\
\hline 89 & 10EA089 & OLH & 0.2399 & 0.0212 & 0.0184 & 0.2003 & 0.3729 & 0.1371 & 0.0102 & 1.1641 & 18.162 & 10 \\
\hline$* 90$ & 10EA090 & $\mathrm{OLH}$ & 0.0750 & 0.0439 & 0.0136 & 0.2872 & 0.4361 & 0.1371 & 0.0070 & 0.6094 & 18.264 & 10 \\
\hline 91 & 10EA091 & $\mathrm{OLH}$ & 0.1154 & 0.0306 & 0.0105 & 0.3533 & 0.3620 & 0.1211 & 0.0071 & 0.8594 & 18.57 & 10 \\
\hline 92 & 10EA092 & OLH & 0.1491 & 0.0195 & 0.0069 & 0.3508 & 0.3724 & 0.0931 & 0.0083 & 0.1914 & \begin{tabular}{|l|}
18.672 \\
\end{tabular} & 10 \\
\hline
\end{tabular}


Table 32. Envelope A Design Matrix for OLI Simulations (cont'd)

\begin{tabular}{|c|c|c|c|c|c|c|c|c|c|c|c|c|}
\hline Run & Test ID & Type & 102 & $\mathrm{O} 3$ & $\mathrm{X}_{\mathrm{F}}$ & $\mathrm{O} 2$ & $\mathrm{OO} 3$ & $\mathrm{OH}$ & $\mathrm{OO4}$ & SB & emp & $\mathrm{Na} \mathrm{M}$ \\
\hline$* 93$ & 10EA093 & $\mathrm{OLH}$ & 173 & 0919 & 0084 & 2373 & 4153 & 1102 & 0195 & 1.0195 & 19.284 & 10 \\
\hline 94 & 10EA094 & OLH & 1130 & 0907 & .0043 & 1710 & 5200 & 0981 & 0030 & 0.5352 & 9.488 & 10 \\
\hline 95 & 10EA095 & $\mathrm{LH}$ & 898 & 0323 & 0215 & 2772 & 3724 & 0925 & 44 & 3203 & 9.998 & 10 \\
\hline 96 & 10EA096 & OLH & 669 & 748 & 031 & 3337 & 3280 & 894 & 10 & 398 & 0.508 & 10 \\
\hline 97 & 10EA097 & OLH & 0.2043 & 0.1181 & 0.0061 & 0.2501 & .3185 & 0.0955 & 0.0074 & 0.1563 & 21.222 & 10 \\
\hline 98 & 10EA098 & DLH & 1058 & 0125 & .0041 & 1956 & 5094 & 1547 & 0.0179 & 1.5977 & 21.426 & 10 \\
\hline 99 & 10EA099 & OLH & 003 & 21 & 0199 & 2472 & 3224 & 0.0956 & 0.0126 & .1719 & 21.732 & 10 \\
\hline 100 & 10EA100 & OLH & 876 & 509 & 0021 & 1938 & 4624 & 0914 & & 6094 & 22.956 & 10 \\
\hline 101 & 0EA101 & OLH & 0.1378 & 0.0348 & 0.0086 & 0.2134 & 0.5015 & 0.0869 & 0.0169 & 0.8633 & 23.466 & 10 \\
\hline 102 & 10EA 102 & $\mathrm{OLH}$ & 185 & 1199 & 0.0182 & 1962 & 4383 & 0.1047 & 0042 & 1.0703 & 24.282 & 10 \\
\hline 103 & 10EA103 & OLH & 916 & 103 & 0056 & 2613 & 31 & 934 & 48 & .0273 & 26.016 & 10 \\
\hline 104 & 10EA104 & $\mathrm{OLH}$ & 1544 & 0814 & .0136 & 1791 & 4433 & 194 & 87 & 1.1758 & 26.22 & 10 \\
\hline *105 & 10EA105 & OLH & 0.2332 & 0.0319 & 0.0046 & 0.2387 & 0.3824 & 0.1014 & 0.0079 & 0.5 & 26.526 & 10 \\
\hline 106 & $10 \mathrm{E} A$ & OLH & 655 & 0487 & 0.0189 & 2203 & 83 & 02 & 0.0 & 695 & 27.036 & 10 \\
\hline 107 & $10 \mathrm{EA}$ & OLH & 505 & 207 & & 818 & & & & 195 & 27.138 & 10 \\
\hline 108 & 10EA108 & OLH & 0.1492 & 0.0373 & 0.0219 & 0.2318 & 0.4468 & 0.0979 & 0.0151 & 1.4414 & 27.24 & 10 \\
\hline 109 & 10EA109 & OLH & 0.1778 & 0.0144 & 0.0032 & 0.2740 & 0.4405 & 0.0866 & 0.0036 & 1.0547 & 28.056 & 10 \\
\hline 110 & $10 \mathrm{E}$ & LH & 498 & 426 & 4 & 232 & 17 & 99 & 0.0 & 461 & 28.26 & 10 \\
\hline 111 & $10 \mathrm{EA}$ & $\mathrm{LH}$ & 0733 & 262 & 7 & 0.2809 & 44 & 29 & & 0977 & 28.464 & 10 \\
\hline$* 112$ & 10EA112 & OLH & 0.1772 & 0.0437 & 0.0069 & 0.2768 & 0.3998 & 0.0841 & 0.0115 & 0.6211 & 28.566 & 10 \\
\hline 113 & 13 & OLH & 12 & 21 & 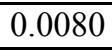 & 4 & 20 & 26 & & 664 & 28.872 & 10 \\
\hline$* 114$ & 4 & LH & 19 & 73 & 2 & 1 & 50 & & 2 & 563 & 29.382 & 10 \\
\hline 115 & 10EA115 & $\mathrm{OLH}$ & 898 & 209 & 0.0170 & 0.2095 & 567 & 16 & & 3867 & 29.484 & 10 \\
\hline$* 116$ & 10EA116 & OLH & 0.1441 & 0.0763 & 0.0138 & 0.2381 & 0.4232 & 0.1 & 0.0 & 1.0078 & 30.198 & 10 \\
\hline 117 & $10 \mathrm{E}$ & $\mathrm{OLH}$ & 307 & 292 & 7 & 27 & 87 & 36 & $\sqrt{3}$ & 711 & 30.504 & 10 \\
\hline 118 & $10 \mathrm{E} A$ & OLH & 601 & 891 & .0172 & 871 & 39 & 78 & 49 & 664 & 30.606 & 10 \\
\hline 119 & 10EA119 & $\mathrm{OLH}$ & 0716 & 0437 & 0.0 & 0.2888 & & 07 & 97 & 953 & 31.422 & 10 \\
\hline *120 & 10EA120 & OLH & 0.1124 & 0.0277 & 0.0204 & 0.2901 & 0.4374 & 0.1046 & 0.0075 & 1.4219 & 31.626 & 10 \\
\hline 121 & $10 \mathrm{E}$ & $\mathrm{OLH}$ & 520 & 487 & 7 & 465 & 510 & 827 & 0. & 3555 & 32.748 & 10 \\
\hline 122 & 10EA122 & OLH & 398 & 0.1680 & 0.0101 & 0.2033 & 0.3637 & 084 & 567 & 2383 & 32.952 & 10 \\
\hline 123 & 10EA123 & $\mathrm{OLH}$ & 0.2073 & 0.0202 & 0.0 & 0.1988 & 32 & & & 28 & 33.36 & 10 \\
\hline 124 & 10EA124 & OLH & 0.0982 & 0.1168 & 0.0127 & 0.3097 & 0.3397 & 0.1029 & 0.0 & 1.5313 & 33.564 & 10 \\
\hline$* 125$ & $10 \mathrm{EA}$ & $\mathrm{OLH}$ & 829 & 37 & 0.0 & 901 & 54 & 98 & 60 & 727 & 33.666 & 10 \\
\hline 126 & 10EA126 & OLH & 0.1996 & 0.0242 & 0.0128 & 0.2253 & 13 & 0.1147 & 0.0 & 0.5195 & 34.176 & 10 \\
\hline 127 & 10EA127 & OLH & 0.2046 & 0.0536 & 0.0149 & 0.1910 & 0.3573 & 683 & 0.0 & 1.9727 & 34.278 & 10 \\
\hline 128 & 10EA128 & OLH & 158 & 555 & 0.0131 & 86 & 49 & 0.1004 & 0.1 & 0.1523 & 34.788 & 10 \\
\hline 129 & 10EA129 & OLH & 0.1708 & 0.0205 & 0.0083 & 0.1925 & 250 & 0.1767 & 62 & 1.668 & 37.032 & 10 \\
\hline 130 & 10EA130 & $\mathrm{OLH}$ & 0.1130 & 0.0351 & 0.0057 & 0.1819 & 0.5027 & 0.1567 & 0.0050 & .9336 & 38.358 & 10 \\
\hline 131 & 10EA131 & OLH & 606 & 0717 & 0.0140 & 0.1845 & 0.4605 & 0.0986 & 0.0100 & 1.4258 & \begin{tabular}{|l|}
39.48 \\
\end{tabular} & 10 \\
\hline$* 132$ & 10EA132 & $\mathrm{OLH}$ & 0.1098 & 0.0634 & 0.0106 & 0.2276 & 0.4297 & 0.1397 & 0.0191 & 0.168 & 39.99 & 10 \\
\hline 133 & 10EA133 & $\mathrm{OLH}$ & 0.3090 & 0.0103 & 0.0189 & 0.1772 & 0.3275 & 0.1534 & 0.0037 & 1.6641 & 40.194 & 10 \\
\hline 134 & 10EA134 & $\mathrm{OLH}$ & 0.1224 & 0.0753 & 0.0109 & 0.1920 & 0.4947 & 0.1004 & 0.0042 & 0.2344 & 40.602 & 10 \\
\hline 135 & 10EA135 & $\mathrm{OLH}$ & 0.0984 & 0.1516 & 0.0077 & 0.1989 & 0.3849 & 0.1472 & 0.0113 & 1.6914 & 41.112 & 10 \\
\hline$* 136$ & 10EA136 & $\mathrm{OLH}$ & 0.1669 & 0.0697 & 0.0017 & 0.2348 & 0.4110 & 0.1045 & 0.0114 & 1.125 & \begin{tabular}{|l|}
41.622 \\
\end{tabular} & 10 \\
\hline 137 & 10EA137 & $\mathrm{OLH}$ & 0.0589 & 0.0346 & 0.0075 & 0.2795 & 0.5124 & 0.0947 & 0.0124 & 0.2813 & 42.744 & 10 \\
\hline$* 138$ & 10EA138 & $\mathrm{OLH}$ & 0.0626 & 291 & 0.0064 & 0.1950 & 0.4996 & 0.0994 & 0.0079 & 1.7813 & 43.152 & 10 \\
\hline 139 & 10EA139 & OLH & 1311 & 039 & 4 & 30 & & 56 & & 92 & 43.56 & 10 \\
\hline
\end{tabular}


Table 32. Envelope A Design Matrix for OLI Simulations (cont'd)

\begin{tabular}{|c|c|c|c|c|c|c|c|c|c|c|c|c|}
\hline $\begin{array}{l}\text { Run } \\
\text { No. }\end{array}$ & Test ID & Type & 102 & $\mathrm{O} 3$ & $\mathrm{X}_{\mathrm{F}}$ & $\mathrm{NO} 2$ & $\mathrm{NO} 3$ & $\mathrm{OH}$ & $\mathrm{OO4}$ & SB & emp & $\mathrm{a} \mathrm{M}$ \\
\hline 140 & EA140 & $\mathrm{OLH}$ & 595 & 0782 & 0088 & 2218 & 4479 & 0805 & 0034 & 0.957 & 43.662 & 10 \\
\hline 141 & OEA141 & OLH & 533 & 0128 & .0022 & 2050 & 5234 & 0881 & .01 & 0.3164 & 43.866 & 10 \\
\hline 142 & EA142 & $\mathrm{LH}$ & 0698 & 153 & $\overline{0170}$ & 3329 & 4729 & .0880 & 0.006 & 7539 & \begin{tabular}{|l|}
44.172 \\
\end{tabular} & 10 \\
\hline *143 & EA143 & OLH & 615 & 301 & 010 & 2107 & 3925 & 951 & 1 & 4375 & 44.274 & 10 \\
\hline 144 & 0EA144 & OLH & 0.1759 & 0.0258 & 0.0155 & 0.2949 & .3976 & 0.0845 & 0.0058 & 0.7617 & 44.376 & 10 \\
\hline 145 & 0EA145 & OLH & 0835 & 155 & .0123 & 0.2952 & 3669 & 1216 & 49 & 0.6758 & 44.58 & 10 \\
\hline$* 146$ & EA146 & $\mathrm{LH}$ & 345 & 261 & 0159 & 3489 & 3629 & 58 & .0159 & .4922 & 45.396 & 10 \\
\hline 147 & $10 \mathrm{EA} 147$ & OLH & 912 & 248 & 0125 & 0.1967 & 4709 & & & 1.168 & 45.6 & 10 \\
\hline 148 & 10EA148 & OLH & 0.1887 & 0.0493 & 0.0034 & 0.2270 & 0.4343 & 0.0792 & 0.0181 & 0.7852 & \begin{tabular}{|l|}
45.702 \\
\end{tabular} & 10 \\
\hline *149 & 0EA149 & OLH & 0686 & 1216 & 0090 & 3445 & 3364 & 1002 & 0197 & 9219 & 45.804 & 10 \\
\hline 150 & $10 \mathrm{EA}$ & OLH & 098 & 921 & 0073 & 1675 & 4174 & 0975 & 33 & 2031 & 46.008 & 10 \\
\hline 151 & 10EA151 & OLH & 1460 & 0424 & .0042 & 0.2977 & 4206 & 0.0856 & 34 & .6289 & 46.518 & 10 \\
\hline 152 & 10EA152 & OLH & 0.2237 & 0.0336 & 0.0172 & 0.1951 & 0.4230 & 0.0982 & 0.0093 & 0.0078 & 47.028 & 10 \\
\hline 153 & $10 \mathrm{E}$ & OLH & 656 & 179 & 62 & 1905 & 56 & 77 & 65 & .2773 & 47.13 & 10 \\
\hline *154 & 0EA & OLH & 154 & 181 & 0060 & 2646 & 3665 & 98 & 96 & 9531 & 47.742 & 10 \\
\hline 155 & 10EA155 & OLH & 0.0801 & 0.0774 & 0.0229 & 0.2804 & 0.3884 & 0.1344 & 0.0164 & 1.6328 & 47.844 & 10 \\
\hline *156 & 10EA156 & OLH & 0.1280 & 0.0508 & 0.0015 & 0.3018 & 0.4202 & 0.0851 & 0.0127 & 1.1289 & 47.946 & 10 \\
\hline 157 & $10 \mathrm{E}$ & $\mathrm{OLH}$ & 177 & 76 & 4 & 628 & 74 & 14 & & 773 & \begin{tabular}{|l|}
48.456 \\
\end{tabular} & 10 \\
\hline 158 & 10EA158 & $\mathrm{OLH}$ & 680 & 0695 & 7 & 0.3174 & 16 & 56 & & 1602 & 48.762 & 10 \\
\hline 159 & 10EA159 & OLH & 0.1811 & 0.0320 & 0.0164 & 0.2236 & 0.3974 & & & 1.1172 & 48.864 & 10 \\
\hline 160 & $10 \mathrm{E}$ & $\mathrm{LH}$ & 595 & 07 & 0.0 & 0.2310 & 22 & 99 & & 45 & 49.782 & 10 \\
\hline 161 & $10 \mathrm{E}$ & $\mathrm{OLH}$ & 32 & 1 & 8 & 75 & & & & 5391 & 50.088 & 10 \\
\hline 162 & 10EA162 & OLH & 318 & 21 & 5 & 0.1808 & 00 & 35 & & 0547 & 51.108 & 10 \\
\hline 163 & 10EA163 & OLH & 0.1505 & 0.1198 & 0.0105 & 0.2479 & 0.3470 & 66 & 0.0 & 1.9258 & 52.026 & 10 \\
\hline 164 & 10 & LH & 540 & 81 & 4 & 58 & 11 & 3 & & 4766 & \begin{tabular}{|l|}
53.454 \\
\end{tabular} & 10 \\
\hline 165 & $10 \mathrm{EA}$ & OLH & 877 & 516 & 38 & 2156 & 39 & 71 & $\overline{04}$ & 0.457 & 53.556 & 10 \\
\hline 166 & 10EA166 & $\mathrm{OLH}$ & 1839 & 375 & 0.0 & 0.2007 & 0.4 & & & 1.2734 & 54.066 & 10 \\
\hline 167 & 10EA167 & OLH & 0.1168 & 0.0904 & 0.0115 & 0.2788 & 0.3790 & 0 . & 0. & 0.625 & 54.27 & 10 \\
\hline 168 & $10 \mathrm{E}$ & $\mathrm{OLH}$ & 445 & 880 & 9 & 786 & 35 & 78 & 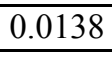 & 1484 & 54.882 & 10 \\
\hline *169 & 10EA169 & OLH & 0.1160 & 887 & 0.0084 & 0.2963 & 542 & 161 & 03 & 1.7695 & 55.902 & 10 \\
\hline 170 & 10EA170 & $\mathrm{OLH}$ & 0.2639 & 0480 & 0 & 0.1958 & & & 0 & 1.918 & 56.514 & 10 \\
\hline 171 & 10EA171 & $\mathrm{OLH}$ & 0.0857 & 0.0219 & 0.0025 & 0.2029 & 0.5554 & 01 & & 0.207 & 57.738 & 10 \\
\hline 172 & $10 \mathrm{E} A$ & $\mathrm{OLH}$ & 944 & 58 & 0 & 378 & 44 & 90 & & .7266 & 58.758 & 10 \\
\hline 173 & 10EA173 & OLH & 0.0711 & 286 & 0.0090 & 0.3841 & 570 & 0.1442 & 0.0 & 1.0898 & 58.86 & 10 \\
\hline 174 & 10EA174 & OLH & 0.0687 & 321 & 0.0055 & 0.2443 & 0.4377 & & & 0.8945 & 60.39 & 10 \\
\hline$* 175$ & 10EA175 & $\mathrm{OLH}$ & 1743 & 0647 & 0.0030 & 0.1795 & 0.4891 & 0.0792 & 0.0104 & 1.6758 & 60.696 & 10 \\
\hline 176 & 10EA176 & OLH & 2841 & 0169 & 0.0098 & 0.1980 & 0.3539 & 0.1186 & 87 & 1.0625 & 60.9 & 10 \\
\hline 177 & 10EA177 & $\mathrm{OLH}$ & 0.1433 & 0.0625 & 0.0156 & 0.2674 & 0.3988 & 0.1082 & 0.0041 & 1.9492 & 61.206 & 10 \\
\hline *178 & 10EA178 & OLH & 261 & 236 & 0.0196 & 0.2022 & 85 & 0.0934 & 0.0167 & 1.7852 & 61.92 & 10 \\
\hline 179 & 10EA179 & $\mathrm{OLH}$ & 1113 & 0.0153 & 0.0137 & 0.2596 & 0.5009 & 0.0841 & 0.0151 & 0.2422 & \begin{tabular}{|l|}
62.022 \\
\end{tabular} & 10 \\
\hline$* 180$ & 10EA180 & $\mathrm{OLH}$ & 0.2326 & 0.0556 & 0.0121 & 0.2253 & 0.3643 & 0.0969 & 0.0132 & 0.4961 & 63.042 & 10 \\
\hline 181 & 10EA181 & $\mathrm{OLH}$ & 0.0911 & 0228 & 0.0120 & 0.2286 & 0.5396 & 0.0948 & 0.0111 & 0.6367 & 63.348 & 10 \\
\hline 182 & 10EA182 & $\mathrm{OLH}$ & 0.1226 & 0.0255 & 0.0083 & 0.2787 & 0.3779 & & 0.0 & 1.4102 & 63.552 & 10 \\
\hline 183 & 10EA183 & $\mathrm{OLH}$ & 0.2295 & 0.0373 & 0.0119 & 0.1679 & 0.4290 & 0.1072 & 0.0171 & 0.4453 & 63.756 & 10 \\
\hline 184 & 10EA184 & $\mathrm{OLH}$ & 0.1460 & 0.0645 & 0.0103 & 0.1800 & 0.4975 & 0.0978 & 0.0040 & 0.1836 & 64.164 & 10 \\
\hline$* 185$ & 10EA185 & OLH & 361 & 601 & 0.0204 & 0.2124 & 0.4612 & 0.1047 & 0.0050 & 1.6563 & 64.47 & 10 \\
\hline 186 & 10EA186 & OLH & 504 & 56 & 4 & 17 & & & & 75 & 64.674 & 10 \\
\hline
\end{tabular}


Table 32. Envelope A Design Matrix for OLI Simulations (cont'd)

\begin{tabular}{|c|c|c|c|c|c|c|c|c|c|c|c|c|}
\hline $\begin{array}{l}\text { Run } \\
\text { No. }\end{array}$ & Test ID & Type & $\mathrm{x}_{\mathrm{AlO} 2}$ & $\mathrm{x}_{\mathrm{CO} 3}$ & $\mathrm{X}_{\mathrm{F}}$ & $\mathrm{x}_{\mathrm{NO} 2}$ & $\mathrm{x}_{\mathrm{NO} 3}$ & $\mathrm{x}_{\mathrm{OH}}$ & $\mathrm{X}_{\mathrm{PO} 4}$ & SBS/Feed & Temp & $\mathrm{Na} \mathrm{M}$ \\
\hline$* 187$ & 10EA187 & $\mathrm{OLH}$ & 0.1310 & 0.0956 & 0.0228 & 0.2471 & 0.4037 & 0.0897 & 0.0101 & 1.7969 & 64.776 & 10 \\
\hline 188 & 10EA188 & OLH & 0.1555 & 0.0348 & 0.0175 & 0.3104 & 0.3612 & 0.1086 & 0.0120 & 1.0508 & 65.388 & 10 \\
\hline 189 & $8 \mathrm{EA001}$ & MPV & 0.0774 & 0.0124 & 0.0011 & 0.2132 & 0.3895 & 0.2810 & 0.0254 & 0 & 15 & 8 \\
\hline 190 & $8 \mathrm{EA002}$ & MPV & 0.0782 & 0.0125 & 0.0011 & 0.2153 & 0.3935 & 0.2956 & 0.0038 & 0 & 15 & 8 \\
\hline 191 & 8EA003 & MPV & 0.0552 & 0.0089 & 0.0008 & 0.3208 & 0.5360 & 0.0756 & 0.0027 & 0 & 15 & 8 \\
\hline 192 & 8EA004 & MPV & 0.0602 & 0.0097 & 0.0214 & 0.1659 & 0.6167 & 0.1232 & 0.0030 & 0 & 15 & 8 \\
\hline 193 & 8EA005 & MPV & 0.0673 & 0.0108 & 0.0240 & 0.3911 & 0.3389 & 0.1501 & 0.0178 & 0 & 15 & 8 \\
\hline 194 & $8 \mathrm{EA006}$ & MPV & 0.0672 & 0.2197 & 0.0239 & 0.1852 & 0.3941 & 0.0920 & 0.0178 & 0 & 15 & 8 \\
\hline 195 & 8EA007 & MPV & 0.2066 & 0.2077 & 0.0009 & 0.1750 & 0.3197 & 0.0870 & 0.0031 & 0 & 15 & 8 \\
\hline 196 & 8EA008 & MPV & 0.1920 & 0.0087 & 0.0008 & 0.1487 & 0.5530 & 0.0791 & 0.0178 & 0 & 15 & 8 \\
\hline 197 & 8EA009 & MPV & 0.2347 & 0.0094 & 0.0009 & 0.3423 & 0.2966 & 0.0967 & 0.0194 & 0 & 15 & 8 \\
\hline 198 & 8EA010 & MPV & 0.3310 & 0.0101 & 0.0225 & 0.1738 & 0.3176 & 0.1420 & 0.0031 & 0 & 15 & 8 \\
\hline 199 & 8EA011 & MPV & 0.0774 & 0.0124 & 0.0011 & 0.2132 & 0.3895 & 0.2810 & 0.0254 & 0 & 15 & 8 \\
\hline 200 & $8 \mathrm{EA} 012$ & MPV & 0.0782 & 0.0125 & 0.0011 & 0.2153 & 0.3935 & 0.2956 & 0.0038 & 0 & 15 & 8 \\
\hline 201 & 8EA013 & MPV & 0.0552 & 0.0089 & 0.0008 & 0.3208 & 0.5360 & 0.0756 & 0.0027 & 0 & 15 & 8 \\
\hline 202 & 8EA014 & MPV & 0.0602 & 0.0097 & 0.0214 & 0.1659 & 0.6167 & 0.1232 & 0.0030 & 0 & 15 & 8 \\
\hline 203 & $8 \mathrm{EA} 015$ & MPV & 0.0673 & 0.0108 & 0.0240 & 0.3911 & 0.3389 & 0.1501 & 0.0178 & 0 & 15 & 8 \\
\hline 204 & \begin{tabular}{|l|} 
8EA016 \\
\end{tabular} & MPV & 0.0672 & 0.2197 & 0.0239 & 0.1852 & 0.3941 & 0.0920 & 0.0178 & 0 & 15 & 8 \\
\hline 205 & $8 \mathrm{EA} 017$ & MPV & 0.2066 & 0.2077 & 0.0009 & 0.1750 & 0.3197 & 0.0870 & 0.0031 & 0 & 15 & 8 \\
\hline 206 & 8EA018 & MPV & 0.1920 & 0.0087 & 0.0008 & 0.1487 & 0.5530 & 0.0791 & 0.0178 & 0 & 15 & 8 \\
\hline 207 & \begin{tabular}{|l|l} 
8EA019 \\
\end{tabular} & MPV & 0.2347 & 0.0094 & 0.0009 & 0.3423 & 0.2966 & 0.0967 & 0.0194 & 0 & 15 & 8 \\
\hline 208 & $8 \mathrm{EA} 020$ & MPV & 0.3310 & 0.0101 & 0.0225 & 0.1738 & 0.3176 & 0.1420 & 0.0031 & 0 & 15 & 8 \\
\hline 209 & 8EA021 & MPV & 0.0774 & 0.0124 & 0.0011 & 0.2132 & 0.3895 & 0.2810 & 0.0254 & 2 & 15 & 8 \\
\hline 210 & 8EA022 & MPV & 0.0782 & 0.0125 & 0.0011 & 0.2153 & 0.3935 & 0.2956 & 0.0038 & 2 & 15 & 8 \\
\hline 211 & $8 \mathrm{EA} 023$ & MPV & 0.0552 & 0.0089 & 0.0008 & 0.3208 & 0.5360 & 0.0756 & 0.0027 & 2 & 15 & 8 \\
\hline 212 & $8 \mathrm{EA024}$ & MPV & 0.0602 & 0.0097 & 0.0214 & 0.1659 & 0.6167 & 0.1232 & 0.0030 & 2 & 15 & 8 \\
\hline 213 & 8EA025 & MPV & 0.0673 & 0.0108 & 0.0240 & 0.3911 & 0.3389 & 0.1501 & 0.0178 & 2 & 15 & 8 \\
\hline 214 & $8 \mathrm{EA} 026$ & MPV & 0.0672 & 0.2197 & 0.0239 & 0.1852 & 0.3941 & 0.0920 & 0.0178 & 2 & 15 & 8 \\
\hline 215 & $8 \mathrm{EA} 027$ & MPV & 0.2066 & 0.2077 & 0.0009 & 0.1750 & 0.3197 & 0.0870 & 0.0031 & 2 & 15 & 8 \\
\hline 216 & 8EA028 & MPV & 0.1920 & 0.0087 & 0.0008 & 0.1487 & 0.5530 & 0.0791 & 0.0178 & 2 & 15 & 8 \\
\hline 217 & 8EA029 & MPV & 0.2347 & 0.0094 & 0.0009 & 0.3423 & 0.2966 & 0.0967 & 0.0194 & 2 & 15 & 8 \\
\hline 218 & $8 \mathrm{EA} 030$ & MPV & 0.3310 & 0.0101 & 0.0225 & 0.1738 & 0.3176 & 0.1420 & 0.0031 & 2 & 15 & 8 \\
\hline 219 & 8EA031 & MPV & 0.0774 & 0.0124 & 0.0011 & 0.2132 & 0.3895 & 0.2810 & 0.0254 & 2 & 15 & 8 \\
\hline 220 & 8EA032 & MPV & 0.0782 & 0.0125 & 0.0011 & 0.2153 & 0.3935 & 0.2956 & 0.0038 & 2 & 15 & 8 \\
\hline 221 & 8EA033 & MPV & 0.0552 & 0.0089 & 0.0008 & 0.3208 & 0.5360 & 0.0756 & 0.0027 & 2 & 15 & 8 \\
\hline 222 & \begin{tabular}{|l|l} 
8EA034 \\
\end{tabular} & MPV & 0.0602 & 0.0097 & 0.0214 & 0.1659 & 0.6167 & 0.1232 & 0.0030 & 2 & 15 & 8 \\
\hline 223 & 8EA035 & MPV & 0.0673 & 0.0108 & 0.0240 & 0.3911 & 0.3389 & 0.1501 & 0.0178 & 2 & 15 & 8 \\
\hline 224 & \begin{tabular}{|l|} 
8EA036 \\
\end{tabular} & MPV & 0.0672 & 0.2197 & 0.0239 & 0.1852 & 0.3941 & 0.0920 & 0.0178 & 2 & 15 & 8 \\
\hline 225 & 8EA037 & MPV & 0.2066 & 0.2077 & 0.0009 & 0.1750 & 0.3197 & 0.0870 & 0.0031 & 2 & 15 & 8 \\
\hline 226 & 8EA038 & MPV & 0.1920 & 0.0087 & 0.0008 & 0.1487 & 0.5530 & 0.0791 & 0.0178 & 2 & 15 & 8 \\
\hline 227 & 8EA039 & MPV & 0.2347 & 0.0094 & 0.0009 & 0.3423 & 0.2966 & 0.0967 & 0.0194 & 2 & 15 & 8 \\
\hline 228 & 8EA040 & MPV & 0.3310 & 0.0101 & 0.0225 & 0.1738 & 0.3176 & 0.1420 & 0.0031 & 2 & 15 & 8 \\
\hline 229 & 8EA041 & MPV & 0.0774 & 0.0124 & 0.0011 & 0.2132 & 0.3895 & 0.2810 & 0.0254 & 0 & 66 & 8 \\
\hline 230 & $8 \mathrm{EA} 042$ & MPV & 0.0782 & 0.0125 & 0.0011 & 0.2153 & 0.3935 & 0.2956 & 0.0038 & 0 & 66 & 8 \\
\hline 231 & 8EA043 & MPV & 0.0552 & 0.0089 & 0.0008 & 0.3208 & 0.5360 & 0.0756 & 0.0027 & 0 & 66 & 8 \\
\hline 232 & 8EA044 & MPV & 0.0602 & 0.0097 & 0.0214 & 0.1659 & 0.6167 & 0.1232 & 0.0030 & 0 & 66 & 8 \\
\hline 233 & $8 \mathrm{EA} 045$ & MPV & 0.0673 & 0.0108 & 0.0240 & 0.3911 & 0.3389 & 0.1501 & 0.0178 & 0 & 66 & 8 \\
\hline
\end{tabular}


Table 32. Envelope A Design Matrix for OLI Simulations (cont'd)

\begin{tabular}{|c|c|c|c|c|c|c|c|c|c|c|c|c|}
\hline $\begin{array}{l}\text { Run } \\
\text { No. }\end{array}$ & Test ID & Type & $\mathrm{x}_{\mathrm{AlO} 2}$ & $\mathrm{x}_{\mathrm{CO} 3}$ & $\mathrm{x}_{\mathrm{F}}$ & $\mathrm{x}_{\mathrm{NO} 2}$ & $\mathrm{x}_{\mathrm{NO} 3}$ & $\mathrm{x}_{\mathrm{OH}}$ & $\mathrm{X}_{\mathrm{PO} 4}$ & SBS/Feed & Temp & $\mathrm{Na} \mathrm{M}$ \\
\hline 234 & 8EA046 & MPV & 0.0672 & 0.2197 & 0.0239 & 1852 & .3941 & 0.0920 & 0.0178 & 0 & 66 & 8 \\
\hline 235 & $8 \mathrm{EA} 047$ & MPV & 0.2066 & 0.2077 & 0.0009 & .1750 & .3197 & .0870 & 0.0031 & 0 & 66 & 8 \\
\hline 236 & 8EA048 & MPV & 0.1920 & 0.0087 & 0.0008 & .1487 & 0.5530 & 0.0791 & 0.0178 & 0 & 66 & 8 \\
\hline 237 & 8EA049 & MPV & 0.2347 & 0.0094 & 0.0009 & 0.3423 & 0.2966 & 0.0967 & 0.0194 & 0 & 66 & 8 \\
\hline 238 & 8EA050 & MPV & 0.3310 & 0.0101 & 0.0225 & 0.1738 & 0.3176 & 0.1420 & \begin{tabular}{|l|}
0.0031 \\
\end{tabular} & 0 & 66 & 8 \\
\hline 239 & 8EA051 & MPV & 0.0774 & 0.0124 & 0.0011 & 0.2132 & 0.3895 & 0.2810 & 0.0254 & 0 & 66 & 8 \\
\hline 240 & 8EA052 & MPV & 0.0782 & 0.0125 & 0.0011 & 0.2153 & 0.3935 & 0.2956 & 0.0038 & 0 & 66 & 8 \\
\hline 241 & 8EA053 & MPV & 0.0552 & 0.0089 & 0.0008 & 0.3208 & 0.5360 & 0.0756 & 0.0027 & 0 & 66 & 8 \\
\hline 242 & 8EA054 & MPV & 0.0602 & 0.0097 & 0.0214 & 0.1659 & 0.6167 & 0.1232 & 0.0030 & 0 & 66 & 8 \\
\hline 243 & 8EA055 & MPV & 0.0673 & 0.0108 & 0.0240 & 0.3911 & 0.3389 & 0.1501 & 0.0178 & 0 & 66 & 8 \\
\hline 244 & 8EA056 & MPV & 0.0672 & 0.2197 & 0.0239 & 0.1852 & 0.3941 & 0.0920 & \begin{tabular}{|l|l|}
0.0178 \\
\end{tabular} & 0 & 66 & 8 \\
\hline 245 & \begin{tabular}{|l|} 
8EA057 \\
\end{tabular} & MPV & 0.2066 & 0.2077 & 0.0009 & 0.1750 & 0.3197 & 0.0870 & 0.0031 & 0 & 66 & 8 \\
\hline 246 & 8EA058 & MPV & 0.1920 & 0.0087 & 0.0008 & 0.1487 & 0.5530 & 0.0791 & 0.0178 & 0 & 66 & 8 \\
\hline 247 & \begin{tabular}{|l|l|} 
8EA059 \\
\end{tabular} & MPV & 0.2347 & 0.0094 & 0.0009 & 0.3423 & 0.2966 & 0.0967 & 0.0194 & 0 & 66 & 8 \\
\hline 248 & 8EA060 & MPV & 0.3310 & 0.0101 & 0.0225 & 0.1738 & 0.3176 & 0.1420 & 0.0031 & 0 & 66 & 8 \\
\hline 249 & \begin{tabular}{|l|} 
8EA061 \\
\end{tabular} & MPV & 0.0774 & 0.0124 & 0.0011 & 0.2132 & 0.3895 & 0.2810 & 0.0254 & 2 & 66 & 8 \\
\hline 250 & 8EA062 & MPV & 0.0782 & 0.0125 & 0.0011 & 0.2153 & 0.3935 & 0.2956 & 0.0038 & 2 & 66 & 8 \\
\hline 251 & 8EA063 & MPV & 0.0552 & 0.0089 & 0.0008 & 0.3208 & 0.5360 & 0.0756 & 0.0027 & 2 & 66 & 8 \\
\hline 252 & 8EA064 & MPV & 0.0602 & 0.0097 & 0.0214 & 0.1659 & 0.6167 & 0.1232 & 0.0030 & 2 & 66 & 8 \\
\hline 253 & 8EA065 & MPV & 0.0673 & 0.0108 & 0.0240 & 0.3911 & \begin{tabular}{|l|l|}
0.3389 \\
\end{tabular} & 0.1501 & 0.0178 & 2 & 66 & 8 \\
\hline 254 & 8EA066 & MPV & 0.0672 & 0.2197 & 0.0239 & 0.1852 & 0.3941 & 0.0920 & 0.0178 & 2 & 66 & 8 \\
\hline 255 & 8EA067 & MPV & 0.2066 & 0.2077 & 0.0009 & 0.1750 & 0.3197 & 0.0870 & \begin{tabular}{|l|}
0.0031 \\
\end{tabular} & 2 & 66 & 8 \\
\hline 256 & \begin{tabular}{|l|} 
8EA068 \\
\end{tabular} & MPV & 0.1920 & 0.0087 & 0.0008 & 0.1487 & 0.5530 & 0.0791 & 0.0178 & 2 & 66 & 8 \\
\hline 257 & 8EA069 & MPV & 0.2347 & 0.0094 & 0.0009 & 0.3423 & 0.2966 & \begin{tabular}{|l|}
0.0967 \\
\end{tabular} & \begin{tabular}{|l|l}
0.0194 \\
\end{tabular} & 2 & 66 & 8 \\
\hline 258 & \begin{tabular}{|l|l} 
8EA070 \\
\end{tabular} & MPV & 0.3310 & 0.0101 & 0.0225 & 0.1738 & 0.3176 & 0.1420 & 0.0031 & 2 & 66 & 8 \\
\hline 259 & \begin{tabular}{|l|} 
8EA071 \\
\end{tabular} & MPV & 0.0774 & 0.0124 & 0.0011 & 0.2132 & 0.3895 & 0.2810 & 0.0254 & 2 & 66 & 8 \\
\hline 260 & \begin{tabular}{|l|} 
8EA072 \\
\end{tabular} & MPV & 0.0782 & 0.0125 & 0.0011 & 0.2153 & 0.3935 & 0.2956 & 0.0038 & 2 & 66 & 8 \\
\hline 261 & 8EA073 & MPV & 0.0552 & 0.0089 & 0.0008 & 0.3208 & 0.5360 & 0.0756 & 0.0027 & 2 & 66 & 8 \\
\hline 262 & 8EA074 & MPV & 0.0602 & 0.0097 & 0.0214 & 0.1659 & 0.6167 & 0.1232 & 0.0030 & 2 & 66 & 8 \\
\hline 263 & 8EA075 & MPV & 0.0673 & 0.0108 & 0.0240 & 0.3911 & 0.3389 & 0.1501 & 0.0178 & 2 & 66 & 8 \\
\hline 264 & 8EA076 & MPV & 0.0672 & 0.2197 & 0.0239 & 0.1852 & 0.3941 & 0.0920 & 0.0178 & 2 & 66 & 8 \\
\hline 265 & $8 \mathrm{EA} 077$ & MPV & 0.2066 & 0.2077 & 0.0009 & 0.1750 & 0.3197 & 0.0870 & 0.0031 & 2 & 66 & 8 \\
\hline 266 & 8EA078 & MPV & 0.1920 & 0.0087 & 0.0008 & 0.1487 & 0.5530 & 0.0791 & 0.0178 & 2 & 66 & 8 \\
\hline 267 & 8EA079 & MPV & 0.2347 & 0.0094 & 0.0009 & 0.3423 & 0.2966 & 0.0967 & 0.0194 & 2 & 66 & 8 \\
\hline 268 & 8EA080 & MPV & 0.3310 & 0.0101 & 0.0225 & 0.1738 & 0.3176 & 0.1420 & 0.0031 & 2 & 66 & 8 \\
\hline 269 & \begin{tabular}{|l|} 
8EA081 \\
\end{tabular} & $\mathrm{OLH}$ & 0.1918 & 0.1138 & 0.0232 & 0.2382 & 0.3215 & 0.0952 & 0.0163 & 0.0391 & 15 & 8 \\
\hline 270 & \begin{tabular}{|l|l} 
8EA082 \\
\end{tabular} & $\mathrm{OLH}$ & 0.1843 & 0.0414 & 0.0223 & 0.2884 & 0.3481 & 0.1047 & 0.0108 & 1.957 & 15.102 & 8 \\
\hline$* 271$ & 8EA083 & $\mathrm{OLH}$ & 0.1542 & 0.0297 & 0.0155 & 0.1729 & 0.5433 & 0.0790 & 0.0053 & 0.7578 & 15.714 & 8 \\
\hline 272 & 8EA084 & $\mathrm{OLH}$ & 0.1966 & 0.0295 & 0.0198 & 0.1654 & 0.4987 & 0.0840 & 0.0060 & 0.3008 & 15.918 & 8 \\
\hline 273 & \begin{tabular}{|l|l|} 
8EA085 \\
\end{tabular} & $\mathrm{OLH}$ & 0.0956 & 0.1002 & 0.0214 & 0.3327 & 0.3348 & 0.0982 & 0.0171 & 0.0039 & 16.122 & 8 \\
\hline 274 & 8EA086 & $\mathrm{OLH}$ & 0.0998 & 0.0861 & 0.0117 & 0.3543 & 0.3352 & 0.1095 & 0.0033 & 1.2305 & 17.04 & 8 \\
\hline$* 275$ & \begin{tabular}{|l|} 
8EA087 \\
\end{tabular} & $\mathrm{OLH}$ & 0.1262 & 0.1404 & 0.0048 & 0.2244 & 0.3738 & 0.1118 & 0.0186 & 1.5898 & 17.55 & 8 \\
\hline 276 & 8EA088 & $\mathrm{OLH}$ & 0.1821 & 0.1572 & 0.0199 & 0.1779 & 0.3597 & 0.0908 & 0.0123 & 0.7734 & 18.06 & 8 \\
\hline 277 & 8EA089 & OLH & 0.2399 & 0.0212 & 0.0184 & 0.2003 & \begin{tabular}{|l|l|}
0.3729 \\
\end{tabular} & \begin{tabular}{|l|l}
0.1371 \\
\end{tabular} & 0.0102 & 1.1641 & 18.162 & 8 \\
\hline 278 & 8EA090 & $\mathrm{OLH}$ & 0.0750 & 0.0439 & 0.0136 & 0.2872 & 0.4361 & 0.1371 & 0.0070 & 0.6094 & 18.264 & 8 \\
\hline$* 279$ & 8EA091 & $\mathrm{OLH}$ & 0.1154 & 0.0306 & 0.0105 & 0.3533 & 0.3620 & 0.1211 & 0.0071 & 0.8594 & 18.57 & 8 \\
\hline 280 & 8EA092 & $\mathrm{OLH}$ & 0.1491 & 0.0195 & 0.0069 & 0.3508 & 0.3724 & 0.0931 & 0.0083 & 0.1914 & 18.672 & 8 \\
\hline
\end{tabular}


Table 32. Envelope A Design Matrix for OLI Simulations (cont'd)

\begin{tabular}{|c|c|c|c|c|c|c|c|c|c|c|c|c|}
\hline $\begin{array}{l}\text { Run } \\
\text { No. }\end{array}$ & Test ID & Type & $\mathrm{x}_{\mathrm{AlO} 2}$ & $\mathrm{CO} 3$ & $\mathrm{x}_{\mathrm{F}}$ & NO2 & $\mathrm{NO} 3$ & $\mathrm{x}_{\mathrm{OH}}$ & $\mathrm{x}_{\mathrm{PO4}}$ & SBS/Feed & Temp & $\mathrm{Na} \mathrm{M}$ \\
\hline 281 & A093 & $\mathrm{LH}$ & 0.1173 & 0.0919 & .0084 & 2373 & 4153 & 1102 & 0.0195 & 1.0195 & 19.284 & 8 \\
\hline 282 & $\overline{\text { EA094 }}$ & $\overline{\mathrm{LH}}$ & 1130 & 0907 & 0.0043 & 1710 & 5200 & 0981 & $\overline{0030}$ & 5352 & 19.4 & 8 \\
\hline$* 283$ & A095 & $\mathrm{OLH}$ & 1898 & 0.0323 & 0.0215 & 2772 & 3724 & 0925 & .0144 & 1.3203 & 19.998 & $\varepsilon$ \\
\hline 284 & 8EA096 & OLH & 0.1669 & 0.0748 & 0.0031 & 0.3337 & .3280 & .0894 & \begin{tabular}{|l|l}
0.0040 \\
\end{tabular} & 1.8398 & 20.508 & 8 \\
\hline 285 & 8EA097 & OLH & 0.2043 & 0.1181 & 0.0061 & 0.2501 & 0.3185 & 0.0955 & \begin{tabular}{|l|l}
0.0074 \\
\end{tabular} & 0.1563 & 21.222 & 8 \\
\hline$* 286$ & 8EA098 & \begin{tabular}{|l|l|} 
OLH \\
\end{tabular} & 0.1058 & 0.0125 & 0.0041 & 1956 & 5094 & 1547 & 0.0179 & 1.5977 & 21.426 & $\varepsilon$ \\
\hline 287 & 8EA099 & OLH & 0.2003 & 0.1021 & 0.0199 & 0.2472 & .3224 & .0956 & \begin{tabular}{|l|l}
0.0126 \\
\end{tabular} & 0.1719 & 21.732 & 8 \\
\hline 288 & 8EA100 & OLH & 0.1876 & 0.0509 & 0.0021 & 0.1938 & 0.4624 & 0.0914 & 0.0119 & 1.6094 & 22.956 & 0 \\
\hline$* 289$ & 8EA101 & OLH & 0.1378 & 0.0348 & 0.0086 & 0.2134 & 0.5015 & 0.0869 & \begin{tabular}{|l}
0.0169 \\
\end{tabular} & 0.8633 & 23.466 & 8 \\
\hline 290 & 8EA102 & OLH & 0.1185 & 0.1199 & 0.0182 & 1962 & 0.4383 & 0.1047 & 0.0042 & 1.0703 & 24.282 & $\varepsilon$ \\
\hline 291 & 8EA103 & OLH & 0.1916 & 0.0103 & 0.0056 & 2613 & 4231 & 0934 & 0.0148 & 1.0273 & 26.016 & 8 \\
\hline$* 292$ & 8EA104 & OLH & 0.1544 & 0.0814 & 0.0136 & 0.1791 & 0.4433 & 0.1194 & 0.0087 & 1.1758 & 26.22 & 8 \\
\hline 293 & 8EA105 & OLH & 0.2332 & 0.0319 & 0.0046 & 0.2387 & 0.3824 & 0.1014 & \begin{tabular}{|l|l|}
0.0079 \\
\end{tabular} & 0.5 & 26.526 & 8 \\
\hline 294 & 8EA106 & $\mathrm{LH}$ & 555 & 0.0487 & 0.0189 & 2203 & 83 & 802 & .0082 & 2695 & 27.036 & $\varepsilon$ \\
\hline 295 & 8EA107 & OLH & 0.2505 & 0.0207 & 0.0017 & 2818 & 3293 & .1081 & \begin{tabular}{|l|l}
0.0077 \\
\end{tabular} & .0195 & 27.138 & 8 \\
\hline 296 & 8EA108 & $\mathrm{OLH}$ & 0.1492 & 0.0373 & 0.0219 & 0.2318 & 0.4468 & 0.0979 & 0.0151 & 1.4414 & 27.24 & 8 \\
\hline 297 & 8EA109 & $\mathrm{LH}$ & 0.1778 & 0.0144 & 0.0032 & 2740 & 4405 & 0866 & 0.0036 & 1.0547 & 28.056 & $\varepsilon$ \\
\hline$* 298$ & $8 \mathrm{EA} 110$ & $\mathrm{OLH}$ & 0.2498 & 0.0426 & 0.0124 & 2232 & 3617 & .1069 & 0.0034 & 1.2461 & 28.26 & 8 \\
\hline 299 & 8EA111 & $\mathrm{OLH}$ & 0.0733 & 0.0262 & 0.0027 & 0.2809 & .5244 & 0.0829 & 0.0097 & 1.0977 & 28.464 & 8 \\
\hline 300 & 8EA112 & OLH & 0.1772 & 0.0437 & 0.0069 & 0.2768 & \begin{tabular}{|l|l}
0.3998 \\
\end{tabular} & 0.0841 & 0.0115 & 0.6211 & 28.566 & 8 \\
\hline 301 & 8EA113 & LH & 0.1312 & 0.0821 & 0.0080 & 3134 & 3320 & 126 & 208 & 1.0664 & 28.872 & 8 \\
\hline 302 & 8EA114 & OLH & 0.1219 & 0.0573 & 0.0032 & 2411 & 4850 & .0883 & 0.0032 & 0.6563 & 29.382 & 8 \\
\hline$* 303$ & 8EA115 & OLH & 0.1898 & 0.0209 & 0.0170 & 0.2095 & 0.4567 & 0.1016 & 0.0045 & 1.3867 & 29.484 & 8 \\
\hline 304 & 8EA116 & OLH & 0.1441 & 0.0763 & 0.0138 & .231 & .4232 & \begin{tabular}{|l|l}
0.1011 \\
\end{tabular} & 0.0035 & 1.0078 & 30.198 & $\gamma$ \\
\hline 305 & $8 \mathrm{EA}$ & $\mathrm{LH}$ & 307 & 92 & 0.0147 & 27 & 87 & 836 & 105 & 1.8711 & 30.504 & 8 \\
\hline 306 & 8EA118 & $\mathrm{OLH}$ & 0.2601 & 0.0891 & 0.0172 & 1871 & 239 & 0.1178 & \begin{tabular}{|l|l|} 
\\
\end{tabular} & 0.5664 & 30.606 & 8 \\
\hline 307 & 8EA119 & $\mathrm{OLH}$ & 0.0716 & 0.0437 & 0.0121 & 0.2888 & 3934 & .1807 & \begin{tabular}{|l|l|}
0.0097 \\
\end{tabular} & 0.6953 & 31.422 & 8 \\
\hline 308 & 8EA120 & $\mathrm{OLH}$ & 0.1124 & 0.0277 & 0.0204 & 0.2901 & 0.4374 & 0.1046 & 0.0075 & 1.4219 & 31.626 & $\pi$ \\
\hline 309 & 8EA121 & $\mathrm{OLH}$ & 0.1520 & 0.0487 & 0.0037 & 2465 & 4610 & 0.0827 & 0.0054 & 1.3555 & 32.748 & 8 \\
\hline 310 & 8EA122 & OLH & 0.1398 & 0.1680 & 0.0101 & 2033 & 3637 & 0.1084 & \begin{tabular}{|l}
0.0067 \\
\end{tabular} & 0.2383 & 32.952 & 8 \\
\hline 311 & 8EA123 & OLH & 0.2073 & 0.0202 & 0.0032 & 0.1988 & 0.4432 & 0.1137 & 0.0136 & 0.6328 & 33.36 & 8 \\
\hline 312 & 8EA124 & OLH & 0.0982 & 0.1168 & 0.0127 & 0.3097 & 3397 & 029 & 0.0201 & 1.5313 & 33.564 & 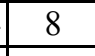 \\
\hline 313 & 8EA125 & OLH & 0.0829 & 0.0137 & 0.0221 & 1901 & 5254 & 0.1598 & 0.0060 & 0.4727 & 33.666 & 8 \\
\hline 314 & 8EA126 & OLH & 0.1996 & 0.0242 & 0.0128 & .2253 & 0.4113 & 0.1147 & 0.0122 & 0.5195 & 34.176 & 8 \\
\hline 315 & 8EA127 & $\mathrm{OLH}$ & 0.2046 & 0.0536 & 0.0149 & .1910 & 0.3573 & 0.1683 & \begin{tabular}{|l|l|} 
\\
\end{tabular} & 1.9727 & 34.278 & - \\
\hline$* 316$ & 8EA128 & $\mathrm{LH}$ & 158 & 0.0555 & 0.0131 & 186 & 3849 & \begin{tabular}{|l|l|l|}
0 \\
\end{tabular} & 0.0118 & 0.1523 & 34.788 & 8 \\
\hline 317 & 8EA129 & $\mathrm{OLH}$ & 0.1708 & 0.0205 & 0.0083 & 0.1925 & 0.4250 & 0.1767 & 0.0062 & 1.668 & 37.032 & 8 \\
\hline 318 & 8EA130 & $\mathrm{OLH}$ & 0.1130 & 0.0351 & 0.0057 & 0.1819 & 0.5027 & 0.1567 & 0.0050 & 1.9336 & 38.358 & 0 \\
\hline 319 & 8EA131 & $\mathrm{LH}$ & 606 & 0.0717 & 0.0140 & 845 & .4605 & 0.0986 & 0.0100 & 1.4258 & \begin{tabular}{|l|}
39.48 \\
\end{tabular} & 8 \\
\hline 320 & 8EA132 & $\mathrm{OLH}$ & 0.1098 & 0.0634 & 0.0106 & 0.2276 & 0.4297 & 0.1397 & 0.0191 & 0.168 & \begin{tabular}{|l|}
39.99 \\
\end{tabular} & 8 \\
\hline$* 321$ & 8EA133 & $\mathrm{OLH}$ & 0.3090 & 0.0103 & 0.0189 & 0.1772 & 0.3275 & 0.1534 & 0.0037 & 1.6641 & 40.194 & 8 \\
\hline 322 & 8EA134 & $\mathrm{OLH}$ & 0.1224 & 0.0753 & 0.0109 & 0.1920 & 0.4947 & 0.1004 & 0.0042 & 0.2344 & 40.602 & 8 \\
\hline 323 & 8EA135 & $\mathrm{OLH}$ & 0.0984 & 0.1516 & 0.0077 & 0.1989 & 0.3849 & 0.1472 & 0.0113 & 1.6914 & 41.112 & 0 \\
\hline 324 & 8EA136 & OLH & 0.1669 & 0.0697 & 0.0017 & 0.2348 & 0.4110 & 0.1045 & 0.0114 & 1.125 & 41.622 & 8 \\
\hline 325 & 8EA137 & OLH & 0.0589 & 0.0346 & 0.0075 & 0.2795 & 0.5124 & 0.0947 & 0.0124 & 0.2813 & 42.744 & 8 \\
\hline 326 & 8EA138 & OLH & 0.0626 & 0.1291 & 0.0064 & 0.1950 & 0.4996 & 0.0994 & 0.0079 & 1.7813 & 43.152 & 8 \\
\hline 327 & 8EA139 & OLH & 0.1311 & 0.1039 & 0.0144 & 0.2730 & 0.3735 & 0.0956 & 0.0085 & 0.6992 & 43.56 & 8 \\
\hline
\end{tabular}


Table 32. Envelope A Design Matrix for OLI Simulations (cont'd)

\begin{tabular}{|c|c|c|c|c|c|c|c|c|c|c|c|c|}
\hline $\begin{array}{l}\text { Run } \\
\text { No. }\end{array}$ & Test ID & Type & $\mathrm{x}_{\mathrm{AlO} 2}$ & $\mathrm{CO} 3$ & $\mathrm{x}_{\mathrm{F}}$ & $\mathrm{N}_{\mathrm{NO} 2}$ & $\mathrm{x}_{\mathrm{NO} 3}$ & $\mathrm{x}_{\mathrm{OH}}$ & $\mathrm{x}_{\mathrm{PO4}}$ & SBS/Feed & Temp & $\mathrm{Na} \mathrm{M}$ \\
\hline 328 & A140 & $\mathrm{OLH}$ & 0.1595 & 0.0782 & .0088 & 2218 & 4479 & .0805 & 0.0034 & 0.957 & 43.662 & 8 \\
\hline$* 329$ & EA141 & $\overline{\mathrm{LH}}$ & 533 & 0128 & .0022 & 2050 & 5234 & 0881 & 0152 & .3164 & 43.866 & 8 \\
\hline 330 & A142 & $\mathrm{OLH}$ & 0.0698 & 0.0153 & 0.0170 & 3329 & 4729 & 0880 & .0043 & 1.7539 & 44.172 & $\varepsilon$ \\
\hline 331 & 8EA143 & OLH & 0.1615 & 0.1301 & 0.0010 & 0.2107 & .3925 & 0.0951 & 0.0091 & 0.4375 & 44.274 & 8 \\
\hline 332 & 8EA144 & OLH & 0.1759 & 0.0258 & 0.0155 & 0.2949 & 0.3976 & 0.0845 & 0.0058 & 0.7617 & 44.376 & 8 \\
\hline 333 & 8EA145 & LH & 0.0835 & 0.1155 & 0.0123 & 2952 & 3669 & 1216 & 0.0049 & 0.6758 & 44.58 & E \\
\hline 334 & 8EA146 & OLH & 0.1345 & 0.0261 & 0.0159 & 0.3489 & 0.3629 & 0.0958 & 0.0159 & 1.4922 & 45.396 & 8 \\
\hline$* 335$ & 8EA147 & OLH & 0.0912 & 0.1248 & 0.0125 & 0.1967 & 0.4709 & 0.0918 & 0.0120 & 1.168 & 45.6 & 0 \\
\hline 336 & 8EA148 & OLH & 0.1887 & 0.0493 & 0.0034 & 0.2270 & 0.4343 & 0.0792 & 0.0181 & 0.7852 & 45.702 & 8 \\
\hline 337 & 8EA149 & $\mathrm{OLH}$ & 0.0686 & 0.1216 & 0.0090 & .3445 & .3364 & 0.1002 & 0.0197 & 0.9219 & 45.804 & $\varepsilon$ \\
\hline 338 & 8EA150 & OLH & 0.2098 & 0.0921 & 0.0073 & 1675 & 4174 & 0.0975 & 0.0083 & 2031 & 46.008 & 8 \\
\hline 339 & 8EA151 & $\mathrm{OLH}$ & 0.1460 & 0.0424 & 0.0042 & 0.2977 & 0.4206 & 0.0856 & 0.0034 & 0.6289 & 46.518 & 8 \\
\hline 340 & 8EA152 & $\mathrm{OLH}$ & 0.2237 & 0.0336 & 0.0172 & 0.1951 & 0.4230 & 0.0982 & 0.0093 & 0.0078 & 47.028 & 8 \\
\hline 341 & 8EA153 & $\mathrm{LH}$ & 556 & 0.0179 & 0.0162 & 1905 & 56 & 377 & 165 & .2773 & 47.13 & $\varepsilon$ \\
\hline 342 & 8EA154 & OLH & 0.1154 & 0.1181 & 0.0060 & 0.2646 & .3665 & 1198 & 0.0096 & 0.9531 & 47.742 & $\stackrel{0}{0}$ \\
\hline 343 & 8EA155 & OLH & 0.0801 & 0.0774 & 0.0229 & 0.2804 & 0.3884 & 0.1344 & 0.0164 & 1.6328 & 47.844 & 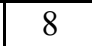 \\
\hline$* 344$ & EA156 & $\mathrm{LH}$ & 0.1280 & 0.0508 & 0.0015 & 3018 & 4202 & 851 & 0127 & 1.1289 & 47.946 & $\varepsilon$ \\
\hline 345 & 8EA157 & OLH & 177 & 0.0376 & 0.0074 & 2628 & .3774 & .0914 & 0.0058 & 1.7773 & 48.456 & 8 \\
\hline$* 346$ & 8EA158 & $\mathrm{OLH}$ & 0.1680 & 0.0695 & 0.0027 & 0.3174 & .3216 & 0.1056 & 0.0151 & 1.1602 & 48.762 & 8 \\
\hline 347 & 8EA159 & OLH & 0.1811 & 0.0320 & 0.0164 & 0.2236 & \begin{tabular}{|l|l|}
0.3974 \\
\end{tabular} & 0.1312 & 0.0182 & 1.1172 & 48.864 & 8 \\
\hline 348 & 8EA160 & $\mathrm{OLH}$ & 595 & 307 & 0.0151 & 2310 & 3962 & 559 & 16 & 1.8945 & 49.782 & 8 \\
\hline 349 & 8EA161 & OLH & 0.0732 & 0.0251 & 0.0068 & 1875 & .5517 & 1364 & 0.0192 & 1.5391 & 50.088 & 8 \\
\hline 350 & 8EA162 & OLH & 0.1318 & 0.1021 & 0.0025 & 0.1808 & 0.4500 & 0.1235 & 0.0094 & 0.0547 & 51.108 & 8 \\
\hline 351 & 8EA163 & OLH & 0.1505 & 0.1198 & 0.0105 & 0.2479 & .3470 & \begin{tabular}{|l}
0.1066 \\
\end{tabular} & 0.0178 & 1.9258 & 52.026 & $\gamma$ \\
\hline 352 & $8 \mathrm{EA}$ & $\mathrm{LH}$ & 540 & 81 & 0.0084 & 58 & 11 & 53 & 73 & 1.4766 & 53.454 & 8 \\
\hline 353 & 8EA165 & $\mathrm{OLH}$ & 0.1877 & 0.1616 & 0.0038 & 0.2156 & 3339 & 0.0871 & 0.0104 & 0.457 & 53.556 & 8 \\
\hline 354 & 8EA166 & $\mathrm{OLH}$ & 0.1839 & 0.0375 & 0.0052 & 0.2007 & .4698 & .0871 & 0.0157 & 1.2734 & 54.066 & 8 \\
\hline$* 355$ & 8EA167 & $\mathrm{OLH}$ & 0.1168 & 0.0904 & 0.0115 & 0.2788 & 0.3790 & 0.1191 & 0.0045 & 0.625 & \begin{tabular}{|l|}
54.27 \\
\end{tabular} & $\pi$ \\
\hline 356 & 8EA168 & $\mathrm{OLH}$ & 0.1445 & 280 & 0.0089 & 1786 & 4385 & 0.0878 & 0.0138 & 1.1484 & 54.882 & 8 \\
\hline 357 & 8EA169 & OLH & 0.1160 & 0.0887 & 0.0084 & .2963 & 5542 & .1161 & 0.0203 & 1.7695 & 55.902 & 8 \\
\hline 358 & 8EA170 & OLH & 0.2639 & 0.0480 & 0.0011 & 0.1958 & 0.3705 & 0.1146 & 0.0061 & 1.918 & 56.514 & 8 \\
\hline$* 359$ & 8EA171 & OLH & 0.0857 & 0.0219 & 0.0025 & 2029 & 5554 & \begin{tabular}{|l|l|}
0.1201 \\
\end{tabular} & 115 & 0.207 & 57.738 & 8 \\
\hline 360 & 8EA172 & OLH & 0.0944 & 0.0558 & 0.0035 & 0.3378 & 0.4044 & 0.0890 & 0.0151 & 1.7266 & 58.758 & 8 \\
\hline 361 & 8EA173 & OLH & 0.0711 & 0.0286 & 0.0090 & 0.3841 & .3570 & 0.1442 & 0.0061 & 1.0898 & \begin{tabular}{|l|}
58.86 \\
\end{tabular} & 8 \\
\hline 362 & 8EA174 & $\mathrm{OLH}$ & 0.0687 & 0.1321 & 0.0055 & 0.2443 & 0.4377 & 0.0954 & 0.0163 & 0.8945 & \begin{tabular}{|l}
60.39 \\
\end{tabular} & -0 \\
\hline$* 363$ & 8EA175 & $\mathrm{LH}$ & 0.1743 & 0.0647 & 0.0030 & 1795 & \begin{tabular}{|l|l|}
0.4891 \\
\end{tabular} & 0.0792 & 0.0104 & 1.6758 & 60.696 & 8 \\
\hline 364 & 8EA176 & $\mathrm{OLH}$ & 0.2841 & 0.0169 & 0.0098 & 0.1980 & 0.3539 & 0.1186 & 0.0187 & 1.0625 & 60.9 & 8 \\
\hline 365 & 8EA177 & $\mathrm{OLH}$ & 0.1433 & 0.0625 & 0.0156 & 0.2674 & 0.3988 & 0.1082 & 0.0041 & 1.9492 & 61.206 & 0 \\
\hline 366 & 8EA178 & $\mathrm{LH}$ & 0.2261 & 236 & 0.0196 & 2022 & 185 & 0.0934 & 0.0167 & 1.7852 & \begin{tabular}{|l|}
61.92 \\
\end{tabular} & 8 \\
\hline 367 & 8EA179 & $\mathrm{OLH}$ & 0.1113 & 0.0153 & 0.0137 & 0.2596 & 0.5009 & 0.0841 & 0.0151 & 0.2422 & 62.022 & 8 \\
\hline 368 & 8EA180 & OLH & 0.2326 & 0.0556 & 0.0121 & 0.2253 & 0.3643 & 0.0969 & 0.0132 & 0.4961 & 63.042 & 8 \\
\hline$* 369$ & 8EA181 & OLH & 0.0911 & 0.0228 & 0.0120 & 0.2286 & 0.5396 & 0.0948 & 0.0111 & 0.6367 & 63.348 & 8 \\
\hline 370 & 8EA182 & $\mathrm{OLH}$ & 0.1226 & 0.0255 & 0.0083 & 0.2787 & 0.3779 & 0.1696 & 0.0173 & 1.4102 & 63.552 & 0 \\
\hline *371 & 8EA183 & OLH & 0.2295 & 0.0373 & 0.0119 & 0.1679 & 0.4290 & 0.1072 & 0.0171 & 0.4453 & 63.756 & 8 \\
\hline 372 & 8EA184 & OLH & 0.1460 & 0.0645 & 0.0103 & 0.1800 & 0.4975 & 0.0978 & 0.0040 & 0.1836 & 64.164 & 8 \\
\hline 373 & 8EA185 & OLH & 0.1361 & 0.0601 & 0.0204 & 0.2124 & 0.4612 & 0.1047 & 0.0050 & 1.6563 & 64.47 & 8 \\
\hline 374 & 8EA186 & $\mathrm{LH}$ & 0.1504 & 0.1056 & 0.0174 & 0.2417 & 0.3505 & \begin{tabular}{|l}
0.1218 \\
\end{tabular} & 0.0126 & 0.1875 & 64.674 & 8 \\
\hline
\end{tabular}


Table 32. Envelope A Design Matrix for OLI Simulations (cont'd)

\begin{tabular}{|c|c|c|c|c|c|c|c|c|c|c|c|c|}
\hline $\begin{array}{l}\text { Run } \\
\text { No. }\end{array}$ & Test ID & Type & $\mathrm{x}_{\mathrm{AlO} 2}$ & $\mathrm{x}_{\mathrm{CO} 3}$ & $\mathrm{X}_{\mathrm{F}}$ & $\mathrm{x}_{\mathrm{NO} 2}$ & $\mathrm{x}_{\mathrm{NO} 3}$ & $\mathrm{x}_{\mathrm{OH}}$ & $\mathrm{X}_{\mathrm{PO} 4}$ & SBS/Feed & Temp & $\mathrm{Na} \mathrm{M}$ \\
\hline$* 375$ & 8EA187 & $\mathrm{OLH}$ & 0.1310 & 0.0956 & 0.0228 & 0.2471 & 0.4037 & 0.0897 & 0.0101 & 1.7969 & 64.776 & 8 \\
\hline 376 & 8EA188 & OLH & 0.1555 & 0.0348 & 0.0175 & 0.3104 & 0.3612 & 0.1086 & 0.0120 & 1.0508 & 65.388 & 8 \\
\hline 377 & 6EA001 & MPV & 0.0774 & 0.0124 & 0.0011 & 0.2132 & 0.3895 & 0.2810 & 0.0254 & 0 & 15 & 6 \\
\hline 378 & 6EA002 & MPV & 0.0782 & 0.0125 & 0.0011 & 0.2153 & 0.3935 & 0.2956 & 0.0038 & 0 & 15 & 6 \\
\hline 379 & 6EA003 & MPV & 0.0552 & 0.0089 & 0.0008 & 0.3208 & 0.5360 & 0.0756 & 0.0027 & 0 & 15 & 6 \\
\hline 380 & 6EA004 & MPV & 0.0602 & 0.0097 & 0.0214 & 0.1659 & 0.6167 & 0.1232 & 0.0030 & 0 & 15 & 6 \\
\hline 381 & 6EA005 & MPV & 0.0673 & 0.0108 & 0.0240 & 0.3911 & 0.3389 & 0.1501 & 0.0178 & 0 & 15 & 6 \\
\hline 382 & 6EA006 & MPV & 0.0672 & 0.2197 & 0.0239 & 0.1852 & 0.3941 & 0.0920 & 0.0178 & 0 & 15 & 6 \\
\hline 383 & 6ЕA007 & MPV & 0.2066 & 0.2077 & 0.0009 & 0.1750 & 0.3197 & 0.0870 & 0.0031 & 0 & 15 & 6 \\
\hline 384 & 6EA008 & MPV & 0.1920 & 0.0087 & 0.0008 & 0.1487 & 0.5530 & 0.0791 & 0.0178 & 0 & 15 & 6 \\
\hline 385 & 6EA009 & MPV & 0.2347 & 0.0094 & 0.0009 & 0.3423 & 0.2966 & 0.0967 & 0.0194 & 0 & 15 & 6 \\
\hline 386 & $6 \mathrm{EA} 010$ & MPV & 0.3310 & 0.0101 & 0.0225 & 0.1738 & 0.3176 & 0.1420 & 0.0031 & 0 & 15 & 6 \\
\hline 387 & 6EA011 & MPV & 0.0774 & 0.0124 & 0.0011 & 0.2132 & 0.3895 & 0.2810 & 0.0254 & 0 & 15 & 6 \\
\hline 388 & 6EA012 & MPV & 0.0782 & 0.0125 & 0.0011 & 0.2153 & 0.3935 & 0.2956 & 0.0038 & 0 & 15 & 6 \\
\hline 389 & 6EA013 & MPV & 0.0552 & 0.0089 & 0.0008 & 0.3208 & 0.5360 & 0.0756 & 0.0027 & 0 & 15 & 6 \\
\hline 390 & 6ЕA014 & MPV & 0.0602 & 0.0097 & 0.0214 & 0.1659 & 0.6167 & 0.1232 & 0.0030 & 0 & 15 & 6 \\
\hline 391 & 6EA015 & MPV & 0.0673 & 0.0108 & 0.0240 & 0.3911 & 0.3389 & 0.1501 & 0.0178 & 0 & 15 & 6 \\
\hline 392 & 6EA016 & MPV & 0.0672 & 0.2197 & 0.0239 & 0.1852 & 0.3941 & 0.0920 & 0.0178 & 0 & 15 & 6 \\
\hline 393 & 6EA017 & MPV & 0.2066 & 0.2077 & 0.0009 & 0.1750 & 0.3197 & 0.0870 & 0.0031 & 0 & 15 & 6 \\
\hline 394 & 6EA018 & MPV & 0.1920 & 0.0087 & 0.0008 & 0.1487 & 0.5530 & 0.0791 & 0.0178 & 0 & 15 & 6 \\
\hline 395 & 6EA019 & MPV & 0.2347 & 0.0094 & 0.0009 & 0.3423 & 0.2966 & 0.0967 & 0.0194 & 0 & 15 & 0 \\
\hline 396 & 6EA020 & MPV & 0.3310 & 0.0101 & 0.0225 & 0.1738 & 0.3176 & 0.1420 & 0.0031 & 0 & 15 & 6 \\
\hline 397 & 6EA021 & MPV & 0.0774 & 0.0124 & 0.0011 & 0.2132 & 0.3895 & 0.2810 & 0.0254 & 2 & 15 & 6 \\
\hline 398 & 6EA022 & MPV & 0.0782 & 0.0125 & 0.0011 & 0.2153 & 0.3935 & 0.2956 & 0.0038 & 2 & 15 & 6 \\
\hline 399 & 6EA023 & MPV & 0.0552 & 0.0089 & 0.0008 & 0.3208 & 0.5360 & 0.0756 & 0.0027 & 2 & 15 & 6 \\
\hline 400 & 6EA024 & MPV & 0.0602 & 0.0097 & 0.0214 & 0.1659 & 0.6167 & 0.1232 & 0.0030 & 2 & 15 & 6 \\
\hline 401 & 6EA025 & MPV & 0.0673 & 0.0108 & 0.0240 & 0.3911 & 0.3389 & 0.1501 & 0.0178 & 2 & 15 & 6 \\
\hline 402 & 6ЕA026 & MPV & 0.0672 & 0.2197 & 0.0239 & 0.1852 & 0.3941 & 0.0920 & 0.0178 & 2 & 15 & 6 \\
\hline 403 & 6EA027 & MPV & 0.2066 & 0.2077 & 0.0009 & 0.1750 & 0.3197 & 0.0870 & 0.0031 & 2 & 15 & 6 \\
\hline 404 & 6ЕA028 & MPV & 0.1920 & 0.0087 & 0.0008 & 0.1487 & 0.5530 & 0.0791 & 0.0178 & 2 & 15 & 6 \\
\hline 405 & 6ЕA029 & MPV & 0.2347 & 0.0094 & 0.0009 & 0.3423 & 0.2966 & 0.0967 & 0.0194 & 2 & 15 & 6 \\
\hline 406 & 6ЕA030 & MPV & 0.3310 & 0.0101 & 0.0225 & 0.1738 & 0.3176 & 0.1420 & 0.0031 & 2 & 15 & 6 \\
\hline 407 & 6EA031 & MPV & 0.0774 & 0.0124 & 0.0011 & 0.2132 & 0.3895 & 0.2810 & 0.0254 & 2 & 15 & 6 \\
\hline 408 & 6EA032 & MPV & 0.0782 & 0.0125 & 0.0011 & 0.2153 & 0.3935 & 0.2956 & 0.0038 & 2 & 15 & 6 \\
\hline 409 & 6EA033 & MPV & 0.0552 & 0.0089 & 0.0008 & 0.3208 & 0.5360 & 0.0756 & 0.0027 & 2 & 15 & 6 \\
\hline 410 & 6EA034 & MPV & 0.0602 & 0.0097 & 0.0214 & 0.1659 & 0.6167 & 0.1232 & 0.0030 & 2 & 15 & 6 \\
\hline 411 & 6EA035 & MPV & 0.0673 & 0.0108 & 0.0240 & 0.3911 & 0.3389 & 0.1501 & 0.0178 & 2 & 15 & 6 \\
\hline 412 & 6ЕA036 & MPV & 0.0672 & 0.2197 & 0.0239 & 0.1852 & 0.3941 & 0.0920 & 0.0178 & 2 & 15 & 6 \\
\hline 413 & 6EA037 & MPV & 0.2066 & 0.2077 & 0.0009 & 0.1750 & 0.3197 & 0.0870 & 0.0031 & 2 & 15 & 6 \\
\hline 414 & 6EA038 & MPV & 0.1920 & 0.0087 & 0.0008 & 0.1487 & 0.5530 & 0.0791 & 0.0178 & 2 & 15 & 6 \\
\hline 415 & 6ЕA039 & MPV & 0.2347 & 0.0094 & 0.0009 & 0.3423 & 0.2966 & 0.0967 & 0.0194 & 2 & 15 & 6 \\
\hline 416 & 6ЕA040 & MPV & 0.3310 & 0.0101 & 0.0225 & 0.1738 & 0.3176 & 0.1420 & 0.0031 & 2 & 15 & 6 \\
\hline 417 & 6ЕA041 & MPV & 0.0774 & 0.0124 & 0.0011 & 0.2132 & 0.3895 & 0.2810 & 0.0254 & 0 & 66 & 6 \\
\hline 418 & 6EA042 & MPV & 0.0782 & 0.0125 & 0.0011 & 0.2153 & 0.3935 & 0.2956 & 0.0038 & 0 & 66 & 6 \\
\hline 419 & 6EA043 & MPV & 0.0552 & 0.0089 & 0.0008 & 0.3208 & 0.5360 & 0.0756 & 0.0027 & 0 & 66 & 6 \\
\hline 420 & 6EA044 & MPV & 0.0602 & 0.0097 & 0.0214 & 0.1659 & 0.6167 & 0.1232 & 0.0030 & 0 & 66 & 6 \\
\hline 421 & 6EA045 & MPV & 0.0673 & 0.0108 & 0.0240 & 0.3911 & 0.3389 & 0.1501 & 0.0178 & 0 & 66 & 6 \\
\hline
\end{tabular}


Table 32. Envelope A Design Matrix for OLI Simulations (cont'd)

\begin{tabular}{|c|c|c|c|c|c|c|c|c|c|c|c|c|}
\hline $\begin{array}{l}\text { Run } \\
\text { No. }\end{array}$ & \begin{tabular}{|l|} 
Test ID \\
\end{tabular} & Type & $\mathrm{x}_{\mathrm{AlO} 2}$ & $\mathrm{x}_{\mathrm{CO} 3}$ & $\mathrm{x}_{\mathrm{F}}$ & $\mathrm{x}_{\mathrm{NO} 2}$ & $\mathrm{x}_{\mathrm{NO} 3}$ & $\mathrm{x}_{\mathrm{OH}}$ & $\mathrm{x}_{\mathrm{PO4}}$ & SBS/Feed & Temp & $\mathrm{Na} \mathrm{M}$ \\
\hline 422 & 6EA046 & MPV & 0.0672 & 0.2197 & 0.0239 & 1852 & .3941 & 0.0920 & 0.0178 & 0 & 66 & 6 \\
\hline 423 & 6ЕA047 & MPV & 0.2066 & 0.2077 & 0.0009 & .1750 & .3197 & .0870 & 0.0031 & 0 & 66 & 6 \\
\hline 424 & 6EA048 & MPV & 0.1920 & 0.0087 & 0.0008 & .1487 & 0.5530 & 0.0791 & 0.0178 & 0 & 66 & 6 \\
\hline 425 & 6ЕA049 & MPV & 0.2347 & 0.0094 & 0.0009 & 0.3423 & 0.2966 & 0.0967 & 0.0194 & 0 & 66 & 6 \\
\hline 426 & 6EA050 & MPV & 0.3310 & 0.0101 & 0.0225 & 0.1738 & 0.3176 & \begin{tabular}{|l|l|l}
0.1420 \\
\end{tabular} & 0.0031 & 0 & 66 & 6 \\
\hline 427 & 6EA051 & MPV & 0.0774 & 0.0124 & 0.0011 & 0.2132 & 0.3895 & 0.2810 & 0.0254 & 0 & 66 & 6 \\
\hline 428 & 6EA052 & MPV & 0.0782 & 0.0125 & 0.0011 & 0.2153 & 0.3935 & 0.2956 & 0.0038 & 0 & 66 & 6 \\
\hline 429 & 6EA053 & MPV & 0.0552 & 0.0089 & 0.0008 & 0.3208 & 0.5360 & 0.0756 & 0.0027 & 0 & 66 & 6 \\
\hline 430 & 6ЕA054 & MPV & 0.0602 & 0.0097 & 0.0214 & 0.1659 & 0.6167 & 0.1232 & 0.0030 & 0 & 66 & 6 \\
\hline 431 & 6EA055 & MPV & 0.0673 & 0.0108 & 0.0240 & 0.3911 & 0.3389 & 0.1501 & 0.0178 & 0 & 66 & 6 \\
\hline 432 & 6ЕA056 & MPV & 0.0672 & 0.2197 & 0.0239 & 0.1852 & 0.3941 & 0.0920 & 0.0178 & 0 & 66 & 6 \\
\hline 433 & 6EA057 & MPV & 0.2066 & 0.2077 & 0.0009 & 0.1750 & 0.3197 & 0.0870 & 0.0031 & 0 & 66 & 6 \\
\hline 434 & 6EA058 & MPV & 0.1920 & 0.0087 & 0.0008 & 0.1487 & 0.5530 & 0.0791 & 0.0178 & 0 & 66 & 6 \\
\hline 435 & 6ЕA059 & MPV & 0.2347 & 0.0094 & 0.0009 & 0.3423 & 0.2966 & 0.0967 & 0.0194 & 0 & 66 & 76 \\
\hline 436 & 6EA060 & MPV & 0.3310 & 0.0101 & 0.0225 & 0.1738 & 0.3176 & 0.1420 & 0.0031 & 0 & 66 & 6 \\
\hline 437 & 6EA061 & MPV & 0.0774 & 0.0124 & 0.0011 & 0.2132 & 0.3895 & 0.2810 & 0.0254 & 2 & 66 & 6 \\
\hline 438 & 6EA062 & MPV & 0.0782 & 0.0125 & 0.0011 & 0.2153 & 0.3935 & 0.2956 & 0.0038 & 2 & 66 & 66 \\
\hline 439 & 6EA063 & MPV & 0.0552 & 0.0089 & 0.0008 & 0.3208 & 0.5360 & 0.0756 & 0.0027 & 2 & 66 & 6 \\
\hline 440 & 6EA064 & MPV & 0.0602 & 0.0097 & 0.0214 & 0.1659 & 0.6167 & 0.1232 & 0.0030 & 2 & 66 & 6 \\
\hline 441 & 6ЕA065 & MPV & 0.0673 & 0.0108 & 0.0240 & 0.3911 & \begin{tabular}{|l|l}
0.3389 \\
\end{tabular} & 0.1501 & 0.0178 & 2 & 66 & 6 \\
\hline 442 & 6ЕA066 & MPV & 0.0672 & 0.2197 & 0.0239 & 0.1852 & 0.3941 & 0.0920 & 0.0178 & 2 & 66 & 6 \\
\hline 443 & 6EA067 & MPV & 0.2066 & 0.2077 & 0.0009 & 0.1750 & 0.3197 & 0.0870 & 0.0031 & 2 & 66 & 6 \\
\hline 444 & 6EA068 & MPV & 0.1920 & 0.0087 & 0.0008 & 0.1487 & 0.5530 & \begin{tabular}{|l|l|}
0.0791 \\
\end{tabular} & 0.0178 & 2 & 66 & 6 \\
\hline 445 & 6ЕA069 & MPV & 0.2347 & 0.0094 & 0.0009 & 0.3423 & 0.2966 & 0.0967 & 0.0194 & 2 & 66 & 6 \\
\hline 446 & 6ЕA070 & MPV & 0.3310 & 0.0101 & 0.0225 & 0.1738 & 0.3176 & 0.1420 & 0.0031 & 2 & 66 & 6 \\
\hline 447 & 6EA071 & MPV & 0.0774 & 0.0124 & 0.0011 & 0.2132 & 0.3895 & 0.2810 & 0.0254 & 2 & 66 & 6 \\
\hline 448 & 6EA072 & MPV & 0.0782 & 0.0125 & 0.0011 & 0.2153 & 0.3935 & 0.2956 & 0.0038 & 2 & 66 & 6 \\
\hline 449 & 6EA073 & MPV & 0.0552 & 0.0089 & 0.0008 & 0.3208 & 0.5360 & 0.0756 & 0.0027 & 2 & 66 & 6 \\
\hline 450 & 6EA074 & MPV & 0.0602 & 0.0097 & 0.0214 & 0.1659 & 0.6167 & 0.1232 & 0.0030 & 2 & 66 & 6 \\
\hline 451 & 6ЕA075 & MPV & 0.0673 & 0.0108 & 0.0240 & 0.3911 & 0.3389 & 0.1501 & 0.0178 & 2 & 66 & 6 \\
\hline 452 & 6EA076 & MPV & 0.0672 & 0.2197 & 0.0239 & 0.1852 & 0.3941 & 0.0920 & 0.0178 & 2 & 66 & 6 \\
\hline 453 & 6ЕA077 & MPV & 0.2066 & 0.2077 & 0.0009 & 0.1750 & 0.3197 & 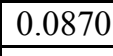 & 0.0031 & 2 & 66 & 6 \\
\hline 454 & 6ЕA078 & MPV & 0.1920 & 0.0087 & 0.0008 & 0.1487 & 0.5530 & \begin{tabular}{|l|l}
0.0791 \\
\end{tabular} & 0.0178 & 2 & 66 & 6 \\
\hline 455 & 6ЕA079 & MPV & 0.2347 & 0.0094 & 0.0009 & 0.3423 & 0.2966 & 0.0967 & 0.0194 & 2 & 66 & 6 \\
\hline 456 & 6ЕA080 & MPV & 0.3310 & 0.0101 & 0.0225 & 0.1738 & 0.3176 & 0.1420 & 0.0031 & 2 & 66 & 6 \\
\hline 457 & 6ЕA081 & OLH & 0.1918 & 0.1138 & 0.0232 & 0.2382 & 0.3215 & 0.0952 & 0.0163 & 0.0391 & 15 & 6 \\
\hline 458 & 6ЕA082 & $\mathrm{OLH}$ & 0.1843 & 0.0414 & 0.0223 & 0.2884 & 0.3481 & 0.1047 & 0.0108 & 1.957 & 15.102 & 6 \\
\hline 459 & 6ЕA083 & $\mathrm{OLH}$ & 0.1542 & 0.0297 & 0.0155 & 0.1729 & 0.5433 & 0.0790 & 0.0053 & 0.7578 & 15.714 & 6 \\
\hline 460 & 6EA084 & $\mathrm{OLH}$ & 0.1966 & 0.0295 & 0.0198 & 0.1654 & 0.4987 & 0.0840 & 0.0060 & 0.3008 & 15.918 & 6 \\
\hline 461 & 6ЕA085 & $\mathrm{OLH}$ & 0.0956 & 0.1002 & 0.0214 & 0.3327 & 0.3348 & 0.0982 & 0.0171 & 0.0039 & 16.122 & 6 \\
\hline$* 462$ & 6EA086 & $\mathrm{OLH}$ & 0.0998 & 0.0861 & 0.0117 & 0.3543 & 0.3352 & 0.1095 & 0.0033 & 1.2305 & \begin{tabular}{|l|}
17.04 \\
\end{tabular} & 6 \\
\hline 463 & 6EA087 & $\mathrm{OLH}$ & 0.1262 & 0.1404 & 0.0048 & 0.2244 & 0.3738 & 0.1118 & 0.0186 & 1.5898 & 17.55 & 6 \\
\hline 464 & 6ЕA088 & $\mathrm{OLH}$ & 0.1821 & 0.1572 & 0.0199 & 0.1779 & 0.3597 & 0.0908 & 0.0123 & 0.7734 & 18.06 & 6 \\
\hline$* 465$ & 6ЕA089 & OLH & 0.2399 & 0.0212 & 0.0184 & 0.2003 & 0.3729 & 0.1371 & 0.0102 & 1.1641 & 18.162 & 6 \\
\hline 466 & 6ЕA090 & OLH & 0.0750 & 0.0439 & 0.0136 & 0.2872 & 0.4361 & 0.1371 & 0.0070 & 0.6094 & 18.264 & 6 \\
\hline 467 & 6ЕA091 & OLH & 0.1154 & 0.0306 & 0.0105 & 0.3533 & 0.3620 & 0.1211 & 0.0071 & 0.8594 & 18.57 & 6 \\
\hline 468 & 6EA092 & OLH & 0.1491 & 0.0195 & 0.0069 & 0.3508 & 0.3724 & \begin{tabular}{|l|l|}
0.0931 \\
\end{tabular} & 0.0083 & 0.1914 & 18.672 & 6 \\
\hline
\end{tabular}


Table 32. Envelope A Design Matrix for OLI Simulations (cont'd)

\begin{tabular}{|c|c|c|c|c|c|c|c|c|c|c|c|c|}
\hline $\begin{array}{l}\text { Run } \\
\text { No. }\end{array}$ & \begin{tabular}{|l|} 
Test ID \\
\end{tabular} & Type & $\mathrm{x}_{\mathrm{AlO} 2}$ & $\mathrm{CO} 3$ & $\mathrm{x}_{\mathrm{F}}$ & NO2 & $\mathrm{NO} 3$ & $\mathrm{x}_{\mathrm{OH}}$ & $\mathrm{x}_{\mathrm{PO4}}$ & SBS/Feed & Temp & $\mathrm{Na} \mathrm{M}$ \\
\hline$* 469$ & A093 & $\mathrm{LH}$ & 0.1173 & 0919 & .0084 & 2373 & 4153 & 1102 & 0.0195 & 1.0195 & 19.284 & 6 \\
\hline 470 & EA094 & $\overline{\mathrm{LH}}$ & 1130 & 0907 & 0.0043 & 1710 & 5200 & 0981 & $\overline{0030}$ & $\overline{5352}$ & |19.4 & 6 \\
\hline 471 & A095 & $\mathrm{OLH}$ & 898 & 0.0323 & 0.0215 & 2772 & 3724 & 0925 & .0144 & 1.3203 & 19.998 & 6 \\
\hline$* 472$ & 6EA096 & OLH & 0.1669 & 0.0748 & 0.0031 & .3337 & .3280 & 0.0894 & 0.0040 & 1.8398 & 20.508 & 6 \\
\hline 473 & 6ЕA097 & OLH & 0.2043 & 0.1181 & 0.0061 & 0.2501 & 0.3185 & 0.0955 & \begin{tabular}{|l|l}
0.0074 \\
\end{tabular} & 0.1563 & 21.222 & 6 \\
\hline 474 & 6ЕA098 & LH & 0.1058 & 0.0125 & 0.0041 & 1956 & 5094 & 1547 & 0.0179 & 1.5977 & 21.426 & 6 \\
\hline 475 & 6ЕA099 & OLH & 0.2003 & 0.1021 & 0.0199 & .2472 & .3224 & .0956 & \begin{tabular}{|l|l}
0.0126 \\
\end{tabular} & 0.1719 & 21.732 & 6 \\
\hline$* 476$ & 6EA100 & OLH & 0.1876 & 0.0509 & 0.0021 & 0.1938 & 0.4624 & 0.0914 & 0.0119 & 1.6094 & 22.956 & 6 \\
\hline 477 & 6EA101 & OLH & 0.1378 & 0.0348 & 0.0086 & 0.2134 & 0.5015 & 0.0869 & \begin{tabular}{|l}
0.0169 \\
\end{tabular} & 0.8633 & 23.466 & 6 \\
\hline 478 & 6EA102 & $\mathrm{OLH}$ & 0.1185 & 0.1199 & 0.0182 & 1962 & 0.4383 & 0.1047 & 0.0042 & 1.0703 & 24.282 & 6 \\
\hline 479 & 6EA103 & OLH & 0.1916 & 0.0103 & 0.0056 & 2613 & 4231 & 0934 & 0.0148 & 1.0273 & 26.016 & 6 \\
\hline 480 & 6EA104 & $\mathrm{OLH}$ & 0.1544 & 0.0814 & 0.0136 & 0.1791 & 0.4433 & .1194 & 0.0087 & 1.1758 & 26.22 & 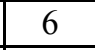 \\
\hline 481 & 6EA105 & OLH & 0.2332 & 0.0319 & 0.0046 & 0.2387 & 0.3824 & 0.1014 & \begin{tabular}{|l|l|}
0.0079 \\
\end{tabular} & 0.5 & 26.526 & 6 \\
\hline 482 & 6EA106 & $\mathrm{LH}$ & 55 & 0.0487 & 0.0189 & 2203 & 83 & 302 & .0082 & 2695 & 27.036 & 6 \\
\hline 483 & 6EA107 & $\mathrm{OLH}$ & 0.2505 & 0.0207 & 0.0017 & .2818 & 3293 & .1081 & 0.0077 & .0195 & 27.138 & 0 \\
\hline$* 484$ & 6EA108 & $\mathrm{OLH}$ & 0.1492 & 0.0373 & 0.0219 & 0.2318 & 0.4468 & 0.0979 & 0.0151 & 1.4414 & 27.24 & 6 \\
\hline 485 & 6EA109 & $\mathrm{LH}$ & 0.1778 & 0.0144 & 0.0032 & 2740 & 4405 & 0866 & 0.0036 & 1.0547 & 28.05 & 6 \\
\hline 486 & 6EA110 & $\mathrm{OLH}$ & 498 & 0.0426 & 0.0124 & 2232 & 3617 & .1069 & 0.0034 & 1.2461 & 28.26 & 6 \\
\hline$* 487$ & 6EA111 & $\mathrm{OLH}$ & 0.0733 & 0.0262 & 0.0027 & 0.2809 & .5244 & 0.0829 & 0.0097 & 1.0977 & 28.464 & 6 \\
\hline 488 & 6EA112 & OLH & 0.1772 & 0.0437 & 0.0069 & 0.2768 & \begin{tabular}{|l|l}
0.3998 \\
\end{tabular} & 0.0841 & 0.0115 & 0.6211 & 28.566 & 6 \\
\hline 489 & 6EAII3 & LH & 0.1312 & 0.0821 & 0.0080 & 3134 & 3320 & 126 & 208 & 1.0664 & 28.872 & 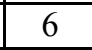 \\
\hline 490 & 6EA114 & OLH & 0.1219 & 0.0573 & 0.0032 & 2411 & 4850 & .0883 & 0.0032 & 0.6563 & 29.382 & 6 \\
\hline$* 491$ & 6EA115 & OLH & 0.1898 & 0.0209 & 0.0170 & 0.2095 & 0.4567 & 0.1016 & 0.0045 & 1.3867 & 29.484 & 6 \\
\hline 492 & 6EA116 & OLH & 0.1441 & 0.0763 & 0.0138 & .231 & .4232 & \begin{tabular}{|l|l}
0.1011 \\
\end{tabular} & 0.0035 & 1.0078 & 30.198 & 6 \\
\hline 493 & 6. & $\mathrm{LH}$ & 307 & 92 & 0.0147 & 27 & 87 & 836 & 105 & 1.8711 & 30.504 & 6 \\
\hline 494 & 6EA118 & $\mathrm{OLH}$ & 0.2601 & 0.0891 & 0.0172 & 1871 & 3239 & 0.1178 & \begin{tabular}{|l|l|} 
\\
\end{tabular} & 0.5664 & 30.606 & 6 \\
\hline 495 & 6EA119 & $\mathrm{OLH}$ & 0.0716 & 0.0437 & 0.0121 & 0.2888 & 3934 & .1807 & \begin{tabular}{|l|l|}
0.0097 \\
\end{tabular} & 0.6953 & 31.422 & 6 \\
\hline 496 & 6EA120 & $\mathrm{OLH}$ & 0.1124 & 0.0277 & 0.0204 & 0.2901 & 0.4374 & 0.1046 & 0.0075 & 1.4219 & 31.626 & $\pi$ \\
\hline 497 & 6EA121 & $\mathrm{OLH}$ & 520 & 0.0487 & 0.0037 & 2465 & 4610 & .0827 & 0.0054 & 1.3555 & 32.748 & 6 \\
\hline 498 & 6EA122 & OLH & 0.1398 & 0.1680 & 0.0101 & 0.2033 & 3637 & 0.1084 & \begin{tabular}{|l}
0.0067 \\
\end{tabular} & 0.2383 & 32.952 & - \\
\hline$* 499$ & 6EA123 & OLH & 0.2073 & 0.0202 & 0.0032 & 0.1988 & 0.4432 & 0.1137 & 0.0136 & 0.6328 & 33.36 & 6 \\
\hline 500 & 6EA124 & OLH & 0.0982 & 0.1168 & 0.0127 & 0.3097 & 3397 & 029 & 201 & 1.5313 & 33.564 & 0 \\
\hline$* 501$ & 6EA125 & OLH & 0.0829 & 0.0137 & 0.0221 & 0.1901 & .5254 & 0.1598 & 0.0060 & 0.4727 & 33.666 & 6 \\
\hline 502 & 6EA126 & OLH & 0.1996 & 0.0242 & 0.0128 & .2253 & 0.4113 & 0.1147 & 0.0122 & 0.5195 & 34.176 & 6 \\
\hline 503 & 6EA127 & $\mathrm{OLH}$ & 0.2046 & 0.0536 & 0.0149 & 0.1910 & 0.3573 & 0.1683 & \begin{tabular}{|l|l|} 
\\
\end{tabular} & 1.9727 & 34.278 & - \\
\hline$* 504$ & 6EA128 & $\mathrm{LH}$ & 158 & 0.0555 & 0.0131 & 186 & 3849 & \begin{tabular}{|l|l|l|}
0 \\
\end{tabular} & 0.0118 & 0.1523 & 34.788 & 6 \\
\hline 505 & 6EA129 & $\mathrm{OLH}$ & 0.1708 & 0.0205 & 0.0083 & 0.1925 & 0.4250 & 0.1767 & 0.0062 & 1.668 & 37.032 & 6 \\
\hline 506 & 6EA130 & $\mathrm{OLH}$ & 0.1130 & 0.0351 & 0.0057 & 0.1819 & 0.5027 & 0.1567 & 0.0050 & 1.9336 & 38.358 & 6 \\
\hline 507 & 6EA131 & $\mathrm{OLH}$ & 606 & 0.0717 & 0.0140 & 845 & 4605 & 0.0986 & 0.0100 & 1.4258 & \begin{tabular}{|l|}
39.48 \\
\end{tabular} & 0 \\
\hline 508 & 6EA132 & $\mathrm{OLH}$ & 0.1098 & 0.0634 & 0.0106 & 0.2276 & 0.4297 & 0.1397 & 0.0191 & 0.168 & \begin{tabular}{|l|}
39.99 \\
\end{tabular} & 6 \\
\hline$* 509$ & 6EA133 & $\mathrm{OLH}$ & 0.3090 & 0.0103 & 0.0189 & 0.1772 & 0.3275 & 0.1534 & 0.0037 & 1.6641 & 40.194 & 6 \\
\hline 510 & 6EA134 & $\mathrm{OLH}$ & 0.1224 & 0.0753 & 0.0109 & 0.1920 & 0.4947 & 0.1004 & 0.0042 & 0.2344 & 40.602 & 6 \\
\hline 511 & 6EA135 & $\mathrm{OLH}$ & 0.0984 & 0.1516 & 0.0077 & 0.1989 & 0.3849 & 0.1472 & 0.0113 & 1.6914 & 41.112 & 6 \\
\hline$* 512$ & 6EA136 & OLH & 0.1669 & 0.0697 & 0.0017 & 0.2348 & 0.4110 & 0.1045 & 0.0114 & 1.125 & 41.622 & 6 \\
\hline 513 & 6EA137 & OLH & 0.0589 & 0.0346 & 0.0075 & 0.2795 & 0.5124 & 0.0947 & 0.0124 & 0.2813 & 42.744 & 6 \\
\hline 514 & 6EA138 & OLH & 0.0626 & 0.1291 & 0.0064 & 0.1950 & 0.4996 & 0.0994 & 0.0079 & 1.7813 & 43.152 & 0 \\
\hline 515 & 6EA139 & $\mathrm{LH}$ & 0.1311 & 0.1039 & 0.0144 & 0.2730 & 0.3735 & 0.0956 & 0.0085 & 0.6992 & 43.56 & 4 \\
\hline
\end{tabular}


Table 32. Envelope A Design Matrix for OLI Simulations (cont'd)

\begin{tabular}{|c|c|c|c|c|c|c|c|c|c|c|c|c|}
\hline $\begin{array}{l}\text { Run } \\
\text { No. }\end{array}$ & Test ID & Type & $\mathrm{x}_{\mathrm{AlO} 2}$ & $\mathrm{CO} 3$ & $\mathrm{x}_{\mathrm{F}}$ & $\mathrm{N}_{\mathrm{NO} 2}$ & $\mathrm{x}_{\mathrm{NO} 3}$ & $\mathrm{x}_{\mathrm{OH}}$ & $\mathrm{x}_{\mathrm{PO4}}$ & SBS/Feed & Temp & $\mathrm{Na} \mathrm{M}$ \\
\hline 516 & A140 & $\mathrm{OLH}$ & 0.1595 & 0.0782 & .0088 & 2218 & 4479 & .0805 & 0.0034 & 0.957 & 43.662 & 6 \\
\hline 517 & A141 & $\overline{\mathrm{LH}}$ & 533 & 0128 & .0022 & 2050 & 5234 & 881 & 0152 & .3164 & 43.866 & 6 \\
\hline$* 518$ & A142 & $\mathrm{OLH}$ & 0.0698 & 0.0153 & 0.0170 & 3329 & 4729 & 0880 & .0043 & 1.7539 & 44.172 & 6 \\
\hline 519 & 6EA143 & OLH & 0.1615 & 0.1301 & 0.0010 & 0.2107 & .3925 & 0.0951 & 0.0091 & 0.4375 & 44.274 & 6 \\
\hline 520 & 6EA144 & OLH & 0.1759 & 0.0258 & 0.0155 & 0.2949 & 0.3976 & 0.0845 & 0.0058 & 0.7617 & 44.376 & 6 \\
\hline 521 & 6EA145 & LH & 0.0835 & 0.1155 & 0.0123 & 2952 & 3669 & .1216 & 0.0049 & 0.6758 & 44.58 & 6 \\
\hline 522 & 6EA146 & OLH & 0.1345 & 0.0261 & 0.0159 & 0.3489 & 0.3629 & 0.0958 & 0.0159 & 1.4922 & 45.396 & 6 \\
\hline$* 523$ & 6EA147 & OLH & 0.0912 & 0.1248 & 0.0125 & 0.1967 & 0.4709 & 0.0918 & 0.0120 & 1.168 & 45.6 & 6 \\
\hline 524 & 6EA148 & OLH & 0.1887 & 0.0493 & 0.0034 & 0.2270 & 0.4343 & 0.0792 & 0.0181 & 0.7852 & 45.702 & 6 \\
\hline$* 525$ & 6EA149 & $\mathrm{OLH}$ & 0.0686 & 0.1216 & 0.0090 & .3445 & 0.3364 & 0.1002 & 0.0197 & 0.9219 & 45.804 & 6 \\
\hline 526 & 6EA150 & OLH & 0.2098 & 0.0921 & 0.0073 & 0.1675 & 4174 & 0.0975 & 0.0083 & .2031 & 46.008 & 6 \\
\hline 527 & 6EA151 & OLH & 0.1460 & 0.0424 & 0.0042 & 0.2977 & 0.4206 & 0.0856 & 0.0034 & 0.6289 & 46.518 & 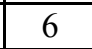 \\
\hline 528 & 6EA152 & $\mathrm{OLH}$ & 0.2237 & 0.0336 & 0.0172 & 0.1951 & 0.4230 & 0.0982 & 0.0093 & 0.0078 & 47.028 & 6 \\
\hline 529 & 6EA153 & $\mathrm{OLH}$ & 656 & 0.0179 & 0.0162 & 1905 & 56 & 377 & 165 & .2773 & 47.13 & 6 \\
\hline 530 & 6EA154 & $\mathrm{OLH}$ & 0.1154 & 0.1181 & 0.0060 & 0.2646 & .3665 & 1198 & 0.0096 & 0.9531 & 47.742 & 0 \\
\hline$* 531$ & 6EA155 & $\mathrm{OLH}$ & 0.0801 & 0.0774 & 0.0229 & 0.2804 & 0.3884 & 0.1344 & 0.0164 & 1.6328 & 47.844 & 6 \\
\hline 532 & $6 \mathrm{EA}$ & $\mathrm{OLH}$ & 0.1280 & 0.0508 & 0.0015 & 3018 & 4202 & 0851 & 0.0127 & 1.1289 & 47.946 & 6 \\
\hline 533 & $6 \mathrm{EA}$ & $\mathrm{OLH}$ & 177 & 0.0376 & 0.0074 & .2628 & 3774 & .0914 & 0.0058 & 1.7773 & 48.456 & 6 \\
\hline 534 & 6EA158 & $\mathrm{OLH}$ & 0.1680 & 0.0695 & 0.0027 & 0.3174 & .3216 & 0.1056 & 0.0151 & 1.1602 & 48.762 & 6 \\
\hline$* 535$ & 6EA159 & OLH & 0.1811 & 0.0320 & 0.0164 & 0.2236 & 0.3974 & 0.1312 & 0.0182 & 1.1172 & 48.864 & 6 \\
\hline 536 & 6EA160 & $\mathrm{OLH}$ & 595 & 0.0307 & 0.0151 & 2310 & 3962 & 559 & 16 & 1.8945 & 49.782 & 6 \\
\hline 537 & 6EA161 & OLH & 0.0732 & 0.0251 & 0.0068 & 1875 & .5517 & .1364 & 0.0192 & 1.5391 & 50.088 & 6 \\
\hline$* 538$ & 6EA162 & OLH & 0.1318 & 0.1021 & 0.0025 & 0.1808 & 0.4500 & 0.1235 & 0.0094 & 0.0547 & 51.108 & 6 \\
\hline 539 & 6EA163 & $\mathrm{OLH}$ & 0.1505 & 0.1198 & 0.0105 & 0.2479 & .3470 & 0.1066 & 0.0178 & 1.9258 & 52.026 & - \\
\hline 540 & 6 & $\mathrm{LH}$ & 540 & 81 & 0.0084 & 58 & 11 & 53 & 173 & 1.4766 & 53.454 & 6 \\
\hline 541 & 6EA165 & $\mathrm{OLH}$ & 0.1877 & 0.1616 & 0.0038 & 0.2156 & .3339 & 0.0871 & 0.0104 & 0.457 & 53.556 & 6 \\
\hline 542 & 6EA166 & $\mathrm{OLH}$ & 0.1839 & 0.0375 & 0.0052 & 0.2007 & .4698 & .0871 & 57 & 1.2734 & 54.066 & 6 \\
\hline 543 & 6EA167 & $\mathrm{OLH}$ & 0.1168 & 0.0904 & 0.0115 & 0.2788 & 0.3790 & 0.1191 & 0.0045 & 0.625 & \begin{tabular}{|l|}
54.27 \\
\end{tabular} & - \\
\hline$* 544$ & 6EA168 & $\mathrm{LH}$ & 0.1445 & 0.1280 & 0.0089 & 1786 & 4385 & 0.0878 & 138 & 1.1484 & 54.882 & 6 \\
\hline 545 & 6EA169 & OLH & 0.1160 & 0.0887 & 0.0084 & 2963 & 542 & .1161 & 03 & 1.7695 & 55.902 & - \\
\hline 546 & 6EA170 & OLH & 0.2639 & 0.0480 & 0.0011 & 0.1958 & 0.3705 & 0.1146 & 0.0061 & 1.918 & 56.514 & 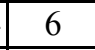 \\
\hline 547 & 6EA171 & OLH & 0.0857 & 0.0219 & 0.0025 & 0.2029 & 5554 & 201 & 115 & 0.207 & 57.738 & 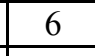 \\
\hline$* 548$ & 6EA172 & OLH & 0.0944 & 0.0558 & 0.0035 & 0.3378 & 0.4044 & 0.0890 & 0.0151 & 1.7266 & 58.758 & 6 \\
\hline 549 & 6EA173 & OLH & 0.0711 & 0.0286 & 0.0090 & 0.3841 & .3570 & 0.1442 & 0.0061 & 1.0898 & \begin{tabular}{|l|}
58.86 \\
\end{tabular} & 6 \\
\hline 550 & 6EA174 & $\mathrm{OLH}$ & 0.0687 & 0.1321 & 0.0055 & 0.2443 & 0.4377 & 0.0954 & 0.0163 & 0.8945 & \begin{tabular}{|l}
60.39 \\
\end{tabular} & - \\
\hline 551 & 6EA175 & $\mathrm{LH}$ & 0.1743 & 0.0647 & 0.0030 & 1795 & \begin{tabular}{|l|l|}
0.4891 \\
\end{tabular} & 0.0792 & 0.0104 & 1.6758 & 60.696 & 6 \\
\hline 552 & 6EA176 & $\mathrm{OLH}$ & 0.2841 & 0.0169 & 0.0098 & 0.1980 & 0.3539 & 0.1186 & 0.0187 & 1.0625 & 60.9 & 6 \\
\hline 553 & 6EA177 & $\mathrm{OLH}$ & 0.1433 & 0.0625 & 0.0156 & 0.2674 & 0.3988 & 0.1082 & 0.0041 & 1.9492 & 61.206 & 6 \\
\hline 554 & 6EA178 & $\mathrm{OLH}$ & 0.2261 & 0.1236 & 0.0196 & 2022 & 0.3185 & 0.0934 & 0.0167 & 1.7852 & \begin{tabular}{|l|}
61.92 \\
\end{tabular} & 0 \\
\hline$* 555$ & 6EA179 & $\mathrm{OLH}$ & 0.1113 & 0.0153 & 0.0137 & 0.2596 & 0.5009 & 0.0841 & 0.0151 & 0.2422 & 62.022 & 6 \\
\hline 556 & 6EA180 & OLH & 0.2326 & 0.0556 & 0.0121 & 0.2253 & 0.3643 & 0.0969 & 0.0132 & 0.4961 & 63.042 & 6 \\
\hline 557 & 6EA181 & $\mathrm{OLH}$ & 0.0911 & 0.0228 & 0.0120 & 0.2286 & 0.5396 & 0.0948 & 0.0111 & 0.6367 & 63.348 & 6 \\
\hline 558 & 6EA182 & $\mathrm{OLH}$ & 0.1226 & 0.0255 & 0.0083 & 0.2787 & 0.3779 & 0.1696 & 0.0173 & 1.4102 & 63.552 & 6 \\
\hline$* 559$ & 6EA183 & OLH & 0.2295 & 0.0373 & 0.0119 & 0.1679 & 0.4290 & 0.1072 & 0.0171 & 0.4453 & 63.756 & 6 \\
\hline 560 & 6EA184 & OLH & 0.1460 & 0.0645 & 0.0103 & 0.1800 & 0.4975 & 0.0978 & 0.0040 & 0.1836 & 64.164 & 6 \\
\hline 561 & 6EA185 & OLH & 0.1361 & 0.0601 & 0.0204 & 0.2124 & 0.4612 & 0.1047 & 0.0050 & 1.6563 & 64.47 & 0 \\
\hline 562 & 6EA186 & $\mathrm{LH}$ & 0.1504 & 0.1056 & 0.0174 & 0.2417 & 0.3505 & \begin{tabular}{|l}
0.1218 \\
\end{tabular} & .0126 & 0.1875 & 4.674 & 6 \\
\hline
\end{tabular}



SRT-RPP-2003-00125, REVISION 0

Table 32. Envelope A Design Matrix for OLI Simulations (cont'd)

\begin{tabular}{|c|c|c|c|c|c|c|c|c|c|c|c|c|}
\hline $\begin{array}{c}\text { Run } \\
\text { No. }\end{array}$ & Test ID & Type & $\mathrm{x}_{\mathrm{AlO} 2}$ & $\mathrm{x}_{\mathrm{CO} 3}$ & $\mathrm{x}_{\mathrm{F}}$ & $\mathrm{x}_{\mathrm{NO} 2}$ & $\mathrm{x}_{\mathrm{NO} 3}$ & $\mathrm{x}_{\mathrm{OH}}$ & $\mathrm{x}_{\mathrm{PO} 4}$ & SBS/Feed & Temp & Na M \\
\hline *563 & 6EA187 & OLH & 0.1310 & 0.0956 & 0.0228 & 0.2471 & 0.4037 & 0.0897 & 0.0101 & 1.7969 & 64.776 & 6 \\
\hline 564 & 6EA188 & OLH & 0.1555 & 0.0348 & 0.0175 & 0.3104 & 0.3612 & 0.1086 & 0.0120 & 1.0508 & 65.388 & 6 \\
\hline
\end{tabular}

* OLH Points used in fits where more points needed to capture nonlinear behavior 
Table 33. Envelope B Design Matrix for OLI Simulations

\begin{tabular}{|c|c|c|c|c|c|c|c|c|c|c|c|}
\hline $\begin{array}{l}\text { Run } \\
\text { No. }\end{array}$ & Test ID & Type & $\mathrm{x}_{\mathrm{AlO} 2}$ & $\mathrm{x}_{\mathrm{CO} 3}$ & $\mathrm{x}_{\mathrm{NO} 3}$ & $\mathrm{x}_{\mathrm{OH}}$ & $\mathrm{x}_{\mathrm{SO} 4}$ & $\mathrm{x}_{\mathrm{C} 2 \mathrm{O} 4}$ & $\overline{\text { SBS/Feed }}$ & Temp & $\begin{array}{l}\mathrm{Na} \\
\mathrm{M} \\
\end{array}$ \\
\hline 1 & 10TB001 & EV & 0.1102 & 0.2529 & 0.3755 & 0.0928 & 0.1438 & 0.0248 & 0 & 15 & 10 \\
\hline 2 & 10TB002 & $\mathrm{EV}$ & 0.0380 & 0.2785 & 0.3755 & 0.0928 & 0.2152 & 0.0000 & 0 & 15 & 10 \\
\hline 3 & $10 \mathrm{~TB} 003$ & EV & 0.0788 & 0.2529 & 0.3603 & 0.0928 & 0.2152 & 0.0000 & 0 & 15 & 10 \\
\hline 4 & 10TB004 & EV & 0.0380 & 0.2529 & 0.3755 & 0.1074 & 0.2014 & 0.0248 & 0 & 15 & 10 \\
\hline 5 & $10 \mathrm{~TB} 005$ & EV & 0.0380 & 0.2785 & 0.3603 & 0.0928 & 0.2056 & 0.0248 & 0 & 15 & 10 \\
\hline 6 & 10 TB006 & EV & 0.1100 & 0.2785 & 0.3603 & 0.1074 & 0.1438 & 0.0000 & 0 & 15 & 10 \\
\hline 7 & 10 TB007 & EV & 0.1102 & 0.2529 & 0.3755 & 0.0928 & 0.1438 & 0.0248 & 0 & 66 & 10 \\
\hline 8 & 10TB008 & EV & 0.0380 & 0.2785 & 0.3755 & 0.0928 & 0.2152 & 0.0000 & 0 & 66 & 10 \\
\hline 9 & 10TB009 & EV & 0.0788 & 0.2529 & 0.3603 & 0.0928 & 0.2152 & 0.0000 & 0 & 66 & 10 \\
\hline 10 & $10 \mathrm{~TB} 010$ & $\mathrm{EV}$ & 0.0380 & 0.2529 & 0.3755 & 0.1074 & 0.2014 & 0.0248 & 0 & 66 & 10 \\
\hline 11 & 10TB011 & EV & 0.0380 & 0.2785 & 0.3603 & 0.0928 & 0.2056 & 0.0248 & 0 & 66 & 10 \\
\hline 12 & $10 \mathrm{~TB} 012$ & $\overline{E V}$ & 0.1100 & 0.2785 & 0.3603 & 0.1074 & 0.1438 & 0.0000 & 0 & 66 & 10 \\
\hline 13 & 10TB013 & $\overline{E V}$ & 0.1102 & 0.2529 & 0.3755 & 0.0928 & 0.1438 & 0.0248 & 2 & 15 & 10 \\
\hline 14 & 10TB014 & $\mathrm{EV}$ & 0.0380 & 0.2785 & 0.3755 & 0.0928 & 0.2152 & 0.0000 & 2 & 15 & 10 \\
\hline 15 & 10 TB015 & $\mathrm{EV}$ & 0.0788 & 0.2529 & 0.3603 & 0.0928 & 0.2152 & 0.0000 & 2 & 15 & 10 \\
\hline 16 & $10 \mathrm{~TB} 016$ & EV & 0.0380 & 0.2529 & 0.3755 & 0.1074 & 0.2014 & 0.0248 & 2 & 15 & 10 \\
\hline 17 & 10 TB017 & EV & 0.0380 & 0.2785 & 0.3603 & 0.0928 & 0.2056 & 0.0248 & 2 & 15 & 10 \\
\hline 18 & 10TB018 & EV & 0.1100 & 0.2785 & 0.3603 & 0.1074 & 0.1438 & 0.0000 & 2 & 15 & 10 \\
\hline 19 & 10TB019 & EV & 0.1102 & 0.2529 & 0.3755 & 0.0928 & 0.1438 & 0.0248 & 2 & 66 & 10 \\
\hline 20 & 10 TB020 & EV & 0.0380 & 0.2785 & 0.3755 & 0.0928 & 0.2152 & 0.0000 & 2 & 66 & 10 \\
\hline 21 & 10TB021 & EV & 0.0788 & 0.2529 & 0.3603 & 0.0928 & 0.2152 & 0.0000 & 2 & 66 & 10 \\
\hline 22 & 10TB022 & EV & 0.0380 & 0.2529 & 0.3755 & 0.1074 & 0.2014 & 0.0248 & 2 & 66 & 10 \\
\hline 23 & $10 \mathrm{~TB} 023$ & $\mathrm{EV}$ & 0.0380 & 0.2785 & 0.3603 & 0.0928 & 0.2056 & 0.0248 & 2 & 66 & 10 \\
\hline 24 & $10 \mathrm{~TB} 024$ & $\mathrm{EV}$ & 0.1100 & 0.2785 & 0.3603 & 0.1074 & 0.1438 & 0.0000 & 2 & 66 & 10 \\
\hline 25 & 10 TB025 & $\mathrm{OLH}$ & 0.0988 & 0.2617 & 0.3635 & 0.0936 & 0.1741 & 0.0083 & 0.9 & 40.1016 & 10 \\
\hline 26 & $10 \mathrm{~TB} 026$ & OLH & 0.0857 & 0.2743 & 0.3637 & 0.0998 & 0.1722 & 0.0043 & 0.1 & 16.9922 & 10 \\
\hline 27 & 10TB027 & OLH & 0.0942 & 0.2583 & 0.3703 & 0.0960 & 0.1752 & 0.0060 & 0.8 & 34.9219 & 10 \\
\hline 28 & 10TB028 & OLH & 0.0954 & 0.2727 & 0.3730 & 0.0930 & 0.1570 & 0.0089 & 0.1 & 33.7266 & 10 \\
\hline 29 & 10TB029 & OLH & 0.1096 & 0.2585 & 0.3611 & 0.1043 & 0.1665 & 0.0000 & 0.6 & 22.5703 & 10 \\
\hline 30 & 10 TB030 & OLH & 0.0948 & 0.2781 & 0.3675 & 0.1041 & 0.1475 & 0.0079 & 0.3 & 34.125 & 10 \\
\hline *31 & 10TB031 & OLH & 0.0761 & 0.2543 & 0.3722 & 0.1024 & 0.1906 & 0.0045 & 0.6 & 28.5469 & 10 \\
\hline 32 & 10TB032 & OLH & 0.0925 & 0.2575 & 0.3603 & 0.0977 & 0.1685 & 0.0234 & 0.6 & 20.9766 & 10 \\
\hline$* 33$ & 10TB033 & $\mathrm{OLH}$ & 0.0976 & 0.2721 & 0.3652 & 0.0953 & 0.1568 & 0.0130 & 0 & 30.1406 & 10 \\
\hline 34 & 10 TB034 & $\mathrm{OLH}$ & 0.0874 & 0.2529 & 0.3728 & 0.0963 & 0.1712 & 0.0194 & 0.1 & 38.1094 & 10 \\
\hline$* 35$ & 10 TB035 & $\mathrm{OLH}$ & 0.0846 & 0.2591 & 0.3654 & 0.1074 & 0.1640 & 0.0196 & 0.3 & 22.9688 & 10 \\
\hline 36 & 10TB036 & $\mathrm{OLH}$ & 0.0931 & 0.2693 & 0.3629 & 0.1027 & 0.1528 & 0.0192 & 0.8 & 36.5156 & 10 \\
\hline$* 37$ & $10 \mathrm{~TB} 037$ & OLH & 0.0982 & 0.2615 & 0.3718 & 0.1048 & 0.1474 & 0.0163 & 0.6 & 31.7344 & 10 \\
\hline 38 & 10TB038 & OLH & 0.0863 & 0.2741 & 0.3700 & 0.1038 & 0.1451 & 0.0207 & 0 & 26.9531 & 10 \\
\hline 39 & 10TB039 & OLH & 0.1101 & 0.2607 & 0.3624 & 0.0969 & 0.1590 & 0.0109 & 1.3 & 18.9844 & 10 \\
\hline 40 & $10 \mathrm{~TB} 040$ & OLH & 0.0886 & 0.2783 & 0.3665 & 0.0931 & 0.1720 & 0.0016 & 1.7 & 28.9453 & 10 \\
\hline 41 & $10 \mathrm{~TB} 041$ & $\mathrm{OLH}$ & 0.0812 & 0.2565 & 0.3709 & 0.0933 & 0.1883 & 0.0099 & 1.5 & 20.5781 & 10 \\
\hline 42 & 10 TB042 & OLH & 0.1005 & 0.2681 & 0.3754 & 0.0966 & 0.1583 & 0.0012 & 1.3 & 25.3594 & 10 \\
\hline 43 & 10 TB043 & OLH & 0.0920 & 0.2537 & 0.3646 & 0.1054 & 0.1767 & 0.0078 & 2 & 20.1797 & 10 \\
\hline 44 & 10TB044 & OLH & 0.1084 & 0.2719 & 0.3607 & 0.1015 & 0.1543 & 0.0033 & 1.4 & 38.5078 & 10 \\
\hline 45 & 10TB045 & OLH & 0.1090 & 0.2601 & 0.3750 & 0.1030 & 0.1458 & 0.0072 & 1.6 & 36.1172 & 10 \\
\hline
\end{tabular}


Table 33. Envelope B Design Matrix for OLI Simulations (cont'd)

\begin{tabular}{|c|c|c|c|c|c|c|c|c|c|c|c|}
\hline $\begin{array}{l}\text { Run } \\
\text { No. }\end{array}$ & Test ID & Type & $\mathrm{x}_{\mathrm{AlO} 2}$ & $\mathrm{x}_{\mathrm{CO} 3}$ & $\mathrm{x}_{\mathrm{NO} 3}$ & $\mathrm{x}_{\mathrm{OH}}$ & $\mathrm{x}_{\mathrm{SO} 4}$ & $\mathrm{x}_{\mathrm{C} 2 \mathrm{O} 4}$ & SBS/Feed & Temp & $\begin{array}{l}\mathrm{Na} \\
\mathrm{M}\end{array}$ \\
\hline 46 & 10TB046 & $\mathrm{LH}$ & 0.0766 & 2603 & 651 & $\overline{0992}$ & 1810 & 0178 & 1.9 & 5234 & 10 \\
\hline$* 47$ & 10TB047 & $\mathrm{OLH}$ & 0.0897 & 0.2665 & .3623 & 0.0937 & .1636 & 0.0242 & 1 & 18.5859 & 10 \\
\hline 48 & 10TB048 & $\mathrm{OLH}$ & 0.1010 & 0.2609 & 0.3711 & 0.0986 & 0.1443 & 0.0240 & 1.6 & 36.9141 & 10 \\
\hline 49 & 10TB049 & $\overline{\mathrm{OLH}}$ & 0.0880 & 0.2751 & 0.3684 & 0.0935 & 0.1567 & 0.0184 & 2 & 37.7109 & 10 \\
\hline$* 50$ & 10TB050 & $\overline{\mathrm{OLH}}$ & 0.0817 & 0.2773 & 0.3613 & 0.1055 & 0.1595 & \begin{tabular}{|l|}
0.0147 \\
\end{tabular} & 1.5 & 19.7813 & 10 \\
\hline 51 & 10TB051 & $\mathrm{OLH}$ & 0.0789 & 0.2769 & 0.3748 & 0.1006 & 0.1443 & 0.0246 & 1.7 & 19.3828 & 10 \\
\hline 52 & 10TB052 & $\mathrm{OLH}$ & 0.1045 & 0.2579 & 0.3632 & 0.0984 & 0.1742 & 0.0019 & 1 & 43.6875 & 10 \\
\hline 53 & 10TB053 & $\mathrm{OLH}$ & 0.0965 & 0.2763 & 0.3617 & 0.0938 & 0.1649 & 0.0068 & 0.1 & 62.8125 & 10 \\
\hline 54 & 10TB054 & OLH & 0.1039 & 0.2577 & 0.3725 & 0.0991 & 0.1641 & 0.0027 & 0.8 & 45.6797 & 10 \\
\hline$* 55$ & 10TB055 & $\mathrm{OLH}$ & 0.0971 & 0.2761 & 0.3742 & 0.0993 & 0.1483 & 0.0050 & 0.7 & 63.6094 & 10 \\
\hline 56 & 10TB056 & OLH & 0.0783 & 0.2639 & 0.3661 & 0.1047 & 0.1845 & 0.0025 & 0.3 & 50.0625 & 10 \\
\hline 57 & 10TB057 & $\mathrm{OLH}$ & 0.0795 & 0.2671 & 0.3614 & 0.1060 & 0.1746 & 0.0114 & 0.8 & 59.2266 & 10 \\
\hline 58 & 10TB058 & $\mathrm{OLH}$ & 0.1056 & 0.2627 & 0.3690 & 0.1046 & 1479 & 0.0103 & 0.5 & 51.2578 & 10 \\
\hline 59 & 10TB059 & $\overline{\mathrm{OLH}}$ & 0.0829 & 0.2767 & 0.3687 & 0.1062 & 0.1568 & 0.0087 & 1 & 42.0938 & 10 \\
\hline$* 60$ & 10TB060 & $\mathrm{OLH}$ & 0.0851 & 0.2635 & 0.3618 & 0.0939 & 0.1803 & 0.0153 & 0.2 & 51.6563 & 10 \\
\hline 61 & 10TB061 & $\mathrm{OLH}$ & 0.0806 & 0.2695 & 0.3673 & 0.0968 & 0.1627 & 0.0231 & 0.6 & 64.8047 & 10 \\
\hline$* 62$ & 10TB062 & OLH & 0.0772 & 0.2555 & 0.3692 & 0.0944 & 1896 & 0.0141 & 0.9 & 64.4063 & 10 \\
\hline 63 & 10TB063 & $\mathrm{OLH}$ & 0.1033 & 0.2667 & 0.3702 & 0.0958 & .1503 & 0.0138 & 0.3 & 52.8516 & 10 \\
\hline 64 & 10TB064 & OLH & 0.0959 & 0.2557 & 0.3615 & 0.1059 & 0.1608 & 0.0202 & 0.3 & 58.8281 & 10 \\
\hline 65 & 10TB065 & $\overline{\mathrm{OLH}}$ & 0.0908 & 0.2549 & 0.3738 & 0.1014 & .1591 & 0.0200 & 0.7 & 50.4609 & 10 \\
\hline 66 & 10TB066 & $\mathrm{OLH}$ & 0.0937 & 0.2613 & 0.3627 & 0.0945 & .1756 & 0.0122 & 1.8 & 48.8672 & 10 \\
\hline 67 & 10TB067 & $\mathrm{OLH}$ & 0.0869 & 0.2725 & 0.3667 & 0.0971 & 0.1758 & 0.0010 & 1.5 & 57.2344 & 10 \\
\hline 68 & 10TB068 & OLH & 0.0800 & 0.2569 & 0.3705 & 0.0994 & .1835 & 0.0097 & 1.8 & 53.6484 & 10 \\
\hline 69 & 10TB069 & $\mathrm{OLH}$ & 0.0993 & 0.2677 & 0.3719 & 0.0946 & 1573 & 0.0091 & 1.6 & 47.6719 & 10 \\
\hline$* 70$ & 10TB070 & $\mathrm{OLH}$ & 0.1016 & 0.2645 & 0.3621 & 0.1051 & 0.1630 & 0.0037 & 1.8 & 66 & 10 \\
\hline 71 & 10TB071 & $\mathrm{OLH}$ & 0.0778 & 0.2753 & 0.3648 & 0.1012 & 0.1716 & 0.0093 & 1.1 & 49.6641 & 10 \\
\hline 72 & 10TB072 & $\mathrm{OLH}$ & 0.0891 & 0.2559 & 0.3686 & 0.1026 & 0.1772 & 0.0066 & 1.2 & 56.8359 & 10 \\
\hline 73 & 10TB073 & $\mathrm{OLH}$ & 0.0755 & 0.2597 & 0.3626 & 0.1000 & 0.1804 & 0.0219 & 1.2 & 63.2109 & 10 \\
\hline$* 74$ & 10TB074 & $\mathrm{OLH}$ & 0.0914 & 0.2661 & 0.3660 & 0.0934 & 1657 & 0.0174 & 1.9 & 41.6953 & 10 \\
\hline 75 & 10TB075 & $\mathrm{OLH}$ & 0.0834 & 0.2567 & 0.3715 & 0.0985 & 0.1763 & 0.0136 & 1.1 & 54.8438 & 10 \\
\hline$* 76$ & 10TB076 & $\mathrm{OLH}$ & 0.0840 & 0.2629 & 0.3678 & 0.1052 & .1592 & 0.0209 & 1.6 & 55.2422 & 10 \\
\hline 77 & 10TB077 & $\mathrm{OLH}$ & 0.0823 & 0.2691 & 0.3609 & 0.1019 & 0.1714 & 0.0143 & 1.8 & 54.4453 & 10 \\
\hline 78 & 10TB078 & $\mathrm{OLH}$ & 0.0749 & 0.2775 & 0.3699 & 0.1003 & 0.1583 & 0.0190 & 1.8 & 57.6328 & 10 \\
\hline$* 79$ & 10TB079 & $\mathrm{OLH}$ & 0.0744 & 0.2657 & 0.3679 & 0.1001 & 0.1796 & 0.0124 & 1 & 40.5 & 10 \\
\hline 80 & 10TB080 & $\mathrm{OLH}$ & 0.0499 & 0.2697 & 0.3723 & 0.1066 & 0.1850 & 0.0165 & 1.1 & 40.8984 & 10 \\
\hline 81 & 10TB081 & $\mathrm{OLH}$ & 0.0630 & 0.2571 & 0.3721 & 0.1004 & 0.1869 & 0.0205 & 1.9 & 64.0078 & 10 \\
\hline 82 & 10TB082 & $\mathrm{OLH}$ & 0.0545 & 0.2731 & 0.3655 & 0.1042 & 0.1839 & 0.0188 & 1.2 & 46.0781 & 10 \\
\hline 83 & 10TB083 & $\mathrm{OLH}$ & 0.0533 & 0.2587 & 0.3628 & 0.1072 & 0.2021 & 0.0159 & 1.9 & 47.2734 & 10 \\
\hline 84 & 10TB084 & $\mathrm{OLH}$ & 0.0391 & 0.2729 & 0.3747 & 0.0959 & 0.1926 & 0.0248 & 1.4 & 58.4297 & 10 \\
\hline 85 & 10TB085 & $\mathrm{OLH}$ & 0.0539 & 0.2533 & 0.3683 & 0.0961 & 0.2116 & 0.0169 & 1.7 & 46.875 & 10 \\
\hline 86 & 10TB086 & $\mathrm{OLH}$ & 0.0727 & 0.2771 & 0.3636 & 0.0978 & 0.1685 & 0.0203 & 1.4 & 52.4531 & 10 \\
\hline 87 & 10TB087 & $\mathrm{OLH}$ & 0.0562 & 0.2739 & 0.3755 & 0.1025 & 0.1906 & 0.0014 & 1.4 & 60.0234 & 10 \\
\hline$* 88$ & 10TB088 & $\mathrm{OLH}$ & 0.0511 & 0.2593 & 0.3706 & 0.1049 & 0.2023 & 0.0118 & 2 & 50.8594 & 10 \\
\hline 89 & 10TB089 & $\mathrm{OLH}$ & 0.0613 & 0.2785 & 0.3630 & 0.1039 & 0.1879 & 0.0054 & 1.9 & 42.8906 & 10 \\
\hline 90 & 10TB090 & $\mathrm{OLH}$ & 0.0641 & 0.2723 & 0.3704 & 0.0928 & 0.1952 & 0.0052 & 1.7 & 58.0313 & 10 \\
\hline$* 91$ & 10TB091 & $\mathrm{OLH}$ & 0.0556 & 0.2621 & 0.3729 & 0.0975 & 0.2063 & 0.0056 & 1.2 & 44.4844 & 10 \\
\hline
\end{tabular}


Table 33. Envelope B Design Matrix for OLI Simulations (cont'd)

\begin{tabular}{|c|c|c|c|c|c|c|c|c|c|c|c|}
\hline $\begin{array}{l}\text { Run } \\
\text { No. }\end{array}$ & Test ID & Type & $\mathrm{x}_{\mathrm{AlO} 2}$ & $\mathrm{x}_{\mathrm{CO} 3}$ & $\mathrm{x}_{\mathrm{NO} 3}$ & $\mathrm{x}_{\mathrm{OH}}$ & $\mathrm{x}_{\mathrm{SO} 4}$ & $\mathrm{x}_{\mathrm{C} 2 \mathrm{O} 4}$ & SBS/Feed & Temp & $\begin{array}{l}\mathrm{Na} \\
\mathrm{M} \\
\end{array}$ \\
\hline 92 & 10TB092 & OLH & 0.0505 & 0.2699 & 0.3640 & 0.0954 & 0.2117 & 0.0085 & 1.4 & 49.2656 & 10 \\
\hline 93 & 10TB093 & OLH & 0.0624 & 0.2573 & 0.3658 & 0.0965 & 0.2140 & 0.0041 & 2 & 54.0469 & 10 \\
\hline$* 94$ & 10TB094 & OLH & 0.0386 & 0.2707 & 0.3734 & 0.1033 & 0.2001 & 0.0140 & 0.7 & 62.0156 & 10 \\
\hline 95 & 10TB095 & $\mathrm{OLH}$ & 0.0602 & 0.2531 & 0.3693 & 0.1071 & 0.1871 & 0.0233 & 0.3 & 52.0547 & 10 \\
\hline *96 & 10TB096 & $\mathrm{OLH}$ & 0.0675 & 0.2749 & 0.3649 & 0.1069 & 0.1708 & 0.0149 & 0.5 & 60.4219 & 10 \\
\hline 97 & 10TB097 & OLH & 0.0482 & 0.2633 & 0.3604 & 0.1036 & 0.2008 & 0.0236 & 0.7 & 55.6406 & 10 \\
\hline 98 & 10TB098 & OLH & 0.0567 & 0.2777 & 0.3712 & 0.0949 & 0.1824 & 0.0171 & 0 & 60.8203 & 10 \\
\hline *99 & 10TB099 & OLH & 0.0403 & 0.2595 & 0.3751 & 0.0987 & 0.2049 & 0.0215 & 0.6 & 42.4922 & 10 \\
\hline 100 & $10 \mathrm{~TB} 100$ & $\mathrm{OLH}$ & 0.0397 & 0.2713 & 0.3608 & 0.0973 & 0.2133 & 0.0176 & 0.4 & 44.8828 & 10 \\
\hline 101 & 10TB101 & $\mathrm{OLH}$ & 0.0721 & 0.2711 & 0.3708 & 0.1010 & 0.1781 & 0.0070 & 0.1 & 46.4766 & 10 \\
\hline *102 & $10 \mathrm{~TB} 102$ & OLH & 0.0590 & 0.2649 & 0.3735 & 0.1065 & 0.1955 & 0.0006 & 1 & 62.4141 & 10 \\
\hline 103 & $10 \mathrm{~TB} 103$ & $\mathrm{OLH}$ & 0.0477 & 0.2705 & 0.3647 & 0.1016 & 0.2148 & 0.0008 & 0.4 & 44.0859 & 10 \\
\hline 104 & 10TB104 & OLH & 0.0607 & 0.2563 & 0.3674 & 0.1067 & 0.2024 & 0.0064 & 0 & 43.2891 & 10 \\
\hline$* 105$ & $10 \mathrm{~TB} 105$ & $\mathrm{OLH}$ & 0.0670 & 0.2541 & 0.3746 & 0.0947 & 0.1996 & 0.0101 & 0.5 & 61.2188 & 10 \\
\hline 106 & $10 \mathrm{~TB} 106$ & OLH & 0.0698 & 0.2545 & 0.3610 & 0.0996 & 0.2149 & 0.0002 & 0.3 & 61.6172 & 10 \\
\hline 107 & 10 TB107 & OLH & 0.0443 & 0.2735 & 0.3727 & 0.1018 & 0.1849 & 0.0229 & 1 & 37.3125 & 10 \\
\hline *108 & 10 TB108 & OLH & 0.0522 & 0.2551 & 0.3741 & 0.1064 & 0.1942 & 0.0180 & 1.9 & 18.1875 & 10 \\
\hline 109 & 10 TB109 & OLH & 0.0448 & 0.2737 & 0.3633 & 0.1011 & 0.1950 & 0.0221 & 1.2 & 35.3203 & 10 \\
\hline 110 & $10 \mathrm{~TB} 110$ & OLH & 0.0516 & 0.2553 & 0.3616 & 0.1009 & 0.2108 & 0.0198 & 1.3 & 17.3906 & 10 \\
\hline 111 & $10 \mathrm{~TB} 111$ & OLH & 0.0704 & 0.2675 & 0.3697 & 0.0955 & 0.1746 & 0.0223 & 1.7 & 30.9375 & 10 \\
\hline 112 & $10 \mathrm{~TB} 112$ & OLH & 0.0692 & 0.2643 & 0.3744 & 0.0942 & 0.1845 & 0.0134 & 1.3 & 21.7734 & 10 \\
\hline 113 & $10 \mathrm{~TB} 113$ & OLH & 0.0431 & 0.2687 & 0.3668 & 0.0957 & 0.2112 & 0.0145 & 1.5 & 29.7422 & 10 \\
\hline$* 114$ & $10 \mathrm{~TB} 114$ & $\mathrm{OLH}$ & 0.0658 & 0.2547 & 0.3671 & 0.0941 & 0.2023 & 0.0161 & 1 & 38.9063 & 10 \\
\hline 115 & $10 \mathrm{~TB} 115$ & OLH & 0.0636 & 0.2679 & 0.3740 & 0.1063 & 0.1788 & 0.0095 & 1.8 & 29.3438 & 10 \\
\hline 116 & $10 \mathrm{~TB} 116$ & $\mathrm{OLH}$ & 0.0681 & 0.2619 & 0.3685 & 0.1034 & 0.1964 & 0.0017 & 1.4 & 16.1953 & 10 \\
\hline$* 117$ & $10 \mathrm{~TB} 117$ & $\mathrm{OLH}$ & 0.0715 & 0.2759 & 0.3666 & 0.1058 & 0.1695 & 0.0107 & 1.1 & 16.5938 & 10 \\
\hline 118 & $10 \mathrm{~TB} 118$ & $\mathrm{OLH}$ & 0.0454 & 0.2647 & 0.3656 & 0.1044 & 0.2088 & 0.0110 & 1.8 & 28.1484 & 10 \\
\hline 119 & 10TB119 & $\mathrm{OLH}$ & 0.0528 & 0.2757 & 0.3743 & 0.0943 & 0.1983 & 0.0047 & 1.7 & 22.1719 & 10 \\
\hline 120 & 10 TB120 & $\mathrm{OLH}$ & 0.0579 & 0.2765 & 0.3620 & 0.0989 & 0.2000 & 0.0048 & 1.3 & 30.5391 & 10 \\
\hline 121 & 10TB121 & OLH & 0.0550 & 0.2701 & 0.3731 & 0.1057 & 0.1835 & 0.0126 & 0.2 & 32.1328 & 10 \\
\hline$* 122$ & $10 \mathrm{~TB} 122$ & OLH & 0.0619 & 0.2589 & 0.3691 & 0.1031 & 0.1833 & 0.0238 & 0.5 & 23.7656 & 10 \\
\hline 123 & $10 \mathrm{~TB} 123$ & $\mathrm{OLH}$ & 0.0687 & 0.2745 & 0.3653 & 0.1008 & 0.1757 & 0.0151 & 0.2 & 27.3516 & 10 \\
\hline 124 & $10 \mathrm{~TB} 124$ & $\mathrm{OLH}$ & 0.0494 & 0.2637 & 0.3639 & 0.1056 & 0.2018 & 0.0157 & 0.4 & 33.3281 & 10 \\
\hline 125 & $10 \mathrm{~TB} 125$ & $\mathrm{OLH}$ & 0.0471 & 0.2669 & 0.3737 & 0.0951 & 0.1961 & 0.0211 & 0.2 & 15 & 10 \\
\hline *126 & $10 \mathrm{~TB} 126$ & $\mathrm{OLH}$ & 0.0709 & 0.2561 & 0.3710 & 0.0990 & 0.1875 & 0.0155 & 0.9 & 31.3359 & 10 \\
\hline 127 & $10 \mathrm{~TB} 127$ & OLH & 0.0596 & 0.2755 & 0.3672 & 0.0976 & 0.1819 & 0.0182 & 0.8 & 24.1641 & 10 \\
\hline *128 & $10 \mathrm{~TB} 128$ & OLH & 0.0732 & 0.2717 & 0.3732 & 0.1002 & 0.1787 & 0.0029 & 0.8 & 17.7891 & 10 \\
\hline 129 & 10TB129 & $\mathrm{OLH}$ & 0.0573 & 0.2653 & 0.3698 & 0.1068 & 0.1934 & 0.0074 & 0.1 & 39.3047 & 10 \\
\hline 130 & 10 TB130 & $\mathrm{OLH}$ & 0.0653 & 0.2747 & 0.3643 & 0.1017 & 0.1828 & 0.0112 & 0.9 & 26.1563 & 10 \\
\hline 131 & $10 \mathrm{~TB} 131$ & $\mathrm{OLH}$ & 0.0647 & 0.2685 & 0.3680 & 0.0950 & 0.1999 & 0.0039 & 0.4 & 25.7578 & 10 \\
\hline 132 & $10 \mathrm{~TB} 132$ & $\mathrm{OLH}$ & 0.0664 & 0.2623 & 0.3749 & 0.0983 & 0.1877 & 0.0105 & 0.2 & 26.5547 & 10 \\
\hline 133 & 10 TB133 & OLH & 0.0738 & 0.2539 & 0.3659 & 0.0999 & 0.2008 & 0.0058 & 0.2 & 23.3672 & 10 \\
\hline 134 & $8 \mathrm{~TB} 001$ & EV & 0.1102 & 0.2529 & 0.3755 & 0.0928 & 0.1438 & 0.0248 & 0 & 15 & 8 \\
\hline 135 & 8TB002 & EV & 0.0380 & 0.2785 & 0.3755 & 0.0928 & 0.2152 & 0.0000 & 0 & 15 & 8 \\
\hline 136 & 8TB003 & EV & 0.0788 & 0.2529 & 0.3603 & 0.0928 & 0.2152 & 0.0000 & 0 & 15 & 8 \\
\hline 137 & 8ТВ004 & EV & 0.0380 & 0.2529 & 0.3755 & 0.1074 & 0.2014 & 0.0248 & 0 & 15 & 8 \\
\hline 138 & $8 \mathrm{~TB} 005$ & EV & 0.0380 & 0.2785 & 0.3603 & 0.0928 & 0.2056 & 0.0248 & 0 & 15 & 8 \\
\hline
\end{tabular}


Table 33. Envelope B Design Matrix for OLI Simulations (cont'd)

\begin{tabular}{|c|c|c|c|c|c|c|c|c|c|c|c|}
\hline $\begin{array}{l}\text { Run } \\
\text { No. }\end{array}$ & Test ID & Type & $\mathrm{x}_{\mathrm{AlO} 2}$ & $\mathrm{x}_{\mathrm{CO} 3}$ & $\mathrm{x}_{\mathrm{NO} 3}$ & $\mathrm{x}_{\mathrm{OH}}$ & $\mathrm{x}_{\mathrm{SO} 4}$ & $\mathrm{x}_{\mathrm{C} 2 \mathrm{O} 4}$ & SBS/Feed & Temp & $\begin{array}{l}\mathrm{Na} \\
\mathrm{M} \\
\end{array}$ \\
\hline 139 & 8TB006 & EV & 0.1100 & 0.2785 & 0.3603 & 0.1074 & 0.1438 & 0.0000 & 0 & 15 & 8 \\
\hline 140 & 8 8TB007 & EV & 0.1102 & 0.2529 & 0.3755 & 0.0928 & 0.1438 & 0.0248 & 0 & 66 & 8 \\
\hline 141 & 8TB008 & EV & 0.0380 & 0.2785 & 0.3755 & 0.0928 & 0.2152 & 0.0000 & 0 & 66 & 8 \\
\hline 142 & 8TB009 & EV & 0.0788 & 0.2529 & 0.3603 & 0.0928 & 0.2152 & 0.0000 & 0 & 66 & 8 \\
\hline 143 & 8 TB010 & EV & 0.0380 & 0.2529 & 0.3755 & 0.1074 & 0.2014 & 0.0248 & 0 & 66 & 8 \\
\hline 144 & 8TB011 & EV & 0.0380 & 0.2785 & 0.3603 & 0.0928 & 0.2056 & 0.0248 & 0 & 66 & 8 \\
\hline 145 & 8TB012 & EV & 0.1100 & 0.2785 & 0.3603 & 0.1074 & 0.1438 & 0.0000 & 0 & 66 & 8 \\
\hline 146 & 8TB013 & EV & 0.1102 & 0.2529 & 0.3755 & 0.0928 & 0.1438 & 0.0248 & 2 & 15 & 8 \\
\hline 147 & 8TB014 & EV & 0.0380 & 0.2785 & 0.3755 & 0.0928 & 0.2152 & 0.0000 & 2 & 15 & 8 \\
\hline 148 & 8TB015 & EV & 0.0788 & 0.2529 & 0.3603 & 0.0928 & 0.2152 & 0.0000 & 2 & 15 & 8 \\
\hline 149 & 8TB016 & EV & 0.0380 & 0.2529 & 0.3755 & 0.1074 & 0.2014 & 0.0248 & 2 & 15 & 8 \\
\hline 150 & 8TB017 & EV & 0.0380 & 0.2785 & 0.3603 & 0.0928 & 0.2056 & 0.0248 & 2 & 15 & 8 \\
\hline 151 & 8TB018 & EV & 0.1100 & 0.2785 & 0.3603 & 0.1074 & 0.1438 & 0.0000 & 2 & 15 & 8 \\
\hline 152 & 8TB019 & EV & 0.1102 & 0.2529 & 0.3755 & 0.0928 & 0.1438 & 0.0248 & 2 & 66 & 8 \\
\hline 153 & 8TB020 & EV & 0.0380 & 0.2785 & 0.3755 & 0.0928 & 0.2152 & 0.0000 & 2 & 66 & 8 \\
\hline 154 & 8TB021 & EV & 0.0788 & 0.2529 & 0.3603 & 0.0928 & 0.2152 & 0.0000 & 2 & 66 & 8 \\
\hline 155 & 8TB022 & EV & 0.0380 & 0.2529 & 0.3755 & 0.1074 & 0.2014 & 0.0248 & 2 & 66 & 8 \\
\hline 156 & $8 \mathrm{~TB} 023$ & EV & 0.0380 & 0.2785 & 0.3603 & 0.0928 & 0.2056 & 0.0248 & 2 & 66 & 8 \\
\hline 157 & 8TB024 & EV & 0.1100 & 0.2785 & 0.3603 & 0.1074 & 0.1438 & 0.0000 & 2 & 66 & 8 \\
\hline 158 & 8 TB025 & $\mathrm{OLH}$ & 0.0988 & 0.2617 & 0.3635 & 0.0936 & 0.1741 & 0.0083 & 0.9 & 40.1016 & 8 \\
\hline 159 & 8TB026 & $\mathrm{OLH}$ & 0.0857 & 0.2743 & 0.3637 & 0.0998 & 0.1722 & 0.0043 & 0.1 & 16.9922 & 8 \\
\hline 160 & 8TB027 & $\mathrm{OLH}$ & 0.0942 & 0.2583 & 0.3703 & 0.0960 & 0.1752 & 0.0060 & 0.8 & 34.9219 & 8 \\
\hline 161 & 8TB028 & $\mathrm{OLH}$ & 0.0954 & 0.2727 & 0.3730 & 0.0930 & 0.1570 & 0.0089 & 0.1 & 33.7266 & 8 \\
\hline *162 & 8TB029 & $\mathrm{OLH}$ & 0.1096 & 0.2585 & 0.3611 & 0.1043 & 0.1665 & 0.0000 & 0.6 & 22.5703 & 8 \\
\hline 163 & 8TB030 & $\mathrm{OLH}$ & 0.0948 & 0.2781 & 0.3675 & 0.1041 & 0.1475 & 0.0079 & 0.3 & 34.125 & 8 \\
\hline 164 & 8TB031 & $\mathrm{OLH}$ & 0.0761 & 0.2543 & 0.3722 & 0.1024 & 0.1906 & 0.0045 & 0.6 & 28.5469 & 8 \\
\hline 165 & 8TB032 & $\mathrm{OLH}$ & 0.0925 & 0.2575 & 0.3603 & 0.0977 & 0.1685 & 0.0234 & 0.6 & 20.9766 & 8 \\
\hline 166 & 8TB033 & $\mathrm{OLH}$ & 0.0976 & 0.2721 & 0.3652 & 0.0953 & 0.1568 & 0.0130 & 0 & 30.1406 & 8 \\
\hline 167 & 8TB034 & $\mathrm{OLH}$ & 0.0874 & 0.2529 & 0.3728 & 0.0963 & 0.1712 & 0.0194 & 0.1 & 38.1094 & 8 \\
\hline 168 & $8 \mathrm{~TB} 035$ & $\mathrm{OLH}$ & 0.0846 & 0.2591 & 0.3654 & 0.1074 & 0.1640 & 0.0196 & 0.3 & 22.9688 & 8 \\
\hline 169 & 8TB036 & $\mathrm{OLH}$ & 0.0931 & 0.2693 & 0.3629 & 0.1027 & 0.1528 & 0.0192 & 0.8 & 36.5156 & 8 \\
\hline *170 & 8 TB037 & $\mathrm{OLH}$ & 0.0982 & 0.2615 & 0.3718 & 0.1048 & 0.1474 & 0.0163 & 0.6 & 31.7344 & 8 \\
\hline 171 & 8TB038 & $\mathrm{OLH}$ & 0.0863 & 0.2741 & 0.3700 & 0.1038 & 0.1451 & 0.0207 & 0 & 26.9531 & 8 \\
\hline$* 172$ & 8TB039 & $\mathrm{OLH}$ & 0.1101 & 0.2607 & 0.3624 & 0.0969 & 0.1590 & 0.0109 & 1.3 & 18.9844 & 8 \\
\hline 173 & 8TB040 & $\mathrm{OLH}$ & 0.0886 & 0.2783 & 0.3665 & 0.0931 & 0.1720 & 0.0016 & 1.7 & 28.9453 & 8 \\
\hline 174 & 8TB041 & $\mathrm{OLH}$ & 0.0812 & 0.2565 & 0.3709 & 0.0933 & 0.1883 & 0.0099 & 1.5 & 20.5781 & 8 \\
\hline 175 & 8TB042 & $\mathrm{OLH}$ & 0.1005 & 0.2681 & 0.3754 & 0.0966 & 0.1583 & 0.0012 & 1.3 & 25.3594 & 8 \\
\hline 176 & 8TB043 & $\mathrm{OLH}$ & 0.0920 & 0.2537 & 0.3646 & 0.1054 & 0.1767 & 0.0078 & 2 & 20.1797 & 8 \\
\hline$* 177$ & 8TB044 & $\mathrm{OLH}$ & 0.1084 & 0.2719 & 0.3607 & 0.1015 & 0.1543 & 0.0033 & 1.4 & 38.5078 & 8 \\
\hline 178 & 8TB045 & $\mathrm{OLH}$ & 0.1090 & 0.2601 & 0.3750 & 0.1030 & 0.1458 & 0.0072 & 1.6 & 36.1172 & 8 \\
\hline$* 179$ & 8TB046 & $\mathrm{OLH}$ & 0.0766 & 0.2603 & 0.3651 & 0.0992 & 0.1810 & 0.0178 & 1.9 & 34.5234 & 8 \\
\hline 180 & 8TB047 & $\mathrm{OLH}$ & 0.0897 & 0.2665 & 0.3623 & 0.0937 & 0.1636 & 0.0242 & 1 & 18.5859 & 8 \\
\hline 181 & 8TB048 & $\mathrm{OLH}$ & 0.1010 & 0.2609 & 0.3711 & 0.0986 & 0.1443 & 0.0240 & 1.6 & 36.9141 & 8 \\
\hline 182 & 8TB049 & $\mathrm{OLH}$ & 0.0880 & 0.2751 & 0.3684 & 0.0935 & 0.1567 & 0.0184 & 2 & 37.7109 & 8 \\
\hline 183 & 8ТВ050 & $\mathrm{OLH}$ & 0.0817 & 0.2773 & 0.3613 & 0.1055 & 0.1595 & 0.0147 & 1.5 & 19.7813 & 8 \\
\hline 184 & 8TB051 & $\mathrm{OLH}$ & 0.0789 & 0.2769 & 0.3748 & 0.1006 & 0.1443 & 0.0246 & 1.7 & 19.3828 & 8 \\
\hline 185 & 8TB052 & $\mathrm{OLH}$ & 0.1045 & 0.2579 & 0.3632 & 0.0984 & 0.1742 & 0.0019 & 1 & 43.6875 & 8 \\
\hline
\end{tabular}


Table 33. Envelope B Design Matrix for OLI Simulations (cont'd)

\begin{tabular}{|c|c|c|c|c|c|c|c|c|c|c|c|}
\hline $\begin{array}{l}\text { Run } \\
\text { No. }\end{array}$ & Test ID & Type & $\mathrm{x}_{\mathrm{AlO} 2}$ & $\mathrm{x}_{\mathrm{CO} 3}$ & $\mathrm{x}_{\mathrm{NO} 3}$ & $\mathrm{x}_{\mathrm{OH}}$ & $\mathrm{x}_{\mathrm{SO} 4}$ & $\mathrm{x}_{\mathrm{C} 2 \mathrm{O} 4}$ & SBS/Feed & Temp & $\begin{array}{l}\mathrm{Na} \\
\mathrm{M} \\
\end{array}$ \\
\hline$* 186$ & $8 \mathrm{~TB} 053$ & $\mathrm{OLH}$ & 0.0965 & 0.2763 & 0.3617 & 0.0938 & 0.1649 & 0.0068 & 0.1 & 62.8125 & 8 \\
\hline 187 & $8 \mathrm{~TB} 054$ & $\mathrm{OLH}$ & 0.1039 & 0.2577 & 0.3725 & 0.0991 & 0.1641 & 0.0027 & 0.8 & 45.6797 & 8 \\
\hline 188 & $8 \mathrm{~TB} 055$ & $\mathrm{OLH}$ & 0.0971 & 0.2761 & 0.3742 & 0.0993 & 0.1483 & 0.0050 & 0.7 & 63.6094 & 8 \\
\hline *189 & 8TB056 & $\mathrm{OLH}$ & 0.0783 & 0.2639 & 0.3661 & 0.1047 & 0.1845 & 0.0025 & 0.3 & 50.0625 & 8 \\
\hline 190 & 8TB057 & $\mathrm{OLH}$ & 0.0795 & 0.2671 & 0.3614 & 0.1060 & 0.1746 & 0.0114 & 0.8 & 59.2266 & 8 \\
\hline 191 & $8 \mathrm{~TB} 058$ & $\mathrm{OLH}$ & 0.1056 & 0.2627 & 0.3690 & 0.1046 & 0.1479 & 0.0103 & 0.5 & 51.2578 & 8 \\
\hline 192 & 8ТВ059 & OLH & 0.0829 & 0.2767 & 0.3687 & 0.1062 & 0.1568 & 0.0087 & 1 & 42.0938 & 8 \\
\hline *193 & 8 ТВ060 & $\mathrm{OLH}$ & 0.0851 & 0.2635 & 0.3618 & 0.0939 & 0.1803 & 0.0153 & 0.2 & 51.6563 & 8 \\
\hline 194 & 8TB061 & $\mathrm{OLH}$ & 0.0806 & 0.2695 & 0.3673 & 0.0968 & 0.1627 & 0.0231 & 0.6 & 64.8047 & 8 \\
\hline *195 & 8TB062 & $\mathrm{OLH}$ & 0.0772 & 0.2555 & 0.3692 & 0.0944 & 0.1896 & 0.0141 & 0.9 & 64.4063 & 8 \\
\hline 196 & 8TB063 & $\mathrm{OLH}$ & 0.1033 & 0.2667 & 0.3702 & 0.0958 & 0.1503 & 0.0138 & 0.3 & 52.8516 & 8 \\
\hline 197 & 8TB064 & $\mathrm{OLH}$ & 0.0959 & 0.2557 & 0.3615 & 0.1059 & 0.1608 & 0.0202 & 0.3 & 58.8281 & 8 \\
\hline 198 & 8 TB065 & OLH & 0.0908 & 0.2549 & 0.3738 & 0.1014 & 0.1591 & 0.0200 & 0.7 & 50.4609 & 8 \\
\hline 199 & 8TB066 & $\mathrm{OLH}$ & 0.0937 & 0.2613 & 0.3627 & 0.0945 & 0.1756 & 0.0122 & 1.8 & 48.8672 & 8 \\
\hline 200 & 8TB067 & $\mathrm{OLH}$ & 0.0869 & 0.2725 & 0.3667 & 0.0971 & 0.1758 & 0.0010 & 1.5 & 57.2344 & 8 \\
\hline 201 & 8TB068 & $\mathrm{OLH}$ & 0.0800 & 0.2569 & 0.3705 & 0.0994 & 0.1835 & 0.0097 & 1.8 & 53.6484 & 8 \\
\hline 202 & 8TB069 & $\mathrm{OLH}$ & 0.0993 & 0.2677 & 0.3719 & 0.0946 & 0.1573 & 0.0091 & 1.6 & 47.6719 & 8 \\
\hline *203 & $8 \mathrm{~TB} 070$ & $\mathrm{OLH}$ & 0.1016 & 0.2645 & 0.3621 & 0.1051 & 0.1630 & 0.0037 & 1.8 & 66 & 8 \\
\hline 204 & 8TB071 & $\mathrm{OLH}$ & 0.0778 & 0.2753 & 0.3648 & 0.1012 & 0.1716 & 0.0093 & 1.1 & 49.6641 & 8 \\
\hline 205 & 8TB072 & $\mathrm{OLH}$ & 0.0891 & 0.2559 & 0.3686 & 0.1026 & 0.1772 & 0.0066 & 1.2 & 56.8359 & 8 \\
\hline 206 & 8TB073 & $\mathrm{OLH}$ & 0.0755 & 0.2597 & 0.3626 & 0.1000 & 0.1804 & 0.0219 & 1.2 & 63.2109 & 8 \\
\hline *207 & 8TB074 & $\mathrm{OLH}$ & 0.0914 & 0.2661 & 0.3660 & 0.0934 & 0.1657 & 0.0174 & 1.9 & 41.6953 & 8 \\
\hline 208 & 8TB075 & $\mathrm{OLH}$ & 0.0834 & 0.2567 & 0.3715 & 0.0985 & 0.1763 & 0.0136 & 1.1 & 54.8438 & 8 \\
\hline *209 & 8TB076 & $\mathrm{OLH}$ & 0.0840 & 0.2629 & 0.3678 & 0.1052 & 0.1592 & 0.0209 & 1.6 & 55.2422 & 8 \\
\hline 210 & 8TB077 & $\mathrm{OLH}$ & 0.0823 & 0.2691 & 0.3609 & 0.1019 & 0.1714 & 0.0143 & 1.8 & 54.4453 & 8 \\
\hline 211 & 8TB078 & $\mathrm{OLH}$ & 0.0749 & 0.2775 & 0.3699 & 0.1003 & 0.1583 & 0.0190 & 1.8 & 57.6328 & 8 \\
\hline 212 & 8TB079 & $\mathrm{OLH}$ & 0.0744 & 0.2657 & 0.3679 & 0.1001 & 0.1796 & 0.0124 & 1 & 40.5 & 8 \\
\hline 213 & 8TB080 & $\mathrm{OLH}$ & 0.0499 & 0.2697 & 0.3723 & 0.1066 & 0.1850 & 0.0165 & 1.1 & 40.8984 & 8 \\
\hline *214 & 8TB081 & $\mathrm{OLH}$ & 0.0630 & 0.2571 & 0.3721 & 0.1004 & 0.1869 & 0.0205 & 1.9 & 64.0078 & 8 \\
\hline 215 & 8TB082 & $\mathrm{OLH}$ & 0.0545 & 0.2731 & 0.3655 & 0.1042 & 0.1839 & 0.0188 & 1.2 & 46.0781 & 8 \\
\hline *216 & 8TB083 & $\mathrm{OLH}$ & 0.0533 & 0.2587 & 0.3628 & 0.1072 & 0.2021 & 0.0159 & 1.9 & 47.2734 & 8 \\
\hline 217 & 8TB084 & $\mathrm{OLH}$ & 0.0391 & 0.2729 & 0.3747 & 0.0959 & 0.1926 & 0.0248 & 1.4 & 58.4297 & 8 \\
\hline *218 & $8 \mathrm{~TB} 085$ & $\mathrm{OLH}$ & 0.0539 & 0.2533 & 0.3683 & 0.0961 & 0.2116 & 0.0169 & 1.7 & 46.875 & 8 \\
\hline 219 & 8ТВ086 & $\mathrm{OLH}$ & 0.0727 & 0.2771 & 0.3636 & 0.0978 & 0.1685 & 0.0203 & 1.4 & 52.4531 & 8 \\
\hline 220 & 8TB087 & OLH & 0.0562 & 0.2739 & 0.3755 & 0.1025 & 0.1906 & 0.0014 & 1.4 & 60.0234 & 8 \\
\hline *221 & 8 TB088 & $\mathrm{OLH}$ & 0.0511 & 0.2593 & 0.3706 & 0.1049 & 0.2023 & 0.0118 & 2 & 50.8594 & 8 \\
\hline 222 & 8TB089 & $\mathrm{OLH}$ & 0.0613 & 0.2785 & 0.3630 & 0.1039 & 0.1879 & 0.0054 & 1.9 & 42.8906 & 8 \\
\hline 223 & 8TB090 & $\mathrm{OLH}$ & 0.0641 & 0.2723 & 0.3704 & 0.0928 & 0.1952 & 0.0052 & 1.7 & 58.0313 & 8 \\
\hline 224 & 8TB091 & $\mathrm{OLH}$ & 0.0556 & 0.2621 & 0.3729 & 0.0975 & 0.2063 & 0.0056 & 1.2 & 44.4844 & 8 \\
\hline 225 & 8TB092 & $\mathrm{OLH}$ & 0.0505 & 0.2699 & 0.3640 & 0.0954 & 0.2117 & 0.0085 & 1.4 & 49.2656 & 8 \\
\hline 226 & 8TB093 & $\mathrm{OLH}$ & 0.0624 & 0.2573 & 0.3658 & 0.0965 & 0.2140 & 0.0041 & 2 & 54.0469 & 8 \\
\hline 227 & 8TB094 & $\mathrm{OLH}$ & 0.0386 & 0.2707 & 0.3734 & 0.1033 & 0.2001 & 0.0140 & 0.7 & 62.0156 & 8 \\
\hline *228 & 8TB095 & $\mathrm{OLH}$ & 0.0602 & 0.2531 & 0.3693 & 0.1071 & 0.1871 & 0.0233 & 0.3 & 52.0547 & 8 \\
\hline 229 & 8TB096 & $\mathrm{OLH}$ & 0.0675 & 0.2749 & 0.3649 & 0.1069 & 0.1708 & 0.0149 & 0.5 & 60.4219 & 8 \\
\hline 230 & 8ТВ097 & $\mathrm{OLH}$ & 0.0482 & 0.2633 & 0.3604 & 0.1036 & 0.2008 & 0.0236 & 0.7 & 55.6406 & 8 \\
\hline *231 & 8TB098 & $\mathrm{OLH}$ & 0.0567 & 0.2777 & 0.3712 & 0.0949 & 0.1824 & 0.0171 & 0 & 60.8203 & 8 \\
\hline 232 & 8TB099 & $\mathrm{OLH}$ & 0.0403 & 0.2595 & 0.3751 & 0.0987 & 0.2049 & 0.0215 & 0.6 & 42.4922 & 8 \\
\hline
\end{tabular}


Table 33. Envelope B Design Matrix for OLI Simulations (cont'd)

\begin{tabular}{|c|c|c|c|c|c|c|c|c|c|c|c|}
\hline $\begin{array}{l}\text { Run } \\
\text { No. }\end{array}$ & Test ID & Type & $\mathrm{x}_{\mathrm{AlO} 2}$ & $\mathrm{x}_{\mathrm{CO} 3}$ & $\mathrm{x}_{\mathrm{NO} 3}$ & $\mathrm{x}_{\mathrm{OH}}$ & $\mathrm{x}_{\mathrm{SO} 4}$ & $\mathrm{x}_{\mathrm{C} 2 \mathrm{O} 4}$ & SBS/Feed & Temp & $\begin{array}{l}\mathrm{Na} \\
\mathrm{M} \\
\end{array}$ \\
\hline 233 & $8 \mathrm{~TB} 100$ & $\mathrm{OLH}$ & 0.0397 & 0.2713 & 0.3608 & 0.0973 & 0.2133 & 0.0176 & 0.4 & 44.8828 & 8 \\
\hline 234 & 8 8TB101 & OLH & 0.0721 & 0.2711 & 0.3708 & 0.1010 & 0.1781 & 0.0070 & 0.1 & 46.4766 & 8 \\
\hline 235 & $8 \mathrm{~TB} 102$ & $\mathrm{OLH}$ & 0.0590 & 0.2649 & 0.3735 & 0.1065 & 0.1955 & 0.0006 & 1 & 62.4141 & 8 \\
\hline 236 & $8 \mathrm{~TB} 103$ & $\mathrm{OLH}$ & 0.0477 & 0.2705 & 0.3647 & 0.1016 & 0.2148 & 0.0008 & 0.4 & 44.0859 & 8 \\
\hline *237 & 8TB104 & $\mathrm{OLH}$ & 0.0607 & 0.2563 & 0.3674 & 0.1067 & 0.2024 & 0.0064 & 0 & 43.2891 & 8 \\
\hline 238 & $8 \mathrm{~TB} 105$ & $\mathrm{OLH}$ & 0.0670 & 0.2541 & 0.3746 & 0.0947 & 0.1996 & 0.0101 & 0.5 & 61.2188 & 8 \\
\hline *239 & 8TB106 & OLH & 0.0698 & 0.2545 & 0.3610 & 0.0996 & 0.2149 & 0.0002 & 0.3 & 61.6172 & 8 \\
\hline 240 & $8 \mathrm{~TB} 107$ & OLH & 0.0443 & 0.2735 & 0.3727 & 0.1018 & 0.1849 & 0.0229 & 1 & 37.3125 & 8 \\
\hline 241 & 8TB108 & $\mathrm{OLH}$ & 0.0522 & 0.2551 & 0.3741 & 0.1064 & 0.1942 & 0.0180 & 1.9 & 18.1875 & 8 \\
\hline$* 242$ & 8TB109 & $\mathrm{OLH}$ & 0.0448 & 0.2737 & 0.3633 & 0.1011 & 0.1950 & 0.0221 & 1.2 & 35.3203 & 8 \\
\hline 243 & $8 \mathrm{~TB} 110$ & $\mathrm{OLH}$ & 0.0516 & 0.2553 & 0.3616 & 0.1009 & 0.2108 & 0.0198 & 1.3 & 17.3906 & 8 \\
\hline 244 & 8TB111 & $\mathrm{OLH}$ & 0.0704 & 0.2675 & 0.3697 & 0.0955 & 0.1746 & 0.0223 & 1.7 & 30.9375 & 8 \\
\hline 245 & $8 \mathrm{~TB} 112$ & OLH & 0.0692 & 0.2643 & 0.3744 & 0.0942 & 0.1845 & 0.0134 & 1.3 & 21.7734 & 8 \\
\hline *246 & $8 \mathrm{~TB} 113$ & $\mathrm{OLH}$ & 0.0431 & 0.2687 & 0.3668 & 0.0957 & 0.2112 & 0.0145 & 1.5 & 29.7422 & 8 \\
\hline 247 & 8TB114 & $\mathrm{OLH}$ & 0.0658 & 0.2547 & 0.3671 & 0.0941 & 0.2023 & 0.0161 & 1 & 38.9063 & 8 \\
\hline 248 & $8 \mathrm{~TB} 115$ & $\mathrm{OLH}$ & 0.0636 & 0.2679 & 0.3740 & 0.1063 & 0.1788 & 0.0095 & 1.8 & 29.3438 & 8 \\
\hline *249 & $8 \mathrm{~TB} 116$ & $\mathrm{OLH}$ & 0.0681 & 0.2619 & 0.3685 & 0.1034 & 0.1964 & 0.0017 & 1.4 & 16.1953 & 8 \\
\hline 250 & $8 \mathrm{~TB} 117$ & $\mathrm{OLH}$ & 0.0715 & 0.2759 & 0.3666 & 0.1058 & 0.1695 & 0.0107 & 1.1 & 16.5938 & 8 \\
\hline 251 & 8TB118 & $\mathrm{OLH}$ & 0.0454 & 0.2647 & 0.3656 & 0.1044 & 0.2088 & 0.0110 & 1.8 & 28.1484 & 8 \\
\hline 252 & 8TB119 & $\mathrm{OLH}$ & 0.0528 & 0.2757 & 0.3743 & 0.0943 & 0.1983 & 0.0047 & 1.7 & 22.1719 & 8 \\
\hline 253 & $8 \mathrm{~TB} 120$ & $\mathrm{OLH}$ & 0.0579 & 0.2765 & 0.3620 & 0.0989 & 0.2000 & 0.0048 & 1.3 & 30.5391 & 8 \\
\hline 254 & 8TB121 & OLH & 0.0550 & 0.2701 & 0.3731 & 0.1057 & 0.1835 & 0.0126 & 0.2 & 32.1328 & 8 \\
\hline 255 & 8TB122 & $\mathrm{OLH}$ & 0.0619 & 0.2589 & 0.3691 & 0.1031 & 0.1833 & 0.0238 & 0.5 & 23.7656 & 8 \\
\hline 256 & $8 \mathrm{~TB} 123$ & $\mathrm{OLH}$ & 0.0687 & 0.2745 & 0.3653 & 0.1008 & 0.1757 & 0.0151 & 0.2 & 27.3516 & 8 \\
\hline 257 & 8TB124 & $\mathrm{OLH}$ & 0.0494 & 0.2637 & 0.3639 & 0.1056 & 0.2018 & 0.0157 & 0.4 & 33.3281 & 8 \\
\hline$* 258$ & $8 \mathrm{~TB} 125$ & $\mathrm{OLH}$ & 0.0471 & 0.2669 & 0.3737 & 0.0951 & 0.1961 & 0.0211 & 0.2 & 15 & 8 \\
\hline 259 & $8 \mathrm{~TB} 126$ & $\mathrm{OLH}$ & 0.0709 & 0.2561 & 0.3710 & 0.0990 & 0.1875 & 0.0155 & 0.9 & 31.3359 & 8 \\
\hline 260 & 8TB127 & $\mathrm{OLH}$ & 0.0596 & 0.2755 & 0.3672 & 0.0976 & 0.1819 & 0.0182 & 0.8 & 24.1641 & 8 \\
\hline 261 & 8TB128 & $\mathrm{OLH}$ & 0.0732 & 0.2717 & 0.3732 & 0.1002 & 0.1787 & 0.0029 & 0.8 & 17.7891 & 8 \\
\hline 262 & 8TB129 & $\mathrm{OLH}$ & 0.0573 & 0.2653 & 0.3698 & 0.1068 & 0.1934 & 0.0074 & 0.1 & 39.3047 & 8 \\
\hline *263 & $8 \mathrm{~TB} 130$ & $\mathrm{OLH}$ & 0.0653 & 0.2747 & 0.3643 & 0.1017 & 0.1828 & 0.0112 & 0.9 & 26.1563 & 8 \\
\hline 264 & 8 TB131 & $\mathrm{OLH}$ & 0.0647 & 0.2685 & 0.3680 & 0.0950 & 0.1999 & 0.0039 & 0.4 & 25.7578 & 8 \\
\hline 265 & 8TB132 & OLH & 0.0664 & 0.2623 & 0.3749 & 0.0983 & 0.1877 & 0.0105 & 0.2 & 26.5547 & 8 \\
\hline 266 & 8TB133 & $\mathrm{OLH}$ & 0.0738 & 0.2539 & 0.3659 & 0.0999 & 0.2008 & 0.0058 & 0.2 & 23.3672 & 8 \\
\hline 267 & 6TB001 & EV & 0.1102 & 0.2529 & 0.3755 & 0.0928 & 0.1438 & 0.0248 & 0 & 15 & 6 \\
\hline 268 & 6TB002 & EV & 0.0380 & 0.2785 & 0.3755 & 0.0928 & 0.2152 & 0.0000 & 0 & 15 & 6 \\
\hline 269 & 6ТВ003 & EV & 0.0788 & 0.2529 & 0.3603 & 0.0928 & 0.2152 & 0.0000 & 0 & 15 & 6 \\
\hline 270 & 6ТВ004 & EV & 0.0380 & 0.2529 & 0.3755 & 0.1074 & 0.2014 & 0.0248 & 0 & 15 & 6 \\
\hline 271 & 6ТВ005 & EV & 0.0380 & 0.2785 & 0.3603 & 0.0928 & 0.2056 & 0.0248 & 0 & 15 & 6 \\
\hline 272 & 6ТВ006 & EV & 0.1100 & 0.2785 & 0.3603 & 0.1074 & 0.1438 & 0.0000 & 0 & 15 & 6 \\
\hline 273 & 6TB007 & EV & 0.1102 & 0.2529 & 0.3755 & 0.0928 & 0.1438 & 0.0248 & 0 & 66 & 6 \\
\hline 274 & 6TB008 & EV & 0.0380 & 0.2785 & 0.3755 & 0.0928 & 0.2152 & 0.0000 & 0 & 66 & 6 \\
\hline 275 & 6ТВ009 & EV & 0.0788 & 0.2529 & 0.3603 & 0.0928 & 0.2152 & 0.0000 & 0 & 66 & 6 \\
\hline 276 & 6ТВ010 & EV & 0.0380 & 0.2529 & 0.3755 & 0.1074 & 0.2014 & 0.0248 & 0 & 66 & 6 \\
\hline 277 & 6ТВ011 & EV & 0.0380 & 0.2785 & 0.3603 & 0.0928 & 0.2056 & 0.0248 & 0 & 66 & 6 \\
\hline 278 & 6TB012 & EV & 0.1100 & 0.2785 & 0.3603 & 0.1074 & 0.1438 & 0.0000 & 0 & 66 & 6 \\
\hline 279 & 6TB013 & EV & 0.1102 & 0.2529 & 0.3755 & 0.0928 & 0.1438 & 0.0248 & 2 & 15 & 6 \\
\hline
\end{tabular}


Table 33. Envelope B Design Matrix for OLI Simulations (cont'd)

\begin{tabular}{|c|c|c|c|c|c|c|c|c|c|c|c|}
\hline $\begin{array}{l}\text { Run } \\
\text { No. }\end{array}$ & Test ID & Type & $\mathrm{x}_{\mathrm{AlO} 2}$ & $\mathrm{x}_{\mathrm{CO} 3}$ & $\mathrm{x}_{\mathrm{NO} 3}$ & $\mathrm{x}_{\mathrm{OH}}$ & $\mathrm{x}_{\mathrm{SO} 4}$ & $\mathrm{x}_{\mathrm{C} 2 \mathrm{O} 4}$ & SBS/Feed & Temp & $\begin{array}{l}\mathrm{Na} \\
\mathrm{M} \\
\end{array}$ \\
\hline 280 & 6TB014 & EV & 0.0380 & 0.2785 & 0.3755 & 0.0928 & 0.2152 & 0.0000 & 2 & 15 & 6 \\
\hline 281 & 6TB015 & EV & 0.0788 & 0.2529 & 0.3603 & 0.0928 & 0.2152 & 0.0000 & 2 & 15 & 6 \\
\hline 282 & 6TB016 & EV & 0.0380 & 0.2529 & 0.3755 & 0.1074 & 0.2014 & 0.0248 & 2 & 15 & 6 \\
\hline 283 & 6TB017 & EV & 0.0380 & 0.2785 & 0.3603 & 0.0928 & 0.2056 & 0.0248 & 2 & 15 & 6 \\
\hline 284 & 6TB018 & EV & 0.1100 & 0.2785 & 0.3603 & 0.1074 & 0.1438 & 0.0000 & 2 & 15 & 6 \\
\hline 285 & 6TB019 & EV & 0.1102 & 0.2529 & 0.3755 & 0.0928 & 0.1438 & 0.0248 & 2 & 66 & 6 \\
\hline 286 & 6TB020 & EV & 0.0380 & 0.2785 & 0.3755 & 0.0928 & 0.2152 & 0.0000 & 2 & 66 & 6 \\
\hline 287 & 6ТВ021 & EV & 0.0788 & 0.2529 & 0.3603 & 0.0928 & 0.2152 & 0.0000 & 2 & 66 & 6 \\
\hline 288 & 6TB022 & EV & 0.0380 & 0.2529 & 0.3755 & 0.1074 & 0.2014 & 0.0248 & 2 & 66 & 6 \\
\hline 289 & 6ТВ023 & EV & 0.0380 & 0.2785 & 0.3603 & 0.0928 & 0.2056 & 0.0248 & 2 & 66 & 6 \\
\hline 290 & 6TB024 & EV & 0.1100 & 0.2785 & 0.3603 & 0.1074 & 0.1438 & 0.0000 & 2 & 66 & 6 \\
\hline 291 & 6TB025 & $\mathrm{OLH}$ & 0.0988 & 0.2617 & 0.3635 & 0.0936 & 0.1741 & 0.0083 & 0.9 & 40.1016 & 6 \\
\hline 292 & 6TB026 & $\mathrm{OLH}$ & 0.0857 & 0.2743 & 0.3637 & 0.0998 & 0.1722 & 0.0043 & 0.1 & 16.9922 & 6 \\
\hline *293 & 6TB027 & $\mathrm{OLH}$ & 0.0942 & 0.2583 & 0.3703 & 0.0960 & 0.1752 & 0.0060 & 0.8 & 34.9219 & 6 \\
\hline 294 & 6TB028 & $\mathrm{OLH}$ & 0.0954 & 0.2727 & 0.3730 & 0.0930 & 0.1570 & 0.0089 & 0.1 & 33.7266 & 6 \\
\hline 295 & 6ТВ029 & $\mathrm{OLH}$ & 0.1096 & 0.2585 & 0.3611 & 0.1043 & 0.1665 & 0.0000 & 0.6 & 22.5703 & 6 \\
\hline 296 & 6TB030 & $\mathrm{OLH}$ & 0.0948 & 0.2781 & 0.3675 & 0.1041 & 0.1475 & 0.0079 & 0.3 & 34.125 & 6 \\
\hline 297 & 6TB031 & $\mathrm{OLH}$ & 0.0761 & 0.2543 & 0.3722 & 0.1024 & 0.1906 & 0.0045 & 0.6 & 28.5469 & 6 \\
\hline *298 & 6TB032 & $\mathrm{OLH}$ & 0.0925 & 0.2575 & 0.3603 & 0.0977 & 0.1685 & 0.0234 & 0.6 & 20.9766 & 6 \\
\hline 299 & 6TB033 & $\mathrm{OLH}$ & 0.0976 & 0.2721 & 0.3652 & 0.0953 & 0.1568 & 0.0130 & 0 & 30.1406 & 6 \\
\hline 300 & 6TB034 & $\mathrm{OLH}$ & 0.0874 & 0.2529 & 0.3728 & 0.0963 & 0.1712 & 0.0194 & 0.1 & 38.1094 & 6 \\
\hline *301 & 6TB035 & $\mathrm{OLH}$ & 0.0846 & 0.2591 & 0.3654 & 0.1074 & 0.1640 & 0.0196 & 0.3 & 22.9688 & 6 \\
\hline 302 & 6ТВ036 & $\mathrm{OLH}$ & 0.0931 & 0.2693 & 0.3629 & 0.1027 & 0.1528 & 0.0192 & 0.8 & 36.5156 & 6 \\
\hline *303 & 6ТВ037 & $\mathrm{OLH}$ & 0.0982 & 0.2615 & 0.3718 & 0.1048 & 0.1474 & 0.0163 & 0.6 & 31.7344 & 6 \\
\hline 304 & 6TB038 & $\mathrm{OLH}$ & 0.0863 & 0.2741 & 0.3700 & 0.1038 & 0.1451 & 0.0207 & 0 & 26.9531 & 6 \\
\hline 305 & 6ТВ039 & $\mathrm{OLH}$ & 0.1101 & 0.2607 & 0.3624 & 0.0969 & 0.1590 & 0.0109 & 1.3 & 18.9844 & 6 \\
\hline *306 & 6TB040 & $\mathrm{OLH}$ & 0.0886 & 0.2783 & 0.3665 & 0.0931 & 0.1720 & 0.0016 & 1.7 & 28.9453 & 6 \\
\hline 307 & 6ТВ041 & $\mathrm{OLH}$ & 0.0812 & 0.2565 & 0.3709 & 0.0933 & 0.1883 & 0.0099 & 1.5 & 20.5781 & 6 \\
\hline *308 & 6TB042 & $\mathrm{OLH}$ & 0.1005 & 0.2681 & 0.3754 & 0.0966 & 0.1583 & 0.0012 & 1.3 & 25.3594 & 6 \\
\hline 309 & 6ТВ043 & $\mathrm{OLH}$ & 0.0920 & 0.2537 & 0.3646 & 0.1054 & 0.1767 & 0.0078 & 2 & 20.1797 & 6 \\
\hline 310 & 6TB044 & $\mathrm{OLH}$ & 0.1084 & 0.2719 & 0.3607 & 0.1015 & 0.1543 & 0.0033 & 1.4 & 38.5078 & 6 \\
\hline 311 & 6TB045 & $\mathrm{OLH}$ & 0.1090 & 0.2601 & 0.3750 & 0.1030 & 0.1458 & 0.0072 & 1.6 & 36.1172 & 6 \\
\hline *312 & 6TB046 & OLH & 0.0766 & 0.2603 & 0.3651 & 0.0992 & 0.1810 & 0.0178 & 1.9 & 34.5234 & 6 \\
\hline 313 & 6ТВ047 & $\mathrm{OLH}$ & 0.0897 & 0.2665 & 0.3623 & 0.0937 & 0.1636 & 0.0242 & 1 & 18.5859 & 6 \\
\hline 314 & 6TB048 & OLH & 0.1010 & 0.2609 & 0.3711 & 0.0986 & 0.1443 & 0.0240 & 1.6 & 36.9141 & 6 \\
\hline 315 & 6ТВ049 & $\mathrm{OLH}$ & 0.0880 & 0.2751 & 0.3684 & 0.0935 & 0.1567 & 0.0184 & 2 & 37.7109 & 6 \\
\hline 316 & 6ТВ050 & $\mathrm{OLH}$ & 0.0817 & 0.2773 & 0.3613 & 0.1055 & 0.1595 & 0.0147 & 1.5 & 19.7813 & 6 \\
\hline 317 & 6ТВ051 & $\mathrm{OLH}$ & 0.0789 & 0.2769 & 0.3748 & 0.1006 & 0.1443 & 0.0246 & 1.7 & 19.3828 & 6 \\
\hline$* 318$ & 6ТВ052 & $\mathrm{OLH}$ & 0.1045 & 0.2579 & 0.3632 & 0.0984 & 0.1742 & 0.0019 & 1 & 43.6875 & 6 \\
\hline 319 & 6TB053 & $\mathrm{OLH}$ & 0.0965 & 0.2763 & 0.3617 & 0.0938 & 0.1649 & 0.0068 & 0.1 & 62.8125 & 6 \\
\hline 320 & 6TB054 & $\mathrm{OLH}$ & 0.1039 & 0.2577 & 0.3725 & 0.0991 & 0.1641 & 0.0027 & 0.8 & 45.6797 & 6 \\
\hline 321 & 6TB055 & $\mathrm{OLH}$ & 0.0971 & 0.2761 & 0.3742 & 0.0993 & 0.1483 & 0.0050 & 0.7 & 63.6094 & 6 \\
\hline 322 & 6TB056 & $\mathrm{OLH}$ & 0.0783 & 0.2639 & 0.3661 & 0.1047 & 0.1845 & 0.0025 & 0.3 & 50.0625 & 6 \\
\hline 323 & 6TB057 & $\mathrm{OLH}$ & 0.0795 & 0.2671 & 0.3614 & 0.1060 & 0.1746 & 0.0114 & 0.8 & 59.2266 & 6 \\
\hline *324 & 6ТВ058 & $\mathrm{OLH}$ & 0.1056 & 0.2627 & 0.3690 & 0.1046 & 0.1479 & 0.0103 & 0.5 & 51.2578 & 6 \\
\hline 325 & 6ТВ059 & $\mathrm{OLH}$ & 0.0829 & 0.2767 & 0.3687 & 0.1062 & 0.1568 & 0.0087 & 1 & 42.0938 & 6 \\
\hline 326 & 6TB060 & $\mathrm{OLH}$ & 0.0851 & 0.2635 & 0.3618 & 0.0939 & 0.1803 & 0.0153 & 0.2 & 51.6563 & 6 \\
\hline
\end{tabular}


Table 33. Envelope B Design Matrix for OLI Simulations (cont'd)

\begin{tabular}{|c|c|c|c|c|c|c|c|c|c|c|c|}
\hline $\begin{array}{l}\text { Run } \\
\text { No. }\end{array}$ & Test ID & Type & $\mathrm{x}_{\mathrm{AlO} 2}$ & $\mathrm{x}_{\mathrm{CO} 3}$ & $\mathrm{x}_{\mathrm{NO} 3}$ & $\mathrm{x}_{\mathrm{OH}}$ & $\mathrm{x}_{\mathrm{SO} 4}$ & $\mathrm{x}_{\mathrm{C} 2 \mathrm{O} 4}$ & SBS/Feed & Temp & $\begin{array}{l}\mathrm{Na} \\
\mathrm{M} \\
\end{array}$ \\
\hline 327 & 6TB061 & $\mathrm{OLH}$ & 0.0806 & 0.2695 & 0.3673 & 0.0968 & 0.1627 & 0.0231 & 0.6 & 64.8047 & 6 \\
\hline 328 & 6TB062 & OLH & 0.0772 & 0.2555 & 0.3692 & 0.0944 & 0.1896 & 0.0141 & 0.9 & 64.4063 & 6 \\
\hline 329 & 6TB063 & $\mathrm{OLH}$ & 0.1033 & 0.2667 & 0.3702 & 0.0958 & 0.1503 & 0.0138 & 0.3 & 52.8516 & 6 \\
\hline 330 & 6TB064 & $\mathrm{OLH}$ & 0.0959 & 0.2557 & 0.3615 & 0.1059 & 0.1608 & 0.0202 & 0.3 & 58.8281 & 6 \\
\hline 331 & 6TB065 & $\mathrm{OLH}$ & 0.0908 & 0.2549 & 0.3738 & 0.1014 & 0.1591 & 0.0200 & 0.7 & 50.4609 & 6 \\
\hline *332 & 6TB066 & $\mathrm{OLH}$ & 0.0937 & 0.2613 & 0.3627 & 0.0945 & 0.1756 & 0.0122 & 1.8 & 48.8672 & 6 \\
\hline 333 & 6TB067 & OLH & 0.0869 & 0.2725 & 0.3667 & 0.0971 & 0.1758 & 0.0010 & 1.5 & 57.2344 & 6 \\
\hline *334 & 6TB068 & $\mathrm{OLH}$ & 0.0800 & 0.2569 & 0.3705 & 0.0994 & 0.1835 & 0.0097 & 1.8 & 53.6484 & 6 \\
\hline 335 & 6TB069 & $\mathrm{OLH}$ & 0.0993 & 0.2677 & 0.3719 & 0.0946 & 0.1573 & 0.0091 & 1.6 & 47.6719 & 6 \\
\hline 336 & 6ТВ070 & $\mathrm{OLH}$ & 0.1016 & 0.2645 & 0.3621 & 0.1051 & 0.1630 & 0.0037 & 1.8 & 66 & 6 \\
\hline *337 & 6TB071 & $\mathrm{OLH}$ & 0.0778 & 0.2753 & 0.3648 & 0.1012 & 0.1716 & 0.0093 & 1.1 & 49.6641 & 6 \\
\hline 338 & 6TB072 & $\mathrm{OLH}$ & 0.0891 & 0.2559 & 0.3686 & 0.1026 & 0.1772 & 0.0066 & 1.2 & 56.8359 & 6 \\
\hline 339 & 6TB073 & OLH & 0.0755 & 0.2597 & 0.3626 & 0.1000 & 0.1804 & 0.0219 & 1.2 & 63.2109 & 6 \\
\hline 340 & 6TB074 & $\mathrm{OLH}$ & 0.0914 & 0.2661 & 0.3660 & 0.0934 & 0.1657 & 0.0174 & 1.9 & 41.6953 & 6 \\
\hline 341 & 6TB075 & $\mathrm{OLH}$ & 0.0834 & 0.2567 & 0.3715 & 0.0985 & 0.1763 & 0.0136 & 1.1 & 54.8438 & 6 \\
\hline 342 & 6TB076 & $\mathrm{OLH}$ & 0.0840 & 0.2629 & 0.3678 & 0.1052 & 0.1592 & 0.0209 & 1.6 & 55.2422 & 6 \\
\hline *343 & 6TB077 & $\mathrm{OLH}$ & 0.0823 & 0.2691 & 0.3609 & 0.1019 & 0.1714 & 0.0143 & 1.8 & 54.4453 & 6 \\
\hline 344 & 6TB078 & $\mathrm{OLH}$ & 0.0749 & 0.2775 & 0.3699 & 0.1003 & 0.1583 & 0.0190 & 1.8 & 57.6328 & 6 \\
\hline *345 & 6TB079 & $\mathrm{OLH}$ & 0.0744 & 0.2657 & 0.3679 & 0.1001 & 0.1796 & 0.0124 & 1 & 40.5 & 6 \\
\hline 346 & 6ТВ080 & $\mathrm{OLH}$ & 0.0499 & 0.2697 & 0.3723 & 0.1066 & 0.1850 & 0.0165 & 1.1 & 40.8984 & 6 \\
\hline 347 & 6TB081 & $\mathrm{OLH}$ & 0.0630 & 0.2571 & 0.3721 & 0.1004 & 0.1869 & 0.0205 & 1.9 & 64.0078 & 6 \\
\hline 348 & 6TB082 & OLH & 0.0545 & 0.2731 & 0.3655 & 0.1042 & 0.1839 & 0.0188 & 1.2 & 46.0781 & 6 \\
\hline 349 & 6ТВ083 & $\mathrm{OLH}$ & 0.0533 & 0.2587 & 0.3628 & 0.1072 & 0.2021 & 0.0159 & 1.9 & 47.2734 & 6 \\
\hline *350 & 6TB084 & $\mathrm{OLH}$ & 0.0391 & 0.2729 & 0.3747 & 0.0959 & 0.1926 & 0.0248 & 1.4 & 58.4297 & 6 \\
\hline 351 & 6ТВ085 & $\mathrm{OLH}$ & 0.0539 & 0.2533 & 0.3683 & 0.0961 & 0.2116 & 0.0169 & 1.7 & 46.875 & 6 \\
\hline 352 & 6ТВ086 & $\mathrm{OLH}$ & 0.0727 & 0.2771 & 0.3636 & 0.0978 & 0.1685 & 0.0203 & 1.4 & 52.4531 & 6 \\
\hline *353 & 6TB087 & $\mathrm{OLH}$ & 0.0562 & 0.2739 & 0.3755 & 0.1025 & 0.1906 & 0.0014 & 1.4 & 60.0234 & 6 \\
\hline 354 & 6ТВ088 & $\mathrm{OLH}$ & 0.0511 & 0.2593 & 0.3706 & 0.1049 & 0.2023 & 0.0118 & 2 & 50.8594 & 6 \\
\hline$* 355$ & 6TB089 & $\mathrm{OLH}$ & 0.0613 & 0.2785 & 0.3630 & 0.1039 & 0.1879 & 0.0054 & 1.9 & 42.8906 & 6 \\
\hline 356 & 6ТВ090 & $\mathrm{OLH}$ & 0.0641 & 0.2723 & 0.3704 & 0.0928 & 0.1952 & 0.0052 & 1.7 & 58.0313 & 6 \\
\hline 357 & 6TB091 & $\mathrm{OLH}$ & 0.0556 & 0.2621 & 0.3729 & 0.0975 & 0.2063 & 0.0056 & 1.2 & 44.4844 & 6 \\
\hline 358 & 6ТВ092 & $\mathrm{OLH}$ & 0.0505 & 0.2699 & 0.3640 & 0.0954 & 0.2117 & 0.0085 & 1.4 & 49.2656 & 6 \\
\hline 359 & 6ТВ093 & OLH & 0.0624 & 0.2573 & 0.3658 & 0.0965 & 0.2140 & 0.0041 & 2 & 54.0469 & 6 \\
\hline$* 360$ & 6ТВ094 & $\mathrm{OLH}$ & 0.0386 & 0.2707 & 0.3734 & 0.1033 & 0.2001 & 0.0140 & 0.7 & 62.0156 & 6 \\
\hline 361 & 6TB095 & OLH & 0.0602 & 0.2531 & 0.3693 & 0.1071 & 0.1871 & 0.0233 & 0.3 & 52.0547 & 6 \\
\hline 362 & 6TB096 & $\mathrm{OLH}$ & 0.0675 & 0.2749 & 0.3649 & 0.1069 & 0.1708 & \begin{tabular}{|l}
0.0149 \\
\end{tabular} & 0.5 & 60.4219 & 6 \\
\hline 363 & 6ТВ097 & $\mathrm{OLH}$ & 0.0482 & 0.2633 & 0.3604 & 0.1036 & 0.2008 & 0.0236 & 0.7 & 55.6406 & 6 \\
\hline 364 & 6ТВ098 & $\mathrm{OLH}$ & 0.0567 & 0.2777 & 0.3712 & 0.0949 & 0.1824 & 0.0171 & 0 & 60.8203 & 6 \\
\hline$* 365$ & 6ТВ099 & $\mathrm{OLH}$ & 0.0403 & 0.2595 & 0.3751 & 0.0987 & 0.2049 & 0.0215 & 0.6 & 42.4922 & 6 \\
\hline 366 & 6TB100 & $\mathrm{OLH}$ & 0.0397 & 0.2713 & 0.3608 & 0.0973 & 0.2133 & 0.0176 & 0.4 & 44.8828 & 6 \\
\hline 367 & 6TB101 & $\mathrm{OLH}$ & 0.0721 & 0.2711 & 0.3708 & 0.1010 & 0.1781 & 0.0070 & 0.1 & 46.4766 & 6 \\
\hline *368 & 6TB102 & $\mathrm{OLH}$ & 0.0590 & 0.2649 & 0.3735 & 0.1065 & 0.1955 & 0.0006 & 1 & 62.4141 & 6 \\
\hline 369 & 6TB103 & $\mathrm{OLH}$ & 0.0477 & 0.2705 & 0.3647 & 0.1016 & 0.2148 & 0.0008 & 0.4 & 44.0859 & 6 \\
\hline 370 & 6TB104 & $\mathrm{OLH}$ & 0.0607 & 0.2563 & 0.3674 & 0.1067 & 0.2024 & 0.0064 & 0 & 43.2891 & 6 \\
\hline 371 & 6TB105 & $\mathrm{OLH}$ & 0.0670 & 0.2541 & 0.3746 & 0.0947 & 0.1996 & 0.0101 & 0.5 & 61.2188 & 6 \\
\hline *372 & 6TB106 & $\mathrm{OLH}$ & 0.0698 & 0.2545 & 0.3610 & 0.0996 & 0.2149 & 0.0002 & 0.3 & 61.6172 & 6 \\
\hline 373 & 6TB107 & $\mathrm{OLH}$ & 0.0443 & 0.2735 & 0.3727 & 0.1018 & 0.1849 & 0.0229 & 1 & 37.3125 & 6 \\
\hline
\end{tabular}


Table 33. Envelope B Design Matrix for OLI Simulations (cont'd)

\begin{tabular}{|c|c|c|c|c|c|c|c|c|c|c|c|}
\hline $\begin{array}{l}\text { Run } \\
\text { No. }\end{array}$ & Test ID & Type & $\mathrm{x}_{\mathrm{AlO} 2}$ & $\mathrm{x}_{\mathrm{CO} 3}$ & $\mathrm{x}_{\mathrm{NO} 3}$ & $\mathrm{x}_{\mathrm{OH}}$ & $\mathrm{x}_{\mathrm{SO} 4}$ & $\mathrm{x}_{\mathrm{C} 2 \mathrm{O} 4}$ & SBS/F & Temp & $\begin{array}{l}\mathrm{Na} \\
\mathrm{M}\end{array}$ \\
\hline 374 & 6TB108 & $\mathrm{OLH}$ & 0.0522 & 0.2551 & 0.3741 & 0.1064 & 0.1942 & 0.0180 & 1.9 & 18.1875 & 6 \\
\hline *375 & 6TB109 & OLH & 0.0448 & 0.2737 & 0.3633 & 0.1011 & 0.1950 & 0.0221 & 1.2 & 35.3203 & 6 \\
\hline 376 & 6TB110 & $\overline{\mathrm{OLH}}$ & 0.0516 & 0.2553 & 0.3616 & 0.1009 & 0.2108 & 0.0198 & 1.3 & 17.3906 & 6 \\
\hline 377 & 6TB111 & OLH & 0.0704 & 0.2675 & 0.3697 & 0.0955 & 0.1746 & 0.0223 & 1.7 & 30.9375 & 6 \\
\hline *378 & 6TB112 & $\overline{\mathrm{OLH}}$ & 0.0692 & 0.2643 & 0.3744 & 0.0942 & 0.1845 & 0.0134 & 1.3 & 21.7734 & 6 \\
\hline 379 & 6TB113 & OLH & 0.0431 & 0.2687 & 0.3668 & 0.0957 & 0.2112 & 0.0145 & 1.5 & 29.7422 & 6 \\
\hline *380 & 6TB114 & $\mathrm{OLH}$ & 0.0658 & 0.2547 & 0.3671 & 0.0941 & 0.2023 & 0.0161 & 1 & 38.9063 & 6 \\
\hline 381 & 6TB115 & $\mathrm{OLH}$ & 0.0636 & 0.2679 & 0.3740 & 0.1063 & 0.1788 & 0.0095 & 1.8 & 29.3438 & 6 \\
\hline 382 & 6TB116 & $\mathrm{OLH}$ & 0.0681 & 0.2619 & 0.3685 & 0.1034 & 0.1964 & 0.0017 & 1.4 & 16.1953 & 6 \\
\hline 383 & 6TB117 & $\mathrm{OLH}$ & 0.0715 & 0.2759 & 0.3666 & 0.1058 & 0.1695 & 0.0107 & 1.1 & 16.5938 & 6 \\
\hline *384 & 6TB118 & $\mathrm{OLH}$ & 0.0454 & 0.2647 & 0.3656 & 0.1044 & 0.2088 & 0.0110 & 1.8 & 28.1484 & 6 \\
\hline 385 & 6TB119 & $\mathrm{OLH}$ & 0.0528 & 0.2757 & 0.3743 & 0.0943 & 0.1983 & 0.0047 & 1.7 & 22.1719 & 6 \\
\hline *386 & 6TB120 & OLH & 0.0579 & 0.2765 & 0.3620 & 0.0989 & 0.2000 & 0.0048 & 1.3 & 30.5391 & 6 \\
\hline 387 & 6TB121 & OLH & 0.0550 & 0.2701 & 0.3731 & 0.1057 & 0.1835 & 0.0126 & 0.2 & 32.1328 & 6 \\
\hline 388 & 6TB122 & OLH & 0.0619 & 0.2589 & 0.3691 & 0.1031 & 0.1833 & 0.0238 & 0.5 & 23.7656 & 6 \\
\hline 389 & 6TB123 & OLH & 0.0687 & 0.2745 & 0.3653 & 0.1008 & 0.1757 & 0.0151 & 0.2 & 27.3516 & 6 \\
\hline 390 & 6TB124 & OLH & 0.0494 & 0.2637 & 0.3639 & 0.1056 & 0.2018 & 0.0157 & 0.4 & 33.3281 & 6 \\
\hline 391 & 6TB125 & $\mathrm{OLH}$ & 0.0471 & 0.2669 & 0.3737 & 0.0951 & 0.1961 & 0.0211 & 0.2 & 15 & 6 \\
\hline 392 & 6TB126 & OLH & 0.0709 & 0.2561 & 0.3710 & 0.0990 & 0.1875 & 0.0155 & 0.9 & 31.3359 & 6 \\
\hline *393 & 6TB127 & $\mathrm{OLH}$ & 0.0596 & 0.2755 & 0.3672 & 0.0976 & 0.1819 & 0.0182 & 0.8 & 24.1641 & 6 \\
\hline 394 & 6TB128 & $\mathrm{OLH}$ & 0.0732 & 0.2717 & 0.3732 & 0.1002 & 0.1787 & 0.0029 & 0.8 & 17.7891 & 6 \\
\hline 395 & 6TB129 & $\mathrm{OLH}$ & 0.0573 & 0.2653 & 0.3698 & 0.1068 & 0.1934 & 0.0074 & 0.1 & 39.3047 & 6 \\
\hline 396 & 6TB130 & $\mathrm{OLH}$ & 0.0653 & 0.2747 & 0.3643 & 0.1017 & 0.1828 & 0.0112 & 0.9 & 26.1563 & 6 \\
\hline 397 & 6TB131 & $\mathrm{OLH}$ & 0.0647 & 0.2685 & 0.3680 & 0.0950 & 0.1999 & 0.0039 & 0.4 & 25.7578 & 6 \\
\hline *398 & 6TB132 & OLH & 0.0664 & 0.2623 & 0.3749 & 0.0983 & 0.1877 & 0.0105 & 0.2 & 26.5547 & 6 \\
\hline 399 & 6TB133 & OLH & 0.0738 & 0.2539 & 0.3659 & 0.0999 & 0.2008 & 0.0058 & 0.2 & 23.3672 & 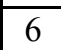 \\
\hline
\end{tabular}

* OLH Points used in fits where more points needed to capture nonlinear behavior 
Table 34. Envelope C Design Matrix for OLI Simulations

\begin{tabular}{|c|c|c|c|c|c|c|c|c|c|c|}
\hline $\begin{array}{l}\text { Run } \\
\text { No. }\end{array}$ & Test ID & Type & $\mathrm{x}_{\mathrm{AlO} 2}$ & $\mathrm{x}_{\mathrm{CO} 3}$ & $\mathrm{x}_{\mathrm{NO} 2}$ & $\mathrm{x}_{\mathrm{OH}}$ & $\mathrm{x}_{\mathrm{SO} 4}$ & SBS/Feed & Temp & $\begin{array}{l}\mathrm{Na} \\
\mathrm{M} \\
\end{array}$ \\
\hline 1 & 10TC001 & EV & 0.1319 & 0.3282 & 0.3927 & 0.0828 & 0.0644 & 0 & 15 & 10 \\
\hline 2 & 10TC002 & $\overline{E V}$ & 0.1258 & 0.2984 & 0.3926 & 0.1099 & 0.0733 & 0 & 15 & 10 \\
\hline 3 & 10TC003 & EV & 0.0413 & 0.2984 & 0.5131 & 0.0828 & 0.0644 & 0 & 15 & 10 \\
\hline 4 & $10 \mathrm{TC} 004$ & $\mathrm{EV}$ & 0.0052 & 0.3282 & 0.4923 & 0.1099 & 0.0644 & 0 & 15 & 10 \\
\hline 5 & $10 \mathrm{TC} 005$ & EV & 0.0052 & 0.3282 & 0.5105 & 0.0828 & 0.0733 & 0 & 15 & 10 \\
\hline 6 & $10 \mathrm{TC} 006$ & EV & 0.1319 & 0.3282 & 0.3927 & 0.0828 & 0.0644 & 0 & 66 & 10 \\
\hline 7 & 10TC007 & EV & 0.1258 & 0.2984 & 0.3926 & 0.1099 & 0.0733 & 0 & 66 & 10 \\
\hline 8 & 10TC008 & EV & 0.0413 & 0.2984 & 0.5131 & 0.0828 & 0.0644 & 0 & 66 & 10 \\
\hline 9 & 10TC009 & EV & 0.0052 & 0.3282 & 0.4923 & 0.1099 & 0.0644 & 0 & 66 & 10 \\
\hline 10 & $10 \mathrm{TC} 010$ & EV & 0.0052 & 0.3282 & 0.5105 & 0.0828 & 0.0733 & 0 & 66 & 10 \\
\hline 11 & 10TC011 & EV & 0.1319 & 0.3282 & 0.3927 & 0.0828 & 0.0644 & 2 & 15 & 10 \\
\hline 12 & 10TC012 & $\overline{E V}$ & 0.1258 & 0.2984 & 0.3926 & 0.1099 & 0.0733 & 2 & 15 & 10 \\
\hline 13 & $10 \mathrm{TC} 013$ & $\overline{E V}$ & 0.0413 & 0.2984 & 0.5131 & 0.0828 & 0.0644 & 2 & 15 & 10 \\
\hline 14 & 10TC014 & EV & 0.0052 & 0.3282 & 0.4923 & 0.1099 & 0.0644 & 2 & 15 & 10 \\
\hline 15 & $10 \mathrm{TC} 015$ & $\mathrm{EV}$ & 0.0052 & 0.3282 & 0.5105 & 0.0828 & 0.0733 & 2 & 15 & 10 \\
\hline 16 & 10TC016 & EV & 0.1319 & 0.3282 & 0.3927 & 0.0828 & 0.0644 & 2 & 66 & 10 \\
\hline 17 & $10 \mathrm{TC} 017$ & $\mathrm{EV}$ & 0.1258 & 0.2984 & 0.3926 & 0.1099 & 0.0733 & 2 & 66 & 10 \\
\hline 18 & 10TC018 & EV & 0.0413 & 0.2984 & 0.5131 & 0.0828 & 0.0644 & 2 & 66 & 10 \\
\hline 19 & 10TC019 & $\overline{E V}$ & 0.0052 & 0.3282 & 0.4923 & 0.1099 & 0.0644 & 2 & 66 & 10 \\
\hline 20 & $10 \mathrm{TC} 020$ & EV & 0.0052 & 0.3282 & 0.5105 & 0.0828 & 0.0733 & 2 & 66 & 10 \\
\hline 21 & $10 \mathrm{TC} 021$ & $\mathrm{OLH}$ & 0.11111 & 0.30864 & 0.42684 & 0.08852 & 0.06489 & 0.672 & 37.3125 & 10 \\
\hline 22 & 10TC022 & OLH & 0.08835 & 0.32331 & 0.43076 & 0.08894 & 0.06864 & 0.344 & 18.1875 & 10 \\
\hline$* 23$ & $10 \mathrm{TC} 023$ & OLH & 0.1032 & 0.30469 & 0.42519 & 0.10058 & 0.06634 & 0.484 & 35.3203 & 10 \\
\hline 24 & $10 \mathrm{TC} 024$ & OLH & 0.10517 & 0.32145 & 0.40338 & 0.10545 & 0.06455 & 0.719 & 17.3906 & 10 \\
\hline 25 & $10 \mathrm{TC} 025$ & OLH & 0.12992 & 0.30492 & 0.40946 & 0.08428 & 0.07142 & 0 & 30.9375 & 10 \\
\hline 26 & $10 \mathrm{TC} 026$ & OLH & 0.10418 & 0.32773 & 0.40108 & 0.09572 & 0.07129 & 0.641 & 21.7734 & 10 \\
\hline$* 27$ & $10 \mathrm{TC} 027$ & OLH & 0.07152 & 0.30003 & 0.45424 & 0.10397 & 0.07024 & 0.359 & 29.7422 & 10 \\
\hline 28 & 10TC028 & OLH & 0.10023 & 0.30376 & 0.44583 & 0.0828 & 0.06738 & 1.891 & 29.3438 & 10 \\
\hline$* 29$ & 10TC029 & OLH & 0.10913 & 0.32075 & 0.41271 & 0.09148 & 0.06593 & 1.047 & 16.1953 & 10 \\
\hline 30 & $10 \mathrm{TC} 030$ & OLH & 0.09132 & 0.2984 & 0.4387 & 0.10503 & 0.06655 & 1.563 & 16.5938 & 10 \\
\hline$* 31$ & $10 \mathrm{TC} 031$ & OLH & 0.08637 & 0.30562 & 0.44281 & 0.0919 & 0.0733 & 1.578 & 22.1719 & 10 \\
\hline 32 & 10TC032 & OLH & 0.10122 & 0.31749 & 0.42339 & 0.08746 & 0.07044 & 1.547 & 35.7188 & 10 \\
\hline 33 & 10TC033 & $\mathrm{OLH}$ & 0.11012 & 0.30841 & 0.40643 & 0.10334 & 0.0717 & 1.313 & 30.5391 & 10 \\
\hline 34 & 10TC034 & OLH & 0.08934 & 0.32308 & 0.41635 & 0.10016 & 0.07107 & 1.672 & 15.3984 & 10 \\
\hline$* 35$ & $10 \mathrm{TC} 035$ & OLH & 0.13091 & 0.30748 & 0.4081 & 0.08661 & 0.0669 & 0.875 & 48.8672 & 10 \\
\hline 36 & $10 \mathrm{TC} 036$ & OLH & 0.0933 & 0.32797 & 0.42032 & 0.09381 & 0.0646 & 0.125 & 57.2344 & 10 \\
\hline 37 & $10 \mathrm{TC} 037$ & $\mathrm{OLH}$ & 0.08043 & 0.30259 & 0.45066 & 0.10164 & 0.06468 & 0.797 & 53.6484 & 10 \\
\hline$* 38$ & 10TC038 & OLH & 0.11408 & 0.31609 & 0.39344 & 0.10969 & 0.0667 & 0.094 & 47.6719 & 10 \\
\hline 39 & 10TC039 & OLH & 0.09924 & 0.29933 & 0.43896 & 0.09042 & 0.07205 & 0.625 & 66 & 10 \\
\hline 40 & 10TC040 & OLH & 0.12794 & 0.32052 & 0.39842 & 0.08344 & 0.06968 & 0.266 & 49.6641 & 10 \\
\hline$* 41$ & $10 \mathrm{TC} 041$ & $\mathrm{OLH}$ & 0.09627 & 0.3275 & 0.40009 & 0.10291 & 0.07323 & 0.938 & 53.25 & 10 \\
\hline 42 & $10 \mathrm{TC} 042$ & OLH & 0.07251 & 0.30701 & 0.46091 & 0.09127 & 0.0683 & 1.438 & 63.2109 & 10 \\
\hline 43 & $10 \mathrm{TC} 043$ & OLH & 0.09528 & 0.31423 & 0.43914 & 0.0864 & 0.06495 & 1.953 & 41.6953 & 10 \\
\hline 44 & 10TC044 & $\mathrm{OLH}$ & 0.11507 & 0.30771 & 0.4072 & 0.10207 & 0.06795 & 1.938 & 54.8438 & 10 \\
\hline 45 & $10 \mathrm{TC} 045$ & OLH & 0.09231 & 0.32424 & 0.42144 & 0.0972 & 0.06481 & 1.484 & 65.2031 & 10 \\
\hline
\end{tabular}


Table 34. Envelope C Design Matrix for OLI Simulations (cont'd)

\begin{tabular}{|c|c|c|c|c|c|c|c|c|c|c|}
\hline $\begin{array}{l}\text { Run } \\
\text { No. }\end{array}$ & Test ID & Type & $\mathrm{x}_{\mathrm{AlO} 2}$ & $\mathrm{x}_{\mathrm{CO} 3}$ & $\mathrm{x}_{\mathrm{NO} 2}$ & $\mathrm{x}_{\mathrm{OH}}$ & $\mathrm{x}_{\mathrm{SO} 4}$ & SBS/Feed & Temp & $\begin{array}{c}\mathrm{Na} \\
\mathrm{M}\end{array}$ \\
\hline$* 46$ & 10TC046 & $\mathrm{LH}$ & 2596 & 027 & 9859 & U & 52 & 1.719 & 2422 & 10 \\
\hline 47 & 10TC047 & OLH & .08142 & 3268 & 43517 & 0.08449 & 0.07212 & 1.188 & 54.4453 & 10 \\
\hline 48 & 10TC048 & OLH & 0.12398 & 0.31144 & 0.39475 & 0.0991 & 0.07073 & 1.391 & 48.4688 & 10 \\
\hline$* 49$ & 10TC049 & OLH & 0.07647 & 0.32634 & 0.41944 & 0.10863 & 0.06912 & 1.984 & 57.6328 & 10 \\
\hline 50 & 10TC050 & OLH & 0.12101 & 0.30422 & 0.41908 & 0.08788 & 0.06781 & 0.156 & 40.1016 & 10 \\
\hline 51 & 10TC051 & OLH & 0.10715 & 0.32564 & 0.41684 & 0.08534 & 0.06503 & 0.547 & 16.9922 & 10 \\
\hline$* 52$ & 10TC052 & OLH & 0.12002 & 0.30399 & 0.40316 & 0.10461 & 0.06822 & 0.219 & 34.9219 & 10 \\
\hline 53 & 10TC053 & OLH & 0.07548 & 0.31121 & 0.44851 & 0.09317 & 0.07163 & 0.203 & 22.5703 & 10 \\
\hline 54 & 10TC054 & OLH & 0.07746 & 0.31493 & 0.45044 & 0.08471 & 0.07246 & 0.922 & 34.125 & 10 \\
\hline$* 55$ & 10TC055 & OLH & 0.12299 & 0.30981 & 0.39738 & 0.09826 & 0.07156 & 0.828 & 28.5469 & 10 \\
\hline 56 & 10TC056 & OLH & 0.0834 & 0.32611 & 0.42013 & 0.09783 & 0.07253 & 0.703 & 39.7031 & 10 \\
\hline 57 & 10TC057 & OLH & 0.08736 & 0.31074 & 0.45126 & 0.08555 & 0.06509 & 1.234 & 20.9766 & 10 \\
\hline 58 & $10 \mathrm{~T}$ & OLH & 0.07944 & 772 & 071 & 0.0 & 0.0 & 1.859 & 30.1406 & 10 \\
\hline 59 & $10 \mathrm{TC}$ & OLH & 0.0735 & $\overline{143}$ & 0.46102 & 0.0 & 0.06537 & 1.141 & 38.1094 & 10 \\
\hline$* 60$ & $10 \mathrm{TC} 060$ & OLH & 0.11903 & 0.31446 & 0.39992 & 0.10037 & 0.06622 & 1.109 & 21.375 & 10 \\
\hline 61 & 10TC061 & OLH & 0.10616 & 0.30166 & 0.43486 & 0.08492 & 0.0724 & 1.625 & 22.9688 & 10 \\
\hline 62 & 10TC062 & OLH & 0.11804 & 215 & 4004 & 0.09021 & 0.0692 & 1.813 & 36.5156 & 10 \\
\hline 63 & 10TC063 & OLH & 0.09726 & 0073 & .42547 & 0.10694 & 0.0696 & 1.609 & 31.7344 & 10 \\
\hline$* 64$ & 10TC064 & OLH & 0.10221 & 0.30818 & 0.43714 & 0.08703 & 0.06544 & 0.984 & 62.0156 & 10 \\
\hline 65 & $10 \mathrm{TC} 065$ & $\mathrm{OLH}$ & 0.09033 & 2122 & 42718 & 0.09423 & 0.06704 & 0.078 & 52.0547 & 10 \\
\hline 66 & 10TC066 & OLH & 0.07845 & $\overline{0306}$ & 44905 & 0.10101 & 0.06843 & 0.781 & 60.4219 & 10 \\
\hline 67 & 10TC067 & OLH & 0.1121 & 0.31563 & 0.40321 & 0.10355 & 0.0 & 0.734 & 55.6406 & 10 \\
\hline 68 & 10TC068 & OLH & 0.11606 & 19 & 415 & 0.0 & 0.0 & 0.297 & 60.8203 & 10 \\
\hline 69 & $10 \mathrm{TC} 069$ & $\mathrm{OLH}$ & 0.07449 & 2448 & 4065 & 0.09085 & 0.06953 & 0.75 & 42.4922 & 10 \\
\hline 70 & 10TC070 & OLH & 0.09429 & 189 & 582 & 0.09762 & 0.07038 & 0.531 & 44.8828 & 10 \\
\hline 71 & $10 \mathrm{TC} 071$ & OLH & 0.07053 & 0.30632 & 755 & 0.08682 & 0.0 & 1.766 & 46.4766 & 10 \\
\hline 72 & $10 \mathrm{TC} 072$ & OLH & 0.09825 & 1377 & 0.43028 & 0.09296 & 0.06474 & 1.406 & 62.4141 & 10 \\
\hline 73 & $10 \mathrm{TC} 073$ & OLH & 0.08439 & 0.30282 & 221 & 0.1027 & 0.06788 & 1.094 & 44.0859 & 10 \\
\hline *74 & 10TC074 & OLH & 0.11309 & 703 & 544 & 0.09677 & 767 & 1.75 & 43.2891 & 10 \\
\hline 75 & $10 \mathrm{TC} 075$ & OLH & 0.08538 & 0.31004 & 0.43646 & 0.09614 & 0.07198 & 1.688 & 56.4375 & 10 \\
\hline 76 & $10 \mathrm{TC} 076$ & OLH & 0.08241 & 0.31726 & 4651 & 0.08386 & 0.06996 & 1.156 & 61.2188 & 10 \\
\hline 77 & 10TC077 & OLH & 0.06954 & 0.32704 & 449 & 0.09995 & 0.06898 & 1.531 & 61.6172 & 10 \\
\hline 78 & $10 \mathrm{TC} 078$ & OLH & 0.06855 & 0.3133 & 0.45295 & 0.09635 & 0.06885 & 1 & 40.5 & 10 \\
\hline 79 & 10TC079 & OLH & 0.02599 & 0.31796 & 0.47906 & 0.10418 & 0.07281 & 1.328 & 43.6875 & 10 \\
\hline 80 & $10 \mathrm{TC} 080$ & $\mathrm{OLH}$ & 0.04875 & 0.30329 & 514 & 0.10376 & 0.06906 & 1.656 & 62.8125 & 10 \\
\hline 81 & 10TC081 & OLH & 0.03391 & 0.32191 & 0.48071 & 0.09212 & 0.07135 & 1.516 & 45.6797 & 10 \\
\hline$* 82$ & $10 \mathrm{TC} 082$ & $\mathrm{OLH}$ & 0.03193 & 0.30515 & 0.50252 & 0.08725 & 0.07315 & 1.281 & 63.6094 & 10 \\
\hline 83 & $10 \mathrm{TC} 083$ & $\mathrm{OLH}$ & 0.00718 & 0.32168 & 0.49644 & 0.10842 & 0.06628 & 2 & 50.0625 & 10 \\
\hline 84 & $10 \mathrm{TC} 084$ & OLH & 0.03292 & 0.29887 & 0.50482 & 0.09699 & 0.0664 & 1.359 & 59.2266 & 10 \\
\hline$* 85$ & $10 \mathrm{TC} 085$ & OLH & 0.06558 & 0.32657 & 0.45166 & 0.08873 & 0.06746 & 1.641 & 51.2578 & 10 \\
\hline 86 & $10 \mathrm{TC} 086$ & OLH & 0.03688 & 0.32285 & 0.46007 & 0.1099 & 0.0703 & 0.109 & 51.6563 & 10 \\
\hline 87 & 10TC087 & OLH & 0.02797 & 0.30585 & 0.49319 & 0.10122 & 0.07177 & 0.953 & 64.8047 & 10 \\
\hline$* 88$ & $10 \mathrm{TC} 088$ & OLH & 0.04578 & 0.3282 & 0.4672 & 0.08767 & 0.07115 & 0.438 & 64.4063 & 10 \\
\hline 89 & 10TC089 & $\mathrm{OLH}$ & 0.05073 & 0.32098 & 0.46309 & 0.1008 & 0.0644 & 0.422 & 58.8281 & 10 \\
\hline 90 & 10TC090 & OLH & 0.03589 & 0.30911 & 0.48251 & 0.10524 & 0.06725 & 0.453 & 45.2813 & 10 \\
\hline
\end{tabular}


Table 34. Envelope C Design Matrix for OLI Simulations (cont'd)

\begin{tabular}{|c|c|c|c|c|c|c|c|c|c|c|}
\hline $\begin{array}{l}\text { Run } \\
\text { No. } \\
\end{array}$ & Test ID & Type & $\mathrm{x}_{\mathrm{AlO} 2}$ & $\mathrm{x}_{\mathrm{CO} 3}$ & $\mathrm{x}_{\mathrm{NO} 2}$ & $\mathrm{x}_{\mathrm{OH}}$ & $\mathrm{x}_{\mathrm{SO} 4}$ & SBS/Feed & Temp & $\begin{array}{l}\mathrm{Na} \\
\mathrm{M} \\
\end{array}$ \\
\hline 91 & 10TC091 & OLH & 0.02698 & 0.31819 & 0.49947 & 0.08936 & 0.066 & 0.688 & 50.4609 & 10 \\
\hline 92 & 10TC092 & OLH & 0.04776 & 0.30352 & 0.48955 & 0.09254 & 0.06663 & 0.328 & 65.6016 & 10 \\
\hline 93 & $10 \mathrm{TC} 093$ & $\mathrm{OLH}$ & 0.00619 & 0.31912 & 0.4978 & 0.10609 & 0.0708 & 1.125 & 32.1328 & 10 \\
\hline 94 & 10TC094 & OLH & 0.0438 & 0.29863 & 0.48558 & 0.09889 & 0.0731 & 1.875 & 23.7656 & 10 \\
\hline$* 95$ & $10 \mathrm{TC} 095$ & OLH & 0.05667 & 0.32401 & 0.45524 & 0.09106 & 0.07302 & 1.203 & 27.3516 & 10 \\
\hline 96 & 10TC096 & OLH & 0.02302 & 0.31051 & 0.51246 & 0.08301 & 0.071 & 1.906 & 33.3281 & 10 \\
\hline *97 & 10TC097 & OLH & 0.03787 & 0.32727 & 0.46694 & 0.10228 & 0.06564 & 1.375 & 15 & 10 \\
\hline 98 & 10TC098 & OLH & 0.00916 & 0.30608 & 0.50748 & 0.10927 & 0.06801 & 1.734 & 31.3359 & 10 \\
\hline 99 & 10TC099 & OLH & 0.04083 & 0.2991 & 0.50581 & 0.08979 & 0.06447 & 1.063 & 27.75 & 10 \\
\hline$* 100$ & $10 \mathrm{TC} 100$ & OLH & 0.06459 & 0.31959 & 0.44499 & 0.10143 & 0.0694 & 0.563 & 17.7891 & 10 \\
\hline 101 & 10TC101 & OLH & 0.04182 & 0.31237 & 0.46676 & 0.1063 & 0.07275 & 0.047 & 39.3047 & 10 \\
\hline 102 & $10 \mathrm{TC} 102$ & $\mathrm{OLH}$ & 0.02203 & 0.31889 & 0.4987 & 0.09063 & 0.06975 & 0.063 & 26.1563 & 10 \\
\hline 103 & 10TC103 & OLH & 0.04479 & 0.30236 & 0.48446 & 0.0955 & 0.07289 & 0.516 & 15.7969 & 10 \\
\hline$* 104$ & $10 \mathrm{TC} 104$ & $\mathrm{OLH}$ & 0.01114 & 0.31633 & 0.50731 & 0.09804 & 0.06718 & 0.281 & 25.7578 & 10 \\
\hline 105 & 10TC105 & OLH & 0.05568 & 0.2998 & 0.47073 & 0.10821 & 0.06558 & 0.813 & 26.5547 & 10 \\
\hline$* 106$ & $10 \mathrm{TC} 106$ & $\mathrm{OLH}$ & 0.01312 & 0.31516 & 0.51115 & 0.0936 & 0.06697 & 0.609 & 32.5313 & 10 \\
\hline 107 & $10 \mathrm{TC} 107$ & $\mathrm{OLH}$ & 0.06063 & 0.30026 & 0.48646 & 0.08407 & 0.06858 & 0.016 & 23.3672 & 10 \\
\hline 108 & $10 \mathrm{TC} 108$ & $\mathrm{OLH}$ & 0.01609 & 0.32238 & 0.48682 & 0.10482 & 0.06989 & 1.844 & 40.8984 & 10 \\
\hline 109 & 10TC109 & $\mathrm{OLH}$ & 0.02995 & 0.30096 & 0.48906 & 0.10736 & 0.07267 & 1.453 & 64.0078 & 10 \\
\hline 110 & 10TC110 & OLH & 0.01708 & 0.32261 & 0.50274 & 0.08809 & 0.06948 & 1.781 & 46.0781 & 10 \\
\hline$* 111$ & 10TC111 & OLH & 0.06162 & 0.3154 & 0.45739 & 0.09953 & 0.06606 & 1.797 & 58.4297 & 10 \\
\hline 112 & 10TC112 & OLH & 0.05964 & 0.31167 & 0.45546 & 0.108 & 0.06523 & 1.078 & 46.875 & 10 \\
\hline$* 113$ & $10 \mathrm{TC} 113$ & OLH & 0.01411 & 0.31679 & 0.50852 & 0.09445 & 0.06613 & 1.172 & 52.4531 & 10 \\
\hline 114 & 10TC114 & OLH & 0.0537 & 0.3005 & 0.48577 & 0.09487 & 0.06516 & 1.297 & 41.2969 & 10 \\
\hline$* 115$ & 10TC115 & $\mathrm{OLH}$ & 0.04974 & 0.31586 & 0.45464 & 0.10715 & 0.07261 & 0.766 & 60.0234 & 10 \\
\hline 116 & $10 \mathrm{TC} 116$ & OLH & 0.05766 & 0.30888 & 0.46519 & 0.09741 & 0.07086 & 0.141 & 50.8594 & 10 \\
\hline 117 & $10 \mathrm{TC} 117$ & $\mathrm{OLH}$ & 0.0636 & 0.32517 & 0.44488 & 0.09402 & 0.07233 & 0.859 & 42.8906 & 10 \\
\hline 118 & $10 \mathrm{TC} 118$ & $\mathrm{OLH}$ & 0.01807 & 0.31214 & 0.50598 & 0.09233 & 0.07148 & 0.891 & 59.625 & 10 \\
\hline 119 & 10TC119 & $\mathrm{OLH}$ & 0.03094 & 0.32494 & 0.47104 & 0.10778 & 0.0653 & 0.375 & 58.0313 & 10 \\
\hline 120 & $10 \mathrm{TC} 120$ & $\mathrm{OLH}$ & 0.01906 & 0.30445 & 0.5055 & 0.10249 & 0.0685 & 0.188 & 44.4844 & 10 \\
\hline *121 & 10TC121 & $\mathrm{OLH}$ & 0.03985 & 0.32587 & 0.48043 & 0.08576 & 0.06809 & 0.391 & 49.2656 & 10 \\
\hline 122 & $10 \mathrm{TC} 122$ & $\mathrm{OLH}$ & 0.0349 & 0.31842 & 0.46876 & 0.10567 & 0.07225 & 1.016 & 18.9844 & 10 \\
\hline 123 & $10 \mathrm{TC} 123$ & OLH & 0.04677 & 0.30538 & 0.47872 & 0.09847 & 0.07066 & 1.922 & 28.9453 & 10 \\
\hline$* 124$ & 10TC124 & $\mathrm{OLH}$ & 0.05865 & 0.32354 & 0.45685 & 0.09169 & 0.06927 & 1.219 & 20.5781 & 10 \\
\hline 125 & $10 \mathrm{TC} 125$ & OLH & 0.025 & 0.31097 & 0.50269 & 0.08915 & 0.07219 & 1.266 & 25.3594 & 10 \\
\hline 126 & 10TC126 & $\mathrm{OLH}$ & 0.02104 & 0.3147 & 0.49175 & 0.10672 & 0.06579 & 1.703 & 20.1797 & 10 \\
\hline 127 & $10 \mathrm{TC} 127$ & $\mathrm{OLH}$ & 0.06261 & 0.30213 & 0.46525 & 0.10186 & 0.06815 & 1.25 & 38.5078 & 10 \\
\hline 128 & 10TC128 & $\mathrm{OLH}$ & 0.04281 & 0.32471 & 0.47008 & 0.09508 & 0.06732 & 1.469 & 36.1172 & 10 \\
\hline 129 & 10TC129 & $\mathrm{OLH}$ & 0.06657 & 0.32028 & 0.43835 & 0.10588 & 0.06892 & 0.234 & 34.5234 & 10 \\
\hline$* 130$ & $10 \mathrm{TC} 130$ & $\mathrm{OLH}$ & 0.03886 & 0.31283 & 0.47562 & 0.09974 & 0.07295 & 0.594 & 18.5859 & 10 \\
\hline 131 & 10TC131 & $\mathrm{OLH}$ & 0.05271 & 0.32378 & 0.46369 & 0.09 & 0.06982 & 0.906 & 36.9141 & 10 \\
\hline$* 132$ & 10TC132 & $\mathrm{OLH}$ & 0.02401 & 0.30958 & 0.50046 & 0.09593 & 0.07002 & 0.25 & 37.7109 & 10 \\
\hline 133 & $10 \mathrm{TC} 133$ & $\mathrm{OLH}$ & 0.05172 & 0.31656 & 0.46944 & 0.09656 & 0.06572 & 0.313 & 24.5625 & 10 \\
\hline$* 134$ & 10TC134 & OLH & 0.05469 & 0.30934 & 0.45939 & 0.10884 & 0.06774 & 0.844 & 19.7813 & 10 \\
\hline 135 & 10TC135 & $\mathrm{OLH}$ & 0.06756 & 0.29956 & 0.47141 & 0.09275 & 0.06872 & 0.469 & 19.3828 & 10 \\
\hline 136 & $8 \mathrm{TC} 001$ & EV & 0.1319 & 0.3282 & 0.3927 & 0.0828 & 0.0644 & 0 & 15 & 8 \\
\hline 137 & $8 \mathrm{TC} 002$ & EV & 0.1258 & 0.2984 & 0.3926 & 0.1099 & 0.0733 & 0 & 15 & 8 \\
\hline
\end{tabular}


Table 34. Envelope C Design Matrix for OLI Simulations (cont'd)

\begin{tabular}{|c|c|c|c|c|c|c|c|c|c|c|}
\hline $\begin{array}{l}\text { Run } \\
\text { No. } \\
\end{array}$ & Test ID & Type & $\mathrm{x}_{\mathrm{AlO} 2}$ & $\mathrm{x}_{\mathrm{CO} 3}$ & $\mathrm{x}_{\mathrm{NO} 2}$ & $\mathrm{x}_{\mathrm{OH}}$ & $\mathrm{x}_{\mathrm{SO} 4}$ & SBS/Feed & Temp & $\begin{array}{l}\mathrm{Na} \\
\mathrm{M} \\
\end{array}$ \\
\hline 138 & $8 \mathrm{TC} 003$ & EV & 0.0413 & 0.2984 & 0.5131 & 0.0828 & 0.0644 & 0 & 15 & 8 \\
\hline 139 & 8TC004 & EV & 0.0052 & 0.3282 & 0.4923 & 0.1099 & 0.0644 & 0 & 15 & 8 \\
\hline 140 & $8 \mathrm{TC} 005$ & $\mathrm{EV}$ & 0.0052 & 0.3282 & 0.5105 & 0.0828 & 0.0733 & 0 & 15 & 8 \\
\hline 141 & $8 \mathrm{TC} 006$ & EV & 0.1319 & 0.3282 & 0.3927 & 0.0828 & 0.0644 & 0 & 66 & 8 \\
\hline 142 & $8 \mathrm{TC} 007$ & EV & 0.1258 & 0.2984 & 0.3926 & 0.1099 & 0.0733 & 0 & 66 & 8 \\
\hline 143 & $8 \mathrm{TC} 008$ & EV & 0.0413 & 0.2984 & 0.5131 & 0.0828 & 0.0644 & 0 & 66 & 8 \\
\hline 144 & 8ТC009 & EV & 0.0052 & 0.3282 & 0.4923 & 0.1099 & 0.0644 & 0 & 66 & 8 \\
\hline 145 & $8 \mathrm{TC} 010$ & EV & 0.0052 & 0.3282 & 0.5105 & 0.0828 & 0.0733 & 0 & 66 & 8 \\
\hline 146 & $8 \mathrm{TC} 011$ & EV & 0.1319 & 0.3282 & 0.3927 & 0.0828 & 0.0644 & 2 & 15 & 8 \\
\hline 147 & $8 \mathrm{TC} 012$ & $\mathrm{EV}$ & 0.1258 & 0.2984 & 0.3926 & 0.1099 & 0.0733 & 2 & 15 & 8 \\
\hline 148 & $8 \mathrm{TC} 013$ & EV & 0.0413 & 0.2984 & 0.5131 & 0.0828 & 0.0644 & 2 & 15 & 8 \\
\hline 149 & 8TC014 & EV & 0.0052 & 0.3282 & 0.4923 & 0.1099 & 0.0644 & 2 & 15 & 8 \\
\hline 150 & $8 \mathrm{TC} 015$ & EV & 0.0052 & 0.3282 & 0.5105 & 0.0828 & 0.0733 & 2 & 15 & 8 \\
\hline 151 & $8 \mathrm{TC} 016$ & EV & 0.1319 & 0.3282 & 0.3927 & 0.0828 & 0.0644 & 2 & 66 & 8 \\
\hline 152 & 8TC017 & EV & 0.1258 & 0.2984 & 0.3926 & 0.1099 & 0.0733 & 2 & 66 & 8 \\
\hline 153 & $8 \mathrm{TC} 018$ & EV & 0.0413 & 0.2984 & 0.5131 & 0.0828 & 0.0644 & 2 & 66 & 8 \\
\hline 154 & $8 \mathrm{TC} 019$ & EV & 0.0052 & 0.3282 & 0.4923 & 0.1099 & 0.0644 & 2 & 66 & 8 \\
\hline 155 & $8 \mathrm{TC} 020$ & EV & 0.0052 & 0.3282 & 0.5105 & 0.0828 & 0.0733 & 2 & 66 & 8 \\
\hline 156 & 8TC021 & $\mathrm{OLH}$ & 0.11111 & 0.30864 & 0.42684 & 0.08852 & 0.06489 & 0.672 & 37.3125 & 8 \\
\hline$* 157$ & $8 \mathrm{TC} 022$ & OLH & 0.08835 & 0.32331 & 0.43076 & 0.08894 & 0.06864 & 0.344 & 18.1875 & 8 \\
\hline 158 & $8 \mathrm{TC} 023$ & OLH & 0.1032 & 0.30469 & 0.42519 & 0.10058 & 0.06634 & 0.484 & 35.3203 & 8 \\
\hline 159 & $8 \mathrm{TC} 024$ & OLH & 0.10517 & 0.32145 & 0.40338 & 0.10545 & 0.06455 & 0.719 & 17.3906 & 8 \\
\hline$* 160$ & $8 \mathrm{TC} 025$ & $\mathrm{OLH}$ & 0.12992 & 0.30492 & 0.40946 & 0.08428 & 0.07142 & 0 & 30.9375 & 8 \\
\hline 161 & $8 \mathrm{TC} 026$ & OLH & 0.10418 & 0.32773 & 0.40108 & 0.09572 & 0.07129 & 0.641 & 21.7734 & 8 \\
\hline 162 & $8 \mathrm{TC} 027$ & $\mathrm{OLH}$ & 0.07152 & 0.30003 & 0.45424 & 0.10397 & 0.07024 & 0.359 & 29.7422 & 8 \\
\hline 163 & 8TC028 & $\mathrm{OLH}$ & 0.10023 & 0.30376 & 0.44583 & 0.0828 & 0.06738 & 1.891 & 29.3438 & 8 \\
\hline$* 164$ & $8 \mathrm{TC} 029$ & $\mathrm{OLH}$ & 0.10913 & 0.32075 & 0.41271 & 0.09148 & 0.06593 & 1.047 & 16.1953 & 8 \\
\hline 165 & $8 \mathrm{TC} 030$ & $\mathrm{OLH}$ & 0.09132 & 0.2984 & 0.4387 & 0.10503 & 0.06655 & 1.563 & 16.5938 & 8 \\
\hline 166 & $8 \mathrm{TC} 031$ & $\mathrm{OLH}$ & 0.08637 & 0.30562 & 0.44281 & 0.0919 & 0.0733 & 1.578 & 22.1719 & 8 \\
\hline 167 & $8 \mathrm{TC} 032$ & $\mathrm{OLH}$ & 0.10122 & 0.31749 & 0.42339 & 0.08746 & 0.07044 & 1.547 & 35.7188 & 8 \\
\hline 168 & $8 \mathrm{TC} 033$ & $\mathrm{OLH}$ & 0.11012 & 0.30841 & 0.40643 & 0.10334 & 0.0717 & 1.313 & 30.5391 & 8 \\
\hline 169 & $8 \mathrm{TC} 034$ & $\mathrm{OLH}$ & 0.08934 & 0.32308 & 0.41635 & 0.10016 & 0.07107 & 1.672 & 15.3984 & 8 \\
\hline$* 170$ & $8 \mathrm{TC} 035$ & $\mathrm{OLH}$ & 0.13091 & 0.30748 & 0.4081 & 0.08661 & 0.0669 & 0.875 & 48.8672 & 8 \\
\hline 171 & 8TC036 & $\mathrm{OLH}$ & 0.0933 & 0.32797 & 0.42032 & 0.09381 & 0.0646 & 0.125 & 57.2344 & 8 \\
\hline 172 & 8TC037 & $\mathrm{OLH}$ & 0.08043 & 0.30259 & 0.45066 & 0.10164 & 0.06468 & 0.797 & 53.6484 & 8 \\
\hline 173 & $8 \mathrm{TC} 038$ & $\mathrm{OLH}$ & 0.11408 & 0.31609 & 0.39344 & 0.10969 & 0.0667 & 0.094 & 47.6719 & 8 \\
\hline 174 & 8TC039 & $\mathrm{OLH}$ & 0.09924 & 0.29933 & 0.43896 & 0.09042 & 0.07205 & 0.625 & 66 & 8 \\
\hline 175 & 8TC040 & $\mathrm{OLH}$ & 0.12794 & 0.32052 & 0.39842 & 0.08344 & 0.06968 & 0.266 & 49.6641 & 8 \\
\hline 176 & 8TC041 & $\mathrm{OLH}$ & 0.09627 & 0.3275 & 0.40009 & 0.10291 & 0.07323 & 0.938 & 53.25 & 8 \\
\hline *177 & 8TC042 & $\mathrm{OLH}$ & 0.07251 & 0.30701 & 0.46091 & 0.09127 & 0.0683 & 1.438 & 63.2109 & 8 \\
\hline 178 & $8 \mathrm{TC} 043$ & $\mathrm{OLH}$ & 0.09528 & 0.31423 & 0.43914 & 0.0864 & 0.06495 & 1.953 & 41.6953 & 8 \\
\hline 179 & $8 \mathrm{TC} 044$ & $\mathrm{OLH}$ & 0.11507 & 0.30771 & 0.4072 & 0.10207 & 0.06795 & 1.938 & 54.8438 & 8 \\
\hline 180 & $8 \mathrm{TC} 045$ & $\mathrm{OLH}$ & 0.09231 & 0.32424 & 0.42144 & 0.0972 & 0.06481 & 1.484 & 65.2031 & 8 \\
\hline$* 181$ & $8 \mathrm{TC} 046$ & $\mathrm{OLH}$ & 0.12596 & 0.31027 & 0.39859 & 0.09466 & 0.07052 & 1.719 & 55.2422 & 8 \\
\hline 182 & 8ТC047 & $\mathrm{OLH}$ & 0.08142 & 0.3268 & 0.43517 & 0.08449 & 0.07212 & 1.188 & 54.4453 & 8 \\
\hline 183 & 8TC048 & OLH & 0.12398 & 0.31144 & 0.39475 & 0.0991 & 0.07073 & 1.391 & 48.4688 & 8 \\
\hline 184 & $8 \mathrm{TC} 049$ & OLH & 0.07647 & 0.32634 & 0.41944 & 0.10863 & 0.06912 & 1.984 & 57.6328 & 8 \\
\hline
\end{tabular}


Table 34. Envelope C Design Matrix for OLI Simulations (cont'd)

\begin{tabular}{|c|c|c|c|c|c|c|c|c|c|c|}
\hline $\begin{array}{l}\text { Run } \\
\text { No. } \\
\end{array}$ & Test ID & Type & $\mathrm{x}_{\mathrm{AlO} 2}$ & $\mathrm{x}_{\mathrm{CO} 3}$ & $\mathrm{x}_{\mathrm{NO} 2}$ & $\mathrm{x}_{\mathrm{OH}}$ & $\mathrm{x}_{\mathrm{SO} 4}$ & SBS/Feed & Temp & $\begin{array}{l}\mathrm{Na} \\
\mathrm{M} \\
\end{array}$ \\
\hline$* 185$ & $8 \mathrm{TC} 050$ & OLH & 0.12101 & 0.30422 & 0.41908 & 0.08788 & 0.06781 & 0.156 & 40.1016 & 8 \\
\hline 186 & $8 \mathrm{TC} 051$ & OLH & 0.10715 & 0.32564 & 0.41684 & 0.08534 & 0.06503 & 0.547 & 16.9922 & 8 \\
\hline 187 & $8 \mathrm{TC} 052$ & $\mathrm{OLH}$ & 0.12002 & 0.30399 & 0.40316 & 0.10461 & 0.06822 & 0.219 & 34.9219 & 8 \\
\hline 188 & $8 \mathrm{TC} 053$ & OLH & 0.07548 & 0.31121 & 0.44851 & 0.09317 & 0.07163 & 0.203 & 22.5703 & 8 \\
\hline 189 & $8 \mathrm{TC} 054$ & OLH & 0.07746 & 0.31493 & 0.45044 & 0.08471 & 0.07246 & 0.922 & 34.125 & 8 \\
\hline 190 & $8 \mathrm{TC} 055$ & OLH & 0.12299 & 0.30981 & 0.39738 & 0.09826 & 0.07156 & 0.828 & 28.5469 & 8 \\
\hline *191 & $8 \mathrm{TC} 056$ & OLH & 0.0834 & 0.32611 & 0.42013 & 0.09783 & 0.07253 & 0.703 & 39.7031 & 8 \\
\hline 192 & 8TC057 & OLH & 0.08736 & 0.31074 & 0.45126 & 0.08555 & 0.06509 & 1.234 & 20.9766 & 8 \\
\hline 193 & $8 \mathrm{TC} 058$ & OLH & 0.07944 & 0.31772 & 0.44071 & 0.09529 & 0.06684 & 1.859 & 30.1406 & 8 \\
\hline 194 & 8TC059 & $\mathrm{OLH}$ & 0.0735 & 0.30143 & 0.46102 & 0.09868 & 0.06537 & 1.141 & 38.1094 & 8 \\
\hline *195 & $8 \mathrm{TC} 060$ & OLH & 0.11903 & 0.31446 & 0.39992 & 0.10037 & 0.06622 & 1.109 & 21.375 & 8 \\
\hline 196 & 8TC061 & $\mathrm{OLH}$ & 0.10616 & 0.30166 & 0.43486 & 0.08492 & 0.0724 & 1.625 & 22.9688 & 8 \\
\hline 197 & $8 \mathrm{TC} 062$ & OLH & 0.11804 & 0.32215 & 0.4004 & 0.09021 & 0.0692 & 1.813 & 36.5156 & 8 \\
\hline 198 & $8 \mathrm{TC} 063$ & $\mathrm{OLH}$ & 0.09726 & 0.30073 & .42547 & 0.10694 & 0.0696 & 1.609 & 31.7344 & 8 \\
\hline 199 & 8TC064 & $\mathrm{OLH}$ & 0.10221 & 0.30818 & 0.43714 & 0.08703 & 0.06544 & 0.984 & 62.0156 & 8 \\
\hline$* 200$ & $8 \mathrm{TC} 065$ & $\mathrm{OLH}$ & 0.09033 & 0.32122 & 0.42718 & 0.09423 & 0.06704 & 0.078 & 52.0547 & 8 \\
\hline 201 & $8 \mathrm{TC} 066$ & $\mathrm{OLH}$ & 0.07845 & 0.30306 & 0.44905 & 0.10101 & 0.06843 & 0.781 & 60.4219 & 8 \\
\hline 202 & $8 \mathrm{TC} 067$ & $\mathrm{OLH}$ & 0.1121 & 0.31563 & 0.40321 & 0.10355 & 0.06551 & 0.734 & 55.6406 & 8 \\
\hline *203 & $8 \mathrm{TC} 068$ & $\mathrm{OLH}$ & 0.11606 & 0.3119 & 0.41415 & 0.08598 & 0.07191 & 0.297 & 60.8203 & 8 \\
\hline 204 & 8ТC069 & OLH & 0.07449 & 0.32448 & 0.44065 & 0.09085 & 0.06953 & 0.75 & 42.4922 & 8 \\
\hline 205 & $8 \mathrm{TC} 070$ & OLH & 0.09429 & 0.30189 & 0.43582 & 0.09762 & 0.07038 & 0.531 & 44.8828 & 8 \\
\hline 206 & $8 \mathrm{TC} 071$ & OLH & 0.07053 & 0.30632 & 0.46755 & 0.08682 & 0.06878 & 1.766 & 46.4766 & 8 \\
\hline 207 & 8TC072 & $\mathrm{OLH}$ & 0.09825 & 0.31377 & 0.43028 & 0.09296 & 0.06474 & 1.406 & 62.4141 & 8 \\
\hline 208 & $8 \mathrm{TC} 073$ & OLH & 0.08439 & 0.30282 & 0.44221 & 0.1027 & 0.06788 & 1.094 & 44.0859 & 8 \\
\hline *209 & $8 \mathrm{TC} 074$ & $\mathrm{OLH}$ & 0.11309 & 0.31703 & 0.40544 & 0.09677 & 0.06767 & 1.75 & 43.2891 & 8 \\
\hline 210 & $8 \mathrm{TC} 075$ & $\mathrm{OLH}$ & 0.08538 & 0.31004 & 0.43646 & 0.09614 & 0.07198 & 1.688 & 56.4375 & 8 \\
\hline 211 & $8 \mathrm{TC} 076$ & $\mathrm{OLH}$ & 0.08241 & 0.31726 & 0.44651 & 0.08386 & 0.06996 & 1.156 & 61.2188 & 8 \\
\hline 212 & $8 \mathrm{TC} 077$ & $\mathrm{OLH}$ & 0.06954 & 0.32704 & 0.43449 & 0.09995 & 0.06898 & 1.531 & 61.6172 & 8 \\
\hline 213 & $8 \mathrm{TC} 078$ & $\mathrm{OLH}$ & 0.06855 & 0.3133 & 0.45295 & 0.09635 & 0.06885 & 1 & 40.5 & 8 \\
\hline *214 & $8 \mathrm{TC} 079$ & $\mathrm{OLH}$ & 0.02599 & 0.31796 & 0.47906 & 0.10418 & 0.07281 & 1.328 & 43.6875 & 8 \\
\hline 215 & $8 \mathrm{TC} 080$ & $\mathrm{OLH}$ & 0.04875 & 0.30329 & 0.47514 & 0.10376 & 0.06906 & 1.656 & 62.8125 & 8 \\
\hline 216 & $8 \mathrm{TC} 081$ & $\mathrm{OLH}$ & 0.03391 & 0.32191 & 0.48071 & 0.09212 & 0.07135 & 1.516 & 45.6797 & 8 \\
\hline 217 & 8TC082 & OLH & 0.03193 & 0.30515 & 0.50252 & 0.08725 & 0.07315 & 1.281 & 63.6094 & 8 \\
\hline 218 & 8TC083 & $\mathrm{OLH}$ & 0.00718 & 0.32168 & 0.49644 & 0.10842 & 0.06628 & 2 & 50.0625 & 8 \\
\hline 219 & 8TC084 & OLH & 0.03292 & 0.29887 & 0.50482 & 0.09699 & 0.0664 & 1.359 & 59.2266 & 8 \\
\hline$* 220$ & $8 \mathrm{TC} 085$ & $\mathrm{OLH}$ & 0.06558 & 0.32657 & 0.45166 & 0.08873 & 0.06746 & 1.641 & 51.2578 & 8 \\
\hline 221 & 8TC086 & $\mathrm{OLH}$ & 0.03688 & 0.32285 & 0.46007 & 0.1099 & 0.0703 & 0.109 & 51.6563 & 8 \\
\hline$* 222$ & 8TC087 & $\mathrm{OLH}$ & 0.02797 & 0.30585 & 0.49319 & 0.10122 & 0.07177 & 0.953 & 64.8047 & 8 \\
\hline 223 & 8TC088 & $\mathrm{OLH}$ & 0.04578 & 0.3282 & 0.4672 & 0.08767 & 0.07115 & 0.438 & 64.4063 & 8 \\
\hline 224 & 8TC089 & $\mathrm{OLH}$ & 0.05073 & 0.32098 & 0.46309 & 0.1008 & 0.0644 & 0.422 & 58.8281 & 8 \\
\hline 225 & $8 \mathrm{TC} 090$ & $\mathrm{OLH}$ & 0.03589 & 0.30911 & 0.48251 & 0.10524 & 0.06725 & 0.453 & 45.2813 & 8 \\
\hline 226 & 8TC091 & $\mathrm{OLH}$ & 0.02698 & 0.31819 & 0.49947 & 0.08936 & 0.066 & 0.688 & 50.4609 & 8 \\
\hline 227 & $8 \mathrm{TC} 092$ & $\mathrm{OLH}$ & 0.04776 & 0.30352 & 0.48955 & 0.09254 & 0.06663 & 0.328 & 65.6016 & 8 \\
\hline 228 & 8TC093 & $\mathrm{OLH}$ & 0.00619 & 0.31912 & 0.4978 & 0.10609 & 0.0708 & 1.125 & 32.1328 & 8 \\
\hline$* 229$ & 8ТC094 & $\mathrm{OLH}$ & 0.0438 & 0.29863 & 0.48558 & 0.09889 & 0.0731 & 1.875 & 23.7656 & 8 \\
\hline 230 & 8ТC095 & $\mathrm{OLH}$ & 0.05667 & 0.32401 & 0.45524 & 0.09106 & 0.07302 & 1.203 & 27.3516 & 8 \\
\hline *231 & $8 \mathrm{TC} 096$ & OLH & 0.02302 & 0.31051 & 0.51246 & 0.08301 & 0.071 & 1.906 & 33.3281 & 8 \\
\hline
\end{tabular}


Table 34. Envelope C Design Matrix for OLI Simulations (cont'd)

\begin{tabular}{|c|c|c|c|c|c|c|c|c|c|c|}
\hline $\begin{array}{l}\text { Run } \\
\text { No. } \\
\end{array}$ & Test ID & Type & $\mathrm{x}_{\mathrm{AlO} 2}$ & $\mathrm{x}_{\mathrm{CO} 3}$ & $\mathrm{x}_{\mathrm{NO} 2}$ & $\mathrm{x}_{\mathrm{OH}}$ & $\mathrm{x}_{\mathrm{SO} 4}$ & SBS/Feed & Temp & $\begin{array}{l}\mathrm{Na} \\
\mathrm{M} \\
\end{array}$ \\
\hline 232 & 8ТC097 & OLH & 0.03787 & 0.32727 & 0.46694 & 0.10228 & 0.06564 & 1.375 & 15 & 8 \\
\hline 233 & 8TC098 & OLH & 0.00916 & 0.30608 & 0.50748 & 0.10927 & 0.06801 & 1.734 & 31.3359 & 8 \\
\hline 234 & 8TC099 & $\mathrm{OLH}$ & 0.04083 & 0.2991 & 0.50581 & 0.08979 & 0.06447 & 1.063 & 27.75 & 8 \\
\hline 235 & $8 \mathrm{TC} 100$ & OLH & 0.06459 & 0.31959 & 0.44499 & 0.10143 & 0.0694 & 0.563 & 17.7891 & 8 \\
\hline 236 & 8TC101 & OLH & 0.04182 & 0.31237 & 0.46676 & 0.1063 & 0.07275 & 0.047 & 39.3047 & 8 \\
\hline 237 & 8TC102 & OLH & 0.02203 & 0.31889 & 0.4987 & 0.09063 & 0.06975 & 0.063 & 26.1563 & 8 \\
\hline 238 & 8TC103 & OLH & 0.04479 & 0.30236 & 0.48446 & 0.0955 & 0.07289 & 0.516 & 15.7969 & 8 \\
\hline 239 & 8TC104 & OLH & 0.01114 & 0.31633 & 0.50731 & 0.09804 & 0.06718 & 0.281 & 25.7578 & 8 \\
\hline$* 240$ & $8 \mathrm{TC} 105$ & OLH & 0.05568 & 0.2998 & 0.47073 & 0.10821 & 0.06558 & 0.813 & 26.5547 & 8 \\
\hline 241 & 8TC106 & OLH & 0.01312 & 0.31516 & 0.51115 & 0.0936 & 0.06697 & 0.609 & 32.5313 & 8 \\
\hline *242 & 8TC107 & OLH & 0.06063 & 0.30026 & 0.48646 & 0.08407 & 0.06858 & 0.016 & 23.3672 & 8 \\
\hline 243 & 8TC108 & $\mathrm{OLH}$ & 0.01609 & 0.32238 & 0.48682 & 0.10482 & 0.06989 & 1.844 & 40.8984 & 8 \\
\hline 244 & 8TC109 & OLH & 0.02995 & 0.30096 & 0.48906 & 0.10736 & 0.07267 & 1.453 & 64.0078 & 8 \\
\hline$* 245$ & $8 \mathrm{TC} 110$ & $\mathrm{OLH}$ & 0.01708 & 0.32261 & 0.50274 & 0.08809 & 0.06948 & 1.781 & 46.0781 & 8 \\
\hline 246 & 8TC111 & OLH & 0.06162 & 0.3154 & 0.45739 & 0.09953 & 0.06606 & 1.797 & 58.4297 & 8 \\
\hline 247 & $8 \mathrm{TC} 112$ & $\mathrm{OLH}$ & 0.05964 & 0.31167 & 0.45546 & 0.108 & 0.06523 & 1.078 & 46.875 & 8 \\
\hline *248 & $8 \mathrm{TC} 113$ & $\mathrm{OLH}$ & 0.01411 & 0.31679 & 0.50852 & 0.09445 & 0.06613 & 1.172 & 52.4531 & 8 \\
\hline 249 & $8 \mathrm{TC} 114$ & $\mathrm{OLH}$ & 0.0537 & 0.3005 & 0.48577 & 0.09487 & 0.06516 & 1.297 & 41.2969 & 8 \\
\hline 250 & $8 \mathrm{TC} 115$ & $\mathrm{OLH}$ & 0.04974 & 0.31586 & 0.45464 & 0.10715 & 0.07261 & 0.766 & 60.0234 & 8 \\
\hline 251 & $8 \mathrm{TC} 116$ & OLH & 0.05766 & 0.30888 & 0.46519 & 0.09741 & 0.07086 & 0.141 & 50.8594 & 8 \\
\hline$* 252$ & $8 \mathrm{TC} 117$ & OLH & 0.0636 & 0.32517 & 0.44488 & 0.09402 & 0.07233 & 0.859 & 42.8906 & 8 \\
\hline 253 & 8TC118 & OLH & 0.01807 & 0.31214 & 0.50598 & 0.09233 & 0.07148 & 0.891 & 59.625 & 8 \\
\hline 254 & $8 \mathrm{TC} 119$ & OLH & 0.03094 & 0.32494 & 0.47104 & 0.10778 & 0.0653 & 0.375 & 58.0313 & 8 \\
\hline 255 & $8 \mathrm{TC} 120$ & OLH & 0.01906 & 0.30445 & 0.5055 & 0.10249 & 0.0685 & 0.188 & 44.4844 & 8 \\
\hline 256 & $8 \mathrm{TC} 121$ & $\mathrm{OLH}$ & 0.03985 & 0.32587 & 0.48043 & 0.08576 & 0.06809 & 0.391 & 49.2656 & 8 \\
\hline 257 & 8TC122 & $\mathrm{OLH}$ & 0.0349 & 0.31842 & 0.46876 & 0.10567 & 0.07225 & 1.016 & 18.9844 & 8 \\
\hline$* 258$ & $8 \mathrm{TC} 123$ & $\mathrm{OLH}$ & 0.04677 & 0.30538 & 0.47872 & 0.09847 & 0.07066 & 1.922 & 28.9453 & 8 \\
\hline 259 & 8TC124 & $\mathrm{OLH}$ & 0.05865 & 0.32354 & 0.45685 & 0.09169 & 0.06927 & 1.219 & 20.5781 & 8 \\
\hline 260 & $8 \mathrm{TC} 125$ & $\mathrm{OLH}$ & 0.025 & 0.31097 & 0.50269 & 0.08915 & 0.07219 & 1.266 & 25.3594 & 8 \\
\hline 261 & $8 \mathrm{TC} 126$ & $\mathrm{OLH}$ & 0.02104 & 0.3147 & 0.49175 & 0.10672 & 0.06579 & 1.703 & 20.1797 & 8 \\
\hline 262 & $8 \mathrm{TC} 127$ & $\mathrm{OLH}$ & 0.06261 & 0.30213 & 0.46525 & 0.10186 & 0.06815 & 1.25 & 38.5078 & 8 \\
\hline 263 & $8 \mathrm{TC} 128$ & $\mathrm{OLH}$ & 0.04281 & 0.32471 & 0.47008 & 0.09508 & 0.06732 & 1.469 & 36.1172 & 8 \\
\hline 264 & 8ТC129 & OLH & 0.06657 & 0.32028 & 0.43835 & 0.10588 & 0.06892 & 0.234 & 34.5234 & 8 \\
\hline$* 265$ & 8TC130 & $\mathrm{OLH}$ & 0.03886 & 0.31283 & 0.47562 & 0.09974 & 0.07295 & 0.594 & 18.5859 & 8 \\
\hline 266 & 8TC131 & $\mathrm{OLH}$ & 0.05271 & 0.32378 & 0.46369 & 0.09 & 0.06982 & 0.906 & 36.9141 & 8 \\
\hline 267 & $8 \mathrm{TC} 132$ & $\mathrm{OLH}$ & 0.02401 & 0.30958 & 0.50046 & 0.09593 & 0.07002 & 0.25 & 37.7109 & 8 \\
\hline$* 268$ & 8TC133 & $\mathrm{OLH}$ & 0.05172 & 0.31656 & 0.46944 & 0.09656 & 0.06572 & 0.313 & 24.5625 & 8 \\
\hline 269 & 8TC134 & $\mathrm{OLH}$ & 0.05469 & 0.30934 & 0.45939 & 0.10884 & 0.06774 & 0.844 & 19.7813 & 8 \\
\hline 270 & $8 \mathrm{TC} 135$ & $\mathrm{OLH}$ & 0.06756 & 0.29956 & 0.47141 & 0.09275 & 0.06872 & 0.469 & 19.3828 & 8 \\
\hline 271 & 6TC001 & EV & 0.1319 & 0.3282 & 0.3927 & 0.0828 & 0.0644 & 0 & 15 & 6 \\
\hline 272 & 6TC002 & EV & 0.1258 & 0.2984 & 0.3926 & 0.1099 & 0.0733 & 0 & 15 & 6 \\
\hline 273 & 6TC003 & EV & 0.0413 & 0.2984 & 0.5131 & 0.0828 & 0.0644 & 0 & 15 & 6 \\
\hline 274 & 6TC004 & EV & 0.0052 & 0.3282 & 0.4923 & 0.1099 & 0.0644 & 0 & 15 & 6 \\
\hline 275 & $6 \mathrm{TC} 005$ & EV & 0.0052 & 0.3282 & 0.5105 & 0.0828 & 0.0733 & 0 & 15 & 6 \\
\hline 276 & $6 \mathrm{TC} 006$ & EV & 0.1319 & 0.3282 & 0.3927 & 0.0828 & 0.0644 & 0 & 66 & 6 \\
\hline 277 & 6TC007 & EV & 0.1258 & 0.2984 & 0.3926 & 0.1099 & 0.0733 & 0 & 66 & 6 \\
\hline 278 & 6TC008 & EV & 0.0413 & 0.2984 & 0.5131 & 0.0828 & 0.0644 & 0 & 66 & 6 \\
\hline
\end{tabular}


Table 34. Envelope C Design Matrix for OLI Simulations (cont'd)

\begin{tabular}{|c|c|c|c|c|c|c|c|c|c|c|}
\hline $\begin{array}{l}\text { Run } \\
\text { No. } \\
\end{array}$ & Test ID & Type & $\mathrm{x}_{\mathrm{AlO} 2}$ & $\mathrm{x}_{\mathrm{CO} 3}$ & $\mathrm{x}_{\mathrm{NO} 2}$ & $\mathrm{x}_{\mathrm{OH}}$ & $\mathrm{x}_{\mathrm{SO} 4}$ & SBS/Feed & Temp & $\begin{array}{l}\mathrm{Na} \\
\mathrm{M} \\
\end{array}$ \\
\hline 279 & 6TC009 & EV & 0.0052 & 0.3282 & 0.4923 & 0.1099 & 0.0644 & 0 & 66 & 6 \\
\hline 280 & 6TC010 & EV & 0.0052 & 0.3282 & 0.5105 & 0.0828 & 0.0733 & 0 & 66 & 6 \\
\hline 281 & 6TC011 & $\mathrm{EV}$ & 0.1319 & 0.3282 & 0.3927 & 0.0828 & 0.0644 & 2 & 15 & 6 \\
\hline 282 & 6TC012 & EV & 0.1258 & 0.2984 & 0.3926 & 0.1099 & 0.0733 & 2 & 15 & 6 \\
\hline 283 & $6 \mathrm{TC} 013$ & EV & 0.0413 & 0.2984 & 0.5131 & 0.0828 & 0.0644 & 2 & 15 & 6 \\
\hline 284 & 6TC014 & EV & 0.0052 & 0.3282 & 0.4923 & 0.1099 & 0.0644 & 2 & 15 & 6 \\
\hline 285 & 6ТC015 & EV & 0.0052 & 0.3282 & 0.5105 & 0.0828 & 0.0733 & 2 & 15 & 6 \\
\hline 286 & 6TC016 & EV & 0.1319 & 0.3282 & 0.3927 & 0.0828 & 0.0644 & 2 & 66 & 6 \\
\hline 287 & 6ТC017 & EV & 0.1258 & 0.2984 & 0.3926 & 0.1099 & 0.0733 & 2 & 66 & 6 \\
\hline 288 & 6TC018 & $\mathrm{EV}$ & 0.0413 & 0.2984 & 0.5131 & 0.0828 & 0.0644 & 2 & 66 & 6 \\
\hline 289 & 6ТC019 & EV & 0.0052 & 0.3282 & 0.4923 & 0.1099 & 0.0644 & 2 & 66 & 6 \\
\hline 290 & 6TC020 & EV & 0.0052 & 0.3282 & 0.5105 & 0.0828 & 0.0733 & 2 & 66 & 6 \\
\hline 291 & 6TC021 & $\mathrm{OLH}$ & 0.11111 & 0.30864 & 0.42684 & 0.08852 & 0.06489 & 0.672 & 37.3125 & 6 \\
\hline 292 & 6TC022 & $\mathrm{OLH}$ & 0.08835 & 0.32331 & 0.43076 & 0.08894 & 0.06864 & 0.344 & 18.1875 & 6 \\
\hline 293 & 6TC023 & $\mathrm{OLH}$ & 0.1032 & 0.30469 & 0.42519 & 0.10058 & 0.06634 & 0.484 & 35.3203 & 6 \\
\hline 294 & 6TC024 & $\mathrm{OLH}$ & 0.10517 & 0.32145 & 0.40338 & 0.10545 & 0.06455 & 0.719 & 17.3906 & 6 \\
\hline 295 & 6TC025 & $\mathrm{OLH}$ & 0.12992 & 0.30492 & 0.40946 & 0.08428 & 0.07142 & 0 & 30.9375 & 6 \\
\hline *296 & 6TC026 & $\mathrm{OLH}$ & 0.10418 & 0.32773 & 0.40108 & 0.09572 & 0.07129 & 0.641 & 21.7734 & 6 \\
\hline 297 & 6TC027 & $\mathrm{OLH}$ & 0.07152 & 0.30003 & 0.45424 & 0.10397 & 0.07024 & 0.359 & 29.7422 & 6 \\
\hline 298 & 6TC028 & OLH & 0.10023 & 0.30376 & 0.44583 & 0.0828 & 0.06738 & 1.891 & 29.3438 & 6 \\
\hline *299 & 6TC029 & OLH & 0.10913 & 0.32075 & 0.41271 & 0.09148 & 0.06593 & 1.047 & 16.1953 & 6 \\
\hline 300 & 6ТC030 & OLH & 0.09132 & 0.2984 & 0.4387 & 0.10503 & 0.06655 & 1.563 & 16.5938 & 6 \\
\hline 301 & 6TC031 & $\mathrm{OLH}$ & 0.08637 & 0.30562 & 0.44281 & 0.0919 & 0.0733 & 1.578 & 22.1719 & 6 \\
\hline *302 & 6ТC032 & OLH & 0.10122 & 0.31749 & 0.42339 & 0.08746 & 0.07044 & 1.547 & 35.7188 & 6 \\
\hline 303 & 6TC033 & $\mathrm{OLH}$ & 0.11012 & 0.30841 & 0.40643 & 0.10334 & 0.0717 & 1.313 & 30.5391 & 6 \\
\hline 304 & 6TC034 & $\mathrm{OLH}$ & 0.08934 & 0.32308 & 0.41635 & 0.10016 & 0.07107 & 1.672 & 15.3984 & 6 \\
\hline 305 & 6TC035 & $\mathrm{OLH}$ & 0.13091 & 0.30748 & 0.4081 & 0.08661 & 0.0669 & 0.875 & 48.8672 & 6 \\
\hline 306 & 6TC036 & $\mathrm{OLH}$ & 0.0933 & 0.32797 & 0.42032 & 0.09381 & 0.0646 & 0.125 & 57.2344 & 6 \\
\hline 307 & 6TC037 & $\mathrm{OLH}$ & 0.08043 & 0.30259 & 0.45066 & 0.10164 & 0.06468 & 0.797 & 53.6484 & 6 \\
\hline 308 & 6TC038 & $\mathrm{OLH}$ & 0.11408 & 0.31609 & 0.39344 & 0.10969 & 0.0667 & 0.094 & 47.6719 & 6 \\
\hline 309 & 6TC039 & $\mathrm{OLH}$ & 0.09924 & 0.29933 & 0.43896 & 0.09042 & 0.07205 & 0.625 & 66 & 6 \\
\hline$* 310$ & 6ТC040 & $\mathrm{OLH}$ & 0.12794 & 0.32052 & 0.39842 & 0.08344 & 0.06968 & 0.266 & 49.6641 & 6 \\
\hline 311 & 6ТC041 & OLH & 0.09627 & 0.3275 & 0.40009 & 0.10291 & 0.07323 & 0.938 & 53.25 & 6 \\
\hline 312 & 6TC042 & $\mathrm{OLH}$ & 0.07251 & 0.30701 & 0.46091 & 0.09127 & 0.0683 & 1.438 & 63.2109 & 6 \\
\hline 313 & 6ТC043 & OLH & 0.09528 & 0.31423 & 0.43914 & 0.0864 & 0.06495 & 1.953 & 41.6953 & 6 \\
\hline 314 & 6TC044 & $\mathrm{OLH}$ & 0.11507 & 0.30771 & 0.4072 & 0.10207 & 0.06795 & 1.938 & 54.8438 & 6 \\
\hline 315 & 6TC045 & $\mathrm{OLH}$ & 0.09231 & 0.32424 & 0.42144 & 0.0972 & 0.06481 & 1.484 & 65.2031 & 6 \\
\hline$* 316$ & 6TC046 & $\mathrm{OLH}$ & 0.12596 & 0.31027 & 0.39859 & 0.09466 & 0.07052 & 1.719 & 55.2422 & 6 \\
\hline 317 & 6TC047 & $\mathrm{OLH}$ & 0.08142 & 0.3268 & 0.43517 & 0.08449 & 0.07212 & 1.188 & 54.4453 & 6 \\
\hline 318 & 6TC048 & $\mathrm{OLH}$ & 0.12398 & 0.31144 & 0.39475 & 0.0991 & 0.07073 & 1.391 & 48.4688 & 6 \\
\hline *319 & 6TC049 & $\mathrm{OLH}$ & 0.07647 & 0.32634 & 0.41944 & 0.10863 & 0.06912 & 1.984 & 57.6328 & 6 \\
\hline 320 & 6TC050 & $\mathrm{OLH}$ & 0.12101 & 0.30422 & 0.41908 & 0.08788 & 0.06781 & 0.156 & 40.1016 & 6 \\
\hline 321 & 6TC051 & $\mathrm{OLH}$ & 0.10715 & 0.32564 & 0.41684 & 0.08534 & 0.06503 & 0.547 & 16.9922 & 6 \\
\hline 322 & 6TC052 & $\mathrm{OLH}$ & 0.12002 & 0.30399 & 0.40316 & 0.10461 & 0.06822 & 0.219 & 34.9219 & 6 \\
\hline 323 & 6TC053 & $\mathrm{OLH}$ & 0.07548 & 0.31121 & 0.44851 & 0.09317 & 0.07163 & 0.203 & 22.5703 & 6 \\
\hline 324 & 6TC054 & $\mathrm{OLH}$ & 0.07746 & 0.31493 & 0.45044 & 0.08471 & 0.07246 & 0.922 & 34.125 & 6 \\
\hline *325 & 6TC055 & OLH & 0.12299 & 0.30981 & 0.39738 & 0.09826 & 0.07156 & 0.828 & 28.5469 & 6 \\
\hline
\end{tabular}


Table 34. Envelope C Design Matrix for OLI Simulations (cont'd)

\begin{tabular}{|c|c|c|c|c|c|c|c|c|c|c|}
\hline $\begin{array}{l}\text { Run } \\
\text { No. } \\
\end{array}$ & Test ID & Type & $\mathrm{x}_{\mathrm{AlO} 2}$ & $\mathrm{x}_{\mathrm{CO} 3}$ & $\mathrm{x}_{\mathrm{NO} 2}$ & $\mathrm{x}_{\mathrm{OH}}$ & $\mathrm{x}_{\mathrm{SO} 4}$ & SBS/Feed & Temp & $\begin{array}{l}\mathrm{Na} \\
\mathrm{M} \\
\end{array}$ \\
\hline 326 & 6TC056 & OLH & 0.0834 & 0.32611 & 0.42013 & 0.09783 & 0.07253 & 0.703 & 39.7031 & 6 \\
\hline$* 327$ & 6TC057 & OLH & 0.08736 & 0.31074 & 0.45126 & 0.08555 & 0.06509 & 1.234 & 20.9766 & 6 \\
\hline 328 & 6TC058 & OLH & 0.07944 & 0.31772 & 0.44071 & 0.09529 & 0.06684 & 1.859 & 30.1406 & 6 \\
\hline 329 & 6TC059 & OLH & 0.0735 & 0.30143 & 0.46102 & 0.09868 & 0.06537 & 1.141 & 38.1094 & 6 \\
\hline 330 & 6ТC060 & $\mathrm{OLH}$ & 0.11903 & 0.31446 & 0.39992 & 0.10037 & 0.06622 & 1.109 & 21.375 & 6 \\
\hline *331 & 6TC061 & OLH & 0.10616 & 0.30166 & 0.43486 & 0.08492 & 0.0724 & 1.625 & 22.9688 & 6 \\
\hline 332 & 6ТC062 & OLH & 0.11804 & 0.32215 & 0.4004 & 0.09021 & 0.0692 & 1.813 & 36.5156 & 6 \\
\hline 333 & 6TC063 & $\mathrm{OLH}$ & 0.09726 & 0.30073 & 0.42547 & 0.10694 & 0.0696 & 1.609 & 31.7344 & 6 \\
\hline 334 & 6ТC064 & $\mathrm{OLH}$ & 0.10221 & 0.30818 & 0.43714 & 0.08703 & 0.06544 & 0.984 & 62.0156 & 6 \\
\hline 335 & $6 \mathrm{TC} 065$ & OLH & 0.09033 & 0.32122 & 0.42718 & 0.09423 & 0.06704 & 0.078 & 52.0547 & 6 \\
\hline 336 & 6ТC066 & $\mathrm{OLH}$ & 0.07845 & 0.30306 & 0.44905 & 0.10101 & 0.06843 & 0.781 & 60.4219 & 6 \\
\hline 337 & 6TC067 & OLH & 0.1121 & 0.31563 & 0.40321 & 0.10355 & 0.06551 & 0.734 & 55.6406 & 6 \\
\hline 338 & 6TC068 & OLH & 0.11606 & 0.3119 & 0.41415 & 0.08598 & 0.07191 & 0.297 & 60.8203 & 6 \\
\hline 339 & 6TC069 & OLH & 0.07449 & 0.32448 & 0.44065 & 0.09085 & 0.06953 & 0.75 & 42.4922 & 6 \\
\hline 340 & 6TC070 & OLH & 0.09429 & 0.30189 & 0.43582 & 0.09762 & 0.07038 & 0.531 & 44.8828 & 6 \\
\hline *341 & 6TC071 & OLH & 0.07053 & 0.30632 & 0.46755 & 0.08682 & 0.06878 & 1.766 & 46.4766 & 6 \\
\hline 342 & 6TC072 & OLH & 0.09825 & 0.31377 & 0.43028 & 0.09296 & 0.06474 & 1.406 & 62.4141 & 6 \\
\hline 343 & 6TC073 & OLH & 0.08439 & 0.30282 & 0.44221 & 0.1027 & 0.06788 & 1.094 & 44.0859 & 6 \\
\hline 344 & 6TC074 & OLH & 0.11309 & 0.31703 & 0.40544 & 0.09677 & 0.06767 & 1.75 & 43.2891 & 6 \\
\hline *345 & 6ТC075 & $\mathrm{OLH}$ & 0.08538 & 0.31004 & 0.43646 & 0.09614 & 0.07198 & 1.688 & 56.4375 & 6 \\
\hline 346 & 6TC076 & OLH & 0.08241 & 0.31726 & 0.44651 & 0.08386 & 0.06996 & 1.156 & 61.2188 & 6 \\
\hline 347 & 6ТC077 & $\mathrm{OLH}$ & 0.06954 & 0.32704 & 0.43449 & 0.09995 & 0.06898 & 1.531 & 61.6172 & 6 \\
\hline 348 & $6 \mathrm{TC} 078$ & $\mathrm{OLH}$ & 0.06855 & 0.3133 & 0.45295 & 0.09635 & 0.06885 & 1 & 40.5 & 6 \\
\hline 349 & 6ТС079 & $\mathrm{OLH}$ & 0.02599 & 0.31796 & 0.47906 & 0.10418 & 0.07281 & 1.328 & 43.6875 & 6 \\
\hline$* 350$ & 6TC080 & OLH & 0.04875 & 0329 & 0.47514 & 0.10376 & 0.06906 & 1.656 & 62.8125 & 6 \\
\hline 351 & 6TC081 & $\mathrm{OLH}$ & 0.03391 & 0.32191 & 0.48071 & 0.09212 & 0.07135 & 1.516 & 45.6797 & 6 \\
\hline 352 & 6TC082 & OLH & 0.03193 & 0.30515 & 0.50252 & 0.08725 & 0.07315 & 1.281 & 63.6094 & 6 \\
\hline$* 353$ & $6 \mathrm{TC} 083$ & OLH & 0.00718 & 0.32168 & 0.49644 & 0.10842 & 0.06628 & 2 & 50.0625 & 6 \\
\hline 354 & 6TC084 & OLH & 0.03292 & 0.29887 & 0.50482 & 0.09699 & 0.0664 & 1.359 & 59.2266 & 6 \\
\hline 355 & 6TC085 & OLH & 0.06558 & 0.32657 & 0.45166 & 0.08873 & 0.06746 & 1.641 & 51.2578 & 6 \\
\hline *356 & 6TC086 & OLH & 0.03688 & 0.32285 & 0.46007 & 0.1099 & 0.0703 & 0.109 & 51.6563 & 6 \\
\hline 357 & 6ТC087 & OLH & 0.02797 & 0.30585 & 0.49319 & 0.10122 & 0.07177 & 0.953 & 64.8047 & 6 \\
\hline 358 & 6ТC088 & $\mathrm{OLH}$ & 0.04578 & 0.3282 & 0.4672 & 0.08767 & 0.07115 & 0.438 & 64.4063 & 6 \\
\hline 359 & 6ТC089 & $\mathrm{OLH}$ & 0.05073 & 0.32098 & 0.46309 & 0.1008 & 0.0644 & 0.422 & 58.8281 & 6 \\
\hline$* 360$ & 6ТC090 & $\mathrm{OLH}$ & 0.03589 & 0.30911 & 0.48251 & 0.10524 & 0.06725 & 0.453 & 45.2813 & 6 \\
\hline 361 & 6TC091 & OLH & 0.02698 & 0.31819 & 0.49947 & 0.08936 & 0.066 & 0.688 & 50.4609 & 6 \\
\hline 362 & 6TC092 & OLH & 0.04776 & 0.30352 & 0.48955 & 0.09254 & 0.06663 & 0.328 & 65.6016 & 6 \\
\hline$* 363$ & 6ТC093 & $\mathrm{OLH}$ & 0.00619 & 0.31912 & 0.4978 & 0.10609 & 0.0708 & 1.125 & 32.1328 & 6 \\
\hline 364 & 6TC094 & OLH & 0.0438 & 0.29863 & 0.48558 & 0.09889 & 0.0731 & 1.875 & 23.7656 & 6 \\
\hline$* 365$ & 6TC095 & OLH & 0.05667 & 0.32401 & 0.45524 & 0.09106 & 0.07302 & 1.203 & 27.3516 & 6 \\
\hline 366 & 6TC096 & OLH & 0.02302 & 0.31051 & 0.51246 & 0.08301 & 0.071 & 1.906 & 33.3281 & 6 \\
\hline 367 & 6TC097 & OLH & 0.03787 & 0.32727 & 0.46694 & 0.10228 & 0.06564 & 1.375 & 15 & 6 \\
\hline$* 368$ & 6ТC098 & OLH & 0.00916 & 0.30608 & 0.50748 & 0.10927 & 0.06801 & 1.734 & 31.3359 & 6 \\
\hline 369 & 6TC099 & OLH & 0.04083 & 0.2991 & 0.50581 & 0.08979 & 0.06447 & 1.063 & 27.75 & 6 \\
\hline 370 & 6TC100 & $\mathrm{OLH}$ & 0.06459 & 0.31959 & 0.44499 & 0.10143 & 0.0694 & 0.563 & 17.7891 & 6 \\
\hline$* 371$ & 6TC101 & OLH & 0.04182 & 0.31237 & 0.46676 & 0.1063 & 0.07275 & 0.047 & 39.3047 & 6 \\
\hline 372 & 6TC102 & OLH & 0.02203 & 0.31889 & 0.4987 & 0.09063 & 0.06975 & 0.063 & 26.1563 & 6 \\
\hline
\end{tabular}


Table 34. Envelope C Design Matrix for OLI Simulations (cont'd)

\begin{tabular}{|c|c|c|c|c|c|c|c|c|c|c|}
\hline $\begin{array}{l}\text { Run } \\
\text { No. } \\
\end{array}$ & Test ID & Type & $\mathrm{x}_{\mathrm{AlO} 2}$ & $\mathrm{x}_{\mathrm{CO} 3}$ & $\mathrm{x}_{\mathrm{NO} 2}$ & $\mathrm{x}_{\mathrm{OH}}$ & $\mathrm{x}_{\mathrm{SO} 4}$ & SBS/Feed & Temp & $\begin{array}{l}\mathrm{Na} \\
\mathrm{M} \\
\end{array}$ \\
\hline 373 & 6TC103 & $\mathrm{OLH}$ & 0.04479 & 0.30236 & 0.48446 & 0.0955 & 0.07289 & 0.516 & 15.7969 & 6 \\
\hline 374 & 6TC104 & OLH & 0.01114 & 0.31633 & .50731 & 0.09804 & 0.06718 & 0.281 & 25.7578 & 6 \\
\hline 375 & 6TC105 & $\mathrm{OLH}$ & 0.05568 & 0.2998 & .47073 & 0.10821 & 0.06558 & 0.813 & 26.5547 & 6 \\
\hline$* 376$ & 6TC106 & OLH & 0.01312 & 0.31516 & 0.51115 & 0.0936 & 0.06697 & 0.609 & 32.5313 & 6 \\
\hline 377 & 6TC107 & OLH & 0.06063 & 0.30026 & 0.48646 & 0.08407 & 0.06858 & 0.016 & 23.3672 & 6 \\
\hline 378 & 6TC108 & OLH & 0.01609 & 0.32238 & 0.48682 & 0.10482 & 0.06989 & 1.844 & 40.8984 & 6 \\
\hline *379 & $6 \mathrm{TC} 109$ & OLH & 0.02995 & 0.30096 & 0.48906 & 0.10736 & 0.07267 & 1.453 & 64.0078 & 6 \\
\hline 380 & $6 \mathrm{TC} 110$ & OLH & 0.01708 & 0.32261 & 0.50274 & 0.08809 & 0.06948 & 1.781 & 46.0781 & 6 \\
\hline 381 & 6TC111 & OLH & 0.06162 & 0.3154 & 0.45739 & 0.09953 & 0.06606 & 1.797 & 58.4297 & 6 \\
\hline 382 & $6 \mathrm{TC} 112$ & OLH & 0.05964 & 0.31167 & 0.45546 & 0.108 & 0.06523 & 1.078 & 46.875 & 6 \\
\hline *383 & $6 \mathrm{TC} 113$ & OLH & 0.01411 & 0.31679 & 0.50852 & 0.09445 & 0.06613 & 1.172 & 52.4531 & 6 \\
\hline 384 & 6TC114 & OLH & 0.0537 & 0.3005 & 0.48577 & 0.09487 & 0.06516 & 1.297 & 41.2969 & 6 \\
\hline 385 & 6TC115 & OLH & 0.04974 & 0.31586 & 0.45464 & 0.10715 & 0.07261 & 0.766 & 60.0234 & 6 \\
\hline 386 & 61 & $\mathrm{OLH}$ & 0.05766 & 0888 & 0.46519 & 0.09741 & 0.07086 & 0.141 & 50.8594 & 6 \\
\hline 387 & 6TC117 & OLH & 0.0636 & 0.32517 & 0.44488 & 0.09402 & 0.07233 & 0.859 & 42.8906 & 6 \\
\hline 388 & 6TC118 & $\mathrm{OLH}$ & 0.01807 & 0.31214 & 0.50598 & 0.09233 & 0.07148 & 0.891 & 59.625 & 6 \\
\hline 389 & 6TC119 & $\mathrm{OLH}$ & 0.03094 & 0.32494 & 0.47104 & 0.10778 & 0.0653 & 0.375 & 58.0313 & 6 \\
\hline 390 & $6 \mathrm{TC} 120$ & $\mathrm{OLH}$ & 0.01906 & 0.30445 & 0.5055 & 0.10249 & 0.0685 & 0.188 & 44.4844 & 6 \\
\hline 391 & $6 \mathrm{TC} 121$ & $\mathrm{OLH}$ & 0.03985 & 0.32587 & 0.48043 & 0.08576 & 0.06809 & 0.391 & 49.2656 & 6 \\
\hline *392 & $6 \mathrm{TC} 122$ & OLH & 0.0349 & 0.31842 & 0.46876 & 0.10567 & 0.07225 & 1.016 & 18.9844 & 6 \\
\hline 393 & $6 \mathrm{TC} 123$ & OLH & 0.04677 & 0.30538 & 0.47872 & 0.09847 & 0.07066 & 1.922 & 28.9453 & 6 \\
\hline 394 & $6 \mathrm{TC} 124$ & OLH & 0.05865 & 0.32354 & 0.45685 & 0.09169 & 0.06927 & 1.219 & 20.5781 & 6 \\
\hline 395 & $6 \mathrm{TC} 125$ & OLH & 0.025 & 0.31097 & 0.50269 & 0.08915 & 0.07219 & 1.266 & 25.3594 & 6 \\
\hline 396 & $6 \mathrm{TC} 126$ & OLH & 0.02104 & 0.3147 & 0.49175 & 0.10672 & 0.06579 & 1.703 & 20.1797 & 6 \\
\hline *397 & 6TC127 & $\mathrm{OLH}$ & 0.06261 & 0.30213 & 0.46525 & 0.10186 & 0.06815 & 1.25 & 38.5078 & 6 \\
\hline 398 & $6 \mathrm{TC} 128$ & OLH & 0.04281 & 0.32471 & 0.47008 & 0.09508 & 0.06732 & 1.469 & 36.1172 & 6 \\
\hline 399 & 6TC129 & $\mathrm{OLH}$ & 0.06657 & 0.32028 & 0.43835 & 0.10588 & 0.06892 & 0.234 & 34.5234 & 6 \\
\hline 400 & $6 \mathrm{TC} 130$ & $\mathrm{OLH}$ & 0.03886 & 0.31283 & 0.47562 & 0.09974 & 0.07295 & 0.594 & 18.5859 & 6 \\
\hline$* 401$ & 6TC131 & $\mathrm{OLH}$ & 0.05271 & 0.32378 & 0.46369 & 0.09 & 0.06982 & 0.906 & 36.9141 & 6 \\
\hline 402 & 6TC132 & $\mathrm{OLH}$ & 0.02401 & 0.30958 & 0.50046 & 0.09593 & 0.07002 & 0.25 & 37.7109 & 6 \\
\hline 403 & 6TC133 & $\mathrm{OLH}$ & 0.05172 & 0.31656 & 0.46944 & 0.09656 & 0.06572 & 0.313 & 24.5625 & 6 \\
\hline 404 & 6TC134 & $\mathrm{OLH}$ & 0.05469 & 0.30934 & 0.45939 & 0.10884 & 0.06774 & 0.844 & 19.7813 & 6 \\
\hline 405 & 6TC135 & OLH & 0.06756 & 0.29956 & 0.47141 & 0.09275 & 0.06872 & 0.469 & 19.3828 & 6 \\
\hline
\end{tabular}

* OLH Points used in fits where more points needed to capture nonlinear behavior 


\section{APPENDIX B. SOLIDS PREDICTIONS FOR ENVELOPES A, B, AND C}

Table 35 through Table 40 lists the predicted solids from the OLI simulations for Envelopes A, $\mathrm{B}$, and $\mathrm{C}$. The Test ID indicates the type of run with the first 1-2 digits represent the target Sodium Molarity (10, 8 or 6) and then EA, TB, or TC for Envelope A, B, and C respectively followed by the 3 digits that represent the run number. No accurate prediction equations for the solubility of the evaporator bottoms stream in terms of the total insoluble solids present could be derived in either linear or nonlinear forms for Envelopes A, B, or C. Several attempts were made to include nonlinear and linear terms in the prediction fits, but the waste feed compositions, SBS to waste Feed ratio, the bottoms temperature, and Na molarity did not provide enough data about this phenomenon. The following tables are included to show what solids OLI predicted for each run. 
Table 35. Predicted Solids for Envelope A Simulations - Part 1

\begin{tabular}{|c|c|c|c|c|c|c|c|c|c|c|c|c|c|c|}
\hline Test ID & SBS/Feed & Temp $\left[{ }^{\circ} \mathrm{C}\right]$ & $\begin{array}{c}\mathrm{Na} \mathrm{M} \\
{[\mathrm{mol} / \mathrm{L}]}\end{array}$ & $\begin{array}{l}\mathrm{ALOH} 3 \\
{[\mathrm{~g}]}\end{array}$ & \begin{tabular}{|c|} 
ANATASE \\
{$[\mathrm{g}]$}
\end{tabular} & $\begin{array}{c}\text { CA3PO42 } \\
{[\mathrm{g}]}\end{array}$ & $\begin{array}{c}\mathrm{CACO} 3 \\
{[\mathrm{~g}]} \\
\end{array}$ & CAF2 $[\mathrm{g}]$ & CANC $[\mathrm{g}]$ & $\begin{array}{c}\mathrm{CAOH} 2 \\
{[\mathrm{~g}]}\end{array}$ & $\begin{array}{c}\text { CATIO3 } \\
{[\mathrm{g}]} \\
\end{array}$ & $\begin{array}{c}\mathrm{CROH} 3 \\
{[\mathrm{~g}]}\end{array}$ & \begin{tabular}{|c|} 
HYDROSOD \\
{$[\mathrm{g}]$}
\end{tabular} & $\begin{array}{c}\mathrm{MGOH} 2 \\
{[\mathrm{~g}]}\end{array}$ \\
\hline 10EA001 & 0 & \begin{tabular}{|l|}
15 \\
\end{tabular} & 10 & 0 & 0 & \begin{tabular}{|c|}
0 \\
\end{tabular} & 0 & \begin{tabular}{l|}
0 \\
\end{tabular} & \begin{tabular}{|l|}
0 \\
\end{tabular} & 0 & 0 & 0 & 0 & 0 \\
\hline 10EA002 & 0 & 15 & 10 & 0 & 0 & 0 & 0 & 0 & 0 & 0 & 0 & 0 & 0 & 0 \\
\hline 10EA003 & 0 & 15 & 10 & 0 & 0 & 0 & 0 & 0 & 0 & 0 & 0 & 0 & 0 & 0 \\
\hline 10EA004 & 0 & 15 & 10 & 0 & 0 & 0 & 0 & 0 & 0 & 0 & 0 & 0 & 0 & 0 \\
\hline 10EA005 & 0 & 15 & 10 & 0 & 0 & 0 & 0 & 0 & 0 & 0 & 0 & 0 & 0 & 0 \\
\hline 10EA006 & 0 & 15 & 10 & 0 & 0 & 0 & 0 & 0 & 0 & 0 & 0 & 0 & 0 & 0 \\
\hline 10EA007 & 0 & 15 & 10 & 0 & 0 & 0 & 0 & 0 & 0 & 0 & 0 & 0 & 0 & 0 \\
\hline 10EA008 & 0 & 15 & 10 & 0 & 0 & 0 & 0 & 0 & 0 & 0 & 0 & 0 & 0 & 0 \\
\hline 10EA009 & 0 & 15 & 10 & 0 & 0 & 0 & 0 & 0 & 0 & 0 & 0 & 0 & 0 & 0 \\
\hline 10EA010 & 0 & 15 & 10 & 0 & 0 & 0 & 0 & 0 & 0 & 0 & 0 & 0 & 0 & 0 \\
\hline 10EA011 & 0 & 15 & 10 & 0 & 0 & 0 & 0 & 0 & 0 & 0 & 0 & 0 & 0 & 0 \\
\hline 10EA012 & 0 & 15 & 10 & 0 & 0 & 0 & 0 & 0 & 0 & 0 & 0 & 0 & 0 & 0 \\
\hline 10EA013 & 0 & 15 & 10 & 0 & 0 & 0 & 0 & 0 & 0 & 0 & 0 & 0 & 0 & 0 \\
\hline 10EA014 & 0 & 15 & 10 & 0 & 0 & 0 & 0 & 0 & 0 & 0 & 0 & 0 & 0 & 0 \\
\hline 10EA015 & 0 & 15 & 10 & 0 & 0 & 0 & 0 & 0 & 0 & 0 & 0 & 0 & 0 & 0 \\
\hline 10EA016 & 0 & 15 & 10 & 0 & 0 & 0 & 0 & 0 & 0 & 0 & 0 & 0 & 0 & 0 \\
\hline 10EA017 & 0 & 15 & 10 & 0 & 0 & 0 & 0 & 0 & 0 & 0 & 0 & 0 & 0 & 0 \\
\hline 10EA018 & 0 & 15 & 10 & 0 & 0 & 0 & 0 & 0 & 0 & 0 & 0 & 0 & 0 & 0 \\
\hline 10EA019 & 0 & 15 & 10 & 0 & 0 & 0 & 0 & 0 & 0 & 0 & 0 & 0 & 0 & 0 \\
\hline 10EA020 & 0 & 15 & 10 & 0 & 0 & 0 & 0 & 0 & 0 & 0 & 0 & 0 & 0 & 0 \\
\hline 10EA021 & 2 & 15 & 10 & 0 & 0 & 0 & 0 & 0 & 0 & $3.15 \mathrm{E}+02$ & $4.97 \mathrm{E}+01$ & 0 & 0 & $7.38 \mathrm{E}+01$ \\
\hline 10EA022 & 2 & 15 & 10 & 0 & 0 & 0 & 0 & 0 & 0 & $3.19 \mathrm{E}+02$ & $5.03 \mathrm{E}+01$ & 0 & 0 & $7.46 \mathrm{E}+01$ \\
\hline 10EA023 & 2 & 15 & 10 & 0 & 0 & 0 & 0 & 0 & 0 & $3.54 \mathrm{E}+02$ & $5.58 \mathrm{E}+01$ & 0 & 0 & $8.28 \mathrm{E}+01$ \\
\hline 10EA024 & 2 & 15 & 10 & 0 & 0 & 0 & 0 & 0 & 0 & $3.41 \mathrm{E}+02$ & $5.37 \mathrm{E}+01$ & 0 & 0 & $7.97 \mathrm{E}+01$ \\
\hline 10EA025 & 2 & 15 & 10 & 0 & 0 & 0 & 0 & 0 & 0 & $3.21 \mathrm{E}+02$ & $5.06 \mathrm{E}+01$ & 0 & 0 & $7.51 \mathrm{E}+01$ \\
\hline 10EA026 & 2 & 15 & 10 & 0 & 0 & 0 & 0 & $3.75 \mathrm{E}+02$ & 0 & 0 & $5.61 \mathrm{E}+01$ & 0 & 0 & $8.33 \mathrm{E}+01$ \\
\hline $10 \mathrm{EA} 027$ & 2 & 15 & 10 & 0 & 0 & 0 & 0 & 0 & 0 & $3.62 \mathrm{E}+02$ & $5.70 \mathrm{E}+01$ & 0 & 0 & $8.47 \mathrm{E}+01$ \\
\hline 10EA028 & 2 & 15 & 10 & 0 & 0 & 0 & 0 & 0 & 0 & $3.60 \mathrm{E}+02$ & $5.68 \mathrm{E}+01$ & 0 & 0 & $8.43 \mathrm{E}+01$ \\
\hline 10EA029 & 2 & 15 & 10 & 0 & 0 & 0 & 0 & 0 & 0 & $3.49 \mathrm{E}+02$ & $5.50 \mathrm{E}+01$ & 0 & 0 & $8.17 \mathrm{E}+01$ \\
\hline 10EA030 & 2 & 15 & 10 & 0 & 0 & 0 & 0 & 0 & 0 & $3.37 \mathrm{E}+02$ & $5.32 \mathrm{E}+01$ & 0 & 0 & $7.89 \mathrm{E}+01$ \\
\hline 10EA031 & 2 & 15 & 10 & 0 & 0 & 0 & 0 & 0 & 0 & $3.15 \mathrm{E}+02$ & $4.97 \mathrm{E}+01$ & 0 & 0 & $7.38 \mathrm{E}+01$ \\
\hline
\end{tabular}


Table 35. Predicted Solids for Envelope A Simulations - Part 1 (cont'd)

\begin{tabular}{|c|c|c|c|c|c|c|c|c|c|c|c|c|c|c|}
\hline Test ID & $\begin{array}{l}\text { SBS/ } \\
\text { Feed } \\
\end{array}$ & $\begin{array}{l}\text { Temp } \\
{\left[{ }^{\circ} \mathrm{C}\right]}\end{array}$ & $\begin{array}{c}\mathrm{Na} \mathrm{M} \\
{[\mathrm{mol} / \mathrm{L}]}\end{array}$ & $\begin{array}{c}\text { ALOH3 } \\
{[\mathrm{g}]}\end{array}$ & $\begin{array}{c}\text { ANATAS } \\
\text { E }[\mathrm{g}] \\
\end{array}$ & $\begin{array}{c}\text { CA3PO42 } \\
{[\mathrm{g}]} \\
\end{array}$ & $\begin{array}{c}\mathrm{CACO} 3 \\
{[\mathrm{~g}]} \\
\end{array}$ & CAF2 [g] & CANC $[\mathrm{g}]$ & $\begin{array}{c}\mathrm{CAOH} 2 \\
{[\mathrm{~g}]}\end{array}$ & $\begin{array}{c}\text { CATIO3 } \\
{[\mathrm{g}]} \\
\end{array}$ & $\begin{array}{c}\mathrm{CROH} 3 \\
{[\mathrm{~g}]}\end{array}$ & $\begin{array}{c}\text { HYDROS } \\
\text { OD [g] }\end{array}$ & $\begin{array}{c}\mathrm{MGOH} 2 \\
{[\mathrm{~g}]}\end{array}$ \\
\hline 10EA032 & 2 & 15 & 10 & 0 & 0 & 0 & 0 & 0 & 0 & $3.19 \mathrm{E}+02$ & $5.03 \mathrm{E}+01$ & 0 & 0 & $7.46 \mathrm{E}+01$ \\
\hline 10EA033 & 2 & 15 & 10 & 0 & 0 & 0 & 0 & 0 & 0 & $3.54 \mathrm{E}+02$ & $5.58 \mathrm{E}+01$ & 0 & 0 & $8.28 \mathrm{E}+01$ \\
\hline 10EA034 & 2 & 15 & 10 & 0 & 0 & 0 & 0 & 0 & 0 & $3.41 \mathrm{E}+02$ & $5.37 \mathrm{E}+01$ & 0 & 0 & $7.97 \mathrm{E}+01$ \\
\hline 10EA035 & 2 & 15 & 10 & 0 & 0 & 0 & 0 & 0 & 0 & $3.21 \mathrm{E}+02$ & $5.06 \mathrm{E}+01$ & 0 & 0 & $7.51 \mathrm{E}+01$ \\
\hline 10EA036 & 2 & 15 & 10 & 0 & 0 & 0 & 0 & $3.75 \mathrm{E}+02$ & 0 & 0 & $5.61 \mathrm{E}+01$ & 0 & 0 & $8.33 \mathrm{E}+01$ \\
\hline 10EA037 & 2 & 15 & 10 & 0 & 0 & 0 & 0 & 0 & 0 & $3.62 \mathrm{E}+02$ & $5.70 \mathrm{E}+01$ & 0 & 0 & $8.47 \mathrm{E}+01$ \\
\hline 10EA039 & 2 & 15 & 10 & 0 & 0 & 0 & 0 & 0 & 0 & $3.49 \mathrm{E}+02$ & $5.50 \mathrm{E}+01$ & 0 & 0 & $8.17 \mathrm{E}+01$ \\
\hline 10EA040 & 2 & 15 & 10 & 0 & 0 & 0 & 0 & 0 & 0 & $3.37 \mathrm{E}+02$ & $5.32 \mathrm{E}+01$ & 0 & 0 & $7.89 \mathrm{E}+01$ \\
\hline 10EA041 & 0 & 66 & 10 & 0 & 0 & 0 & 0 & 0 & 0 & 0 & 0 & 0 & 0 & 0 \\
\hline 10EA042 & 0 & 66 & 10 & 0 & 0 & 0 & 0 & 0 & 0 & 0 & 0 & 0 & 0 & 0 \\
\hline $10 \mathrm{EA} 043$ & 0 & 66 & 10 & 0 & 0 & 0 & 0 & 0 & 0 & 0 & 0 & 0 & 0 & 0 \\
\hline 10EA044 & 0 & 66 & 10 & 0 & 0 & 0 & 0 & 0 & 0 & 0 & 0 & 0 & 0 & 0 \\
\hline 10EA045 & 0 & 66 & 10 & 0 & 0 & 0 & 0 & 0 & 0 & 0 & 0 & 0 & 0 & 0 \\
\hline 10EA047 & 0 & 66 & 10 & 0 & 0 & 0 & 0 & 0 & 0 & 0 & 0 & 0 & 0 & 0 \\
\hline 10EA048 & 0 & 66 & 10 & 0 & 0 & 0 & 0 & 0 & 0 & 0 & 0 & 0 & 0 & 0 \\
\hline 10EA049 & 0 & 66 & 10 & 0 & 0 & 0 & 0 & 0 & 0 & 0 & 0 & 0 & 0 & 0 \\
\hline 10EA050 & 0 & 66 & 10 & 0 & 0 & 0 & 0 & 0 & 0 & 0 & 0 & 0 & 0 & 0 \\
\hline 10EA051 & 0 & 66 & 10 & 0 & 0 & 0 & 0 & 0 & 0 & 0 & 0 & 0 & 0 & 0 \\
\hline 10EA052 & 0 & 66 & 10 & 0 & 0 & 0 & 0 & 0 & 0 & 0 & 0 & 0 & 0 & 0 \\
\hline 10EA053 & 0 & 66 & 10 & 0 & 0 & 0 & 0 & 0 & 0 & 0 & 0 & 0 & 0 & 0 \\
\hline 10EA054 & 0 & 66 & 10 & 0 & 0 & 0 & 0 & 0 & 0 & 0 & 0 & 0 & 0 & 0 \\
\hline 10EA055 & 0 & 66 & 10 & 0 & 0 & 0 & 0 & 0 & 0 & 0 & 0 & 0 & 0 & 0 \\
\hline 10EA056 & 0 & 66 & 10 & 0 & 0 & 0 & 0 & 0 & 0 & 0 & 0 & 0 & 0 & 0 \\
\hline 10EA057 & 0 & 66 & 10 & 0 & 0 & 0 & 0 & 0 & 0 & 0 & 0 & 0 & 0 & 0 \\
\hline 10EA058 & 0 & 66 & 10 & 0 & 0 & 0 & 0 & 0 & 0 & 0 & 0 & 0 & 0 & 0 \\
\hline 10EA059 & 0 & 66 & 10 & 0 & 0 & 0 & 0 & 0 & 0 & 0 & 0 & 0 & 0 & 0 \\
\hline $10 \mathrm{EA} 060$ & 0 & 66 & 10 & 0 & 0 & 0 & 0 & 0 & 0 & 0 & 0 & 0 & 0 & 0 \\
\hline 10EA063 & 2 & 66 & 10 & 0 & 0 & 0 & 0 & 0 & 0 & $3.52 \mathrm{E}+02$ & $5.58 \mathrm{E}+01$ & 0 & 0 & $8.28 \mathrm{E}+01$ \\
\hline
\end{tabular}


Table 35. Predicted Solids for Envelope A Simulations - Part 1 (cont'd)

\begin{tabular}{|c|c|c|c|c|c|c|c|c|c|c|c|c|c|c|}
\hline Test ID & $\begin{array}{l}\text { SBS/ } \\
\text { Feed }\end{array}$ & $\begin{array}{l}\text { Temp } \\
{\left[{ }^{\circ} \mathrm{C}\right]} \\
\end{array}$ & $\begin{array}{c}\mathrm{Na} \mathrm{M} \\
{[\mathrm{mol} / \mathrm{L}]}\end{array}$ & $\begin{array}{c}\mathrm{ALOH} 3 \\
{[\mathrm{~g}]}\end{array}$ & $\begin{array}{c}\text { ANATAS } \\
\text { E }[\mathrm{g}]\end{array}$ & $\begin{array}{c}\text { CA3PO42 } \\
{[\mathrm{g}]} \\
\end{array}$ & $\begin{array}{c}\mathrm{CACO} 3 \\
{[\mathrm{~g}]}\end{array}$ & CAF2 [g] & CANC [g] & $\begin{array}{c}\mathrm{CAOH} 2 \\
{[\mathrm{~g}]}\end{array}$ & $\begin{array}{c}\text { CATIO3 } \\
{[\mathrm{g}]}\end{array}$ & $\begin{array}{c}\mathrm{CROH} 3 \\
{[\mathrm{~g}]} \\
\end{array}$ & $\begin{array}{c}\text { HYDROS } \\
\text { OD [g] }\end{array}$ & $\begin{array}{c}\mathrm{MGOH} 2 \\
{[\mathrm{~g}]}\end{array}$ \\
\hline 10EA064 & 2 & 66 & 10 & 0 & 0 & 0 & 0 & 0 & 0 & $3.40 \mathrm{E}+02$ & $5.37 \mathrm{E}+01$ & 0 & 0 & $7.97 \mathrm{E}+01$ \\
\hline 10EA065 & 2 & 66 & 10 & 0 & 0 & 0 & 0 & 0 & 0 & $3.20 \mathrm{E}+02$ & $5.06 \mathrm{E}+01$ & 0 & 0 & $7.51 \mathrm{E}+01$ \\
\hline 10EA067 & 2 & 66 & 10 & 0 & 0 & 0 & 0 & 0 & 0 & $3.61 \mathrm{E}+02$ & $5.70 \mathrm{E}+01$ & 0 & 0 & $8.47 \mathrm{E}+01$ \\
\hline 10EA068 & 2 & 66 & 10 & 0 & 0 & $4.98 \mathrm{E}+02$ & 0 & 0 & 0 & 0 & $5.68 \mathrm{E}+01$ & 0 & 0 & $8.43 \mathrm{E}+01$ \\
\hline 10EA069 & 2 & 66 & 10 & 0 & 0 & $4.83 \mathrm{E}+02$ & 0 & 0 & 0 & 0 & $5.50 \mathrm{E}+01$ & 0 & 0 & $8.17 \mathrm{E}+01$ \\
\hline 10EA071 & 2 & 66 & 10 & 0 & 0 & 0 & 0 & 0 & 0 & $3.15 \mathrm{E}+02$ & $4.97 \mathrm{E}+01$ & 0 & 0 & $7.38 \mathrm{E}+01$ \\
\hline 10EA072 & 2 & 66 & 10 & 0 & 0 & 0 & 0 & 0 & 0 & $3.19 \mathrm{E}+02$ & $5.03 \mathrm{E}+01$ & 0 & 0 & $7.46 \mathrm{E}+01$ \\
\hline 10EA073 & 2 & 66 & 10 & 0 & 0 & 0 & 0 & 0 & 0 & $3.52 \mathrm{E}+02$ & $5.58 \mathrm{E}+01$ & 0 & 0 & $8.28 \mathrm{E}+01$ \\
\hline 10EA074 & 2 & 66 & 10 & 0 & 0 & 0 & 0 & 0 & 0 & $3.40 \mathrm{E}+02$ & $5.37 \mathrm{E}+01$ & 0 & 0 & $7.97 \mathrm{E}+01$ \\
\hline 10EA075 & 2 & 66 & 10 & 0 & 0 & 0 & 0 & 0 & 0 & $3.20 \mathrm{E}+02$ & $5.06 \mathrm{E}+01$ & 0 & 0 & $7.51 \mathrm{E}+01$ \\
\hline 10EA076 & 2 & 66 & 10 & 0 & 0 & 0 & 0 & $3.73 \mathrm{E}+02$ & 0 & 0 & $5.61 \mathrm{E}+01$ & $4.69 \mathrm{E}+00$ & 0 & $8.33 \mathrm{E}+01$ \\
\hline 10EA077 & 2 & 66 & 10 & 0 & 0 & 0 & 0 & 0 & 0 & $3.61 \mathrm{E}+02$ & $5.70 \mathrm{E}+01$ & 0 & 0 & $8.47 \mathrm{E}+01$ \\
\hline 10EA079 & 2 & 66 & 10 & 0 & 0 & $4.83 \mathrm{E}+02$ & 0 & 0 & 0 & 0 & $5.50 \mathrm{E}+01$ & 0 & 0 & $8.17 \mathrm{E}+01$ \\
\hline 10EA080 & 2 & 66 & 10 & 0 & 0 & 0 & 0 & 0 & 0 & $3.37 \mathrm{E}+02$ & $5.32 \mathrm{E}+01$ & 0 & 0 & $7.89 \mathrm{E}+01$ \\
\hline 10EA081 & 0.0391 & 15 & 10 & 0 & 0 & 0 & 0 & 0 & 0 & $6.51 \mathrm{E}+00$ & $1.07 \mathrm{E}+00$ & 0 & 0 & $1.58 \mathrm{E}+00$ \\
\hline 10EA082 & 1.957 & 15.102 & 10 & 0 & 0 & 0 & 0 & 0 & 0 & $3.35 \mathrm{E}+02$ & $5.29 \mathrm{E}+01$ & 0 & 0 & $7.85 \mathrm{E}+01$ \\
\hline 10EA083 & 0.7578 & 15.714 & 10 & 0 & 0 & 0 & 0 & 0 & 0 & $1.35 \mathrm{E}+02$ & $2.14 \mathrm{E}+01$ & 0 & 0 & $3.17 \mathrm{E}+01$ \\
\hline 10EA084 & 0.3008 & 15.918 & 10 & 0 & 0 & 0 & 0 & 0 & 0 & $5.28 \mathrm{E}+01$ & $8.34 \mathrm{E}+00$ & 0 & 0 & $1.24 \mathrm{E}+01$ \\
\hline 10EA085 & 0.0039 & 16.122 & 10 & 0 & 0 & 0 & 0 & 0 & 0 & $2.80 \mathrm{E}-01$ & $1.07 \mathrm{E}-01$ & 0 & 0 & $1.59 \mathrm{E}-01$ \\
\hline 10EA086 & 1.2305 & 17.04 & 10 & 0 & 0 & 0 & 0 & 0 & 0 & $2.16 \mathrm{E}+02$ & $3.41 \mathrm{E}+01$ & 0 & 0 & $5.06 \mathrm{E}+01$ \\
\hline 10EA087 & 1.5898 & 17.55 & 10 & 0 & 0 & 0 & 0 & 0 & 0 & $2.85 \mathrm{E}+02$ & $4.50 \mathrm{E}+01$ & 0 & 0 & $6.68 \mathrm{E}+01$ \\
\hline 10EA088 & 0.7734 & 18.06 & 10 & 0 & 0 & 0 & 0 & 0 & 0 & $1.37 \mathrm{E}+02$ & $2.17 \mathrm{E}+01$ & 0 & 0 & $3.22 \mathrm{E}+01$ \\
\hline 10EA089 & 1.1641 & 18.162 & 10 & 0 & 0 & 0 & 0 & 0 & 0 & $1.99 \mathrm{E}+02$ & $3.14 \mathrm{E}+01$ & 0 & 0 & $4.66 \mathrm{E}+01$ \\
\hline 10EA090 & 0.6094 & 18.264 & 10 & 0 & 0 & 0 & 0 & 0 & 0 & $1.04 \mathrm{E}+02$ & $1.65 \mathrm{E}+01$ & 0 & 0 & $2.45 \mathrm{E}+01$ \\
\hline 10EA091 & 0.8594 & 18.57 & 10 & 0 & 0 & 0 & 0 & 0 & 0 & $1.50 \mathrm{E}+02$ & $2.36 \mathrm{E}+01$ & 0 & 0 & $3.51 \mathrm{E}+01$ \\
\hline 10EA092 & 0.1914 & 18.672 & 10 & 0 & 0 & 0 & 0 & 0 & 0 & $3.34 \mathrm{E}+01$ & $5.31 \mathrm{E}+00$ & 0 & 0 & $7.89 \mathrm{E}+00$ \\
\hline 10EA095 & 1.3203 & 19.998 & 10 & 0 & 0 & 0 & 0 & 0 & 0 & $2.26 \mathrm{E}+02$ & $3.56 \mathrm{E}+01$ & 0 & 0 & $5.29 \mathrm{E}+01$ \\
\hline 10EA096 & 1.8398 & 20.508 & 10 & 0 & 0 & 0 & 0 & 0 & 0 & $3.26 \mathrm{E}+02$ & $5.14 \mathrm{E}+01$ & 0 & 0 & $7.62 \mathrm{E}+01$ \\
\hline 10EA097 & 0.1563 & 21.222 & 10 & 0 & 0 & 0 & 0 & 0 & 0 & $2.74 \mathrm{E}+01$ & $4.39 \mathrm{E}+00$ & 0 & 0 & $6.51 \mathrm{E}+00$ \\
\hline
\end{tabular}


Table 35. Predicted Solids for Envelope A Simulations - Part 1 (cont'd)

\begin{tabular}{|c|c|c|c|c|c|c|c|c|c|c|c|c|c|c|}
\hline Test ID & $\begin{array}{l}\text { SBS/ } \\
\text { Feed }\end{array}$ & $\begin{array}{l}\text { Temp } \\
{\left[{ }^{\circ} \mathrm{C}\right]} \\
\end{array}$ & $\begin{array}{c}\mathrm{Na} \mathrm{M} \\
{[\mathrm{mol} / \mathrm{L}]}\end{array}$ & $\begin{array}{c}\mathrm{ALOH} 3 \\
{[\mathrm{~g}]}\end{array}$ & $\begin{array}{c}\text { ANATAS } \\
\text { E }[\mathrm{g}] \\
\end{array}$ & $\begin{array}{c}\text { CA3PO42 } \\
{[\mathrm{g}]} \\
\end{array}$ & $\begin{array}{c}\mathrm{CACO} 3 \\
{[\mathrm{~g}]}\end{array}$ & CAF2 [g] & CANC [g] & $\begin{array}{c}\mathrm{CAOH} 2 \\
{[\mathrm{~g}]}\end{array}$ & $\begin{array}{c}\text { CATIO3 } \\
{[\mathrm{g}]}\end{array}$ & $\begin{array}{c}\text { CROH3 } \\
{[\mathrm{g}]}\end{array}$ & $\begin{array}{c}\text { HYDROS } \\
\text { OD [g] }\end{array}$ & $\begin{array}{c}\mathrm{MGOH} 2 \\
{[\mathrm{~g}]}\end{array}$ \\
\hline 10EA098 & 1.5977 & 21.426 & 10 & 0 & 0 & 0 & 0 & 0 & 0 & $2.74 \mathrm{E}+02$ & $4.32 \mathrm{E}+01$ & 0 & 0 & $6.42 \mathrm{E}+01$ \\
\hline 10EA099 & 0.1719 & 21.732 & 10 & 0 & 0 & 0 & 0 & 0 & 0 & $2.97 \mathrm{E}+01$ & $4.73 E+00$ & 0 & 0 & $7.02 \mathrm{E}+00$ \\
\hline 10EA101 & 0.8633 & 23.466 & 10 & 0 & 0 & 0 & 0 & 0 & 0 & $1.53 \mathrm{E}+02$ & $2.41 \mathrm{E}+01$ & 0 & 0 & $3.58 \mathrm{E}+01$ \\
\hline 10EA102 & 1.0703 & 24.282 & 10 & 0 & 0 & 0 & 0 & 0 & 0 & $1.91 \mathrm{E}+02$ & $3.02 \mathrm{E}+01$ & 0 & 0 & $4.48 \mathrm{E}+01$ \\
\hline 10EA103 & 1.0273 & 26.016 & 10 & 0 & 0 & 0 & 0 & 0 & 0 & $1.80 \mathrm{E}+02$ & $2.84 \mathrm{E}+01$ & 0 & 0 & $4.22 \mathrm{E}+01$ \\
\hline 10EA105 & 0.5 & 26.526 & 10 & 0 & 0 & 0 & 0 & 0 & 0 & $8.79 \mathrm{E}+01$ & $1.39 \mathrm{E}+01$ & 0 & 0 & $2.06 \mathrm{E}+01$ \\
\hline 10EA106 & 1.2695 & 27.036 & 10 & 0 & 0 & 0 & 0 & 0 & 0 & $2.24 \mathrm{E}+02$ & $3.53 \mathrm{E}+01$ & 0 & 0 & $5.25 \mathrm{E}+01$ \\
\hline 10EA107 & 0.0195 & 27.138 & 10 & 0 & 0 & 0 & 0 & 0 & 0 & $3.08 \mathrm{E}+00$ & 5.39E-01 & 0 & 0 & $8.00 \mathrm{E}-01$ \\
\hline 10EA108 & 1.4414 & 27.24 & 10 & 0 & 0 & 0 & 0 & 0 & 0 & $2.48 \mathrm{E}+02$ & $3.92 \mathrm{E}+01$ & 0 & 0 & $5.82 \mathrm{E}+01$ \\
\hline 10EA109 & 1.0547 & 28.056 & 10 & 0 & 0 & 0 & 0 & 0 & 0 & $1.87 \mathrm{E}+02$ & $2.94 \mathrm{E}+01$ & 0 & 0 & $4.37 \mathrm{E}+01$ \\
\hline 10EA110 & 1.2461 & 28.26 & 10 & 0 & 0 & 0 & 0 & 0 & 0 & $2.19 \mathrm{E}+02$ & $3.45 \mathrm{E}+01$ & 0 & 0 & $5.12 \mathrm{E}+01$ \\
\hline 10EA111 & 1.0977 & 28.464 & 10 & 0 & 0 & 0 & 0 & 0 & 0 & $1.95 \mathrm{E}+02$ & $3.09 \mathrm{E}+01$ & 0 & 0 & $4.58 \mathrm{E}+01$ \\
\hline 10EA113 & 1.0664 & 28.872 & 10 & 0 & 0 & 0 & 0 & 0 & 0 & $1.86 \mathrm{E}+02$ & $2.94 \mathrm{E}+01$ & 0 & 0 & $4.37 \mathrm{E}+01$ \\
\hline 10EA114 & 0.6563 & 29.382 & 10 & 0 & 0 & 0 & 0 & 0 & 0 & $1.17 \mathrm{E}+02$ & $1.85 \mathrm{E}+01$ & 0 & 0 & $2.75 \mathrm{E}+01$ \\
\hline 10EA115 & 1.3867 & 29.484 & 10 & 0 & 0 & 0 & 0 & 0 & 0 & $2.43 \mathrm{E}+02$ & $3.83 \mathrm{E}+01$ & 0 & 0 & $5.69 \mathrm{E}+01$ \\
\hline 10EA116 & 1.0078 & 30.198 & 10 & 0 & 0 & 0 & 0 & 0 & 0 & $1.79 \mathrm{E}+02$ & $2.82 \mathrm{E}+01$ & 0 & 0 & $4.19 \mathrm{E}+01$ \\
\hline 10EA117 & 1.8711 & 30.504 & 10 & 0 & 0 & 0 & 0 & 0 & 0 & $3.28 \mathrm{E}+02$ & $5.17 \mathrm{E}+01$ & 0 & 0 & $7.68 \mathrm{E}+01$ \\
\hline 10EA118 & 0.5664 & 30.606 & 10 & 0 & 0 & 0 & 0 & 0 & 0 & $9.88 \mathrm{E}+01$ & $1.56 \mathrm{E}+01$ & 0 & 0 & $2.32 \mathrm{E}+01$ \\
\hline 10EA119 & 0.6953 & 31.422 & 10 & 0 & 0 & 0 & 0 & 0 & 0 & $1.15 \mathrm{E}+02$ & $1.82 \mathrm{E}+01$ & 0 & 0 & $2.70 \mathrm{E}+01$ \\
\hline 10EA120 & 1.4219 & 31.626 & 10 & 0 & 0 & 0 & 0 & 0 & 0 & $2.48 \mathrm{E}+02$ & $3.91 \mathrm{E}+01$ & 0 & 0 & $5.81 \mathrm{E}+01$ \\
\hline 10EA121 & 1.3555 & 32.748 & 10 & 0 & 0 & 0 & 0 & 0 & 0 & $2.42 \mathrm{E}+02$ & $3.82 \mathrm{E}+01$ & 0 & 0 & $5.67 \mathrm{E}+01$ \\
\hline 10EA122 & 0.2383 & 32.952 & 10 & 0 & 0 & 0 & 0 & 0 & 0 & $4.23 \mathrm{E}+01$ & $6.75 \mathrm{E}+00$ & 0 & 0 & $1.00 \mathrm{E}+01$ \\
\hline 10EA123 & 0.6328 & 33.36 & 10 & 0 & 0 & 0 & 0 & 0 & 0 & $1.11 \mathrm{E}+02$ & $1.77 \mathrm{E}+01$ & 0 & 0 & $2.62 \mathrm{E}+01$ \\
\hline 10EA124 & 1.5313 & 33.564 & 10 & 0 & 0 & 0 & 0 & 0 & 0 & $2.68 \mathrm{E}+02$ & $4.23 \mathrm{E}+01$ & 0 & 0 & $6.28 \mathrm{E}+01$ \\
\hline 10EA125 & 0.4727 & 33.666 & 10 & 0 & 0 & 0 & 0 & 0 & 0 & $7.85 \mathrm{E}+01$ & $1.24 \mathrm{E}+01$ & 0 & 0 & $1.84 \mathrm{E}+01$ \\
\hline 10EA126 & 0.5195 & 34.176 & 10 & 0 & 0 & 0 & 0 & 0 & 0 & $9.03 \mathrm{E}+01$ & $1.43 \mathrm{E}+01$ & 0 & 0 & $2.12 \mathrm{E}+01$ \\
\hline 10EA129 & 1.668 & 37.032 & 10 & 0 & 0 & 0 & 0 & 0 & 0 & $2.90 \mathrm{E}+02$ & $4.58 \mathrm{E}+01$ & 0 & 0 & $6.80 \mathrm{E}+01$ \\
\hline 10EA130 & 1.9336 & 38.358 & 10 & 0 & 0 & 0 & 0 & 0 & 0 & $3.36 \mathrm{E}+02$ & $5.30 \mathrm{E}+01$ & 0 & 0 & $7.86 \mathrm{E}+01$ \\
\hline 10EA131 & 1.4258 & 39.48 & 10 & 0 & 0 & 0 & 0 & 0 & 0 & $2.53 \mathrm{E}+02$ & $4.00 \mathrm{E}+01$ & 0 & 0 & $5.94 \mathrm{E}+01$ \\
\hline
\end{tabular}


Table 35. Predicted Solids for Envelope A Simulations - Part 1 (cont'd)

\begin{tabular}{|c|c|c|c|c|c|c|c|c|c|c|c|c|c|c|}
\hline Test ID & $\begin{array}{l}\text { SBS/ } \\
\text { Feed }\end{array}$ & $\begin{array}{l}\text { Temp } \\
{\left[{ }^{\circ} \mathrm{C}\right]} \\
\end{array}$ & $\begin{array}{c}\mathrm{Na} \mathrm{M} \\
{[\mathrm{mol} / \mathrm{L}]}\end{array}$ & $\begin{array}{c}\mathrm{ALOH} 3 \\
{[\mathrm{~g}]}\end{array}$ & $\begin{array}{c}\text { ANATAS } \\
\text { E }[\mathrm{g}]\end{array}$ & $\begin{array}{c}\text { CA3PO42 } \\
{[\mathrm{g}]} \\
\end{array}$ & $\begin{array}{c}\mathrm{CACO} 3 \\
{[\mathrm{~g}]}\end{array}$ & CAF2 [g] & CANC [g] & $\begin{array}{c}\mathrm{CAOH} 2 \\
{[\mathrm{~g}]}\end{array}$ & $\begin{array}{c}\text { CATIO3 } \\
{[\mathrm{g}]}\end{array}$ & $\begin{array}{c}\mathrm{CROH} 3 \\
{[\mathrm{~g}]}\end{array}$ & $\begin{array}{c}\text { HYDROS } \\
\text { OD }[\mathrm{g}] \\
\end{array}$ & $\begin{array}{c}\mathrm{MGOH} 2 \\
{[\mathrm{~g}]}\end{array}$ \\
\hline 10EA132 & 0.168 & 39.99 & 10 & 0 & 0 & 0 & 0 & 0 & 0 & $2.84 \mathrm{E}+01$ & $4.56 \mathrm{E}+00$ & 0 & 0 & $6.78 \mathrm{E}+00$ \\
\hline 10EA133 & 1.6641 & 40.194 & 10 & 0 & 0 & 0 & 0 & 0 & 0 & $2.83 \mathrm{E}+02$ & $4.46 \mathrm{E}+01$ & 0 & 0 & $6.63 \mathrm{E}+01$ \\
\hline 10EA135 & 1.6914 & 41.112 & 10 & 0 & 0 & 0 & 0 & 0 & 0 & $2.97 \mathrm{E}+02$ & $4.69 \mathrm{E}+01$ & 0 & 0 & $6.97 \mathrm{E}+01$ \\
\hline 10EA136 & 1.125 & 41.622 & 10 & 0 & 0 & 0 & 0 & 0 & 0 & $2.00 \mathrm{E}+02$ & $3.16 \mathrm{E}+01$ & 0 & 0 & $4.69 \mathrm{E}+01$ \\
\hline 10EA137 & 0.2813 & 42.744 & 10 & 0 & 0 & 0 & 0 & 0 & 0 & $4.80 \mathrm{E}+01$ & $7.79 \mathrm{E}+00$ & 0 & 0 & $1.16 \mathrm{E}+01$ \\
\hline 10EA139 & \begin{tabular}{|l}
0.6992 \\
\end{tabular} & 43.56 & 10 & 0 & 0 & 0 & 0 & 0 & 0 & $1.23 \mathrm{E}+02$ & $1.96 \mathrm{E}+01$ & 0 & 0 & $2.91 \mathrm{E}+01$ \\
\hline 10EA140 & 0.957 & 43.662 & 10 & 0 & 0 & 0 & 0 & 0 & 0 & $1.71 \mathrm{E}+02$ & $2.70 \mathrm{E}+01$ & 0 & 0 & $4.01 \mathrm{E}+01$ \\
\hline 10EA141 & \begin{tabular}{|l|}
0.3164 \\
\end{tabular} & 43.866 & 10 & 0 & 0 & 0 & 0 & 0 & 0 & $5.51 \mathrm{E}+01$ & $8.94 \mathrm{E}+00$ & 0 & 0 & $1.33 \mathrm{E}+01$ \\
\hline 10EA142 & 1.7539 & 44.172 & 10 & 0 & 0 & 0 & 0 & $3.22 \mathrm{E}+02$ & 0 & 0 & $4.82 \mathrm{E}+01$ & 0 & 0 & $7.16 \mathrm{E}+01$ \\
\hline 10EA143 & 0.4375 & 44.274 & 10 & 0 & 0 & 0 & 0 & 0 & 0 & $7.76 \mathrm{E}+01$ & $1.24 \mathrm{E}+01$ & 0 & 0 & $1.84 \mathrm{E}+01$ \\
\hline 10EA144 & 0.7617 & 44.376 & 10 & 0 & 0 & 0 & 0 & 0 & 0 & $1.33 \mathrm{E}+02$ & $2.11 \mathrm{E}+01$ & 0 & 0 & $3.13 \mathrm{E}+01$ \\
\hline 10EA145 & 0.6758 & 44.58 & 10 & 0 & 0 & 0 & 0 & 0 & 0 & $1.18 \mathrm{E}+02$ & $1.86 \mathrm{E}+01$ & 0 & 0 & $2.77 \mathrm{E}+01$ \\
\hline 10EA147 & 1.168 & 45.6 & 10 & 0 & 0 & 0 & 0 & $2.21 \mathrm{E}+02$ & 0 & 0 & $3.33 \mathrm{E}+01$ & 0 & 0 & $4.94 \mathrm{E}+01$ \\
\hline 10EA148 & 0.7852 & 45.702 & 10 & 0 & 0 & 0 & 0 & 0 & 0 & $1.38 \mathrm{E}+02$ & $2.21 \mathrm{E}+01$ & 0 & 0 & $3.27 \mathrm{E}+01$ \\
\hline 10EA149 & \begin{tabular}{|l}
0.9219 \\
\end{tabular} & 45.804 & 10 & 0 & 0 & 0 & 0 & $1.68 \mathrm{E}+02$ & 0 & 0 & $2.54 \mathrm{E}+01$ & 0 & 0 & $3.77 \mathrm{E}+01$ \\
\hline 10EA150 & 1.2031 & 46.008 & 10 & 0 & 0 & 0 & 0 & 0 & 0 & $2.14 \mathrm{E}+02$ & $3.39 \mathrm{E}+01$ & 0 & 0 & $5.03 \mathrm{E}+01$ \\
\hline 10EA151 & 0.6289 & 46.518 & 10 & 0 & 0 & 0 & 0 & 0 & 0 & $1.11 \mathrm{E}+02$ & $1.76 \mathrm{E}+01$ & 0 & 0 & $2.61 \mathrm{E}+01$ \\
\hline 10EA152 & 0.0078 & 47.028 & 10 & 0 & 0 & 0 & 0 & 0 & 0 & $7.87 \mathrm{E}-01$ & $2.14 \mathrm{E}-01$ & 0 & 0 & $3.18 \mathrm{E}-01$ \\
\hline 10EA153 & 1.2773 & 47.13 & 10 & 0 & 0 & 0 & 0 & 0 & 0 & $2.20 \mathrm{E}+02$ & $3.47 \mathrm{E}+01$ & 0 & 0 & $5.15 \mathrm{E}+01$ \\
\hline 10EA154 & 0.9531 & 47.742 & 10 & 0 & 0 & 0 & 0 & 0 & 0 & $1.68 \mathrm{E}+02$ & $2.67 \mathrm{E}+01$ & 0 & 0 & $3.97 \mathrm{E}+01$ \\
\hline 10EA155 & 1.6328 & 47.844 & 10 & 0 & 0 & 0 & 0 & 0 & 0 & $2.73 \mathrm{E}+02$ & $4.32 \mathrm{E}+01$ & 0 & 0 & $6.41 \mathrm{E}+01$ \\
\hline 10EA156 & 1.1289 & 47.946 & 10 & 0 & 0 & 0 & 0 & 0 & 0 & $1.99 \mathrm{E}+02$ & $3.18 \mathrm{E}+01$ & 0 & 0 & $4.71 \mathrm{E}+01$ \\
\hline 10EA157 & 1.7773 & 48.456 & 10 & 0 & 0 & 0 & 0 & 0 & 0 & $3.13 \mathrm{E}+02$ & $4.94 \mathrm{E}+01$ & 0 & 0 & $7.34 \mathrm{E}+01$ \\
\hline 10EA158 & \begin{tabular}{|l|}
1.1602 \\
\end{tabular} & 48.762 & 10 & 0 & 0 & 0 & 0 & 0 & 0 & $2.04 \mathrm{E}+02$ & $3.24 \mathrm{E}+01$ & 0 & 0 & $4.81 \mathrm{E}+01$ \\
\hline 10EA159 & \begin{tabular}{|l}
1.1172 \\
\end{tabular} & 48.864 & 10 & 0 & 0 & 0 & 0 & 0 & 0 & $1.91 \mathrm{E}+02$ & $3.02 \mathrm{E}+01$ & 0 & 0 & $4.49 \mathrm{E}+01$ \\
\hline 10EA160 & 1.8945 & 49.782 & 10 & 0 & 0 & 0 & 0 & 0 & 0 & $3.27 \mathrm{E}+02$ & $5.16 \mathrm{E}+01$ & 0 & 0 & $7.67 \mathrm{E}+01$ \\
\hline 10EA163 & 1.9258 & 52.026 & 10 & 0 & 0 & 0 & 0 & 0 & 0 & $3.38 \mathrm{E}+02$ & $5.36 \mathrm{E}+01$ & 0 & 0 & $7.96 \mathrm{E}+01$ \\
\hline 10EA164 & \begin{tabular}{|l}
1.4766 \\
\end{tabular} & 53.454 & 10 & 0 & 0 & 0 & 0 & 0 & 0 & $2.56 \mathrm{E}+02$ & $4.05 \mathrm{E}+01$ & 0 & 0 & $6.02 \mathrm{E}+01$ \\
\hline 10EA165 & 0.457 & 53.556 & 10 & 0 & 0 & 0 & 0 & 0 & 0 & $8.05 \mathrm{E}+01$ & $1.30 \mathrm{E}+01$ & 0 & 0 & $1.93 \mathrm{E}+01$ \\
\hline
\end{tabular}


Table 35. Predicted Solids for Envelope A Simulations - Part 1 (cont'd)

\begin{tabular}{|c|c|c|c|c|c|c|c|c|c|c|c|c|c|c|}
\hline Test ID & $\begin{array}{l}\text { SBS/ } \\
\text { Feed }\end{array}$ & $\begin{array}{l}\text { Temp } \\
{\left[{ }^{\circ} \mathrm{C}\right]}\end{array}$ & $\begin{array}{c}\mathrm{Na} \mathrm{M} \\
{[\mathrm{mol} / \mathrm{L}]}\end{array}$ & $\begin{array}{c}\mathrm{ALOH} 3 \\
{[\mathrm{~g}]}\end{array}$ & $\begin{array}{c}\text { ANATAS } \\
\text { E [g] }\end{array}$ & $\begin{array}{c}\text { CA3PO42 } \\
{[\mathrm{g}]}\end{array}$ & $\begin{array}{c}\mathrm{CACO} 3 \\
{[\mathrm{~g}]}\end{array}$ & CAF2 [g] & CANC $[\mathrm{g}]$ & $\begin{array}{c}\mathrm{CAOH} 2 \\
{[\mathrm{~g}]}\end{array}$ & $\begin{array}{c}\text { CATIO3 } \\
{[\mathrm{g}]}\end{array}$ & $\begin{array}{c}\text { CROH3 } \\
{[\mathrm{g}]}\end{array}$ & $\begin{array}{c}\text { HYDROS } \\
\text { OD [g] }\end{array}$ & $\begin{array}{c}\mathrm{MGOH} 2 \\
{[\mathrm{~g}]}\end{array}$ \\
\hline 10EA166 & 1.2734 & 54.066 & 10 & 0 & 0 & 0 & 0 & 0 & 0 & $2.24 \mathrm{E}+02$ & $3.57 \mathrm{E}+01$ & 0 & 0 & $5.30 \mathrm{E}+01$ \\
\hline 10EA167 & 0.625 & 54.27 & 10 & 0 & 0 & 0 & 0 & 0 & 0 & $1.10 \mathrm{E}+02$ & $1.74 \mathrm{E}+01$ & 0 & 0 & $2.59 \mathrm{E}+01$ \\
\hline 10EA169 & 1.7695 & 55.902 & 10 & 0 & 0 & 0 & 0 & 0 & 0 & $3.08 \mathrm{E}+02$ & $4.89 \mathrm{E}+01$ & 0 & 0 & $7.25 \mathrm{E}+01$ \\
\hline 10EA170 & 1.918 & 56.514 & 10 & 0 & 0 & 0 & 0 & 0 & 0 & $3.38 \mathrm{E}+02$ & $5.33 \mathrm{E}+01$ & 0 & 0 & $7.92 \mathrm{E}+01$ \\
\hline 10EA171 & 0.207 & 57.738 & 10 & 0 & 0 & 0 & 0 & 0 & 0 & $3.48 \mathrm{E}+01$ & $5.75 \mathrm{E}+00$ & 0 & 0 & $8.53 \mathrm{E}+00$ \\
\hline 10EA173 & \begin{tabular}{|l|}
1.0898 \\
\end{tabular} & 58.86 & 10 & 0 & 0 & 0 & 0 & 0 & 0 & $1.84 \mathrm{E}+02$ & $2.91 \mathrm{E}+01$ & 0 & 0 & $4.32 \mathrm{E}+01$ \\
\hline 10EA174 & 0.8945 & 60.39 & 10 & 0 & 0 & 0 & 0 & $1.65 \mathrm{E}+02$ & 0 & 0 & $2.52 \mathrm{E}+01$ & 0 & 0 & $3.75 \mathrm{E}+01$ \\
\hline 10EA175 & 1.6758 & 60.696 & 10 & 0 & 0 & 0 & 0 & 0 & 0 & $2.99 \mathrm{E}+02$ & $4.76 \mathrm{E}+01$ & 0 & 0 & $7.06 \mathrm{E}+01$ \\
\hline 10EA176 & 1.0625 & 60.9 & 10 & 0 & 0 & 0 & 0 & 0 & 0 & $1.82 \mathrm{E}+02$ & $2.88 \mathrm{E}+01$ & 0 & 0 & $4.27 \mathrm{E}+01$ \\
\hline 10EA177 & 1.9492 & 61.206 & 10 & 0 & 0 & 0 & 0 & 0 & 0 & $3.43 \mathrm{E}+02$ & $5.43 \mathrm{E}+01$ & 0 & 0 & $8.06 \mathrm{E}+01$ \\
\hline 10EA178 & 1.7852 & 61.92 & 10 & 0 & 0 & 0 & 0 & 0 & 0 & $3.10 \mathrm{E}+02$ & $4.91 \mathrm{E}+01$ & 0 & 0 & $7.29 \mathrm{E}+01$ \\
\hline 10EA179 & 0.2422 & 62.022 & 10 & 0 & 0 & 0 & 0 & 0 & 0 & $4.05 \mathrm{E}+01$ & $6.72 \mathrm{E}+00$ & 0 & 0 & $9.98 \mathrm{E}+00$ \\
\hline 10EA181 & 0.6367 & 63.348 & 10 & 0 & 0 & 0 & 0 & 0 & 0 & $1.11 \mathrm{E}+02$ & $1.78 \mathrm{E}+01$ & 0 & 0 & $2.65 \mathrm{E}+01$ \\
\hline 10EA182 & 1.4102 & 63.552 & 10 & 0 & 0 & 0 & 0 & 0 & 0 & $2.37 \mathrm{E}+02$ & $3.76 \mathrm{E}+01$ & 0 & 0 & $5.58 \mathrm{E}+01$ \\
\hline 10EA183 & 0.4453 & 63.756 & 10 & 0 & 0 & 0 & 0 & 0 & 0 & $7.64 \mathrm{E}+01$ & $1.22 \mathrm{E}+01$ & 0 & 0 & $1.81 \mathrm{E}+01$ \\
\hline 10EA184 & 0.1836 & 64.164 & 10 & 0 & 0 & 0 & 0 & 0 & 0 & $3.19 \mathrm{E}+01$ & $5.18 \mathrm{E}+00$ & 0 & 0 & $7.69 \mathrm{E}+00$ \\
\hline 10EA185 & 1.6563 & 64.47 & 10 & 0 & 0 & 0 & 0 & 0 & 0 & $2.91 \mathrm{E}+02$ & $4.60 \mathrm{E}+01$ & 0 & 0 & $6.83 \mathrm{E}+01$ \\
\hline 10EA186 & 0.1875 & 64.674 & 10 & 0 & 0 & 0 & 0 & 0 & 0 & $3.15 \mathrm{E}+01$ & $5.20 \mathrm{E}+00$ & 0 & 0 & $7.72 \mathrm{E}+00$ \\
\hline 10EA187 & \begin{tabular}{|l|}
1.7969 \\
\end{tabular} & 64.776 & 10 & 0 & 0 & 0 & 0 & $3.31 \mathrm{E}+02$ & 0 & 0 & $4.99 \mathrm{E}+01$ & 0 & 0 & $7.41 \mathrm{E}+01$ \\
\hline 10EA188 & 1.0508 & 65.388 & 10 & 0 & 0 & 0 & 0 & 0 & 0 & $1.80 \mathrm{E}+02$ & $2.87 \mathrm{E}+01$ & 0 & 0 & $4.26 \mathrm{E}+01$ \\
\hline 8EA001 & 0 & 15 & 8 & 0 & 0 & 0 & 0 & 0 & 0 & 0 & 0 & 0 & 0 & 0 \\
\hline 8EA002 & 0 & 15 & 8 & 0 & 0 & 0 & 0 & 0 & 0 & 0 & 0 & 0 & 0 & 0 \\
\hline 8EA003 & 0 & 15 & 8 & 0 & 0 & 0 & 0 & 0 & 0 & 0 & 0 & 0 & 0 & 0 \\
\hline 8EA004 & 0 & 15 & 8 & 0 & 0 & 0 & 0 & 0 & 0 & 0 & 0 & 0 & 0 & 0 \\
\hline 8EA005 & 0 & 15 & 8 & 0 & 0 & 0 & 0 & 0 & 0 & 0 & 0 & 0 & 0 & 0 \\
\hline 8EA006 & 0 & 15 & 8 & 0 & 0 & 0 & 0 & 0 & 0 & 0 & 0 & 0 & 0 & 0 \\
\hline 8EA009 & 0 & 15 & 8 & 0 & 0 & 0 & 0 & 0 & 0 & 0 & 0 & 0 & 0 & 0 \\
\hline 8EA010 & 0 & 15 & 8 & 0 & 0 & 0 & 0 & 0 & 0 & 0 & 0 & 0 & 0 & 0 \\
\hline 8EA011 & 0 & 15 & 8 & 0 & 0 & 0 & 0 & 0 & 0 & 0 & 0 & 0 & 0 & 0 \\
\hline
\end{tabular}


Table 35. Predicted Solids for Envelope A Simulations - Part 1 (cont'd)

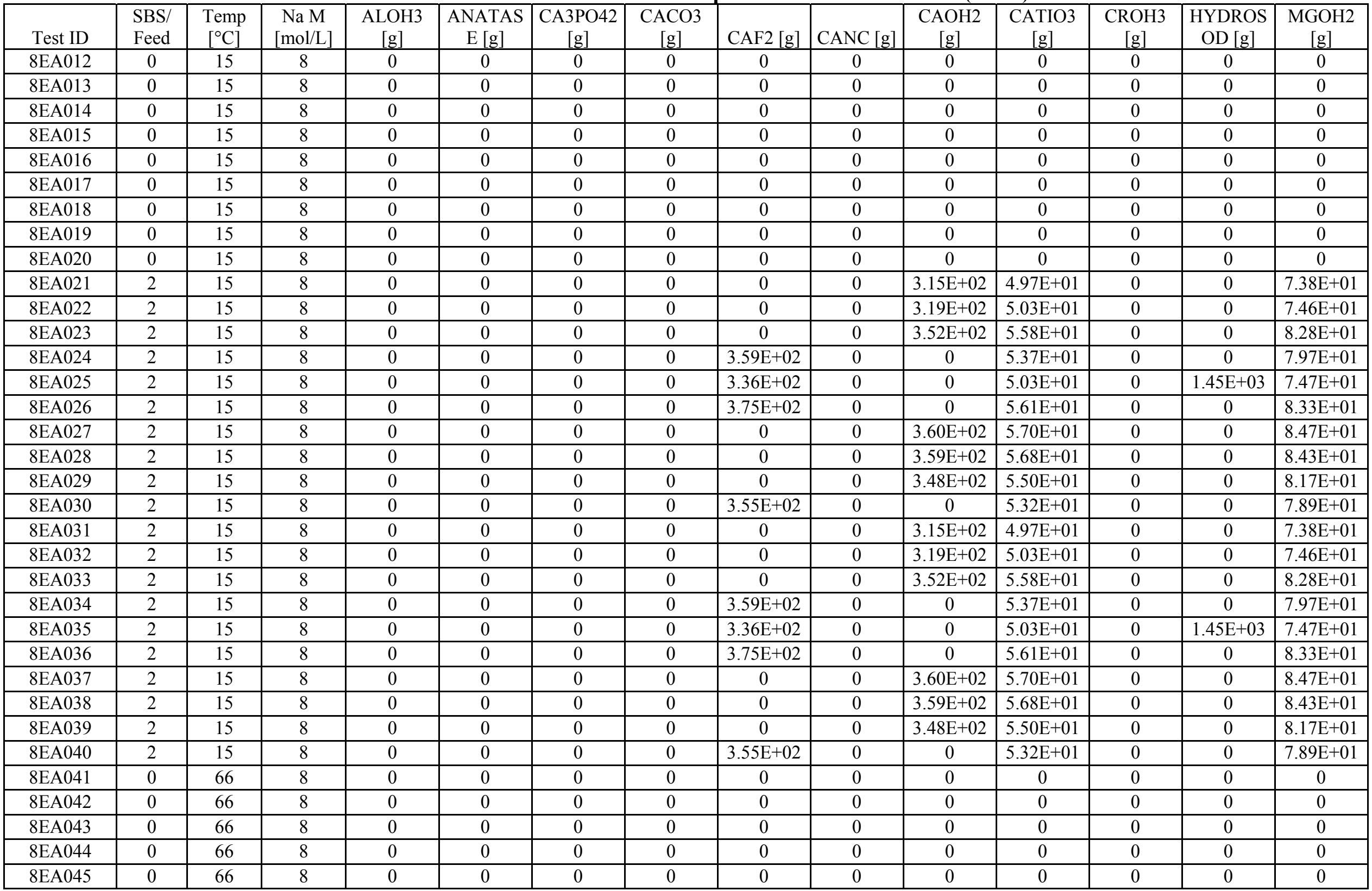


Table 35. Predicted Solids for Envelope A Simulations - Part 1 (cont'd)

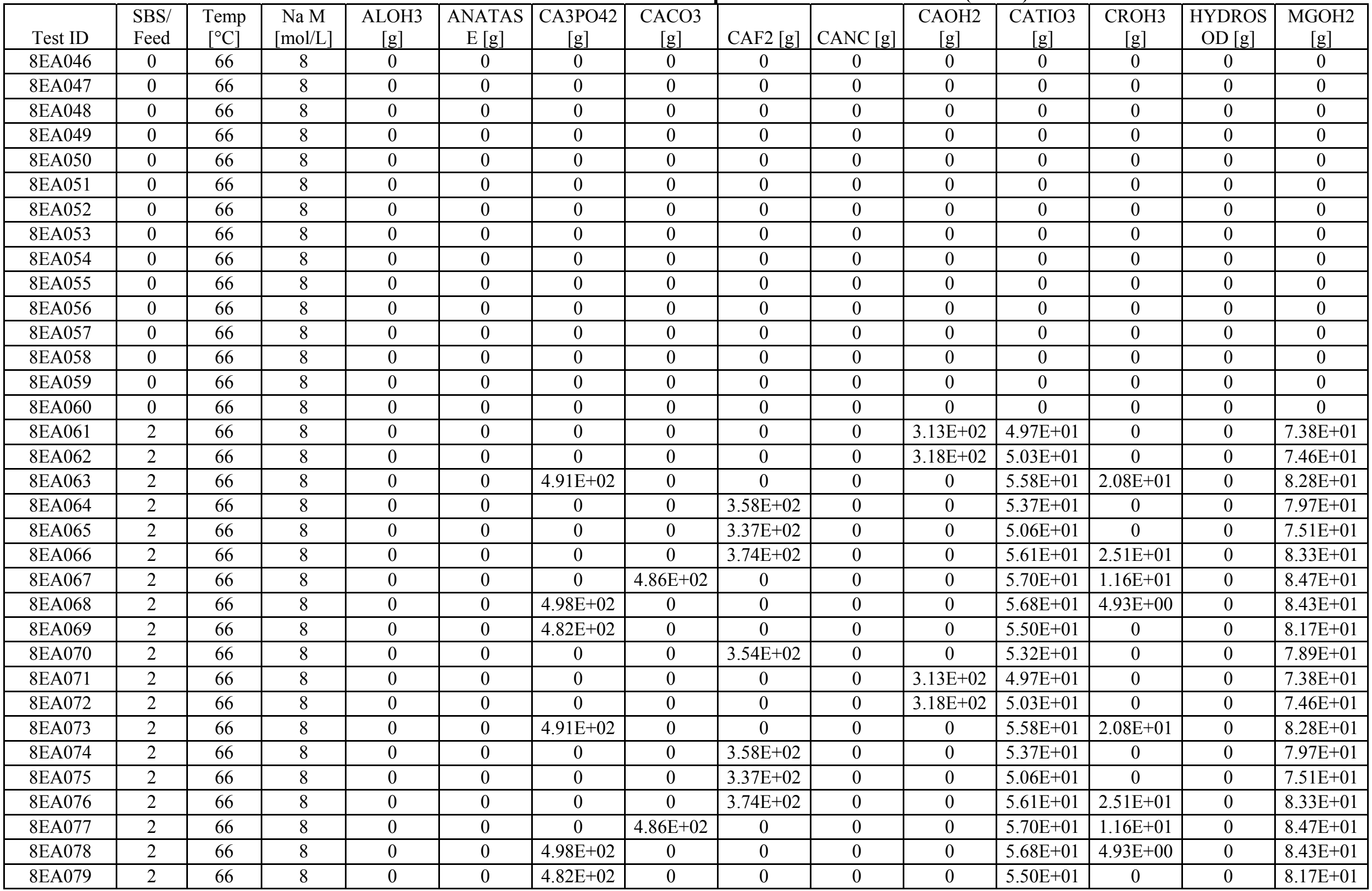


Table 35. Predicted Solids for Envelope A Simulations - Part 1 (cont'd)

\begin{tabular}{|c|c|c|c|c|c|c|c|c|c|c|c|c|c|c|}
\hline Test ID & $\begin{array}{l}\text { SBS/ } \\
\text { Feed } \\
\end{array}$ & $\begin{array}{l}\text { Temp } \\
{\left[{ }^{\circ} \mathrm{C}\right]} \\
\end{array}$ & $\begin{array}{c}\mathrm{Na} \mathrm{M} \\
{[\mathrm{mol} / \mathrm{L}]}\end{array}$ & $\begin{array}{c}\text { ALOH3 } \\
{[\mathrm{g}]}\end{array}$ & $\begin{array}{c}\text { ANATAS } \\
\text { E }[\mathrm{g}] \\
\end{array}$ & $\begin{array}{c}\text { CA3PO42 } \\
{[\mathrm{g}]} \\
\end{array}$ & $\begin{array}{c}\mathrm{CACO} 3 \\
{[\mathrm{~g}]}\end{array}$ & CAF2 [g] & CANC [g] & $\begin{array}{c}\mathrm{CAOH} 2 \\
{[\mathrm{~g}]}\end{array}$ & $\begin{array}{c}\text { CATIO3 } \\
{[\mathrm{g}]}\end{array}$ & $\begin{array}{c}\mathrm{CROH} 3 \\
{[\mathrm{~g}]}\end{array}$ & $\begin{array}{c}\text { HYDROS } \\
\text { OD [g] } \\
\end{array}$ & $\begin{array}{c}\mathrm{MGOH} 2 \\
{[\mathrm{~g}]}\end{array}$ \\
\hline $8 \mathrm{EA} 080$ & 2 & 66 & 8 & 0 & 0 & 0 & 0 & $3.54 \mathrm{E}+02$ & 0 & 0 & $5.32 \mathrm{E}+01$ & 0 & 0 & $7.89 \mathrm{E}+01$ \\
\hline 8EA081 & 0.0391 & 15 & 8 & 0 & 0 & 0 & 0 & $6.86 \mathrm{E}+00$ & 0 & 0 & $1.07 \mathrm{E}+00$ & 0 & 0 & $1.58 \mathrm{E}+00$ \\
\hline 8EA083 & 0.7578 & 15.714 & 8 & 0 & 0 & 0 & 0 & $1.43 \mathrm{E}+02$ & 0 & 0 & $2.14 \mathrm{E}+01$ & 0 & 0 & $3.17 \mathrm{E}+01$ \\
\hline 8EA084 & 0.3008 & 15.918 & 8 & 0 & 0 & 0 & 0 & $5.54 \mathrm{E}+01$ & 0 & 0 & $8.34 \mathrm{E}+00$ & 0 & 0 & $1.24 \mathrm{E}+01$ \\
\hline 8EA085 & 0.0039 & 16.122 & 8 & 0 & 0 & 0 & 0 & $4.43 \mathrm{E}-01$ & 0 & 0 & $1.07 \mathrm{E}-01$ & 0 & 0 & $1.59 \mathrm{E}-01$ \\
\hline 8EA087 & 1.5898 & 17.55 & 8 & 0 & 0 & 0 & 0 & $3.00 \mathrm{E}+02$ & 0 & 0 & $4.50 \mathrm{E}+01$ & 0 & 0 & $6.68 \mathrm{E}+01$ \\
\hline $8 \mathrm{EA} 088$ & 0.7734 & 18.06 & 8 & 0 & 0 & 0 & 0 & $1.45 \mathrm{E}+02$ & 0 & 0 & $2.17 \mathrm{E}+01$ & 0 & 0 & $3.22 \mathrm{E}+01$ \\
\hline 8EA089 & 1.1641 & 18.162 & 8 & 0 & 0 & 0 & 0 & $2.09 \mathrm{E}+02$ & 0 & 0 & $3.14 \mathrm{E}+01$ & 0 & 0 & $4.66 \mathrm{E}+01$ \\
\hline 8EA090 & 0.6094 & 18.264 & 8 & 0 & 0 & 0 & 0 & $1.10 \mathrm{E}+02$ & 0 & 0 & $1.65 \mathrm{E}+01$ & 0 & 0 & $2.45 \mathrm{E}+01$ \\
\hline 8EA091 & 0.8594 & 18.57 & 8 & 0 & 0 & 0 & 0 & $1.58 \mathrm{E}+02$ & 0 & 0 & $2.36 \mathrm{E}+01$ & 0 & 0 & $3.51 \mathrm{E}+01$ \\
\hline 8EA092 & 0.1914 & 18.672 & 8 & 0 & 0 & 0 & 0 & $3.52 \mathrm{E}+01$ & 0 & 0 & $5.31 \mathrm{E}+00$ & 0 & 0 & $7.89 \mathrm{E}+00$ \\
\hline 8EA093 & 1.0195 & 19.284 & 8 & 0 & 0 & 0 & 0 & $1.90 \mathrm{E}+02$ & 0 & 0 & $2.84 \mathrm{E}+01$ & 0 & 0 & $4.22 \mathrm{E}+01$ \\
\hline 8EA095 & 1.3203 & 19.998 & 8 & 0 & 0 & 0 & 0 & $2.38 \mathrm{E}+02$ & 0 & 0 & $3.56 \mathrm{E}+01$ & 0 & 0 & $5.29 \mathrm{E}+01$ \\
\hline 8EA096 & 1.8398 & 20.508 & 8 & 0 & 0 & 0 & 0 & 0 & 0 & $3.24 \mathrm{E}+02$ & $5.14 \mathrm{E}+01$ & 0 & 0 & $7.62 \mathrm{E}+01$ \\
\hline $8 \mathrm{EA} 097$ & 0.1563 & 21.222 & 8 & 0 & 0 & 0 & 0 & 0 & 0 & $2.62 \mathrm{E}+01$ & $4.39 \mathrm{E}+00$ & 0 & 0 & $6.51 \mathrm{E}+00$ \\
\hline 8EA098 & 1.5977 & 21.426 & 8 & 0 & 0 & 0 & 0 & 0 & 0 & $2.73 \mathrm{E}+02$ & $4.32 \mathrm{E}+01$ & 0 & 0 & $6.42 \mathrm{E}+01$ \\
\hline 8EA099 & 0.1719 & 21.732 & 8 & 0 & 0 & 0 & 0 & $3.13 \mathrm{E}+01$ & 0 & 0 & $4.73 \mathrm{E}+00$ & 0 & 0 & $7.02 \mathrm{E}+00$ \\
\hline 8EA100 & 1.6094 & 22.956 & 8 & 0 & 0 & 0 & 0 & 0 & 0 & $2.85 \mathrm{E}+02$ & $4.54 \mathrm{E}+01$ & 0 & 0 & $6.74 \mathrm{E}+01$ \\
\hline 8EA101 & 0.8633 & 23.466 & 8 & 0 & 0 & 0 & 0 & $1.60 \mathrm{E}+02$ & 0 & 0 & $2.41 \mathrm{E}+01$ & 0 & 0 & $3.58 \mathrm{E}+01$ \\
\hline 8EA102 & 1.0703 & 24.282 & 8 & 0 & 0 & 0 & 0 & $2.02 \mathrm{E}+02$ & 0 & 0 & $3.02 \mathrm{E}+01$ & 0 & 0 & $4.48 \mathrm{E}+01$ \\
\hline 8EA103 & 1.0273 & 26.016 & 8 & 0 & 0 & 0 & 0 & 0 & 0 & $1.78 \mathrm{E}+02$ & $2.84 \mathrm{E}+01$ & 0 & 0 & $4.22 \mathrm{E}+01$ \\
\hline 8EA104 & 1.1758 & 26.22 & 8 & 0 & 0 & 0 & 0 & $2.20 \mathrm{E}+02$ & 0 & 0 & $3.30 \mathrm{E}+01$ & 0 & 0 & $4.90 \mathrm{E}+01$ \\
\hline 8EA105 & 0.5 & 26.526 & 8 & 0 & 0 & 0 & 0 & 0 & 0 & $8.66 \mathrm{E}+01$ & $1.39 \mathrm{E}+01$ & 0 & 0 & $2.06 \mathrm{E}+01$ \\
\hline 8EA106 & 1.2695 & 27.036 & 8 & 0 & 0 & 0 & 0 & $2.36 \mathrm{E}+02$ & 0 & 0 & $3.53 \mathrm{E}+01$ & 0 & 0 & $5.25 \mathrm{E}+01$ \\
\hline 8 EA107 & 0.0195 & 27.138 & 8 & 0 & 0 & 0 & 0 & 0 & 0 & $1.87 \mathrm{E}+00$ & 5.39E-01 & 0 & 0 & $8.00 \mathrm{E}-01$ \\
\hline 8EA108 & 1.4414 & 27.24 & 8 & 0 & 0 & 0 & 0 & $2.62 \mathrm{E}+02$ & 0 & 0 & $3.92 \mathrm{E}+01$ & 0 & 0 & $5.82 \mathrm{E}+01$ \\
\hline 8EA111 & 1.0977 & 28.464 & 8 & 0 & 0 & 0 & 0 & 0 & 0 & $1.91 \mathrm{E}+02$ & $3.09 \mathrm{E}+01$ & 0 & 0 & $4.58 \mathrm{E}+01$ \\
\hline 8EA112 & 0.6211 & 28.566 & 8 & 0 & 0 & 0 & 0 & $1.14 \mathrm{E}+02$ & 0 & 0 & $1.74 \mathrm{E}+01$ & 0 & 0 & $2.58 \mathrm{E}+01$ \\
\hline 8EA113 & 1.0664 & 28.872 & 8 & 0 & 0 & 0 & 0 & $4.02 \mathrm{E}+01$ & 0 & $1.46 \mathrm{E}+02$ & $2.94 \mathrm{E}+01$ & 0 & 0 & $4.37 \mathrm{E}+01$ \\
\hline
\end{tabular}


Table 35. Predicted Solids for Envelope A Simulations - Part 1 (cont'd)

\begin{tabular}{|c|c|c|c|c|c|c|c|c|c|c|c|c|c|c|}
\hline Test ID & $\begin{array}{l}\text { SBS/ } \\
\text { Feed }\end{array}$ & $\begin{array}{l}\text { Temp } \\
{\left[{ }^{\circ} \mathrm{C}\right]} \\
\end{array}$ & $\begin{array}{c}\mathrm{Na} \mathrm{M} \\
{[\mathrm{mol} / \mathrm{L}]}\end{array}$ & $\begin{array}{c}\text { ALOH3 } \\
{[\mathrm{g}]}\end{array}$ & $\begin{array}{c}\text { ANATAS } \\
\text { E [g] }\end{array}$ & $\begin{array}{c}\text { CA3PO42 } \\
{[\mathrm{g}]} \\
\end{array}$ & $\begin{array}{c}\mathrm{CACO} 3 \\
{[\mathrm{~g}]}\end{array}$ & CAF2 [g] & CANC [g] & $\begin{array}{c}\mathrm{CAOH} 2 \\
{[\mathrm{~g}]}\end{array}$ & $\begin{array}{c}\text { CATIO3 } \\
{[\mathrm{g}]}\end{array}$ & $\begin{array}{c}\mathrm{CROH} 3 \\
{[\mathrm{~g}]}\end{array}$ & $\begin{array}{c}\text { HYDROS } \\
\text { OD [g] } \\
\end{array}$ & $\begin{array}{c}\mathrm{MGOH} 2 \\
{[\mathrm{~g}]}\end{array}$ \\
\hline 8EA114 & 0.6563 & 29.382 & 8 & 0 & 0 & 0 & 0 & 0 & 0 & $1.16 \mathrm{E}+02$ & $1.85 \mathrm{E}+01$ & 0 & 0 & $2.75 \mathrm{E}+01$ \\
\hline 8EA115 & 1.3867 & 29.484 & 8 & 0 & 0 & 0 & 0 & $2.56 \mathrm{E}+02$ & 0 & 0 & $3.83 \mathrm{E}+01$ & 0 & 0 & $5.69 \mathrm{E}+01$ \\
\hline 8EA117 & 1.8711 & 30.504 & 8 & 0 & 0 & 0 & 0 & $3.46 \mathrm{E}+02$ & 0 & 0 & $5.17 \mathrm{E}+01$ & 0 & 0 & $7.68 \mathrm{E}+01$ \\
\hline 8EA118 & 0.5664 & 30.606 & 8 & 0 & 0 & 0 & 0 & $1.04 \mathrm{E}+02$ & 0 & 0 & $1.56 \mathrm{E}+01$ & 0 & 0 & $2.32 \mathrm{E}+01$ \\
\hline 8EA119 & 0.6953 & 31.422 & 8 & 0 & 0 & 0 & 0 & $1.21 \mathrm{E}+02$ & 0 & 0 & $1.82 \mathrm{E}+01$ & 0 & 0 & $2.70 \mathrm{E}+01$ \\
\hline 8EA121 & 1.3555 & 32.748 & 8 & 0 & 0 & 0 & 0 & 0 & 0 & $2.39 \mathrm{E}+02$ & $3.82 \mathrm{E}+01$ & 0 & 0 & $5.67 \mathrm{E}+01$ \\
\hline $8 \mathrm{EA} 122$ & 0.2383 & 32.952 & 8 & 0 & 0 & 0 & 0 & $4.44 \mathrm{E}+01$ & 0 & 0 & $6.75 \mathrm{E}+00$ & 0 & 0 & $1.00 \mathrm{E}+01$ \\
\hline 8EA123 & 0.6328 & 33.36 & 8 & 0 & 0 & 0 & 0 & 0 & 0 & $1.09 \mathrm{E}+02$ & $1.77 \mathrm{E}+01$ & 0 & 0 & $2.62 \mathrm{E}+01$ \\
\hline 8EA124 & 1.5313 & 33.564 & 8 & 0 & 0 & 0 & 0 & $2.82 \mathrm{E}+02$ & 0 & 0 & $4.23 \mathrm{E}+01$ & 0 & 0 & $6.28 \mathrm{E}+01$ \\
\hline 8EA125 & 0.4727 & 33.666 & 8 & 0 & 0 & 0 & 0 & $8.23 \mathrm{E}+01$ & 0 & 0 & $1.24 \mathrm{E}+01$ & 0 & 0 & $1.84 \mathrm{E}+01$ \\
\hline 8EA126 & 0.5195 & 34.176 & 8 & 0 & 0 & 0 & 0 & $9.49 \mathrm{E}+01$ & 0 & 0 & $1.43 \mathrm{E}+01$ & 0 & 0 & $2.12 \mathrm{E}+01$ \\
\hline 8EA127 & 1.9727 & 34.278 & 8 & 0 & 0 & 0 & 0 & $3.60 \mathrm{E}+02$ & 0 & 0 & $5.39 \mathrm{E}+01$ & 0 & 0 & $8.00 \mathrm{E}+01$ \\
\hline 8EA129 & 1.668 & 37.032 & 8 & 0 & 0 & 0 & 0 & 0 & 0 & $2.89 \mathrm{E}+02$ & $4.58 \mathrm{E}+01$ & 0 & 0 & $6.80 \mathrm{E}+01$ \\
\hline 8EA130 & 1.9336 & 38.358 & 8 & 0 & 0 & 0 & 0 & 0 & 0 & $3.34 \mathrm{E}+02$ & $5.30 \mathrm{E}+01$ & 0 & 0 & $7.86 \mathrm{E}+01$ \\
\hline 8 EA131 & 1.4258 & 39.48 & 8 & 0 & 0 & 0 & 0 & $2.67 \mathrm{E}+02$ & 0 & 0 & $4.00 \mathrm{E}+01$ & 0 & 0 & $5.94 \mathrm{E}+01$ \\
\hline 8EA132 & 0.168 & 39.99 & 8 & 0 & 0 & 0 & 0 & $2.85 \mathrm{E}+01$ & 0 & 0 & $4.56 \mathrm{E}+00$ & 0 & 0 & $6.78 \mathrm{E}+00$ \\
\hline 8EA133 & 1.6641 & 40.194 & 8 & 0 & 0 & 0 & 0 & $2.98 \mathrm{E}+02$ & 0 & 0 & $4.46 \mathrm{E}+01$ & 0 & 0 & $6.63 \mathrm{E}+01$ \\
\hline 8EA134 & 0.2344 & 40.602 & 8 & 0 & 0 & 0 & 0 & $4.38 \mathrm{E}+01$ & 0 & 0 & $6.62 \mathrm{E}+00$ & 0 & 0 & $9.83 \mathrm{E}+00$ \\
\hline 8EA135 & 1.6914 & 41.112 & 8 & 0 & 0 & 0 & 0 & $3.13 \mathrm{E}+02$ & 0 & 0 & $4.69 \mathrm{E}+01$ & 0 & 0 & $6.97 \mathrm{E}+01$ \\
\hline 8EA136 & 1.125 & 41.622 & 8 & 0 & 0 & $1.56 \mathrm{E}+02$ & 0 & 0 & 0 & $8.36 \mathrm{E}+01$ & $3.16 \mathrm{E}+01$ & 0 & 0 & $4.69 \mathrm{E}+01$ \\
\hline 8EA137 & 0.2813 & 42.744 & 8 & 0 & 0 & 0 & 0 & $5.10 \mathrm{E}+01$ & 0 & 0 & $7.79 \mathrm{E}+00$ & 0 & 0 & $1.16 \mathrm{E}+01$ \\
\hline 8EA138 & 1.7813 & 43.152 & 8 & 0 & 0 & 0 & 0 & $3.35 \mathrm{E}+02$ & 0 & 0 & $5.02 \mathrm{E}+01$ & 0 & 0 & $7.45 \mathrm{E}+01$ \\
\hline 8EA139 & 0.6992 & 43.56 & 8 & 0 & 0 & 0 & 0 & $1.31 \mathrm{E}+02$ & 0 & 0 & $1.96 \mathrm{E}+01$ & 0 & 0 & $2.91 \mathrm{E}+01$ \\
\hline 8EA140 & 0.957 & 43.662 & 8 & 0 & 0 & 0 & 0 & $1.80 \mathrm{E}+02$ & 0 & 0 & $2.70 \mathrm{E}+01$ & 0 & 0 & $4.01 \mathrm{E}+01$ \\
\hline 8 EA141 & 0.3164 & 43.866 & 8 & 0 & 0 & $7.18 \mathrm{E}+01$ & 0 & 0 & 0 & 0 & $8.94 \mathrm{E}+00$ & 0 & 0 & $1.33 \mathrm{E}+01$ \\
\hline 8EA142 & 1.7539 & 44.172 & 8 & 0 & 0 & 0 & 0 & $3.22 \mathrm{E}+02$ & 0 & 0 & $4.82 \mathrm{E}+01$ & 0 & 0 & $7.16 \mathrm{E}+01$ \\
\hline 8EA145 & 0.6758 & 44.58 & 8 & 0 & 0 & 0 & 0 & $1.24 \mathrm{E}+02$ & 0 & 0 & $1.86 \mathrm{E}+01$ & 0 & 0 & $2.77 \mathrm{E}+01$ \\
\hline 8EA146 & 1.4922 & 45.396 & 8 & 0 & 0 & 0 & 0 & $2.72 \mathrm{E}+02$ & 0 & 0 & $4.07 \mathrm{E}+01$ & 0 & 0 & $6.05 \mathrm{E}+01$ \\
\hline 8EA147 & 1.168 & 45.6 & 8 & 0 & 0 & 0 & 0 & $2.22 \mathrm{E}+02$ & 0 & 0 & $3.33 \mathrm{E}+01$ & 0 & 0 & $4.94 \mathrm{E}+01$ \\
\hline
\end{tabular}


Table 35. Predicted Solids for Envelope A Simulations - Part 1 (cont'd)

\begin{tabular}{|c|c|c|c|c|c|c|c|c|c|c|c|c|c|c|}
\hline Test ID & $\begin{array}{l}\text { SBS/ } \\
\text { Feed }\end{array}$ & $\begin{array}{l}\text { Temp } \\
{\left[{ }^{\circ} \mathrm{C}\right]} \\
\end{array}$ & $\begin{array}{c}\mathrm{Na} \mathrm{M} \\
{[\mathrm{mol} / \mathrm{L}]}\end{array}$ & $\begin{array}{c}\text { ALOH3 } \\
{[\mathrm{g}]}\end{array}$ & $\begin{array}{c}\text { ANATAS } \\
\text { E [g] }\end{array}$ & $\begin{array}{c}\text { CA3PO42 } \\
{[\mathrm{g}]} \\
\end{array}$ & $\begin{array}{c}\mathrm{CACO} 3 \\
{[\mathrm{~g}]}\end{array}$ & CAF2 [g] & CANC [g] & $\begin{array}{c}\mathrm{CAOH} 2 \\
{[\mathrm{~g}]}\end{array}$ & $\begin{array}{c}\text { CATIO3 } \\
{[\mathrm{g}]}\end{array}$ & $\begin{array}{c}\mathrm{CROH} 3 \\
{[\mathrm{~g}]}\end{array}$ & $\begin{array}{c}\text { HYDROS } \\
\text { OD [g] } \\
\end{array}$ & $\begin{array}{c}\mathrm{MGOH} 2 \\
{[\mathrm{~g}]}\end{array}$ \\
\hline 8EA148 & 0.7852 & 45.702 & 8 & 0 & 0 & $1.88 \mathrm{E}+02$ & 0 & 0 & 0 & 0 & $2.21 \mathrm{E}+01$ & 0 & 0 & $3.27 \mathrm{E}+01$ \\
\hline 8EA149 & 0.9219 & 45.804 & 8 & 0 & 0 & 0 & 0 & $1.68 \mathrm{E}+02$ & 0 & 0 & $2.54 \mathrm{E}+01$ & 0 & 0 & $3.77 \mathrm{E}+01$ \\
\hline 8EA151 & 0.6289 & 46.518 & 8 & 0 & 0 & 0 & 0 & $1.16 \mathrm{E}+02$ & 0 & 0 & $1.76 \mathrm{E}+01$ & 0 & 0 & $2.61 \mathrm{E}+01$ \\
\hline 8EA152 & 0.0078 & 47.028 & 8 & 0 & 0 & 0 & 0 & $4.74 \mathrm{E}-01$ & 0 & 0 & $2.14 \mathrm{E}-01$ & 0 & 0 & $3.18 \mathrm{E}-01$ \\
\hline 8EA153 & 1.2773 & 47.13 & 8 & 0 & 0 & 0 & 0 & $2.31 \mathrm{E}+02$ & 0 & 0 & $3.47 \mathrm{E}+01$ & 0 & 0 & $5.15 \mathrm{E}+01$ \\
\hline 8EA155 & 1.6328 & 47.844 & 8 & 0 & 0 & 0 & 0 & $2.88 \mathrm{E}+02$ & 0 & 0 & $4.32 \mathrm{E}+01$ & 0 & 0 & $6.41 \mathrm{E}+01$ \\
\hline 8 EA156 & 1.1289 & 47.946 & 8 & 0 & 0 & $2.75 \mathrm{E}+02$ & 0 & 0 & 0 & 0 & $3.18 \mathrm{E}+01$ & 0 & 0 & $4.71 \mathrm{E}+01$ \\
\hline 8EA157 & 1.7773 & 48.456 & 8 & 0 & 0 & 0 & 0 & $3.30 \mathrm{E}+02$ & 0 & 0 & $4.94 \mathrm{E}+01$ & 0 & 0 & $7.34 \mathrm{E}+01$ \\
\hline 8EA158 & 1.1602 & 48.762 & 8 & 0 & 0 & $2.80 \mathrm{E}+02$ & 0 & 0 & 0 & 0 & $3.24 \mathrm{E}+01$ & 0 & 0 & $4.81 \mathrm{E}+01$ \\
\hline 8EA159 & 1.1172 & 48.864 & 8 & 0 & 0 & 0 & 0 & $2.01 \mathrm{E}+02$ & 0 & 0 & $3.02 \mathrm{E}+01$ & 0 & 0 & $4.49 \mathrm{E}+01$ \\
\hline 8EA160 & 1.8945 & 49.782 & 8 & 0 & 0 & 0 & 0 & $3.44 \mathrm{E}+02$ & 0 & 0 & $5.16 \mathrm{E}+01$ & 0 & 0 & $7.67 \mathrm{E}+01$ \\
\hline 8EA161 & 1.5391 & 50.088 & 8 & 0 & 0 & 0 & 0 & $2.75 \mathrm{E}+02$ & 0 & 0 & $4.15 \mathrm{E}+01$ & 0 & 0 & $6.16 \mathrm{E}+01$ \\
\hline 8EA163 & 1.9258 & 52.026 & 8 & 0 & 0 & 0 & 0 & $3.57 \mathrm{E}+02$ & 0 & 0 & $5.36 \mathrm{E}+01$ & 0 & 0 & $7.96 \mathrm{E}+01$ \\
\hline 8EA164 & 1.4766 & 53.454 & 8 & 0 & 0 & 0 & 0 & $2.69 \mathrm{E}+02$ & 0 & 0 & $4.05 \mathrm{E}+01$ & 0 & 0 & $6.02 \mathrm{E}+01$ \\
\hline 8 EA165 & 0.457 & 53.556 & 8 & 0 & 0 & $1.09 \mathrm{E}+02$ & 0 & 0 & 0 & 0 & $1.30 \mathrm{E}+01$ & 0 & 0 & $1.93 \mathrm{E}+01$ \\
\hline 8EA166 & 1.2734 & 54.066 & 8 & 0 & 0 & $4.78 \mathrm{E}+01$ & 0 & $1.98 \mathrm{E}+02$ & 0 & 0 & $3.57 \mathrm{E}+01$ & 0 & 0 & $5.30 \mathrm{E}+01$ \\
\hline 8EA167 & 0.625 & 54.27 & 8 & 0 & 0 & 0 & 0 & $1.16 \mathrm{E}+02$ & 0 & 0 & $1.74 \mathrm{E}+01$ & 0 & 0 & $2.59 \mathrm{E}+01$ \\
\hline 8EA168 & 1.1484 & 54.882 & 8 & 0 & 0 & 0 & 0 & $2.17 \mathrm{E}+02$ & 0 & 0 & $3.27 \mathrm{E}+01$ & 0 & 0 & $4.85 \mathrm{E}+01$ \\
\hline 8EA169 & 1.7695 & 55.902 & 8 & 0 & 0 & 0 & 0 & $3.25 \mathrm{E}+02$ & 0 & 0 & $4.89 \mathrm{E}+01$ & 0 & 0 & $7.25 \mathrm{E}+01$ \\
\hline 8EA170 & 1.918 & 56.514 & 8 & 0 & 0 & 0 & 0 & 0 & 0 & $3.35 \mathrm{E}+02$ & $5.33 \mathrm{E}+01$ & 0 & 0 & $7.92 \mathrm{E}+01$ \\
\hline 8EA171 & 0.207 & 57.738 & 8 & 0 & 0 & $4.54 \mathrm{E}+01$ & 0 & 0 & 0 & 0 & $5.75 \mathrm{E}+00$ & 0 & 0 & $8.53 \mathrm{E}+00$ \\
\hline 8EA172 & 1.7266 & 58.758 & 8 & 0 & 0 & $4.24 \mathrm{E}+02$ & 0 & 0 & 0 & 0 & $4.85 \mathrm{E}+01$ & 0 & 0 & $7.20 \mathrm{E}+01$ \\
\hline 8EA173 & 1.0898 & 58.86 & 8 & 0 & 0 & 0 & 0 & $1.93 \mathrm{E}+02$ & 0 & 0 & $2.91 \mathrm{E}+01$ & 0 & 0 & $4.32 \mathrm{E}+01$ \\
\hline 8EA174 & 0.8945 & 60.39 & 8 & 0 & 0 & $2.18 \mathrm{E}+02$ & 0 & 0 & 0 & 0 & $2.52 \mathrm{E}+01$ & 0 & 0 & $3.75 \mathrm{E}+01$ \\
\hline 8 EA175 & 1.6758 & 60.696 & 8 & 0 & 0 & $4.16 \mathrm{E}+02$ & 0 & 0 & 0 & 0 & $4.76 \mathrm{E}+01$ & 0 & 0 & $7.06 \mathrm{E}+01$ \\
\hline 8EA176 & 1.0625 & 60.9 & 8 & 0 & 0 & 0 & 0 & $1.91 \mathrm{E}+02$ & 0 & 0 & $2.88 \mathrm{E}+01$ & 0 & 0 & $4.27 \mathrm{E}+01$ \\
\hline 8EA179 & 0.2422 & 62.022 & 8 & 0 & 0 & 0 & 0 & $4.36 \mathrm{E}+01$ & 0 & 0 & $6.72 \mathrm{E}+00$ & 0 & 0 & $9.98 \mathrm{E}+00$ \\
\hline 8EA180 & 0.4961 & 63.042 & 8 & 0 & 0 & 0 & 0 & $9.00 \mathrm{E}+01$ & 0 & 0 & $1.37 \mathrm{E}+01$ & 0 & 0 & $2.03 \mathrm{E}+01$ \\
\hline 8EA181 & 0.6367 & 63.348 & 8 & 0 & 0 & 0 & 0 & $1.18 \mathrm{E}+02$ & 0 & 0 & $1.78 \mathrm{E}+01$ & 0 & 0 & $2.65 \mathrm{E}+01$ \\
\hline
\end{tabular}


Table 35. Predicted Solids for Envelope A Simulations - Part 1 (cont'd)

\begin{tabular}{|c|c|c|c|c|c|c|c|c|c|c|c|c|c|c|}
\hline Test ID & $\begin{array}{l}\text { SBS/ } \\
\text { Feed }\end{array}$ & $\begin{array}{l}\text { Temp } \\
{\left[{ }^{\circ} \mathrm{C}\right]} \\
\end{array}$ & $\begin{array}{c}\mathrm{Na} \mathrm{M} \\
{[\mathrm{mol} / \mathrm{L}]}\end{array}$ & $\begin{array}{c}\text { ALOH3 } \\
{[\mathrm{g}]}\end{array}$ & $\begin{array}{c}\text { ANATAS } \\
\text { E }[\mathrm{g}] \\
\end{array}$ & $\begin{array}{c}\text { CA3PO42 } \\
{[\mathrm{g}]} \\
\end{array}$ & $\begin{array}{c}\mathrm{CACO} 3 \\
{[\mathrm{~g}]}\end{array}$ & CAF2 [g] & CANC $[\mathrm{g}]$ & $\begin{array}{c}\mathrm{CAOH} 2 \\
{[\mathrm{~g}]}\end{array}$ & $\begin{array}{c}\text { CATIO3 } \\
{[\mathrm{g}]}\end{array}$ & $\begin{array}{c}\mathrm{CROH} 3 \\
{[\mathrm{~g}]}\end{array}$ & $\begin{array}{c}\text { HYDROS } \\
\text { OD }[\mathrm{g}] \\
\end{array}$ & $\begin{array}{c}\mathrm{MGOH} 2 \\
{[\mathrm{~g}]}\end{array}$ \\
\hline 8EA182 & 1.4102 & 63.552 & 8 & 0 & 0 & 0 & 0 & $2.48 \mathrm{E}+02$ & 0 & 0 & $3.76 \mathrm{E}+01$ & 0 & 0 & $5.58 \mathrm{E}+01$ \\
\hline 8EA183 & 0.4453 & 63.756 & 8 & 0 & 0 & 0 & 0 & $8.02 \mathrm{E}+01$ & 0 & 0 & $1.22 \mathrm{E}+01$ & 0 & 0 & $1.81 \mathrm{E}+01$ \\
\hline 8 EA185 & 1.6563 & 64.47 & 8 & 0 & 0 & 0 & 0 & $3.06 \mathrm{E}+02$ & 0 & 0 & $4.59 \mathrm{E}+01$ & 0 & $1.32 \mathrm{E}+03$ & $6.81 \mathrm{E}+01$ \\
\hline 8EA186 & 0.1875 & 64.674 & 8 & 0 & 0 & 0 & 0 & $3.33 \mathrm{E}+01$ & 0 & 0 & $5.20 \mathrm{E}+00$ & 0 & 0 & $7.72 \mathrm{E}+00$ \\
\hline $8 \mathrm{EA} 187$ & 1.7969 & 64.776 & 8 & 0 & 0 & 0 & 0 & $3.32 \mathrm{E}+02$ & 0 & 0 & $4.99 \mathrm{E}+01$ & $2.98 \mathrm{E}+00$ & 0 & $7.41 \mathrm{E}+01$ \\
\hline 6EA001 & 0 & 15 & 6 & 0 & 0 & 0 & 0 & 0 & 0 & 0 & 0 & 0 & 0 & 0 \\
\hline 6EA002 & 0 & 15 & 6 & 0 & 0 & 0 & 0 & 0 & 0 & 0 & 0 & 0 & 0 & 0 \\
\hline 6EA003 & 0 & 15 & 6 & 0 & 0 & 0 & 0 & 0 & 0 & 0 & 0 & 0 & 0 & 0 \\
\hline 6EA004 & 0 & 15 & 6 & 0 & 0 & 0 & 0 & 0 & 0 & 0 & 0 & 0 & 0 & 0 \\
\hline $6 \mathrm{EA} 005$ & 0 & 15 & 6 & 0 & 0 & 0 & 0 & 0 & 0 & 0 & 0 & 0 & 0 & 0 \\
\hline 6EA006 & 0 & 15 & 6 & 0 & 0 & 0 & 0 & 0 & 0 & 0 & 0 & 0 & 0 & 0 \\
\hline 6EA007 & 0 & 15 & 6 & $2.84 \mathrm{E}+03$ & 0 & 0 & 0 & 0 & 0 & 0 & 0 & 0 & 0 & 0 \\
\hline 6ЕA009 & 0 & 15 & 6 & $4.56 \mathrm{E}+03$ & 0 & 0 & 0 & 0 & 0 & 0 & 0 & 0 & 0 & 0 \\
\hline 6EA010 & 0 & 15 & 6 & $6.82 \mathrm{E}+03$ & 0 & 0 & 0 & 0 & 0 & 0 & 0 & 0 & 0 & 0 \\
\hline 6EA011 & 0 & 15 & 6 & 0 & 0 & 0 & 0 & 0 & 0 & 0 & 0 & 0 & 0 & 0 \\
\hline 6EA012 & 0 & 15 & 6 & 0 & 0 & 0 & 0 & 0 & 0 & 0 & 0 & 0 & 0 & 0 \\
\hline 6EA013 & 0 & 15 & 6 & 0 & 0 & 0 & 0 & 0 & 0 & 0 & 0 & 0 & 0 & 0 \\
\hline 6EA014 & 0 & 15 & 6 & 0 & 0 & 0 & 0 & 0 & 0 & 0 & 0 & 0 & 0 & 0 \\
\hline 6EA015 & 0 & 15 & 6 & 0 & 0 & 0 & 0 & 0 & 0 & 0 & 0 & 0 & 0 & 0 \\
\hline 6EA016 & 0 & 15 & 6 & 0 & 0 & 0 & 0 & 0 & 0 & 0 & 0 & 0 & 0 & 0 \\
\hline 6EA017 & 0 & 15 & 6 & $2.84 \mathrm{E}+03$ & 0 & 0 & 0 & 0 & 0 & 0 & 0 & 0 & 0 & 0 \\
\hline 6ЕA018 & 0 & 15 & 6 & $2.82 \mathrm{E}+03$ & 0 & 0 & 0 & 0 & 0 & 0 & 0 & 0 & 0 & 0 \\
\hline 6EA019 & 0 & 15 & 6 & $4.56 \mathrm{E}+03$ & 0 & 0 & 0 & 0 & 0 & 0 & 0 & 0 & 0 & 0 \\
\hline 6EA020 & 0 & 15 & 6 & $6.82 \mathrm{E}+03$ & 0 & 0 & 0 & 0 & 0 & 0 & 0 & 0 & 0 & 0 \\
\hline 6EA021 & 2 & 15 & 6 & 0 & 0 & 0 & 0 & 0 & 0 & $3.14 \mathrm{E}+02$ & $4.97 \mathrm{E}+01$ & 0 & 0 & $7.38 \mathrm{E}+01$ \\
\hline 6EA022 & 2 & 15 & 6 & 0 & 0 & 0 & 0 & 0 & 0 & $3.17 \mathrm{E}+02$ & $5.03 \mathrm{E}+01$ & 0 & 0 & $7.46 \mathrm{E}+01$ \\
\hline 6EA023 & 2 & 15 & 6 & 0 & 0 & $3.08 \mathrm{E}+02$ & 0 & $1.32 \mathrm{E}+02$ & 0 & 0 & $5.58 \mathrm{E}+01$ & 0 & 0 & $8.28 \mathrm{E}+01$ \\
\hline $6 \mathrm{EA} 025$ & 2 & 15 & 6 & 0 & 0 & 0 & 0 & $3.38 \mathrm{E}+02$ & 0 & 0 & $5.06 \mathrm{E}+01$ & 0 & 0 & $7.51 \mathrm{E}+01$ \\
\hline 6EA026 & 2 & 15 & 6 & 0 & 0 & 0 & 0 & $3.75 \mathrm{E}+02$ & 0 & 0 & $5.61 \mathrm{E}+01$ & 0 & 0 & $8.33 \mathrm{E}+01$ \\
\hline 6EA027 & 2 & 15 & 6 & $6.42 \mathrm{E}+03$ & 0 & 0 & $4.84 \mathrm{E}+02$ & 0 & 0 & 0 & $5.70 \mathrm{E}+01$ & 0 & 0 & $8.47 \mathrm{E}+01$ \\
\hline
\end{tabular}


Table 35. Predicted Solids for Envelope A Simulations - Part 1 (cont'd)

\begin{tabular}{|c|c|c|c|c|c|c|c|c|c|c|c|c|c|c|}
\hline Test ID & $\begin{array}{l}\text { SBS/ } \\
\text { Feed }\end{array}$ & $\begin{array}{l}\text { Temp } \\
{\left[{ }^{\circ} \mathrm{C}\right]}\end{array}$ & $\begin{array}{c}\mathrm{Na} \mathrm{M} \\
{[\mathrm{mol} / \mathrm{L}]}\end{array}$ & $\begin{array}{c}\mathrm{ALOH} 3 \\
{[\mathrm{~g}]}\end{array}$ & $\begin{array}{c}\text { ANATAS } \\
\text { E }[\mathrm{g}]\end{array}$ & $\begin{array}{c}\text { CA3PO42 } \\
{[\mathrm{g}]} \\
\end{array}$ & $\begin{array}{c}\mathrm{CACO} 3 \\
{[\mathrm{~g}]}\end{array}$ & CAF2 [g] & CANC $[\mathrm{g}]$ & $\begin{array}{c}\mathrm{CAOH} 2 \\
{[\mathrm{~g}]}\end{array}$ & $\begin{array}{c}\text { CATIO3 } \\
{[\mathrm{g}]}\end{array}$ & $\begin{array}{c}\mathrm{CROH} 3 \\
{[\mathrm{~g}]}\end{array}$ & $\begin{array}{c}\text { HYDROS } \\
\text { OD [g] }\end{array}$ & $\begin{array}{c}\mathrm{MGOH} 2 \\
{[\mathrm{~g}]}\end{array}$ \\
\hline 6EA028 & 2 & 15 & 6 & $7.12 \mathrm{E}+03$ & 0 & 0 & 0 & 0 & 0 & $3.52 \mathrm{E}+02$ & $5.68 \mathrm{E}+01$ & 0 & 0 & $8.43 \mathrm{E}+01$ \\
\hline 6EA029 & 2 & 15 & 6 & $8.88 \mathrm{E}+03$ & 0 & 0 & 0 & 0 & 0 & $3.43 \mathrm{E}+02$ & $5.50 \mathrm{E}+01$ & 0 & 0 & $8.17 \mathrm{E}+01$ \\
\hline 6EA031 & 2 & 15 & 6 & 0 & 0 & 0 & 0 & 0 & 0 & $3.14 \mathrm{E}+02$ & $4.97 \mathrm{E}+01$ & 0 & 0 & $7.38 \mathrm{E}+01$ \\
\hline 6EA032 & 2 & 15 & 6 & 0 & 0 & 0 & 0 & 0 & 0 & $3.17 \mathrm{E}+02$ & $5.03 \mathrm{E}+01$ & 0 & 0 & $7.46 \mathrm{E}+01$ \\
\hline 6EA033 & 2 & 15 & 6 & 0 & 0 & $3.08 \mathrm{E}+02$ & 0 & $1.32 \mathrm{E}+02$ & 0 & 0 & $5.58 \mathrm{E}+01$ & 0 & 0 & $8.28 \mathrm{E}+01$ \\
\hline 6EA035 & 2 & 15 & 6 & 0 & 0 & 0 & 0 & $3.38 \mathrm{E}+02$ & 0 & 0 & $5.06 \mathrm{E}+01$ & 0 & 0 & $7.51 \mathrm{E}+01$ \\
\hline 6EA036 & 2 & 15 & 6 & 0 & 0 & 0 & 0 & $3.75 \mathrm{E}+02$ & 0 & 0 & $5.61 \mathrm{E}+01$ & 0 & 0 & $8.33 \mathrm{E}+01$ \\
\hline 6EA037 & 2 & 15 & 6 & $6.42 \mathrm{E}+03$ & 0 & 0 & $4.84 \mathrm{E}+02$ & 0 & 0 & 0 & $5.70 \mathrm{E}+01$ & 0 & 0 & $8.47 \mathrm{E}+01$ \\
\hline 6EA038 & 2 & 15 & 6 & $7.12 \mathrm{E}+03$ & 0 & 0 & 0 & 0 & 0 & $3.52 \mathrm{E}+02$ & $5.68 \mathrm{E}+01$ & 0 & 0 & $8.43 \mathrm{E}+01$ \\
\hline 6EA039 & 2 & 15 & 6 & $8.88 \mathrm{E}+03$ & 0 & 0 & 0 & 0 & 0 & $3.43 \mathrm{E}+02$ & $5.50 \mathrm{E}+01$ & 0 & 0 & $8.17 \mathrm{E}+01$ \\
\hline 6EA040 & 2 & 15 & 6 & $1.09 \mathrm{E}+04$ & 0 & 0 & 0 & $3.55 \mathrm{E}+02$ & 0 & 0 & $5.32 \mathrm{E}+01$ & 0 & 0 & $7.89 \mathrm{E}+01$ \\
\hline 6EA041 & 0 & 66 & 6 & 0 & 0 & 0 & 0 & 0 & 0 & 0 & 0 & 0 & 0 & 0 \\
\hline 6EA043 & 0 & 66 & 6 & 0 & 0 & 0 & 0 & 0 & 0 & 0 & 0 & 0 & 0 & 0 \\
\hline 6EA044 & 0 & 66 & 6 & 0 & 0 & 0 & 0 & 0 & 0 & 0 & 0 & 0 & 0 & 0 \\
\hline 6EA045 & 0 & 66 & 6 & 0 & 0 & 0 & 0 & 0 & 0 & 0 & 0 & 0 & 0 & 0 \\
\hline 6EA046 & 0 & 66 & 6 & 0 & 0 & 0 & 0 & 0 & 0 & 0 & 0 & 0 & 0 & 0 \\
\hline 6EA047 & 0 & 66 & 6 & 0 & 0 & 0 & 0 & 0 & 0 & 0 & 0 & 0 & 0 & 0 \\
\hline 6EA048 & 0 & 66 & 6 & 0 & 0 & 0 & 0 & 0 & 0 & 0 & 0 & 0 & 0 & 0 \\
\hline 6EA049 & 0 & 66 & 6 & 0 & 0 & 0 & 0 & 0 & 0 & 0 & 0 & 0 & 0 & 0 \\
\hline $6 \mathrm{EA} 050$ & 0 & 66 & 6 & 0 & 0 & 0 & 0 & 0 & 0 & 0 & 0 & 0 & 0 & 0 \\
\hline $6 \mathrm{EA} 051$ & 0 & 66 & 6 & 0 & 0 & 0 & 0 & 0 & 0 & 0 & 0 & 0 & 0 & 0 \\
\hline 6ЕA052 & 0 & 66 & 6 & 0 & 0 & 0 & 0 & 0 & 0 & 0 & 0 & 0 & 0 & 0 \\
\hline 6EA053 & 0 & 66 & 6 & 0 & 0 & 0 & 0 & 0 & 0 & 0 & 0 & 0 & 0 & 0 \\
\hline 6EA054 & 0 & 66 & 6 & 0 & 0 & 0 & 0 & 0 & 0 & 0 & 0 & 0 & 0 & 0 \\
\hline $6 \mathrm{EA} 055$ & 0 & 66 & 6 & 0 & 0 & 0 & 0 & 0 & 0 & 0 & 0 & 0 & 0 & 0 \\
\hline 6EA056 & 0 & 66 & 6 & 0 & 0 & 0 & 0 & 0 & 0 & 0 & 0 & 0 & 0 & 0 \\
\hline 6EA057 & 0 & 66 & 6 & 0 & 0 & 0 & 0 & 0 & 0 & 0 & 0 & 0 & 0 & 0 \\
\hline 6EA059 & 0 & 66 & 6 & 0 & 0 & 0 & 0 & 0 & 0 & 0 & 0 & 0 & 0 & 0 \\
\hline 6EA060 & 0 & 66 & 6 & 0 & 0 & 0 & 0 & 0 & 0 & 0 & 0 & 0 & 0 & 0 \\
\hline 6EA061 & 2 & 66 & 6 & 0 & 0 & $4.35 \mathrm{E}+02$ & 0 & 0 & 0 & 0 & $4.97 \mathrm{E}+01$ & 0 & 0 & $7.38 \mathrm{E}+01$ \\
\hline
\end{tabular}


Table 35. Predicted Solids for Envelope A Simulations - Part 1 (cont'd)

\begin{tabular}{|c|c|c|c|c|c|c|c|c|c|c|c|c|c|c|}
\hline Test ID & $\begin{array}{l}\text { SBS/ } \\
\text { Feed }\end{array}$ & $\begin{array}{l}\text { Temp } \\
{\left[{ }^{\circ} \mathrm{C}\right]} \\
\end{array}$ & $\begin{array}{c}\mathrm{Na} \mathrm{M} \\
{[\mathrm{mol} / \mathrm{L}]}\end{array}$ & $\begin{array}{c}\mathrm{ALOH} 3 \\
{[\mathrm{~g}]}\end{array}$ & $\begin{array}{c}\text { ANATAS } \\
\text { E [g] }\end{array}$ & $\begin{array}{c}\text { CA3PO42 } \\
{[\mathrm{g}]} \\
\end{array}$ & $\begin{array}{c}\mathrm{CACO} 3 \\
{[\mathrm{~g}]}\end{array}$ & CAF2 $[\mathrm{g}]$ & CANC [g] & $\begin{array}{c}\mathrm{CAOH} 2 \\
{[\mathrm{~g}]}\end{array}$ & $\begin{array}{c}\text { CATIO3 } \\
{[\mathrm{g}]}\end{array}$ & $\begin{array}{c}\mathrm{CROH} 3 \\
{[\mathrm{~g}]}\end{array}$ & $\begin{array}{c}\text { HYDROS } \\
\text { OD [g] }\end{array}$ & $\begin{array}{c}\mathrm{MGOH} 2 \\
{[\mathrm{~g}]}\end{array}$ \\
\hline $6 \mathrm{EA} 062$ & 2 & 66 & 6 & 0 & 0 & $4.40 \mathrm{E}+02$ & 0 & 0 & 0 & 0 & $5.03 \mathrm{E}+01$ & 0 & 0 & $7.46 \mathrm{E}+01$ \\
\hline 6EA063 & 2 & 66 & 6 & 0 & 0 & $4.90 \mathrm{E}+02$ & 0 & 0 & 0 & 0 & $5.58 \mathrm{E}+01$ & $3.50 \mathrm{E}+01$ & 0 & $8.28 \mathrm{E}+01$ \\
\hline $6 \mathrm{EA} 065$ & 2 & 66 & 6 & 0 & 0 & 0 & 0 & $3.38 \mathrm{E}+02$ & 0 & 0 & $5.06 \mathrm{E}+01$ & $9.15 \mathrm{E}+00$ & 0 & $7.51 \mathrm{E}+01$ \\
\hline 6ЕA066 & 2 & 66 & 6 & 0 & 0 & 0 & 0 & $3.75 \mathrm{E}+02$ & 0 & 0 & $5.61 \mathrm{E}+01$ & $3.70 \mathrm{E}+01$ & 0 & $8.33 \mathrm{E}+01$ \\
\hline $6 \mathrm{EA} 067$ & 2 & 66 & 6 & 0 & 0 & 0 & $4.86 \mathrm{E}+02$ & 0 & 0 & 0 & $5.70 \mathrm{E}+01$ & $2.79 \mathrm{E}+01$ & 0 & $8.47 \mathrm{E}+01$ \\
\hline 6EA069 & 2 & 66 & 6 & 0 & 0 & $4.81 \mathrm{E}+02$ & 0 & 0 & 0 & 0 & $5.50 \mathrm{E}+01$ & $1.65 \mathrm{E}+01$ & 0 & $8.17 \mathrm{E}+01$ \\
\hline 6ЕA070 & 2 & 66 & 6 & 0 & 0 & 0 & 0 & $3.55 \mathrm{E}+02$ & 0 & 0 & $5.32 \mathrm{E}+01$ & 0 & 0 & $7.89 \mathrm{E}+01$ \\
\hline 6ЕA071 & 2 & 66 & 6 & 0 & 0 & $4.35 \mathrm{E}+02$ & 0 & 0 & 0 & 0 & $4.97 \mathrm{E}+01$ & 0 & 0 & $7.38 \mathrm{E}+01$ \\
\hline 6EA072 & 2 & 66 & 6 & 0 & 0 & $4.40 \mathrm{E}+02$ & 0 & 0 & 0 & 0 & $5.03 \mathrm{E}+01$ & 0 & 0 & $7.46 \mathrm{E}+01$ \\
\hline $6 \mathrm{EA} 073$ & 2 & 66 & 6 & 0 & 0 & $4.90 \mathrm{E}+02$ & 0 & 0 & 0 & 0 & $5.58 \mathrm{E}+01$ & $3.50 \mathrm{E}+01$ & 0 & $8.28 \mathrm{E}+01$ \\
\hline 6EA074 & 2 & 66 & 6 & 0 & 0 & 0 & 0 & $3.59 \mathrm{E}+02$ & 0 & 0 & $5.37 \mathrm{E}+01$ & $1.81 \mathrm{E}+01$ & 0 & $7.97 \mathrm{E}+01$ \\
\hline 6ЕA075 & 2 & 66 & 6 & 0 & 0 & 0 & 0 & $3.38 \mathrm{E}+02$ & 0 & 0 & $5.06 \mathrm{E}+01$ & $9.15 \mathrm{E}+00$ & 0 & $7.51 \mathrm{E}+01$ \\
\hline 6EA077 & 2 & 66 & 6 & 0 & 0 & 0 & $4.86 \mathrm{E}+02$ & 0 & 0 & 0 & $5.70 \mathrm{E}+01$ & $2.79 \mathrm{E}+01$ & 0 & $8.47 \mathrm{E}+01$ \\
\hline 6EA078 & 2 & 66 & 6 & 0 & 0 & $4.96 \mathrm{E}+02$ & 0 & 0 & 0 & 0 & $5.68 \mathrm{E}+01$ & $2.44 \mathrm{E}+01$ & 0 & $8.43 \mathrm{E}+01$ \\
\hline 6ЕA079 & 2 & 66 & 6 & 0 & 0 & $4.81 \mathrm{E}+02$ & 0 & 0 & 0 & 0 & $5.50 \mathrm{E}+01$ & $1.65 \mathrm{E}+01$ & 0 & $8.17 \mathrm{E}+01$ \\
\hline 6EA080 & 2 & 66 & 6 & 0 & 0 & 0 & 0 & $3.55 \mathrm{E}+02$ & 0 & 0 & $5.32 \mathrm{E}+01$ & 0 & 0 & $7.89 \mathrm{E}+01$ \\
\hline 6EA081 & 0.0391 & 15 & 6 & $3.83 \mathrm{E}+03$ & 0 & 0 & 0 & $6.98 \mathrm{E}+00$ & 0 & 0 & $1.07 \mathrm{E}+00$ & 0 & 0 & $1.58 \mathrm{E}+00$ \\
\hline 6EA082 & 1.957 & 15.102 & 6 & $7.90 \mathrm{E}+03$ & 0 & 0 & 0 & $3.54 \mathrm{E}+02$ & 0 & 0 & $5.29 \mathrm{E}+01$ & 0 & 0 & $7.85 \mathrm{E}+01$ \\
\hline 6EA083 & 0.7578 & 15.714 & 6 & $3.90 \mathrm{E}+03$ & 0 & 0 & 0 & $1.43 \mathrm{E}+02$ & 0 & 0 & $2.14 \mathrm{E}+01$ & 0 & 0 & $3.17 \mathrm{E}+01$ \\
\hline 6EA084 & 0.3008 & 15.918 & 6 & $3.32 \mathrm{E}+03$ & 0 & 0 & 0 & $5.56 \mathrm{E}+01$ & 0 & 0 & $8.34 \mathrm{E}+00$ & 0 & 0 & $1.24 \mathrm{E}+01$ \\
\hline $6 \mathrm{EA} 085$ & 0.0039 & 16.122 & 6 & $2.42 \mathrm{E}+02$ & 0 & 0 & 0 & 5.49E-01 & 0 & 0 & $1.07 \mathrm{E}-01$ & 0 & 0 & $1.59 \mathrm{E}-01$ \\
\hline 6ЕA086 & 1.2305 & 17.04 & 6 & 0 & 0 & 0 & 0 & $2.28 \mathrm{E}+02$ & 0 & 0 & $3.41 \mathrm{E}+01$ & 0 & 0 & $5.06 \mathrm{E}+01$ \\
\hline $6 \mathrm{EA} 087$ & 1.5898 & 17.55 & 6 & $3.96 \mathrm{E}+03$ & 0 & 0 & 0 & $2.99 \mathrm{E}+02$ & 0 & 0 & $4.50 \mathrm{E}+01$ & 0 & 0 & $6.68 \mathrm{E}+01$ \\
\hline 6EA088 & 0.7734 & 18.06 & 6 & $2.06 \mathrm{E}+03$ & 0 & 0 & 0 & $1.45 \mathrm{E}+02$ & 0 & 0 & $2.17 \mathrm{E}+01$ & 0 & 0 & $3.22 \mathrm{E}+01$ \\
\hline 6ЕA089 & 1.1641 & 18.162 & 6 & $4.04 \mathrm{E}+03$ & 0 & 0 & 0 & $2.10 \mathrm{E}+02$ & 0 & 0 & $3.14 \mathrm{E}+01$ & 0 & 0 & $4.66 \mathrm{E}+01$ \\
\hline 6EA090 & 0.6094 & 18.264 & 6 & 0 & 0 & 0 & 0 & $1.10 \mathrm{E}+02$ & 0 & 0 & $1.65 \mathrm{E}+01$ & 0 & 0 & $2.45 \mathrm{E}+01$ \\
\hline 6EA093 & 1.0195 & 19.284 & 6 & $1.55 \mathrm{E}+03$ & 0 & 0 & 0 & $1.89 \mathrm{E}+02$ & 0 & 0 & $2.84 \mathrm{E}+01$ & 0 & 0 & $4.22 \mathrm{E}+01$ \\
\hline 6EA094 & 0.5352 & 19.488 & 6 & $1.21 \mathrm{E}+02$ & 0 & 0 & 0 & $1.01 \mathrm{E}+02$ & 0 & 0 & $1.52 \mathrm{E}+01$ & 0 & 0 & $2.26 \mathrm{E}+01$ \\
\hline 6EA095 & 1.3203 & 19.998 & 6 & $1.76 \mathrm{E}+03$ & 0 & 0 & 0 & $2.38 \mathrm{E}+02$ & 0 & 0 & $3.56 \mathrm{E}+01$ & 0 & 0 & $5.29 \mathrm{E}+01$ \\
\hline
\end{tabular}


Table 35. Predicted Solids for Envelope A Simulations - Part 1 (cont'd)

\begin{tabular}{|c|c|c|c|c|c|c|c|c|c|c|c|c|c|c|}
\hline Test ID & $\begin{array}{l}\text { SBS/ } \\
\text { Feed }\end{array}$ & $\begin{array}{l}\text { Temp } \\
{\left[{ }^{\circ} \mathrm{C}\right]} \\
\end{array}$ & $\begin{array}{c}\mathrm{Na} \mathrm{M} \\
{[\mathrm{mol} / \mathrm{L}]}\end{array}$ & $\begin{array}{c}\text { ALOH3 } \\
{[\mathrm{g}]}\end{array}$ & $\begin{array}{c}\text { ANATAS } \\
\text { E }[\mathrm{g}] \\
\end{array}$ & $\begin{array}{c}\text { CA3PO42 } \\
{[\mathrm{g}]}\end{array}$ & $\begin{array}{c}\mathrm{CACO} 3 \\
{[\mathrm{~g}]}\end{array}$ & CAF2 [g] & CANC [g] & $\begin{array}{c}\mathrm{CAOH} 2 \\
{[\mathrm{~g}]}\end{array}$ & $\begin{array}{c}\text { CATIO3 } \\
{[\mathrm{g}]}\end{array}$ & $\begin{array}{c}\mathrm{CROH} 3 \\
{[\mathrm{~g}]}\end{array}$ & $\begin{array}{c}\text { HYDROS } \\
\text { OD [g] } \\
\end{array}$ & $\begin{array}{c}\mathrm{MGOH} 2 \\
{[\mathrm{~g}]}\end{array}$ \\
\hline 6EA096 & 1.8398 & 20.508 & 6 & $2.31 \mathrm{E}+03$ & 0 & 0 & 0 & $3.41 \mathrm{E}+02$ & 0 & 0 & $5.14 \mathrm{E}+01$ & 0 & 0 & $7.62 \mathrm{E}+01$ \\
\hline 6EA097 & 0.1563 & 21.222 & 6 & 0 & 0 & 0 & 0 & $2.83 \mathrm{E}+01$ & 0 & 0 & $4.39 \mathrm{E}+00$ & 0 & 0 & $6.51 \mathrm{E}+00$ \\
\hline 6ЕA099 & 0.1719 & 21.732 & 6 & 0 & 0 & 0 & 0 & $3.14 \mathrm{E}+01$ & 0 & 0 & $4.73 \mathrm{E}+00$ & 0 & 0 & $7.02 \mathrm{E}+00$ \\
\hline 6EA100 & 1.6094 & 22.956 & 6 & 0 & 0 & $3.75 \mathrm{E}+02$ & 0 & $1.12 \mathrm{E}+01$ & 0 & 0 & $4.54 \mathrm{E}+01$ & 0 & 0 & $6.74 \mathrm{E}+01$ \\
\hline 6EA101 & 0.8633 & 23.466 & 6 & 0 & 0 & 0 & 0 & $1.61 \mathrm{E}+02$ & 0 & 0 & $2.41 \mathrm{E}+01$ & 0 & 0 & $3.58 \mathrm{E}+01$ \\
\hline 6EA103 & 1.0273 & 26.016 & 6 & 0 & 0 & 0 & 0 & $1.88 \mathrm{E}+02$ & 0 & 0 & $2.84 \mathrm{E}+01$ & 0 & 0 & $4.22 \mathrm{E}+01$ \\
\hline 6EA104 & 1.1758 & 26.22 & 6 & 0 & 0 & 0 & 0 & $2.20 \mathrm{E}+02$ & 0 & 0 & $3.30 \mathrm{E}+01$ & 0 & 0 & $4.90 \mathrm{E}+01$ \\
\hline 6EA105 & 0.5 & 26.526 & 6 & 0 & 0 & 0 & 0 & $9.05 \mathrm{E}+01$ & 0 & 0 & $1.39 \mathrm{E}+01$ & 0 & 0 & $2.06 \mathrm{E}+01$ \\
\hline 6EA106 & 1.2695 & 27.036 & 6 & 0 & 0 & 0 & 0 & $2.36 \mathrm{E}+02$ & 0 & 0 & $3.53 \mathrm{E}+01$ & 0 & 0 & $5.25 \mathrm{E}+01$ \\
\hline 6EA107 & 0.0195 & 27.138 & 6 & 0 & 0 & 0 & 0 & 0 & 0 & 0 & $5.39 \mathrm{E}-01$ & 0 & 0 & $8.00 \mathrm{E}-01$ \\
\hline 6EA108 & 1.4414 & 27.24 & 6 & 0 & 0 & 0 & 0 & $2.62 \mathrm{E}+02$ & 0 & 0 & $3.92 \mathrm{E}+01$ & 0 & 0 & $5.82 \mathrm{E}+01$ \\
\hline 6EA109 & 1.0547 & 28.056 & 6 & 0 & 0 & 0 & 0 & $1.94 \mathrm{E}+02$ & 0 & 0 & $2.94 \mathrm{E}+01$ & 0 & 0 & $4.37 \mathrm{E}+01$ \\
\hline 6EA111 & 1.0977 & 28.464 & 6 & 0 & 0 & $4.78 \mathrm{E}+01$ & 0 & $1.62 \mathrm{E}+02$ & 0 & 0 & $3.09 \mathrm{E}+01$ & 0 & 0 & $4.58 \mathrm{E}+01$ \\
\hline 6EA112 & 0.6211 & 28.566 & 6 & 0 & 0 & 0 & 0 & $1.15 \mathrm{E}+02$ & 0 & 0 & $1.74 \mathrm{E}+01$ & 0 & 0 & $2.58 \mathrm{E}+01$ \\
\hline $6 \mathrm{EA} 113$ & 1.0664 & 28.872 & 6 & 0 & 0 & 0 & 0 & $1.95 \mathrm{E}+02$ & 0 & 0 & $2.94 \mathrm{E}+01$ & 0 & 0 & $4.37 \mathrm{E}+01$ \\
\hline 6EA114 & 0.6563 & 29.382 & 6 & 0 & 0 & 0 & 0 & $1.22 \mathrm{E}+02$ & 0 & 0 & $1.85 \mathrm{E}+01$ & 0 & 0 & $2.75 \mathrm{E}+01$ \\
\hline 6EA115 & 1.3867 & 29.484 & 6 & 0 & 0 & 0 & 0 & $2.56 \mathrm{E}+02$ & 0 & 0 & $3.83 \mathrm{E}+01$ & 0 & 0 & $5.69 \mathrm{E}+01$ \\
\hline 6EA116 & 1.0078 & 30.198 & 6 & 0 & 0 & 0 & 0 & $1.89 \mathrm{E}+02$ & 0 & 0 & $2.82 \mathrm{E}+01$ & 0 & 0 & $4.19 \mathrm{E}+01$ \\
\hline 6EA117 & 1.8711 & 30.504 & 6 & 0 & 0 & 0 & 0 & $3.46 \mathrm{E}+02$ & 0 & 0 & $5.17 \mathrm{E}+01$ & 0 & 0 & $7.68 \mathrm{E}+01$ \\
\hline 6EA118 & 0.5664 & 30.606 & 6 & 0 & 0 & 0 & 0 & $1.04 \mathrm{E}+02$ & 0 & 0 & $1.56 \mathrm{E}+01$ & 0 & 0 & $2.32 \mathrm{E}+01$ \\
\hline 6EA119 & 0.6953 & 31.422 & 6 & 0 & 0 & 0 & 0 & $1.21 \mathrm{E}+02$ & 0 & 0 & $1.82 \mathrm{E}+01$ & 0 & 0 & $2.70 \mathrm{E}+01$ \\
\hline 6EA120 & 1.4219 & 31.626 & 6 & 0 & 0 & 0 & 0 & $2.61 \mathrm{E}+02$ & 0 & 0 & $3.91 \mathrm{E}+01$ & 0 & 0 & $5.81 \mathrm{E}+01$ \\
\hline 6EA121 & 1.3555 & 32.748 & 6 & 0 & 0 & 0 & 0 & $2.52 \mathrm{E}+02$ & 0 & 0 & $3.82 \mathrm{E}+01$ & 0 & 0 & $5.67 \mathrm{E}+01$ \\
\hline 6EA122 & 0.2383 & 32.952 & 6 & 0 & 0 & 0 & 0 & $4.46 \mathrm{E}+01$ & 0 & 0 & $6.75 \mathrm{E}+00$ & 0 & 0 & $1.00 \mathrm{E}+01$ \\
\hline 6EA123 & 0.6328 & 33.36 & 6 & 0 & 0 & $1.46 \mathrm{E}+02$ & 0 & 0 & 0 & 0 & $1.77 \mathrm{E}+01$ & 0 & 0 & $2.62 \mathrm{E}+01$ \\
\hline 6EA124 & 1.5313 & 33.564 & 6 & 0 & 0 & 0 & 0 & $2.82 \mathrm{E}+02$ & 0 & 0 & $4.23 \mathrm{E}+01$ & 0 & 0 & $6.28 \mathrm{E}+01$ \\
\hline 6EA127 & 1.9727 & 34.278 & 6 & 0 & 0 & 0 & 0 & $3.60 \mathrm{E}+02$ & 0 & 0 & $5.39 \mathrm{E}+01$ & 0 & 0 & $8.00 \mathrm{E}+01$ \\
\hline 6EA128 & 0.1523 & 34.788 & 6 & 0 & 0 & 0 & 0 & $2.77 \mathrm{E}+01$ & 0 & 0 & $4.23 \mathrm{E}+00$ & 0 & 0 & $6.28 \mathrm{E}+00$ \\
\hline 6EA129 & 1.668 & 37.032 & 6 & 0 & 0 & 0 & 0 & $3.04 \mathrm{E}+02$ & 0 & 0 & $4.58 \mathrm{E}+01$ & 0 & 0 & $6.80 \mathrm{E}+01$ \\
\hline
\end{tabular}


Table 35. Predicted Solids for Envelope A Simulations - Part 1 (cont'd)

\begin{tabular}{|c|c|c|c|c|c|c|c|c|c|c|c|c|c|c|}
\hline Test ID & $\begin{array}{l}\text { SBS/ } \\
\text { Feed }\end{array}$ & $\begin{array}{l}\text { Temp } \\
{\left[{ }^{\circ} \mathrm{C}\right]} \\
\end{array}$ & $\begin{array}{c}\mathrm{Na} \mathrm{M} \\
{[\mathrm{mol} / \mathrm{L}]}\end{array}$ & $\begin{array}{c}\text { ALOH3 } \\
{[\mathrm{g}]}\end{array}$ & $\begin{array}{c}\text { ANATAS } \\
\text { E [g] }\end{array}$ & $\begin{array}{c}\text { CA3PO42 } \\
{[\mathrm{g}]} \\
\end{array}$ & $\begin{array}{c}\mathrm{CACO} 3 \\
{[\mathrm{~g}]}\end{array}$ & CAF2 [g] & CANC [g] & $\begin{array}{c}\mathrm{CAOH} 2 \\
{[\mathrm{~g}]}\end{array}$ & $\begin{array}{c}\text { CATIO3 } \\
{[\mathrm{g}]}\end{array}$ & $\begin{array}{c}\mathrm{CROH} 3 \\
{[\mathrm{~g}]}\end{array}$ & $\begin{array}{c}\text { HYDROS } \\
\text { OD [g] } \\
\end{array}$ & $\begin{array}{c}\mathrm{MGOH} 2 \\
{[\mathrm{~g}]}\end{array}$ \\
\hline 6EA130 & 1.9336 & 38.358 & 6 & 0 & 0 & 0 & 0 & $3.52 \mathrm{E}+02$ & 0 & 0 & $5.30 \mathrm{E}+01$ & 0 & 0 & $7.86 \mathrm{E}+01$ \\
\hline 6EA131 & 1.4258 & 39.48 & 6 & 0 & 0 & 0 & 0 & $2.67 \mathrm{E}+02$ & 0 & 0 & $4.00 \mathrm{E}+01$ & 0 & 0 & $5.94 \mathrm{E}+01$ \\
\hline 6EA133 & 1.6641 & 40.194 & 6 & 0 & 0 & 0 & 0 & $2.98 \mathrm{E}+02$ & 0 & 0 & $4.46 \mathrm{E}+01$ & 0 & 0 & $6.63 \mathrm{E}+01$ \\
\hline 6EA134 & 0.2344 & 40.602 & 6 & 0 & 0 & 0 & 0 & $4.39 \mathrm{E}+01$ & 0 & 0 & $6.62 \mathrm{E}+00$ & 0 & 0 & $9.83 \mathrm{E}+00$ \\
\hline 6EA135 & 1.6914 & 41.112 & 6 & 0 & 0 & 0 & 0 & $3.11 \mathrm{E}+02$ & 0 & 0 & $4.69 \mathrm{E}+01$ & 0 & 0 & $6.97 \mathrm{E}+01$ \\
\hline 6EA137 & 0.2813 & 42.744 & 6 & 0 & 0 & 0 & 0 & $4.98 \mathrm{E}+01$ & 0 & 0 & $7.79 \mathrm{E}+00$ & 0 & 0 & $1.16 \mathrm{E}+01$ \\
\hline 6EA138 & 1.7813 & 43.152 & 6 & 0 & 0 & 0 & 0 & $3.33 \mathrm{E}+02$ & 0 & 0 & $5.02 \mathrm{E}+01$ & 0 & 0 & $7.45 \mathrm{E}+01$ \\
\hline 6EA139 & 0.6992 & 43.56 & 6 & 0 & 0 & 0 & 0 & $1.31 \mathrm{E}+02$ & 0 & 0 & $1.96 \mathrm{E}+01$ & 0 & 0 & $2.91 \mathrm{E}+01$ \\
\hline 6EA140 & 0.957 & 43.662 & 6 & 0 & 0 & 0 & 0 & $1.80 \mathrm{E}+02$ & 0 & 0 & $2.70 \mathrm{E}+01$ & 0 & 0 & $4.01 \mathrm{E}+01$ \\
\hline 6EA141 & 0.3164 & 43.866 & 6 & 0 & 0 & $6.99 \mathrm{E}+01$ & 0 & 0 & 0 & 0 & $8.94 \mathrm{E}+00$ & 0 & 0 & $1.33 \mathrm{E}+01$ \\
\hline 6EA142 & 1.7539 & 44.172 & 6 & 0 & 0 & 0 & 0 & $3.22 \mathrm{E}+02$ & 0 & 0 & $4.82 \mathrm{E}+01$ & 0 & 0 & $7.16 \mathrm{E}+01$ \\
\hline 6EA143 & 0.4375 & 44.274 & 6 & 0 & 0 & $1.02 \mathrm{E}+02$ & 0 & 0 & 0 & 0 & $1.24 \mathrm{E}+01$ & 0 & 0 & $1.84 \mathrm{E}+01$ \\
\hline 6EA145 & 0.6758 & 44.58 & 6 & 0 & 0 & 0 & 0 & $1.24 \mathrm{E}+02$ & 0 & 0 & $1.86 \mathrm{E}+01$ & 0 & 0 & $2.77 \mathrm{E}+01$ \\
\hline 6EA146 & 1.4922 & 45.396 & 6 & 0 & 0 & 0 & 0 & $2.72 \mathrm{E}+02$ & 0 & 0 & $4.07 \mathrm{E}+01$ & 0 & 0 & $6.05 \mathrm{E}+01$ \\
\hline 6EA147 & 1.168 & 45.6 & 6 & 0 & 0 & 0 & 0 & $2.22 \mathrm{E}+02$ & 0 & 0 & $3.33 \mathrm{E}+01$ & 0 & 0 & $4.94 \mathrm{E}+01$ \\
\hline 6EA148 & 0.7852 & 45.702 & 6 & 0 & 0 & $1.86 \mathrm{E}+02$ & 0 & 0 & 0 & 0 & $2.21 \mathrm{E}+01$ & 0 & 0 & $3.27 \mathrm{E}+01$ \\
\hline 6EA149 & 0.9219 & 45.804 & 6 & 0 & 0 & 0 & 0 & $1.67 \mathrm{E}+02$ & 0 & 0 & $2.54 \mathrm{E}+01$ & 0 & 0 & $3.77 \mathrm{E}+01$ \\
\hline 6EA150 & 1.2031 & 46.008 & 6 & 0 & 0 & 0 & 0 & $2.25 \mathrm{E}+02$ & 0 & 0 & $3.39 \mathrm{E}+01$ & 0 & 0 & $5.03 \mathrm{E}+01$ \\
\hline 6EA151 & 0.6289 & 46.518 & 6 & 0 & 0 & 0 & 0 & $1.15 \mathrm{E}+02$ & 0 & 0 & $1.76 \mathrm{E}+01$ & 0 & 0 & $2.61 \mathrm{E}+01$ \\
\hline 6EA152 & 0.0078 & 47.028 & 6 & 0 & 0 & 0 & 0 & $9.47 \mathrm{E}-01$ & 0 & 0 & $2.14 \mathrm{E}-01$ & 0 & 0 & $3.18 \mathrm{E}-01$ \\
\hline 6EA153 & 1.2773 & 47.13 & 6 & 0 & 0 & 0 & 0 & $2.32 \mathrm{E}+02$ & 0 & 0 & $3.47 \mathrm{E}+01$ & 0 & 0 & $5.15 \mathrm{E}+01$ \\
\hline 6EA154 & 0.9531 & 47.742 & 6 & 0 & 0 & 0 & 0 & $1.75 \mathrm{E}+02$ & 0 & 0 & $2.67 \mathrm{E}+01$ & 0 & 0 & $3.97 \mathrm{E}+01$ \\
\hline 6EA155 & 1.6328 & 47.844 & 6 & 0 & 0 & 0 & 0 & $2.88 \mathrm{E}+02$ & 0 & 0 & $4.32 \mathrm{E}+01$ & 0 & 0 & $6.41 \mathrm{E}+01$ \\
\hline 6EA156 & 1.1289 & 47.946 & 6 & 0 & 0 & $2.73 \mathrm{E}+02$ & 0 & 0 & 0 & 0 & $3.18 \mathrm{E}+01$ & 0 & 0 & $4.71 \mathrm{E}+01$ \\
\hline 6EA157 & 1.7773 & 48.456 & 6 & 0 & 0 & 0 & 0 & $3.29 \mathrm{E}+02$ & 0 & 0 & $4.94 \mathrm{E}+01$ & 0 & 0 & $7.34 \mathrm{E}+01$ \\
\hline 6EA158 & 1.1602 & 48.762 & 6 & 0 & 0 & $2.78 \mathrm{E}+02$ & 0 & 0 & 0 & 0 & $3.24 \mathrm{E}+01$ & 0 & 0 & $4.81 \mathrm{E}+01$ \\
\hline 6EA161 & 1.5391 & 50.088 & 6 & 0 & 0 & 0 & 0 & $2.72 \mathrm{E}+02$ & 0 & 0 & $4.15 \mathrm{E}+01$ & 0 & 0 & $6.16 \mathrm{E}+01$ \\
\hline 6EA162 & 0.0547 & 51.108 & 6 & 0 & 0 & $6.48 \mathrm{E}+00$ & 0 & 0 & 0 & 0 & $1.55 \mathrm{E}+00$ & 0 & 0 & $2.30 \mathrm{E}+00$ \\
\hline 6EA163 & 1.9258 & 52.026 & 6 & 0 & 0 & 0 & 0 & $3.57 \mathrm{E}+02$ & 0 & 0 & $5.36 \mathrm{E}+01$ & 0 & 0 & $7.96 \mathrm{E}+01$ \\
\hline
\end{tabular}


Table 35. Predicted Solids for Envelope A Simulations - Part 1 (cont'd)

\begin{tabular}{|c|c|c|c|c|c|c|c|c|c|c|c|c|c|c|}
\hline Test ID & $\begin{array}{l}\text { SBS/ } \\
\text { Feed }\end{array}$ & $\begin{array}{l}\text { Temp } \\
{\left[{ }^{\circ} \mathrm{C}\right]}\end{array}$ & $\begin{array}{c}\mathrm{Na} \mathrm{M} \\
{[\mathrm{mol} / \mathrm{L}]}\end{array}$ & $\begin{array}{c}\mathrm{ALOH} 3 \\
{[\mathrm{~g}]}\end{array}$ & $\begin{array}{c}\text { ANATAS } \\
\text { E }[\mathrm{g}]\end{array}$ & $\begin{array}{c}\text { CA3PO42 } \\
{[\mathrm{g}]} \\
\end{array}$ & $\begin{array}{c}\mathrm{CACO} 3 \\
{[\mathrm{~g}]}\end{array}$ & CAF2 [g] & CANC [g] & $\begin{array}{c}\mathrm{CAOH} 2 \\
{[\mathrm{~g}]}\end{array}$ & $\begin{array}{c}\text { CATIO3 } \\
{[\mathrm{g}]}\end{array}$ & $\begin{array}{c}\mathrm{CROH} 3 \\
{[\mathrm{~g}]}\end{array}$ & $\begin{array}{c}\text { HYDROS } \\
\text { OD }[\mathrm{g}] \\
\end{array}$ & $\begin{array}{c}\mathrm{MGOH} 2 \\
{[\mathrm{~g}]}\end{array}$ \\
\hline 6EA164 & 1.4766 & 53.454 & 6 & 0 & 0 & 0 & 0 & $2.68 \mathrm{E}+02$ & 0 & 0 & $4.05 \mathrm{E}+01$ & 0 & 0 & $6.02 \mathrm{E}+01$ \\
\hline 6EA165 & 0.457 & 53.556 & 6 & 0 & 0 & $1.08 \mathrm{E}+02$ & 0 & 0 & 0 & 0 & $1.30 \mathrm{E}+01$ & 0 & 0 & $1.93 \mathrm{E}+01$ \\
\hline 6EA167 & 0.625 & 54.27 & 6 & 0 & 0 & 0 & 0 & $1.16 \mathrm{E}+02$ & 0 & 0 & $1.74 \mathrm{E}+01$ & 0 & 0 & $2.59 \mathrm{E}+01$ \\
\hline 6EA168 & 1.1484 & 54.882 & 6 & 0 & 0 & 0 & 0 & $2.15 \mathrm{E}+02$ & 0 & 0 & $3.27 \mathrm{E}+01$ & 0 & 0 & $4.85 \mathrm{E}+01$ \\
\hline 6EA169 & 1.7695 & 55.902 & 6 & 0 & 0 & 0 & 0 & $3.22 \mathrm{E}+02$ & 0 & 0 & $4.89 \mathrm{E}+01$ & 0 & 0 & $7.25 \mathrm{E}+01$ \\
\hline 6EA171 & 0.207 & 57.738 & 6 & 0 & 0 & $4.41 \mathrm{E}+01$ & 0 & 0 & 0 & 0 & $5.75 \mathrm{E}+00$ & 0 & 0 & $8.53 \mathrm{E}+00$ \\
\hline 6EA172 & 1.7266 & 58.758 & 6 & 0 & 0 & $4.23 \mathrm{E}+02$ & 0 & 0 & 0 & 0 & $4.85 \mathrm{E}+01$ & 0 & 0 & $7.20 \mathrm{E}+01$ \\
\hline 6EA173 & 1.0898 & 58.86 & 6 & 0 & 0 & 0 & 0 & $1.92 \mathrm{E}+02$ & 0 & 0 & $2.91 \mathrm{E}+01$ & 0 & 0 & $4.32 \mathrm{E}+01$ \\
\hline 6EA174 & 0.8945 & 60.39 & 6 & 0 & 0 & $2.17 \mathrm{E}+02$ & 0 & 0 & 0 & 0 & $2.52 \mathrm{E}+01$ & 0 & 0 & $3.75 \mathrm{E}+01$ \\
\hline $6 \mathrm{EA} 175$ & 1.6758 & 60.696 & 6 & 0 & 0 & $4.15 \mathrm{E}+02$ & 0 & 0 & 0 & 0 & $4.76 \mathrm{E}+01$ & 0 & 0 & $7.06 \mathrm{E}+01$ \\
\hline 6EA176 & 1.0625 & 60.9 & 6 & 0 & 0 & 0 & 0 & $1.90 \mathrm{E}+02$ & 0 & 0 & $2.88 \mathrm{E}+01$ & 0 & 0 & $4.27 \mathrm{E}+01$ \\
\hline 6EA177 & 1.9492 & 61.206 & 6 & 0 & 0 & 0 & 0 & $3.63 \mathrm{E}+02$ & 0 & 0 & $5.43 \mathrm{E}+01$ & $2.91 \mathrm{E}+00$ & 0 & $8.06 \mathrm{E}+01$ \\
\hline 6EA179 & 0.2422 & 62.022 & 6 & 0 & 0 & 0 & 0 & $4.38 \mathrm{E}+01$ & 0 & 0 & $6.72 \mathrm{E}+00$ & 0 & 0 & $9.98 \mathrm{E}+00$ \\
\hline 6EA180 & 0.4961 & 63.042 & 6 & 0 & 0 & 0 & 0 & $8.99 \mathrm{E}+01$ & 0 & 0 & $1.37 \mathrm{E}+01$ & 0 & 0 & $2.03 \mathrm{E}+01$ \\
\hline 6EA181 & 0.6367 & 63.348 & 6 & 0 & 0 & 0 & 0 & $1.18 \mathrm{E}+02$ & 0 & 0 & $1.78 \mathrm{E}+01$ & 0 & 0 & $2.65 \mathrm{E}+01$ \\
\hline 6EA182 & 1.4102 & 63.552 & 6 & 0 & 0 & $3.27 \mathrm{E}+02$ & 0 & 0 & 0 & 0 & $3.76 \mathrm{E}+01$ & 0 & 0 & $5.58 \mathrm{E}+01$ \\
\hline 6EA183 & 0.4453 & 63.756 & 6 & 0 & 0 & 0 & 0 & $8.00 \mathrm{E}+01$ & 0 & 0 & $1.22 \mathrm{E}+01$ & 0 & 0 & $1.81 \mathrm{E}+01$ \\
\hline 6EA184 & 0.1836 & 64.164 & 6 & 0 & 0 & 0 & 0 & $3.35 \mathrm{E}+01$ & 0 & 0 & $5.18 \mathrm{E}+00$ & 0 & 0 & $7.69 \mathrm{E}+00$ \\
\hline 6EA185 & 1.6563 & 64.47 & 6 & 0 & 0 & 0 & 0 & $3.07 \mathrm{E}+02$ & 0 & 0 & $4.60 \mathrm{E}+01$ & $8.66 \mathrm{E}+00$ & 0 & $6.83 \mathrm{E}+01$ \\
\hline 6EA186 & 0.1875 & 64.674 & 6 & 0 & 0 & 0 & 0 & $3.39 \mathrm{E}+01$ & 0 & 0 & $5.20 \mathrm{E}+00$ & 0 & 0 & $7.72 \mathrm{E}+00$ \\
\hline 6EA187 & 1.7969 & 64.776 & 6 & 0 & 0 & 0 & 0 & $3.33 \mathrm{E}+02$ & 0 & 0 & $4.99 \mathrm{E}+01$ & $2.03 \mathrm{E}+01$ & 0 & $7.41 \mathrm{E}+01$ \\
\hline 6EA188 & 1.0508 & 65.388 & 6 & 0 & 0 & 0 & 0 & $1.91 \mathrm{E}+02$ & 0 & 0 & $2.87 \mathrm{E}+01$ & 0 & 0 & $4.26 \mathrm{E}+01$ \\
\hline
\end{tabular}


Table 36. Predicted Solids for Envelope A Simulations - Part 2

\begin{tabular}{|c|c|c|c|c|c|c|c|c|c|c|c|c|c|c|c|}
\hline Test ID & $\mathrm{NA} 2 \mathrm{C} 2 \mathrm{O} 4$ & \begin{tabular}{|c|}
$\mathrm{NA} 2 \mathrm{CO} 3.1$ \\
$\mathrm{H} 2 \mathrm{O}$ \\
\end{tabular} & $\mathrm{NA} 2 \mathrm{U} 2 \mathrm{O} 7$ & NA3FSO4 & \begin{tabular}{|c|} 
NA6SO42 \\
CO3
\end{tabular} & NAF & $\begin{array}{c}\text { NAFPO4.1 } \\
9 \mathrm{H} 2 \mathrm{O}\end{array}$ & NANO3 & $\begin{array}{c}\text { NAPHOH. } \\
12 \mathrm{H} 2 \mathrm{O} \\
\end{array}$ & $\begin{array}{c}\text { NASGEL. } \\
15.5 \mathrm{H} 2 \mathrm{O} \\
\end{array}$ & $\mathrm{ZRO} 2$ & \begin{tabular}{|c|} 
ZRSO42.4 \\
$\mathrm{H} 2 \mathrm{O}$ \\
\end{tabular} & $\begin{array}{c}\text { Total } \\
\text { Solids } \\
\end{array}$ & $\begin{array}{c}\text { Total Soln } \\
\text { Mass }\end{array}$ & $\begin{array}{c}\text { Solids } \% \\
\text { of Total } \\
\text { Soln } \\
\end{array}$ \\
\hline 10EA001 & $4.26 \mathrm{E}+02$ & 0 & 0 & 0 & 0 & 0 & 0 & $5.68 \mathrm{E}+02$ & $6.75 \mathrm{E}+03$ & 0 & 0 & 0 & $7.74 \mathrm{E}+03$ & $1.04 \mathrm{E}+06$ & $0.74 \%$ \\
\hline 10EA002 & $4.07 \mathrm{E}+02$ & 0 & 0 & 0 & 0 & 0 & 0 & 0 & $1.21 \mathrm{E}+03$ & 0 & 0 & 0 & $1.62 \mathrm{E}+03$ & $1.06 \mathrm{E}+06$ & $0.15 \%$ \\
\hline 10EA003 & $1.25 \mathrm{E}+03$ & 0 & 0 & 0 & 0 & 0 & 0 & $6.85 \mathrm{E}+04$ & 0 & 0 & 0 & 0 & $6.98 \mathrm{E}+04$ & $1.04 \mathrm{E}+06$ & $6.69 \%$ \\
\hline 10EA004 & $9.85 \mathrm{E}+02$ & 0 & 0 & 0 & 0 & $9.20 \mathrm{E}+03$ & 0 & $1.55 \mathrm{E}+05$ & $6.75 \mathrm{E}+02$ & 0 & 0 & 0 & $1.66 \mathrm{E}+05$ & $9.41 \mathrm{E}+05$ & $17.67 \%$ \\
\hline 10EA005 & $7.42 \mathrm{E}+02$ & 0 & 0 & 0 & 0 & $7.30 \mathrm{E}+03$ & 0 & 0 & $1.12 \mathrm{E}+03$ & 0 & 0 & 0 & $9.17 \mathrm{E}+03$ & $1.02 \mathrm{E}+06$ & $0.90 \%$ \\
\hline 10EA006 & $1.61 \mathrm{E}+03$ & 0 & 7.13E-05 & 0 & 0 & $1.11 \mathrm{E}+04$ & 0 & 0 & $6.34 \mathrm{E}+03$ & 0 & 0 & 0 & $1.90 \mathrm{E}+04$ & $1.05 \mathrm{E}+06$ & $1.81 \%$ \\
\hline 10EA007 & $1.42 \mathrm{E}+03$ & 0 & $3.10 \mathrm{E}-05$ & 0 & 0 & 0 & 0 & 0 & 0 & 0 & 0 & 0 & $1.42 \mathrm{E}+03$ & $1.10 \mathrm{E}+06$ & $0.13 \%$ \\
\hline 10EA008 & $1.17 \mathrm{E}+03$ & 0 & 0 & 0 & 0 & 0 & 0 & $1.47 \mathrm{E}+05$ & $2.19 \mathrm{E}+04$ & 0 & 0 & 0 & $1.70 \mathrm{E}+05$ & $9.91 \mathrm{E}+05$ & $17.18 \%$ \\
\hline 10EA009 & $9.40 \mathrm{E}+02$ & 0 & 0 & 0 & 0 & 0 & 0 & 0 & $1.60 \mathrm{E}+04$ & 0 & 0 & 0 & $1.70 \mathrm{E}+04$ & $1.08 \mathrm{E}+06$ & $1.56 \%$ \\
\hline 10EA010 & $6.95 \mathrm{E}+02$ & 0 & 0 & 0 & 0 & $7.34 \mathrm{E}+03$ & 0 & 0 & $2.07 \mathrm{E}+02$ & 0 & 0 & 0 & $8.24 \mathrm{E}+03$ & $1.05 \mathrm{E}+06$ & $0.79 \%$ \\
\hline 10EA011 & $4.26 \mathrm{E}+02$ & 0 & 0 & 0 & 0 & 0 & 0 & $5.68 \mathrm{E}+02$ & $6.75 \mathrm{E}+03$ & 0 & 0 & 0 & $7.74 \mathrm{E}+03$ & $1.04 \mathrm{E}+06$ & $0.74 \%$ \\
\hline 10EA012 & $4.07 \mathrm{E}+02$ & 0 & 0 & 0 & 0 & 0 & 0 & 0 & $1.21 \mathrm{E}+03$ & 0 & 0 & 0 & $1.62 \mathrm{E}+03$ & $1.06 \mathrm{E}+06$ & $0.15 \%$ \\
\hline 10EA013 & $1.25 \mathrm{E}+03$ & 0 & 0 & 0 & 0 & 0 & 0 & $6.85 \mathrm{E}+04$ & 0 & 0 & 0 & 0 & $6.98 \mathrm{E}+04$ & $1.04 \mathrm{E}+06$ & $6.69 \%$ \\
\hline 10EA014 & $9.85 \mathrm{E}+02$ & 0 & 0 & 0 & 0 & $9.20 \mathrm{E}+03$ & 0 & $1.55 \mathrm{E}+05$ & $6.75 \mathrm{E}+02$ & 0 & 0 & 0 & $1.66 \mathrm{E}+05$ & $9.41 \mathrm{E}+05$ & $17.67 \%$ \\
\hline 10EA015 & $7.42 \mathrm{E}+02$ & 0 & 0 & 0 & 0 & $7.30 \mathrm{E}+03$ & 0 & 0 & $1.12 \mathrm{E}+03$ & 0 & 0 & 0 & $9.17 \mathrm{E}+03$ & $1.02 \mathrm{E}+06$ & $0.90 \%$ \\
\hline 10EA016 & $1.61 \mathrm{E}+03$ & 0 & 7.13E-05 & 0 & 0 & $1.11 \mathrm{E}+04$ & 0 & 0 & $6.34 \mathrm{E}+03$ & 0 & 0 & 0 & $1.90 \mathrm{E}+04$ & $1.05 \mathrm{E}+06$ & $1.81 \%$ \\
\hline $10 \mathrm{EA} 017$ & $1.42 \mathrm{E}+03$ & 0 & $3.10 \mathrm{E}-05$ & 0 & 0 & 0 & 0 & 0 & 0 & 0 & 0 & 0 & $1.42 \mathrm{E}+03$ & $1.10 \mathrm{E}+06$ & $0.13 \%$ \\
\hline 10EA018 & $1.17 \mathrm{E}+03$ & 0 & 0 & 0 & 0 & 0 & 0 & $1.47 \mathrm{E}+05$ & $2.19 \mathrm{E}+04$ & 0 & 0 & 0 & $1.70 \mathrm{E}+05$ & $9.91 \mathrm{E}+05$ & $17.18 \%$ \\
\hline 10EA019 & $9.40 \mathrm{E}+02$ & 0 & 0 & 0 & 0 & 0 & 0 & 0 & $1.60 \mathrm{E}+04$ & 0 & 0 & 0 & $1.70 \mathrm{E}+04$ & $1.08 \mathrm{E}+06$ & $1.56 \%$ \\
\hline 10EA020 & $6.95 \mathrm{E}+02$ & 0 & 0 & 0 & 0 & $7.34 \mathrm{E}+03$ & 0 & 0 & $2.07 \mathrm{E}+02$ & 0 & 0 & 0 & $8.24 \mathrm{E}+03$ & $1.05 \mathrm{E}+06$ & $0.79 \%$ \\
\hline 10EA021 & $4.19 \mathrm{E}+02$ & 0 & 0 & 0 & 0 & 0 & 0 & 0 & $6.43 \mathrm{E}+03$ & 0 & 0 & 0 & $7.28 \mathrm{E}+03$ & $1.09 \mathrm{E}+06$ & $0.67 \%$ \\
\hline 10EA022 & $4.01 \mathrm{E}+02$ & 0 & 0 & 0 & 0 & 0 & 0 & 0 & $9.10 \mathrm{E}+02$ & 0 & 0 & 0 & $1.75 \mathrm{E}+03$ & $1.11 \mathrm{E}+06$ & $0.16 \%$ \\
\hline 10EA023 & $1.20 \mathrm{E}+03$ & 0 & 0 & 0 & 0 & 0 & 0 & $3.59 \mathrm{E}+04$ & 0 & 0 & 0 & 0 & $3.76 \mathrm{E}+04$ & $1.11 \mathrm{E}+06$ & $3.38 \%$ \\
\hline 10EA024 & $9.65 \mathrm{E}+02$ & 0 & 0 & 0 & 0 & $9.43 \mathrm{E}+03$ & 0 & $1.33 \mathrm{E}+05$ & 0 & 0 & 0 & 0 & $1.43 \mathrm{E}+05$ & $1.00 \mathrm{E}+06$ & $14.33 \%$ \\
\hline 10EA025 & $7.27 \mathrm{E}+02$ & 0 & 0 & 0 & 0 & $7.54 \mathrm{E}+03$ & 0 & 0 & $4.42 \mathrm{E}+02$ & 0 & 0 & 0 & $9.16 \mathrm{E}+03$ & $1.06 \mathrm{E}+06$ & $0.86 \%$ \\
\hline 10EA026 & $1.58 \mathrm{E}+03$ & 0 & 7.12E-05 & 0 & 0 & $1.09 \mathrm{E}+04$ & 0 & 0 & $5.22 \mathrm{E}+03$ & 0 & 0 & 0 & $1.82 \mathrm{E}+04$ & $1.10 \mathrm{E}+06$ & $1.65 \%$ \\
\hline 10EA027 & $1.39 \mathrm{E}+03$ & 0 & 3.09E-05 & 0 & 0 & 0 & 0 & 0 & 0 & 0 & 0 & 0 & $1.90 \mathrm{E}+03$ & $1.15 \mathrm{E}+06$ & $0.17 \%$ \\
\hline 10EA028 & $1.15 \mathrm{E}+03$ & 0 & 0 & 0 & 0 & 0 & 0 & $1.20 \mathrm{E}+05$ & $2.02 \mathrm{E}+04$ & 0 & 0 & 0 & $1.42 \mathrm{E}+05$ & $1.06 \mathrm{E}+06$ & $13.45 \%$ \\
\hline 10EA029 & $9.21 \mathrm{E}+02$ & 0 & 0 & 0 & 0 & 0 & 0 & 0 & $1.52 \mathrm{E}+04$ & 0 & 0 & 0 & $1.66 \mathrm{E}+04$ & $1.13 \mathrm{E}+06$ & $1.47 \%$ \\
\hline 10EA030 & $6.85 \mathrm{E}+02$ & 0 & 0 & 0 & 0 & $7.65 \mathrm{E}+03$ & 0 & 0 & 0 & 0 & 0 & 0 & $8.80 \mathrm{E}+03$ & $1.09 \mathrm{E}+06$ & $0.81 \%$ \\
\hline 10EA031 & $4.19 \mathrm{E}+02$ & 0 & 0 & 0 & 0 & 0 & 0 & 0 & $6.43 \mathrm{E}+03$ & 0 & 0 & 0 & $7.28 \mathrm{E}+03$ & $1.09 \mathrm{E}+06$ & $0.67 \%$ \\
\hline
\end{tabular}


Table 36. Predicted Solids for Envelope A Simulations - Part 2 (cont'd)

\begin{tabular}{|c|c|c|c|c|c|c|c|c|c|c|c|c|c|c|c|}
\hline Test ID & $\begin{array}{c}\text { NA2C2O } \\
4 \\
\end{array}$ & $\begin{array}{c}\text { NA2CO3. } \\
1 \mathrm{H} 2 \mathrm{O}\end{array}$ & $\begin{array}{c}\text { NA2U2O } \\
7 \\
\end{array}$ & NA3FSO4 & $\begin{array}{c}\mathrm{NA6SO} 42 \\
\mathrm{CO} 3 \\
\end{array}$ & NAF & $\begin{array}{c}\text { NAFPO4. } \\
19 \mathrm{H} 2 \mathrm{O}\end{array}$ & NANO3 & \begin{tabular}{|c} 
NAPHOH \\
$.12 \mathrm{H} 2 \mathrm{O}$ \\
\end{tabular} & \begin{tabular}{|c|} 
NASGEL. \\
$15.5 \mathrm{H} 2 \mathrm{O}$ \\
\end{tabular} & ZRO2 & $\begin{array}{c}\text { ZRSO42. } \\
4 \mathrm{H} 2 \mathrm{O}\end{array}$ & $\begin{array}{c}\text { Total } \\
\text { Solids } \\
\end{array}$ & $\begin{array}{c}\text { Total Soln } \\
\text { Mass } \\
\end{array}$ & $\begin{array}{c}\text { Solids } \% \\
\text { of Total } \\
\text { Soln } \\
\end{array}$ \\
\hline 10EA032 & $4.01 \mathrm{E}+02$ & 0 & 0 & 0 & 0 & 0 & 0 & 0 & $9.10 \mathrm{E}+02$ & 0 & 0 & 0 & $1.75 \mathrm{E}+03$ & $1.11 \mathrm{E}+06$ & $0.16 \%$ \\
\hline 10EA033 & $1.20 \mathrm{E}+03$ & 0 & 0 & 0 & 0 & 0 & 0 & $3.59 \mathrm{E}+04$ & 0 & 0 & 0 & 0 & $3.76 \mathrm{E}+04$ & $1.11 \mathrm{E}+06$ & $3.38 \%$ \\
\hline 10EA034 & $9.65 \mathrm{E}+02$ & 0 & 0 & 0 & 0 & $9.43 \mathrm{E}+03$ & 0 & $1.33 \mathrm{E}+05$ & 0 & 0 & 0 & 0 & $1.43 \mathrm{E}+05$ & $1.00 \mathrm{E}+06$ & $14.33 \%$ \\
\hline 10EA035 & $7.27 \mathrm{E}+02$ & 0 & 0 & 0 & 0 & $7.54 \mathrm{E}+03$ & 0 & 0 & $4.42 \mathrm{E}+02$ & 0 & 0 & 0 & $9.16 \mathrm{E}+03$ & $1.06 \mathrm{E}+06$ & $0.86 \%$ \\
\hline 10EA036 & $1.58 \mathrm{E}+03$ & 0 & 7.12E-05 & 0 & 0 & $1.09 \mathrm{E}+04$ & 0 & 0 & $5.22 \mathrm{E}+03$ & 0 & 0 & 0 & $1.82 \mathrm{E}+04$ & $1.10 \mathrm{E}+06$ & $1.65 \%$ \\
\hline 10EA037 & $1.39 \mathrm{E}+03$ & 0 & $3.09 \mathrm{E}-05$ & 0 & 0 & 0 & 0 & 0 & 0 & 0 & 0 & 0 & $1.90 \mathrm{E}+03$ & $1.15 \mathrm{E}+06$ & $0.17 \%$ \\
\hline 10EA038 & $1.15 \mathrm{E}+03$ & 0 & 0 & 0 & 0 & 0 & 0 & $1.20 \mathrm{E}+05$ & $2.02 \mathrm{E}+04$ & 0 & 0 & 0 & $1.42 \mathrm{E}+05$ & $1.06 \mathrm{E}+06$ & $13.45 \%$ \\
\hline 10EA039 & $9.21 \mathrm{E}+02$ & 0 & 0 & 0 & 0 & 0 & 0 & 0 & $1.52 \mathrm{E}+04$ & 0 & 0 & 0 & $1.66 \mathrm{E}+04$ & $1.13 \mathrm{E}+06$ & $1.47 \%$ \\
\hline 10EA040 & $6.85 \mathrm{E}+02$ & 0 & 0 & 0 & 0 & $7.65 \mathrm{E}+03$ & 0 & 0 & 0 & 0 & 0 & 0 & $8.80 \mathrm{E}+03$ & $1.09 \mathrm{E}+06$ & $0.81 \%$ \\
\hline 10EA041 & $2.35 \mathrm{E}+02$ & 0 & 0 & 0 & $1.19 \mathrm{E}+04$ & 0 & 0 & 0 & 0 & 0 & 0 & 0 & $1.21 \mathrm{E}+04$ & $9.90 \mathrm{E}+05$ & $1.22 \%$ \\
\hline 10EA042 & $2.22 \mathrm{E}+02$ & 0 & 0 & 0 & $1.19 \mathrm{E}+04$ & 0 & 0 & 0 & 0 & 0 & 0 & 0 & $1.22 \mathrm{E}+04$ & $1.01 \mathrm{E}+06$ & $1.21 \%$ \\
\hline 10EA043 & $5.79 \mathrm{E}+02$ & 0 & 0 & 0 & $1.46 \mathrm{E}+03$ & 0 & 0 & 0 & 0 & 0 & 0 & 0 & $2.04 \mathrm{E}+03$ & $1.03 \mathrm{E}+06$ & $0.20 \%$ \\
\hline 10EA044 & $5.24 \mathrm{E}+02$ & 0 & 0 & 0 & $3.26 \mathrm{E}+03$ & $7.62 \mathrm{E}+03$ & 0 & 0 & 0 & 0 & 0 & 0 & $1.14 \mathrm{E}+04$ & $1.00 \mathrm{E}+06$ & $1.14 \%$ \\
\hline 10EA045 & $4.18 \mathrm{E}+02$ & 0 & 0 & 0 & $3.33 \mathrm{E}+03$ & $6.20 \mathrm{E}+03$ & 0 & 0 & 0 & 0 & 0 & 0 & $9.95 \mathrm{E}+03$ & $9.68 \mathrm{E}+05$ & $1.03 \%$ \\
\hline 10EA046 & $1.03 \mathrm{E}+03$ & $4.22 \mathrm{E}+02$ & 0 & 0 & $1.39 \mathrm{E}+04$ & $9.85 \mathrm{E}+03$ & 0 & 0 & 0 & 0 & 0 & 0 & $2.52 \mathrm{E}+04$ & $9.86 \mathrm{E}+05$ & $2.55 \%$ \\
\hline 10EA047 & $1.02 \mathrm{E}+03$ & $3.10 \mathrm{E}+04$ & 0 & 0 & $1.45 \mathrm{E}+04$ & 0 & 0 & 0 & 0 & 0 & 0 & 0 & $4.66 \mathrm{E}+04$ & $9.86 \mathrm{E}+05$ & $4.72 \%$ \\
\hline 10EA048 & $6.45 \mathrm{E}+02$ & 0 & 0 & 0 & $5.50 \mathrm{E}+03$ & 0 & 0 & 0 & 0 & 0 & 0 & 0 & $6.15 \mathrm{E}+03$ & $1.04 \mathrm{E}+06$ & $0.59 \%$ \\
\hline 10EA049 & $5.72 \mathrm{E}+02$ & 0 & 0 & 0 & $7.97 \mathrm{E}+03$ & 0 & 0 & 0 & 0 & 0 & 0 & 0 & $8.54 \mathrm{E}+03$ & $1.02 \mathrm{E}+06$ & $0.83 \%$ \\
\hline 10EA050 & $4.43 \mathrm{E}+02$ & 0 & 0 & 0 & $5.30 \mathrm{E}+03$ & $6.35 \mathrm{E}+03$ & 0 & 0 & 0 & 0 & 0 & 0 & $1.21 \mathrm{E}+04$ & $9.94 \mathrm{E}+05$ & $1.22 \%$ \\
\hline 10EA051 & $2.35 \mathrm{E}+02$ & 0 & 0 & 0 & $1.19 \mathrm{E}+04$ & 0 & 0 & 0 & 0 & 0 & 0 & 0 & $1.21 \mathrm{E}+04$ & $9.90 \mathrm{E}+05$ & $1.22 \%$ \\
\hline 10EA052 & $2.22 \mathrm{E}+02$ & 0 & 0 & 0 & $1.19 \mathrm{E}+04$ & 0 & 0 & 0 & 0 & 0 & 0 & 0 & $1.22 \mathrm{E}+04$ & $1.01 \mathrm{E}+06$ & $1.21 \%$ \\
\hline 10EA053 & $5.79 E+02$ & 0 & 0 & 0 & $1.46 \mathrm{E}+03$ & 0 & 0 & 0 & 0 & 0 & 0 & 0 & $2.04 \mathrm{E}+03$ & $1.03 \mathrm{E}+06$ & $0.20 \%$ \\
\hline 10EA054 & $5.24 \mathrm{E}+02$ & 0 & 0 & 0 & $3.26 \mathrm{E}+03$ & $7.62 \mathrm{E}+03$ & 0 & 0 & 0 & 0 & 0 & 0 & $1.14 \mathrm{E}+04$ & $1.00 \mathrm{E}+06$ & $1.14 \%$ \\
\hline 10EA055 & $4.18 \mathrm{E}+02$ & 0 & 0 & 0 & $3.33 \mathrm{E}+03$ & $6.20 \mathrm{E}+03$ & 0 & 0 & 0 & 0 & 0 & 0 & $9.95 \mathrm{E}+03$ & $9.68 \mathrm{E}+05$ & $1.03 \%$ \\
\hline 10EA056 & $1.03 \mathrm{E}+03$ & $4.22 \mathrm{E}+02$ & 0 & 0 & $1.39 \mathrm{E}+04$ & $9.85 \mathrm{E}+03$ & 0 & 0 & 0 & 0 & 0 & 0 & $2.52 \mathrm{E}+04$ & $9.86 \mathrm{E}+05$ & $2.55 \%$ \\
\hline 10EA057 & $1.02 \mathrm{E}+03$ & $3.10 \mathrm{E}+04$ & 0 & 0 & $1.45 \mathrm{E}+04$ & 0 & 0 & 0 & 0 & 0 & 0 & 0 & $4.66 \mathrm{E}+04$ & $9.86 \mathrm{E}+05$ & $4.72 \%$ \\
\hline 10EA058 & $6.45 \mathrm{E}+02$ & 0 & 0 & 0 & $5.50 \mathrm{E}+03$ & 0 & 0 & 0 & 0 & 0 & 0 & 0 & $6.15 \mathrm{E}+03$ & $1.04 \mathrm{E}+06$ & $0.59 \%$ \\
\hline 10EA059 & $5.72 \mathrm{E}+02$ & 0 & 0 & 0 & $7.97 \mathrm{E}+03$ & 0 & 0 & 0 & 0 & 0 & 0 & 0 & $8.54 \mathrm{E}+03$ & $1.02 \mathrm{E}+06$ & $0.83 \%$ \\
\hline 10EA060 & $4.43 \mathrm{E}+02$ & 0 & 0 & 0 & $5.30 \mathrm{E}+03$ & $6.35 \mathrm{E}+03$ & 0 & 0 & 0 & 0 & 0 & 0 & $1.21 \mathrm{E}+04$ & $9.94 \mathrm{E}+05$ & $1.22 \%$ \\
\hline 10EA061 & $2.14 \mathrm{E}+02$ & 0 & 0 & 0 & $1.39 \mathrm{E}+04$ & 0 & 0 & 0 & 0 & 0 & 0 & 0 & $1.45 \mathrm{E}+04$ & $1.02 \mathrm{E}+06$ & $1.42 \%$ \\
\hline 10EA062 & $2.02 \mathrm{E}+02$ & 0 & 0 & 0 & $1.40 \mathrm{E}+04$ & 0 & 0 & 0 & 0 & 0 & 0 & 0 & $1.46 \mathrm{E}+04$ & $1.04 \mathrm{E}+06$ & $1.41 \%$ \\
\hline
\end{tabular}


Table 36. Predicted Solids for Envelope A Simulations - Part 2 (cont'd)

\begin{tabular}{|c|c|c|c|c|c|c|c|c|c|c|c|c|c|c|c|}
\hline Test ID & $\begin{array}{c}\text { NA2C } 2 \mathrm{O} \\
4 \\
\end{array}$ & $\begin{array}{c}\text { NA2CO3. } \\
1 \mathrm{H} 2 \mathrm{O}\end{array}$ & $\begin{array}{c}\text { NA2U2O } \\
7 \\
\end{array}$ & NA3FSO4 & $\begin{array}{c}\mathrm{NA} 6 \mathrm{SO} 42 \\
\mathrm{CO} 3\end{array}$ & NAF & $\begin{array}{c}\text { NAFPO4. } \\
19 \mathrm{H} 2 \mathrm{O}\end{array}$ & NANO3 & $\begin{array}{c}\text { NAPHOH } \\
.12 \mathrm{H} 2 \mathrm{O} \\
\end{array}$ & $\begin{array}{c}\text { NASGEL. } \\
15.5 \mathrm{H} 2 \mathrm{O} \\
\end{array}$ & $\mathrm{ZRO} 2$ & $\begin{array}{c}\text { ZRSO42. } \\
4 \mathrm{H} 2 \mathrm{O}\end{array}$ & $\begin{array}{c}\text { Total } \\
\text { Solids } \\
\end{array}$ & $\begin{array}{c}\text { Total Soln } \\
\text { Mass } \\
\end{array}$ & $\begin{array}{c}\text { Solids \% } \\
\text { of Total } \\
\text { Soln } \\
\end{array}$ \\
\hline 10EA063 & $4.94 \mathrm{E}+02$ & 0 & 0 & 0 & $2.51 \mathrm{E}+03$ & 0 & 0 & 0 & 0 & 0 & $2.76 \mathrm{E}+00$ & 0 & $3.50 \mathrm{E}+03$ & $1.08 \mathrm{E}+06$ & $0.33 \%$ \\
\hline 10EA064 & $4.71 \mathrm{E}+02$ & 0 & 0 & 0 & $4.97 \mathrm{E}+03$ & $7.85 \mathrm{E}+03$ & 0 & 0 & 0 & 0 & $1.52 \mathrm{E}+00$ & 0 & $1.38 \mathrm{E}+04$ & $1.04 \mathrm{E}+06$ & $1.33 \%$ \\
\hline 10EA065 & $3.81 \mathrm{E}+02$ & 0 & 0 & 0 & $5.42 \mathrm{E}+03$ & $6.44 \mathrm{E}+03$ & 0 & 0 & 0 & 0 & 7.97E-01 & 0 & $1.27 \mathrm{E}+04$ & $1.00 \mathrm{E}+06$ & $1.26 \%$ \\
\hline 10EA066 & $9.69 \mathrm{E}+02$ & 0 & 0 & 0 & $1.75 \mathrm{E}+04$ & $9.69 \mathrm{E}+03$ & 0 & 0 & 0 & 0 & $2.35 \mathrm{E}+00$ & 0 & $2.87 \mathrm{E}+04$ & $1.02 \mathrm{E}+06$ & $2.80 \%$ \\
\hline 10EA067 & $9.31 \mathrm{E}+02$ & $1.69 \mathrm{E}+04$ & 0 & 0 & $1.82 \mathrm{E}+04$ & 0 & 0 & 0 & 0 & 0 & $1.68 \mathrm{E}+00$ & 0 & $3.64 \mathrm{E}+04$ & $1.04 \mathrm{E}+06$ & $3.50 \%$ \\
\hline 10EA068 & $5.84 \mathrm{E}+02$ & 0 & 0 & 0 & $7.05 \mathrm{E}+03$ & 0 & 0 & 0 & 0 & 0 & $1.89 \mathrm{E}+00$ & 0 & $8.28 \mathrm{E}+03$ & $1.08 \mathrm{E}+06$ & $0.77 \%$ \\
\hline 10EA069 & $5.28 \mathrm{E}+02$ & 0 & 0 & 0 & $9.77 \mathrm{E}+03$ & 0 & 0 & 0 & 0 & 0 & $1.29 \mathrm{E}+00$ & 0 & $1.09 \mathrm{E}+04$ & $1.06 \mathrm{E}+06$ & $1.03 \%$ \\
\hline 10EA070 & $4.14 \mathrm{E}+02$ & 0 & 0 & 0 & \begin{tabular}{|l|}
$7.76 \mathrm{E}+03$ \\
\end{tabular} & $6.63 \mathrm{E}+03$ & 0 & 0 & 0 & 0 & $1.33 \mathrm{E}-01$ & 0 & $1.53 \mathrm{E}+04$ & $1.03 \mathrm{E}+06$ & $1.48 \%$ \\
\hline 10EA071 & $2.14 \mathrm{E}+02$ & 0 & 0 & 0 & $1.39 \mathrm{E}+04$ & 0 & 0 & 0 & 0 & 0 & 0 & 0 & $1.45 \mathrm{E}+04$ & $1.02 \mathrm{E}+06$ & $1.42 \%$ \\
\hline 10EA072 & $2.02 \mathrm{E}+02$ & 0 & 0 & 0 & $1.40 \mathrm{E}+04$ & 0 & 0 & 0 & 0 & 0 & 0 & 0 & $1.46 \mathrm{E}+04$ & $1.04 \mathrm{E}+06$ & $1.41 \%$ \\
\hline 10EA073 & $4.94 \mathrm{E}+02$ & 0 & 0 & 0 & $2.51 \mathrm{E}+03$ & 0 & 0 & 0 & 0 & 0 & $2.76 \mathrm{E}+00$ & 0 & $3.50 \mathrm{E}+03$ & $1.08 \mathrm{E}+06$ & $0.33 \%$ \\
\hline 10EA074 & $4.71 \mathrm{E}+02$ & 0 & 0 & 0 & $4.97 \mathrm{E}+03$ & $7.85 \mathrm{E}+03$ & 0 & 0 & 0 & 0 & $1.52 \mathrm{E}+00$ & 0 & $1.38 \mathrm{E}+04$ & $1.04 \mathrm{E}+06$ & $1.33 \%$ \\
\hline 10EA075 & $3.81 \mathrm{E}+02$ & 0 & 0 & 0 & $5.42 \mathrm{E}+03$ & $6.44 \mathrm{E}+03$ & 0 & 0 & 0 & 0 & 7.97E-01 & 0 & $1.27 \mathrm{E}+04$ & $1.00 \mathrm{E}+06$ & $1.26 \%$ \\
\hline 10EA076 & $9.69 \mathrm{E}+02$ & 0 & 0 & 0 & $1.75 \mathrm{E}+04$ & $9.69 \mathrm{E}+03$ & 0 & 0 & 0 & 0 & $2.35 \mathrm{E}+00$ & 0 & $2.87 \mathrm{E}+04$ & $1.02 \mathrm{E}+06$ & $2.80 \%$ \\
\hline 10EA077 & $9.31 \mathrm{E}+02$ & $1.69 \mathrm{E}+04$ & 0 & 0 & $1.82 \mathrm{E}+04$ & 0 & 0 & 0 & 0 & 0 & $1.68 \mathrm{E}+00$ & 0 & $3.64 \mathrm{E}+04$ & $1.04 \mathrm{E}+06$ & $3.50 \%$ \\
\hline 10EA078 & $5.84 \mathrm{E}+02$ & 0 & 0 & 0 & $7.05 \mathrm{E}+03$ & 0 & 0 & 0 & 0 & 0 & $1.89 \mathrm{E}+00$ & 0 & $8.28 \mathrm{E}+03$ & $1.08 \mathrm{E}+06$ & $0.77 \%$ \\
\hline 10EA079 & $5.28 \mathrm{E}+02$ & 0 & 0 & 0 & $9.77 \mathrm{E}+03$ & 0 & 0 & 0 & 0 & 0 & $1.29 \mathrm{E}+00$ & 0 & $1.09 \mathrm{E}+04$ & $1.06 \mathrm{E}+06$ & $1.03 \%$ \\
\hline 10EA080 & $4.14 \mathrm{E}+02$ & 0 & 0 & 0 & $7.76 \mathrm{E}+03$ & $6.63 \mathrm{E}+03$ & 0 & 0 & 0 & 0 & 1.33E-01 & 0 & $1.53 \mathrm{E}+04$ & $1.03 \mathrm{E}+06$ & $1.48 \%$ \\
\hline 10EA081 & $1.19 \mathrm{E}+03$ & 0 & $4.22 \mathrm{E}-06$ & 0 & 0 & $9.61 \mathrm{E}+03$ & 0 & 0 & $3.96 \mathrm{E}+03$ & 0 & 0 & 0 & $1.48 \mathrm{E}+04$ & $1.05 \mathrm{E}+06$ & $1.41 \%$ \\
\hline 10EA082 & $9.98 \mathrm{E}+02$ & 0 & 0 & 0 & 0 & $9.27 \mathrm{E}+03$ & 0 & 0 & $1.17 \mathrm{E}+03$ & 0 & 0 & 0 & $1.19 \mathrm{E}+04$ & $1.09 \mathrm{E}+06$ & $1.09 \%$ \\
\hline 10EA083 & $1.24 \mathrm{E}+03$ & 0 & $1.43 \mathrm{E}-07$ & 0 & 0 & $9.78 \mathrm{E}+03$ & 0 & $9.34 \mathrm{E}+04$ & $8.16 \mathrm{E}+02$ & 0 & 0 & 0 & $1.05 \mathrm{E}+05$ & $1.03 \mathrm{E}+06$ & $10.29 \%$ \\
\hline 10EA084 & $1.13 \mathrm{E}+03$ & 0 & 0 & 0 & 0 & $9.98 \mathrm{E}+03$ & 0 & $7.81 \mathrm{E}+04$ & $2.55 \mathrm{E}+03$ & 0 & 0 & 0 & $9.18 \mathrm{E}+04$ & $1.01 \mathrm{E}+06$ & $9.08 \%$ \\
\hline 10EA085 & $1.26 \mathrm{E}+03$ & 0 & 4.49E-06 & 0 & 0 & $9.96 \mathrm{E}+03$ & 0 & 0 & $2.69 \mathrm{E}+03$ & 0 & 0 & 0 & $1.39 \mathrm{E}+04$ & $1.05 \mathrm{E}+06$ & $1.32 \%$ \\
\hline 10EA086 & $1.15 \mathrm{E}+03$ & 0 & $1.94 \mathrm{E}-06$ & 0 & 0 & $5.38 \mathrm{E}+03$ & 0 & 0 & 0 & 0 & 0 & 0 & $6.83 \mathrm{E}+03$ & $1.11 \mathrm{E}+06$ & $0.62 \%$ \\
\hline 10EA087 & $1.29 \mathrm{E}+03$ & 0 & $8.92 \mathrm{E}-06$ & 0 & 0 & $2.14 \mathrm{E}+01$ & $1.18 \mathrm{E}+04$ & 0 & 0 & 0 & 0 & 0 & $1.35 \mathrm{E}+04$ & $1.12 \mathrm{E}+06$ & $1.20 \%$ \\
\hline 10EA088 & $1.34 \mathrm{E}+03$ & 0 & $1.42 \mathrm{E}-05$ & 0 & 0 & $1.02 \mathrm{E}+04$ & $3.08 \mathrm{E}+03$ & 0 & 0 & 0 & 0 & 0 & $1.48 \mathrm{E}+04$ & $1.08 \mathrm{E}+06$ & $1.37 \%$ \\
\hline 10EA089 & $8.15 \mathrm{E}+02$ & 0 & 0 & 0 & 0 & $8.24 \mathrm{E}+03$ & $6.17 \mathrm{E}+02$ & 0 & 0 & 0 & 0 & 0 & $9.95 \mathrm{E}+03$ & $1.07 \mathrm{E}+06$ & $0.93 \%$ \\
\hline 10EA090 & $9.26 \mathrm{E}+02$ & 0 & 0 & 0 & 0 & $6.29 \mathrm{E}+03$ & $1.14 \mathrm{E}+03$ & 0 & 0 & 0 & 0 & 0 & $8.50 \mathrm{E}+03$ & $1.08 \mathrm{E}+06$ & $0.79 \%$ \\
\hline 10EA091 & $9.98 \mathrm{E}+02$ & 0 & 0 & 0 & 0 & $4.62 \mathrm{E}+03$ & $8.68 \mathrm{E}+02$ & 0 & 0 & 0 & 0 & 0 & $6.69 \mathrm{E}+03$ & $1.09 \mathrm{E}+06$ & $0.61 \%$ \\
\hline 10EA092 & $1.07 \mathrm{E}+03$ & 0 & 0 & 0 & 0 & $2.33 \mathrm{E}+03$ & $2.03 \mathrm{E}+03$ & 0 & 0 & 0 & 0 & 0 & $5.48 \mathrm{E}+03$ & $1.09 \mathrm{E}+06$ & $0.50 \%$ \\
\hline 10EA093 & $1.20 \mathrm{E}+03$ & 0 & $2.59 \mathrm{E}-06$ & 0 & 0 & $2.41 \mathrm{E}+03$ & $6.55 \mathrm{E}+03$ & 0 & 0 & 0 & 0 & 0 & $1.04 \mathrm{E}+04$ & $1.09 \mathrm{E}+06$ & $0.95 \%$ \\
\hline 10EA094 & $1.28 \mathrm{E}+03$ & 0 & $3.50 \mathrm{E}-06$ & 0 & 0 & $6.42 \mathrm{E}+02$ & 0 & $5.52 \mathrm{E}+03$ & 0 & 0 & 0 & 0 & $7.58 \mathrm{E}+03$ & $1.10 \mathrm{E}+06$ & $0.69 \%$ \\
\hline 10EA095 & $1.00 \mathrm{E}+03$ & 0 & 0 & 0 & 0 & $9.17 \mathrm{E}+03$ & $7.47 \mathrm{E}+02$ & 0 & 0 & 0 & 0 & 0 & $1.12 \mathrm{E}+04$ & $1.07 \mathrm{E}+06$ & $1.05 \%$ \\
\hline
\end{tabular}


Table 36. Predicted Solids for Envelope A Simulations - Part 2 (cont'd)

\begin{tabular}{|c|c|c|c|c|c|c|c|c|c|c|c|c|c|c|c|}
\hline Test ID & $\begin{array}{c}\mathrm{NA} 2 \mathrm{C} 2 \mathrm{O} \\
4\end{array}$ & $\begin{array}{c}\mathrm{NA} 2 \mathrm{CO} 3 . \\
1 \mathrm{H} 2 \mathrm{O}\end{array}$ & $\begin{array}{c}\text { NA2U2O } \\
7\end{array}$ & NA3FSO4 & $\begin{array}{c}\mathrm{NA} 6 \mathrm{SO} 42 \\
\mathrm{CO} 3\end{array}$ & NAF & $\begin{array}{c}\text { NAFPO4. } \\
19 \mathrm{H} 2 \mathrm{O}\end{array}$ & NANO3 & $\begin{array}{c}\text { NAPHOH } \\
.12 \mathrm{H} 2 \mathrm{O}\end{array}$ & $\begin{array}{c}\text { NASGEL. } \\
15.5 \mathrm{H} 2 \mathrm{O}\end{array}$ & ZRO2 & $\begin{array}{c}\text { ZRSO42. } \\
4 \mathrm{H} 2 \mathrm{O}\end{array}$ & $\begin{array}{l}\text { Total } \\
\text { Solids }\end{array}$ & $\begin{array}{c}\text { Total Soln } \\
\text { Mass }\end{array}$ & $\begin{array}{c}\text { Solids } \% \\
\text { of Total } \\
\text { Soln }\end{array}$ \\
\hline 10EA096 & $1.12 \mathrm{E}+03$ & 0 & $1.45 \mathrm{E}-06$ & $9.41 \mathrm{E}+03$ & 0 & 0 & 0 & 0 & 0 & 0 & 0 & 0 & $1.10 \mathrm{E}+04$ & $1.12 \mathrm{E}+06$ & $0.98 \%$ \\
\hline 10EA097 & $1.18 \mathrm{E}+03$ & 0 & $4.38 \mathrm{E}-06$ & $1.36 \mathrm{E}+04$ & 0 & 0 & $2.22 \mathrm{E}+02$ & 0 & 0 & 0 & 0 & 0 & $1.50 \mathrm{E}+04$ & $1.07 \mathrm{E}+06$ & $1.40 \%$ \\
\hline 10EA098 & $8.23 \mathrm{E}+02$ & 0 & 0 & $1.05 \mathrm{E}+04$ & 0 & 0 & 0 & $4.02 \mathrm{E}+04$ & $2.90 \mathrm{E}+03$ & 0 & 0 & 0 & $5.48 \mathrm{E}+04$ & $1.06 \mathrm{E}+06$ & $5.16 \%$ \\
\hline 10EA099 & $1.14 \mathrm{E}+03$ & 0 & $2.86 \mathrm{E}-06$ & $1.36 \mathrm{E}+04$ & 0 & $6.23 \mathrm{E}+03$ & $3.09 \mathrm{E}+03$ & 0 & 0 & 0 & 0 & 0 & $2.41 \mathrm{E}+04$ & $1.04 \mathrm{E}+06$ & $2.32 \%$ \\
\hline 10EA100 & $1.09 \mathrm{E}+03$ & 0 & $4.24 \mathrm{E}-07$ & $6.89 \mathrm{E}+03$ & 0 & 0 & 0 & 0 & 0 & 0 & 0 & 0 & $8.38 \mathrm{E}+03$ & $\mid 1.11 \mathrm{E}+06$ & $0.75 \%$ \\
\hline 10EA101 & $1.16 \mathrm{E}+03$ & 0 & $2.05 \mathrm{E}-07$ & $1.61 \mathrm{E}+04$ & 0 & 0 & $4.94 \mathrm{E}+03$ & $2.27 \mathrm{E}+04$ & 0 & 0 & 0 & 0 & $4.51 \mathrm{E}+04$ & $1.05 \mathrm{E}+06$ & $4.28 \%$ \\
\hline 10EA102 & $1.27 \mathrm{E}+03$ & 0 & $6.73 \mathrm{E}-06$ & $1.65 \mathrm{E}+04$ & 0 & $6.06 \mathrm{E}+03$ & 0 & 0 & 0 & 0 & 0 & 0 & $2.41 \mathrm{E}+04$ & $1.07 \mathrm{E}+06$ & $2.24 \%$ \\
\hline 10EA103 & $9.74 \mathrm{E}+02$ & 0 & 0 & $1.43 \mathrm{E}+04$ & 0 & 0 & $8.32 \mathrm{E}+01$ & 0 & 0 & 0 & 0 & 0 & $1.56 \mathrm{E}+04$ & $1.08 \mathrm{E}+06$ & $1.45 \%$ \\
\hline 10EA104 & $1.09 \mathrm{E}+03$ & 0 & $1.24 \mathrm{E}-06$ & $\mid 1.68 \mathrm{E}+04$ & 0 & $2.71 \mathrm{E}+03$ & $3.41 \mathrm{E}+03$ & 0 & 0 & 0 & 0 & 0 & $2.43 \mathrm{E}+04$ & $\mid 1.08 \mathrm{E}+06$ & $2.26 \%$ \\
\hline 10EA105 & $9.40 \mathrm{E}+02$ & 0 & 0 & $1.26 \mathrm{E}+04$ & 0 & 0 & 0 & 0 & 0 & 0 & 0 & 0 & $1.37 \mathrm{E}+04$ & $1.07 \mathrm{E}+06$ & $1.27 \%$ \\
\hline 10EA106 & $1.14 \mathrm{E}+03$ & 0 & 5.43E-07 & $1.60 \mathrm{E}+04$ & 0 & $6.32 \mathrm{E}+03$ & $1.28 \mathrm{E}+03$ & 0 & 0 & 0 & 0 & 0 & $2.51 \mathrm{E}+04$ & $1.06 \mathrm{E}+06$ & $2.36 \%$ \\
\hline 10EA107 & $8.64 \mathrm{E}+02$ & 0 & 0 & $4.57 \mathrm{E}+03$ & 0 & 0 & 0 & 0 & 0 & 0 & 0 & 0 & $5.44 \mathrm{E}+03$ & $1.07 \mathrm{E}+06$ & $0.51 \%$ \\
\hline 10EA108 & $1.04 \mathrm{E}+03$ & 0 & 0 & $1.52 \mathrm{E}+04$ & 0 & $6.00 \mathrm{E}+03$ & $9.49 \mathrm{E}+02$ & 0 & 0 & 0 & 0 & 0 & $2.36 \mathrm{E}+04$ & $1.05 \mathrm{E}+06$ & $2.25 \%$ \\
\hline 10EA109 & $1.00 \mathrm{E}+03$ & 0 & 0 & $9.67 \mathrm{E}+03$ & 0 & 0 & 0 & 0 & 0 & 0 & 0 & 0 & $1.09 \mathrm{E}+04$ & $1.09 \mathrm{E}+06$ & $1.00 \%$ \\
\hline 10EA110 & $9.07 \mathrm{E}+02$ & 0 & 0 & $1.71 \mathrm{E}+04$ & 0 & $2.37 \mathrm{E}+03$ & 0 & 0 & 0 & 0 & 0 & 0 & $2.07 \mathrm{E}+04$ & $1.08 \mathrm{E}+06$ & $1.92 \%$ \\
\hline 10EA111 & $1.15 \mathrm{E}+03$ & 0 & 0 & $7.99 \mathrm{E}+03$ & 0 & 0 & 0 & 0 & 0 & 0 & 0 & 0 & $9.41 \mathrm{E}+03$ & $1.09 \mathrm{E}+06$ & $0.86 \%$ \\
\hline 10EA112 & $1.08 \mathrm{E}+03$ & 0 & $3.15 \mathrm{E}-07$ & $1.49 \mathrm{E}+04$ & 0 & 0 & $3.64 \mathrm{E}+03$ & 0 & 0 & 0 & 0 & 0 & $1.98 \mathrm{E}+04$ & $\mid 1.07 \mathrm{E}+06$ & $1.86 \%$ \\
\hline 10EA113 & $1.08 \mathrm{E}+03$ & 0 & $1.49 \mathrm{E}-06$ & $1.52 \mathrm{E}+04$ & 0 & 0 & $4.12 \mathrm{E}+03$ & 0 & 0 & 0 & 0 & 0 & $2.06 \mathrm{E}+04$ & $\mid 1.06 \mathrm{E}+06$ & $1.94 \%$ \\
\hline 10EA114 & $1.16 \mathrm{E}+03$ & 0 & $9.66 \mathrm{E}-07$ & $8.99 \mathrm{E}+03$ & 0 & 0 & 0 & 0 & 0 & 0 & 0 & 0 & $1.03 \mathrm{E}+04$ & $1.08 \mathrm{E}+06$ & $0.96 \%$ \\
\hline 10EA115 & $9.54 \mathrm{E}+02$ & 0 & 0 & $1.47 \mathrm{E}+04$ & 0 & $5.89 \mathrm{E}+03$ & 0 & 0 & 0 & 0 & 0 & 0 & $2.18 \mathrm{E}+04$ & $1.07 \mathrm{E}+06$ & $2.05 \%$ \\
\hline 10EA116 & $1.11 \mathrm{E}+03$ & 0 & 1.48E-06 & $1.64 \mathrm{E}+04$ & 0 & $3.26 \mathrm{E}+03$ & 0 & 0 & 0 & 0 & 0 & 0 & $2.10 \mathrm{E}+04$ & $1.07 \mathrm{E}+06$ & $1.97 \%$ \\
\hline 10EA117 & $1.07 \mathrm{E}+03$ & 0 & 0 & $1.78 \mathrm{E}+04$ & 0 & $3.81 \mathrm{E}+03$ & $9.42 \mathrm{E}+02$ & 0 & 0 & 0 & 0 & 0 & $2.40 \mathrm{E}+04$ & $1.08 \mathrm{E}+06$ & $2.23 \%$ \\
\hline 10EA118 & $9.35 \mathrm{E}+02$ & 0 & 9.35E-07 & $1.33 \mathrm{E}+04$ & 0 & $5.17 \mathrm{E}+03$ & $7.26 \mathrm{E}+02$ & 0 & 0 & 0 & 0 & 0 & $2.03 \mathrm{E}+04$ & $1.05 \mathrm{E}+06$ & $1.93 \%$ \\
\hline 10EA119 & $6.77 \mathrm{E}+02$ & 0 & 0 & $1.42 \mathrm{E}+04$ & 0 & $7.14 \mathrm{E}+02$ & $1.75 \mathrm{E}+03$ & 0 & 0 & 0 & 0 & 0 & $1.75 \mathrm{E}+04$ & $1.04 \mathrm{E}+06$ & $1.68 \%$ \\
\hline 10EA120 & $1.03 \mathrm{E}+03$ & 0 & 0 & $1.54 \mathrm{E}+04$ & 0 & $6.10 \mathrm{E}+03$ & 0 & 0 & 0 & 0 & 0 & 0 & $2.28 \mathrm{E}+04$ & $1.06 \mathrm{E}+06$ & $2.15 \%$ \\
\hline 10EA121 & $1.09 \mathrm{E}+03$ & 0 & $5.38 \mathrm{E}-07$ & $1.06 \mathrm{E}+04$ & $1.30 \mathrm{E}+03$ & 0 & 0 & 0 & 0 & 0 & 0 & 0 & $1.34 \mathrm{E}+04$ & $1.09 \mathrm{E}+06$ & $1.23 \%$ \\
\hline 10EA122 & $1.26 \mathrm{E}+03$ & 0 & $1.50 \mathrm{E}-05$ & $1.50 \mathrm{E}+04$ & 0 & $3.12 \mathrm{E}+02$ & $9.56 \mathrm{E}+02$ & 0 & 0 & 0 & 0 & 0 & $1.75 \mathrm{E}+04$ & $\mid 1.06 \mathrm{E}+06$ & $1.66 \%$ \\
\hline 10EA123 & $8.96 \mathrm{E}+02$ & 0 & 0 & $8.47 \mathrm{E}+03$ & $3.05 \mathrm{E}+03$ & 0 & 0 & 0 & 0 & 0 & 0 & 0 & $1.26 \mathrm{E}+04$ & $1.07 \mathrm{E}+06$ & $1.17 \%$ \\
\hline 10EA124 & $1.16 \mathrm{E}+03$ & 0 & $6.15 \mathrm{E}-06$ & $1.71 \mathrm{E}+04$ & 0 & $9.41 \mathrm{E}+02$ & $4.35 \mathrm{E}+03$ & 0 & 0 & 0 & 0 & 0 & $2.40 \mathrm{E}+04$ & $1.06 \mathrm{E}+06$ & $2.25 \%$ \\
\hline 10EA125 & $7.17 \mathrm{E}+02$ & 0 & 0 & $1.03 \mathrm{E}+04$ & 0 & $5.13 \mathrm{E}+03$ & 0 & $6.53 \mathrm{E}+03$ & 0 & 0 & 0 & 0 & $2.28 \mathrm{E}+04$ & $1.02 \mathrm{E}+06$ & $2.24 \%$ \\
\hline 10EA126 & $8.91 \mathrm{E}+02$ & 0 & 0 & $1.58 \mathrm{E}+04$ & 0 & $2.28 \mathrm{E}+03$ & $7.51 \mathrm{E}+02$ & 0 & 0 & 0 & 0 & 0 & $1.98 \mathrm{E}+04$ & $1.05 \mathrm{E}+06$ & $1.90 \%$ \\
\hline 10EA127 & $7.82 \mathrm{E}+02$ & 0 & 0 & $1.67 \mathrm{E}+04$ & 0 & $2.35 \mathrm{E}+03$ & $9.77 \mathrm{E}+02$ & 0 & 0 & 0 & 0 & 0 & $2.12 \mathrm{E}+04$ & $1.08 \mathrm{E}+06$ & $1.98 \%$ \\
\hline 10EA128 & $1.08 \mathrm{E}+03$ & 0 & $6.50 \mathrm{E}-07$ & $1.49 \mathrm{E}+04$ & 0 & $2.57 \mathrm{E}+03$ & $1.83 \mathrm{E}+03$ & 0 & 0 & 0 & 0 & 0 & $2.04 \mathrm{E}+04$ & $1.04 \mathrm{E}+06$ & $1.96 \%$ \\
\hline
\end{tabular}


Table 36. Predicted Solids for Envelope A Simulations - Part 2 (cont'd)

\begin{tabular}{|c|c|c|c|c|c|c|c|c|c|c|c|c|c|c|c|}
\hline Test ID & $\begin{array}{c}\mathrm{NA} 2 \mathrm{C} 2 \mathrm{O} \\
4 \\
\end{array}$ & $\begin{array}{c}\text { NA2CO3. } \\
1 \mathrm{H} 2 \mathrm{O}\end{array}$ & $\begin{array}{c}\text { NA2U2O } \\
7 \\
\end{array}$ & NA3FSO4 & \begin{tabular}{|c|}
$\mathrm{NA} 6 \mathrm{SO} 42$ \\
$\mathrm{CO} 3$ \\
\end{tabular} & NAF & $\begin{array}{c}\text { NAFPO4. } \\
19 \mathrm{H} 2 \mathrm{O}\end{array}$ & NANO3 & $\begin{array}{c}\text { NAPHOH } \\
.12 \mathrm{H} 2 \mathrm{O}\end{array}$ & $\begin{array}{c}\text { NASGEL. } \\
15.5 \mathrm{H} 2 \mathrm{O}\end{array}$ & ZRO2 & $\begin{array}{c}\text { ZRSO42. } \\
4 \mathrm{H} 2 \mathrm{O}\end{array}$ & $\begin{array}{c}\text { Total } \\
\text { Solids } \\
\end{array}$ & $\begin{array}{c}\text { Total Soln } \\
\text { Mass }\end{array}$ & $\begin{array}{c}\text { Solids \% } \\
\text { of Total } \\
\text { Soln }\end{array}$ \\
\hline 10EA129 & $7.23 \mathrm{E}+02$ & 0 & 0 & $1.73 \mathrm{E}+04$ & 0 & 0 & 0 & 0 & 0 & 0 & 0 & 0 & $1.84 \mathrm{E}+04$ & $1.08 \mathrm{E}+06$ & $1.71 \%$ \\
\hline 10EA130 & $7.61 \mathrm{E}+02$ & 0 & 0 & $1.51 \mathrm{E}+04$ & 0 & 0 & 0 & 0 & 0 & 0 & 0 & 0 & $1.63 \mathrm{E}+04$ & $1.08 \mathrm{E}+06$ & $1.50 \%$ \\
\hline 10EA131 & $1.04 \mathrm{E}+03$ & 0 & $1.16 \mathrm{E}-06$ & $1.71 \mathrm{E}+04$ & 0 & $3.20 \mathrm{E}+03$ & 0 & 0 & 0 & 0 & 0 & 0 & $2.17 \mathrm{E}+04$ & $1.06 \mathrm{E}+06$ & $2.04 \%$ \\
\hline 10EA132 & $8.70 \mathrm{E}+02$ & 0 & $3.54 \mathrm{E}-07$ & $1.50 \mathrm{E}+04$ & 0 & 0 & $1.39 \mathrm{E}+02$ & 0 & 0 & 0 & 0 & 0 & $1.61 \mathrm{E}+04$ & $1.03 \mathrm{E}+06$ & $1.56 \%$ \\
\hline 10EA133 & $6.21 \mathrm{E}+02$ & 0 & 0 & 0 & 7.79E+03 & $7.24 \mathrm{E}+03$ & 0 & 0 & 0 & 0 & 0 & 0 & $1.60 \mathrm{E}+04$ & $1.06 \mathrm{E}+06$ & $1.52 \%$ \\
\hline 10EA134 & $1.10 \mathrm{E}+03$ & 0 & $1.68 \mathrm{E}-06$ & 0 & $1.27 \mathrm{E}+04$ & $4.86 \mathrm{E}+03$ & 0 & 0 & 0 & 0 & 0 & 0 & $1.87 \mathrm{E}+04$ & $1.04 \mathrm{E}+06$ & $1.79 \%$ \\
\hline 10EA135 & $1.00 \mathrm{E}+03$ & $2.06 \mathrm{E}+04$ & $4.67 \mathrm{E}-06$ & 0 & $1.69 \mathrm{E}+04$ & $1.99 \mathrm{E}+03$ & $2.67 \mathrm{E}+03$ & 0 & 0 & 0 & 0 & 0 & $4.36 \mathrm{E}+04$ & $1.05 \mathrm{E}+06$ & $4.17 \%$ \\
\hline 10EA136 & $9.77 \mathrm{E}+02$ & 0 & $8.65 \mathrm{E}-07$ & 0 & $\mid 1.46 \mathrm{E}+04$ & 0 & 0 & 0 & 0 & 0 & 0 & 0 & $1.58 \mathrm{E}+04$ & $1.07 \mathrm{E}+06$ & $1.48 \%$ \\
\hline 10EA137 & $9.79 \mathrm{E}+02$ & 0 & $1.81 \mathrm{E}-07$ & 0 & $9.94 \mathrm{E}+03$ & $2.39 \mathrm{E}+03$ & 0 & 0 & 0 & 0 & 0 & 0 & $1.34 \mathrm{E}+04$ & $1.04 \mathrm{E}+06$ & $1.28 \%$ \\
\hline 10EA138 & $1.11 \mathrm{E}+03$ & 0 & $8.69 \mathrm{E}-06$ & 0 & $1.54 \mathrm{E}+04$ & $1.19 \mathrm{E}+03$ & 0 & 0 & 0 & 0 & 0 & 0 & $1.82 \mathrm{E}+04$ & $1.08 \mathrm{E}+06$ & $1.69 \%$ \\
\hline 10EA139 & $1.10 \mathrm{E}+03$ & 0 & $4.24 \mathrm{E}-06$ & 0 & $1.40 \mathrm{E}+04$ & $6.88 \mathrm{E}+03$ & 0 & 0 & 0 & 0 & 0 & 0 & $2.21 \mathrm{E}+04$ & $1.04 \mathrm{E}+06$ & $2.12 \%$ \\
\hline 10EA140 & $1.06 \mathrm{E}+03$ & 0 & $2.15 \mathrm{E}-06$ & 0 & $1.38 \mathrm{E}+04$ & $3.52 \mathrm{E}+03$ & 0 & 0 & 0 & 0 & 0 & 0 & $1.86 \mathrm{E}+04$ & $1.06 \mathrm{E}+06$ & $1.76 \%$ \\
\hline 10EA141 & $9.55 \mathrm{E}+02$ & 0 & 0 & 0 & $7.18 \mathrm{E}+03$ & 0 & 0 & 0 & 0 & 0 & 0 & 0 & $8.21 \mathrm{E}+03$ & $1.06 \mathrm{E}+06$ & $0.77 \%$ \\
\hline 10EA142 & $9.13 \mathrm{E}+02$ & 0 & 0 & 0 & $5.68 \mathrm{E}+03$ & $8.87 \mathrm{E}+03$ & 0 & 0 & 0 & 0 & 0 & 0 & $1.59 \mathrm{E}+04$ & $1.07 \mathrm{E}+06$ & $1.49 \%$ \\
\hline 10EA143 & $1.10 \mathrm{E}+03$ & 0 & $7.12 \mathrm{E}-06$ & 0 & $\mid 1.41 \mathrm{E}+04$ & 0 & 0 & 0 & 0 & 0 & 0 & 0 & $1.53 \mathrm{E}+04$ & $1.05 \mathrm{E}+06$ & $1.46 \%$ \\
\hline 10EA144 & $9.20 \mathrm{E}+02$ & 0 & 0 & 0 & $1.03 \mathrm{E}+04$ & $8.27 \mathrm{E}+03$ & 0 & 0 & 0 & 0 & 0 & 0 & $1.96 \mathrm{E}+04$ & $1.04 \mathrm{E}+06$ & $1.88 \%$ \\
\hline 10EA145 & $9.70 \mathrm{E}+02$ & 0 & $3.05 \mathrm{E}-06$ & 0 & $1.45 \mathrm{E}+04$ & $4.99 \mathrm{E}+03$ & 0 & 0 & 0 & 0 & 0 & 0 & $2.06 \mathrm{E}+04$ & $1.04 \mathrm{E}+06$ & $1.98 \%$ \\
\hline 10EA146 & $8.87 \mathrm{E}+02$ & 0 & 0 & 0 & $1.15 \mathrm{E}+04$ & $7.69 \mathrm{E}+03$ & 0 & 0 & 0 & 0 & 0 & 0 & $2.04 \mathrm{E}+04$ & $1.04 \mathrm{E}+06$ & $1.96 \%$ \\
\hline 10EA147 & $1.19 \mathrm{E}+03$ & 0 & $1.06 \mathrm{E}-05$ & 0 & $1.45 \mathrm{E}+04$ & $5.37 \mathrm{E}+03$ & 0 & 0 & 0 & 0 & 0 & 0 & $2.14 \mathrm{E}+04$ & $1.06 \mathrm{E}+06$ & $2.03 \%$ \\
\hline 10EA148 & $9.68 \mathrm{E}+02$ & 0 & $4.43 \mathrm{E}-07$ & 0 & $\mid 1.31 \mathrm{E}+04$ & 0 & 0 & 0 & 0 & 0 & 0 & 0 & $1.43 \mathrm{E}+04$ & $1.06 \mathrm{E}+06$ & $1.35 \%$ \\
\hline 10EA149 & $1.04 \mathrm{E}+03$ & 0 & $5.94 \mathrm{E}-06$ & 0 & $\mid 1.44 \mathrm{E}+04$ & $2.24 \mathrm{E}+03$ & 0 & 0 & 0 & 0 & 0 & 0 & $1.79 \mathrm{E}+04$ & $1.04 \mathrm{E}+06$ & $1.72 \%$ \\
\hline 10EA150 & $9.62 \mathrm{E}+02$ & 0 & $1.74 \mathrm{E}-06$ & 0 & $\mid 1.56 \mathrm{E}+04$ & $2.27 \mathrm{E}+03$ & 0 & 0 & 0 & 0 & 0 & 0 & $1.91 \mathrm{E}+04$ & $1.06 \mathrm{E}+06$ & $1.80 \%$ \\
\hline 10EA151 & $9.41 \mathrm{E}+02$ & 0 & $2.98 \mathrm{E}-07$ & 0 & $1.18 \mathrm{E}+04$ & $1.02 \mathrm{E}+02$ & 0 & 0 & 0 & 0 & 0 & 0 & $1.30 \mathrm{E}+04$ & $1.06 \mathrm{E}+06$ & $1.23 \%$ \\
\hline 10EA152 & $8.41 \mathrm{E}+02$ & 0 & $7.55 \mathrm{E}-08$ & 0 & $9.53 \mathrm{E}+03$ & $8.49 \mathrm{E}+03$ & 0 & 0 & 0 & 0 & 0 & 0 & $1.89 \mathrm{E}+04$ & $1.02 \mathrm{E}+06$ & $1.86 \%$ \\
\hline 10EA153 & $7.53 \mathrm{E}+02$ & 0 & 0 & 0 & $\mid 1.06 \mathrm{E}+04$ & $7.01 \mathrm{E}+03$ & 0 & 0 & 0 & 0 & 0 & 0 & $1.86 \mathrm{E}+04$ & $1.04 \mathrm{E}+06$ & $1.80 \%$ \\
\hline 10EA154 & $9.86 \mathrm{E}+02$ & 0 & $3.36 \mathrm{E}-06$ & 0 & $1.51 \mathrm{E}+04$ & $9.26 \mathrm{E}+02$ & 0 & 0 & 0 & 0 & 0 & 0 & $1.73 \mathrm{E}+04$ & $1.06 \mathrm{E}+06$ & $1.64 \%$ \\
\hline 10EA155 & $7.77 \mathrm{E}+02$ & 0 & $5.35 \mathrm{E}-07$ & 0 & $1.24 \mathrm{E}+04$ & $8.14 \mathrm{E}+03$ & 0 & 0 & 0 & 0 & 0 & 0 & $2.17 \mathrm{E}+04$ & $1.02 \mathrm{E}+06$ & $2.12 \%$ \\
\hline 10EA156 & $9.64 \mathrm{E}+02$ & 0 & $5.61 \mathrm{E}-07$ & 0 & \begin{tabular}{|l|}
$1.29 \mathrm{E}+04$ \\
\end{tabular} & 0 & 0 & 0 & 0 & 0 & 0 & 0 & $1.42 \mathrm{E}+04$ & $1.06 \mathrm{E}+06$ & $1.33 \%$ \\
\hline 10EA157 & $8.19 \mathrm{E}+02$ & 0 & 0 & 0 & $1.47 \mathrm{E}+04$ & $2.50 \mathrm{E}+03$ & 0 & 0 & 0 & 0 & 0 & 0 & $1.85 \mathrm{E}+04$ & $1.07 \mathrm{E}+06$ & $1.73 \%$ \\
\hline 10EA158 & $8.94 \mathrm{E}+02$ & 0 & $6.70 \mathrm{E}-07$ & 0 & $\mid 1.51 \mathrm{E}+04$ & 0 & 0 & 0 & 0 & 0 & 0 & 0 & $1.63 \mathrm{E}+04$ & $1.06 \mathrm{E}+06$ & $1.53 \%$ \\
\hline 10EA159 & $7.54 \mathrm{E}+02$ & 0 & 0 & 0 & $1.22 \mathrm{E}+04$ & $6.92 \mathrm{E}+03$ & 0 & 0 & 0 & 0 & 0 & 0 & $2.02 \mathrm{E}+04$ & $1.03 \mathrm{E}+06$ & $1.97 \%$ \\
\hline 10EA160 & $7.04 \mathrm{E}+02$ & 0 & 0 & 0 & $1.39 \mathrm{E}+04$ & $6.22 \mathrm{E}+03$ & 0 & 0 & 0 & 0 & 0 & 0 & $2.13 \mathrm{E}+04$ & $1.05 \mathrm{E}+06$ & $2.03 \%$ \\
\hline 10EA161 & $6.96 \mathrm{E}+02$ & 0 & 0 & 0 & $1.33 \mathrm{E}+04$ & $1.12 \mathrm{E}+03$ & 0 & 0 & 0 & 0 & 0 & 0 & $1.55 \mathrm{E}+04$ & $1.05 \mathrm{E}+06$ & $1.47 \%$ \\
\hline
\end{tabular}


Table 36. Predicted Solids for Envelope A Simulations - Part 2 (cont'd)

\begin{tabular}{|c|c|c|c|c|c|c|c|c|c|c|c|c|c|c|c|}
\hline Test ID & $\begin{array}{c}\text { NA2C } 2 \mathrm{O} \\
4 \\
\end{array}$ & $\begin{array}{c}\text { NA2CO3. } \\
1 \mathrm{H} 2 \mathrm{O}\end{array}$ & $\begin{array}{c}\text { NA2U2O } \\
7 \\
\end{array}$ & NA3FSO4 & $\begin{array}{c}\mathrm{NA} 6 \mathrm{SO} 42 \\
\mathrm{CO} 3\end{array}$ & NAF & $\begin{array}{c}\text { NAFPO4. } \\
19 \mathrm{H} 2 \mathrm{O}\end{array}$ & NANO3 & $\begin{array}{c}\text { NAPHOH } \\
.12 \mathrm{H} 2 \mathrm{O} \\
\end{array}$ & $\begin{array}{c}\text { NASGEL. } \\
15.5 \mathrm{H} 2 \mathrm{O} \\
\end{array}$ & ZRO2 & $\begin{array}{c}\text { ZRSO42. } \\
4 \mathrm{H} 2 \mathrm{O}\end{array}$ & $\begin{array}{c}\text { Total } \\
\text { Solids } \\
\end{array}$ & $\begin{array}{c}\text { Total Soln } \\
\text { Mass }\end{array}$ & $\begin{array}{c}\text { Solids } \% \\
\text { of Total } \\
\text { Soln } \\
\end{array}$ \\
\hline 10EA162 & $9.47 \mathrm{E}+02$ & 0 & $1.92 \mathrm{E}-06$ & 0 & $1.37 \mathrm{E}+04$ & 0 & 0 & 0 & 0 & 0 & 0 & 0 & $1.47 \mathrm{E}+04$ & $1.04 \mathrm{E}+06$ & $1.41 \%$ \\
\hline 10EA163 & $9.43 \mathrm{E}+02$ & 0 & $2.50 \mathrm{E}-06$ & 0 & $1.70 \mathrm{E}+04$ & $3.52 \mathrm{E}+03$ & 0 & 0 & 0 & 0 & 0 & 0 & $2.20 \mathrm{E}+04$ & $1.05 \mathrm{E}+06$ & $2.08 \%$ \\
\hline 10EA164 & $7.26 \mathrm{E}+02$ & 0 & $1.76 \mathrm{E}-07$ & 0 & $1.64 \mathrm{E}+04$ & $2.19 \mathrm{E}+03$ & 0 & 0 & 0 & 0 & 0 & 0 & $1.96 \mathrm{E}+04$ & $1.04 \mathrm{E}+06$ & $1.88 \%$ \\
\hline 10EA165 & $1.08 \mathrm{E}+03$ & $1.00 \mathrm{E}+04$ & $1.00 \mathrm{E}-05$ & 0 & $1.48 \mathrm{E}+04$ & 0 & 0 & 0 & 0 & 0 & 0 & 0 & $2.60 \mathrm{E}+04$ & $1.03 \mathrm{E}+06$ & $2.52 \%$ \\
\hline 10EA166 & $8.23 \mathrm{E}+02$ & 0 & $1.19 \mathrm{E}-07$ & 0 & $1.36 \mathrm{E}+04$ & $5.47 \mathrm{E}+02$ & 0 & 0 & 0 & 0 & 0 & 0 & $1.53 \mathrm{E}+04$ & $1.06 \mathrm{E}+06$ & $1.44 \%$ \\
\hline 10EA167 & $8.75 \mathrm{E}+02$ & 0 & $9.68 \mathrm{E}-07$ & 0 & $1.44 \mathrm{E}+04$ & $4.44 \mathrm{E}+03$ & 0 & 0 & 0 & 0 & 0 & 0 & $1.99 \mathrm{E}+04$ & $1.03 \mathrm{E}+06$ & $1.93 \%$ \\
\hline 10EA168 & $1.02 \mathrm{E}+03$ & 0 & $3.00 \mathrm{E}-06$ & 0 & $1.54 \mathrm{E}+04$ & $2.67 \mathrm{E}+03$ & 0 & 0 & 0 & 0 & 0 & 0 & $1.93 \mathrm{E}+04$ & $1.05 \mathrm{E}+06$ & $1.84 \%$ \\
\hline 10EA169 & $8.30 \mathrm{E}+02$ & 0 & $3.30 \mathrm{E}-07$ & 0 & $1.64 \mathrm{E}+04$ & $1.90 \mathrm{E}+03$ & 0 & 0 & 0 & 0 & 0 & 0 & $1.95 \mathrm{E}+04$ & $1.05 \mathrm{E}+06$ & $1.86 \%$ \\
\hline 10EA170 & $6.64 \mathrm{E}+02$ & 0 & 0 & 0 & $1.69 \mathrm{E}+04$ & 0 & 0 & 0 & 0 & 0 & 0 & 0 & $1.80 \mathrm{E}+04$ & $1.07 \mathrm{E}+06$ & $1.68 \%$ \\
\hline 10EA171 & $7.07 \mathrm{E}+02$ & 0 & 0 & 0 & $1.03 \mathrm{E}+04$ & 0 & 0 & 0 & 0 & 0 & 0 & 0 & $1.11 \mathrm{E}+04$ & $1.04 \mathrm{E}+06$ & $1.07 \%$ \\
\hline 10EA172 & $8.14 \mathrm{E}+02$ & 0 & 0 & 0 & $1.41 \mathrm{E}+04$ & 0 & 0 & 0 & 0 & 0 & $5.55 \mathrm{E}-01$ & 0 & $1.55 \mathrm{E}+04$ & $1.06 \mathrm{E}+06$ & $1.46 \%$ \\
\hline 10EA173 & $5.64 \mathrm{E}+02$ & 0 & 0 & 0 & $1.34 \mathrm{E}+04$ & $2.51 \mathrm{E}+03$ & 0 & 0 & 0 & 0 & 0 & 0 & $1.67 \mathrm{E}+04$ & $1.04 \mathrm{E}+06$ & $1.61 \%$ \\
\hline 10EA174 & $9.10 \mathrm{E}+02$ & 0 & 0 & 0 & $1.47 \mathrm{E}+04$ & $7.25 \mathrm{E}+01$ & 0 & 0 & 0 & 0 & 0 & 0 & $1.59 \mathrm{E}+04$ & $1.04 \mathrm{E}+06$ & $1.52 \%$ \\
\hline 10EA175 & $7.88 \mathrm{E}+02$ & 0 & 0 & 0 & $1.53 \mathrm{E}+04$ & 0 & 0 & 0 & 0 & 0 & $2.43 \mathrm{E}-01$ & 0 & $1.65 \mathrm{E}+04$ & $1.07 \mathrm{E}+06$ & $1.55 \%$ \\
\hline 10EA176 & $5.59 \mathrm{E}+02$ & 0 & 0 & 0 & $1.31 \mathrm{E}+04$ & $2.78 \mathrm{E}+03$ & 0 & 0 & 0 & 0 & 0 & 0 & $1.67 \mathrm{E}+04$ & $1.03 \mathrm{E}+06$ & $1.63 \%$ \\
\hline 10EA177 & $7.27 \mathrm{E}+02$ & 0 & 0 & 0 & $1.60 \mathrm{E}+04$ & $7.58 \mathrm{E}+03$ & 0 & 0 & 0 & 0 & 8.41E-01 & 0 & $2.48 \mathrm{E}+04$ & $1.05 \mathrm{E}+06$ & $2.37 \%$ \\
\hline 10EA178 & $7.95 \mathrm{E}+02$ & 0 & 0 & 0 & $1.61 \mathrm{E}+04$ & $8.78 \mathrm{E}+03$ & 0 & 0 & 0 & 0 & $1.11 \mathrm{E}-02$ & 0 & $2.61 \mathrm{E}+04$ & $1.02 \mathrm{E}+06$ & $2.55 \%$ \\
\hline 10EA179 & \begin{tabular}{|l|}
$7.36 \mathrm{E}+02$ \\
\end{tabular} & 0 & 0 & 0 & $7.05 \mathrm{E}+03$ & $6.26 \mathrm{E}+03$ & 0 & 0 & 0 & 0 & 0 & 0 & $1.41 \mathrm{E}+04$ & $1.02 \mathrm{E}+06$ & $1.39 \%$ \\
\hline 10EA180 & $6.81 \mathrm{E}+02$ & 0 & 0 & 0 & $1.42 \mathrm{E}+04$ & $4.78 \mathrm{E}+03$ & 0 & 0 & 0 & 0 & 0 & 0 & $1.98 \mathrm{E}+04$ & $1.02 \mathrm{E}+06$ & $1.95 \%$ \\
\hline 10EA181 & $7.11 \mathrm{E}+02$ & 0 & 0 & 0 & $9.67 \mathrm{E}+03$ & $5.27 \mathrm{E}+03$ & 0 & 0 & 0 & 0 & 0 & 0 & $1.58 \mathrm{E}+04$ & $1.03 \mathrm{E}+06$ & $1.53 \%$ \\
\hline 10EA182 & $4.77 \mathrm{E}+02$ & 0 & 0 & 0 & $1.44 \mathrm{E}+04$ & $1.34 \mathrm{E}+03$ & 0 & 0 & 0 & 0 & 0 & 0 & $1.65 \mathrm{E}+04$ & $1.03 \mathrm{E}+06$ & $1.60 \%$ \\
\hline 10EA183 & $6.28 \mathrm{E}+02$ & 0 & 0 & 0 & $1.35 \mathrm{E}+04$ & $4.41 \mathrm{E}+03$ & 0 & 0 & 0 & 0 & 0 & 0 & $1.86 \mathrm{E}+04$ & $1.01 \mathrm{E}+06$ & $1.84 \%$ \\
\hline 10EA184 & $7.50 \mathrm{E}+02$ & 0 & 0 & 0 & $1.29 \mathrm{E}+04$ & $3.92 \mathrm{E}+03$ & 0 & 0 & 0 & 0 & 0 & 0 & $1.76 \mathrm{E}+04$ & $1.02 \mathrm{E}+06$ & $1.72 \%$ \\
\hline 10EA185 & $6.89 \mathrm{E}+02$ & 0 & 0 & 0 & $1.40 \mathrm{E}+04$ & $9.00 \mathrm{E}+03$ & 0 & 0 & 0 & 0 & $6.79 \mathrm{E}-01$ & 0 & $2.41 \mathrm{E}+04$ & $1.03 \mathrm{E}+06$ & $2.33 \%$ \\
\hline 10EA186 & $7.62 \mathrm{E}+02$ & 0 & 0 & 0 & $1.43 \mathrm{E}+04$ & $7.46 \mathrm{E}+03$ & 0 & 0 & 0 & 0 & 0 & 0 & $2.25 \mathrm{E}+04$ & $1.00 \mathrm{E}+06$ & $2.25 \%$ \\
\hline 10EA187 & $7.71 \mathrm{E}+02$ & 0 & 0 & 0 & $1.62 \mathrm{E}+04$ & $9.02 \mathrm{E}+03$ & 0 & 0 & 0 & 0 & $1.38 \mathrm{E}+00$ & 0 & $2.64 \mathrm{E}+04$ & $1.03 \mathrm{E}+06$ & $2.57 \%$ \\
\hline 10EA188 & $6.18 \mathrm{E}+02$ & 0 & 0 & 0 & $1.13 \mathrm{E}+04$ & $7.85 \mathrm{E}+03$ & 0 & 0 & 0 & 0 & 0 & 0 & $2.01 \mathrm{E}+04$ & $1.02 \mathrm{E}+06$ & $1.97 \%$ \\
\hline 8EA001 & $3.72 \mathrm{E}+02$ & 0 & 0 & 0 & 0 & 0 & 0 & 0 & $7.42 \mathrm{E}+03$ & 0 & 0 & 0 & $7.79 \mathrm{E}+03$ & $1.25 \mathrm{E}+06$ & $0.63 \%$ \\
\hline 8EA002 & $3.54 \mathrm{E}+02$ & 0 & 0 & 0 & 0 & 0 & 0 & 0 & $1.87 \mathrm{E}+03$ & 0 & 0 & 0 & $2.22 \mathrm{E}+03$ & $1.27 \mathrm{E}+06$ & $0.18 \%$ \\
\hline 8EA003 & $1.06 \mathrm{E}+03$ & 0 & 0 & 0 & 0 & 0 & 0 & 0 & 0 & 0 & 0 & 0 & $1.06 \mathrm{E}+03$ & $1.30 \mathrm{E}+06$ & $0.08 \%$ \\
\hline 8EA004 & $8.31 \mathrm{E}+02$ & 0 & 0 & 0 & 0 & $7.22 \mathrm{E}+03$ & 0 & 0 & 0 & 0 & 0 & 0 & $8.05 \mathrm{E}+03$ & $1.25 \mathrm{E}+06$ & $0.65 \%$ \\
\hline 8EA005 & $6.47 \mathrm{E}+02$ & 0 & 0 & 0 & 0 & $5.89 \mathrm{E}+03$ & 0 & 0 & $2.36 \mathrm{E}+03$ & 0 & 0 & 0 & $8.89 \mathrm{E}+03$ & $1.22 \mathrm{E}+06$ & $0.73 \%$ \\
\hline 8EA006 & $1.45 \mathrm{E}+03$ & $1.23 \mathrm{E}+04$ & $7.08 \mathrm{E}-05$ & 0 & 0 & $9.38 \mathrm{E}+03$ & 0 & 0 & $6.32 \mathrm{E}+03$ & 0 & 0 & 0 & $2.94 \mathrm{E}+04$ & $1.25 \mathrm{E}+06$ & $2.35 \%$ \\
\hline
\end{tabular}


Table 36. Predicted Solids for Envelope A Simulations - Part 2 (cont'd)

\begin{tabular}{|c|c|c|c|c|c|c|c|c|c|c|c|c|c|c|c|}
\hline Test ID & $\begin{array}{c}\text { NA2C } 2 \mathrm{O} \\
4 \\
\end{array}$ & $\begin{array}{c}\text { NA2CO3. } \\
1 \mathrm{H} 2 \mathrm{O}\end{array}$ & $\begin{array}{c}\text { NA2U2O } \\
7 \\
\end{array}$ & NA3FSO4 & $\begin{array}{c}\mathrm{NA} 6 \mathrm{SO} 42 \\
\mathrm{CO} 3\end{array}$ & NAF & $\begin{array}{c}\text { NAFPO4. } \\
19 \mathrm{H} 2 \mathrm{O}\end{array}$ & NANO3 & $\begin{array}{c}\text { NAPHOH } \\
.12 \mathrm{H} 2 \mathrm{O} \\
\end{array}$ & $\begin{array}{c}\text { NASGEL. } \\
15.5 \mathrm{H} 2 \mathrm{O} \\
\end{array}$ & ZRO2 & $\begin{array}{c}\text { ZRSO42. } \\
4 \mathrm{H} 2 \mathrm{O}\end{array}$ & $\begin{array}{c}\text { Total } \\
\text { Solids } \\
\end{array}$ & $\begin{array}{c}\text { Total Soln } \\
\text { Mass } \\
\end{array}$ & $\begin{array}{c}\text { Solids \% } \\
\text { of Total } \\
\text { Soln } \\
\end{array}$ \\
\hline $8 \mathrm{EA} 007$ & $1.29 \mathrm{E}+03$ & $1.66 \mathrm{E}+04$ & $3.07 \mathrm{E}-05$ & 0 & 0 & 0 & 0 & 0 & 0 & 0 & 0 & 0 & $1.79 \mathrm{E}+04$ & $1.30 \mathrm{E}+06$ & $1.37 \%$ \\
\hline 8EA008 & $1.01 \mathrm{E}+03$ & 0 & 0 & 0 & 0 & 0 & 0 & 0 & $1.96 \mathrm{E}+04$ & 0 & 0 & 0 & $2.06 \mathrm{E}+04$ & $1.29 \mathrm{E}+06$ & $1.60 \%$ \\
\hline 8EA009 & $8.37 \mathrm{E}+02$ & 0 & 0 & 0 & 0 & 0 & 0 & 0 & $1.76 \mathrm{E}+04$ & 0 & 0 & 0 & $1.84 \mathrm{E}+04$ & $1.29 \mathrm{E}+06$ & $1.43 \%$ \\
\hline 8EA010 & $6.26 \mathrm{E}+02$ & 0 & 0 & 0 & 0 & $6.21 \mathrm{E}+03$ & 0 & 0 & $1.12 \mathrm{E}+03$ & 0 & 0 & 0 & $7.95 \mathrm{E}+03$ & $1.24 \mathrm{E}+06$ & $0.64 \%$ \\
\hline 8EA011 & $3.72 \mathrm{E}+02$ & 0 & 0 & 0 & 0 & 0 & 0 & 0 & $7.42 \mathrm{E}+03$ & 0 & 0 & 0 & $7.79 \mathrm{E}+03$ & $1.25 \mathrm{E}+06$ & $0.63 \%$ \\
\hline 8EA012 & $3.54 \mathrm{E}+02$ & 0 & 0 & 0 & 0 & 0 & 0 & 0 & $1.87 \mathrm{E}+03$ & 0 & 0 & 0 & $2.22 \mathrm{E}+03$ & $1.27 \mathrm{E}+06$ & $0.18 \%$ \\
\hline 8EA013 & $1.06 \mathrm{E}+03$ & 0 & 0 & 0 & 0 & 0 & 0 & 0 & 0 & 0 & 0 & 0 & $1.06 \mathrm{E}+03$ & $1.30 \mathrm{E}+06$ & $0.08 \%$ \\
\hline 8EA014 & $8.31 \mathrm{E}+02$ & 0 & 0 & 0 & 0 & $7.22 \mathrm{E}+03$ & 0 & 0 & 0 & 0 & 0 & 0 & $8.05 \mathrm{E}+03$ & $1.25 \mathrm{E}+06$ & $0.65 \%$ \\
\hline 8EA015 & $6.47 \mathrm{E}+02$ & 0 & 0 & 0 & 0 & $5.89 \mathrm{E}+03$ & 0 & 0 & $2.36 \mathrm{E}+03$ & 0 & 0 & 0 & $8.89 \mathrm{E}+03$ & $1.22 \mathrm{E}+06$ & $0.73 \%$ \\
\hline 8EA016 & $1.45 \mathrm{E}+03$ & $1.23 \mathrm{E}+04$ & $7.08 \mathrm{E}-05$ & 0 & 0 & $9.38 \mathrm{E}+03$ & 0 & 0 & $6.32 \mathrm{E}+03$ & 0 & 0 & 0 & $2.94 \mathrm{E}+04$ & $1.25 \mathrm{E}+06$ & $2.35 \%$ \\
\hline 8EA017 & $1.29 \mathrm{E}+03$ & $1.66 \mathrm{E}+04$ & $3.07 \mathrm{E}-05$ & 0 & 0 & 0 & 0 & 0 & 0 & 0 & 0 & 0 & $1.79 \mathrm{E}+04$ & $1.30 \mathrm{E}+06$ & $1.37 \%$ \\
\hline 8EA018 & $1.01 \mathrm{E}+03$ & 0 & 0 & 0 & 0 & 0 & 0 & 0 & $1.96 \mathrm{E}+04$ & 0 & 0 & 0 & $2.06 \mathrm{E}+04$ & $1.29 \mathrm{E}+06$ & $1.60 \%$ \\
\hline 8EA019 & $8.37 \mathrm{E}+02$ & 0 & 0 & 0 & 0 & 0 & 0 & 0 & $1.76 \mathrm{E}+04$ & 0 & 0 & 0 & $1.84 \mathrm{E}+04$ & $1.29 \mathrm{E}+06$ & $1.43 \%$ \\
\hline 8EA020 & $6.26 \mathrm{E}+02$ & 0 & 0 & 0 & 0 & $6.21 \mathrm{E}+03$ & 0 & 0 & $1.12 \mathrm{E}+03$ & 0 & 0 & 0 & $7.95 \mathrm{E}+03$ & $1.24 \mathrm{E}+06$ & $0.64 \%$ \\
\hline 8EA021 & $3.56 \mathrm{E}+02$ & 0 & 0 & 0 & 0 & 0 & 0 & 0 & $7.11 \mathrm{E}+03$ & 0 & 0 & 0 & $7.90 \mathrm{E}+03$ & $1.29 \mathrm{E}+06$ & $0.61 \%$ \\
\hline $8 \mathrm{EA} 022$ & $3.39 \mathrm{E}+02$ & 0 & 0 & 0 & 0 & 0 & 0 & 0 & $1.57 \mathrm{E}+03$ & 0 & 0 & 0 & $2.35 \mathrm{E}+03$ & $1.32 \mathrm{E}+06$ & $0.18 \%$ \\
\hline $8 \mathrm{EA} 023$ & $1.01 \mathrm{E}+03$ & 0 & 0 & 0 & 0 & 0 & 0 & 0 & 0 & \begin{tabular}{|l|}
$2.30 \mathrm{E}+02$ \\
\end{tabular} & 0 & 0 & $1.73 \mathrm{E}+03$ & $1.35 \mathrm{E}+06$ & $0.13 \%$ \\
\hline 8EA024 & $7.97 \mathrm{E}+02$ & 0 & 0 & 0 & 0 & $6.91 \mathrm{E}+03$ & 0 & 0 & 0 & 0 & 0 & 0 & $8.20 \mathrm{E}+03$ & $1.30 \mathrm{E}+06$ & $0.63 \%$ \\
\hline 8EA025 & $6.07 \mathrm{E}+02$ & 0 & 0 & 0 & 0 & $5.53 \mathrm{E}+03$ & 0 & 0 & $1.69 \mathrm{E}+03$ & 0 & 0 & 0 & $9.74 \mathrm{E}+03$ & $1.26 \mathrm{E}+06$ & $0.77 \%$ \\
\hline $8 \mathrm{EA} 026$ & $1.37 \mathrm{E}+03$ & 0 & $6.98 \mathrm{E}-05$ & 0 & 0 & $8.86 \mathrm{E}+03$ & 0 & 0 & $4.86 \mathrm{E}+03$ & $5.46 \mathrm{E}+02$ & 0 & 0 & $1.61 \mathrm{E}+04$ & $1.31 \mathrm{E}+06$ & $1.23 \%$ \\
\hline 8EA027 & $1.22 \mathrm{E}+03$ & $5.02 \mathrm{E}+03$ & $3.04 \mathrm{E}-05$ & 0 & 0 & 0 & 0 & 0 & 0 & $4.73 \mathrm{E}+02$ & 0 & 0 & $7.22 \mathrm{E}+03$ & $1.36 \mathrm{E}+06$ & $0.53 \%$ \\
\hline 8EA028 & $9.71 \mathrm{E}+02$ & 0 & 0 & 0 & 0 & 0 & 0 & 0 & $1.85 \mathrm{E}+04$ & $1.56 \mathrm{E}+02$ & 0 & 0 & $2.02 \mathrm{E}+04$ & $1.35 \mathrm{E}+06$ & $1.50 \%$ \\
\hline 8EA029 & $8.03 \mathrm{E}+02$ & 0 & 0 & 0 & 0 & 0 & 0 & 0 & $1.68 \mathrm{E}+04$ & $8.53 \mathrm{E}+01$ & 0 & 0 & $1.81 \mathrm{E}+04$ & $1.34 \mathrm{E}+06$ & $1.35 \%$ \\
\hline 8EA030 & $6.05 \mathrm{E}+02$ & 0 & 0 & 0 & 0 & $5.97 \mathrm{E}+03$ & 0 & 0 & $6.42 \mathrm{E}+02$ & 0 & 0 & 0 & $7.71 \mathrm{E}+03$ & $1.29 \mathrm{E}+06$ & $0.60 \%$ \\
\hline 8EA031 & $3.56 \mathrm{E}+02$ & 0 & 0 & 0 & 0 & 0 & 0 & 0 & $7.11 \mathrm{E}+03$ & 0 & 0 & 0 & $7.90 \mathrm{E}+03$ & $1.29 \mathrm{E}+06$ & $0.61 \%$ \\
\hline 8EA032 & $3.39 \mathrm{E}+02$ & 0 & 0 & 0 & 0 & 0 & 0 & 0 & $1.57 \mathrm{E}+03$ & 0 & 0 & 0 & $2.35 \mathrm{E}+03$ & $1.32 \mathrm{E}+06$ & $0.18 \%$ \\
\hline 8EA033 & $1.01 \mathrm{E}+03$ & 0 & 0 & 0 & 0 & 0 & 0 & 0 & 0 & $2.30 \mathrm{E}+02$ & 0 & 0 & $1.73 \mathrm{E}+03$ & $1.35 \mathrm{E}+06$ & $0.13 \%$ \\
\hline 8EA034 & $7.97 \mathrm{E}+02$ & 0 & 0 & 0 & 0 & $6.91 \mathrm{E}+03$ & 0 & 0 & 0 & 0 & 0 & 0 & $8.20 \mathrm{E}+03$ & $1.30 \mathrm{E}+06$ & $0.63 \%$ \\
\hline $8 \mathrm{EA} 035$ & $6.07 \mathrm{E}+02$ & 0 & 0 & 0 & 0 & $5.53 \mathrm{E}+03$ & 0 & 0 & $1.69 \mathrm{E}+03$ & 0 & 0 & 0 & $9.74 \mathrm{E}+03$ & $1.26 \mathrm{E}+06$ & $0.77 \%$ \\
\hline 8EA036 & $1.37 \mathrm{E}+03$ & 0 & $6.98 \mathrm{E}-05$ & 0 & 0 & $8.86 \mathrm{E}+03$ & 0 & 0 & $4.86 \mathrm{E}+03$ & $5.46 \mathrm{E}+02$ & 0 & 0 & $1.61 \mathrm{E}+04$ & $1.31 \mathrm{E}+06$ & $1.23 \%$ \\
\hline 8EA037 & $1.22 \mathrm{E}+03$ & $5.02 \mathrm{E}+03$ & $3.04 \mathrm{E}-05$ & 0 & 0 & 0 & 0 & 0 & 0 & $4.73 \mathrm{E}+02$ & 0 & 0 & $7.22 \mathrm{E}+03$ & $1.36 \mathrm{E}+06$ & $0.53 \%$ \\
\hline 8EA038 & $9.71 \mathrm{E}+02$ & 0 & 0 & 0 & 0 & 0 & 0 & 0 & $1.85 \mathrm{E}+04$ & $1.56 \mathrm{E}+02$ & 0 & 0 & $2.02 \mathrm{E}+04$ & $1.35 \mathrm{E}+06$ & $1.50 \%$ \\
\hline 8EA039 & $8.03 \mathrm{E}+02$ & 0 & 0 & 0 & 0 & 0 & 0 & 0 & $1.68 \mathrm{E}+04$ & $8.53 \mathrm{E}+01$ & 0 & 0 & $1.81 \mathrm{E}+04$ & $1.34 \mathrm{E}+06$ & $1.35 \%$ \\
\hline
\end{tabular}


Table 36. Predicted Solids for Envelope A Simulations - Part 2 (cont'd)

\begin{tabular}{|c|c|c|c|c|c|c|c|c|c|c|c|c|c|c|c|}
\hline Test ID & $\begin{array}{c}\text { NA2C } 2 \mathrm{O} \\
4 \\
\end{array}$ & $\begin{array}{c}\text { NA2CO3. } \\
1 \mathrm{H} 2 \mathrm{O}\end{array}$ & $\begin{array}{c}\mathrm{NA} 2 \mathrm{U} 2 \mathrm{O} \\
7 \\
\end{array}$ & NA3FSO4 & $\begin{array}{c}\mathrm{NA} 6 \mathrm{SO} 42 \\
\mathrm{CO} 3\end{array}$ & NAF & $\begin{array}{c}\text { NAFPO4. } \\
19 \mathrm{H} 2 \mathrm{O}\end{array}$ & NANO3 & $\begin{array}{c}\text { NAPHOH } \\
.12 \mathrm{H} 2 \mathrm{O} \\
\end{array}$ & $\begin{array}{c}\text { NASGEL. } \\
15.5 \mathrm{H} 2 \mathrm{O} \\
\end{array}$ & ZRO2 & $\begin{array}{c}\text { ZRSO42. } \\
4 \mathrm{H} 2 \mathrm{O}\end{array}$ & $\begin{array}{c}\text { Total } \\
\text { Solids } \\
\end{array}$ & $\begin{array}{c}\text { Total Soln } \\
\text { Mass } \\
\end{array}$ & $\begin{array}{c}\text { Solids \% } \\
\text { of Total } \\
\text { Soln } \\
\end{array}$ \\
\hline $8 \mathrm{EA} 040$ & $6.05 \mathrm{E}+02$ & 0 & 0 & 0 & 0 & $5.97 \mathrm{E}+03$ & 0 & 0 & $6.42 \mathrm{E}+02$ & 0 & 0 & 0 & $7.71 \mathrm{E}+03$ & $1.29 \mathrm{E}+06$ & $0.60 \%$ \\
\hline 8EA041 & 0 & 0 & 0 & 0 & $2.45 \mathrm{E}+03$ & 0 & 0 & 0 & 0 & 0 & 0 & 0 & $2.45 \mathrm{E}+03$ & $1.20 \mathrm{E}+06$ & $0.20 \%$ \\
\hline 8EA042 & 0 & 0 & 0 & 0 & $2.64 \mathrm{E}+03$ & 0 & 0 & 0 & 0 & 0 & 0 & 0 & $2.64 \mathrm{E}+03$ & $1.22 \mathrm{E}+06$ & $0.22 \%$ \\
\hline 8EA043 & $7.06 \mathrm{E}+01$ & 0 & 0 & 0 & 0 & 0 & 0 & 0 & 0 & 0 & 0 & 0 & $7.06 \mathrm{E}+01$ & $1.24 \mathrm{E}+06$ & $0.01 \%$ \\
\hline 8EA044 & $6.70 \mathrm{E}+01$ & 0 & 0 & 0 & 0 & $5.12 \mathrm{E}+03$ & 0 & 0 & 0 & 0 & 0 & 0 & $5.19 \mathrm{E}+03$ & $1.21 \mathrm{E}+06$ & $0.43 \%$ \\
\hline 8EA045 & $2.95 \mathrm{E}+01$ & 0 & 0 & 0 & 0 & $3.92 \mathrm{E}+03$ & 0 & 0 & 0 & 0 & 0 & 0 & $3.95 \mathrm{E}+03$ & $1.17 \mathrm{E}+06$ & $0.34 \%$ \\
\hline 8EA046 & $4.15 \mathrm{E}+02$ & 0 & 0 & 0 & $5.73 \mathrm{E}+03$ & $7.11 \mathrm{E}+03$ & 0 & 0 & 0 & 0 & 0 & 0 & $1.33 \mathrm{E}+04$ & $1.20 \mathrm{E}+06$ & $1.10 \%$ \\
\hline $8 \mathrm{EA047}$ & $3.85 \mathrm{E}+02$ & 0 & 0 & 0 & $7.75 \mathrm{E}+03$ & 0 & 0 & 0 & 0 & 0 & 0 & 0 & $8.14 \mathrm{E}+03$ & $1.23 \mathrm{E}+06$ & $0.66 \%$ \\
\hline 8EA048 & $1.54 \mathrm{E}+02$ & 0 & 0 & 0 & 0 & 0 & 0 & 0 & 0 & 0 & 0 & 0 & $1.54 \mathrm{E}+02$ & $1.25 \mathrm{E}+06$ & $0.01 \%$ \\
\hline 8EA049 & $1.41 \mathrm{E}+02$ & 0 & 0 & 0 & 0 & 0 & 0 & 0 & 0 & 0 & 0 & 0 & $1.41 \mathrm{E}+02$ & $1.24 \mathrm{E}+06$ & $0.01 \%$ \\
\hline $8 \mathrm{EA} 050$ & $9.05 \mathrm{E}+01$ & 0 & 0 & 0 & 0 & $4.26 \mathrm{E}+03$ & 0 & 0 & 0 & 0 & 0 & 0 & $4.35 \mathrm{E}+03$ & $1.20 \mathrm{E}+06$ & $0.36 \%$ \\
\hline $8 \mathrm{EA} 051$ & 0 & 0 & 0 & 0 & $2.45 \mathrm{E}+03$ & 0 & 0 & 0 & 0 & 0 & 0 & 0 & $2.45 \mathrm{E}+03$ & $1.20 \mathrm{E}+06$ & $0.20 \%$ \\
\hline 8EA052 & 0 & 0 & 0 & 0 & $2.64 \mathrm{E}+03$ & 0 & 0 & 0 & 0 & 0 & 0 & 0 & $2.64 \mathrm{E}+03$ & $1.22 \mathrm{E}+06$ & $0.22 \%$ \\
\hline 8EA053 & $7.06 \mathrm{E}+01$ & 0 & 0 & 0 & 0 & 0 & 0 & 0 & 0 & 0 & 0 & 0 & $7.06 \mathrm{E}+01$ & $1.24 \mathrm{E}+06$ & $0.01 \%$ \\
\hline 8EA054 & $6.70 \mathrm{E}+01$ & 0 & 0 & 0 & 0 & $5.12 \mathrm{E}+03$ & 0 & 0 & 0 & 0 & 0 & 0 & $5.19 \mathrm{E}+03$ & $1.21 \mathrm{E}+06$ & $0.43 \%$ \\
\hline 8EA055 & $2.95 \mathrm{E}+01$ & 0 & 0 & 0 & 0 & $3.92 \mathrm{E}+03$ & 0 & 0 & 0 & 0 & 0 & 0 & $3.95 \mathrm{E}+03$ & $1.17 \mathrm{E}+06$ & $0.34 \%$ \\
\hline 8EA056 & $4.15 \mathrm{E}+02$ & 0 & 0 & 0 & $5.73 \mathrm{E}+03$ & $7.11 \mathrm{E}+03$ & 0 & 0 & 0 & 0 & 0 & 0 & $1.33 \mathrm{E}+04$ & $1.20 \mathrm{E}+06$ & $1.10 \%$ \\
\hline 8EA057 & $3.85 \mathrm{E}+02$ & 0 & 0 & 0 & $7.75 \mathrm{E}+03$ & 0 & 0 & 0 & 0 & 0 & 0 & 0 & $8.14 \mathrm{E}+03$ & $1.23 \mathrm{E}+06$ & $0.66 \%$ \\
\hline 8EA058 & $1.54 \mathrm{E}+02$ & 0 & 0 & 0 & 0 & 0 & 0 & 0 & 0 & 0 & 0 & 0 & $1.54 \mathrm{E}+02$ & $1.25 \mathrm{E}+06$ & $0.01 \%$ \\
\hline 8EA059 & $1.41 \mathrm{E}+02$ & 0 & 0 & 0 & 0 & 0 & 0 & 0 & 0 & 0 & 0 & 0 & $1.41 \mathrm{E}+02$ & $1.24 \mathrm{E}+06$ & $0.01 \%$ \\
\hline 8EA060 & $9.05 \mathrm{E}+01$ & 0 & 0 & 0 & 0 & $4.26 \mathrm{E}+03$ & 0 & 0 & 0 & 0 & 0 & 0 & $4.35 \mathrm{E}+03$ & $1.20 \mathrm{E}+06$ & $0.36 \%$ \\
\hline 8EA061 & 0 & 0 & 0 & 0 & $4.08 \mathrm{E}+03$ & 0 & 0 & 0 & 0 & 0 & 0 & 0 & $4.52 \mathrm{E}+03$ & $1.24 \mathrm{E}+06$ & $0.36 \%$ \\
\hline 8EA062 & 0 & 0 & 0 & 0 & $4.34 \mathrm{E}+03$ & 0 & 0 & 0 & 0 & 0 & 0 & 0 & $4.78 \mathrm{E}+03$ & $1.26 \mathrm{E}+06$ & $0.38 \%$ \\
\hline 8EA063 & 0 & 0 & 0 & 0 & 0 & 0 & 0 & 0 & 0 & 0 & $2.40 \mathrm{E}+00$ & 0 & $6.53 \mathrm{E}+02$ & $1.29 \mathrm{E}+06$ & $0.05 \%$ \\
\hline 8EA064 & 0 & 0 & 0 & 0 & 0 & $4.79 \mathrm{E}+03$ & 0 & 0 & 0 & 0 & 8.19E-01 & 0 & $5.29 \mathrm{E}+03$ & $1.25 \mathrm{E}+06$ & $0.42 \%$ \\
\hline $8 \mathrm{EA} 065$ & 0 & 0 & 0 & 0 & 0 & $3.63 \mathrm{E}+03$ & 0 & 0 & 0 & 0 & $6.49 \mathrm{E}-02$ & 0 & $4.10 \mathrm{E}+03$ & $1.21 \mathrm{E}+06$ & $0.34 \%$ \\
\hline $8 \mathrm{EA066}$ & $3.32 \mathrm{E}+02$ & 0 & 0 & 0 & $8.82 \mathrm{E}+03$ & $6.83 \mathrm{E}+03$ & 0 & 0 & 0 & $5.42 \mathrm{E}+02$ & $2.09 \mathrm{E}+00$ & 0 & $1.71 \mathrm{E}+04$ & $1.24 \mathrm{E}+06$ & $1.37 \%$ \\
\hline 8EA067 & $3.09 \mathrm{E}+02$ & 0 & 0 & 0 & $1.10 \mathrm{E}+04$ & 0 & 0 & 0 & 0 & 0 & $1.20 \mathrm{E}+00$ & 0 & $1.19 \mathrm{E}+04$ & $1.28 \mathrm{E}+06$ & $0.93 \%$ \\
\hline $8 \mathrm{EA} 068$ & $5.13 \mathrm{E}+01$ & 0 & 0 & 0 & 0 & 0 & 0 & 0 & 0 & 0 & $1.29 \mathrm{E}+00$ & 0 & $6.96 \mathrm{E}+02$ & $1.30 \mathrm{E}+06$ & $0.05 \%$ \\
\hline 8EA069 & $6.00 \mathrm{E}+01$ & 0 & 0 & 0 & 0 & 0 & 0 & 0 & 0 & 0 & $6.31 \mathrm{E}-01$ & 0 & $6.79 \mathrm{E}+02$ & $1.28 \mathrm{E}+06$ & $0.05 \%$ \\
\hline 8EA070 & $2.74 \mathrm{E}+01$ & 0 & 0 & 0 & 0 & $3.99 \mathrm{E}+03$ & 0 & 0 & 0 & 0 & 0 & 0 & $4.50 \mathrm{E}+03$ & $1.25 \mathrm{E}+06$ & $0.36 \%$ \\
\hline 8EA071 & 0 & 0 & 0 & 0 & $4.08 \mathrm{E}+03$ & 0 & 0 & 0 & 0 & 0 & 0 & 0 & $4.52 \mathrm{E}+03$ & $1.24 \mathrm{E}+06$ & $0.36 \%$ \\
\hline 8EA072 & 0 & 0 & 0 & 0 & $4.34 \mathrm{E}+03$ & 0 & 0 & 0 & 0 & 0 & 0 & 0 & $4.78 \mathrm{E}+03$ & $1.26 \mathrm{E}+06$ & $0.38 \%$ \\
\hline
\end{tabular}


Table 36. Predicted Solids for Envelope A Simulations - Part 2 (cont'd)

\begin{tabular}{|c|c|c|c|c|c|c|c|c|c|c|c|c|c|c|c|}
\hline Test ID & $\begin{array}{c}\text { NA2C } 2 \mathrm{O} \\
4 \\
\end{array}$ & $\begin{array}{c}\text { NA2CO3. } \\
1 \mathrm{H} 2 \mathrm{O}\end{array}$ & $\begin{array}{c}\text { NA2U2O } \\
7 \\
\end{array}$ & NA3FSO4 & $\begin{array}{c}\mathrm{NA} 6 \mathrm{SO} 42 \\
\mathrm{CO} 3\end{array}$ & NAF & $\begin{array}{c}\text { NAFPO4. } \\
19 \mathrm{H} 2 \mathrm{O}\end{array}$ & NANO3 & $\begin{array}{c}\text { NAPHOH } \\
.12 \mathrm{H} 2 \mathrm{O} \\
\end{array}$ & $\begin{array}{c}\text { NASGEL. } \\
15.5 \mathrm{H} 2 \mathrm{O} \\
\end{array}$ & ZRO2 & $\begin{array}{c}\text { ZRSO42. } \\
4 \mathrm{H} 2 \mathrm{O}\end{array}$ & $\begin{array}{c}\text { Total } \\
\text { Solids } \\
\end{array}$ & $\begin{array}{c}\text { Total Soln } \\
\text { Mass } \\
\end{array}$ & $\begin{array}{c}\text { Solids \% } \\
\text { of Total } \\
\text { Soln } \\
\end{array}$ \\
\hline $8 \mathrm{EA} 073$ & 0 & 0 & 0 & 0 & 0 & 0 & 0 & 0 & 0 & 0 & $2.40 \mathrm{E}+00$ & 0 & $6.53 \mathrm{E}+02$ & $1.29 \mathrm{E}+06$ & $0.05 \%$ \\
\hline $8 \mathrm{EA} 074$ & 0 & 0 & 0 & 0 & 0 & $4.79 \mathrm{E}+03$ & 0 & 0 & 0 & 0 & 8.19E-01 & 0 & $5.29 \mathrm{E}+03$ & $1.25 \mathrm{E}+06$ & $0.42 \%$ \\
\hline $8 \mathrm{EA} 075$ & 0 & 0 & 0 & 0 & 0 & $3.63 \mathrm{E}+03$ & 0 & 0 & 0 & 0 & 6.49E-02 & 0 & $4.10 \mathrm{E}+03$ & $1.21 \mathrm{E}+06$ & $0.34 \%$ \\
\hline 8EA076 & $3.32 \mathrm{E}+02$ & 0 & 0 & 0 & $8.82 \mathrm{E}+03$ & $6.83 \mathrm{E}+03$ & 0 & 0 & 0 & $5.42 \mathrm{E}+02$ & $2.09 \mathrm{E}+00$ & 0 & $1.71 \mathrm{E}+04$ & $1.24 \mathrm{E}+06$ & $1.37 \%$ \\
\hline 8EA077 & $3.09 \mathrm{E}+02$ & 0 & 0 & 0 & $1.10 \mathrm{E}+04$ & 0 & 0 & 0 & 0 & 0 & $1.20 \mathrm{E}+00$ & 0 & $1.19 \mathrm{E}+04$ & $1.28 \mathrm{E}+06$ & $0.93 \%$ \\
\hline 8EA078 & $5.13 \mathrm{E}+01$ & 0 & 0 & 0 & 0 & 0 & 0 & 0 & 0 & 0 & $1.29 \mathrm{E}+00$ & 0 & $6.96 \mathrm{E}+02$ & $1.30 \mathrm{E}+06$ & $0.05 \%$ \\
\hline 8EA079 & $6.00 \mathrm{E}+01$ & 0 & 0 & 0 & 0 & 0 & 0 & 0 & 0 & 0 & $6.31 \mathrm{E}-01$ & 0 & $6.79 \mathrm{E}+02$ & $1.28 \mathrm{E}+06$ & $0.05 \%$ \\
\hline 8EA080 & $2.74 \mathrm{E}+01$ & 0 & 0 & 0 & 0 & $3.99 \mathrm{E}+03$ & 0 & 0 & 0 & 0 & 0 & 0 & $4.50 \mathrm{E}+03$ & $1.25 \mathrm{E}+06$ & $0.36 \%$ \\
\hline 8EA081 & $1.05 \mathrm{E}+03$ & 0 & 4.17E-06 & 0 & 0 & $7.99 \mathrm{E}+03$ & 0 & 0 & $4.36 \mathrm{E}+03$ & 0 & 0 & 0 & $1.34 \mathrm{E}+04$ & $1.25 \mathrm{E}+06$ & $1.07 \%$ \\
\hline 8EA082 & $8.68 \mathrm{E}+02$ & 0 & 0 & 0 & 0 & $7.17 \mathrm{E}+03$ & 0 & 0 & $2.43 \mathrm{E}+03$ & $1.64 \mathrm{E}+02$ & 0 & 0 & $1.11 \mathrm{E}+04$ & $1.30 \mathrm{E}+06$ & $0.86 \%$ \\
\hline $8 \mathrm{EA} 083$ & $1.06 \mathrm{E}+03$ & 0 & $1.41 \mathrm{E}-07$ & 0 & 0 & $7.48 \mathrm{E}+03$ & 0 & 0 & 0 & 0 & 0 & 0 & $8.74 \mathrm{E}+03$ & $1.30 \mathrm{E}+06$ & $0.67 \%$ \\
\hline 8EA084 & $9.72 \mathrm{E}+02$ & 0 & 0 & 0 & 0 & $8.02 \mathrm{E}+03$ & 0 & 0 & $1.85 \mathrm{E}+03$ & 0 & 0 & 0 & $1.09 \mathrm{E}+04$ & $1.27 \mathrm{E}+06$ & $0.86 \%$ \\
\hline 8EA085 & $1.11 \mathrm{E}+03$ & 0 & $4.43 \mathrm{E}-06$ & 0 & 0 & $8.18 \mathrm{E}+03$ & 0 & 0 & $3.34 \mathrm{E}+03$ & 0 & 0 & 0 & $1.26 \mathrm{E}+04$ & $1.25 \mathrm{E}+06$ & $1.01 \%$ \\
\hline 8EA086 & $9.81 \mathrm{E}+02$ & 0 & $1.89 \mathrm{E}-06$ & 0 & 0 & $3.24 \mathrm{E}+03$ & 0 & 0 & 0 & 0 & 0 & 0 & $4.54 \mathrm{E}+03$ & $1.32 \mathrm{E}+06$ & $0.34 \%$ \\
\hline 8EA087 & $1.11 \mathrm{E}+03$ & 0 & $8.70 \mathrm{E}-06$ & 0 & 0 & 0 & 0 & 0 & $1.25 \mathrm{E}+04$ & 0 & 0 & 0 & $1.40 \mathrm{E}+04$ & $1.34 \mathrm{E}+06$ & $1.05 \%$ \\
\hline 8EA088 & $1.16 \mathrm{E}+03$ & 0 & $1.39 \mathrm{E}-05$ & 0 & 0 & $8.24 \mathrm{E}+03$ & $2.89 \mathrm{E}+03$ & 0 & 0 & 0 & 0 & 0 & $1.25 \mathrm{E}+04$ & $1.29 \mathrm{E}+06$ & $0.97 \%$ \\
\hline 8EA089 & $7.10 \mathrm{E}+02$ & 0 & 0 & 0 & 0 & $6.51 \mathrm{E}+03$ & $1.34 \mathrm{E}+03$ & 0 & 0 & 0 & 0 & 0 & $8.84 \mathrm{E}+03$ & $1.28 \mathrm{E}+06$ & $0.69 \%$ \\
\hline 8EA090 & $7.91 \mathrm{E}+02$ & 0 & 0 & 0 & 0 & $4.44 \mathrm{E}+03$ & $1.96 \mathrm{E}+03$ & 0 & 0 & 0 & 0 & 0 & $7.35 \mathrm{E}+03$ & $1.29 \mathrm{E}+06$ & $0.57 \%$ \\
\hline 8EA091 & $8.53 \mathrm{E}+02$ & 0 & 0 & 0 & 0 & $2.61 \mathrm{E}+03$ & $1.76 \mathrm{E}+03$ & 0 & 0 & 0 & 0 & 0 & $5.44 \mathrm{E}+03$ & $1.31 \mathrm{E}+06$ & $0.42 \%$ \\
\hline 8EA092 & $9.22 \mathrm{E}+02$ & 0 & 0 & 0 & 0 & $3.89 \mathrm{E}+02$ & $3.28 \mathrm{E}+03$ & 0 & 0 & 0 & 0 & 0 & $4.64 \mathrm{E}+03$ & $1.30 \mathrm{E}+06$ & $0.36 \%$ \\
\hline 8EA093 & $1.02 \mathrm{E}+03$ & 0 & $2.52 \mathrm{E}-06$ & 0 & 0 & $3.04 \mathrm{E}+02$ & $7.02 \mathrm{E}+03$ & 0 & 0 & 0 & 0 & 0 & $8.61 \mathrm{E}+03$ & $1.30 \mathrm{E}+06$ & $0.66 \%$ \\
\hline 8EA094 & $1.10 \mathrm{E}+03$ & 0 & $3.41 \mathrm{E}-06$ & 0 & 0 & 0 & 0 & 0 & 0 & 0 & 0 & 0 & $1.24 \mathrm{E}+03$ & $1.31 \mathrm{E}+06$ & $0.09 \%$ \\
\hline 8EA095 & $8.52 \mathrm{E}+02$ & 0 & 0 & 0 & 0 & $7.09 \mathrm{E}+03$ & $1.68 \mathrm{E}+03$ & 0 & 0 & 0 & 0 & 0 & $9.95 \mathrm{E}+03$ & $1.27 \mathrm{E}+06$ & $0.78 \%$ \\
\hline 8EA096 & $9.40 \mathrm{E}+02$ & 0 & $1.39 \mathrm{E}-06$ & $5.54 \mathrm{E}+03$ & 0 & 0 & 0 & 0 & 0 & $1.27 \mathrm{E}+02$ & 0 & 0 & $7.06 \mathrm{E}+03$ & $1.33 \mathrm{E}+06$ & $0.53 \%$ \\
\hline 8EA097 & $1.01 \mathrm{E}+03$ & 0 & $4.26 \mathrm{E}-06$ & $9.38 \mathrm{E}+03$ & 0 & 0 & $7.02 \mathrm{E}+02$ & 0 & 0 & 0 & 0 & 0 & $1.11 \mathrm{E}+04$ & $1.28 \mathrm{E}+06$ & $0.87 \%$ \\
\hline 8EA098 & $6.79 \mathrm{E}+02$ & 0 & 0 & $7.43 \mathrm{E}+03$ & 0 & 0 & 0 & 0 & $4.26 \mathrm{E}+03$ & 0 & 0 & 0 & $1.28 \mathrm{E}+04$ & $1.30 \mathrm{E}+06$ & $0.98 \%$ \\
\hline 8EA099 & $9.83 \mathrm{E}+02$ & 0 & $2.77 \mathrm{E}-06$ & $1.17 \mathrm{E}+04$ & 0 & $4.94 \mathrm{E}+03$ & $3.27 \mathrm{E}+03$ & 0 & 0 & 0 & 0 & 0 & $2.09 \mathrm{E}+04$ & $1.24 \mathrm{E}+06$ & $1.68 \%$ \\
\hline $8 \mathrm{EA} 100$ & $9.07 \mathrm{E}+02$ & 0 & 4.04E-07 & $3.39 \mathrm{E}+03$ & 0 & 0 & $1.29 \mathrm{E}+03$ & 0 & 0 & 0 & 0 & 0 & $5.99 \mathrm{E}+03$ & $1.33 \mathrm{E}+06$ & $0.45 \%$ \\
\hline 8EA101 & $9.73 \mathrm{E}+02$ & 0 & $1.97 \mathrm{E}-07$ & $1.23 \mathrm{E}+04$ & 0 & 0 & $4.70 \mathrm{E}+03$ & 0 & 0 & 0 & 0 & 0 & $1.82 \mathrm{E}+04$ & $1.28 \mathrm{E}+06$ & $1.42 \%$ \\
\hline 8EA102 & $1.07 \mathrm{E}+03$ & 0 & $6.39 \mathrm{E}-06$ & $1.41 \mathrm{E}+04$ & 0 & $4.42 \mathrm{E}+03$ & 0 & 0 & 0 & 0 & 0 & 0 & $1.99 \mathrm{E}+04$ & $1.28 \mathrm{E}+06$ & $1.55 \%$ \\
\hline 8EA103 & $8.06 \mathrm{E}+02$ & 0 & 0 & $9.69 \mathrm{E}+03$ & 0 & 0 & $3.76 \mathrm{E}+03$ & 0 & 0 & 0 & 0 & 0 & $1.45 \mathrm{E}+04$ & $1.28 \mathrm{E}+06$ & $1.13 \%$ \\
\hline 8EA104 & $9.08 \mathrm{E}+02$ & 0 & $1.16 \mathrm{E}-06$ & $1.49 \mathrm{E}+04$ & 0 & $1.05 \mathrm{E}+03$ & $4.01 \mathrm{E}+03$ & 0 & 0 & 0 & 0 & 0 & $2.11 \mathrm{E}+04$ & $1.29 \mathrm{E}+06$ & $1.64 \%$ \\
\hline 8EA105 & $7.80 \mathrm{E}+02$ & 0 & 0 & $8.61 \mathrm{E}+03$ & 0 & 0 & $9.28 \mathrm{E}+02$ & 0 & 0 & 0 & 0 & 0 & $1.04 \mathrm{E}+04$ & $1.28 \mathrm{E}+06$ & $0.81 \%$ \\
\hline
\end{tabular}


Table 36. Predicted Solids for Envelope A Simulations - Part 2 (cont'd)

\begin{tabular}{|c|c|c|c|c|c|c|c|c|c|c|c|c|c|c|c|}
\hline Test ID & $\begin{array}{c}\text { NA2C } 2 \mathrm{O} \\
4 \\
\end{array}$ & $\begin{array}{c}\text { NA2CO3. } \\
1 \mathrm{H} 2 \mathrm{O}\end{array}$ & $\begin{array}{c}\text { NA2U2O } \\
7 \\
\end{array}$ & NA3FSO4 & $\begin{array}{c}\text { NA6SO42 } \\
\mathrm{CO} 3 \\
\end{array}$ & NAF & $\begin{array}{c}\text { NAFPO4. } \\
19 \mathrm{H} 2 \mathrm{O}\end{array}$ & NANO3 & $\begin{array}{c}\text { NAPHOH } \\
.12 \mathrm{H} 2 \mathrm{O} \\
\end{array}$ & $\begin{array}{c}\text { NASGEL. } \\
15.5 \mathrm{H} 2 \mathrm{O} \\
\end{array}$ & $\mathrm{ZRO} 2$ & $\begin{array}{c}\text { ZRSO42. } \\
4 \mathrm{H} 2 \mathrm{O}\end{array}$ & $\begin{array}{c}\text { Total } \\
\text { Solids } \\
\end{array}$ & $\begin{array}{c}\text { Total Soln } \\
\text { Mass } \\
\end{array}$ & $\begin{array}{c}\text { Solids \% } \\
\text { of Total } \\
\text { Soln } \\
\end{array}$ \\
\hline 8EA106 & $9.35 \mathrm{E}+02$ & 0 & $5.01 \mathrm{E}-07$ & $1.39 \mathrm{E}+04$ & 0 & $4.51 \mathrm{E}+03$ & $2.50 \mathrm{E}+03$ & 0 & 0 & 0 & 0 & 0 & $2.21 \mathrm{E}+04$ & $1.27 \mathrm{E}+06$ & $1.74 \%$ \\
\hline 8EA107 & $7.15 \mathrm{E}+02$ & 0 & 0 & $2.05 \mathrm{E}+03$ & 0 & 0 & 0 & 0 & 0 & 0 & 0 & 0 & $2.77 \mathrm{E}+03$ & $1.28 \mathrm{E}+06$ & $0.22 \%$ \\
\hline 8EA108 & $8.49 \mathrm{E}+02$ & 0 & 0 & $1.33 \mathrm{E}+04$ & 0 & $4.20 \mathrm{E}+03$ & $2.17 \mathrm{E}+03$ & 0 & 0 & 0 & 0 & 0 & $2.09 \mathrm{E}+04$ & $1.25 \mathrm{E}+06$ & $1.66 \%$ \\
\hline 8EA109 & $8.04 \mathrm{E}+02$ & 0 & 0 & $5.86 \mathrm{E}+03$ & 0 & 0 & 0 & 0 & 0 & 0 & 0 & 0 & $6.92 \mathrm{E}+03$ & $1.30 \mathrm{E}+06$ & $0.53 \%$ \\
\hline 8EA110 & $7.41 \mathrm{E}+02$ & 0 & 0 & $1.54 \mathrm{E}+04$ & 0 & $7.57 \mathrm{E}+02$ & 0 & 0 & 0 & 0 & 0 & 0 & $1.72 \mathrm{E}+04$ & $1.29 \mathrm{E}+06$ & $1.34 \%$ \\
\hline 8EA111 & $9.13 \mathrm{E}+02$ & 0 & 0 & $3.72 \mathrm{E}+03$ & 0 & 0 & 0 & 0 & 0 & 0 & 0 & 0 & $4.90 \mathrm{E}+03$ & $1.31 \mathrm{E}+06$ & $0.38 \%$ \\
\hline 8EA112 & $8.84 \mathrm{E}+02$ & 0 & $2.89 \mathrm{E}-07$ & $1.06 \mathrm{E}+04$ & 0 & 0 & $4.62 \mathrm{E}+03$ & 0 & 0 & 0 & 0 & 0 & $1.62 \mathrm{E}+04$ & $1.28 \mathrm{E}+06$ & $1.27 \%$ \\
\hline 8EA113 & $8.72 \mathrm{E}+02$ & 0 & $1.34 \mathrm{E}-06$ & \begin{tabular}{|l|}
$1.07 \mathrm{E}+04$ \\
\end{tabular} & 0 & 0 & $4.88 \mathrm{E}+03$ & 0 & 0 & 0 & 0 & 0 & $1.67 \mathrm{E}+04$ & $1.27 \mathrm{E}+06$ & $1.32 \%$ \\
\hline 8EA114 & $9.29 \mathrm{E}+02$ & 0 & $8.73 \mathrm{E}-07$ & $4.68 \mathrm{E}+03$ & 0 & 0 & 0 & 0 & 0 & 0 & 0 & 0 & $5.77 \mathrm{E}+03$ & $1.29 \mathrm{E}+06$ & $0.45 \%$ \\
\hline $8 \mathrm{EA} 115$ & $7.69 \mathrm{E}+02$ & 0 & 0 & $1.28 \mathrm{E}+04$ & 0 & $4.14 \mathrm{E}+03$ & 0 & 0 & 0 & 0 & 0 & 0 & $1.81 \mathrm{E}+04$ & $1.28 \mathrm{E}+06$ & $1.42 \%$ \\
\hline 8EA116 & $8.95 \mathrm{E}+02$ & 0 & $1.31 \mathrm{E}-06$ & $1.43 \mathrm{E}+04$ & 0 & $1.55 \mathrm{E}+03$ & 0 & 0 & 0 & 0 & 0 & 0 & $1.70 \mathrm{E}+04$ & $1.28 \mathrm{E}+06$ & $1.33 \%$ \\
\hline $8 \mathrm{EA} 117$ & $8.38 \mathrm{E}+02$ & 0 & 0 & $1.55 \mathrm{E}+04$ & 0 & $1.73 \mathrm{E}+03$ & $2.38 \mathrm{E}+03$ & 0 & 0 & $1.54 \mathrm{E}+02$ & 0 & 0 & $2.11 \mathrm{E}+04$ & $1.29 \mathrm{E}+06$ & $1.64 \%$ \\
\hline 8EA118 & $7.62 \mathrm{E}+02$ & 0 & $8.30 \mathrm{E}-07$ & $1.17 \mathrm{E}+04$ & 0 & $3.74 \mathrm{E}+03$ & $9.70 \mathrm{E}+02$ & 0 & 0 & 0 & 0 & 0 & $1.73 \mathrm{E}+04$ & $1.26 \mathrm{E}+06$ & $1.38 \%$ \\
\hline 8EA119 & $5.22 \mathrm{E}+02$ & 0 & 0 & $1.24 \mathrm{E}+04$ & 0 & 0 & $1.99 \mathrm{E}+03$ & 0 & 0 & 0 & 0 & 0 & $1.50 \mathrm{E}+04$ & $1.25 \mathrm{E}+06$ & $1.20 \%$ \\
\hline 8EA120 & $8.07 \mathrm{E}+02$ & 0 & 0 & $1.33 \mathrm{E}+04$ & 0 & $4.17 \mathrm{E}+03$ & $1.39 \mathrm{E}+03$ & 0 & 0 & 0 & 0 & 0 & $2.00 \mathrm{E}+04$ & $1.27 \mathrm{E}+06$ & $1.58 \%$ \\
\hline 8EA121 & $8.35 \mathrm{E}+02$ & 0 & $4.36 \mathrm{E}-07$ & $6.38 \mathrm{E}+03$ & 0 & 0 & 0 & 0 & 0 & 0 & 0 & 0 & $7.55 \mathrm{E}+03$ & $1.30 \mathrm{E}+06$ & $0.58 \%$ \\
\hline 8EA122 & $1.00 \mathrm{E}+03$ & 0 & $1.27 \mathrm{E}-05$ & $1.17 \mathrm{E}+04$ & 0 & 0 & $6.63 \mathrm{E}+01$ & 0 & 0 & 0 & 0 & 0 & $1.29 \mathrm{E}+04$ & $1.27 \mathrm{E}+06$ & $1.01 \%$ \\
\hline 8EA123 & $7.01 \mathrm{E}+02$ & 0 & 0 & $5.06 \mathrm{E}+03$ & 0 & 0 & $3.01 \mathrm{E}+03$ & 0 & 0 & 0 & 0 & 0 & $8.93 \mathrm{E}+03$ & $1.28 \mathrm{E}+06$ & $0.70 \%$ \\
\hline 8EA124 & $8.94 \mathrm{E}+02$ & 0 & $4.70 \mathrm{E}-06$ & $1.41 \mathrm{E}+04$ & 0 & 0 & $3.96 \mathrm{E}+03$ & 0 & 0 & 0 & 0 & 0 & $1.93 \mathrm{E}+04$ & $1.27 \mathrm{E}+06$ & $1.52 \%$ \\
\hline $8 \mathrm{EA} 125$ & $5.46 \mathrm{E}+02$ & 0 & 0 & $8.77 \mathrm{E}+03$ & 0 & $3.66 \mathrm{E}+03$ & $5.74 \mathrm{E}+02$ & 0 & 0 & 0 & 0 & 0 & $1.37 \mathrm{E}+04$ & $1.23 \mathrm{E}+06$ & $1.11 \%$ \\
\hline 8EA126 & $7.01 \mathrm{E}+02$ & 0 & 0 & $1.41 \mathrm{E}+04$ & 0 & $6.70 \mathrm{E}+02$ & $2.05 \mathrm{E}+03$ & 0 & 0 & 0 & 0 & 0 & $1.76 \mathrm{E}+04$ & $1.25 \mathrm{E}+06$ & $1.41 \%$ \\
\hline 8EA127 & $6.04 \mathrm{E}+02$ & 0 & 0 & $1.51 \mathrm{E}+04$ & 0 & $5.62 \mathrm{E}+02$ & $1.54 \mathrm{E}+03$ & 0 & 0 & 0 & 0 & 0 & $1.83 \mathrm{E}+04$ & $1.28 \mathrm{E}+06$ & $1.42 \%$ \\
\hline 8EA128 & $8.44 \mathrm{E}+02$ & 0 & $5.26 \mathrm{E}-07$ & $1.27 \mathrm{E}+04$ & 0 & $9.21 \mathrm{E}+02$ & $2.93 \mathrm{E}+03$ & 0 & 0 & 0 & 0 & 0 & $1.75 \mathrm{E}+04$ & $1.25 \mathrm{E}+06$ & $1.40 \%$ \\
\hline 8EA129 & $5.37 \mathrm{E}+02$ & 0 & 0 & $1.41 \mathrm{E}+04$ & 0 & 0 & 0 & 0 & 0 & 0 & 0 & 0 & $1.51 \mathrm{E}+04$ & $1.29 \mathrm{E}+06$ & $1.17 \%$ \\
\hline 8EA130 & $5.36 \mathrm{E}+02$ & 0 & 0 & $1.09 \mathrm{E}+04$ & 0 & 0 & 0 & 0 & 0 & 0 & 0 & 0 & $1.19 \mathrm{E}+04$ & $1.30 \mathrm{E}+06$ & $0.92 \%$ \\
\hline 8EA131 & $7.59 \mathrm{E}+02$ & 0 & $5.76 \mathrm{E}-07$ & $1.49 \mathrm{E}+04$ & 0 & $1.27 \mathrm{E}+03$ & 0 & 0 & 0 & 0 & 0 & 0 & $1.73 \mathrm{E}+04$ & $1.28 \mathrm{E}+06$ & $1.35 \%$ \\
\hline 8 EA132 & $6.37 \mathrm{E}+02$ & 0 & $2.00 \mathrm{E}-07$ & $1.21 \mathrm{E}+04$ & 0 & 0 & 0 & 0 & 0 & 0 & 0 & 0 & $1.28 \mathrm{E}+04$ & $1.23 \mathrm{E}+06$ & $1.04 \%$ \\
\hline 8EA133 & $4.32 \mathrm{E}+02$ & 0 & 0 & 0 & 0 & $5.20 \mathrm{E}+03$ & 0 & 0 & 0 & 0 & 0 & 0 & $6.04 \mathrm{E}+03$ & $1.27 \mathrm{E}+06$ & $0.47 \%$ \\
\hline 8EA134 & $7.74 \mathrm{E}+02$ & 0 & $8.59 \mathrm{E}-07$ & 0 & 0 & $2.50 \mathrm{E}+03$ & 0 & 0 & 0 & 0 & 0 & 0 & $3.33 \mathrm{E}+03$ & $1.27 \mathrm{E}+06$ & $0.26 \%$ \\
\hline 8EA135 & $6.57 \mathrm{E}+02$ & 0 & $1.09 \mathrm{E}-06$ & 0 & $6.45 \mathrm{E}+03$ & 0 & $1.52 \mathrm{E}+03$ & 0 & 0 & 0 & 0 & 0 & $9.06 \mathrm{E}+03$ & $1.30 \mathrm{E}+06$ & $0.70 \%$ \\
\hline 8EA136 & $6.63 \mathrm{E}+02$ & 0 & $2.61 \mathrm{E}-07$ & 0 & $3.71 \mathrm{E}+02$ & 0 & 0 & 0 & 0 & 0 & 0 & 0 & $1.35 \mathrm{E}+03$ & $1.30 \mathrm{E}+06$ & $0.10 \%$ \\
\hline 8EA137 & $6.41 \mathrm{E}+02$ & 0 & $4.00 \mathrm{E}-08$ & 0 & 0 & 0 & 0 & 0 & 0 & 0 & 0 & 0 & $7.12 \mathrm{E}+02$ & $1.27 \mathrm{E}+06$ & $0.06 \%$ \\
\hline 8EA138 & $7.05 \mathrm{E}+02$ & 0 & 0 & 0 & $3.80 \mathrm{E}+02$ & 0 & 0 & 0 & 0 & 0 & 0 & 0 & $1.54 \mathrm{E}+03$ & $1.31 \mathrm{E}+06$ & $0.12 \%$ \\
\hline
\end{tabular}


Table 36. Predicted Solids for Envelope A Simulations - Part 2 (cont'd)

\begin{tabular}{|c|c|c|c|c|c|c|c|c|c|c|c|c|c|c|c|}
\hline Test ID & $\begin{array}{c}\text { NA2C } 2 \mathrm{O} \\
4 \\
\end{array}$ & $\begin{array}{c}\text { NA2CO3. } \\
1 \mathrm{H} 2 \mathrm{O}\end{array}$ & $\begin{array}{c}\text { NA2U2O } \\
7 \\
\end{array}$ & NA3FSO4 & $\begin{array}{c}\mathrm{NA} 6 \mathrm{SO} 42 \\
\mathrm{CO} 3\end{array}$ & NAF & $\begin{array}{c}\text { NAFPO4. } \\
19 \mathrm{H} 2 \mathrm{O}\end{array}$ & NANO3 & $\begin{array}{c}\text { NAPHOH } \\
.12 \mathrm{H} 2 \mathrm{O} \\
\end{array}$ & $\begin{array}{c}\text { NASGEL. } \\
15.5 \mathrm{H} 2 \mathrm{O} \\
\end{array}$ & $\mathrm{ZRO} 2$ & $\begin{array}{c}\text { ZRSO42. } \\
4 \mathrm{H} 2 \mathrm{O}\end{array}$ & $\begin{array}{c}\text { Total } \\
\text { Solids } \\
\end{array}$ & $\begin{array}{c}\text { Total Soln } \\
\text { Mass }\end{array}$ & $\begin{array}{c}\text { Solids } \% \\
\text { of Total } \\
\text { Soln } \\
\end{array}$ \\
\hline 8EA139 & $7.36 \mathrm{E}+02$ & 0 & $3.16 \mathrm{E}-07$ & 0 & $4.55 \mathrm{E}+02$ & $4.33 \mathrm{E}+03$ & 0 & 0 & 0 & 0 & 0 & 0 & $5.70 \mathrm{E}+03$ & $1.27 \mathrm{E}+06$ & $0.45 \%$ \\
\hline $8 \mathrm{EA} 140$ & $6.92 \mathrm{E}+02$ & 0 & 0 & 0 & 0 & $8.49 \mathrm{E}+02$ & 0 & 0 & 0 & 0 & 0 & 0 & $1.79 \mathrm{E}+03$ & $1.29 \mathrm{E}+06$ & $0.14 \%$ \\
\hline 8EA141 & $6.46 \mathrm{E}+02$ & 0 & 0 & 0 & 0 & 0 & 0 & 0 & 0 & 0 & 0 & 0 & $7.40 \mathrm{E}+02$ & $1.28 \mathrm{E}+06$ & $0.06 \%$ \\
\hline 8EA142 & $5.63 \mathrm{E}+02$ & 0 & 0 & 0 & 0 & $6.37 \mathrm{E}+03$ & 0 & 0 & 0 & 0 & 0 & 0 & $7.38 \mathrm{E}+03$ & $1.28 \mathrm{E}+06$ & $0.57 \%$ \\
\hline 8EA143 & $7.53 \mathrm{E}+02$ & 0 & $7.37 \mathrm{E}-07$ & 0 & $2.67 \mathrm{E}+03$ & 0 & 0 & 0 & 0 & 0 & 0 & 0 & $3.55 \mathrm{E}+03$ & $1.28 \mathrm{E}+06$ & $0.28 \%$ \\
\hline $8 \mathrm{EA} 144$ & $6.00 \mathrm{E}+02$ & 0 & 0 & 0 & 0 & $5.79 \mathrm{E}+03$ & 0 & 0 & 0 & 0 & 0 & 0 & $6.58 \mathrm{E}+03$ & $1.27 \mathrm{E}+06$ & $0.52 \%$ \\
\hline 8EA145 & $6.28 \mathrm{E}+02$ & 0 & 0 & 0 & $2.97 \mathrm{E}+03$ & $2.54 \mathrm{E}+03$ & 0 & 0 & 0 & 0 & 0 & 0 & $6.31 \mathrm{E}+03$ & $1.27 \mathrm{E}+06$ & $0.50 \%$ \\
\hline $8 \mathrm{EA} 146$ & $5.48 \mathrm{E}+02$ & 0 & 0 & 0 & 0 & $4.99 \mathrm{E}+03$ & 0 & 0 & 0 & 0 & 0 & 0 & $5.91 \mathrm{E}+03$ & $1.27 \mathrm{E}+06$ & $0.47 \%$ \\
\hline 8EA147 & \begin{tabular}{|l}
$7.60 \mathrm{E}+02$ \\
\end{tabular} & 0 & 0 & 0 & 0 & $2.78 \mathrm{E}+03$ & 0 & 0 & 0 & 0 & 0 & 0 & $3.84 \mathrm{E}+03$ & $1.29 \mathrm{E}+06$ & $0.30 \%$ \\
\hline 8EA148 & $6.17 \mathrm{E}+02$ & 0 & 0 & 0 & 0 & 0 & 0 & 0 & 0 & 0 & 0 & 0 & $8.60 \mathrm{E}+02$ & $1.28 \mathrm{E}+06$ & $0.07 \%$ \\
\hline 8EA149 & $6.50 \mathrm{E}+02$ & 0 & 0 & 0 & $1.35 \mathrm{E}+03$ & 0 & 0 & 0 & 0 & 0 & 0 & 0 & $2.23 \mathrm{E}+03$ & $1.27 \mathrm{E}+06$ & $0.18 \%$ \\
\hline $8 \mathrm{EA} 150$ & $6.14 \mathrm{E}+02$ & 0 & 0 & 0 & $4.13 \mathrm{E}+03$ & 0 & 0 & 0 & 0 & 0 & 0 & 0 & $5.05 \mathrm{E}+03$ & $1.29 \mathrm{E}+06$ & $0.39 \%$ \\
\hline $8 \mathrm{EA} 151$ & $5.84 \mathrm{E}+02$ & 0 & 0 & 0 & 0 & 0 & 0 & 0 & 0 & 0 & 0 & 0 & $7.43 \mathrm{E}+02$ & $1.28 \mathrm{E}+06$ & $0.06 \%$ \\
\hline $8 \mathrm{EA} 152$ & $5.44 \mathrm{E}+02$ & 0 & 0 & 0 & 0 & $6.35 \mathrm{E}+03$ & 0 & 0 & 0 & 0 & 0 & 0 & $6.90 \mathrm{E}+03$ & $1.23 \mathrm{E}+06$ & $0.56 \%$ \\
\hline 8EA153 & $4.56 \mathrm{E}+02$ & 0 & 0 & 0 & 0 & $4.60 \mathrm{E}+03$ & 0 & 0 & 0 & 0 & 0 & 0 & $5.37 \mathrm{E}+03$ & $1.26 \mathrm{E}+06$ & $0.43 \%$ \\
\hline $8 \mathrm{EA} 154$ & $6.17 \mathrm{E}+02$ & 0 & 0 & 0 & $4.17 \mathrm{E}+03$ & 0 & 0 & 0 & 0 & 0 & 0 & 0 & $5.03 \mathrm{E}+03$ & $1.28 \mathrm{E}+06$ & $0.39 \%$ \\
\hline 8EA155 & $4.39 \mathrm{E}+02$ & 0 & 0 & 0 & $1.15 \mathrm{E}+03$ & $5.55 \mathrm{E}+03$ & 0 & 0 & 0 & 0 & 0 & 0 & $7.54 \mathrm{E}+03$ & $1.24 \mathrm{E}+06$ & $0.61 \%$ \\
\hline 8EA156 & $5.72 \mathrm{E}+02$ & 0 & 0 & 0 & 0 & 0 & 0 & 0 & 0 & 0 & 0 & 0 & $9.26 \mathrm{E}+02$ & $1.29 \mathrm{E}+06$ & $0.07 \%$ \\
\hline 8 EA157 & $4.55 \mathrm{E}+02$ & 0 & 0 & 0 & 0 & 0 & 0 & 0 & 0 & 0 & 0 & 0 & $9.07 \mathrm{E}+02$ & $1.30 \mathrm{E}+06$ & $0.07 \%$ \\
\hline $8 \mathrm{EA} 158$ & $5.40 \mathrm{E}+02$ & 0 & 0 & 0 & $2.89 \mathrm{E}+03$ & 0 & 0 & 0 & 0 & 0 & 0 & 0 & $3.79 \mathrm{E}+03$ & $1.28 \mathrm{E}+06$ & $0.30 \%$ \\
\hline 8EA159 & $4.40 \mathrm{E}+02$ & 0 & 0 & 0 & 0 & $4.50 \mathrm{E}+03$ & 0 & 0 & 0 & 0 & 0 & 0 & $5.22 \mathrm{E}+03$ & $1.25 \mathrm{E}+06$ & $0.42 \%$ \\
\hline $8 \mathrm{EA} 160$ & $3.80 \mathrm{E}+02$ & 0 & 0 & 0 & $2.44 \mathrm{E}+02$ & $3.59 \mathrm{E}+03$ & 0 & 0 & 0 & 0 & 0 & 0 & $4.69 \mathrm{E}+03$ & $1.28 \mathrm{E}+06$ & $0.37 \%$ \\
\hline 8EA161 & $3.42 \mathrm{E}+02$ & 0 & 0 & 0 & 0 & 0 & 0 & 0 & 0 & 0 & 0 & 0 & $7.20 \mathrm{E}+02$ & $1.27 \mathrm{E}+06$ & $0.06 \%$ \\
\hline $8 \mathrm{EA} 162$ & $5.74 \mathrm{E}+02$ & 0 & 0 & 0 & $3.73 \mathrm{E}+03$ & 0 & 0 & 0 & 0 & 0 & 0 & 0 & $4.32 \mathrm{E}+03$ & $1.26 \mathrm{E}+06$ & $0.34 \%$ \\
\hline $8 \mathrm{EA} 163$ & $5.22 \mathrm{E}+02$ & 0 & 0 & 0 & $6.52 \mathrm{E}+03$ & $6.21 \mathrm{E}+02$ & 0 & 0 & 0 & 0 & 0 & 0 & $8.15 \mathrm{E}+03$ & $1.28 \mathrm{E}+06$ & $0.64 \%$ \\
\hline 8EA164 & $3.78 \mathrm{E}+02$ & 0 & 0 & 0 & $6.63 \mathrm{E}+03$ & 0 & 0 & 0 & 0 & 0 & 0 & 0 & $7.38 \mathrm{E}+03$ & $1.27 \mathrm{E}+06$ & $0.58 \%$ \\
\hline $8 \mathrm{EA} 165$ & $6.19 \mathrm{E}+02$ & 0 & 0 & 0 & $5.72 \mathrm{E}+03$ & 0 & 0 & 0 & 0 & 0 & 0 & 0 & $6.48 \mathrm{E}+03$ & $1.26 \mathrm{E}+06$ & $0.51 \%$ \\
\hline 8EA166 & $3.99 \mathrm{E}+02$ & 0 & 0 & 0 & 0 & 0 & 0 & 0 & 0 & 0 & 0 & 0 & $7.33 \mathrm{E}+02$ & $1.28 \mathrm{E}+06$ & $0.06 \%$ \\
\hline $8 \mathrm{EA} 167$ & $4.61 \mathrm{E}+02$ & 0 & 0 & 0 & $3.85 \mathrm{E}+03$ & $1.83 \mathrm{E}+03$ & 0 & 0 & 0 & 0 & 0 & 0 & $6.30 \mathrm{E}+03$ & $1.26 \mathrm{E}+06$ & $0.50 \%$ \\
\hline 8EA168 & $5.32 \mathrm{E}+02$ & 0 & 0 & 0 & $4.21 \mathrm{E}+03$ & $8.01 \mathrm{E}+00$ & 0 & 0 & 0 & 0 & 0 & 0 & $5.05 \mathrm{E}+03$ & $1.28 \mathrm{E}+06$ & $0.40 \%$ \\
\hline 8EA169 & $3.91 \mathrm{E}+02$ & 0 & 0 & 0 & $5.49 \mathrm{E}+03$ & 0 & 0 & 0 & 0 & 0 & 0 & 0 & $6.33 \mathrm{E}+03$ & $1.27 \mathrm{E}+06$ & $0.50 \%$ \\
\hline $8 \mathrm{EA} 170$ & $2.91 \mathrm{E}+02$ & 0 & 0 & 0 & $6.65 \mathrm{E}+03$ & 0 & 0 & 0 & 0 & 0 & 0 & 0 & $7.41 \mathrm{E}+03$ & $1.29 \mathrm{E}+06$ & $0.57 \%$ \\
\hline 8EA171 & $2.89 \mathrm{E}+02$ & 0 & 0 & 0 & 0 & 0 & 0 & 0 & 0 & 0 & 0 & 0 & $3.49 \mathrm{E}+02$ & $1.26 \mathrm{E}+06$ & $0.03 \%$ \\
\hline
\end{tabular}


Table 36. Predicted Solids for Envelope A Simulations - Part 2 (cont'd)

\begin{tabular}{|c|c|c|c|c|c|c|c|c|c|c|c|c|c|c|c|}
\hline Test ID & $\begin{array}{c}\text { NA2C } 2 \mathrm{O} \\
4 \\
\end{array}$ & $\begin{array}{c}\text { NA2CO3. } \\
1 \mathrm{H} 2 \mathrm{O}\end{array}$ & $\begin{array}{c}\text { NA2U2O } \\
7 \\
\end{array}$ & NA3FSO4 & $\begin{array}{c}\mathrm{NA} 6 \mathrm{SO} 42 \\
\mathrm{CO} 3\end{array}$ & NAF & $\begin{array}{c}\text { NAFPO4. } \\
19 \mathrm{H} 2 \mathrm{O}\end{array}$ & NANO3 & $\begin{array}{c}\text { NAPHOH } \\
.12 \mathrm{H} 2 \mathrm{O} \\
\end{array}$ & $\begin{array}{c}\text { NASGEL. } \\
15.5 \mathrm{H} 2 \mathrm{O} \\
\end{array}$ & ZRO2 & $\begin{array}{c}\text { ZRSO42. } \\
4 \mathrm{H} 2 \mathrm{O}\end{array}$ & $\begin{array}{c}\text { Total } \\
\text { Solids } \\
\end{array}$ & $\begin{array}{c}\text { Total Soln } \\
\text { Mass } \\
\end{array}$ & $\begin{array}{c}\text { Solids \% } \\
\text { of Total } \\
\text { Soln } \\
\end{array}$ \\
\hline $8 \mathrm{EA} 172$ & $2.98 \mathrm{E}+02$ & 0 & 0 & 0 & 0 & 0 & 0 & 0 & 0 & 0 & $4.63 \mathrm{E}-02$ & 0 & $8.43 \mathrm{E}+02$ & $1.29 \mathrm{E}+06$ & $0.07 \%$ \\
\hline $8 \mathrm{EA} 173$ & $1.53 \mathrm{E}+02$ & 0 & 0 & 0 & 0 & 0 & 0 & 0 & 0 & 0 & 0 & 0 & $4.19 \mathrm{E}+02$ & $1.26 \mathrm{E}+06$ & $0.03 \%$ \\
\hline 8EA174 & $3.71 \mathrm{E}+02$ & 0 & 0 & 0 & $4.10 \mathrm{E}+03$ & 0 & 0 & 0 & 0 & 0 & 0 & 0 & $4.75 \mathrm{E}+03$ & $1.26 \mathrm{E}+06$ & $0.38 \%$ \\
\hline 8EA175 & $2.68 \mathrm{E}+02$ & 0 & 0 & 0 & $2.70 \mathrm{E}+03$ & 0 & 0 & 0 & 0 & 0 & 0 & 0 & $3.50 \mathrm{E}+03$ & $1.29 \mathrm{E}+06$ & $0.27 \%$ \\
\hline 8EA176 & $1.66 \mathrm{E}+02$ & 0 & 0 & 0 & 0 & $2.21 \mathrm{E}+02$ & 0 & 0 & 0 & 0 & 0 & 0 & $6.50 \mathrm{E}+02$ & $1.25 \mathrm{E}+06$ & $0.05 \%$ \\
\hline 8EA177 & $2.34 \mathrm{E}+02$ & 0 & 0 & 0 & $4.33 \mathrm{E}+03$ & $4.48 \mathrm{E}+03$ & 0 & 0 & 0 & 0 & $1.79 \mathrm{E}-01$ & 0 & $9.54 \mathrm{E}+03$ & $1.28 \mathrm{E}+06$ & $0.75 \%$ \\
\hline 8EA178 & $3.23 \mathrm{E}+02$ & 0 & 0 & 0 & $8.42 \mathrm{E}+03$ & $5.91 \mathrm{E}+03$ & 0 & 0 & 0 & 0 & 0 & 0 & $1.51 \mathrm{E}+04$ & $1.24 \mathrm{E}+06$ & $1.22 \%$ \\
\hline 8EA179 & $2.49 \mathrm{E}+02$ & 0 & 0 & 0 & 0 & $3.51 \mathrm{E}+03$ & 0 & 0 & 0 & 0 & 0 & 0 & $3.82 \mathrm{E}+03$ & $1.23 \mathrm{E}+06$ & $0.31 \%$ \\
\hline 8EA180 & $2.29 \mathrm{E}+02$ & 0 & 0 & 0 & $4.41 \mathrm{E}+03$ & $2.18 \mathrm{E}+03$ & 0 & 0 & 0 & 0 & 0 & 0 & $6.95 \mathrm{E}+03$ & $1.23 \mathrm{E}+06$ & $0.56 \%$ \\
\hline 8EA181 & $1.87 \mathrm{E}+02$ & 0 & 0 & 0 & 0 & $2.35 \mathrm{E}+03$ & 0 & 0 & 0 & 0 & 0 & 0 & $2.70 \mathrm{E}+03$ & $1.25 \mathrm{E}+06$ & $0.22 \%$ \\
\hline 8EA182 & $6.35 \mathrm{E}+01$ & 0 & 0 & 0 & $2.59 \mathrm{E}+03$ & 0 & 0 & 0 & 0 & 0 & 0 & 0 & $2.99 \mathrm{E}+03$ & $1.25 \mathrm{E}+06$ & $0.24 \%$ \\
\hline 8EA183 & $1.76 \mathrm{E}+02$ & 0 & 0 & 0 & $2.45 \mathrm{E}+03$ & $1.84 \mathrm{E}+03$ & 0 & 0 & 0 & 0 & 0 & 0 & $4.58 \mathrm{E}+03$ & $1.23 \mathrm{E}+06$ & $0.37 \%$ \\
\hline 8EA184 & $2.20 \mathrm{E}+02$ & 0 & 0 & 0 & $1.68 \mathrm{E}+03$ & $1.16 \mathrm{E}+03$ & 0 & 0 & 0 & 0 & 0 & 0 & $3.10 \mathrm{E}+03$ & $1.25 \mathrm{E}+06$ & $0.25 \%$ \\
\hline $8 \mathrm{EA} 185$ & $1.64 \mathrm{E}+02$ & 0 & 0 & 0 & $2.64 \mathrm{E}+03$ & $5.90 \mathrm{E}+03$ & 0 & 0 & 0 & 0 & $5.75 \mathrm{E}-02$ & 0 & $1.05 \mathrm{E}+04$ & $1.25 \mathrm{E}+06$ & $0.83 \%$ \\
\hline 8EA186 & $2.84 \mathrm{E}+02$ & 0 & 0 & 0 & $6.46 \mathrm{E}+03$ & $4.92 \mathrm{E}+03$ & 0 & 0 & 0 & 0 & 0 & 0 & $1.17 \mathrm{E}+04$ & $1.22 \mathrm{E}+06$ & $0.96 \%$ \\
\hline 8EA187 & $2.12 \mathrm{E}+02$ & 0 & 0 & 0 & $5.76 \mathrm{E}+03$ & $6.22 \mathrm{E}+03$ & 0 & 0 & 0 & 0 & 9.59E-01 & 0 & $1.26 \mathrm{E}+04$ & $1.25 \mathrm{E}+06$ & $1.01 \%$ \\
\hline 8 EA188 & $1.24 \mathrm{E}+02$ & 0 & 0 & 0 & 0 & $4.97 \mathrm{E}+03$ & 0 & 0 & 0 & 0 & 0 & 0 & $5.36 \mathrm{E}+03$ & $1.24 \mathrm{E}+06$ & $0.43 \%$ \\
\hline 6EA001 & $1.89 \mathrm{E}+02$ & 0 & 0 & 0 & 0 & 0 & 0 & 0 & $6.87 \mathrm{E}+03$ & 0 & 0 & 0 & $7.06 \mathrm{E}+03$ & $1.57 \mathrm{E}+06$ & $0.45 \%$ \\
\hline 6EA002 & $1.72 \mathrm{E}+02$ & 0 & 0 & 0 & 0 & 0 & 0 & 0 & $1.32 \mathrm{E}+03$ & 0 & 0 & 0 & $1.50 \mathrm{E}+03$ & $1.61 \mathrm{E}+06$ & $0.09 \%$ \\
\hline 6EA003 & $6.59 \mathrm{E}+02$ & 0 & 0 & 0 & 0 & 0 & 0 & 0 & 0 & 0 & 0 & 0 & $6.59 \mathrm{E}+02$ & $1.64 \mathrm{E}+06$ & $0.04 \%$ \\
\hline 6EA004 & $5.09 \mathrm{E}+02$ & 0 & 0 & 0 & 0 & $3.38 \mathrm{E}+03$ & 0 & 0 & 0 & 0 & 0 & 0 & $3.89 \mathrm{E}+03$ & $1.59 \mathrm{E}+06$ & $0.24 \%$ \\
\hline 6EA005 & $3.61 \mathrm{E}+02$ & 0 & 0 & 0 & 0 & $2.35 \mathrm{E}+03$ & 0 & 0 & $1.39 \mathrm{E}+03$ & 0 & 0 & 0 & $4.09 \mathrm{E}+03$ & $1.55 \mathrm{E}+06$ & $0.26 \%$ \\
\hline 6ЕA006 & $1.04 \mathrm{E}+03$ & $2.71 \mathrm{E}+04$ & $6.82 \mathrm{E}-05$ & 0 & 0 & $5.56 \mathrm{E}+03$ & 0 & 0 & $4.28 \mathrm{E}+03$ & 0 & 0 & 0 & $3.80 \mathrm{E}+04$ & $1.57 \mathrm{E}+06$ & $2.43 \%$ \\
\hline 6EA007 & $9.66 \mathrm{E}+02$ & $3.69 \mathrm{E}+04$ & $3.00 \mathrm{E}-05$ & 0 & 0 & 0 & 0 & 0 & 0 & 0 & 0 & 0 & $4.07 \mathrm{E}+04$ & $1.60 \mathrm{E}+06$ & $2.54 \%$ \\
\hline 6ЕA008 & $6.89 \mathrm{E}+02$ & 0 & 0 & 0 & 0 & 0 & 0 & 0 & $1.92 \mathrm{E}+04$ & 0 & 0 & 0 & $2.27 \mathrm{E}+04$ & $1.63 \mathrm{E}+06$ & $1.40 \%$ \\
\hline 6EA009 & $5.47 \mathrm{E}+02$ & 0 & 0 & 0 & 0 & 0 & 0 & 0 & $1.68 \mathrm{E}+04$ & 0 & 0 & 0 & $2.19 \mathrm{E}+04$ & $1.62 \mathrm{E}+06$ & $1.35 \%$ \\
\hline 6EA010 & $4.04 \mathrm{E}+02$ & 0 & 0 & 0 & 0 & $3.05 \mathrm{E}+03$ & 0 & 0 & $4.45 \mathrm{E}+02$ & 0 & 0 & 0 & $1.07 \mathrm{E}+04$ & $1.58 \mathrm{E}+06$ & $0.68 \%$ \\
\hline 6EA011 & $1.89 \mathrm{E}+02$ & 0 & 0 & 0 & 0 & 0 & 0 & 0 & $6.87 \mathrm{E}+03$ & 0 & 0 & 0 & $7.06 \mathrm{E}+03$ & $1.57 \mathrm{E}+06$ & $0.45 \%$ \\
\hline 6EA012 & $1.72 \mathrm{E}+02$ & 0 & 0 & 0 & 0 & 0 & 0 & 0 & $1.32 \mathrm{E}+03$ & 0 & 0 & 0 & $1.50 \mathrm{E}+03$ & $1.61 \mathrm{E}+06$ & $0.09 \%$ \\
\hline 6EA013 & $6.59 \mathrm{E}+02$ & 0 & 0 & 0 & 0 & 0 & 0 & 0 & 0 & 0 & 0 & 0 & $6.59 \mathrm{E}+02$ & $1.64 \mathrm{E}+06$ & $0.04 \%$ \\
\hline 6EA014 & $5.09 \mathrm{E}+02$ & 0 & 0 & 0 & 0 & $3.38 \mathrm{E}+03$ & 0 & 0 & 0 & 0 & 0 & 0 & $3.89 \mathrm{E}+03$ & $1.59 \mathrm{E}+06$ & $0.24 \%$ \\
\hline 6EA015 & $3.61 \mathrm{E}+02$ & 0 & 0 & 0 & 0 & $2.35 \mathrm{E}+03$ & 0 & 0 & $1.39 \mathrm{E}+03$ & 0 & 0 & 0 & $4.09 \mathrm{E}+03$ & $1.55 \mathrm{E}+06$ & $0.26 \%$ \\
\hline 6EA016 & $1.04 \mathrm{E}+03$ & $2.71 \mathrm{E}+04$ & $6.82 \mathrm{E}-05$ & 0 & 0 & $5.56 \mathrm{E}+03$ & 0 & 0 & $4.28 \mathrm{E}+03$ & 0 & 0 & 0 & $3.80 \mathrm{E}+04$ & $1.57 \mathrm{E}+06$ & $2.43 \%$ \\
\hline
\end{tabular}


Table 36. Predicted Solids for Envelope A Simulations - Part 2 (cont'd)

\begin{tabular}{|c|c|c|c|c|c|c|c|c|c|c|c|c|c|c|c|}
\hline Test ID & $\begin{array}{c}\mathrm{NA} 2 \mathrm{C} 2 \mathrm{O} \\
4\end{array}$ & $\begin{array}{c}\text { NA2CO3. } \\
1 \mathrm{H} 2 \mathrm{O}\end{array}$ & $\begin{array}{c}\mathrm{NA} 2 \mathrm{U} 2 \mathrm{O} \\
7\end{array}$ & NA3FSO4 & $\begin{array}{c}\mathrm{NA} 6 \mathrm{SO} 42 \\
\mathrm{CO} 3\end{array}$ & NAF & $\begin{array}{c}\text { NAFPO4. } \\
19 \mathrm{H} 2 \mathrm{O}\end{array}$ & NANO3 & $\begin{array}{c}\text { NAPHOH } \\
.12 \mathrm{H} 2 \mathrm{O}\end{array}$ & $\begin{array}{c}\text { NASGEL. } \\
15.5 \mathrm{H} 2 \mathrm{O}\end{array}$ & ZRO2 & $\begin{array}{c}\text { ZRSO42. } \\
4 \mathrm{H} 2 \mathrm{O}\end{array}$ & $\begin{array}{l}\text { Total } \\
\text { Solids }\end{array}$ & $\begin{array}{c}\text { Total Soln } \\
\text { Mass }\end{array}$ & $\begin{array}{c}\text { Solids } \% \\
\text { of Total } \\
\text { Soln }\end{array}$ \\
\hline 6EA017 & $9.66 \mathrm{E}+02$ & $3.69 \mathrm{E}+04$ & $3.00 \mathrm{E}-05$ & 0 & 0 & 0 & 0 & 0 & 0 & 0 & 0 & 0 & $4.07 \mathrm{E}+04$ & $1.60 \mathrm{E}+06$ & $2.54 \%$ \\
\hline 6EA018 & $6.89 \mathrm{E}+02$ & 0 & 0 & 0 & 0 & 0 & 0 & 0 & $1.92 \mathrm{E}+04$ & 0 & 0 & 0 & $2.27 \mathrm{E}+04$ & $1.63 \mathrm{E}+06$ & $1.40 \%$ \\
\hline 6ЕA019 & $5.47 \mathrm{E}+02$ & 0 & 0 & 0 & 0 & 0 & 0 & 0 & $1.68 \mathrm{E}+04$ & 0 & 0 & 0 & $2.19 \mathrm{E}+04$ & $1.62 \mathrm{E}+06$ & $1.35 \%$ \\
\hline 6ЕA020 & $4.04 \mathrm{E}+02$ & 0 & 0 & 0 & 0 & $3.05 \mathrm{E}+03$ & 0 & 0 & $4.45 \mathrm{E}+02$ & 0 & 0 & 0 & $1.07 \mathrm{E}+04$ & $1.58 \mathrm{E}+06$ & $0.68 \%$ \\
\hline 6EA021 & $1.51 \mathrm{E}+02$ & 0 & 0 & 0 & 0 & 0 & 0 & 0 & $6.45 \mathrm{E}+03$ & 0 & 0 & 0 & $7.04 \mathrm{E}+03$ & $1.63 \mathrm{E}+06$ & $0.43 \%$ \\
\hline 6EA022 & $1.35 \mathrm{E}+02$ & 0 & 0 & 0 & 0 & 0 & 0 & 0 & $9.15 \mathrm{E}+02$ & 0 & 0 & 0 & $1.49 \mathrm{E}+03$ & $1.66 \mathrm{E}+06$ & $0.09 \%$ \\
\hline 6EA023 & $5.79 \mathrm{E}+02$ & 0 & 0 & 0 & 0 & 0 & 0 & 0 & 0 & $1.77 \mathrm{E}+03$ & 0 & 0 & $2.93 \mathrm{E}+03$ & $1.70 \mathrm{E}+06$ & $0.17 \%$ \\
\hline 6EA024 & $4.44 \mathrm{E}+02$ & 0 & 0 & 0 & 0 & $2.83 \mathrm{E}+03$ & 0 & 0 & 0 & $1.47 \mathrm{E}+03$ & 0 & 0 & $5.24 \mathrm{E}+03$ & $1.65 \mathrm{E}+06$ & $0.32 \%$ \\
\hline 6ЕA025 & $3.03 \mathrm{E}+02$ & 0 & 0 & 0 & 0 & $1.84 \mathrm{E}+03$ & 0 & 0 & $5.98 \mathrm{E}+02$ & $1.01 \mathrm{E}+03$ & 0 & 0 & $4.22 \mathrm{E}+03$ & $1.61 \mathrm{E}+06$ & $0.26 \%$ \\
\hline 6EA026 & $8.93 \mathrm{E}+02$ & $1.30 \mathrm{E}+04$ & $6.33 \mathrm{E}-05$ & 0 & 0 & $4.58 \mathrm{E}+03$ & 0 & 0 & $2.22 \mathrm{E}+03$ & $1.73 \mathrm{E}+03$ & 0 & 0 & $2.30 \mathrm{E}+04$ & $1.65 \mathrm{E}+06$ & $1.39 \%$ \\
\hline 6EA027 & $8.61 \mathrm{E}+02$ & $2.53 \mathrm{E}+04$ & $2.90 \mathrm{E}-05$ & 0 & 0 & 0 & 0 & 0 & 0 & $1.51 \mathrm{E}+03$ & 0 & 0 & $3.47 \mathrm{E}+04$ & $1.68 \mathrm{E}+06$ & $2.06 \%$ \\
\hline 6EA028 & $6.27 \mathrm{E}+02$ & 0 & 0 & 0 & 0 & 0 & 0 & 0 & $1.83 \mathrm{E}+04$ & $1.50 \mathrm{E}+03$ & 0 & 0 & $2.80 \mathrm{E}+04$ & $1.69 \mathrm{E}+06$ & $1.65 \%$ \\
\hline 6ЕA029 & $4.93 \mathrm{E}+02$ & 0 & 0 & 0 & 0 & 0 & 0 & 0 & $1.60 \mathrm{E}+04$ & $1.44 \mathrm{E}+03$ & 0 & 0 & $2.73 \mathrm{E}+04$ & $1.68 \mathrm{E}+06$ & $1.63 \%$ \\
\hline 6EA030 & $3.63 \mathrm{E}+02$ & 0 & 0 & 0 & 0 & $2.61 \mathrm{E}+03$ & 0 & 0 & 0 & $1.33 \mathrm{E}+03$ & 0 & 0 & $1.57 \mathrm{E}+04$ & $1.64 \mathrm{E}+06$ & $0.95 \%$ \\
\hline 6EA031 & $1.51 \mathrm{E}+02$ & 0 & 0 & 0 & 0 & 0 & 0 & 0 & $6.45 \mathrm{E}+03$ & 0 & 0 & 0 & $7.04 \mathrm{E}+03$ & $1.63 \mathrm{E}+06$ & $0.43 \%$ \\
\hline 6ЕA032 & $1.35 \mathrm{E}+02$ & 0 & 0 & 0 & 0 & 0 & 0 & 0 & $9.15 \mathrm{E}+02$ & 0 & 0 & 0 & $1.49 \mathrm{E}+03$ & $1.66 \mathrm{E}+06$ & $0.09 \%$ \\
\hline 6EA033 & $5.79 \mathrm{E}+02$ & 0 & 0 & 0 & 0 & 0 & 0 & 0 & 0 & $1.77 \mathrm{E}+03$ & 0 & 0 & $2.93 \mathrm{E}+03$ & $1.70 \mathrm{E}+06$ & $0.17 \%$ \\
\hline 6ЕA034 & $4.44 \mathrm{E}+02$ & 0 & 0 & 0 & 0 & $2.83 \mathrm{E}+03$ & 0 & 0 & 0 & $1.47 \mathrm{E}+03$ & 0 & 0 & $5.24 \mathrm{E}+03$ & $1.65 \mathrm{E}+06$ & $0.32 \%$ \\
\hline 6EA035 & $3.03 \mathrm{E}+02$ & 0 & 0 & 0 & 0 & $1.84 \mathrm{E}+03$ & 0 & 0 & $5.98 \mathrm{E}+02$ & $1.01 \mathrm{E}+03$ & 0 & 0 & $4.22 \mathrm{E}+03$ & $1.61 \mathrm{E}+06$ & $0.26 \%$ \\
\hline 6EA036 & $8.93 \mathrm{E}+02$ & $1.30 \mathrm{E}+04$ & $6.33 \mathrm{E}-05$ & 0 & 0 & $4.58 \mathrm{E}+03$ & 0 & 0 & $2.22 \mathrm{E}+03$ & $1.73 \mathrm{E}+03$ & 0 & 0 & $2.30 \mathrm{E}+04$ & $1.65 \mathrm{E}+06$ & $1.39 \%$ \\
\hline 6EA037 & $8.61 \mathrm{E}+02$ & $2.53 \mathrm{E}+04$ & $2.90 \mathrm{E}-05$ & 0 & 0 & 0 & 0 & 0 & 0 & $1.51 \mathrm{E}+03$ & 0 & 0 & $3.47 \mathrm{E}+04$ & $1.68 \mathrm{E}+06$ & $2.06 \%$ \\
\hline 6EA038 & $6.27 \mathrm{E}+02$ & 0 & 0 & 0 & 0 & 0 & 0 & 0 & $1.83 \mathrm{E}+04$ & $1.50 \mathrm{E}+03$ & 0 & 0 & $2.80 \mathrm{E}+04$ & $1.69 \mathrm{E}+06$ & $1.65 \%$ \\
\hline 6ЕA039 & $4.93 \mathrm{E}+02$ & 0 & 0 & 0 & 0 & 0 & 0 & 0 & $1.60 \mathrm{E}+04$ & $1.44 \mathrm{E}+03$ & 0 & 0 & $2.73 \mathrm{E}+04$ & $1.68 \mathrm{E}+06$ & $1.63 \%$ \\
\hline 6EA040 & $3.63 \mathrm{E}+02$ & 0 & 0 & 0 & 0 & $2.61 \mathrm{E}+03$ & 0 & 0 & 0 & $1.33 \mathrm{E}+03$ & 0 & 0 & $1.57 \mathrm{E}+04$ & $1.64 \mathrm{E}+06$ & $0.95 \%$ \\
\hline 6ЕA041 & 0 & 0 & 0 & 0 & 0 & 0 & 0 & 0 & 0 & 0 & 0 & 0 & $0.00 \mathrm{E}+00$ & $1.53 \mathrm{E}+06$ & $0.00 \%$ \\
\hline 6ЕA042 & 0 & 0 & 0 & 0 & 0 & 0 & 0 & 0 & 0 & 0 & 0 & 0 & $0.00 \mathrm{E}+00$ & $1.56 \mathrm{E}+06$ & $0.00 \%$ \\
\hline 6EA043 & 0 & 0 & 0 & 0 & 0 & 0 & 0 & 0 & 0 & 0 & 0 & 0 & $0.00 \mathrm{E}+00$ & $1.57 \mathrm{E}+06$ & $0.00 \%$ \\
\hline 6EA044 & 0 & 0 & 0 & 0 & 0 & 0 & 0 & 0 & 0 & 0 & 0 & 0 & $0.00 \mathrm{E}+00$ & $1.55 \mathrm{E}+06$ & $0.00 \%$ \\
\hline $6 \mathrm{EA} 045$ & 0 & 0 & 0 & 0 & 0 & 0 & 0 & 0 & 0 & 0 & 0 & 0 & $0.00 \mathrm{E}+00$ & $1.50 \mathrm{E}+06$ & $0.00 \%$ \\
\hline 6EA046 & 0 & 0 & 0 & 0 & 0 & $1.00 \mathrm{E}+03$ & 0 & 0 & 0 & 0 & 0 & 0 & $1.00 \mathrm{E}+03$ & $1.56 \mathrm{E}+06$ & $0.06 \%$ \\
\hline 6EA047 & 0 & 0 & 0 & 0 & 0 & 0 & 0 & 0 & 0 & 0 & 0 & 0 & $0.00 \mathrm{E}+00$ & $1.58 \mathrm{E}+06$ & $0.00 \%$ \\
\hline 6ЕA048 & 0 & 0 & 0 & 0 & 0 & 0 & 0 & 0 & 0 & 0 & 0 & 0 & $0.00 \mathrm{E}+00$ & $1.58 \mathrm{E}+06$ & $0.00 \%$ \\
\hline 6ЕA049 & 0 & 0 & 0 & 0 & 0 & 0 & 0 & 0 & 0 & 0 & 0 & 0 & $0.00 \mathrm{E}+00$ & $1.57 \mathrm{E}+06$ & $0.00 \%$ \\
\hline
\end{tabular}


Table 36. Predicted Solids for Envelope A Simulations - Part 2 (cont'd)

\begin{tabular}{|c|c|c|c|c|c|c|c|c|c|c|c|c|c|c|c|}
\hline Test ID & \begin{tabular}{|c|} 
NA2C2O \\
4 \\
\end{tabular} & $\begin{array}{c}\text { NA2CO3. } \\
1 \mathrm{H} 2 \mathrm{O}\end{array}$ & $\begin{array}{c}\text { NA2U2O } \\
7 \\
\end{array}$ & NA3FSO4 & $\begin{array}{c}\text { NA6SO42 } \\
\mathrm{CO} 3 \\
\end{array}$ & NAF & $\begin{array}{c}\text { NAFPO4. } \\
19 \mathrm{H} 2 \mathrm{O}\end{array}$ & NANO3 & $\begin{array}{c}\text { NAPHOH } \\
.12 \mathrm{H} 2 \mathrm{O} \\
\end{array}$ & $\begin{array}{c}\text { NASGEL. } \\
15.5 \mathrm{H} 2 \mathrm{O}\end{array}$ & ZRO2 & $\begin{array}{c}\text { ZRSO42. } \\
4 \mathrm{H} 2 \mathrm{O}\end{array}$ & $\begin{array}{c}\text { Total } \\
\text { Solids } \\
\end{array}$ & $\begin{array}{c}\text { Total Soln } \\
\text { Mass }\end{array}$ & $\begin{array}{c}\text { Solids } \% \\
\text { of Total } \\
\text { Soln } \\
\end{array}$ \\
\hline $6 \mathrm{EA} 050$ & 0 & 0 & 0 & 0 & 0 & 0 & 0 & 0 & 0 & 0 & 0 & 0 & $0.00 \mathrm{E}+00$ & $1.54 \mathrm{E}+06$ & $0.00 \%$ \\
\hline 6EA051 & 0 & 0 & 0 & 0 & 0 & 0 & 0 & 0 & 0 & 0 & 0 & 0 & $0.00 \mathrm{E}+00$ & $1.53 \mathrm{E}+06$ & $0.00 \%$ \\
\hline 6EA052 & 0 & 0 & 0 & 0 & 0 & 0 & 0 & 0 & 0 & 0 & 0 & 0 & $0.00 \mathrm{E}+00$ & $1.56 \mathrm{E}+06$ & $0.00 \%$ \\
\hline 6EA053 & 0 & 0 & 0 & 0 & 0 & 0 & 0 & 0 & 0 & 0 & 0 & 0 & $0.00 \mathrm{E}+00$ & $1.57 \mathrm{E}+06$ & $0.00 \%$ \\
\hline 6EA054 & 0 & 0 & 0 & 0 & 0 & 0 & 0 & 0 & 0 & 0 & 0 & 0 & $0.00 \mathrm{E}+00$ & $1.55 \mathrm{E}+06$ & $0.00 \%$ \\
\hline $6 \mathrm{EA} 055$ & 0 & 0 & 0 & 0 & 0 & 0 & 0 & 0 & 0 & 0 & 0 & 0 & $0.00 \mathrm{E}+00$ & $1.50 \mathrm{E}+06$ & $0.00 \%$ \\
\hline 6EA056 & 0 & 0 & 0 & 0 & 0 & $1.00 \mathrm{E}+03$ & 0 & 0 & 0 & 0 & 0 & 0 & $1.00 \mathrm{E}+03$ & $1.56 \mathrm{E}+06$ & $0.06 \%$ \\
\hline 6EA057 & 0 & 0 & 0 & 0 & 0 & 0 & 0 & 0 & 0 & 0 & 0 & 0 & $0.00 \mathrm{E}+00$ & $1.58 \mathrm{E}+06$ & $0.00 \%$ \\
\hline 6EA058 & 0 & 0 & 0 & 0 & 0 & 0 & 0 & 0 & 0 & 0 & 0 & 0 & $0.00 \mathrm{E}+00$ & $1.58 \mathrm{E}+06$ & $0.00 \%$ \\
\hline 6EA059 & 0 & 0 & 0 & 0 & 0 & 0 & 0 & 0 & 0 & 0 & 0 & 0 & $0.00 \mathrm{E}+00$ & $1.57 \mathrm{E}+06$ & $0.00 \%$ \\
\hline 6EA060 & 0 & 0 & 0 & 0 & 0 & 0 & 0 & 0 & 0 & 0 & 0 & 0 & $0.00 \mathrm{E}+00$ & $1.54 \mathrm{E}+06$ & $0.00 \%$ \\
\hline 6EA061 & 0 & 0 & 0 & 0 & 0 & 0 & 0 & 0 & 0 & 0 & 0 & 0 & $5.58 \mathrm{E}+02$ & $1.58 \mathrm{E}+06$ & $0.04 \%$ \\
\hline 6EA062 & 0 & 0 & 0 & 0 & 0 & 0 & 0 & 0 & 0 & 0 & 0 & 0 & $5.65 \mathrm{E}+02$ & $1.61 \mathrm{E}+06$ & $0.04 \%$ \\
\hline $6 \mathrm{EA} 063$ & 0 & 0 & 0 & 0 & 0 & 0 & 0 & 0 & 0 & $1.31 \mathrm{E}+03$ & $2.03 \mathrm{E}+00$ & 0 & $1.98 \mathrm{E}+03$ & $1.63 \mathrm{E}+06$ & $0.12 \%$ \\
\hline 6EA064 & 0 & 0 & 0 & 0 & 0 & 0 & 0 & 0 & 0 & $7.33 \mathrm{E}+02$ & 1.83E-01 & 0 & $1.24 \mathrm{E}+03$ & $1.60 \mathrm{E}+06$ & $0.08 \%$ \\
\hline 6EA065 & 0 & 0 & 0 & 0 & 0 & 0 & 0 & 0 & 0 & $1.52 \mathrm{E}+02$ & 0 & 0 & $6.25 \mathrm{E}+02$ & $1.55 \mathrm{E}+06$ & $0.04 \%$ \\
\hline 6EA066 & 0 & 0 & 0 & 0 & 0 & $4.18 \mathrm{E}+02$ & 0 & 0 & 0 & $1.48 \mathrm{E}+03$ & $1.81 \mathrm{E}+00$ & 0 & $2.45 \mathrm{E}+03$ & $1.62 \mathrm{E}+06$ & $0.15 \%$ \\
\hline 6ЕA067 & 0 & 0 & 0 & 0 & 0 & 0 & 0 & 0 & 0 & $1.38 \mathrm{E}+03$ & 7.78E-01 & 0 & $2.04 \mathrm{E}+03$ & $1.64 \mathrm{E}+06$ & $0.12 \%$ \\
\hline 6EA068 & 0 & 0 & 0 & 0 & 0 & 0 & 0 & 0 & 0 & $1.20 \mathrm{E}+03$ & 7.34E-01 & 0 & $1.86 \mathrm{E}+03$ & $1.64 \mathrm{E}+06$ & $0.11 \%$ \\
\hline 6EA069 & 0 & 0 & 0 & 0 & 0 & 0 & 0 & 0 & 0 & $1.10 \mathrm{E}+03$ & 2.89E-02 & 0 & $1.73 \mathrm{E}+03$ & $1.62 \mathrm{E}+06$ & $0.11 \%$ \\
\hline 6EA070 & 0 & 0 & 0 & 0 & 0 & 0 & 0 & 0 & 0 & $6.64 \mathrm{E}+02$ & 0 & 0 & $1.15 \mathrm{E}+03$ & $1.59 \mathrm{E}+06$ & $0.07 \%$ \\
\hline 6EA071 & 0 & 0 & 0 & 0 & 0 & 0 & 0 & 0 & 0 & 0 & 0 & 0 & $5.58 \mathrm{E}+02$ & $1.58 \mathrm{E}+06$ & $0.04 \%$ \\
\hline 6EA072 & 0 & 0 & 0 & 0 & 0 & 0 & 0 & 0 & 0 & 0 & 0 & 0 & $5.65 \mathrm{E}+02$ & $1.61 \mathrm{E}+06$ & $0.04 \%$ \\
\hline 6EA073 & 0 & 0 & 0 & 0 & 0 & 0 & 0 & 0 & 0 & $1.31 \mathrm{E}+03$ & $2.03 \mathrm{E}+00$ & 0 & $1.98 \mathrm{E}+03$ & $1.63 \mathrm{E}+06$ & $0.12 \%$ \\
\hline 6EA074 & 0 & 0 & 0 & 0 & 0 & 0 & 0 & 0 & 0 & $7.33 \mathrm{E}+02$ & 1.83E-01 & 0 & $1.24 \mathrm{E}+03$ & $1.60 \mathrm{E}+06$ & $0.08 \%$ \\
\hline 6EA075 & 0 & 0 & 0 & 0 & 0 & 0 & 0 & 0 & 0 & $1.52 \mathrm{E}+02$ & 0 & 0 & $6.25 \mathrm{E}+02$ & $1.55 \mathrm{E}+06$ & $0.04 \%$ \\
\hline 6EA076 & 0 & 0 & 0 & 0 & 0 & $4.18 \mathrm{E}+02$ & 0 & 0 & 0 & $1.48 \mathrm{E}+03$ & $1.81 \mathrm{E}+00$ & 0 & $2.45 \mathrm{E}+03$ & $1.62 \mathrm{E}+06$ & $0.15 \%$ \\
\hline 6EA077 & 0 & 0 & 0 & 0 & 0 & 0 & 0 & 0 & 0 & $1.38 \mathrm{E}+03$ & 7.78E-01 & 0 & $2.04 \mathrm{E}+03$ & $1.64 \mathrm{E}+06$ & $0.12 \%$ \\
\hline 6EA078 & 0 & 0 & 0 & 0 & 0 & 0 & 0 & 0 & 0 & $1.20 \mathrm{E}+03$ & 7.34E-01 & 0 & $1.86 \mathrm{E}+03$ & $1.64 \mathrm{E}+06$ & $0.11 \%$ \\
\hline 6EA079 & 0 & 0 & 0 & 0 & 0 & 0 & 0 & 0 & 0 & $1.10 \mathrm{E}+03$ & 2.89E-02 & 0 & $1.73 \mathrm{E}+03$ & $1.62 \mathrm{E}+06$ & $0.11 \%$ \\
\hline 6ЕA080 & 0 & 0 & 0 & 0 & 0 & 0 & 0 & 0 & 0 & $6.64 \mathrm{E}+02$ & 0 & 0 & $1.15 \mathrm{E}+03$ & $1.59 \mathrm{E}+06$ & $0.07 \%$ \\
\hline 6EA081 & $6.85 \mathrm{E}+02$ & 0 & $3.78 \mathrm{E}-06$ & 0 & 0 & $4.10 \mathrm{E}+03$ & 0 & 0 & $2.69 \mathrm{E}+03$ & 0 & 0 & 0 & $1.13 \mathrm{E}+04$ & $1.59 \mathrm{E}+06$ & $0.71 \%$ \\
\hline 6EA082 & $5.20 \mathrm{E}+02$ & 0 & 0 & 0 & 0 & $3.20 \mathrm{E}+03$ & 0 & 0 & $1.33 \mathrm{E}+03$ & $1.39 \mathrm{E}+03$ & 0 & 0 & $1.48 \mathrm{E}+04$ & $1.65 \mathrm{E}+06$ & $0.90 \%$ \\
\hline
\end{tabular}


Table 36. Predicted Solids for Envelope A Simulations - Part 2 (cont'd)

\begin{tabular}{|c|c|c|c|c|c|c|c|c|c|c|c|c|c|c|c|}
\hline Test ID & $\begin{array}{c}\mathrm{NA} 2 \mathrm{C} 2 \mathrm{O} \\
4\end{array}$ & $\begin{array}{c}\text { NA2 } 2 \mathrm{CO} 3 . \\
1 \mathrm{H} 2 \mathrm{O}\end{array}$ & $\begin{array}{c}\text { NA2U2O } \\
7\end{array}$ & NA3FSO4 & $\begin{array}{c}\mathrm{NA} 6 \mathrm{SO} 42 \\
\mathrm{CO} 3\end{array}$ & NAF & $\begin{array}{c}\text { NAFPO4. } \\
19 \mathrm{H} 2 \mathrm{O}\end{array}$ & NANO3 & $\begin{array}{c}\text { NAPHOH } \\
.12 \mathrm{H} 2 \mathrm{O}\end{array}$ & $\begin{array}{c}\text { NASGEL. } \\
15.5 \mathrm{H} 2 \mathrm{O}\end{array}$ & ZRO2 & $\begin{array}{c}\text { ZRSO42. } \\
4 \mathrm{H} 2 \mathrm{O}\end{array}$ & $\begin{array}{l}\text { Total } \\
\text { Solids }\end{array}$ & $\begin{array}{c}\text { Total Soln } \\
\text { Mass }\end{array}$ & $\begin{array}{c}\text { Solids \% } \\
\text { of Total } \\
\text { Soln }\end{array}$ \\
\hline 6EA083 & $6.66 \mathrm{E}+02$ & 0 & $1.25 \mathrm{E}-07$ & 0 & 0 & $3.26 \mathrm{E}+03$ & 0 & 0 & 0 & $3.91 \mathrm{E}+02$ & 0 & 0 & $8.41 \mathrm{E}+03$ & $1.65 \mathrm{E}+06$ & $0.51 \%$ \\
\hline 6EA084 & $6.18 \mathrm{E}+02$ & 0 & 0 & 0 & 0 & $4.04 \mathrm{E}+03$ & 0 & 0 & $8.18 \mathrm{E}+02$ & 0 & 0 & 0 & $8.87 \mathrm{E}+03$ & $1.61 \mathrm{E}+06$ & $0.55 \%$ \\
\hline 6EA085 & $6.80 \mathrm{E}+02$ & 0 & $3.92 \mathrm{E}-06$ & 0 & 0 & $4.00 \mathrm{E}+03$ & 0 & 0 & $1.06 \mathrm{E}+03$ & 0 & 0 & 0 & $5.99 \mathrm{E}+03$ & $1.60 \mathrm{E}+06$ & $0.38 \%$ \\
\hline 6EA086 & $5.43 \mathrm{E}+02$ & 0 & $1.55 \mathrm{E}-06$ & 0 & 0 & 0 & 0 & 0 & 0 & $9.60 \mathrm{E}+02$ & 0 & 0 & $1.81 \mathrm{E}+03$ & $1.67 \mathrm{E}+06$ & $0.11 \%$ \\
\hline 6EA087 & $6.66 \mathrm{E}+02$ & 0 & $7.27 \mathrm{E}-06$ & 0 & 0 & 0 & 0 & 0 & $1.00 \mathrm{E}+04$ & $1.17 \mathrm{E}+03$ & 0 & 0 & $1.62 \mathrm{E}+04$ & $1.68 \mathrm{E}+06$ & $0.97 \%$ \\
\hline 6EA088 & $6.92 \mathrm{E}+02$ & 0 & $1.17 \mathrm{E}-05$ & 0 & 0 & $4.08 \mathrm{E}+03$ & $5.50 \mathrm{E}+02$ & 0 & 0 & $4.22 \mathrm{E}+02$ & 0 & 0 & $8.00 \mathrm{E}+03$ & $1.64 \mathrm{E}+06$ & $0.49 \%$ \\
\hline 6ЕA089 & $3.98 \mathrm{E}+02$ & 0 & 0 & 0 & 0 & $2.88 \mathrm{E}+03$ & $1.41 \mathrm{E}+02$ & 0 & 0 & $7.01 \mathrm{E}+02$ & 0 & 0 & $8.45 \mathrm{E}+03$ & $1.63 \mathrm{E}+06$ & $0.52 \%$ \\
\hline 6EA090 & $4.08 \mathrm{E}+02$ & 0 & 0 & 0 & 0 & $4.53 \mathrm{E}+02$ & $4.82 \mathrm{E}+02$ & 0 & 0 & $1.36 \mathrm{E}+02$ & 0 & 0 & $1.63 \mathrm{E}+03$ & $1.64 \mathrm{E}+06$ & $0.10 \%$ \\
\hline 6EA091 & $4.52 \mathrm{E}+02$ & 0 & 0 & 0 & 0 & 0 & 0 & 0 & 0 & $5.73 \mathrm{E}+02$ & 0 & 0 & $1.24 \mathrm{E}+03$ & $1.66 \mathrm{E}+06$ & $0.07 \%$ \\
\hline 6EA092 & $5.20 \mathrm{E}+02$ & 0 & 0 & 0 & 0 & 0 & 0 & 0 & 0 & 0 & 0 & 0 & $1.51 \mathrm{E}+03$ & $1.64 \mathrm{E}+06$ & $0.09 \%$ \\
\hline 6EA093 & $5.75 \mathrm{E}+02$ & 0 & $2.00 \mathrm{E}-06$ & 0 & 0 & 0 & $2.71 \mathrm{E}+03$ & 0 & 0 & $6.67 \mathrm{E}+02$ & 0 & 0 & $5.76 \mathrm{E}+03$ & $1.65 \mathrm{E}+06$ & $0.35 \%$ \\
\hline 6EA094 & $6.36 \mathrm{E}+02$ & 0 & $2.77 \mathrm{E}-06$ & 0 & 0 & 0 & 0 & 0 & 0 & $2.38 \mathrm{E}+02$ & 0 & 0 & $1.13 \mathrm{E}+03$ & $1.66 \mathrm{E}+06$ & $0.07 \%$ \\
\hline 6EA095 & $4.41 \mathrm{E}+02$ & 0 & 0 & 0 & 0 & $3.01 \mathrm{E}+03$ & $3.94 \mathrm{E}+02$ & 0 & 0 & $9.21 \mathrm{E}+02$ & 0 & 0 & $6.86 \mathrm{E}+03$ & $1.62 \mathrm{E}+06$ & $0.42 \%$ \\
\hline 6EA096 & $4.68 \mathrm{E}+02$ & 0 & $9.76 \mathrm{E}-07$ & 0 & 0 & 0 & 0 & 0 & 0 & \begin{tabular}{|l|}
$1.44 \mathrm{E}+03$ \\
\end{tabular} & 0 & 0 & $4.69 \mathrm{E}+03$ & $1.69 \mathrm{E}+06$ & $0.28 \%$ \\
\hline 6EA097 & $5.41 \mathrm{E}+02$ & 0 & $3.23 \mathrm{E}-06$ & 0 & 0 & 0 & 0 & 0 & 0 & 0 & 0 & 0 & $5.80 \mathrm{E}+02$ & $1.64 \mathrm{E}+06$ & $0.04 \%$ \\
\hline 6EA098 & $3.01 \mathrm{E}+02$ & 0 & 0 & 0 & 0 & 0 & 0 & 0 & $2.48 \mathrm{E}+03$ & $\mid 1.11 \mathrm{E}+03$ & 0 & 0 & $4.29 \mathrm{E}+03$ & $1.65 \mathrm{E}+06$ & $0.26 \%$ \\
\hline 6EA099 & $5.28 \mathrm{E}+02$ & 0 & $2.06 \mathrm{E}-06$ & $5.84 \mathrm{E}+03$ & 0 & $2.19 \mathrm{E}+03$ & $1.51 \mathrm{E}+03$ & 0 & 0 & 0 & 0 & 0 & $1.01 \mathrm{E}+04$ & $1.59 \mathrm{E}+06$ & $0.64 \%$ \\
\hline 6EA100 & $4.29 \mathrm{E}+02$ & 0 & $2.46 \mathrm{E}-07$ & 0 & 0 & 0 & 0 & 0 & 0 & $\mid 1.23 \mathrm{E}+03$ & 0 & 0 & $2.16 \mathrm{E}+03$ & $1.68 \mathrm{E}+06$ & $0.13 \%$ \\
\hline 6EA101 & $4.73 \mathrm{E}+02$ & 0 & $1.25 \mathrm{E}-07$ & $1.76 \mathrm{E}+03$ & 0 & 0 & $2.92 \mathrm{E}+03$ & 0 & 0 & $5.25 \mathrm{E}+02$ & 0 & 0 & $5.90 \mathrm{E}+03$ & $1.63 \mathrm{E}+06$ & $0.36 \%$ \\
\hline 6EA102 & $5.14 \mathrm{E}+02$ & 0 & $3.71 \mathrm{E}-06$ & $7.80 \mathrm{E}+03$ & 0 & $1.26 \mathrm{E}+03$ & 0 & 0 & 0 & $7.29 \mathrm{E}+02$ & 0 & 0 & $1.06 \mathrm{E}+04$ & $1.64 \mathrm{E}+06$ & $0.65 \%$ \\
\hline 6EA103 & $3.23 \mathrm{E}+02$ & 0 & 0 & 0 & 0 & 0 & $2.82 \mathrm{E}+03$ & 0 & 0 & $6.26 \mathrm{E}+02$ & 0 & 0 & $4.03 \mathrm{E}+03$ & $1.64 \mathrm{E}+06$ & $0.25 \%$ \\
\hline 6EA104 & $3.91 \mathrm{E}+02$ & 0 & $5.09 \mathrm{E}-07$ & $7.30 \mathrm{E}+03$ & 0 & 0 & $1.84 \mathrm{E}+03$ & 0 & 0 & \begin{tabular}{|l|}
$7.63 \mathrm{E}+02$ \\
\end{tabular} & 0 & 0 & $1.06 \mathrm{E}+04$ & $1.64 \mathrm{E}+06$ & $0.65 \%$ \\
\hline 6EA105 & $3.14 \mathrm{E}+02$ & 0 & 0 & 0 & 0 & 0 & 0 & 0 & 0 & $8.93 \mathrm{E}+01$ & 0 & 0 & $5.28 \mathrm{E}+02$ & $1.64 \mathrm{E}+06$ & $0.03 \%$ \\
\hline 6EA106 & $3.80 \mathrm{E}+02$ & 0 & $1.68 \mathrm{E}-07$ & \begin{tabular}{|c|}
$7.97 \mathrm{E}+03$ \\
\end{tabular} & 0 & $1.29 \mathrm{E}+03$ & $1.21 \mathrm{E}+03$ & 0 & 0 & \begin{tabular}{|l|}
$8.89 \mathrm{E}+02$ \\
\end{tabular} & 0 & 0 & $1.21 \mathrm{E}+04$ & $1.63 \mathrm{E}+06$ & $0.74 \%$ \\
\hline 6EA107 & $2.88 \mathrm{E}+02$ & 0 & 0 & 0 & 0 & 0 & 0 & 0 & 0 & 0 & 0 & 0 & $2.90 \mathrm{E}+02$ & $1.62 \mathrm{E}+06$ & $0.02 \%$ \\
\hline 6EA108 & $3.26 \mathrm{E}+02$ & 0 & 0 & $7.68 \mathrm{E}+03$ & 0 & $1.06 \mathrm{E}+03$ & $9.77 \mathrm{E}+02$ & 0 & 0 & \begin{tabular}{|l|}
$1.01 \mathrm{E}+03$ \\
\end{tabular} & 0 & 0 & $1.14 \mathrm{E}+04$ & $1.60 \mathrm{E}+06$ & $0.71 \%$ \\
\hline 6EA109 & $2.80 \mathrm{E}+02$ & 0 & 0 & 0 & 0 & 0 & 0 & 0 & 0 & $6.50 \mathrm{E}+02$ & 0 & 0 & $1.20 \mathrm{E}+03$ & $1.65 \mathrm{E}+06$ & $0.07 \%$ \\
\hline 6EA110 & $2.60 \mathrm{E}+02$ & 0 & 0 & $8.06 \mathrm{E}+03$ & 0 & 0 & 0 & 0 & 0 & $7.74 \mathrm{E}+02$ & 0 & 0 & $9.41 \mathrm{E}+03$ & $1.64 \mathrm{E}+06$ & $0.57 \%$ \\
\hline 6EA111 & $3.18 \mathrm{E}+02$ & 0 & 0 & 0 & 0 & 0 & 0 & 0 & 0 & $7.24 \mathrm{E}+02$ & 0 & 0 & $1.33 \mathrm{E}+03$ & $1.66 \mathrm{E}+06$ & $0.08 \%$ \\
\hline 6EA112 & $3.20 \mathrm{E}+02$ & 0 & $7.23 \mathrm{E}-08$ & 0 & 0 & 0 & $2.91 \mathrm{E}+03$ & 0 & 0 & $2.42 \mathrm{E}+02$ & 0 & 0 & $3.63 \mathrm{E}+03$ & $1.64 \mathrm{E}+06$ & $0.22 \%$ \\
\hline 6EA113 & $2.94 \mathrm{E}+02$ & 0 & $1.74 \mathrm{E}-07$ & 0 & 0 & 0 & $2.73 \mathrm{E}+03$ & 0 & 0 & $6.61 \mathrm{E}+02$ & 0 & 0 & $3.95 \mathrm{E}+03$ & $1.63 \mathrm{E}+06$ & $0.24 \%$ \\
\hline 6EA114 & $3.33 \mathrm{E}+02$ & 0 & $1.52 \mathrm{E}-07$ & 0 & 0 & 0 & 0 & 0 & 0 & $2.83 \mathrm{E}+02$ & 0 & 0 & $7.84 \mathrm{E}+02$ & $1.64 \mathrm{E}+06$ & $0.05 \%$ \\
\hline 6EA115 & $2.45 \mathrm{E}+02$ & 0 & 0 & $7.45 \mathrm{E}+03$ & 0 & $8.55 \mathrm{E}+02$ & 0 & 0 & 0 & $9.23 \mathrm{E}+02$ & 0 & 0 & $9.83 \mathrm{E}+03$ & $1.63 \mathrm{E}+06$ & $0.60 \%$ \\
\hline
\end{tabular}


Table 36. Predicted Solids for Envelope A Simulations - Part 2 (cont'd)

\begin{tabular}{|c|c|c|c|c|c|c|c|c|c|c|c|c|c|c|c|}
\hline Test ID & \begin{tabular}{|c|}
$\mathrm{NA} 2 \mathrm{C} 2 \mathrm{O}$ \\
4 \\
\end{tabular} & $\begin{array}{c}\text { NA2CO3. } \\
1 \mathrm{H} 2 \mathrm{O}\end{array}$ & $\begin{array}{c}\text { NA2U2O } \\
7 \\
\end{array}$ & NA3FSO4 & $\begin{array}{c}\text { NA6SO42 } \\
\text { CO3 }\end{array}$ & NAF & $\begin{array}{c}\text { NAFPO4. } \\
19 \mathrm{H} 2 \mathrm{O}\end{array}$ & NANO3 & \begin{tabular}{|c} 
NAPHOH \\
$.12 \mathrm{H} 2 \mathrm{O}$ \\
\end{tabular} & $\begin{array}{c}\text { NASGEL. } \\
15.5 \mathrm{H} 2 \mathrm{O}\end{array}$ & ZRO2 & $\begin{array}{c}\text { ZRSO42. } \\
4 \mathrm{H} 2 \mathrm{O}\end{array}$ & $\begin{array}{c}\text { Total } \\
\text { Solids } \\
\end{array}$ & \begin{tabular}{|c|} 
Total Soln \\
Mass
\end{tabular} & $\begin{array}{c}\text { Solids } \% \\
\text { of Total } \\
\text { Soln }\end{array}$ \\
\hline 6EA116 & $2.94 \mathrm{E}+02$ & 0 & 0 & $6.78 \mathrm{E}+03$ & 0 & 0 & 0 & 0 & 0 & $6.02 \mathrm{E}+02$ & 0 & 0 & $7.94 \mathrm{E}+03$ & $1.64 \mathrm{E}+06$ & $0.49 \%$ \\
\hline 6EA117 & $2.19 \mathrm{E}+02$ & 0 & 0 & $8.25 \mathrm{E}+03$ & 0 & 0 & $3.95 \mathrm{E}+02$ & 0 & 0 & $1.47 \mathrm{E}+03$ & 0 & 0 & $1.08 \mathrm{E}+04$ & $1.65 \mathrm{E}+06$ & $0.66 \%$ \\
\hline 6EA118 & $2.45 \mathrm{E}+02$ & 0 & 0 & $6.45 \mathrm{E}+03$ & 0 & $7.07 \mathrm{E}+02$ & 0 & 0 & 0 & $8.82 \mathrm{E}+01$ & 0 & 0 & $7.64 \mathrm{E}+03$ & $1.61 \mathrm{E}+06$ & $0.47 \%$ \\
\hline 6EA119 & $5.38 \mathrm{E}+01$ & 0 & 0 & $4.57 \mathrm{E}+03$ & 0 & 0 & 0 & 0 & 0 & 0 & 0 & 0 & $4.79 \mathrm{E}+03$ & $1.60 \mathrm{E}+06$ & $0.30 \%$ \\
\hline 6EA120 & $2.05 \mathrm{E}+02$ & 0 & 0 & $7.72 \mathrm{E}+03$ & 0 & $7.58 \mathrm{E}+02$ & $1.26 \mathrm{E}+02$ & 0 & 0 & $9.88 \mathrm{E}+02$ & 0 & 0 & $1.02 \mathrm{E}+04$ & $1.62 \mathrm{E}+06$ & $0.63 \%$ \\
\hline 6EA121 & $1.87 \mathrm{E}+02$ & 0 & 0 & 0 & 0 & 0 & 0 & 0 & 0 & $9.33 \mathrm{E}+02$ & 0 & 0 & $1.47 \mathrm{E}+03$ & $1.66 \mathrm{E}+06$ & $0.09 \%$ \\
\hline 6EA122 & $3.02 \mathrm{E}+02$ & 0 & 0 & $1.17 \mathrm{E}+03$ & 0 & 0 & 0 & 0 & 0 & 0 & 0 & 0 & $1.53 \mathrm{E}+03$ & $1.63 \mathrm{E}+06$ & $0.09 \%$ \\
\hline $6 \mathrm{EA} 123$ & $1.57 \mathrm{E}+02$ & 0 & 0 & 0 & 0 & 0 & $1.01 \mathrm{E}+03$ & 0 & 0 & $1.12 \mathrm{E}+02$ & 0 & 0 & $1.47 \mathrm{E}+03$ & $1.63 \mathrm{E}+06$ & $0.09 \%$ \\
\hline 6EA124 & $1.81 \mathrm{E}+02$ & 0 & 0 & $4.08 \mathrm{E}+03$ & 0 & 0 & $2.52 \mathrm{E}+02$ & 0 & 0 & $1.10 \mathrm{E}+03$ & 0 & 0 & $6.00 \mathrm{E}+03$ & $1.63 \mathrm{E}+06$ & $0.37 \%$ \\
\hline $6 \mathrm{EA} 125$ & $4.87 \mathrm{E}+01$ & 0 & 0 & $4.03 \mathrm{E}+03$ & 0 & $5.41 \mathrm{E}+02$ & 0 & 0 & 0 & 0 & 0 & 0 & $4.73 \mathrm{E}+03$ & $1.57 \mathrm{E}+06$ & $0.30 \%$ \\
\hline $6 \mathrm{EA} 126$ & $1.48 \mathrm{E}+02$ & 0 & 0 & $6.85 \mathrm{E}+03$ & 0 & 0 & 0 & 0 & 0 & $1.45 \mathrm{E}+01$ & 0 & 0 & $7.14 \mathrm{E}+03$ & $1.60 \mathrm{E}+06$ & $0.45 \%$ \\
\hline 6EA127 & $7.45 \mathrm{E}+01$ & 0 & 0 & $8.12 \mathrm{E}+03$ & 0 & 0 & 0 & 0 & 0 & $1.33 \mathrm{E}+03$ & 0 & 0 & $1.00 \mathrm{E}+04$ & $1.64 \mathrm{E}+06$ & $0.61 \%$ \\
\hline 6EA128 & $1.84 \mathrm{E}+02$ & 0 & 0 & $4.87 \mathrm{E}+03$ & 0 & 0 & $8.72 \mathrm{E}+01$ & 0 & 0 & 0 & 0 & 0 & $5.18 \mathrm{E}+03$ & $1.60 \mathrm{E}+06$ & $0.32 \%$ \\
\hline 6EA129 & 0 & 0 & 0 & $4.00 \mathrm{E}+03$ & 0 & 0 & 0 & 0 & 0 & $9.75 \mathrm{E}+02$ & 0 & 0 & $5.39 \mathrm{E}+03$ & $1.65 \mathrm{E}+06$ & $0.33 \%$ \\
\hline 6EA130 & 0 & 0 & 0 & 0 & 0 & 0 & 0 & 0 & 0 & $1.20 \mathrm{E}+03$ & 0 & 0 & $1.68 \mathrm{E}+03$ & $1.66 \mathrm{E}+06$ & $0.10 \%$ \\
\hline 6EA131 & $7.72 \mathrm{E}+00$ & 0 & 0 & $7.11 \mathrm{E}+03$ & 0 & 0 & 0 & 0 & 0 & $9.15 \mathrm{E}+02$ & 0 & 0 & $8.40 \mathrm{E}+03$ & $1.63 \mathrm{E}+06$ & $0.51 \%$ \\
\hline 6EA132 & 0 & 0 & 0 & $2.61 \mathrm{E}+03$ & 0 & 0 & 0 & 0 & 0 & 0 & 0 & 0 & $2.65 \mathrm{E}+03$ & $1.58 \mathrm{E}+06$ & $0.17 \%$ \\
\hline 6EA133 & 0 & 0 & 0 & 0 & 0 & $8.93 \mathrm{E}+02$ & 0 & 0 & 0 & $8.97 \mathrm{E}+02$ & 0 & 0 & $2.20 \mathrm{E}+03$ & $1.62 \mathrm{E}+06$ & $0.14 \%$ \\
\hline 6EA134 & $7.84 \mathrm{E}+00$ & 0 & 0 & 0 & 0 & 0 & 0 & 0 & 0 & 0 & 0 & 0 & $6.82 \mathrm{E}+01$ & $1.62 \mathrm{E}+06$ & $0.00 \%$ \\
\hline 6EA135 & 0 & 0 & 0 & 0 & 0 & 0 & 0 & 0 & 0 & $1.02 \mathrm{E}+03$ & 0 & 0 & $1.45 \mathrm{E}+03$ & $1.66 \mathrm{E}+06$ & $0.09 \%$ \\
\hline 6EA136 & 0 & 0 & 0 & 0 & 0 & 0 & 0 & 0 & 0 & $5.81 \mathrm{E}+02$ & 0 & 0 & $9.31 \mathrm{E}+02$ & $1.64 \mathrm{E}+06$ & $0.06 \%$ \\
\hline 6EA137 & 0 & 0 & 0 & 0 & 0 & 0 & 0 & 0 & 0 & 0 & 0 & 0 & $6.91 \mathrm{E}+01$ & $1.61 \mathrm{E}+06$ & $0.00 \%$ \\
\hline 6EA138 & 0 & 0 & 0 & 0 & 0 & 0 & 0 & 0 & 0 & $1.23 \mathrm{E}+03$ & 0 & 0 & $1.69 \mathrm{E}+03$ & $1.66 \mathrm{E}+06$ & $0.10 \%$ \\
\hline 6EA139 & 0 & 0 & 0 & 0 & 0 & 0 & 0 & 0 & 0 & $2.47 \mathrm{E}+02$ & 0 & 0 & $4.27 \mathrm{E}+02$ & $1.63 \mathrm{E}+06$ & $0.03 \%$ \\
\hline 6EA140 & 0 & 0 & 0 & 0 & 0 & 0 & 0 & 0 & 0 & $4.78 \mathrm{E}+02$ & 0 & 0 & $7.25 \mathrm{E}+02$ & $1.64 \mathrm{E}+06$ & $0.04 \%$ \\
\hline 6EA141 & 0 & 0 & 0 & 0 & 0 & 0 & 0 & 0 & 0 & 0 & 0 & 0 & $9.21 \mathrm{E}+01$ & $1.62 \mathrm{E}+06$ & $0.01 \%$ \\
\hline 6EA142 & 0 & 0 & 0 & 0 & 0 & $9.31 \mathrm{E}+02$ & 0 & 0 & 0 & $1.22 \mathrm{E}+03$ & 0 & 0 & $2.59 \mathrm{E}+03$ & $1.64 \mathrm{E}+06$ & $0.16 \%$ \\
\hline 6EA143 & 0 & 0 & 0 & 0 & 0 & 0 & 0 & 0 & 0 & 0 & 0 & 0 & $1.33 \mathrm{E}+02$ & $1.62 \mathrm{E}+06$ & $0.01 \%$ \\
\hline 6EA144 & 0 & 0 & 0 & 0 & 0 & $6.12 \mathrm{E}+02$ & 0 & 0 & 0 & $2.54 \mathrm{E}+02$ & 0 & 0 & $1.06 \mathrm{E}+03$ & $1.62 \mathrm{E}+06$ & $0.07 \%$ \\
\hline 6EA145 & 0 & 0 & 0 & 0 & 0 & 0 & 0 & 0 & 0 & $5.37 \mathrm{E}+01$ & 0 & 0 & $2.24 \mathrm{E}+02$ & $1.62 \mathrm{E}+06$ & $0.01 \%$ \\
\hline $6 \mathrm{EA} 146$ & 0 & 0 & 0 & 0 & 0 & 0 & 0 & 0 & 0 & $9.59 \mathrm{E}+02$ & 0 & 0 & $1.33 \mathrm{E}+03$ & $1.62 \mathrm{E}+06$ & $0.08 \%$ \\
\hline 6EA147 & 0 & 0 & 0 & 0 & 0 & 0 & 0 & 0 & 0 & $7.36 \mathrm{E}+02$ & 0 & 0 & $1.04 \mathrm{E}+03$ & $1.64 \mathrm{E}+06$ & $0.06 \%$ \\
\hline 6EA148 & 0 & 0 & 0 & 0 & 0 & 0 & 0 & 0 & 0 & $2.42 \mathrm{E}+02$ & 0 & 0 & $4.83 \mathrm{E}+02$ & $1.62 \mathrm{E}+06$ & $0.03 \%$ \\
\hline
\end{tabular}


Table 36. Predicted Solids for Envelope A Simulations - Part 2 (cont'd)

\begin{tabular}{|c|c|c|c|c|c|c|c|c|c|c|c|c|c|c|c|}
\hline Test ID & $\begin{array}{c}\mathrm{NA} 2 \mathrm{C} 2 \mathrm{O} \\
4\end{array}$ & $\begin{array}{c}\text { NA2CO3. } \\
1 \mathrm{H} 2 \mathrm{O}\end{array}$ & $\begin{array}{c}\mathrm{NA} 2 \mathrm{U} 2 \mathrm{O} \\
7\end{array}$ & NA3FSO4 & $\begin{array}{c}\mathrm{NA} 6 \mathrm{SO} 42 \\
\mathrm{CO} 3\end{array}$ & NAF & $\begin{array}{c}\text { NAFPO4. } \\
19 \mathrm{H} 2 \mathrm{O}\end{array}$ & NANO3 & $\begin{array}{c}\text { NAPHOH } \\
.12 \mathrm{H} 2 \mathrm{O}\end{array}$ & $\begin{array}{c}\text { NASGEL. } \\
15.5 \mathrm{H} 2 \mathrm{O}\end{array}$ & $\mathrm{ZRO} 2$ & $\begin{array}{c}\text { ZRSO42. } \\
4 \mathrm{H} 2 \mathrm{O}\end{array}$ & $\begin{array}{l}\text { Total } \\
\text { Solids }\end{array}$ & $\begin{array}{c}\text { Total Soln } \\
\text { Mass }\end{array}$ & $\begin{array}{c}\text { Solids } \% \\
\text { of Total } \\
\text { Soln }\end{array}$ \\
\hline 6EA149 & 0 & 0 & 0 & 0 & 0 & 0 & 0 & 0 & 0 & $3.64 \mathrm{E}+02$ & 0 & 0 & $5.94 \mathrm{E}+02$ & $\mid 1.61 \mathrm{E}+06$ & $0.04 \%$ \\
\hline $6 \mathrm{EA} 150$ & 0 & 0 & 0 & 0 & 0 & 0 & 0 & 0 & 0 & $6.11 \mathrm{E}+02$ & 0 & 0 & $9.20 \mathrm{E}+02$ & $1.64 \mathrm{E}+06$ & $0.06 \%$ \\
\hline 6EA151 & 0 & 0 & 0 & 0 & 0 & 0 & 0 & 0 & 0 & $1.15 \mathrm{E}+02$ & 0 & 0 & $2.74 \mathrm{E}+02$ & $1.62 \mathrm{E}+06$ & $0.02 \%$ \\
\hline 6EA152 & 0 & 0 & 0 & 0 & 0 & $1.44 \mathrm{E}+03$ & 0 & 0 & 0 & 0 & 0 & 0 & $1.44 \mathrm{E}+03$ & $1.58 \mathrm{E}+06$ & $0.09 \%$ \\
\hline 6EA153 & 0 & 0 & 0 & 0 & 0 & 0 & 0 & 0 & 0 & $5.81 \mathrm{E}+02$ & 0 & 0 & $8.99 \mathrm{E}+02$ & $1.61 \mathrm{E}+06$ & $0.06 \%$ \\
\hline 6EA154 & 0 & 0 & 0 & 0 & 0 & 0 & 0 & 0 & 0 & $3.90 \mathrm{E}+02$ & 0 & 0 & $6.31 \mathrm{E}+02$ & $1.63 \mathrm{E}+06$ & $0.04 \%$ \\
\hline 6EA155 & 0 & 0 & 0 & 0 & 0 & $4.59 \mathrm{E}+02$ & 0 & 0 & 0 & $8.53 \mathrm{E}+02$ & 0 & 0 & $1.71 \mathrm{E}+03$ & $1.59 \mathrm{E}+06$ & $0.11 \%$ \\
\hline 6EA156 & 0 & 0 & 0 & 0 & 0 & 0 & 0 & 0 & 0 & $6.22 \mathrm{E}+02$ & 0 & 0 & $9.74 \mathrm{E}+02$ & $1.63 \mathrm{E}+06$ & $0.06 \%$ \\
\hline 6EA157 & 0 & 0 & 0 & 0 & 0 & 0 & 0 & 0 & 0 & $1.14 \mathrm{E}+03$ & 0 & 0 & $1.59 \mathrm{E}+03$ & $1.65 \mathrm{E}+06$ & $0.10 \%$ \\
\hline 6EA158 & 0 & 0 & 0 & 0 & 0 & 0 & 0 & 0 & 0 & $5.70 \mathrm{E}+02$ & 0 & 0 & $9.29 \mathrm{E}+02$ & $1.63 \mathrm{E}+06$ & $0.06 \%$ \\
\hline 6EA159 & 0 & 0 & 0 & 0 & 0 & 0 & 0 & 0 & 0 & $4.25 \mathrm{E}+02$ & 0 & 0 & $7.01 \mathrm{E}+02$ & $1.59 \mathrm{E}+06$ & $0.04 \%$ \\
\hline 6EA160 & 0 & 0 & 0 & 0 & 0 & 0 & 0 & 0 & 0 & $1.12 \mathrm{E}+03$ & 0 & 0 & $1.59 \mathrm{E}+03$ & $1.63 \mathrm{E}+06$ & $0.10 \%$ \\
\hline 6EA161 & 0 & 0 & 0 & 0 & 0 & 0 & 0 & 0 & 0 & $4.34 \mathrm{E}+02$ & 0 & 0 & $8.09 \mathrm{E}+02$ & $1.61 \mathrm{E}+06$ & $0.05 \%$ \\
\hline 6EA162 & 0 & 0 & 0 & 0 & 0 & 0 & 0 & 0 & 0 & 0 & 0 & 0 & $1.03 \mathrm{E}+01$ & $1.60 \mathrm{E}+06$ & $0.00 \%$ \\
\hline 6EA163 & 0 & 0 & 0 & 0 & 0 & 0 & 0 & 0 & 0 & $1.33 \mathrm{E}+03$ & 0 & 0 & $1.82 \mathrm{E}+03$ & $1.64 \mathrm{E}+06$ & $0.11 \%$ \\
\hline 6EA164 & 0 & 0 & 0 & 0 & 0 & 0 & 0 & 0 & 0 & $6.75 \mathrm{E}+02$ & 0 & 0 & $1.04 \mathrm{E}+03$ & $1.61 \mathrm{E}+06$ & $0.06 \%$ \\
\hline 6EA165 & 0 & 0 & 0 & 0 & 0 & 0 & 0 & 0 & 0 & 0 & 0 & 0 & $1.40 \mathrm{E}+02$ & $1.61 \mathrm{E}+06$ & $0.01 \%$ \\
\hline 6EA166 & 0 & 0 & 0 & 0 & 0 & 0 & 0 & 0 & 0 & $6.25 \mathrm{E}+02$ & 0 & 0 & $1.02 \mathrm{E}+03$ & $1.62 \mathrm{E}+06$ & $0.06 \%$ \\
\hline 6EA167 & 0 & 0 & 0 & 0 & 0 & 0 & 0 & 0 & 0 & $1.82 \mathrm{E}+01$ & 0 & 0 & $1.77 \mathrm{E}+02$ & $1.61 \mathrm{E}+06$ & $0.01 \%$ \\
\hline 6EA168 & 0 & 0 & 0 & 0 & 0 & 0 & 0 & 0 & 0 & $6.19 \mathrm{E}+02$ & 0 & 0 & $9.16 \mathrm{E}+02$ & $1.63 \mathrm{E}+06$ & $0.06 \%$ \\
\hline 6EA169 & 0 & 0 & 0 & 0 & 0 & 0 & 0 & 0 & 0 & $1.13 \mathrm{E}+03$ & 0 & 0 & $1.57 \mathrm{E}+03$ & $1.62 \mathrm{E}+06$ & $0.10 \%$ \\
\hline 6EA170 & 0 & 0 & 0 & 0 & 0 & 0 & 0 & 0 & 0 & $1.04 \mathrm{E}+03$ & 0 & 0 & $1.64 \mathrm{E}+03$ & $1.64 \mathrm{E}+06$ & $0.10 \%$ \\
\hline 6EA171 & 0 & 0 & 0 & 0 & 0 & 0 & 0 & 0 & 0 & 0 & 0 & 0 & $5.84 \mathrm{E}+01$ & $1.59 \mathrm{E}+06$ & $0.00 \%$ \\
\hline 6EA172 & 0 & 0 & 0 & 0 & 0 & 0 & 0 & 0 & 0 & $1.18 \mathrm{E}+03$ & 0 & 0 & $1.72 \mathrm{E}+03$ & $1.64 \mathrm{E}+06$ & $0.11 \%$ \\
\hline 6EA173 & 0 & 0 & 0 & 0 & 0 & 0 & 0 & 0 & 0 & 0 & 0 & 0 & $2.65 \mathrm{E}+02$ & $1.60 \mathrm{E}+06$ & $0.02 \%$ \\
\hline 6EA174 & 0 & 0 & 0 & 0 & 0 & 0 & 0 & 0 & 0 & $2.82 \mathrm{E}+02$ & 0 & 0 & $5.62 \mathrm{E}+02$ & $1.61 \mathrm{E}+06$ & $0.03 \%$ \\
\hline 6EA175 & 0 & 0 & 0 & 0 & 0 & 0 & 0 & 0 & 0 & $1.03 \mathrm{E}+03$ & 0 & 0 & $1.57 \mathrm{E}+03$ & $1.64 \mathrm{E}+06$ & $0.10 \%$ \\
\hline 6EA176 & 0 & 0 & 0 & 0 & 0 & 0 & 0 & 0 & 0 & $6.91 \mathrm{E}+01$ & 0 & 0 & $3.30 \mathrm{E}+02$ & $1.58 \mathrm{E}+06$ & $0.02 \%$ \\
\hline 6EA177 & 0 & 0 & 0 & 0 & 0 & 0 & 0 & 0 & 0 & $1.27 \mathrm{E}+03$ & 0 & 0 & $1.77 \mathrm{E}+03$ & $1.64 \mathrm{E}+06$ & $0.11 \%$ \\
\hline 6EA178 & 0 & 0 & 0 & 0 & 0 & $7.02 \mathrm{E}+01$ & 0 & 0 & 0 & $1.07 \mathrm{E}+03$ & 0 & 0 & $1.59 \mathrm{E}+03$ & $1.61 \mathrm{E}+06$ & $0.10 \%$ \\
\hline 6EA179 & 0 & 0 & 0 & 0 & 0 & 0 & 0 & 0 & 0 & 0 & 0 & 0 & $6.05 \mathrm{E}+01$ & $1.57 \mathrm{E}+06$ & $0.00 \%$ \\
\hline 6EA180 & 0 & 0 & 0 & 0 & 0 & 0 & 0 & 0 & 0 & 0 & 0 & 0 & $1.24 \mathrm{E}+02$ & $1.58 \mathrm{E}+06$ & $0.01 \%$ \\
\hline 6EA181 & 0 & 0 & 0 & 0 & 0 & 0 & 0 & 0 & 0 & 0 & 0 & 0 & $1.62 \mathrm{E}+02$ & $1.59 \mathrm{E}+06$ & $0.01 \%$ \\
\hline
\end{tabular}


Table 36. Predicted Solids for Envelope A Simulations - Part 2 (cont'd)

\begin{tabular}{|c|c|c|c|c|c|c|c|c|c|c|c|c|c|c|c|}
\hline Test ID & $\begin{array}{c}\mathrm{NA} 2 \mathrm{C} 2 \mathrm{O} \\
4\end{array}$ & $\begin{array}{c}\text { NA2 } 2 \mathrm{CO} 3 . \\
1 \mathrm{H} 2 \mathrm{O}\end{array}$ & $\begin{array}{c}\text { NA2U2O } \\
7\end{array}$ & NA3FSO4 & $\begin{array}{c}\mathrm{NA} 6 \mathrm{SO} 42 \\
\mathrm{CO} 3\end{array}$ & NAF & $\begin{array}{c}\text { NAFPO4. } \\
19 \mathrm{H} 2 \mathrm{O}\end{array}$ & NANO3 & $\begin{array}{c}\text { NAPHOH } \\
.12 \mathrm{H} 2 \mathrm{O}\end{array}$ & $\begin{array}{c}\text { NASGEL. } \\
15.5 \mathrm{H} 2 \mathrm{O}\end{array}$ & ZRO2 & $\begin{array}{c}\text { ZRSO42. } \\
4 \mathrm{H} 2 \mathrm{O}\end{array}$ & $\begin{array}{c}\text { Total } \\
\text { Solids }\end{array}$ & $\begin{array}{c}\text { Total Soln } \\
\text { Mass }\end{array}$ & $\begin{array}{c}\text { Solids } \% \\
\text { of Total } \\
\text { Soln }\end{array}$ \\
\hline 6EA182 & 0 & 0 & 0 & 0 & 0 & 0 & 0 & 0 & 0 & $3.23 \mathrm{E}+02$ & 0 & 0 & $7.44 \mathrm{E}+02$ & $1.59 \mathrm{E}+06$ & $0.05 \%$ \\
\hline 6EA183 & 0 & 0 & 0 & 0 & 0 & 0 & 0 & 0 & 0 & 0 & 0 & 0 & $1.10 \mathrm{E}+02$ & $1.57 \mathrm{E}+06$ & $0.01 \%$ \\
\hline 6EA184 & 0 & 0 & 0 & 0 & 0 & 0 & 0 & 0 & 0 & 0 & 0 & 0 & $4.64 \mathrm{E}+01$ & $1.59 \mathrm{E}+06$ & $0.00 \%$ \\
\hline 6EA185 & 0 & 0 & 0 & 0 & 0 & 0 & 0 & 0 & 0 & $9.56 \mathrm{E}+02$ & 0 & 0 & $1.39 \mathrm{E}+03$ & $1.61 \mathrm{E}+06$ & $0.09 \%$ \\
\hline 6EA186 & 0 & 0 & 0 & 0 & 0 & 0 & 0 & 0 & 0 & 0 & 0 & 0 & $4.68 \mathrm{E}+01$ & $1.58 \mathrm{E}+06$ & $0.00 \%$ \\
\hline 6EA187 & 0 & 0 & 0 & 0 & 0 & $6.19 \mathrm{E}+01$ & 0 & 0 & 0 & $1.19 \mathrm{E}+03$ & $5.46 \mathrm{E}-01$ & 0 & $1.73 \mathrm{E}+03$ & $1.61 \mathrm{E}+06$ & $0.11 \%$ \\
\hline 6EA188 & 0 & 0 & 0 & 0 & 0 & 0 & 0 & 0 & 0 & $3.06 \mathrm{E}+02$ & 0 & 0 & $5.69 \mathrm{E}+02$ & $1.59 \mathrm{E}+06$ & $0.04 \%$ \\
\hline
\end{tabular}


Table 37. Predicted Solids for Envelope B Simulations - Part 1

\begin{tabular}{|c|c|c|c|c|c|c|c|c|c|c|c|c|c|c|c|}
\hline Test ID & $\begin{array}{l}\text { SBS/ } \\
\text { Feed }\end{array}$ & $\begin{array}{l}\text { Temp } \\
{\left[{ }^{\circ} \mathrm{C}\right]}\end{array}$ & $\begin{array}{c}\mathrm{Na} \mathrm{M} \\
{[\mathrm{mol} / \mathrm{L}]}\end{array}$ & ALOH3 & $\begin{array}{c}\text { ANATA } \\
\text { SE }\end{array}$ & \begin{tabular}{|c|} 
CA3PO4 \\
2 \\
\end{tabular} & $\mathrm{CACO} 3$ & CAF2 & CANC & $\mathrm{CAOH} 2$ & CATIO3 & $\mathrm{CROH} 3$ & \begin{tabular}{|c|} 
FEIIIOH \\
3 \\
\end{tabular} & $\begin{array}{c}\text { HYDRO } \\
\text { SOD } \\
\end{array}$ & MGOH2 \\
\hline 10TB001 & 0 & 15 & 10 & 0 & 0 & 0 & 0 & 0 & 0 & 0 & 0 & 0 & 0 & 0 & 0 \\
\hline 10TB002 & 0 & 15 & 10 & 0 & 0 & 0 & 0 & 0 & 0 & 0 & 0 & 0 & 0 & 0 & 0 \\
\hline 10TB003 & 0 & 15 & 10 & 0 & 0 & 0 & 0 & 0 & 0 & 0 & 0 & 0 & 0 & 0 & 0 \\
\hline 10TB004 & 0 & 15 & 10 & 0 & 0 & 0 & 0 & 0 & 0 & 0 & 0 & 0 & 0 & 0 & 0 \\
\hline 10TB005 & 0 & 15 & 10 & 0 & 0 & 0 & 0 & 0 & 0 & 0 & 0 & 0 & 0 & 0 & 0 \\
\hline 10TB006 & 0 & 15 & 10 & 0 & 0 & 0 & 0 & 0 & 0 & 0 & 0 & 0 & 0 & 0 & 0 \\
\hline 10TB007 & 0 & 66 & 10 & 0 & 0 & 0 & 0 & 0 & 0 & 0 & 0 & 0 & 0 & 0 & 0 \\
\hline 10TB008 & 0 & 66 & 10 & 0 & 0 & 0 & 0 & 0 & 0 & 0 & 0 & 0 & 0 & 0 & 0 \\
\hline 10TB009 & 0 & 66 & 10 & 0 & 0 & 0 & 0 & 0 & 0 & 0 & 0 & 0 & 0 & 0 & 0 \\
\hline 10TB010 & 0 & 66 & 10 & 0 & 0 & 0 & 0 & 0 & 0 & 0 & 0 & 0 & 0 & 0 & 0 \\
\hline 10TB011 & 0 & 66 & 10 & 0 & 0 & 0 & 0 & 0 & 0 & 0 & 0 & 0 & 0 & 0 & 0 \\
\hline 10TB012 & 0 & 66 & 10 & 0 & 0 & 0 & 0 & 0 & 0 & 0 & 0 & 0 & 0 & 0 & 0 \\
\hline 10TB013 & 2 & 15 & 10 & 0 & 0 & 0 & 0 & $4.24 \mathrm{E}+00$ & 0 & 0 & $1.21 \mathrm{E}+00$ & 0 & 0 & 0 & $9.74 \mathrm{E}+00$ \\
\hline 10TB014 & 2 & 15 & 10 & 0 & 0 & 0 & 0 & $4.27 \mathrm{E}+00$ & 0 & 0 & $1.21 \mathrm{E}+00$ & 0 & 0 & 0 & $9.81 \mathrm{E}+00$ \\
\hline 10TB015 & 2 & 15 & 10 & 0 & 0 & 0 & 0 & $4.29 \mathrm{E}+00$ & 0 & 0 & $1.22 \mathrm{E}+00$ & 0 & 0 & 0 & $9.84 \mathrm{E}+00$ \\
\hline 10TB016 & 2 & 15 & 10 & 0 & 0 & 0 & 0 & $4.23 \mathrm{E}+00$ & 0 & 0 & $1.20 \mathrm{E}+00$ & 0 & 0 & 0 & $9.71 \mathrm{E}+00$ \\
\hline 10TB017 & 2 & 15 & 10 & 0 & 0 & 0 & 0 & $4.26 \mathrm{E}+00$ & 0 & 0 & $1.21 \mathrm{E}+00$ & 0 & 0 & 0 & $9.78 \mathrm{E}+00$ \\
\hline 10TB018 & 2 & 15 & 10 & 0 & 0 & 0 & 0 & $4.24 \mathrm{E}+00$ & 0 & 0 & $1.21 \mathrm{E}+00$ & 0 & 0 & 0 & $9.75 \mathrm{E}+00$ \\
\hline 10TB019 & 2 & 66 & 10 & 0 & 0 & 0 & 0 & $4.20 \mathrm{E}+00$ & 0 & 0 & $1.21 \mathrm{E}+00$ & 0 & 0 & 0 & $9.74 \mathrm{E}+00$ \\
\hline 10TB020 & 2 & 66 & 10 & 0 & 0 & 0 & 0 & $4.23 \mathrm{E}+00$ & 0 & 0 & $1.21 \mathrm{E}+00$ & 0 & 0 & 0 & $9.81 \mathrm{E}+00$ \\
\hline 10TB021 & 2 & 66 & 10 & 0 & 0 & 0 & 0 & $4.24 \mathrm{E}+00$ & 0 & 0 & $1.22 \mathrm{E}+00$ & 0 & 0 & 0 & $9.84 \mathrm{E}+00$ \\
\hline 10TB022 & 2 & 66 & 10 & 0 & 0 & 0 & 0 & $4.18 \mathrm{E}+00$ & 0 & 0 & $1.20 \mathrm{E}+00$ & 0 & 0 & 0 & $9.71 \mathrm{E}+00$ \\
\hline 10TB023 & 2 & 66 & 10 & 0 & 0 & 0 & 0 & $4.21 \mathrm{E}+00$ & 0 & 0 & $1.21 \mathrm{E}+00$ & 0 & 0 & 0 & $9.78 \mathrm{E}+00$ \\
\hline 10TB024 & 2 & 66 & 10 & 0 & 0 & 0 & 0 & $4.18 \mathrm{E}+00$ & 0 & 0 & $1.21 \mathrm{E}+00$ & 0 & 0 & 0 & $9.75 \mathrm{E}+00$ \\
\hline 10TB025 & 0.9 & 40.1016 & 10 & 0 & 0 & 0 & 0 & $1.90 \mathrm{E}+00$ & 0 & 0 & $5.47 \mathrm{E}-01$ & 0 & 0 & 0 & $4.42 \mathrm{E}+00$ \\
\hline 10TB026 & 0.1 & 16.9922 & 10 & 0 & 0 & 0 & 0 & $1.97 \mathrm{E}-01$ & 0 & 0 & $6.07 \mathrm{E}-02$ & 0 & 0 & 0 & $4.90 \mathrm{E}-01$ \\
\hline 10TB027 & 0.8 & 34.9219 & 10 & 0 & 0 & 0 & 0 & $1.43 \mathrm{E}+00$ & 0 & 0 & $4.86 \mathrm{E}-01$ & 0 & 0 & 0 & $3.92 \mathrm{E}+00$ \\
\hline 10TB028 & 0.1 & 33.7266 & 10 & 0 & 0 & 0 & 0 & $2.65 \mathrm{E}-02$ & 0 & 0 & $6.07 \mathrm{E}-02$ & 0 & 0 & 0 & $4.91 \mathrm{E}-01$ \\
\hline 10TB029 & 0.6 & 22.5703 & 10 & 0 & 0 & 0 & 0 & 9.32E-01 & 0 & 0 & $3.63 \mathrm{E}-01$ & 0 & 0 & 0 & $2.93 \mathrm{E}+00$ \\
\hline
\end{tabular}




\begin{tabular}{|c|c|c|c|c|c|c|c|c|c|c|c|c|c|c|c|}
\hline \multicolumn{16}{|c|}{ Table 37. Predicted Solids for Envelope B Simulation - Part 1 (cont'd) } \\
\hline Test ID & $\begin{array}{l}\text { SBS/ } \\
\text { Feed }\end{array}$ & $\begin{array}{l}\text { Temp } \\
{\left[{ }^{\circ} \mathrm{C}\right]} \\
\end{array}$ & $\begin{array}{c}\mathrm{NaM} \\
{[\mathrm{mol} / \mathrm{L}]}\end{array}$ & ALOH3 & \begin{tabular}{|c|} 
ANATA \\
SE \\
\end{tabular} & $\begin{array}{c}\text { CA3PO4 } \\
2 \\
\end{array}$ & $\mathrm{CACO} 3$ & CAF2 & CANC & $\mathrm{CAOH} 2$ & CATIO3 & $\mathrm{CROH} 3$ & $\begin{array}{c}\text { FEIIIOH } \\
3 \\
\end{array}$ & $\begin{array}{c}\text { HYDRO } \\
\text { SOD } \\
\end{array}$ & MGOH2 \\
\hline 10TB030 & 0.3 & 34.125 & 10 & 0 & 0 & 0 & 0 & $4.15 \mathrm{E}-01$ & 0 & 0 & $1.82 \mathrm{E}-01$ & 0 & 0 & 0 & $1.47 \mathrm{E}+00$ \\
\hline 10TB032 & 0.6 & 20.9766 & 10 & 0 & 0 & 0 & 0 & $9.98 \mathrm{E}-01$ & 0 & 0 & $3.63 \mathrm{E}-01$ & 0 & 0 & 0 & $2.93 \mathrm{E}+00$ \\
\hline 10TB033 & 0 & 30.1406 & 10 & 0 & 0 & 0 & 0 & 0 & 0 & 0 & 0 & 0 & 0 & 0 & 0 \\
\hline 10TB034 & 0.1 & 38.1094 & 10 & 0 & 0 & 0 & 0 & 0 & 0 & 0 & \begin{tabular}{|l|}
$6.07 \mathrm{E}-02$ \\
\end{tabular} & 0 & 0 & 0 & \begin{tabular}{|l|}
$4.90 \mathrm{E}-01$ \\
\end{tabular} \\
\hline 10TB037 & 0.6 & 31.7344 & 10 & 0 & 0 & 0 & 0 & $1.07 \mathrm{E}+00$ & 0 & 0 & \begin{tabular}{|l|}
$3.63 \mathrm{E}-01$ \\
\end{tabular} & 0 & 0 & 0 & $2.93 \mathrm{E}+00$ \\
\hline 10TB038 & 0 & 26.9531 & 10 & 0 & 0 & 0 & 0 & 0 & 0 & 0 & 0 & 0 & 0 & 0 & 0 \\
\hline 10TB039 & 1.3 & 18.9844 & 10 & 0 & 0 & 0 & 0 & $2.76 \mathrm{E}+00$ & 0 & 0 & \begin{tabular}{|l|}
$7.88 \mathrm{E}-01$ \\
\end{tabular} & 0 & 0 & 0 & $6.37 \mathrm{E}+00$ \\
\hline 10TB040 & 1.7 & 28.9453 & 10 & 0 & 0 & 0 & 0 & $3.39 \mathrm{E}+00$ & 0 & 0 & $1.03 \mathrm{E}+00$ & 0 & 0 & 0 & $8.34 \mathrm{E}+00$ \\
\hline 10TB041 & 1.5 & 20.5781 & 10 & 0 & 0 & 0 & 0 & $2.95 \mathrm{E}+00$ & 0 & 0 & 9.14E-01 & 0 & 0 & 0 & $7.38 \mathrm{E}+00$ \\
\hline 10TB042 & 1.3 & 25.3594 & 10 & 0 & 0 & 0 & 0 & $2.58 \mathrm{E}+00$ & 0 & 0 & \begin{tabular}{|l|}
$7.88 \mathrm{E}-01$ \\
\end{tabular} & 0 & 0 & 0 & $6.36 \mathrm{E}+00$ \\
\hline 10TB047 & 1 & 18.5859 & 10 & 0 & 0 & 0 & 0 & $2.12 \mathrm{E}+00$ & 0 & 0 & 6.05E-01 & 0 & 0 & 0 & $4.89 \mathrm{E}+00$ \\
\hline 10TB048 & 1.6 & 36.9141 & 10 & 0 & 0 & 0 & 0 & $3.20 \mathrm{E}+00$ & 0 & 0 & \begin{tabular}{|l|}
$9.65 \mathrm{E}-01$ \\
\end{tabular} & 0 & 0 & 0 & $7.79 \mathrm{E}+00$ \\
\hline 10TB049 & 2 & 37.7109 & 10 & 0 & 0 & 0 & 0 & $4.05 \mathrm{E}+00$ & 0 & 0 & $1.21 \mathrm{E}+00$ & 0 & 0 & 0 & $9.81 \mathrm{E}+00$ \\
\hline $10 \mathrm{~TB} 050$ & 1.5 & 19.7813 & 10 & 0 & 0 & 0 & 0 & $3.19 \mathrm{E}+00$ & 0 & 0 & $9.10 \mathrm{E}-01$ & 0 & 0 & 0 & $7.35 \mathrm{E}+00$ \\
\hline 10TB051 & 1.7 & 19.3828 & 10 & 0 & 0 & 0 & 0 & $3.61 \mathrm{E}+00$ & 0 & 0 & $1.03 \mathrm{E}+00$ & 0 & 0 & 0 & $8.29 \mathrm{E}+00$ \\
\hline 10TB052 & 1 & 43.6875 & 10 & 0 & 0 & 0 & 0 & $2.11 \mathrm{E}+00$ & 0 & 0 & \begin{tabular}{|l|}
$6.06 \mathrm{E}-01$ \\
\end{tabular} & 0 & 0 & 0 & $4.90 \mathrm{E}+00$ \\
\hline 10TB053 & 0.1 & 62.8125 & 10 & 0 & 0 & 0 & 0 & \begin{tabular}{|l|}
$1.52 \mathrm{E}-01$ \\
\end{tabular} & 0 & 0 & \begin{tabular}{|l|}
$6.07 \mathrm{E}-02$ \\
\end{tabular} & 0 & 0 & 0 & \begin{tabular}{|l|}
$4.91 \mathrm{E}-01$ \\
\end{tabular} \\
\hline 10TB054 & 0.8 & 45.6797 & 10 & 0 & 0 & 0 & 0 & $1.68 \mathrm{E}+00$ & 0 & 0 & \begin{tabular}{|l|}
$4.85 \mathrm{E}-01$ \\
\end{tabular} & 0 & 0 & 0 & $3.91 \mathrm{E}+00$ \\
\hline 10TB055 & 0.7 & 63.6094 & 10 & 0 & 0 & 0 & 0 & $1.44 \mathrm{E}+00$ & 0 & 0 & \begin{tabular}{|l|}
$4.24 \mathrm{E}-01$ \\
\end{tabular} & 0 & 0 & 0 & $3.43 \mathrm{E}+00$ \\
\hline 10TB056 & 0.3 & 50.0625 & 10 & 0 & 0 & 0 & 0 & \begin{tabular}{|l|}
$6.03 \mathrm{E}-01$ \\
\end{tabular} & 0 & 0 & \begin{tabular}{|l|}
$1.82 \mathrm{E}-01$ \\
\end{tabular} & 0 & 0 & 0 & $1.47 \mathrm{E}+00$ \\
\hline 10TB057 & 0.8 & 59.2266 & 10 & 0 & 0 & 0 & 0 & $1.66 \mathrm{E}+00$ & 0 & 0 & \begin{tabular}{|l|}
$4.86 \mathrm{E}-01$ \\
\end{tabular} & 0 & 0 & 0 & $3.92 \mathrm{E}+00$ \\
\hline
\end{tabular}




\begin{tabular}{|c|c|c|c|c|c|c|c|c|c|c|c|c|c|c|c|}
\hline \multicolumn{16}{|c|}{ Table 37. Predicted Solids for Envelope B Simulation - Part 1 (cont'd) } \\
\hline Test ID & $\begin{array}{l}\text { SBS/ } \\
\text { Feed }\end{array}$ & $\begin{array}{l}\text { Temp } \\
{\left[{ }^{\circ} \mathrm{C}\right]}\end{array}$ & $\begin{array}{c}\mathrm{Na} \mathrm{M} \\
{[\mathrm{mol} / \mathrm{L}]}\end{array}$ & $\mathrm{ALOH} 3$ & \begin{tabular}{|c|} 
ANATA \\
SE \\
\end{tabular} & \begin{tabular}{|c|} 
CA3PO4 \\
2 \\
\end{tabular} & $\mathrm{CACO} 3$ & CAF2 & CANC & $\mathrm{CAOH} 2$ & CATIO3 & $\mathrm{CROH} 3$ & \begin{tabular}{|c} 
FEIIIOH \\
3 \\
\end{tabular} & \begin{tabular}{|c|c|} 
HYDRO \\
SOD
\end{tabular} & MGOH2 \\
\hline 10TB062 & 0.9 & 64.4063 & 10 & 0 & 0 & 0 & 0 & $1.88 \mathrm{E}+00$ & 0 & \begin{tabular}{|l|l|}
0 \\
\end{tabular} & 5.49E-01 & 0 & 0 & 0 & $4.43 \mathrm{E}+00$ \\
\hline 10TB064 & 0.3 & 58.8281 & 10 & 0 & 0 & 0 & 0 & $5.81 \mathrm{E}-01$ & 0 & 0 & $1.81 \mathrm{E}-01$ & 0 & 0 & 0 & $1.46 \mathrm{E}+00$ \\
\hline \begin{tabular}{|l} 
10TB065 \\
\end{tabular} & 0.7 & \begin{tabular}{|l|}
50.4609 \\
\end{tabular} & 10 & 0 & 0 & 0 & 0 & $1.46 \mathrm{E}+00$ & 0 & 0 & 4.24E-01 & 0 & 0 & 0 & $3.42 \mathrm{E}+00$ \\
\hline 10TB066 & 1.8 & 48.8672 & 10 & 0 & 0 & 0 & 0 & $3.83 \mathrm{E}+00$ & 0 & 0 & $1.09 \mathrm{E}+00$ & 0 & 0 & 0 & $8.84 \mathrm{E}+00$ \\
\hline \begin{tabular}{|l|} 
10TB069 \\
\end{tabular} & 1.6 & 47.6719 & 10 & 0 & 0 & 0 & 0 & $3.39 \mathrm{E}+00$ & 0 & 0 & 9.71E-01 & 0 & 0 & 0 & $7.85 \mathrm{E}+00$ \\
\hline \begin{tabular}{|l|} 
10TB070 \\
\end{tabular} & 1.8 & 66 & 10 & 0 & 0 & 0 & 0 & $3.77 \mathrm{E}+00$ & 0 & 0 & $1.09 \mathrm{E}+00$ & 0 & 0 & 0 & $8.80 \mathrm{E}+00$ \\
\hline \begin{tabular}{|l|} 
10TB071 \\
\end{tabular} & 1.1 & 49.6641 & 10 & 0 & 0 & 0 & 0 & $2.32 \mathrm{E}+00$ & 0 & 0 & $6.68 \mathrm{E}-01$ & 0 & 0 & 0 & $5.40 \mathrm{E}+00$ \\
\hline \begin{tabular}{|l|} 
10TB072 \\
\end{tabular} & 1.2 & \begin{tabular}{|l|}
56.8359 \\
\end{tabular} & 10 & 0 & 0 & 0 & 0 & $2.52 \mathrm{E}+00$ & 0 & 0 & 7.28E-01 & 0 & 0 & 0 & $5.88 \mathrm{E}+00$ \\
\hline 10TB073 & 1.2 & 63.2109 & 10 & 0 & 0 & 0 & 0 & $2.51 \mathrm{E}+00$ & 0 & 0 & 7.27E-01 & 0 & 0 & 0 & $5.88 \mathrm{E}+00$ \\
\hline 10TB074 & 1.9 & 41.6953 & 10 & 0 & 0 & 0 & 0 & $4.05 \mathrm{E}+00$ & 0 & 0 & $1.15 \mathrm{E}+00$ & 0 & 0 & 0 & $9.33 \mathrm{E}+00$ \\
\hline \begin{tabular}{|l|} 
10TB079 \\
\end{tabular} & 1 & 40.5 & 10 & 0 & 0 & 0 & 0 & $2.12 \mathrm{E}+00$ & 0 & 0 & $6.08 \mathrm{E}-01$ & 0 & 0 & 0 & $4.92 \mathrm{E}+00$ \\
\hline 10TB080 & 1.1 & 40.8984 & 10 & 0 & 0 & 0 & 0 & $2.32 \mathrm{E}+00$ & 0 & 0 & $6.65 \mathrm{E}-01$ & 0 & 0 & 0 & $5.38 \mathrm{E}+00$ \\
\hline 10TB081 & 1.9 & 64.0078 & 10 & 0 & 0 & 0 & 0 & $4.01 \mathrm{E}+00$ & 0 & 0 & $1.15 \mathrm{E}+00$ & 0 & 0 & 0 & $9.31 \mathrm{E}+00$ \\
\hline 10TB082 & 1.2 & 46.0781 & 10 & 0 & 0 & 0 & 0 & $2.53 \mathrm{E}+00$ & 0 & 0 & $7.26 \mathrm{E}-01$ & 0 & 0 & 0 & $5.87 \mathrm{E}+00$ \\
\hline \begin{tabular}{|l|} 
10TB083 \\
\end{tabular} & 1.9 & \begin{tabular}{|l|}
47.2734 \\
\end{tabular} & 10 & 0 & 0 & 0 & 0 & $4.02 \mathrm{E}+00$ & 0 & 0 & $1.15 \mathrm{E}+00$ & 0 & 0 & 0 & $9.30 \mathrm{E}+00$ \\
\hline \begin{tabular}{|l|} 
10TB084 \\
\end{tabular} & 1.4 & \begin{tabular}{|l|}
58.4297 \\
\end{tabular} & 10 & 0 & 0 & 0 & 0 & $2.94 \mathrm{E}+00$ & 0 & 0 & \begin{tabular}{|l|}
$8.46 \mathrm{E}-01$ \\
\end{tabular} & 0 & 0 & 0 & $6.83 \mathrm{E}+00$ \\
\hline 10TB085 & 1.7 & 46.875 & 10 & 0 & 0 & 0 & 0 & $3.62 \mathrm{E}+00$ & 0 & 0 & $1.04 \mathrm{E}+00$ & 0 & 0 & 0 & $8.36 \mathrm{E}+00$ \\
\hline \begin{tabular}{|l|} 
10TB086 \\
\end{tabular} & 1.4 & 52.4531 & 10 & 0 & 0 & 0 & 0 & $2.96 \mathrm{E}+00$ & 0 & 0 & $8.50 \mathrm{E}-01$ & 0 & 0 & 0 & $6.86 \mathrm{E}+00$ \\
\hline \begin{tabular}{|l|} 
10TB087 \\
\end{tabular} & 1.4 & 60.0234 & 10 & 0 & 0 & 0 & 0 & $2.94 \mathrm{E}+00$ & 0 & 0 & \begin{tabular}{|l|}
$8.48 \mathrm{E}-01$ \\
\end{tabular} & 0 & 0 & 0 & $6.85 \mathrm{E}+00$ \\
\hline \begin{tabular}{|l|} 
10TB088 \\
\end{tabular} & 2 & 50.8594 & 10 & 0 & 0 & 0 & 0 & $4.24 \mathrm{E}+00$ & 0 & 0 & $1.21 \mathrm{E}+00$ & 0 & 0 & 0 & $9.79 \mathrm{E}+00$ \\
\hline \begin{tabular}{|l|} 
10TB089 \\
\end{tabular} & 1.9 & 42.8906 & 10 & 0 & 0 & 0 & 0 & $4.03 \mathrm{E}+00$ & 0 & 0 & $1.15 \mathrm{E}+00$ & 0 & 0 & 0 & $9.31 \mathrm{E}+00$ \\
\hline
\end{tabular}




\begin{tabular}{|c|c|c|c|c|c|c|c|c|c|c|c|c|c|c|c|}
\hline \multicolumn{16}{|c|}{ Table 37. Predicted Solids for Envelope B Simulation - Part 1 (cont'd) } \\
\hline Test ID & $\begin{array}{l}\text { SBS/ } \\
\text { Feed }\end{array}$ & $\begin{array}{l}\text { Temp } \\
{\left[{ }^{\circ} \mathrm{C}\right]}\end{array}$ & $\begin{array}{c}\mathrm{Na} \mathrm{M} \\
{[\mathrm{mol} / \mathrm{L}]}\end{array}$ & $\mathrm{ALOH} 3$ & \begin{tabular}{|c|} 
ANATA \\
SE \\
\end{tabular} & \begin{tabular}{|c|} 
CA3PO4 \\
2 \\
\end{tabular} & $\mathrm{CACO} 3$ & CAF2 & CANC & $\mathrm{CAOH} 2$ & CATIO3 & $\mathrm{CROH} 3$ & \begin{tabular}{|c} 
FEIIIOH \\
3 \\
\end{tabular} & \begin{tabular}{|c|c|} 
HYDRO \\
SOD
\end{tabular} & MGOH2 \\
\hline 10TB094 & 0.7 & 62.0156 & 10 & 0 & 0 & 0 & 0 & $1.44 \mathrm{E}+00$ & 0 & \begin{tabular}{|l|l|}
0 \\
\end{tabular} & $4.24 \mathrm{E}-01$ & 0 & 0 & 0 & $3.42 \mathrm{E}+00$ \\
\hline 10TB096 & 0.5 & 60.4219 & 10 & 0 & 0 & 0 & 0 & $1.01 \mathrm{E}+00$ & 0 & 0 & $3.03 \mathrm{E}-01$ & 0 & 0 & 0 & $2.45 \mathrm{E}+00$ \\
\hline \begin{tabular}{|l|} 
10TB097 \\
\end{tabular} & 0.7 & 55.6406 & 10 & 0 & 0 & 0 & 0 & $1.44 \mathrm{E}+00$ & 0 & 0 & 4.22E-01 & 0 & 0 & 0 & $3.41 \mathrm{E}+00$ \\
\hline 10TB098 & 0 & 60.8203 & 10 & 0 & 0 & 0 & 0 & 0 & 0 & 0 & 0 & 0 & 0 & 0 & 0 \\
\hline 10TB101 & 0.1 & 46.4766 & 10 & 0 & 0 & 0 & 0 & $1.82 \mathrm{E}-01$ & 0 & 0 & 6.08E-02 & 0 & 0 & 0 & $4.91 \mathrm{E}-01$ \\
\hline \begin{tabular}{|l|} 
10TB102 \\
\end{tabular} & 1 & 62.4141 & 10 & 0 & 0 & 0 & 0 & $2.08 \mathrm{E}+00$ & 0 & 0 & 6.05E-01 & 0 & 0 & 0 & $4.89 \mathrm{E}+00$ \\
\hline \begin{tabular}{|l|}
$10 \mathrm{~TB} 103$ \\
\end{tabular} & 0.4 & \begin{tabular}{|l|}
44.0859 \\
\end{tabular} & 10 & 0 & 0 & 0 & 0 & $8.24 \mathrm{E}-01$ & 0 & 0 & $2.42 \mathrm{E}-01$ & 0 & 0 & 0 & $1.96 \mathrm{E}+00$ \\
\hline 10TB104 & 0 & 43.2891 & 10 & 0 & 0 & 0 & 0 & 0 & 0 & 0 & 0 & 0 & 0 & 0 & 0 \\
\hline 10TB105 & 0.5 & 61.2188 & 10 & 0 & 0 & 0 & 0 & $1.03 \mathrm{E}+00$ & 0 & 0 & $3.05 \mathrm{E}-01$ & 0 & 0 & 0 & $2.46 \mathrm{E}+00$ \\
\hline 10TB106 & 0.3 & 61.6172 & 10 & 0 & 0 & 0 & 0 & $5.85 \mathrm{E}-01$ & 0 & 0 & $1.83 \mathrm{E}-01$ & 0 & 0 & 0 & $1.48 \mathrm{E}+00$ \\
\hline \begin{tabular}{|l|} 
10TB111 \\
\end{tabular} & 1.7 & 30.9375 & 10 & 0 & 0 & 0 & 0 & $3.40 \mathrm{E}+00$ & 0 & 0 & $1.03 \mathrm{E}+00$ & 0 & 0 & 0 & $8.34 \mathrm{E}+00$ \\
\hline 10TB112 & 1.3 & 21.7734 & 10 & 0 & 0 & 0 & 0 & $2.57 \mathrm{E}+00$ & 0 & 0 & 7.93E-01 & 0 & 0 & 0 & $6.40 \mathrm{E}+00$ \\
\hline 10TB113 & 1.5 & 29.7422 & 10 & 0 & 0 & 0 & 0 & $2.84 \mathrm{E}+00$ & 0 & 0 & 9.13E-01 & 0 & 0 & 0 & $7.37 \mathrm{E}+00$ \\
\hline 10TB114 & 1 & 38.9063 & 10 & 0 & 0 & 0 & 0 & $1.77 \mathrm{E}+00$ & 0 & 0 & $6.11 \mathrm{E}-01$ & 0 & 0 & 0 & $4.93 \mathrm{E}+00$ \\
\hline \begin{tabular}{|l|} 
10TB115 \\
\end{tabular} & 1.8 & 29.3438 & 10 & 0 & 0 & 0 & 0 & $3.55 \mathrm{E}+00$ & 0 & 0 & $1.09 \mathrm{E}+00$ & 0 & 0 & 0 & $8.81 \mathrm{E}+00$ \\
\hline \begin{tabular}{|l|}
$10 \mathrm{~TB} 116$ \\
\end{tabular} & 1.4 & 16.1953 & 10 & 0 & 0 & 0 & 0 & $2.98 \mathrm{E}+00$ & 0 & 0 & $8.50 \mathrm{E}-01$ & 0 & 0 & 0 & $6.87 \mathrm{E}+00$ \\
\hline 10TB117 & 1.1 & 16.5938 & 10 & 0 & 0 & 0 & 0 & $2.34 \mathrm{E}+00$ & 0 & 0 & $6.67 \mathrm{E}-01$ & 0 & 0 & 0 & $5.39 \mathrm{E}+00$ \\
\hline \begin{tabular}{|l|} 
10TB118 \\
\end{tabular} & 1.8 & 28.1484 & 10 & 0 & 0 & 0 & 0 & $3.38 \mathrm{E}+00$ & 0 & 0 & $1.09 \mathrm{E}+00$ & 0 & 0 & 0 & $8.81 \mathrm{E}+00$ \\
\hline \begin{tabular}{|l|} 
10TB119 \\
\end{tabular} & 1.7 & 22.1719 & 10 & 0 & 0 & 0 & 0 & $3.38 \mathrm{E}+00$ & 0 & 0 & $1.03 \mathrm{E}+00$ & 0 & 0 & 0 & $8.35 \mathrm{E}+00$ \\
\hline 10TB120 & 1.3 & 30.5391 & 10 & 0 & 0 & 0 & 0 & $2.37 \mathrm{E}+00$ & 0 & 0 & \begin{tabular}{|l|}
$7.90 \mathrm{E}-01$ \\
\end{tabular} & 0 & 0 & 0 & $6.38 \mathrm{E}+00$ \\
\hline \begin{tabular}{|l|} 
10TB121 \\
\end{tabular} & 0.2 & 32.1328 & 10 & 0 & 0 & 0 & 0 & $1.12 \mathrm{E}-01$ & 0 & 0 & $1.21 \mathrm{E}-01$ & 0 & 0 & 0 & 9.79E-01 \\
\hline
\end{tabular}




\begin{tabular}{|c|c|c|c|c|c|c|c|c|c|c|c|c|c|c|c|}
\hline \multicolumn{16}{|c|}{ Table 37. Predicted Solids for Envelope B Simulation - Part 1 (cont'd) } \\
\hline Test ID & $\begin{array}{l}\text { SBS/ } \\
\text { Feed }\end{array}$ & $\begin{array}{l}\text { Temp } \\
{\left[{ }^{\circ} \mathrm{C}\right]}\end{array}$ & $\begin{array}{c}\mathrm{Na} \mathrm{M} \\
{[\mathrm{mol} / \mathrm{L}]}\end{array}$ & ALOH3 & $\begin{array}{c}\text { ANATA } \\
\text { SE }\end{array}$ & \begin{tabular}{|c|} 
CA3PO4 \\
2 \\
\end{tabular} & $\mathrm{CACO} 3$ & CAF2 & CANC & $\mathrm{CAOH} 2$ & CATIO3 & $\mathrm{CROH} 3$ & $\begin{array}{c}\text { FEIIIOH } \\
3 \\
\end{array}$ & \begin{tabular}{|c|}
$\begin{array}{c}\text { HYDRO } \\
\text { SOD }\end{array}$ \\
\end{tabular} & MGOH2 \\
\hline $10 \mathrm{~TB} 126$ & 0.9 & 31.3359 & 10 & 0 & 0 & 0 & 0 & $1.64 \mathrm{E}+00$ & 0 & 0 & 5.48E-01 & 0 & 0 & 0 & $4.43 \mathrm{E}+00$ \\
\hline $10 \mathrm{~TB} 128$ & 0.8 & 17.7891 & 10 & 0 & 0 & 0 & 0 & $1.70 \mathrm{E}+00$ & 0 & 0 & 4.86E-01 & 0 & 0 & 0 & $3.93 \mathrm{E}+00$ \\
\hline 10TB129 & 0.1 & \begin{tabular}{|l|}
39.3047 \\
\end{tabular} & 10 & 0 & 0 & 0 & 0 & 0 & 0 & 0 & \begin{tabular}{|l|}
$6.06 \mathrm{E}-02$ \\
\end{tabular} & 0 & 0 & 0 & \begin{tabular}{|l|}
$4.89 \mathrm{E}-01$ \\
\end{tabular} \\
\hline $10 \mathrm{~TB} 130$ & 0.9 & 26.1563 & 10 & 0 & 0 & 0 & 0 & $1.60 \mathrm{E}+00$ & 0 & 0 & 5.47E-01 & 0 & 0 & 0 & $4.42 \mathrm{E}+00$ \\
\hline $10 \mathrm{~TB} 133$ & 0.2 & \begin{tabular}{|l|}
23.3672 \\
\end{tabular} & 10 & 0 & 0 & 0 & 0 & \begin{tabular}{|l|}
$4.05 \mathrm{E}-02$ \\
\end{tabular} & 0 & 0 & 1.22E-01 & 0 & 0 & 0 & $9.83 \mathrm{E}-01$ \\
\hline 8TB001 & 0 & 15 & 8 & 0 & 0 & 0 & 0 & 0 & 0 & 0 & 0 & 0 & 0 & 0 & 0 \\
\hline 8TB002 & 0 & 15 & 8 & 0 & 0 & 0 & 0 & 0 & 0 & 0 & 0 & 0 & 0 & 0 & 0 \\
\hline 8TB003 & 0 & 15 & 8 & 0 & 0 & 0 & 0 & 0 & 0 & 0 & 0 & 0 & 0 & 0 & 0 \\
\hline 8TB004 & 0 & 15 & 8 & 0 & 0 & 0 & 0 & 0 & 0 & 0 & 0 & 0 & 0 & 0 & 0 \\
\hline 8TB005 & 0 & 15 & 8 & 0 & 0 & 0 & 0 & 0 & 0 & 0 & 0 & 0 & 0 & 0 & 0 \\
\hline 8TB010 & 0 & 66 & 8 & 0 & 0 & 0 & 0 & 0 & 0 & 0 & 0 & 0 & 0 & 0 & 0 \\
\hline 8TB011 & 0 & 66 & 8 & 0 & 0 & 0 & 0 & 0 & 0 & 0 & 0 & 0 & 0 & 0 & 0 \\
\hline 8TB012 & 0 & 66 & 8 & 0 & 0 & 0 & 0 & 0 & 0 & 0 & 0 & 0 & 0 & 0 & 0 \\
\hline $8 \mathrm{~TB} 013$ & 2 & 15 & 8 & 0 & 0 & 0 & 0 & $4.24 \mathrm{E}+00$ & 0 & 0 & $1.21 \mathrm{E}+00$ & 0 & 0 & 0 & $9.74 \mathrm{E}+00$ \\
\hline 8TB014 & 2 & 15 & 8 & 0 & 0 & 0 & 0 & $4.28 \mathrm{E}+00$ & 0 & 0 & $1.21 \mathrm{E}+00$ & 0 & 0 & 0 & $9.81 \mathrm{E}+00$ \\
\hline $8 \mathrm{~TB} 015$ & 2 & 15 & 8 & 0 & 0 & 0 & 0 & $4.29 \mathrm{E}+00$ & 0 & 0 & $1.22 \mathrm{E}+00$ & 0 & 0 & 0 & $9.84 \mathrm{E}+00$ \\
\hline 8TB016 & 2 & 15 & 8 & 0 & 0 & 0 & 0 & $4.23 \mathrm{E}+00$ & 0 & 0 & $1.20 \mathrm{E}+00$ & 0 & 0 & 0 & $9.71 \mathrm{E}+00$ \\
\hline 8TB017 & 2 & 15 & 8 & 0 & 0 & 0 & 0 & $4.26 \mathrm{E}+00$ & 0 & 0 & $1.21 \mathrm{E}+00$ & 0 & 0 & 0 & $9.78 \mathrm{E}+00$ \\
\hline 8TB018 & 2 & 15 & 8 & 0 & 0 & 0 & 0 & \begin{tabular}{|l|}
$4.24 \mathrm{E}+00$ \\
\end{tabular} & 0 & 0 & \begin{tabular}{|l|}
$1.21 \mathrm{E}+00$ \\
\end{tabular} & 0 & 0 & 0 & $9.75 \mathrm{E}+00$ \\
\hline 8TB019 & 2 & 66 & 8 & 0 & 0 & 0 & 0 & \begin{tabular}{|l|}
$4.18 \mathrm{E}+00$ \\
\end{tabular} & 0 & 0 & \begin{tabular}{|l|}
$1.21 \mathrm{E}+00$ \\
\end{tabular} & 0 & 0 & 0 & $9.74 \mathrm{E}+00$ \\
\hline 8TB020 & 2 & 66 & 8 & 0 & 0 & 0 & 0 & \begin{tabular}{|l|}
$4.21 \mathrm{E}+00$ \\
\end{tabular} & 0 & 0 & $1.21 \mathrm{E}+00$ & 0 & 0 & 0 & $9.81 \mathrm{E}+00$ \\
\hline
\end{tabular}




\begin{tabular}{|c|c|c|c|c|c|c|c|c|c|c|c|c|c|c|c|}
\hline \multicolumn{16}{|c|}{ Table 37. Predicted Solids for Envelope B Simulation - Part 1 (cont'd) } \\
\hline Test ID & $\begin{array}{l}\text { SBS/ } \\
\text { Feed }\end{array}$ & $\begin{array}{l}\text { Temp } \\
{\left[{ }^{\circ} \mathrm{C}\right]}\end{array}$ & $\begin{array}{c}\mathrm{Na} \mathrm{M} \\
{[\mathrm{mol} / \mathrm{L}]}\end{array}$ & ALOH3 & \begin{tabular}{|c|} 
ANATA \\
SE \\
\end{tabular} & \begin{tabular}{|c} 
CA3PO4 \\
2 \\
\end{tabular} & CACO3 & CAF2 & CANC & $\mathrm{CAOH} 2$ & CATIO3 & $\mathrm{CROH} 3$ & \begin{tabular}{|c} 
FEIIIOH \\
3 \\
\end{tabular} & \begin{tabular}{|c|c|} 
HYDRO \\
SOD
\end{tabular} & MGOH2 \\
\hline $8 \mathrm{~TB} 025$ & 0.9 & 40.1016 & 8 & 0 & 0 & 0 & \begin{tabular}{|l|l|}
0 \\
\end{tabular} & $1.90 \mathrm{E}+00$ & 0 & \begin{tabular}{|l|l|}
0 \\
\end{tabular} & $5.47 \mathrm{E}-01$ & 0 & 0 & 0 & $4.42 \mathrm{E}+00$ \\
\hline $8 \mathrm{~TB} 027$ & 0.8 & 34.9219 & 8 & 0 & 0 & 0 & 0 & $1.60 \mathrm{E}+00$ & 0 & 0 & $4.86 \mathrm{E}-01$ & 0 & 0 & 0 & $3.92 \mathrm{E}+00$ \\
\hline $8 \mathrm{~TB} 028$ & 0.1 & 33.7266 & 8 & 0 & 0 & 0 & 0 & $1.30 \mathrm{E}-01$ & 0 & 0 & $6.07 \mathrm{E}-02$ & 0 & 0 & 0 & 4.91E-01 \\
\hline $8 \mathrm{~TB} 029$ & 0.6 & 22.5703 & 8 & 0 & 0 & 0 & 0 & $1.15 \mathrm{E}+00$ & 0 & 0 & $3.63 \mathrm{E}-01$ & 0 & 0 & 0 & $2.93 \mathrm{E}+00$ \\
\hline $8 \mathrm{~TB} 032$ & 0.6 & 20.9766 & 8 & 0 & 0 & 0 & 0 & $1.17 \mathrm{E}+00$ & 0 & 0 & 3.63E-01 & 0 & 0 & 0 & $2.93 \mathrm{E}+00$ \\
\hline $8 \mathrm{~TB} 033$ & 0 & 30.1406 & 8 & 0 & 0 & 0 & 0 & 0 & 0 & 0 & 0 & 0 & 0 & 0 & 0 \\
\hline $8 \mathrm{~TB} 034$ & 0.1 & 38.1094 & 8 & 0 & 0 & 0 & 0 & 1.09E-01 & 0 & 0 & $6.07 \mathrm{E}-02$ & 0 & 0 & 0 & 4.90E-01 \\
\hline $8 \mathrm{~TB} 035$ & 0.3 & 22.9688 & 8 & 0 & 0 & 0 & 0 & 5.28E-01 & 0 & 0 & 1.81E-01 & 0 & 0 & 0 & $1.47 \mathrm{E}+00$ \\
\hline $8 \mathrm{~TB} 036$ & 0.8 & 36.5156 & 8 & 0 & 0 & 0 & 0 & $1.60 \mathrm{E}+00$ & 0 & 0 & 4.84E-01 & 0 & 0 & 0 & $3.91 \mathrm{E}+00$ \\
\hline $8 \mathrm{~TB} 037$ & 0.6 & 31.7344 & 8 & 0 & 0 & 0 & 0 & $1.19 \mathrm{E}+00$ & 0 & 0 & 3.63E-01 & 0 & 0 & 0 & $2.93 \mathrm{E}+00$ \\
\hline $8 \mathrm{~TB} 042$ & 1.3 & 25.3594 & 8 & 0 & 0 & 0 & 0 & $2.69 \mathrm{E}+00$ & 0 & 0 & 7.88E-01 & 0 & 0 & 0 & $6.36 \mathrm{E}+00$ \\
\hline $8 \mathrm{~TB} 043$ & 2 & 20.1797 & 8 & 0 & 0 & 0 & 0 & $4.14 \mathrm{E}+00$ & 0 & 0 & $1.21 \mathrm{E}+00$ & 0 & 0 & 0 & $9.80 \mathrm{E}+00$ \\
\hline 8TB044 & 1.4 & 38.5078 & 8 & 0 & 0 & 0 & 0 & $2.87 \mathrm{E}+00$ & 0 & 0 & $8.47 \mathrm{E}-01$ & 0 & 0 & 0 & $6.84 \mathrm{E}+00$ \\
\hline $8 \mathrm{~TB} 045$ & 1.6 & 36.1172 & 8 & 0 & 0 & 0 & 0 & $3.32 \mathrm{E}+00$ & 0 & 0 & 9.68E-01 & 0 & 0 & 0 & $7.82 \mathrm{E}+00$ \\
\hline $8 \mathrm{~TB} 046$ & 1.9 & 34.5234 & 8 & 0 & 0 & 0 & 0 & $3.95 \mathrm{E}+00$ & 0 & 0 & $1.15 \mathrm{E}+00$ & 0 & 0 & 0 & $9.33 \mathrm{E}+00$ \\
\hline $8 \mathrm{~TB} 047$ & 1 & 18.5859 & 8 & 0 & 0 & 0 & 0 & $2.12 \mathrm{E}+00$ & 0 & 0 & 6.05E-01 & 0 & 0 & 0 & $4.89 \mathrm{E}+00$ \\
\hline 8TB048 & 1.6 & 36.9141 & 8 & 0 & 0 & 0 & 0 & $3.31 \mathrm{E}+00$ & 0 & 0 & $9.65 \mathrm{E}-01$ & 0 & 0 & 0 & $7.79 \mathrm{E}+00$ \\
\hline 8TB049 & 2 & 37.7109 & 8 & 0 & 0 & 0 & 0 & $4.19 \mathrm{E}+00$ & 0 & 0 & $1.21 \mathrm{E}+00$ & 0 & 0 & 0 & $9.81 \mathrm{E}+00$ \\
\hline $8 \mathrm{~TB} 050$ & 1.5 & 19.7813 & 8 & 0 & 0 & 0 & 0 & $3.20 \mathrm{E}+00$ & 0 & 0 & 9.10E-01 & 0 & 0 & 0 & $7.35 \mathrm{E}+00$ \\
\hline $8 \mathrm{~TB} 051$ & 1.7 & 19.3828 & 8 & 0 & 0 & 0 & 0 & $3.61 \mathrm{E}+00$ & 0 & 0 & $1.03 \mathrm{E}+00$ & 0 & 0 & 0 & $8.29 \mathrm{E}+00$ \\
\hline $8 \mathrm{~TB} 052$ & 1 & 43.6875 & 8 & 0 & 0 & 0 & 0 & $2.10 \mathrm{E}+00$ & 0 & 0 & 6.06E-01 & 0 & 0 & 0 & $4.90 \mathrm{E}+00$ \\
\hline
\end{tabular}




\begin{tabular}{|c|c|c|c|c|c|c|c|c|c|c|c|c|c|c|c|}
\hline \multicolumn{16}{|c|}{ Table 37. Predicted Solids for Envelope B Simulation - Part 1 (cont'd) } \\
\hline Test ID & $\begin{array}{l}\text { SBS/ } \\
\text { Feed }\end{array}$ & $\begin{array}{l}\text { Temp } \\
{\left[{ }^{\circ} \mathrm{C}\right]}\end{array}$ & $\begin{array}{c}\mathrm{Na} \mathrm{M} \\
{[\mathrm{mol} / \mathrm{L}]}\end{array}$ & ALOH3 & $\begin{array}{c}\text { ANATA } \\
\text { SE }\end{array}$ & \begin{tabular}{|c|} 
CA3PO4 \\
2 \\
\end{tabular} & $\mathrm{CACO} 3$ & CAF2 & CANC & $\mathrm{CAOH} 2$ & CATIO3 & $\mathrm{CROH} 3$ & $\begin{array}{c}\text { FEIIIOH } \\
3 \\
\end{array}$ & \begin{tabular}{|c|}
$\begin{array}{c}\text { HYDRO } \\
\text { SOD }\end{array}$ \\
\end{tabular} & MGOH2 \\
\hline $8 \mathrm{~TB} 057$ & 0.8 & 59.2266 & 8 & 0 & 0 & 0 & 0 & $1.63 \mathrm{E}+00$ & 0 & 0 & 4.86E-01 & 0 & 0 & 0 & $3.92 \mathrm{E}+00$ \\
\hline $8 \mathrm{~TB} 059$ & 1 & 42.0938 & 8 & 0 & 0 & 0 & 0 & $2.10 \mathrm{E}+00$ & 0 & 0 & 6.06E-01 & 0 & 0 & 0 & $4.90 \mathrm{E}+00$ \\
\hline 8 TB060 & 0.2 & \begin{tabular}{|l|}
51.6563 \\
\end{tabular} & 8 & 0 & 0 & 0 & 0 & \begin{tabular}{|l|}
$3.65 \mathrm{E}-01$ \\
\end{tabular} & 0 & 0 & \begin{tabular}{|l|}
$1.22 \mathrm{E}-01$ \\
\end{tabular} & 0 & 0 & 0 & 9.84E-01 \\
\hline 8TB061 & 0.6 & 64.8047 & 8 & 0 & 0 & 0 & 0 & $1.18 \mathrm{E}+00$ & 0 & 0 & 3.63E-01 & 0 & 0 & 0 & $2.93 \mathrm{E}+00$ \\
\hline 8 8TB064 & 0.3 & \begin{tabular}{|l|}
58.8281 \\
\end{tabular} & 8 & 0 & 0 & 0 & 0 & \begin{tabular}{|l|}
$5.50 \mathrm{E}-01$ \\
\end{tabular} & 0 & 0 & \begin{tabular}{|l|}
$1.81 \mathrm{E}-01$ \\
\end{tabular} & 0 & 0 & 0 & $1.46 \mathrm{E}+00$ \\
\hline 8 TB065 & 0.7 & \begin{tabular}{|l|}
50.4609 \\
\end{tabular} & 8 & 0 & 0 & 0 & 0 & $1.44 \mathrm{E}+00$ & 0 & 0 & \begin{tabular}{|l|}
$4.24 \mathrm{E}-01$ \\
\end{tabular} & 0 & 0 & 0 & $3.42 \mathrm{E}+00$ \\
\hline 8TB066 & 1.8 & \begin{tabular}{|l|}
48.8672 \\
\end{tabular} & 8 & 0 & 0 & 0 & 0 & $3.82 \mathrm{E}+00$ & 0 & 0 & $1.09 \mathrm{E}+00$ & 0 & 0 & 0 & $8.84 \mathrm{E}+00$ \\
\hline 8TB067 & 1.5 & \begin{tabular}{|l|}
57.2344 \\
\end{tabular} & 8 & 0 & 0 & 0 & 0 & $3.15 \mathrm{E}+00$ & 0 & 0 & \begin{tabular}{|c|}
$9.11 \mathrm{E}-01$ \\
\end{tabular} & 0 & 0 & 0 & $7.36 \mathrm{E}+00$ \\
\hline 8TB068 & 1.8 & 53.6484 & 8 & 0 & 0 & 0 & 0 & $3.81 \mathrm{E}+00$ & 0 & 0 & $1.09 \mathrm{E}+00$ & 0 & 0 & 0 & $8.84 \mathrm{E}+00$ \\
\hline 8TB069 & 1.6 & 47.6719 & 8 & 0 & 0 & 0 & 0 & $3.39 \mathrm{E}+00$ & 0 & 0 & \begin{tabular}{|l|}
$9.71 \mathrm{E}-01$ \\
\end{tabular} & 0 & 0 & 0 & $7.85 \mathrm{E}+00$ \\
\hline 8TB074 & 1.9 & \begin{tabular}{|l|}
41.6953 \\
\end{tabular} & 8 & 0 & 0 & 0 & 0 & $4.04 \mathrm{E}+00$ & 0 & 0 & $1.15 \mathrm{E}+00$ & 0 & 0 & 0 & $9.33 \mathrm{E}+00$ \\
\hline 8 TB075 & 1.1 & 54.8438 & 8 & 0 & 0 & 0 & 0 & $2.30 \mathrm{E}+00$ & 0 & 0 & 6.69E-01 & 0 & 0 & 0 & $5.41 \mathrm{E}+00$ \\
\hline 8TB076 & 1.6 & 55.2422 & 8 & 0 & 0 & 0 & 0 & $3.36 \mathrm{E}+00$ & 0 & 0 & 9.67E-01 & 0 & 0 & 0 & $7.81 \mathrm{E}+00$ \\
\hline 8TB077 & 1.8 & 54.4453 & 8 & 0 & 0 & 0 & 0 & $3.81 \mathrm{E}+00$ & 0 & 0 & $1.09 \mathrm{E}+00$ & 0 & 0 & 0 & $8.84 \mathrm{E}+00$ \\
\hline 8TB078 & 1.8 & \begin{tabular}{|l|}
57.6328 \\
\end{tabular} & 8 & 0 & 0 & 0 & 0 & $3.79 \mathrm{E}+00$ & 0 & 0 & $1.09 \mathrm{E}+00$ & 0 & 0 & 0 & $8.82 \mathrm{E}+00$ \\
\hline 8TB079 & 1 & 40.5 & 8 & 0 & 0 & 0 & 0 & $2.11 \mathrm{E}+00$ & 0 & 0 & \begin{tabular}{|l|}
$6.08 \mathrm{E}-01$ \\
\end{tabular} & 0 & 0 & 0 & $4.92 \mathrm{E}+00$ \\
\hline $8 \mathrm{~TB} 080$ & 1.1 & \begin{tabular}{|l|}
40.8984 \\
\end{tabular} & 8 & 0 & 0 & 0 & 0 & $2.31 \mathrm{E}+00$ & 0 & 0 & \begin{tabular}{|l|}
$6.65 \mathrm{E}-01$ \\
\end{tabular} & 0 & 0 & 0 & $5.38 \mathrm{E}+00$ \\
\hline 8TB081 & 1.9 & \begin{tabular}{|l|}
64.0078 \\
\end{tabular} & 8 & 0 & 0 & 0 & 0 & $3.99 \mathrm{E}+00$ & 0 & 0 & $1.15 \mathrm{E}+00$ & 0 & 0 & 0 & $9.31 \mathrm{E}+00$ \\
\hline 8 TR082 & 1.2 & \begin{tabular}{|l|}
46.0781 \\
\end{tabular} & 8 & 0 & 0 & 0 & 0 & $2.52 \mathrm{E}+00$ & 0 & 0 & \begin{tabular}{|l|}
$7.26 \mathrm{E}-01$ \\
\end{tabular} & 0 & 0 & 0 & $5.87 \mathrm{E}+00$ \\
\hline 8 TB083 & 1.9 & \begin{tabular}{|l|}
47.2734 \\
\end{tabular} & 8 & 0 & 0 & 0 & 0 & $4.02 \mathrm{E}+00$ & 0 & 0 & $1.15 \mathrm{E}+00$ & 0 & 0 & 0 & $9.30 \mathrm{E}+00$ \\
\hline 8TB084 & 1.4 & \begin{tabular}{|l|}
58.4297 \\
\end{tabular} & 8 & 0 & 0 & 0 & 0 & $2.92 \mathrm{E}+00$ & 0 & 0 & \begin{tabular}{|l|}
$8.46 \mathrm{E}-01$ \\
\end{tabular} & 0 & 0 & 0 & $6.83 \mathrm{E}+00$ \\
\hline
\end{tabular}




\begin{tabular}{|c|c|c|c|c|c|c|c|c|c|c|c|c|c|c|c|}
\hline \multicolumn{16}{|c|}{ Table 37. Predicted Solids for Envelope B Simulation - Part 1 (cont'd) } \\
\hline Test ID & $\begin{array}{l}\text { SBS/ } \\
\text { Feed }\end{array}$ & $\begin{array}{l}\text { Temp } \\
{\left[{ }^{\circ} \mathrm{C}\right]}\end{array}$ & $\begin{array}{c}\mathrm{Na} \mathrm{M} \\
{[\mathrm{mol} / \mathrm{L}]}\end{array}$ & ALOH3 & \begin{tabular}{|c|} 
ANATA \\
SE \\
\end{tabular} & \begin{tabular}{|c|} 
CA3PO4 \\
2 \\
\end{tabular} & CACO3 & CAF2 & CANC & $\mathrm{CAOH} 2$ & CATIO3 & $\mathrm{CROH} 3$ & \begin{tabular}{|c} 
FEIIIOH \\
3 \\
\end{tabular} & \begin{tabular}{|c|c|} 
HYDRO \\
SOD
\end{tabular} & MGOH2 \\
\hline $8 \mathrm{~TB} 089$ & 1.9 & 42.8906 & 8 & 0 & 0 & 0 & \begin{tabular}{|l|l|}
0 \\
\end{tabular} & $4.03 \mathrm{E}+00$ & 0 & \begin{tabular}{|l|l|}
0 \\
\end{tabular} & $1.15 \mathrm{E}+00$ & 0 & 0 & 0 & $9.31 \mathrm{E}+00$ \\
\hline $8 \mathrm{~TB} 091$ & 1.2 & 44.4844 & 8 & 0 & 0 & 0 & 0 & $2.54 \mathrm{E}+00$ & 0 & 0 & $7.30 \mathrm{E}-01$ & 0 & 0 & 0 & $5.90 \mathrm{E}+00$ \\
\hline 8TB092 & 1.4 & 49.2656 & 8 & 0 & 0 & 0 & 0 & $2.96 \mathrm{E}+00$ & 0 & 0 & \begin{tabular}{|l|}
$8.52 \mathrm{E}-01$ \\
\end{tabular} & 0 & 0 & 0 & $6.88 \mathrm{E}+00$ \\
\hline 8TB093 & 2 & \begin{tabular}{|l|}
54.0469 \\
\end{tabular} & 8 & 0 & 0 & 0 & 0 & $4.25 \mathrm{E}+00$ & 0 & 0 & $1.22 \mathrm{E}+00$ & 0 & 0 & 0 & $9.84 \mathrm{E}+00$ \\
\hline 8 8TB096 & 0.5 & 60.4219 & 8 & 0 & 0 & 0 & 0 & 9.79E-01 & 0 & 0 & 3.03E-01 & 0 & 0 & 0 & $2.45 \mathrm{E}+00$ \\
\hline 8TB097 & 0.7 & 55.6406 & 8 & 0 & 0 & 0 & 0 & $1.42 \mathrm{E}+00$ & 0 & 0 & 4.22E-01 & 0 & 0 & 0 & $3.41 \mathrm{E}+00$ \\
\hline $8 \mathrm{~TB} 098$ & 0 & 60.8203 & 8 & 0 & 0 & 0 & 0 & 0 & 0 & 0 & 0 & 0 & 0 & 0 & 0 \\
\hline 8TB099 & 0.6 & 42.4922 & 8 & 0 & 0 & 0 & 0 & $1.24 \mathrm{E}+00$ & 0 & 0 & 3.63E-01 & 0 & 0 & 0 & $2.93 \mathrm{E}+00$ \\
\hline $8 \mathrm{~TB} 100$ & 0.4 & 44.8828 & 8 & 0 & 0 & 0 & 0 & $8.11 \mathrm{E}-01$ & 0 & 0 & $2.43 \mathrm{E}-01$ & 0 & 0 & 0 & $1.96 \mathrm{E}+00$ \\
\hline $8 \mathrm{~TB} 101$ & 0.1 & 46.4766 & 8 & 0 & 0 & 0 & 0 & $1.62 \mathrm{E}-01$ & 0 & 0 & $6.08 \mathrm{E}-02$ & 0 & 0 & 0 & 4.91E-01 \\
\hline $8 \mathrm{~TB} 106$ & 0.3 & 61.6172 & 8 & 0 & 0 & 0 & 0 & 5.47E-01 & 0 & 0 & $1.83 \mathrm{E}-01$ & 0 & 0 & 0 & $1.48 \mathrm{E}+00$ \\
\hline $8 \mathrm{~TB} 107$ & 1 & 37.3125 & 8 & 0 & 0 & 0 & 0 & $2.01 \mathrm{E}+00$ & 0 & 0 & 6.03E-01 & 0 & 0 & 0 & $4.87 \mathrm{E}+00$ \\
\hline $8 \mathrm{~TB} 108$ & 1.9 & 18.1875 & 8 & 0 & 0 & 0 & 0 & $4.04 \mathrm{E}+00$ & 0 & 0 & $1.15 \mathrm{E}+00$ & 0 & 0 & 0 & $9.29 \mathrm{E}+00$ \\
\hline $8 \mathrm{~TB} 109$ & 1.2 & 35.3203 & 8 & 0 & 0 & 0 & 0 & $2.41 \mathrm{E}+00$ & 0 & 0 & $7.25 \mathrm{E}-01$ & 0 & 0 & 0 & $5.86 \mathrm{E}+00$ \\
\hline $8 \mathrm{~TB} 110$ & 1.3 & 17.3906 & 8 & 0 & 0 & 0 & 0 & $2.77 \mathrm{E}+00$ & 0 & 0 & 7.88E-01 & 0 & 0 & 0 & $6.37 \mathrm{E}+00$ \\
\hline $8 \mathrm{~TB} 111$ & 1.7 & 30.9375 & 8 & 0 & 0 & 0 & 0 & $3.54 \mathrm{E}+00$ & 0 & 0 & $1.03 \mathrm{E}+00$ & 0 & 0 & 0 & $8.34 \mathrm{E}+00$ \\
\hline 8TB112 & 1.3 & 21.7734 & 8 & 0 & 0 & 0 & 0 & $2.69 \mathrm{E}+00$ & 0 & 0 & 7.93E-01 & 0 & 0 & 0 & $6.40 \mathrm{E}+00$ \\
\hline $8 \mathrm{~TB} 113$ & 1.5 & 29.7422 & 8 & 0 & 0 & 0 & 0 & $3.08 \mathrm{E}+00$ & 0 & 0 & 9.13E-01 & 0 & 0 & 0 & $7.37 \mathrm{E}+00$ \\
\hline $8 \mathrm{~TB} 114$ & 1 & 38.9063 & 8 & 0 & 0 & 0 & 0 & $2.02 \mathrm{E}+00$ & 0 & 0 & $6.11 \mathrm{E}-01$ & 0 & 0 & 0 & $4.93 \mathrm{E}+00$ \\
\hline $8 \mathrm{~TB} 115$ & 1.8 & 29.3438 & 8 & 0 & 0 & 0 & 0 & $3.73 \mathrm{E}+00$ & 0 & 0 & $1.09 \mathrm{E}+00$ & 0 & 0 & 0 & $8.81 \mathrm{E}+00$ \\
\hline $8 \mathrm{~TB} 116$ & 1.4 & 16.1953 & 8 & 0 & 0 & 0 & 0 & $2.99 \mathrm{E}+00$ & 0 & 0 & \begin{tabular}{|l|}
$8.50 \mathrm{E}-01$ \\
\end{tabular} & 0 & 0 & 0 & $6.87 \mathrm{E}+00$ \\
\hline
\end{tabular}




\begin{tabular}{|c|c|c|c|c|c|c|c|c|c|c|c|c|c|c|c|}
\hline \multicolumn{16}{|c|}{ Table 37. Predicted Solids for Envelope B Simulation - Part 1 (cont'd) } \\
\hline Test ID & $\begin{array}{l}\text { SBS/ } \\
\text { Feed }\end{array}$ & $\begin{array}{l}\text { Temp } \\
{\left[{ }^{\circ} \mathrm{C}\right]}\end{array}$ & $\begin{array}{c}\mathrm{Na} \mathrm{M} \\
{[\mathrm{mol} / \mathrm{L}]}\end{array}$ & ALOH3 & $\begin{array}{c}\text { ANATA } \\
\text { SE }\end{array}$ & \begin{tabular}{|c|} 
CA3PO4 \\
2 \\
\end{tabular} & $\mathrm{CACO} 3$ & CAF2 & CANC & $\mathrm{CAOH} 2$ & CATIO3 & $\mathrm{CROH} 3$ & $\begin{array}{c}\text { FEIIIOH } \\
3 \\
\end{array}$ & \begin{tabular}{|c|}
$\begin{array}{c}\text { HYDRO } \\
\text { SOD }\end{array}$ \\
\end{tabular} & MGOH2 \\
\hline $8 \mathrm{~TB} 121$ & 0.2 & 32.1328 & 8 & 0 & 0 & 0 & 0 & 3.07E-01 & 0 & 0 & $1.21 \mathrm{E}-01$ & 0 & 0 & 0 & 9.79E-01 \\
\hline $8 \mathrm{~TB} 123$ & 0.2 & 27.3516 & 8 & 0 & 0 & 0 & 0 & $3.20 \mathrm{E}-01$ & 0 & 0 & $1.22 \mathrm{E}-01$ & 0 & 0 & 0 & $9.83 \mathrm{E}-01$ \\
\hline $8 \mathrm{~TB} 124$ & 0.4 & \begin{tabular}{|l|}
33.3281 \\
\end{tabular} & 8 & 0 & 0 & 0 & 0 & 6.93E-01 & 0 & 0 & \begin{tabular}{|l|}
$2.42 \mathrm{E}-01$ \\
\end{tabular} & 0 & 0 & 0 & $1.96 \mathrm{E}+00$ \\
\hline $8 \mathrm{~TB} 125$ & 0.2 & 15 & 8 & 0 & 0 & 0 & 0 & $4.15 \mathrm{E}-01$ & 0 & 0 & $1.21 \mathrm{E}-01$ & 0 & 0 & 0 & $9.80 \mathrm{E}-01$ \\
\hline $8 \mathrm{~TB} 128$ & 0.8 & \begin{tabular}{|l|}
17.7891 \\
\end{tabular} & 8 & 0 & 0 & 0 & 0 & $1.70 \mathrm{E}+00$ & 0 & 0 & \begin{tabular}{|l|}
$4.86 \mathrm{E}-01$ \\
\end{tabular} & 0 & 0 & 0 & $3.93 \mathrm{E}+00$ \\
\hline 8 TB129 & 0.1 & 39.3047 & 8 & 0 & 0 & 0 & 0 & $6.13 \mathrm{E}-02$ & 0 & 0 & 6.06E-02 & 0 & 0 & 0 & 4.89E-01 \\
\hline $8 \mathrm{~TB} 130$ & 0.9 & \begin{tabular}{|l|}
26.1563 \\
\end{tabular} & 8 & 0 & 0 & 0 & 0 & $1.81 \mathrm{E}+00$ & 0 & 0 & \begin{tabular}{|l|}
$5.47 \mathrm{E}-01$ \\
\end{tabular} & 0 & 0 & 0 & $4.42 \mathrm{E}+00$ \\
\hline 8 TB131 & 0.4 & \begin{tabular}{|l|}
25.7578 \\
\end{tabular} & 8 & 0 & 0 & 0 & 0 & \begin{tabular}{|l|}
$7.34 \mathrm{E}-01$ \\
\end{tabular} & 0 & 0 & \begin{tabular}{|l|}
$2.44 \mathrm{E}-01$ \\
\end{tabular} & 0 & 0 & 0 & $1.97 \mathrm{E}+00$ \\
\hline 8 TB132 & 0.2 & 26.5547 & 8 & 0 & 0 & 0 & 0 & $3.20 \mathrm{E}-01$ & 0 & 0 & 1.22E-01 & 0 & 0 & 0 & 9.84E-01 \\
\hline $8 \mathrm{~TB} 133$ & 0.2 & \begin{tabular}{|l|}
23.3672 \\
\end{tabular} & 8 & 0 & 0 & 0 & 0 & 2.83E-01 & 0 & 0 & \begin{tabular}{|l|}
$1.22 \mathrm{E}-01$ \\
\end{tabular} & 0 & 0 & 0 & \begin{tabular}{|l|}
$9.83 \mathrm{E}-01$ \\
\end{tabular} \\
\hline 6TB005 & 0 & 15 & 6 & 0 & 0 & 0 & 0 & 0 & 0 & 0 & 0 & 0 & 0 & 0 & 0 \\
\hline 6TB006 & 0 & 15 & 6 & $3.77 \mathrm{E}+02$ & 0 & 0 & 0 & 0 & 0 & 0 & 0 & 0 & 0 & 0 & 0 \\
\hline 6TB007 & 0 & 66 & 6 & 0 & 0 & 0 & 0 & 0 & 0 & 0 & 0 & 0 & 0 & 0 & 0 \\
\hline 6TB008 & 0 & 66 & 6 & 0 & 0 & 0 & 0 & 0 & 0 & 0 & 0 & 0 & 0 & 0 & 0 \\
\hline 6TB009 & 0 & 66 & 6 & 0 & 0 & 0 & 0 & 0 & 0 & 0 & 0 & 0 & 0 & 0 & 0 \\
\hline 6TB010 & 0 & 66 & 6 & 0 & 0 & 0 & 0 & 0 & 0 & 0 & 0 & 0 & 0 & 0 & 0 \\
\hline 6TB011 & 0 & 66 & 6 & 0 & 0 & 0 & 0 & 0 & 0 & 0 & 0 & 0 & 0 & 0 & 0 \\
\hline 6TB012 & 0 & 66 & 6 & 0 & 0 & 0 & 0 & 0 & 0 & 0 & 0 & 0 & 0 & 0 & 0 \\
\hline 6TB013 & 2 & 15 & 6 & $7.14 \mathrm{E}+02$ & 0 & 0 & 0 & $4.23 \mathrm{E}+00$ & 0 & 0 & \begin{tabular}{|l|}
$1.21 \mathrm{E}+00$ \\
\end{tabular} & 0 & 0 & 0 & $9.74 \mathrm{E}+00$ \\
\hline 6TB014 & 2 & 15 & 6 & 0 & 0 & 0 & 0 & $4.26 \mathrm{E}+00$ & 0 & 0 & \begin{tabular}{|l|}
$1.21 \mathrm{E}+00$ \\
\end{tabular} & 0 & 0 & 0 & $9.81 \mathrm{E}+00$ \\
\hline 6TB015 & 2 & 15 & 6 & $6.67 \mathrm{E}+02$ & 0 & 0 & 0 & $4.27 \mathrm{E}+00$ & 0 & 0 & $1.22 \mathrm{E}+00$ & 0 & 0 & 0 & $9.84 \mathrm{E}+00$ \\
\hline
\end{tabular}




\begin{tabular}{|c|c|c|c|c|c|c|c|c|c|c|c|c|c|c|c|}
\hline \multicolumn{16}{|c|}{ Table 37. Predicted Solids for Envelope B Simulation - Part 1 (cont'd) } \\
\hline Test ID & $\begin{array}{l}\text { SBS/ } \\
\text { Feed }\end{array}$ & $\begin{array}{l}\text { Temp } \\
{\left[{ }^{\circ} \mathrm{C}\right]}\end{array}$ & $\begin{array}{c}\mathrm{Na} \mathrm{M} \\
{[\mathrm{mol} / \mathrm{L}]}\end{array}$ & $\mathrm{ALOH3}$ & $\begin{array}{c}\text { ANATA } \\
\text { SE }\end{array}$ & \begin{tabular}{|c|} 
CA3PO4 \\
2 \\
\end{tabular} & $\mathrm{CACO} 3$ & CAF2 & CANC & $\mathrm{CAOH} 2$ & CATIO3 & $\mathrm{CROH} 3$ & $\begin{array}{c}\text { FEIIIOH } \\
3 \\
\end{array}$ & \begin{tabular}{|c|}
$\begin{array}{c}\text { HYDRO } \\
\text { SOD }\end{array}$ \\
\end{tabular} & MGOH2 \\
\hline 6TB020 & 2 & 66 & 6 & 0 & 0 & 0 & 0 & $4.09 \mathrm{E}+00$ & 0 & 0 & $1.21 \mathrm{E}+00$ & 0 & 0 & 0 & $9.81 \mathrm{E}+00$ \\
\hline 6TB022 & 2 & 66 & 6 & 0 & 0 & 0 & 0 & $4.03 \mathrm{E}+00$ & 0 & 0 & $1.20 \mathrm{E}+00$ & 0 & 0 & 0 & $9.71 \mathrm{E}+00$ \\
\hline 6TB023 & 2 & 66 & 6 & 0 & 0 & 0 & 0 & $4.07 \mathrm{E}+00$ & 0 & 0 & $1.21 \mathrm{E}+00$ & 0 & 0 & 0 & $9.78 \mathrm{E}+00$ \\
\hline 6TB024 & 2 & 66 & 6 & 0 & 0 & 0 & 0 & $4.04 \mathrm{E}+00$ & 0 & 0 & $1.21 \mathrm{E}+00$ & 0 & 0 & 0 & $9.75 \mathrm{E}+00$ \\
\hline 6TB027 & 0.8 & \begin{tabular}{|l|}
34.9219 \\
\end{tabular} & 6 & 0 & 0 & 0 & 0 & $1.63 \mathrm{E}+00$ & 0 & 0 & \begin{tabular}{|l|}
$4.86 \mathrm{E}-01$ \\
\end{tabular} & 0 & 0 & 0 & $3.92 \mathrm{E}+00$ \\
\hline 6TB028 & 0.1 & \begin{tabular}{|l|}
33.7266 \\
\end{tabular} & 6 & 0 & 0 & 0 & 0 & \begin{tabular}{|l|}
$1.31 \mathrm{E}-01$ \\
\end{tabular} & 0 & 0 & \begin{tabular}{|l|}
$6.07 \mathrm{E}-02$ \\
\end{tabular} & 0 & 0 & 0 & \begin{tabular}{|l|}
$4.91 \mathrm{E}-01$ \\
\end{tabular} \\
\hline 6TB029 & 0.6 & \begin{tabular}{|l|}
22.5703 \\
\end{tabular} & 6 & 0 & 0 & 0 & 0 & $1.20 \mathrm{E}+00$ & 0 & 0 & \begin{tabular}{|l|}
$3.63 \mathrm{E}-01$ \\
\end{tabular} & 0 & 0 & 0 & $2.93 \mathrm{E}+00$ \\
\hline 6TB030 & 0.3 & \begin{tabular}{|l|}
34.125 \\
\end{tabular} & 6 & 0 & 0 & 0 & 0 & \begin{tabular}{|l|}
$5.58 \mathrm{E}-01$ \\
\end{tabular} & 0 & 0 & \begin{tabular}{|l|}
$1.82 \mathrm{E}-01$ \\
\end{tabular} & 0 & 0 & 0 & $1.47 \mathrm{E}+00$ \\
\hline 6TB031 & 0.6 & 28.5469 & 6 & 0 & 0 & 0 & 0 & $1.20 \mathrm{E}+00$ & 0 & 0 & 3.65E-01 & 0 & 0 & 0 & $2.95 \mathrm{E}+00$ \\
\hline 6TB032 & 0.6 & 20.9766 & 6 & 0 & 0 & 0 & 0 & $1.21 \mathrm{E}+00$ & 0 & 0 & 3.63E-01 & 0 & 0 & 0 & $2.93 \mathrm{E}+00$ \\
\hline 6TB037 & 0.6 & 31.7344 & 6 & 0 & 0 & 0 & 0 & $1.21 \mathrm{E}+00$ & 0 & 0 & 3.63E-01 & 0 & 0 & 0 & $2.93 \mathrm{E}+00$ \\
\hline 6TB038 & 0 & 26.9531 & 6 & 0 & 0 & 0 & 0 & 0 & 0 & 0 & 0 & 0 & 0 & 0 & 0 \\
\hline 6TB039 & 1.3 & 18.9844 & 6 & $1.92 \mathrm{E}+02$ & 0 & 0 & 0 & $2.75 \mathrm{E}+00$ & 0 & 0 & \begin{tabular}{|l|}
$7.88 \mathrm{E}-01$ \\
\end{tabular} & 0 & 0 & 0 & $6.37 \mathrm{E}+00$ \\
\hline 6TB040 & 1.7 & 28.9453 & 6 & 0 & 0 & 0 & 0 & $3.57 \mathrm{E}+00$ & 0 & 0 & $1.03 \mathrm{E}+00$ & 0 & 0 & 0 & $8.34 \mathrm{E}+00$ \\
\hline 6TB041 & 1.5 & \begin{tabular}{|l|}
20.5781 \\
\end{tabular} & 6 & $6.13 \mathrm{E}+01$ & 0 & 0 & 0 & $3.15 \mathrm{E}+00$ & 0 & 0 & \begin{tabular}{|l|}
$9.14 \mathrm{E}-01$ \\
\end{tabular} & 0 & 0 & 0 & $7.38 \mathrm{E}+00$ \\
\hline 6TB042 & 1.3 & \begin{tabular}{|l|}
25.3594 \\
\end{tabular} & 6 & 0 & 0 & 0 & 0 & $2.71 \mathrm{E}+00$ & 0 & 0 & \begin{tabular}{|l|}
$7.88 \mathrm{E}-01$ \\
\end{tabular} & 0 & 0 & 0 & $6.36 \mathrm{E}+00$ \\
\hline 6TB043 & 2 & 20.1797 & 6 & $1.87 \mathrm{E}+02$ & 0 & 0 & 0 & $4.20 \mathrm{E}+00$ & 0 & 0 & $1.21 \mathrm{E}+00$ & 0 & 0 & 0 & $9.80 \mathrm{E}+00$ \\
\hline 6TB044 & 1.4 & \begin{tabular}{|l|}
38.5078 \\
\end{tabular} & 6 & 0 & 0 & 0 & 0 & $2.90 \mathrm{E}+00$ & 0 & 0 & 8.47E-01 & 0 & 0 & 0 & $6.84 \mathrm{E}+00$ \\
\hline 6TB045 & 1.6 & \begin{tabular}{|l|}
36.1172 \\
\end{tabular} & 6 & 0 & 0 & 0 & 0 & $3.33 \mathrm{E}+00$ & 0 & 0 & \begin{tabular}{|l|}
$9.68 \mathrm{E}-01$ \\
\end{tabular} & 0 & 0 & 0 & $7.82 \mathrm{E}+00$ \\
\hline 6TB046 & 1.9 & 34.5234 & 6 & 0 & 0 & 0 & 0 & $3.98 \mathrm{E}+00$ & 0 & 0 & $1.15 \mathrm{E}+00$ & 0 & 0 & 0 & $9.33 \mathrm{E}+00$ \\
\hline 6TB047 & 1 & \begin{tabular}{|l|}
18.5859 \\
\end{tabular} & 6 & $1.62 \mathrm{E}+02$ & 0 & 0 & 0 & $2.10 \mathrm{E}+00$ & 0 & 0 & \begin{tabular}{|l|}
$6.05 \mathrm{E}-01$ \\
\end{tabular} & 0 & 0 & 0 & $4.89 \mathrm{E}+00$ \\
\hline
\end{tabular}




\begin{tabular}{|c|c|c|c|c|c|c|c|c|c|c|c|c|c|c|c|}
\hline \multicolumn{16}{|c|}{ Table 37. Predicted Solids for Envelope B Simulation - Part 1 (cont'd) } \\
\hline Test ID & $\begin{array}{l}\text { SBS/ } \\
\text { Feed }\end{array}$ & $\begin{array}{l}\text { Temp } \\
{\left[{ }^{\circ} \mathrm{C}\right]}\end{array}$ & $\begin{array}{c}\mathrm{Na} \mathrm{M} \\
{[\mathrm{mol} / \mathrm{L}]}\end{array}$ & ALOH3 & $\begin{array}{c}\text { ANATA } \\
\text { SE }\end{array}$ & \begin{tabular}{|c|} 
CA3PO4 \\
2 \\
\end{tabular} & $\mathrm{CACO} 3$ & CAF2 & CANC & $\mathrm{CAOH} 2$ & CATIO3 & $\mathrm{CROH} 3$ & $\begin{array}{c}\text { FEIIIOH } \\
3 \\
\end{array}$ & \begin{tabular}{|c|}
$\begin{array}{c}\text { HYDRO } \\
\text { SOD }\end{array}$ \\
\end{tabular} & MGOH2 \\
\hline 6TB052 & 1 & 43.6875 & 6 & 0 & 0 & 0 & 0 & $2.03 \mathrm{E}+00$ & 0 & 0 & 6.06E-01 & 0 & 0 & 0 & $4.90 \mathrm{E}+00$ \\
\hline 6TB054 & 0.8 & 45.6797 & 6 & 0 & 0 & 0 & 0 & $1.59 \mathrm{E}+00$ & 0 & 0 & 4.85E-01 & 0 & 0 & 0 & $3.91 \mathrm{E}+00$ \\
\hline 6TB055 & 0.7 & \begin{tabular}{|l|}
63.6094 \\
\end{tabular} & 6 & 0 & 0 & 0 & 0 & $1.25 \mathrm{E}+00$ & 0 & 0 & \begin{tabular}{|l|}
$4.24 \mathrm{E}-01$ \\
\end{tabular} & 0 & 0 & 0 & $3.43 \mathrm{E}+00$ \\
\hline 6TB056 & 0.3 & \begin{tabular}{|l|}
50.0625 \\
\end{tabular} & 6 & 0 & 0 & 0 & 0 & \begin{tabular}{|l|}
$4.83 \mathrm{E}-01$ \\
\end{tabular} & 0 & 0 & \begin{tabular}{|l|}
$1.82 \mathrm{E}-01$ \\
\end{tabular} & 0 & 0 & 0 & $1.47 \mathrm{E}+00$ \\
\hline 6TB059 & 1 & \begin{tabular}{|l|}
42.0938 \\
\end{tabular} & 6 & 0 & 0 & 0 & 0 & $2.04 \mathrm{E}+00$ & 0 & 0 & \begin{tabular}{|l|}
$6.06 \mathrm{E}-01$ \\
\end{tabular} & 0 & 0 & 0 & $4.90 \mathrm{E}+00$ \\
\hline 6TB060 & 0.2 & \begin{tabular}{|l|}
51.6563 \\
\end{tabular} & 6 & 0 & 0 & 0 & 0 & \begin{tabular}{|l|}
$2.57 \mathrm{E}-01$ \\
\end{tabular} & 0 & 0 & \begin{tabular}{|l|}
$1.22 \mathrm{E}-01$ \\
\end{tabular} & 0 & 0 & 0 & \begin{tabular}{|l|}
$9.84 \mathrm{E}-01$ \\
\end{tabular} \\
\hline 6TB061 & 0.6 & \begin{tabular}{|l|}
64.8047 \\
\end{tabular} & 6 & 0 & 0 & 0 & 0 & $1.02 \mathrm{E}+00$ & 0 & 0 & \begin{tabular}{|l|}
$3.63 \mathrm{E}-01$ \\
\end{tabular} & 0 & 0 & 0 & $2.93 \mathrm{E}+00$ \\
\hline 6TB062 & 0.9 & \begin{tabular}{|l|}
64.4063 \\
\end{tabular} & 6 & 0 & 0 & 0 & 0 & $1.69 \mathrm{E}+00$ & 0 & 0 & \begin{tabular}{|l|}
$5.49 \mathrm{E}-01$ \\
\end{tabular} & 0 & 0 & 0 & $4.43 \mathrm{E}+00$ \\
\hline 6TB063 & 0.3 & 52.8516 & 6 & 0 & 0 & 0 & 0 & 4.64E-01 & 0 & 0 & 1.82E-01 & 0 & 0 & 0 & $1.47 \mathrm{E}+00$ \\
\hline 6TB064 & 0.3 & 58.8281 & 6 & 0 & 0 & 0 & 0 & 4.08E-01 & 0 & 0 & \begin{tabular}{|l|}
$1.81 \mathrm{E}-01$ \\
\end{tabular} & 0 & 0 & 0 & $1.46 \mathrm{E}+00$ \\
\hline 6TB069 & 1.6 & \begin{tabular}{|l|}
47.6719 \\
\end{tabular} & 6 & 0 & 0 & 0 & 0 & $3.32 \mathrm{E}+00$ & 0 & 0 & 9.71E-01 & 0 & 0 & 0 & $7.85 \mathrm{E}+00$ \\
\hline 6TB070 & 1.8 & 66 & 6 & 0 & 0 & 0 & 0 & $3.62 \mathrm{E}+00$ & 0 & 0 & $1.09 \mathrm{E}+00$ & 0 & 0 & 0 & $8.80 \mathrm{E}+00$ \\
\hline 6TB071 & 1.1 & 49.6641 & 6 & 0 & 0 & 0 & 0 & $2.23 \mathrm{E}+00$ & 0 & 0 & 6.68E-01 & 0 & 0 & 0 & $5.40 \mathrm{E}+00$ \\
\hline 6TB072 & 1.2 & \begin{tabular}{|l|}
56.8359 \\
\end{tabular} & 6 & 0 & 0 & 0 & 0 & $2.40 \mathrm{E}+00$ & 0 & 0 & 7.28E-01 & 0 & 0 & 0 & $5.88 \mathrm{E}+00$ \\
\hline 6TB073 & 1.2 & \begin{tabular}{|l|}
63.2109 \\
\end{tabular} & 6 & 0 & 0 & 0 & 0 & $2.35 \mathrm{E}+00$ & 0 & 0 & \begin{tabular}{|l|}
$7.27 \mathrm{E}-01$ \\
\end{tabular} & 0 & 0 & 0 & $5.88 \mathrm{E}+00$ \\
\hline 6TB074 & 1.9 & \begin{tabular}{|l|}
41.6953 \\
\end{tabular} & 6 & 0 & 0 & 0 & 0 & $3.99 \mathrm{E}+00$ & 0 & 0 & $1.15 \mathrm{E}+00$ & 0 & 0 & 0 & $9.33 \mathrm{E}+00$ \\
\hline 6TB075 & 1.1 & \begin{tabular}{|l|}
54.8438 \\
\end{tabular} & 6 & 0 & 0 & 0 & 0 & $2.20 \mathrm{E}+00$ & 0 & 0 & 6.69E-01 & 0 & 0 & 0 & $5.41 \mathrm{E}+00$ \\
\hline 6TB076 & 1.6 & \begin{tabular}{|l|}
55.2422 \\
\end{tabular} & 6 & 0 & 0 & 0 & 0 & $3.27 \mathrm{E}+00$ & 0 & 0 & 9.67E-01 & 0 & 0 & 0 & $7.81 \mathrm{E}+00$ \\
\hline 6TB077 & 1.8 & \begin{tabular}{|l|}
54.4453 \\
\end{tabular} & 6 & 0 & 0 & 0 & 0 & $3.72 \mathrm{E}+00$ & 0 & 0 & \begin{tabular}{|l|}
$1.09 \mathrm{E}+00$ \\
\end{tabular} & 0 & 0 & 0 & $8.84 \mathrm{E}+00$ \\
\hline 6TB078 & 1.8 & \begin{tabular}{|l|}
57.6328 \\
\end{tabular} & 6 & 0 & 0 & 0 & 0 & $3.70 \mathrm{E}+00$ & 0 & 0 & $1.09 \mathrm{E}+00$ & 0 & 0 & 0 & $8.82 \mathrm{E}+00$ \\
\hline 6TB079 & 1 & 40.5 & 6 & 0 & 0 & 0 & 0 & $2.06 \mathrm{E}+00$ & 0 & 0 & \begin{tabular}{|l|}
$6.08 \mathrm{E}-01$ \\
\end{tabular} & 0 & 0 & 0 & $4.92 \mathrm{E}+00$ \\
\hline
\end{tabular}




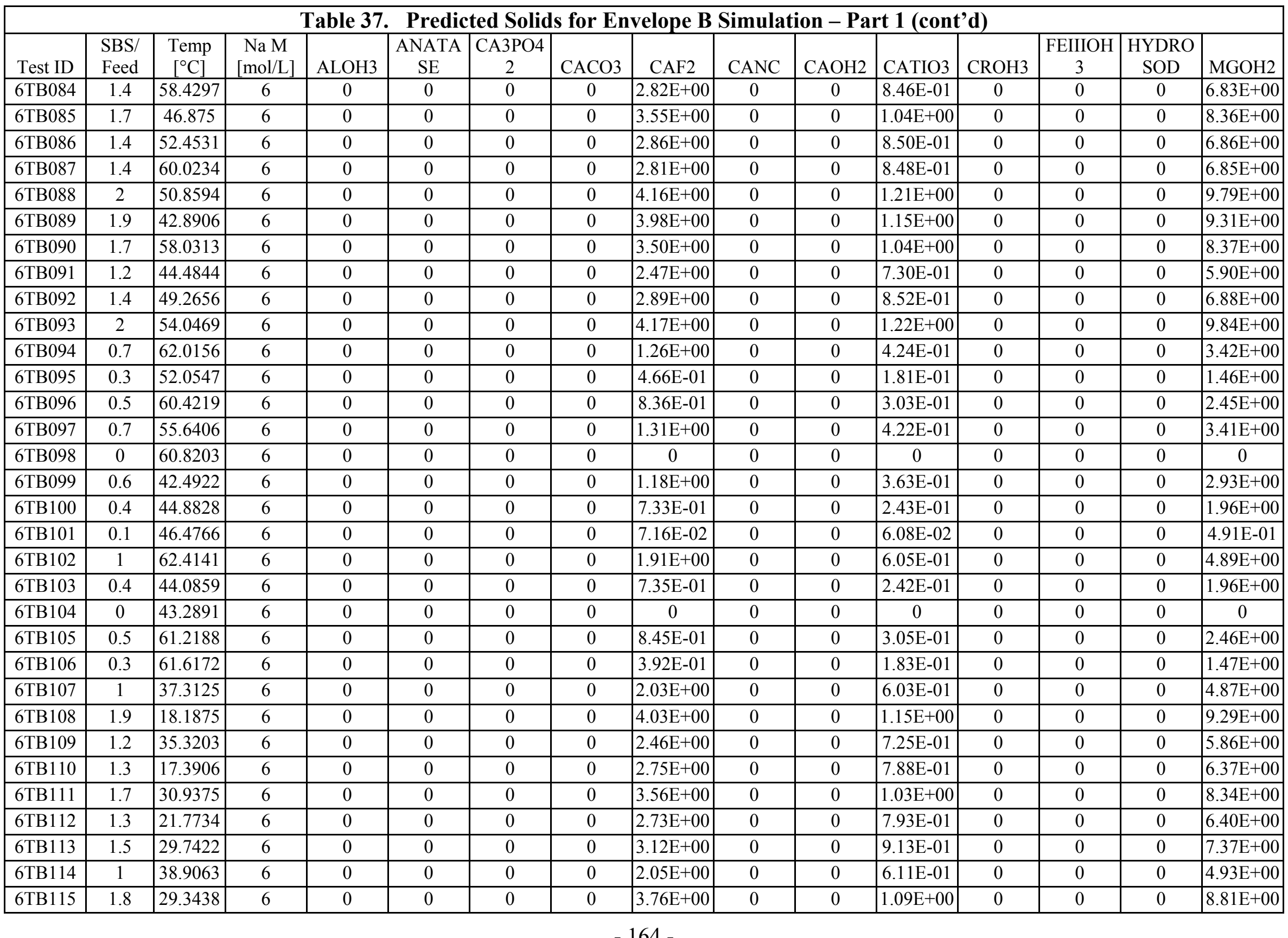




\begin{tabular}{|c|c|c|c|c|c|c|c|c|c|c|c|c|c|c|c|}
\hline \multicolumn{16}{|c|}{ Table 37. Predicted Solids for Envelope B Simulation - Part 1 (cont'd) } \\
\hline Test ID & $\begin{array}{l}\text { SBS/ } \\
\text { Feed }\end{array}$ & $\begin{array}{l}\text { Temp } \\
{\left[{ }^{\circ} \mathrm{C}\right]} \\
\end{array}$ & $\begin{array}{c}\mathrm{Na} \mathrm{M} \\
{[\mathrm{mol} / \mathrm{L}]}\end{array}$ & ALOH3 & $\begin{array}{c}\text { ANATA } \\
\text { SE }\end{array}$ & \begin{tabular}{|c|} 
CA3PO4 \\
2 \\
\end{tabular} & $\mathrm{CACO} 3$ & CAF2 & CANC & $\mathrm{CAOH} 2$ & CATIO3 & $\mathrm{CROH} 3$ & $\begin{array}{c}\text { FEIIIOH } \\
3 \\
\end{array}$ & $\begin{array}{c}\text { HYDRO } \\
\text { SOD } \\
\end{array}$ & MGOH2 \\
\hline 6TB116 & 1.4 & 16.1953 & 6 & $1.74 \mathrm{E}+02$ & 0 & 0 & 0 & $2.97 \mathrm{E}+00$ & 0 & 0 & $8.50 \mathrm{E}-01$ & 0 & 0 & 0 & $6.87 \mathrm{E}+00$ \\
\hline 6TB117 & 1.1 & 16.5938 & 6 & $1.59 \mathrm{E}+02$ & 0 & 0 & 0 & $2.32 \mathrm{E}+00$ & 0 & 0 & $6.67 \mathrm{E}-01$ & 0 & 0 & 0 & $5.39 \mathrm{E}+00$ \\
\hline 6TB118 & 1.8 & 28.1484 & 6 & 0 & 0 & 0 & 0 & $3.74 \mathrm{E}+00$ & 0 & 0 & $1.09 \mathrm{E}+00$ & 0 & 0 & 0 & $8.81 \mathrm{E}+00$ \\
\hline 6TB119 & 1.7 & 22.1719 & 6 & 0 & 0 & 0 & 0 & $3.57 \mathrm{E}+00$ & 0 & 0 & $1.03 \mathrm{E}+00$ & 0 & 0 & 0 & $8.35 \mathrm{E}+00$ \\
\hline 6TB120 & 1.3 & 30.5391 & 6 & 0 & 0 & 0 & 0 & $2.69 \mathrm{E}+00$ & 0 & 0 & \begin{tabular}{|l|}
$7.90 \mathrm{E}-01$ \\
\end{tabular} & 0 & 0 & 0 & $6.38 \mathrm{E}+00$ \\
\hline 6TB121 & 0.2 & 32.1328 & 6 & 0 & 0 & 0 & 0 & $3.40 \mathrm{E}-01$ & 0 & 0 & $1.21 \mathrm{E}-01$ & 0 & 0 & 0 & 9.79E-01 \\
\hline 6TB122 & 0.5 & 23.7656 & 6 & 0 & 0 & 0 & 0 & 9.89E-01 & 0 & 0 & \begin{tabular}{|l|}
$3.02 \mathrm{E}-01$ \\
\end{tabular} & 0 & 0 & 0 & $2.44 \mathrm{E}+00$ \\
\hline 6TB123 & 0.2 & 27.3516 & 6 & 0 & 0 & 0 & 0 & $3.54 \mathrm{E}-01$ & 0 & 0 & $1.22 \mathrm{E}-01$ & 0 & 0 & 0 & $9.83 \mathrm{E}-01$ \\
\hline 6TB124 & 0.4 & 33.3281 & 6 & 0 & 0 & 0 & 0 & \begin{tabular}{|l|} 
7.47E-01 \\
\end{tabular} & 0 & 0 & \begin{tabular}{|l|}
$2.42 \mathrm{E}-01$ \\
\end{tabular} & 0 & 0 & 0 & $1.96 \mathrm{E}+00$ \\
\hline 6TB125 & 0.2 & 15 & 6 & 0 & 0 & 0 & 0 & 3.93E-01 & 0 & 0 & \begin{tabular}{|l|}
$1.21 \mathrm{E}-01$ \\
\end{tabular} & 0 & 0 & 0 & \begin{tabular}{|l|}
$9.80 \mathrm{E}-01$ \\
\end{tabular} \\
\hline 6TB126 & 0.9 & 31.3359 & 6 & 0 & 0 & 0 & 0 & $1.85 \mathrm{E}+00$ & 0 & 0 & \begin{tabular}{|c|}
$5.48 \mathrm{E}-01$ \\
\end{tabular} & 0 & 0 & 0 & $4.43 \mathrm{E}+00$ \\
\hline 6TB127 & 0.8 & 24.1641 & 6 & 0 & 0 & 0 & 0 & $1.64 \mathrm{E}+00$ & 0 & 0 & \begin{tabular}{|l|}
$4.86 \mathrm{E}-01$ \\
\end{tabular} & 0 & 0 & 0 & $3.93 \mathrm{E}+00$ \\
\hline 6TB128 & 0.8 & 17.7891 & 6 & $2.04 \mathrm{E}+02$ & 0 & 0 & 0 & $1.68 \mathrm{E}+00$ & 0 & 0 & \begin{tabular}{|l|}
$4.86 \mathrm{E}-01$ \\
\end{tabular} & 0 & 0 & 0 & $3.93 \mathrm{E}+00$ \\
\hline 6TB129 & 0.1 & 39.3047 & 6 & 0 & 0 & 0 & 0 & \begin{tabular}{|l|}
$1.03 \mathrm{E}-01$ \\
\end{tabular} & 0 & 0 & \begin{tabular}{|l|}
$6.06 \mathrm{E}-02$ \\
\end{tabular} & 0 & 0 & 0 & \begin{tabular}{|l|}
$4.89 \mathrm{E}-01$ \\
\end{tabular} \\
\hline 6TB130 & 0.9 & 26.1563 & 6 & 0 & 0 & 0 & 0 & $1.85 \mathrm{E}+00$ & 0 & 0 & \begin{tabular}{|l|}
$5.47 \mathrm{E}-01$ \\
\end{tabular} & 0 & 0 & 0 & $4.42 \mathrm{E}+00$ \\
\hline 6TB131 & 0.4 & 25.7578 & 6 & 0 & 0 & 0 & 0 & 7.76E-01 & 0 & 0 & \begin{tabular}{|l|}
$2.44 \mathrm{E}-01$ \\
\end{tabular} & 0 & 0 & 0 & $1.97 \mathrm{E}+00$ \\
\hline 6TB132 & 0.2 & 26.5547 & 6 & 0 & 0 & 0 & 0 & 3.52E-01 & 0 & 0 & \begin{tabular}{|l|}
$1.22 \mathrm{E}-01$ \\
\end{tabular} & 0 & 0 & 0 & $9.84 \mathrm{E}-01$ \\
\hline 6TB133 & 0.2 & 23.3672 & 6 & 0 & 0 & 0 & 0 & $3.41 \mathrm{E}-01$ & 0 & 0 & $1.22 \mathrm{E}-01$ & 0 & 0 & 0 & $9.83 \mathrm{E}-01$ \\
\hline
\end{tabular}


Table 38. Predicted Solids for Envelope B Simulations - Part 2

\begin{tabular}{|c|c|c|c|c|c|c|c|c|c|c|c|c|c|c|c|}
\hline Test ID & $\mathrm{NA} 2 \mathrm{C} 2 \mathrm{O} 4$ & $\begin{array}{c}\text { NA2CO3. } \\
1 \mathrm{H} 2 \mathrm{O}\end{array}$ & $\mathrm{NA} 2 \mathrm{U} 2 \mathrm{O} 7$ & NA3FSO4 & \begin{tabular}{|c|} 
NA6SO42 \\
$\mathrm{CO} 3$ \\
\end{tabular} & NAF & $\begin{array}{c}\text { NAFPO4. } \\
19 \mathrm{H} 2 \mathrm{O}\end{array}$ & NANO3 & $\begin{array}{c}\text { NAPHOH. } \\
12 \mathrm{H} 2 \mathrm{O}\end{array}$ & \begin{tabular}{|c|} 
NASGEL. \\
$15.5 \mathrm{H} 2 \mathrm{O}$
\end{tabular} & $\mathrm{NIOH} 2$ & ZRO2 & $\begin{array}{c}\text { Total } \\
\text { Solids } \\
\end{array}$ & \begin{tabular}{|c|} 
Total Soln \\
Mass
\end{tabular} & $\begin{array}{c}\text { Solids } \% \\
\text { of Total } \\
\text { Soln } \\
\end{array}$ \\
\hline 10TB001 & $8.30 \mathrm{E}+02$ & 0 & 0 & 0 & 0 & $5.83 \mathrm{E}+02$ & 0 & 0 & 0 & 0 & 0 & 0 & $1.41 \mathrm{E}+03$ & $2.91 \mathrm{E}+05$ & $0.49 \%$ \\
\hline 10TB002 & 0 & 0 & 0 & 0 & 0 & $5.22 \mathrm{E}+02$ & 0 & 0 & 0 & 0 & 0 & 0 & $5.22 \mathrm{E}+02$ & $2.94 \mathrm{E}+05$ & $0.18 \%$ \\
\hline 10TB003 & 0 & 0 & 0 & 0 & 0 & $5.76 \mathrm{E}+02$ & 0 & 0 & 0 & 0 & 0 & 0 & $5.76 \mathrm{E}+02$ & $2.94 \mathrm{E}+05$ & $0.20 \%$ \\
\hline 10TB004 & $8.33 \mathrm{E}+02$ & 0 & 0 & 0 & 0 & $5.92 \mathrm{E}+02$ & 0 & 0 & 0 & 0 & 0 & 0 & $1.42 \mathrm{E}+03$ & $2.90 \mathrm{E}+05$ & $0.49 \%$ \\
\hline $10 \mathrm{~TB} 005$ & $8.78 \mathrm{E}+02$ & 0 & 0 & 0 & 0 & $5.65 \mathrm{E}+02$ & 0 & 0 & 0 & 0 & 0 & 0 & $1.44 \mathrm{E}+03$ & $2.91 \mathrm{E}+05$ & $0.50 \%$ \\
\hline $10 \mathrm{~TB} 006$ & 0 & 0 & 0 & 0 & 0 & $6.54 \mathrm{E}+02$ & 0 & 0 & 0 & 0 & 0 & 0 & $6.54 \mathrm{E}+02$ & $2.93 \mathrm{E}+05$ & $0.22 \%$ \\
\hline 10TB007 & 0 & 0 & 0 & 0 & 0 & 0 & 0 & 0 & 0 & 0 & 0 & 0 & 0 & $2.79 \mathrm{E}+05$ & 0 \\
\hline 10TB008 & 0 & 0 & 0 & 0 & 0 & 0 & 0 & 0 & 0 & 0 & 0 & 0 & 0 & $2.81 \mathrm{E}+05$ & 0 \\
\hline 10TB009 & 0 & 0 & 0 & 0 & 0 & $3.80 \mathrm{E}+01$ & 0 & 0 & 0 & 0 & 0 & 0 & $3.80 \mathrm{E}+01$ & $2.81 \mathrm{E}+05$ & $0.01 \%$ \\
\hline $10 \mathrm{~TB} 010$ & 0 & 0 & 0 & 0 & 0 & $3.27 \mathrm{E}+01$ & 0 & 0 & 0 & 0 & 0 & 0 & $3.27 \mathrm{E}+01$ & $2.79 \mathrm{E}+05$ & $0.01 \%$ \\
\hline 10TB011 & $6.65 \mathrm{E}+01$ & 0 & 0 & 0 & 0 & $3.83 \mathrm{E}+01$ & 0 & 0 & 0 & 0 & 0 & 0 & $1.05 \mathrm{E}+02$ & $2.79 \mathrm{E}+05$ & $0.04 \%$ \\
\hline 10TB012 & 0 & 0 & 0 & 0 & 0 & $1.44 \mathrm{E}+02$ & 0 & 0 & 0 & 0 & 0 & 0 & $1.44 \mathrm{E}+02$ & $2.80 \mathrm{E}+05$ & $0.05 \%$ \\
\hline $10 \mathrm{~TB} 014$ & \begin{tabular}{|l|}
0 \\
\end{tabular} & 0 & 0 & 0 & 0 & $9.41 \mathrm{E}+02$ & 0 & 0 & 0 & 0 & 0 & 0 & $9.57 \mathrm{E}+02$ & $3.05 \mathrm{E}+05$ & $0.31 \%$ \\
\hline 10TB015 & 0 & 0 & 0 & 0 & 0 & $9.98 \mathrm{E}+02$ & 0 & 0 & 0 & 0 & 0 & 0 & $1.01 \mathrm{E}+03$ & $3.05 \mathrm{E}+05$ & $0.33 \%$ \\
\hline $10 \mathrm{~TB} 016$ & $7.98 \mathrm{E}+02$ & 0 & 0 & 0 & 0 & $1.01 \mathrm{E}+03$ & 0 & 0 & 0 & 0 & 0 & 0 & $1.82 \mathrm{E}+03$ & $3.01 \mathrm{E}+05$ & $0.60 \%$ \\
\hline 10TB017 & $8.42 \mathrm{E}+02$ & 0 & 0 & 0 & 0 & $9.83 \mathrm{E}+02$ & 0 & 0 & 0 & 0 & 0 & 0 & $1.84 \mathrm{E}+03$ & $3.02 \mathrm{E}+05$ & $0.61 \%$ \\
\hline 10TB018 & 0 & 0 & 0 & 0 & 0 & $1.08 \mathrm{E}+03$ & 0 & 0 & 0 & 0 & 0 & 0 & $1.09 \mathrm{E}+03$ & $3.03 \mathrm{E}+05$ & $0.36 \%$ \\
\hline 10TB019 & 0 & 0 & 0 & 0 & 0 & $4.05 \mathrm{E}+02$ & 0 & 0 & 0 & 0 & $1.09 \mathrm{E}+00$ & 0 & $4.21 \mathrm{E}+02$ & $2.89 \mathrm{E}+05$ & $0.15 \%$ \\
\hline 10TB020 & 0 & 0 & 0 & 0 & 0 & $3.75 \mathrm{E}+02$ & 0 & 0 & 0 & 0 & $1.13 \mathrm{E}+00$ & 0 & $3.91 \mathrm{E}+02$ & $2.91 \mathrm{E}+05$ & $0.13 \%$ \\
\hline 10TB021 & 0 & 0 & 0 & 0 & 0 & $4.51 \mathrm{E}+02$ & 0 & 0 & 0 & 0 & $1.12 \mathrm{E}+00$ & 0 & $4.67 \mathrm{E}+02$ & $2.91 \mathrm{E}+05$ & $0.16 \%$ \\
\hline 10TB022 & 0 & 0 & 0 & 0 & 0 & $4.40 \mathrm{E}+02$ & 0 & 0 & 0 & 0 & $1.09 \mathrm{E}+00$ & 0 & $4.56 \mathrm{E}+02$ & $2.89 \mathrm{E}+05$ & $0.16 \%$ \\
\hline 10TB023 & 0 & 0 & 0 & 0 & 0 & $4.48 \mathrm{E}+02$ & 0 & 0 & 0 & 0 & $1.13 \mathrm{E}+00$ & 0 & $4.64 \mathrm{E}+02$ & $2.89 \mathrm{E}+05$ & $0.16 \%$ \\
\hline 10TB024 & 0 & 0 & 0 & 0 & 0 & $5.55 \mathrm{E}+02$ & 0 & 0 & 0 & 0 & $1.08 \mathrm{E}+00$ & 0 & $5.71 \mathrm{E}+02$ & $2.90 \mathrm{E}+05$ & $0.20 \%$ \\
\hline 10TB025 & 0 & 0 & 0 & 0 & 0 & $4.41 \mathrm{E}+02$ & 0 & 0 & 0 & 0 & 0 & 0 & $4.48 \mathrm{E}+02$ & $2.91 \mathrm{E}+05$ & $0.15 \%$ \\
\hline 10TB026 & 0 & 0 & 0 & 0 & 0 & $5.93 \mathrm{E}+02$ & 0 & 0 & 0 & 0 & 0 & 0 & $5.93 \mathrm{E}+02$ & $2.93 \mathrm{E}+05$ & $0.20 \%$ \\
\hline 10TB029 & 0 & 0 & 0 & $6.81 \mathrm{E}+03$ & 0 & 0 & 0 & 0 & 0 & 0 & 0 & 0 & $6.81 \mathrm{E}+03$ & $2.86 \mathrm{E}+05$ & $2.39 \%$ \\
\hline
\end{tabular}

$-166-$ 
Table 38. Predicted Solids for Envelope B Simulations - Part 2 (cont'd)

\begin{tabular}{|c|c|c|c|c|c|c|c|c|c|c|c|c|c|c|c|}
\hline Test ID & $\begin{array}{c}\mathrm{NA} 2 \mathrm{C} 2 \mathrm{O} \\
4 \\
\end{array}$ & $\begin{array}{c}\text { NA2 } 2 \mathrm{CO} 3 . \\
1 \mathrm{H} 2 \mathrm{O}\end{array}$ & NA2U2O7 & $\begin{array}{c}\text { NA3FSO } \\
4 \\
\end{array}$ & $\begin{array}{c}\mathrm{NA} 6 \mathrm{SO} 4 \\
2 \mathrm{CO} 3 \\
\end{array}$ & NAF & $\begin{array}{c}\text { NAFPO4. } \\
19 \mathrm{H} 2 \mathrm{O}\end{array}$ & NANO3 & \begin{tabular}{|c} 
NAPHOH \\
$.12 \mathrm{H} 2 \mathrm{O}$ \\
\end{tabular} & $\begin{array}{l}\text { NASGEL. } \\
15.5 \mathrm{H} 2 \mathrm{O}\end{array}$ & $\mathrm{NIOH} 2$ & ZRO2 & $\begin{array}{c}\text { Total } \\
\text { Solids } \\
\end{array}$ & $\begin{array}{c}\text { Total Soln } \\
\text { Mass }\end{array}$ & $\begin{array}{c}\text { Solids } \% \\
\text { of Total } \\
\text { Soln }\end{array}$ \\
\hline 10TB030 & 0 & 0 & 0 & $5.83 \mathrm{E}+03$ & 0 & 0 & 0 & 0 & 0 & 0 & 0 & 0 & $5.83 \mathrm{E}+03$ & $2.81 \mathrm{E}+05$ & $2.07 \%$ \\
\hline 10TB031 & 0 & 0 & 0 & $6.66 \mathrm{E}+03$ & 0 & 0 & 0 & 0 & 0 & 0 & 0 & 0 & $6.66 \mathrm{E}+03$ & $2.85 \mathrm{E}+05$ & $2.34 \%$ \\
\hline \begin{tabular}{|l|} 
10TB032 \\
\end{tabular} & \begin{tabular}{|l|}
$7.27 \mathrm{E}+02$ \\
\end{tabular} & 0 & 0 & $6.76 \mathrm{E}+03$ & 0 & 0 & 0 & 0 & 0 & 0 & 0 & 0 & $\begin{array}{l}7.50 \mathrm{E}+03 \\
\end{array}$ & $2.84 \mathrm{E}+05$ & $2.64 \%$ \\
\hline 10TB033 & $3.96 \mathrm{E}+02$ & 0 & 0 & $5.65 \mathrm{E}+03$ & 0 & 0 & 0 & 0 & 0 & 0 & 0 & 0 & $6.05 \mathrm{E}+03$ & $2.81 \mathrm{E}+05$ & $2.15 \%$ \\
\hline 10TB034 & $3.40 \mathrm{E}+02$ & 0 & 0 & $5.60 \mathrm{E}+03$ & 0 & 0 & 0 & 0 & 0 & 0 & 0 & 0 & $5.94 \mathrm{E}+03$ & $2.79 \mathrm{E}+05$ & $2.13 \%$ \\
\hline \begin{tabular}{|l|}
$10 \mathrm{~TB} 035$ \\
\end{tabular} & $6.94 \mathrm{E}+02$ & 0 & 0 & $6.37 \mathrm{E}+03$ & 0 & 0 & 0 & 0 & 0 & 0 & 0 & 0 & $7.07 \mathrm{E}+03$ & $2.83 \mathrm{E}+05$ & $2.50 \%$ \\
\hline 10TB036 & $4.26 \mathrm{E}+02$ & 0 & 0 & $6.44 \mathrm{E}+03$ & 0 & 0 & 0 & 0 & 0 & 0 & 0 & 0 & $6.87 \mathrm{E}+03$ & $2.82 \mathrm{E}+05$ & $2.44 \%$ \\
\hline 10TB037 & $4.95 \mathrm{E}+02$ & 0 & 0 & $6.17 \mathrm{E}+03$ & 0 & 0 & 0 & 0 & 0 & 0 & 0 & 0 & $6.67 \mathrm{E}+03$ & $2.83 \mathrm{E}+05$ & $2.36 \%$ \\
\hline 10TB038 & $6.18 \mathrm{E}+02$ & 0 & 0 & $5.58 \mathrm{E}+03$ & 0 & 0 & 0 & 0 & 0 & 0 & 0 & 0 & $6.20 \mathrm{E}+03$ & $2.81 \mathrm{E}+05$ & $2.21 \%$ \\
\hline 10TB039 & $4.51 \mathrm{E}+02$ & 0 & 0 & 0 & 0 & $8.29 \mathrm{E}+02$ & 0 & 0 & 0 & 0 & 0 & 0 & $1.29 \mathrm{E}+03$ & $2.98 \mathrm{E}+05$ & $0.43 \%$ \\
\hline 10TB040 & 0 & 0 & 0 & $7.65 \mathrm{E}+03$ & 0 & 0 & 0 & 0 & 0 & 0 & 0 & 0 & $7.66 \mathrm{E}+03$ & $2.89 \mathrm{E}+05$ & $2.65 \%$ \\
\hline \begin{tabular}{|l|} 
10TB041 \\
\end{tabular} & $2.59 \mathrm{E}+02$ & 0 & 0 & $7.73 \mathrm{E}+03$ & 0 & 0 & 0 & 0 & 0 & 0 & 0 & 0 & $8.00 \mathrm{E}+03$ & $2.91 \mathrm{E}+05$ & $2.75 \%$ \\
\hline \begin{tabular}{|l|} 
10TB042 \\
\end{tabular} & 0 & 0 & 0 & $7.13 \mathrm{E}+03$ & 0 & 0 & 0 & 0 & 0 & 0 & 0 & 0 & $7.14 \mathrm{E}+03$ & $2.88 \mathrm{E}+05$ & $2.47 \%$ \\
\hline 10TB043 & $1.60 \mathrm{E}+02$ & 0 & 0 & $8.39 \mathrm{E}+03$ & 0 & 0 & 0 & 0 & 0 & 0 & 0 & 0 & $8.57 \mathrm{E}+03$ & $2.92 \mathrm{E}+05$ & $2.93 \%$ \\
\hline 10TB044 & 0 & 0 & 0 & $7.14 \mathrm{E}+03$ & 0 & 0 & 0 & 0 & 0 & 0 & 0 & 0 & $7.15 \mathrm{E}+03$ & $2.85 \mathrm{E}+05$ & $2.51 \%$ \\
\hline 10TB045 & 0 & 0 & 0 & $7.09 \mathrm{E}+03$ & 0 & 0 & 0 & 0 & 0 & 0 & 0 & 0 & $7.10 \mathrm{E}+03$ & $2.87 \mathrm{E}+05$ & $2.48 \%$ \\
\hline 10TB046 & $4.05 \mathrm{E}+02$ & 0 & 0 & $7.88 \mathrm{E}+03$ & 0 & 0 & 0 & 0 & 0 & 0 & $2.14 \mathrm{E}-01$ & 0 & $8.30 \mathrm{E}+03$ & $2.87 \mathrm{E}+05$ & $2.89 \%$ \\
\hline 10TB047 & $7.73 \mathrm{E}+02$ & 0 & 0 & 0 & 0 & $7.49 \mathrm{E}+02$ & 0 & 0 & 0 & 0 & 0 & 0 & $1.53 \mathrm{E}+03$ & $2.95 \mathrm{E}+05$ & $0.52 \%$ \\
\hline 10TB048 & $3.27 \mathrm{E}+02$ & 0 & 0 & $7.00 \mathrm{E}+03$ & 0 & 0 & 0 & 0 & 0 & 0 & $6.13 \mathrm{E}-02$ & 0 & $7.34 \mathrm{E}+03$ & $2.85 \mathrm{E}+05$ & $2.58 \%$ \\
\hline 10TB049 & $3.04 \mathrm{E}+02$ & 0 & 0 & $7.54 \mathrm{E}+03$ & 0 & 0 & 0 & 0 & 0 & 0 & $4.56 \mathrm{E}-01$ & 0 & $7.86 \mathrm{E}+03$ & $2.87 \mathrm{E}+05$ & $2.74 \%$ \\
\hline \begin{tabular}{|l|} 
10TB050 \\
\end{tabular} & $7.33 \mathrm{E}+02$ & 0 & 0 & 0 & 0 & $8.75 \mathrm{E}+02$ & 0 & 0 & 0 & 0 & 0 & 0 & $1.62 \mathrm{E}+03$ & $2.99 \mathrm{E}+05$ & $0.54 \%$ \\
\hline \begin{tabular}{|l|} 
10TB051 \\
\end{tabular} & $7.30 \mathrm{E}+02$ & 0 & 0 & 0 & 0 & $8.70 \mathrm{E}+02$ & 0 & 0 & 0 & 0 & 0 & 0 & $1.61 \mathrm{E}+03$ & $2.98 \mathrm{E}+05$ & $0.54 \%$ \\
\hline 10TB052 & 0 & 0 & 0 & 0 & 0 & $4.55 \mathrm{E}+02$ & 0 & 0 & 0 & 0 & 0 & 0 & $4.63 \mathrm{E}+02$ & $2.90 \mathrm{E}+05$ & $0.16 \%$ \\
\hline \begin{tabular}{|l|} 
10TB053 \\
\end{tabular} & 0 & 0 & 0 & 0 & 0 & $1.02 \mathrm{E}+02$ & 0 & 0 & 0 & 0 & 0 & 0 & $1.03 \mathrm{E}+02$ & $2.81 \mathrm{E}+05$ & $0.04 \%$ \\
\hline \begin{tabular}{|l|}
$10 \mathrm{~TB} 054$ \\
\end{tabular} & 0 & 0 & 0 & 0 & 0 & $3.57 \mathrm{E}+02$ & 0 & 0 & 0 & 0 & 0 & 0 & $3.63 \mathrm{E}+02$ & $2.89 \mathrm{E}+05$ & $0.13 \%$ \\
\hline 10TB055 & 0 & 0 & 0 & 0 & 0 & $1.94 \mathrm{E}+02$ & 0 & 0 & 0 & 0 & $2.26 \mathrm{E}-01$ & 0 & $1.99 \mathrm{E}+02$ & $2.84 \mathrm{E}+05$ & $0.07 \%$ \\
\hline \begin{tabular}{|l|}
$10 \mathrm{~TB} 056$ \\
\end{tabular} & 0 & 0 & 0 & 0 & 0 & $2.50 \mathrm{E}+02$ & 0 & 0 & 0 & 0 & 0 & 0 & $2.53 \mathrm{E}+02$ & $2.85 \mathrm{E}+05$ & $0.09 \%$ \\
\hline \begin{tabular}{|l|}
$10 \mathrm{~TB} 057$ \\
\end{tabular} & 0 & 0 & 0 & 0 & 0 & $3.10 \mathrm{E}+02$ & 0 & 0 & 0 & 0 & $2.27 \mathrm{E}-01$ & 0 & $3.17 \mathrm{E}+02$ & $2.86 \mathrm{E}+05$ & $0.11 \%$ \\
\hline 10TB058 & 0 & 0 & 0 & 0 & 0 & $2.90 \mathrm{E}+02$ & 0 & 0 & 0 & 0 & 0 & 0 & $2.94 \mathrm{E}+02$ & $2.85 \mathrm{E}+05$ & $0.10 \%$ \\
\hline 10TB059 & 0 & 0 & 0 & 0 & 0 & $4.74 \mathrm{E}+02$ & 0 & 0 & 0 & 0 & 0 & 0 & $4.82 \mathrm{E}+02$ & $2.90 \mathrm{E}+05$ & $0.17 \%$ \\
\hline
\end{tabular}


Table 38. Predicted Solids for Envelope B Simulations - Part 2 (cont'd)

\begin{tabular}{|c|c|c|c|c|c|c|c|c|c|c|c|c|c|c|c|}
\hline Test ID & $\begin{array}{c}\mathrm{NA} 2 \mathrm{C} 2 \mathrm{O} \\
4\end{array}$ & $\begin{array}{c}\text { NA2CO3. } \\
1 \mathrm{H} 2 \mathrm{O}\end{array}$ & NA2U2O7 & $\begin{array}{c}\text { NA3FSO } \\
4\end{array}$ & $\begin{array}{c}\mathrm{NA6SO} 4 \\
2 \mathrm{CO} 3\end{array}$ & NAF & $\begin{array}{c}\text { NAFPO4. } \\
19 \mathrm{H} 2 \mathrm{O}\end{array}$ & NANO3 & $\begin{array}{c}\text { NAPHOH } \\
.12 \mathrm{H} 2 \mathrm{O}\end{array}$ & $\begin{array}{c}\text { NASGEL. } \\
15.5 \mathrm{H} 2 \mathrm{O}\end{array}$ & NIOH2 & ZRO2 & $\begin{array}{l}\text { Total } \\
\text { Solids }\end{array}$ & $\begin{array}{c}\text { Total Soln } \\
\text { Mass }\end{array}$ & $\begin{array}{c}\text { Solids } \% \\
\text { of Total } \\
\text { Soln }\end{array}$ \\
\hline 10TB060 & $1.94 \mathrm{E}+02$ & 0 & 0 & 0 & 0 & $1.85 \mathrm{E}+02$ & 0 & 0 & 0 & 0 & 0 & 0 & $3.81 \mathrm{E}+02$ & $2.84 \mathrm{E}+05$ & $0.13 \%$ \\
\hline 10TB061 & 0 & 0 & 0 & 0 & 0 & $1.71 \mathrm{E}+02$ & 0 & 0 & 0 & 0 & $1.88 \mathrm{E}-01$ & 0 & $1.75 \mathrm{E}+02$ & $2.83 \mathrm{E}+05$ & $0.06 \%$ \\
\hline 10TB062 & 0 & 0 & 0 & 0 & 0 & $1.98 \mathrm{E}+02$ & 0 & 0 & 0 & 0 & $3.87 \mathrm{E}-01$ & 0 & $2.05 \mathrm{E}+02$ & $2.86 \mathrm{E}+05$ & $0.07 \%$ \\
\hline 10TB063 & $3.48 \mathrm{E}+01$ & 0 & 0 & 0 & 0 & $1.85 \mathrm{E}+02$ & 0 & 0 & 0 & 0 & 0 & 0 & $2.22 \mathrm{E}+02$ & $2.84 \mathrm{E}+05$ & $0.08 \%$ \\
\hline 10TB064 & $1.33 \mathrm{E}+02$ & 0 & 0 & 0 & 0 & $2.20 \mathrm{E}+02$ & 0 & 0 & 0 & 0 & 0 & 0 & $3.56 \mathrm{E}+02$ & $2.82 \mathrm{E}+05$ & $0.13 \%$ \\
\hline 10TB065 & $1.32 \mathrm{E}+02$ & 0 & 0 & 0 & 0 & $2.86 \mathrm{E}+02$ & 0 & 0 & 0 & 0 & 0 & 0 & $4.23 \mathrm{E}+02$ & $2.86 \mathrm{E}+05$ & $0.15 \%$ \\
\hline 10TB066 & 0 & 0 & 0 & 0 & 0 & $5.43 \mathrm{E}+02$ & 0 & 0 & 0 & 0 & 6.91E-01 & 0 & $5.57 \mathrm{E}+02$ & $2.93 \mathrm{E}+05$ & $0.19 \%$ \\
\hline 10TB067 & 0 & 0 & 0 & 0 & 0 & $4.11 \mathrm{E}+02$ & 0 & 0 & 0 & 0 & $6.68 \mathrm{E}-01$ & 0 & $4.23 \mathrm{E}+02$ & $2.90 \mathrm{E}+05$ & $0.15 \%$ \\
\hline 10TB068 & 0 & 0 & 0 & 0 & 0 & $4.79 \mathrm{E}+02$ & 0 & 0 & 0 & 0 & $8.00 \mathrm{E}-01$ & 0 & $4.93 \mathrm{E}+02$ & $2.92 \mathrm{E}+05$ & $0.17 \%$ \\
\hline 10TB069 & 0 & 0 & 0 & 0 & 0 & $4.80 \mathrm{E}+02$ & 0 & 0 & 0 & 0 & $5.17 \mathrm{E}-01$ & 0 & $4.93 \mathrm{E}+02$ & $2.92 \mathrm{E}+05$ & $0.17 \%$ \\
\hline 10TB070 & 0 & 0 & 0 & 0 & 0 & $4.83 \mathrm{E}+02$ & 0 & 0 & 0 & 0 & $9.56 \mathrm{E}-01$ & 0 & $4.98 \mathrm{E}+02$ & $2.89 \mathrm{E}+05$ & $0.17 \%$ \\
\hline 10TB071 & 0 & 0 & 0 & 0 & 0 & $4.10 \mathrm{E}+02$ & 0 & 0 & 0 & 0 & $2.44 \mathrm{E}-01$ & 0 & $4.19 \mathrm{E}+02$ & $2.89 \mathrm{E}+05$ & $0.14 \%$ \\
\hline 10TB072 & 0 & 0 & 0 & 0 & 0 & $3.64 \mathrm{E}+02$ & 0 & 0 & 0 & 0 & $4.46 \mathrm{E}-01$ & 0 & $3.74 \mathrm{E}+02$ & $2.88 \mathrm{E}+05$ & $0.13 \%$ \\
\hline 10TB073 & $2.64 \mathrm{E}+01$ & 0 & 0 & 0 & 0 & $3.30 \mathrm{E}+02$ & 0 & 0 & 0 & 0 & $5.57 \mathrm{E}-01$ & 0 & $3.66 \mathrm{E}+02$ & $2.86 \mathrm{E}+05$ & $0.13 \%$ \\
\hline 10ТВ074 & $2.78 \mathrm{E}+02$ & 0 & 0 & 0 & 0 & $6.15 \mathrm{E}+02$ & 0 & 0 & 0 & 0 & $5.47 \mathrm{E}-01$ & 0 & $9.08 \mathrm{E}+02$ & $2.95 \mathrm{E}+05$ & $0.31 \%$ \\
\hline 10TB075 & 0 & 0 & 0 & 0 & 0 & $3.19 \mathrm{E}+02$ & 0 & 0 & 0 & 0 & $3.61 \mathrm{E}-01$ & 0 & $3.27 \mathrm{E}+02$ & $2.89 \mathrm{E}+05$ & $0.11 \%$ \\
\hline 10TB076 & $9.07 \mathrm{E}+01$ & 0 & 0 & 0 & 0 & $4.76 \mathrm{E}+02$ & 0 & 0 & 0 & 0 & $6.75 \mathrm{E}-01$ & 0 & $5.80 \mathrm{E}+02$ & $2.89 \mathrm{E}+05$ & $0.20 \%$ \\
\hline 10TB077 & $8.87 \mathrm{E}+01$ & 0 & 0 & 0 & 0 & $5.37 \mathrm{E}+02$ & 0 & 0 & 0 & 0 & $8.07 \mathrm{E}-01$ & 0 & $6.40 \mathrm{E}+02$ & $2.92 \mathrm{E}+05$ & $0.22 \%$ \\
\hline 10TB078 & $3.17 \mathrm{E}+01$ & 0 & 0 & 0 & 0 & $4.65 \mathrm{E}+02$ & 0 & 0 & 0 & 0 & $8.74 \mathrm{E}-01$ & 0 & $5.11 \mathrm{E}+02$ & $2.91 \mathrm{E}+05$ & $0.18 \%$ \\
\hline 10TB079 & $1.18 \mathrm{E}+02$ & 0 & 0 & 0 & 0 & $4.53 \mathrm{E}+02$ & 0 & 0 & 0 & 0 & 0 & 0 & $5.79 \mathrm{E}+02$ & $2.91 \mathrm{E}+05$ & $0.20 \%$ \\
\hline 10TB080 & $3.37 \mathrm{E}+02$ & 0 & 0 & 0 & 0 & $4.78 \mathrm{E}+02$ & 0 & 0 & 0 & 0 & 0 & 0 & $8.24 \mathrm{E}+02$ & $2.91 \mathrm{E}+05$ & $0.28 \%$ \\
\hline 10TB081 & 0 & 0 & 0 & 0 & 0 & $4.19 \mathrm{E}+02$ & 0 & 0 & 0 & 0 & $1.03 \mathrm{E}+00$ & 0 & $4.34 \mathrm{E}+02$ & $2.90 \mathrm{E}+05$ & $0.15 \%$ \\
\hline 10TB082 & $2.70 \mathrm{E}+02$ & 0 & 0 & 0 & 0 & $4.66 \mathrm{E}+02$ & 0 & 0 & 0 & 0 & $2.05 \mathrm{E}-01$ & 0 & $7.45 \mathrm{E}+02$ & $2.90 \mathrm{E}+05$ & $0.26 \%$ \\
\hline 10TB083 & $2.44 \mathrm{E}+02$ & 0 & 0 & 0 & 0 & $6.17 \mathrm{E}+02$ & 0 & 0 & 0 & 0 & $6.91 \mathrm{E}-01$ & 0 & $8.77 \mathrm{E}+02$ & $2.93 \mathrm{E}+05$ & $0.30 \%$ \\
\hline 10TB084 & 0 & 0 & 0 & 0 & 0 & $3.13 \mathrm{E}+02$ & 0 & 0 & 0 & 0 & $6.39 \mathrm{E}-01$ & 0 & $3.25 \mathrm{E}+02$ & $2.88 \mathrm{E}+05$ & $0.11 \%$ \\
\hline 10TB085 & $1.78 \mathrm{E}+02$ & 0 & 0 & 0 & 0 & $4.93 \mathrm{E}+02$ & 0 & 0 & 0 & 0 & $6.07 \mathrm{E}-01$ & 0 & $6.84 \mathrm{E}+02$ & $2.93 \mathrm{E}+05$ & $0.23 \%$ \\
\hline 10TB086 & $1.49 \mathrm{E}+02$ & 0 & 0 & 0 & 0 & $4.36 \mathrm{E}+02$ & 0 & 0 & 0 & 0 & 5.25E-01 & 0 & $5.96 \mathrm{E}+02$ & $2.89 \mathrm{E}+05$ & $0.21 \%$ \\
\hline 10TB087 & 0 & 0 & 0 & 0 & 0 & $3.43 \mathrm{E}+02$ & 0 & 0 & 0 & 0 & $6.46 \mathrm{E}-01$ & 0 & $3.54 \mathrm{E}+02$ & $2.89 \mathrm{E}+05$ & $0.12 \%$ \\
\hline 10TB088 & 0 & 0 & 0 & 0 & 0 & $5.58 \mathrm{E}+02$ & 0 & 0 & 0 & 0 & $8.63 \mathrm{E}-01$ & 0 & $5.74 \mathrm{E}+02$ & $2.94 \mathrm{E}+05$ & $0.20 \%$ \\
\hline 10TB089 & 0 & 0 & 0 & 0 & 0 & $6.51 \mathrm{E}+02$ & 0 & 0 & 0 & 0 & $5.73 \mathrm{E}-01$ & 0 & $6.67 \mathrm{E}+02$ & $2.95 \mathrm{E}+05$ & $0.23 \%$ \\
\hline 10TB090 & 0 & 0 & 0 & 0 & 0 & $3.88 \mathrm{E}+02$ & 0 & 0 & 0 & 0 & $8.44 \mathrm{E}-01$ & 0 & $4.01 \mathrm{E}+02$ & $2.91 \mathrm{E}+05$ & $0.14 \%$ \\
\hline
\end{tabular}


Table 38. Predicted Solids for Envelope B Simulations - Part 2 (cont'd)

\begin{tabular}{|c|c|c|c|c|c|c|c|c|c|c|c|c|c|c|c|}
\hline Test ID & $\begin{array}{c}\mathrm{NA} 2 \mathrm{C} 2 \mathrm{O} \\
4\end{array}$ & $\begin{array}{c}\text { NA2CO3. } \\
1 \mathrm{H} 2 \mathrm{O}\end{array}$ & NA2U2O7 & $\begin{array}{c}\text { NA3FSO } \\
4\end{array}$ & $\begin{array}{c}\mathrm{NA} 6 \mathrm{SO} 4 \\
2 \mathrm{CO} 3\end{array}$ & NAF & $\begin{array}{c}\text { NAFPO4. } \\
19 \mathrm{H} 2 \mathrm{O}\end{array}$ & NANO3 & $\begin{array}{c}\text { NAPHOH } \\
.12 \mathrm{H} 2 \mathrm{O}\end{array}$ & $\begin{array}{c}\text { NASGEL. } \\
15.5 \mathrm{H} 2 \mathrm{O}\end{array}$ & NIOH2 & ZRO2 & $\begin{array}{l}\text { Total } \\
\text { Solids }\end{array}$ & $\begin{array}{c}\text { Total Soln } \\
\text { Mass }\end{array}$ & $\begin{array}{c}\text { Solids } \% \\
\text { of Total } \\
\text { Soln }\end{array}$ \\
\hline 10TB091 & 0 & 0 & 0 & 0 & 0 & $4.05 \mathrm{E}+02$ & 0 & 0 & 0 & 0 & $1.95 \mathrm{E}-01$ & 0 & $4.15 \mathrm{E}+02$ & $2.92 \mathrm{E}+05$ & $0.14 \%$ \\
\hline 10TB092 & 0 & 0 & 0 & 0 & 0 & $4.31 \mathrm{E}+02$ & 0 & 0 & 0 & 0 & $4.73 \mathrm{E}-01$ & 0 & $4.42 \mathrm{E}+02$ & $2.91 \mathrm{E}+05$ & $0.15 \%$ \\
\hline 10TB093 & 0 & 0 & 0 & 0 & 0 & $5.12 \mathrm{E}+02$ & 0 & 0 & 0 & 0 & $9.63 \mathrm{E}-01$ & 0 & $5.28 \mathrm{E}+02$ & $2.94 \mathrm{E}+05$ & $0.18 \%$ \\
\hline 10TB094 & 0 & 0 & 0 & 0 & 0 & $1.91 \mathrm{E}+02$ & 0 & 0 & 0 & 0 & $2.17 \mathrm{E}-01$ & 0 & $1.97 \mathrm{E}+02$ & $2.85 \mathrm{E}+05$ & $0.07 \%$ \\
\hline 10TB095 & $1.74 \mathrm{E}+02$ & 0 & 0 & 0 & 0 & $2.22 \mathrm{E}+02$ & 0 & 0 & 0 & 0 & 0 & 0 & $3.99 \mathrm{E}+02$ & $2.83 \mathrm{E}+05$ & $0.14 \%$ \\
\hline 10TB096 & $1.09 \mathrm{E}+02$ & 0 & 0 & 0 & 0 & $2.29 \mathrm{E}+02$ & 0 & 0 & 0 & 0 & $5.21 \mathrm{E}-02$ & 0 & $3.42 \mathrm{E}+02$ & $2.84 \mathrm{E}+05$ & $0.12 \%$ \\
\hline 10TB097 & $1.66 \mathrm{E}+02$ & 0 & 0 & 0 & 0 & $2.97 \mathrm{E}+02$ & 0 & 0 & 0 & 0 & $1.09 \mathrm{E}-01$ & 0 & $4.68 \mathrm{E}+02$ & $2.84 \mathrm{E}+05$ & $0.16 \%$ \\
\hline 10TB098 & $3.57 \mathrm{E}+01$ & 0 & 0 & 0 & 0 & $2.83 \mathrm{E}+01$ & 0 & 0 & 0 & 0 & 0 & 0 & $6.40 \mathrm{E}+01$ & $2.81 \mathrm{E}+05$ & $0.02 \%$ \\
\hline 10TB099 & $2.71 \mathrm{E}+02$ & 0 & 0 & 0 & 0 & $2.96 \mathrm{E}+02$ & 0 & 0 & 0 & 0 & 0 & 0 & $5.71 \mathrm{E}+02$ & $2.88 \mathrm{E}+05$ & $0.20 \%$ \\
\hline $10 \mathrm{~TB} 100$ & $3.25 \mathrm{E}+02$ & 0 & 0 & 0 & 0 & $2.88 \mathrm{E}+02$ & 0 & 0 & 0 & 0 & 0 & 0 & $6.16 \mathrm{E}+02$ & $2.86 \mathrm{E}+05$ & $0.22 \%$ \\
\hline 10TB101 & 0 & 0 & 0 & 0 & 0 & $2.00 \mathrm{E}+02$ & 0 & 0 & 0 & 0 & 0 & 0 & $2.01 \mathrm{E}+02$ & $2.85 \mathrm{E}+05$ & $0.07 \%$ \\
\hline 10TB102 & 0 & 0 & 0 & 0 & 0 & $2.72 \mathrm{E}+02$ & 0 & 0 & 0 & 0 & 4.07E-01 & 0 & $2.80 \mathrm{E}+02$ & $2.86 \mathrm{E}+05$ & $0.10 \%$ \\
\hline $10 \mathrm{~TB} 103$ & 0 & 0 & 0 & 0 & 0 & $3.05 \mathrm{E}+02$ & 0 & 0 & 0 & 0 & 0 & 0 & $3.08 \mathrm{E}+02$ & $2.87 \mathrm{E}+05$ & $0.11 \%$ \\
\hline 10TB104 & 0 & 0 & 0 & 0 & 0 & $2.45 \mathrm{E}+02$ & 0 & 0 & 0 & 0 & 0 & 0 & $2.45 \mathrm{E}+02$ & $2.85 \mathrm{E}+05$ & $0.09 \%$ \\
\hline $10 \mathrm{~TB} 105$ & 0 & 0 & 0 & 0 & 0 & $1.00 \mathrm{E}+02$ & 0 & 0 & 0 & 0 & $9.17 \mathrm{E}-02$ & 0 & $1.04 \mathrm{E}+02$ & $2.85 \mathrm{E}+05$ & $0.04 \%$ \\
\hline 10TB106 & 0 & 0 & 0 & 0 & 0 & $1.50 \mathrm{E}+02$ & 0 & 0 & 0 & 0 & 0 & 0 & $1.53 \mathrm{E}+02$ & $2.83 \mathrm{E}+05$ & $0.05 \%$ \\
\hline $10 \mathrm{~TB} 107$ & $3.53 \mathrm{E}+02$ & 0 & 0 & $6.78 \mathrm{E}+03$ & 0 & 0 & 0 & 0 & 0 & 0 & 0 & 0 & $7.14 \mathrm{E}+03$ & $2.82 \mathrm{E}+05$ & $2.53 \%$ \\
\hline $10 \mathrm{~TB} 108$ & $7.49 \mathrm{E}+02$ & 0 & 0 & 0 & 0 & $9.38 \mathrm{E}+02$ & 0 & 0 & 0 & 0 & 0 & 0 & $1.70 \mathrm{E}+03$ & $3.01 \mathrm{E}+05$ & $0.57 \%$ \\
\hline 10TB109 & $4.31 \mathrm{E}+02$ & 0 & 0 & $7.25 \mathrm{E}+03$ & 0 & 0 & 0 & 0 & 0 & 0 & 0 & 0 & $7.69 \mathrm{E}+03$ & $2.83 \mathrm{E}+05$ & $2.72 \%$ \\
\hline $10 \mathrm{~TB} 110$ & $7.96 \mathrm{E}+02$ & 0 & 0 & 0 & 0 & $8.33 \mathrm{E}+02$ & 0 & 0 & 0 & 0 & 0 & 0 & $1.64 \mathrm{E}+03$ & $2.98 \mathrm{E}+05$ & $0.55 \%$ \\
\hline 10TB111 & $4.63 \mathrm{E}+02$ & 0 & 0 & $7.55 \mathrm{E}+03$ & 0 & 0 & 0 & 0 & 0 & 0 & 0 & 0 & $8.02 \mathrm{E}+03$ & $2.87 \mathrm{E}+05$ & $2.79 \%$ \\
\hline $10 \mathrm{~TB} 112$ & $4.95 \mathrm{E}+02$ & 0 & 0 & $7.40 \mathrm{E}+03$ & 0 & 0 & 0 & 0 & 0 & 0 & 0 & 0 & $7.90 \mathrm{E}+03$ & $2.89 \mathrm{E}+05$ & $2.73 \%$ \\
\hline 10TB113 & $4.24 \mathrm{E}+02$ & 0 & 0 & $7.69 \mathrm{E}+03$ & 0 & 0 & 0 & 0 & 0 & 0 & 0 & 0 & $8.13 E+03$ & $2.87 \mathrm{E}+05$ & $2.83 \%$ \\
\hline $10 \mathrm{~TB} 114$ & $3.12 \mathrm{E}+02$ & 0 & 0 & $6.89 \mathrm{E}+03$ & 0 & 0 & 0 & 0 & 0 & 0 & 0 & 0 & $7.21 \mathrm{E}+03$ & $2.83 \mathrm{E}+05$ & $2.55 \%$ \\
\hline 10TB115 & $6.00 \mathrm{E}+01$ & 0 & 0 & $7.83 \mathrm{E}+03$ & 0 & 0 & 0 & 0 & 0 & 0 & 0 & 0 & $7.91 \mathrm{E}+03$ & $2.89 \mathrm{E}+05$ & $2.74 \%$ \\
\hline 10TB116 & 0 & 0 & 0 & 0 & 0 & $8.68 \mathrm{E}+02$ & 0 & 0 & 0 & 0 & 0 & 0 & $8.79 \mathrm{E}+02$ & $3.01 \mathrm{E}+05$ & $0.29 \%$ \\
\hline $10 \mathrm{~TB} 117$ & $4.55 \mathrm{E}+02$ & 0 & 0 & 0 & 0 & $8.18 \mathrm{E}+02$ & 0 & 0 & 0 & 0 & 0 & 0 & $1.28 \mathrm{E}+03$ & $2.98 \mathrm{E}+05$ & $0.43 \%$ \\
\hline 10TB118 & $2.30 \mathrm{E}+02$ & 0 & 0 & $8.14 \mathrm{E}+03$ & 0 & 0 & 0 & 0 & 0 & 0 & 0 & 0 & $8.38 \mathrm{E}+03$ & $2.89 \mathrm{E}+05$ & $2.90 \%$ \\
\hline 10TB119 & 0 & 0 & 0 & $7.90 \mathrm{E}+03$ & 0 & 0 & 0 & 0 & 0 & 0 & 0 & 0 & $7.92 \mathrm{E}+03$ & $2.91 \mathrm{E}+05$ & $2.72 \%$ \\
\hline $10 \mathrm{~TB} 120$ & 0 & 0 & 0 & $7.49 \mathrm{E}+03$ & 0 & 0 & 0 & 0 & 0 & 0 & 0 & 0 & $7.50 \mathrm{E}+03$ & $2.87 \mathrm{E}+05$ & $2.62 \%$ \\
\hline 10TB121 & $2.96 \mathrm{E}+02$ & 0 & 0 & $6.09 \mathrm{E}+03$ & 0 & 0 & 0 & 0 & 0 & 0 & 0 & 0 & $6.39 \mathrm{E}+03$ & $2.81 \mathrm{E}+05$ & $2.27 \%$ \\
\hline
\end{tabular}


Table 38. Predicted Solids for Envelope B Simulations - Part 2 (cont'd)

\begin{tabular}{|c|c|c|c|c|c|c|c|c|c|c|c|c|c|c|c|}
\hline Test ID & $\begin{array}{c}\mathrm{NA} 2 \mathrm{C} 2 \mathrm{O} \\
4 \\
\end{array}$ & $\begin{array}{c}\text { NA2CO3. } \\
1 \mathrm{H} 2 \mathrm{O}\end{array}$ & NA2U2O7 & $\begin{array}{c}\text { NA3FSO } \\
4 \\
\end{array}$ & $\begin{array}{c}\text { NA6SO4 } \\
2 \mathrm{CO} 3 \\
\end{array}$ & NAF & $\begin{array}{c}\text { NAFPO4. } \\
19 \mathrm{H} 2 \mathrm{O} \\
\end{array}$ & NANO3 & $\begin{array}{c}\text { NAPHOH } \\
.12 \mathrm{H} 2 \mathrm{O} \\
\end{array}$ & $\begin{array}{c}\text { NASGEL. } \\
15.5 \mathrm{H} 2 \mathrm{O}\end{array}$ & $\mathrm{NIOH} 2$ & $\mathrm{ZRO} 2$ & $\begin{array}{c}\text { Total } \\
\text { Solids } \\
\end{array}$ & $\begin{array}{c}\text { Total Soln } \\
\text { Mass }\end{array}$ & $\begin{array}{c}\text { Solids } \% \\
\text { of Total } \\
\text { Soln } \\
\end{array}$ \\
\hline 10TB122 & $6.66 \mathrm{E}+02$ & 0 & 0 & $6.63 \mathrm{E}+03$ & 0 & 0 & 0 & 0 & 0 & 0 & 0 & 0 & $7.30 \mathrm{E}+03$ & $2.83 \mathrm{E}+05$ & $2.58 \%$ \\
\hline 10TB123 & $6.05 \mathrm{E}+02$ & 0 & 0 & $6.16 \mathrm{E}+03$ & 0 & 0 & 0 & 0 & 0 & 0 & 0 & 0 & $6.77 \mathrm{E}+03$ & $2.82 \mathrm{E}+05$ & $2.40 \%$ \\
\hline 10TB124 & $5.05 \mathrm{E}+02$ & 0 & 0 & $6.54 \mathrm{E}+03$ & 0 & 0 & 0 & 0 & 0 & 0 & 0 & 0 & $7.05 \mathrm{E}+03$ & $2.81 \mathrm{E}+05$ & $2.51 \%$ \\
\hline $10 \mathrm{~TB} 125$ & $8.61 \mathrm{E}+02$ & 0 & 0 & 0 & 0 & $5.88 \mathrm{E}+02$ & 0 & 0 & 0 & 0 & 0 & 0 & $1.45 \mathrm{E}+03$ & $2.93 \mathrm{E}+05$ & $0.50 \%$ \\
\hline $10 \mathrm{~TB} 126$ & $4.85 \mathrm{E}+02$ & 0 & 0 & $6.84 \mathrm{E}+03$ & 0 & 0 & 0 & 0 & 0 & 0 & 0 & 0 & $7.33 \mathrm{E}+03$ & $2.85 \mathrm{E}+05$ & $2.58 \%$ \\
\hline 10TB127 & $6.63 \mathrm{E}+02$ & 0 & 0 & $6.88 \mathrm{E}+03$ & 0 & 0 & 0 & 0 & 0 & 0 & 0 & 0 & $7.55 \mathrm{E}+03$ & $2.85 \mathrm{E}+05$ & $2.64 \%$ \\
\hline 10TB128 & 0 & 0 & 0 & 0 & 0 & $6.97 \mathrm{E}+02$ & 0 & 0 & 0 & 0 & 0 & 0 & $7.03 \mathrm{E}+02$ & $2.97 \mathrm{E}+05$ & $0.24 \%$ \\
\hline 10TB129 & 0 & 0 & 0 & $5.99 \mathrm{E}+03$ & 0 & 0 & 0 & 0 & 0 & 0 & 0 & 0 & $5.99 \mathrm{E}+03$ & $2.79 \mathrm{E}+05$ & $2.15 \%$ \\
\hline $10 \mathrm{~TB} 130$ & $3.08 \mathrm{E}+02$ & 0 & 0 & $7.03 \mathrm{E}+03$ & 0 & 0 & 0 & 0 & 0 & 0 & 0 & 0 & $7.35 \mathrm{E}+03$ & $2.86 \mathrm{E}+05$ & $2.57 \%$ \\
\hline 10TB131 & 0 & 0 & 0 & $6.53 \mathrm{E}+03$ & 0 & 0 & 0 & 0 & 0 & 0 & 0 & 0 & $6.53 \mathrm{E}+03$ & $2.85 \mathrm{E}+05$ & $2.29 \%$ \\
\hline 10TB132 & $1.97 \mathrm{E}+02$ & 0 & 0 & $6.15 \mathrm{E}+03$ & 0 & 0 & 0 & 0 & 0 & 0 & 0 & 0 & $6.35 \mathrm{E}+03$ & $2.83 \mathrm{E}+05$ & $2.24 \%$ \\
\hline 10TB133 & 0 & 0 & 0 & $6.46 \mathrm{E}+03$ & 0 & 0 & 0 & 0 & 0 & 0 & 0 & 0 & $6.47 \mathrm{E}+03$ & $2.84 \mathrm{E}+05$ & $2.27 \%$ \\
\hline 8TB001 & $7.67 \mathrm{E}+02$ & 0 & 0 & 0 & 0 & 0 & 0 & 0 & 0 & 0 & 0 & 0 & $7.67 \mathrm{E}+02$ & $3.43 \mathrm{E}+05$ & $0.22 \%$ \\
\hline $8 \mathrm{~TB} 002$ & 0 & 0 & 0 & 0 & 0 & 0 & 0 & 0 & 0 & 0 & 0 & 0 & 0 & $3.47 \mathrm{E}+05$ & $0.00 \%$ \\
\hline $8 \mathrm{~TB} 003$ & 0 & 0 & 0 & 0 & 0 & 0 & 0 & 0 & 0 & 0 & 0 & 0 & 0 & $3.47 \mathrm{E}+05$ & $0.00 \%$ \\
\hline $8 \mathrm{~TB} 004$ & $7.58 \mathrm{E}+02$ & 0 & 0 & 0 & 0 & 0 & 0 & 0 & 0 & 0 & 0 & 0 & $7.58 \mathrm{E}+02$ & $3.43 \mathrm{E}+05$ & $0.22 \%$ \\
\hline $8 \mathrm{~TB} 005$ & $7.95 \mathrm{E}+02$ & 0 & 0 & 0 & 0 & 0 & 0 & 0 & 0 & 0 & 0 & 0 & $7.95 \mathrm{E}+02$ & $3.44 \mathrm{E}+05$ & $0.23 \%$ \\
\hline $8 \mathrm{~TB} 006$ & 0 & 0 & 0 & 0 & 0 & $2.67 \mathrm{E}+00$ & 0 & 0 & 0 & 0 & 0 & 0 & $2.67 \mathrm{E}+00$ & $3.46 \mathrm{E}+05$ & $0.00 \%$ \\
\hline $8 \mathrm{~TB} 007$ & 0 & 0 & 0 & 0 & 0 & 0 & 0 & 0 & 0 & 0 & 0 & 0 & 0 & $3.30 \mathrm{E}+05$ & $0.00 \%$ \\
\hline $8 \mathrm{~TB} 008$ & 0 & 0 & 0 & 0 & 0 & 0 & 0 & 0 & 0 & 0 & 0 & 0 & 0 & $3.32 \mathrm{E}+05$ & $0.00 \%$ \\
\hline 8TB009 & 0 & 0 & 0 & 0 & 0 & 0 & 0 & 0 & 0 & 0 & 0 & 0 & 0 & $3.33 \mathrm{E}+05$ & $0.00 \%$ \\
\hline $8 \mathrm{~TB} 010$ & 0 & 0 & 0 & 0 & 0 & 0 & 0 & 0 & 0 & 0 & 0 & 0 & 0 & $3.30 \mathrm{E}+05$ & $0.00 \%$ \\
\hline 8TB011 & 0 & 0 & 0 & 0 & 0 & 0 & 0 & 0 & 0 & 0 & 0 & 0 & 0 & $3.31 \mathrm{E}+05$ & $0.00 \%$ \\
\hline 8TB012 & 0 & 0 & 0 & 0 & 0 & 0 & 0 & 0 & 0 & 0 & 0 & 0 & 0 & $3.32 \mathrm{E}+05$ & $0.00 \%$ \\
\hline $8 \mathrm{~TB} 013$ & $7.28 \mathrm{E}+02$ & 0 & 0 & 0 & 0 & $3.25 \mathrm{E}+02$ & 0 & 0 & 0 & 0 & 0 & 0 & $1.07 \mathrm{E}+03$ & $3.55 \mathrm{E}+05$ & $0.30 \%$ \\
\hline $8 \mathrm{~TB} 014$ & 0 & 0 & 0 & 0 & 0 & $2.38 \mathrm{E}+02$ & 0 & 0 & 0 & 0 & 0 & 0 & $2.54 \mathrm{E}+02$ & $3.59 \mathrm{E}+05$ & $0.07 \%$ \\
\hline $8 \mathrm{~TB} 015$ & 0 & 0 & 0 & 0 & 0 & $2.96 \mathrm{E}+02$ & 0 & 0 & 0 & 0 & 0 & 0 & $3.12 \mathrm{E}+02$ & $3.60 \mathrm{E}+05$ & $0.09 \%$ \\
\hline $8 \mathrm{~TB} 016$ & $7.19 \mathrm{E}+02$ & 0 & 0 & 0 & 0 & $3.26 \mathrm{E}+02$ & 0 & 0 & 0 & 0 & 0 & 0 & $1.06 \mathrm{E}+03$ & $3.55 \mathrm{E}+05$ & $0.30 \%$ \\
\hline $8 \mathrm{~TB} 017$ & $7.55 \mathrm{E}+02$ & 0 & 0 & 0 & 0 & $2.82 \mathrm{E}+02$ & 0 & 0 & 0 & 0 & 0 & 0 & $1.05 \mathrm{E}+03$ & $3.56 \mathrm{E}+05$ & $0.30 \%$ \\
\hline 8TB018 & 0 & 0 & 0 & 0 & 0 & $3.92 \mathrm{E}+02$ & 0 & 0 & 0 & 0 & 0 & 0 & $4.07 \mathrm{E}+02$ & $3.58 \mathrm{E}+05$ & $0.11 \%$ \\
\hline $8 \mathrm{~TB} 019$ & 0 & 0 & 0 & 0 & 0 & 0 & 0 & 0 & 0 & 0 & $1.15 \mathrm{E}+00$ & 0 & $1.63 \mathrm{E}+01$ & $3.43 \mathrm{E}+05$ & $0.00 \%$ \\
\hline
\end{tabular}


Table 38. Predicted Solids for Envelope B Simulations - Part 2 (cont'd)

\begin{tabular}{|c|c|c|c|c|c|c|c|c|c|c|c|c|c|c|c|}
\hline Test ID & $\begin{array}{c}\text { NA2C2O } \\
4 \\
\end{array}$ & $\begin{array}{c}\text { NA2CO3. } \\
1 \mathrm{H} 2 \mathrm{O} \\
\end{array}$ & NA2U2O7 & $\begin{array}{c}\text { NA3FSO } \\
4 \\
\end{array}$ & $\begin{array}{c}\text { NA6SO4 } \\
2 \mathrm{CO} 3 \\
\end{array}$ & NAF & $\begin{array}{c}\text { NAFPO4. } \\
19 \mathrm{H} 2 \mathrm{O} \\
\end{array}$ & NANO3 & $\begin{array}{c}\text { NAPHOH } \\
.12 \mathrm{H} 2 \mathrm{O} \\
\end{array}$ & $\begin{array}{c}\text { NASGEL. } \\
15.5 \mathrm{H} 2 \mathrm{O}\end{array}$ & $\mathrm{NIOH} 2$ & ZRO2 & $\begin{array}{c}\text { Total } \\
\text { Solids } \\
\end{array}$ & $\begin{array}{c}\text { Total Soln } \\
\text { Mass } \\
\end{array}$ & $\begin{array}{c}\text { Solids } \% \\
\text { of Total } \\
\text { Soln } \\
\end{array}$ \\
\hline $8 \mathrm{~TB} 020$ & 0 & 0 & 0 & 0 & 0 & 0 & 0 & 0 & 0 & 0 & $1.18 \mathrm{E}+00$ & 0 & $1.64 \mathrm{E}+01$ & $3.45 \mathrm{E}+05$ & $0.00 \%$ \\
\hline 8TB021 & 0 & 0 & 0 & 0 & 0 & 0 & 0 & 0 & 0 & 0 & $1.18 \mathrm{E}+00$ & 0 & $1.65 \mathrm{E}+01$ & $3.46 \mathrm{E}+05$ & $0.00 \%$ \\
\hline $8 \mathrm{~TB} 022$ & 0 & 0 & 0 & 0 & 0 & 0 & 0 & 0 & 0 & 0 & $1.15 \mathrm{E}+00$ & 0 & $1.62 \mathrm{E}+01$ & $3.43 \mathrm{E}+05$ & $0.00 \%$ \\
\hline $8 \mathrm{~TB} 023$ & 0 & 0 & 0 & 0 & 0 & 0 & 0 & 0 & 0 & 0 & $1.17 \mathrm{E}+00$ & $4.12 \mathrm{E}-03$ & $1.64 \mathrm{E}+01$ & $3.43 \mathrm{E}+05$ & $0.00 \%$ \\
\hline $8 \mathrm{~TB} 024$ & 0 & 0 & 0 & 0 & 0 & 0 & 0 & 0 & 0 & 0 & $1.14 \mathrm{E}+00$ & 0 & $1.63 \mathrm{E}+01$ & $3.44 \mathrm{E}+05$ & $0.00 \%$ \\
\hline $8 \mathrm{~TB} 025$ & 0 & 0 & 0 & 0 & 0 & 0 & 0 & 0 & 0 & 0 & $6.75 \mathrm{E}-02$ & 0 & $6.93 \mathrm{E}+00$ & $3.44 \mathrm{E}+05$ & $0.00 \%$ \\
\hline $8 \mathrm{~TB} 026$ & 0 & 0 & 0 & 0 & 0 & 0 & 0 & 0 & 0 & 0 & 0 & 0 & $7.50 \mathrm{E}-01$ & $3.47 \mathrm{E}+05$ & $0.00 \%$ \\
\hline $8 \mathrm{~TB} 027$ & 0 & 0 & 0 & $4.35 \mathrm{E}+03$ & 0 & 0 & 0 & 0 & 0 & 0 & 0 & 0 & $4.36 \mathrm{E}+03$ & $3.38 \mathrm{E}+05$ & $1.29 \%$ \\
\hline $8 \mathrm{~TB} 028$ & 0 & 0 & 0 & $3.12 \mathrm{E}+03$ & 0 & 0 & 0 & 0 & 0 & 0 & 0 & 0 & $3.12 \mathrm{E}+03$ & $3.35 \mathrm{E}+05$ & $0.93 \%$ \\
\hline 8TB029 & 0 & 0 & 0 & $5.17 \mathrm{E}+03$ & 0 & 0 & 0 & 0 & 0 & 0 & 0 & 0 & $5.17 \mathrm{E}+03$ & $3.38 \mathrm{E}+05$ & $1.53 \%$ \\
\hline $8 \mathrm{~TB} 030$ & 0 & 0 & 0 & $3.42 \mathrm{E}+03$ & 0 & 0 & 0 & 0 & 0 & 0 & 0 & 0 & $3.43 \mathrm{E}+03$ & $3.36 \mathrm{E}+05$ & $1.02 \%$ \\
\hline $8 \mathrm{~TB} 031$ & 0 & 0 & 0 & $4.85 \mathrm{E}+03$ & 0 & 0 & 0 & 0 & 0 & 0 & 0 & 0 & $4.86 \mathrm{E}+03$ & $3.37 \mathrm{E}+05$ & $1.44 \%$ \\
\hline 8TB032 & $6.39 \mathrm{E}+02$ & 0 & 0 & $5.15 \mathrm{E}+03$ & 0 & 0 & 0 & 0 & 0 & 0 & 0 & 0 & $5.79 \mathrm{E}+03$ & $3.36 \mathrm{E}+05$ & $1.72 \%$ \\
\hline $8 \mathrm{~TB} 033$ & $2.58 \mathrm{E}+02$ & 0 & 0 & $3.47 \mathrm{E}+03$ & 0 & 0 & 0 & 0 & 0 & 0 & 0 & 0 & $3.73 \mathrm{E}+03$ & $3.35 \mathrm{E}+05$ & $1.11 \%$ \\
\hline $8 \mathrm{~TB} 034$ & $1.60 \mathrm{E}+02$ & 0 & 0 & $3.19 \mathrm{E}+03$ & 0 & 0 & 0 & 0 & 0 & 0 & 0 & 0 & $3.36 \mathrm{E}+03$ & $3.33 \mathrm{E}+05$ & $1.01 \%$ \\
\hline 8TB035 & $5.94 \mathrm{E}+02$ & 0 & 0 & $4.65 \mathrm{E}+03$ & 0 & 0 & 0 & 0 & 0 & 0 & 0 & 0 & $5.24 \mathrm{E}+03$ & $3.35 \mathrm{E}+05$ & $1.56 \%$ \\
\hline $8 \mathrm{~TB} 036$ & $2.38 \mathrm{E}+02$ & 0 & 0 & $4.03 \mathrm{E}+03$ & 0 & 0 & 0 & 0 & 0 & 0 & 0 & 0 & $4.27 \mathrm{E}+03$ & $3.36 \mathrm{E}+05$ & $1.27 \%$ \\
\hline $8 \mathrm{~TB} 037$ & $3.52 \mathrm{E}+02$ & 0 & 0 & $3.92 \mathrm{E}+03$ & 0 & 0 & 0 & 0 & 0 & 0 & 0 & 0 & $4.27 \mathrm{E}+03$ & $3.37 \mathrm{E}+05$ & $1.27 \%$ \\
\hline 8TB038 & $5.01 \mathrm{E}+02$ & 0 & 0 & $3.46 \mathrm{E}+03$ & 0 & 0 & 0 & 0 & 0 & 0 & 0 & 0 & $3.96 \mathrm{E}+03$ & $3.34 \mathrm{E}+05$ & $1.18 \%$ \\
\hline 8TB039 & $3.53 \mathrm{E}+02$ & 0 & 0 & 0 & 0 & $1.15 \mathrm{E}+02$ & 0 & 0 & 0 & 0 & 0 & 0 & $4.78 \mathrm{E}+02$ & $3.53 \mathrm{E}+05$ & $0.14 \%$ \\
\hline 8TB040 & 0 & 0 & 0 & $5.64 \mathrm{E}+03$ & 0 & 0 & 0 & 0 & 0 & 0 & $2.43 \mathrm{E}-01$ & 0 & $5.65 \mathrm{E}+03$ & $3.43 \mathrm{E}+05$ & $1.65 \%$ \\
\hline $8 \mathrm{~TB} 041$ & $1.78 \mathrm{E}+02$ & 0 & 0 & $6.03 \mathrm{E}+03$ & 0 & 0 & 0 & 0 & 0 & 0 & 0 & 0 & $6.22 \mathrm{E}+03$ & $3.50 \mathrm{E}+05$ & $1.78 \%$ \\
\hline $8 \mathrm{~TB} 042$ & 0 & 0 & 0 & $5.22 \mathrm{E}+03$ & 0 & 0 & 0 & 0 & 0 & 0 & 0 & 0 & $5.23 \mathrm{E}+03$ & $3.42 \mathrm{E}+05$ & $1.53 \%$ \\
\hline 8TB043 & $7.37 \mathrm{E}+01$ & 0 & 0 & $6.91 \mathrm{E}+03$ & 0 & 0 & 0 & 0 & 0 & 0 & 0 & 0 & $7.00 \mathrm{E}+03$ & $3.45 \mathrm{E}+05$ & $2.03 \%$ \\
\hline 8ТВ044 & 0 & 0 & 0 & $4.71 \mathrm{E}+03$ & 0 & 0 & 0 & 0 & 0 & 0 & $3.06 \mathrm{E}-01$ & 0 & $4.73 \mathrm{E}+03$ & $3.39 \mathrm{E}+05$ & $1.39 \%$ \\
\hline $8 \mathrm{~TB} 045$ & 0 & 0 & 0 & $4.64 \mathrm{E}+03$ & 0 & 0 & 0 & 0 & 0 & 0 & $3.52 \mathrm{E}-01$ & 0 & $4.66 \mathrm{E}+03$ & $3.41 \mathrm{E}+05$ & $1.36 \%$ \\
\hline 8TB046 & $2.33 \mathrm{E}+02$ & 0 & 0 & $5.74 \mathrm{E}+03$ & 0 & 0 & 0 & 0 & 0 & 0 & 5.79E-01 & 0 & $5.99 \mathrm{E}+03$ & $3.42 \mathrm{E}+05$ & $1.75 \%$ \\
\hline 8TB047 & $6.81 \mathrm{E}+02$ & 0 & 0 & 0 & 0 & $4.20 \mathrm{E}+01$ & 0 & 0 & 0 & 0 & 0 & 0 & $7.30 \mathrm{E}+02$ & $3.49 \mathrm{E}+05$ & $0.21 \%$ \\
\hline $8 \mathrm{~TB} 048$ & $1.55 \mathrm{E}+02$ & 0 & 0 & $4.47 \mathrm{E}+03$ & 0 & 0 & 0 & 0 & 0 & 0 & 4.09E-01 & 0 & $4.64 \mathrm{E}+03$ & $3.39 \mathrm{E}+05$ & $1.37 \%$ \\
\hline 8TB049 & $1.19 \mathrm{E}+02$ & 0 & 0 & $5.00 \mathrm{E}+03$ & 0 & 0 & 0 & 0 & 0 & 0 & $7.54 \mathrm{E}-01$ & 0 & $5.13 \mathrm{E}+03$ & $3.43 \mathrm{E}+05$ & $1.50 \%$ \\
\hline $8 \mathrm{~TB} 050$ & $6.24 \mathrm{E}+02$ & 0 & 0 & 0 & 0 & $1.46 \mathrm{E}+02$ & 0 & 0 & 0 & 0 & 0 & 0 & $7.82 \mathrm{E}+02$ & $3.53 \mathrm{E}+05$ & $0.22 \%$ \\
\hline
\end{tabular}


Table 38. Predicted Solids for Envelope B Simulations - Part 2 (cont'd)

\begin{tabular}{|c|c|c|c|c|c|c|c|c|c|c|c|c|c|c|c|}
\hline Test ID & $\begin{array}{c}\mathrm{NA} 2 \mathrm{C} 2 \mathrm{O} \\
4\end{array}$ & $\begin{array}{c}\text { NA2CO3. } \\
1 \mathrm{H} 2 \mathrm{O}\end{array}$ & NA2U2O7 & $\begin{array}{c}\text { NA3FSO } \\
4\end{array}$ & $\begin{array}{c}\text { NA6SO4 } \\
2 \mathrm{CO} 3\end{array}$ & NAF & $\begin{array}{c}\text { NAFPO4. } \\
19 \mathrm{H} 2 \mathrm{O}\end{array}$ & NANO3 & $\begin{array}{c}\text { NAPHOH } \\
.12 \mathrm{H} 2 \mathrm{O}\end{array}$ & $\begin{array}{c}\text { NASGEL. } \\
15.5 \mathrm{H} 2 \mathrm{O}\end{array}$ & $\mathrm{NIOH} 2$ & $\mathrm{ZRO} 2$ & $\begin{array}{l}\text { Total } \\
\text { Solids }\end{array}$ & $\begin{array}{c}\text { Total Soln } \\
\text { Mass }\end{array}$ & $\begin{array}{c}\text { Solids \% } \\
\text { of Total } \\
\text { Soln }\end{array}$ \\
\hline $8 \mathrm{~TB} 051$ & $6.40 \mathrm{E}+02$ & 0 & 0 & 0 & 0 & $1.49 \mathrm{E}+02$ & 0 & 0 & 0 & 0 & 0 & 0 & $8.02 \mathrm{E}+02$ & $3.53 \mathrm{E}+05$ & $0.23 \%$ \\
\hline $8 \mathrm{~TB} 052$ & 0 & 0 & 0 & 0 & 0 & 0 & 0 & 0 & 0 & 0 & $1.98 \mathrm{E}-01$ & 0 & $7.80 \mathrm{E}+00$ & $3.44 \mathrm{E}+05$ & $0.00 \%$ \\
\hline $8 \mathrm{~TB} 053$ & 0 & 0 & 0 & 0 & 0 & 0 & 0 & 0 & 0 & 0 & 0 & 0 & 6.56E-01 & $3.34 \mathrm{E}+05$ & $0.00 \%$ \\
\hline 8TB054 & 0 & 0 & 0 & 0 & 0 & 0 & 0 & 0 & 0 & 0 & 1.09E-01 & 0 & $6.17 \mathrm{E}+00$ & $3.42 \mathrm{E}+05$ & $0.00 \%$ \\
\hline $8 \mathrm{~TB} 055$ & 0 & 0 & 0 & 0 & 0 & 0 & 0 & 0 & 0 & 0 & $2.99 \mathrm{E}-01$ & 0 & $5.55 \mathrm{E}+00$ & $3.37 \mathrm{E}+05$ & $0.00 \%$ \\
\hline $8 \mathrm{~TB} 056$ & 0 & 0 & 0 & 0 & 0 & 0 & 0 & 0 & 0 & 0 & 0 & 0 & $2.24 \mathrm{E}+00$ & $3.38 \mathrm{E}+05$ & $0.00 \%$ \\
\hline $8 \mathrm{~TB} 057$ & 0 & 0 & 0 & 0 & 0 & 0 & 0 & 0 & 0 & 0 & $3.23 \mathrm{E}-01$ & 0 & $6.37 \mathrm{E}+00$ & $3.39 \mathrm{E}+05$ & $0.00 \%$ \\
\hline $8 \mathrm{~TB} 058$ & 0 & 0 & 0 & 0 & 0 & 0 & 0 & 0 & 0 & 0 & $5.20 \mathrm{E}-03$ & 0 & $3.76 \mathrm{E}+00$ & $3.38 \mathrm{E}+05$ & $0.00 \%$ \\
\hline 8TB059 & 0 & 0 & 0 & 0 & 0 & 0 & 0 & 0 & 0 & 0 & 1.64E-01 & 0 & $7.77 \mathrm{E}+00$ & $3.44 \mathrm{E}+05$ & $0.00 \%$ \\
\hline $8 \mathrm{~TB} 060$ & 0 & 0 & 0 & 0 & 0 & 0 & 0 & 0 & 0 & 0 & 0 & 0 & $1.47 \mathrm{E}+00$ & $3.37 \mathrm{E}+05$ & $0.00 \%$ \\
\hline 8TB061 & 0 & 0 & 0 & 0 & 0 & 0 & 0 & 0 & 0 & 0 & $2.52 \mathrm{E}-01$ & 0 & $4.73 \mathrm{E}+00$ & $3.35 \mathrm{E}+05$ & $0.00 \%$ \\
\hline 8TB062 & 0 & 0 & 0 & 0 & 0 & 0 & 0 & 0 & 0 & 0 & $4.50 \mathrm{E}-01$ & 0 & $7.27 \mathrm{E}+00$ & $3.39 \mathrm{E}+05$ & $0.00 \%$ \\
\hline 8TB063 & 0 & 0 & 0 & 0 & 0 & 0 & 0 & 0 & 0 & 0 & 0 & 0 & $2.23 \mathrm{E}+00$ & $3.37 \mathrm{E}+05$ & $0.00 \%$ \\
\hline 8TB064 & 0 & 0 & 0 & 0 & 0 & 0 & 0 & 0 & 0 & 0 & 0 & 0 & $2.20 \mathrm{E}+00$ & $3.35 \mathrm{E}+05$ & $0.00 \%$ \\
\hline $8 \mathrm{~TB} 065$ & 0 & 0 & 0 & 0 & 0 & 0 & 0 & 0 & 0 & 0 & $1.40 \mathrm{E}-01$ & 0 & $5.43 \mathrm{E}+00$ & $3.39 \mathrm{E}+05$ & $0.00 \%$ \\
\hline $8 \mathrm{~TB} 066$ & 0 & 0 & 0 & 0 & 0 & 0 & 0 & 0 & 0 & 0 & $8.53 \mathrm{E}-01$ & 0 & $1.46 \mathrm{E}+01$ & $3.48 \mathrm{E}+05$ & $0.00 \%$ \\
\hline 8 TB067 & 0 & 0 & 0 & 0 & 0 & 0 & 0 & 0 & 0 & 0 & $7.67 \mathrm{E}-01$ & 0 & $1.22 \mathrm{E}+01$ & $3.44 \mathrm{E}+05$ & $0.00 \%$ \\
\hline 8TB068 & 0 & 0 & 0 & 0 & 0 & 0 & 0 & 0 & 0 & 0 & $9.22 \mathrm{E}-01$ & 0 & $1.47 \mathrm{E}+01$ & $3.47 \mathrm{E}+05$ & $0.00 \%$ \\
\hline 8TB069 & 0 & 0 & 0 & 0 & 0 & 0 & 0 & 0 & 0 & 0 & $6.95 \mathrm{E}-01$ & 0 & $1.29 \mathrm{E}+01$ & $3.47 \mathrm{E}+05$ & $0.00 \%$ \\
\hline $8 \mathrm{~TB} 070$ & 0 & 0 & 0 & 0 & 0 & 0 & 0 & 0 & 0 & 0 & $1.02 \mathrm{E}+00$ & 0 & $1.47 \mathrm{E}+01$ & $3.43 \mathrm{E}+05$ & $0.00 \%$ \\
\hline 8TB071 & 0 & 0 & 0 & 0 & 0 & 0 & 0 & 0 & 0 & 0 & $4.05 \mathrm{E}-01$ & 0 & $8.78 \mathrm{E}+00$ & $3.43 \mathrm{E}+05$ & $0.00 \%$ \\
\hline $8 \mathrm{~TB} 072$ & 0 & 0 & 0 & 0 & 0 & 0 & 0 & 0 & 0 & 0 & $5.56 \mathrm{E}-01$ & 0 & $9.67 \mathrm{E}+00$ & $3.42 \mathrm{E}+05$ & $0.00 \%$ \\
\hline $8 \mathrm{~TB} 073$ & 0 & 0 & 0 & 0 & 0 & 0 & 0 & 0 & 0 & 0 & $6.26 \mathrm{E}-01$ & 0 & $9.71 \mathrm{E}+00$ & $3.39 \mathrm{E}+05$ & $0.00 \%$ \\
\hline 8TB074 & $1.05 \mathrm{E}+01$ & 0 & 0 & 0 & 0 & 0 & 0 & 0 & 0 & 0 & $7.85 \mathrm{E}-01$ & 0 & $2.59 \mathrm{E}+01$ & $3.50 \mathrm{E}+05$ & $0.01 \%$ \\
\hline $8 \mathrm{~TB} 075$ & 0 & 0 & 0 & 0 & 0 & 0 & 0 & 0 & 0 & 0 & $4.78 \mathrm{E}-01$ & 0 & $8.85 \mathrm{E}+00$ & $3.42 \mathrm{E}+05$ & $0.00 \%$ \\
\hline $8 \mathrm{~TB} 076$ & 0 & 0 & 0 & 0 & 0 & 0 & 0 & 0 & 0 & 0 & $7.93 \mathrm{E}-01$ & 0 & $1.29 \mathrm{E}+01$ & $3.44 \mathrm{E}+05$ & $0.00 \%$ \\
\hline $8 \mathrm{~TB} 077$ & 0 & 0 & 0 & 0 & 0 & 0 & 0 & 0 & 0 & 0 & $9.26 \mathrm{E}-01$ & 0 & $1.47 \mathrm{E}+01$ & $3.46 \mathrm{E}+05$ & $0.00 \%$ \\
\hline $8 \mathrm{~TB} 078$ & 0 & 0 & 0 & 0 & 0 & 0 & 0 & 0 & 0 & 0 & $9.68 \mathrm{E}-01$ & 0 & $1.47 \mathrm{E}+01$ & $3.45 \mathrm{E}+05$ & $0.00 \%$ \\
\hline 8TB079 & 0 & 0 & 0 & 0 & 0 & 0 & 0 & 0 & 0 & 0 & $1.56 \mathrm{E}-01$ & 0 & $7.79 \mathrm{E}+00$ & $3.45 \mathrm{E}+05$ & $0.00 \%$ \\
\hline $8 \mathrm{~TB} 080$ & $7.74 \mathrm{E}+01$ & 0 & 0 & 0 & 0 & 0 & 0 & 0 & 0 & 0 & $2.08 \mathrm{E}-01$ & 0 & $8.60 \mathrm{E}+01$ & $3.45 \mathrm{E}+05$ & $0.02 \%$ \\
\hline $8 \mathrm{~TB} 081$ & 0 & 0 & 0 & 0 & 0 & 0 & 0 & 0 & 0 & 0 & $1.09 \mathrm{E}+00$ & 0 & $1.55 \mathrm{E}+01$ & $3.44 \mathrm{E}+05$ & $0.00 \%$ \\
\hline
\end{tabular}


Table 38. Predicted Solids for Envelope B Simulations - Part 2 (cont'd)

\begin{tabular}{|c|c|c|c|c|c|c|c|c|c|c|c|c|c|c|c|}
\hline Test ID & $\begin{array}{c}\mathrm{NA} 2 \mathrm{C} 2 \mathrm{O} \\
4\end{array}$ & $\begin{array}{c}\mathrm{NA} 2 \mathrm{CO} 3 . \\
1 \mathrm{H} 2 \mathrm{O}\end{array}$ & NA2U2O7 & $\begin{array}{c}\text { NA3FSO } \\
4\end{array}$ & $\begin{array}{c}\mathrm{NA} 6 \mathrm{SO} 4 \\
2 \mathrm{CO} 3\end{array}$ & NAF & $\begin{array}{c}\text { NAFPO4. } \\
19 \mathrm{H} 2 \mathrm{O}\end{array}$ & NANO3 & $\begin{array}{c}\text { NAPHOH } \\
.12 \mathrm{H} 2 \mathrm{O}\end{array}$ & $\begin{array}{c}\text { NASGEL. } \\
15.5 \mathrm{H} 2 \mathrm{O}\end{array}$ & NIOH2 & ZRO2 & $\begin{array}{l}\text { Total } \\
\text { Solids }\end{array}$ & $\begin{array}{c}\text { Total Soln } \\
\text { Mass }\end{array}$ & $\begin{array}{c}\text { Solids } \% \\
\text { of Total } \\
\text { Soln }\end{array}$ \\
\hline $8 \mathrm{~TB} 082$ & 0 & 0 & 0 & 0 & 0 & 0 & 0 & 0 & 0 & 0 & $4.01 \mathrm{E}-01$ & 0 & $9.51 \mathrm{E}+00$ & $3.44 \mathrm{E}+05$ & $0.00 \%$ \\
\hline $8 \mathrm{~TB} 083$ & 0 & 0 & 0 & 0 & 0 & 0 & 0 & 0 & 0 & 0 & $8.73 \mathrm{E}-01$ & 0 & $1.53 \mathrm{E}+01$ & $3.48 \mathrm{E}+05$ & $0.00 \%$ \\
\hline 8ТВ084 & 0 & 0 & 0 & 0 & 0 & 0 & 0 & 0 & 0 & 0 & 7.24E-01 & 0 & $1.13 \mathrm{E}+01$ & $3.41 \mathrm{E}+05$ & $0.00 \%$ \\
\hline $8 \mathrm{~TB} 085$ & 0 & 0 & 0 & 0 & 0 & 0 & 0 & 0 & 0 & 0 & 7.75E-01 & 0 & $1.38 \mathrm{E}+01$ & $3.48 \mathrm{E}+05$ & $0.00 \%$ \\
\hline 8ТВ086 & 0 & 0 & 0 & 0 & 0 & 0 & 0 & 0 & 0 & 0 & $6.53 \mathrm{E}-01$ & 0 & $1.13 \mathrm{E}+01$ & $3.43 \mathrm{E}+05$ & $0.00 \%$ \\
\hline $8 \mathrm{~TB} 087$ & 0 & 0 & 0 & 0 & 0 & 0 & 0 & 0 & 0 & 0 & $7.29 \mathrm{E}-01$ & 0 & $1.14 \mathrm{E}+01$ & $3.42 \mathrm{E}+05$ & $0.00 \%$ \\
\hline 8TB088 & 0 & 0 & 0 & 0 & 0 & 0 & 0 & 0 & 0 & 0 & $1.01 \mathrm{E}+00$ & 0 & $1.62 \mathrm{E}+01$ & $3.48 \mathrm{E}+05$ & $0.00 \%$ \\
\hline 8TB089 & 0 & 0 & 0 & 0 & 0 & 0 & 0 & 0 & 0 & 0 & 7.99E-01 & 0 & $1.53 \mathrm{E}+01$ & $3.50 \mathrm{E}+05$ & $0.00 \%$ \\
\hline 8ТВ090 & 0 & 0 & 0 & 0 & 0 & 0 & 0 & 0 & 0 & 0 & $9.28 \mathrm{E}-01$ & 0 & $1.39 \mathrm{E}+01$ & $3.45 \mathrm{E}+05$ & $0.00 \%$ \\
\hline 8TB091 & 0 & 0 & 0 & 0 & 0 & 0 & 0 & 0 & 0 & 0 & $3.96 \mathrm{E}-01$ & 0 & $9.56 \mathrm{E}+00$ & $3.45 \mathrm{E}+05$ & $0.00 \%$ \\
\hline 8TB092 & 0 & 0 & 0 & 0 & 0 & 0 & 0 & 0 & 0 & 0 & $6.20 \mathrm{E}-01$ & 0 & $1.13 \mathrm{E}+01$ & $3.45 \mathrm{E}+05$ & $0.00 \%$ \\
\hline 8ТВ093 & 0 & 0 & 0 & 0 & 0 & 0 & 0 & 0 & 0 & 0 & $1.07 \mathrm{E}+00$ & 0 & $1.64 \mathrm{E}+01$ & $3.48 \mathrm{E}+05$ & $0.00 \%$ \\
\hline 8ТВ094 & 0 & 0 & 0 & 0 & 0 & 0 & 0 & 0 & 0 & 0 & $2.92 \mathrm{E}-01$ & 0 & $5.55 \mathrm{E}+00$ & $3.37 \mathrm{E}+05$ & $0.00 \%$ \\
\hline 8TB095 & 0 & 0 & 0 & 0 & 0 & 0 & 0 & 0 & 0 & 0 & 0 & 0 & $2.22 \mathrm{E}+00$ & $3.36 \mathrm{E}+05$ & $0.00 \%$ \\
\hline 8TB096 & 0 & 0 & 0 & 0 & 0 & 0 & 0 & 0 & 0 & 0 & $1.41 \mathrm{E}-01$ & 0 & $3.87 \mathrm{E}+00$ & $3.37 \mathrm{E}+05$ & $0.00 \%$ \\
\hline 8ТВ097 & 0 & 0 & 0 & 0 & 0 & 0 & 0 & 0 & 0 & 0 & $2.23 \mathrm{E}-01$ & 0 & $5.48 \mathrm{E}+00$ & $3.37 \mathrm{E}+05$ & $0.00 \%$ \\
\hline 8TB098 & 0 & 0 & 0 & 0 & 0 & 0 & 0 & 0 & 0 & 0 & 0 & 0 & 0 & $3.33 \mathrm{E}+05$ & $0.00 \%$ \\
\hline 8ТВ099 & $2.54 \mathrm{E}+00$ & 0 & 0 & 0 & 0 & 0 & 0 & 0 & 0 & 0 & 0 & 0 & $7.08 \mathrm{E}+00$ & $3.41 \mathrm{E}+05$ & $0.00 \%$ \\
\hline $8 \mathrm{~TB} 100$ & $1.91 \mathrm{E}+01$ & 0 & 0 & 0 & 0 & 0 & 0 & 0 & 0 & 0 & 0 & 0 & $2.21 \mathrm{E}+01$ & $3.39 \mathrm{E}+05$ & $0.01 \%$ \\
\hline 8TB101 & 0 & 0 & 0 & 0 & 0 & 0 & 0 & 0 & 0 & 0 & 0 & 0 & 7.14E-01 & $3.38 \mathrm{E}+05$ & $0.00 \%$ \\
\hline $8 \mathrm{~TB} 102$ & 0 & 0 & 0 & 0 & 0 & 0 & 0 & 0 & 0 & 0 & $4.83 \mathrm{E}-01$ & 0 & $8.03 \mathrm{E}+00$ & $3.39 \mathrm{E}+05$ & $0.00 \%$ \\
\hline $8 \mathrm{~TB} 103$ & 0 & 0 & 0 & 0 & 0 & 0 & 0 & 0 & 0 & 0 & 0 & 0 & $3.01 \mathrm{E}+00$ & $3.40 \mathrm{E}+05$ & $0.00 \%$ \\
\hline $8 \mathrm{~TB} 104$ & 0 & 0 & 0 & 0 & 0 & 0 & 0 & 0 & 0 & 0 & 0 & 0 & 0 & $3.38 \mathrm{E}+05$ & $0.00 \%$ \\
\hline $8 \mathrm{~TB} 105$ & 0 & 0 & 0 & 0 & 0 & 0 & 0 & 0 & 0 & 0 & $1.68 \mathrm{E}-01$ & 0 & $3.93 \mathrm{E}+00$ & $3.37 \mathrm{E}+05$ & $0.00 \%$ \\
\hline $8 \mathrm{~TB} 106$ & 0 & 0 & 0 & 0 & 0 & 0 & 0 & 0 & 0 & 0 & $3.25 \mathrm{E}-02$ & 0 & $2.24 \mathrm{E}+00$ & $3.36 \mathrm{E}+05$ & $0.00 \%$ \\
\hline 8 TB107 & $1.66 \mathrm{E}+02$ & 0 & 0 & $4.51 \mathrm{E}+03$ & 0 & 0 & 0 & 0 & 0 & 0 & $6.19 \mathrm{E}-02$ & 0 & $4.68 \mathrm{E}+03$ & $3.36 \mathrm{E}+05$ & $1.39 \%$ \\
\hline 8TB108 & $6.57 \mathrm{E}+02$ & 0 & 0 & 0 & 0 & $2.23 \mathrm{E}+02$ & 0 & 0 & 0 & 0 & 0 & 0 & $8.94 \mathrm{E}+02$ & $3.56 \mathrm{E}+05$ & $0.25 \%$ \\
\hline $8 \mathrm{~TB} 109$ & $2.44 \mathrm{E}+02$ & 0 & 0 & $5.18 \mathrm{E}+03$ & 0 & 0 & 0 & 0 & 0 & 0 & $1.36 \mathrm{E}-01$ & 0 & $5.43 \mathrm{E}+03$ & $3.37 \mathrm{E}+05$ & $1.61 \%$ \\
\hline $8 \mathrm{~TB} 110$ & $7.00 \mathrm{E}+02$ & 0 & 0 & 0 & 0 & $1.27 \mathrm{E}+02$ & 0 & 0 & 0 & 0 & 0 & 0 & $8.37 \mathrm{E}+02$ & $3.52 \mathrm{E}+05$ & $0.24 \%$ \\
\hline 8TB111 & $3.29 \mathrm{E}+02$ & 0 & 0 & $5.45 \mathrm{E}+03$ & 0 & 0 & 0 & 0 & 0 & 0 & $3.49 \mathrm{E}-01$ & 0 & $5.79 \mathrm{E}+03$ & $3.41 \mathrm{E}+05$ & $1.70 \%$ \\
\hline $8 \mathrm{~TB} 112$ & $4.21 \mathrm{E}+02$ & 0 & 0 & $5.77 \mathrm{E}+03$ & 0 & 0 & 0 & 0 & 0 & 0 & 0 & 0 & $6.20 \mathrm{E}+03$ & $3.42 \mathrm{E}+05$ & $1.81 \%$ \\
\hline
\end{tabular}


Table 38. Predicted Solids for Envelope B Simulations - Part 2 (cont'd)

\begin{tabular}{|c|c|c|c|c|c|c|c|c|c|c|c|c|c|c|c|}
\hline Test ID & $\begin{array}{c}\mathrm{NA} 2 \mathrm{C} 2 \mathrm{O} \\
4\end{array}$ & $\begin{array}{c}\text { NA2CO3. } \\
1 \mathrm{H} 2 \mathrm{O}\end{array}$ & NA2U2O7 & $\begin{array}{c}\text { NA3FSO } \\
4\end{array}$ & $\begin{array}{c}\text { NA6SO4 } \\
2 \mathrm{CO} 3\end{array}$ & NAF & $\begin{array}{c}\text { NAFPO4. } \\
19 \mathrm{H} 2 \mathrm{O}\end{array}$ & NANO3 & $\begin{array}{c}\text { NAPHOH } \\
.12 \mathrm{H} 2 \mathrm{O}\end{array}$ & $\begin{array}{c}\text { NASGEL. } \\
15.5 \mathrm{H} 2 \mathrm{O}\end{array}$ & $\mathrm{NIOH} 2$ & $\mathrm{ZRO} 2$ & $\begin{array}{l}\text { Total } \\
\text { Solids }\end{array}$ & $\begin{array}{c}\text { Total Soln } \\
\text { Mass }\end{array}$ & $\begin{array}{c}\text { Solids \% } \\
\text { of Total } \\
\text { Soln }\end{array}$ \\
\hline $8 \mathrm{~TB} 113$ & $2.84 \mathrm{E}+02$ & 0 & 0 & $5.90 \mathrm{E}+03$ & 0 & 0 & 0 & 0 & 0 & 0 & $1.78 \mathrm{E}-01$ & 0 & $6.19 \mathrm{E}+03$ & $3.41 \mathrm{E}+05$ & $1.82 \%$ \\
\hline $8 \mathrm{~TB} 114$ & $1.11 \mathrm{E}+02$ & 0 & 0 & $4.66 \mathrm{E}+03$ & 0 & 0 & 0 & 0 & 0 & 0 & $1.47 \mathrm{E}-01$ & 0 & $4.78 \mathrm{E}+03$ & $3.37 \mathrm{E}+05$ & $1.42 \%$ \\
\hline $8 \mathrm{~TB} 115$ & 0 & 0 & 0 & $5.90 \mathrm{E}+03$ & 0 & 0 & 0 & 0 & 0 & 0 & $2.72 \mathrm{E}-01$ & 0 & $5.91 \mathrm{E}+03$ & $3.43 \mathrm{E}+05$ & $1.72 \%$ \\
\hline 8 TB116 & 0 & 0 & 0 & 0 & 0 & $1.72 \mathrm{E}+02$ & 0 & 0 & 0 & 0 & 0 & 0 & $1.83 \mathrm{E}+02$ & $3.55 \mathrm{E}+05$ & $0.05 \%$ \\
\hline 8 TB117 & $3.65 \mathrm{E}+02$ & 0 & 0 & 0 & 0 & $1.24 \mathrm{E}+02$ & 0 & 0 & 0 & 0 & 0 & 0 & $4.98 \mathrm{E}+02$ & $3.52 \mathrm{E}+05$ & $0.14 \%$ \\
\hline $8 \mathrm{~TB} 118$ & $9.34 \mathrm{E}+01$ & 0 & 0 & $6.48 \mathrm{E}+03$ & 0 & 0 & 0 & 0 & 0 & 0 & $2.33 \mathrm{E}-01$ & 0 & $6.59 \mathrm{E}+03$ & $3.42 \mathrm{E}+05$ & $1.93 \%$ \\
\hline $8 \mathrm{~TB} 119$ & 0 & 0 & 0 & $6.32 \mathrm{E}+03$ & 0 & 0 & 0 & 0 & 0 & 0 & 0 & 0 & $6.33 \mathrm{E}+03$ & $3.44 \mathrm{E}+05$ & $1.84 \%$ \\
\hline $8 \mathrm{~TB} 120$ & 0 & 0 & 0 & $5.63 \mathrm{E}+03$ & 0 & 0 & 0 & 0 & 0 & 0 & $4.47 \mathrm{E}-02$ & 0 & $5.64 \mathrm{E}+03$ & $3.40 \mathrm{E}+05$ & $1.66 \%$ \\
\hline 8 TB121 & $1.43 \mathrm{E}+02$ & 0 & 0 & $4.04 \mathrm{E}+03$ & 0 & 0 & 0 & 0 & 0 & 0 & 0 & 0 & $4.19 \mathrm{E}+03$ & $3.35 \mathrm{E}+05$ & $1.25 \%$ \\
\hline $8 \mathrm{~TB} 122$ & $5.68 \mathrm{E}+02$ & 0 & 0 & $4.97 \mathrm{E}+03$ & 0 & 0 & 0 & 0 & 0 & 0 & 0 & 0 & $5.54 \mathrm{E}+03$ & $3.35 \mathrm{E}+05$ & $1.65 \%$ \\
\hline $8 \mathrm{~TB} 123$ & $4.79 \mathrm{E}+02$ & 0 & 0 & $4.24 \mathrm{E}+03$ & 0 & 0 & 0 & 0 & 0 & 0 & 0 & 0 & $4.72 \mathrm{E}+03$ & $3.35 \mathrm{E}+05$ & $1.41 \%$ \\
\hline 8 TB124 & $3.32 \mathrm{E}+02$ & 0 & 0 & $4.65 \mathrm{E}+03$ & 0 & 0 & 0 & 0 & 0 & 0 & 0 & 0 & $4.99 \mathrm{E}+03$ & $3.34 \mathrm{E}+05$ & $1.49 \%$ \\
\hline 8 TB125 & $7.89 \mathrm{E}+02$ & 0 & 0 & 0 & 0 & 0 & 0 & 0 & 0 & 0 & 0 & 0 & $7.91 \mathrm{E}+02$ & $3.46 \mathrm{E}+05$ & $0.23 \%$ \\
\hline $8 \mathrm{~TB} 126$ & $3.46 \mathrm{E}+02$ & 0 & 0 & $4.85 \mathrm{E}+03$ & 0 & 0 & 0 & 0 & 0 & 0 & 0 & 0 & $5.20 \mathrm{E}+03$ & $3.38 \mathrm{E}+05$ & $1.54 \%$ \\
\hline $8 \mathrm{~TB} 127$ & $5.62 \mathrm{E}+02$ & 0 & 0 & $5.13 \mathrm{E}+03$ & 0 & 0 & 0 & 0 & 0 & 0 & 0 & 0 & $5.69 \mathrm{E}+03$ & $3.38 \mathrm{E}+05$ & $1.68 \%$ \\
\hline $8 \mathrm{~TB} 128$ & 0 & 0 & 0 & 0 & 0 & 0 & 0 & 0 & 0 & 0 & 0 & 0 & $6.11 \mathrm{E}+00$ & $3.51 \mathrm{E}+05$ & $0.00 \%$ \\
\hline 8 TB129 & 0 & 0 & 0 & $3.78 \mathrm{E}+03$ & 0 & 0 & 0 & 0 & 0 & 0 & 0 & 0 & $3.78 \mathrm{E}+03$ & $3.33 \mathrm{E}+05$ & $1.14 \%$ \\
\hline $8 \mathrm{~TB} 130$ & $1.86 \mathrm{E}+02$ & 0 & 0 & $5.24 \mathrm{E}+03$ & 0 & 0 & 0 & 0 & 0 & 0 & 0 & 0 & $5.43 \mathrm{E}+03$ & $3.39 \mathrm{E}+05$ & $1.60 \%$ \\
\hline 8TB131 & 0 & 0 & 0 & $4.82 \mathrm{E}+03$ & 0 & 0 & 0 & 0 & 0 & 0 & 0 & 0 & $4.82 \mathrm{E}+03$ & $3.37 \mathrm{E}+05$ & $1.43 \%$ \\
\hline $8 \mathrm{~TB} 132$ & $9.12 \mathrm{E}+01$ & 0 & 0 & $4.33 \mathrm{E}+03$ & 0 & 0 & 0 & 0 & 0 & 0 & 0 & 0 & $4.43 \mathrm{E}+03$ & $3.36 \mathrm{E}+05$ & $1.32 \%$ \\
\hline 8TB133 & 0 & 0 & 0 & $4.93 \mathrm{E}+03$ & 0 & 0 & 0 & 0 & 0 & 0 & 0 & 0 & $4.94 \mathrm{E}+03$ & $3.36 \mathrm{E}+05$ & $1.47 \%$ \\
\hline 6TB001 & $6.08 \mathrm{E}+02$ & 0 & 0 & 0 & 0 & 0 & 0 & 0 & 0 & 0 & 0 & 0 & $9.49 \mathrm{E}+02$ & $4.28 \mathrm{E}+05$ & $0.22 \%$ \\
\hline 6TB002 & 0 & 0 & 0 & 0 & 0 & 0 & 0 & 0 & 0 & 0 & 0 & 0 & 0 & $4.32 \mathrm{E}+05$ & $0.00 \%$ \\
\hline 6ТВ003 & 0 & 0 & 0 & 0 & 0 & 0 & 0 & 0 & 0 & 0 & 0 & 0 & $2.93 \mathrm{E}+02$ & $4.32 \mathrm{E}+05$ & $0.07 \%$ \\
\hline 6TB004 & $5.94 \mathrm{E}+02$ & 0 & 0 & 0 & 0 & 0 & 0 & 0 & 0 & 0 & 0 & 0 & $5.94 \mathrm{E}+02$ & $4.27 \mathrm{E}+05$ & $0.14 \%$ \\
\hline 6TB005 & $6.26 \mathrm{E}+02$ & 0 & 0 & 0 & 0 & 0 & 0 & 0 & 0 & 0 & 0 & 0 & $6.26 \mathrm{E}+02$ & $4.28 \mathrm{E}+05$ & $0.15 \%$ \\
\hline 6TB006 & 0 & 0 & 0 & 0 & 0 & 0 & 0 & 0 & 0 & 0 & 0 & 0 & $3.77 \mathrm{E}+02$ & $4.31 \mathrm{E}+05$ & $0.09 \%$ \\
\hline 6TB007 & 0 & 0 & 0 & 0 & 0 & 0 & 0 & 0 & 0 & 0 & 0 & 0 & 0 & $4.14 \mathrm{E}+05$ & $0.00 \%$ \\
\hline 6TB008 & 0 & 0 & 0 & 0 & 0 & 0 & 0 & 0 & 0 & 0 & 0 & 0 & 0 & $4.17 \mathrm{E}+05$ & $0.00 \%$ \\
\hline 6TB009 & 0 & 0 & 0 & 0 & 0 & 0 & 0 & 0 & 0 & 0 & 0 & 0 & 0 & $4.17 \mathrm{E}+05$ & $0.00 \%$ \\
\hline 6TB010 & 0 & 0 & 0 & 0 & 0 & 0 & 0 & 0 & 0 & 0 & 0 & 0 & 0 & $4.14 \mathrm{E}+05$ & $0.00 \%$ \\
\hline
\end{tabular}


Table 38. Predicted Solids for Envelope B Simulations - Part 2 (cont'd)

\begin{tabular}{|c|c|c|c|c|c|c|c|c|c|c|c|c|c|c|c|}
\hline Test ID & $\begin{array}{c}\mathrm{NA} 2 \mathrm{C} 2 \mathrm{O} \\
4\end{array}$ & $\begin{array}{c}\text { NA2CO3. } \\
1 \mathrm{H} 2 \mathrm{O}\end{array}$ & NA2U2O7 & $\begin{array}{c}\text { NA3FSO } \\
4\end{array}$ & $\begin{array}{c}\text { NA6SO4 } \\
2 \mathrm{CO} 3\end{array}$ & NAF & $\begin{array}{c}\text { NAFPO4. } \\
19 \mathrm{H} 2 \mathrm{O}\end{array}$ & NANO3 & $\begin{array}{c}\text { NAPHOH } \\
.12 \mathrm{H} 2 \mathrm{O}\end{array}$ & $\begin{array}{c}\text { NASGEL. } \\
15.5 \mathrm{H} 2 \mathrm{O}\end{array}$ & $\mathrm{NIOH} 2$ & ZRO2 & $\begin{array}{l}\text { Total } \\
\text { Solids }\end{array}$ & $\begin{array}{c}\text { Total Soln } \\
\text { Mass }\end{array}$ & $\begin{array}{c}\text { Solids \% } \\
\text { of Total } \\
\text { Soln }\end{array}$ \\
\hline 6TB011 & 0 & 0 & 0 & 0 & 0 & 0 & 0 & 0 & 0 & 0 & 0 & 0 & 0 & $4.15 \mathrm{E}+05$ & $0.00 \%$ \\
\hline 6TB012 & 0 & 0 & 0 & 0 & 0 & 0 & 0 & 0 & 0 & 0 & 0 & 0 & 0 & $4.16 \mathrm{E}+05$ & $0.00 \%$ \\
\hline 6TB013 & $5.67 \mathrm{E}+02$ & 0 & 0 & 0 & 0 & 0 & 0 & 0 & 0 & 0 & $8.13 \mathrm{E}-02$ & 0 & $1.30 \mathrm{E}+03$ & $4.44 \mathrm{E}+05$ & $0.29 \%$ \\
\hline 6TB014 & 0 & 0 & 0 & 0 & 0 & 0 & 0 & 0 & 0 & 0 & $2.85 \mathrm{E}-01$ & 0 & $1.56 \mathrm{E}+01$ & $4.48 \mathrm{E}+05$ & $0.00 \%$ \\
\hline 6TB015 & 0 & 0 & 0 & 0 & 0 & 0 & 0 & 0 & 0 & 0 & $2.01 \mathrm{E}-01$ & 0 & $6.83 \mathrm{E}+02$ & $4.48 \mathrm{E}+05$ & $0.15 \%$ \\
\hline 6TB016 & $5.49 \mathrm{E}+02$ & 0 & 0 & 0 & 0 & 0 & 0 & 0 & 0 & 0 & $1.10 \mathrm{E}-01$ & 0 & $5.64 \mathrm{E}+02$ & $4.43 \mathrm{E}+05$ & $0.13 \%$ \\
\hline 6TB017 & $5.79 \mathrm{E}+02$ & 0 & 0 & 0 & 0 & 0 & 0 & 0 & 0 & 0 & $2.82 \mathrm{E}-01$ & 0 & $5.95 \mathrm{E}+02$ & $4.44 \mathrm{E}+05$ & $0.13 \%$ \\
\hline 6TB018 & 0 & 0 & 0 & 0 & 0 & 0 & 0 & 0 & 0 & 0 & 0 & 0 & $7.74 \mathrm{E}+02$ & $4.47 \mathrm{E}+05$ & $0.17 \%$ \\
\hline 6TB019 & 0 & 0 & 0 & 0 & 0 & 0 & 0 & 0 & 0 & 0 & $1.19 \mathrm{E}+00$ & 0 & $1.62 \mathrm{E}+01$ & $4.30 \mathrm{E}+05$ & $0.00 \%$ \\
\hline 6TB020 & 0 & 0 & 0 & 0 & 0 & 0 & 0 & 0 & 0 & 0 & $1.21 \mathrm{E}+00$ & $3.58 \mathrm{E}-02$ & $1.64 \mathrm{E}+01$ & $4.32 \mathrm{E}+05$ & $0.00 \%$ \\
\hline 6TB021 & 0 & 0 & 0 & 0 & 0 & 0 & 0 & 0 & 0 & 0 & $1.21 \mathrm{E}+00$ & 7.30E-04 & $1.64 \mathrm{E}+01$ & $4.33 \mathrm{E}+05$ & $0.00 \%$ \\
\hline 6TB022 & 0 & 0 & 0 & 0 & 0 & 0 & 0 & 0 & 0 & 0 & $1.18 \mathrm{E}+00$ & 0 & $1.61 \mathrm{E}+01$ & $4.29 \mathrm{E}+05$ & $0.00 \%$ \\
\hline 6TB023 & 0 & 0 & 0 & 0 & 0 & 0 & 0 & 0 & 0 & 0 & $1.21 \mathrm{E}+00$ & $3.78 \mathrm{E}-02$ & $1.63 \mathrm{E}+01$ & $4.30 \mathrm{E}+05$ & $0.00 \%$ \\
\hline 6ТВ024 & 0 & 0 & 0 & 0 & 0 & 0 & 0 & 0 & 0 & 0 & $1.18 \mathrm{E}+00$ & 0 & $1.62 \mathrm{E}+01$ & $4.32 \mathrm{E}+05$ & $0.00 \%$ \\
\hline 6TB025 & 0 & 0 & 0 & 0 & 0 & 0 & 0 & 0 & 0 & 0 & $2.40 \mathrm{E}-01$ & 0 & $7.04 \mathrm{E}+00$ & $4.31 \mathrm{E}+05$ & $0.00 \%$ \\
\hline 6TB026 & 0 & 0 & 0 & 0 & 0 & 0 & 0 & 0 & 0 & 0 & 0 & 0 & $1.47 \mathrm{E}+02$ & $4.32 \mathrm{E}+05$ & $0.03 \%$ \\
\hline 6TB027 & 0 & 0 & 0 & $7.46 \mathrm{E}+02$ & 0 & 0 & 0 & 0 & 0 & 0 & $6.80 \mathrm{E}-02$ & 0 & $7.52 \mathrm{E}+02$ & $4.30 \mathrm{E}+05$ & $0.18 \%$ \\
\hline 6ТВ028 & 0 & 0 & 0 & 0 & 0 & 0 & 0 & 0 & 0 & 0 & 0 & 0 & $6.82 \mathrm{E}-01$ & $4.26 \mathrm{E}+05$ & $0.00 \%$ \\
\hline 6TB029 & 0 & 0 & 0 & $1.80 \mathrm{E}+03$ & 0 & 0 & 0 & 0 & 0 & 0 & 0 & 0 & $1.81 \mathrm{E}+03$ & $4.29 \mathrm{E}+05$ & $0.42 \%$ \\
\hline 6TB030 & 0 & 0 & 0 & 0 & 0 & 0 & 0 & 0 & 0 & 0 & 0 & 0 & $2.21 \mathrm{E}+00$ & $4.28 \mathrm{E}+05$ & $0.00 \%$ \\
\hline 6TB031 & 0 & 0 & 0 & $1.57 \mathrm{E}+03$ & 0 & 0 & 0 & 0 & 0 & 0 & 0 & 0 & $1.57 \mathrm{E}+03$ & $4.28 \mathrm{E}+05$ & $0.37 \%$ \\
\hline 6TB032 & $4.47 \mathrm{E}+02$ & 0 & 0 & $1.80 \mathrm{E}+03$ & 0 & 0 & 0 & 0 & 0 & 0 & 0 & 0 & $2.25 \mathrm{E}+03$ & $4.27 \mathrm{E}+05$ & $0.53 \%$ \\
\hline 6TB033 & $1.92 \mathrm{E}+01$ & 0 & 0 & 0 & 0 & 0 & 0 & 0 & 0 & 0 & 0 & 0 & $1.92 \mathrm{E}+01$ & $4.27 \mathrm{E}+05$ & $0.00 \%$ \\
\hline 6TB034 & 0 & 0 & 0 & 0 & 0 & 0 & 0 & 0 & 0 & 0 & 0 & 0 & $6.65 \mathrm{E}-01$ & $4.24 \mathrm{E}+05$ & $0.00 \%$ \\
\hline 6TB035 & $3.92 \mathrm{E}+02$ & 0 & 0 & $1.20 \mathrm{E}+03$ & 0 & 0 & 0 & 0 & 0 & 0 & 0 & 0 & $1.59 \mathrm{E}+03$ & $4.26 \mathrm{E}+05$ & $0.37 \%$ \\
\hline 6TB036 & 0 & 0 & 0 & $8.34 \mathrm{E}+01$ & 0 & 0 & 0 & 0 & 0 & 0 & $8.46 \mathrm{E}-02$ & 0 & $8.96 \mathrm{E}+01$ & $4.30 \mathrm{E}+05$ & $0.02 \%$ \\
\hline 6TB037 & $1.04 \mathrm{E}+02$ & 0 & 0 & $1.13 \mathrm{E}+02$ & 0 & 0 & 0 & 0 & 0 & 0 & 0 & 0 & $2.22 \mathrm{E}+02$ & $4.30 \mathrm{E}+05$ & $0.05 \%$ \\
\hline 6TB038 & $2.80 \mathrm{E}+02$ & 0 & 0 & 0 & 0 & 0 & 0 & 0 & 0 & 0 & 0 & 0 & $2.80 \mathrm{E}+02$ & $4.26 \mathrm{E}+05$ & $0.07 \%$ \\
\hline 6TB039 & $1.68 \mathrm{E}+02$ & 0 & 0 & 0 & 0 & 0 & 0 & 0 & 0 & 0 & 0 & 0 & $3.70 \mathrm{E}+02$ & $4.40 \mathrm{E}+05$ & $0.08 \%$ \\
\hline 6TB040 & 0 & 0 & 0 & $2.14 \mathrm{E}+03$ & 0 & 0 & 0 & 0 & 0 & 0 & $5.40 \mathrm{E}-01$ & 0 & $2.16 \mathrm{E}+03$ & $4.35 \mathrm{E}+05$ & $0.50 \%$ \\
\hline 6TB041 & $6.62 \mathrm{E}+00$ & 0 & 0 & $3.05 \mathrm{E}+03$ & 0 & 0 & 0 & 0 & 0 & 0 & $1.27 \mathrm{E}-01$ & 0 & $3.13 \mathrm{E}+03$ & $4.35 \mathrm{E}+05$ & $0.72 \%$ \\
\hline
\end{tabular}


Table 38. Predicted Solids for Envelope B Simulations - Part 2 (cont'd)

\begin{tabular}{|c|c|c|c|c|c|c|c|c|c|c|c|c|c|c|c|}
\hline Test ID & $\begin{array}{c}\mathrm{NA} 2 \mathrm{C} 2 \mathrm{O} \\
4\end{array}$ & $\begin{array}{c}\text { NA2CO3. } \\
1 \mathrm{H} 2 \mathrm{O}\end{array}$ & NA2U2O7 & $\begin{array}{c}\text { NA3FSO } \\
4\end{array}$ & $\begin{array}{c}\text { NA6SO4 } \\
2 \mathrm{CO} 3\end{array}$ & NAF & $\begin{array}{c}\text { NAFPO4. } \\
19 \mathrm{H} 2 \mathrm{O}\end{array}$ & NANO3 & $\begin{array}{c}\text { NAPHOH } \\
.12 \mathrm{H} 2 \mathrm{O}\end{array}$ & $\begin{array}{c}\text { NASGEL. } \\
15.5 \mathrm{H} 2 \mathrm{O}\end{array}$ & $\mathrm{NIOH} 2$ & $\mathrm{ZRO} 2$ & $\begin{array}{l}\text { Total } \\
\text { Solids }\end{array}$ & $\begin{array}{c}\text { Total Soln } \\
\text { Mass }\end{array}$ & $\begin{array}{c}\text { Solids \% } \\
\text { of Total } \\
\text { Soln }\end{array}$ \\
\hline 6TB042 & 0 & 0 & 0 & $1.70 \mathrm{E}+03$ & 0 & 0 & 0 & 0 & 0 & 0 & $1.15 \mathrm{E}-01$ & 0 & $1.71 \mathrm{E}+03$ & $4.34 \mathrm{E}+05$ & $0.39 \%$ \\
\hline 6TB043 & 0 & 0 & 0 & $3.71 \mathrm{E}+03$ & 0 & 0 & 0 & 0 & 0 & 0 & $3.36 \mathrm{E}-01$ & 0 & $3.92 \mathrm{E}+03$ & $4.37 \mathrm{E}+05$ & $0.90 \%$ \\
\hline 6TB044 & 0 & 0 & 0 & $7.66 \mathrm{E}+02$ & 0 & 0 & 0 & 0 & 0 & 0 & $5.08 \mathrm{E}-01$ & 0 & $7.77 \mathrm{E}+02$ & $4.33 \mathrm{E}+05$ & $0.18 \%$ \\
\hline 6TB045 & 0 & 0 & 0 & $7.79 \mathrm{E}+02$ & 0 & 0 & 0 & 0 & 0 & 0 & 5.82E-01 & 0 & $7.91 \mathrm{E}+02$ & $4.35 \mathrm{E}+05$ & $0.18 \%$ \\
\hline 6TB046 & 0 & 0 & 0 & $2.19 \mathrm{E}+03$ & 0 & 0 & 0 & 0 & 0 & 0 & $8.01 \mathrm{E}-01$ & 0 & $2.20 \mathrm{E}+03$ & $4.34 \mathrm{E}+05$ & $0.51 \%$ \\
\hline 6ТВ047 & $5.01 \mathrm{E}+02$ & 0 & 0 & 0 & 0 & 0 & 0 & 0 & 0 & 0 & 0 & 0 & $6.71 \mathrm{E}+02$ & $4.35 \mathrm{E}+05$ & $0.15 \%$ \\
\hline 6TB048 & 0 & 0 & 0 & $5.55 \mathrm{E}+02$ & 0 & 0 & 0 & 0 & 0 & 0 & $6.18 \mathrm{E}-01$ & 0 & $5.67 \mathrm{E}+02$ & $4.33 \mathrm{E}+05$ & $0.13 \%$ \\
\hline 6ТВ049 & 0 & 0 & 0 & $1.10 \mathrm{E}+03$ & 0 & 0 & 0 & 0 & 0 & 0 & $9.35 \mathrm{E}-01$ & 0 & $1.12 \mathrm{E}+03$ & $4.37 \mathrm{E}+05$ & $0.26 \%$ \\
\hline 6TB050 & $4.31 \mathrm{E}+02$ & 0 & 0 & 0 & 0 & 0 & 0 & 0 & 0 & 0 & $1.46 \mathrm{E}-02$ & 0 & $5.86 \mathrm{E}+02$ & $4.41 \mathrm{E}+05$ & $0.13 \%$ \\
\hline 6TB051 & $4.59 \mathrm{E}+02$ & 0 & 0 & 0 & 0 & 0 & 0 & 0 & 0 & 0 & $1.63 \mathrm{E}-01$ & 0 & $7.04 \mathrm{E}+02$ & $4.40 \mathrm{E}+05$ & $0.16 \%$ \\
\hline 6TB052 & 0 & 0 & 0 & 0 & 0 & 0 & 0 & 0 & 0 & 0 & $3.46 \mathrm{E}-01$ & 0 & $7.88 \mathrm{E}+00$ & $4.30 \mathrm{E}+05$ & $0.00 \%$ \\
\hline 6TB053 & 0 & 0 & 0 & 0 & 0 & 0 & 0 & 0 & 0 & 0 & 0 & 0 & 5.51E-01 & $4.18 \mathrm{E}+05$ & $0.00 \%$ \\
\hline 6TB054 & 0 & 0 & 0 & 0 & 0 & 0 & 0 & 0 & 0 & 0 & $2.43 \mathrm{E}-01$ & 0 & $6.23 \mathrm{E}+00$ & $4.28 \mathrm{E}+05$ & $0.00 \%$ \\
\hline 6TB055 & 0 & 0 & 0 & 0 & 0 & 0 & 0 & 0 & 0 & 0 & $3.45 \mathrm{E}-01$ & 0 & $5.44 \mathrm{E}+00$ & $4.23 \mathrm{E}+05$ & $0.00 \%$ \\
\hline 6TB056 & 0 & 0 & 0 & 0 & 0 & 0 & 0 & 0 & 0 & 0 & 0 & 0 & $2.14 \mathrm{E}+00$ & $4.23 \mathrm{E}+05$ & $0.00 \%$ \\
\hline 6TB057 & 0 & 0 & 0 & 0 & 0 & 0 & 0 & 0 & 0 & 0 & $3.83 \mathrm{E}-01$ & 0 & $6.30 \mathrm{E}+00$ & $4.25 \mathrm{E}+05$ & $0.00 \%$ \\
\hline 6TB058 & 0 & 0 & 0 & 0 & 0 & 0 & 0 & 0 & 0 & 0 & $1.08 \mathrm{E}-01$ & 0 & $3.76 \mathrm{E}+00$ & $4.24 \mathrm{E}+05$ & $0.00 \%$ \\
\hline 6TB059 & 0 & 0 & 0 & 0 & 0 & 0 & 0 & 0 & 0 & 0 & $3.24 \mathrm{E}-01$ & 0 & $7.87 \mathrm{E}+00$ & $4.31 \mathrm{E}+05$ & $0.00 \%$ \\
\hline 6ТВ060 & 0 & 0 & 0 & 0 & 0 & 0 & 0 & 0 & 0 & 0 & 0 & 0 & $1.36 \mathrm{E}+00$ & $4.22 \mathrm{E}+05$ & $0.00 \%$ \\
\hline 6TB061 & 0 & 0 & 0 & 0 & 0 & 0 & 0 & 0 & 0 & 0 & $2.92 \mathrm{E}-01$ & 0 & $4.61 \mathrm{E}+00$ & $4.20 \mathrm{E}+05$ & $0.00 \%$ \\
\hline 6TB062 & 0 & 0 & 0 & 0 & 0 & 0 & 0 & 0 & 0 & 0 & 4.89E-01 & 0 & $7.16 \mathrm{E}+00$ & $4.25 \mathrm{E}+05$ & $0.00 \%$ \\
\hline 6TB063 & 0 & 0 & 0 & 0 & 0 & 0 & 0 & 0 & 0 & 0 & $8.02 \mathrm{E}-03$ & 0 & $2.12 \mathrm{E}+00$ & $4.22 \mathrm{E}+05$ & $0.00 \%$ \\
\hline 6TB064 & 0 & 0 & 0 & 0 & 0 & 0 & 0 & 0 & 0 & 0 & $4.92 \mathrm{E}-02$ & 0 & $2.10 \mathrm{E}+00$ & $4.19 \mathrm{E}+05$ & $0.00 \%$ \\
\hline 6TB065 & 0 & 0 & 0 & 0 & 0 & 0 & 0 & 0 & 0 & 0 & $2.40 \mathrm{E}-01$ & 0 & $5.43 \mathrm{E}+00$ & $4.25 \mathrm{E}+05$ & $0.00 \%$ \\
\hline 6TB066 & 0 & 0 & 0 & 0 & 0 & 0 & 0 & 0 & 0 & 0 & $9.53 \mathrm{E}-01$ & 0 & $1.46 \mathrm{E}+01$ & $4.35 \mathrm{E}+05$ & $0.00 \%$ \\
\hline 6TB067 & 0 & 0 & 0 & 0 & 0 & 0 & 0 & 0 & 0 & 0 & $8.29 \mathrm{E}-01$ & 0 & $1.21 \mathrm{E}+01$ & $4.31 \mathrm{E}+05$ & $0.00 \%$ \\
\hline 6TB068 & 0 & 0 & 0 & 0 & 0 & 0 & 0 & 0 & 0 & 0 & 9.97E-01 & 0 & $1.47 \mathrm{E}+01$ & $4.34 \mathrm{E}+05$ & $0.00 \%$ \\
\hline 6ТВ069 & 0 & 0 & 0 & 0 & 0 & 0 & 0 & 0 & 0 & 0 & 8.04E-01 & 0 & $1.29 \mathrm{E}+01$ & $4.34 \mathrm{E}+05$ & $0.00 \%$ \\
\hline 6TB070 & 0 & 0 & 0 & 0 & 0 & 0 & 0 & 0 & 0 & 0 & $1.06 \mathrm{E}+00$ & 0 & $1.46 \mathrm{E}+01$ & $4.30 \mathrm{E}+05$ & $0.00 \%$ \\
\hline 6TB071 & 0 & 0 & 0 & 0 & 0 & 0 & 0 & 0 & 0 & 0 & 5.03E-01 & 0 & $8.80 \mathrm{E}+00$ & $4.30 \mathrm{E}+05$ & $0.00 \%$ \\
\hline 6TB072 & 0 & 0 & 0 & 0 & 0 & 0 & 0 & 0 & 0 & 0 & $6.24 \mathrm{E}-01$ & 0 & $9.63 \mathrm{E}+00$ & $4.28 \mathrm{E}+05$ & $0.00 \%$ \\
\hline
\end{tabular}


Table 38. Predicted Solids for Envelope B Simulations - Part 2 (cont'd)

\begin{tabular}{|c|c|c|c|c|c|c|c|c|c|c|c|c|c|c|c|}
\hline Test ID & $\begin{array}{c}\mathrm{NA} 2 \mathrm{C} 2 \mathrm{O} \\
4\end{array}$ & $\begin{array}{c}\text { NA2CO3. } \\
1 \mathrm{H} 2 \mathrm{O}\end{array}$ & NA2U2O7 & $\begin{array}{c}\text { NA3FSO } \\
4\end{array}$ & $\begin{array}{c}\text { NA6SO4 } \\
2 \mathrm{CO} 3\end{array}$ & NAF & $\begin{array}{c}\text { NAFPO4. } \\
19 \mathrm{H} 2 \mathrm{O}\end{array}$ & NANO3 & $\begin{array}{c}\text { NAPHOH } \\
.12 \mathrm{H} 2 \mathrm{O}\end{array}$ & $\begin{array}{c}\text { NASGEL. } \\
15.5 \mathrm{H} 2 \mathrm{O}\end{array}$ & $\mathrm{NIOH} 2$ & $\mathrm{ZRO} 2$ & $\begin{array}{l}\text { Total } \\
\text { Solids }\end{array}$ & $\begin{array}{c}\text { Total Soln } \\
\text { Mass }\end{array}$ & $\begin{array}{c}\text { Solids \% } \\
\text { of Total } \\
\text { Soln }\end{array}$ \\
\hline 6TB073 & 0 & 0 & 0 & 0 & 0 & 0 & 0 & 0 & 0 & 0 & $6.70 \mathrm{E}-01$ & 0 & $9.62 \mathrm{E}+00$ & $4.25 \mathrm{E}+05$ & $0.00 \%$ \\
\hline 6TB074 & 0 & 0 & 0 & 0 & 0 & 0 & 0 & 0 & 0 & 0 & $9.31 \mathrm{E}-01$ & 0 & $1.54 \mathrm{E}+01$ & $4.38 \mathrm{E}+05$ & $0.00 \%$ \\
\hline 6TB075 & 0 & 0 & 0 & 0 & 0 & 0 & 0 & 0 & 0 & 0 & 5.52E-01 & 0 & $8.83 \mathrm{E}+00$ & $4.28 \mathrm{E}+05$ & $0.00 \%$ \\
\hline 6TB076 & 0 & 0 & 0 & 0 & 0 & 0 & 0 & 0 & 0 & 0 & $8.67 \mathrm{E}-01$ & 0 & $1.29 \mathrm{E}+01$ & $4.30 \mathrm{E}+05$ & $0.00 \%$ \\
\hline 6TB077 & 0 & 0 & 0 & 0 & 0 & 0 & 0 & 0 & 0 & 0 & $1.00 \mathrm{E}+00$ & 0 & $1.47 \mathrm{E}+01$ & $4.34 \mathrm{E}+05$ & $0.00 \%$ \\
\hline 6TB078 & 0 & 0 & 0 & 0 & 0 & 0 & 0 & 0 & 0 & 0 & $1.03 \mathrm{E}+00$ & 0 & $1.46 \mathrm{E}+01$ & $4.32 \mathrm{E}+05$ & $0.00 \%$ \\
\hline 6TB079 & 0 & 0 & 0 & 0 & 0 & 0 & 0 & 0 & 0 & 0 & $3.20 \mathrm{E}-01$ & 0 & $7.90 \mathrm{E}+00$ & $4.32 \mathrm{E}+05$ & $0.00 \%$ \\
\hline 6TB080 & 0 & 0 & 0 & 0 & 0 & 0 & 0 & 0 & 0 & 0 & $3.74 \mathrm{E}-01$ & 0 & $8.67 \mathrm{E}+00$ & $4.31 \mathrm{E}+05$ & $0.00 \%$ \\
\hline 6TB081 & 0 & 0 & 0 & 0 & 0 & 0 & 0 & 0 & 0 & 0 & $1.13 \mathrm{E}+00$ & 0 & $1.55 \mathrm{E}+01$ & $4.31 \mathrm{E}+05$ & $0.00 \%$ \\
\hline 6TB082 & 0 & 0 & 0 & 0 & 0 & 0 & 0 & 0 & 0 & 0 & $5.22 \mathrm{E}-01$ & 0 & $9.57 \mathrm{E}+00$ & $4.30 \mathrm{E}+05$ & $0.00 \%$ \\
\hline 6TB083 & 0 & 0 & 0 & 0 & 0 & 0 & 0 & 0 & 0 & 0 & $9.86 \mathrm{E}-01$ & 0 & $1.54 \mathrm{E}+01$ & $4.36 \mathrm{E}+05$ & $0.00 \%$ \\
\hline 6TB084 & 0 & 0 & 0 & 0 & 0 & 0 & 0 & 0 & 0 & 0 & $7.77 \mathrm{E}-01$ & 0 & $1.13 \mathrm{E}+01$ & $4.27 \mathrm{E}+05$ & $0.00 \%$ \\
\hline 6TB085 & 0 & 0 & 0 & 0 & 0 & 0 & 0 & 0 & 0 & 0 & $8.80 \mathrm{E}-01$ & 0 & $1.38 \mathrm{E}+01$ & $4.35 \mathrm{E}+05$ & $0.00 \%$ \\
\hline 6TB086 & 0 & 0 & 0 & 0 & 0 & 0 & 0 & 0 & 0 & 0 & $7.32 \mathrm{E}-01$ & 0 & $1.13 \mathrm{E}+01$ & $4.30 \mathrm{E}+05$ & $0.00 \%$ \\
\hline 6TB087 & 0 & 0 & 0 & 0 & 0 & 0 & 0 & 0 & 0 & 0 & $7.81 \mathrm{E}-01$ & 0 & $1.13 \mathrm{E}+01$ & $4.29 \mathrm{E}+05$ & $0.00 \%$ \\
\hline 6TB088 & 0 & 0 & 0 & 0 & 0 & 0 & 0 & 0 & 0 & 0 & $1.10 \mathrm{E}+00$ & 0 & $1.63 \mathrm{E}+01$ & $4.36 \mathrm{E}+05$ & $0.00 \%$ \\
\hline 6TB089 & 0 & 0 & 0 & 0 & 0 & 0 & 0 & 0 & 0 & 0 & 9.39E-01 & 0 & $1.54 \mathrm{E}+01$ & $4.37 \mathrm{E}+05$ & $0.00 \%$ \\
\hline 6TB090 & 0 & 0 & 0 & 0 & 0 & 0 & 0 & 0 & 0 & 0 & 9.81E-01 & 0 & $1.39 \mathrm{E}+01$ & $4.32 \mathrm{E}+05$ & $0.00 \%$ \\
\hline 6ТВ091 & 0 & 0 & 0 & 0 & 0 & 0 & 0 & 0 & 0 & 0 & $5.20 \mathrm{E}-01$ & 0 & $9.62 \mathrm{E}+00$ & $4.32 \mathrm{E}+05$ & $0.00 \%$ \\
\hline 6TB092 & 0 & 0 & 0 & 0 & 0 & 0 & 0 & 0 & 0 & 0 & $7.11 \mathrm{E}-01$ & 0 & $1.13 \mathrm{E}+01$ & $4.32 \mathrm{E}+05$ & $0.00 \%$ \\
\hline 6TB093 & 0 & 0 & 0 & 0 & 0 & 0 & 0 & 0 & 0 & 0 & $1.14 \mathrm{E}+00$ & 0 & $1.64 \mathrm{E}+01$ & $4.36 \mathrm{E}+05$ & $0.00 \%$ \\
\hline 6ТВ094 & 0 & 0 & 0 & 0 & 0 & 0 & 0 & 0 & 0 & 0 & $3.40 \mathrm{E}-01$ & 0 & $5.45 \mathrm{E}+00$ & $4.23 \mathrm{E}+05$ & $0.00 \%$ \\
\hline 6TB095 & 0 & 0 & 0 & 0 & 0 & 0 & 0 & 0 & 0 & 0 & 0 & 0 & $2.11 \mathrm{E}+00$ & $4.20 \mathrm{E}+05$ & $0.00 \%$ \\
\hline 6TB096 & 0 & 0 & 0 & 0 & 0 & 0 & 0 & 0 & 0 & 0 & $1.97 \mathrm{E}-01$ & 0 & $3.79 \mathrm{E}+00$ & $4.22 \mathrm{E}+05$ & $0.00 \%$ \\
\hline 6TB097 & 0 & 0 & 0 & 0 & 0 & 0 & 0 & 0 & 0 & 0 & $2.94 \mathrm{E}-01$ & 0 & $5.44 \mathrm{E}+00$ & $4.23 \mathrm{E}+05$ & $0.00 \%$ \\
\hline 6TB098 & 0 & 0 & 0 & 0 & 0 & 0 & 0 & 0 & 0 & 0 & 0 & 0 & 0 & $4.18 \mathrm{E}+05$ & $0.00 \%$ \\
\hline 6ТВ099 & 0 & 0 & 0 & 0 & 0 & 0 & 0 & 0 & 0 & 0 & 9.43E-02 & 0 & $4.57 \mathrm{E}+00$ & $4.26 \mathrm{E}+05$ & $0.00 \%$ \\
\hline 6TB100 & 0 & 0 & 0 & 0 & 0 & 0 & 0 & 0 & 0 & 0 & $2.89 \mathrm{E}-03$ & 0 & $2.94 \mathrm{E}+00$ & $4.25 \mathrm{E}+05$ & $0.00 \%$ \\
\hline 6TB101 & 0 & 0 & 0 & 0 & 0 & 0 & 0 & 0 & 0 & 0 & 0 & 0 & $6.23 \mathrm{E}-01$ & $4.23 \mathrm{E}+05$ & $0.00 \%$ \\
\hline 6TB102 & 0 & 0 & 0 & 0 & 0 & 0 & 0 & 0 & 0 & 0 & $5.31 \mathrm{E}-01$ & 0 & $7.94 \mathrm{E}+00$ & $4.25 \mathrm{E}+05$ & $0.00 \%$ \\
\hline 6TB103 & 0 & 0 & 0 & 0 & 0 & 0 & 0 & 0 & 0 & 0 & 0 & 0 & $2.94 \mathrm{E}+00$ & $4.25 \mathrm{E}+05$ & $0.00 \%$ \\
\hline
\end{tabular}


Table 38. Predicted Solids for Envelope B Simulations - Part 2 (cont'd)

\begin{tabular}{|c|c|c|c|c|c|c|c|c|c|c|c|c|c|c|c|}
\hline Test ID & $\begin{array}{c}\mathrm{NA} 2 \mathrm{C} 2 \mathrm{O} \\
4\end{array}$ & $\begin{array}{c}\mathrm{NA} 2 \mathrm{CO} 3 . \\
1 \mathrm{H} 2 \mathrm{O}\end{array}$ & NA2U2O7 & $\begin{array}{c}\text { NA3FSO } \\
4\end{array}$ & $\begin{array}{c}\text { NA6SO4 } \\
2 \mathrm{CO} 3\end{array}$ & NAF & $\begin{array}{c}\text { NAFPO4. } \\
19 \mathrm{H} 2 \mathrm{O}\end{array}$ & NANO3 & $\begin{array}{c}\text { NAPHOH } \\
.12 \mathrm{H} 2 \mathrm{O}\end{array}$ & $\begin{array}{c}\text { NASGEL. } \\
15.5 \mathrm{H} 2 \mathrm{O}\end{array}$ & $\mathrm{NIOH} 2$ & ZRO2 & $\begin{array}{l}\text { Total } \\
\text { Solids }\end{array}$ & $\begin{array}{c}\text { Total Soln } \\
\text { Mass }\end{array}$ & $\begin{array}{c}\text { Solids } \% \\
\text { of Total } \\
\text { Soln }\end{array}$ \\
\hline 6TB104 & 0 & 0 & 0 & 0 & 0 & 0 & 0 & 0 & 0 & 0 & 0 & 0 & 0 & $4.23 \mathrm{E}+05$ & $0.00 \%$ \\
\hline 6TB105 & 0 & 0 & 0 & 0 & 0 & 0 & 0 & 0 & 0 & 0 & $2.16 \mathrm{E}-01$ & 0 & $3.83 \mathrm{E}+00$ & $4.22 \mathrm{E}+05$ & $0.00 \%$ \\
\hline 6TB106 & 0 & 0 & 0 & 0 & 0 & 0 & 0 & 0 & 0 & 0 & $8.25 \mathrm{E}-02$ & 0 & $2.13 \mathrm{E}+00$ & $4.21 \mathrm{E}+05$ & $0.00 \%$ \\
\hline 6TB107 & 0 & 0 & 0 & $8.47 \mathrm{E}+02$ & 0 & 0 & 0 & 0 & 0 & 0 & $2.58 \mathrm{E}-01$ & 0 & $8.55 \mathrm{E}+02$ & $4.28 \mathrm{E}+05$ & $0.20 \%$ \\
\hline 6TB108 & $4.76 \mathrm{E}+02$ & 0 & 0 & 0 & 0 & 0 & 0 & 0 & 0 & 0 & $2.24 \mathrm{E}-01$ & 0 & $4.91 \mathrm{E}+02$ & $4.44 \mathrm{E}+05$ & $0.11 \%$ \\
\hline 6TB109 & 0 & 0 & 0 & $1.66 \mathrm{E}+03$ & 0 & 0 & 0 & 0 & 0 & 0 & $3.53 \mathrm{E}-01$ & 0 & $1.67 \mathrm{E}+03$ & $4.29 \mathrm{E}+05$ & $0.39 \%$ \\
\hline 6TB110 & $5.21 \mathrm{E}+02$ & 0 & 0 & 0 & 0 & 0 & 0 & 0 & 0 & 0 & 0 & 0 & $5.30 \mathrm{E}+02$ & $4.39 \mathrm{E}+05$ & $0.12 \%$ \\
\hline 6TB111 & $1.04 \mathrm{E}+02$ & 0 & 0 & $1.93 \mathrm{E}+03$ & 0 & 0 & 0 & 0 & 0 & 0 & $6.07 \mathrm{E}-01$ & 0 & $2.05 \mathrm{E}+03$ & $4.33 \mathrm{E}+05$ & $0.47 \%$ \\
\hline 6TB112 & $2.38 \mathrm{E}+02$ & 0 & 0 & $2.51 \mathrm{E}+03$ & 0 & 0 & 0 & 0 & 0 & 0 & $6.54 \mathrm{E}-02$ & 0 & $2.76 \mathrm{E}+03$ & $4.33 \mathrm{E}+05$ & $0.64 \%$ \\
\hline 6TB113 & $6.48 \mathrm{E}+01$ & 0 & 0 & $2.71 \mathrm{E}+03$ & 0 & 0 & 0 & 0 & 0 & 0 & $4.52 \mathrm{E}-01$ & 0 & $2.78 \mathrm{E}+03$ & $4.32 \mathrm{E}+05$ & $0.64 \%$ \\
\hline 6TB114 & 0 & 0 & 0 & $1.11 \mathrm{E}+03$ & 0 & 0 & 0 & 0 & 0 & 0 & $3.18 \mathrm{E}-01$ & 0 & $1.12 \mathrm{E}+03$ & $4.30 \mathrm{E}+05$ & $0.26 \%$ \\
\hline 6TB115 & 0 & 0 & 0 & $2.48 \mathrm{E}+03$ & 0 & 0 & 0 & 0 & 0 & 0 & $5.80 \mathrm{E}-01$ & 0 & $2.49 \mathrm{E}+03$ & $4.35 \mathrm{E}+05$ & $0.57 \%$ \\
\hline 6TB116 & 0 & 0 & 0 & 0 & 0 & 0 & 0 & 0 & 0 & 0 & 0 & 0 & \begin{tabular}{|l|}
$1.84 \mathrm{E}+02$ \\
\end{tabular} & $4.42 \mathrm{E}+05$ & $0.04 \%$ \\
\hline 6TB117 & $1.86 \mathrm{E}+02$ & 0 & 0 & 0 & 0 & 0 & 0 & 0 & 0 & 0 & 0 & 0 & $3.54 \mathrm{E}+02$ & $4.40 \mathrm{E}+05$ & $0.08 \%$ \\
\hline 6TB118 & 0 & 0 & 0 & $3.35 \mathrm{E}+03$ & 0 & 0 & 0 & 0 & 0 & 0 & $5.55 \mathrm{E}-01$ & 0 & $3.37 \mathrm{E}+03$ & $4.33 \mathrm{E}+05$ & $0.78 \%$ \\
\hline 6TB119 & 0 & 0 & 0 & $3.18 \mathrm{E}+03$ & 0 & 0 & 0 & 0 & 0 & 0 & $3.62 \mathrm{E}-01$ & 0 & $3.19 \mathrm{E}+03$ & $4.35 \mathrm{E}+05$ & $0.73 \%$ \\
\hline 6TB120 & 0 & 0 & 0 & $2.32 \mathrm{E}+03$ & 0 & 0 & 0 & 0 & 0 & 0 & $3.21 \mathrm{E}-01$ & 0 & $2.33 \mathrm{E}+03$ & $4.31 \mathrm{E}+05$ & $0.54 \%$ \\
\hline $6 \mathrm{~TB} 121$ & 0 & 0 & 0 & $5.08 \mathrm{E}+02$ & 0 & 0 & 0 & 0 & 0 & 0 & 0 & 0 & $5.10 \mathrm{E}+02$ & $4.26 \mathrm{E}+05$ & $0.12 \%$ \\
\hline 6TB122 & $3.71 \mathrm{E}+02$ & 0 & 0 & $1.70 \mathrm{E}+03$ & 0 & 0 & 0 & 0 & 0 & 0 & 0 & 0 & $2.08 \mathrm{E}+03$ & $4.25 \mathrm{E}+05$ & $0.49 \%$ \\
\hline 6TB123 & $2.56 \mathrm{E}+02$ & 0 & 0 & $7.33 \mathrm{E}+02$ & 0 & 0 & 0 & 0 & 0 & 0 & 0 & 0 & $9.91 \mathrm{E}+02$ & $4.27 \mathrm{E}+05$ & $0.23 \%$ \\
\hline 6TB124 & $7.13 \mathrm{E}+01$ & 0 & 0 & $1.26 \mathrm{E}+03$ & 0 & 0 & 0 & 0 & 0 & 0 & 0 & 0 & $1.33 \mathrm{E}+03$ & $4.25 \mathrm{E}+05$ & $0.31 \%$ \\
\hline $6 \mathrm{~TB} 125$ & $6.24 \mathrm{E}+02$ & 0 & 0 & 0 & 0 & 0 & 0 & 0 & 0 & 0 & 0 & 0 & $6.26 \mathrm{E}+02$ & $4.31 \mathrm{E}+05$ & $0.15 \%$ \\
\hline $6 \mathrm{~TB} 126$ & $1.18 \mathrm{E}+02$ & 0 & 0 & $1.43 \mathrm{E}+03$ & 0 & 0 & 0 & 0 & 0 & 0 & $6.93 \mathrm{E}-02$ & 0 & $1.56 \mathrm{E}+03$ & $4.30 \mathrm{E}+05$ & $0.36 \%$ \\
\hline 6TB127 & $3.60 \mathrm{E}+02$ & 0 & 0 & $1.77 \mathrm{E}+03$ & 0 & 0 & 0 & 0 & 0 & 0 & 0 & 0 & $2.14 \mathrm{E}+03$ & $4.29 \mathrm{E}+05$ & $0.50 \%$ \\
\hline 6TB128 & 0 & 0 & 0 & 0 & 0 & 0 & 0 & 0 & 0 & 0 & 0 & 0 & $2.10 \mathrm{E}+02$ & $4.37 \mathrm{E}+05$ & $0.05 \%$ \\
\hline 6TB129 & 0 & 0 & 0 & $1.11 \mathrm{E}+02$ & 0 & 0 & 0 & 0 & 0 & 0 & 0 & 0 & $1.12 \mathrm{E}+02$ & $4.24 \mathrm{E}+05$ & $0.03 \%$ \\
\hline $6 \mathrm{~TB} 130$ & 0 & 0 & 0 & $1.84 \mathrm{E}+03$ & 0 & 0 & 0 & 0 & 0 & 0 & 0 & 0 & $1.85 \mathrm{E}+03$ & $4.31 \mathrm{E}+05$ & $0.43 \%$ \\
\hline 6TB131 & 0 & 0 & 0 & $1.62 \mathrm{E}+03$ & 0 & 0 & 0 & 0 & 0 & 0 & 0 & 0 & $1.62 \mathrm{E}+03$ & $4.28 \mathrm{E}+05$ & $0.38 \%$ \\
\hline 6TB132 & 0 & 0 & 0 & $1.00 \mathrm{E}+03$ & 0 & 0 & 0 & 0 & 0 & 0 & 0 & 0 & $1.00 \mathrm{E}+03$ & $4.27 \mathrm{E}+05$ & $0.23 \%$ \\
\hline 6TB133 & 0 & 0 & 0 & $1.83 \mathrm{E}+03$ & 0 & 0 & 0 & 0 & 0 & 0 & 0 & 0 & $1.83 \mathrm{E}+03$ & $4.26 \mathrm{E}+05$ & $0.43 \%$ \\
\hline
\end{tabular}


Table 39. Predicted Solids for Envelope C Simulations - Part 1

\begin{tabular}{|c|c|c|c|c|c|c|c|c|c|c|c|c|c|c|c|}
\hline Test ID & $\begin{array}{l}\text { SBS/ } \\
\text { Feed }\end{array}$ & $\begin{array}{l}\text { Temp } \\
{\left[{ }^{\circ} \mathrm{C}\right]}\end{array}$ & $\begin{array}{c}\mathrm{Na} \mathrm{M} \\
{[\mathrm{mol} / \mathrm{L}]}\end{array}$ & ALOH3 & BASO4 & CA3PO42 & $\mathrm{CACO} 3$ & CAF2 & $\mathrm{CAOH} 2$ & CATIO3 & $\mathrm{CROH} 3$ & FEIIIOH3 & $\begin{array}{c}\text { HYDROS } \\
\text { OD }\end{array}$ & MGF2 & MGOH2 \\
\hline $10 \mathrm{TC} 001$ & 0 & 15 & 10 & 0 & 0 & 0 & 0 & 0 & 0 & 0 & 0 & 0 & 0 & 0 & 0 \\
\hline $10 \mathrm{TC} 002$ & 0 & 15 & 10 & 0 & 0 & 0 & 0 & 0 & 0 & 0 & 0 & 0 & 0 & 0 & 0 \\
\hline $10 \mathrm{TC} 003$ & 0 & 15 & 10 & 0 & 0 & 0 & 0 & 0 & 0 & 0 & 0 & 0 & 0 & 0 & 0 \\
\hline $10 \mathrm{TC} 004$ & 0 & 15 & 10 & 0 & 0 & 0 & 0 & 0 & 0 & 0 & 0 & 0 & 0 & 0 & 0 \\
\hline $10 \mathrm{TC} 005$ & 0 & 15 & 10 & 0 & 0 & 0 & 0 & 0 & 0 & 0 & 0 & 0 & 0 & 0 & 0 \\
\hline $10 \mathrm{TC} 006$ & 0 & 66 & 10 & 0 & 0 & 0 & 0 & 0 & 0 & 0 & 0 & 0 & 0 & 0 & 0 \\
\hline $10 \mathrm{TC} 007$ & 0 & 66 & 10 & 0 & 0 & 0 & 0 & 0 & 0 & 0 & 0 & 0 & 0 & 0 & 0 \\
\hline $10 \mathrm{TC} 008$ & 0 & 66 & 10 & 0 & 0 & 0 & 0 & 0 & 0 & 0 & 0 & 0 & 0 & 0 & 0 \\
\hline $10 \mathrm{TC} 009$ & 0 & 66 & 10 & 0 & 0 & 0 & 0 & 0 & 0 & 0 & 0 & 0 & 0 & 0 & 0 \\
\hline $10 \mathrm{TC} 010$ & 0 & 66 & 10 & 0 & 0 & 0 & 0 & 0 & 0 & 0 & 0 & 0 & 0 & 0 & 0 \\
\hline $10 \mathrm{TC} 011$ & 2 & 15 & 10 & 0 & 0 & 0 & 0 & $2.04 \mathrm{E}+03$ & 0 & $2.89 \mathrm{E}+02$ & 0 & $4.71 \mathrm{E}+02$ & $4.11 \mathrm{E}+03$ & 0 & $3.74 \mathrm{E}+02$ \\
\hline $10 \mathrm{TC} 012$ & 2 & 15 & 10 & 0 & 0 & 0 & 0 & $2.03 \mathrm{E}+03$ & 0 & $2.88 \mathrm{E}+02$ & 0 & $4.29 \mathrm{E}+02$ & \begin{tabular}{|l|}
$3.74 \mathrm{E}+03$ \\
\end{tabular} & 0 & $3.72 \mathrm{E}+02$ \\
\hline $10 \mathrm{TC} 013$ & 2 & 15 & 10 & 0 & 0 & 0 & 0 & $2.01 \mathrm{E}+03$ & 0 & $2.86 \mathrm{E}+02$ & 0 & $4.94 \mathrm{E}+02$ & $4.31 \mathrm{E}+03$ & $2.01 \mathrm{E}+02$ & $3.70 \mathrm{E}+02$ \\
\hline $10 \mathrm{TC} 014$ & 2 & 15 & 10 & 0 & 0 & 0 & 0 & $1.99 \mathrm{E}+03$ & 0 & $2.83 \mathrm{E}+02$ & 0 & $4.61 \mathrm{E}+02$ & $4.02 \mathrm{E}+03$ & 0 & $3.65 \mathrm{E}+02$ \\
\hline $10 \mathrm{TC} 015$ & 2 & 15 & 10 & 0 & 0 & 0 & 0 & $2.01 \mathrm{E}+03$ & 0 & $2.85 \mathrm{E}+02$ & 0 & $5.17 \mathrm{E}+02$ & $4.51 \mathrm{E}+03$ & $5.30 \mathrm{E}+02$ & $3.68 \mathrm{E}+02$ \\
\hline $10 \mathrm{TC} 016$ & 2 & 66 & 10 & 0 & $3.47 \mathrm{E}+00$ & 0 & 0 & $2.04 \mathrm{E}+03$ & 0 & $2.89 \mathrm{E}+02$ & $2.64 \mathrm{E}+02$ & 0 & 0 & $1.23 \mathrm{E}+03$ & $3.74 \mathrm{E}+02$ \\
\hline $10 \mathrm{TC} 017$ & 2 & 66 & 10 & 0 & $3.47 \mathrm{E}+00$ & 0 & 0 & $2.03 \mathrm{E}+03$ & 0 & \begin{tabular}{|l|}
$2.88 \mathrm{E}+02$ \\
\end{tabular} & $2.59 \mathrm{E}+02$ & 0 & 0 & $1.04 \mathrm{E}+03$ & $3.72 \mathrm{E}+02$ \\
\hline $10 \mathrm{TC} 018$ & 2 & 66 & 10 & 0 & $3.55 \mathrm{E}+00$ & 0 & 0 & $2.01 \mathrm{E}+03$ & 0 & $2.86 \mathrm{E}+02$ & $2.64 \mathrm{E}+02$ & 0 & 0 & $1.28 \mathrm{E}+03$ & $3.70 \mathrm{E}+02$ \\
\hline $10 \mathrm{TC} 019$ & 2 & 66 & 10 & 0 & $3.42 \mathrm{E}+00$ & 0 & 0 & $1.99 \mathrm{E}+03$ & 0 & \begin{tabular}{|l|}
$2.83 \mathrm{E}+02$ \\
\end{tabular} & $2.58 \mathrm{E}+02$ & 0 & 0 & $1.13 \mathrm{E}+03$ & $3.65 \mathrm{E}+02$ \\
\hline $10 \mathrm{TC} 020$ & 2 & 66 & 10 & 0 & $3.53 \mathrm{E}+00$ & 0 & 0 & $2.01 \mathrm{E}+03$ & 0 & $2.85 \mathrm{E}+02$ & $2.66 \mathrm{E}+02$ & 0 & 0 & $1.36 \mathrm{E}+03$ & $3.68 \mathrm{E}+02$ \\
\hline $10 \mathrm{TC} 021$ & 0.672 & 37.3125 & 10 & 0 & $2.52 \mathrm{E}-01$ & $4.87 \mathrm{E}+02$ & $2.10 \mathrm{E}+02$ & $1.48 \mathrm{E}+02$ & 0 & $9.70 \mathrm{E}+01$ & 0 & 0 & 0 & 0 & $1.25 \mathrm{E}+02$ \\
\hline $10 \mathrm{TC} 022$ & 0.344 & 18.1875 & 10 & 0 & 0 & 0 & 0 & \begin{tabular}{|l|}
$3.50 \mathrm{E}+02$ \\
\end{tabular} & 0 & \begin{tabular}{|l|}
$4.97 \mathrm{E}+01$ \\
\end{tabular} & 0 & 0 & 0 & 0 & $6.42 \mathrm{E}+01$ \\
\hline $10 \mathrm{TC} 023$ & 0.484 & 35.3203 & 10 & 0 & 0 & 0 & 0 & $2.23 \mathrm{E}+02$ & $2.53 \mathrm{E}+02$ & $6.98 \mathrm{E}+01$ & 0 & 0 & 0 & 0 & $9.02 \mathrm{E}+01$ \\
\hline $10 \mathrm{TC} 024$ & 0.719 & 17.3906 & 10 & 0 & 0 & 0 & 0 & $7.30 \mathrm{E}+02$ & 0 & $1.04 \mathrm{E}+02$ & 0 & 0 & 0 & 0 & $1.34 \mathrm{E}+02$ \\
\hline $10 \mathrm{TC} 025$ & 0 & 30.9375 & 10 & 0 & 0 & 0 & 0 & 0 & 0 & 0 & 0 & 0 & 0 & 0 & 0 \\
\hline $10 \mathrm{TC} 026$ & 0.641 & 21.7734 & 10 & 0 & $1.78 \mathrm{E}-01$ & 0 & 0 & \begin{tabular}{|l|}
$8.54 \mathrm{E}+01$ \\
\end{tabular} & $5.36 \mathrm{E}+02$ & $9.27 \mathrm{E}+01$ & 0 & 0 & 0 & 0 & $1.20 \mathrm{E}+02$ \\
\hline $10 \mathrm{TC} 027$ & 0.359 & 29.7422 & 10 & 0 & 0 & 0 & 0 & $9.79 \mathrm{E}+01$ & \begin{tabular}{|l|}
$2.49 \mathrm{E}+02$ \\
\end{tabular} & $5.15 \mathrm{E}+01$ & 0 & 0 & 0 & 0 & $6.65 \mathrm{E}+01$ \\
\hline $10 \mathrm{TC} 028$ & 1.891 & 29.3438 & 10 & 0 & $3.05 \mathrm{E}+00$ & $2.32 \mathrm{E}+02$ & $2.23 \mathrm{E}+03$ & 0 & 0 & \begin{tabular}{|l|}
$2.73 \mathrm{E}+02$ \\
\end{tabular} & $3.09 \mathrm{E}+01$ & $2.75 \mathrm{E}+02$ & $2.40 \mathrm{E}+03$ & 0 & $3.52 \mathrm{E}+02$ \\
\hline $10 \mathrm{TC} 029$ & 1.047 & 16.1953 & 10 & 0 & 0 & 0 & 0 & $1.06 \mathrm{E}+03$ & 0 & $1.51 \mathrm{E}+02$ & 0 & $1.09 \mathrm{E}+02$ & $9.55 \mathrm{E}+02$ & 0 & $1.95 \mathrm{E}+02$ \\
\hline
\end{tabular}


Table 39. Predicted Solids for Envelope C Simulations - Part 1 (cont'd)

\begin{tabular}{|c|c|c|c|c|c|c|c|c|c|c|c|c|c|c|c|c|}
\hline Test ID & $\begin{array}{l}\text { SBS/ } \\
\text { Feed }\end{array}$ & $\begin{array}{l}\text { Temp } \\
{\left[{ }^{\circ} \mathrm{C}\right]}\end{array}$ & $\begin{array}{c}\mathrm{Na} \mathrm{M} \\
{[\mathrm{mol} / \mathrm{L}]}\end{array}$ & ALOH3 & BASO4 & CA3PO42 & $\mathrm{CACO} 3$ & CAF2 & CANC & $\mathrm{CAOH} 2$ & CATIO3 & $\mathrm{CROH} 3$ & FEIIIOH3 & $\begin{array}{c}\text { HYDROS } \\
\text { OD }\end{array}$ & MGF2 & MGOH2 \\
\hline 10TC030 & 1.563 & 16.5938 & 10 & 0 & 0 & 0 & 0 & $1.58 \mathrm{E}+03$ & 0 & 0 & $2.25 \mathrm{E}+02$ & 0 & \begin{tabular}{|l|}
$2.72 \mathrm{E}+02$ \\
\end{tabular} & $2.37 \mathrm{E}+03$ & 0 & $2.90 \mathrm{E}+02$ \\
\hline 10TC031 & 1.578 & 22.1719 & 10 & 0 & $2.45 \mathrm{E}+00$ & 0 & $2.05 \mathrm{E}+03$ & 0 & 0 & 0 & $2.28 \mathrm{E}+02$ & 0 & $2.35 \mathrm{E}+02$ & $2.05 \mathrm{E}+03$ & 0 & $2.94 \mathrm{E}+02$ \\
\hline $10 \mathrm{TC} 032$ & 1.547 & 35.7188 & 10 & 0 & $2.38 \mathrm{E}+00$ & $4.63 \mathrm{E}+02$ & $1.57 \mathrm{E}+03$ & 0 & 0 & 0 & $2.24 \mathrm{E}+02$ & $3.40 \mathrm{E}+01$ & 0 & 0 & 0 & $2.89 \mathrm{E}+02$ \\
\hline $10 \mathrm{TC} 033$ & 1.313 & 30.5391 & 10 & 0 & $1.85 \mathrm{E}+00$ & $2.25 \mathrm{E}+02$ & $1.49 \mathrm{E}+03$ & 0 & 0 & 0 & $1.89 \mathrm{E}+02$ & 0 & 0 & 0 & 0 & $2.45 \mathrm{E}+02$ \\
\hline $10 \mathrm{TC} 034$ & 1.672 & 15.3984 & 10 & 0 & 0 & 0 & 0 & $1.70 \mathrm{E}+03$ & 0 & 0 & $2.41 \mathrm{E}+02$ & 0 & $3.41 \mathrm{E}+02$ & $2.97 \mathrm{E}+03$ & 0 & $3.11 \mathrm{E}+02$ \\
\hline 10TC035 & 0.875 & 48.8672 & 10 & 0 & $8.60 \mathrm{E}-01$ & 0 & 0 & $8.88 \mathrm{E}+02$ & 0 & 0 & $1.26 \mathrm{E}+02$ & $3.35 \mathrm{E}+01$ & 0 & 0 & 0 & $1.63 \mathrm{E}+02$ \\
\hline $10 \mathrm{TC} 036$ & 0.125 & 57.2344 & 10 & 0 & 0 & 0 & 0 & $1.27 \mathrm{E}+02$ & 0 & 0 & $1.81 \mathrm{E}+01$ & 0 & 0 & 0 & 0 & $2.33 \mathrm{E}+01$ \\
\hline $10 \mathrm{TC} 037$ & 0.797 & 53.6484 & 10 & 0 & $6.74 \mathrm{E}-01$ & 0 & 0 & $8.05 \mathrm{E}+02$ & 0 & 0 & $1.14 \mathrm{E}+02$ & $4.93 \mathrm{E}+01$ & 0 & 0 & 0 & $1.48 \mathrm{E}+02$ \\
\hline $10 \mathrm{TC} 038$ & 0.094 & 47.6719 & 10 & 0 & 0 & 0 & 0 & $9.46 \mathrm{E}+01$ & 0 & 0 & $1.35 \mathrm{E}+01$ & 0 & 0 & 0 & 0 & $1.75 \mathrm{E}+01$ \\
\hline 10TC039 & 0.625 & 66 & 10 & 0 & $2.39 \mathrm{E}-01$ & 0 & 0 & $6.34 \mathrm{E}+02$ & 0 & 0 & $9.02 \mathrm{E}+01$ & $6.70 \mathrm{E}+01$ & 0 & 0 & 0 & $1.16 \mathrm{E}+02$ \\
\hline $10 \mathrm{TC} 040$ & 0.266 & 49.6641 & 10 & 0 & 0 & 0 & 0 & $2.70 \mathrm{E}+02$ & 0 & 0 & $3.84 \mathrm{E}+01$ & 0 & 0 & 0 & 0 & $4.96 \mathrm{E}+01$ \\
\hline 10TC041 & 0.938 & 53.25 & 10 & 0 & $9.78 \mathrm{E}-01$ & 0 & 0 & $9.52 \mathrm{E}+02$ & 0 & 0 & $1.35 \mathrm{E}+02$ & $6.99 \mathrm{E}+01$ & 0 & 0 & 0 & $1.75 \mathrm{E}+02$ \\
\hline 10TC042 & 1.438 & 63.2109 & 10 & 0 & $2.19 \mathrm{E}+00$ & 0 & 0 & $1.45 \mathrm{E}+03$ & 0 & 0 & $2.07 \mathrm{E}+02$ & $1.80 \mathrm{E}+02$ & 0 & 0 & $3.46 \mathrm{E}+02$ & $2.67 \mathrm{E}+02$ \\
\hline $10 \mathrm{TC} 043$ & 1.953 & 41.6953 & 10 & 0 & $3.31 \mathrm{E}+00$ & 0 & 0 & $1.98 \mathrm{E}+03$ & 0 & 0 & $2.82 \mathrm{E}+02$ & $1.85 \mathrm{E}+02$ & $1.76 \mathrm{E}+01$ & $1.53 \mathrm{E}+02$ & $7.47 \mathrm{E}+02$ & $3.64 \mathrm{E}+02$ \\
\hline $10 \mathrm{TC} 044$ & 1.938 & 54.8438 & 10 & 0 & $3.34 \mathrm{E}+00$ & 0 & 0 & $1.97 \mathrm{E}+03$ & 0 & 0 & $2.79 \mathrm{E}+02$ & $2.29 \mathrm{E}+02$ & 0 & 0 & $7.76 \mathrm{E}+02$ & $3.61 \mathrm{E}+02$ \\
\hline $10 \mathrm{TC} 045$ & 1.484 & 65.2031 & 10 & 0 & $2.25 \mathrm{E}+00$ & 0 & 0 & $1.51 \mathrm{E}+03$ & 0 & 0 & $2.14 \mathrm{E}+02$ & $1.89 \mathrm{E}+02$ & 0 & 0 & $4.30 \mathrm{E}+02$ & $2.77 \mathrm{E}+02$ \\
\hline 10TC046 & 1.719 & 55.2422 & 10 & 0 & $2.83 \mathrm{E}+00$ & 0 & 0 & $1.75 \mathrm{E}+03$ & 0 & 0 & $2.48 \mathrm{E}+02$ & $1.99 \mathrm{E}+02$ & 0 & 0 & $4.69 \mathrm{E}+02$ & $3.20 \mathrm{E}+02$ \\
\hline 10TC047 & 1.188 & 54.4453 & 10 & 0 & $1.62 \mathrm{E}+00$ & 0 & 0 & $1.21 \mathrm{E}+03$ & 0 & 0 & $1.72 \mathrm{E}+02$ & $1.26 \mathrm{E}+02$ & 0 & 0 & 0 & $2.22 \mathrm{E}+02$ \\
\hline $10 \mathrm{TC} 048$ & 1.391 & 48.4688 & 10 & 0 & $2.04 \mathrm{E}+00$ & 0 & 0 & $1.41 \mathrm{E}+03$ & 0 & 0 & $2.01 \mathrm{E}+02$ & $1.10 \mathrm{E}+02$ & 0 & 0 & 0 & $2.59 \mathrm{E}+02$ \\
\hline $10 \mathrm{TC} 049$ & 1.984 & 57.6328 & 10 & 0 & $3.40 \mathrm{E}+00$ & 0 & 0 & $2.00 \mathrm{E}+03$ & 0 & 0 & $2.85 \mathrm{E}+02$ & $2.43 \mathrm{E}+02$ & 0 & 0 & $9.11 \mathrm{E}+02$ & $3.68 \mathrm{E}+02$ \\
\hline 10TC050 & 0.156 & 40.1016 & 10 & 0 & 0 & 0 & 0 & $1.58 \mathrm{E}+02$ & 0 & 0 & $2.26 \mathrm{E}+01$ & 0 & 0 & 0 & 0 & $2.91 \mathrm{E}+01$ \\
\hline 10TC051 & 0.547 & 16.9922 & 10 & 0 & 0 & 0 & 0 & $5.56 \mathrm{E}+02$ & 0 & 0 & $7.91 \mathrm{E}+01$ & 0 & 0 & 0 & 0 & $1.02 \mathrm{E}+02$ \\
\hline 10TC052 & 0.219 & 34.9219 & 10 & 0 & 0 & 0 & 0 & $1.13 \mathrm{E}+02$ & 0 & $1.02 \mathrm{E}+02$ & $3.15 \mathrm{E}+01$ & 0 & 0 & 0 & 0 & $4.07 \mathrm{E}+01$ \\
\hline $10 \mathrm{TC} 053$ & 0.203 & 22.5703 & 10 & 0 & 0 & 0 & 0 & $2.04 \mathrm{E}+02$ & 0 & 0 & \begin{tabular}{|l|}
$2.93 \mathrm{E}+01$ \\
\end{tabular} & 0 & 0 & 0 & 0 & $3.78 \mathrm{E}+01$ \\
\hline $10 \mathrm{TC} 054$ & 0.922 & 34.125 & 10 & 0 & $9.37 \mathrm{E}-01$ & $3.08 \mathrm{E}+02$ & $9.00 \mathrm{E}+02$ & 0 & 0 & 0 & $1.33 \mathrm{E}+02$ & 0 & 0 & 0 & 0 & $1.72 \mathrm{E}+02$ \\
\hline $10 \mathrm{TC} 055$ & 0.828 & 28.5469 & 10 & 0 & $6.51 \mathrm{E}-01$ & 0 & 0 & 0 & 0 & $7.96 \mathrm{E}+02$ & $1.20 \mathrm{E}+02$ & 0 & 0 & 0 & 0 & $1.54 \mathrm{E}+02$ \\
\hline 10TC056 & 0.703 & 39.7031 & 10 & 0 & $3.85 \mathrm{E}-01$ & $5.39 \mathrm{E}+02$ & $3.91 \mathrm{E}+02$ & 0 & 0 & 0 & $1.01 \mathrm{E}+02$ & 0 & 0 & 0 & 0 & $1.31 \mathrm{E}+02$ \\
\hline
\end{tabular}


Table 39. Predicted Solids for Envelope C Simulations - Part 1 (cont'd)

\begin{tabular}{|c|c|c|c|c|c|c|c|c|c|c|c|c|c|c|c|c|}
\hline Test ID & $\begin{array}{l}\text { SBS/ } \\
\text { Feed }\end{array}$ & $\begin{array}{l}\text { Temp } \\
{\left[{ }^{\circ} \mathrm{C}\right]}\end{array}$ & $\left.\begin{array}{c}\mathrm{Na} \mathrm{M} \\
{[\mathrm{mol} / \mathrm{L}]}\end{array}\right]$ & ALOH3 & BASO4 & CA3PO42 & $\mathrm{CACO} 3$ & CAF2 & CANC & $\mathrm{CAOH} 2$ & CATIO3 & $\mathrm{CROH} 3$ & FEIIIOH3 & \begin{tabular}{|c|} 
HYDROS \\
OD \\
\end{tabular} & MGF2 & MGOH2 \\
\hline 10TC057 & 1.234 & 20.9766 & 10 & 0 & $1.66 \mathrm{E}+00$ & 0 & $1.48 \mathrm{E}+03$ & $9.43 \mathrm{E}+01$ & 0 & 0 & $1.78 \mathrm{E}+02$ & 0 & $1.34 \mathrm{E}+02$ & $1.17 \mathrm{E}+03$ & 0 & $2.30 \mathrm{E}+02$ \\
\hline $10 \mathrm{TC} 058$ & 1.859 & 30.1406 & 10 & 0 & $3.02 \mathrm{E}+00$ & $2.11 \mathrm{E}+02$ & $2.21 \mathrm{E}+03$ & 0 & 0 & 0 & $2.68 \mathrm{E}+02$ & $6.44 \mathrm{E}+00$ & $2.05 \mathrm{E}+02$ & $1.79 \mathrm{E}+03$ & 0 & $3.46 \mathrm{E}+02$ \\
\hline $10 \mathrm{TC} 059$ & 1.141 & 38.1094 & 10 & 0 & $1.42 \mathrm{E}+00$ & $6.97 \mathrm{E}+02$ & $7.99 \mathrm{E}+02$ & 0 & 0 & 0 & $1.64 \mathrm{E}+02$ & 0 & 0 & 0 & 0 & $2.11 \mathrm{E}+02$ \\
\hline $10 \mathrm{TC} 060$ & 1.109 & 21.375 & 10 & 0 & $1.31 \mathrm{E}+00$ & 0 & 0 & 0 & 0 & $1.07 \mathrm{E}+03$ & $1.60 \mathrm{E}+02$ & 0 & $3.14 \mathrm{E}+01$ & $2.74 \mathrm{E}+02$ & 0 & $2.07 \mathrm{E}+02$ \\
\hline 10TC061 & 1.625 & 22.9688 & 10 & 0 & $2.54 \mathrm{E}+00$ & 0 & $2.11 \mathrm{E}+03$ & 0 & 0 & 0 & $2.35 \mathrm{E}+02$ & 0 & $2.44 \mathrm{E}+02$ & $2.13 \mathrm{E}+03$ & 0 & $3.03 \mathrm{E}+02$ \\
\hline 10TC062 & 1.813 & 36.5156 & 10 & 0 & $2.97 \mathrm{E}+00$ & $5.11 \mathrm{E}+02$ & $1.87 \mathrm{E}+03$ & 0 & 0 & 0 & $2.62 \mathrm{E}+02$ & \begin{tabular}{|l|}
$8.70 \mathrm{E}+01$ \\
\end{tabular} & 0 & 0 & 0 & $3.38 \mathrm{E}+02$ \\
\hline $10 \mathrm{TC} 063$ & 1.609 & 31.7344 & 10 & 0 & $2.51 \mathrm{E}+00$ & $4.38 \mathrm{E}+02$ & $1.66 \mathrm{E}+03$ & 0 & 0 & 0 & $2.32 \mathrm{E}+02$ & 0 & 0 & 0 & 0 & $2.99 \mathrm{E}+02$ \\
\hline $10 \mathrm{TC} 065$ & 0.078 & 52.0547 & 10 & 0 & 0 & 0 & 0 & $7.89 \mathrm{E}+01$ & 0 & 0 & $1.13 \mathrm{E}+01$ & 0 & 0 & 0 & 0 & $1.46 \mathrm{E}+01$ \\
\hline 10TC066 & 0.781 & 60.4219 & 10 & 0 & $6.16 \mathrm{E}-01$ & 0 & 0 & $7.89 \mathrm{E}+02$ & 0 & 0 & $1.12 \mathrm{E}+02$ & \begin{tabular}{|l|}
$7.46 \mathrm{E}+01$ \\
\end{tabular} & 0 & 0 & 0 & $1.45 \mathrm{E}+02$ \\
\hline $10 \mathrm{TC} 067$ & 0.734 & 55.6406 & 10 & 0 & $4.87 \mathrm{E}-01$ & 0 & 0 & $7.45 \mathrm{E}+02$ & 0 & 0 & $1.06 \mathrm{E}+02$ & $4.75 \mathrm{E}+01$ & 0 & 0 & 0 & $1.37 \mathrm{E}+02$ \\
\hline 10TC068 & 0.297 & 60.8203 & 10 & 0 & 0 & 0 & 0 & \begin{tabular}{|l|}
$3.01 \mathrm{E}+02$ \\
\end{tabular} & 0 & 0 & $4.29 \mathrm{E}+01$ & $6.20 \mathrm{E}+00$ & 0 & 0 & 0 & $5.54 \mathrm{E}+01$ \\
\hline 10TC069 & 0.75 & 42.4922 & 10 & 0 & $5.81 \mathrm{E}-01$ & 0 & 0 & $7.61 \mathrm{E}+02$ & 0 & 0 & $1.08 \mathrm{E}+02$ & 0 & 0 & 0 & 0 & $1.40 \mathrm{E}+02$ \\
\hline $10 \mathrm{TC} 070$ & 0.531 & 44.8828 & 10 & 0 & $8.42 \mathrm{E}-02$ & 0 & 0 & \begin{tabular}{|l|}
$5.39 \mathrm{E}+02$ \\
\end{tabular} & 0 & 0 & $7.66 \mathrm{E}+01$ & 0 & 0 & 0 & 0 & $9.89 \mathrm{E}+01$ \\
\hline 10TC071 & 1.766 & 46.4766 & 10 & 0 & $2.93 \mathrm{E}+00$ & 0 & 0 & $1.79 \mathrm{E}+03$ & 0 & 0 & $2.54 \mathrm{E}+02$ & \begin{tabular}{|l|}
$1.84 \mathrm{E}+02$ \\
\end{tabular} & 0 & 0 & 0 & $3.28 \mathrm{E}+02$ \\
\hline $10 \mathrm{TC} 072$ & 1.406 & 62.4141 & 10 & 0 & $2.10 \mathrm{E}+00$ & 0 & 0 & $1.43 \mathrm{E}+03$ & 0 & 0 & $2.03 \mathrm{E}+02$ & $1.73 \mathrm{E}+02$ & 0 & 0 & $2.50 \mathrm{E}+02$ & $2.62 \mathrm{E}+02$ \\
\hline 10TC074 & 1.75 & 43.2891 & 10 & 0 & $2.86 \mathrm{E}+00$ & 0 & 0 & $1.78 \mathrm{E}+03$ & 0 & 0 & $2.53 \mathrm{E}+02$ & $1.40 \mathrm{E}+02$ & 0 & 0 & 0 & $3.26 \mathrm{E}+02$ \\
\hline $10 \mathrm{TC} 075$ & 1.688 & 56.4375 & 10 & 0 & $2.78 \mathrm{E}+00$ & 0 & 0 & $1.71 \mathrm{E}+03$ & 0 & 0 & $2.43 \mathrm{E}+02$ & $2.01 \mathrm{E}+02$ & 0 & 0 & 0 & $3.14 \mathrm{E}+02$ \\
\hline 10TC076 & 1.156 & 61.2188 & 10 & 0 & $1.55 \mathrm{E}+00$ & 0 & 0 & $1.18 \mathrm{E}+03$ & 0 & 0 & $1.67 \mathrm{E}+02$ & \begin{tabular}{|l|}
$1.38 \mathrm{E}+02$ \\
\end{tabular} & 0 & 0 & 0 & $2.16 \mathrm{E}+02$ \\
\hline $10 \mathrm{TC} 077$ & 1.531 & 61.6172 & 10 & 0 & $2.36 \mathrm{E}+00$ & 0 & 0 & $1.55 \mathrm{E}+03$ & 0 & 0 & $2.20 \mathrm{E}+02$ & $1.88 \mathrm{E}+02$ & 0 & 0 & $3.84 \mathrm{E}+02$ & $2.84 \mathrm{E}+02$ \\
\hline $10 \mathrm{TC} 078$ & 1 & 40.5 & 10 & 0 & $1.14 \mathrm{E}+00$ & 0 & 0 & $1.01 \mathrm{E}+03$ & 0 & 0 & $1.44 \mathrm{E}+02$ & 0 & 0 & 0 & 0 & $1.85 \mathrm{E}+02$ \\
\hline $10 \mathrm{TC} 079$ & 1.328 & 43.6875 & 10 & 0 & $1.87 \mathrm{E}+00$ & 0 & 0 & $1.33 \mathrm{E}+03$ & 0 & 0 & $1.89 \mathrm{E}+02$ & \begin{tabular}{|l|}
$6.85 \mathrm{E}+01$ \\
\end{tabular} & 0 & 0 & 0 & $2.44 \mathrm{E}+02$ \\
\hline 10TC080 & 1.656 & 62.8125 & 10 & 0 & $2.66 \mathrm{E}+00$ & 0 & 0 & $1.66 \mathrm{E}+03$ & 0 & 0 & $2.36 \mathrm{E}+02$ & \begin{tabular}{|l|}
$2.06 \mathrm{E}+02$ \\
\end{tabular} & 0 & 0 & 0 & $3.05 \mathrm{E}+02$ \\
\hline 10TC081 & 1.516 & 45.6797 & 10 & 0 & $2.31 \mathrm{E}+00$ & 0 & 0 & $1.52 \mathrm{E}+03$ & 0 & 0 & $2.17 \mathrm{E}+02$ & \begin{tabular}{|l|}
$1.32 \mathrm{E}+02$ \\
\end{tabular} & 0 & 0 & $6.73 \mathrm{E}+01$ & $2.80 \mathrm{E}+02$ \\
\hline 10TC082 & 1.281 & 63.6094 & 10 & 0 & $1.83 \mathrm{E}+00$ & 0 & 0 & $1.29 \mathrm{E}+03$ & 0 & 0 & $1.83 \mathrm{E}+02$ & \begin{tabular}{|l|}
$1.58 \mathrm{E}+02$ \\
\end{tabular} & 0 & 0 & $1.53 \mathrm{E}+02$ & $2.36 \mathrm{E}+02$ \\
\hline 10TC083 & 2 & 50.0625 & 10 & 0 & $3.39 \mathrm{E}+00$ & 0 & 0 & $1.99 \mathrm{E}+03$ & 0 & 0 & $2.83 \mathrm{E}+02$ & $2.25 \mathrm{E}+02$ & 0 & 0 & $8.54 \mathrm{E}+02$ & $3.65 \mathrm{E}+02$ \\
\hline $10 \mathrm{TC} 084$ & 1.359 & 59.2266 & 10 & 0 & $2.00 \mathrm{E}+00$ & 0 & 0 & $1.36 \mathrm{E}+03$ & 0 & 0 & $1.94 \mathrm{E}+02$ & \begin{tabular}{|l|}
$1.58 \mathrm{E}+02$ \\
\end{tabular} & 0 & 0 & $4.47 \mathrm{E}+01$ & $2.50 \mathrm{E}+02$ \\
\hline 10TC085 & 1.641 & 51.2578 & 10 & 0 & $2.64 \mathrm{E}+00$ & 0 & 0 & $1.66 \mathrm{E}+03$ & 0 & 0 & $2.36 \mathrm{E}+02$ & $1.83 \mathrm{E}+02$ & 0 & 0 & 0 & $3.05 \mathrm{E}+02$ \\
\hline $10 \mathrm{TC} 086$ & 0.109 & 51.6563 & 10 & 0 & 0 & 0 & 0 & $1.09 \mathrm{E}+02$ & 0 & 0 & $1.56 \mathrm{E}+01$ & 0 & 0 & 0 & 0 & $2.01 \mathrm{E}+01$ \\
\hline
\end{tabular}


Table 39. Predicted Solids for Envelope C Simulations - Part 1 (cont'd)

\begin{tabular}{|c|c|c|c|c|c|c|c|c|c|c|c|c|c|c|c|c|}
\hline Test ID & $\begin{array}{l}\text { SBS/ } \\
\text { Feed }\end{array}$ & $\begin{array}{l}\text { Temp } \\
{\left[{ }^{\circ} \mathrm{C}\right]}\end{array}$ & $\left.\begin{array}{c}\mathrm{Na} \mathrm{M} \\
{[\mathrm{mol} / \mathrm{L}]}\end{array}\right]$ & ALOH3 & BASO4 & CA3PO42 & $\mathrm{CACO} 3$ & CAF2 & CANC & $\mathrm{CAOH} 2$ & CATIO3 & $\mathrm{CROH} 3$ & FEIIIOH3 & \begin{tabular}{|c|} 
HYDROS \\
OD \\
\end{tabular} & MGF2 & MGOH2 \\
\hline 10TC087 & 0.953 & 64.8047 & 10 & 0 & $9.96 \mathrm{E}-01$ & 0 & 0 & $9.53 \mathrm{E}+02$ & 0 & 0 & $1.36 \mathrm{E}+02$ & \begin{tabular}{|l|}
$1.10 \mathrm{E}+02$ \\
\end{tabular} & 0 & 0 & 0 & $1.75 \mathrm{E}+02$ \\
\hline $10 \mathrm{TC} 088$ & 0.438 & 64.4063 & 10 & 0 & 0 & 0 & 0 & $4.41 \mathrm{E}+02$ & 0 & 0 & $6.28 \mathrm{E}+01$ & $3.87 \mathrm{E}+01$ & 0 & 0 & 0 & $8.11 \mathrm{E}+01$ \\
\hline $10 \mathrm{TC} 089$ & 0.422 & 58.8281 & 10 & 0 & 0 & 0 & 0 & \begin{tabular}{|l|}
$4.24 \mathrm{E}+02$ \\
\end{tabular} & 0 & 0 & $6.03 \mathrm{E}+01$ & $1.61 \mathrm{E}+01$ & 0 & 0 & 0 & 7.79E +01 \\
\hline $10 \mathrm{TC} 090$ & 0.453 & 45.2813 & 10 & 0 & 0 & 0 & 0 & $4.53 \mathrm{E}+02$ & 0 & 0 & $6.45 \mathrm{E}+01$ & 0 & 0 & 0 & 0 & $8.32 \mathrm{E}+01$ \\
\hline 10TC091 & 0.688 & 50.4609 & 10 & 0 & $4.39 \mathrm{E}-01$ & 0 & 0 & $6.90 \mathrm{E}+02$ & 0 & 0 & $9.81 \mathrm{E}+01$ & \begin{tabular}{|l|}
$2.59 \mathrm{E}+01$ \\
\end{tabular} & 0 & 0 & 0 & $1.27 \mathrm{E}+02$ \\
\hline 10TC092 & 0.328 & 65.6016 & 10 & 0 & 0 & 0 & 0 & \begin{tabular}{|l|}
$3.29 \mathrm{E}+02$ \\
\end{tabular} & 0 & 0 & $4.69 \mathrm{E}+01$ & \begin{tabular}{|l|}
$2.33 \mathrm{E}+01$ \\
\end{tabular} & 0 & 0 & 0 & $6.06 \mathrm{E}+01$ \\
\hline $10 \mathrm{TC} 093$ & 1.125 & 32.1328 & 10 & 0 & $1.33 \mathrm{E}+00$ & $2.89 \mathrm{E}+02$ & $1.15 \mathrm{E}+03$ & 0 & 0 & 0 & $1.59 \mathrm{E}+02$ & 0 & 0 & 0 & 0 & $2.06 \mathrm{E}+02$ \\
\hline $10 \mathrm{TC} 095$ & 1.203 & 27.3516 & 10 & 0 & $1.57 \mathrm{E}+00$ & 0 & $1.56 \mathrm{E}+03$ & 0 & 0 & 0 & $1.73 \mathrm{E}+02$ & 0 & 0 & 0 & 0 & $2.23 \mathrm{E}+02$ \\
\hline 10TC096 & 1.906 & 33.3281 & 10 & 0 & $3.07 \mathrm{E}+00$ & $4.81 \mathrm{E}+02$ & $1.99 \mathrm{E}+03$ & 0 & 0 & 0 & $2.72 \mathrm{E}+02$ & $1.24 \mathrm{E}+02$ & $2.67 \mathrm{E}+02$ & $2.33 \mathrm{E}+03$ & 0 & $3.52 \mathrm{E}+02$ \\
\hline $10 \mathrm{TC} 097$ & 1.375 & 15 & 10 & 0 & 0 & 0 & 0 & $1.38 \mathrm{E}+03$ & 0 & 0 & $1.96 \mathrm{E}+02$ & 0 & $2.37 \mathrm{E}+02$ & $2.07 \mathrm{E}+03$ & 0 & $2.53 \mathrm{E}+02$ \\
\hline 10TC098 & 1.734 & 31.3359 & 10 & 0 & $2.69 \mathrm{E}+00$ & $4.44 \mathrm{E}+02$ & $1.78 \mathrm{E}+03$ & 0 & 0 & 0 & $2.45 \mathrm{E}+02$ & 0 & $9.01 \mathrm{E}+01$ & \begin{tabular}{|l|}
$7.86 \mathrm{E}+02$ \\
\end{tabular} & 0 & $3.17 \mathrm{E}+02$ \\
\hline 10TC099 & 1.063 & 27.75 & 10 & 0 & $1.19 \mathrm{E}+00$ & $1.14 \mathrm{E}+02$ & $1.08 \mathrm{E}+03$ & \begin{tabular}{|l|}
$1.39 \mathrm{E}+02$ \\
\end{tabular} & 0 & 0 & $1.52 \mathrm{E}+02$ & 0 & 0 & 0 & 0 & $1.96 \mathrm{E}+02$ \\
\hline $10 \mathrm{TC} 100$ & 0.563 & 17.7891 & 10 & 0 & 0 & 0 & 0 & $5.68 \mathrm{E}+02$ & 0 & 0 & $8.07 \mathrm{E}+01$ & 0 & 0 & 0 & 0 & $1.04 \mathrm{E}+02$ \\
\hline 10TC101 & 0.047 & 39.3047 & 10 & 0 & 0 & 0 & 0 & $4.55 \mathrm{E}+01$ & 0 & 0 & $6.68 \mathrm{E}+00$ & 0 & 0 & 0 & 0 & $8.63 \mathrm{E}+00$ \\
\hline $10 \mathrm{TC} 102$ & 0.063 & 26.1563 & 10 & 0 & 0 & 0 & 0 & $6.19 \mathrm{E}+01$ & 0 & 0 & $8.91 \mathrm{E}+00$ & 0 & 0 & 0 & 0 & $1.15 \mathrm{E}+01$ \\
\hline 10TC104 & 0.281 & 25.7578 & 10 & 0 & 0 & 0 & 0 & $2.80 \mathrm{E}+02$ & 0 & 0 & $3.99 \mathrm{E}+01$ & 0 & 0 & 0 & 0 & $5.15 \mathrm{E}+01$ \\
\hline $10 \mathrm{TC} 105$ & 0.813 & 26.5547 & 10 & 0 & $5.14 \mathrm{E}-01$ & 0 & 0 & $1.27 \mathrm{E}+02$ & 0 & $6.51 \mathrm{E}+02$ & $1.16 \mathrm{E}+02$ & 0 & 0 & 0 & 0 & $1.50 \mathrm{E}+02$ \\
\hline 10TC106 & 0.609 & 32.5313 & 10 & 0 & $1.15 \mathrm{E}-01$ & $2.15 \mathrm{E}+02$ & $1.35 \mathrm{E}+02$ & \begin{tabular}{|l|}
$3.39 \mathrm{E}+02$ \\
\end{tabular} & 0 & 0 & $8.66 \mathrm{E}+01$ & 0 & 0 & 0 & 0 & $1.12 \mathrm{E}+02$ \\
\hline $10 \mathrm{TC} 107$ & 0.016 & 23.3672 & 10 & 0 & 0 & 0 & 0 & $1.51 \mathrm{E}+01$ & 0 & 0 & $2.25 \mathrm{E}+00$ & 0 & 0 & 0 & 0 & $2.90 \mathrm{E}+00$ \\
\hline 10TC108 & 1.844 & 40.8984 & 10 & 0 & $2.99 \mathrm{E}+00$ & 0 & 0 & \begin{tabular}{|l|}
$1.84 \mathrm{E}+03$ \\
\end{tabular} & 0 & 0 & $2.62 \mathrm{E}+02$ & \begin{tabular}{|l|}
$1.42 \mathrm{E}+02$ \\
\end{tabular} & 0 & 0 & $3.60 \mathrm{E}+02$ & $3.38 \mathrm{E}+02$ \\
\hline 10TC109 & 1.453 & 64.0078 & 10 & 0 & $2.17 \mathrm{E}+00$ & 0 & 0 & $1.45 \mathrm{E}+03$ & 0 & 0 & $2.06 \mathrm{E}+02$ & \begin{tabular}{|l|}
$1.78 \mathrm{E}+02$ \\
\end{tabular} & 0 & 0 & $2.04 \mathrm{E}+02$ & $2.66 \mathrm{E}+02$ \\
\hline $10 \mathrm{TC} 110$ & 1.781 & 46.0781 & 10 & 0 & $2.91 \mathrm{E}+00$ & 0 & 0 & $1.79 \mathrm{E}+03$ & 0 & 0 & $2.54 \mathrm{E}+02$ & \begin{tabular}{|l|}
$1.88 \mathrm{E}+02$ \\
\end{tabular} & 0 & 0 & 0 & $3.28 \mathrm{E}+02$ \\
\hline 10TC111 & 1.797 & 58.4297 & 10 & 0 & $3.00 \mathrm{E}+00$ & 0 & 0 & $1.81 \mathrm{E}+03$ & 0 & 0 & $2.58 \mathrm{E}+02$ & $2.20 \mathrm{E}+02$ & 0 & 0 & $7.18 \mathrm{E}+02$ & $3.32 \mathrm{E}+02$ \\
\hline 10TC112 & 1.078 & 46.875 & 10 & 0 & $1.30 \mathrm{E}+00$ & 0 & 0 & $1.08 \mathrm{E}+03$ & 0 & 0 & $1.54 \mathrm{E}+02$ & \begin{tabular}{|l|}
$4.41 \mathrm{E}+01$ \\
\end{tabular} & 0 & 0 & 0 & $1.99 \mathrm{E}+02$ \\
\hline 10TC113 & 1.172 & 52.4531 & 10 & 0 & $1.55 \mathrm{E}+00$ & 0 & 0 & $1.17 \mathrm{E}+03$ & 0 & 0 & $1.67 \mathrm{E}+02$ & $1.11 \mathrm{E}+02$ & 0 & 0 & 0 & $2.15 \mathrm{E}+02$ \\
\hline $10 \mathrm{TC} 114$ & 1.297 & 41.2969 & 10 & 0 & $1.82 \mathrm{E}+00$ & 0 & 0 & $1.30 \mathrm{E}+03$ & 0 & 0 & $1.86 \mathrm{E}+02$ & \begin{tabular}{|l|}
$4.60 \mathrm{E}+01$ \\
\end{tabular} & 0 & 0 & 0 & $2.40 \mathrm{E}+02$ \\
\hline $10 \mathrm{TC} 115$ & 0.766 & 60.0234 & 10 & 0 & $5.41 \mathrm{E}-01$ & 0 & 0 & $7.69 \mathrm{E}+02$ & 0 & 0 & $1.09 \mathrm{E}+02$ & $6.96 \mathrm{E}+01$ & 0 & 0 & 0 & $1.41 \mathrm{E}+02$ \\
\hline $10 \mathrm{TC} 116$ & 0.141 & 50.8594 & 10 & 0 & 0 & 0 & 0 & $1.41 \mathrm{E}+02$ & 0 & 0 & $2.01 \mathrm{E}+01$ & 0 & 0 & 0 & 0 & $2.60 \mathrm{E}+01$ \\
\hline
\end{tabular}


Table 39. Predicted Solids for Envelope C Simulations - Part 1 (cont'd)

\begin{tabular}{|c|c|c|c|c|c|c|c|c|c|c|c|c|c|c|c|c|}
\hline Test ID & $\begin{array}{l}\text { SBS/ } \\
\text { Feed }\end{array}$ & $\begin{array}{l}\text { Temp } \\
{\left[{ }^{\circ} \mathrm{C}\right]}\end{array}$ & $\begin{array}{c}\mathrm{NaM} \\
{[\mathrm{mol} / \mathrm{L}]}\end{array}$ & ALOH3 & BASO4 & CA3PO42 & $\mathrm{CACO} 3$ & CAF2 & CANC & $\mathrm{CAOH} 2$ & CATIO3 & $\mathrm{CROH} 3$ & FEIIIOH3 & \begin{tabular}{|c|} 
HYDROS \\
OD
\end{tabular} & MGF2 & MGOH2 \\
\hline 10TC117 & 0.859 & 42.8906 & 10 & 0 & $8.24 \mathrm{E}-01$ & 0 & 0 & $8.69 \mathrm{E}+02$ & 0 & 0 & $1.24 \mathrm{E}+02$ & 0 & 0 & 0 & 0 & $1.60 \mathrm{E}+02$ \\
\hline $10 \mathrm{TC} 118$ & 0.891 & 59.625 & 10 & 0 & $9.01 \mathrm{E}-01$ & 0 & 0 & $8.92 \mathrm{E}+02$ & 0 & 0 & $1.27 \mathrm{E}+02$ & $9.27 \mathrm{E}+01$ & 0 & 0 & 0 & $1.64 \mathrm{E}+02$ \\
\hline 10TC119 & 0.375 & 58.0313 & 10 & 0 & 0 & 0 & 0 & $3.75 \mathrm{E}+02$ & 0 & 0 & $5.33 \mathrm{E}+01$ & $2.75 \mathrm{E}+00$ & 0 & 0 & 0 & $6.89 \mathrm{E}+01$ \\
\hline $10 \mathrm{TC} 120$ & 0.188 & 44.4844 & 10 & 0 & 0 & 0 & 0 & $1.87 \mathrm{E}+02$ & 0 & 0 & $2.66 \mathrm{E}+01$ & 0 & 0 & 0 & 0 & $3.43 \mathrm{E}+01$ \\
\hline 10TC121 & 0.391 & 49.2656 & 10 & 0 & 0 & 0 & 0 & $3.94 \mathrm{E}+02$ & 0 & 0 & $5.60 \mathrm{E}+01$ & 0 & 0 & 0 & 0 & $7.23 \mathrm{E}+01$ \\
\hline $10 \mathrm{TC} 122$ & 1.016 & 18.9844 & 10 & 0 & 0 & 0 & 0 & $1.02 \mathrm{E}+03$ & 0 & 0 & $1.45 \mathrm{E}+02$ & 0 & \begin{tabular}{|l|}
$4.16 \mathrm{E}+01$ \\
\end{tabular} & $3.63 \mathrm{E}+02$ & 0 & $1.87 \mathrm{E}+02$ \\
\hline $10 \mathrm{TC} 123$ & 1.922 & 28.9453 & 10 & 0 & $3.07 \mathrm{E}+00$ & $2.83 \mathrm{E}+02$ & $2.20 \mathrm{E}+03$ & 0 & 0 & 0 & $2.75 \mathrm{E}+02$ & 0 & $2.41 \mathrm{E}+02$ & $2.11 \mathrm{E}+03$ & 0 & $3.54 \mathrm{E}+02$ \\
\hline $10 \mathrm{TC} 125$ & 1.266 & 25.3594 & 10 & 0 & $1.70 \mathrm{E}+00$ & 0 & $1.62 \mathrm{E}+03$ & 0 & 0 & 0 & $1.81 \mathrm{E}+02$ & 0 & $7.75 \mathrm{E}+01$ & $6.76 \mathrm{E}+02$ & 0 & $2.33 \mathrm{E}+02$ \\
\hline $10 \mathrm{TC} 126$ & 1.703 & 20.1797 & 10 & 0 & $2.68 \mathrm{E}+00$ & 0 & $2.17 \mathrm{E}+03$ & 0 & 0 & 0 & $2.42 \mathrm{E}+02$ & 0 & $2.81 \mathrm{E}+02$ & $2.45 \mathrm{E}+03$ & 0 & $3.12 \mathrm{E}+02$ \\
\hline $10 \mathrm{TC} 127$ & 1.25 & 38.5078 & 10 & 0 & $1.70 \mathrm{E}+00$ & $7.36 \mathrm{E}+02$ & $8.98 \mathrm{E}+02$ & 0 & 0 & 0 & $1.79 \mathrm{E}+02$ & 0 & 0 & 0 & 0 & $2.31 \mathrm{E}+02$ \\
\hline $10 \mathrm{TC} 128$ & 1.469 & 36.1172 & 10 & 0 & $2.16 \mathrm{E}+00$ & $4.63 \mathrm{E}+02$ & $1.44 \mathrm{E}+03$ & 0 & 0 & 0 & $2.10 \mathrm{E}+02$ & $1.87 \mathrm{E}+01$ & 0 & 0 & 0 & $2.71 \mathrm{E}+02$ \\
\hline 10TC129 & 0.234 & 34.5234 & 10 & 0 & 0 & 0 & 0 & $2.09 \mathrm{E}+02$ & 0 & \begin{tabular}{|l|}
$2.43 \mathrm{E}+01$ \\
\end{tabular} & $3.36 \mathrm{E}+01$ & 0 & 0 & 0 & 0 & $4.34 \mathrm{E}+01$ \\
\hline $10 \mathrm{TC} 130$ & 0.594 & 18.5859 & 10 & 0 & 0 & 0 & 0 & $5.96 \mathrm{E}+02$ & 0 & 0 & $8.47 \mathrm{E}+01$ & 0 & 0 & 0 & 0 & $1.09 \mathrm{E}+02$ \\
\hline 10TC131 & 0.906 & 36.9141 & 10 & 0 & $8.54 \mathrm{E}-01$ & $4.26 \mathrm{E}+02$ & $7.58 \mathrm{E}+02$ & 0 & 0 & 0 & $1.30 \mathrm{E}+02$ & 0 & 0 & 0 & 0 & $1.68 \mathrm{E}+02$ \\
\hline $10 \mathrm{TC} 132$ & 0.25 & 37.7109 & 10 & 0 & 0 & 0 & 0 & $2.48 \mathrm{E}+02$ & 0 & 0 & $3.56 \mathrm{E}+01$ & 0 & 0 & 0 & 0 & $4.59 \mathrm{E}+01$ \\
\hline $10 \mathrm{TC} 134$ & 0.844 & 19.7813 & 10 & 0 & 0 & 0 & 0 & $8.47 \mathrm{E}+02$ & 0 & 0 & $1.20 \mathrm{E}+02$ & 0 & 0 & 0 & 0 & $1.55 \mathrm{E}+02$ \\
\hline $10 \mathrm{TC} 135$ & 0.469 & 19.3828 & 10 & 0 & 0 & 0 & 0 & $4.73 \mathrm{E}+02$ & 0 & 0 & $6.73 \mathrm{E}+01$ & 0 & 0 & 0 & 0 & $8.69 \mathrm{E}+01$ \\
\hline $8 \mathrm{TC} 001$ & 0 & 15 & 8 & 0 & 0 & 0 & 0 & 0 & 0 & 0 & 0 & 0 & 0 & 0 & 0 & 0 \\
\hline $8 \mathrm{TC} 002$ & 0 & 15 & 8 & 0 & 0 & 0 & 0 & 0 & 0 & 0 & 0 & 0 & 0 & 0 & 0 & 0 \\
\hline $8 \mathrm{TC} 003$ & 0 & 15 & 8 & 0 & 0 & 0 & 0 & 0 & 0 & 0 & 0 & 0 & 0 & 0 & 0 & 0 \\
\hline $8 \mathrm{TC} 004$ & 0 & 15 & 8 & 0 & 0 & 0 & 0 & 0 & 0 & 0 & 0 & 0 & 0 & 0 & 0 & 0 \\
\hline $8 \mathrm{TC} 005$ & 0 & 15 & 8 & 0 & 0 & 0 & 0 & 0 & 0 & 0 & 0 & 0 & 0 & 0 & 0 & 0 \\
\hline $8 \mathrm{TC} 006$ & 0 & 66 & 8 & 0 & 0 & 0 & 0 & 0 & 0 & 0 & 0 & 0 & 0 & 0 & 0 & 0 \\
\hline $8 \mathrm{TC} 007$ & 0 & 66 & 8 & 0 & 0 & 0 & 0 & 0 & 0 & 0 & 0 & 0 & 0 & 0 & 0 & 0 \\
\hline 8TC008 & 0 & 66 & 8 & 0 & 0 & 0 & 0 & 0 & 0 & 0 & 0 & 0 & 0 & 0 & 0 & 0 \\
\hline 8TC009 & 0 & 66 & 8 & 0 & 0 & 0 & 0 & 0 & 0 & 0 & 0 & 0 & 0 & 0 & 0 & 0 \\
\hline $8 \mathrm{TC} 010$ & 0 & 66 & 8 & 0 & 0 & 0 & 0 & 0 & 0 & 0 & 0 & 0 & 0 & 0 & 0 & 0 \\
\hline $8 \mathrm{TC} 011$ & 2 & 15 & 8 & $1.51 \mathrm{E}+03$ & $1.05 \mathrm{E}+00$ & 0 & 0 & $2.04 \mathrm{E}+03$ & 0 & 0 & $2.89 \mathrm{E}+02$ & 0 & $5.12 \mathrm{E}+02$ & $4.47 \mathrm{E}+03$ & 0 & $3.74 \mathrm{E}+02$ \\
\hline
\end{tabular}


Table 39. Predicted Solids for Envelope C Simulations - Part 1 (cont'd)

\begin{tabular}{|c|c|c|c|c|c|c|c|c|c|c|c|c|c|c|c|c|}
\hline Test ID & $\begin{array}{l}\text { SBS/ } \\
\text { Feed }\end{array}$ & $\begin{array}{l}\text { Temp } \\
{\left[{ }^{\circ} \mathrm{C}\right]}\end{array}$ & $\left.\begin{array}{c}\mathrm{Na} \mathrm{M} \\
{[\mathrm{mol} / \mathrm{L}]}\end{array}\right]$ & ALOH3 & BASO4 & CA3PO42 & $\mathrm{CACO} 3$ & CAF2 & CANC & $\mathrm{CAOH} 2$ & CATIO3 & $\mathrm{CROH} 3$ & FEIIIOH3 & \begin{tabular}{|c|} 
HYDROS \\
OD \\
\end{tabular} & MGF2 & MGOH2 \\
\hline $8 \mathrm{TC} 012$ & 2 & 15 & 8 & $1.16 \mathrm{E}+03$ & $8.13 \mathrm{E}-01$ & 0 & 0 & $2.03 \mathrm{E}+03$ & 0 & 0 & $2.88 \mathrm{E}+02$ & 0 & $4.87 \mathrm{E}+02$ & $4.25 \mathrm{E}+03$ & 0 & \begin{tabular}{|l|}
$3.72 \mathrm{E}+02$ \\
\end{tabular} \\
\hline $8 \mathrm{TC} 013$ & 2 & 15 & 8 & 0 & $8.57 \mathrm{E}-01$ & 0 & 0 & $2.01 \mathrm{E}+03$ & 0 & 0 & $2.86 \mathrm{E}+02$ & 0 & $5.28 \mathrm{E}+02$ & $4.61 \mathrm{E}+03$ & 0 & $3.70 \mathrm{E}+02$ \\
\hline $8 \mathrm{TC} 014$ & 2 & 15 & 8 & 0 & $1.20 \mathrm{E}+00$ & 0 & 0 & $1.99 \mathrm{E}+03$ & 0 & 0 & $2.83 \mathrm{E}+02$ & 0 & $5.03 \mathrm{E}+02$ & $4.39 \mathrm{E}+03$ & 0 & $3.65 \mathrm{E}+02$ \\
\hline $8 \mathrm{TC} 015$ & 2 & 15 & 8 & 0 & $3.62 \mathrm{E}-01$ & 0 & 0 & $2.01 \mathrm{E}+03$ & 0 & 0 & $2.85 \mathrm{E}+02$ & $5.80 \mathrm{E}+01$ & $5.40 \mathrm{E}+02$ & $4.71 \mathrm{E}+03$ & $1.01 \mathrm{E}+02$ & $3.68 \mathrm{E}+02$ \\
\hline $8 \mathrm{TC} 016$ & 2 & 66 & 8 & 0 & $3.44 \mathrm{E}+00$ & 0 & 0 & $2.03 \mathrm{E}+03$ & 0 & 0 & $2.89 \mathrm{E}+02$ & $2.68 \mathrm{E}+02$ & 0 & 0 & $2.48 \mathrm{E}+02$ & $3.74 \mathrm{E}+02$ \\
\hline $8 \mathrm{TC} 017$ & 2 & 66 & 8 & 0 & $3.48 \mathrm{E}+00$ & 0 & 0 & $2.03 \mathrm{E}+03$ & 0 & 0 & $2.88 \mathrm{E}+02$ & $2.65 \mathrm{E}+02$ & 0 & 0 & $7.29 \mathrm{E}+01$ & \begin{tabular}{|l|}
$3.72 \mathrm{E}+02$ \\
\end{tabular} \\
\hline $8 \mathrm{TC} 018$ & 2 & 66 & 8 & 0 & $3.37 \mathrm{E}+00$ & 0 & 0 & $2.01 \mathrm{E}+03$ & 0 & 0 & $2.86 \mathrm{E}+02$ & $2.67 \mathrm{E}+02$ & 0 & 0 & $3.15 \mathrm{E}+02$ & $3.70 \mathrm{E}+02$ \\
\hline $8 \mathrm{TC} 020$ & 2 & 66 & 8 & 0 & $3.32 \mathrm{E}+00$ & 0 & 0 & $2.01 \mathrm{E}+03$ & 0 & 0 & $2.85 \mathrm{E}+02$ & $2.68 \mathrm{E}+02$ & 0 & 0 & $4.00 \mathrm{E}+02$ & $3.68 \mathrm{E}+02$ \\
\hline $8 \mathrm{TC} 021$ & 0.672 & 37.3125 & 8 & 0 & 0 & 0 & 0 & $6.81 \mathrm{E}+02$ & 0 & 0 & $9.70 \mathrm{E}+01$ & 0 & 0 & 0 & 0 & $1.25 \mathrm{E}+02$ \\
\hline $8 \mathrm{TC} 022$ & 0.344 & 18.1875 & 8 & 0 & 0 & 0 & 0 & $3.50 \mathrm{E}+02$ & 0 & 0 & $4.97 \mathrm{E}+01$ & 0 & 0 & 0 & 0 & $6.42 \mathrm{E}+01$ \\
\hline $8 \mathrm{TC} 023$ & 0.484 & 35.3203 & 8 & 0 & 0 & 0 & 0 & \begin{tabular}{|l|}
$4.90 \mathrm{E}+02$ \\
\end{tabular} & 0 & 0 & $6.98 \mathrm{E}+01$ & 0 & 0 & 0 & 0 & $9.02 \mathrm{E}+01$ \\
\hline 8TC024 & 0.719 & 17.3906 & 8 & 0 & 0 & 0 & 0 & \begin{tabular}{|l|}
$7.30 \mathrm{E}+02$ \\
\end{tabular} & 0 & 0 & $1.04 \mathrm{E}+02$ & 0 & $5.47 \mathrm{E}+01$ & $4.77 \mathrm{E}+02$ & 0 & $1.34 \mathrm{E}+02$ \\
\hline $8 \mathrm{TC} 025$ & 0 & 30.9375 & 8 & 0 & 0 & 0 & 0 & 0 & 0 & 0 & 0 & 0 & 0 & \begin{tabular}{|l|}
0 \\
\end{tabular} & 0 & \begin{tabular}{|l|}
0 \\
\end{tabular} \\
\hline 8TC026 & 0.641 & 21.7734 & 8 & 0 & $1.85 \mathrm{E}-01$ & 0 & 0 & $6.51 \mathrm{E}+02$ & 0 & 0 & $9.27 \mathrm{E}+01$ & 0 & 0 & 0 & 0 & $1.20 \mathrm{E}+02$ \\
\hline $8 \mathrm{TC} 027$ & 0.359 & 29.7422 & 8 & 0 & 0 & 0 & 0 & $3.62 \mathrm{E}+02$ & 0 & 0 & $5.15 \mathrm{E}+01$ & 0 & 0 & 0 & 0 & $6.65 \mathrm{E}+01$ \\
\hline 8TC029 & 1.047 & 16.1953 & 8 & 0 & $3.66 \mathrm{E}-01$ & 0 & 0 & $1.06 \mathrm{E}+03$ & 0 & 0 & $1.51 \mathrm{E}+02$ & 0 & $1.91 \mathrm{E}+02$ & $1.66 \mathrm{E}+03$ & 0 & \begin{tabular}{|l|}
$1.95 \mathrm{E}+02$ \\
\end{tabular} \\
\hline $8 \mathrm{TC} 030$ & 1.563 & 16.5938 & 8 & 0 & 7.47E-01 & 0 & 0 & $1.58 \mathrm{E}+03$ & 0 & 0 & $2.25 \mathrm{E}+02$ & 0 & $3.46 \mathrm{E}+02$ & \begin{tabular}{|l|}
$3.02 \mathrm{E}+03$ \\
\end{tabular} & 0 & $2.90 \mathrm{E}+02$ \\
\hline $8 \mathrm{TC} 031$ & 1.578 & 22.1719 & 8 & 0 & $1.88 \mathrm{E}+00$ & 0 & 0 & $1.60 \mathrm{E}+03$ & 0 & 0 & $2.28 \mathrm{E}+02$ & 0 & $3.28 \mathrm{E}+02$ & $2.86 \mathrm{E}+03$ & 0 & \begin{tabular}{|l|}
$2.94 \mathrm{E}+02$ \\
\end{tabular} \\
\hline $8 \mathrm{TC} 032$ & 1.547 & 35.7188 & 8 & 0 & $1.47 \mathrm{E}+00$ & $8.24 \mathrm{E}+01$ & $1.18 \mathrm{E}+02$ & $1.42 \mathrm{E}+03$ & 0 & 0 & $2.24 \mathrm{E}+02$ & $1.05 \mathrm{E}+02$ & $1.36 \mathrm{E}+02$ & $1.19 \mathrm{E}+03$ & 0 & $2.89 \mathrm{E}+02$ \\
\hline $8 \mathrm{TC} 033$ & 1.313 & 30.5391 & 8 & 0 & $1.28 \mathrm{E}+00$ & 0 & 0 & $1.33 \mathrm{E}+03$ & 0 & 0 & $1.89 \mathrm{E}+02$ & 0 & $7.02 \mathrm{E}+01$ & \begin{tabular}{|l|}
$6.12 \mathrm{E}+02$ \\
\end{tabular} & 0 & $2.45 \mathrm{E}+02$ \\
\hline $8 \mathrm{TC} 034$ & 1.672 & 15.3984 & 8 & $5.85 \mathrm{E}+01$ & 7.34E-01 & 0 & 0 & $1.70 \mathrm{E}+03$ & 0 & 0 & $2.41 \mathrm{E}+02$ & 0 & $4.01 \mathrm{E}+02$ & $3.49 \mathrm{E}+03$ & 0 & $3.11 \mathrm{E}+02$ \\
\hline $8 \mathrm{TC} 035$ & 0.875 & 48.8672 & 8 & 0 & 0 & 0 & 0 & $8.89 \mathrm{E}+02$ & 0 & 0 & $1.26 \mathrm{E}+02$ & $6.67 \mathrm{E}+01$ & 0 & 0 & 0 & $1.63 \mathrm{E}+02$ \\
\hline $8 \mathrm{TC} 036$ & 0.125 & 57.2344 & 8 & 0 & 0 & 0 & 0 & $1.27 \mathrm{E}+02$ & 0 & 0 & $1.81 \mathrm{E}+01$ & 0 & 0 & 0 & 0 & $2.33 \mathrm{E}+01$ \\
\hline $8 \mathrm{TC} 037$ & 0.797 & 53.6484 & 8 & 0 & $3.84 \mathrm{E}-02$ & 0 & 0 & $8.05 \mathrm{E}+02$ & 0 & 0 & $1.14 \mathrm{E}+02$ & $7.15 \mathrm{E}+01$ & 0 & 0 & 0 & $1.48 \mathrm{E}+02$ \\
\hline $8 \mathrm{TC} 038$ & 0.094 & 47.6719 & 8 & 0 & 0 & 0 & 0 & $9.48 \mathrm{E}+01$ & 0 & 0 & $1.35 \mathrm{E}+01$ & 0 & 0 & 0 & 0 & $1.75 \mathrm{E}+01$ \\
\hline $8 \mathrm{TC} 039$ & 0.625 & 66 & 8 & 0 & $5.55 \mathrm{E}-02$ & 0 & 0 & $6.34 \mathrm{E}+02$ & 0 & 0 & $9.02 \mathrm{E}+01$ & $7.37 \mathrm{E}+01$ & 0 & 0 & 0 & $1.16 \mathrm{E}+02$ \\
\hline $8 \mathrm{TC} 040$ & 0.266 & 49.6641 & 8 & 0 & 0 & 0 & 0 & $2.70 \mathrm{E}+02$ & 0 & 0 & $3.84 \mathrm{E}+01$ & 0 & 0 & 0 & 0 & $4.96 \mathrm{E}+01$ \\
\hline $8 \mathrm{TC} 041$ & 0.938 & 53.25 & 8 & 0 & 4.02E-01 & 0 & 0 & $9.53 \mathrm{E}+02$ & 0 & 0 & $1.35 \mathrm{E}+02$ & $9.20 \mathrm{E}+01$ & 0 & 0 & 0 & $1.75 \mathrm{E}+02$ \\
\hline
\end{tabular}


Table 39. Predicted Solids for Envelope C Simulations - Part 1 (cont'd)

\begin{tabular}{|c|c|c|c|c|c|c|c|c|c|c|c|c|c|c|c|c|}
\hline Test ID & $\begin{array}{l}\text { SBS/ } \\
\text { Feed }\end{array}$ & $\begin{array}{l}\text { Temp } \\
{\left[{ }^{\circ} \mathrm{C}\right]} \\
\end{array}$ & $\left.\begin{array}{c}\mathrm{Na} \mathrm{M} \\
{[\mathrm{mol} / \mathrm{L}]}\end{array}\right]$ & ALOH3 & BASO4 & CA3PO42 & $\mathrm{CACO} 3$ & CAF2 & CANC & $\mathrm{CAOH} 2$ & CATIO3 & $\mathrm{CROH} 3$ & FEIIIOH3 & \begin{tabular}{|c|} 
HYDROS \\
OD \\
\end{tabular} & MGF2 & MGOH2 \\
\hline $8 \mathrm{TC} 042$ & 1.438 & 63.2109 & 8 & 0 & $1.95 \mathrm{E}+00$ & 0 & 0 & $1.45 \mathrm{E}+03$ & 0 & 0 & $2.07 \mathrm{E}+02$ & $1.86 \mathrm{E}+02$ & 0 & 0 & 0 & $2.67 \mathrm{E}+02$ \\
\hline $8 \mathrm{TC} 043$ & 1.953 & 41.6953 & 8 & 0 & $1.19 \mathrm{E}+00$ & 0 & 0 & $1.99 \mathrm{E}+03$ & 0 & 0 & $2.82 \mathrm{E}+02$ & $2.18 \mathrm{E}+02$ & $2.36 \mathrm{E}+02$ & $2.06 \mathrm{E}+03$ & $4.99 \mathrm{E}+01$ & $3.64 \mathrm{E}+02$ \\
\hline $8 \mathrm{TC} 044$ & 1.938 & 54.8438 & 8 & 0 & $2.86 \mathrm{E}+00$ & 0 & 0 & $1.97 \mathrm{E}+03$ & 0 & 0 & $2.79 \mathrm{E}+02$ & $2.43 \mathrm{E}+02$ & 0 & 0 & 0 & \begin{tabular}{|l|}
$3.61 \mathrm{E}+02$ \\
\end{tabular} \\
\hline $8 \mathrm{TC} 045$ & 1.484 & 65.2031 & 8 & 0 & $2.14 \mathrm{E}+00$ & 0 & 0 & $1.51 \mathrm{E}+03$ & 0 & 0 & $2.14 \mathrm{E}+02$ & $1.94 \mathrm{E}+02$ & 0 & 0 & 0 & $2.77 \mathrm{E}+02$ \\
\hline $8 \mathrm{TC} 046$ & 1.719 & 55.2422 & 8 & 0 & $2.36 \mathrm{E}+00$ & 0 & 0 & $1.75 \mathrm{E}+03$ & 0 & 0 & $2.48 \mathrm{E}+02$ & $2.13 \mathrm{E}+02$ & 0 & 0 & 0 & $3.20 \mathrm{E}+02$ \\
\hline $8 \mathrm{TC} 047$ & 1.188 & 54.4453 & 8 & 0 & $9.28 \mathrm{E}-01$ & 0 & 0 & $1.21 \mathrm{E}+03$ & 0 & 0 & $1.72 \mathrm{E}+02$ & $1.40 \mathrm{E}+02$ & 0 & 0 & 0 & $2.22 \mathrm{E}+02$ \\
\hline $8 \mathrm{TC} 048$ & 1.391 & 48.4688 & 8 & 0 & $1.19 \mathrm{E}+00$ & 0 & 0 & $1.41 \mathrm{E}+03$ & 0 & 0 & $2.01 \mathrm{E}+02$ & $1.41 \mathrm{E}+02$ & 0 & 0 & 0 & $2.59 \mathrm{E}+02$ \\
\hline $8 \mathrm{TC} 049$ & 1.984 & 57.6328 & 8 & 0 & $3.11 \mathrm{E}+00$ & 0 & 0 & $2.00 \mathrm{E}+03$ & 0 & 0 & $2.85 \mathrm{E}+02$ & $2.54 \mathrm{E}+02$ & 0 & 0 & \begin{tabular}{|l|}
$4.59 \mathrm{E}+01$ \\
\end{tabular} & \begin{tabular}{|l|}
$3.68 \mathrm{E}+02$ \\
\end{tabular} \\
\hline $8 \mathrm{TC} 050$ & 0.156 & 40.1016 & 8 & 0 & 0 & 0 & 0 & $1.58 \mathrm{E}+02$ & 0 & 0 & $2.26 \mathrm{E}+01$ & 0 & 0 & 0 & 0 & $2.91 \mathrm{E}+01$ \\
\hline $8 \mathrm{TC} 051$ & 0.547 & 16.9922 & 8 & 0 & 0 & 0 & 0 & $5.56 \mathrm{E}+02$ & 0 & 0 & $7.91 \mathrm{E}+01$ & 0 & $2.20 \mathrm{E}+01$ & $1.92 \mathrm{E}+02$ & 0 & $1.02 \mathrm{E}+02$ \\
\hline $8 \mathrm{TC} 052$ & 0.219 & 34.9219 & 8 & 0 & 0 & 0 & 0 & $2.21 \mathrm{E}+02$ & 0 & 0 & $3.15 \mathrm{E}+01$ & 0 & 0 & 0 & 0 & $4.07 \mathrm{E}+01$ \\
\hline $8 \mathrm{TC} 053$ & 0.203 & 22.5703 & 8 & 0 & 0 & 0 & 0 & $2.05 \mathrm{E}+02$ & 0 & 0 & $2.93 \mathrm{E}+01$ & 0 & 0 & 0 & 0 & \begin{tabular}{|l|}
$3.78 \mathrm{E}+01$ \\
\end{tabular} \\
\hline $8 \mathrm{TC} 054$ & 0.922 & 34.125 & 8 & 0 & $4.50 \mathrm{E}-01$ & 0 & 0 & $9.35 \mathrm{E}+02$ & 0 & 0 & $1.33 \mathrm{E}+02$ & 0 & 0 & 0 & 0 & $1.72 \mathrm{E}+02$ \\
\hline $8 \mathrm{TC} 055$ & 0.828 & 28.5469 & 8 & 0 & 4.54E-01 & 0 & 0 & $8.40 \mathrm{E}+02$ & 0 & 0 & $1.20 \mathrm{E}+02$ & 0 & 0 & 0 & 0 & \begin{tabular}{|l|}
$1.54 \mathrm{E}+02$ \\
\end{tabular} \\
\hline $8 \mathrm{TC} 056$ & 0.703 & 39.7031 & 8 & 0 & 0 & 0 & 0 & $7.12 \mathrm{E}+02$ & 0 & 0 & $1.01 \mathrm{E}+02$ & 0 & 0 & 0 & 0 & $1.31 \mathrm{E}+02$ \\
\hline $8 \mathrm{TC} 057$ & 1.234 & 20.9766 & 8 & 0 & $1.47 \mathrm{E}+00$ & 0 & 0 & $1.25 \mathrm{E}+03$ & 0 & 0 & $1.78 \mathrm{E}+02$ & 0 & $2.28 \mathrm{E}+02$ & $1.99 \mathrm{E}+03$ & 0 & $2.30 \mathrm{E}+02$ \\
\hline $8 \mathrm{TC} 058$ & 1.859 & 30.1406 & 8 & 0 & $2.05 \mathrm{E}+00$ & 0 & $1.76 \mathrm{E}+02$ & $1.74 \mathrm{E}+03$ & 0 & 0 & $2.68 \mathrm{E}+02$ & $1.05 \mathrm{E}+02$ & $3.39 \mathrm{E}+02$ & \begin{tabular}{|l|}
$2.95 \mathrm{E}+03$ \\
\end{tabular} & 0 & \begin{tabular}{|l|}
$3.46 \mathrm{E}+02$ \\
\end{tabular} \\
\hline $8 \mathrm{TC} 059$ & 1.141 & 38.1094 & 8 & 0 & $9.12 \mathrm{E}-01$ & 0 & 0 & $1.15 \mathrm{E}+03$ & 0 & 0 & $1.64 \mathrm{E}+02$ & $3.70 \mathrm{E}+01$ & 0 & 0 & 0 & $2.11 \mathrm{E}+02$ \\
\hline $8 \mathrm{TC} 060$ & 1.109 & 21.375 & 8 & 0 & $1.26 \mathrm{E}+00$ & 0 & 0 & $1.13 \mathrm{E}+03$ & 0 & 0 & $1.60 \mathrm{E}+02$ & 0 & $1.39 \mathrm{E}+02$ & $1.21 \mathrm{E}+03$ & 0 & $2.07 \mathrm{E}+02$ \\
\hline $8 \mathrm{TC} 061$ & 1.625 & 22.9688 & 8 & 0 & $1.89 \mathrm{E}+00$ & 0 & 0 & $1.65 \mathrm{E}+03$ & 0 & 0 & $2.35 \mathrm{E}+02$ & 0 & $3.39 \mathrm{E}+02$ & $2.96 \mathrm{E}+03$ & 0 & \begin{tabular}{|l|}
$3.03 \mathrm{E}+02$ \\
\end{tabular} \\
\hline $8 \mathrm{TC} 062$ & 1.813 & 36.5156 & 8 & 0 & $2.18 \mathrm{E}+00$ & $1.43 \mathrm{E}+02$ & $7.92 \mathrm{E}+02$ & $1.12 \mathrm{E}+03$ & 0 & 0 & $2.62 \mathrm{E}+02$ & $1.51 \mathrm{E}+02$ & $2.08 \mathrm{E}+02$ & $1.81 \mathrm{E}+03$ & 0 & $3.38 \mathrm{E}+02$ \\
\hline $8 \mathrm{TC} 063$ & 1.609 & 31.7344 & 8 & 0 & $1.72 \mathrm{E}+00$ & \begin{tabular}{|l|}
$7.29 \mathrm{E}+01$ \\
\end{tabular} & $2.46 \mathrm{E}+01$ & $1.55 \mathrm{E}+03$ & 0 & 0 & $2.32 \mathrm{E}+02$ & $3.97 \mathrm{E}+01$ & $1.64 \mathrm{E}+02$ & $1.43 \mathrm{E}+03$ & 0 & $2.99 \mathrm{E}+02$ \\
\hline $8 \mathrm{TC} 064$ & 0.984 & 62.0156 & 8 & 0 & $8.16 \mathrm{E}-01$ & 0 & 0 & $9.99 \mathrm{E}+02$ & 0 & 0 & $1.42 \mathrm{E}+02$ & $1.20 \mathrm{E}+02$ & 0 & 0 & 0 & $1.84 \mathrm{E}+02$ \\
\hline $8 \mathrm{TC} 065$ & 0.078 & 52.0547 & 8 & 0 & 0 & 0 & 0 & $7.90 \mathrm{E}+01$ & 0 & 0 & $1.13 \mathrm{E}+01$ & 0 & 0 & 0 & 0 & $1.46 \mathrm{E}+01$ \\
\hline $8 \mathrm{TC} 066$ & 0.781 & 60.4219 & 8 & 0 & $2.85 \mathrm{E}-01$ & 0 & 0 & $7.89 \mathrm{E}+02$ & 0 & 0 & $1.12 \mathrm{E}+02$ & $8.63 \mathrm{E}+01$ & 0 & 0 & 0 & $1.45 \mathrm{E}+02$ \\
\hline $8 \mathrm{TC} 067$ & 0.734 & 55.6406 & 8 & 0 & $3.18 \mathrm{E}-02$ & 0 & 0 & $7.45 \mathrm{E}+02$ & 0 & 0 & $1.06 \mathrm{E}+02$ & $6.73 \mathrm{E}+01$ & 0 & 0 & 0 & $1.37 \mathrm{E}+02$ \\
\hline $8 \mathrm{TC} 068$ & 0.297 & 60.8203 & 8 & 0 & 0 & 0 & 0 & $3.01 \mathrm{E}+02$ & 0 & 0 & $4.29 \mathrm{E}+01$ & $1.92 \mathrm{E}+01$ & 0 & 0 & 0 & $5.54 \mathrm{E}+01$ \\
\hline $8 \mathrm{TC} 069$ & 0.75 & 42.4922 & 8 & 0 & 0 & 0 & 0 & $7.61 \mathrm{E}+02$ & 0 & 0 & $1.08 \mathrm{E}+02$ & $1.75 \mathrm{E}+01$ & 0 & 0 & 0 & $1.40 \mathrm{E}+02$ \\
\hline $8 \mathrm{TC} 070$ & 0.531 & 44.8828 & 8 & 0 & 0 & 0 & 0 & $5.39 \mathrm{E}+02$ & 0 & 0 & $7.66 \mathrm{E}+01$ & 0 & 0 & 0 & 0 & $9.89 \mathrm{E}+01$ \\
\hline $8 \mathrm{TC} 071$ & 1.766 & 46.4766 & 8 & 0 & $1.44 \mathrm{E}+00$ & 0 & 0 & $1.79 \mathrm{E}+03$ & 0 & 0 & $2.54 \mathrm{E}+02$ & $2.06 \mathrm{E}+02$ & $5.52 \mathrm{E}+01$ & $4.82 \mathrm{E}+02$ & 0 & $3.28 \mathrm{E}+02$ \\
\hline
\end{tabular}


Table 39. Predicted Solids for Envelope C Simulations - Part 1 (cont'd)

\begin{tabular}{|c|c|c|c|c|c|c|c|c|c|c|c|c|c|c|c|c|}
\hline Test ID & $\begin{array}{l}\text { SBS/ } \\
\text { Feed }\end{array}$ & $\begin{array}{l}\text { Temp } \\
{\left[{ }^{\circ} \mathrm{C}\right]} \\
\end{array}$ & $\left.\begin{array}{c}\mathrm{Na} \mathrm{M} \\
{[\mathrm{mol} / \mathrm{L}]}\end{array}\right]$ & ALOH3 & BASO4 & CA3PO42 & $\mathrm{CACO} 3$ & CAF2 & CANC & $\mathrm{CAOH} 2$ & CATIO3 & $\mathrm{CROH} 3$ & FEIIIOH3 & \begin{tabular}{|c|} 
HYDROS \\
OD \\
\end{tabular} & MGF2 & MGOH2 \\
\hline $8 \mathrm{TC} 072$ & 1.406 & 62.4141 & 8 & 0 & $1.87 \mathrm{E}+00$ & 0 & 0 & $1.43 \mathrm{E}+03$ & 0 & 0 & $2.03 \mathrm{E}+02$ & $1.81 \mathrm{E}+02$ & 0 & 0 & 0 & $2.62 \mathrm{E}+02$ \\
\hline $8 \mathrm{TC} 073$ & 1.094 & 44.0859 & 8 & 0 & $3.49 \mathrm{E}-02$ & 0 & 0 & $1.11 \mathrm{E}+03$ & 0 & 0 & $1.57 \mathrm{E}+02$ & $7.26 \mathrm{E}+01$ & 0 & 0 & 0 & $2.03 \mathrm{E}+02$ \\
\hline $8 \mathrm{TC} 074$ & 1.75 & 43.2891 & 8 & 0 & $1.40 \mathrm{E}+00$ & 0 & 0 & $1.78 \mathrm{E}+03$ & 0 & 0 & $2.53 \mathrm{E}+02$ & $1.79 \mathrm{E}+02$ & $3.40 \mathrm{E}+00$ & \begin{tabular}{|l|}
$2.97 \mathrm{E}+01$ \\
\end{tabular} & 0 & $3.26 \mathrm{E}+02$ \\
\hline $8 \mathrm{TC} 075$ & 1.688 & 56.4375 & 8 & 0 & $2.29 \mathrm{E}+00$ & 0 & 0 & $1.71 \mathrm{E}+03$ & 0 & 0 & $2.43 \mathrm{E}+02$ & $2.13 \mathrm{E}+02$ & 0 & 0 & 0 & $3.14 \mathrm{E}+02$ \\
\hline $8 \mathrm{TC} 076$ & 1.156 & 61.2188 & 8 & 0 & $1.19 \mathrm{E}+00$ & 0 & 0 & $1.18 \mathrm{E}+03$ & 0 & 0 & $1.67 \mathrm{E}+02$ & $1.46 \mathrm{E}+02$ & 0 & 0 & 0 & $2.16 \mathrm{E}+02$ \\
\hline $8 \mathrm{TC} 077$ & 1.531 & 61.6172 & 8 & 0 & $2.14 \mathrm{E}+00$ & 0 & 0 & $1.55 \mathrm{E}+03$ & 0 & 0 & $2.20 \mathrm{E}+02$ & $1.96 \mathrm{E}+02$ & 0 & 0 & 0 & $2.84 \mathrm{E}+02$ \\
\hline $8 \mathrm{TC} 078$ & 1 & 40.5 & 8 & 0 & 0 & 0 & 0 & $1.01 \mathrm{E}+03$ & 0 & 0 & $1.44 \mathrm{E}+02$ & $3.85 \mathrm{E}+01$ & 0 & 0 & 0 & $1.85 \mathrm{E}+02$ \\
\hline $8 \mathrm{TC} 079$ & 1.328 & 43.6875 & 8 & 0 & $4.01 \mathrm{E}-01$ & 0 & 0 & $1.33 \mathrm{E}+03$ & 0 & 0 & $1.89 \mathrm{E}+02$ & $1.09 \mathrm{E}+02$ & 0 & 0 & 0 & $2.44 \mathrm{E}+02$ \\
\hline $8 \mathrm{TC} 080$ & 1.656 & 62.8125 & 8 & 0 & $2.47 \mathrm{E}+00$ & 0 & 0 & $1.66 \mathrm{E}+03$ & 0 & 0 & $2.36 \mathrm{E}+02$ & $2.13 \mathrm{E}+02$ & 0 & 0 & 0 & $3.05 \mathrm{E}+02$ \\
\hline $8 \mathrm{TC} 081$ & 1.516 & 45.6797 & 8 & 0 & $8.78 \mathrm{E}-01$ & 0 & 0 & $1.52 \mathrm{E}+03$ & 0 & 0 & $2.17 \mathrm{E}+02$ & $1.59 \mathrm{E}+02$ & 0 & 0 & 0 & $2.80 \mathrm{E}+02$ \\
\hline $8 \mathrm{TC} 082$ & 1.281 & 63.6094 & 8 & 0 & $1.55 \mathrm{E}+00$ & 0 & 0 & $1.29 \mathrm{E}+03$ & 0 & 0 & $1.83 \mathrm{E}+02$ & $1.64 \mathrm{E}+02$ & 0 & 0 & 0 & $2.36 \mathrm{E}+02$ \\
\hline $8 \mathrm{TC} 083$ & 2 & 50.0625 & 8 & 0 & $2.39 \mathrm{E}+00$ & 0 & 0 & $1.99 \mathrm{E}+03$ & 0 & 0 & $2.83 \mathrm{E}+02$ & $2.41 \mathrm{E}+02$ & 0 & 0 & $1.73 \mathrm{E}+01$ & \begin{tabular}{|l|}
$3.65 \mathrm{E}+02$ \\
\end{tabular} \\
\hline $8 \mathrm{TC} 084$ & 1.359 & 59.2266 & 8 & 0 & $1.57 \mathrm{E}+00$ & 0 & 0 & $1.36 \mathrm{E}+03$ & 0 & 0 & $1.94 \mathrm{E}+02$ & $1.67 \mathrm{E}+02$ & 0 & 0 & 0 & $2.50 \mathrm{E}+02$ \\
\hline $8 \mathrm{TC} 085$ & 1.641 & 51.2578 & 8 & 0 & $1.75 \mathrm{E}+00$ & 0 & 0 & $1.66 \mathrm{E}+03$ & 0 & 0 & $2.36 \mathrm{E}+02$ & $1.99 \mathrm{E}+02$ & 0 & 0 & 0 & \begin{tabular}{|l|}
$3.05 \mathrm{E}+02$ \\
\end{tabular} \\
\hline $8 \mathrm{TC} 086$ & 0.109 & 51.6563 & 8 & 0 & 0 & 0 & 0 & $1.09 \mathrm{E}+02$ & 0 & 0 & $1.56 \mathrm{E}+01$ & 0 & 0 & 0 & 0 & $2.01 \mathrm{E}+01$ \\
\hline $8 \mathrm{TC} 087$ & 0.953 & 64.8047 & 8 & 0 & $8.03 \mathrm{E}-01$ & 0 & 0 & $9.53 \mathrm{E}+02$ & 0 & 0 & $1.36 \mathrm{E}+02$ & $1.16 \mathrm{E}+02$ & 0 & 0 & 0 & $1.75 \mathrm{E}+02$ \\
\hline $8 \mathrm{TC} 088$ & 0.438 & 64.4063 & 8 & 0 & 0 & 0 & 0 & $4.41 \mathrm{E}+02$ & 0 & 0 & $6.28 \mathrm{E}+01$ & $4.62 \mathrm{E}+01$ & 0 & 0 & 0 & $8.11 \mathrm{E}+01$ \\
\hline $8 \mathrm{TC} 089$ & 0.422 & 58.8281 & 8 & 0 & 0 & 0 & 0 & $4.24 \mathrm{E}+02$ & 0 & 0 & $6.03 \mathrm{E}+01$ & $3.16 \mathrm{E}+01$ & 0 & 0 & 0 & $7.79 \mathrm{E}+01$ \\
\hline $8 \mathrm{TC} 090$ & 0.453 & 45.2813 & 8 & 0 & 0 & 0 & 0 & $4.53 \mathrm{E}+02$ & 0 & 0 & $6.45 \mathrm{E}+01$ & 0 & 0 & 0 & 0 & $8.32 \mathrm{E}+01$ \\
\hline $8 \mathrm{TC} 091$ & 0.688 & 50.4609 & 8 & 0 & 0 & 0 & 0 & $6.90 \mathrm{E}+02$ & 0 & 0 & $9.81 \mathrm{E}+01$ & $5.04 \mathrm{E}+01$ & 0 & 0 & 0 & $1.27 \mathrm{E}+02$ \\
\hline $8 \mathrm{TC} 092$ & 0.328 & 65.6016 & 8 & 0 & 0 & 0 & 0 & $3.29 \mathrm{E}+02$ & 0 & 0 & $4.69 \mathrm{E}+01$ & $3.12 \mathrm{E}+01$ & 0 & 0 & 0 & $6.06 \mathrm{E}+01$ \\
\hline $8 \mathrm{TC} 093$ & 1.125 & 32.1328 & 8 & 0 & $8.78 \mathrm{E}-01$ & 0 & 0 & $1.12 \mathrm{E}+03$ & 0 & 0 & $1.59 \mathrm{E}+02$ & 0 & 0 & 0 & 0 & $2.06 \mathrm{E}+02$ \\
\hline $8 \mathrm{TC} 094$ & 1.875 & 23.7656 & 8 & 0 & $2.13 \mathrm{E}+00$ & 0 & 0 & $1.88 \mathrm{E}+03$ & 0 & 0 & $2.68 \mathrm{E}+02$ & $3.18 \mathrm{E}+00$ & $4.00 \mathrm{E}+02$ & $3.49 \mathrm{E}+03$ & 0 & $3.45 \mathrm{E}+02$ \\
\hline $8 \mathrm{TC} 095$ & 1.203 & 27.3516 & 8 & 0 & $1.09 \mathrm{E}+00$ & 0 & 0 & $1.21 \mathrm{E}+03$ & 0 & 0 & $1.73 \mathrm{E}+02$ & 0 & $1.34 \mathrm{E}+02$ & $1.16 \mathrm{E}+03$ & 0 & $2.23 \mathrm{E}+02$ \\
\hline $8 \mathrm{TC} 096$ & 1.906 & 33.3281 & 8 & 0 & $1.82 \mathrm{E}+00$ & $9.00 \mathrm{E}+01$ & $2.78 \mathrm{E}+02$ & $1.63 \mathrm{E}+03$ & 0 & 0 & $2.72 \mathrm{E}+02$ & $1.74 \mathrm{E}+02$ & $3.72 \mathrm{E}+02$ & $3.24 \mathrm{E}+03$ & 0 & $3.52 \mathrm{E}+02$ \\
\hline $8 \mathrm{TC} 097$ & 1.375 & 15 & 8 & 0 & $8.33 \mathrm{E}-01$ & 0 & 0 & $1.38 \mathrm{E}+03$ & 0 & 0 & $1.96 \mathrm{E}+02$ & 0 & $3.00 \mathrm{E}+02$ & $2.62 \mathrm{E}+03$ & 0 & $2.53 \mathrm{E}+02$ \\
\hline $8 \mathrm{TC} 098$ & 1.734 & 31.3359 & 8 & 0 & $1.79 \mathrm{E}+00$ & 0 & 0 & $1.72 \mathrm{E}+03$ & 0 & 0 & $2.45 \mathrm{E}+02$ & $7.27 \mathrm{E}+01$ & $2.39 \mathrm{E}+02$ & $2.09 \mathrm{E}+03$ & 0 & \begin{tabular}{|l|}
$3.17 \mathrm{E}+02$ \\
\end{tabular} \\
\hline $8 \mathrm{TC} 099$ & 1.063 & 27.75 & 8 & 0 & $9.17 \mathrm{E}-01$ & 0 & 0 & $1.07 \mathrm{E}+03$ & 0 & 0 & $1.52 \mathrm{E}+02$ & 0 & $7.35 \mathrm{E}+01$ & $6.41 \mathrm{E}+02$ & 0 & $1.96 \mathrm{E}+02$ \\
\hline $8 \mathrm{TC} 100$ & 0.563 & 17.7891 & 8 & 0 & 0 & 0 & 0 & $5.68 \mathrm{E}+02$ & 0 & 0 & $8.07 \mathrm{E}+01$ & 0 & $4.17 \mathrm{E}+00$ & $3.64 \mathrm{E}+01$ & 0 & \begin{tabular}{|l|}
$1.04 \mathrm{E}+02$ \\
\end{tabular} \\
\hline $8 \mathrm{TC} 101$ & 0.047 & 39.3047 & 8 & 0 & 0 & 0 & 0 & $4.62 \mathrm{E}+01$ & 0 & 0 & $6.68 \mathrm{E}+00$ & 0 & 0 & 0 & 0 & $8.63 \mathrm{E}+00$ \\
\hline
\end{tabular}


Table 39. Predicted Solids for Envelope C Simulations - Part 1 (cont'd)

\begin{tabular}{|c|c|c|c|c|c|c|c|c|c|c|c|c|c|c|c|c|}
\hline Test ID & $\begin{array}{l}\text { SBS/ } \\
\text { Feed }\end{array}$ & $\begin{array}{l}\text { Temp } \\
{\left[{ }^{\circ} \mathrm{C}\right]}\end{array}$ & $\left.\begin{array}{c}\mathrm{Na} \mathrm{M} \\
{[\mathrm{mol} / \mathrm{L}]}\end{array}\right]$ & ALOH3 & BASO4 & CA3PO42 & $\mathrm{CACO} 3$ & CAF2 & CANC & $\mathrm{CAOH} 2$ & CATIO3 & $\mathrm{CROH} 3$ & FEIIIOH3 & \begin{tabular}{|c|} 
HYDROS \\
OD \\
\end{tabular} & MGF2 & MGOH2 \\
\hline $8 \mathrm{TC} 102$ & 0.063 & 26.1563 & 8 & 0 & 0 & 0 & 0 & $6.23 \mathrm{E}+01$ & 0 & 0 & $8.91 \mathrm{E}+00$ & 0 & 0 & 0 & 0 & \begin{tabular}{|l|}
$1.15 \mathrm{E}+01$ \\
\end{tabular} \\
\hline $8 \mathrm{TC} 103$ & 0.516 & 15.7969 & 8 & 0 & 0 & 0 & 0 & $5.18 \mathrm{E}+02$ & 0 & 0 & $7.37 \mathrm{E}+01$ & 0 & $1.72 \mathrm{E}+01$ & $1.50 \mathrm{E}+02$ & 0 & $9.51 \mathrm{E}+01$ \\
\hline $8 \mathrm{TC} 104$ & 0.281 & 25.7578 & 8 & 0 & 0 & 0 & 0 & $2.80 \mathrm{E}+02$ & 0 & 0 & $3.99 \mathrm{E}+01$ & 0 & 0 & 0 & 0 & $5.15 \mathrm{E}+01$ \\
\hline $8 \mathrm{TC} 105$ & 0.813 & 26.5547 & 8 & 0 & $4.72 \mathrm{E}-01$ & 0 & 0 & $8.14 \mathrm{E}+02$ & 0 & 0 & $1.16 \mathrm{E}+02$ & 0 & 0 & 0 & 0 & $1.50 \mathrm{E}+02$ \\
\hline $8 \mathrm{TC} 106$ & 0.609 & 32.5313 & 8 & 0 & 0 & 0 & 0 & $6.09 \mathrm{E}+02$ & 0 & 0 & $8.66 \mathrm{E}+01$ & 0 & 0 & 0 & 0 & $1.12 \mathrm{E}+02$ \\
\hline $8 \mathrm{TC} 107$ & 0.016 & 23.3672 & 8 & 0 & 0 & 0 & 0 & $1.54 \mathrm{E}+01$ & 0 & 0 & $2.25 \mathrm{E}+00$ & 0 & 0 & 0 & 0 & $2.90 \mathrm{E}+00$ \\
\hline $8 \mathrm{TC} 108$ & 1.844 & 40.8984 & 8 & 0 & $9.23 \mathrm{E}-01$ & 0 & 0 & $1.84 \mathrm{E}+03$ & 0 & 0 & $2.62 \mathrm{E}+02$ & $1.81 \mathrm{E}+02$ & $1.22 \mathrm{E}+02$ & $1.06 \mathrm{E}+03$ & 0 & $3.38 \mathrm{E}+02$ \\
\hline $8 \mathrm{TC} 110$ & 1.781 & 46.0781 & 8 & 0 & $1.32 \mathrm{E}+00$ & 0 & 0 & $1.79 \mathrm{E}+03$ & 0 & 0 & $2.54 \mathrm{E}+02$ & $2.07 \mathrm{E}+02$ & $9.43 \mathrm{E}+01$ & $8.23 \mathrm{E}+02$ & 0 & $3.28 \mathrm{E}+02$ \\
\hline $8 \mathrm{TC} 111$ & 1.797 & 58.4297 & 8 & 0 & $2.63 \mathrm{E}+00$ & 0 & 0 & $1.81 \mathrm{E}+03$ & 0 & 0 & $2.58 \mathrm{E}+02$ & $2.29 \mathrm{E}+02$ & \begin{tabular}{|l|}
0 \\
\end{tabular} & 0 & 0 & $3.32 \mathrm{E}+02$ \\
\hline $8 \mathrm{TC} 112$ & 1.078 & 46.875 & 8 & 0 & $2.89 \mathrm{E}-01$ & 0 & 0 & $1.08 \mathrm{E}+03$ & 0 & 0 & $1.54 \mathrm{E}+02$ & $8.25 \mathrm{E}+01$ & 0 & 0 & 0 & $1.99 \mathrm{E}+02$ \\
\hline $8 \mathrm{TC} 113$ & 1.172 & 52.4531 & 8 & 0 & $6.96 \mathrm{E}-01$ & 0 & 0 & $1.17 \mathrm{E}+03$ & 0 & 0 & $1.67 \mathrm{E}+02$ & $1.28 \mathrm{E}+02$ & 0 & 0 & 0 & \begin{tabular}{|l|}
$2.15 \mathrm{E}+02$ \\
\end{tabular} \\
\hline $8 \mathrm{TC} 114$ & 1.297 & 41.2969 & 8 & 0 & 0 & 0 & 0 & $1.31 \mathrm{E}+03$ & 0 & 0 & $1.86 \mathrm{E}+02$ & $9.63 \mathrm{E}+01$ & 0 & 0 & 0 & $2.40 \mathrm{E}+02$ \\
\hline $8 \mathrm{TC} 115$ & 0.766 & 60.0234 & 8 & 0 & $2.36 \mathrm{E}-01$ & 0 & 0 & $7.69 \mathrm{E}+02$ & 0 & 0 & $1.09 \mathrm{E}+02$ & $8.18 \mathrm{E}+01$ & 0 & 0 & 0 & $1.41 \mathrm{E}+02$ \\
\hline $8 \mathrm{TC} 116$ & 0.141 & 50.8594 & 8 & 0 & 0 & 0 & 0 & $1.41 \mathrm{E}+02$ & 0 & 0 & $2.01 \mathrm{E}+01$ & 0 & 0 & 0 & 0 & $2.60 \mathrm{E}+01$ \\
\hline $8 \mathrm{TC} 117$ & 0.859 & 42.8906 & 8 & 0 & 0 & 0 & 0 & $8.69 \mathrm{E}+02$ & 0 & 0 & $1.24 \mathrm{E}+02$ & $3.55 \mathrm{E}+01$ & 0 & 0 & 0 & $1.60 \mathrm{E}+02$ \\
\hline $8 \mathrm{TC} 119$ & 0.375 & 58.0313 & 8 & 0 & 0 & 0 & 0 & $3.75 \mathrm{E}+02$ & 0 & 0 & $5.33 \mathrm{E}+01$ & $2.12 \mathrm{E}+01$ & 0 & 0 & 0 & $6.89 \mathrm{E}+01$ \\
\hline $8 \mathrm{TC} 120$ & 0.188 & 44.4844 & 8 & 0 & 0 & 0 & 0 & $1.87 \mathrm{E}+02$ & 0 & 0 & $2.66 \mathrm{E}+01$ & 0 & 0 & 0 & 0 & $3.43 \mathrm{E}+01$ \\
\hline $8 \mathrm{TC} 121$ & 0.391 & 49.2656 & 8 & 0 & 0 & 0 & 0 & $3.94 \mathrm{E}+02$ & 0 & 0 & $5.60 \mathrm{E}+01$ & $2.09 \mathrm{E}+00$ & 0 & 0 & 0 & $7.23 \mathrm{E}+01$ \\
\hline $8 \mathrm{TC} 122$ & 1.016 & 18.9844 & 8 & 0 & 0 & 0 & 0 & $1.02 \mathrm{E}+03$ & 0 & 0 & $1.45 \mathrm{E}+02$ & 0 & $1.40 \mathrm{E}+02$ & $1.22 \mathrm{E}+03$ & 0 & $1.87 \mathrm{E}+02$ \\
\hline $8 \mathrm{TC} 123$ & 1.922 & 28.9453 & 8 & 0 & $2.10 \mathrm{E}+00$ & 0 & $2.54 \mathrm{E}+02$ & $1.73 \mathrm{E}+03$ & 0 & 0 & $2.75 \mathrm{E}+02$ & $9.43 \mathrm{E}+01$ & $3.63 \mathrm{E}+02$ & $3.17 \mathrm{E}+03$ & 0 & $3.54 \mathrm{E}+02$ \\
\hline $8 \mathrm{TC} 124$ & 1.219 & 20.5781 & 8 & 0 & $1.41 \mathrm{E}+00$ & 0 & 0 & $1.23 \mathrm{E}+03$ & 0 & 0 & $1.75 \mathrm{E}+02$ & 0 & $2.16 \mathrm{E}+02$ & $1.88 \mathrm{E}+03$ & 0 & $2.26 \mathrm{E}+02$ \\
\hline $8 \mathrm{TC} 125$ & 1.266 & 25.3594 & 8 & 0 & $1.24 \mathrm{E}+00$ & 0 & 0 & $1.27 \mathrm{E}+03$ & 0 & 0 & $1.81 \mathrm{E}+02$ & 0 & $1.89 \mathrm{E}+02$ & \begin{tabular}{|l|}
$1.65 \mathrm{E}+03$ \\
\end{tabular} & 0 & $2.33 \mathrm{E}+02$ \\
\hline $8 \mathrm{TC} 126$ & 1.703 & 20.1797 & 8 & 0 & $2.30 \mathrm{E}+00$ & 0 & 0 & $1.70 \mathrm{E}+03$ & 0 & 0 & $2.42 \mathrm{E}+02$ & 0 & $3.61 \mathrm{E}+02$ & $3.15 \mathrm{E}+03$ & 0 & $3.12 \mathrm{E}+02$ \\
\hline $8 \mathrm{TC} 127$ & 1.25 & 38.5078 & 8 & 0 & $1.05 \mathrm{E}+00$ & 0 & 0 & $1.26 \mathrm{E}+03$ & 0 & 0 & $1.79 \mathrm{E}+02$ & $5.62 \mathrm{E}+01$ & 0 & 0 & 0 & $2.31 \mathrm{E}+02$ \\
\hline $8 \mathrm{TC} 128$ & 1.469 & 36.1172 & 8 & 0 & $1.35 \mathrm{E}+00$ & 0 & 0 & $1.48 \mathrm{E}+03$ & 0 & 0 & $2.10 \mathrm{E}+02$ & $8.81 \mathrm{E}+01$ & $7.95 \mathrm{E}+01$ & \begin{tabular}{|l|}
$6.93 \mathrm{E}+02$ \\
\end{tabular} & 0 & $2.71 \mathrm{E}+02$ \\
\hline $8 \mathrm{TC} 129$ & 0.234 & 34.5234 & 8 & 0 & 0 & 0 & 0 & $2.36 \mathrm{E}+02$ & 0 & 0 & $3.36 \mathrm{E}+01$ & 0 & 0 & 0 & 0 & $4.34 \mathrm{E}+01$ \\
\hline $8 \mathrm{TC} 130$ & 0.594 & 18.5859 & 8 & 0 & 0 & 0 & 0 & $5.96 \mathrm{E}+02$ & 0 & 0 & $8.47 \mathrm{E}+01$ & 0 & $9.09 \mathrm{E}+00$ & \begin{tabular}{|l|}
$7.93 \mathrm{E}+01$ \\
\end{tabular} & 0 & $1.09 \mathrm{E}+02$ \\
\hline $8 \mathrm{TC} 131$ & 0.906 & 36.9141 & 8 & 0 & 4.10E-01 & 0 & 0 & $9.14 \mathrm{E}+02$ & 0 & 0 & $1.30 \mathrm{E}+02$ & 0 & 0 & 0 & 0 & $1.68 \mathrm{E}+02$ \\
\hline
\end{tabular}


Table 39. Predicted Solids for Envelope C Simulations - Part 1 (cont'd)

\begin{tabular}{|c|c|c|c|c|c|c|c|c|c|c|c|c|c|c|c|c|}
\hline Test ID & $\begin{array}{l}\text { SBS/ } \\
\text { Feed }\end{array}$ & $\begin{array}{l}\text { Temp } \\
{\left[{ }^{\circ} \mathrm{C}\right]} \\
\end{array}$ & $\left.\begin{array}{c}\mathrm{Na} \mathrm{M} \\
{[\mathrm{mol} / \mathrm{L}]}\end{array}\right]$ & ALOH3 & BASO4 & CA3PO42 & $\mathrm{CACO} 3$ & CAF2 & CANC & $\mathrm{CAOH} 2$ & CATIO3 & $\mathrm{CROH} 3$ & FEIIIOH3 & \begin{tabular}{|c|} 
HYDROS \\
OD \\
\end{tabular} & MGF2 & MGOH2 \\
\hline $8 \mathrm{TC} 132$ & 0.25 & 37.7109 & 8 & 0 & 0 & 0 & 0 & $2.50 \mathrm{E}+02$ & 0 & 0 & $3.56 \mathrm{E}+01$ & 0 & 0 & 0 & 0 & $4.59 \mathrm{E}+01$ \\
\hline $8 \mathrm{TC} 133$ & 0.313 & 24.5625 & 8 & 0 & 0 & 0 & 0 & $3.14 \mathrm{E}+02$ & 0 & 0 & $4.47 \mathrm{E}+01$ & 0 & 0 & 0 & 0 & $5.78 \mathrm{E}+01$ \\
\hline $8 \mathrm{TC} 134$ & 0.844 & 19.7813 & 8 & 0 & 0 & 0 & 0 & \begin{tabular}{|l|}
$8.47 \mathrm{E}+02$ \\
\end{tabular} & 0 & 0 & $1.20 \mathrm{E}+02$ & 0 & $6.39 \mathrm{E}+01$ & $5.58 \mathrm{E}+02$ & 0 & $1.55 \mathrm{E}+02$ \\
\hline $8 \mathrm{TC} 135$ & 0.469 & 19.3828 & 8 & 0 & 0 & 0 & 0 & $4.73 \mathrm{E}+02$ & 0 & 0 & $6.73 \mathrm{E}+01$ & 0 & 0 & 0 & 0 & $8.69 \mathrm{E}+01$ \\
\hline 6TC001 & 0 & 15 & 6 & $3.71 \mathrm{E}+02$ & 0 & 0 & 0 & 0 & 0 & 0 & 0 & 0 & 0 & 0 & 0 & 0 \\
\hline 6TC002 & 0 & 15 & 6 & $5.15 \mathrm{E}+02$ & 0 & 0 & 0 & 0 & 0 & 0 & 0 & 0 & 0 & 0 & 0 & 0 \\
\hline 6TC003 & 0 & 15 & 6 & 0 & 0 & 0 & 0 & 0 & 0 & 0 & 0 & 0 & 0 & 0 & 0 & 0 \\
\hline 6TC004 & 0 & 15 & 6 & 0 & 0 & 0 & 0 & 0 & 0 & 0 & 0 & 0 & 0 & 0 & 0 & 0 \\
\hline 6TC005 & 0 & 15 & 6 & 0 & 0 & 0 & 0 & 0 & 0 & 0 & 0 & 0 & 0 & 0 & 0 & 0 \\
\hline 6TC006 & 0 & 66 & 6 & 0 & 0 & 0 & 0 & 0 & 0 & 0 & 0 & 0 & 0 & 0 & 0 & 0 \\
\hline 6TC007 & 0 & 66 & 6 & 0 & 0 & 0 & 0 & 0 & 0 & 0 & 0 & 0 & 0 & 0 & 0 & 0 \\
\hline 6TC008 & 0 & 66 & 6 & 0 & 0 & 0 & 0 & 0 & 0 & 0 & 0 & 0 & 0 & 0 & 0 & 0 \\
\hline 6TC009 & 0 & 66 & 6 & 0 & 0 & 0 & 0 & 0 & 0 & 0 & 0 & 0 & 0 & 0 & 0 & 0 \\
\hline 6TC010 & 0 & 66 & 6 & 0 & 0 & 0 & 0 & 0 & 0 & 0 & 0 & 0 & 0 & 0 & 0 & 0 \\
\hline 6TC011 & 2 & 15 & 6 & $3.18 \mathrm{E}+03$ & $3.82 \mathrm{E}+00$ & 0 & 0 & $2.04 \mathrm{E}+03$ & 0 & 0 & $2.89 \mathrm{E}+02$ & 0 & $5.37 \mathrm{E}+02$ & $4.68 \mathrm{E}+03$ & 0 & \begin{tabular}{|l|}
$3.74 \mathrm{E}+02$ \\
\end{tabular} \\
\hline 6TC012 & 2 & 15 & 6 & $3.39 \mathrm{E}+03$ & $3.80 \mathrm{E}+00$ & 0 & 0 & $2.03 \mathrm{E}+03$ & 0 & 0 & $2.88 \mathrm{E}+02$ & 0 & $5.20 \mathrm{E}+02$ & $4.54 \mathrm{E}+03$ & 0 & $3.72 \mathrm{E}+02$ \\
\hline 6TC013 & 2 & 15 & 6 & 0 & $3.74 \mathrm{E}+00$ & 0 & 0 & $2.01 \mathrm{E}+03$ & 0 & 0 & $2.86 \mathrm{E}+02$ & $9.38 \mathrm{E}+01$ & $5.47 \mathrm{E}+02$ & $4.77 \mathrm{E}+03$ & 0 & \begin{tabular}{|l|}
$3.70 \mathrm{E}+02$ \\
\end{tabular} \\
\hline 6TC014 & 2 & 15 & 6 & 0 & $3.73 \mathrm{E}+00$ & 0 & 0 & $1.99 \mathrm{E}+03$ & 0 & 0 & $2.83 \mathrm{E}+02$ & $1.77 \mathrm{E}+01$ & $5.29 \mathrm{E}+02$ & $4.61 \mathrm{E}+03$ & 0 & $3.65 \mathrm{E}+02$ \\
\hline 6TC015 & 2 & 15 & 6 & 0 & $3.66 \mathrm{E}+00$ & 0 & 0 & $2.01 \mathrm{E}+03$ & 0 & 0 & $2.85 \mathrm{E}+02$ & $1.36 \mathrm{E}+02$ & $5.52 \mathrm{E}+02$ & $4.82 \mathrm{E}+03$ & 0 & $3.68 \mathrm{E}+02$ \\
\hline 6TC016 & 2 & 66 & 6 & 0 & $2.56 \mathrm{E}+00$ & 0 & 0 & $2.03 \mathrm{E}+03$ & 0 & 0 & $2.89 \mathrm{E}+02$ & $2.71 \mathrm{E}+02$ & 0 & 0 & 0 & \begin{tabular}{|l|}
$3.74 \mathrm{E}+02$ \\
\end{tabular} \\
\hline 6TC017 & 2 & 66 & 6 & 0 & $2.59 \mathrm{E}+00$ & 0 & 0 & $2.03 \mathrm{E}+03$ & 0 & 0 & $2.88 \mathrm{E}+02$ & $2.68 \mathrm{E}+02$ & 0 & 0 & 0 & $3.72 \mathrm{E}+02$ \\
\hline 6TC018 & 2 & 66 & 6 & 0 & $2.53 \mathrm{E}+00$ & 0 & 0 & $2.01 \mathrm{E}+03$ & 0 & 0 & $2.86 \mathrm{E}+02$ & $2.69 \mathrm{E}+02$ & 0 & 0 & 0 & $3.70 \mathrm{E}+02$ \\
\hline 6TC019 & 2 & 66 & 6 & 0 & $2.56 \mathrm{E}+00$ & 0 & 0 & $1.99 \mathrm{E}+03$ & 0 & 0 & $2.83 \mathrm{E}+02$ & $2.64 \mathrm{E}+02$ & 0 & 0 & 0 & $3.65 \mathrm{E}+02$ \\
\hline 6TC020 & 2 & 66 & 6 & 0 & $2.36 \mathrm{E}+00$ & 0 & 0 & $2.00 \mathrm{E}+03$ & 0 & 0 & $2.85 \mathrm{E}+02$ & $2.69 \mathrm{E}+02$ & $1.18 \mathrm{E}+02$ & \begin{tabular}{|l|}
$1.03 \mathrm{E}+03$ \\
\end{tabular} & 0 & $3.68 \mathrm{E}+02$ \\
\hline 6TC021 & 0.672 & 37.3125 & 6 & 0 & $4.71 \mathrm{E}-02$ & 0 & 0 & $6.82 \mathrm{E}+02$ & 0 & 0 & $9.70 \mathrm{E}+01$ & $2.41 \mathrm{E}+00$ & 0 & 0 & 0 & $1.25 \mathrm{E}+02$ \\
\hline 6TC022 & 0.344 & 18.1875 & 6 & $4.04 \mathrm{E}+02$ & 0 & 0 & 0 & $3.50 \mathrm{E}+02$ & 0 & 0 & $4.97 \mathrm{E}+01$ & 0 & $5.50 \mathrm{E}+00$ & $4.79 \mathrm{E}+01$ & 0 & $6.42 \mathrm{E}+01$ \\
\hline $6 \mathrm{TC} 023$ & 0.484 & 35.3203 & 6 & 0 & 0 & 0 & 0 & $4.91 \mathrm{E}+02$ & 0 & 0 & $6.98 \mathrm{E}+01$ & 0 & 0 & 0 & 0 & $9.02 \mathrm{E}+01$ \\
\hline 6TC024 & 0.719 & 17.3906 & 6 & $1.15 \mathrm{E}+03$ & 7.93E-01 & 0 & 0 & \begin{tabular}{|l|}
$7.30 \mathrm{E}+02$ \\
\end{tabular} & 0 & 0 & $1.04 \mathrm{E}+02$ & 0 & $1.14 \mathrm{E}+02$ & $9.91 \mathrm{E}+02$ & 0 & $1.34 \mathrm{E}+02$ \\
\hline 6TC025 & 0 & 30.9375 & 6 & 0 & 0 & 0 & 0 & 0 & 0 & 0 & 0 & 0 & 0 & 0 & 0 & 0 \\
\hline 6TC026 & 0.641 & 21.7734 & 6 & $1.47 \mathrm{E}+02$ & $4.43 \mathrm{E}-01$ & 0 & 0 & $6.52 \mathrm{E}+02$ & 0 & 0 & $9.27 \mathrm{E}+01$ & 0 & $6.49 \mathrm{E}+01$ & $5.67 \mathrm{E}+02$ & 0 & $1.20 \mathrm{E}+02$ \\
\hline
\end{tabular}


Table 39. Predicted Solids for Envelope C Simulations - Part 1 (cont'd)

\begin{tabular}{|c|c|c|c|c|c|c|c|c|c|c|c|c|c|c|c|c|}
\hline Test ID & $\begin{array}{l}\text { SBS/ } \\
\text { Feed }\end{array}$ & $\begin{array}{l}\text { Temp } \\
{\left[{ }^{\circ} \mathrm{C}\right]}\end{array}$ & $\begin{array}{c}\mathrm{NaM} \\
{[\mathrm{mol} / \mathrm{L}]}\end{array}$ & ALOH3 & BASO4 & CA3PO42 & $\mathrm{CACO} 3$ & CAF2 & CANC & $\mathrm{CAOH} 2$ & CATIO3 & $\mathrm{CROH} 3$ & FEIIIOH3 & \begin{tabular}{|c|} 
HYDROS \\
OD \\
\end{tabular} & MGF2 & MGOH2 \\
\hline 6TC027 & 0.359 & 29.7422 & 6 & 0 & 0 & 0 & 0 & \begin{tabular}{|l|}
$3.62 \mathrm{E}+02$ \\
\end{tabular} & 0 & 0 & $5.15 \mathrm{E}+01$ & 0 & 0 & 0 & 0 & $6.65 \mathrm{E}+01$ \\
\hline $6 \mathrm{TC} 028$ & 1.891 & 29.3438 & 6 & $1.32 \mathrm{E}+03$ & $2.91 \mathrm{E}+00$ & 0 & 0 & $1.92 \mathrm{E}+03$ & 0 & 0 & $2.73 \mathrm{E}+02$ & $1.69 \mathrm{E}+02$ & $4.40 \mathrm{E}+02$ & $3.84 \mathrm{E}+03$ & 0 & $3.52 \mathrm{E}+02$ \\
\hline $6 \mathrm{TC} 029$ & 1.047 & 16.1953 & 6 & $1.79 \mathrm{E}+03$ & $1.60 \mathrm{E}+00$ & 0 & 0 & $1.06 \mathrm{E}+03$ & 0 & 0 & $1.51 \mathrm{E}+02$ & 0 & $2.32 \mathrm{E}+02$ & $2.03 \mathrm{E}+03$ & 0 & $1.95 \mathrm{E}+02$ \\
\hline 6TC030 & 1.563 & 16.5938 & 6 & $1.50 \mathrm{E}+03$ & $2.76 \mathrm{E}+00$ & 0 & 0 & $1.58 \mathrm{E}+03$ & 0 & 0 & $2.25 \mathrm{E}+02$ & 0 & $3.85 \mathrm{E}+02$ & $3.36 \mathrm{E}+03$ & 0 & $2.90 \mathrm{E}+02$ \\
\hline 6TC031 & 1.578 & 22.1719 & 6 & $1.39 \mathrm{E}+03$ & $2.59 \mathrm{E}+00$ & 0 & 0 & $1.60 \mathrm{E}+03$ & 0 & 0 & $2.28 \mathrm{E}+02$ & $2.79 \mathrm{E}+01$ & $3.75 \mathrm{E}+02$ & $3.27 \mathrm{E}+03$ & 0 & $2.94 \mathrm{E}+02$ \\
\hline 6TC032 & 1.547 & 35.7188 & 6 & 0 & $1.93 \mathrm{E}+00$ & 0 & 0 & $1.57 \mathrm{E}+03$ & 0 & 0 & $2.24 \mathrm{E}+02$ & $1.45 \mathrm{E}+02$ & $2.54 \mathrm{E}+02$ & $2.22 \mathrm{E}+03$ & 0 & $2.89 \mathrm{E}+02$ \\
\hline 6TC033 & 1.313 & 30.5391 & 6 & 0 & $1.70 \mathrm{E}+00$ & 0 & 0 & \begin{tabular}{|l|}
$1.33 \mathrm{E}+03$ \\
\end{tabular} & 0 & 0 & $1.89 \mathrm{E}+02$ & $4.54 \mathrm{E}+01$ & $1.90 \mathrm{E}+02$ & $1.66 \mathrm{E}+03$ & 0 & $2.45 \mathrm{E}+02$ \\
\hline 6TC035 & 0.875 & 48.8672 & 6 & 0 & $2.74 \mathrm{E}-01$ & 0 & 0 & $8.88 \mathrm{E}+02$ & 0 & 0 & $1.26 \mathrm{E}+02$ & $8.54 \mathrm{E}+01$ & 0 & 0 & 0 & $1.63 \mathrm{E}+02$ \\
\hline 6TC036 & 0.125 & 57.2344 & 6 & 0 & 0 & 0 & 0 & $1.26 \mathrm{E}+02$ & 0 & 0 & $1.81 \mathrm{E}+01$ & 0 & 0 & 0 & 0 & $2.33 \mathrm{E}+01$ \\
\hline 6TC037 & 0.797 & 53.6484 & 6 & 0 & $1.32 \mathrm{E}-01$ & 0 & 0 & $8.04 \mathrm{E}+02$ & 0 & 0 & $1.14 \mathrm{E}+02$ & $8.43 \mathrm{E}+01$ & 0 & 0 & 0 & $1.48 \mathrm{E}+02$ \\
\hline 6TC038 & 0.094 & 47.6719 & 6 & 0 & 0 & 0 & 0 & $9.43 \mathrm{E}+01$ & 0 & 0 & $1.35 \mathrm{E}+01$ & 0 & 0 & 0 & 0 & $1.75 \mathrm{E}+01$ \\
\hline 6TC039 & 0.625 & 66 & 6 & 0 & 0 & 0 & 0 & $6.33 \mathrm{E}+02$ & 0 & 0 & $9.02 \mathrm{E}+01$ & $7.76 \mathrm{E}+01$ & 0 & 0 & 0 & $1.16 \mathrm{E}+02$ \\
\hline 6TC040 & 0.266 & 49.6641 & 6 & 0 & 0 & 0 & 0 & \begin{tabular}{|l|}
$2.69 \mathrm{E}+02$ \\
\end{tabular} & 0 & 0 & $3.84 \mathrm{E}+01$ & 0 & 0 & 0 & 0 & $4.96 \mathrm{E}+01$ \\
\hline 6TC041 & 0.938 & 53.25 & 6 & 0 & $3.47 \mathrm{E}-01$ & 0 & 0 & $9.52 \mathrm{E}+02$ & 0 & 0 & $1.35 \mathrm{E}+02$ & $1.05 \mathrm{E}+02$ & 0 & 0 & 0 & $1.75 \mathrm{E}+02$ \\
\hline 6TC042 & 1.438 & 63.2109 & 6 & 0 & $1.44 \mathrm{E}+00$ & 0 & 0 & $1.45 \mathrm{E}+03$ & 0 & 0 & $2.07 \mathrm{E}+02$ & $1.89 \mathrm{E}+02$ & 0 & 0 & 0 & $2.67 \mathrm{E}+02$ \\
\hline 6TC044 & 1.938 & 54.8438 & 6 & 0 & $2.01 \mathrm{E}+00$ & 0 & 0 & $1.97 \mathrm{E}+03$ & 0 & 0 & $2.79 \mathrm{E}+02$ & $2.51 \mathrm{E}+02$ & 0 & 0 & 0 & \begin{tabular}{|l|}
$3.61 \mathrm{E}+02$ \\
\end{tabular} \\
\hline 6TC045 & 1.484 & 65.2031 & 6 & 0 & $1.65 \mathrm{E}+00$ & 0 & 0 & $1.51 \mathrm{E}+03$ & 0 & 0 & $2.14 \mathrm{E}+02$ & $1.97 \mathrm{E}+02$ & 0 & 0 & 0 & $2.77 \mathrm{E}+02$ \\
\hline 6TC046 & 1.719 & 55.2422 & 6 & 0 & $1.66 \mathrm{E}+00$ & 0 & 0 & $1.74 \mathrm{E}+03$ & 0 & 0 & $2.48 \mathrm{E}+02$ & $2.21 \mathrm{E}+02$ & 0 & 0 & 0 & $3.20 \mathrm{E}+02$ \\
\hline 6TC047 & 1.188 & 54.4453 & 6 & 0 & $7.66 \mathrm{E}-01$ & 0 & 0 & $1.21 \mathrm{E}+03$ & 0 & 0 & $1.72 \mathrm{E}+02$ & $1.48 \mathrm{E}+02$ & 0 & 0 & 0 & $2.22 \mathrm{E}+02$ \\
\hline 6TC048 & 1.391 & 48.4688 & 6 & 0 & $1.18 \mathrm{E}+00$ & 0 & 0 & $1.41 \mathrm{E}+03$ & 0 & 0 & $2.01 \mathrm{E}+02$ & $1.59 \mathrm{E}+02$ & 0 & 0 & 0 & $2.59 \mathrm{E}+02$ \\
\hline 6TC049 & 1.984 & 57.6328 & 6 & 0 & $2.12 \mathrm{E}+00$ & 0 & 0 & $2.00 \mathrm{E}+03$ & 0 & 0 & $2.85 \mathrm{E}+02$ & $2.59 \mathrm{E}+02$ & 0 & 0 & 0 & $3.68 \mathrm{E}+02$ \\
\hline 6TC050 & 0.156 & 40.1016 & 6 & 0 & 0 & 0 & 0 & $1.58 \mathrm{E}+02$ & 0 & 0 & $2.26 \mathrm{E}+01$ & 0 & 0 & 0 & 0 & $2.91 \mathrm{E}+01$ \\
\hline 6TC051 & 0.547 & 16.9922 & 6 & $9.26 \mathrm{E}+02$ & $3.74 \mathrm{E}-01$ & 0 & 0 & $5.56 \mathrm{E}+02$ & 0 & 0 & $7.91 \mathrm{E}+01$ & 0 & $7.51 \mathrm{E}+01$ & \begin{tabular}{|l|}
$6.56 \mathrm{E}+02$ \\
\end{tabular} & 0 & $1.02 \mathrm{E}+02$ \\
\hline $6 \mathrm{TC} 052$ & 0.219 & 34.9219 & 6 & 0 & 0 & 0 & 0 & $2.21 \mathrm{E}+02$ & 0 & 0 & $3.15 \mathrm{E}+01$ & 0 & 0 & 0 & 0 & $4.07 \mathrm{E}+01$ \\
\hline 6TC053 & 0.203 & 22.5703 & 6 & 0 & 0 & 0 & 0 & $2.06 \mathrm{E}+02$ & 0 & 0 & $2.93 \mathrm{E}+01$ & 0 & 0 & 0 & 0 & $3.78 \mathrm{E}+01$ \\
\hline 6TC054 & 0.922 & 34.125 & 6 & 0 & $6.43 \mathrm{E}-01$ & 0 & 0 & $9.36 \mathrm{E}+02$ & 0 & 0 & $1.33 \mathrm{E}+02$ & $3.59 \mathrm{E}+01$ & $5.62 \mathrm{E}+01$ & \begin{tabular}{|l|}
$4.90 \mathrm{E}+02$ \\
\end{tabular} & 0 & $1.72 \mathrm{E}+02$ \\
\hline 6TC055 & 0.828 & 28.5469 & 6 & 0 & $6.76 \mathrm{E}-01$ & 0 & 0 & $8.41 \mathrm{E}+02$ & 0 & 0 & $1.20 \mathrm{E}+02$ & 0 & $4.31 \mathrm{E}+01$ & \begin{tabular}{|l|}
$3.76 \mathrm{E}+02$ \\
\end{tabular} & 0 & \begin{tabular}{|l|}
$1.54 \mathrm{E}+02$ \\
\end{tabular} \\
\hline 6TC056 & 0.703 & 39.7031 & 6 & 0 & $3.14 \mathrm{E}-02$ & 0 & 0 & $7.13 \mathrm{E}+02$ & 0 & 0 & $1.01 \mathrm{E}+02$ & $2.13 \mathrm{E}+01$ & 0 & 0 & 0 & $1.31 \mathrm{E}+02$ \\
\hline
\end{tabular}


Table 39. Predicted Solids for Envelope C Simulations - Part 1 (cont'd)

\begin{tabular}{|c|c|c|c|c|c|c|c|c|c|c|c|c|c|c|c|c|}
\hline Test ID & $\begin{array}{l}\text { SBS/ } \\
\text { Feed }\end{array}$ & $\begin{array}{l}\text { Temp } \\
{\left[{ }^{\circ} \mathrm{C}\right]}\end{array}$ & $\left.\begin{array}{c}\mathrm{Na} \mathrm{M} \\
{[\mathrm{mol} / \mathrm{L}]}\end{array}\right]$ & ALOH3 & BASO4 & CA3PO42 & $\mathrm{CACO} 3$ & CAF2 & CANC & $\mathrm{CAOH} 2$ & CATIO3 & $\mathrm{CROH} 3$ & FEIIIOH3 & \begin{tabular}{|c|} 
HYDROS \\
OD \\
\end{tabular} & MGF2 & MGOH2 \\
\hline 6TC057 & 1.234 & 20.9766 & 6 & $1.34 \mathrm{E}+03$ & $1.87 \mathrm{E}+00$ & 0 & 0 & $1.25 \mathrm{E}+03$ & 0 & 0 & $1.78 \mathrm{E}+02$ & 0 & $2.76 \mathrm{E}+02$ & $2.41 \mathrm{E}+03$ & 0 & \begin{tabular}{|l|}
$2.30 \mathrm{E}+02$ \\
\end{tabular} \\
\hline $6 \mathrm{TC} 058$ & 1.859 & 30.1406 & 6 & 0 & $2.83 \mathrm{E}+00$ & 0 & 0 & $1.88 \mathrm{E}+03$ & 0 & 0 & $2.68 \mathrm{E}+02$ & $1.62 \mathrm{E}+02$ & $4.15 \mathrm{E}+02$ & $3.62 \mathrm{E}+03$ & 0 & $3.46 \mathrm{E}+02$ \\
\hline 6TC059 & 1.141 & 38.1094 & 6 & 0 & $1.07 \mathrm{E}+00$ & 0 & 0 & $1.15 \mathrm{E}+03$ & 0 & 0 & $1.64 \mathrm{E}+02$ & $8.13 \mathrm{E}+01$ & $2.85 \mathrm{E}+01$ & $2.48 \mathrm{E}+02$ & 0 & $2.11 \mathrm{E}+02$ \\
\hline 6TC060 & 1.109 & 21.375 & 6 & $9.59 \mathrm{E}+02$ & $1.60 \mathrm{E}+00$ & 0 & 0 & $1.13 \mathrm{E}+03$ & 0 & 0 & $1.60 \mathrm{E}+02$ & 0 & $2.10 \mathrm{E}+02$ & $1.83 \mathrm{E}+03$ & 0 & $2.07 \mathrm{E}+02$ \\
\hline 6TC061 & 1.625 & 22.9688 & 6 & $1.64 \mathrm{E}+03$ & $2.66 \mathrm{E}+00$ & 0 & 0 & $1.65 \mathrm{E}+03$ & 0 & 0 & $2.35 \mathrm{E}+02$ & \begin{tabular}{|l|}
$4.76 \mathrm{E}+01$ \\
\end{tabular} & $3.86 \mathrm{E}+02$ & $3.36 \mathrm{E}+03$ & 0 & $3.03 \mathrm{E}+02$ \\
\hline 6TC062 & 1.813 & 36.5156 & 6 & 0 & $2.44 \mathrm{E}+00$ & 0 & 0 & $1.84 \mathrm{E}+03$ & 0 & 0 & $2.62 \mathrm{E}+02$ & \begin{tabular}{|l|}
$1.87 \mathrm{E}+02$ \\
\end{tabular} & $3.26 \mathrm{E}+02$ & $2.85 \mathrm{E}+03$ & 0 & \begin{tabular}{|l|}
$3.38 \mathrm{E}+02$ \\
\end{tabular} \\
\hline 6TC063 & 1.609 & 31.7344 & 6 & 0 & $2.29 \mathrm{E}+00$ & 0 & 0 & $1.63 \mathrm{E}+03$ & 0 & 0 & $2.32 \mathrm{E}+02$ & $1.08 \mathrm{E}+02$ & $2.79 \mathrm{E}+02$ & $2.43 \mathrm{E}+03$ & 0 & $2.99 \mathrm{E}+02$ \\
\hline 6TC065 & 0.078 & 52.0547 & 6 & 0 & 0 & 0 & 0 & $7.84 \mathrm{E}+01$ & 0 & 0 & $1.13 \mathrm{E}+01$ & 0 & 0 & 0 & 0 & $1.46 \mathrm{E}+01$ \\
\hline 6TC066 & 0.781 & 60.4219 & 6 & 0 & $1.19 \mathrm{E}-01$ & 0 & 0 & $7.88 \mathrm{E}+02$ & 0 & 0 & $1.12 \mathrm{E}+02$ & $9.30 \mathrm{E}+01$ & 0 & 0 & 0 & $1.45 \mathrm{E}+02$ \\
\hline 6TC067 & 0.734 & 55.6406 & 6 & 0 & $4.29 \mathrm{E}-03$ & 0 & 0 & $7.44 \mathrm{E}+02$ & 0 & 0 & $1.06 \mathrm{E}+02$ & \begin{tabular}{|l|}
$7.86 \mathrm{E}+01$ \\
\end{tabular} & 0 & 0 & 0 & $1.37 \mathrm{E}+02$ \\
\hline 6TC068 & 0.297 & 60.8203 & 6 & 0 & 0 & 0 & 0 & \begin{tabular}{|l|}
$3.00 \mathrm{E}+02$ \\
\end{tabular} & 0 & 0 & $4.29 \mathrm{E}+01$ & \begin{tabular}{|l|}
$2.64 \mathrm{E}+01$ \\
\end{tabular} & 0 & 0 & 0 & \begin{tabular}{|l|}
$5.54 \mathrm{E}+01$ \\
\end{tabular} \\
\hline 6ТC069 & 0.75 & 42.4922 & 6 & 0 & $6.14 \mathrm{E}-02$ & 0 & 0 & $7.60 \mathrm{E}+02$ & 0 & 0 & $1.08 \mathrm{E}+02$ & $4.82 \mathrm{E}+01$ & 0 & 0 & 0 & $1.40 \mathrm{E}+02$ \\
\hline 6TC070 & 0.531 & 44.8828 & 6 & 0 & 0 & 0 & 0 & \begin{tabular}{|l|}
$5.38 \mathrm{E}+02$ \\
\end{tabular} & 0 & 0 & $7.66 \mathrm{E}+01$ & \begin{tabular}{|l|}
$1.96 \mathrm{E}+01$ \\
\end{tabular} & 0 & 0 & 0 & $9.89 \mathrm{E}+01$ \\
\hline 6TC071 & 1.766 & 46.4766 & 6 & 0 & $1.69 \mathrm{E}+00$ & 0 & 0 & $1.79 \mathrm{E}+03$ & 0 & 0 & $2.54 \mathrm{E}+02$ & \begin{tabular}{|l|}
$2.18 \mathrm{E}+02$ \\
\end{tabular} & $2.13 \mathrm{E}+02$ & \begin{tabular}{|l|}
$1.86 \mathrm{E}+03$ \\
\end{tabular} & 0 & \begin{tabular}{|l|}
$3.28 \mathrm{E}+02$ \\
\end{tabular} \\
\hline 6TC072 & 1.406 & 62.4141 & 6 & 0 & $1.42 \mathrm{E}+00$ & 0 & 0 & $1.43 \mathrm{E}+03$ & 0 & 0 & $2.03 \mathrm{E}+02$ & $1.85 \mathrm{E}+02$ & 0 & 0 & 0 & $2.62 \mathrm{E}+02$ \\
\hline 6TC074 & 1.75 & 43.2891 & 6 & 0 & $1.83 \mathrm{E}+00$ & 0 & 0 & $1.78 \mathrm{E}+03$ & 0 & 0 & $2.53 \mathrm{E}+02$ & $2.01 \mathrm{E}+02$ & $1.81 \mathrm{E}+02$ & $1.58 \mathrm{E}+03$ & 0 & \begin{tabular}{|l|}
$3.26 \mathrm{E}+02$ \\
\end{tabular} \\
\hline 6TC075 & 1.688 & 56.4375 & 6 & 0 & $1.61 \mathrm{E}+00$ & 0 & 0 & $1.71 \mathrm{E}+03$ & 0 & 0 & $2.43 \mathrm{E}+02$ & \begin{tabular}{|l|}
$2.19 \mathrm{E}+02$ \\
\end{tabular} & 0 & 0 & 0 & $3.14 \mathrm{E}+02$ \\
\hline 6TC076 & 1.156 & 61.2188 & 6 & 0 & $8.43 \mathrm{E}-01$ & 0 & 0 & $1.17 \mathrm{E}+03$ & 0 & 0 & $1.67 \mathrm{E}+02$ & $1.50 \mathrm{E}+02$ & 0 & 0 & 0 & $2.16 \mathrm{E}+02$ \\
\hline 6TC077 & 1.531 & 61.6172 & 6 & 0 & $1.56 \mathrm{E}+00$ & 0 & 0 & $1.55 \mathrm{E}+03$ & 0 & 0 & $2.20 \mathrm{E}+02$ & $2.00 \mathrm{E}+02$ & 0 & 0 & 0 & $2.84 \mathrm{E}+02$ \\
\hline 6TC078 & 1 & 40.5 & 6 & 0 & $6.16 \mathrm{E}-01$ & 0 & 0 & $1.01 \mathrm{E}+03$ & 0 & 0 & $1.44 \mathrm{E}+02$ & \begin{tabular}{|l|}
$7.40 \mathrm{E}+01$ \\
\end{tabular} & 0 & 0 & 0 & $1.85 \mathrm{E}+02$ \\
\hline 6TC079 & 1.328 & 43.6875 & 6 & 0 & $1.08 \mathrm{E}+00$ & 0 & 0 & $1.33 \mathrm{E}+03$ & 0 & 0 & $1.89 \mathrm{E}+02$ & \begin{tabular}{|l|}
$1.34 \mathrm{E}+02$ \\
\end{tabular} & 0 & 0 & 0 & $2.44 \mathrm{E}+02$ \\
\hline 6TC080 & 1.656 & 62.8125 & 6 & 0 & $1.81 \mathrm{E}+00$ & 0 & 0 & $1.66 \mathrm{E}+03$ & 0 & 0 & $2.36 \mathrm{E}+02$ & \begin{tabular}{|l|}
$2.17 \mathrm{E}+02$ \\
\end{tabular} & 0 & 0 & 0 & $3.05 \mathrm{E}+02$ \\
\hline 6TC081 & 1.516 & 45.6797 & 6 & 0 & $1.32 \mathrm{E}+00$ & 0 & 0 & $1.52 \mathrm{E}+03$ & 0 & 0 & $2.17 \mathrm{E}+02$ & \begin{tabular}{|l|}
$1.75 \mathrm{E}+02$ \\
\end{tabular} & $8.51 \mathrm{E}+01$ & 7.42E+02 & 0 & $2.80 \mathrm{E}+02$ \\
\hline 6TC082 & 1.281 & 63.6094 & 6 & 0 & $1.09 \mathrm{E}+00$ & 0 & 0 & $1.29 \mathrm{E}+03$ & 0 & 0 & $1.83 \mathrm{E}+02$ & \begin{tabular}{|l|}
$1.67 \mathrm{E}+02$ \\
\end{tabular} & 0 & 0 & 0 & $2.36 \mathrm{E}+02$ \\
\hline 6TC083 & 2 & 50.0625 & 6 & 0 & $2.05 \mathrm{E}+00$ & 0 & 0 & $1.99 \mathrm{E}+03$ & 0 & 0 & $2.83 \mathrm{E}+02$ & $2.50 \mathrm{E}+02$ & $1.67 \mathrm{E}+02$ & $1.45 \mathrm{E}+03$ & 0 & $3.65 \mathrm{E}+02$ \\
\hline 6TC084 & 1.359 & 59.2266 & 6 & 0 & $1.22 \mathrm{E}+00$ & 0 & 0 & $1.36 \mathrm{E}+03$ & 0 & 0 & $1.94 \mathrm{E}+02$ & $1.73 \mathrm{E}+02$ & 0 & 0 & 0 & $2.50 \mathrm{E}+02$ \\
\hline 6TC085 & 1.641 & 51.2578 & 6 & 0 & $1.53 \mathrm{E}+00$ & 0 & 0 & $1.66 \mathrm{E}+03$ & 0 & 0 & $2.36 \mathrm{E}+02$ & $2.07 \mathrm{E}+02$ & $5.66 \mathrm{E}+01$ & $4.94 \mathrm{E}+02$ & 0 & \begin{tabular}{|l|}
$3.05 \mathrm{E}+02$ \\
\end{tabular} \\
\hline 6TC086 & 0.109 & 51.6563 & 6 & 0 & 0 & 0 & 0 & $1.09 \mathrm{E}+02$ & 0 & 0 & $1.56 \mathrm{E}+01$ & 0 & 0 & 0 & 0 & $2.01 \mathrm{E}+01$ \\
\hline
\end{tabular}


Table 39. Predicted Solids for Envelope C Simulations - Part 1 (cont'd)

\begin{tabular}{|c|c|c|c|c|c|c|c|c|c|c|c|c|c|c|c|c|}
\hline Test ID & $\begin{array}{l}\text { SBS/ } \\
\text { Feed }\end{array}$ & $\begin{array}{l}\text { Temp } \\
{\left[{ }^{\circ} \mathrm{C}\right]}\end{array}$ & $\left.\begin{array}{c}\mathrm{Na} \mathrm{M} \\
{[\mathrm{mol} / \mathrm{L}]}\end{array}\right]$ & ALOH3 & BASO4 & CA3PO42 & $\mathrm{CACO} 3$ & CAF2 & CANC & $\mathrm{CAOH} 2$ & CATIO3 & CROH3 & FEIIIOH3 & \begin{tabular}{|c|} 
HYDROS \\
OD \\
\end{tabular} & MGF2 & MGOH2 \\
\hline 6TC087 & 0.953 & 64.8047 & 6 & 0 & $5.15 \mathrm{E}-01$ & 0 & 0 & $9.52 \mathrm{E}+02$ & 0 & 0 & $1.36 \mathrm{E}+02$ & \begin{tabular}{|l|}
$1.20 \mathrm{E}+02$ \\
\end{tabular} & 0 & 0 & 0 & \begin{tabular}{|l|}
$1.75 \mathrm{E}+02$ \\
\end{tabular} \\
\hline $6 \mathrm{TC} 088$ & 0.438 & 64.4063 & 6 & 0 & 0 & 0 & 0 & $4.40 \mathrm{E}+02$ & 0 & 0 & $6.28 \mathrm{E}+01$ & $5.06 \mathrm{E}+01$ & 0 & 0 & 0 & $8.11 \mathrm{E}+01$ \\
\hline 6TC089 & 0.422 & 58.8281 & 6 & 0 & 0 & 0 & 0 & $4.23 \mathrm{E}+02$ & 0 & 0 & $6.03 \mathrm{E}+01$ & $4.01 \mathrm{E}+01$ & 0 & 0 & 0 & $7.79 \mathrm{E}+01$ \\
\hline 6TC090 & 0.453 & 45.2813 & 6 & 0 & 0 & 0 & 0 & $4.53 \mathrm{E}+02$ & 0 & 0 & $6.45 \mathrm{E}+01$ & $5.45 \mathrm{E}+00$ & 0 & 0 & 0 & $8.32 \mathrm{E}+01$ \\
\hline 6TC091 & 0.688 & 50.4609 & 6 & 0 & 0 & 0 & 0 & $6.89 \mathrm{E}+02$ & 0 & 0 & $9.81 \mathrm{E}+01$ & \begin{tabular}{|l|}
$6.50 \mathrm{E}+01$ \\
\end{tabular} & 0 & 0 & 0 & $1.27 \mathrm{E}+02$ \\
\hline 6TC092 & 0.328 & 65.6016 & 6 & 0 & 0 & 0 & 0 & \begin{tabular}{|l|}
$3.28 \mathrm{E}+02$ \\
\end{tabular} & 0 & 0 & $4.69 \mathrm{E}+01$ & \begin{tabular}{|l|}
$3.55 \mathrm{E}+01$ \\
\end{tabular} & 0 & 0 & 0 & $6.06 \mathrm{E}+01$ \\
\hline 6TC093 & 1.125 & 32.1328 & 6 & 0 & $1.14 \mathrm{E}+00$ & 0 & 0 & \begin{tabular}{|l|}
$1.12 \mathrm{E}+03$ \\
\end{tabular} & 0 & 0 & $1.59 \mathrm{E}+02$ & $3.31 \mathrm{E}+01$ & $1.11 \mathrm{E}+02$ & $9.68 \mathrm{E}+02$ & 0 & $2.06 \mathrm{E}+02$ \\
\hline 6TC095 & 1.203 & 27.3516 & 6 & 0 & $1.50 \mathrm{E}+00$ & 0 & 0 & $1.22 \mathrm{E}+03$ & 0 & 0 & $1.73 \mathrm{E}+02$ & $2.10 \mathrm{E}+01$ & $2.17 \mathrm{E}+02$ & $1.90 \mathrm{E}+03$ & 0 & $2.23 \mathrm{E}+02$ \\
\hline 6TC096 & 1.906 & 33.3281 & 6 & 0 & $2.61 \mathrm{E}+00$ & 0 & 0 & $1.91 \mathrm{E}+03$ & 0 & 0 & $2.72 \mathrm{E}+02$ & $2.05 \mathrm{E}+02$ & $4.36 \mathrm{E}+02$ & $3.80 \mathrm{E}+03$ & 0 & \begin{tabular}{|l|}
$3.52 \mathrm{E}+02$ \\
\end{tabular} \\
\hline 6TC097 & 1.375 & 15 & 6 & 0 & $2.37 \mathrm{E}+00$ & 0 & 0 & $1.38 \mathrm{E}+03$ & 0 & 0 & $1.96 \mathrm{E}+02$ & 0 & $3.38 \mathrm{E}+02$ & $2.95 \mathrm{E}+03$ & 0 & $2.53 \mathrm{E}+02$ \\
\hline 6TC098 & 1.734 & 31.3359 & 6 & 0 & $2.47 \mathrm{E}+00$ & 0 & 0 & \begin{tabular}{|l|}
$1.72 \mathrm{E}+03$ \\
\end{tabular} & 0 & 0 & $2.45 \mathrm{E}+02$ & \begin{tabular}{|l|}
$1.31 \mathrm{E}+02$ \\
\end{tabular} & \begin{tabular}{|l|}
$3.31 \mathrm{E}+02$ \\
\end{tabular} & $2.89 \mathrm{E}+03$ & 0 & \begin{tabular}{|l|}
$3.17 \mathrm{E}+02$ \\
\end{tabular} \\
\hline 6TC099 & 1.063 & 27.75 & 6 & 0 & $1.19 \mathrm{E}+00$ & 0 & 0 & \begin{tabular}{|l|}
$1.07 \mathrm{E}+03$ \\
\end{tabular} & 0 & 0 & $1.52 \mathrm{E}+02$ & 0 & $1.64 \mathrm{E}+02$ & \begin{tabular}{|l|}
$1.43 \mathrm{E}+03$ \\
\end{tabular} & 0 & $1.96 \mathrm{E}+02$ \\
\hline 6TC100 & 0.563 & 17.7891 & 6 & 0 & $3.75 \mathrm{E}-01$ & 0 & 0 & \begin{tabular}{|l|}
$5.67 \mathrm{E}+02$ \\
\end{tabular} & 0 & 0 & $8.07 \mathrm{E}+01$ & 0 & $6.74 \mathrm{E}+01$ & $5.88 \mathrm{E}+02$ & 0 & $1.04 \mathrm{E}+02$ \\
\hline 6TC101 & 0.047 & 39.3047 & 6 & 0 & 0 & 0 & 0 & $4.64 \mathrm{E}+01$ & 0 & 0 & $6.68 \mathrm{E}+00$ & 0 & 0 & 0 & 0 & $8.63 \mathrm{E}+00$ \\
\hline 6TC102 & 0.063 & 26.1563 & 6 & 0 & 0 & 0 & 0 & $6.24 \mathrm{E}+01$ & 0 & 0 & $8.91 \mathrm{E}+00$ & 0 & 0 & 0 & 0 & $1.15 \mathrm{E}+01$ \\
\hline 6TC104 & 0.281 & 25.7578 & 6 & 0 & 0 & 0 & 0 & $2.81 \mathrm{E}+02$ & 0 & 0 & $3.99 \mathrm{E}+01$ & 0 & 0 & 0 & 0 & \begin{tabular}{|l}
$5.15 \mathrm{E}+01$ \\
\end{tabular} \\
\hline 6TC105 & 0.813 & 26.5547 & 6 & 0 & $6.95 \mathrm{E}-01$ & 0 & 0 & $8.15 \mathrm{E}+02$ & 0 & 0 & $1.16 \mathrm{E}+02$ & 0 & $5.61 \mathrm{E}+01$ & $4.90 \mathrm{E}+02$ & 0 & $1.50 \mathrm{E}+02$ \\
\hline 6TC106 & 0.609 & 32.5313 & 6 & 0 & 0 & 0 & 0 & $6.09 \mathrm{E}+02$ & 0 & 0 & $8.66 \mathrm{E}+01$ & 0 & 0 & 0 & 0 & $1.12 \mathrm{E}+02$ \\
\hline 6TC107 & 0.016 & 23.3672 & 6 & 0 & 0 & 0 & 0 & $1.55 \mathrm{E}+01$ & 0 & 0 & $2.25 \mathrm{E}+00$ & 0 & 0 & 0 & 0 & $2.90 \mathrm{E}+00$ \\
\hline 6TC108 & 1.844 & 40.8984 & 6 & 0 & $1.96 \mathrm{E}+00$ & 0 & 0 & \begin{tabular}{|l|}
$1.84 \mathrm{E}+03$ \\
\end{tabular} & 0 & 0 & $2.62 \mathrm{E}+02$ & $2.04 \mathrm{E}+02$ & $2.59 \mathrm{E}+02$ & \begin{tabular}{|l|}
$2.26 \mathrm{E}+03$ \\
\end{tabular} & 0 & $3.38 \mathrm{E}+02$ \\
\hline 6TC109 & 1.453 & 64.0078 & 6 & 0 & $1.46 \mathrm{E}+00$ & 0 & 0 & $1.45 \mathrm{E}+03$ & 0 & 0 & $2.06 \mathrm{E}+02$ & \begin{tabular}{|l|}
$1.88 \mathrm{E}+02$ \\
\end{tabular} & 0 & 0 & 0 & $2.66 \mathrm{E}+02$ \\
\hline 6TC110 & 1.781 & 46.0781 & 6 & 0 & $1.68 \mathrm{E}+00$ & 0 & 0 & $1.79 \mathrm{E}+03$ & 0 & 0 & $2.54 \mathrm{E}+02$ & $2.18 \mathrm{E}+02$ & $2.33 \mathrm{E}+02$ & \begin{tabular}{|l|}
$2.03 \mathrm{E}+03$ \\
\end{tabular} & 0 & $3.28 \mathrm{E}+02$ \\
\hline 6TC111 & 1.797 & 58.4297 & 6 & 0 & $1.92 \mathrm{E}+00$ & 0 & 0 & $1.81 \mathrm{E}+03$ & 0 & 0 & $2.58 \mathrm{E}+02$ & $2.34 \mathrm{E}+02$ & 0 & 0 & 0 & $3.32 \mathrm{E}+02$ \\
\hline 6TC112 & 1.078 & 46.875 & 6 & 0 & 7.14E-01 & 0 & 0 & $1.08 \mathrm{E}+03$ & 0 & 0 & $1.54 \mathrm{E}+02$ & \begin{tabular}{|l|}
$1.05 \mathrm{E}+02$ \\
\end{tabular} & 0 & 0 & 0 & $1.99 \mathrm{E}+02$ \\
\hline 6TC113 & 1.172 & 52.4531 & 6 & 0 & 7.95E-01 & 0 & 0 & $1.17 \mathrm{E}+03$ & 0 & 0 & $1.67 \mathrm{E}+02$ & $1.38 \mathrm{E}+02$ & 0 & 0 & 0 & $2.15 \mathrm{E}+02$ \\
\hline 6TC114 & 1.297 & 41.2969 & 6 & 0 & $1.17 \mathrm{E}+00$ & 0 & 0 & $1.30 \mathrm{E}+03$ & 0 & 0 & $1.86 \mathrm{E}+02$ & \begin{tabular}{|l|}
$1.25 \mathrm{E}+02$ \\
\end{tabular} & $5.01 \mathrm{E}+01$ & \begin{tabular}{|l|}
$4.37 \mathrm{E}+02$ \\
\end{tabular} & 0 & $2.40 \mathrm{E}+02$ \\
\hline 6TC115 & 0.766 & 60.0234 & 6 & 0 & 4.92E-02 & 0 & 0 & $7.68 \mathrm{E}+02$ & 0 & 0 & $1.09 \mathrm{E}+02$ & $8.90 \mathrm{E}+01$ & 0 & 0 & 0 & \begin{tabular}{|l|}
$1.41 \mathrm{E}+02$ \\
\end{tabular} \\
\hline 6TC116 & 0.141 & 50.8594 & 6 & 0 & 0 & 0 & 0 & $1.41 \mathrm{E}+02$ & 0 & 0 & $2.01 \mathrm{E}+01$ & 0 & 0 & 0 & 0 & $2.60 \mathrm{E}+01$ \\
\hline
\end{tabular}


Table 39. Predicted Solids for Envelope C Simulations - Part 1 (cont'd)

\begin{tabular}{|c|c|c|c|c|c|c|c|c|c|c|c|c|c|c|c|c|}
\hline Test ID & $\begin{array}{l}\text { SBS/ } \\
\text { Feed }\end{array}$ & $\begin{array}{l}\text { Temp } \\
{\left[{ }^{\circ} \mathrm{C}\right]} \\
\end{array}$ & $\begin{array}{c}\mathrm{Na} \mathrm{M} \\
{[\mathrm{mol} / \mathrm{L}]}\end{array}$ & ALOH3 & BASO4 & CA3PO42 & $\mathrm{CACO} 3$ & CAF2 & CANC & $\mathrm{CAOH} 2$ & CATIO3 & CROH3 & FEIIIOH3 & $\begin{array}{c}\text { HYDROS } \\
\text { OD }\end{array}$ & MGF2 & $\mathrm{MGOH} 2$ \\
\hline 6TC117 & 0.859 & 42.8906 & 6 & 0 & $2.52 \mathrm{E}-01$ & 0 & 0 & \begin{tabular}{|l|}
$8.69 \mathrm{E}+02$ \\
\end{tabular} & 0 & 0 & $1.24 \mathrm{E}+02$ & \begin{tabular}{|l|}
$6.49 \mathrm{E}+01$ \\
\end{tabular} & 0 & 0 & 0 & $1.60 \mathrm{E}+02$ \\
\hline 6TC118 & 0.891 & 59.625 & 6 & 0 & $2.75 \mathrm{E}-01$ & 0 & 0 & $8.91 \mathrm{E}+02$ & 0 & 0 & $1.27 \mathrm{E}+02$ & $1.08 \mathrm{E}+02$ & 0 & 0 & 0 & $1.64 \mathrm{E}+02$ \\
\hline 6TC119 & 0.375 & 58.0313 & 6 & 0 & 0 & 0 & 0 & \begin{tabular}{|l|}
$3.74 \mathrm{E}+02$ \\
\end{tabular} & 0 & 0 & $5.33 \mathrm{E}+01$ & \begin{tabular}{|l|}
$3.09 \mathrm{E}+01$ \\
\end{tabular} & 0 & 0 & 0 & $6.89 \mathrm{E}+01$ \\
\hline 6TC120 & 0.188 & 44.4844 & 6 & 0 & 0 & 0 & 0 & $1.86 \mathrm{E}+02$ & 0 & 0 & $2.66 \mathrm{E}+01$ & \begin{tabular}{|l|}
0 \\
\end{tabular} & 0 & 0 & 0 & $3.43 \mathrm{E}+01$ \\
\hline 6TC121 & 0.391 & 49.2656 & 6 & 0 & 0 & 0 & 0 & \begin{tabular}{|l|}
$3.93 \mathrm{E}+02$ \\
\end{tabular} & 0 & 0 & $5.60 \mathrm{E}+01$ & \begin{tabular}{|l|}
$1.98 \mathrm{E}+01$ \\
\end{tabular} & 0 & 0 & 0 & $7.23 \mathrm{E}+01$ \\
\hline 6TC122 & 1.016 & 18.9844 & 6 & 0 & $1.36 \mathrm{E}+00$ & 0 & 0 & $1.02 \mathrm{E}+03$ & 0 & 0 & $1.45 \mathrm{E}+02$ & 0 & \begin{tabular}{|l|}
$1.99 \mathrm{E}+02$ \\
\end{tabular} & \begin{tabular}{|l|}
$1.74 \mathrm{E}+03$ \\
\end{tabular} & 0 & $1.87 \mathrm{E}+02$ \\
\hline 6TC123 & 1.922 & 28.9453 & 6 & 0 & $2.96 \mathrm{E}+00$ & 0 & 0 & \begin{tabular}{|l|}
$1.93 \mathrm{E}+03$ \\
\end{tabular} & 0 & 0 & $2.75 \mathrm{E}+02$ & \begin{tabular}{|l|}
$1.57 \mathrm{E}+02$ \\
\end{tabular} & \begin{tabular}{|l|}
$4.35 \mathrm{E}+02$ \\
\end{tabular} & $3.80 \mathrm{E}+03$ & 0 & $3.54 \mathrm{E}+02$ \\
\hline 6TC125 & 1.266 & 25.3594 & 6 & 0 & $1.69 \mathrm{E}+00$ & 0 & 0 & \begin{tabular}{|l|}
$1.27 \mathrm{E}+03$ \\
\end{tabular} & 0 & 0 & $1.81 \mathrm{E}+02$ & \begin{tabular}{|l|}
$1.68 \mathrm{E}+01$ \\
\end{tabular} & $2.56 \mathrm{E}+02$ & $2.24 \mathrm{E}+03$ & 0 & $2.33 \mathrm{E}+02$ \\
\hline 6TC126 & 1.703 & 20.1797 & 6 & 0 & $2.93 \mathrm{E}+00$ & 0 & 0 & $1.70 \mathrm{E}+03$ & 0 & 0 & $2.42 \mathrm{E}+02$ & \begin{tabular}{|l|}
$8.53 \mathrm{E}-01$ \\
\end{tabular} & $4.10 \mathrm{E}+02$ & \begin{tabular}{|l|}
$3.57 \mathrm{E}+03$ \\
\end{tabular} & 0 & $3.12 \mathrm{E}+02$ \\
\hline 6TC127 & 1.25 & 38.5078 & 6 & 0 & $1.28 \mathrm{E}+00$ & 0 & 0 & $1.26 \mathrm{E}+03$ & 0 & 0 & $1.79 \mathrm{E}+02$ & \begin{tabular}{|l|}
$9.83 \mathrm{E}+01$ \\
\end{tabular} & \begin{tabular}{|l|}
$5.31 \mathrm{E}+01$ \\
\end{tabular} & $4.64 \mathrm{E}+02$ & 0 & $2.31 \mathrm{E}+02$ \\
\hline 6TC128 & 1.469 & 36.1172 & 6 & 0 & $1.75 \mathrm{E}+00$ & 0 & 0 & $1.48 \mathrm{E}+03$ & 0 & 0 & $2.10 \mathrm{E}+02$ & \begin{tabular}{|l|}
$1.29 \mathrm{E}+02$ \\
\end{tabular} & \begin{tabular}{|l|}
$2.06 \mathrm{E}+02$ \\
\end{tabular} & $1.80 \mathrm{E}+03$ & 0 & $2.71 \mathrm{E}+02$ \\
\hline 6TC129 & 0.234 & 34.5234 & 6 & 0 & 0 & 0 & 0 & \begin{tabular}{|l|}
$2.36 \mathrm{E}+02$ \\
\end{tabular} & 0 & 0 & \begin{tabular}{|l|}
$3.36 \mathrm{E}+01$ \\
\end{tabular} & 0 & 0 & 0 & 0 & $4.34 \mathrm{E}+01$ \\
\hline 6TC130 & 0.594 & 18.5859 & 6 & 0 & $4.03 \mathrm{E}-01$ & 0 & 0 & \begin{tabular}{|l|}
$5.96 \mathrm{E}+02$ \\
\end{tabular} & 0 & 0 & $8.47 \mathrm{E}+01$ & 0 & \begin{tabular}{|l|}
$7.28 \mathrm{E}+01$ \\
\end{tabular} & $6.35 \mathrm{E}+02$ & 0 & $1.09 \mathrm{E}+02$ \\
\hline 6TC131 & 0.906 & 36.9141 & 6 & 0 & $5.27 \mathrm{E}-01$ & 0 & 0 & \begin{tabular}{|l|}
$9.15 \mathrm{E}+02$ \\
\end{tabular} & 0 & 0 & $1.30 \mathrm{E}+02$ & $4.52 \mathrm{E}+01$ & 0 & 0 & 0 & $1.68 \mathrm{E}+02$ \\
\hline 6TC132 & 0.25 & 37.7109 & 6 & 0 & 0 & 0 & 0 & $2.50 \mathrm{E}+02$ & 0 & 0 & $3.56 \mathrm{E}+01$ & 0 & 0 & 0 & 0 & $4.59 \mathrm{E}+01$ \\
\hline 6TC134 & 0.844 & 19.7813 & 6 & 0 & $9.72 \mathrm{E}-01$ & 0 & 0 & $8.47 \mathrm{E}+02$ & 0 & 0 & $1.20 \mathrm{E}+02$ & 0 & \begin{tabular}{|l|}
$1.34 \mathrm{E}+02$ \\
\end{tabular} & \begin{tabular}{|l|}
$1.17 \mathrm{E}+03$ \\
\end{tabular} & 0 & $1.55 \mathrm{E}+02$ \\
\hline 6TC135 & 0.469 & 19.3828 & 6 & 0 & $9.74 \mathrm{E}-02$ & 0 & 0 & $4.73 \mathrm{E}+02$ & 0 & 0 & $6.73 \mathrm{E}+01$ & 0 & $3.35 \mathrm{E}+01$ & $2.93 \mathrm{E}+02$ & 0 & $8.69 \mathrm{E}+01$ \\
\hline
\end{tabular}


Table 40. Predicted Solids for Envelope C Simulations - Part 2

\begin{tabular}{|c|c|c|c|c|c|c|c|c|c|c|c|c|c|}
\hline Test ID & NA2C2O4 & NA2U2O7 & NA3FSO4 & $\begin{array}{c}\text { NA6SO42 } \\
\text { CO3 }\end{array}$ & NAF & NANO3 & $\begin{array}{c}\text { NASGEL. } 1 \\
5.5 \mathrm{H} 2 \mathrm{O}\end{array}$ & $\mathrm{NIOH} 2$ & SRCO3 & $\mathrm{ZRO} 2$ & $\begin{array}{c}\text { Total } \\
\text { Solids } \\
\end{array}$ & \begin{tabular}{|c|} 
Total Soln \\
Mass
\end{tabular} & $\begin{array}{c}\text { Solids \% of } \\
\text { Total Soln } \\
\end{array}$ \\
\hline $10 \mathrm{TC} 001$ & $1.17 \mathrm{E}+03$ & 0 & 0 & 0 & $1.69 \mathrm{E}+03$ & 0 & 0 & 0 & 0 & 0 & $2.85 \mathrm{E}+03$ & $6.29 \mathrm{E}+05$ & $0.45 \%$ \\
\hline $10 \mathrm{TC} 002$ & $1.10 \mathrm{E}+03$ & 0 & 0 & 0 & $1.74 \mathrm{E}+03$ & $2.71 \mathrm{E}+03$ & 0 & 0 & 0 & 0 & $5.55 \mathrm{E}+03$ & $6.27 \mathrm{E}+05$ & $0.89 \%$ \\
\hline $10 \mathrm{TC} 003$ & $1.14 \mathrm{E}+03$ & 0 & 0 & 0 & $1.65 \mathrm{E}+03$ & 0 & 0 & 0 & 0 & 0 & $2.79 \mathrm{E}+03$ & $6.28 \mathrm{E}+05$ & $0.44 \%$ \\
\hline $10 \mathrm{TC} 004$ & $1.06 \mathrm{E}+03$ & 0 & 0 & 0 & $1.68 \mathrm{E}+03$ & 0 & 0 & 0 & 0 & 0 & $2.75 \mathrm{E}+03$ & $6.25 \mathrm{E}+05$ & $0.44 \%$ \\
\hline $10 \mathrm{TC} 005$ & $1.14 \mathrm{E}+03$ & 0 & 0 & 0 & $1.64 \mathrm{E}+03$ & 0 & 0 & 0 & 0 & 0 & $2.78 \mathrm{E}+03$ & $6.27 \mathrm{E}+05$ & $0.44 \%$ \\
\hline $10 \mathrm{TC} 006$ & $6.73 \mathrm{E}+02$ & 0 & 0 & $8.46 \mathrm{E}+03$ & $9.86 \mathrm{E}+02$ & 0 & 0 & 0 & 0 & 0 & $1.01 \mathrm{E}+04$ & $5.91 \mathrm{E}+05$ & $1.71 \%$ \\
\hline $10 \mathrm{TC} 007$ & $6.48 \mathrm{E}+02$ & 0 & 0 & $9.84 \mathrm{E}+03$ & $1.04 \mathrm{E}+03$ & 0 & 0 & 0 & 0 & 0 & $1.15 \mathrm{E}+04$ & $5.90 \mathrm{E}+05$ & $1.95 \%$ \\
\hline $10 \mathrm{TC} 008$ & $6.29 \mathrm{E}+02$ & 0 & 0 & $8.07 \mathrm{E}+03$ & $9.55 \mathrm{E}+02$ & 0 & 0 & 0 & 0 & 0 & $9.65 \mathrm{E}+03$ & $5.89 \mathrm{E}+05$ & $1.64 \%$ \\
\hline $10 \mathrm{TC} 009$ & $5.98 \mathrm{E}+02$ & 0 & 0 & $7.84 \mathrm{E}+03$ & $9.68 \mathrm{E}+02$ & 0 & 0 & 0 & 0 & 0 & $9.40 \mathrm{E}+03$ & $5.88 \mathrm{E}+05$ & $1.60 \%$ \\
\hline $10 \mathrm{TC} 010$ & $6.39 \mathrm{E}+02$ & 0 & 0 & $9.54 \mathrm{E}+03$ & $9.48 \mathrm{E}+02$ & 0 & 0 & 0 & 0 & 0 & $1.11 \mathrm{E}+04$ & $5.87 \mathrm{E}+05$ & $1.90 \%$ \\
\hline 10TC011 & $1.16 \mathrm{E}+03$ & 0 & 0 & 0 & $1.23 \mathrm{E}+03$ & 0 & $7.34 \mathrm{E}+03$ & $5.53 \mathrm{E}+01$ & $4.85 \mathrm{E}+00$ & $4.48 \mathrm{E}+01$ & $1.71 \mathrm{E}+04$ & $7.11 \mathrm{E}+05$ & $2.41 \%$ \\
\hline 10TC012 & $1.09 \mathrm{E}+03$ & 0 & 0 & 0 & $1.28 \mathrm{E}+03$ & 0 & $7.02 \mathrm{E}+03$ & $5.40 \mathrm{E}+01$ & $4.82 \mathrm{E}+00$ & $4.09 \mathrm{E}+01$ & $1.63 \mathrm{E}+04$ & $7.10 \mathrm{E}+05$ & $2.30 \%$ \\
\hline $10 \mathrm{TC} 013$ & $1.13 \mathrm{E}+03$ & 0 & 0 & 0 & $1.06 \mathrm{E}+03$ & 0 & $7.07 \mathrm{E}+03$ & $5.54 \mathrm{E}+01$ & $4.80 \mathrm{E}+00$ & $4.72 \mathrm{E}+01$ & $1.70 \mathrm{E}+04$ & $7.09 \mathrm{E}+05$ & $2.40 \%$ \\
\hline $10 \mathrm{TC} 014$ & $1.04 \mathrm{E}+03$ & 0 & 0 & 0 & $1.19 \mathrm{E}+03$ & 0 & $2.40 \mathrm{E}+03$ & $5.40 \mathrm{E}+01$ & $4.74 \mathrm{E}+00$ & $4.38 \mathrm{E}+01$ & $1.19 \mathrm{E}+04$ & $7.06 \mathrm{E}+05$ & $1.68 \%$ \\
\hline $10 \mathrm{TC} 015$ & $1.12 \mathrm{E}+03$ & 0 & 0 & 0 & $7.78 \mathrm{E}+02$ & 0 & $3.40 \mathrm{E}+03$ & $5.58 \mathrm{E}+01$ & $4.79 \mathrm{E}+00$ & $4.93 \mathrm{E}+01$ & $1.36 \mathrm{E}+04$ & $7.09 \mathrm{E}+05$ & $1.92 \%$ \\
\hline $10 \mathrm{TC} 016$ & $6.86 \mathrm{E}+02$ & 0 & 0 & $2.70 \mathrm{E}+04$ & 0 & 0 & $7.34 \mathrm{E}+03$ & $5.80 \mathrm{E}+01$ & $4.83 \mathrm{E}+00$ & $5.56 \mathrm{E}+01$ & $3.93 \mathrm{E}+04$ & $6.46 \mathrm{E}+05$ & $6.09 \%$ \\
\hline 10TC017 & $6.57 \mathrm{E}+02$ & 0 & 0 & $2.82 \mathrm{E}+04$ & $6.80 \mathrm{E}+01$ & 0 & $6.96 \mathrm{E}+03$ & $5.77 \mathrm{E}+01$ & $4.79 \mathrm{E}+00$ & $5.52 \mathrm{E}+01$ & $4.00 \mathrm{E}+04$ & $6.44 \mathrm{E}+05$ & $6.21 \%$ \\
\hline $10 \mathrm{TC} 018$ & $6.37 \mathrm{E}+02$ & 0 & 0 & $2.63 \mathrm{E}+04$ & 0 & 0 & $7.12 \mathrm{E}+03$ & $5.74 \mathrm{E}+01$ & $4.77 \mathrm{E}+00$ & $5.51 \mathrm{E}+01$ & $3.84 \mathrm{E}+04$ & $6.43 \mathrm{E}+05$ & $5.97 \%$ \\
\hline $10 \mathrm{TC} 019$ & $5.62 \mathrm{E}+02$ & 0 & 0 & $2.57 \mathrm{E}+04$ & 0 & 0 & $2.53 \mathrm{E}+03$ & $5.67 \mathrm{E}+01$ & $4.72 \mathrm{E}+00$ & $5.44 \mathrm{E}+01$ & $3.29 \mathrm{E}+04$ & $6.42 \mathrm{E}+05$ & $5.13 \%$ \\
\hline $10 \mathrm{TC} 020$ & $6.09 \mathrm{E}+02$ & 0 & 0 & $2.76 \mathrm{E}+04$ & 0 & 0 & $3.42 \mathrm{E}+03$ & $5.72 \mathrm{E}+01$ & $4.77 \mathrm{E}+00$ & $5.50 \mathrm{E}+01$ & $3.60 \mathrm{E}+04$ & $6.41 \mathrm{E}+05$ & $5.62 \%$ \\
\hline 10TC021 & $9.98 \mathrm{E}+02$ & 0 & $1.35 \mathrm{E}+04$ & $\begin{array}{l}0 \\
\end{array}$ & 0 & 0 & $1.01 \mathrm{E}+03$ & $1.79 \mathrm{E}+01$ & $1.56 \mathrm{E}+00$ & $1.51 \mathrm{E}+01$ & $1.66 \mathrm{E}+04$ & $6.27 \mathrm{E}+05$ & $2.65 \%$ \\
\hline $10 \mathrm{TC} 022$ & $1.15 \mathrm{E}+03$ & 0 & 0 & 0 & $1.55 \mathrm{E}+03$ & 0 & 0 & $5.31 \mathrm{E}+00$ & 7.59E-01 & 0 & $3.17 \mathrm{E}+03$ & $6.41 \mathrm{E}+05$ & $0.49 \%$ \\
\hline $10 \mathrm{TC} 023$ & $9.95 \mathrm{E}+02$ & 0 & $1.22 \mathrm{E}+04$ & 0 & 0 & 0 & 0 & $1.21 \mathrm{E}+01$ & $1.09 \mathrm{E}+00$ & $8.82 \mathrm{E}+00$ & $1.39 \mathrm{E}+04$ & $6.23 \mathrm{E}+05$ & $2.23 \%$ \\
\hline $10 \mathrm{TC} 024$ & $1.11 \mathrm{E}+03$ & 0 & 0 & 0 & $1.51 \mathrm{E}+03$ & 0 & $1.14 \mathrm{E}+03$ & $1.58 \mathrm{E}+01$ & $1.68 \mathrm{E}+00$ & $2.97 \mathrm{E}+00$ & $4.74 \mathrm{E}+03$ & $6.57 \mathrm{E}+05$ & $0.72 \%$ \\
\hline $10 \mathrm{TC} 025$ & $1.06 \mathrm{E}+03$ & 0 & $1.01 \mathrm{E}+04$ & 0 & 0 & 0 & 0 & 0 & 0 & 0 & $1.12 \mathrm{E}+04$ & $6.10 \mathrm{E}+05$ & $1.84 \%$ \\
\hline $10 \mathrm{TC} 026$ & $1.12 \mathrm{E}+03$ & 0 & $1.40 \mathrm{E}+04$ & 0 & 0 & 0 & $8.92 \mathrm{E}+02$ & $1.49 \mathrm{E}+01$ & $1.49 \mathrm{E}+00$ & $6.74 \mathrm{E}+00$ & $1.69 \mathrm{E}+04$ & $6.35 \mathrm{E}+05$ & $2.66 \%$ \\
\hline $10 \mathrm{TC} 027$ & $1.02 \mathrm{E}+03$ & 0 & $1.21 \mathrm{E}+04$ & 0 & 0 & 0 & 0 & $7.46 \mathrm{E}+00$ & $7.74 \mathrm{E}-01$ & $2.66 \mathrm{E}+00$ & $1.36 \mathrm{E}+04$ & $6.20 \mathrm{E}+05$ & $2.19 \%$ \\
\hline $10 \mathrm{TC} 028$ & $1.08 \mathrm{E}+03$ & 0 & $2.10 \mathrm{E}+04$ & 0 & 0 & 0 & $6.92 \mathrm{E}+03$ & $5.36 \mathrm{E}+01$ & $4.57 \mathrm{E}+00$ & $4.96 \mathrm{E}+01$ & $3.49 \mathrm{E}+04$ & $6.70 \mathrm{E}+05$ & $5.20 \%$ \\
\hline $10 \mathrm{TC} 029$ & $1.14 \mathrm{E}+03$ & 0 & 0 & 0 & $1.43 \mathrm{E}+03$ & 0 & $2.90 \mathrm{E}+03$ & $2.61 \mathrm{E}+01$ & $2.49 \mathrm{E}+00$ & $1.37 \mathrm{E}+01$ & $8.00 \mathrm{E}+03$ & $6.71 \mathrm{E}+05$ & $1.19 \%$ \\
\hline
\end{tabular}


Table 40. Predicted Solids for Envelope C Simulations - Part 2 (cont'd)

\begin{tabular}{|c|c|c|c|c|c|c|c|c|c|c|c|c|c|}
\hline Test ID & $\mathrm{NA} 2 \mathrm{C} 2 \mathrm{O} 4$ & NA2U2O7 & NA3FSO4 & $\begin{array}{c}\text { NA6SO42 } \\
\text { CO3 }\end{array}$ & NAF & NANO3 & $\begin{array}{c}\text { NASGEL. } 1 \\
5.5 \mathrm{H} 2 \mathrm{O}\end{array}$ & $\mathrm{NIOH} 2$ & SRCO3 & ZRO2 & Total Solids & $\begin{array}{c}\text { Total Soln } \\
\text { Mass }\end{array}$ & $\begin{array}{c}\text { Solids \% of } \\
\text { Total Soln }\end{array}$ \\
\hline 10TC030 & $1.09 \mathrm{E}+03$ & 0 & 0 & 0 & $1.33 \mathrm{E}+03$ & 0 & $5.05 \mathrm{E}+03$ & $4.13 \mathrm{E}+01$ & $3.73 \mathrm{E}+00$ & $2.99 \mathrm{E}+01$ & $1.23 \mathrm{E}+04$ & $6.91 \mathrm{E}+05$ & $1.78 \%$ \\
\hline $10 \mathrm{TC} 031$ & $1.12 \mathrm{E}+03$ & 0 & $1.96 \mathrm{E}+04$ & 0 & 0 & 0 & $5.38 \mathrm{E}+03$ & $4.34 \mathrm{E}+01$ & $3.79 \mathrm{E}+00$ & $3.70 \mathrm{E}+01$ & $3.10 \mathrm{E}+04$ & $6.65 \mathrm{E}+05$ & $4.67 \%$ \\
\hline 10TC032 & $1.04 \mathrm{E}+03$ & 0 & $1.90 \mathrm{E}+04$ & $1.53 \mathrm{E}+03$ & 0 & 0 & $5.30 \mathrm{E}+03$ & $4.38 \mathrm{E}+01$ & $3.72 \mathrm{E}+00$ & $4.06 \mathrm{E}+01$ & $2.95 \mathrm{E}+04$ & $6.53 \mathrm{E}+05$ & $4.52 \%$ \\
\hline $10 \mathrm{TC} 033$ & $1.04 \mathrm{E}+03$ & 0 & $1.82 \mathrm{E}+04$ & 0 & 0 & 0 & $3.88 \mathrm{E}+03$ & $3.60 \mathrm{E}+01$ & $3.13 \mathrm{E}+00$ & $3.15 \mathrm{E}+01$ & $2.53 \mathrm{E}+04$ & $6.50 \mathrm{E}+05$ & $3.89 \%$ \\
\hline 10TC034 & $1.14 \mathrm{E}+03$ & 0 & 0 & 0 & $1.31 \mathrm{E}+03$ & 0 & $5.72 \mathrm{E}+03$ & $4.49 \mathrm{E}+01$ & $4.03 \mathrm{E}+00$ & $3.33 \mathrm{E}+01$ & $1.38 \mathrm{E}+04$ & $6.97 \mathrm{E}+05$ & $1.98 \%$ \\
\hline 10TC035 & $8.90 \mathrm{E}+02$ & 0 & 0 & $1.64 \mathrm{E}+04$ & $1.16 \mathrm{E}+03$ & 0 & $1.99 \mathrm{E}+03$ & $2.46 \mathrm{E}+01$ & $2.05 \mathrm{E}+00$ & $2.27 \mathrm{E}+01$ & $2.17 \mathrm{E}+04$ & $6.24 \mathrm{E}+05$ & $3.47 \%$ \\
\hline $10 \mathrm{TC} 036$ & $7.99 \mathrm{E}+02$ & 0 & 0 & $9.10 \mathrm{E}+03$ & $1.12 \mathrm{E}+03$ & 0 & 0 & $3.04 \mathrm{E}+00$ & $2.05 \mathrm{E}-01$ & $2.02 \mathrm{E}+00$ & $1.12 \mathrm{E}+04$ & $5.99 \mathrm{E}+05$ & $1.87 \%$ \\
\hline 10TC037 & $8.02 \mathrm{E}+02$ & 0 & 0 & $1.51 \mathrm{E}+04$ & $1.09 \mathrm{E}+03$ & 0 & $1.36 \mathrm{E}+03$ & $2.24 \mathrm{E}+01$ & $1.84 \mathrm{E}+00$ & $2.07 \mathrm{E}+01$ & $1.95 \mathrm{E}+04$ & $6.18 \mathrm{E}+05$ & $3.16 \%$ \\
\hline 10TC038 & $8.89 \mathrm{E}+02$ & 0 & 0 & $8.83 \mathrm{E}+03$ & $1.31 \mathrm{E}+03$ & 0 & 0 & $1.50 \mathrm{E}+00$ & $1.18 \mathrm{E}-01$ & 0 & $1.12 \mathrm{E}+04$ & $6.03 \mathrm{E}+05$ & $1.85 \%$ \\
\hline 10TC039 & $6.49 \mathrm{E}+02$ & 0 & 0 & $1.55 \mathrm{E}+04$ & $9.11 \mathrm{E}+02$ & 0 & $9.71 \mathrm{E}+02$ & $1.78 \mathrm{E}+01$ & $1.43 \mathrm{E}+00$ & $1.67 \mathrm{E}+01$ & $1.90 \mathrm{E}+04$ & $6.06 \mathrm{E}+05$ & $3.14 \%$ \\
\hline $10 \mathrm{TC} 040$ & $8.97 \mathrm{E}+02$ & 0 & 0 & $1.13 \mathrm{E}+04$ & $1.23 \mathrm{E}+03$ & 0 & 0 & $6.84 \mathrm{E}+00$ & $5.52 \mathrm{E}-01$ & $5.34 \mathrm{E}+00$ & $1.38 \mathrm{E}+04$ & $6.06 \mathrm{E}+05$ & $2.27 \%$ \\
\hline 10TC041 & $8.46 \mathrm{E}+02$ & 0 & 0 & $1.80 \mathrm{E}+04$ & $1.09 \mathrm{E}+03$ & 0 & $2.23 \mathrm{E}+03$ & $2.66 \mathrm{E}+01$ & $2.20 \mathrm{E}+00$ & $2.48 \mathrm{E}+01$ & $2.36 \mathrm{E}+04$ & $6.22 \mathrm{E}+05$ & $3.79 \%$ \\
\hline 10TC042 & $6.97 \mathrm{E}+02$ & 0 & 0 & $2.21 \mathrm{E}+04$ & $6.07 \mathrm{E}+02$ & 0 & $4.72 \mathrm{E}+03$ & $4.13 \mathrm{E}+01$ & $3.42 \mathrm{E}+00$ & $3.95 \mathrm{E}+01$ & $3.06 \mathrm{E}+04$ & $6.30 \mathrm{E}+05$ & $4.87 \%$ \\
\hline $10 \mathrm{TC} 044$ & $8.20 \mathrm{E}+02$ & 0 & 0 & $2.63 \mathrm{E}+04$ & $4.12 \mathrm{E}+02$ & 0 & $6.84 \mathrm{E}+03$ & $5.58 \mathrm{E}+01$ & $4.65 \mathrm{E}+00$ & $5.32 \mathrm{E}+01$ & $3.81 \mathrm{E}+04$ & $6.50 \mathrm{E}+05$ & $5.87 \%$ \\
\hline $10 \mathrm{TC} 045$ & $6.79 \mathrm{E}+02$ & 0 & 0 & $2.20 \mathrm{E}+04$ & $5.16 \mathrm{E}+02$ & 0 & $4.98 \mathrm{E}+03$ & $4.28 \mathrm{E}+01$ & $3.55 \mathrm{E}+00$ & $4.09 \mathrm{E}+01$ & $3.09 \mathrm{E}+04$ & $6.31 \mathrm{E}+05$ & $4.89 \%$ \\
\hline 10TC046 & $8.23 \mathrm{E}+02$ & 0 & 0 & $2.50 \mathrm{E}+04$ & $6.45 \mathrm{E}+02$ & 0 & $5.89 \mathrm{E}+03$ & $4.95 \mathrm{E}+01$ & $4.12 \mathrm{E}+00$ & $4.71 \mathrm{E}+01$ & $3.55 \mathrm{E}+04$ & $6.43 \mathrm{E}+05$ & $5.52 \%$ \\
\hline 10TC047 & $8.59 \mathrm{E}+02$ & 0 & 0 & $2.02 \mathrm{E}+04$ & $1.01 \mathrm{E}+03$ & 0 & $3.84 \mathrm{E}+03$ & $3.41 \mathrm{E}+01$ & $2.83 \mathrm{E}+00$ & $3.23 \mathrm{E}+01$ & $2.78 \mathrm{E}+04$ & $6.28 \mathrm{E}+05$ & $4.42 \%$ \\
\hline 10TC048 & $8.94 \mathrm{E}+02$ & 0 & 0 & $2.16 \mathrm{E}+04$ & $1.11 \mathrm{E}+03$ & 0 & $4.30 \mathrm{E}+03$ & $3.96 \mathrm{E}+01$ & $3.31 \mathrm{E}+00$ & $3.73 \mathrm{E}+01$ & $3.00 \mathrm{E}+04$ & $6.37 \mathrm{E}+05$ & $4.71 \%$ \\
\hline 10TC049 & $7.74 \mathrm{E}+02$ & 0 & 0 & $2.68 \mathrm{E}+04$ & $2.60 \mathrm{E}+02$ & 0 & $6.82 \mathrm{E}+03$ & $5.69 \mathrm{E}+01$ & $4.75 \mathrm{E}+00$ & $5.44 \mathrm{E}+01$ & $3.86 \mathrm{E}+04$ & $6.48 \mathrm{E}+05$ & $5.95 \%$ \\
\hline $10 \mathrm{TC} 050$ & $9.79 \mathrm{E}+02$ & 0 & 0 & $9.19 \mathrm{E}+03$ & $1.39 \mathrm{E}+03$ & 0 & 0 & $2.88 \mathrm{E}+00$ & $2.76 \mathrm{E}-01$ & $5.18 \mathrm{E}-01$ & $1.18 \mathrm{E}+04$ & $6.10 \mathrm{E}+05$ & $1.93 \%$ \\
\hline 10TC051 & $1.15 \mathrm{E}+03$ & 0 & 0 & 0 & $1.52 \mathrm{E}+03$ & 0 & $5.83 \mathrm{E}+02$ & $1.12 \mathrm{E}+01$ & $1.26 \mathrm{E}+00$ & 0 & $4.01 \mathrm{E}+03$ & $6.50 \mathrm{E}+05$ & $0.62 \%$ \\
\hline 10TC052 & $9.95 \mathrm{E}+02$ & 0 & $1.09 \mathrm{E}+04$ & 0 & 0 & 0 & 0 & $3.92 \mathrm{E}+00$ & $4.31 \mathrm{E}-01$ & $3.57 \mathrm{E}-01$ & $1.22 \mathrm{E}+04$ & $6.14 \mathrm{E}+05$ & $1.99 \%$ \\
\hline $10 \mathrm{TC} 053$ & $1.11 \mathrm{E}+03$ & 0 & $1.10 \mathrm{E}+04$ & 0 & 0 & 0 & 0 & $1.67 \mathrm{E}+00$ & $4.04 \mathrm{E}-01$ & 0 & $1.24 \mathrm{E}+04$ & $6.21 \mathrm{E}+05$ & $2.00 \%$ \\
\hline $10 \mathrm{TC} 054$ & $1.05 \mathrm{E}+03$ & 0 & $1.59 \mathrm{E}+04$ & 0 & 0 & 0 & $2.42 \mathrm{E}+03$ & $2.53 \mathrm{E}+01$ & $2.18 \mathrm{E}+00$ & $2.22 \mathrm{E}+01$ & $2.10 \mathrm{E}+04$ & $6.36 \mathrm{E}+05$ & $3.30 \%$ \\
\hline 10TC055 & $1.05 \mathrm{E}+03$ & 0 & $1.55 \mathrm{E}+04$ & 0 & 0 & 0 & $1.68 \mathrm{E}+03$ & $2.15 \mathrm{E}+01$ & $1.94 \mathrm{E}+00$ & $1.64 \mathrm{E}+01$ & $1.93 \mathrm{E}+04$ & $6.36 \mathrm{E}+05$ & $3.03 \%$ \\
\hline $10 \mathrm{TC} 056$ & $9.79 \mathrm{E}+02$ & 0 & $1.45 \mathrm{E}+04$ & 0 & 0 & 0 & $1.01 \mathrm{E}+03$ & $1.90 \mathrm{E}+01$ & $1.64 \mathrm{E}+00$ & $1.64 \mathrm{E}+01$ & $1.77 \mathrm{E}+04$ & $6.25 \mathrm{E}+05$ & $2.83 \%$ \\
\hline $10 \mathrm{TC} 057$ & $1.14 \mathrm{E}+03$ & 0 & $1.71 \mathrm{E}+04$ & 0 & 0 & 0 & $3.90 \mathrm{E}+03$ & $3.31 \mathrm{E}+01$ & $2.95 \mathrm{E}+00$ & $2.60 \mathrm{E}+01$ & $2.55 \mathrm{E}+04$ & $6.55 \mathrm{E}+05$ & $3.89 \%$ \\
\hline 10TC059 & $9.61 \mathrm{E}+02$ & 0 & $1.67 \mathrm{E}+04$ & 0 & 0 & 0 & $2.94 \mathrm{E}+03$ & $3.16 \mathrm{E}+01$ & $2.69 \mathrm{E}+00$ & $2.87 \mathrm{E}+01$ & $2.25 \mathrm{E}+04$ & $6.39 \mathrm{E}+05$ & $3.53 \%$ \\
\hline 10TC060 & $1.10 \mathrm{E}+03$ & 0 & $1.68 \mathrm{E}+04$ & 0 & 0 & 0 & $3.09 \mathrm{E}+03$ & $2.88 \mathrm{E}+01$ & $2.64 \mathrm{E}+00$ & $2.07 \mathrm{E}+01$ & $2.27 \mathrm{E}+04$ & $6.50 \mathrm{E}+05$ & $3.50 \%$ \\
\hline
\end{tabular}


Table 40. Predicted Solids for Envelope C Simulations - Part 2 (cont'd)

\begin{tabular}{|c|c|c|c|c|c|c|c|c|c|c|c|c|c|}
\hline Test ID & NA2C2O4 & NA2U2O7 & NA3FSO4 & $\begin{array}{c}\text { NA6SO42 } \\
\text { CO3 }\end{array}$ & NAF & NANO3 & $\begin{array}{c}\text { NASGEL.1 } \\
5.5 \mathrm{H} 2 \mathrm{O}\end{array}$ & $\mathrm{NIOH} 2$ & SRCO3 & $\mathrm{ZRO} 2$ & Total Solids & $\begin{array}{c}\text { Total Soln } \\
\text { Mass }\end{array}$ & $\begin{array}{l}\text { Solids \% of } \\
\text { Total Soln }\end{array}$ \\
\hline 10TC061 & $1.12 \mathrm{E}+03$ & 0 & $1.98 \mathrm{E}+04$ & 0 & 0 & 0 & $5.63 \mathrm{E}+03$ & $4.50 \mathrm{E}+01$ & $3.91 \mathrm{E}+00$ & $3.90 \mathrm{E}+01$ & $3.17 \mathrm{E}+04$ & $6.66 \mathrm{E}+05$ & $4.76 \%$ \\
\hline 10TC062 & $1.02 \mathrm{E}+03$ & 0 & $2.01 \mathrm{E}+04$ & $2.80 \mathrm{E}+03$ & 0 & 0 & $6.42 \mathrm{E}+03$ & $5.16 \mathrm{E}+01$ & $4.38 \mathrm{E}+00$ & $4.83 \mathrm{E}+01$ & $3.35 \mathrm{E}+04$ & $6.60 \mathrm{E}+05$ & $5.07 \%$ \\
\hline 10TC063 & $1.02 \mathrm{E}+03$ & 0 & $1.95 \mathrm{E}+04$ & $7.04 \mathrm{E}+02$ & 0 & 0 & $5.20 \mathrm{E}+03$ & $4.48 \mathrm{E}+01$ & $3.85 \mathrm{E}+00$ & $4.06 \mathrm{E}+01$ & $2.91 \mathrm{E}+04$ & $6.58 \mathrm{E}+05$ & $4.43 \%$ \\
\hline 10TC064 & $7.21 \mathrm{E}+02$ & 0 & 0 & $1.76 \mathrm{E}+04$ & $9.22 \mathrm{E}+02$ & 0 & $2.73 \mathrm{E}+03$ & $2.82 \mathrm{E}+01$ & $2.32 \mathrm{E}+00$ & $2.67 \mathrm{E}+01$ & $2.35 \mathrm{E}+04$ & $6.19 \mathrm{E}+05$ & $3.79 \%$ \\
\hline 10TC065 & $8.65 \mathrm{E}+02$ & 0 & 0 & $8.86 \mathrm{E}+03$ & $1.21 \mathrm{E}+03$ & 0 & 0 & $1.45 \mathrm{E}+00$ & $8.74 \mathrm{E}-02$ & $2.26 \mathrm{E}-01$ & $1.10 \mathrm{E}+04$ & $6.00 \mathrm{E}+05$ & $1.84 \%$ \\
\hline 10TC066 & $7.15 \mathrm{E}+02$ & 0 & 0 & $1.59 \mathrm{E}+04$ & $9.84 \mathrm{E}+02$ & 0 & $1.34 \mathrm{E}+03$ & $2.21 \mathrm{E}+01$ & $1.80 \mathrm{E}+00$ & $2.07 \mathrm{E}+01$ & $2.01 \mathrm{E}+04$ & $6.13 \mathrm{E}+05$ & $3.28 \%$ \\
\hline 10TC067 & $7.95 \mathrm{E}+02$ & 0 & 0 & $1.50 \mathrm{E}+04$ & $1.08 \mathrm{E}+03$ & 0 & $1.16 \mathrm{E}+03$ & $2.07 \mathrm{E}+01$ & $1.70 \mathrm{E}+00$ & $1.91 \mathrm{E}+01$ & $1.91 \mathrm{E}+04$ & $6.16 \mathrm{E}+05$ & $3.10 \%$ \\
\hline 10TC068 & $7.44 \mathrm{E}+02$ & 0 & 0 & $1.24 \mathrm{E}+04$ & $1.04 \mathrm{E}+03$ & 0 & 0 & $8.17 \mathrm{E}+00$ & $6.25 \mathrm{E}-01$ & $7.15 \mathrm{E}+00$ & $1.46 \mathrm{E}+04$ & $6.00 \mathrm{E}+05$ & $2.43 \%$ \\
\hline 10TC069 & $9.66 \mathrm{E}+02$ & 0 & 0 & $1.47 \mathrm{E}+04$ & $1.24 \mathrm{E}+03$ & 0 & $1.42 \mathrm{E}+03$ & $2.07 \mathrm{E}+01$ & $1.74 \mathrm{E}+00$ & $1.85 \mathrm{E}+01$ & $1.94 \mathrm{E}+04$ & $6.24 \mathrm{E}+05$ & $3.11 \%$ \\
\hline $10 \mathrm{TC} 070$ & $9.22 \mathrm{E}+02$ & 0 & 0 & $1.32 \mathrm{E}+04$ & $1.26 \mathrm{E}+03$ & 0 & $2.93 \mathrm{E}+02$ & $1.43 \mathrm{E}+01$ & $1.20 \mathrm{E}+00$ & $1.24 \mathrm{E}+01$ & $1.64 \mathrm{E}+04$ & $6.16 \mathrm{E}+05$ & $2.66 \%$ \\
\hline 10TC071 & $9.28 \mathrm{E}+02$ & 0 & 0 & $2.42 \mathrm{E}+04$ & $1.05 \mathrm{E}+03$ & 0 & $6.30 \mathrm{E}+03$ & $5.06 \mathrm{E}+01$ & $4.23 \mathrm{E}+00$ & $4.81 \mathrm{E}+01$ & $3.51 \mathrm{E}+04$ & $6.49 \mathrm{E}+05$ & $5.41 \%$ \\
\hline 10TC072 & $7.20 \mathrm{E}+02$ & 0 & 0 & $2.13 \mathrm{E}+04$ & $6.96 \mathrm{E}+02$ & 0 & $4.65 \mathrm{E}+03$ & $4.05 \mathrm{E}+01$ & $3.36 \mathrm{E}+00$ & $3.86 \mathrm{E}+01$ & $2.97 \mathrm{E}+04$ & $6.30 \mathrm{E}+05$ & $4.72 \%$ \\
\hline $10 \mathrm{TC} 073$ & $9.16 \mathrm{E}+02$ & 0 & 0 & $1.77 \mathrm{E}+04$ & $1.20 \mathrm{E}+03$ & 0 & $2.78 \mathrm{E}+03$ & $3.06 \mathrm{E}+01$ & $2.57 \mathrm{E}+00$ & $2.82 \mathrm{E}+01$ & $2.42 \mathrm{E}+04$ & $6.32 \mathrm{E}+05$ & $3.83 \%$ \\
\hline $10 \mathrm{TC} 075$ & $8.07 \mathrm{E}+02$ & 0 & 0 & $2.48 \mathrm{E}+04$ & $9.37 \mathrm{E}+02$ & 0 & $5.85 \mathrm{E}+03$ & $4.85 \mathrm{E}+01$ & $4.04 \mathrm{E}+00$ & $4.63 \mathrm{E}+01$ & $3.50 \mathrm{E}+04$ & $6.40 \mathrm{E}+05$ & $5.47 \%$ \\
\hline 10TC076 & $7.53 \mathrm{E}+02$ & 0 & 0 & $1.99 \mathrm{E}+04$ & $9.07 \mathrm{E}+02$ & 0 & $3.71 \mathrm{E}+03$ & $3.33 \mathrm{E}+01$ & $2.75 \mathrm{E}+00$ & $3.17 \mathrm{E}+01$ & $2.70 \mathrm{E}+04$ & $6.23 \mathrm{E}+05$ & $4.34 \%$ \\
\hline 10TC077 & $7.23 \mathrm{E}+02$ & 0 & 0 & $2.29 \mathrm{E}+04$ & $6.01 \mathrm{E}+02$ & 0 & $4.92 \mathrm{E}+03$ & $4.39 \mathrm{E}+01$ & $3.65 \mathrm{E}+00$ & $4.19 \mathrm{E}+01$ & $3.19 \mathrm{E}+04$ & $6.33 \mathrm{E}+05$ & $5.03 \%$ \\
\hline 10TC078 & $9.60 \mathrm{E}+02$ & 0 & 0 & $1.66 \mathrm{E}+04$ & $1.24 \mathrm{E}+03$ & 0 & $2.34 \mathrm{E}+03$ & $2.77 \mathrm{E}+01$ & $2.34 \mathrm{E}+00$ & $2.52 \mathrm{E}+01$ & $2.25 \mathrm{E}+04$ & $6.32 \mathrm{E}+05$ & $3.57 \%$ \\
\hline 10TC079 & $8.87 \mathrm{E}+02$ & 0 & 0 & $2.02 \mathrm{E}+04$ & $1.14 \mathrm{E}+03$ & 0 & $2.28 \mathrm{E}+03$ & $3.71 \mathrm{E}+01$ & $3.11 \mathrm{E}+00$ & $3.46 \mathrm{E}+01$ & $2.64 \mathrm{E}+04$ & $6.36 \mathrm{E}+05$ & $4.15 \%$ \\
\hline 10TC080 & $6.69 \mathrm{E}+02$ & 0 & 0 & $2.39 \mathrm{E}+04$ & $8.29 \mathrm{E}+02$ & 0 & $4.99 \mathrm{E}+03$ & $4.72 \mathrm{E}+01$ & $3.91 \mathrm{E}+00$ & $4.51 \mathrm{E}+01$ & $3.29 \mathrm{E}+04$ & $6.34 \mathrm{E}+05$ & $5.19 \%$ \\
\hline 10TC081 & $9.06 \mathrm{E}+02$ & 0 & 0 & $2.20 \mathrm{E}+04$ & $1.03 \mathrm{E}+03$ & 0 & $4.23 \mathrm{E}+03$ & $4.29 \mathrm{E}+01$ & $3.59 \mathrm{E}+00$ & $4.06 \mathrm{E}+01$ & $3.05 \mathrm{E}+04$ & $6.41 \mathrm{E}+05$ & $4.75 \%$ \\
\hline 10TC082 & $6.67 \mathrm{E}+02$ & 0 & 0 & $2.13 \mathrm{E}+04$ & $7.33 \mathrm{E}+02$ & 0 & $3.35 \mathrm{E}+03$ & $3.66 \mathrm{E}+01$ & $3.01 \mathrm{E}+00$ & $3.49 \mathrm{E}+01$ & $2.82 \mathrm{E}+04$ & $6.23 \mathrm{E}+05$ & $4.52 \%$ \\
\hline 10TC083 & $7.85 \mathrm{E}+02$ & 0 & 0 & $2.52 \mathrm{E}+04$ & $3.45 \mathrm{E}+02$ & 0 & $2.85 \mathrm{E}+03$ & $5.65 \mathrm{E}+01$ & $4.72 \mathrm{E}+00$ & $5.39 \mathrm{E}+01$ & $3.30 \mathrm{E}+04$ & $6.52 \mathrm{E}+05$ & $5.06 \%$ \\
\hline 10TC084 & $7.06 \mathrm{E}+02$ & 0 & 0 & $2.05 \mathrm{E}+04$ & $8.71 \mathrm{E}+02$ & 0 & $3.26 \mathrm{E}+03$ & $3.86 \mathrm{E}+01$ & $3.19 \mathrm{E}+00$ & $3.67 \mathrm{E}+01$ & $2.75 \mathrm{E}+04$ & $6.28 \mathrm{E}+05$ & $4.37 \%$ \\
\hline 10TC085 & $8.79 \mathrm{E}+02$ & 0 & 0 & $2.32 \mathrm{E}+04$ & $9.91 \mathrm{E}+02$ & 0 & $5.67 \mathrm{E}+03$ & $4.71 \mathrm{E}+01$ & $3.93 \mathrm{E}+00$ & $4.48 \mathrm{E}+01$ & $3.32 \mathrm{E}+04$ & $6.42 \mathrm{E}+05$ & $5.17 \%$ \\
\hline $10 \mathrm{TC} 086$ & $8.11 \mathrm{E}+02$ & 0 & 0 & $9.49 \mathrm{E}+03$ & $1.22 \mathrm{E}+03$ & 0 & 0 & $2.20 \mathrm{E}+00$ & $1.53 \mathrm{E}-01$ & $8.00 \mathrm{E}-01$ & $1.17 \mathrm{E}+04$ & $5.99 \mathrm{E}+05$ & $1.95 \%$ \\
\hline $10 \mathrm{TC} 087$ & $6.14 \mathrm{E}+02$ & 0 & 0 & $1.79 \mathrm{E}+04$ & $8.64 \mathrm{E}+02$ & 0 & $9.69 \mathrm{E}+02$ & $2.70 \mathrm{E}+01$ & $2.20 \mathrm{E}+00$ & $2.55 \mathrm{E}+01$ & $2.18 \mathrm{E}+04$ & $6.13 \mathrm{E}+05$ & $3.56 \%$ \\
\hline $10 \mathrm{TC} 088$ & $6.67 \mathrm{E}+02$ & 0 & 0 & $1.33 \mathrm{E}+04$ & $9.25 \mathrm{E}+02$ & 0 & 0 & $1.23 \mathrm{E}+01$ & $9.70 \mathrm{E}-01$ & $1.13 \mathrm{E}+01$ & $1.55 \mathrm{E}+04$ & $6.01 \mathrm{E}+05$ & $2.59 \%$ \\
\hline 10TC090 & $8.66 \mathrm{E}+02$ & 0 & 0 & $1.16 \mathrm{E}+04$ & $1.25 \mathrm{E}+03$ & 0 & 0 & $1.18 \mathrm{E}+01$ & $9.86 \mathrm{E}-01$ & $9.83 \mathrm{E}+00$ & $1.44 \mathrm{E}+04$ & $6.12 \mathrm{E}+05$ & $2.35 \%$ \\
\hline 10TC091 & $8.31 \mathrm{E}+02$ & 0 & 0 & $1.38 \mathrm{E}+04$ & $1.10 \mathrm{E}+03$ & 0 & $5.34 \mathrm{E}+00$ & $1.91 \mathrm{E}+01$ & $1.57 \mathrm{E}+00$ & $1.75 \mathrm{E}+01$ & $1.68 \mathrm{E}+04$ & $6.16 \mathrm{E}+05$ & $2.72 \%$ \\
\hline 10TC092 & $6.24 \mathrm{E}+02$ & 0 & 0 & $1.15 \mathrm{E}+04$ & $9.29 \mathrm{E}+02$ & 0 & 0 & $9.10 \mathrm{E}+00$ & $6.89 \mathrm{E}-01$ & $8.20 \mathrm{E}+00$ & $1.35 \mathrm{E}+04$ & $5.98 \mathrm{E}+05$ & $2.26 \%$ \\
\hline
\end{tabular}


Table 40. Predicted Solids for Envelope C Simulations - Part 2 (cont'd)

\begin{tabular}{|c|c|c|c|c|c|c|c|c|c|c|c|c|c|}
\hline Test ID & $\mathrm{NA} 2 \mathrm{C} 2 \mathrm{O} 4$ & NA2U2O7 & NA3FSO4 & $\begin{array}{c}\text { NA6SO42 } \\
\text { CO3 }\end{array}$ & NAF & NANO3 & $\begin{array}{c}\text { NASGEL.1 } \\
5.5 \mathrm{H} 2 \mathrm{O}\end{array}$ & $\mathrm{NIOH} 2$ & SRCO3 & $\mathrm{ZRO} 2$ & Total Solids & $\begin{array}{c}\text { Total Soln } \\
\text { Mass }\end{array}$ & $\begin{array}{l}\text { Solids \% of } \\
\text { Total Soln }\end{array}$ \\
\hline $10 \mathrm{TC} 093$ & $9.55 \mathrm{E}+02$ & 0 & $1.67 \mathrm{E}+04$ & 0 & 0 & 0 & 0 & $3.03 \mathrm{E}+01$ & $2.62 \mathrm{E}+00$ & $2.64 \mathrm{E}+01$ & $1.96 \mathrm{E}+04$ & $6.40 \mathrm{E}+05$ & $3.06 \%$ \\
\hline 10TC094 & $1.06 \mathrm{E}+03$ & 0 & $2.09 \mathrm{E}+04$ & 0 & 0 & 0 & $5.84 \mathrm{E}+03$ & $5.17 \mathrm{E}+01$ & $4.47 \mathrm{E}+00$ & $4.60 \mathrm{E}+01$ & $3.39 \mathrm{E}+04$ & $6.71 \mathrm{E}+05$ & $5.06 \%$ \\
\hline 10TC095 & $1.08 \mathrm{E}+03$ & 0 & $1.75 \mathrm{E}+04$ & 0 & 0 & 0 & $3.26 \mathrm{E}+03$ & $3.27 \mathrm{E}+01$ & $2.86 \mathrm{E}+00$ & $2.80 \mathrm{E}+01$ & $2.39 \mathrm{E}+04$ & $6.48 \mathrm{E}+05$ & $3.68 \%$ \\
\hline 10TC096 & $1.02 \mathrm{E}+03$ & 0 & $2.06 \mathrm{E}+04$ & $1.68 \mathrm{E}+03$ & 0 & 0 & $5.84 \mathrm{E}+03$ & $5.39 \mathrm{E}+01$ & $4.56 \mathrm{E}+00$ & $5.08 \mathrm{E}+01$ & $3.51 \mathrm{E}+04$ & $6.64 \mathrm{E}+05$ & $5.29 \%$ \\
\hline 10TC097 & $1.09 \mathrm{E}+03$ & 0 & 0 & 0 & $1.36 \mathrm{E}+03$ & 0 & $3.16 \mathrm{E}+03$ & $3.53 \mathrm{E}+01$ & $3.26 \mathrm{E}+00$ & $2.25 \mathrm{E}+01$ & $9.81 \mathrm{E}+03$ & $6.82 \mathrm{E}+05$ & $1.44 \%$ \\
\hline 10TC098 & $9.43 \mathrm{E}+02$ & 0 & $1.99 \mathrm{E}+04$ & 0 & 0 & 0 & $1.99 \mathrm{E}+03$ & $4.78 \mathrm{E}+01$ & $4.08 \mathrm{E}+00$ & $4.38 \mathrm{E}+01$ & $2.66 \mathrm{E}+04$ & $6.59 \mathrm{E}+05$ & $4.03 \%$ \\
\hline 10TC099 & $1.04 \mathrm{E}+03$ & 0 & $1.58 \mathrm{E}+04$ & 0 & 0 & 0 & $2.13 \mathrm{E}+03$ & $2.84 \mathrm{E}+01$ & $2.49 \mathrm{E}+00$ & $2.39 \mathrm{E}+01$ & $2.07 \mathrm{E}+04$ & $6.43 \mathrm{E}+05$ & $3.22 \%$ \\
\hline 10TC100 & $1.10 \mathrm{E}+03$ & 0 & 0 & 0 & $1.51 \mathrm{E}+03$ & 0 & 0 & $1.14 \mathrm{E}+01$ & $1.28 \mathrm{E}+00$ & 0 & $3.37 \mathrm{E}+03$ & $6.49 \mathrm{E}+05$ & $0.52 \%$ \\
\hline 10TC101 & $9.35 \mathrm{E}+02$ & 0 & $1.00 \mathrm{E}+04$ & 0 & 0 & 0 & 0 & 0 & 5.99E-03 & 0 & $1.10 \mathrm{E}+04$ & $6.04 \mathrm{E}+05$ & $1.82 \%$ \\
\hline 10TC102 & $1.06 \mathrm{E}+03$ & 0 & $9.99 \mathrm{E}+03$ & 0 & 0 & 0 & 0 & 0 & $5.58 \mathrm{E}-02$ & 0 & $1.11 \mathrm{E}+04$ & $6.13 \mathrm{E}+05$ & $1.82 \%$ \\
\hline 10TC103 & $1.10 \mathrm{E}+03$ & 0 & 0 & 0 & $1.54 \mathrm{E}+03$ & 0 & 0 & $9.55 \mathrm{E}+00$ & $1.16 \mathrm{E}+00$ & 0 & $3.33 \mathrm{E}+03$ & $6.48 \mathrm{E}+05$ & $0.51 \%$ \\
\hline 10TC104 & $1.03 \mathrm{E}+03$ & 0 & $1.08 \mathrm{E}+04$ & 0 & 0 & 0 & 0 & $4.50 \mathrm{E}+00$ & $5.82 \mathrm{E}-01$ & 0 & $1.22 \mathrm{E}+04$ & $6.20 \mathrm{E}+05$ & $1.97 \%$ \\
\hline $10 \mathrm{TC} 105$ & $1.01 \mathrm{E}+03$ & 0 & $1.44 \mathrm{E}+04$ & 0 & 0 & 0 & $6.45 \mathrm{E}+02$ & $2.04 \mathrm{E}+01$ & $1.87 \mathrm{E}+00$ & $1.46 \mathrm{E}+01$ & $1.72 \mathrm{E}+04$ & $6.36 \mathrm{E}+05$ & $2.70 \%$ \\
\hline 10TC107 & $1.11 \mathrm{E}+03$ & 0 & $9.81 \mathrm{E}+03$ & 0 & 0 & 0 & 0 & 0 & 0 & 0 & $1.09 \mathrm{E}+04$ & $6.14 \mathrm{E}+05$ & $1.78 \%$ \\
\hline 10TC108 & $9.05 \mathrm{E}+02$ & 0 & 0 & $2.37 \mathrm{E}+04$ & $8.45 \mathrm{E}+02$ & 0 & $3.75 \mathrm{E}+03$ & $5.18 \mathrm{E}+01$ & $4.36 \mathrm{E}+00$ & $4.89 \mathrm{E}+01$ & $3.23 \mathrm{E}+04$ & $6.53 \mathrm{E}+05$ & $4.94 \%$ \\
\hline 10TC109 & $6.26 \mathrm{E}+02$ & 0 & 0 & $2.25 \mathrm{E}+04$ & $6.90 \mathrm{E}+02$ & 0 & $3.15 \mathrm{E}+03$ & $4.12 \mathrm{E}+01$ & $3.40 \mathrm{E}+00$ & $3.93 \mathrm{E}+01$ & $2.94 \mathrm{E}+04$ & $6.26 \mathrm{E}+05$ & $4.69 \%$ \\
\hline 10TC110 & $8.93 \mathrm{E}+02$ & 0 & 0 & $2.39 \mathrm{E}+04$ & $1.01 \mathrm{E}+03$ & 0 & $4.56 \mathrm{E}+03$ & $5.06 \mathrm{E}+01$ & $4.23 \mathrm{E}+00$ & $4.82 \mathrm{E}+01$ & $3.30 \mathrm{E}+04$ & $6.48 \mathrm{E}+05$ & $5.10 \%$ \\
\hline 10TC111 & $7.55 \mathrm{E}+02$ & 0 & 0 & $2.46 \mathrm{E}+04$ & $3.84 \mathrm{E}+02$ & 0 & $6.01 \mathrm{E}+03$ & $5.14 \mathrm{E}+01$ & $4.28 \mathrm{E}+00$ & $4.92 \mathrm{E}+01$ & $3.52 \mathrm{E}+04$ & $6.43 \mathrm{E}+05$ & $5.47 \%$ \\
\hline 10TC112 & $8.61 \mathrm{E}+02$ & 0 & 0 & $1.72 \mathrm{E}+04$ & $1.15 \mathrm{E}+03$ & 0 & $2.15 \mathrm{E}+03$ & $3.01 \mathrm{E}+01$ & $2.51 \mathrm{E}+00$ & $2.78 \mathrm{E}+01$ & $2.29 \mathrm{E}+04$ & $6.29 \mathrm{E}+05$ & $3.63 \%$ \\
\hline $10 \mathrm{TC} 113$ & $7.87 \mathrm{E}+02$ & 0 & 0 & $1.83 \mathrm{E}+04$ & $1.00 \mathrm{E}+03$ & 0 & $1.20 \mathrm{E}+03$ & $3.30 \mathrm{E}+01$ & $2.74 \mathrm{E}+00$ & $3.11 \mathrm{E}+01$ & $2.30 \mathrm{E}+04$ & $6.27 \mathrm{E}+05$ & $3.67 \%$ \\
\hline 10TC114 & $9.34 \mathrm{E}+02$ & 0 & 0 & $1.86 \mathrm{E}+04$ & $1.18 \mathrm{E}+03$ & 0 & $3.54 \mathrm{E}+03$ & $3.63 \mathrm{E}+01$ & $3.06 \mathrm{E}+00$ & $3.38 \mathrm{E}+01$ & $2.61 \mathrm{E}+04$ & $6.40 \mathrm{E}+05$ & $4.07 \%$ \\
\hline 10TC115 & $7.02 \mathrm{E}+02$ & 0 & 0 & $1.63 \mathrm{E}+04$ & $9.86 \mathrm{E}+02$ & 0 & $5.51 \mathrm{E}+02$ & $2.15 \mathrm{E}+01$ & $1.75 \mathrm{E}+00$ & $2.01 \mathrm{E}+01$ & $1.97 \mathrm{E}+04$ & $6.11 \mathrm{E}+05$ & $3.22 \%$ \\
\hline 10TC116 & $8.45 \mathrm{E}+02$ & 0 & 0 & $9.95 \mathrm{E}+03$ & $1.22 \mathrm{E}+03$ & 0 & 0 & $3.17 \mathrm{E}+00$ & $2.32 \mathrm{E}-01$ & $1.82 \mathrm{E}+00$ & $1.22 \mathrm{E}+04$ & $6.01 \mathrm{E}+05$ & $2.03 \%$ \\
\hline 10TC117 & $9.50 \mathrm{E}+02$ & 0 & 0 & $1.62 \mathrm{E}+04$ & $1.22 \mathrm{E}+03$ & 0 & $1.69 \mathrm{E}+03$ & $2.38 \mathrm{E}+01$ & $2.01 \mathrm{E}+00$ & $2.16 \mathrm{E}+01$ & $2.12 \mathrm{E}+04$ & $6.26 \mathrm{E}+05$ & $3.39 \%$ \\
\hline 10TC118 & $6.99 \mathrm{E}+02$ & 0 & 0 & $1.71 \mathrm{E}+04$ & $9.38 \mathrm{E}+02$ & 0 & $4.27 \mathrm{E}+02$ & $2.51 \mathrm{E}+01$ & $2.06 \mathrm{E}+00$ & $2.37 \mathrm{E}+01$ & $2.05 \mathrm{E}+04$ & $6.14 \mathrm{E}+05$ & $3.34 \%$ \\
\hline 10TC119 & $7.21 \mathrm{E}+02$ & 0 & 0 & $1.13 \mathrm{E}+04$ & $1.06 \mathrm{E}+03$ & 0 & 0 & $1.02 \mathrm{E}+01$ & $8.00 \mathrm{E}-01$ & $8.91 \mathrm{E}+00$ & $1.36 \mathrm{E}+04$ & $6.03 \mathrm{E}+05$ & $2.26 \%$ \\
\hline 10TC120 & $8.72 \mathrm{E}+02$ & 0 & 0 & $9.39 \mathrm{E}+03$ & $1.30 \mathrm{E}+03$ & 0 & 0 & $4.04 \mathrm{E}+00$ & 3.39E-01 & $2.13 \mathrm{E}+00$ & $1.18 \mathrm{E}+04$ & $6.05 \mathrm{E}+05$ & $1.95 \%$ \\
\hline 10TC122 & $1.05 \mathrm{E}+03$ & 0 & 0 & 0 & $1.39 \mathrm{E}+03$ & 0 & $1.07 \mathrm{E}+03$ & $2.52 \mathrm{E}+01$ & $2.37 \mathrm{E}+00$ & $1.50 \mathrm{E}+01$ & $5.31 \mathrm{E}+03$ & $6.65 \mathrm{E}+05$ & $0.80 \%$ \\
\hline 10TC123 & $1.03 \mathrm{E}+03$ & 0 & $2.11 \mathrm{E}+04$ & 0 & 0 & 0 & $6.21 \mathrm{E}+03$ & $5.37 \mathrm{E}+01$ & $4.59 \mathrm{E}+00$ & $4.93 \mathrm{E}+01$ & $3.39 \mathrm{E}+04$ & $6.69 \mathrm{E}+05$ & $5.07 \%$ \\
\hline 10TC124 & $1.12 \mathrm{E}+03$ & 0 & $1.74 \mathrm{E}+04$ & 0 & 0 & 0 & $3.32 \mathrm{E}+03$ & $3.22 \mathrm{E}+01$ & $2.90 \mathrm{E}+00$ & $2.46 \mathrm{E}+01$ & $2.51 \mathrm{E}+04$ & $6.53 \mathrm{E}+05$ & $3.84 \%$ \\
\hline
\end{tabular}


Table 40. Predicted Solids for Envelope C Simulations - Part 2 (cont'd)

\begin{tabular}{|c|c|c|c|c|c|c|c|c|c|c|c|c|c|}
\hline Test ID & NA2C2O4 & NA2U2O7 & NA3FSO4 & $\begin{array}{c}\text { NA6SO42 } \\
\text { CO3 }\end{array}$ & NAF & NANO3 & $\begin{array}{c}\text { NASGEL. } 1 \\
5.5 \mathrm{H} 2 \mathrm{O}\end{array}$ & $\mathrm{NIOH} 2$ & $\mathrm{SRCO} 3$ & ZRO2 & Total Solids & $\begin{array}{c}\text { Total Soln } \\
\text { Mass }\end{array}$ & $\begin{array}{l}\text { Solids \% of } \\
\text { Total Soln }\end{array}$ \\
\hline $10 \mathrm{TC} 125$ & $1.06 \mathrm{E}+03$ & 0 & $1.77 \mathrm{E}+04$ & 0 & 0 & 0 & $2.49 \mathrm{E}+03$ & $3.42 \mathrm{E}+01$ & $2.99 \mathrm{E}+00$ & $2.92 \mathrm{E}+01$ & $2.41 \mathrm{E}+04$ & $6.50 \mathrm{E}+05$ & $3.71 \%$ \\
\hline 10TC126 & $1.05 \mathrm{E}+03$ & 0 & $1.97 \mathrm{E}+04$ & 0 & 0 & 0 & $3.32 \mathrm{E}+03$ & $4.58 \mathrm{E}+01$ & $4.03 \mathrm{E}+00$ & $3.80 \mathrm{E}+01$ & $2.96 \mathrm{E}+04$ & $6.67 \mathrm{E}+05$ & $4.44 \%$ \\
\hline 10TC127 & $9.45 \mathrm{E}+02$ & 0 & $1.74 \mathrm{E}+04$ & $1.43 \mathrm{E}+02$ & 0 & 0 & $3.18 \mathrm{E}+03$ & $3.47 \mathrm{E}+01$ & $2.95 \mathrm{E}+00$ & $3.17 \mathrm{E}+01$ & $2.38 \mathrm{E}+04$ & $6.42 \mathrm{E}+05$ & $3.71 \%$ \\
\hline 10TC128 & $9.86 \mathrm{E}+02$ & 0 & $1.85 \mathrm{E}+04$ & $2.78 \mathrm{E}+02$ & 0 & 0 & $4.10 \mathrm{E}+03$ & $4.11 \mathrm{E}+01$ & $3.49 \mathrm{E}+00$ & $3.79 \mathrm{E}+01$ & $2.64 \mathrm{E}+04$ & $6.50 \mathrm{E}+05$ & $4.06 \%$ \\
\hline 10TC129 & $9.91 \mathrm{E}+02$ & 0 & $1.07 \mathrm{E}+04$ & 0 & 0 & 0 & 0 & $4.39 \mathrm{E}+00$ & $4.70 \mathrm{E}-01$ & $8.05 \mathrm{E}-01$ & $1.20 \mathrm{E}+04$ & $6.14 \mathrm{E}+05$ & $1.96 \%$ \\
\hline $10 \mathrm{TC} 130$ & $1.07 \mathrm{E}+03$ & 0 & 0 & 0 & $1.49 \mathrm{E}+03$ & 0 & 0 & $1.25 \mathrm{E}+01$ & $1.35 \mathrm{E}+00$ & $1.49 \mathrm{E}+00$ & $3.37 \mathrm{E}+03$ & $6.49 \mathrm{E}+05$ & $0.52 \%$ \\
\hline 10TC131 & $9.97 \mathrm{E}+02$ & 0 & $1.55 \mathrm{E}+04$ & 0 & 0 & 0 & $1.78 \mathrm{E}+03$ & $2.48 \mathrm{E}+01$ & $2.13 \mathrm{E}+00$ & $2.20 \mathrm{E}+01$ & $1.99 \mathrm{E}+04$ & $6.32 \mathrm{E}+05$ & $3.14 \%$ \\
\hline 10TC132 & $9.51 \mathrm{E}+02$ & 0 & $1.08 \mathrm{E}+04$ & 0 & 0 & 0 & 0 & $5.36 \mathrm{E}+00$ & $5.04 \mathrm{E}-01$ & $2.65 \mathrm{E}+00$ & $1.21 \mathrm{E}+04$ & $6.12 \mathrm{E}+05$ & $1.97 \%$ \\
\hline 10TC133 & $1.07 \mathrm{E}+03$ & 0 & $1.09 \mathrm{E}+04$ & 0 & 0 & 0 & 0 & $5.31 \mathrm{E}+00$ & $6.67 \mathrm{E}-01$ & 0 & $1.24 \mathrm{E}+04$ & $6.23 \mathrm{E}+05$ & $1.98 \%$ \\
\hline 10TC134 & $1.05 \mathrm{E}+03$ & 0 & 0 & 0 & $1.44 \mathrm{E}+03$ & 0 & $7.57 \mathrm{E}+02$ & $2.00 \mathrm{E}+01$ & $1.95 \mathrm{E}+00$ & $9.77 \mathrm{E}+00$ & $4.39 \mathrm{E}+03$ & $6.58 \mathrm{E}+05$ & $0.67 \%$ \\
\hline 10TC135 & $1.10 \mathrm{E}+03$ & 0 & 0 & 0 & $1.51 \mathrm{E}+03$ & 0 & 0 & $9.20 \mathrm{E}+00$ & $1.05 \mathrm{E}+00$ & 0 & $3.24 \mathrm{E}+03$ & $6.45 \mathrm{E}+05$ & $0.50 \%$ \\
\hline $8 \mathrm{TC} 001$ & $1.02 \mathrm{E}+03$ & 0 & 0 & 0 & $3.64 \mathrm{E}+02$ & 0 & 0 & 0 & 0 & 0 & $1.38 \mathrm{E}+03$ & $7.56 \mathrm{E}+05$ & $0.18 \%$ \\
\hline $8 \mathrm{TC} 002$ & $9.60 \mathrm{E}+02$ & 0 & 0 & 0 & $4.41 \mathrm{E}+02$ & 0 & 0 & 0 & 0 & 0 & $1.40 \mathrm{E}+03$ & $7.55 \mathrm{E}+05$ & $0.19 \%$ \\
\hline 8TC004 & $9.20 \mathrm{E}+02$ & 0 & 0 & 0 & $3.81 \mathrm{E}+02$ & 0 & 0 & 0 & 0 & 0 & $1.30 \mathrm{E}+03$ & $7.52 \mathrm{E}+05$ & $0.17 \%$ \\
\hline $8 \mathrm{TC} 005$ & $9.90 \mathrm{E}+02$ & 0 & 0 & 0 & $2.84 \mathrm{E}+02$ & 0 & 0 & 0 & 0 & 0 & $1.27 \mathrm{E}+03$ & $7.54 \mathrm{E}+05$ & $0.17 \%$ \\
\hline $8 \mathrm{TC} 006$ & $2.46 \mathrm{E}+02$ & 0 & 0 & $3.10 \mathrm{E}+02$ & 0 & 0 & 0 & 0 & 0 & 0 & $5.55 \mathrm{E}+02$ & $7.22 \mathrm{E}+05$ & $0.08 \%$ \\
\hline $8 \mathrm{TC} 007$ & $2.38 \mathrm{E}+02$ & 0 & 0 & $1.98 \mathrm{E}+03$ & 0 & 0 & 0 & 0 & 0 & 0 & $2.22 \mathrm{E}+03$ & $7.19 \mathrm{E}+05$ & $0.31 \%$ \\
\hline $8 \mathrm{TC} 008$ & $2.05 \mathrm{E}+02$ & 0 & 0 & 0 & 0 & 0 & 0 & 0 & 0 & 0 & $2.05 \mathrm{E}+02$ & $7.20 \mathrm{E}+05$ & $0.03 \%$ \\
\hline 8TC009 & $1.81 \mathrm{E}+02$ & 0 & 0 & 0 & 0 & 0 & 0 & 0 & 0 & 0 & $1.81 \mathrm{E}+02$ & $7.18 \mathrm{E}+05$ & $0.03 \%$ \\
\hline $8 \mathrm{TC} 010$ & $2.08 \mathrm{E}+02$ & 0 & 0 & $1.00 \mathrm{E}+03$ & 0 & 0 & 0 & 0 & 0 & 0 & $1.21 \mathrm{E}+03$ & $7.18 \mathrm{E}+05$ & $0.17 \%$ \\
\hline $8 \mathrm{TC} 011$ & $9.85 \mathrm{E}+02$ & 0 & 0 & 0 & 0 & 0 & $7.59 \mathrm{E}+03$ & $5.63 \mathrm{E}+01$ & $4.85 \mathrm{E}+00$ & $4.36 \mathrm{E}+01$ & $1.79 \mathrm{E}+04$ & $8.47 \mathrm{E}+05$ & $2.11 \%$ \\
\hline $8 \mathrm{TC} 012$ & $9.29 \mathrm{E}+02$ & 0 & 0 & 0 & 0 & 0 & $7.48 \mathrm{E}+03$ & $5.55 \mathrm{E}+01$ & $4.82 \mathrm{E}+00$ & $3.97 \mathrm{E}+01$ & $1.71 \mathrm{E}+04$ & $8.46 \mathrm{E}+05$ & $2.02 \%$ \\
\hline $8 \mathrm{TC} 013$ & $9.47 \mathrm{E}+02$ & 0 & 0 & 0 & 0 & 0 & $7.47 \mathrm{E}+03$ & $5.62 \mathrm{E}+01$ & $4.80 \mathrm{E}+00$ & $4.70 \mathrm{E}+01$ & $1.63 \mathrm{E}+04$ & $8.44 \mathrm{E}+05$ & $1.94 \%$ \\
\hline $8 \mathrm{TC} 014$ & $8.64 \mathrm{E}+02$ & 0 & 0 & 0 & 0 & 0 & $3.15 \mathrm{E}+03$ & $5.51 \mathrm{E}+01$ & $4.74 \mathrm{E}+00$ & $4.31 \mathrm{E}+01$ & $1.16 \mathrm{E}+04$ & $8.41 \mathrm{E}+05$ & $1.38 \%$ \\
\hline $8 \mathrm{TC} 015$ & $9.32 \mathrm{E}+02$ & 0 & 0 & 0 & 0 & 0 & $3.61 \mathrm{E}+03$ & $5.64 \mathrm{E}+01$ & $4.79 \mathrm{E}+00$ & $4.90 \mathrm{E}+01$ & $1.27 \mathrm{E}+04$ & $8.44 \mathrm{E}+05$ & $1.51 \%$ \\
\hline $8 \mathrm{TC} 016$ & $2.50 \mathrm{E}+02$ & 0 & 0 & $1.85 \mathrm{E}+04$ & 0 & 0 & $7.64 \mathrm{E}+03$ & $5.80 \mathrm{E}+01$ & $4.83 \mathrm{E}+00$ & $5.56 \mathrm{E}+01$ & $2.97 \mathrm{E}+04$ & $7.77 \mathrm{E}+05$ & $3.83 \%$ \\
\hline $8 \mathrm{TC} 017$ & $2.40 \mathrm{E}+02$ & 0 & 0 & $2.00 \mathrm{E}+04$ & 0 & 0 & $7.51 \mathrm{E}+03$ & $5.78 \mathrm{E}+01$ & $4.79 \mathrm{E}+00$ & $5.52 \mathrm{E}+01$ & $3.09 \mathrm{E}+04$ & $7.74 \mathrm{E}+05$ & $3.99 \%$ \\
\hline $8 \mathrm{TC} 020$ & $1.67 \mathrm{E}+02$ & 0 & 0 & $1.82 \mathrm{E}+04$ & 0 & 0 & $3.56 \mathrm{E}+03$ & $5.72 \mathrm{E}+01$ & $4.77 \mathrm{E}+00$ & $5.50 \mathrm{E}+01$ & $2.54 \mathrm{E}+04$ & $7.73 \mathrm{E}+05$ & $3.28 \%$ \\
\hline $8 \mathrm{TC} 021$ & $7.55 \mathrm{E}+02$ & 0 & $8.02 \mathrm{E}+03$ & 0 & 0 & 0 & $2.09 \mathrm{E}+03$ & $1.86 \mathrm{E}+01$ & $1.56 \mathrm{E}+00$ & $1.48 \mathrm{E}+01$ & $1.18 \mathrm{E}+04$ & $7.56 \mathrm{E}+05$ & $1.56 \%$ \\
\hline
\end{tabular}


Table 40. Predicted Solids for Envelope C Simulations - Part 2 (cont'd)

\begin{tabular}{|c|c|c|c|c|c|c|c|c|c|c|c|c|c|}
\hline Test ID & NA2C2O4 & NA2U2O7 & NA3FSO4 & $\begin{array}{c}\text { NA6SO42 } \\
\mathrm{CO} 3\end{array}$ & NAF & NANO3 & $\begin{array}{c}\text { NASGEL. } 1 \\
5.5 \mathrm{H} 2 \mathrm{O} \\
\end{array}$ & $\mathrm{NIOH} 2$ & SRCO3 & ZRO2 & Total Solids & $\begin{array}{c}\text { Total Soln } \\
\text { Mass }\end{array}$ & \begin{tabular}{|c|} 
Solids $\%$ of \\
Total Soln
\end{tabular} \\
\hline $8 \mathrm{TC} 022$ & $9.84 \mathrm{E}+02$ & 0 & 0 & 0 & $1.51 \mathrm{E}+02$ & 0 & $7.62 \mathrm{E}+02$ & $7.28 \mathrm{E}+00$ & $7.65 \mathrm{E}-01$ & 0 & $2.37 \mathrm{E}+03$ & $7.70 \mathrm{E}+05$ & $0.31 \%$ \\
\hline $8 \mathrm{TC} 023$ & $7.73 \mathrm{E}+02$ & 0 & $7.65 \mathrm{E}+03$ & 0 & 0 & 0 & $1.24 \mathrm{E}+03$ & $1.28 \mathrm{E}+01$ & $1.09 \mathrm{E}+00$ & $8.40 \mathrm{E}+00$ & $1.03 \mathrm{E}+04$ & $7.50 \mathrm{E}+05$ & $1.38 \%$ \\
\hline $8 \mathrm{TC} 024$ & $9.50 \mathrm{E}+02$ & 0 & 0 & 0 & $1.14 \mathrm{E}+02$ & 0 & $2.24 \mathrm{E}+03$ & $1.79 \mathrm{E}+01$ & $1.68 \mathrm{E}+00$ & $2.19 \mathrm{E}+00$ & $4.83 \mathrm{E}+03$ & $7.86 \mathrm{E}+05$ & $0.61 \%$ \\
\hline $8 \mathrm{TC} 025$ & $8.57 \mathrm{E}+02$ & 0 & $6.46 \mathrm{E}+03$ & 0 & 0 & 0 & 0 & 0 & 0 & 0 & $7.32 \mathrm{E}+03$ & $7.34 \mathrm{E}+05$ & $1.00 \%$ \\
\hline $8 \mathrm{TC} 026$ & $9.51 \mathrm{E}+02$ & 0 & $8.71 \mathrm{E}+03$ & 0 & 0 & 0 & $1.94 \mathrm{E}+03$ & $1.64 \mathrm{E}+01$ & $1.50 \mathrm{E}+00$ & $5.80 \mathrm{E}+00$ & $1.25 \mathrm{E}+04$ & $7.65 \mathrm{E}+05$ & $1.63 \%$ \\
\hline $8 \mathrm{TC} 027$ & $8.18 \mathrm{E}+02$ & 0 & $7.67 \mathrm{E}+03$ & 0 & 0 & 0 & $4.53 \mathrm{E}+02$ & $8.65 \mathrm{E}+00$ & 7.79E-01 & $2.23 \mathrm{E}+00$ & $9.43 \mathrm{E}+03$ & $7.47 \mathrm{E}+05$ & $1.26 \%$ \\
\hline $8 \mathrm{TC} 028$ & $8.37 \mathrm{E}+02$ & 0 & $1.05 \mathrm{E}+04$ & 0 & 0 & 0 & $7.22 \mathrm{E}+03$ & $5.41 \mathrm{E}+01$ & $4.57 \mathrm{E}+00$ & $4.94 \mathrm{E}+01$ & $2.51 \mathrm{E}+04$ & $8.11 \mathrm{E}+05$ & $3.09 \%$ \\
\hline $8 \mathrm{TC} 029$ & $9.75 \mathrm{E}+02$ & 0 & 0 & 0 & 0 & 0 & $3.69 \mathrm{E}+03$ & $2.79 \mathrm{E}+01$ & $2.50 \mathrm{E}+00$ & $1.31 \mathrm{E}+01$ & $7.97 \mathrm{E}+03$ & $8.03 \mathrm{E}+05$ & $0.99 \%$ \\
\hline $8 \mathrm{TC} 030$ & $9.21 \mathrm{E}+02$ & 0 & 0 & 0 & 0 & 0 & $5.72 \mathrm{E}+03$ & $4.29 \mathrm{E}+01$ & $3.74 \mathrm{E}+00$ & $2.94 \mathrm{E}+01$ & $1.22 \mathrm{E}+04$ & $8.24 \mathrm{E}+05$ & $1.48 \%$ \\
\hline 8TC031 & $9.23 \mathrm{E}+02$ & 0 & $1.02 \mathrm{E}+04$ & 0 & 0 & 0 & $5.90 \mathrm{E}+03$ & $4.44 \mathrm{E}+01$ & $3.80 \mathrm{E}+00$ & $3.66 \mathrm{E}+01$ & $2.24 \mathrm{E}+04$ & $8.03 \mathrm{E}+05$ & $2.79 \%$ \\
\hline $8 \mathrm{TC} 032$ & $7.67 \mathrm{E}+02$ & 0 & $1.05 \mathrm{E}+04$ & 0 & 0 & 0 & $5.79 \mathrm{E}+03$ & $4.43 \mathrm{E}+01$ & $3.73 \mathrm{E}+00$ & $4.04 \mathrm{E}+01$ & $2.07 \mathrm{E}+04$ & $7.91 \mathrm{E}+05$ & $2.61 \%$ \\
\hline $8 \mathrm{TC} 033$ & $8.15 \mathrm{E}+02$ & 0 & $9.91 \mathrm{E}+03$ & 0 & 0 & 0 & $4.71 \mathrm{E}+03$ & $3.68 \mathrm{E}+01$ & $3.13 \mathrm{E}+00$ & $3.11 \mathrm{E}+01$ & $1.80 \mathrm{E}+04$ & $7.85 \mathrm{E}+05$ & $2.29 \%$ \\
\hline $8 \mathrm{TC} 034$ & $9.63 \mathrm{E}+02$ & 0 & 0 & 0 & 0 & 0 & $6.24 \mathrm{E}+03$ & $4.64 \mathrm{E}+01$ & $4.03 \mathrm{E}+00$ & $3.28 \mathrm{E}+01$ & $1.35 \mathrm{E}+04$ & $8.31 \mathrm{E}+05$ & $1.62 \%$ \\
\hline $8 \mathrm{TC} 036$ & $4.18 \mathrm{E}+02$ & 0 & 0 & 0 & 0 & 0 & 0 & $3.27 \mathrm{E}+00$ & $2.10 \mathrm{E}-01$ & $1.91 \mathrm{E}+00$ & $5.91 \mathrm{E}+02$ & $7.33 \mathrm{E}+05$ & $0.08 \%$ \\
\hline $8 \mathrm{TC} 037$ & $4.45 \mathrm{E}+02$ & 0 & 0 & $4.41 \mathrm{E}+03$ & 0 & 0 & $2.47 \mathrm{E}+03$ & $2.26 \mathrm{E}+01$ & $1.84 \mathrm{E}+00$ & $2.05 \mathrm{E}+01$ & $8.51 \mathrm{E}+03$ & $7.56 \mathrm{E}+05$ & $1.13 \%$ \\
\hline $8 \mathrm{TC} 038$ & $5.78 \mathrm{E}+02$ & 0 & 0 & 0 & 0 & 0 & 0 & $1.99 \mathrm{E}+00$ & $1.25 \mathrm{E}-01$ & 0 & $7.06 \mathrm{E}+02$ & $7.38 \mathrm{E}+05$ & $0.10 \%$ \\
\hline $8 \mathrm{TC} 039$ & $2.32 \mathrm{E}+02$ & 0 & 0 & $6.99 \mathrm{E}+03$ & 0 & 0 & $1.92 \mathrm{E}+03$ & $1.79 \mathrm{E}+01$ & $1.43 \mathrm{E}+00$ & $1.66 \mathrm{E}+01$ & $1.01 \mathrm{E}+04$ & $7.37 \mathrm{E}+05$ & $1.37 \%$ \\
\hline $8 \mathrm{TC} 040$ & $5.54 \mathrm{E}+02$ & 0 & 0 & 0 & 0 & 0 & $3.94 \mathrm{E}+02$ & $7.19 \mathrm{E}+00$ & $5.58 \mathrm{E}-01$ & $5.17 \mathrm{E}+00$ & $1.32 \mathrm{E}+03$ & $7.45 \mathrm{E}+05$ & $0.18 \%$ \\
\hline $8 \mathrm{TC} 041$ & $4.89 \mathrm{E}+02$ & 0 & 0 & $7.50 \mathrm{E}+03$ & 0 & 0 & $3.17 \mathrm{E}+03$ & $2.68 \mathrm{E}+01$ & $2.21 \mathrm{E}+00$ & $2.46 \mathrm{E}+01$ & $1.26 \mathrm{E}+04$ & $7.58 \mathrm{E}+05$ & $1.66 \%$ \\
\hline $8 \mathrm{TC} 042$ & $2.80 \mathrm{E}+02$ & 0 & 0 & $1.28 \mathrm{E}+04$ & 0 & 0 & $5.25 \mathrm{E}+03$ & $4.14 \mathrm{E}+01$ & $3.42 \mathrm{E}+00$ & $3.94 \mathrm{E}+01$ & $2.06 \mathrm{E}+04$ & $7.63 \mathrm{E}+05$ & $2.70 \%$ \\
\hline $8 \mathrm{TC} 043$ & $6.53 \mathrm{E}+02$ & 0 & 0 & $8.79 \mathrm{E}+03$ & 0 & 0 & $7.48 \mathrm{E}+03$ & $5.63 \mathrm{E}+01$ & $4.72 \mathrm{E}+00$ & $5.31 \mathrm{E}+01$ & $2.22 \mathrm{E}+04$ & $8.08 \mathrm{E}+05$ & $2.75 \%$ \\
\hline $8 \mathrm{TC} 044$ & $4.46 \mathrm{E}+02$ & 0 & 0 & $1.58 \mathrm{E}+04$ & 0 & 0 & $7.31 \mathrm{E}+03$ & $5.59 \mathrm{E}+01$ & $4.66 \mathrm{E}+00$ & $5.31 \mathrm{E}+01$ & $2.65 \mathrm{E}+04$ & $7.86 \mathrm{E}+05$ & $3.37 \%$ \\
\hline $8 \mathrm{TC} 045$ & $2.52 \mathrm{E}+02$ & 0 & 0 & $1.34 \mathrm{E}+04$ & 0 & 0 & $5.49 \mathrm{E}+03$ & $4.29 \mathrm{E}+01$ & $3.55 \mathrm{E}+00$ & $4.09 \mathrm{E}+01$ & $2.14 \mathrm{E}+04$ & $7.63 \mathrm{E}+05$ & $2.81 \%$ \\
\hline $8 \mathrm{TC} 046$ & $4.48 \mathrm{E}+02$ & 0 & 0 & $1.46 \mathrm{E}+04$ & 0 & 0 & $6.43 \mathrm{E}+03$ & $4.96 \mathrm{E}+01$ & $4.13 \mathrm{E}+00$ & $4.70 \mathrm{E}+01$ & $2.41 \mathrm{E}+04$ & $7.78 \mathrm{E}+05$ & $3.10 \%$ \\
\hline $8 \mathrm{TC} 047$ & $4.77 \mathrm{E}+02$ & 0 & 0 & $9.14 \mathrm{E}+03$ & 0 & 0 & $4.35 \mathrm{E}+03$ & $3.43 \mathrm{E}+01$ & $2.84 \mathrm{E}+00$ & $3.22 \mathrm{E}+01$ & $1.58 \mathrm{E}+04$ & $7.65 \mathrm{E}+05$ & $2.06 \%$ \\
\hline $8 \mathrm{TC} 048$ & $5.56 \mathrm{E}+02$ & 0 & 0 & $9.72 \mathrm{E}+03$ & 0 & 0 & $5.05 \mathrm{E}+03$ & $3.99 \mathrm{E}+01$ & $3.32 \mathrm{E}+00$ & $3.71 \mathrm{E}+01$ & $1.74 \mathrm{E}+04$ & $7.77 \mathrm{E}+05$ & $2.24 \%$ \\
\hline 8ТC049 & $3.88 \mathrm{E}+02$ & 0 & 0 & $1.70 \mathrm{E}+04$ & 0 & 0 & $7.36 \mathrm{E}+03$ & $5.70 \mathrm{E}+01$ & $4.75 \mathrm{E}+00$ & $5.43 \mathrm{E}+01$ & $2.79 \mathrm{E}+04$ & $7.82 \mathrm{E}+05$ & $3.56 \%$ \\
\hline $8 \mathrm{TC} 052$ & $7.76 \mathrm{E}+02$ & 0 & $6.91 \mathrm{E}+03$ & 0 & 0 & 0 & $5.44 \mathrm{E}+01$ & $4.93 \mathrm{E}+00$ & $4.37 \mathrm{E}-01$ & $6.83 \mathrm{E}-02$ & $8.04 \mathrm{E}+03$ & $7.40 \mathrm{E}+05$ & $1.09 \%$ \\
\hline $8 \mathrm{TC} 053$ & $9.34 \mathrm{E}+02$ & 0 & $7.28 \mathrm{E}+03$ & 0 & 0 & 0 & 0 & $3.51 \mathrm{E}+00$ & $4.10 \mathrm{E}-01$ & 0 & $8.49 \mathrm{E}+03$ & $7.47 \mathrm{E}+05$ & $1.14 \%$ \\
\hline
\end{tabular}


Table 40. Predicted Solids for Envelope C Simulations - Part 2 (cont'd)

\begin{tabular}{|c|c|c|c|c|c|c|c|c|c|c|c|c|c|}
\hline Test ID & $\mathrm{NA} 2 \mathrm{C} 2 \mathrm{O} 4$ & NA2U2O7 & NA3FSO4 & $\begin{array}{c}\text { NA6SO42 } \\
\text { CO3 }\end{array}$ & NAF & NANO3 & $\begin{array}{c}\text { NASGEL.1 } \\
5.5 \mathrm{H} 2 \mathrm{O}\end{array}$ & $\mathrm{NIOH} 2$ & $\mathrm{SRCO} 3$ & ZRO2 & Total Solids & $\begin{array}{c}\text { Total Soln } \\
\text { Mass }\end{array}$ & $\begin{array}{l}\text { Solids \% of } \\
\text { Total Soln }\end{array}$ \\
\hline $8 \mathrm{TC} 054$ & $8.08 \mathrm{E}+02$ & 0 & $9.00 \mathrm{E}+03$ & 0 & 0 & 0 & $3.19 \mathrm{E}+03$ & $2.59 \mathrm{E}+01$ & $2.18 \mathrm{E}+00$ & $2.19 \mathrm{E}+01$ & $1.43 \mathrm{E}+04$ & $7.67 \mathrm{E}+05$ & $1.86 \%$ \\
\hline $8 \mathrm{TC} 055$ & $8.53 \mathrm{E}+02$ & 0 & $9.19 \mathrm{E}+03$ & 0 & 0 & 0 & $2.68 \mathrm{E}+03$ & $2.24 \mathrm{E}+01$ & $1.95 \mathrm{E}+00$ & $1.56 \mathrm{E}+01$ & $1.39 \mathrm{E}+04$ & $7.67 \mathrm{E}+05$ & $1.81 \%$ \\
\hline $8 \mathrm{TC} 056$ & $7.22 \mathrm{E}+02$ & 0 & $8.38 \mathrm{E}+03$ & 0 & 0 & 0 & $2.15 \mathrm{E}+03$ & $1.96 \mathrm{E}+01$ & $1.64 \mathrm{E}+00$ & $1.62 \mathrm{E}+01$ & $1.22 \mathrm{E}+04$ & $7.55 \mathrm{E}+05$ & $1.62 \%$ \\
\hline $8 \mathrm{TC} 057$ & $9.53 \mathrm{E}+02$ & 0 & $9.46 \mathrm{E}+03$ & 0 & 0 & 0 & $4.51 \mathrm{E}+03$ & $3.42 \mathrm{E}+01$ & $2.96 \mathrm{E}+00$ & $2.56 \mathrm{E}+01$ & $1.89 \mathrm{E}+04$ & $7.90 \mathrm{E}+05$ & $2.39 \%$ \\
\hline $8 \mathrm{TC} 058$ & $8.15 \mathrm{E}+02$ & 0 & $1.07 \mathrm{E}+04$ & 0 & 0 & 0 & $7.00 \mathrm{E}+03$ & $5.30 \mathrm{E}+01$ & $4.48 \mathrm{E}+00$ & $4.81 \mathrm{E}+01$ & $2.45 \mathrm{E}+04$ & $8.08 \mathrm{E}+05$ & $3.04 \%$ \\
\hline $8 \mathrm{TC} 059$ & $7.03 \mathrm{E}+02$ & 0 & $9.07 \mathrm{E}+03$ & 0 & 0 & 0 & $3.89 \mathrm{E}+03$ & $3.21 \mathrm{E}+01$ & $2.69 \mathrm{E}+00$ & $2.84 \mathrm{E}+01$ & $1.53 \mathrm{E}+04$ & $7.72 \mathrm{E}+05$ & $1.98 \%$ \\
\hline $8 \mathrm{TC} 060$ & $9.24 \mathrm{E}+02$ & 0 & $9.54 \mathrm{E}+03$ & 0 & 0 & 0 & $3.86 \mathrm{E}+03$ & $3.00 \mathrm{E}+01$ & $2.64 \mathrm{E}+00$ & $1.94 \mathrm{E}+01$ & $1.72 \mathrm{E}+04$ & $7.84 \mathrm{E}+05$ & $2.20 \%$ \\
\hline $8 \mathrm{TC} 061$ & $9.16 \mathrm{E}+02$ & 0 & $1.02 \mathrm{E}+04$ & 0 & 0 & 0 & $6.11 \mathrm{E}+03$ & $4.59 \mathrm{E}+01$ & $3.92 \mathrm{E}+00$ & $3.86 \mathrm{E}+01$ & $2.28 \mathrm{E}+04$ & $8.05 \mathrm{E}+05$ & $2.83 \%$ \\
\hline $8 \mathrm{TC} 062$ & $7.53 \mathrm{E}+02$ & 0 & $1.30 \mathrm{E}+04$ & 0 & 0 & 0 & $6.86 \mathrm{E}+03$ & $5.20 \mathrm{E}+01$ & $4.38 \mathrm{E}+00$ & $4.80 \mathrm{E}+01$ & $2.55 \mathrm{E}+04$ & $7.98 \mathrm{E}+05$ & $3.20 \%$ \\
\hline $8 \mathrm{TC} 063$ & $7.80 \mathrm{E}+02$ & 0 & $1.04 \mathrm{E}+04$ & 0 & 0 & 0 & $5.90 \mathrm{E}+03$ & $4.55 \mathrm{E}+01$ & $3.85 \mathrm{E}+00$ & $4.02 \mathrm{E}+01$ & $2.10 \mathrm{E}+04$ & $7.96 \mathrm{E}+05$ & $2.64 \%$ \\
\hline $8 \mathrm{TC} 064$ & $3.13 \mathrm{E}+02$ & 0 & 0 & $8.31 \mathrm{E}+03$ & 0 & 0 & $3.44 \mathrm{E}+03$ & $2.84 \mathrm{E}+01$ & $2.32 \mathrm{E}+00$ & $2.66 \mathrm{E}+01$ & $1.36 \mathrm{E}+04$ & $7.53 \mathrm{E}+05$ & $1.80 \%$ \\
\hline $8 \mathrm{TC} 065$ & $5.19 \mathrm{E}+02$ & 0 & 0 & 0 & 0 & 0 & 0 & $1.78 \mathrm{E}+00$ & $9.29 \mathrm{E}-02$ & $9.20 \mathrm{E}-02$ & $6.26 \mathrm{E}+02$ & $7.35 \mathrm{E}+05$ & $0.09 \%$ \\
\hline $8 \mathrm{TC} 066$ & $3.27 \mathrm{E}+02$ & 0 & 0 & $6.66 \mathrm{E}+03$ & 0 & 0 & $2.41 \mathrm{E}+03$ & $2.23 \mathrm{E}+01$ & $1.80 \mathrm{E}+00$ & $2.06 \mathrm{E}+01$ & $1.06 \mathrm{E}+04$ & $7.47 \mathrm{E}+05$ & $1.42 \%$ \\
\hline $8 \mathrm{TC} 068$ & $3.48 \mathrm{E}+02$ & 0 & 0 & $3.22 \mathrm{E}+03$ & 0 & 0 & $5.39 \mathrm{E}+02$ & $8.34 \mathrm{E}+00$ & $6.29 \mathrm{E}-01$ & $7.04 \mathrm{E}+00$ & $4.54 \mathrm{E}+03$ & $7.33 \mathrm{E}+05$ & $0.62 \%$ \\
\hline $8 \mathrm{TC} 069$ & $6.46 \mathrm{E}+02$ & 0 & 0 & $2.58 \mathrm{E}+02$ & 0 & 0 & $2.40 \mathrm{E}+03$ & $2.11 \mathrm{E}+01$ & $1.75 \mathrm{E}+00$ & $1.83 \mathrm{E}+01$ & $4.37 \mathrm{E}+03$ & $7.69 \mathrm{E}+05$ & $0.57 \%$ \\
\hline $8 \mathrm{TC} 070$ & $6.05 \mathrm{E}+02$ & 0 & 0 & $1.94 \mathrm{E}+01$ & 0 & 0 & $1.49 \mathrm{E}+03$ & $1.47 \mathrm{E}+01$ & $1.20 \mathrm{E}+00$ & $1.21 \mathrm{E}+01$ & $2.85 \mathrm{E}+03$ & $7.59 \mathrm{E}+05$ & $0.38 \%$ \\
\hline $8 \mathrm{TC} 071$ & $5.75 \mathrm{E}+02$ & 0 & 0 & $1.01 \mathrm{E}+04$ & 0 & 0 & $6.65 \mathrm{E}+03$ & $5.08 \mathrm{E}+01$ & $4.24 \mathrm{E}+00$ & $4.80 \mathrm{E}+01$ & $2.05 \mathrm{E}+04$ & $7.93 \mathrm{E}+05$ & $2.58 \%$ \\
\hline $8 \mathrm{TC} 072$ & $3.07 \mathrm{E}+02$ & 0 & 0 & $1.21 \mathrm{E}+04$ & 0 & 0 & $5.18 \mathrm{E}+03$ & $4.06 \mathrm{E}+01$ & $3.36 \mathrm{E}+00$ & $3.86 \mathrm{E}+01$ & $1.97 \mathrm{E}+04$ & $7.64 \mathrm{E}+05$ & $2.58 \%$ \\
\hline $8 \mathrm{TC} 073$ & $5.97 \mathrm{E}+02$ & 0 & 0 & $4.12 \mathrm{E}+03$ & 0 & 0 & $3.74 \mathrm{E}+03$ & $3.10 \mathrm{E}+01$ & $2.58 \mathrm{E}+00$ & $2.80 \mathrm{E}+01$ & $1.01 \mathrm{E}+04$ & $7.76 \mathrm{E}+05$ & $1.30 \%$ \\
\hline $8 \mathrm{TC} 074$ & $6.30 \mathrm{E}+02$ & 0 & 0 & $9.56 \mathrm{E}+03$ & 0 & 0 & $6.58 \mathrm{E}+03$ & $5.03 \mathrm{E}+01$ & $4.21 \mathrm{E}+00$ & $4.70 \mathrm{E}+01$ & $1.94 \mathrm{E}+04$ & $7.97 \mathrm{E}+05$ & $2.44 \%$ \\
\hline $8 \mathrm{TC} 075$ & $4.21 \mathrm{E}+02$ & 0 & 0 & $1.43 \mathrm{E}+04$ & 0 & 0 & $6.31 \mathrm{E}+03$ & $4.87 \mathrm{E}+01$ & $4.04 \mathrm{E}+00$ & $4.62 \mathrm{E}+01$ & $2.36 \mathrm{E}+04$ & $7.76 \mathrm{E}+05$ & $3.04 \%$ \\
\hline $8 \mathrm{TC} 076$ & $3.41 \mathrm{E}+02$ & 0 & 0 & $1.02 \mathrm{E}+04$ & 0 & 0 & $4.22 \mathrm{E}+03$ & $3.34 \mathrm{E}+01$ & $2.75 \mathrm{E}+00$ & $3.16 \mathrm{E}+01$ & $1.65 \mathrm{E}+04$ & $7.58 \mathrm{E}+05$ & $2.18 \%$ \\
\hline $8 \mathrm{TC} 077$ & $3.16 \mathrm{E}+02$ & 0 & 0 & $1.38 \mathrm{E}+04$ & 0 & 0 & $5.53 \mathrm{E}+03$ & $4.40 \mathrm{E}+01$ & $3.65 \mathrm{E}+00$ & $4.18 \mathrm{E}+01$ & $2.20 \mathrm{E}+04$ & $7.66 \mathrm{E}+05$ & $2.87 \%$ \\
\hline $8 \mathrm{TC} 078$ & $6.51 \mathrm{E}+02$ & 0 & 0 & $1.28 \mathrm{E}+03$ & 0 & 0 & $3.32 \mathrm{E}+03$ & $2.82 \mathrm{E}+01$ & $2.35 \mathrm{E}+00$ & $2.49 \mathrm{E}+01$ & $6.69 \mathrm{E}+03$ & $7.79 \mathrm{E}+05$ & $0.86 \%$ \\
\hline $8 \mathrm{TC} 079$ & $5.67 \mathrm{E}+02$ & 0 & 0 & $6.20 \mathrm{E}+03$ & 0 & 0 & $3.70 \mathrm{E}+03$ & $3.74 \mathrm{E}+01$ & $3.12 \mathrm{E}+00$ & $3.43 \mathrm{E}+01$ & $1.24 \mathrm{E}+04$ & $7.80 \mathrm{E}+05$ & $1.59 \%$ \\
\hline $8 \mathrm{TC} 080$ & $2.62 \mathrm{E}+02$ & 0 & 0 & $1.48 \mathrm{E}+04$ & 0 & 0 & $5.75 \mathrm{E}+03$ & $4.73 \mathrm{E}+01$ & $3.92 \mathrm{E}+00$ & $4.50 \mathrm{E}+01$ & $2.34 \mathrm{E}+04$ & $7.67 \mathrm{E}+05$ & $3.05 \%$ \\
\hline $8 \mathrm{TC} 081$ & $5.64 \mathrm{E}+02$ & 0 & 0 & $8.11 \mathrm{E}+03$ & 0 & 0 & $5.11 \mathrm{E}+03$ & $4.32 \mathrm{E}+01$ & $3.60 \mathrm{E}+00$ & $4.04 \mathrm{E}+01$ & $1.60 \mathrm{E}+04$ & $7.85 \mathrm{E}+05$ & $2.05 \%$ \\
\hline $8 \mathrm{TC} 084$ & $3.15 \mathrm{E}+02$ & 0 & 0 & $1.06 \mathrm{E}+04$ & 0 & 0 & $4.27 \mathrm{E}+03$ & $3.87 \mathrm{E}+01$ & $3.19 \mathrm{E}+00$ & $3.66 \mathrm{E}+01$ & $1.72 \mathrm{E}+04$ & $7.63 \mathrm{E}+05$ & $2.26 \%$ \\
\hline $8 \mathrm{TC} 085$ & $5.07 \mathrm{E}+02$ & 0 & 0 & $1.11 \mathrm{E}+04$ & 0 & 0 & $6.10 \mathrm{E}+03$ & $4.72 \mathrm{E}+01$ & $3.93 \mathrm{E}+00$ & $4.47 \mathrm{E}+01$ & $2.02 \mathrm{E}+04$ & $7.83 \mathrm{E}+05$ & $2.58 \%$ \\
\hline
\end{tabular}


Table 40. Predicted Solids for Envelope C Simulations - Part 2 (cont'd)

\begin{tabular}{|c|c|c|c|c|c|c|c|c|c|c|c|c|c|}
\hline Test ID & NA2C2O4 & NA2U2O7 & NA3FSO4 & $\begin{array}{c}\text { NA6SO42 } \\
\text { CO3 }\end{array}$ & NAF & NANO3 & $\begin{array}{c}\text { NASGEL.1 } \\
5.5 \mathrm{H} 2 \mathrm{O}\end{array}$ & $\mathrm{NIOH} 2$ & $\mathrm{SRCO} 3$ & ZRO2 & Total Solids & $\begin{array}{c}\text { Total Soln } \\
\text { Mass }\end{array}$ & $\begin{array}{l}\text { Solids \% of } \\
\text { Total Soln }\end{array}$ \\
\hline $8 \mathrm{TC} 086$ & $4.71 \mathrm{E}+02$ & 0 & 0 & 0 & 0 & 0 & 0 & $2.57 \mathrm{E}+00$ & $1.60 \mathrm{E}-01$ & $6.30 \mathrm{E}-01$ & $6.20 \mathrm{E}+02$ & $7.34 \mathrm{E}+05$ & $0.08 \%$ \\
\hline $8 \mathrm{TC} 087$ & $2.07 \mathrm{E}+02$ & 0 & 0 & $9.34 \mathrm{E}+03$ & 0 & 0 & $2.31 \mathrm{E}+03$ & $2.71 \mathrm{E}+01$ & $2.20 \mathrm{E}+00$ & $2.54 \mathrm{E}+01$ & $1.33 \mathrm{E}+04$ & $7.45 \mathrm{E}+05$ & $1.78 \%$ \\
\hline $8 \mathrm{TC} 088$ & $2.50 \mathrm{E}+02$ & 0 & 0 & $4.52 \mathrm{E}+03$ & 0 & 0 & $8.58 \mathrm{E}+02$ & $1.24 \mathrm{E}+01$ & $9.72 \mathrm{E}-01$ & $1.12 \mathrm{E}+01$ & $6.28 \mathrm{E}+03$ & $7.33 \mathrm{E}+05$ & $0.86 \%$ \\
\hline $8 \mathrm{TC} 089$ & $3.40 \mathrm{E}+02$ & 0 & 0 & $2.21 \mathrm{E}+03$ & 0 & 0 & $6.13 \mathrm{E}+02$ & $1.18 \mathrm{E}+01$ & $9.25 \mathrm{E}-01$ & $1.03 \mathrm{E}+01$ & $3.78 \mathrm{E}+03$ & $7.39 \mathrm{E}+05$ & $0.51 \%$ \\
\hline $8 \mathrm{TC} 090$ & $5.49 \mathrm{E}+02$ & 0 & 0 & 0 & 0 & 0 & $2.91 \mathrm{E}+02$ & $1.23 \mathrm{E}+01$ & $9.94 \mathrm{E}-01$ & $9.62 \mathrm{E}+00$ & $1.46 \mathrm{E}+03$ & $7.52 \mathrm{E}+05$ & $0.19 \%$ \\
\hline 8TC091 & $4.80 \mathrm{E}+02$ & 0 & 0 & $1.79 \mathrm{E}+03$ & 0 & 0 & $1.42 \mathrm{E}+03$ & $1.93 \mathrm{E}+01$ & $1.58 \mathrm{E}+00$ & $1.73 \mathrm{E}+01$ & $4.70 \mathrm{E}+03$ & $7.56 \mathrm{E}+05$ & $0.62 \%$ \\
\hline $8 \mathrm{TC} 092$ & $2.01 \mathrm{E}+02$ & 0 & 0 & $2.83 \mathrm{E}+03$ & 0 & 0 & $3.12 \mathrm{E}+02$ & $9.22 \mathrm{E}+00$ & $6.92 \mathrm{E}-01$ & $8.11 \mathrm{E}+00$ & $3.83 \mathrm{E}+03$ & $7.30 \mathrm{E}+05$ & $0.52 \%$ \\
\hline $8 \mathrm{TC} 093$ & $7.22 \mathrm{E}+02$ & 0 & $9.23 \mathrm{E}+03$ & 0 & 0 & 0 & $9.17 \mathrm{E}+02$ & $3.09 \mathrm{E}+01$ & $2.62 \mathrm{E}+00$ & $2.60 \mathrm{E}+01$ & $1.24 \mathrm{E}+04$ & $7.73 \mathrm{E}+05$ & $1.60 \%$ \\
\hline 8TC094 & $8.47 \mathrm{E}+02$ & 0 & $1.04 \mathrm{E}+04$ & 0 & 0 & 0 & $6.67 \mathrm{E}+03$ & $5.25 \mathrm{E}+01$ & $4.47 \mathrm{E}+00$ & $4.55 \mathrm{E}+01$ & $2.44 \mathrm{E}+04$ & $8.12 \mathrm{E}+05$ & $3.00 \%$ \\
\hline $8 \mathrm{TC} 095$ & $8.65 \mathrm{E}+02$ & 0 & $9.62 \mathrm{E}+03$ & 0 & 0 & 0 & $4.15 \mathrm{E}+03$ & $3.35 \mathrm{E}+01$ & $2.86 \mathrm{E}+00$ & $2.76 \mathrm{E}+01$ & $1.76 \mathrm{E}+04$ & $7.82 \mathrm{E}+05$ & $2.25 \%$ \\
\hline $8 \mathrm{TC} 096$ & $7.51 \mathrm{E}+02$ & 0 & $1.12 \mathrm{E}+04$ & 0 & 0 & 0 & $6.52 \mathrm{E}+03$ & $5.42 \mathrm{E}+01$ & $4.56 \mathrm{E}+00$ & $5.05 \mathrm{E}+01$ & $2.50 \mathrm{E}+04$ & $8.04 \mathrm{E}+05$ & $3.11 \%$ \\
\hline $8 \mathrm{TC} 097$ & $9.30 \mathrm{E}+02$ & 0 & 0 & 0 & 0 & 0 & $4.40 \mathrm{E}+03$ & $3.70 \mathrm{E}+01$ & $3.26 \mathrm{E}+00$ & $2.17 \mathrm{E}+01$ & $1.01 \mathrm{E}+04$ & $8.15 \mathrm{E}+05$ & $1.24 \%$ \\
\hline $8 \mathrm{TC} 098$ & $7.02 \mathrm{E}+02$ & 0 & $9.95 \mathrm{E}+03$ & 0 & 0 & 0 & $3.22 \mathrm{E}+03$ & $4.84 \mathrm{E}+01$ & $4.08 \mathrm{E}+00$ & $4.34 \mathrm{E}+01$ & $1.87 \mathrm{E}+04$ & $7.98 \mathrm{E}+05$ & $2.34 \%$ \\
\hline $8 \mathrm{TC} 100$ & $9.34 \mathrm{E}+02$ & 0 & 0 & 0 & $1.23 \mathrm{E}+02$ & 0 & $1.35 \mathrm{E}+03$ & $1.33 \mathrm{E}+01$ & $1.29 \mathrm{E}+00$ & 0 & $3.22 \mathrm{E}+03$ & $7.78 \mathrm{E}+05$ & $0.41 \%$ \\
\hline 8TC101 & $6.97 \mathrm{E}+02$ & 0 & $6.18 \mathrm{E}+03$ & 0 & 0 & 0 & 0 & $2.08 \mathrm{E}-01$ & $1.15 \mathrm{E}-02$ & 0 & $6.94 \mathrm{E}+03$ & $7.29 \mathrm{E}+05$ & $0.95 \%$ \\
\hline $8 \mathrm{TC} 102$ & $8.74 \mathrm{E}+02$ & 0 & $6.31 \mathrm{E}+03$ & 0 & 0 & 0 & 0 & 0 & $6.21 \mathrm{E}-02$ & 0 & $7.27 \mathrm{E}+03$ & $7.38 \mathrm{E}+05$ & $0.98 \%$ \\
\hline $8 \mathrm{TC} 103$ & $9.43 \mathrm{E}+02$ & 0 & 0 & 0 & $1.53 \mathrm{E}+02$ & 0 & $9.60 \mathrm{E}+02$ & $1.17 \mathrm{E}+01$ & $1.17 \mathrm{E}+00$ & 0 & $2.92 \mathrm{E}+03$ & $7.77 \mathrm{E}+05$ & $0.38 \%$ \\
\hline $8 \mathrm{TC} 104$ & $8.44 \mathrm{E}+02$ & 0 & $6.99 \mathrm{E}+03$ & 0 & 0 & 0 & 0 & $6.01 \mathrm{E}+00$ & $5.89 \mathrm{E}-01$ & 0 & $8.21 \mathrm{E}+03$ & $7.46 \mathrm{E}+05$ & $1.10 \%$ \\
\hline $8 \mathrm{TC} 105$ & $8.23 \mathrm{E}+02$ & 0 & $8.75 \mathrm{E}+03$ & 0 & 0 & 0 & $2.13 \mathrm{E}+03$ & $2.15 \mathrm{E}+01$ & $1.88 \mathrm{E}+00$ & $1.37 \mathrm{E}+01$ & $1.28 \mathrm{E}+04$ & $7.66 \mathrm{E}+05$ & $1.67 \%$ \\
\hline $8 \mathrm{TC} 106$ & $7.59 \mathrm{E}+02$ & 0 & $7.81 \mathrm{E}+03$ & 0 & 0 & 0 & $1.30 \mathrm{E}+02$ & $1.63 \mathrm{E}+01$ & $1.38 \mathrm{E}+00$ & $1.17 \mathrm{E}+01$ & $9.54 \mathrm{E}+03$ & $7.54 \mathrm{E}+05$ & $1.26 \%$ \\
\hline $8 \mathrm{TC} 107$ & $9.33 \mathrm{E}+02$ & 0 & $6.19 \mathrm{E}+03$ & 0 & 0 & 0 & 0 & 0 & 0 & 0 & $7.15 \mathrm{E}+03$ & $7.40 \mathrm{E}+05$ & $0.97 \%$ \\
\hline $8 \mathrm{TC} 108$ & $5.86 \mathrm{E}+02$ & 0 & 0 & $7.88 \mathrm{E}+03$ & 0 & 0 & $4.86 \mathrm{E}+03$ & $5.21 \mathrm{E}+01$ & $4.37 \mathrm{E}+00$ & $4.87 \mathrm{E}+01$ & $1.72 \mathrm{E}+04$ & $8.01 \mathrm{E}+05$ & $2.15 \%$ \\
\hline 8TC109 & $2.22 \mathrm{E}+02$ & 0 & 0 & $1.38 \mathrm{E}+04$ & 0 & 0 & $4.32 \mathrm{E}+03$ & $4.13 \mathrm{E}+01$ & $3.40 \mathrm{E}+00$ & $3.92 \mathrm{E}+01$ & $2.05 \mathrm{E}+04$ & $7.59 \mathrm{E}+05$ & $2.71 \%$ \\
\hline $8 \mathrm{TC} 110$ & $5.41 \mathrm{E}+02$ & 0 & 0 & $9.51 \mathrm{E}+03$ & 0 & 0 & $5.33 \mathrm{E}+03$ & $5.08 \mathrm{E}+01$ & $4.24 \mathrm{E}+00$ & $4.80 \mathrm{E}+01$ & $1.90 \mathrm{E}+04$ & $7.93 \mathrm{E}+05$ & $2.39 \%$ \\
\hline $8 \mathrm{TC} 111$ & $3.61 \mathrm{E}+02$ & 0 & 0 & $1.46 \mathrm{E}+04$ & 0 & 0 & $6.57 \mathrm{E}+03$ & $5.16 \mathrm{E}+01$ & $4.29 \mathrm{E}+00$ & $4.91 \mathrm{E}+01$ & $2.43 \mathrm{E}+04$ & $7.77 \mathrm{E}+05$ & $3.12 \%$ \\
\hline $8 \mathrm{TC} 112$ & $5.35 \mathrm{E}+02$ & 0 & 0 & $4.76 \mathrm{E}+03$ & 0 & 0 & $3.38 \mathrm{E}+03$ & $3.04 \mathrm{E}+01$ & $2.52 \mathrm{E}+00$ & $2.76 \mathrm{E}+01$ & $1.03 \mathrm{E}+04$ & $7.71 \mathrm{E}+05$ & $1.33 \%$ \\
\hline $8 \mathrm{TC} 113$ & $4.23 \mathrm{E}+02$ & 0 & 0 & $6.61 \mathrm{E}+03$ & 0 & 0 & $2.51 \mathrm{E}+03$ & $3.32 \mathrm{E}+01$ & $2.74 \mathrm{E}+00$ & $3.10 \mathrm{E}+01$ & $1.13 \mathrm{E}+04$ & $7.66 \mathrm{E}+05$ & $1.47 \%$ \\
\hline $8 \mathrm{TC} 116$ & $5.04 \mathrm{E}+02$ & 0 & 0 & 0 & 0 & 0 & 0 & $3.52 \mathrm{E}+00$ & $2.39 \mathrm{E}-01$ & $1.67 \mathrm{E}+00$ & $6.97 \mathrm{E}+02$ & $7.37 \mathrm{E}+05$ & $0.09 \%$ \\
\hline $8 \mathrm{TC} 117$ & $6.31 \mathrm{E}+02$ & 0 & 0 & $1.99 \mathrm{E}+03$ & 0 & 0 & $2.73 \mathrm{E}+03$ & $2.42 \mathrm{E}+01$ & $2.01 \mathrm{E}+00$ & $2.13 \mathrm{E}+01$ & $6.58 \mathrm{E}+03$ & $7.70 \mathrm{E}+05$ & $0.85 \%$ \\
\hline
\end{tabular}


Table 40. Predicted Solids for Envelope C Simulations - Part 2 (cont'd)

\begin{tabular}{|c|c|c|c|c|c|c|c|c|c|c|c|c|c|}
\hline Test ID & $\mathrm{NA} 2 \mathrm{C} 2 \mathrm{O} 4$ & NA2U2O7 & NA3FSO4 & $\begin{array}{c}\text { NA6SO42 } \\
\text { CO3 }\end{array}$ & NAF & NANO3 & $\begin{array}{c}\text { NASGEL.1 } \\
5.5 \mathrm{H} 2 \mathrm{O}\end{array}$ & $\mathrm{NIOH} 2$ & $\mathrm{SRCO} 3$ & ZRO2 & Total Solids & $\begin{array}{c}\text { Total Soln } \\
\text { Mass }\end{array}$ & $\begin{array}{l}\text { Solids \% of } \\
\text { Total Soln }\end{array}$ \\
\hline $8 \mathrm{TC} 118$ & $3.09 \mathrm{E}+02$ & 0 & 0 & $7.32 \mathrm{E}+03$ & 0 & 0 & $1.78 \mathrm{E}+03$ & $2.52 \mathrm{E}+01$ & $2.06 \mathrm{E}+00$ & $2.36 \mathrm{E}+01$ & $1.07 \mathrm{E}+04$ & $7.49 \mathrm{E}+05$ & $1.43 \%$ \\
\hline $8 \mathrm{TC} 119$ & $3.36 \mathrm{E}+02$ & 0 & 0 & $1.81 \mathrm{E}+03$ & 0 & 0 & 0 & $1.04 \mathrm{E}+01$ & $8.04 \mathrm{E}-01$ & $8.78 \mathrm{E}+00$ & $2.69 \mathrm{E}+03$ & $7.37 \mathrm{E}+05$ & $0.36 \%$ \\
\hline $8 \mathrm{TC} 120$ & $5.72 \mathrm{E}+02$ & 0 & 0 & 0 & 0 & 0 & 0 & $4.57 \mathrm{E}+00$ & $3.47 \mathrm{E}-01$ & $1.93 \mathrm{E}+00$ & $8.26 \mathrm{E}+02$ & $7.41 \mathrm{E}+05$ & $0.11 \%$ \\
\hline $8 \mathrm{TC} 121$ & $5.28 \mathrm{E}+02$ & 0 & 0 & 0 & 0 & 0 & $5.64 \mathrm{E}+02$ & $1.08 \mathrm{E}+01$ & $8.60 \mathrm{E}-01$ & $8.89 \mathrm{E}+00$ & $1.64 \mathrm{E}+03$ & $7.48 \mathrm{E}+05$ & $0.22 \%$ \\
\hline $8 \mathrm{TC} 122$ & $8.82 \mathrm{E}+02$ & 0 & 0 & 0 & 0 & 0 & $2.68 \mathrm{E}+03$ & $2.67 \mathrm{E}+01$ & $2.38 \mathrm{E}+00$ & $1.41 \mathrm{E}+01$ & $6.32 \mathrm{E}+03$ & $7.96 \mathrm{E}+05$ & $0.79 \%$ \\
\hline $8 \mathrm{TC} 123$ & $7.94 \mathrm{E}+02$ & 0 & $1.11 \mathrm{E}+04$ & 0 & 0 & 0 & $6.93 \mathrm{E}+03$ & $5.43 \mathrm{E}+01$ & $4.59 \mathrm{E}+00$ & $4.90 \mathrm{E}+01$ & $2.52 \mathrm{E}+04$ & $8.09 \mathrm{E}+05$ & $3.11 \%$ \\
\hline 8TC124 & $9.38 \mathrm{E}+02$ & 0 & $9.57 \mathrm{E}+03$ & 0 & 0 & 0 & $4.22 \mathrm{E}+03$ & $3.34 \mathrm{E}+01$ & $2.90 \mathrm{E}+00$ & $2.41 \mathrm{E}+01$ & $1.85 \mathrm{E}+04$ & $7.88 \mathrm{E}+05$ & $2.35 \%$ \\
\hline $8 \mathrm{TC} 125$ & $8.56 \mathrm{E}+02$ & 0 & $9.64 \mathrm{E}+03$ & 0 & 0 & 0 & $3.76 \mathrm{E}+03$ & $3.50 \mathrm{E}+01$ & $2.99 \mathrm{E}+00$ & $2.87 \mathrm{E}+01$ & $1.78 \mathrm{E}+04$ & $7.85 \mathrm{E}+05$ & $2.27 \%$ \\
\hline $8 \mathrm{TC} 126$ & $8.60 \mathrm{E}+02$ & 0 & $1.00 \mathrm{E}+04$ & 0 & 0 & 0 & $4.78 \mathrm{E}+03$ & $4.68 \mathrm{E}+01$ & $4.03 \mathrm{E}+00$ & $3.72 \mathrm{E}+01$ & $2.15 \mathrm{E}+04$ & $8.06 \mathrm{E}+05$ & $2.67 \%$ \\
\hline $8 \mathrm{TC} 127$ & $6.83 \mathrm{E}+02$ & 0 & $9.36 \mathrm{E}+03$ & 0 & 0 & 0 & $4.21 \mathrm{E}+03$ & $3.52 \mathrm{E}+01$ & $2.95 \mathrm{E}+00$ & $3.14 \mathrm{E}+01$ & $1.61 \mathrm{E}+04$ & $7.76 \mathrm{E}+05$ & $2.07 \%$ \\
\hline $8 \mathrm{TC} 128$ & $7.21 \mathrm{E}+02$ & 0 & $9.56 \mathrm{E}+03$ & 0 & 0 & 0 & $5.02 \mathrm{E}+03$ & $4.15 \mathrm{E}+01$ & $3.49 \mathrm{E}+00$ & $3.77 \mathrm{E}+01$ & $1.82 \mathrm{E}+04$ & $7.86 \mathrm{E}+05$ & $2.32 \%$ \\
\hline $8 \mathrm{TC} 129$ & $7.69 \mathrm{E}+02$ & 0 & $6.82 \mathrm{E}+03$ & 0 & 0 & 0 & 0 & $5.38 \mathrm{E}+00$ & $4.76 \mathrm{E}-01$ & $5.75 \mathrm{E}-01$ & $7.91 \mathrm{E}+03$ & $7.40 \mathrm{E}+05$ & $1.07 \%$ \\
\hline $8 \mathrm{TC} 130$ & $9.07 \mathrm{E}+02$ & 0 & 0 & 0 & $7.85 \mathrm{E}+01$ & 0 & $1.09 \mathrm{E}+03$ & $1.44 \mathrm{E}+01$ & $1.36 \mathrm{E}+00$ & $8.24 \mathrm{E}-01$ & $2.97 \mathrm{E}+03$ & $7.78 \mathrm{E}+05$ & $0.38 \%$ \\
\hline $8 \mathrm{TC} 132$ & $7.10 \mathrm{E}+02$ & 0 & $6.74 \mathrm{E}+03$ & 0 & 0 & 0 & 0 & $6.11 \mathrm{E}+00$ & $5.08 \mathrm{E}-01$ & $2.48 \mathrm{E}+00$ & $7.79 \mathrm{E}+03$ & $7.37 \mathrm{E}+05$ & $1.06 \%$ \\
\hline 8TC133 & $8.86 \mathrm{E}+02$ & 0 & $7.08 \mathrm{E}+03$ & 0 & 0 & 0 & $1.39 \mathrm{E}+02$ & $6.89 \mathrm{E}+00$ & $6.73 \mathrm{E}-01$ & 0 & $8.53 \mathrm{E}+03$ & $7.50 \mathrm{E}+05$ & $1.14 \%$ \\
\hline 8TC134 & $8.76 \mathrm{E}+02$ & 0 & 0 & 0 & $1.63 \mathrm{E}+01$ & 0 & $2.29 \mathrm{E}+03$ & $2.17 \mathrm{E}+01$ & $1.96 \mathrm{E}+00$ & $8.92 \mathrm{E}+00$ & $4.96 \mathrm{E}+03$ & $7.88 \mathrm{E}+05$ & $0.63 \%$ \\
\hline $8 \mathrm{TC} 135$ & $9.28 \mathrm{E}+02$ & 0 & 0 & 0 & $9.66 \mathrm{E}+01$ & 0 & $1.09 \mathrm{E}+03$ & $1.10 \mathrm{E}+01$ & $1.06 \mathrm{E}+00$ & 0 & $2.76 \mathrm{E}+03$ & $7.73 \mathrm{E}+05$ & $0.36 \%$ \\
\hline 6TC001 & $6.87 \mathrm{E}+02$ & 0 & 0 & 0 & 0 & 0 & 0 & 0 & 0 & 0 & $1.06 \mathrm{E}+03$ & $9.55 \mathrm{E}+05$ & $0.11 \%$ \\
\hline 6TC002 & $6.47 \mathrm{E}+02$ & 0 & 0 & 0 & 0 & 0 & 0 & 0 & 0 & 0 & $1.16 \mathrm{E}+03$ & $9.54 \mathrm{E}+05$ & $0.12 \%$ \\
\hline 6TC003 & $6.51 \mathrm{E}+02$ & 0 & 0 & 0 & 0 & 0 & 0 & 0 & 0 & 0 & $6.51 \mathrm{E}+02$ & $9.53 \mathrm{E}+05$ & $0.07 \%$ \\
\hline 6ТC004 & $5.97 \mathrm{E}+02$ & 0 & 0 & 0 & 0 & 0 & 0 & 0 & 0 & 0 & $5.97 \mathrm{E}+02$ & $9.51 \mathrm{E}+05$ & $0.06 \%$ \\
\hline $6 \mathrm{TC} 005$ & $6.49 \mathrm{E}+02$ & 0 & 0 & 0 & 0 & 0 & 0 & 0 & 0 & 0 & $6.49 \mathrm{E}+02$ & $9.52 \mathrm{E}+05$ & $0.07 \%$ \\
\hline 6TC006 & 0 & 0 & 0 & 0 & 0 & 0 & 0 & 0 & 0 & 0 & 0 & $9.16 \mathrm{E}+05$ & $0.00 \%$ \\
\hline 6TC007 & 0 & 0 & 0 & 0 & 0 & 0 & 0 & 0 & 0 & 0 & 0 & $9.16 \mathrm{E}+05$ & $0.00 \%$ \\
\hline 6TC008 & 0 & 0 & 0 & 0 & 0 & 0 & 0 & 0 & 0 & 0 & 0 & $9.14 \mathrm{E}+05$ & $0.00 \%$ \\
\hline 6TC009 & 0 & 0 & 0 & 0 & 0 & 0 & 0 & 0 & 0 & 0 & 0 & $9.12 \mathrm{E}+05$ & $0.00 \%$ \\
\hline 6TC010 & 0 & 0 & 0 & 0 & 0 & 0 & 0 & 0 & 0 & 0 & 0 & $9.13 \mathrm{E}+05$ & $0.00 \%$ \\
\hline 6TC013 & $5.59 \mathrm{E}+02$ & 0 & 0 & 0 & 0 & 0 & $7.61 \mathrm{E}+03$ & $5.67 \mathrm{E}+01$ & $4.76 \mathrm{E}+00$ & $4.73 \mathrm{E}+01$ & $1.64 \mathrm{E}+04$ & $1.06 \mathrm{E}+06$ & $1.55 \%$ \\
\hline 6TC014 & $4.83 \mathrm{E}+02$ & 0 & 0 & 0 & 0 & 0 & $3.43 \mathrm{E}+03$ & $5.58 \mathrm{E}+01$ & $4.71 \mathrm{E}+00$ & $4.32 \mathrm{E}+01$ & $1.18 \mathrm{E}+04$ & $1.05 \mathrm{E}+06$ & $1.12 \%$ \\
\hline
\end{tabular}


Table 40. Predicted Solids for Envelope C Simulations - Part 2 (cont'd)

\begin{tabular}{|c|c|c|c|c|c|c|c|c|c|c|c|c|c|}
\hline Test ID & NA2C2O4 & NA2U2O7 & NA3FSO4 & $\begin{array}{c}\text { NA6SO42 } \\
\text { CO3 }\end{array}$ & NAF & NANO3 & $\begin{array}{c}\text { NASGEL. } 1 \\
5.5 \mathrm{H} 2 \mathrm{O} \\
\end{array}$ & $\mathrm{NIOH} 2$ & $\mathrm{SRCO} 3$ & $\mathrm{ZRO} 2$ & Total Solids & $\begin{array}{c}\text { Total Soln } \\
\text { Mass } \\
\end{array}$ & $\begin{array}{l}\text { Solids } \% \text { of } \\
\text { Total Soln } \\
\end{array}$ \\
\hline 6TC015 & $5.31 \mathrm{E}+02$ & 0 & 0 & 0 & 0 & 0 & $3.68 \mathrm{E}+03$ & $5.67 \mathrm{E}+01$ & $4.76 \mathrm{E}+00$ & $4.90 \mathrm{E}+01$ & $1.25 \mathrm{E}+04$ & $1.06 \mathrm{E}+06$ & $1.18 \%$ \\
\hline $6 \mathrm{TC} 016$ & 0 & 0 & 0 & 0 & 0 & 0 & $7.70 \mathrm{E}+03$ & $5.81 \mathrm{E}+01$ & $4.83 \mathrm{E}+00$ & $5.55 \mathrm{E}+01$ & $1.08 \mathrm{E}+04$ & $1.01 \mathrm{E}+06$ & $1.06 \%$ \\
\hline 6ТC017 & 0 & 0 & 0 & $1.04 \mathrm{E}+03$ & 0 & 0 & $7.61 \mathrm{E}+03$ & $5.78 \mathrm{E}+01$ & $4.79 \mathrm{E}+00$ & $5.51 \mathrm{E}+01$ & $1.17 \mathrm{E}+04$ & $1.01 \mathrm{E}+06$ & $1.16 \%$ \\
\hline $6 \mathrm{TC} 018$ & 0 & 0 & 0 & 0 & 0 & 0 & $7.49 \mathrm{E}+03$ & $5.75 \mathrm{E}+01$ & $4.77 \mathrm{E}+00$ & $5.51 \mathrm{E}+01$ & $1.05 \mathrm{E}+04$ & $1.01 \mathrm{E}+06$ & $1.04 \%$ \\
\hline 6TC019 & 0 & 0 & 0 & 0 & 0 & 0 & $3.21 \mathrm{E}+03$ & $5.68 \mathrm{E}+01$ & $4.71 \mathrm{E}+00$ & $5.42 \mathrm{E}+01$ & $6.23 \mathrm{E}+03$ & $1.01 \mathrm{E}+06$ & $0.62 \%$ \\
\hline $6 \mathrm{TC} 020$ & 0 & 0 & 0 & 0 & 0 & 0 & $3.57 \mathrm{E}+03$ & $5.73 \mathrm{E}+01$ & $4.76 \mathrm{E}+00$ & $5.50 \mathrm{E}+01$ & $7.77 \mathrm{E}+03$ & $1.01 \mathrm{E}+06$ & $0.77 \%$ \\
\hline 6TC021 & $1.57 \mathrm{E}+02$ & 0 & 0 & 0 & 0 & 0 & $2.37 \mathrm{E}+03$ & $1.89 \mathrm{E}+01$ & $1.56 \mathrm{E}+00$ & $1.47 \mathrm{E}+01$ & $3.47 \mathrm{E}+03$ & $9.72 \mathrm{E}+05$ & $0.36 \%$ \\
\hline $6 \mathrm{TC} 022$ & $6.14 \mathrm{E}+02$ & 0 & 0 & 0 & 0 & 0 & $1.09 \mathrm{E}+03$ & $8.35 \mathrm{E}+00$ & $7.51 \mathrm{E}-01$ & 0 & $2.63 \mathrm{E}+03$ & $9.70 \mathrm{E}+05$ & $0.27 \%$ \\
\hline 6TC023 & $2.15 \mathrm{E}+02$ & 0 & 0 & 0 & 0 & 0 & $1.60 \mathrm{E}+03$ & $1.33 \mathrm{E}+01$ & $1.09 \mathrm{E}+00$ & $8.33 \mathrm{E}+00$ & $2.48 \mathrm{E}+03$ & $9.64 \mathrm{E}+05$ & $0.26 \%$ \\
\hline 6ТC024 & $5.93 \mathrm{E}+02$ & 0 & 0 & 0 & 0 & 0 & $2.53 \mathrm{E}+03$ & $1.90 \mathrm{E}+01$ & $1.66 \mathrm{E}+00$ & $2.17 \mathrm{E}+00$ & $6.38 \mathrm{E}+03$ & $9.90 \mathrm{E}+05$ & $0.64 \%$ \\
\hline $6 \mathrm{TC} 025$ & $3.63 \mathrm{E}+02$ & 0 & 0 & 0 & 0 & 0 & 0 & 0 & 0 & 0 & $3.63 \mathrm{E}+02$ & $9.43 \mathrm{E}+05$ & $0.04 \%$ \\
\hline $6 \mathrm{TC} 026$ & $5.25 \mathrm{E}+02$ & 0 & $6.17 \mathrm{E}+02$ & 0 & 0 & 0 & $2.27 \mathrm{E}+03$ & $1.73 \mathrm{E}+01$ & $1.49 \mathrm{E}+00$ & $6.02 \mathrm{E}+00$ & $5.08 \mathrm{E}+03$ & $9.81 \mathrm{E}+05$ & $0.52 \%$ \\
\hline 6TC027 & $3.31 \mathrm{E}+02$ & 0 & 0 & 0 & 0 & 0 & $9.79 \mathrm{E}+02$ & $9.31 \mathrm{E}+00$ & $7.76 \mathrm{E}-01$ & $2.19 \mathrm{E}+00$ & $1.80 \mathrm{E}+03$ & $9.61 \mathrm{E}+05$ & $0.19 \%$ \\
\hline 6ТC029 & $6.15 \mathrm{E}+02$ & 0 & 0 & 0 & 0 & 0 & $3.86 \mathrm{E}+03$ & $2.88 \mathrm{E}+01$ & $2.47 \mathrm{E}+00$ & $1.28 \mathrm{E}+01$ & $9.99 E+03$ & $1.01 \mathrm{E}+06$ & $0.99 \%$ \\
\hline 6TC030 & $5.52 \mathrm{E}+02$ & 0 & 0 & 0 & 0 & 0 & $5.88 \mathrm{E}+03$ & $4.37 \mathrm{E}+01$ & $3.72 \mathrm{E}+00$ & $2.91 \mathrm{E}+01$ & $1.38 \mathrm{E}+04$ & $1.03 \mathrm{E}+06$ & $1.34 \%$ \\
\hline 6TC031 & $4.77 \mathrm{E}+02$ & 0 & $2.92 \mathrm{E}+03$ & 0 & 0 & 0 & $6.01 \mathrm{E}+03$ & $4.49 \mathrm{E}+01$ & $3.79 \mathrm{E}+00$ & $3.63 \mathrm{E}+01$ & $1.67 \mathrm{E}+04$ & $1.02 \mathrm{E}+06$ & $1.63 \%$ \\
\hline 6TC032 & $1.56 \mathrm{E}+02$ & 0 & $2.62 \mathrm{E}+03$ & 0 & 0 & 0 & $5.92 \mathrm{E}+03$ & $4.45 \mathrm{E}+01$ & $3.72 \mathrm{E}+00$ & $4.03 \mathrm{E}+01$ & $1.35 \mathrm{E}+04$ & $1.01 \mathrm{E}+06$ & $1.33 \%$ \\
\hline $6 \mathrm{TC} 033$ & $2.90 \mathrm{E}+02$ & 0 & $2.56 \mathrm{E}+03$ & 0 & 0 & 0 & $4.94 \mathrm{E}+03$ & $3.73 \mathrm{E}+01$ & $3.13 \mathrm{E}+00$ & $3.11 \mathrm{E}+01$ & $1.15 \mathrm{E}+04$ & $1.00 \mathrm{E}+06$ & $1.15 \%$ \\
\hline $6 \mathrm{TC} 034$ & $5.96 \mathrm{E}+02$ & 0 & 0 & 0 & 0 & 0 & $6.34 \mathrm{E}+03$ & $4.71 \mathrm{E}+01$ & $4.00 \mathrm{E}+00$ & $3.23 \mathrm{E}+01$ & $1.55 \mathrm{E}+04$ & $1.04 \mathrm{E}+06$ & $1.49 \%$ \\
\hline $6 \mathrm{TC} 035$ & 0 & 0 & 0 & 0 & 0 & 0 & $3.16 \mathrm{E}+03$ & $2.51 \mathrm{E}+01$ & $2.05 \mathrm{E}+00$ & $2.24 \mathrm{E}+01$ & $4.47 \mathrm{E}+03$ & $9.73 \mathrm{E}+05$ & $0.46 \%$ \\
\hline $6 \mathrm{TC} 036$ & 0 & 0 & 0 & 0 & 0 & 0 & $1.19 \mathrm{E}+02$ & $3.39 \mathrm{E}+00$ & $2.04 \mathrm{E}-01$ & $1.82 \mathrm{E}+00$ & $2.92 \mathrm{E}+02$ & $9.29 \mathrm{E}+05$ & $0.03 \%$ \\
\hline 6ТC037 & 0 & 0 & 0 & 0 & 0 & 0 & $2.75 \mathrm{E}+03$ & $2.27 \mathrm{E}+01$ & $1.84 \mathrm{E}+00$ & $2.04 \mathrm{E}+01$ & $3.94 \mathrm{E}+03$ & $9.64 \mathrm{E}+05$ & $0.41 \%$ \\
\hline 6ТC038 & 0 & 0 & 0 & 0 & 0 & 0 & 0 & $2.25 \mathrm{E}+00$ & $1.22 \mathrm{E}-01$ & 0 & $1.28 \mathrm{E}+02$ & $9.35 \mathrm{E}+05$ & $0.01 \%$ \\
\hline $6 \mathrm{TC} 039$ & 0 & 0 & 0 & 0 & 0 & 0 & $2.12 \mathrm{E}+03$ & $1.80 \mathrm{E}+01$ & $1.42 \mathrm{E}+00$ & $1.65 \mathrm{E}+01$ & $3.08 \mathrm{E}+03$ & $9.46 \mathrm{E}+05$ & $0.33 \%$ \\
\hline $6 \mathrm{TC} 040$ & 0 & 0 & 0 & 0 & 0 & 0 & $7.02 \mathrm{E}+02$ & $7.37 \mathrm{E}+00$ & $5.54 \mathrm{E}-01$ & $5.08 \mathrm{E}+00$ & $1.07 \mathrm{E}+03$ & $9.43 \mathrm{E}+05$ & $0.11 \%$ \\
\hline 6TC041 & 0 & 0 & 0 & 0 & 0 & 0 & $3.39 \mathrm{E}+03$ & $2.70 \mathrm{E}+01$ & $2.20 \mathrm{E}+00$ & $2.45 \mathrm{E}+01$ & $4.81 \mathrm{E}+03$ & $9.73 \mathrm{E}+05$ & $0.49 \%$ \\
\hline 6TC042 & 0 & 0 & 0 & 0 & 0 & 0 & $5.36 \mathrm{E}+03$ & $4.15 \mathrm{E}+01$ & $3.42 \mathrm{E}+00$ & $3.93 \mathrm{E}+01$ & $7.56 \mathrm{E}+03$ & $9.87 \mathrm{E}+05$ & $0.77 \%$ \\
\hline 6ТC045 & 0 & 0 & 0 & 0 & 0 & 0 & $5.60 \mathrm{E}+03$ & $4.30 \mathrm{E}+01$ & $3.55 \mathrm{E}+00$ & $4.08 \mathrm{E}+01$ & $7.88 \mathrm{E}+03$ & $9.89 \mathrm{E}+05$ & $0.80 \%$ \\
\hline $6 \mathrm{TC} 046$ & 0 & 0 & 0 & 0 & 0 & 0 & $6.54 \mathrm{E}+03$ & $4.97 \mathrm{E}+01$ & $4.12 \mathrm{E}+00$ & $4.69 \mathrm{E}+01$ & $9.18 \mathrm{E}+03$ & $1.01 \mathrm{E}+06$ & $0.91 \%$ \\
\hline
\end{tabular}


Table 40. Predicted Solids for Envelope C Simulations - Part 2 (cont'd)

\begin{tabular}{|c|c|c|c|c|c|c|c|c|c|c|c|c|c|}
\hline Test ID & NA2C2O4 & NA2U2O7 & NA3FSO4 & $\begin{array}{c}\text { NA6SO42 } \\
\text { CO3 }\end{array}$ & NAF & NANO3 & $\begin{array}{c}\text { NASGEL.1 } \\
5.5 \mathrm{H} 2 \mathrm{O}\end{array}$ & $\mathrm{NIOH} 2$ & SRCO3 & ZRO2 & Total Solids & $\begin{array}{c}\text { Total Soln } \\
\text { Mass }\end{array}$ & $\begin{array}{l}\text { Solids \% of } \\
\text { Total Soln }\end{array}$ \\
\hline 6TC047 & 0 & 0 & 0 & 0 & 0 & 0 & $4.47 \mathrm{E}+03$ & $3.44 \mathrm{E}+01$ & $2.83 \mathrm{E}+00$ & $3.22 \mathrm{E}+01$ & $6.29 \mathrm{E}+03$ & $9.84 \mathrm{E}+05$ & $0.64 \%$ \\
\hline 6TC048 & 0 & 0 & 0 & 0 & 0 & 0 & $5.22 \mathrm{E}+03$ & $4.01 \mathrm{E}+01$ & $3.32 \mathrm{E}+00$ & $3.70 \mathrm{E}+01$ & $7.34 \mathrm{E}+03$ & $9.99 \mathrm{E}+05$ & $0.73 \%$ \\
\hline 6TC049 & 0 & 0 & 0 & 0 & 0 & 0 & $7.49 \mathrm{E}+03$ & $5.71 \mathrm{E}+01$ & $4.75 \mathrm{E}+00$ & $5.42 \mathrm{E}+01$ & $1.05 \mathrm{E}+04$ & $1.02 \mathrm{E}+06$ & $1.03 \%$ \\
\hline $6 \mathrm{TC} 050$ & $1.24 \mathrm{E}+02$ & 0 & 0 & 0 & 0 & 0 & $2.69 \mathrm{E}+02$ & $3.93 \mathrm{E}+00$ & $2.83 \mathrm{E}-01$ & $2.19 \mathrm{E}-01$ & $6.07 \mathrm{E}+02$ & $9.44 \mathrm{E}+05$ & $0.06 \%$ \\
\hline 6TC051 & $6.29 \mathrm{E}+02$ & 0 & 0 & 0 & 0 & 0 & $1.89 \mathrm{E}+03$ & $1.42 \mathrm{E}+01$ & $1.25 \mathrm{E}+00$ & 0 & $4.92 \mathrm{E}+03$ & $9.82 \mathrm{E}+05$ & $0.50 \%$ \\
\hline 6TC052 & $2.42 \mathrm{E}+02$ & 0 & 0 & 0 & 0 & 0 & $5.00 \mathrm{E}+02$ & $5.47 \mathrm{E}+00$ & $4.34 \mathrm{E}-01$ & 0 & $1.04 \mathrm{E}+03$ & $9.51 \mathrm{E}+05$ & $0.11 \%$ \\
\hline $6 \mathrm{TC} 053$ & $5.15 \mathrm{E}+02$ & 0 & 0 & 0 & 0 & 0 & $4.59 \mathrm{E}+02$ & $4.47 \mathrm{E}+00$ & $4.04 \mathrm{E}-01$ & 0 & $1.25 \mathrm{E}+03$ & $9.59 \mathrm{E}+05$ & $0.13 \%$ \\
\hline 6TC054 & $2.31 \mathrm{E}+02$ & 0 & $1.13 \mathrm{E}+03$ & 0 & 0 & 0 & $3.41 \mathrm{E}+03$ & $2.62 \mathrm{E}+01$ & $2.18 \mathrm{E}+00$ & $2.19 \mathrm{E}+01$ & $6.64 \mathrm{E}+03$ & $9.85 \mathrm{E}+05$ & $0.67 \%$ \\
\hline $6 \mathrm{TC} 055$ & $3.62 \mathrm{E}+02$ & 0 & $1.39 \mathrm{E}+03$ & 0 & 0 & 0 & $2.99 \mathrm{E}+03$ & $2.31 \mathrm{E}+01$ & $1.94 \mathrm{E}+00$ & $1.57 \mathrm{E}+01$ & $6.32 \mathrm{E}+03$ & $9.83 \mathrm{E}+05$ & $0.64 \%$ \\
\hline 6TC056 & $9.24 \mathrm{E}+01$ & 0 & $4.00 \mathrm{E}+02$ & 0 & 0 & 0 & $2.45 \mathrm{E}+03$ & $1.99 \mathrm{E}+01$ & $1.63 \mathrm{E}+00$ & $1.61 \mathrm{E}+01$ & $3.95 \mathrm{E}+03$ & $9.71 \mathrm{E}+05$ & $0.41 \%$ \\
\hline $6 \mathrm{TC} 057$ & $5.21 \mathrm{E}+02$ & 0 & $1.60 \mathrm{E}+03$ & 0 & 0 & 0 & $4.65 \mathrm{E}+03$ & $3.48 \mathrm{E}+01$ & $2.95 \mathrm{E}+00$ & $2.54 \mathrm{E}+01$ & $1.25 \mathrm{E}+04$ & $1.01 \mathrm{E}+06$ & $1.24 \%$ \\
\hline 6TC058 & $2.66 \mathrm{E}+02$ & 0 & $3.08 \mathrm{E}+03$ & 0 & 0 & 0 & $7.13 \mathrm{E}+03$ & $5.33 \mathrm{E}+01$ & $4.47 \mathrm{E}+00$ & $4.80 \mathrm{E}+01$ & $1.73 \mathrm{E}+04$ & $1.03 \mathrm{E}+06$ & $1.68 \%$ \\
\hline $6 \mathrm{TC} 059$ & $8.96 \mathrm{E}+01$ & 0 & $1.41 \mathrm{E}+03$ & 0 & 0 & 0 & $4.16 \mathrm{E}+03$ & $3.24 \mathrm{E}+01$ & $2.69 \mathrm{E}+00$ & $2.83 \mathrm{E}+01$ & $7.61 \mathrm{E}+03$ & $9.90 \mathrm{E}+05$ & $0.77 \%$ \\
\hline 6TC061 & $4.62 \mathrm{E}+02$ & 0 & $3.04 \mathrm{E}+03$ & 0 & 0 & 0 & $6.20 \mathrm{E}+03$ & $4.63 \mathrm{E}+01$ & $3.91 \mathrm{E}+00$ & $3.82 \mathrm{E}+01$ & $1.74 \mathrm{E}+04$ & $1.03 \mathrm{E}+06$ & $1.70 \%$ \\
\hline 6TC062 & $1.18 \mathrm{E}+02$ & 0 & $3.17 \mathrm{E}+03$ & 0 & 0 & 0 & $6.97 \mathrm{E}+03$ & $5.23 \mathrm{E}+01$ & $4.37 \mathrm{E}+00$ & $4.80 \mathrm{E}+01$ & $1.62 \mathrm{E}+04$ & $1.02 \mathrm{E}+06$ & $1.58 \%$ \\
\hline 6TC063 & $2.36 \mathrm{E}+02$ & 0 & $3.08 \mathrm{E}+03$ & 0 & 0 & 0 & $6.10 \mathrm{E}+03$ & $4.59 \mathrm{E}+01$ & $3.85 \mathrm{E}+00$ & $4.02 \mathrm{E}+01$ & $1.45 \mathrm{E}+04$ & $1.02 \mathrm{E}+06$ & $1.43 \%$ \\
\hline 6TC064 & 0 & 0 & 0 & 0 & 0 & 0 & $3.60 \mathrm{E}+03$ & $2.84 \mathrm{E}+01$ & $2.31 \mathrm{E}+00$ & $2.66 \mathrm{E}+01$ & $5.10 \mathrm{E}+03$ & $9.67 \mathrm{E}+05$ & $0.53 \%$ \\
\hline 6TC065 & 0 & 0 & 0 & 0 & 0 & 0 & 0 & $1.95 \mathrm{E}+00$ & $8.84 \mathrm{E}-02$ & $7.64 \mathrm{E}-03$ & $1.06 \mathrm{E}+02$ & $9.31 \mathrm{E}+05$ & $0.01 \%$ \\
\hline 6TC066 & 0 & 0 & 0 & 0 & 0 & 0 & $2.66 \mathrm{E}+03$ & $2.24 \mathrm{E}+01$ & $1.80 \mathrm{E}+00$ & $2.05 \mathrm{E}+01$ & $3.85 \mathrm{E}+03$ & $9.58 \mathrm{E}+05$ & $0.40 \%$ \\
\hline $6 \mathrm{TC} 067$ & 0 & 0 & 0 & 0 & 0 & 0 & $2.56 \mathrm{E}+03$ & $2.10 \mathrm{E}+01$ & $1.70 \mathrm{E}+00$ & $1.88 \mathrm{E}+01$ & $3.66 \mathrm{E}+03$ & $9.60 \mathrm{E}+05$ & $0.38 \%$ \\
\hline 6TC068 & 0 & 0 & 0 & 0 & 0 & 0 & $8.06 \mathrm{E}+02$ & $8.43 \mathrm{E}+00$ & $6.22 \mathrm{E}-01$ & $6.94 \mathrm{E}+00$ & $1.25 \mathrm{E}+03$ & $9.35 \mathrm{E}+05$ & $0.13 \%$ \\
\hline $6 \mathrm{TC} 069$ & $7.42 \mathrm{E}+00$ & 0 & 0 & 0 & 0 & 0 & $2.65 \mathrm{E}+03$ & $2.13 \mathrm{E}+01$ & $1.75 \mathrm{E}+00$ & $1.82 \mathrm{E}+01$ & $3.75 \mathrm{E}+03$ & $9.72 \mathrm{E}+05$ & $0.39 \%$ \\
\hline $6 \mathrm{TC} 070$ & 0 & 0 & 0 & 0 & 0 & 0 & $1.77 \mathrm{E}+03$ & $1.50 \mathrm{E}+01$ & $1.20 \mathrm{E}+00$ & $1.20 \mathrm{E}+01$ & $2.54 \mathrm{E}+03$ & $9.59 \mathrm{E}+05$ & $0.26 \%$ \\
\hline 6TC071 & 0 & 0 & 0 & 0 & 0 & 0 & $6.74 \mathrm{E}+03$ & $5.09 \mathrm{E}+01$ & $4.23 \mathrm{E}+00$ & $4.79 \mathrm{E}+01$ & $1.15 \mathrm{E}+04$ & $1.02 \mathrm{E}+06$ & $1.13 \%$ \\
\hline 6TC072 & 0 & 0 & 0 & 0 & 0 & 0 & $5.29 \mathrm{E}+03$ & $4.07 \mathrm{E}+01$ & $3.35 \mathrm{E}+00$ & $3.85 \mathrm{E}+01$ & $7.45 \mathrm{E}+03$ & $9.87 \mathrm{E}+05$ & $0.75 \%$ \\
\hline $6 \mathrm{TC} 073$ & 0 & 0 & 0 & 0 & 0 & 0 & $3.98 \mathrm{E}+03$ & $3.12 \mathrm{E}+01$ & $2.57 \mathrm{E}+00$ & $2.79 \mathrm{E}+01$ & $5.61 \mathrm{E}+03$ & $9.87 \mathrm{E}+05$ & $0.57 \%$ \\
\hline 6TC074 & 0 & 0 & 0 & 0 & 0 & 0 & $6.70 \mathrm{E}+03$ & $5.05 \mathrm{E}+01$ & $4.21 \mathrm{E}+00$ & $4.69 \mathrm{E}+01$ & $1.11 \mathrm{E}+04$ & $1.02 \mathrm{E}+06$ & $1.09 \%$ \\
\hline $6 \mathrm{TC} 076$ & 0 & 0 & 0 & 0 & 0 & 0 & $4.33 \mathrm{E}+03$ & $3.35 \mathrm{E}+01$ & $2.75 \mathrm{E}+00$ & $3.16 \mathrm{E}+01$ & $6.10 \mathrm{E}+03$ & $9.76 \mathrm{E}+05$ & $0.62 \%$ \\
\hline 6ТC077 & 0 & 0 & 0 & 0 & 0 & 0 & $5.68 \mathrm{E}+03$ & $4.41 \mathrm{E}+01$ & $3.65 \mathrm{E}+00$ & $4.18 \mathrm{E}+01$ & $8.02 \mathrm{E}+03$ & $9.93 \mathrm{E}+05$ & $0.81 \%$ \\
\hline $6 \mathrm{TC} 078$ & $3.01 \mathrm{E}+01$ & 0 & 0 & 0 & 0 & 0 & $3.59 \mathrm{E}+03$ & $2.84 \mathrm{E}+01$ & $2.35 \mathrm{E}+00$ & $2.48 \mathrm{E}+01$ & $5.09 \mathrm{E}+03$ & $9.85 \mathrm{E}+05$ & $0.52 \%$ \\
\hline
\end{tabular}


Table 40. Predicted Solids for Envelope C Simulations - Part 2 (cont'd)

\begin{tabular}{|c|c|c|c|c|c|c|c|c|c|c|c|c|c|}
\hline Test ID & NA2C2O4 & NA2U2O7 & NA3FSO4 & $\begin{array}{c}\text { NA6SO42 } \\
\text { CO3 }\end{array}$ & NAF & NANO3 & $\begin{array}{c}\text { NASGEL. } 1 \\
5.5 \mathrm{H} 2 \mathrm{O}\end{array}$ & $\mathrm{NIOH} 2$ & SRCO3 & $\mathrm{ZRO} 2$ & Total Solids & $\begin{array}{c}\text { Total Soln } \\
\text { Mass }\end{array}$ & \begin{tabular}{|l|} 
Solids $\%$ of \\
Total Soln \\
\end{tabular} \\
\hline 6ТС079 & 0 & 0 & 0 & 0 & 0 & 0 & $4.23 \mathrm{E}+03$ & $3.76 \mathrm{E}+01$ & $3.12 \mathrm{E}+00$ & $3.42 \mathrm{E}+01$ & $6.20 \mathrm{E}+03$ & $9.97 \mathrm{E}+05$ & $0.62 \%$ \\
\hline 6ТC080 & 0 & 0 & 0 & 0 & 0 & 0 & $5.95 \mathrm{E}+03$ & $4.74 \mathrm{E}+01$ & $3.91 \mathrm{E}+00$ & $4.49 \mathrm{E}+01$ & $8.47 \mathrm{E}+03$ & $9.96 \mathrm{E}+05$ & $0.85 \%$ \\
\hline 6TC081 & 0 & 0 & 0 & 0 & 0 & 0 & $5.39 \mathrm{E}+03$ & $4.33 \mathrm{E}+01$ & $3.60 \mathrm{E}+00$ & $4.03 \mathrm{E}+01$ & $8.50 \mathrm{E}+03$ & $1.01 \mathrm{E}+06$ & $0.85 \%$ \\
\hline 6TC082 & 0 & 0 & 0 & 0 & 0 & 0 & $4.37 \mathrm{E}+03$ & $3.67 \mathrm{E}+01$ & $3.01 \mathrm{E}+00$ & $3.47 \mathrm{E}+01$ & $6.32 \mathrm{E}+03$ & $9.77 \mathrm{E}+05$ & $0.65 \%$ \\
\hline 6TC083 & 0 & 0 & 0 & 0 & 0 & 0 & $3.78 \mathrm{E}+03$ & $5.67 \mathrm{E}+01$ & $4.72 \mathrm{E}+00$ & $5.36 \mathrm{E}+01$ & $8.41 \mathrm{E}+03$ & $1.02 \mathrm{E}+06$ & $0.82 \%$ \\
\hline 6TC084 & 0 & 0 & 0 & 0 & 0 & 0 & $4.58 \mathrm{E}+03$ & $3.87 \mathrm{E}+01$ & $3.19 \mathrm{E}+00$ & $3.65 \mathrm{E}+01$ & $6.63 \mathrm{E}+03$ & $9.84 \mathrm{E}+05$ & $0.67 \%$ \\
\hline $6 \mathrm{TC} 085$ & 0 & 0 & 0 & 0 & 0 & 0 & $6.21 \mathrm{E}+03$ & $4.73 \mathrm{E}+01$ & $3.93 \mathrm{E}+00$ & $4.47 \mathrm{E}+01$ & $9.27 \mathrm{E}+03$ & $1.01 \mathrm{E}+06$ & $0.92 \%$ \\
\hline 6ТC086 & 0 & 0 & 0 & 0 & 0 & 0 & 0 & $2.76 \mathrm{E}+00$ & $1.56 \mathrm{E}-01$ & $5.27 \mathrm{E}-01$ & $1.48 \mathrm{E}+02$ & $9.30 \mathrm{E}+05$ & $0.02 \%$ \\
\hline 6TC087 & 0 & 0 & 0 & 0 & 0 & 0 & $2.72 \mathrm{E}+03$ & $2.71 \mathrm{E}+01$ & $2.19 \mathrm{E}+00$ & $2.53 \mathrm{E}+01$ & $4.15 \mathrm{E}+03$ & $9.60 \mathrm{E}+05$ & $0.43 \%$ \\
\hline 6TC088 & 0 & 0 & 0 & 0 & 0 & 0 & $1.15 \mathrm{E}+03$ & $1.25 \mathrm{E}+01$ & $9.65 \mathrm{E}-01$ & $1.11 \mathrm{E}+01$ & $1.81 \mathrm{E}+03$ & $9.37 \mathrm{E}+05$ & $0.19 \%$ \\
\hline 6ТC089 & 0 & 0 & 0 & 0 & 0 & 0 & $1.02 \mathrm{E}+03$ & $1.19 \mathrm{E}+01$ & $9.19 \mathrm{E}-01$ & $1.02 \mathrm{E}+01$ & $1.65 \mathrm{E}+03$ & $9.40 \mathrm{E}+05$ & $0.18 \%$ \\
\hline 6TC090 & 0 & 0 & 0 & 0 & 0 & 0 & $9.41 \mathrm{E}+02$ & $1.25 \mathrm{E}+01$ & $9.91 \mathrm{E}-01$ & $9.49 \mathrm{E}+00$ & $1.57 \mathrm{E}+03$ & $9.52 \mathrm{E}+05$ & $0.16 \%$ \\
\hline 6ТC091 & 0 & 0 & 0 & 0 & 0 & 0 & $1.89 \mathrm{E}+03$ & $1.95 \mathrm{E}+01$ & $1.57 \mathrm{E}+00$ & $1.72 \mathrm{E}+01$ & $2.91 \mathrm{E}+03$ & $9.60 \mathrm{E}+05$ & $0.30 \%$ \\
\hline 6ТC093 & $1.81 \mathrm{E}+02$ & 0 & $1.57 \mathrm{E}+03$ & 0 & 0 & 0 & $1.71 \mathrm{E}+03$ & $3.13 \mathrm{E}+01$ & $2.62 \mathrm{E}+00$ & $2.59 \mathrm{E}+01$ & $6.12 \mathrm{E}+03$ & $9.92 \mathrm{E}+05$ & $0.62 \%$ \\
\hline 6ТC094 & $3.79 \mathrm{E}+02$ & 0 & $3.41 \mathrm{E}+03$ & 0 & 0 & 0 & $6.96 \mathrm{E}+03$ & $5.30 \mathrm{E}+01$ & $4.47 \mathrm{E}+00$ & $4.55 \mathrm{E}+01$ & $1.79 \mathrm{E}+04$ & $1.03 \mathrm{E}+06$ & $1.73 \%$ \\
\hline 6TC095 & $3.62 \mathrm{E}+02$ & 0 & $1.97 \mathrm{E}+03$ & 0 & 0 & 0 & $4.43 \mathrm{E}+03$ & $3.40 \mathrm{E}+01$ & $2.86 \mathrm{E}+00$ & $2.76 \mathrm{E}+01$ & $1.06 \mathrm{E}+04$ & $1.00 \mathrm{E}+06$ & $1.06 \%$ \\
\hline 6TC096 & $1.55 \mathrm{E}+02$ & 0 & $3.08 \mathrm{E}+03$ & 0 & 0 & 0 & $6.79 \mathrm{E}+03$ & $5.44 \mathrm{E}+01$ & $4.55 \mathrm{E}+00$ & $5.05 \mathrm{E}+01$ & $1.71 \mathrm{E}+04$ & $1.03 \mathrm{E}+06$ & $1.67 \%$ \\
\hline 6TC097 & $5.68 \mathrm{E}+02$ & 0 & 0 & 0 & 0 & 0 & $4.88 \mathrm{E}+03$ & $3.79 \mathrm{E}+01$ & $3.23 \mathrm{E}+00$ & $2.22 \mathrm{E}+01$ & $1.06 \mathrm{E}+04$ & $1.02 \mathrm{E}+06$ & $1.04 \%$ \\
\hline 6TC098 & $1.59 \mathrm{E}+02$ & 0 & $2.85 \mathrm{E}+03$ & 0 & 0 & 0 & $3.74 \mathrm{E}+03$ & $4.87 \mathrm{E}+01$ & $4.08 \mathrm{E}+00$ & $4.32 \mathrm{E}+01$ & $1.25 \mathrm{E}+04$ & $1.02 \mathrm{E}+06$ & $1.23 \%$ \\
\hline 6TC099 & $3.33 \mathrm{E}+02$ & 0 & $1.15 \mathrm{E}+03$ & 0 & 0 & 0 & $3.72 \mathrm{E}+03$ & $2.97 \mathrm{E}+01$ & $2.49 \mathrm{E}+00$ & $2.34 \mathrm{E}+01$ & $8.27 \mathrm{E}+03$ & $9.94 \mathrm{E}+05$ & $0.83 \%$ \\
\hline 6TC100 & $5.70 \mathrm{E}+02$ & 0 & 0 & 0 & 0 & 0 & $1.83 \mathrm{E}+03$ & $1.45 \mathrm{E}+01$ & $1.27 \mathrm{E}+00$ & 0 & $3.82 \mathrm{E}+03$ & $9.80 \mathrm{E}+05$ & $0.39 \%$ \\
\hline 6TC101 & $1.16 \mathrm{E}+02$ & 0 & 0 & 0 & 0 & 0 & 0 & $6.41 \mathrm{E}-01$ & $8.62 \mathrm{E}-03$ & 0 & $1.79 \mathrm{E}+02$ & $9.37 \mathrm{E}+05$ & $0.02 \%$ \\
\hline 6TC102 & $4.22 \mathrm{E}+02$ & 0 & 0 & 0 & 0 & 0 & 0 & $5.94 \mathrm{E}-01$ & $5.85 \mathrm{E}-02$ & 0 & $5.05 \mathrm{E}+02$ & $9.47 \mathrm{E}+05$ & $0.05 \%$ \\
\hline $6 \mathrm{TC} 103$ & $5.96 \mathrm{E}+02$ & 0 & 0 & 0 & 0 & 0 & $1.54 \mathrm{E}+03$ & $1.30 \mathrm{E}+01$ & $1.14 \mathrm{E}+00$ & 0 & $3.52 \mathrm{E}+03$ & $9.78 \mathrm{E}+05$ & $0.36 \%$ \\
\hline $6 \mathrm{TC} 104$ & $3.91 \mathrm{E}+02$ & 0 & 0 & 0 & 0 & 0 & 0 & $6.82 \mathrm{E}+00$ & $5.85 \mathrm{E}-01$ & 0 & $7.70 \mathrm{E}+02$ & $9.58 \mathrm{E}+05$ & $0.08 \%$ \\
\hline $6 \mathrm{TC} 105$ & $3.59 \mathrm{E}+02$ & 0 & $8.75 \mathrm{E}+02$ & 0 & 0 & 0 & $2.68 \mathrm{E}+03$ & $2.22 \mathrm{E}+01$ & $1.87 \mathrm{E}+00$ & $1.37 \mathrm{E}+01$ & $5.58 \mathrm{E}+03$ & $9.83 \mathrm{E}+05$ & $0.57 \%$ \\
\hline 6TC106 & $2.23 \mathrm{E}+02$ & 0 & 0 & 0 & 0 & 0 & $1.03 \mathrm{E}+03$ & $1.67 \mathrm{E}+01$ & $1.38 \mathrm{E}+00$ & $1.15 \mathrm{E}+01$ & $2.09 \mathrm{E}+03$ & $9.70 \mathrm{E}+05$ & $0.22 \%$ \\
\hline 6TC109 & 0 & 0 & 0 & 0 & 0 & 0 & $4.67 \mathrm{E}+03$ & $4.13 \mathrm{E}+01$ & $3.40 \mathrm{E}+00$ & $3.91 \mathrm{E}+01$ & $6.87 \mathrm{E}+03$ & $9.84 \mathrm{E}+05$ & $0.70 \%$ \\
\hline $6 \mathrm{TC} 110$ & 0 & 0 & 0 & 0 & 0 & 0 & $5.62 \mathrm{E}+03$ & $5.08 \mathrm{E}+01$ & $4.23 \mathrm{E}+00$ & $4.79 \mathrm{E}+01$ & $1.06 \mathrm{E}+04$ & $1.02 \mathrm{E}+06$ & $1.04 \%$ \\
\hline
\end{tabular}


Table 40. Predicted Solids for Envelope C Simulations - Part 2 (cont'd)

\begin{tabular}{|c|c|c|c|c|c|c|c|c|c|c|c|c|c|}
\hline Test ID & NA2C2O4 & NA2U2O7 & NA3FSO4 & $\begin{array}{c}\text { NA6SO42 } \\
\text { CO3 }\end{array}$ & NAF & NANO3 & $\begin{array}{c}\text { NASGEL. } 1 \\
5.5 \mathrm{H} 2 \mathrm{O} \\
\end{array}$ & $\mathrm{NIOH} 2$ & $\mathrm{SRCO} 3$ & ZRO2 & Total Solids & $\begin{array}{c}\text { Total Soln } \\
\text { Mass }\end{array}$ & \begin{tabular}{|c|c|} 
Solids $\%$ of \\
Total Soln
\end{tabular} \\
\hline 6TC111 & 0 & 0 & 0 & 0 & 0 & 0 & $6.70 \mathrm{E}+03$ & $5.16 \mathrm{E}+01$ & $4.28 \mathrm{E}+00$ & $4.90 \mathrm{E}+01$ & $9.44 \mathrm{E}+03$ & $1.01 \mathrm{E}+06$ & $0.94 \%$ \\
\hline 6TC112 & 0 & 0 & 0 & 0 & 0 & 0 & $3.73 \mathrm{E}+03$ & $3.06 \mathrm{E}+01$ & $2.52 \mathrm{E}+00$ & $2.75 \mathrm{E}+01$ & $5.34 \mathrm{E}+03$ & $9.82 \mathrm{E}+05$ & $0.54 \%$ \\
\hline 6TC113 & 0 & 0 & 0 & 0 & 0 & 0 & $3.01 \mathrm{E}+03$ & $3.33 \mathrm{E}+01$ & $2.74 \mathrm{E}+00$ & $3.09 \mathrm{E}+01$ & $4.76 \mathrm{E}+03$ & $9.81 \mathrm{E}+05$ & $0.49 \%$ \\
\hline 6TC114 & 0 & 0 & 0 & 0 & 0 & 0 & $4.70 \mathrm{E}+03$ & $3.69 \mathrm{E}+01$ & $3.06 \mathrm{E}+00$ & $3.34 \mathrm{E}+01$ & $7.12 \mathrm{E}+03$ & $9.98 \mathrm{E}+05$ & $0.71 \%$ \\
\hline $6 \mathrm{TC} 115$ & 0 & 0 & 0 & 0 & 0 & 0 & $2.34 \mathrm{E}+03$ & $2.18 \mathrm{E}+01$ & $1.75 \mathrm{E}+00$ & $1.98 \mathrm{E}+01$ & $3.49 \mathrm{E}+03$ & $9.56 \mathrm{E}+05$ & $0.37 \%$ \\
\hline $6 \mathrm{TC} 116$ & 0 & 0 & 0 & 0 & 0 & 0 & 0 & $3.71 \mathrm{E}+00$ & $2.35 \mathrm{E}-01$ & $1.58 \mathrm{E}+00$ & $1.92 \mathrm{E}+02$ & $9.34 \mathrm{E}+05$ & $0.02 \%$ \\
\hline 6TC117 & 0 & 0 & 0 & 0 & 0 & 0 & $3.02 \mathrm{E}+03$ & $2.44 \mathrm{E}+01$ & $2.01 \mathrm{E}+00$ & $2.13 \mathrm{E}+01$ & $4.28 \mathrm{E}+03$ & $9.76 \mathrm{E}+05$ & $0.44 \%$ \\
\hline 6TC118 & 0 & 0 & 0 & 0 & 0 & 0 & $2.25 \mathrm{E}+03$ & $2.53 \mathrm{E}+01$ & $2.05 \mathrm{E}+00$ & $2.35 \mathrm{E}+01$ & $3.59 \mathrm{E}+03$ & $9.61 \mathrm{E}+05$ & $0.37 \%$ \\
\hline 6TC119 & 0 & 0 & 0 & 0 & 0 & 0 & $4.35 \mathrm{E}+02$ & $1.05 \mathrm{E}+01$ & $7.98 \mathrm{E}-01$ & $8.67 \mathrm{E}+00$ & $9.82 \mathrm{E}+02$ & $9.38 \mathrm{E}+05$ & $0.10 \%$ \\
\hline 6TC120 & 0 & 0 & 0 & 0 & 0 & 0 & 0 & $4.85 \mathrm{E}+00$ & $3.44 \mathrm{E}-01$ & $1.85 \mathrm{E}+00$ & $2.54 \mathrm{E}+02$ & $9.39 \mathrm{E}+05$ & $0.03 \%$ \\
\hline 6TC121 & 0 & 0 & 0 & 0 & 0 & 0 & $9.69 \mathrm{E}+02$ & $1.10 \mathrm{E}+01$ & $8.56 \mathrm{E}-01$ & $8.81 \mathrm{E}+00$ & $1.53 \mathrm{E}+03$ & $9.47 \mathrm{E}+05$ & $0.16 \%$ \\
\hline 6TC122 & $4.99 \mathrm{E}+02$ & 0 & 0 & 0 & 0 & 0 & $3.33 \mathrm{E}+03$ & $2.76 \mathrm{E}+01$ & $2.37 \mathrm{E}+00$ & $1.44 \mathrm{E}+01$ & $7.15 \mathrm{E}+03$ & $1.00 \mathrm{E}+06$ & $0.71 \%$ \\
\hline 6TC123 & $2.63 \mathrm{E}+02$ & 0 & $3.42 \mathrm{E}+03$ & 0 & 0 & 0 & $7.17 \mathrm{E}+03$ & $5.46 \mathrm{E}+01$ & $4.59 \mathrm{E}+00$ & $4.89 \mathrm{E}+01$ & $1.79 \mathrm{E}+04$ & $1.03 \mathrm{E}+06$ & $1.74 \%$ \\
\hline $6 \mathrm{TC} 125$ & $3.76 \mathrm{E}+02$ & 0 & $2.00 \mathrm{E}+03$ & 0 & 0 & 0 & $4.29 \mathrm{E}+03$ & $3.55 \mathrm{E}+01$ & $2.99 \mathrm{E}+00$ & $2.87 \mathrm{E}+01$ & $1.09 \mathrm{E}+04$ & $1.00 \mathrm{E}+06$ & $1.09 \%$ \\
\hline 6TC126 & $4.34 \mathrm{E}+02$ & 0 & $2.59 \mathrm{E}+03$ & 0 & 0 & 0 & $5.49 \mathrm{E}+03$ & $4.75 \mathrm{E}+01$ & $4.02 \mathrm{E}+00$ & $3.72 \mathrm{E}+01$ & $1.48 \mathrm{E}+04$ & $1.03 \mathrm{E}+06$ & $1.44 \%$ \\
\hline 6TC127 & $6.53 \mathrm{E}+01$ & 0 & $1.89 \mathrm{E}+03$ & 0 & 0 & 0 & $4.52 \mathrm{E}+03$ & $3.55 \mathrm{E}+01$ & $2.95 \mathrm{E}+00$ & $3.14 \mathrm{E}+01$ & $8.83 \mathrm{E}+03$ & $9.94 \mathrm{E}+05$ & $0.89 \%$ \\
\hline 6TC128 & $1.15 \mathrm{E}+02$ & 0 & $2.16 \mathrm{E}+03$ & 0 & 0 & 0 & $5.33 \mathrm{E}+03$ & $4.18 \mathrm{E}+01$ & $3.49 \mathrm{E}+00$ & $3.76 \mathrm{E}+01$ & $1.18 \mathrm{E}+04$ & $1.01 \mathrm{E}+06$ & $1.17 \%$ \\
\hline 6TC129 & $2.33 \mathrm{E}+02$ & 0 & 0 & 0 & 0 & 0 & $4.22 \mathrm{E}+02$ & $5.90 \mathrm{E}+00$ & $4.73 \mathrm{E}-01$ & 4.99E-01 & $9.75 \mathrm{E}+02$ & $9.51 \mathrm{E}+05$ & $0.10 \%$ \\
\hline 6TC130 & $5.36 \mathrm{E}+02$ & 0 & 0 & 0 & 0 & 0 & $1.75 \mathrm{E}+03$ & $1.54 \mathrm{E}+01$ & $1.34 \mathrm{E}+00$ & $1.15 \mathrm{E}+00$ & $3.80 \mathrm{E}+03$ & $9.80 \mathrm{E}+05$ & $0.39 \%$ \\
\hline 6TC131 & $1.38 \mathrm{E}+02$ & 0 & $7.86 \mathrm{E}+02$ & 0 & 0 & 0 & $3.18 \mathrm{E}+03$ & $2.56 \mathrm{E}+01$ & $2.13 \mathrm{E}+00$ & $2.17 \mathrm{E}+01$ & $5.42 \mathrm{E}+03$ & $9.81 \mathrm{E}+05$ & $0.55 \%$ \\
\hline 6TC132 & $1.29 \mathrm{E}+02$ & 0 & 0 & 0 & 0 & 0 & $1.04 \mathrm{E}+01$ & $6.51 \mathrm{E}+00$ & $5.05 \mathrm{E}-01$ & $2.44 \mathrm{E}+00$ & $4.81 \mathrm{E}+02$ & $9.48 \mathrm{E}+05$ & $0.05 \%$ \\
\hline 6TC133 & $4.46 \mathrm{E}+02$ & 0 & 0 & 0 & 0 & 0 & $7.40 \mathrm{E}+02$ & $7.72 \mathrm{E}+00$ & $6.68 \mathrm{E}-01$ & 0 & $1.61 \mathrm{E}+03$ & $9.62 \mathrm{E}+05$ & $0.17 \%$ \\
\hline 6TC134 & $4.94 \mathrm{E}+02$ & 0 & 0 & 0 & 0 & 0 & $2.83 \mathrm{E}+03$ & $2.27 \mathrm{E}+01$ & $1.95 \mathrm{E}+00$ & $9.17 \mathrm{E}+00$ & $5.78 \mathrm{E}+03$ & $9.92 \mathrm{E}+05$ & $0.58 \%$ \\
\hline 6TC135 & $5.50 \mathrm{E}+02$ & 0 & 0 & 0 & 0 & 0 & $1.51 \mathrm{E}+03$ & $1.20 \mathrm{E}+01$ & $1.05 \mathrm{E}+00$ & 0 & $3.02 \mathrm{E}+03$ & $9.74 \mathrm{E}+05$ & $0.31 \%$ \\
\hline
\end{tabular}


This page intentionally left blank. 